\title{
Alexander Iben
}

\section{Staatlicher Schutz vor Meinungsrobotern}

(Verfassungs-)Rechtliche Überlegungen zu einer staatlichen Schutzpflicht vor Einflüssen von Meinungsrobotern auf die politische Willensbildung in sozialen Netzwerken 
Herausgegeben von

Prof. Dr. Philip Kunig, Freie Universität Berlin

Prof. Dr. Gerhard Robbers, Universität Trier

Prof. Dr. Andreas Voßkuhle, Universität Freiburg 
Alexander Iben

\section{Staatlicher Schutz vor Meinungsrobotern}

(Verfassungs-)Rechtliche Überlegungen zu einer staatlichen Schutzpflicht vor Einflüssen von Meinungsrobotern auf die politische Willensbildung in sozialen Netzwerken

Nomos Verlagsgesellschaft 
Alexander Iben. Geboren 1993 in Lübeck; Studium der Rechtswissenschaften an der Universität Greifswald; Erstes Staatsexamen 2017; wissenschaftlicher Mitarbeiter am Lehrstubl für Öffentliches Recht, Europa- und Völkerrecht (Prof. Dr. iur. Claus Dieter Classen) an der Universität Greifswald (2017-2020); Promotion 2021.

Die Deutsche Nationalbibliothek verzeichnet diese Publikation in der Deutschen Nationalbibliografie; detaillierte bibliografische Daten sind im Internet über bttp://dnb.d-nb.de abrufbar.

Zugl.: Greifswald, Univ., Diss., 2021

1. Auflage 2021

(C) Alexander Iben

Publiziert von

Nomos Verlagsgesellschaft mbH \& Co. KG

Waldseestraße 3-5 | 76530 Baden-Baden

www.nomos.de

Gesamtherstellung:

Nomos Verlagsgesellschaft mbH \& Co. KG

Waldseestraße 3-5 I 76530 Baden-Baden

ISBN (Print): $\quad$ 978-3-8487-8234-5

ISBN (ePDF): 978-3-7489-2656-6

DOI: https://doi.org/10.5771/9783748926566

Onlineversion Nomos eLibrary

\section{(c) (i) (2)}

Dieses Werk ist lizenziert unter einer Creative Commons Namensnennung Weitergabe unter gleichen Bedingungen 4.0 International Lizenz. 


\section{Vorwort}

Die Arbeit wurde im Wintersemester 2020/2021 von der Rechts- und Staatswissenschaftlichen Fakultät als Promotion angenommen. Sie entstand während meiner Tätigkeit am Lehrstuhl für Öffentliches Recht, Europa- und Völkerrecht von Prof. Dr. Claus Dieter Classen an der Universität Greifswald. Sie befindet sich auf dem Stand von Mai 2021.

Mein besonderer Dank gilt zunächst meinem Doktorvater Prof. Dr. Claus Dieter Classen. Er hat die Arbeit von seiner Idee bis zum Abschluss begleitet, stand für ergiebige Gespräche zur Verfügung und hat mir neben wertvollen Hinweisen vor allem den notwendigen Freiraum für die Entwicklung eigener Argumente und Gedanken gegeben. Ihm war es zudem auch ein persönliches Anliegen, dass die Arbeit zu einem erfolgreichen Ende findet.

Ebenso danke ich Prof. Dr. Axel Beater für die zügige Erstellung des Zweitgutachtens. Auch er stand mir für fruchtbare Gespräche und Diskussionen zur Verfügung und hat das Entstehen der Arbeit stets interessiert verfolgt.

Von meinen Kolleginnen und Kollegen am Lehrstuhl gilt mein Dank neben Ass. jur. Nadja Reimold für zahlreiche wertvolle Gespräche und die angenehme Arbeitsatmosphäre vor allem auch Dr. Christopher Bilz, der mich zudem bei den Korrekturarbeiten am Manuskript vor Drucklegung unterstützt hat.

Den Herausgebern und dem Nomos-Verlag danke ich für die Aufnahme in die Schriftenreihe Neue Schriften zum Staatsrecht. Dr. Marco Ganzhorn danke ich für die Beratung und Betreuung bei der Drucklegung des Manuskripts.

Von meinen Freunden gilt Richard Krahnstöver besonderer Dank nicht nur für die Durchsicht des Manuskripts vor Abgabe, sondern vor allem auch für zahlreiche Gespräche und persönliche Einschätzungen zur Arbeit. Meiner Schwester, Katharina Iben, danke ich für die Zeit, die Sie in das Lektorieren der Arbeit investiert hat. Meiner Lebensgefährtin Judith König danke ich für die Geduld und die stets aufbauenden, motivierenden und zusprechenden Worte, die so maßgeblich zu dem erfolgreichen Abschluss der Arbeit beigetragen hat.

Der größte Dank gilt aber meinen Eltern, Matthias und Renate Iben, die mich stets auf jede erdenkliche Weise unterstützt haben. Ihnen ist die Arbeit gewidmet. 


\section{Inhaltsverzeichnis}

Abkürzungsverzeichnis

2. Teil: Grundlagen - Soziale Netzwerke, politische Willensbildung und Meinungsroboter

A. Soziale Netzwerke:

I. Annäherung an den Begriff des sozialen Netzwerks

II. Facebook

1. Personalisierung - zwischen Verbesserung des Dienstes und ökonomischer Rationalität

2. Der News Feed als Beispiel algorithmenbasierter Personalisierung

III. Twitter

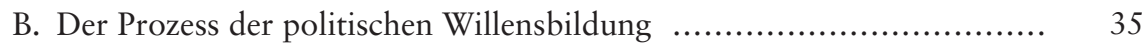

I. Makro-Ebene ..................................................... 37

II. Meso-Ebene ..................................................... 39

III. Mikro-Ebene .................................................... 40

1. Allgemeines zum Prozess der Meinungsbildung ............... 40

2. Informationsaufnahme und -verarbeitung als rationaler Vorgang? .................................................. 41

3. Bedeutung für die politische Willensbildung .................... 43

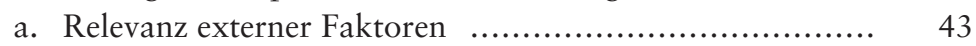

b. Individuelle Verzerrungsfaktoren und Tendenzen .......... 45

c. Herausforderungen und Veränderungen der Meinungsbildung im digitalen Zeitalter

C. Meinungsroboter und ihr Einfluss auf die politische Willensbildung der Nutzer

I. Herleitung des Begriffs der Meinungsroboter ..................... 51

II. Technische Umsetzung - Grundlagen .......................... 53 
III. Verwendungsstrategien und abstraktes Einflusspotenzial ......... 56

1. Informationsdiffusion, Verursachen von Trends .............. 56

2. Verbreitung desinformativer Inhalte ......................... 58

3. Störung von Diskussionen ................................... 60

4. Veränderung des Meinungsklimas ............................ 61

5. Sekundäre, mittelbare Einflüsse in der Offline-Sphäre ........ 62

6. Fazit: Abstraktes Einflusspotential ......................... 63

IV. Konkretes Einflusspotenzial - Empirische Forschungsergebnisse und Beweisschwierigkeiten ..................................... 63

V. Relevante Faktoren für den konkreten Wirkungsgrad ............. 67

VI. Unterschiede zwischen bot-gestützter und herkömmlicher Einflussnahme

1. Identitätstäuschung und Täuschung über quantitative Legitimität ............................................... 70

2. Strukturell divergierende Reichweite ....................... 71

3. Ausnutzung netzwerkinterner Effekte ......................... 72

a. Nutzergemeinschaft als Trendbestimmer ................. 72

b. Personalisierung, Filterblasen Echokammer ................ 72

4. Fazit ..................................................... 74

VII. (Verfassungs-)Rechtliche Bedeutung der bisherigen Befunde? .... 74

3. Teil: Die Wandlung der Rolle des Staates im digitalen Zeitalter Der Staat als Garant (manipulationsfreier) politischer Willensbildung?

1. Kapitel: Die Begründung einer staatlichen Verantwortung für die kommunikationsgrundrechtlichen Schutzgüter in digitalen Kommunikationsräumen

A. Der rundfunkrechtliche Regelungsauftrag des Gesetzgebers ............ 81

I. Entwicklung ................................................... 81

II. Die verfassungsdogmatischen Aussagen des BVerfG ............. 84

III. Kritische Rezeption der Rechtsprechung ...................... 85

B. Grundrechtliche Schutzpflichten des Staates ........................ 90

I. Grundlagen grundrechtlicher Schutzpflichten ................... 91

II. Voraussetzungen und Inhalt der Schutzpflichten ................. 96

1. Tatbestandliche Voraussetzungen ............................ 97 
a. Einschränkungsversuche in der Literatur ................. 99

b. Die Rechtsprechung des BVerfG: ......................... 102

c. Fazit: Gefahrenschwelle als Minimalkonsens ............... 105

2. Die Rechtsfolge - Inhalt und Ausgestaltung staatlicher Schutzpflichten .............................................. 105

a. Allgemeine Grenzen ..................................... 106

b. Spezifische Vorgabe der Schutzpflichtendimension ......... 107 aa. Ausgangspunkt Gestaltungsspielraum des Staates .... 107 bb. Untermaßverbot als Direktive der Effektivität ........ 110 aa. Kontrolle durch das Bundesverfassungsgericht ........ 111 cc. Operable Formel des Untermaßverbots ................ 113

c. Fazit: Untermaßverbot als rechtspraktische verfassungsrechtliche Grenze ........................... 116

2. Kapitel: Aktivierung grundrechtlicher Schutzpflichten durch Meinungsroboter in digitalen Kommunikationsräumen?

A. Schutzpflichten im Verhältnis (Bot-)Verwender-Staat-Nutzer

I. „Unschärfe“ im bisherigen Umgang mit der Schutzpflichten-

Dogmatik im Kontext der Meinungsroboter

II. Relevanter Übergriff auf grundrechtliche Schutzgüter?

1. Übergriff auf die Meinungsäußerungsfreiheit

a. Übergriff durch „psychische Zwangswirkung“? ............

b. Überschreitung der Bagatellgrenze - Einsatz von Meinungsrobotern als bloße Belästigung?

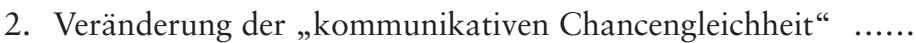

a. Begründbarkeit einer Schutzpflicht zugunsten der „kommunikativen Chancengleichheit“?

b. Inhaltliche Reichweite des Schutzes kommunikativer Chancengleichheit

c. Bloße Bagatelle oder beachtlicher Übergriff?

3. Übergriff auf die Informations- und Meinungsbildungsfreiheit

4. Übergriff auf die Freiheit der Wahlentscheidung

5. Fazit: Übergriff auf die kommunikationsgrundrechtlichen Güter

III. Fakultative Risikovorsorge oder obligatorische Gefahrenabwehr?

1. Bedeutung der betroffenen Grundrechtsgüter

2. Potentielles Schadensausmaß 
a. Allgemeine Bedeutung der Netzwerke als digitale Informations- und Kommunikationsräume ................. 143

b. Meinungsäußerungsfreiheit .................................. 144

c. Kommunikative Chancengleichheit ........................ 145

d. Informations- und Meinungsbildungsfreiheit ............... 146

e. Wechselseitige Verstärkungen der Effekte und das übergeordnete Schutzgut der Kommunikation .............. 148

f. Zwischenfazit .................................................. 150

3. Die Eintrittswahrscheinlichkeit - empirische Unsicherheiten und Rationalitätsanforderungen an die Schutzpflicht

4. Obligatorische Risikovorsorge und Gefahrenabwehr? ......... 158

IV. Fazit: Schutzpflicht wegen einer zumindest abstrakten

Gefährlichkeit

3. Kapitel: Rechtsfolgen der Schutzpflicht - Schutzbedarf und Handlungsoptionen des Staates

A. Das Übermaßverbot und kollidierende Grundrechte als relative Grenze der Schutzverantwortung

I. Der grundrechtliche Schutz der Meinungsroboter unter der

Meinungsfreiheit

1. Automatisierte Äußerungen als geschützte

Meinungsäußerung?

a. Zurechnung aufgrund der Verantwortungs- und Herrschaftssphäre als einfach-rechtlicher Grundsatz .......

b. Bedeutung für die Frage einer grundrechtlichen

Zurechnung der Äußerungen

2. Sachlicher Schutz der Inhalte

3. Restriktion des Schutzbereichs im Falle von

Meinungsrobotern?

a. Allgemeines zur Konzeption von

Schutzbereichsausnahmen

b. Übertragung auf politische Bots .............................. 180

aa. Identitätstäuschung

(I.) Identitätstäuschung als Bestandteil der

Modalitätsfreiheit 
(II.) Berücksichtigung der Rechtsprechung bei unrichtigen Zitaten .............................. 183

(III.) Widerspruch zum Schutzzweck? .................. 184

bb. Meinungsmacht - quantitative Legitimität ........... 187

cc. Manipulative Zielsetzung .............................. 188

(I.) „Prima-facie-Beweis“ einer manipulativen Absicht?

(II.) Materielle Beweislast des Grundrechtsträgers? .. 191

(1.) Negative Grundregel der Beweislast ......... 192

(2.) Normentheorie .............................. 193

(3.) Grundrechtsimmanente Beurteilung der Beweislastverteilung

(4.) Fazit: keine materielle Beweislast des Grundrechtsträgers

4. Fazit: Schutz der Meinungsroboter durch die

Meinungsfreiheit

II. Grundrechtlicher Schutz der Interessen der Netzwerkbetreiber ... 196

III. Kommunikationsfreiheiten der Nutzer ........................... 200

B. (Spezifische) Grenzen der grundrechtlichen Schutzpflicht im Falle der Meinungsroboter

I. Vorbehalt der Möglichkeit staatlichen Schutzes

1. Dichotome Gesetzgebungszuständigkeiten - Adressat der Schutzpflichten

2. Unionskompetenzen, sekundärrechtliche Medienregulierung und Herkunftslandprinzip

3. Faktische Grenzen staatlicher Regulierung

II. Die Schutzbedürftigkeit der Nutzer als Grundprämisse und Grenze staatlichen Schutzes

1. Schutzbedarf der Meinungsäußerungs-, Informations- und Meinungsbildungsfreiheit

a. Kein prinzipieller Widerspruch zur grundrechtlichen Autonomie

b. Schutzbedarf der Meinungsäußerungsfreiheit

c. Schutzbedarf der Informationsfreiheit bzw. Meinungsbildung

aa. Täuschungswirkung

(I.) Vergleichbare, einfach-rechtliche Konstellationen

(II.) Vergleichbarkeit und Konsequenzen für die Täuschungswirkung von Meinungsrobotern ..... 
(III.) Fazit: Insbesondere Förderung von Transparenz und Medienkompetenz .......................... 219

bb. Desinformative Inhalte .................................. 220

cc. Einseitige Informationsrezeption ....................... 221

d. Fazit: Transparenz, Medienkompetenz als wesentliche Schutzmaximen

2. Schutzbedarf der kommunikativen Chancengleichheit

3. Kumulation der Wirkungsweisen und Schutzbedarf des Kommunikationsprozesses in toto

C. Untermaßverbot - Erfüllung und Konkretisierung der staatlichen Schutzpflicht?

I. Das bisherige Schutzkonzept des Staates

1. Beobachtung, Überwachung und Erkenntnissicherung durch den (Bundes-)Gesetzgeber

2. Allgemeine rechtliche Vorgaben für den Einsatz von Meinungsrobotern:

a. Vertragsrechtliche Vorgaben

aa. Wirksamkeit der Nutzungsbedingungen

(I.) Anwendbarkeit des deutschen Vertragsrechts? ...

(II.) Transparenzerfordernis des $\$ 305$ Abs. 2 BGB ...

(III.) Inhaltskontrolle der AGBs - Unwirksamkeit der Regelungen?

bb. Vertragliche Sanktionen bei Vertragsverstößen

b. Gesetzliches Schuldrecht - (Quasi-)negatorischer Unterlassungs- und Beseitigungsanspruch, $\mathbb{S} \mathbb{S} 1004$ Abs. 1, 823 Abs. 1 BGB

c. Urheberrecht:

d. Strafrecht

aa. Das Verändern statistischer Daten als tatbestandliche Handlung der $\mathbb{S} 303 \mathrm{a}, \mathrm{b}$ StGB?

(I.) Datenveränderung, $\$ 303$ a StGB

(II.) Computersabotage, $\mathbb{} 303$ b Abs. 1, 2 StGB $\ldots . . .238$

bb. Grenzen für Beitragsinhalte .............................. 240

e. Spezialfall: völkerrechtliches Interventionsverbot …...... 242

f. Fazit: Betreiber als (ineffektive) Garanten konkreten Schutzes

3. Spezifische Schutzmechanismen für die Informationsfreiheit

a. Soft-law Regularien

aa. Maßnahmen der Betreiber

bb. EU-Verhaltenskodex 
(I.) Allgemeines …................................. 246

(II.) Würdigung und spezifische Probleme $\ldots . . . \ldots \ldots \ldots . . .246$

b. Medienkompetenzvermittlung .............................. 248

aa. Bildung der Medienkompetenz im schulischen Bereich 249

bb. Medienkompetenz älterer Nutzerschichten ........... 251

cc. Fazit: ................................................ 252

c. Transparenzvorgaben des Medienrechts, insbesondere

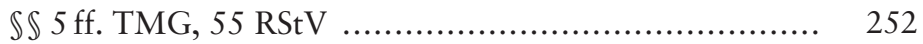

aa. Kennzeichnungspflicht nach $\mathbb{} 5$ Abs. 1 TMG $\ldots . . . . . .253$

bb. Kennzeichnungspflicht des $₫ 55$ Abs. 1 RStV ......... 257

cc. Durchsetzung der Kennzeichnungspflicht .............. 260

dd. Fazit: Lückenhafte Transparenzvorgaben des

Medienrechts ............................................. 261

d. Datenschutzrechtliche Vorgaben ............................. 261

e. Fazit: Primär selbstregulatorischer Schutz durch Betreiber 266

4. Spezifische Schutzmechanismen für die

Meinungsäußerungsfreiheit

a. Einfach-rechtlicher Schutz vor Beeinflussung der

Willensbetätigung

aa. Schutz der allgemeinen freien Willensbetätigung, $\$ 240$ StGB

bb. Schutz der politischen Willensbetätigung im Rahmen von Wahlen

cc. Schutz der politischen Meinungsäußerung durch das Wahlrecht

b. Spezifischer verfassungsrechtlicher Schutz der

Wahläußerung vor Einflussnahme

aa. Einsatz durch staatliche Akteure: ........................ 270

bb. Einsatz durch private Akteure

(I.) Vergleichbarkeit der Charakteristika der Meinungsroboter

(II.) Kumulation von Äußerungsinhalten und Verbreitungsform bei Desinformation?

cc. Besonderheiten beim Einsatz durch Parteien .......... 277

(I.) Bedeutung und Funktion der Parteien ............. 277

(II.) Verfassungsunmittelbare Grenzen des Einsatzes? 278

c. Fazit: Kein spezifischer Schutz der

Meinungsäußerungsfreiheit

5. Bewertung des bisherigen Schutzkonzeptes im Lichte des Untermaßverbots 
II. Die Neuregelung des RStV durch den MStV …................. 287

1. Spezifische Kennzeichnungspflicht für Meinungsroboter ...... 288

a. Telemediendienstanbieter .................................... 290

b. Definition eines Meinungsroboters ......................... 290

aa. Begrenzung auf eine doppelte Täuschungswirkung? .. 290

bb. Begrenzung auf allein meinungsbildungsrelevante Inhalte?

c. Soziales Netzwerk .................................................. 294

d. Fazit: Meinungsroboter als zentraler Regulierungsgegenstand .................................... 296

2. Vorgaben für Medienintermediäre, $\mathbb{S} \mathbb{S} 91-96 \mathrm{MStV} \quad \ldots \ldots \ldots \ldots . .296$

a. Begriff des Medienintermediärs ............................ 296

b. Allgemeine Regulierungsvorgaben für Medienintermediäre

c. Sorgetragungspflicht für Betreiber sozialer Netzwerke nach $\mathbb{9} 93$ Abs. $4 \mathrm{MStV}$

aa. Die Bedeutung der Sorgetragungspflicht des $\$ 93$

Abs. $4 \mathrm{MStV}$

(I.) National-rechtliche Auslegungscanones ......... 301

(1.) Wortlaut „Sorge tragen“ “..................... 301

(2.) Systematik ................................... 302

(3.) Normgenese ................................... 303

(4.) Telos der Norm ................................. 304

(5.) Zwischenfazit: Infrastrukturelle

Verantwortung und regulierte

Selbstregulierung im Kampf gegen

Meinungsroboter

(II.) Verfassungs- bzw. grundrechtliche Implikationen 305

(1.) Interessen der Betreiber, insbes. Art. 12 Abs. 1 GG

(2.) Schutzbedarf der Kommunikationsfreiheiten der Nutzer

(3.) Weitere kollidierende grundrechtliche Interessen

(4.) Zwischenfazit: Zugangskontrolle als Mindestschutz, präventive Überwachung als zumutbare Anforderung möglich

(III.) Auslegung im Lichte der E-Commerce-RL

(1.) Unproblematische Fälle:

Identitätskontrollen,

Beschwerdemanagement, Zugangskontrollen 
(2.) Proaktive Überwachung der Datenströme und eigenständige Kennzeichnung

(a.) Überzeugende Einwände gegen eine Kollision mit Art. 15 Abs. 1 ECRL? .....

(b.) Richtlinienkonforme Ausnahme im Falle der Meinungsroboter?

(aa.) \ 93 Abs. $4 \mathrm{MStV}$ als „spezifischer Fall"?

(bb.) Bereichsausnahme des Haftungsprivilegs im Falle von $\mathbb{} 93$ Abs. 4 MStV?

(cc.) Art 1 Abs. 6 ECRL als „Öffnungsklausel“ für $\$ 93$ Abs. $4 \mathrm{MStV}$ ? ...

(c.) Fazit: Vereinbarkeit mit der

E-Commerce-RL

(IV.) Vereinbarkeit einer proaktiven Pflicht mit der DS-GVO?

(V.) Fazit: präventive Kontrolle als Mindestmaß, proaktive Überwachung als Verantwortung meinungsrelevanter Betreiber

bb. Verhältnis von $\mathbb{S} 93$ Abs. $4 \mathrm{MStV}$ zu den Regelungen der Netzwerkbetreiber

d. Ergänzende Satzungsbefugnis der Medienanstalten, $\mathbb{\int} 96$ MStV

e. Kontrollbefugnisse und Durchsetzungsmöglichkeiten des Staates

aa. Ordnungswidrigkeitentatbestände

bb. Aufsichtsrechtliche Befugnisse und Maßnahmen der Landesmedienanstalten

f. Vereinbarkeit mit den europäischen Vorgaben im Übrigen

aa. Vereinbarkeit mit der P2B-Verordnung

bb. Marktortprinzip d. $\mathbb{\int} 1$ Abs. $8 \mathrm{MStV}$ - Verstoß gegen das Herkunftslandprinzip d. Art. 3 ECRL?

cc. Vereinbarkeit mit der Dienstleistungsfreiheit

g. Fazit: Zulässiges, tranzparenzerhöhendes Schutzkonzept der Länder

3. Bewertung des Schutzkonzeptes anhand des Untermaßverbots

a. Bewertung der allgemeinen Intermediärsvorgaben

aa. Vereinbarkeit mit den Grundsätzen „Effektivität“ und „Erforderlichkeit“ (3. Stufe)? 
bb. Angemessener Ausgleich zwischen Schutzbedürfnissen und Abwehrrechten (4. Stufe)

b. Bewertung des Schutzkonzepts gegen Einflüsse der

Meinungsroboter

aa. Ausgleich zwischen „Effektivität“ und „Erforderlichkeit“ (3. Stufe)

(I.) Fehlende Berücksichtigung akkumulierter Netzwerkdaten

(II.) Fehlende präventive Wirkung?

(III.) Faktische Wirksamkeitshemmung durch ökonomisch-rationale Herangehensweise der Betreiber?

(IV.) Faktische Wirksamkeitshemmung durch das Herkunftslandprinzip des $\mathbb{1} 1$ Abs. $7 \mathrm{MStV}$ ?

(V.) Zwischenergebnis:

bb. Angemessener Ausgleich zwischen grundrechtlichen Schutzbedürfnissen und grundrechtlichen Abwehrrechten (4. Stufe)

(I.) Kennzeichnungspflicht des $\mathbb{} 18$ Abs. $3 \mathrm{MStV}$

(II.) Sorgetragungspflicht der Netzwerkbetreiber, \93 Abs. 4 MStV

(1.) Beeinträchtigung der unternehmerischen Freiheit, Art. 12 Abs. 1 GG

(2.) Privatisierung staatlicher Kontrollverantwortung?

(3.) Die Meinungsfreiheit betroffener Nutzer bei falsch-positiven Kennzeichnungen bzw. Sperrungen

(a.) Vergleichbare Problemlagen

(b.) Vertragsrechtliches Problem oder grundrechtliche Relevanz?

(c.) Verfahrensrechtliche Absicherung als vom Übermaßverbot geforderter Schutz?

(d.) Verfassungswidrigkeit des MStV?

(e.) Zukünftige Verfahrensrechtliche Ausgestaltung

(4.) Zwischenfazit: Abgestuftes Schutzkonzept als Kompromiss zwischen Schutz- und Abwehrrechten

4. Bilanz des Medienstaatsvertrages 
III. Fazit: Prinzipiell wirksame Konkretisierung der

Schutzverantwortung durch die Länder

4. Kapitel: Ausblick auf die zukünftige (Rechts-)Entwicklung

A. Berichtspflichten als Maßnahme der Sensibilisierung und Stärkung der Nutzerautonomie

B. Veränderungen der Nutzeroberfläche oder des Algorithmus

C. Spezielles Einsatzverbot für bestimmte Urheber?

D. Forschungszugang zu sozialen Netzwerken

I. Grundrechtliche Implikationen

II. Perspektive für eine (gesetzliche) Ausgestaltung

III. Spezieller Zugriff zur Implementation von Meinungsrobotern? .

E. Klarnamenpflicht für soziale Netzwerke

I. Notwendigkeit einer entsprechende Identifizierungspflicht

1. (Faktische) Anonymität als Auslöser rechtswidriger Äußerungen?

2. Spezifische Gefahrenlage und fehlende Effektivität bestehender Regelungen?

3. Kostenrisiko als eigentliches Problem? ....................... 402

II. Verhältnismäßigkeitserwägungen ................................ 402

III. Fazit: Einführung einer relativen Klarnamenpflicht als rechtspolitisches Problem

F. Medienkompetenz

G. Ergänzender Schutz vor Desinformation

I. Wahlrechtliche Maßnahmen nach dem Vorbild des „loi relative à la lutte contre la manipulation de l'information “ Frankreichs

II. Strafrechtliches Verbot von Desinformation?

1. Wahlrechtspezifischer Schutz

2. Kollektiver Schutz des Kommunikationsprozesses

3. Fazit: Strafrechtlicher Schutz vor allem eine rechtspolitische Frage

III. Alternative (medienrechtliche) Ansätze 
IV. Fazit: Kein auswegloser, aber ein zurückhaltender Kampf ist möglich

H. Handlungsbedarf auf europäischer Ebene

I. Fazit: Stärkung der Eigenverantwortung als vorrangiges

Regulierungsziel

4. Teil: Zusammenfassung

Literaturverzeichnis 


\section{Abkürzungsverzeichnis}

a.a.O.

$\mathrm{AcP}$

AEUV

AfP

AGB

ABl.

AöR

API

$\mathrm{B} 2 \mathrm{~B}$

B2C

B-W

BB

Bd.

Beck-OK

BeckRS

Beschl.

BGB

BGH

BGHSt

BGHZ

BKartA

BK-GG

bspw.

BT-Drs.

BVerfG

BVerfGE

BVerwG

BVerwGE

BWahlG

bzw.

Ch.

CR

ComSoc

ders. am angegebenen Ort

Archiv für die civilistische Praxis

Vertrag über die Arbeitsweise der Europäischen Union

Zeitschrift für das gesamte Medienrecht

Allgemeine Geschäftsbedingungen

Amtsblatt der Europäischen Union

Archiv des öffentlichen Rechts

Application programming interface

Business-to-Business

Business-to-Customer

Baden-Württemberg

Betriebsberater

Band

Beck'scher Online-Kommentar

beck-online.RECHTSPRECHUNG

Beschluss

Bürgerliches Gesetzbuch

Bundesgerichtshof

Entscheidungen des Bundesgerichtshofs in Strafsachen

Entscheidungen des Bundesgerichtshofs in Zivilsachen

Bundeskartellamt

Bonner Kommentar zum Grundgesetz

beispielsweise

Bundestag Drucksachen

Bundesverfassungsgericht

Entscheidungen des Bundesverfassungsgerichts

Bundesverwaltungsgericht

Entscheidungen des Bundesverwaltungsgerichts

Bundeswahlgesetz

beziehungsweise

Chapter

Computer und Recht

CommunicationSocialis

derselbe 


\begin{tabular}{|c|c|}
\hline dies. & dieselbe/n \\
\hline DÖV & Die öffentliche Verwaltung \\
\hline DS-GVO & Datenschutz-Grundverordnung \\
\hline DuD & Datenschutz und Datensicherheit \\
\hline DVBl. & Deutsches Verwaltungsblatt \\
\hline ebd. & ebenda \\
\hline ECRL & E-Commerce-Richtlinie 2000/31/EG \\
\hline EDPB & European Data Protection Board \\
\hline EGMR & Europäischer Gerichtshof für Menschenrechte \\
\hline EGV & Vertrag zur Gründung der Europäischen Gemeinschaft \\
\hline EJoC & European Journal of Communication \\
\hline ERGA & $\begin{array}{l}\text { European Regulators Group forAudio-visual Media Ser- } \\
\text { vices }\end{array}$ \\
\hline Erwgr. & Erwägungsgrund \\
\hline $\mathrm{EuGrCh}$ & Charta der Grundrechte der Europäischen Union \\
\hline EUV & Vertrag über die Europäische Union \\
\hline EuZW & Europäische Zeitschrift für Wirtschaftsrecht \\
\hline FAZ & Frankfurter Allgemeine Zeitung \\
\hline Fn. & Fußnote \\
\hline FS & Festschrift \\
\hline GewArch & GewerbeArchiv \\
\hline GG & Grundgesetz \\
\hline GRUR & Gewerblicher Rechtsschutz und Urheberrecht \\
\hline GRUR-Prax & $\begin{array}{l}\text { Gewerblicher Rechtsschutz und Urheberrecht - Praxis im } \\
\text { Immaterialgüter- und Wettbewerbsrecht }\end{array}$ \\
\hline GRUR-RR & $\begin{array}{l}\text { Gewerblicher Rechtsschutz und Urheberrecht - Rechtspre- } \\
\text { chungs-Report }\end{array}$ \\
\hline GRUR-RS & $\begin{array}{l}\text { Gewerblicher Rechtsschutz und Urheberrecht - Rechtspre- } \\
\text { chungssammlung }\end{array}$ \\
\hline $\mathrm{Hb}$ & Handbuch \\
\hline HbGR & Handbuch der Grundrechte \\
\hline HbStaatskirchenR & Handbuch des Staatskirchenrechts \\
\hline $\mathrm{HbStR}$ & Handbuch des Staatsrechts \\
\hline Hb VerfR & Handbuch des Verfassungsrechts \\
\hline Hervorh. d. Aut. & Hervorhebung des Autors \\
\hline HRN & Hamburger Rechtsnotizen \\
\hline
\end{tabular}


I.E.

IEEE

InTeR

i.S.d.

IWRZ

JuS

jurisPR-ITR

JZ

Kap.

KG

KMK

K\&R

LG

LT B-W Drs.

MMR

MüKo

MStV

m.w.N.

MV

NJ

NJW

NStZ

NStZ-RR

NordÖR

$\mathrm{Nr}$.

NRW

NVwZ

OLG

P2B-VO

PinG

PNAS

POR

PraxisHb im Ergebnis

Institute of Electrical and Electronics Engineers

Innovations- und Technikrecht

im Sinne des

Zeitschrift für Internationales Wirtschaftsrecht

Juristische Schulung

juris PraxisReport IT-Recht

Juuristenzeitung

Kapitel

Kammergericht

Kultusministerkonferenz

Kommunikation und Recht

Landgericht

Drucksachen des Landtages Baden-Württemberg

Multimedia und Recht

Münchener Kommentar

Medienstaatsvertrag

mit weiteren Nachweisen

Mecklenburg-Vorpommern

Neue Justiz

Neue Juristische Wochenschrift

Neue Zeitschrift für Strafrecht

Neue Zeitschrift für Strafrecht - Rechtsprechungs-Report

Zeitschrift für Öffentliches Recht in Norddeutschland

Nummer

Nord-Rhein-Westfalen

Neue Zeitschrift für Verwaltungsrecht

Oberlandesgericht

Platform-to-Business-Verordnung (EU) 2019/1150

PinG - Privacy in Germany

Proceedings of the National Academy of Sciences of the

United States of America

Polizei- und Ordnungsrecht

Praxishandbuch 


\begin{tabular}{|c|c|}
\hline RabelsZ & $\begin{array}{l}\text { Rabels Zeitschrift für ausländisches und internationales Pri- } \\
\text { vatrecht }\end{array}$ \\
\hline Rechtshb & Rechtshandbuch \\
\hline RiBVerfG & Richter am Bundesverfassungsgericht \\
\hline RiW & Recht der Internationalen Wirtschaft \\
\hline RL & Richtlinie \\
\hline Rn. & Randnummer \\
\hline Rs. & Rechtssache \\
\hline Rspr. & Rechtsprechung \\
\hline $\mathrm{RStV}$ & Rundfunkstaatsvertrag \\
\hline RW & Rechtswissenschaft \\
\hline S. & Seite \\
\hline sog. & sogenannte/r \\
\hline Staat & Der Staat \\
\hline StGB & Strafgesetzbuch \\
\hline SV & Sondervotum \\
\hline TMG & Telemediengesetz \\
\hline TKG & Telekommunikationsgesetz \\
\hline u.a. & und andere \\
\hline UAbs. & Unterabsatz \\
\hline UFITA & $\begin{array}{l}\text { Archiv für Medienrecht und Medienwissenschaft; ehemals } \\
\text { Archiv für Urheber-, Film-, Funk- und Theaterrecht }\end{array}$ \\
\hline UrhG & Urhebergesetz \\
\hline Urt. & Urteil \\
\hline u.U. & unter Umständen \\
\hline u.v.m. & und vieles mehr \\
\hline UWG & Gesetz gegen den unlauteren Wettbewerb \\
\hline VersG & Versammlungsgesetz \\
\hline VG & Verwaltungsgericht \\
\hline Vgl. & Vergleiche \\
\hline VO & Verordnung \\
\hline Vol. & Volume \\
\hline VuR & Verbraucher und Recht \\
\hline WRP & Wettbewerb in Recht und Praxis \\
\hline WWW & World Wide Web \\
\hline $\mathrm{ZD}$ & Zeitschrift für Datenschutz \\
\hline
\end{tabular}


ZEuP

ZJS

ZRP

ZStW

ZUM
Zeitschrift für europäisches Privatrecht

Zeitschrift für das Juristische Studium

Zeitschrift für Rechtspolitik

Zeitschrift für die gesamte Strafrechtswissenschaft

Zeitschrift für Urheber und Medienrecht 


\section{Teil: Einleitung}

Soziale Netzwerke sind neben Suchmaschinen die Erfolgsgeschichte des Internets der letzten 15 Jahre. Klein angefangen erreichen die großen Anbieter Facebook und Twitter mittlerweile Milliarden Nutzer weltweit. Bedeutsam war ihre Entwicklung auch aus demokratischer Sicht. Denn dort, wo der Bürger vorher bloßer Rezipient in einer Medienöffentlichkeit war, wird er nun zum aktiven Teilnehmer im demokratischen Kommunikationsprozess. Er kann sich über territoriale Grenzen hinweg vernetzen und dabei mit einer breiten Öffentlichkeit kommunizieren. Diese deliberative Wirkung sozialer Netzwerke wurde zu Beginn als (digitale) Demokratisierung durchaus positiv bewertet. ${ }^{1}$ Und diese Funktion schienen die Dienste auch zu erfüllen. Der arabische Frühling soll maßgeblich von ihnen profitiert haben. ${ }^{2}$ Doch mittlerweile hat sich die anfängliche „Euphorie“ in akute Skepsis gewandelt. ${ }^{3}$ Den Netzwerken wird nicht nur eine Mitschuld an der Verrohung der Debattenkultur im Internet gegeben. Ihnen wird vielmehr vorgeworfen, aufgrund einer weitreichenden Überwachung und Verarbeitung der Daten der Nutzer den Informationszugang zu personalisieren und dadurch den Kommunikationsprozess zu segregieren. Das hat seit geraumer Zeit eine interdisziplinäre Diskussion um die tatsächlichen Auswirkungen - Stichworte: Filterblase und Echokammer - entstehen lassen. Angesichts der zunehmenden Bedeutung für das Medienkonsumverhalten entfachte das auch eine Debatte darum, ob und inwiefern die neuen Dienste - ähnlich wie der herkömmliche Rundfunk - mit Blick auf Aspekte der kommunikativen Chancengleichheit und Meinungsvielfalt zu regulieren sind. ${ }^{4}$

Die Diskussion gewann sicherlich auch 2016 dadurch an Gewicht, dass allen Vorhersagen entgegen - der republikanische Präsidentschaftskandidat Donald J. Trump die US-amerikanische Präsidentschaftswahl gewann. Schon wäh-

$1 \mathrm{Zu}$ den Vorteilen digitaler Öffentlichkeiten siehe etwa Westphal, in: Binder/Drerup, Demokratieerziehung und die Bildung digitaler Öffentlichkeit, S. 1 (19ff.). Ausführliche, positive Eigenschaften unter Hinweis auf entsprechende empirische Ergebnisse finden sich etwa bei Schmidt u.a., Zur Relevanz von Online-Intermediären für die Meinungsbildung, S. 25. Reinbacher, JZ 2020, 558 sieht sie als „gewichtigen Beitrag zur Demokratisierung der Informationsbeschaffung “ an und Nolte, ZUM 2017, 552 (553) spricht - allerdings mit Blick auf das Internet insgesamt - von einer „Demokratisierung des Kommunikationsprozesses.

2 Hierzu etwa El Difraoui, Die Rolle der neuen Medien im Arabischen Frühling.

3 Diesen Wandel im Verständnis der sozialen Netzwerke betonen auch Volkmann, in: Unger/v. UngernSternberg, Demokratie und künstliche Intelligenz, S. 51 f.; Hegelich, Die Politische Meinung 543 (2017), 32 (34) und mit einer ausführlichen Darstellung positiver und negativer Begleiterscheinungen Drerup, in: Binder/ders., Demokratieerziehung und Bildung digitaler Öffentlichkeit, S. 29 (35 ff.). Kritisch zur Internetkommunikation etwa Habermas, Communication Theory 16 (2006), 411 (423).

4 Diesen Fragen widmen sich umfassend u.a. Bock, Die Übertragung der Kommunikationsfreiheiten des Art. 5 GG auf das Internet; Hartl, Suchmaschinen, Algorithmen und Meinungsmacht; Heidtke, Meinungsbildung und Medienintermediäre; Kellner, Die Regulierung der Meinungsmacht von Internetintermediären und Pille, Meinungsmacht sozialer Netzwerke. 
rend seines Wahlkampfes nutzte er aktiv soziale Netzwerke, um Wähler zu gewinnen. Das führte schnell zu Spekulationen darüber, ob und in welchem Umfang auch soziale Netzwerke seine Wahl begünstigt haben. In Nachbetrachtungen fielen Forschern aber vor allem auch zahlreiche Nutzerprofile auf, die miteinander stark vernetzt waren und überdurchschnittlich viele Inhalte binnen kurzer Zeit verbreitet haben. Dies führte zu dem Verdacht, dass die von ihnen veröffentlichten Inhalte keines rein menschlichen Ursprungs waren, sondern die Profile vielmehr automatisiert gesteuert und eingesetzt wurden, um Inhalte zu veröffentlichen, ihre Reichweite zu vergrößern und die Informationsvermittlung in sozialen Netzwerke zu den eigenen politischen Zwecken auszunutzen. ${ }^{5}$ Aber nicht nur während der US-Wahl ${ }^{6}$, sondern auch zu anderen Zeitpunkten und Begebenheiten - u.a. während des Ukraine-Konflikts ${ }^{7}$, des Brexit-Referendums ${ }^{8}$, der französischen Präsidentschaftswahl, ${ }^{9}$ zu anderen politischen oder gesellschaftlichen Ereignissen in der Welt ${ }^{10}$ und jüngst während der Covid-19-Pandemie ${ }^{11}$ wurden entsprechende Phänomene beobachtet. Das führte seitdem erneut zu interdisziplinären Diskussionen, weil man den automatisierten Profilen eine potentielle Einflussnahme auf die politischen Debatten innerhalb der Netzwerke ${ }^{12}$ und dadurch bedingt einen Einfluss auf die Informationsaufnahme, -verarbeitung und Willensbildung der Nutzer nachsagt, wodurch - möglicherweise - auch die konkrete Wahlentscheidung der Nutzer mitbeeinflusst worden sein könnte. Wenig verwunderlich wurde daher auch vor der Bundestagswahl 2017 befürchtet,

5 Siehe hierzu etwa Bessi/Ferrara, First Monday Vol. 21 Nr. 11 oder Boichak u.a., in: Proceedings of the $13^{\text {th }}$ International Conference, 2018, S. $17 \mathrm{ff}$.

6 Hierzu etwa Bessi/Ferrara, First Monday Vol. 21 (2016) Nr. 11; Howard u.a., Junk News and Bots during the US Election, 2017.

7 Hegelich/Janetzko, in: AAAI, 10th International AAI Conference on Web and Social Media, S. $579 \mathrm{ff}$.

8 Siehe etwa Bastos/Mercea, Social Science Computer Review 37 (2019), 38 ff.; Howard/Kollanyi, Computational Propaganda during UK-EU Referendum.

9 Ferrara, First Monday Vol. 22 (2017) Nr. 8.

10 Siehe etwa zu Venezuela Forelle u.a., Political Bots and the Manipulation of Public Opinion in Venezuela, 2015; zu Japan Schäfer/Evert/Heinrich, Big Data Vol. 5 (2017) Nr. 5, zu den USA Bessil Ferrara, First Monday Vol. 21 (2016) Nr.11; Howard u.a., Junk News and Bots during the US Election, 2017; zu Mexiko Suárez-Serrato u.a., in: Spiro/Ahn, SocInfo 2016: Social Informatics, S. 269ff, und Treré, Trípodos, Número 39 (2016), 35 (39ff.); zum Iran Thieltges u.a., Effects of Social Bots in the Iran-Debate on Twitter, 2018; zu Brasilien vgl. Department of public policy analysis, Bots and Brazil's Electoral legal system, Policy Paper 3, 2019; zum katalanischen Referendum Stella/Ferraral Domenico, PNAS Vol. 115 (2018), 12435 ff. Weitere Nachweise auch bei Woolley, First Monday Vol. 21 (2016) Nr. 4. Zu Deutschland in Ansätzen Institute for Strategic Dialogue, Make Germany Great Again, S. 18 f.; Pfaffenberger/Adrian/Heinrich, in: Holtz-Bacha, Die (Massen-)Medien im Wahlkampf, S. 97 (99); Grimme u.a., PropStop Bericht Bundestagswahl 2017. Mit Blick auf entsprechende Phänomene während der Covid-19-Pandemie u.a. Ahmed u.a., J Med Internet Res 2020:22(5):e19458; Cinelli u.a., Scientific Reports 10 (2020), 16598; Ferrara, First Monday Vol. 25 (2020) Nr. 6; Gallotti u.a., Nature Human Behaviour 2020; Yang/Torres-Lugo/Menczer, Prevalence of Low-Credibility Information on Twitter During the COVID-19 Outbreak, 2020; und Uyheng/Carley, Journal of Computational Social Sciences 2020.

11 Ferrara, First Monday Vol. 25 (2020) Nr. 6; Cinelli u.a., Scientific Reports 10 (2020), 16598.

12 Zum Teil wird sogar davon ausgegangen, dass ausländische Staaten durch Bots versuchen auf politische Debatten in anderen Staaten Einfluss zu nehmen, so Bötticher, Kriminalistik 2017, 16 (17f.). 
dass sie durch den Einsatz solcher „Bots“ korrumpiert werden könnte. ${ }^{13}$ Seitdem entstand ein breites Forschungsfeld, dass sich der Identifizierung und Erkennung von „Bot-Profilen“, der Untersuchung ihres Einflusses und ihrer Auswirkungen und potentieller Gegenmaßnahmen widmet.

Aus rechtswissenschaftlicher Sicht geht es nicht nur um die Frage, wie das neue Phänomen (grund-)rechtlich einzuordnen ist. Angesichts der in den vergangenen Jahren zunehmenden Verlagerung politischer Kommunikation in digitale Diskursräume, die durch ökonomisch-rational agierende Dienstleister bereit gehalten werden, wird schon seit Langem darüber diskutiert, ob und inwieweit den Staat eine Schutzverantwortung dafür trifft, den Kommunikationsprozess innerhalb dieser Diskursräume vor potentiellen Beeinflussungen und Risiken zu schützen, die einerseits von den Betreibern, ${ }^{14}$ andererseits aber auch von Dritten ${ }^{15}$ ausgehen können. Letztere Frage aktualisiert sich im Fall der automatisiert agierenden Nutzerprofile gerade in einem speziellen Fall. Trägt der Staat hier eine (Schutz-)Verantwortung für den Kommunikationsprozess dergestalt, dass er auch potentiellen Risiken für den Kommunikationsprozess durch automatisierte Nutzerprofile begegnen muss? Und wenn dies der Fall wäre, begegnet er den Risiken bereits in einer geeigneten, vertretbaren und verfassungsrechtlich nicht angreifbaren Weise? Die schon in Ansätzen im rechtswissenschaftlichen Schrifttum begonnene Diskussion hierüber ${ }^{16}$ leistete Antrieb für eine vertiefte Auseinandersetzung mit diesen vor allem verfassungsrechtlichen Fragen.

Wie so oft können diese aber nicht ohne einen Blick auf andere wissenschaftliche Ergebnisse beantwortet werden. Schon gedanklich kann die Frage nach einer staatlichen Verantwortung nur unter Berücksichtigung der tatsächlichen Gegebenheiten beantwortet werden: $\mathrm{Ob}$ und inwieweit Schutz für die politische Willensbildung gewährt werden muss, hängt maßgeblich davon $a b$, ob, wie und in welchem Umfang die automatisierten Profile überhaupt die politische Willensbil-

13 Vgl. MMR-Aktuell 2017, 394727. Deshalb haben etwa die Parteien - nach anfänglichem Zögern auch die AfD - entsprechende Selbstverpflichtungen abgegeben, Meinungsroboter nicht einzusetzen, so etwa Bündnis 90/Die Grünen, siehe MMR-Aktuell 2017, 387207.

14 Siehe hierzu etwa Hartl, Suchmaschinen, Algorithmen und Meinungsmacht; Kellner, Die Regulierung der Meinungsmacht von Internetintermediären; oder Pille, Meinungsmacht sozialer Netzwerke.

15 Dieser Frage widmet sich etwa Schimmele, Staatliche Verantwortung für diskursive Integrität, mit Blick auf zunehmende Probleme hinsichtlich der Diskursqualität u.a. angesichts von Propaganda Hate Speech und Fake-News.

16 In diesem Kontext - neben anderen Fragen - etwa Brings-Wiesen, JuWiss-Blog Nr. 93/2016; Dankert/ Dreyer, K\&R 2017, 73 ff.; Hostacna, AL 2018, $1 \mathrm{ff.;} \mathrm{Klaas,} \mathrm{MMR} \mathrm{2018,} 84$ ff.; Löber/Roßnagel, MMR 2019, 493 ff.; Milker, ZUM 2017, 216 ff.; ders., JuWiss-Blog Nr. 91/2016; ders., InTeR 2017, 199 ff.; Steinbach, ZRP 2017, 101 ff. Ebenfalls zu Meinungsrobotern wenn auch in anderem Zusammenhang etwa Bötticher, Kriminalistik 2017, 16 ff.; Dankert, in: Hoffmann-Riem, Big Data - Regulative Herausforderungen, S. 157 ff.; Gasser/Kraatz, VerfBlog, 2017/1/16; Krüper, in: Unger/v. UngernSternberg, Demokratie und künstliche Intelligenz, S. 67 ff.; Oehmer/Pedrazzi, UFITA 2020, 7 ff.; Schröder, DVBl. 2018, 465 ff.; Zumkeller-Quast, JuWiss-Blog Nr. 2/2017. Zu ausgewählten Fragen der Strafbarkeit Volkmann, MMR 2018, 58 ff.; zu urheberrechtlichen Implikationen Wolf, WRP 2019, $440 \mathrm{ff}$. 
dung der Netzwerknutzer beeinflussen können. Insoweit gilt es zunächst grundlegende, empirische Fragen zu klären. Dazu gehört nicht nur ein kurzer Einblick in die Funktionsweise und Bedeutung der sozialen Netzwerke selbst, sondern vor allem auch eine grundlegende Betrachtung des politischen Willensbildungsprozesses. Zentrales Augenmerk liegt hierbei freilich auf der individuellen Sphäre und der Frage, wie sich der Einzelne eine Meinung bildet, welche Faktoren hierfür maßgeblich sind und welche Verzerrungen dabei auf ihn einwirken. Erst danach können die automatisierten Profile selbst betrachtet werden. Neben einer Annäherung an den Begriff der „Meinungsroboter“, steht dabei vor allem auch die technische Umsetzung im Vordergrund, um verstehen zu können, wie Meinungsroboter funktionieren. Hierfür werden auch zentrale Einsatzstrategien aufgezeigt, um das Einflusspotential der Meinungsroboter mit Blick auf die individuelle Willensbildungssphäre bewerten und Unterschiede zu herkömmlichen Einflussversuchen aufzeigen zu können (2. Teil).

So denn hierbei gewisse Einflusspotentiale aufgezeigt werden können, stellt sich die Frage, inwieweit diese Einfluss auf die Rolle des Staates haben. Wird er dadurch zum Garanten für einen (manipulations-)freien politischen Willensbildungsprozess (3. Teil)? Insoweit muss aber erstmal eine abstrakte Verantwortung des Staates für den demokratischen Kommunikationsprozess insgesamt oder zumindest für Teilbereiche begründet werden können (1. Kapitel), bevor sich Aussagen über die konkrete Verantwortung im Falle der Meinungsroboter treffen lassen (2. Kapitel). Selbst wenn hiernach eine Schutzverantwortung dem Grunde nach bestehen würde, bedeutet das freilich nicht, dass umfangreiche eingriffsintensive Regelungen gefordert wären. Schon aus verfassungsrechtlicher Sicht bestehen spezifische Schranken, die es zu berücksichtigen gilt. Insoweit kann die Schutzverantwortung auch nicht losgelöst von den bisherigen normativen und faktischen Regelungen betrachtet werden. Angesichts erheblicher rechtlicher Veränderungen seit Beginn der Untersuchung im Sommer 2017 und vor ihrer Beendigung im Winter 2020 werden dabei zunächst die allgemeinen Regelungsmechanismen betrachtet, die (mittelbar) Auswirkungen auf den Untersuchungsgegenstand haben, und diese vor dem Hintergrund der staatlichen Schutzverantwortung bewertet. Erst danach wird sich intensiv dem Medienstaatsvertrag der Länder gewidmet, der gerade auch mit Blick auf Meinungsroboter zahlreiche, auch europarechtliche Fragen aufwirft. Auch hier schließt die Darstellung mit einer umfangreichen Bewertung der regulativen Maßnahmen im Lichte der staatlichen Schutzpflicht (3. Kapitel). Perspektivisch lässt sich auf die weitere Entwicklung blicken, die auch für den Bereich der Meinungsroboter relevant werden (4. Kapitel).

Die Arbeit schließt sodann mit einer Zusammenfassung der wesentlichen Ergebnisse (4. Teil). 


\section{Teil: Grundlagen - Soziale Netzwerke, politische Willensbildung und Meinungsroboter}

Um die Frage nach einer staatlichen Schutzverantwortung für die neuen Phänomene in den digitalen Kommunikationsräumen der sozialen Netzwerke überhaupt beantworten zu können, müssen zunächst erstmal grundlegende Begriffe und Zusammenhänge erklärt werden. Das gilt nicht nur hinsichtlich der sozialen Netzwerke und ihrer Funktionsweise (A.), sondern auch für die politische Willensbildung, insbesondere der Nutzer (B.) sowie für die Meinungsroboter und ihre Funktions- und Wirkungsweise im politischen Willensbildungsprozess (C.).

\section{A. Soziale Netzwerke:}

Soziale Medien revolutionierten den Kommunikationsprozess in der digitalen Welt. Sie stellen eine öffentliche Plattform zur Verfügung, auf der jeder Teilnehmer sich äußern und potentiell gehört werden kann. Dort, wo die Internetnutzer vorher weit überwiegend Rezipient der von anderen bereitgestellten Informationen waren, ${ }^{17}$ verschaffen soziale Medien ihnen eine Stimme und eine aktive Rolle innerhalb des Kommunikationsprozesses. Das Erfolgs- und Geschäftsmodell sozialer Netzwerke basiert - wie schon der Name erahnen lässt - auf der sozialen Interaktion und der Aktivität der registrierten Nutzer. Dabei legen es die Betreiber gerade auch darauf an, Diskussions-, Kommunikations- und Informationsplattform zu sein. Durch den Erfolg der sozialen Medien haben diese auch an Attraktivität für andere, nicht-private Akteure gewonnen. Neben wirtschaftlichen Unternehmen haben auch staatliche Institutionen, Politiker, Parteien und andere gesellschaftliche Verbände und Akteure den Weg in die sozialen Medien gefunden. Dadurch werden die ansonsten so fern wirkenden medialen und politischen Debatten in die virtuelle Gemeinschaft getragen und die sozialen Netzwerke für die gesellschaftliche und politische Kommunikation relevanter. Wegen dieser neuen Möglichkeiten sind soziale Medien über die Jahre hinweg neben Suchmaschinen zu einem zentralen Dienst für Internetnutzer geworden. $56 \%$ der Deutschen nutzen sie. ${ }^{18}$

17 Schwartmann/Ohr, in: Schwartmann, PraxisHb MedienR, 11 Rn. 1.

18 Statistisches Bundesamt, Private Haushalte in der Informationsgesellschaft, 2018, S. 16. 
I. Annäherung an den Begriff des sozialen Netzwerks

Der Begriff des sozialen Mediums ist aber nur ein Oberbegriff für eine Vielzahl unterschiedlicher Angebote, ${ }^{19}$ von einfachen Medienaustauschdiensten wie Snapchat, Instagram und YouTube, über spezielle berufsbezogene Plattformen wie etwa Xing und LinkedIn bis hin zu (reinen) Kommunikationsdiensten wie WhatsApp oder Telegram. ${ }^{20}$ Für die hiesige Abhandlung ist allein eine Unterkategorie der sozialen Medien von Interesse: die sog. online social networks (ONS), ${ }^{21}$ die sozialen Netzwerke im engeren Sinne. Kennzeichnend für diese sind zwei wesentliche Attribute: das Prinzip des Networking und eine soziale interaktive Komponente. ${ }^{22}$ Die soziale Komponente resultiert maßgeblich aus dem Aufbau der entsprechenden Plattformen selbst. Sie sind darauf angelegt die soziale Interaktion zwischen den Nutzern zu ermöglichen. Nutzer sind nicht bloß passive Rezipienten, sondern aktive Gestalter der Plattformen, indem sie Inhalte unterschiedlichster Art veröffentlichen, mit anderen teilen und diesen die Möglichkeit geben, auf sie zu reagieren. Dies dient letztlich auch der Vernetzung. Die Interaktionen sollen nicht allein der Unterhaltung dienen, vielmehr sollen die Nutzer die Offline-Beziehungen in die Online-Welt tragen und darüber hinaus mit anderen Nutzern in Kontakt treten, um so ein neues, weitreichenderes Freundes- und Bekanntennetzwerk zu erschaffen. Hierfür stellen die Netzwerke die Infrastruktur zur Verfügung, die neben einer öffentlichen auch eine private Kommunikation und Interaktion ermöglicht. Dadurch sind die Netzwerke Medienaustausch-, Kommunikations-, Interaktions- und auch kommerzieller Dienst in einem. ${ }^{23}$ Das macht nicht selten die Abgrenzungen zu den eingangs erwähnten anderen sozialen Medien schwierig. ${ }^{24}$ Für die vorliegenden Zwecke sind vor allem soziale Netzwerke ohne eine irgendwie geartete spezifische Ausrichtung relevant, weil diese auch für die politische Willensbildung insgesamt bedeutsamer sind (sog. Universalnetzwerke) ${ }^{25}$. Unberücksichtigt bleiben also spezifischen

19 Taddicken/Schmidt, in: dies., Hb Soziale Medien, S. 3 (4); Schwartmann/Ohr, in: Schwartmann, PraxisHb Medienrecht, Kap. 11 Rn. 1. Und auch der BKartA, Beschl. v. 06.02.2019 - B6-22/16 = BeckRS 2019, 4895 Rn. 168 bezeichnet die Begriffe als unscharf und weist darauf hin, dass sie ganz unterschiedliche Verwendung finden. Eine umfassendere Liste der Angebote samt Erklärung eben dieser gibt das BKartA ebenfalls a.a.O. Rn. $177 \mathrm{ff}$.

20 Zur weiteren Ausdifferenzierung der unterschiedlichen Dienstangebote etwa Schmidt, Social Media, S. $12 \mathrm{ff}$.

21 Vgl. BKartA, Beschl. v. 06.02.2019 - B6-22/16 = BeckRS 2019, 4895 Rn. 170.

22 Zum Begriff der sozialen Netzwerke siehe auch Schwartmann/Ohr, in: Schwartmann, PraxisHb Medienrecht, Kap. 11 Rn. $12 \mathrm{ff}$.

23 Vgl. Schwartmann/Ohr, in: Schwartmann, PraxisHb Medienrecht, Kap. 11 Rn. 14.

24 Diese Abgrenzungsschwierigkeit betont auch Webster, Northwestern Law Review 104 (2010), 593 (595).

25 Pille, Meinungsmacht sozialer Netzwerke, S. 92 f. 
Zwecken dienende Netzwerke (Xing, LinkedIn oder Partnervermittlungen), ${ }^{26}$ Medienaustauschdienste ${ }^{27}$ oder bloße Kommunikationsdienste, die eher eine individuelle Ausrichtung aufweisen (WhatsApp usw.), ${ }^{28}$ denen also die „öffentliche Sphäre" fehlt. ${ }^{29}$ Zentraler Anbieter in diesem Bereich ist Facebook. Aber auch Twitter $^{30}$ wird in Deutschland zunehmend bedeutsamer. Daneben mag es auch noch weitere Anbieter geben. ${ }^{31}$ In der grundlegenden Funktionsweise unterscheiden sich die meisten Netzwerke aber kaum, sodass die Darstellungen auf die beiden zentralen Anbieter beschränkt bleiben sollen.

\section{Facebook}

Facebook dominiert seit vielen Jahren mit einem Marktanteil zwischen 70 und $90 \%$ den Markt. ${ }^{32}$ Das Unternehmen selbst sieht seine Aufgabe darin, „den Menschen die Möglichkeit zu geben, Gemeinschaften zu bilden, und die Welt näher zusammenzubringen. "33 In der Praxis funktioniert das wie folgt: Facebook ist - mit Ausnahme der Seiten - ein nach außen abgeschottetes Netzwerk; eine Registrierung ist also notwendige Voraussetzung der Inanspruchnahme des Dienstes. Diese setzt die Angabe einiger weniger personenbezogener Daten neben dem Namen eine Emailadresse und das Geburtsdatum - voraus. Nach erfolgreicher Registrierung erhält jeder Nutzer ein individuelles Nutzerprofil, eine Subseite des Netzwerks, welches in gewissen Grenzen selbst gestaltet werden kann. ${ }^{34}$ Vor allem können weitere persönliche Informationen, und Interessen hinzugefügt und durch ein Selbstporträt das Profil vervollständigt werden. Für andere Nutzer ist man unter dem gewählten Profilnamen auffindbar. Insoweit

26 Schwartmann/Ohr, in: Schwartmann, PraxisHb Medienrecht, Kap. 11 Rn. 13. Auch der BKartA, Beschl. v. 06.02.2019 - B6-22/16 = BeckRS 2019, 4895 Rn. 277 ff. hat solche Angebote bei der Analyse des Marktes sozialer Netzwerke nicht berücksichtigt.

27 Zu Snapchat etwa BKartA, a.a.O., Rn. 295 ff., zu YouTube Rn. 309 ff., zu Instagram Rn. 334 ff.

28 Vgl. hierzu auch BKartA, Beschl. v. 06.02.2019 - B6-22/16 = BeckRS 2019, 4895 Rn. 266 ff.

$29 \mathrm{Zu}$ dieser Differenzierung zwischen originären sozialen Netzwerken und Multimediaplattformen etwa auch Schmidt, Social Media, S. 13. Und dieser Ansatz liegt auch der Legaldefinition des $\mathbb{} 1$ Abs. 1 NetzDG zugrunde, wenn dort soziale Netzwerke definiert werden als „Telemediendiensteanbieter, die mit Gewinnerzielungsabsicht Plattformen im Internet betreiben, die dazu bestimmt sind, dass Nutzer beliebige Inhalte mit anderen Nutzern teilen oder der Öffentlichkeit zugänglich machen.“

30 Auch wenn Twitter eher ein Microbloggingdienst ist, so etwa Schwartmann/Ohr, in: Schwartmann, PraxisHb Medienrecht, Kap. 11 Rn. 22 mit Fn. 36, wird er hier als soziales Netzwerk verstanden, weil er eben auch auf der Verbindung von Nutzern dient, auch wenn diese deutlich weniger Bedeutung haben mag als etwa bei Facebook. Zum potentiellen Verständnis als soziales Netzwerk vgl. BKartA, Beschl. v. 06.02.2019 - B6-22/16 = BeckRS 2019, 4895 Rn. 170.

31 Hierzu zählen etwa auch Reddit und bis vor kurzem auch Google+. Der Dienst wurde aber - mangels Interesses an der Nutzung - eingestellt. Das gleiche Schicksal mussten auch die ursprünglichen Begründer Myspace, Schüler- bzw. StudiVz und Netlog über sich ergehen lassen.

32 BKartA, Beschl. v. 06.02.2019 - B6-22/16 = BeckRS 2019, 4895 Rn. 395.

33 So die Aussage zu Beginn der Nutzungsbedingungen unter Nr. 1, abrufbar unter https://www.facebook .com/legal/terms? ref=pf (Stand: 30.05.2021).

$34 \mathrm{Zu}$ dieser Gestaltung siehe auch BKartA, Beschl. v. 06.02.2019 - B6-22/16 = BeckRS 2019, 4895 Rn. 21, 259. 
entsteht eine eigenständige „Online-Identität“35. Für Nutzer, die das Netzwerk (überwiegend) nicht für private Zwecke nutzen - hierzu gehören etwa Politiker, Parteien und (Medien-)Unternehmen - gibt es die Möglichkeit sog. FacebookSeiten anzulegen. In beiden Fällen ${ }^{36}$ ermöglichen die angelegten Profile/Seiten, sich mit anderen Nutzern zusammenzuschließen. Nutzer können sich gegenseitig Freundschaftsanfragen schicken, sich abonnieren, Beiträge auf den Profilseiten des anderen veröffentlichen, Beiträge anderer kommentieren, teilen und vieles mehr. Entsprechendes gilt auch im Verhältnis von Nutzerprofilen zu den Facebook-Seiten. Nutzer können so in Kontakt mit Unternehmen, Organisationen oder Personen des öffentlichen Lebens treten, sie abonnieren, die Beiträge von ihnen kommentieren oder mit ihnen interagieren. Durch Freundschaftsanfragen und Abonnements entsteht ein individuelles soziales Netzwerk. Damit jeder Nutzer erfährt, welche Neuigkeiten es gibt, bietet Facebook auf der Startseite den sog. News Feed an. Er enthält eine Übersicht relevanter Beiträge und Interaktionen anderer Nutzer aus dem individuellen Netzwerk.

Auch wenn die sozialen Interaktionen oftmals innerhalb des individuellen Netzwerkes erfolgen, besteht gleichwohl die Möglichkeit, auch mit Nutzern und Inhalten außerhalb des eigenen Netzwerks in Kontakt zu kommen. Allen voran die Kommentarfunktion unter einem Beitrag macht es möglich, mit anderen Nutzern zu kommunizieren. Und durch die Interaktionen von Angehörigen des individuellen Netzwerkes mit netzwerkfremden Inhalten oder Nutzern gelangen auch netzwerkfremde Inhalte in den News Feed. Allgemeines Ziel dieser Interaktionsund Kommunikationsstrukturen ist es, den Nutzer möglichst lange innerhalb des Netzwerks zu fangen. Um dies zu erreichen werden barrierefreie Nutzerinterfaces bereitgehalten, ${ }^{37}$ die eine unkomplizierte und schnelle Interaktion und Reaktion der Nutzer ermöglichen. Der „Gefällt-mir-Button“ ist das Symbol dieser „Usability“.

\section{Personalisierung - zwischen Verbesserung des Dienstes und ökonomischer Rationalität}

Es ist kein Geheimnis, dass die Betreiber das Nutzerverhalten beobachten und dabei vor allem die „Usability“ der Nutzerinterfaces die Auswertung ,automatischer, impulsiver Reaktionen “38 deutlich erleichtert. Offiziell setzt Facebook alles daran, den Dienst an die individuellen Bedürfnisse anzupassen und so jedem

35 So BKartA, Beschl. v. 06.02.2019 - B6-22/16 = BeckRS 2019, 4895 Rn. 259.

36 Ausführlicher zu den Funktionen für die unterschiedlichen Gruppen der Nutzer BKartA, Beschl. v. 06.02.2019 - B6-22/16 = BeckRS 2019, 4895 Rn. $19 \mathrm{ff}$.

37 Zum „Captology-Design“ siehe auch Lischka, AfP 2018, 388 (389).

38 Lischka, AfP 2018, 388 (389). 
Nutzer ein personalisiertes Erlebnis zu ermöglichen. ${ }^{39}$ Diese Personalisierungsmaßnahmen dienen aber nicht nur dem Interesse des Nutzers, einen möglichst relevanten Ausschnitt der netzwerkinternen Informationen zu erhalten, sondern sie ermöglichen Facebook auch eine höherpreisige Vermarktung von Werbeplätzen innerhalb des Netzwerkes. ${ }^{40}$ Mithilfe der Personalisierungsalgorithmen können potentielle Interessenten und Konsumenten zielgenau angesprochen werden. ${ }^{41}$ Diese Algorithmen liegen nicht nur den Werbeflächen zugrunde, sondern dem gesamten Angebot, allen voran auch dem News Feed. Und auch die Beiträge in den Kommentarspalten werden mithilfe solcher Algorithmen angeordnet ${ }^{42}$. Das dahinterstehende Prinzip ist schnell erklärt: Über Nutzerprofile und andere Identifikatoren ${ }^{43}$ lässt sich das individuelle Nutzerverhalten beobachten (sog. WebTracking). Dabei werden verschiedenste Daten erhoben und gespeichert. ${ }^{44}$ Diese Daten stammen einerseits aus dem Netzwerk selbst ${ }^{45}$ aber auch von Drittseiten, die bspw. über die Social-Plugins - den Gefällt-mir oder den Teilen-Button - der Netzwerkanbieter verfügen. Durch die Social-Plugins werden auch Serververbindungen zum Plug-in-Anbieter hergestellt und Nutzerdaten übermittelt. ${ }^{46}$ Und in sog. (Tracking-) Cookies $^{47}$ werden ebenfalls Informationen über das Surfverhalten, verwendete Geräte der Nutzer u.v.m. auf Drittseiten zusammengefasst und in den Webbrowsern oder auf den PCs der Nutzer gespeichert. ${ }^{48}$ Insbesondere mit Hilfe von Third-Party-Cookies können die Anbieter auf gesammelte Daten

39 Siehe hierzu die Ausführungen in den Nutzungsbedingungen unter Nr. 1, abrufbar unter https://de-de.f acebook.com/legal/terms?ref=pf , (Stand: 30.05.2021).

40 Zu diesem „doppelten Vorteil“ siehe etwa Heldt, CR 2018, 494 (495); Schneiders, Das ist doch Diskriminierung, S. 3 m.w.N. Ausführlicher zur Finanzierung der Netzwerke und Intermediäre etwa Tief, Kommunikation auf Facebook, Twitter \&YouTube, S. 30 f.

41 Vgl. hierzu die Ausführungen des EuGH zu sog. Fan-Pages von Facebook, EuGH, Urt. v. 05.06.2018 C-210/16 = NJW 2018, 2537 Rn. 35 ff. - ULD/Wirtschaftsakademie Schleswig-Holstein. Siehe auch Ingold, Staat 56 (2017), 491 (508 f.).

42 Siehe hierzu https://de-de.facebook.com/help/539680519386145 ?helpref=uf_permalink, (Stand: 30.05.2021).

43 Hierzu kann vor allem auch die IP-Adresse gehören, vgl. Schmitz, in: Hoeren/Sieber/Holznagel, Hb Multimedia-Recht, Teil 16.2 Rn. 107.

44 Ausführlich zu den Datenquellen BKartA, Beschl. v. 06.02.2019 - B6-22/16 = BeckRS 2019, 4895 Rn. $483 \mathrm{ff}$.

45 Gerade wegen der nutzerfreundlichen Interfaces, die eine schnelle Eingabe von Likes ermöglichen, stellen die Nutzer immer genauere Daten etwa darüber zur Verfügung, welche Inhalte Ihnen zusagen, vgl. Maschewski/Nosthoff, in: Mühlhoff/Breljak/Slaby, Affekt, Macht, Netz, S. 55 (63 f.).

46 Hierzu u.a. Schlussantrag des GA Bobek in der Rs. C-40/17, Rn. 1, 17 - Fashion-ID; Kunnert, DuD 2019, 257; detaillierter BKartA, Beschl. v. 06.02.2019 - B6-22/16 = BeckRS 2019, 4895 Rn. 55 ff., 139 ff. Insbesondere Facebook geriet durch diese Praxis in die Kritik, weil es auch von nicht registrierten Nutzern sog. Schattenprofile über die Plug-ins anlegen konnte, u.a. hierzu Fanta, Facebook legt Schattenprofile über alle an, netzpolitik.org.

47 Ausführlicher zu den unterschiedlichen Arten der Cookies Mankowski, in: Fezer/Büscher/Obergfell, UWG, Bd. I, S 12 Rn. 233a.

48 Boelke, in: Hoeren/Sieber/Holznagel, Multimedia-Recht, Teil 11 Rn.100; Konrad/Hausen, in: AuerReinsdorff/Conrad, Hb IT- und DatenschutzR, $\mathbb{3 6}$ Rn. 95; Krüger/Peintinger, in: Martinek/Semler/ Flohr, Hb VertriebsR, $\$ 36$ Rn. 337; Mankowski, a.a.O., Rn. 233. Hierzu siehe auch die Ausführungen des BKartA, Beschl. v. 06.02.2019 - B6-22/16 = BeckRS 2019, 4895 Rn. 146 ff. 
Dritter zugreifen. ${ }^{49}$ Hierüber haben sie Zugriff auf personenbezogene Informationen, die außerhalb des Netzwerks erhoben wurden. Inhaltliche Grenzen für die gesammelten Daten gibt es also kaum, wofür nicht zuletzt der Begriff „Big Data " ${ }^{50}$ symbolisch steht. Anhand der gesammelten Daten lassen sich bestimmte Rückschlüsse u.a. über soziale, psychologische und politische Eigenschaften des jeweiligen Nutzers ziehen und demografisch-sozialpsychologische Nutzerprofile anlegen. Auf die so ermittelten Eigenschaften und Interessen wird der Dienst zugeschnitten. Je mehr Daten verarbeitet werden, desto präziser können dabei die Voraussagen der Algorithmen werden. ${ }^{51}$

\section{Der News Feed als Beispiel algorithmenbasierter Personalisierung}

Insbesondere der News Feed ${ }^{52}$ ist ein Produkt solcher Algorithmen. Zur Funktionsweise gibt das Unternehmen nur sporadische Informationen: ${ }^{53} \mathrm{Ihm}$ liegt wie auch der Suchmaschine von Google- ${ }^{54}$ ein Rankingverfahren zugrunde, das Voraussagen über die Wahrscheinlichkeit trifft, mit der sich ein Nutzer für einen bestimmten Inhalt oder eine bestimmte Interaktion aus seinem Netzwerk interessieren und auf diesen in irgendeiner Weise reagieren wird. Dadurch soll die Verweildauer der Nutzer innerhalb des Netzwerks und so die Nutzerbindung erhöht werden. ${ }^{55}$ In einem ersten Schritt, dem inventory, werden hierfür alle Inhalte ermittelt, die ein Nutzer bisher noch nicht gesehen hat. Sodann werden sog. signals erfasst, die das Ranking beeinflussen. Hierzu zählen u.a. die Affinität zum Urheber, das Gewicht des Inhalts, welches u.a. anhand der Reaktion, der Bewertung oder der Relevanz für andere Nutzer ermittelt wird, und der Aktualität eines Inhaltes. ${ }^{56}$ Auf Grundlage dieser Informationen soll der Algorithmus nun die Inhalte auswählen, bei denen eine Reaktion des Nutzers mit großer Wahrscheinlichkeit zu erwarten ist (predictions). Jeder Inhalt erhält dadurch einen

49 Zu Third-Party-Cookies siehe etwa Jandt, ZD 2018, 405 (408); Conrad/Hausen, a.a.O., Rn. 96; hierauf auch kurz eingehend Krüger/Peintinger, ebd.

50 Zum Begriff siehe u.a. Bender, 300 Keywords Informationsethik, S. 19 ff.; Ohrtmann/Schwiering, NJW 2014, $2984 \mathrm{f}$.

51 Heldt, CR 2018, 494 (496).

$52 \mathrm{Zu}$ diesem im Kontext der Filter- und Personalisierungsdebatte siehe u.a. Kaiser/Reiling, in: Unger/v. Ungern-Sternberg, Demokratie und künstliche Intelligenz, S. 85 (87 ff.).

53 Hierzu etwa ein Video, indem ein Mitarbeiter, Adam Mosseri, die Funktionsweise des Newsfeeds erklärt, abrufbar unter https://newsroom.fb.com/news/2018/05/inside-feed-news-feed-ranking/, (Stand: 30.05.2021).

54 Eine umfassende Darstellung der Funktionsweise des PageRank Algorithmus findet sich bei Page u.a., PageRank Citation Ranking, oder bei Lewandowski, Suchmaschinen verstehen, S. $89 \mathrm{ff}$.

55 Vgl. v. Ungern-Sternberg, in: Unger/Dies., Demokratie und künstliche Intelligenz, S. 3 (9); Kaiser/ Reiling, in: Unger/v. Ungern-Sternberg, Demokratie und künstliche Intelligenz, S. 85 (88); Ladeur/ Gostomzyk, K\&R 2018, 686 (687) oder Heldt, CR 2018, 494 (495).

56 Hierzu (ausführlicher) siehe auch BKartA, Beschl. v. 06.02.2019 - B6-22/16 = BeckRS 2019, 4895 Rn. 489 ff. 
relevancy score. Je höher dieser ist, desto priorisierter wird er im News-Feed angezeigt.

\section{Twitter}

Twitter konnte in den vergangenen Jahren seine Nutzerzahlen auch hierzulande steigern, ist aber Facebook immer noch deutlich unterlegen. Schätzungen gehen von ca. 5 Millionen Nutzern in Deutschland aus. ${ }^{57}$ Anders als bei Facebook erhalten auch nicht registrierte Nutzer Zugang zum Netzwerk und können so Informationen innerhalb des Netzwerks suchen und konsumieren. Die Dunkelziffer der Rezipienten kann also deutlich höher sein. Anders als Facebook dient Twitter nicht primär dem Networking. Originär ist Twitter ein Kurznachrichtendienst, der es ermöglicht, auf 140 Textzeichen begrenzte Beiträge und Multimediainhalten unter einem Profilnamen zu veröffentlichen. Dabei besteht auch hier die Möglichkeit, sich mit anderen zu verbinden (sog. Follower). So entsteht auch hier ein individuelles Netzwerk. Zudem ermöglicht auch Twitter, Inhalte anderer mit dem eigenen Umfeld zu teilen (retweet) oder sich über eine Kommentarfunktion zu Beiträgen anderer zu äußern. Twitter bietet ebenfalls eine Übersicht über aktuelle Beiträge (sog. Timeline) aus dem eigenen Netzwerk an. Auch hier kommen ähnliche personalisierte Rankingverfahren zum Einsatz. Zudem findet sich eine (personalisierte) Übersicht über aktuelle Themen (Trending Topics). ${ }^{58}$ Dabei erkennt Twitter anhand des einschlägigen Hashtags - einem Raute-Zeichen gefolgt von einer Zeichenfolge, mit der das Thema überwiegend in Verbindung gebracht wird $-^{59}$ wie beliebt ein aktuelles Thema im Netzwerk ist. Zentral hierfür ist vor allem die Anzahl der Tweets, die den entsprechenden Hashtag verwenden. ${ }^{60}$

\section{B. Der Prozess der politischen Willensbildung}

Politische Willensbildung ist nicht allein ein individueller, kognitiver Prozess. Schon das Grundgesetz selbst unterscheidet zwischen einer staatlichen politischen Willensbildung (Art.20 Abs.2 GG), der Willensbildung des Volkes (vgl.

57 So Statista, Anzahl der monatlich aktiven Twitter-Nutzer in Deutschland, abrufbar unter https://de.st atista.com/statistik/daten/studie/370490/umfrage/anzahl-der-aktiven-twitter-nutzer-in-deutschland/, (Stand: 31.05.2021).

58 Shao u.a., IEEE Access Vol. 6 (2018), 75327 (75335).

59 Siehe hierzu u.a. Shao u.a., IEEE Access Vol. 6 (2018), 75327 (75334f.) und Gertler, in: Friedrichsen/ Kohn, Digitale Politikvermittlung, S. 83 (97). „\#Covid19“ ist etwa ein Schlüsselbegriff für die Diskussion und Information währen der Corona-Pandemie, nach dem durch Polizeigewalt verursachten Tod von George Floyd war etwa „\#BlackLivesMatter" kennzeichnend für die anschließende Debatte um (rassistische) Polizeigewalt.

60 Ausführlichere Information finden sich unter https://help.twitter.com/de/using-twitter/twitter-trending -faqs, (Stand: 31.05.2021). 
Art. 21 Abs. 1 GG) ${ }^{61}$ Auch wenn diese beiden Sphären prinzipiell auseinandergehalten werden, so ergeben sich praktisch gleichwohl „vielfältig[e] und tagtäglich[e] Wechselwirkungen“62 und mit der Wahl „fällt die Äußerung des Volkswillens mit der Bildung des Staatswillens zusammen. “63 Für die vorliegenden Zwecke liegt der Fokus zwar auf der individuellen Willensbildung der Nutzer sozialer Netzwerke. Wegen vielgestaltiger Interdependenzen und Einflüssen lässt sich die individuelle Willensbildung allerdings - weder aus verfassungsrechtlicher noch aus empirischer Sicht - isoliert betrachten.

Aus verfassungsrechtlicher Sicht ist die individuelle Freiheit des Einzelnen Ausgangspunkt der politischen Willensbildung. ${ }^{64}$ Insbesondere die Kommunikationsgrundrechte dienen - trotz tendenziell anderslautende Konnotation - ${ }^{65}$ zuvörderst der individuellen Freiheitsentfaltungen der eigenen Person in einer komplexen gesellschaftlichen Sphäre. ${ }^{66}$ Die grundrechtlichen Freiheiten gewähren dem mündigen Bürger das Recht, frei und selbstbestimmt Informationen zu konsumieren (Art. 5 Abs. 1 S. 1, 2. HS), diese zu verarbeiten und sich dadurch eine Meinung zu bilden und diese in unterschiedlichen Formen zum Ausdruck zu bringen (Art. 5 Abs. 1, S. 1, S. 2 GG und Art. 38 Abs. 1 S. 1, 3. Var. GG). Insbesondere Art. 5 Abs.1 GG schützt aber (implizit) auch die öffentliche, gesellschaftliche Sphäre der Kommunikation. ${ }^{67}$ Dort, wo individuelle Meinungen und mediale Berichterstattung aufeinander treffen, wo diskutiert und um das sachlich stärkste Argument gestritten wird, entsteht öffentliche Kommunikation, ${ }^{68}$ und nur dort kann sich eine öffentliche Meinung - der maßgebliche Einflussfaktor auf den Staatswillen - ${ }^{69}$ formen. ${ }^{70}$ Insoweit gehört die öffentliche Meinung und

61 Vgl. BVerfGE 8, 104 (113) - Volksbefragung; 20, 56 (99) - Parteifinanzierung I; Klein, in: Maunz/ Dürig, GG, Art. 21 Rn. 154; Rogg, Demokratie und Internet, S. 134.

62 BVerfGE 44, 25 (139f.) - Öffentlichkeitsarbeit der Bundesregierung; 138, 102 Rn. 32 - Wahlkampfäußerungen von Regierungsmitgliedern.

63 BVerfGE 20, 56 (98) - Parteifinanzierung I.

64 Ähnlich Vesting, JZ 2020, 975 (976).

65 Oftmals wird nämlich neben dem individuell-rechtlichen Charakter auch der objektiv-rechtliche Charakter der Kommunikationsfreiheiten betont, zur Meinungsfreiheit BVerfGE 7, 198 (208) - Lüth; zur Informationsfreiheit BVerfGE 27, 71 (81) - Leipziger Volkszeitung; zur Versammlungsfreiheit siehe nur BVerfGE 69, 315 (343 f.) - Brokdorf; zur Pressefreiheit BVerfGE 20, 162 (174) - Spiegel; und mit Blick auf Art. 5 Abs. 1 GG insgesamt BVerfGE 20, 56 (97) - Parteifinanzierung I. Im Falle der Rundfunkfreiheit prävaliert gar der objektiv-rechtliche Charakter, wenn hier die „dienende“ Funktion besonders betont und Grundlage einer eigenständigen Dogmatik ist, hierzu etwa nur BVerfGE, 57, 295. (320) - 3. Rundfunkentscheidung. Ausführlicher zur Rundfunkfreiheit noch unten, 3. Teil, 1. Kapitel, A.

66 BVerfGE 7, 198 (208) - Lüth, zur Meinungsfreiheit; BVerfGE 27, 71 (81f.) - Leipziger Volkszeitung, zur Informationsfreiheit.

67 BVerfGE 20, 56 (98) - Parteifinanzierung I.

68 Vgl. BVerfGE 7, 198 (208) - Lüth; 89, 155 (185) - Maastricht.

69 Vgl. BVerfGE 20, 56 (98 f.) - Parteifinanzierung I; 89, 155 (185) - Maastricht.

70 Vgl. BVerfGE 5, 85 (205) - KPD-Verbot; 7, 198 (219) - Lüth. Zum Zusammenhang individueller Freiheit und der Herrschaft der öffentlichen Meinung auch Volkmann, in: Unger/ v. Ungern-Sternberg, Demokratie und künstliche Intelligenz, S. 51 (62 f.). 
das Prinzip der Öffentlichkeit zum Kernbestand des Demokratieprinzips des Art. 20 Abs. 2 GG. ${ }^{71}$

Zwischen individueller Freiheit und öffentlicher Sphäre bedarf es aber auch aus verfassungsrechtlicher Sicht Vermittler. Das zeigt sich zunächst an der besonderen Gewährleistung der medialen Freiheiten von Rundfunk, Presse und Film in Art. 5 Abs. 1 S. 2 GG. ${ }^{72}$ Daneben treten aber auch Gewährleistungen für bürgerliche Zusammenschlüsse, die in ihrer kollektiven Wirkungssphäre besonders geschützt werden. Neben der Vereinigungs- (Art. 9 GG) zählt vor allem auch die Versammlungsfreiheit (Art. 8 GG) hierzu. Der Schutz einer Meso-Ebene kommt in Art. 21 Abs. 1 GG zum Ausdruck, wenn dort den Parteien ein besonderer Mitwirkungsauftrag an der Willensbildung erteilt wird und ihnen insoweit eine doppelte Vermittlerfunktion zukommt: die Vermittlung zwischen den Bürgern, ${ }^{73}$ aber auch zwischen Staat und Volk. ${ }^{74}$

Auch aus empirischer Sicht kann die individuelle Willensbildung nicht ohne einen Blick auf das gesamte Konstrukt der politischen Willensbildung betrachtet werden. Die verfassungsrechtliche Dreiteilung findet hier sein Pendant in der Aufteilung des Kommunikations- und Willensbildungsprozesses in drei koexistierende und sich wechselseitig beeinflussenden Ebenen: Makro-, Meso- und Mikro-Ebene. ${ }^{75}$

\section{Makro-Ebene}

Auf der Makro-Ebene geht es um die gesellschaftliche Sphäre politischer Kommunikation und Willensbildung als konstitutive Voraussetzung des demokratischen Staates. Wesentliches Ziel gesellschaftlicher Kommunikation ist die Bildung der öffentlichen Meinung als ein mehrheitlicher Konsens divergierender Einzelmeinungen. ${ }^{76}$ Die Bildung öffentlicher Meinung setzt notwendiger Weise Öffentlichkeit voraus. ${ }^{77}$ Aus historischer Sicht wurde diese besonders durch das

71 Vgl. BVerfGE 30, 318 (344) - Stabilisierungsmechanismusgesetz. Zum demokratischen Prinzip der Öffentlichkeit siehe etwa BVerfGE 70, 324 (358) - Haushaltskontrolle der Nachrichtendienste und zur öffentlichen Meinung als demokratische Instanz BVerfGE 20, 56 (98) - Parteifinanzierung I oder BVerfGE 123, 267 (358f.) - Lissabon.

72 Zur Bedeutung der Presse als Vermittlerinstanz etwa BVerfGE 20, 162 (174) - Spiegel.

73 Siehe etwa nur BVerfGE 85, 264 (285 f.) - Parteifinazierung II.

74 Hierzu etwa nur BVerfGE 20, 56 (99) - Parteifinazierung I.

75 Auf diese Unterscheidung im juristischen Kontext der Meinungsbildung greift auch Pille, Meinungsmacht sozialer Netzwerke, S. 37, zurück.

76 So etwa Noelle-Neumann, Öffentlichkeit als Bedrohung, S. 173. Vgl. hierzu auch BVerfGE, 44, 125 (143) - Öffentlichkeitsarbeit, das nicht ausdrücklich von der öffentlichen Meinung spricht, aber „die Entscheidung der Mehrheit bei Ausübung von Staatsgewalt als Wille der Gesamtheit gelten und nach der Idee der freien Selbstbestimmung aller Bürger Verpflichtungskraft für alle [zuspricht]“; vgl. auch BVerfGE 138, 102 (109) - Äußerungen von Regierungsmitgliedern. Ausführlicher zum Begriff der öffentlichen Meinung Kloepfer, in: Isensee/Kirchhof, HbStR III, $\mathbb{} 42$ Rn. $11 \mathrm{ff}$.

77 Ausführlicher zum Begriff von Öffentlichkeit im kommunikativen Zusammenhang etwa Pille, Meinungsmacht sozialer Netzwerke, S. $30 \mathrm{ff}$. 
öffentliche Forum der Antike bzw. die griechische Agora gewährleistet. ${ }^{78}$ Bis heute dient der offene und plurale Meinungsmarkt, der einen „ungehinderten Wettbewerb von Ideen und Meinungen “79 gewährleisten soll, ${ }^{80}$ als Beschreibung dieses Idealbilds. ${ }^{81}$ Auf ihm streiten divergierende Meinungen gegeneinander in einem stetigen Prozess um das bessere Argument. ${ }^{82}$ Auch wenn aus empirischer Sicht divergierende Ansichten darüber bestehen, was Öffentlichkeit eigentlich bedeutet, ${ }^{83}$ so sind zentrale Funktionen dieser unbestritten: Transparenz, Integration, Legitimation, Offenheit und Diskursivität. ${ }^{84}$

Seit der Agora unterliegt die Öffentlichkeit aber einem anhaltenden Wandel, ${ }^{85}$ der zunächst eine gewisse Meinungselite hervorgebracht hat. Durch die Entwicklung der Massenmedien wurde der Bürger dann von einem ursprünglich aktiven Partizipanten zu einem rein passiven Rezipienten. Diese Rolle verändert sich unter dem Einfluss des Internets und sozialer Medien wieder in eine aktivere. ${ }^{86}$ Aber nicht nur die Rolle der Bürger und verschiedener Teilnehmer im Kommunikationsprozess haben sich gewandelt. Auch besteht die Tendenz, dass sich durch einseitigen Medienkonsum, Personalisierungsmaßnahmen der Medienintermediäre im Internet, dezentrale Kommunikationsstrukturen und der Vermischung von

78 Die Agora wird deshalb auch als das Idealbild einer demokratischen Öffentlichkeit bezeichnet, so etwa Zeitelhack, in: Friedrichsen/Kohn, Digitale Politikvermittlung, S. 169 (170); Schliesky, NVwZ 2019, 693 (696) Boehme-Neßler, Das Ende der Demokratie?, S. 42. An diesem Leitbild orientiert sich jedenfalls auch das BVerfG, wenn es von einem „öffentlichen Forum“ spricht und dieses dadurch charakterisiert, „dass auf ihm eine Vielzahl von verschiedenen Tätigkeiten und Anliegen verfolgt werden kann und hierdurch ein vielseitiges und offenes Kommunikationsgeflecht entsteht.“, BVerfGE 128, 226 (253) - Fraport.

79 So Klein, in: Maunz/Dürig, GG, Art. 21 Rn. 485.

80 BVerfGE 44, 125 (139) - Öffentlichkeitsarbeit.

81 So bezeichnend u.a. Andresen, HRN 2017, 9 (10); Mafi-Gudarzi, ZRP 2019, 65; Paal, Medienvielfalt und Wettbewerbsrecht, S. 27; Pille, Meinungsmacht sozialer Netzwerke, S. 45. Der US Supreme Court spricht hinsichtlich des First Amendments (Freedom of speech) von einem "Marketplace of ideas", Red Lion Broadcasting v. FCC, 395 U.S. 367, 390 (1969). In diese Richtung letztlich auch das BVerfG, dass teilweise vom „Wettbewerb der Meinungen“, BVerfGE 25, 256 (268) - Blinkfüer, von einer „geistige[n] Auseinandersetzung, den Kampf der Meinungen“, BVerfGE 7, 198 (208) - Lüth, von einem „geistigen Kampf“, BVerfGE 5, 85 (205) - KPD-Verbot; 12, 113 (125) - Schmid-Spiegel, oder im Kontext der Rundfunkfreiheit von einem „Meinungsmarkt“ spricht, siehe nur BVerfGE 57, 295 (323) - 4. Rundfunkentscheidung. So etwa auch Bullinger, AöR 108 (1983), $161 \mathrm{ff}$.

82 BVerfGE 5, 85 (135) - KPD-Verbot; 7, 198 (208) - Lüth; 20, 56 (98) - Parteifinanzierung I.

83 Vgl. Pille, Meinungsmacht sozialer Netzwerke, S. 30 f. Zu den unterschiedlichen Ansätzen von Öffentlichkeit und der jeweiligen Bedeutung von Meinungsrobotern für sie Keller/Klinger, Political Communication Vol. 36 (2019), 171 (173 ff.).

84 Hindelang, Freiheit und Kommunikation, S. $28 \mathrm{ff}$. Siehe hierzu auch Brinkmann, UFITA 2019, 364 (366 f.); Habermas, Communication Theory 16 (2006), 411 (413) und Drerup, in: Binder/ders., Demokratieerziehung und Bildung digitaler Öffentlichkeit, S. 29 (31 ff.). Besonders zur Integrationsfunktion BVerfGE 8, 104 (113) - Volksbefragung.

85 Grundlegend und ausführlich zu diesem Strukturwandel etwa Habermas, Strukturwandel der Öffentlichkeit. Siehe daneben und überblicksweise Zeitelhack, in: Friedrichsen/Kohn, Digitale Politikvermittlung, S. 169 (170 ff.) und zum digitalen Wandel etwa Drerup, in: Binder/ders., Demokratieerziehung und Bildung digitaler Öffentlichkeit, S. 29 (34 f.).

86 Hegelich, Die Politische Meinung, Nr. 543 (2017), 32 (34), spricht insoweit von einem „disruptiven Umbruch". 
privater und öffentlicher Kommunikation ${ }^{87}$ in sozialen Netzwerken Teilöffentlichkeiten bilden. ${ }^{88}$ Eine (Medien-)Öffentlichkeit lässt sich mittlerweile nur schwer ausmachen. ${ }^{89}$ Dass man sich damit immer weiter vom ursprünglichen Idealbild entfernt, ist offensichtlich. Weil dies aber auch notwendige Begleiterscheinung einer immer heterogeneren und größeren Gesellschaft ist und zumindest mit dem digitalen Wandel mehr Informationen bereitstehen und eine partizipative Öffentlichkeit geschaffen wird, ${ }^{90}$ ist dies nicht per se problematisch. ${ }^{91}$ Vielmehr mag das gerade den Staat dazu anhalten oder gar verpflichten, wesentliche Voraussetzungen der öffentlichen Kommunikation als Grundvoraussetzung der Demokratie abzusichern.

\section{Meso-Ebene}

Auf der Meso-Ebene geht es um die bereits angesprochenen Vermittler zwischen Individuum und Gesellschaft. ${ }^{92}$ Neben den Medien $^{93}$ und Parteien zählen hier auch andere gesellschaftliche Akteure und Verbände zu, ${ }^{44}$ die interessenspezifisch einen Willen bilden und diesen sowohl gegenüber der Gesellschaft, dem Staat als auch dem Einzelnen artikulieren. Nichts anderes gilt auch für die politischen Parteien (vgl. nur Art. 21 Abs. 1 S. 1 GG), ${ }^{95}$ wobei diese noch wesentlich stärker eine Verbindung zwischen dem Volkswillen und dem Staatswillen aufweisen. Sie tragen die Meinungen des Volkes in das Parlament und in die sonstigen staatlichen Organe. ${ }^{96}$

87 Brinkmann, UFITA 2019, 364 (366); Drerup, in: Binder/ders., Demokratieerziehung und Bildung digitaler Öffentlichkeit, S. 29 (36). Zu den verschiedenen Formen von privater und (teil-)öffentlicher Kommunikation ausführlicher Pille, Meinungsmacht sozialer Netzwerke, S. $131 \mathrm{ff}$.

88 Brinkmann, UFITA 2019, 364 (375f.); Gräfe, PinG 2019, 5 (6); Krüger, in: Hooffakker/Kenntemich/ Kulisch, Die neue Öffentlichkeit, S. 9 (15); Quack, in: Friedrichsen/Kohn, Digitale Politikvermittlung, S. 127 (138); Pille, Meinungsmacht sozialer Netzwerke, S. 81 f.; Schaal, in: Bedford-Strohm/Höhne/ Zeyher-Quattlender, Digitaler Strukturwandel der Öffentlichkeit, S. 121 (127); Vesting, in: Unger/v. Ungern-Sternberg, Demokratie und künstliche Intelligenz, S. 33 (45). Mitsch, DVBl. 2019, (812) spricht insoweit von einem „Paradigmenwechsel“.

89 Vgl. Steinbach, JZ 2017, 653 (658). Ob dies allerdings nicht der Normalfall von Öffentlichkeit ist und auch schon in analogen Zeiten verschiedene „Filterblasen“ oder „Echokammern“ existierten, ist jedenfalls nicht unumstritten, hierzu etwa Drerup, in: Binder/ders., Demokratieerziehung und Bildung digitaler Öffentlichkeit, S. 29 (36).

$90 \mathrm{Zu}$ diesen positiven Auswirkungen siehe etwa auch Brinkmann, UFITA 2019, 364 (367).

91 Vgl. Kersten, JuS 2014, 673 (676f.). Zu diesem Spannungsverhältnis zwischen negativen und positiven Auswirkungen mit Blick auf das Prinzip „Öffentlichkeit“ etwa Westphal, in: Binder/Drerup, Demokratieerziehung und die Bildung digitaler Öffentlichkeit, S. 13.

92 Jarren/Donges, Politische Kommunikation, S. 32.

93 Zur Presse etwa BVerfGE 20, 162 (174 f.) - Spiegel.

94 Vgl. BVerfGE 85, 264 (284) - Parteifinanzierung II; 89, 155 (185) - Maastricht.

95 Ähnlich BVerfGE 44, 125 (140) - Öffentlichkeitsarbeit; 85, 264 (284) - Parteifinanzierung II.

96 BVerfGE 20, 56 (99) - Parteifinanzierung $I$. 
III. Mikro-Ebene

Wesentliche Voraussetzung für das Entstehen einer öffentlichen Meinung oder den Erfolg von Vermittlern ist aber der Beitrag jedes Einzelnen. Wenig verwunderlich ist deshalb auch, dass die Mikroebene wesentliches Ziel ganz unterschiedlicher Beeinflussungsversuche ist. Bereits klassische Medien erlangen hier wesentliche Bedeutung, ${ }^{97}$ was auch das BVerfG frühzeitig erkannte ${ }^{98}$. Durch die redaktionelle Aufarbeitung von Themen liefern sie Informationen (Informationsfunktion), geben relevante Themen vor (Agenda-Setting), binden die Themen in einen politischen und sachlichen Kontext ein (Framing) und helfen bei ihrer Bewertung (Priming). ${ }^{99} \mathrm{Um}$ begreifen zu können, warum Medien allgemein und hier eben besonders soziale Netzwerke und die dort agierenden Meinungsroboter die Willensbildung überhaupt beeinflussen können, muss der individuelle Willensbildungsprozess der Nutzer näher betrachtet werden. Wie entsteht also eine Meinung? Und welche Faktoren sind hierfür wesentlich? Ziel ist es freilich nicht, dies vollumfängliche darzustellen, ${ }^{100}$ sondern nur die hierfür relevanten Grundlagen zu skizzieren.

\section{Allgemeines zum Prozess der Meinungsbildung}

Spricht man von Meinung, so ist damit die Einstellung eines Individuums zu einem bestimmten Objekt gemeint. Eine Einstellung weist neben kognitiven auch affektive und verhaltensbedingte Komponenten auf, die sich wechselseitig beeinflussen. ${ }^{101}$ Besonderes Gewicht kommt der affektiven Komponente zu, weil sie durch Emotionen und persönliche Erfahrungen geprägt ist. ${ }^{102}$ Die Reize werden durch das Gehirn verarbeitet und im Idealfall nicht nur im Kurz-, sondern im Langzeitgedächtnis dauerhaft gespeichert und dort zum Abruf bereitgehalten. ${ }^{103}$ Unterschiedliche themenspezifische Reize führen meist zu einer Verbindung der unterschiedlichen Informationen. Je nachdem, ob diese Reize negativ oder positiv

97 Hierzu ausführlicher Kloepfer, in: Isensee/Kirchhof, HbStR III, $\mathbb{} 42$ Rn. 35 ff.; Zippelius, Allgemeine Staatrechtslehre, $\mathbb{2} 8 \mathrm{IV}$; hinsichtlich der Meinungsbildung Neidhardt, in: Krause/Fretwurst/Vogelgesang, FS-Erbringer, 2007, S. 19 (28); Paal/Hennemann, JZ 2017, 641 (642).

98 Allgemein zu den Freiheiten des Art. 5 GG BVerfGE 20, 56 (97) - Parteifinanzierung I; zum Rundfunk siehe nur BVerfGE 12, 205 (260) - 1.Rundfunkentscheidung; zur Presse BVerfGE 20, 162 (174) - Spiegel.

99 Ausführlicher hierzu etwa Jäckel/Fröhlich/Röder, Medienwirkung kompakt, S. 51 ff.

100 Hierzu u.a. Meffert, in: Zmerli/Feldman, Politische Psychologie, S. 85 ff. oder Schoen, Politische Vierteljahresschrift 47 (2006), $89 \mathrm{ff}$.

101 Hierzu ausführlicher Schweiger, Der (des)informierter Bürger, S. 114 f.; Pürer/Eichhorn, in: Pürer, Kommunikationswissenschaft als Sozialwissenschaft, S. 91.

102 Vgl. Meffert, in: Zmerli/Feldman, Politische Psychologie, S. 85 (94). Neue vorhandene Reize aktivieren zunächst auch immer diese affektive Komponente und erst dann andere Kognitionen, Meffert, ebd., S. 98.

103 Ausführlicher hierzu Schoen, Politische Vierteljahresschrift 47 (2006), 89 (90 f.); Meffert, in: Zmerli/ Feldman, Politische Psychologie, S. 85 (87 ff.). 
konnotiert sind, entsteht auch eine entsprechende Voreinstellung. Bereits wenige Reize reichen aus, um auf heuristischen Annahmen beruhende Schlüsse zu ziehen. ${ }^{104}$

Wurden bekannte Informationen und Reize gespeichert und miteinander als Reizmuster verknüpft, kann jeder weitere Reiz dieses Muster reaktivieren und dadurch verfestigen oder widerlegen. ${ }^{105}$ Es findet also ein Wechselspiel zwischen externen Reizen und intern abgespeicherten Informationen statt. ${ }^{106}$ Insoweit handelt es sich sowohl bei Voreinstellungen als auch bei gefestigten Einstellungen nicht um absolute Größen, auch wenn es mit zunehmendem Grad der Verfestigung immer schwieriger wird, diese zu ändern. ${ }^{107}$ Der Grad der Verfestigung oder Änderung einer Meinung hängt aber maßgeblich davon ab, welche Informationen bereitstehen und welche tatsächlich konsumiert und verarbeitet werden. Eine unabdingbare Voraussetzung hierfür ist eine Aktivität in zweierlei Sinne: Einerseits muss überhaupt ein themenspezifisches Informationsinteresse bestehen und zweitens muss dann auch aktiv nach Informationen gesucht und diese konsumiert werden. ${ }^{108}$

\section{Informationsaufnahme und -verarbeitung als rationaler Vorgang?}

Das wirft jedenfalls dann keine wesentlichen Probleme auf, wenn man von einem rationalen Vorgang ausgeht, also den Informationsvorgang von der Aufnahme bis hin zur Verarbeitung als eine an rationalen und objektiven Kriterien orientierte Auswahlentscheidung versteht. Dieses Verständnis lag etwa dem verhaltensökonomischen Verbraucherbild des Homo oeconomicus zugrunde, anhand dessen versucht wurde, die wirtschaftliche Entscheidungsfindung von Verbrauchern zu beschreiben. Danach weist der homo oeconomicus drei zentrale Charakteristika auf: Rationalität, Informiertheit und Egoismus. ${ }^{109}$ Der Verbraucher treffe allein anhand wirtschaftlicher, rationaler Gesichtspunkte die Entscheidung, strebe dabei stets nach dem größtmöglichen Nutzen für sich selbst, informiere sich vollumfänglich und agiere in Kenntnis der wirtschaftlichen Entscheidungsmöglichkeiten und deren Folgen. ${ }^{110}$ Was im Ausgangspunkt so plausibel klang, ${ }^{111}$ wurde

104 Schoen, Politische Vierteljahresschrift 47 (2006), 89 (92 f.). Zur Bedeutung von Heuristik bei der Informationsverarbeitung Schweiger, Der (des)informierter Bürger, S. $74 \mathrm{ff}$.

105 Meffert, in: Zmerli/Feldman, Politische Psychologie, S. 85 (87).

106 Ähnlich Meffert, in: Zmerli/Feldman, Politische Psychologie, S. 85 (86).

107 Pürer/Eichhorn, in: Pürer, Kommunikationswissenschaft als Sozialwissenschaft, S. 97.

108 Schoen, Politische Vierteljahresschrift 47 (2006), 89 (92 f.).

109 H. Beck, Behavioral Economics, S. 2; Achilles, Vom Homo Oeconomicus zum Differenzierten Verbraucher, S. $64 \mathrm{f}$.

110 Hierzu Achilles, Vom Homo Oeconomicus zum Differenzierten Verbraucher, S. 63 ff.; Eidenmüller, JZ 2011, 814 (816); Weber/Schäfer, Staat 56 (2017), 561 (564 f.); Beck, Behavioral Economics, S. 1.

111 Auch wenn Kritik berechtigt ist, zeigt H. Beck, Behavioral Economics, S. $1 \mathrm{ff}$., dass ein solches Modell als Ausgangspunkt dennoch seine Berechtigung hat. 
aber immer weiter dadurch in Zweifel gezogen, ${ }^{112}$ dass tatsächlich getroffene Entscheidungen von dem theoretischen Leitbild abwichen und so nicht plausibel erklärt werden konnten. Vielmehr kommt es zu Verzerrungen, die die Entscheidungsfindung beeinflussen und $\mathrm{zu}$ abweichenden Ergebnissen führen und die letztlich auf die nur beschränkte kognitive Verarbeitungskapazität zurückgeführt werden können (sog. bounded rationality). ${ }^{113}$

Entsprechende Verzerrungen sind schon Resultat der menschlichen Denkweise. Nach der Dual-Process-Theorie existieren zwei verschiedene Denksysteme. ${ }^{114}$ System I arbeitet unterbewusst und automatisch und ist für schnell zu treffende, unterbewusste Entscheidungen zuständig. System II hingegen ist für komplexe Entscheidungen zuständig; Denkprozesse finden hier bewusste und konzentriert statt. Wegen der begrenzten kognitiven Ressourcen kommt aber dem System I wesentliche Bedeutung für die Verarbeitung und -Aufnahme neuer Informationen insbesondere dort zu, wo eine Entscheidungsfindung nicht als wichtig erachtet wird oder in Bereichen, in denen man eine grundlegende Kenntnis über das Entscheidungsob- bzw. -subjekt besitzt. ${ }^{115}$ Das System II kann zwar Entscheidungen in System I überprüfen und verändern. Weil aber überwiegend nur System I angesprochen wird, kommt es dieser Aufgabe nur unzureichend nach. ${ }^{116}$ Problematisch hieran ist, dass Entscheidungen, die innerhalb des ersten Systems getroffen werden, anfälliger für Verzerrungen oder Fehlschlüsse sind, was u.a. schon daran liegt, dass im System I auf Grundlage heuristischer Annahmen schnelle Schlüsse getroffen werden. ${ }^{117}$ Komplexere Entscheidungsfindungen werden dadurch auf einfache „Daumenregeln“118 herunter gebrochen, ${ }^{119}$ die nicht immer zu den richtigen Schlüssen führen. Diese Heuristiken und andere Verzerrungen konterkarieren insbesondere die Rationalitätserwartungen einer Entscheidung. So werden Wahrscheinlichkeiten über- oder unterschätzt (bspw. Hindsight-Bias, Verfügbarkeitsheuristik), oder Entscheidungen in Abhängigkeit von der Präsentation einer Informationen getroffen (Framing-Effekt, Ankereffekt).

112 Allgemeinere Kritik findet sich etwa bei Achilles, Vom Homo Oeconomicus zum Differenzierten Verbraucher, S. 67.

113 Ebers, MMR 2018, 423 (434); vgl. Hill, DÖV 2020, 205.

114 Vgl. hierzu Meffert, in: Zmerli/Feldman, Politische Psychologie, S. 85 (88 f.). Ausführlicher zur DualProcess-Theorie etwa S. Wagner, in: Greve u.a., Der digitalisierte Staat, S. 17 ff. m.w.N.; ders., RW 2020, 344 (345 ff.) m.w.N.

115 Vgl. Meffert, in: Zmerli/Feldman, Politische Psychologie, S. 85 (87f.). Zur situations- und motivationsabhängigen Tiefe und Umfang etwa der kritischen Rezeption von Inhalten Metzger u.a., Journal of Communication Vol. 60 (2010), 413 (416f.).

116 S. Wagner, in: Greve u.a., Greve u.a., Der digitalisierte Staat, S. 15 (16); ders., RW 2020, 344 (345).

117 S. Wagner, a.a.O., S. 18 f.; ders., RW 2020, 344 (346).

118 Eidenmüller, JZ 2011, 814 (816); H. Beck, Behavioral Economics, S. 26.

119 H. Beck, ebd. 


\section{Bedeutung für die politische Willensbildung}

Inwieweit die verhaltensökonomischen Befunde auch auf nicht rein wirtschaftliche Entscheidungen übertragbar sind, ist zwar noch nicht hinreichend geklärt. ${ }^{120}$ Vieles spricht gleichwohl dafür, dass der Einzelne auch hier anfällig für Verzerrungen ist. ${ }^{121}$

\section{a. Relevanz externer Faktoren}

Wenn und weil Informationen zentral für die politische Willensbildung sind, kommt vor allem den Medien ein maßgeblicher Einfluss auf die Willensbildung zu: ${ }^{122}$ Sie liefern dem Einzelnen nicht nur Informationen, sondern nehmen kritische und bewertende Analysen vor und legen so die zentralen, öffentlich relevanten Themen fest. Sie bieten die Grundlage dafür, sich eine fundierte und faktenbasierte Meinung zu bilden. Vor allem auch durch die Vorgabe der Themen-Agenda ${ }^{123}$ (Agenda-setting) haben sie Einfluss auf die interpersonale Kommunikation. ${ }^{124}$ Dabei kann besonders die Art der Präsentation und die Einbindung der Information ihre Verarbeitung und die Meinungsbildung beeinflussen (Framing, Priming). ${ }^{125}$ Daneben führt auch die Kommunikation mit anderen zu einem gewissen Informationsaustausch. Hierbei unterwirft man die eigene Meinung einer kritischen Überprüfung, infolge derer die eigene Meinung gestärkt oder geschwächt wird oder sich verändern kann. ${ }^{126}$ Dieser personale Kontakt kann wirkungsvoller sein als der Einfluss der Massen-Medien. ${ }^{127}$ Wie stark der Einfluss ist, hängt dabei vom Einzelfall ab. So können sog. Opinion Leader,

120 Drexl, ZUM 2017, 529 (534). Vgl. zu einer solchen Übertragung etwa auch Volkmann, in: Unger/v. Ungern-Sternberg, Demokratie und künstliche Intelligenz, S. 51 (57).

121 In diese Richtung auch Fehling/Leymann, AfP 2020, 110 Rn. 5, 7, die auch darauf hinweisen, dass das BVerfG in seiner Entscheidung zum Rundfunkbeitrag, BVerfGE 149, 222 insbeS. Rn. 79 f., hiervon ausging, wenn es gerade die Notwendigkeit eines professionellen Vorfilters betone.

122 Hiermit beschäftigen sich die Kommunikationswissenschaften deutlich umfangreicher, so etwa Jäckel, Medienwirkungen kompakt oder Schweiger/Fahr, Handbuch Medienwirkungsforschung.

123 Welche Themen aufbereitet werden, hängt stark vom Nachrichtenwert ab (sog. Nachrichtenwerttheorie, hierzu ausführlich Sommer u.a., Publizistik 57 (2012), 381 (382ff.)). Dieser ergibt sich neben der Aktualität u.a. auch aus dem Grad der Überraschung, der Personenbezogenheit, der politischen Kontroverse, dem Schaden und dem Nutzen eines BeitragS. Themen, die neben der Aktualität mehrere Faktoren erfüllen gelangen eher in die Medien und werden dadurch auch häufiger Gegenstand interpersonaler Kommunikation. Wenn man so will, spiegelt sich in der Orientierung am Nachrichtenwert gerade die journalistisch-redaktionelle Arbeit und Sorgfalt wider, vgl. Beater, NJ 2019, 365 (368 f.) der als Voraussetzung journalistisch-redaktioneller Gestaltung gerade die Auswahl nach der gesellschaftlichen Relevanz begreift.

$124 \mathrm{Zu}$ diesen Funktionen ausführlicher Schmidt, Social Media, S. $62 \mathrm{ff}$.

125 K. Beck, Kommunikationswissenschaften, S. 187, 215; Jäckel/Fröhlich/Röder, Medienwirkungen kompakt, S. $56 \mathrm{f}$.

126 Vgl. BVerfGE 27, 71 (82) - Leipziger Volkszeitung.

127 Vgl. Katz/Lazarsfeld, Personal Influence, S. 32 f., 40; als wichtige Rolle bezeichnend Scheufele, in: Zerfaß/Piwinger, Hb Unternehmenskommunikation, S. 105 (130). Ausführlicher hierzu Campus, EJoC Vol. 27, 46 (46 ff.). 
Personen, die überdurchschnittlich häufig Medien nutzen und ihr Umfeld so mit Informationen versorgen, einen potentiell größeren kommunikativen Einfluss haben als andere. ${ }^{128}$ Sowohl von der Präsentation von Informationen als auch von der Kommunikation mit anderen gehen zudem persuasive Effekte aus. Die Stärke hängt dabei von Merkmalen des Kommunikators, der medialen Botschaft und des Rezipienten ab. ${ }^{129}$ Neben dem strukturellen Aufbau einer Botschaft, der Frage der argumentativen Sichtweise, ist die Kompetenz, die Vertrauenswürdigkeit, die Attraktivität und die Ähnlichkeit zum Rezipienten entscheidend. ${ }^{130}$

Aber nicht nur für die Informationsverarbeitung und eigentliche Meinungsbildung werden externe Faktoren relevant, sondern auch für die Meinungsäußerung. Man befindet sich mit seinen wahrgenommenen Reizen und den gebildeten Einstellungen nicht in einem Vakuum, sondern ist in ein soziales Gerüst aus Regeln und Konventionen eingebunden. Sich gegen diese zu erheben, kann zu einer sozialen Isolation führen. Deshalb können derlei gemeinsam geteilte Regeln einer Gruppe unmerklich die eigenen Entscheidungen beeinflussen. Auch ohne dass erheblicher Druck seitens einer Gruppe besteht, versucht man sich konform zu verhalten, um nicht aus der Gruppe ausgeschlossen zu werden. ${ }^{131}$ Mit derlei Verhaltensanpassungen kann auch eine Anpassung der Einstellung einhergehen. Das Mehrheitsverhalten ist die Leitlinie, eine Daumenregel, die in unklaren Situationen bedeutsam wird. Diesen Zusammenhang zwischen Makround Mikro-Ebene versucht auch die Theorie der Schweigespirale zu erklären. ${ }^{132}$ Nach ihr sind Personen, die die Mehrheit der Bevölkerung hinter sich zu wissen glauben, eher bereit, ihre Meinung öffentlich zu äußern, als diejenigen, die sich in einer Minderheitsposition wähnen. Menschen seien in der Lage die öffentliche Meinung genau zu erfassen und wüssten, wann sie eine abweichende und in Bezug auf bestimmte Themen „unpassende“ Meinung haben. Aus Angst vor sozialer Isolation und Stigmatisierung werden sie eher schweigen, als ihre Meinung zu äußern. Diese Wirkung kann sowohl von der Makro-Ebene insgesamt oder nur von Teilen, wie etwa den Medien oder dem sozialen Umfeld, ausgehen.

128 Vgl. Katz/Lazarsfeld, Personal Influence, S. 32 f., 44 f., 82, ausgehend hiervon siehe auch Gertler, in: Friedrichsen/Kohn, Digitale Politikvermittlung, S. 83 (87f.).

129 Ähnlich Pürer/Eichhorn, in: Pürer, Kommunikationswissenschaft als Sozialwissenschaft, S. 99.

130 Vgl. Pürer/Eichhorn, in: Pürer, Kommunikationswissenschaft als Sozialwissenschaft, S. 99 f.

131 Ash konnte mit seinem Experiment - eine Testperson sollte in einer Gruppe mit anderen instruierten Personen die Längste dreier Linien bestimmen und beugte sich letztlich der falschen Ansicht der instruierten Personen - diesen Hang zur Konformität empirisch belegen, vgl. hierzu ausführlicher Stroebe/Hewstone/Jonas, in: dies., Sozialpsychologie, S. 1 (8). Dieses Phänomen kann aber auch mit dem Prinzip des Social Proofs, der sozialen Bewährtheit, erklärt werden. Dabei handelt es sich um eine heuristische Annahme in unklaren Situationen: Das Verhalten, das Viele in einer bestimmten Situation zeigen, wird als richtig unterstellt und sich diesem angepasst. Hierzu Graber/Lindemann, in: Sachs-Homburg/Zywietz, Fake News, Hashtags \& Social Bots, S. 51 (59 ff.).

132 Ausführlich hierzu Noelle-Neumann, Die Schweigespirale: öffentliche Meinung - unsere soziale Haut. Ihre Theorie hat international viel Anerkennung gefunden, wurde aber auch oftmals kritisiert, hierzu u.a. K. Beck, Kommunikationswissenschaften, S. 127 f.; zu den empirischen Studien und ihrer Diskussion Pürer, in: ders., Kommunikationswissenschaften als Sozialwissenschaften, S. $126 \mathrm{ff}$. 
Die öffentliche Meinung fungiere so als eine „soziale Haut“133. Ihre Wirkung entfaltet sie vor allem dort, wo es kein richtig und falsch gibt. ${ }^{134}$ Damit wird die Schweigespirale also vor allem auch bei (rechts-)politischen Fragen relevant.

Obwohl man denken könnte, dass das Internet bzw. soziale Netzwerke eine befreiende Wirkung auf die Äußerungsbereitschaft haben, weil hier u.a. die faktische Anonymität einen zumindest gefühlten Schutz bietet, ${ }^{135}$ zeigen Studien auch ein anderes Bild auf. Die Äußerungsbereitschaft bei Diskussionen in sozialen Netzwerken folgt scheinbar keinen anderen Regeln. Auch hier kann die Schweigespirale ihre Wirkung entfalten ${ }^{136}$ und kann tendenziell sogar stärker als in Offline-Diskussionen sein, ${ }^{137}$ was auch an den zusätzlichen (unkalkulierbaren) Risiken liegt, die in der Online-Umgebung bestehen. ${ }^{138}$

\section{b. Individuelle Verzerrungsfaktoren und Tendenzen}

Neben den externen Faktoren werden aber vor allem interne Verzerrungen bei der Informationsaufnahme und -Verarbeitung relevant, von denen einige ausgewählte und im vorliegenden Kontext bedeutsame angesprochen werden sollen. ${ }^{139}$ Wegen der Unmengen an Informationen und der nur geringen Zeit, diese zu verarbeiten, setzen Menschen auf methodische, logische Schlüsse, um bestimmte Probleme mit vergleichsweise geringem Aufwand zu bewältigen und zu praktikablen Ergebnissen zu gelangen, sogenannte Heuristiken. ${ }^{140}$ Dem oben erwähnten Denksystem I kommt deshalb eine wesentliche Funktion zu, wenn es um die Bewertung der Relevanz als Kriterium der Auswahlentscheidung von Informationen geht. Während in Offline-Medien Redaktionen die Auswahl interessanter Beiträge vornehmen, obliegt es in sozialen Netzwerken den Nutzern, aus den

133 So schon die Bezeichnung von Noelle-Neumann, Die Schweigespirale: öffentliche Meinung - unsere soziale Haut.

134 Umfassend zur Theorie der Schweigespirale Noelle-Neumann, ebd.

135 Vgl. Hampton u.a., Social Media and the ,Spiral of Silence', S. 3. Ausführlicher zu den Umständen, die die Effekte theoretisch schwächen könnten Matthes/Knoll/v. Sikorski, Communication Research Vol. 45, 3 (7). Siehe auch Schweiger, Der (des)informierter Bürger, S. $61 \mathrm{ff}$.

136 Siehe hierzu etwa Neubaum/Krämer, Communication Research 45 (2018), 139 (157f.); Hampton u.a., Social Media and the ,Spiral of Silence', S. 3; Matthes/Knoll/v. Sikorski, Communication Research Vol. 45, 3 ff. und ergänzend auch Schweiger, Der (des)informierter Bürger, S. 126 f., m.w.N.

137 Hierzu Hampton u.a., Social Media and the ,Spiral of Silence', S. 4; Matthes/Knoll/v. Sikorski, Communication Research Vol. 45, 3 (22 f.).

138 Hierzu gehört neben der unbegrenzten Reichweite vor allem das Problem der fehlenden Kontrolle über die Auswirkungen einer Äußerung, siehe hierzu ausführlich Neubaum/Krämer, Communication Research 45 (2018), 139 (158ff.). Andeutend auch Hampton u.a., Social Media and the ,Spiral of Silence', S. 8.

139 Ausführlicher zu den Verzerrungstendenzen etwa Weber/Knorr, in: Appel, Psychologie des Postfaktischen, S. $103 \mathrm{ff}$.

140 Meffert, in: Zmerli/Feldman, Politische Psychologie, S. 85 (93). Metzger u.a., Journal of Communication Vol. 60 (2010), 413 (417). 
angezeigten Inhalten relevante herauszufiltern. ${ }^{141}$ Dabei wird oftmals der „sozialen Bewährtheit“ bzw. der „Schwarmintelligenz“ gefolgt: In unübersichtlichen Situationen neigt man eher dazu, dem Urteil oder dem Verhalten anderer zu folgen. ${ }^{142}$ In Teilgruppen, in denen Zugehörige vergleichbare ethnische, religiöse oder politische Merkmale aufweisen, bestehen zudem stärkere Orientierungsund Mitläufereffekte als bei heterogeneren Teilgruppen. ${ }^{143}$ In sozialen Netzwerken übernehmen diese Orientierungsfunktion für die Bewertung der Kredibilität und Relevanz von Inhalten die statistischen Daten (Likes, Abonnements etc.) oder Kommentare anderer Nutzer. ${ }^{144}$

Daneben neigen Menschen aber auch dazu, eher meinungskonforme Informationen auszuwählen und zu verarbeiten (sog. selective exposure bzw. confirmation bias). ${ }^{145}$ Dadurch vermeiden sie letztlich auch das Entstehen kognitiver Dissonanzen, einen inneren störenden Zustand zwischen eigener Einstellung und tatsächlichem Verhalten. ${ }^{146}$ Teilweise tendiert man gar dazu, sich erst eine Meinung zurechtzulegen und dann nach entsprechenden Informationen zu suchen. ${ }^{147}$ Und meinungskonforme Argumente werden in der Glaubwürdigkeit und Überzeugungskraft stärker eingeschätzt. ${ }^{148}$

Aber auch mit Blick auf die kognitive Informationsverarbeitung bestehen Verzerrungstendenzen. Das gilt etwa dort, wo es um die Bewertung der Glaubwürdigkeit oder Relevanz bestimmter Informationen geht. Je häufiger ein Nutzer mit einer bestimmten Information konfrontiert wird, desto höher wird der Wahrheitsgehalt und dementsprechend die Relevanz eingeschätzt (Illusory-Truth-

141 So auch BVerfGE 149, 222 Rn. 80 - Rundfunkbeitrag. Zur dadurch bedingten Anfälligkeit für Manipulationen siehe etwa Thieltges/Hegelich, ZfP 64 (2017), 493 (498).

142 Hierzu Graber/Lindemann, in: Sachs-Hombach/Zywietz, Fake News, Hashtags \&Social Bots, S. 51 (60); Ripperger/Appel, in: Appel, Psychologie des Postfaktischen, S. 167 (169). Vgl. zur „Wisdom of crowds“ auch Webster, Northwestern Law Review 104 (2010), 593 (606f.), m.w.N. Dies konnten etwa Metzger/Flanagin/Medders, Journal of Communication Vol. 60 (2010), 413 (420 f.) in einer Studie bestätigen.

143 Weber/Knorr, in: Appel, Psychologie des Postfaktischen, S. 103 (108).

144 Steinebach u.a., Desinformation aufdecken und wirksam bekämpfen, S. 79. Im Zusammenhang mit dem Influencer-Marketing und mit Blick auf die Kommentare Lettmann, GRUR 2018, 1206 (1209). Maschewski/Nosthoff, in: Mühlhoff/Breljak/Slaby, Affekt, Macht, Netz, S. 55 (69) meinen etwa, dass schon der News Feed von Facebook selbst eben diese Schwarmintelligenz adressiert.

145 Schweiger, Der (des)informierter Bürger, S. 96, 130; Drexl, ZUM 2017, 529 (534); Weber/Knorr, in: Appel, Psychologie des Postfaktischen, S. 103 (105 f.); Boehme-Neßler, Das Ende der Demokratie, S. 36 f. Aus empirischer Sicht etwa Zollo u.a., PLoS ONE 12 (7): e0181821, S. 8 und in diese Richtung auch Metzger/Flanagin/Medders, Journal of Communication Vol. 60 (2010), 413 (423 f.).

146 So auch Schmidt, Social Media, S. 67. Pariser, Filterbubble, S. 88: „Confirmation Bias is a conservative mental Force helping to shore up our schemata against erosion." Zur Informationsaufnahme siehe etwa Drexl, ZUM 2017, 529 (534) oder Weber/Knorr, in: Appel, Psychologie des Postfaktischen, S. 103 (106).

147 Drexl, ZUM 2017, 529 (534); Weber/Knorr, in: Appel, Psychologie des Postfaktischen, S. 103 (105). Vgl. auch Meffert, in: Zmerli/Feldman, Politische Psychologie, S. 85 (97).

148 Weber/Knorr, in: Appel, Psychologie des Postfaktischen, S. 103 (106); Meffert, in: Zmerli/Feldman, Politische Psychologie, S. 85 (98). 
Effect). ${ }^{149}$ Gleichzeitig führt diese Art der Wiederholung zum Eindruck einer Vertrautheit mit dem Thema, was zu einer Überbewertung des eigenen Wissenstandes führen kann. ${ }^{150}$ Daneben sind Menschen auch empfänglicher für Informationen, die auf einer emotionalen Ebene wirken. ${ }^{151}$ So bleiben negative Informationen eher im Gedächtnis als positive (Negativity Bias), ${ }^{152}$ oder werden höher gewichtet. ${ }^{153}$ Und auch die Art und Weise der Präsentation kann Einfluss auf die Bewertung von Inhalten haben. In einer Informationsreihenfolge wird etwa den ersten Informationen mehr Bedeutung beigemessen oder mehr Aufmerksamkeit zu teil (Primacy Effect).

\section{c. Herausforderungen und Veränderungen der Meinungsbildung im digitalen Zeitalter}

Diese Verzerrungstendenzen bei der Informationsaufnahme und -Verarbeitung bestehen zwar immer und sind keine Besonderheit der digitalen Welt. ${ }^{154}$ Gleichwohl führt der digitale Wandel zu Problemen, der unmittelbare Auswirkungen auch für die Meinungsbildung und die Wirkungsweise der Verzerrungstendenzen haben. Ausgangspunkt hierfür ist vor allem auch ein struktureller Medienwandel. Nicht nur wegen der starken Konkurrenz und der „Kostenlos-Kultur“155 im Internet stieg der (wirtschaftliche) Druck auf herkömmliche Medien. ${ }^{156}$ Wesentlich für die Medienwirkung und damit die Erfüllung der den Medien zugewiesenen Funktionen ist das Vertrauen in sie und ihre objektive, unabhängige Arbeit. ${ }^{157}$ In jüngerer Vergangenheit ist dieses aber zurückgegangen. ${ }^{158}$ Der Begriff

149 Weber/Knorr, in: Appel, Psychologie des Postfaktischen, S. 103 (106). Zur Wirkung von Fake News etwa Lazer u.a., Science Vol. 359 (2018), 1094 (1095) und Steinebach u.a., Desinformation aufdecken und wirksam bekämpfen, S. 80 .

150 Vgl. Schäfer, Computers in Human Behavior 103 (2020), 1 (2).

151 Meffert, in: Zmerli/Feldman, Politische Psychologie, S. 85 (94f.). Das mag auch daran liegen, dass externe Reize primär vor allem die bereits bestehenden affektiven Reize aktivieren und erst dann kognitive hinzutreten, vgl. Meffert, ebd., S. 98.

152 Weber/Knorr, in: Appel, Psychologie des Postfaktischen, S. 103 (107).

153 Meffert, in: Zmerli/Feldman, Politische Psychologie, S. 85 (91).

154 Von menschlich sprechend Weber/Knorr, in: Appel, Psychologie des Postfaktischen, S. 103 (113).

155 Ausführlicher hierzu etwa Pille, Meinungsmacht sozialer Netzwerke, S. 62.

156 Hierzu umfassender Schweiger, Der (des)informierter Bürger, S. 14 ff. Zu dieser Entwicklung auch Brinkmann, UFITA 2019, 364 (371).

$157 \mathrm{Zu}$ diesem Vertrauen ausführlich Kohring, in: Akademie für Technikfolgenabschätzung B-W, Arbeitsberichte, Nr. 196, S. 9 ff. und Gertler, in: Friedrichsen/Kohn, Digitale Politikvermittlung, S. 83 (90). Vgl. auch Quandt, EJoC 27 (2012), 7 (8 f., 12) und Coleman, EJoC 27 (2012), 35 f. Wohl gemerkt: es handelt sich freilich nicht um eine absolute Objektivität der Berichterstattung, weil eine solche gar nicht zu leisten ist. Insoweit ist das Gefühl von objektiven Medien schon von vornherein ein subjektives, vgl. hierzu Schweiger, Der (des)informierter Bürger, S. 12 f. Zu dem Wirkungszusammenhang von geringem Vertrauen und geringer Nutzung herkömmlicher Medien und der daraus entstehenden „Spiralwirkung“ Schweiger, a.a.O., S. 111. Allgemein zum Vertrauen als wesentliche Voraussetzung der Demokratie Boehme-Neßler, NVwZ 2017, 528 (530 f.).

1582015 führte Infratest Dimap eine Umfrage durch, bei der 37\% der Befragten angaben, dass ihr Vertrauen in die Medien jedenfalls gesunken sei. $42 \%$ gaben an, dass sie die Informationen in den deut- 
der Lügenpresse ist hierfür symptomatisch. ${ }^{159}$ Das gilt zwar nicht allgemein, sondern nur für Teile der Bevölkerung. ${ }^{160}$ Gleichwohl handelt es sich hierbei nicht um eine numerisch zu vernachlässigende Größe. ${ }^{161}$ Weil aber für die Funktion der Medien das ihnen entgegengebrachte Vertrauen entscheidend ist, stellen sich neue Probleme: ${ }^{162}$ Dort, wo das Vertrauen in herkömmliche Medien zurückgeht, entsteht Raum für (pseudo-journalistische) Alternativen. ${ }^{163}$ Die digitalen Vermittlungsalternativen verändern aber schon die Art der Informationsweitergabe, die mehr einer ökonomischen denn journalistischen Rationalität folgt. ${ }^{164}$ Zudem ermöglichen insbesondere soziale Netzwerke einen schnellen Einstieg in den virtuellen Meinungsmarkt. ${ }^{165}$ Die Einrichtung alternativer Medienangebote ist so wenig aufwendig. Bei diesen Alternativen ist aber die Einhaltung der - wie es nun

schen Medien für nicht glaubwürdig halten, lediglich $52 \%$ waren anderer Meinung, Infratest dimap, Glaubwürdigkeit der Medien, 2015. Aktuellere Zahlen aus 2018 belegen zwar, dass das Vertrauen wieder gestiegen ist $-65 \%$ halten die Informationen für glaubwürdig, ein Zuwachs um $13 \%$, Infratest dimap, Glaubwürdigkeit der Medien, 2018. Allerdings kann das auch daran liegen, dass zur Zeit der Umfrage die politische Lage ruhiger war als 2015. Siehe auch Golding/Sousa/van Zonen, EJoC 27 (2012), 3 (4) für den EU-weiten Vertrauensverlust. Schweiger, Der (des)informierter Bürger, S. 4 spricht auch von Medienverdrossenheit.

159 Ähnlich Hagen, ComSoc 48 (2015), 152 (158). Dem Begriff liegt meist eine verschwörungstheoretische Annahme zugrunde: Medien seien nicht mehr neutral-objektiv, sondern ergreifen Partei zugunsten der Regierenden (hierzu Zoll, ComSoc 48 (2015), 126 (127)), des „Establishments - so der Begriff, der im US-amerikanischen Wahlkampf verwendet wurde. Meist handelt es sich hierbei um ein propagandistisches Mittel, um Desinformation zu fördern, Ungewissheit heraufzubeschwören und sich letztlich selbst als Verfechter der Wahrheit zu präsentieren, obwohl nur alternative Begründungsansätze und Argumente gegeben werden, die einer wahren Tatsachengrundlage entbehren (so Zoll, ComSoc 48 (2015), 126 (127)). Insoweit geht es meist nicht um eine berechtigte Kritik an der Auswahl oder Präsentation von Nachrichten, sondern um die Diskreditierung der Medien und die fundamentale Leugnung ihrer Neutralität.

160 Das gilt vor allem für Personen in Milieus, „die mit der Demokratie und den herrschenden Verhältnissen unzufrieden sind, wirtschaftliche Zukunftsangst haben und populistische Einstellungen vertreten " und damit insbesondere bei rechtsgerichteten Personen, so Prochazka/Schweiger, Media Perspektiven 2020, 196 m.w.N.

161 Schmidt, in: Steinbrecher/Rager, Wenn Maschinen Meinung machen, S. 102 (111), weist beispielsweise in diesem Kontext daraufhin, dass entsprechende Personen falsche Schlüsse aus der Verbreitung dieser Meinung ziehen.

162 Andeutend auch Quandt, EJoC 27 (2012), 7 (18). Freilich gab es schon immer Glaubwürdigkeitskrisen, die die Medien oder vereinzelte Medienunternehmen zu überwinden hatten. Dernbach, in: dies./ Meyer, Vertrauen und Glaubwürdigkeit, S. 135 (136f.) zeigt hierfür einige historische Beispiele auf. Aus jüngster Zeit kann hier wohl die Krise des Magazins Spiegel angeführt werden. Ein ehemaliger Journalist, Claas Relotius, hatte über einen längeren Zeitraum Beiträge verfasst, denen ein reales Thema zugrunde liegt, die aber nicht auf Fakten beruhten, sondern viel eher im Wege einer „freien Dichtung “ entstanden sind. Zum Fall Relotius Fichtner, Manipulation durch Reporter, Spiegel-Online v. 19.12.2018.

163 Teilweise wird auch die veränderte Mediennutzung und die Nutzung von alternativen Medienangeboten jedenfalls als potentiell kausal für das sinkende Vertrauen in die Massenmedien gehalten, in diese Richtung etwa Prochazka/Schweiger, Media Perspektiven 2020, 196 m.w.N.

164 Vgl. Ladeur/Gostomzyk, K\&R 2018, 686 (687); Ingold, in: Unger/v. Ungern-Sternberg, Demokratie und künstliche Intelligenz, S. 183 (187) oder Maschewski/Nosthoff, in: Mühlhoff/Breljak/Slaby, Affekt, Macht, Netz, S. 55 (72).

165 Vgl. Ingold, Staat 56 (2017), 491 (507). Schliesky, NVwZ 2019, 693 (697) sieht dadurch letztlich auch eine neue Anfälligkeit für die öffentliche Meinung gegeben, weil dadurch FakeNews und ,andere Abartigkeiten“ in den Meinungsbildungsprozess gelangen. 
\6 Abs. 1 S. 1 MStV so treffend formuliert - ,anerkannten journalistischen Grundsätze“, insbesondere der Sachlichkeit (\$6 Abs. 1 S. 2 MStV), Wahrheit und journalistischen Sorgfalt ( $\mathbb{S} 6$ Abs. 1 S. $3 \mathrm{MStV}$ ), nicht in einem vergleichbaren Umfang gewährleistet. ${ }^{166}$

Parallel wird die Online Nutzung immer wichtiger. Die Dienste nehmen eine immer größer werdende Rolle im Alltag, insbesondere auch bei der Informationsbeschaffung, ${ }^{167}$ ein, ${ }^{168}$ auch wenn das Vertrauen in die Informationen deutlich geringer als bei herkömmlichen Medien ist. ${ }^{169}$ Diese Entwicklung wird aber durchaus kritisch betrachtet. ${ }^{170}$ Neben der personalisierten Vermittlungsstruktur und dem Rückgriff auf ökonomische Verwertungslogiken, die die Ausrichtung an journalistischen Qualitätskriterien substituieren, ${ }^{171}$ liegt das vor allem auch daran, dass die digitalen Angebote die Art und Weise der Informationsaufnahme verändern. Weil das Internet und auch soziale Netzwerke Unmengen an Beiträgen und Informationen bereithalten, ist es nicht mehr möglich, diese Masse sinnvoll zu bearbeiten. ${ }^{172}$ Dort, wo Redaktionen Wichtiges von Unwichtigem getrennt haben, steht nun der Nutzer. ${ }^{173}$ Wegen der nur begrenzten kognitiven Kapazitäten ${ }^{174}$ kommt der Selektion wesentlich mehr Bedeutung zu. ${ }^{175}$ Insgesamt geht es um eine Kosten-Nutzen-Rechnung: mit möglichst geringem Aufwand

166 Diesen Vergleich stellen auch Paal/Hennemann, JZ 2017, 641 f. an. Auf die fehlende Qualitätssicherung weist auch Schliesky, NVwZ 2019, 693 (697) hin; siehe hierzu auch Ungern-Sternberg, in: Unger/Dies., Demokratie und künstliche Intelligenz, S. 3 (8) oder Brinkmann, UFITA 2019, 364 (372). Siehe daneben auch Bodring, Hass im Netz, S. 125 f. Allgemeiner zum Internet Holznagel, NordÖR 2011, 205 (209).

167 Die Nutzung sozialer Medien für Nachrichtenzwecke ist seit 2013 von 18\% auf 31\% im Jahr 2018 gestiegen. Zeitgleich hat sich der Konsum von Printmedien fast halbiert, hierzu Hölig/Hasebrink, in: Reuters Institute, Digital News Report 2018, S. 81.

168 Hierauf ebenfalls hinweisend Ungern-Sternberg, in: Unger/Dies., Demokratie und künstliche Intelligenz, S. $3(4,8)$.

169 Siehe auch hierzu Hölig/Hasebrink, in: Reuters Institute, Digital News Report 2018, S. 81.

170 Vgl. Mitsch, DVBl. 2019, 811 (812). Diesen Wandel skizzieren u.a. auch Brinkmann, UFITA 2019, 364 (366); Pille, Meinungsmacht sozialer Netzwerke, S. 80 ff. und Gorwa/Guilbeault, Policy \& Internet $12(2020), 225$ (226).

171 Dogruel u.a., Medien Perspektiven 2020, 139 (140); Ingold, in: Unger/v. Ungern-Sternberg, Demokratie und künstliche Intelligenz, S. 183 (190); Liesem, AfP 2020, 277 Rn. 3. Ähnlich auch Maschewski/Nosthoff, in: Mühlhoff/Breljak/Slaby, Affekt, Macht, Netz, S. 55 (72).

172 Diederich, in: Friedrichsen/Kohn, Digitale Politikvermittlung, S. 161 (163); zumindest andeutend Ingold, Staat 56 (2017), 491 (508). Mitsch, DVBl. 2019, 811 (812) spricht von einer „kognitiven Überforderung “. Vgl. auch Paal/Hennemann, JZ 2017, 641 (643); Schäfer, Computers in Human Behavior 103 (2020), 1 (2) oder Hill, DÖV 2020, 205 (206).

173 BVerfGE 149, 222 Rn. 80 - Rundfunkbeitrag.

174 Webster, Northwestern Law Review 104 (2010), 593 (594); Schneiders, Das ist doch Diskriminierung, S. 2 m.w.N., der zudem darauf hinweist, dass im Kampf um Aufmerksamkeit gerade auch die Tendenz besteht, dass Anbieter ihre Inhalte der Plattformlogik anpassen (a.a.O., S. 4). Zu diesem Wettbewerb auch Brinkmann, UFITA 2019, 364 (371); Wiedel, in: Litschka/Krainer, Der Mensch im digitalen Zeitalter, S. 49 (54). Sog. Clickbaiting (dt. Klickköder) ist ein Auswuchs dieses Wettbewerbs um Aufmerksamkeit. näher Zur Bedeutung etwa Bendel, 300 Keywords Informationsethik, S. 28 f. aus der gerichtlichen Praxis siehe etwa OLG Köln, GRUR-RR 2019, 396.

175 Vgl. BVerfGE 149, 222 Rn. 80 - Rundfunkbeitrag. 
möglichst breit informiert sein. ${ }^{176}$ Die Nutzeroberfläche der Netzwerke begünstigt dabei eine nur oberflächliche Informationsaufnahme, ${ }^{177}$ weil einerseits viele Informationen präsentiert und teilweise nur mit kurzen Inhaltsangaben versehen werden ${ }^{178}$ und andererseits die Online-Kommunikation wesentlich schneller abläuft. ${ }^{179}$ Man scannt Beiträge nach relevanten Informationen und konzentriert sich dabei auf Schlagwörter. ${ }^{180}$ Für eine deutlich intensivere Informationsaufnahme wäre das Anklicken entsprechender Inhalte und die Lektüre der vollständigen Artikel notwendig, die auf den verlinkten (Nachrichten-)Seiten bereitgehalten werden. Dies erfolgt aber nur selten, ${ }^{181}$ was von den Nutzern aber nicht unbedingt wahrgenommen wird; tatsächliche und wahrgenommene Informiertheit können divergieren. ${ }^{182}$

Daneben werden hier gerade die bereits angesprochenen Auswahlheuristiken und Verzerrungstendenzen relevant. Auch dadurch kommt es oftmals nur zu einer eingeschränkten Überprüfung der Plausibilität und Glaubwürdigkeit; der Nutzer kann oftmals nicht zwischen wahr und unwahr unterscheiden. ${ }^{183}$ Das liegt auch daran, dass bei der Plausibilitätskontrolle das Votum der Nutzergemeinschaft relevanter wird. ${ }^{184}$ Entscheidend für die Wahrnehmung der Relevanz und Vertrauenswürdigkeit ist damit das soziale Umfeld und die Frage, wie andere Nutzer auf bestimmte Beiträge reagieren. ${ }^{185}$ Auch hierin zeigt sich die Notwendigkeit der Medien als professioneller Filter, um die schwierige Abgrenzung „zwischen Fakten und Meinung, Inhalt und Werbung“ und die Bewertung der „Glaubwürdigkeit von Quellen und Wertungen“ zu übernehmen. ${ }^{186}$ Dies kann auch dafür verantwortlich sein, dass sich Fake-News wesentlich schneller

176 Allgemein zur Meinungsbildung und Informationsverarbeitung Schoen, Politische Vierteljahresschrift 47 (2006), 89 (92), Meffert, in: Zmerli/Feldman, Politische Psychologie, S. 85 (93); Metzger/Flanagin/ Medders, Journal of Communication Vol. 60 (2010), 413 (417).

177 Vgl. Müller/Denner, Was tun gegen „Fake News“?, S. 12; hierzu auch Schäfer, Computers in Human Behavior 103 (2020), 1 f.; Costera Meijer/Groot Kormelink, Digital Journalism 3 (2015), 664 (669 ff.).

178 Müller/Denner, Was tun gegen „Fake News“?, S. $12 \mathrm{f}$. Ausführlicher zu der Wirkung von „snack news“ als „Illusion of knowledge“ Schäfer, Computers in Human Behavior 103 (2020), 1 ff. m.w.N.

179 Vgl. Pille, Meinungsmacht sozialer Netzwerke, S. $306 \mathrm{f}$.

180 Scheibel, (Weiter)Empfehlungen, S. 32; vgl. auch Schweiger, Der (des)informierte Bürger, S. 17.

181 Nach Bakshy u.a., Science 348 (2015), 1130 (1131) haben Nutzer nur auf 7\% tatsächlicher Nachrichten („,hard content") auch tatsächlich geklickt.

182 Ausführlicher zu diesem Aspekt Schäfer, Computers in Human Behavior 103 (2020), 1 ff. m.w.N.

183 Schweiger, Der (des)informierte Bürger, S. 17; Prochazka/Schweiger, Media Perspektiven 2020, 196 (197), die zudem auch das Überangebot und die Vermengung von herkömmlichen, medialen und alternativen Angeboten hierfür verantwortlich machen. Vgl. hierzu auch Müller/Denner, Was tun gegen „Fake News“?, S. 13. Darauf weist auch das BVerfG am Rande hin, vgl. BVerfGE 149, 222 Rn. 80 - Rundfunkbeitrag. Brinkmann, UFITA 2019, 364 (368, 369, 375) führt das auch auf die Unsicherheit der Nutzer bedingt durch die Intransparenz der Kommunikationslandschaft zurück.

184 Ähnlich auch Hines, Houston Law Review 57 (2019), 405 (407).

185 In diese Richtung auch Pfaffenberger/Adrian/Heinrich, in: Holtz-Bacha, (Massen-) Medien im Wahlkampf, S. 97 (98). Zum Zusammenhang von Popularität und Glaubwürdigkeit etwa Silva/Proksch, American Political Science Review 2020, 1.

186 So jedenfalls das BVerfGE 149, 222 Rn. 80 - Rundfunkbeitrag. 
verbreiten als wahre Nachrichten. ${ }^{187}$ Letztlich könnten soziale Netzwerke auch zu einer Segregation von Personen führen. ${ }^{188}$ Zwar existierten schon in analogen Zeiten homogene Gruppen von Personen, die einen engeren Austausch miteinander pflegten. ${ }^{189}$ In sozialen Netzwerken wird diese Segregation aber durch Personalisierungsmaßnahmen deutlich stärker gefördert, weil Inhalte anderer Nutzer und Akteure auch in Abhängigkeit von den Nutzerinteressen angezeigt werden. Deshalb kann es hier zu einer Replikation der eigenen Meinungen kommen. Das mögliche Ergebnis: Filterblasen und Echokammern entstehen. ${ }^{190}$

\section{Meinungsroboter und ihr Einfluss auf die politische Willensbildung der Nutzer}

Wenn ohnehin verschiedene externe Faktoren und interne Verzerrungstendenzen ganz unabhängig von den genauen Umständen auf die individuelle Meinungsbildung einwirken, so stellt sich die Frage, ob und inwieweit automatisierte Nutzerprofile in sozialen Netzwerken so besonders sind, dass eine eigenständige Untersuchung gerechtfertigt ist. Hierfür ist zunächst ein grundlegendes auch technisches Verständnis für das hier als Meinungsroboter bezeichnete Phänomen sog. Social Bots notwendig, bevor die konkreten Einsatzstrategien und ihre Bedeutung für die Meinungsbildung beleuchtet werden können. Erst dann lassen sich Unterschiede zu herkömmlichen Beeinflussungsversuchen aufzeigen.

\section{Herleitung des Begriffs der Meinungsroboter}

Schon die Rechtsprechung hatte sich mit dem Phänomen der Bots auseinanderzusetzen. Dabei ging es aber um sog. Cheat-Bots, also Computerprogramme, die Spielcharaktere in einem Onlinespiel (World of Warcraft) in der Abwesenheit des Spielers steuerten und so für einen stetigen Spielfortschritt sorgten. ${ }^{191}$ Daneben

187 Zu dieser unterschiedlichen Verbreitung Vosoughi/Roy/Aral, Science 359 (2018), 1146 (1147ff.).

188 Vgl. Ingold, Staat 56 (2017), 491 (509); Brinkmann, UFITA 2019, 364 (369 ff.).

189 Hierzu Schweiger, Der (des)informierte Bürger, S. 89 f. Im Kontext der Filterblasenmetaphorik deshalb relativierend Kaiser/Reiling, in: Unger/v. Ungern-Sternberg, Demokratie und künstliche Intelligenz, S. 85 (89).

190 So etwa Schweiger, Der (des)informierte Bürger, S. 88 ff.; siehe auch Mitsch, DVBl. 2019, 811 (812 f.); Brinkmann, UFITA 2019, 364 (369 ff.). In jüngerer Zeit wird aber auch Kritik angesichts der fehlenden und den entgegenstehenden, empirischen Befunden an diesem Narrativ geübt, so etwa Cornils, AfP 2018, 377 (381). Auch wenn die empirischen Ergebnisse dieses Narrativ nicht belegen können, besteht gleichwohl die latente Möglichkeit - um nicht Gefahr zu sagen -, dass eine solche Fragmentierung entsteht. Die wesentlichen Grundlagen und Möglichkeiten hierfür besitzen die Netzwerkbetreiber mit ihrem auf Personalisierung ausgerichteten Angebot jedenfalls. Zu diesen Risiken ausführlich Pille, Meinungsmacht sozialer Netzwerke, S. 322 ff., der hieraus gerade diese besondere "Gefahrgeneigtheit" ableitet (S. 345).

191 OLG Hamburg, GRUR-RR, 2015, 110. Zu der Frage des unlauteren Wettbewerbs durch Betrieb eines Forums in dem u.a. mit Bot-Programme gehandelt wurden, OLG Hamburg, Urt. v. 17.10.2012 - 
sind seit geraumer Zeit auch Chatbots in der kommerziellen und Unternehmenskommunikation zu beobachten. ${ }^{192}$ Auch wenn bislang noch keine einheitliche Definition für die hier relevanten „Social Bots“ gefunden wurde, ${ }^{193}$ so lassen sich doch spezifische Charakteristika ausmachen, die zu ihrer Bezeichnung führen. Wie auch andere Bots werden sie durch ein Computerprogramm (teil-)automatisiert gesteuert. ${ }^{194}$ Ihre Bezeichnung als Social Bots verdanken sie ihrem Einsatzort- und Zweck, der Interaktion innerhalb sozialer Netzwerke und mit ihren Nutzern. ${ }^{195}$ Sie teilen, kommentieren oder liken Inhalte oder folgen anderen Nutzern. ${ }^{196}$ Hierfür verwenden sie herkömmliche Nutzerprofile, ohne dabei - anders als andere Bots bei Twitter $-^{197}$ den Umstand der Automatisierung transparent zu machen, sodass für andere Nutzer der Eindruck eines echten Nutzers entstehen kann. ${ }^{198}$ Insoweit unterscheiden sie sich von Chat- und Servicebots einerseits dadurch, dass sie nicht auf eine direkte One-to-one-Konversation beschränkt sind, etwa um Kundenanfragen oder sonstige Anliegen unkompliziert und schnell zu klären, ${ }^{199}$ und andererseits dadurch, dass hier regelmäßig eine neue persönliche Identität erstellt wird.

Das Interesse der (Rechts-)Wissenschaft an ihnen ist vor allem seit der US-Präsidentschaftswahl 2016 exponentiell gestiegen. Seitdem geht es vor allem um den politisch motivierten Einsatz von Social Bots. ${ }^{200}$ Weil sie hierfür politische Inhalte

5 U 168/11 = MMR 2013, 453. Auch im Übrigen ging es um wettbewerbs- oder vertragswidrige Einsätze von solchen Programmen, LG Hamburg, Urt. v. 19.07.2012 - 312 O 322/12 = WRP 2013, 394 (unlauterer Wettbewerb); AG Charlottenburg, Urt. v. 04.05.2012 - 208 C-42/11 = MMR 2012, 598 (vertragswidriger Gebrauch).

$192 \mathrm{Zu}$ diesen aus rechtswissenschaftlicher Sicht etwa Franck/Müller-Peltzer, in: Taeger, Recht 4.0, S. $241 \mathrm{ff}$.

193 So etwa Stieglitz u.a., Do social bots dream of electric sheep?, 2017, S. 3.

194 Vgl. Thieltges/Hegelich, in: Blätte u.a.., Computational Social Science, S. 357 (358 f.); Hines, Houston Law Review 57 (2019), 405 (408).

195 Howard/Woolley/Calo, Journal of Information Technology \& Politics Vol. 15 (2018), 81 (83).

196 Das sehen Stieglitz u.a., Do social bots dream of electric sheep?, 2017, S. 6 als maßgebliches Kriterium für einen „social bot”.

197 Coleman, Journal of Media Ethics Vol. 33 (2018), 120 (121) bezeichnet solche als „Mechanic bots”.

198 Siehe u.a. Abulaish/Fazil, IEEE Technology and Society Magazine 2020, 52 (53); Coleman, Journal of Media Ethics Vol. 33 (2018), 120 (121); Ferrara u.a., Communications of the ACM 59 (2016), 96; Keller/Klinger, Political Communication Vol. 36 (2019), 171; Howard/Woolley/Calo, Journal of Information Technology \& Politics Vol. 15 (2018), 81 (83); Oebmer/Pedrazzi, UFITA 2020, 7 (10); Steiglitz u.a., Do social bots dream of electric sheep?, 2017, S. 6. Andere Nutzer nehmen diese auch als reale Nutzer wahr, so u.a. auch Bötticher, Kriminalistik 2017, 16; Ferrara u.a.., Communication of the ACM, Vol. 59 (2016), 96 (99); Gasser/Kraatz, VerfBlog, 2017/1/16; Kind u.a.., Social Bots, S. 4; Lewke, InTeR 2017, 207 (209). Aus empirischer Sicht zur Wahrnehmung von Botaccounts als normale Nutzer etwa Edwards u.a., Computers in Human Behaviour 33 (2014), 372 ff.

199 Zur Abgrenzung der unterschiedlichen Erscheinungsformen der Bots siehe auch Oehmer/Pedrazzi, UFITA 2020, 7 (10) oder Gorwa/Guilbeault, Policy \& Internet Vol. 12 (2020), 225 (229 ff.).

200 Hierzu aus rechtswissenschaftlicher Perspektive: Brings-Wiesen, JuWiss-Blog Nr. 93/2016; Milker, JuWiss-Blog Nr. 91/2016; ders., InTeR 2017, 199 ff.; Zumkeller-Quast, JuWiss-Blog Nr. 2/2017; Dankert/Dreyer, K\&R 2017, 73 ff.; Andresen, HRN 2017, 9 ff.; Steinbach, ZRP 2017,101 ff. Aus österreichischer Perspektive Egli/Rechsteiner, APJ 2017, 249 ff. Aus Sicht der Computational Social Science etwa Uyheng/Carley, Journal of Computational social Sciences 2020, S. 7 oder Thieltges/ Hegelich, in: Blätte u.a., Computational Social Science, S. 357 ff. 
verbreiten oder auf andere Weise in den politischen Meinungskampf eingreifen wollen, werden sie auch als „political Bots“201 oder - wie vorliegend - als Meinungsroboter ${ }^{202}$ bezeichnet.

Mit den Meinungsrobotern werden vor allem sog. Internet-Trolle vermengt oder zumindest in einem Atemzug genannt. Internettrolle unterschieden sich von ihnen regelmäßig nur in einer Eigenschaft: Die (Fake-)Accounts werden nicht automatisiert gesteuert, sondern von echten Menschen bedient, die mehrere Accounts steuern können, untereinander vernetzt sind und so Einfluss in sozialen Medien nehmen wollen. ${ }^{203}$ Trolle sind also nichts anderes als menschliche „Servicekräfte“. ${ }^{204}$ Insbesondere zu politischen Entscheidungen wurden mehrere Trollfarmen bekannt. ${ }^{205}$ Die Abgrenzung im Einzelfall ist allerdings schwer, weil Trolle und Social Bots gewisse Ähnlichkeiten in den Datenspuren, die sie in den Netzwerken hinterlassen, aufweisen. Vor allem das Nutzerverhalten und damit verbundene Metadaten (Zahl der Posts, Zeitpunkt des Uploads etc.) können ähnlich sein.

\section{Technische Umsetzung - Grundlagen}

Um zu verstehen, wie Meinungsroboter funktionieren und um auch mögliche Maßnahmen später evaluieren zu können, müssen auch die technischen Grundlagen und die erforderlichen Bedingungen für einen Einsatz zumindest in der notwendigen Kürze aufgezeigt werden: ${ }^{206}$ Ausgangspunkt ist das soziale Netzwerk. Wegen der niedrigschwelligen Registrierung wird Verwendern von Meinungsrobotern zumindest das Anlegen der notwendigen Nutzerprofile vergleichsweise einfach gemacht. Sie registrieren eine Vielzahl von fiktiven Nutzern, wobei sie variierende Emailadressen vorher selbst anlegen müssen. Teilweise stehen hierfür spezielle Dienste bereit, bei denen man nur temporäre Adressen (massenhaft)

201 So Gorwa/Guilbeault, Policy \& Internet Vol. 12 (2020), 225 (232); Haeg, Journal of Practical Ethics, 5 (2017), 85 (86); Howard/Woolley/Calo, Journal of Information Technology \& Politics Vol. 15 (2018), 81 (85); Pfaffenberg/Adrian/Heinrich, in: Holtz-Bacha, (Massen-) Medien im Wahlkampf, S. 97 (98).

202 Zuvor etwa auch schon Iben, in: Greve u.a., Der digitalisierte Staat, S. 153; Milker, ZUM 2017, 216; Neis/Mara, in: Appel, Psychologie des Postfaktischen, S. 189 oder Wolf, WRP 2019, 440.

203 Gorwa/Guilbeault, Policy \& Internet Vol. 12. (2020), 225 (233).

204 So Thieltges/Hegelich, ZfP 2017, 493 (495, Fn. 1).

205 Im US-amerikanischen Wahlkampf sollen russische Trolle unter der Aufsicht der Internet Research Agency Einfluss genommen haben, hierzu Mueller, Report on the Investigation into Russian Interference in the 2016 Presidential Election, S. 22 ff. Vor der Bundestagswahl soll die Gruppe Reconquista Germanica als Trollarmee tätig geworden sein, hierzu etwa Rieger u.a., in: Appel, Die Psychologie des Postfaktischen, S. 45 (52).

206 Zur technischen Umsetzung der Social Bots in Netzwerken siehe auch Li/Yun/Hao/Zhang/Cui/Wang, Modeling Social Engineering Botnet Dynamics across Multiple Social Networks, in: Gritzlis/Furnell/ Theoharidou (Hrsg.), Information Security and Privacy Research, IFIP Advances in Information and Communication Technology, Vol. 376, 2012, S. 264 f. Allerdings geht es hierbei um die Abläufe zur massenhaften Weiterleitung schadhafter Nachrichten (sog. trap messages). 
registrieren kann oder wo zumindest keine Limitierung für Registrierungen für einen Nutzer mit einer bestimmten IP-Adresse vorgesehen ist. ${ }^{207}$ Zwar verpflichten die Nutzungsbedingungen von Facebook die Nutzer dazu, sich mit ihrem bürgerlichen Namen zu registrieren (Klarnamenpflicht). ${ }^{208}$ Die Einhaltung dieser Pflicht wird aber bei der Registrierung und auch im späteren Verlauf nicht auf Initiative der Betreiber hin kontrolliert. ${ }^{209}$ Hat man die Profile angelegt, müssen die falschen Identitäten erstellt werden, indem man den verschiedenen Profilen (fiktive) personenbezogene Daten und Informationen hinzufügt. Dies ist die Grundvoraussetzung dafür, dass die falschen Profile nicht als solche erkannt werden.

Notwendiger zweiter Schritt ist das Erschaffen eines Computerprogramms, welches dann die Profile steuert, also etwa bestimmt unter welchen Umständen welcher Beitrag an welchem Ort veröffentlicht werden soll, wem Freundschaftsanfragen zugesendet oder welche Inhalte geteilt oder "geliked“ werden sollen. Das Programm enthält dabei die Befehle und Leitlinien in Form sog. Algorithmen. Ein Algorithmus besteht dabei aus endlich vielen einzelnen Schritten, um ein konkretes Problem zu lösen. Die ungefähre Funktionsweise konnte schon anhand des Rankingalgorithmus aufgezeigt werden. Im Falle der Meinungsroboter ist das „zu lösende“ Problem letztlich die konkrete Interaktion, die der Meinungsroboter in einer bestimmten Situation, zu einem bestimmten Zeitpunkt vornehmen soll. Mit dem notwendigen Vorwissen können die Programme komplexer sein, etwa auch mit Datenbanken und Linguistikprogrammen verknüpft werden, sodass Bots auf spezifische Inhalte anderer Nutzer inhaltlich und sachlich zutreffend reagieren können. ${ }^{210}$ Das dies recht überzeugend funktioniert, zeigen die zurzeit schon eingesetzten Chat Bots zahlreicher Unternehmen, die einfachere Serviceaufgaben und -Anfragen von Kunden bearbeiten und recht genau beantworten können. Gleichwohl handelt es sich bei Bots nicht um ein vollständig autarkes System, dass in vollständiger Autonomie Entscheidungen trifft. Es ist an die programmatischen Vorgaben des Verwenders gebunden und kann sich- jedenfalls zurzeit - nur innerhalb dieses Rahmens bewegen. ${ }^{211}$

207 Boshmaf u.a., in: Proceedings of the $27^{\text {th }}$ annual Computer Security Applications Conference, S. 93 (95).

208 Siehe hierzu die AGB bzw. Nutzungsbedingung Nr. 3.1, https://www.facebook.com/legal/terms?ref=p f, (Stand 31.05.2021).

209 Einen wirksamen Kontrollmechanismus gibt es gerade nicht. Profile können allerdings von anderen Nutzern gemeldet werden, wodurch Facebook in Kenntnis über mögliche Irregularitäten gesetzt wird und gegebenenfalls Maßnahmen ergreifen kann. Hinsichtlich des Datenschutzes gestaltet sich die Klarnamenpflicht jedenfalls als konfliktträchtig, siehe hierzu Stadler, ZD 2011, 57 oder Caspar, ZUM 2015, 233.

210 Hierzu ausführlicher Lehmann/Thellmann, in: PwC, Social Bots, S. 13 ff. Zu dieser Unterscheidung zwischen einfachen und komplexen Bots siehe auch Howard/Woolley/Calo, Journal of Information Technology \& Politics Vol. 15 (2018), 81 (84).

211 Oehmer/Pedrazzi, UFITA 2020, 7 (10). 
Die Bots müssen aber auf die Netzwerke zugreifen können. Ein solcher Zugriff kann in zweierlei Weise erfolgen: über sogenannte Programmierschnittstellen, die Applikation Interfaces (API) oder durch browserähnliche Zugriffe. ${ }^{212}$ Die APIs ${ }^{213}$ dienen der Anbindung externer Programme und Inhalte an die sozialen Netzwerke. Durch sie können netzwerkinterne Daten gezielt ausgelesen oder Daten in die Netzwerke geschrieben werden. So lassen sich etwa automatisiert Beiträge zu einem bestimmten Thema anhand des Hashtags ausfindig machen. Der Zugriff auf die Schnittstellen setzt oftmals eine Autorisierung voraus. Netzwerke vergeben hierfür sog. access token. Die mit ihnen erteilten Zugriffsrechte können dabei stark variieren. ${ }^{214}$ Der zugewiesene access token wird dem Programm implementiert, sodass sich dieses beim Zugriff auf die API autorisieren kann.

Eine zweite Möglichkeit abseits der API besteht durch einen „browserähnlichen“ Zugriff. Für gewöhnlich verwenden Nutzer Webbrowser, um bestimmte Seiten aufzurufen und im World Wide Web zu navigieren. Zwischen dem Browser des Nutzer-PC und des Servers werden hierfür Datenpakete ausgetauscht. Dabei wird von dem Nutzer-Browser meist eine Hyper-Text-Protocol-Anfrage an den Server der Netzwerkbetreiber gesendet, bestimmte Daten bereitzustellen oder zu empfangen. Wenn im Browsersuchfeld eine URL eingegeben wird, dann wird eine Anfrage an den entsprechenden Server gestellt, die für die Anzeige der Seite relevanten Daten bereitzustellen und zu senden (HTTP-Request). Der Server antwortet dann, indem er die angeforderten Daten übersendet (HTTP-Response). Dasselbe Prinzip können sich auch Social Bots zu eigen machen. Sie gerieren sich also als normale Nutzer und versuchen so entweder Daten vom Server zu empfangen oder selbst dort zu platzieren. ${ }^{215}$

Die Netzwerkbetreiber sehen allerdings Schutzmaßnahmen vor, um solch unberechtigte Zugriffe zu vermeiden. Dort, wo etwa von einer bestimmten IPAdresse viele HTTP-Anfragen binnen kurzer Zeit gestellt werden, werden etwa Captchas (completely automated public Turing test to tell computers and humans apart) vorgehalten. ${ }^{216}$ Hierbei müssen meist visuelle Aufgaben erfüllt werden, an denen automatisierte Zugriffe bisweilen scheitern. Und Facebook betreibt das sog. Facebook Immune System, das sämtliche Aktivitäten innerhalb des

212 Vgl. Boshmaf u.a., in: Proceedings of the $27^{\text {th }}$ annual ComputerSecurity Applications Conference, S. 93 (95).

213 Ausführlicher zu diesen etwa Steinebach u.a., Desinformation aufdecken und bekämpfen, S. 115.

214 Bei Twitter sind die vergebenen Berechtigungen insgesamt größer als bei Facebook. Facebook beispielsweise vergibt für unterschiedliche Anliegen bzw. Motivationslagen unterschiedliche Zugriffsschlüssel, mit denen dann unterschiedliche Zugriffsrechte verbunden sind. Nähere Informationen hält Facebook unter https://developers.facebook.com/docs/facebook-login/access-tokens?locale=de_DE, (Stand 31.05.2021) bereit. Deshalb ist Twitter auch ein Sammelbecken aller möglichen Arten von BotS. So auch PwC, Social Bots, S.10. Zu den vorzufindenden unterschiedlichen Bots nur Veale/ Cook, Twitterbots, S. $29 \mathrm{ff}$.

215 Vgl. Boshmaf u.a., Computer Networks 57 (2013), 556 (573f.).

216 Boshmaf u.a., Computer Networks 57 (2013), 556 (560). 
Netzwerkes auf Unregelmäßigkeiten kontrolliert und so u.a. Fake-Accounts und Spam erkennen soll. ${ }^{217}$

Weil aber letztlich dem Programm gewisse „menschliche“ Verhaltensmuster implementiert werden können - gemeint sind etwa die Einhaltung von Ruhepausen, eine begrenzte Anzahl von Anfragen pro Stunde, eine gewisse Varianz der Inhalte und angesprochenen Themen u.a. Parameter -, wird die Erkennung dieser Automatisierungen vor besondere Herausforderungen gestellt, die sich mit zunehmendem Fortschritt in diesem Bereich ausprägen werden. Insoweit läuft vieles auf ein Katz-und-Maus-Spiel zwischen Betreibern und Verwendern hinaus. ${ }^{218}$

III. Verwendungsstrategien und abstraktes Einflusspotenzial

Welches Einflusspotential von den Meinungsrobotern mit Blick auf die politische Kommunikation und Willensbildung ausgeht, lässt sich kaum ohne Einblicke in die theoretischen und tatsächlichen Verwendungsstrategien bestimmen. Zentral wird es hierbei neben der Informationsdiffusion, auch desinformativer Inhalte, vor allem auch um die Störung von Diskussionen und das Verändern des Meinungsklimas gehen. ${ }^{219}$

\section{Informationsdiffusion, Verursachen von Trends}

Wesentliche Grundlage der Informationsweitergabe der Netzwerke sind die Inhalte der Nutzer (sog. User-generated-Content). Die Netzwerkbetreiber sind Host-Provider, vermitteln also lediglich den Zugang zu den von anderen Nutzern bereitgestellten Inhalten und ergreifen „nur“ inhaltsmoderierende Maßnahmen. Weil sich Meinungsroboter als normale Nutzer gerieren, sind sie Bestandteil der Netzwerköffentlichkeit und haben also im Ausgangspunkt denselben Einfluss auf die Informationsdiffusion wie jeder andere Nutzer auch. ${ }^{220}$ Indem sie aber massenhaft Inhalte $\mathrm{zu}$ einem bestimmten Thema veröffentlichen, können sie

217 Vgl. Boshmaf u.a., in: Proceedings of the $27^{\text {th }}$ annual Computer Security Applications Conference, S. 93 (94). Ausführlicher zu diesem System und seiner Funktionsweise etwa He u.a., Security and Communication Networks 9 (2016), 2157 (2160) oder Stein/Chen/Mangla, Facebook Immune System, 2011.

218 Abolaish/Fazil, IEEE Technology and Society Magazine 2020, 52 (58). He u.a., Security and Communication Networks 9 (2016), 2157 (2159) sprechen von einem „arms race“.

219 Vgl. Kind u.a.., Social Bots, S. 8; Bastos/Mercea, Social Science Computer Review 37 (2019), 38 (40).

220 Zum Einsatz von Social Bots zum Zwecke der Informationsdiffusion unabhängig von den konkreten Intentionen oder Inhalten siehe u.a. Brachten u.a., Social Bots in Social Media Crisis Communication, S. 4 f.; Hagen u.a., Social Science Computer Review, 1 ff. vor allem mit Blick auf die Verbreitung alternativer Sichtweisen; Shao u.a., IEEE Access Vol. 6 (2018), 75327 (753334). 
deren Relevanz signifikant steigern. ${ }^{221}$ Das gilt umso mehr, wenn auch andere statistische Daten, die Aufschluss über die Relevanz, die Bewertung oder Popularität eines Beitrags, einer Person oder einer Institution geben, verändert werden. Dem Netzwerk und seinen Algorithmen selbst wird ein Trend suggeriert (sog. Astroturfing). ${ }^{22}$ Insoweit sorgen Meinungsroboter also dafür, dass bestimmte Inhalte überhaupt sichtbar werden. ${ }^{223}$

Das kann aber nicht nur als externer Faktor Auswirkung auf die Weitergabe einer Information durch den Algorithmus haben. Dadurch ließen sich auch heuristische Denkprozesse und Verzerrungen auf Rezipientenseite aktivieren.224 Den Rezipienten wird eine gewisse soziale Stärke suggeriert, was - ebenso wie eine priorisierte Weitergabe (Primacy-Effekt) - Einfluss auf die Bewertung der Relevanz und der Glaubwürdigkeit des Inhaltes selbst haben kann (soziale Bewährtheit). ${ }^{225}$ Das gilt nochmal mehr, wenn viele ähnliche Inhalte veröffentlicht werden (Illusory-Truth-Effect). ${ }^{226}$ Vergleichbares lässt sich auch mit Profilen oder Seiten bewerkstelligen, wenn die Anzahl der Follower oder Abonnenten von Politikern, Parteien oder anderen Akteuren verändert werden und so eine höhere Beliebtheit suggeriert wird. Bedeutsam kann hierfür auch die Vernetzung innerhalb der Netzwerke werden. Denn die Ähnlichkeit zu anderen Nutzern oder die Zugehörigkeit zu einer bestimmbaren Teilgruppe kann einen größeren - wenn auch nur auf eben diese Teilgruppe beschränkten - Einfluss zeitigen.

Diese primäre Wirkung wird dadurch ergänzt, dass die Inhalte oder geschaffenen Trends selbst wieder Reaktionen anderer echter Nutzer hervorrufen. Wenn sie auf diese reagieren, dann tragen sie zur Erhaltung oder Verstärkung der Relevanz bei. ${ }^{227} \mathrm{Da}$ diese Reaktionen wiederrum auch anderen Nutzern in dem News

221 Vgl. Oehemer/Pedrazzi, UFITA 2020, 7 (13) oder Hines, Houston Law Review 57 (2019), 405 (411), der von einem „bandwagon effect“ spricht. Zu diesem Effekt - im Kontext von Fake News Tandoc/Lim/Ling, Digital Journalism Vol. 6 (2018), 137 (139).

222 Zum Astroturfing als Verwendungsstrategie u.a. Hegelich, Die Politische Meinung, Nr. 543 (2017), 32 (36); Howard/Woolley/Calo, Journal of Information Technology \& Politics Vol. 15 (2018), 81 (86); Oberer/Erkollar/Stein, in: Stumpf (Hrsg.), Digitalisierung und Kommunikation, S. 311 (316); Schäfer/Evert/Heinrich, Big Data Vol. 5 (2017), 294 (306). Siehe auch Thieltges/Hegelich, in: Blätter u.a., Computational Social Science, S. 357 (360); Oehmer/Pedrazzi, UFITA 2020, 7 (13) m.w.N. zu empirischen Ergebnissen. Ein Versuch von Grimme u.a., Big Data Vol. 5 (2017), 279 ff. zeigt diese Möglichkeit zumindest auf.

223 Mehrens, in: Steinbrecher/Rager, Wenn Maschinen Meinung machen, S. 20 (25); Paal/Hennemann, ZRP 2017, 76 (77). Vgl. Varol/Uluturk, First Monday Vol. 23 Nr. 5 (2018). Aus theoretischer Sicht anhand eines Netzwerkmodells etwa Lou/Flammini/Menczer, Information Pollution by Social Bots.

224 Hierzu auch Oehmer/Pedrazzi, UFITA 2020, 7 (12 f.).

225 Die Bedeutung der sozialen Bewährtheit im Kontext der Bots betonen auch Graber/Lindemann, in: Sachs-Hombach/Zywietz, Fake News, Hashtags \& Social Bots, S. 51 (59ff.); Neis/Mara, in: Appel, Psychologie des Postfaktischen, S. 189 (198f.) sowie Oehmer/Pedrazzi, UFITA 2020, 7 (12 f.). Allgemein zur Relevanz der „Wisdom of crowds“ Webster, Northwestern Law Review 104 (2010), 593 (606).

226 Vgl. hierzu in Bezug auf Meinungsroboter Hagen u.a., Social Science Computer Review, 1 (3); Oberer/Erkollar/Stein, in: Stumpf, Digitalisierung und Kommunikation, S. 311 (324).

227 Mehrens, in: Steinbrecher/Rager, Wenn Maschinen Meinung machen, S. 20 (26); Oehmer/Pedrazzi, UFITA 2020, 7 (14). Vgl. zu Fake-News Müller/Denner, Was tun gegen „Fake News“?, S. 8 f. Abok- 
Feed angezeigt werden können, können sich die Trends und Inhalte noch stärker weiterverbreiten, als die eigentliche Reichweite der Meinungsroboter selbst reicht (Schneeball-Effekt $)^{228}$.

\section{Verbreitung desinformativer Inhalte}

Besondere Bedeutung kommt vor allem den Verzerrungstendenzen dort zu, wo es um desinformative Inhalte geht. Oftmals wird für diesen Teilbereich der Begriff „Fake News“ verwendet. Diesem Begriff wird aber kontextabhängig eine unterschiedliche Bedeutung beigemessen und er tendiert immer mehr dazu, Instrument zur Denunzierung politischer Gegner und etablierter Medien - Stichwort: Lügenpresse - zu werden, sodass er weiter an Konturenschärfe einbüßt. ${ }^{229}$ Der Bereich der desinformativen Inhalte umfasst verschiedene Arten. ${ }^{230}$ Klassischerweise geht es hierbei um Desinformation im eigentlichen Sinne, also um bewusst geäußerte, erwiesen unwahre Informationen. Daneben werden aber auch Inhalte erfasst, in denen Informationen unrichtig oder dekontextualisiert wiedergegeben werden. ${ }^{231}$ Besonders suggestiv ist etwa die dekontextualisierte Verwendung von Bildern oder Videosequenzen oder die Verbreitung technisch veränderter Bild-, Videooder Audiodateien. In jüngerer Zeit sind vor allem sog. Deep Fakes, täuschend echt aussehende, künstlich erzeugte Bild oder Videodateien prominent geworden. ${ }^{232}$

Beispiele solcher Inhalte finden sich reichlich: So wurde Hillary Clinton beschuldigt, an einem Pädophilenring in Washington beteiligt gewesen zu sein (sog. Pizzagate-Affäre). ${ }^{233}$ In Deutschland wurde etwa fälschlich behauptet, dass ein Flüchtling, der sich zuvor mit Angela Merkel fotografierte, am Berliner Attentat

hodair/Yoo/McDonald, in: ACM, Proceedings of the 18th ACM Conference on Computer Supported Cooperative Work \& Social Computing, S. 839 (848), ist aufgefallen, dass die meisten Reaktionen auf Beiträge von Bots eher von realen Nutzern kamen, als von anderen BotS.

228 Mafi-Gudarzi, ZRP 2019, 65; Holznagel, MMR 2018, 18 (19).

229 Vgl. hierzu Steinbach, JZ 2017, 653 (654); Vosoughi/Roy/Arai, Science 359, 1146 sprechen deshalb von "true“ oder "false“ newS. Ausführlicher zum Phänomen der Fake News im politischen Bereich etwa Zywietz, in: Sachs-Hombach/ ders., Fake News, Hashtags \& Social Bots, S. 97 ff.

230 Eine ausführliche Einordnung und Differenzierung findet sich etwa bei Möller/Hamaleers, in: dies./ Ferreau, Typen von Desinformation und Misinformation, S. 10 (12 ff.) oder bei Tandoc/Lim/Ling, Digital Journalism Vol. 6 (2018), 137 (141 ff.).

231 Vgl. hierzu Graber/Lindemann, in: Sachs-Hombach/Zywietz, Fake News, Hashtags \& Social Bots, S. 51 (58).

232 Zu diesen Deep-Fakes (aus rechtlicher Sicht) etwa Lantwin, MMR 2019, 574 ff. oder ders., MMR 2020, $78 \mathrm{ff}$. Kalifornien hat ein entsprechendes Gesetz erlassen, welches zumindest Deep-Fakes von Politikern verbietet, Assembly Bill Nr. 730, hierzu MMR-Aktuell 2019, 421493.

233 Siehe hierzu Rehfeld, Verleumdungsaktion „Pizzagate“, FAZ v. 09.12.2016; auf diese Verschwörung auch hinweisend, Mitsch, DVBl. 2019, 811 (813). 
im Dezember 2016 beteiligt gewesen sein soll. ${ }^{234}$ Daneben finden sich aber auch etliche weitere Beispiele. ${ }^{235}$

Bedeutsam werden Meinungsroboter ${ }^{236}$ auch hier nicht nur deshalb, weil sie die Sichtbarkeit solcher Inhalte erhöhen, sondern auch weil sie die Informationsaufnahme, -Verarbeitung und -Bewertung beeinflussen können. Besondere Bedeutung kommt neben der sozialen Bewährtheit vor allem auch dem Illusory-Truth-Effect zu. ${ }^{237}$ Dadurch dass entsprechende Inhalte oft reproduziert oder geteilt werden, könnten sie glaubwürdiger wirken. Und weil desinformative Inhalte - wie im Falle der Pizzagate-Affäre oder des Selfies - besonders auf der affektiven und emotionalen Ebene wirken und zudem noch meist negativ konnotiert sind, kann ihnen auch mehr Aufmerksamkeit zuteilwerden.

Das tückische an solchen Inhalten: Sie verbreiten sich - auch unabhängig von der Beteiligung von Meinungsrobotern - deutlich schneller und weiter als wahre Nachrichten; ${ }^{238}$ Nutzer werden durch sie eher angesprochen. ${ }^{239}$ Entsteht ein viraler Trend, so ist die Verbreitung der Information unkontrollierbar. Es kann nicht gewährleistet werden, dass gegenläufige, korrigierende Informationen auch jeden Konsumenten der Fehlinformation erreichen. ${ }^{240}$ Im Falle von Pizzagate führte die Verbreitung sogar dazu, dass ein bewaffneter junger Mann den angeblichen Treffpunkt, eine Pizzeria in Washington D.C., betrat und um sich schoss. ${ }^{241}$ Durch Meinungsroboter kann die Verbreitung wesentlich beschleunigt werden, indem man eigenständig und ohne Hilfe von anderen Nutzern einen bestimmten Relevanzschwellenwert überschreitet, der sowohl für die Weitergabe durch die

234 Diese falsche Darstellung führte auch zu einer Auseinandersetzung des Opfers dieser Behauptung mit dem Netzwerkbetreiber vor dem LG Würzburg, ZUM 2017, $437 \mathrm{ff}$.

235 Siehe hierzu nur Gensing, Schneller als die Polizei dementieren kann, tagesschau.de vom 7.01.2019 oder Schmid u.a., in: Sachs-Hombach/Zywietz, Fake News, Hashtags \& Social Bots, S. 69 (82 ff.), auch zur Rezeption und den erfolgten Gegenmaßnahmen.

236 Zur Beteiligung von Meinungsrobotern im Feld der Desinformation, siehe u.a. Shao u.a., IEEE Access Vol. 6 (2018), 75327 (75337); Shao u.a., Nature Communications (2018)9:4787. Aus theoretischer Sicht anhand eines Netzwerkmodells etwa Lou/Flammini/Menczer, Information Pollution by Social Bots.

237 So auch Neis/Mara, in: Appel, Psychologie des Postfaktischen, S. 189 (198). Siehe hierzu auch Mehrens, in: Steinbrecher/Rager, Wenn Maschinen Meinung machen, S. 20 (24).

238 So das Ergebnis von Vosoughi/Roy/Arai, Science 359, 1146 (1147). Bei der Analyse der Ergebnisse wurde versucht, mögliche Profile von Bots aufzufinden und aus den gesammelten Daten auszuscheiden (1150).

239 Das gilt vor allem für Leute, die solchen Nachrichten stärker und häufiger ausgesetzt sind als echten, harten Nachrichten. Sie halten Fake News eher für realistisch, so Balmas, Communication Research Vol. 41 (2014), S. 430 (434). Teilweise werden Fake News nicht als Grund für eine Verunsicherung und ein anderes Weltbild angesehen, sondern als Resultat. Sie werden produziert und aufgenommen, weil eine spezifische Voreinstellung schon besteht, Zywietz, in: Sachs-Hombach/ders., Fake News, Hashtags \& Social Bots, S. 97 (108).

240 So auch Mehrens, in: Steinbrecher/Rager, Wenn Maschinen Meinung machen, S. 20 (28). In Deutschland haben bspw. die Parteien spezielle Teams gebildet, um die Wähler vor Fake News zu warnen und schnell auf sie reagieren zu können, hierzu Schmid u.a., in: Sachs-Hombach/Zywietz, Fake News, Hashtags \& Social Bots, S. 85. Anschaulich hierzu auch Brodnig, Hass im Netz, S. 137 ff. und aus empirischer Sicht etwa Zollo u.a., PLoS ONE 12 (7): e018121, S. 4 ff.

241 Hierzu etwa Fisher/Woodrow Cox/Hermann, Pizzagate, The Washington Post (online) v. 06.12.2016. 
Algorithmen als auch für die Rezeption durch andere Nutzer notwendig ist. Danach kann sich die Information (viral) innerhalb des Netzwerkes verbreiten.

Besonders prekär kann dies in Krisenzeiten oder mit Blick auf bestimmte Nutzergruppen, die eher staatskritisch eingestellt sind, sein. In Krisenzeiten sind richtige Informationen elementar, um ein Gefühl von Sicherheit zu verbreiten. Desinformative Inhalte sorgen aber für gegenläufige widersprüchliche Informationen, sodass keine sichere, faktische Grundlage mehr besteht. So kursierten während der Covid-19-Pandemie etwa verschiedene, fehlerhafte Informationen und Verschwörungstheorien in den sozialen Netzwerken, auf die sich oftmals Kritiker der strikten Infektionsschutzmaßnahmen stützten. ${ }^{242}$ Von der dadurch entstehenden Bestätigungstendenz sind vor allem die Nutzer betroffen, die schon zuvor eine gewisse kritische (Vor-)Einstellung besaßen (confirmation bias). Auch dies betrifft oftmals staatskritische Gruppen ${ }^{243}$, die auch klassischen Medien, insbesondere den öffentlich-rechtlichen Medien, kritisch gegenüberstehen. Das kann also extreme Meinungen befördern und zu einer Radikalisierung führen.

\section{Störung von Diskussionen}

Daneben können Meinungsroboter auch dafür sorgen, dass bestimmte Inhalte nicht mehr veröffentlicht oder wahrgenommen werden, indem Diskussionen und ein unmittelbarer Informationsaustausch gestört werden. Wegen der dezentralen Struktur sozialer Netzwerke und die gerade nicht kongruenten individuellen Netzwerke existiert nicht ein zentraler Ort für eine Diskussion, wie etwa bei Internetforen. ${ }^{244}$ Jeder Inhalt kann durch die Kommentarfunktion zum Ausgangspunkt von Diskussionen werden. Macht man etwa themenspezifische Hashtags ${ }^{245}$ ausfindig, lassen sich sämtliche Beiträge und damit potentielle Diskussionspunkte innerhalb eines Netzwerkes ausfindig machen und durch gezielte Intervention stören. ${ }^{246}$ Eine solche Intervention kann durch das zahlreiche Verbreiten von

242 Zum Einsatz von Meinungsrobotern in diesem Kontext siehe etwa Ferrara, First Monday Vol. 25 (2020) Nr. 6 und Yang/Torres-Lugo/Menczer, Prevalence of Low-Credibility Information on Twitter During the COVID-19 Outbreak, S. 2 f. Daneben zur Verbreitung von Hate Speech auch durch Meinungsroboter Uybeng/Carley, Journal of Computational Social Sciences 2020, S. 7.

243 Zur mutmaßlich deutlich stärkeren Unterstützung radikaler Akteure des politisch rechten Spektrums etwa Silva/Proksch, American Political Science Review 2020, $1 \mathrm{ff}$.

244 Diese Foren zeichnen sich dadurch aus, dass dort verschiedene Diskussionsunterforen zu je ganz themenspezifischen Fragen entstehen (sog. Threads), die regelmäßig nicht nur für registrierte Nutzer, sondern auch für Außenstehende einsehbar sind. Zu solchen Internetforen siehe etwa Czauderna, Lernen als soziale Praxis im Internet, S. 19 ff.

245 Zur Bedeutung der Hashtags siehe etwa auch Shao u.a., IEEE Access Vol. 6 (2018), 75327 (75334f.).

246 Coleman, Journal of Media Ethics Vol. 33 (2018), 120 (125); Kaufhold/Reuter/Stefan, in: Burghardt/ Wimmer/Womser-Hacker, Mensch und Computer 2017, S. 51 (56) Lamo/Calo, UCLA Law Review 66 (2019), 988 (999); Mehrens, in: Steinbrecher/Rager, Wenn Maschinen Meinung machen, S. 20 (25); Varol/Uluturk, First Monday Vol. 23 Nr. 5 (2018). Eine ähnliche Strategie konnten etwa Schäfer/Evert/Heinrich, Big Data Vol. 5 (2017), 294 (302 f., 305) in Japan nachweisen. 
mehrheitskonträren Inhalten (Spamming), ${ }^{247}$ durch die Zweckentfremdung der Schlagworte zum Verbreiten nicht themenspezifischer Inhalte (Dekontextualisierung), ${ }^{248}$ durch Verbreitung beleidigender oder sinnloser Beiträge, oder durch das Erschaffen neuer und Verdrängen alter Diskussionstrends erfolgen. ${ }^{249}$ Die Folgen können je nach Verhalten variieren: von Veränderung des Meinungsklimas, über das Verhindern einer Partizipation anderer Nutzer, der Verursachung eines Aufmerksamkeitsverlustes bis hin zum vollständigen Erstarren der Diskussion ist vieles denkbar. ${ }^{250}$

Hier aktivieren die Meinungsroboter weniger Heuristiken oder Verzerrungstendenzen, sondern könnten vielmehr als externer Faktor wirken. Wenn sie als Teil der Netzwerköffentlichkeit wahrgenommen werden, können sie die Effekte der Schweigespirale herbeiführen; andere Nutzer könnten also von der Kundgabe ihrer Meinung abgehalten werden. Auch wenn das auf den ersten Blick eher für die Meinungsäußerung als für die Meinungsbildung relevant ist, so führt das doch dazu, dass bisherige Beiträge in dem von Meinungsrobotern erzeugten Rauschen untergehen, mehrheitskonträre Inhalte nicht kundgegeben werden und damit auch nicht rezipiert werden können. Zudem kann das zeitgleich Nutzer darin bestärken, Minderheitsmeinungen kundzugeben.

\section{Veränderung des Meinungsklimas}

Dadurch kann auch das nach außen wahrnehmbare Meinungsklima beeinflusst werden. ${ }^{251}$ Das gilt nicht nur mit Blick auf die Mehrheiten in Diskussionen, sondern auch für die Veränderung der Followerzahlen ${ }^{252}$ bzw. andere „Popularitätsindikatoren " 253 oder durch die mehrheitliche Veröffentlichung negativ konno-

247 Hierzu Shao u.a., IEEE Access Vol. 6 (2018), 75327 (75334f.).

248 Hierzu siehe u.a. Shao u.a., IEEE Access Vol. 6 (2018), 75327 (75336). Vgl. auch Lamo/Calo, UCLA Law Review 66 (2019), 988 (999) und R. Weaver, International Journal of Digital and Data Law 2020, 21 (22) zu einem US-amerikanische Beispiel nach einem Highschool-Attentat, m.w.N.

249 Siehe hierzu etwa Mehrens, in: Steinbrecher/Rager, Wenn Maschinen Meinung machen, S. 20 (24). Aus empirischer Sicht zu diesem Einsatz etwa Abokhodair u.a., in: ACM, Proceedings of the 18th ACM Conference on Computer Supported Cooperative Work \& Social Computing, S. 839 (849); Lou/Flammini/Menczer, Information Pollution by Social Bots, S.17, oder Suárez-Serrato u.a., in: Spiro/Ahn, SocInfo 2016: Social Informatics, S. 269 (275 ff.).

$250 \mathrm{Zu}$ diesen Strategien siehe etwa Suárez-Serrato u.a., in: Spiro/Ahn, SocInfo 2016: Social Informatics, S. 269 ff. In Ansätzen vgl. auch Kaerlein, in: Hug/Pallaver, Talk with the Bots, S. 17 (22) oder Mehrens, in: Steinbrecher/Rager, Wenn Maschinen Meinung machen, S. 20 (26). Mit Blick auf den Ton in Diskussionen etwa Hagen u.a., Social Science Computer Review, 1 (16f.).

251 Hierzu aus empirischer Sicht etwa Thieltges u.a., Effects of Social Bots in the Iran-Debate on Twitter, S. 7. In der Theorie haben das neben Cheng/Luo/Yu, Physica A 2020, 124163 auch Ross u.a., European Journal of Information Systems 2019, S. 394 ff. bestätigt.

252 Howard/Woolley/Calo, Journal of Information Technology \& Politics Vol. 15 (2018), 81 (86, 87); Lamo/Calo, UCLA Law Review 66 (2019), 988 (1000); Thieltges/Hegelich, in: Blätter u.a., Computational Social Science, S. 357 (361 f.); Silva/Proksch, American Political Science Review 2020, $1 \mathrm{ff}$.

253 Oehmer/Pedrazzi, UFITA 2020, 7 (12). 
tierter, themenspezifischer Inhalte. ${ }^{254}$ Der Einsatz von Meinungsrobotern kann hier - legt man die Theorie der Schweigespirale zugrunde - einen erheblichen Einfluss haben; schon wenige Meinungsroboter könnten so ausreichen um das Meinungsklima zu verändern. ${ }^{255}$ Durch die Effekte der Schweigespirale könnten so bestimmte Personengruppen gezielt eingeschüchtert und von weiteren Äußerungen abgehalten werden. ${ }^{256}$ Dort, wo der Umgangston schärfer wird, mag das auch die Stimmung insgesamt nachhaltig stören oder eine Radikalisierung befördern, indem etwa beleidigende, diffamierende oder rassistische Inhalte veröffentlicht werden oder zum Hass aufgestachelt wird. ${ }^{257}$

\section{Sekundäre, mittelbare Einflüsse in der Offline-Sphäre}

Sekundäre Einflüsse haben Meinungsroboter vor allem durch die Mobilisierung anderer Nutzer. Denn die Entscheidung, ob Informationen anderer für so relevant gehalten werden, dass man sie nicht nur selbst zur Kenntnis nimmt, sondern auch weiterleitet, trifft jeder Nutzer selbst. Es kommt also auf die Überzeugungskraft der Meinungsroboter an. ${ }^{258}$ Das dahinterstehende Modell weist wesentliche Parallelen zu epidemiologische Infektionsmodellen auf. Für die „Infektionsrate“ ist auch der Kontakt mit anderen „infizierten“ Nutzern entscheidend, ${ }^{259}$ was im Wesentlichen bereits dem Prinzip sozialer Bewährtheit entspricht. ${ }^{260}$

Daneben sind soziale Netzwerke oftmals Ausgangspunkt für bestimmte Handlungen in der Offline-Welt. Das gilt nicht nur für das potentielle Agenda-Setting der Netzwerke, wodurch etwa die interpersonale Kommunikation beeinflusst wird. Weil soziale Netzwerke und das dortige Meinungsklima mittlerweile auch relevante Orientierungsgröße sind, kann eine Orientierung von Politikern, Medien und anderen „Meinungsführern“ hieran zu Trugschlüssen führen. ${ }^{261}$

254 Vgl. Mehrens, in: Steinbrecher/Rager, Wenn Maschinen Meinung machen, S. 20 (26).

255 Siehe hierzu insbesondere Cheng/Luo/Yu, Physica A 2020, 124163 und Ross u.a., European Journal of Information Systems 2019. Zu einem solchen Einfluss siehe auch Hagen u.a., Social Science Computer Review, 1 (16f.) und vgl. auch Lou/Flammini/Menczer, Information Pollution by Social Bots, S. 17 m.w.N.

256 Lamo/Calo, UCLA Law Review 66 (2019), 988 (999); Cheng/Luo/Yu, Physica A 2020, 124163, S. 3.

257 Siehe hierzu etwa die Studie zu einem Netzwerk-Modell von Ross u.a.., European Journal of Information Systems, Vol. 28 (2019), $394 \mathrm{ff}$.

258 Zur Bedeutung des „social capital“ etwa Murthy u.a., IJoC 10 (2016), 4952. Zur Notwendigkeit einer Verbindung mit anderen Nutzern daneben auch Lou/Falmmini/Menczer, Imformation Pollution by Social Bots, S. 14 ff.; Abulaish/Fazil, IEEE Technology and Society Magazine 2020, 52 (58).

259 Zu diesem Modell siehe die Darstellung von Thieltges/Hegelich, ZfP 2017, 493 (505). Ausführlicher zum SIR-Modell bei der Informationsdiffusion im virtuellen Raum Woo/Chen, SpringerPlus 5:66 (2016), S. 5 f.

260 Auf dieser einfachen Grundannahme beruht ja letztlich auch (u.a.) der Rankingalgorithmus von Facebook (s.o.) oder auch der Pagerank von Google, hierzu Page u.a., The PageRank Citation Ranking, 2001; Lewandowski, Suchmaschinen verstehen, S. $101 \mathrm{ff}$.

261 Hegelich, Die Politische Meinung, Nr. 543 (2017), 32 (36). Hierin sehen etwa Haser/Hoofacker, Journalistik 2019, 140 (149f.) auch die aktuelle, maßgebliche Gefahr. 


\section{Fazit: Abstraktes Einflusspotential}

Meinungsrobotern kommt also ein abstraktes Einflusspotential nicht nur als externe Faktoren bei der Informationsweitergabe zu. Sie nutzen vielmehr bereits vorhandene Denkheuristiken ${ }^{262}$ und Verzerrungstendenzen aus, mithilfe derer auf die tatsächliche Wahrnehmung und Verarbeitung der Informationen eingewirkt werden kann. In Anbetracht der Tatsache, dass sich die verschiedenen Einsatzarten ohne Weiteres kombinieren lassen, sich teilweise sogar sinnvoll ergänzen, besteht Grund zu der Annahme, dass damit ein nicht unerheblicher Einfluss denkbar sein kann.

\section{Konkretes Einflusspotenzial - Empirische Forschungsergebnisse und} Beweisschwierigkeiten

Weitestgehend konnten empirische Studien ${ }^{263}$ die oben aufgezeigten Verwendungsstrategien in verschiedenen Konstellationen nachweisen, vor allem mit Blick auf die (Des-)Informationsdiffusion ${ }^{264}$ und die Veränderung des Meinungsklimas ${ }^{265}$ bzw. die Störung von Diskussionen. ${ }^{266}$ Diese Studien haben aber zwei entscheidende Schwächen: Erstens ist die (zutreffende) Identifizierung von Meinungsrobotern notwendige Voraussetzung für die Beurteilung eines konkreten Einflusses. ${ }^{267}$ Manche Studien greifen hierbei allerdings auf wenig überzeugende

262 Vgl. Coleman, Journal of Media Ethics Vol. 33 (2018), 120 (122).

263 Ohne Anspruch auf Vollständigkeit: zur US-amerikanischen Präsidentschaftswahl Bessi/Ferrara, First Monday Vol. 21 Nr. 11; Boichak u.a., in: Proceedings of the $13^{\text {th }}$ International Conference, 2018, S. $17 \mathrm{ff}$. oder Howard u.a., Journal of Information Technology \& Politics 15 (2018), $81 \mathrm{ff} . ;$ zum Brexit Murthy u.a.., IJoC 10 (2016), 4952 ff. und Bastos/Mercea, Social Science Computer Review 37 (2019), 38ff; zur Präsidentenwahl in Frankreich Ferrara, First Monday Vol. 22 Nr. 8 (2017); zur deutschen Bundespräsidentenwahl Neudert u.a.., Junk News und Bots bei der Bundespräsidentenwahl, 2017; zur \#YaMe-Canse Debatte in Mexiko Súarez-Serrato u.a., SocInfo 2016, S. 269 ff.; zur politische Debatte in Venezuela Forelle u.a., Political Bots and the Manipulation of the Public Opinion in Venezuela, 2015; zur Wahl in Japan Schäfer/Evert/Heinrich, Big Data 5 (2017), 294ff; zur Verbreitung von Informationen während der Covid-19-Pandemie etwa Ferrara, First Monday Vol. 25 (2020) Nr. 6 oder Uyheng/Carley, Journal of Computational Social Sciences 2020.

264 Vélasquez/Yazdani/Suárez Serrato, Socialbots supporting Human Rights, 2017. Insbesondere mit Blick auf desinformative Inhalte Ferrara, First Monday Vol. 25 (2020) Nr. 6; Shao u.a., IEEE Access Vol. 6 (2018), 75327 (75334ff.); Yang/Torres-Lugo/Menczer, Prevalence of Low-Credibility Information on Twitter During the COVID-19 Outbreak, S.2f. Zur Präsidentschaftswahl in Japan, Schäfer/Evert/Heinrich, Big Data 5 (2017), 294 ff.

265 Jedenfalls als theoretische Studie unter Berücksichtigung der Theorie der Schweigespirale Ross u.a., European Journal of Information Systems 2019, $394 \mathrm{ff}$. Mit Blick auf die Verbreitung von Hate Speech etwa Uyheng/Carley, Journal of Computational social Sciences 2020.

266 Hierzu etwa Suárez-Serrato u.a., in: Spiro/Ahn, SocInfo 2016: Social Informatics, S. 269 (275 ff.) und Abokhodair u.a., in: ACM, Proceedings of the 18th ACM Conference on Computer Supported Cooperative Work \& Social Computing, S. 839 (849). Siehe aber auch Shao u.a., IEEE Access Vol. 6 (2018), 75327 (75335 f.).

267 Schon Neudert wies im Ausschuss des Bundestages für Digitale Agenda daraufhin, dass genaue Ergebnisse nur schwer zu liefern seien, insbesondere die Erkennung von Bots dabei das Hauptproblem 
und fehleranfällige Methoden zurück. ${ }^{268}$ Insbesondere heuristische Modelle, bei denen vom Vorliegen bestimmter (vermeintlicher) Charakteristika auf eine Automatisierung geschlossen wird, zählen hierzu. ${ }^{269}$ Das gilt vor allem dann, wenn etwa von der Anzahl der Tweets pro Tag auf einen hohen Automatisierungsgrad geschlossen wird. ${ }^{270}$ Besonders aktive Nutzer können so fälschlich als Bots eingeordnet werden. ${ }^{271}$ Überzeugender ist es dann eher, wenn verschiedene Faktoren miteinander kombiniert werden, um die Fehleranfälligkeit zumindest zu reduzieren. ${ }^{272}$ Genauere Resultate mag die (zusätzliche) Analyse der Netzwerkdaten liefern. ${ }^{273}$ Dabei wird nicht selten auf Erkennungssoftware zurückgegriffen, die die entsprechenden Daten auswertet. Besonders Botometer (ehemals BotorNot) wird hierfür häufig verwendet. ${ }^{274}$ Mit dem Dienst kann jeder Twitter-Account anhand der öffentlich zugänglichen Profildaten überprüft werden. ${ }^{275}$ Der Algorithmus wertet verschiedenste Daten - Metadaten der Nutzer, das Sentiment, die Freundesstruktur, die Netzwerkstruktur sowie inhaltliche und zeitliche Aspekte

darstelle, Ausschuss-DrS. 19(23)046, S. 3 f. Bock, Übertragung der Kommunikationsfreiheiten, S. 195 weist deshalb richtiger Weise darauf hin, dass Studien mit Vorbehalten zu begegnen sei.

268 Umfassende Kritik an zahlreichen Studien etwa bei Kreil, The army that never existed, 2019.

269 Forelle u.a., Political Bots and the Manipulation of Public Opinion in Venezuela, 2015, S. 3, schließen etwa von der Verwendung bestimmter Plattformen zur Äußerung auf Twitter auf die Boteigenschaft. Kritisch zu derlei heuristischen Modellen Thieltges u.a., Effects of Social Bots in the Iran-Debate on Twitter, 2018, S. 3. Varol u.a., in: Proceedings of the Eleventh International AAAI Conference on Web and Social Media, S. 280 (283) betonen: „In general, however, there is no simple set of rules to assess wether an account is human or bot".

270 So etwa vorzufinden bei Neudert, Computational Propaganda in Germany, S. 10 wo „a high level of automation" angenommen wurde, wenn ein Profil mehr als 50-mal pro Tag Inhalte unter Verwendung bestimmter Hashtags veröffentlichte. Kritisch deshalb neben Kreil, The Army that never existed, 2019, etwa auch Bastos/Mercea, Social Science Computer Review 37 (2019), 38 (42).

271 Kritisch deshalb auch Keller/Klinger, Political Communication Vol. 36 (2019), 171 (177).

272 So etwa bei Bastos/Mercea, Social Science Computer Review 37 (2019), 38 (43). Hier wurden viele Kriterien berücksichtigt: tweets to user, mean tweet to retweet, common words in the username, use of web interface to relay content, ratio of outbound to inbound @-mentions, ratio of inbound to outbound retweets, account creation date, retweet reciprocity and retweet cascade mean time.

273 Oberer/Erkollar/Stein, in: Stumpf (Hrsg.), Digitalisierung und Kommunikation, S. 311 (318). Kritisch hierzu indes Cresci, Communication of the ACM 2020, 72 (76).

274 So u.a. bei Duh/Rupnik/Korošak, Big Data 6 (2018), 113 ff.; Shao u.a., IEEE Access Vol. 6 (2018), 75327 ff.; Ferrara, First Monday Vol. 25 (2020) Nr.6. Yang/Torres-Lugo/Menczer, Prevalence of Low-Credibility Information on Twitter During the COVID-19 Outbreak, S. 2; Brachten u.a., Social Bots in Social Media Crisis Communication, S. 3; Hagen u.a., Social Science Computer Review, 1 (6); Keller/Klinger, Political Communication Vol. 36 (2019), $171 \mathrm{ff}$. Aber auch andere vergleichbare Systeme kommen zum Einsatz, so etwa DeBot, siehe etwa Yuan/Schuchard/Crooks, Social Media + Society 2019, 1 ff. Das System legt den Fokus auf die Erkennung von boshaften Aktivitäten der Nutzer und überprüft weitflächig die Netzwerkdaten nach Korrelationen, etwa inhaltlicher Art, zwischen den Nutzern, um so Automatisierungsprozesse zu entdecken. Wenn etwa ein ähnlicher Inhalt innerhalb von Sekunden(bruchteilen) veröffentlicht und dann Retweetet wird, kann das ein Anzeichen sein. Ausführlicher zur Funktionsweise Chavoshi/Hamooni/Mueen, DeBot: Twitter Bot Detection via Warped Correlation, 2017.

275 Näher zur Funktionsweise von Botometer neben Graber/Lindemann, in Sachs-Hombach/Zywietz, Fake News Hashtags \& Social Bots, S. 52 (63 f.) und Yang, Hum. Behav. and Emerg. Tech. 1 (2019), 48 (51) vor allem Varol u.a., in: Proceedings of the Eleventh International AAAI Conference on Web and Social Media, S. $280 \mathrm{ff}$. 
des Nutzerverhaltens - aus und berechnet anhand dieser eine Automatisierungswahrscheinlichkeit von gering (0) bis sehr hoch (5). ${ }^{276}$.

Aber auch diese Systeme arbeiten nicht fehlerfrei. ${ }^{277}$ Eine frühere Version ordnete etwa institutionelle und staatliche Profile als automatisiert ein. ${ }^{278}$ Und auch andere Nutzerkonten werden immer wieder fälschlich als Bots klassifiziert, ${ }^{279}$ sodass Studien, die diesen Dienst verwenden, ebenfalls zu falschen Schlüssen kommen können. Zudem ist der Dienst primär für englisch-sprachige Profile gedacht. ${ }^{280}$ Auch wenn berechtigte Kritik an den Studien und Verfahren geübt wird, so darf man nicht vergessen, dass Meinungsroboter per definitionem menschliches Verhalten simulieren sollen und schon deshalb Ungenauigkeiten bestehen, die sich mit zunehmender Komplexität tendenziell verstärken können. ${ }^{281}$ Insoweit sind gerade auch falsch-negative Ergebnisse durchaus vorstellbar. ${ }^{282}$ Zudem zeigen Studien, die Bots eigenhändig erstellt und betrieben haben, nicht nur, dass sie anderen Nutzern als solche nicht auffallen, ${ }^{283}$ sondern dass sie gerade auch Einfluss haben können. ${ }^{284}$ Problematisch ist zudem auch, dass bei Twitter nur ein eingeschränkter Zugriff auf Netzwerk- und Profildaten zugelassen wird und bei Facebook ein solcher Zugriff überhaupt nicht möglich ist. ${ }^{285}$ Insoweit verfügen die Betreiber selbst über einen wesentlich breiteren Blick auf die Dinge. ${ }^{286}$ Sie selbst löschen auch seit einigen Jahren zunehmend auffällige Nutzerprofile, ohne dass aber nach außen kommuniziert wird, welchen Charakter diese eigentlich

276 Ausführlich hierzu siehe Varol u.a., in: Proceedings of the Eleventh International AAAI Conference on Web and Social Media, S. 280 (281 ff.).

277 Das geben schon die ursprünglichen Begründer des Dienstes zu, Varol u.a., in: Proceedings of the Eleventh International AAAI Conference on Web and Social Media, S. 280 (284). Und auch neuere Studien belegen dies, so etwa Rauchfleisch/Kaiser, The False Positive Problem of Automatic Bot Detection in Social Science Research, 2020. Illustrativ zu der Fehleranfälligkeit etwa auch Weber, Roboterjournalismus, Chatbots \& Co., S. $85 \mathrm{ff}$.

278 Kritisch deshalb etwa Gallwitz/Kreil, Die Mär von „Social Bots“,Tagesspiegel. Eine ausführliche kritische Evaluation verschiedenster Studien nehmen dies., The Rise and Fall of „Social Bot“ Research, vor.

279 Exemplarisch etwa Gallwitz/Kreil, Die Mär von „Social Bots“, Tagesspiegel.

280 Kritisch deshalb allgemein zu sprachbasierten Erkennungsdiensten Silva/Proksch, American Political Science Review 2020, 1 (2).

281 In diese Richtung auch Rauchfleisch/Kaiser, The False Positive Problem of Automatic Bot Detection in Social Science Research, 2020, S. 18.

282 Vgl. Varol u.a., in: Proceedings of the Eleventh International AAAI Conference on Web and Social Media, S. 280 (288); Lou/Flammini/Menczer, Information pollution by Social Bots, S. 2. Das belegen auch Rauchfleisch/Kaiser, The False Positive Problem of Automatic Bot Detection in Social Science Research, 2020, S. 18.

283 Hierzu etwa Edwards u.a., Computers in Human Behavior 33 (2014), $372 \mathrm{ff}$.

284 Etwa Aiello u.a., in: Proceedings of the $6^{\text {th }}$ International AAAI Conference on Weblogs and Social Media, S. $10 \mathrm{ff} . ;$ Murthy u.a., IJoC 10 (2016), $4952 \mathrm{ff}$.

285 Kritisch mit Blick auf die Erforschung der Filterblasenproblematik deshalb etwa auch Rau/Stier, ZfVP 2019, 399 (409). Neudert spricht im Ausschuss Digitale Agenda deshalb davon, „dass es anhand der Daten, die die sozialen Netzwerke öffentlich zugänglich machen, fast unmöglich ist, mit Sicherheit zu sagen, ob eine Debatte durch Bots beeinflusst wird.", Ausschussdrucksache 19(23)046, S. 3 .

286 So auch Steinbach u.a., Desinformation aufdecken und bekämpfen, S. 112. 
haben. Das mag zwar wegen der unternehmerischen Interessen an der Geheimhaltung durchaus verständlich sein, verhindert aber eine genauere Evaluation und Analyse potentiell bestehender Probleme.

Zweitens lässt sich zwar ein (prozentualer) Einfluss in Diskussionen und bei der Informationsdiffusion nachweisen. ${ }^{287}$ Allerdings lassen sich daraus weder Rückschlüsse auf die tatsächliche Rezeption noch auf die konkrete kognitive Verarbeitung der Nutzer ziehen. ${ }^{288}$ Und selbst dort, wo ein Input nachgewiesen werden könnte, ${ }^{289}$ ist unklar welche Auswirkungen dieser auf den geistig-internen Meinungsbildungsprozess und den letztendlichen Output hat. ${ }^{290}$ Aber auch in anderen Bereichen lassen sich selten genaue Aussagen über die Wirkung eines bestimmten Mediums, eines Beitrages oder einer Aussage von Regierungsmitgliedern im Wahlkampf treffen oder gar ein bestimmter numerischer Einflusswert ermitteln. Dafür wirken zu viele nicht isolierbare Faktoren auf den Einzelnen ein, die in ihrer Stärke variieren. ${ }^{291}$ Auch dort sind vielmehr allgemeine Wirkungsmechanismen herausgearbeitet worden, die maßgeblich an die Funktion der Medien und die Verarbeitung auf Nutzerseite anknüpfen. ${ }^{292}$ Der wesentliche Unterschied liegt hier höchstens in der Erkennbarkeit dieser Einflüsse, weil der Nachweis eines Medienkonsums wesentlich leichter zu erbringen ist. Wenn und weil aber soziale Medien ähnlich wie herkömmliche Medien wirken und Meinungsroboter Einflüsse auf die Informationsdiffusion und -rezeption haben können, kann auch ein Einflusspotential nicht von der Hand gewiesen werden. Das gilt umso mehr, wenn Studien, die selbst Bots implementieren, nachweisen können, dass die Bots als solche nicht erkannt werden, ${ }^{293}$ Nutzer direkt beeinflussen, ${ }^{294}$ oder zumindest in einer Diskussion partizipieren können. ${ }^{295}$

287 So etwa Murthy u.a., IJoC 10 (2016), 4952 (4963 ff.) zur Informationsdiffusion.

288 So Hagen u.a., Social Science Computer Review, 1 (4) mit Blick auf die „public opinion“ oder Thieltges/Hegelich, in: Blätter, Computational Social Science, S. 357 (373). Allgemein hinsichtlich des Impacts Howard/Woolley/Calo, Journal of Information Technology \& Politics Vol. 15 (2018), 81 (85).

289 Das könnte etwa auch hier durch eine einfach heuristische Annahme erfolgen: Wer auf einen Inhalt in irgendeiner Form reagiert, oder dessen Verweildauer eine bestimmte zeitliche Schwelle überschreitet, der rezipiert den Inhalt in irgendeiner Form.

290 Die interne Informationsverarbeitung bleibt also eine „Black Box“, so Meffert, in: Zmerli/Feldman, Politische Psychologie, S. 85 (86).

291 So auch mit Blick auf die allgemeinen Folgen der Digitalisierung auf den demokratischen Kommunikationsprozess Brinkmann, UFITA 2019, 364 (376). Und Ponce, Democracia en la sociedad postfactual, S. 8 meint, dass die Wirkung auf die Wahlentscheidung wegen der vielseitigen Einflüsse jedenfalls in Frage gestellt werden kann.

292 Ausführlicher zur Medienwirkungsforschung etwa Jäckel/Fröhlich/Röder, Medienwirkungen kompakt.

293 Hierzu Edwards u.a., Computers in Human Behavior Vol 33 (2014), $372 \mathrm{ff}$.

294 So etwa bei Aiello u.a., in: Proceedings of the $6^{\text {th }}$ International AAAI Conference on Weblogs and Social Media, S. $10 \mathrm{ff}$., auch wenn es hierbei um ein spezielles Netzwerk für Literaturliebhaber ging. Freitas u.a., Social Network Analysis and Mining (2016), 6:23, zum Einfluss und Popularität a.a.O. S. $8 \mathrm{ff}$.

295 Etwa Murthy u.a., IJoC 10 (2016), $4952 \mathrm{ff}$. 
Angesichts zunehmender Digitalisierung und technischer Fortschritte, vor allem im Bereich des Machine- und Deep-Learning, besteht Grund zu der Annahme, dass auch Bots noch wesentlich komplexer und intelligenter werden. ${ }^{296}$ Und wenn es um die politische Willensbildung geht, so ergeben sich wesentliche Erkenntnisse meist nur innerhalb des Wahlkampfes oder besonders bedeutender politischer Krisen, weil Meinungsroboter auf einen Kulminationspunkt angewiesen sind, ${ }^{297}$ sodass sie auch tendenziell eher dann aktiv sein werden. ${ }^{298}$ Entwicklungen lassen sich also nur in größeren zeitlichen Abständen nachweisen. Insoweit ist Vorsicht in zweierlei Richtungen geboten: einerseits dürfen Ergebnisse nicht überbewertet werden und kritische Fragen müssen gestellt werden, andererseits sollte man auch vor einer pauschalen Verharmlosung warnen, solange noch keine weitreichenderen Wirkungszusammenhänge und Erkenntnisse gewonnen worden sind. Ein grundsätzliches Einflusspotential und die Möglichkeit der Implementierung entsprechender komplexer Botprofile in sozialen Netzwerken kann immerhin aufgezeigt werden und relativiert insoweit die bestehenden Forschungslücken. ${ }^{299}$

\section{Relevante Faktoren für den konkreten Wirkungsgrad}

Ohnehin ist der konkrete Wirkungsgrad der Meinungsroboter situationsspezifisch in dem Sinne, als dass er im Wesentlichen von drei Faktoren beeinflusst wird: nutzer-, botbezogenen und netzwerkinterne Faktoren. Auf Seiten der Nutzer $^{300}$ kommt zuvorderst deren Aktivität in sozialen Netzwerken und der konkreten Nutzung Bedeutung zu. Je öfter und je länger sich ein Nutzer in der virtuellen Welt aufhält, desto häufiger kann er in Kontakt mit Bots kommen. ${ }^{301}$ Nutzt man soziale Netzwerke aktiv und auch zu politischen Zwecken, sei es um sich einen Überblick über Nachrichten oder über aktuelle Themen zu verschaf-

296 In diese Richtung auch TAB, TAB-Fokus Nr. 16, S. 2; Thieltges/Hegelich, in: Blätter u.a., Computer Social Science, S. 357 (373 f.). Im Ansatz weist auch Neudert hierauf hin, AusschussDrS. 19(23)046, S. 4. Siehe hierzu auch dies., MIT Technology Review 121 (2018); Varol/Ulturk, First Monday Vol. 23 Nr. 5 (2018). Unabhängig von den Fortschritten im Bereich der KI hält auch Mehrens, in: Steinbrecher/Rager, Wenn Maschinen Meinung machen, S.20 (31) es für wahrscheinlich, dass die Bots weiterhin aktiv bleiben und „die digitale Arena [nicht] plötzlich wieder verlassen werden“. Graber/ Lindemann, in: Sachs-Hombach/Zywietz, Fake News Hashtags \& Social Bots, S. 52 (65) geben auch zu bedenken, dass die Erkennbarkeit dadurch erschwert werden wird.

297 Kind u.a., Social Bots - TA-Vorstudie, S. 36.

298 In diese Richtung jedenfalls auch Forelle u.a.., Political Bots in Venezuela, 2015, S. 6; Pfaffenberger/ Adrian/Heinrich, in: Holtz-Bacha, (Massen-) Medien im Wahlkampf, S. 97 (104). Mit Blick auf einen Anstieg zumindest der Followerzahlen. Von Parteien im Wahlkampf etwa Keller/Klinger, Political Communication Vol. 36 (2019), 171 (180 ff.).

299 Ähnlich Thieltges/Hegelich, in: Blätter u.a., Computational Social Science, S. 357 (373).

300 Diese individuellen Faktoren sind auch für die Wirkung herkömmlicher Medien entscheidend. Mit Blick auf den Rundfunk vgl. etwa Pille, Meinungsmacht sozialer Netzwerke, S. 235.

301 Pille, Meinungsmacht sozialer Netzwerke, S. 309, begründet mit der Verweildauer eine intensivere Auseinandersetzung mit den Inhalten dort als mit Inhalten klassischer Medien. 
fen, kann der Einfluss deutlich größer sein als bei Nutzern, die die Netzwerke lediglich begrenzt etwa zur Kommunikation mit Bekannten oder aus Vergnügen benutzen. ${ }^{302}$ Und je weniger man andere Medien konsumiert, desto weniger stark können die aufgenommenen Informationen verifiziert oder falsifiziert werden. ${ }^{303}$ Entscheidend wäre zudem auch die Frage, wie ausgeprägt die eigene politische Einstellung bzw. das politische Interesse, und das Selbstbewusstsein ist ${ }^{304}$ und wie stark andere Medien genutzt werden. Besteht ein großes politisches Interesse, so werden auch eher umfangreich und aktiv Informationen gesucht und verarbeitet. ${ }^{305}$ Ist eine politische Einstellung bereits vorhanden und stärker ausgeprägt, so wird es nur schwer möglich sein, Veränderungen zu bewirken; dissonante Informationen werden von den Nutzern oftmals weniger stark berücksichtigt. ${ }^{306}$ Eher wird durch meinungskonforme Inhalte das bereits vorhandene Meinungsbild verstärkt (confirmation bias). Einfacher wäre die Beeinflussung Unentschlossener. ${ }^{307} \mathrm{Da}$ sie selbst noch nicht entschieden haben, welche Einstellung sie zu bestimmten politischen Themen haben, sind sie empfänglicher für alle Arten der Beeinflussung, angefangen von der Aufnahme konsonanter und dissonanter Informationen, über persuasive Kommunikation bis hin zu den Einflüssen des (virtuellen) sozialen Umfelds. Vergleichbares dürfte auch für wechselhafte Wähler gelten. Bei Nichtwählern dürfte es auf die Motivation ankommen: Liegt der Entscheidung ein fehlendes politisches Interesse zugrunde, so wird man sie nur schwer erreichen und überzeugen können. Anders ist es dann, wenn die Entscheidung nicht zu wählen einer allgemeinen Politikverdrossenheit entspringt, aber ein grundsätzliches politisches Interesse besteht. ${ }^{308}$ Letztlich kommt auch der Medienkompetenz sowohl hinsichtlich der Bewertung der Urheber von Informationen als auch hinsichtlich der Informationen selbst eine elementare Rolle zu. Je eher das Wissen um die Funktionsweise der sozialen Netzwerke, Verzerrungstendenzen, Meinungsroboter und desinformative Inhalte und ihre Erkennbarkeit vorhanden ist, desto eher können Informationen auch kritisch rezipiert werden. ${ }^{309}$

302 Im Ansatz ähnlich Thieltges/Hegelich, ZfP 2017, 493 (498). Im Kontext des Filterblasennarrativs auch Kaiser/Reiling, in: Unger/v. Ungern-Sternberg, Demokratie und künstliche Intelligenz, S. 85 (89).

303 So hinsichtlich der Auswirkung von Filterblasen Kaiser/Reiling, ebd.

304 So schon Katz/Lazarsfeld, Personal Influence, S. 133.

305 Vgl. Meffert, in: Zmerli/Feldman, Politische Psychologie, S. 85 (93).

306 Meffert, in: Zmerli/Feldman, Politische Psychologie, S. 85 (98).

307 Auf diese Strategie weisen etwa auch Kaufhold/Reuter/Stefan, in: Burghardt/Wimmer/Womser-Hacker, Mensch und Computer 2017, S. 51 (52) hin.

308 Vgl. hierzu Woo/Chen, SpringerPlus 2016 5:66, S. 5 mit Blick auf die Verbreitung von Informationen.

309 Vgl. hierzu Brinkmann, UFITA 2019, 364 (374) der unabhängig von Meinungsrobotern von einer „Kluft zwischen den findigen und kundigen Nutzern [...] und den weniger kritischen und weniger erfahrenen Nutzern, für die Orientierung und Verlässlichkeit der Netzangebote zum Hauptproblem werden“, spricht. Vgl. auch Hegelich, Die Politische Meinung, Nr. 543 (2017), 32 (36). 
Auf Seiten der Meinungsroboter wird neben der Gestaltung der Profile auch das jeweilige Botprogramm relevant. Ersteres gewährleistet, dass das Profil nicht als falsch entlarvt werden kann. Je konsistenter und je realer die angegebenen Daten nach außen wirken, desto schwieriger ist die Entlarvung als Fake-Profil. Weil aber das Programm die genauen Interaktionen steuert, wird auch der jeweilige Quellcode und damit die Fähigkeiten des Urhebers relevant. Zudem gewährleistet erst der Quellcode, dass das konkrete Verhalten dadurch menschlich wirkt, dass gewisse Ruhezeiten eingehalten werden, eine vielseitige menschliche Interaktion erfolgt oder die Profile überzeugend kommunizieren. Neben diesen technischen und formellen Aspekten kommt auch der Position innerhalb des Netzwerks Bedeutung zu. Erst durch die Verbindung zu anderen Nutzern generieren die Meinungsroboter auch tatsächlich Reichweite und Einfluss innerhalb des Netzwerkes und bauen so ihre Glaubwürdigkeit auf. ${ }^{310}$

Und netzwerkseitig kommt der algorithmenbasierten Informationsdiffusion dadurch Bedeutung zu, dass sie nicht nur auf Grundlage von ermittelten Nutzerprofilen, sondern eben auch auf Grundlage manipulierter statistischer Daten erfolgt. Die Betreiber respektive die Algorithmen bewerten oder gewichten dann Inhalte auf Grundlage dieser Daten. Für die eigentliche Anzeige der Inhalte und ihre Reihenfolge sind also primär die Netzwerkbetreiber verantwortlich. Und auch die Struktur einzelner individueller Netzwerke wird relevant. Je homogener und abgeschlossener die jeweiligen Teilgemeinschaften sind, desto weniger stark dringen meinungskonträre Impulse in diese ein. Meinungskonforme Inhalte haben es dann noch leichter, Anklang zu finden. ${ }^{311}$

\section{Unterschiede zwischen bot-gestützter und herkömmlicher Einflussnahme}

Wenn aber allgemein Verzerrungstendenzen wesentlicher Bestandteil eines jeden Willensbildungsprozesses sind, so fragt sich freilich, worin die Besonderheit von Meinungsrobotern gegenüber anderen, klassischen Arten der Einflussnahme besteht. $^{312}$ Was unterscheidet eine fehlerhafte Berichterstattung in den Medien von der Verbreitung derselben Information durch Meinungsroboter? Warum sollen Meinungsroboter „risikobehafteter“ sein als falsche Wahlkampfaussagen von Politikern oder Parteien? Und weshalb macht es einen Unterschied, ob der Präsi-

310 Zur Relevanz des „social capitals“ vgl. auch Murthy u.a., IJoC 10 (2016), 4952 (4966 ff.). Dass Meinungsroboter einen solchen Einfluss haben können, zeigen etwa Hagen u.a., Social Science Computer Review, 1 (13). Zur notwendigen Verbindung als Voraussetzung effektiver „Infiltration“ siehe auch Lou/Flammini/Menczer, Information Pollution by Social Bots, S. 14ff; Abulaish/Fazil, IEEE Technology and Society Magazine 2020, 52 (58).

311 Hinsichtlich der Abgeschlossenheit Thieltges/Hegelich, ZfP 2017, 493 (506).

312 Ähnlich auch Volkmann, in: Unger/v. Ungern-Sternberg, Demokratie und künstliche Intelligenz, S. 51 (52) hinsichtlich der Kritik an den neuen, digitalen Beeinflussungen. 
dent der USA desinformative Inhalte auf Twitter verbreitet oder Meinungsroboter? Maßgeblich hierfür sind verschiedene Aspekte: ${ }^{313}$

\section{Identitätstäuschung und Täuschung über quantitative Legitimität}

Herkömmliche Wahlbeeinflussung lässt sich einem bestimmten Herausgeber zuordnen: der Hirtenbrief ${ }^{314}$ der Religionsgemeinschaft oder dem Pfarrer, der Gewerkschafsbrief der Gewerkschaft, ${ }^{315}$ das Rundschreiben im Unternehmen dem Arbeitgeber, ${ }^{316}$ Wahlwerbespots und -Plakate den Parteien oder Politikern. Ihre Identität ist für die Rezipienten erkennbar und eine Kontextualisierung der Motive, Einstellungen oder Ideologien ist möglich. ${ }^{317}$ Meinungsroboter hingegen verschleiern nicht nur ihren wahren Charakter, sie verschleiern auch die Identität der Urheber. Objektiv betrachtet, machen sie den Eindruck eines gewöhnlichen Nutzers, wodurch eine „richtige“ Kontextualisierung der Inhalte erschwert wird. Hierfür ist nämlich nicht allein der Äußerungsinhalt bedeutsam, sondern auch die Person des Äußernden ${ }^{318}$ und gegebenenfalls weitere äußerungsbezogene Informationen. Freilich wird das auch bei anderen Nutzern etwa durch anonyme oder pseudonyme Äußerungen erschwert. ${ }^{319}$ Sind keine Profilinformationen vorhanden, kann sich der Kontext nur aus der Äußerung und ihren Umständen etwa durch die Verwendung bestimmten Vokabulars oder bestimmter prägnanter Hashtags - ergeben. Für gewöhnlich besteht aber ein gewisses Vertrauen darin, dass keine versteckte politische Agenda verfolgt wird. ${ }^{320}$ Dieses Vertrauen nutzen Bots gerade aus. Es wird gerade nicht offenbart, dass eine politische Agenda verfolgt und eine gezielte und gemeinsam mit anderen vorgenommene Beeinflussung stattfinden soll. Dieser Umstand ist ohne tiefergehende Analysen nach außen kaum ersichtlich, aber maßgeblich für die Bewertung der Relevanz und Glaubwürdigkeit einer Aussage. Zudem können Profilinformationen auch bewusst so gewählt werden, dass andere in die Irre geführt werden, indem etwa erkennbar Konservative liberale Inhalte verbreiten und andersherum. ${ }^{321}$ Im US-Amerikanischen Wahlkampf soll gar die ethnische Zugehörigkeit von Nutzern ausgenutzt

\footnotetext{
313 Auch Wolf, WRP 2019, 440 Rn. 7 ff., vergleicht den „Bot-,Effekt“ “ mit konventionellen Medien.

314 Siehe etwa BVerwGE 19, $14 \mathrm{ff}$.

315 So etwa bei BVerfGE 42, $133 \mathrm{ff}$ - Gewerkschaftlicher Aufruf zur Kommunalwahl.

316 Hierzu BVerfGE 66, 369 ff. - Wablbeeinflussung durch Unternehmer.

317 Gasser/Kraatz, VerfBlog, 2017/1/16, sprechen mit Blick auf den Unterschied zu herkömmlichen Medien nicht nur von einer Identitäts-, sondern auch von einer Herkunftstäuschung.

318 Vgl. BVerfGE 97, 391 (398f.) - Missbrauchsbezichtigung.

319 Vgl. Quandt, EJoC 27 (2012), 7 (15).

320 Vgl. Quandt, EJoC 27 (2012), 7 (15 f.).

321 Ähnlich Mehrens, in: Steinbrecher/Rager, Wenn Maschinen Meinung machen, S. 20 (29).
} 
worden sein: Profile mit spanischen Namen sollen überdurchschnittlich positive Inhalte zugunsten von Donald J. Trump verbreitet haben. ${ }^{322}$

Aber nicht nur die Kontextualisierung des einzelnen Inhalts wird erschwert. Weil gerade nicht erkennbar ist, dass es um eine politische Agenda geht, die von einer Vielzahl von zusammenagierenden Profilen verfolgt wird, werden sie als Teil der Netzwerköffentlichkeit wahrgenommen und können so die Verzerrungstendenzen und Anomalien ausnutzen und auf individueller und kollektiver Ebene Wirkung entfalten. ${ }^{323}$ Auch hier kann das soeben genannte Beispiel aus dem USamerikanischen Wahlkampf zur Verdeutlichung dienen: ${ }^{324}$ Sie suggerieren eine Netzwerköffentlichkeit und einen gewissen Rückhalt von Ansichten in dieser (quantitative Legitimität) ${ }^{325}$, die in Wahrheit gar nicht besteht.

\section{Strukturell divergierende Reichweite}

Auch mit Blick auf die potentielle Reichweite ergeben sich (deutliche) Unterschiede zu herkömmlichen Nutzerprofilen. Während sich herkömmliche Nutzer ihre Reichweite mühsam erarbeiten müssen, können Botprofile durch den Zusammenschluss diesen Aufwand umgehen oder jedenfalls mindern. ${ }^{326}$ Wenn jedes Profil nur über zehn echte Follower verfügt, potenziert sich die Reichweite mit der Anzahl der zusammenagierenden Profile. Und durch mittelbare Einflüsse auf Nutzer außerhalb dieser Netzwerke - etwa durch den News Feed - können diese Wirkungen noch verstärkt werden. Die eigene unmittelbare Reichweite mag zwar im Vergleich zu der von Politikern oder anderen Personen des öffentlichen Lebens deutlich geringer sein. Nicht zu vergessen ist aber, dass diesen gerade wegen ihrer Funktion auch eine deutlich stärkere und kritischere Gegenöffentlichkeit gegenübersteht. Wenn etwa Donald Trump etwas bei Twitter veröffentlicht, wird das nicht selten von anderen Akteuren aufgegriffen und richtiggestellt. Und nutzerseitige "Shitstorms“ können hierdurch auch begründet werden. Bei Meinungsrobotern lässt sich aber nicht ein Profil als Initiator ausfindig machen, sondern viele Profile können auf unterschiedlicher Weise für den Erfolg einer Information kausal werden. Das Wesen der Reichweite unterscheidet sich also auch strukturell.

322 Hierzu etwa Woolley/Howard, Bots Unite to Automate the Presidential Election; Howard/Wooley/Calo, Journal of Technology \& Politics Vol. 15 (2018), 81.

323 Diese individuellen und kollektiven Risiken zeigen etwa auch Thieltges/Hegelich, ZfP 2017, 493 (507 ff.) auf. Zu den Auswirkungen auf die „public sphere“ siehe etwa Keller/Klinger, Political Communication Vol. 36 (2019), 171 (173 ff.). Hinsichtlich der Einschüchterungswirkung durch das Prinzip der Schweigespirale siehe Cheng/Luo/Yu, Physica A 2020, 124163 und Ross u.a., European Journal of Information Systems Vol. 28 (2019), $394 \mathrm{ff.}$

324 In diese Richtung auch Thieltges/Hegelich, ZfP 2017, 493 (498).

$325 \mathrm{Zu}$ dieser quantitativen Legitimität vor allem Brings-Wiesen, JuWiss-Blog Nr. 93/2016.

326 Vgl. Thieltges/Hegelich, in: Blätte u.a., Computational Social Science, S. 357 f. 


\section{Ausnutzung netzwerkinterner Effekte}

Besonders zeigt sich dieser Unterschied auch mit Blick auf die netzwerkinternen Effekte, die „systematisch“ ausgenutzt werden. ${ }^{327}$

\section{a. Nutzergemeinschaft als Trendbestimmer}

Denn wesentliche Größe u.a. für die Bewertung von Relevanz und Glaubwürdigkeit ist nicht nur auf Nutzerseite die Nutzergemeinschaft. Auch die Selektionsund Sortieralgorithmen beruhen zu einem wesentlichen Teil auf der Auswertung des Klick- und Nutzungsverhaltens der Nutzergemeinschaft. ${ }^{328}$ Während normale Nutzer also darauf angewiesen sind, dass andere Nutzer ihres Netzwerkes Inhalte von ihnen bedeutsam oder attraktiv finden, und dies entweder durch eine entsprechende Verweildauer oder durch die verfügbaren Reaktionsbuttons kennzeichnen, können Meinungsroboter eigenständig die Überschreitung der Relevanzschwelle herbeiführen. Auch hier mag zwar die Reichweite von Influencern, Politikern und Co. größer sein. Sie beruht allerdings auf der Überzeugungskraft und Attraktivität des Angebots, der Person oder der Inhalte und wurde damit eigenständig und über einen längeren Zeitraum erarbeitet. ${ }^{329}$

\section{b. Personalisierung, Filterblasen Echokammer}

Daneben kann auch strukturellen Netzwerkeffekten u.U. Bedeutung zukommen. Denn die Algorithmen sortieren auch individuell anhand des Nutzerprofils. Insoweit kann es dazu kommen, dass bestimmten Nutzergruppen mit vergleichbaren Nutzerprofilen ähnliche Inhalte eher und priorisiert angezeigt bekommen als andere. ${ }^{330}$ Insofern könnte die Weitergabe meinungskonformer Inhalte begünstigt werden, was unmittelbar Einfluss auf die Vielfalt der Inhalte haben kann. Das kann die Entstehung von sog. Filterblasen begünstigen. ${ }^{331}$ Zeitgleich könnten

327 Diese Ausnutzung betonen etwa auch Oehmer/Pedrazzi, UFITA 2020, 7 (11f., 18). Vgl. auch Lee/ Kerr, First Monday Vol. 25 (2020) Nr. 6 zur Ausnutzung dieser Strukturen durch Trolle.

328 Siehe hierzu Lischka, AfP 2019, 388 (389). Zu dem daraus entstehenden „popularity bias“ siehe etwa Schneiders, Das ist doch Diskriminierung, S. 3 oder Webster, Northwestern Law Review 104 (2010), 593 (606).

329 Diese eigenständige Überzeugungskraft wird später noch bedeutsam sein, wenn es um die Frage der kommunikativen Chancengleichheit geht, siehe hierzu 3. Teil, 2. Kapitel A.II.2. Probleme mögen hier anderweitig entstehen, nämlich wenn Influencer und Co. ihre Attraktivität selbst dadurch steigern, dass sie auf Botprofile zurückgreifen, um ihre Abonnentenzahl und damit Reichweite künstlich zu erhöhen, vgl. hierzu Jahnke, in: dies., Influencer-Marketing, S. 1 (10) und Hellenkemper, in: Schach/ Lommatzsch, Influencer Relations, S. 201 (220). Wegen des vor allem auch kommerziellen Charakters, stellen sich dann aber womöglich eher wettbewerbsrechtliche Fragen.

330 So in Bezug auf die personalisierten Trending Topics bei Twitter etwa Shao u.a., IEEE Access Vol. 6 (2018), 75327 (75335f.).

331 Der Begriff geht auf den US-amerikanischen Autor Eli Pariser mit seinem gleichnamigen Buch „Filterbubble - What the Internet is hiding from you“ zurück. Ausführlicher zu dem Phänomen etwa Mes- 
homogene Netzwerke zu einer Reproduktion und Bestätigung eigener Ansichten führen (sog. Echokammern) ${ }^{332}$. Andere, gegenläufige Meinungen haben es schwerer in diese Netzwerke vorzustoßen. Vor allem im politisch rechten Bereich sind solche Gebilde oft vorzufinden. Dort unterhalten sich die Mitglieder abgeschottet und folgen oftmals spezifischen Seiten. ${ }^{333}$

Inwieweit derlei Phänomene auch tatsächlich bestehen, ist - trotz der verbreiteten Narrative $-{ }^{334}$ nicht hinreichend geklärt. ${ }^{335}$ Das liegt auch daran, dass die individuellen Netzwerke heterogen sind, weil nicht nur zahlreiche Verbindungen zu Nutzern mit ähnlichen Interessen und Gemeinsamkeiten (strong ties) sondern auch zu solchen, mit denen er weniger gemein hat (weak ties), haben wird. ${ }^{336}$ Potentiell kann also auch der News Feed meinungskonträre, plurale Informationen anzeigen. ${ }^{337}$ Insoweit könnte eine Isolation im eigenen Informationskosmos vor allem durch die autonome Auswahl meinungskonformer Inhalte durch die Nutzer selbst bedingt sein. ${ }^{338}$ Aber selbst wenn man davon ausgeht, dass die Algorithmen keine entscheidende Wirkung auf das Entstehen der Filterblasen und

singschlager/Holtz, in: Appel, Die Psychologie des Postfaktischen, S. 91 ff. Daneben siehe auch Heldt, CR 2018, 494 (495); Brinkmann, UFITA 2019, 364 (370); Kaiser/Reiling, in: Unger/v. Ungern-Sternberg, Demokratie und künstliche Intelligenz, S. 85 (88 ff.).

332 Zu den Echokammern Schmidt, in: Steinbrecher/Rager, Wenn Maschinen Meinung machen, S. $102 \mathrm{ff}$.

333 Hierzu Butterwegge/Hentges/Wiegel, Rechtspopulisten im Parlament, S. 209 f.; Zollo u.a., PLoS ONE 12(7): e0181821, S. $3 \mathrm{ff}$.

334 Beginnend mit Pariser, Filterbubble hat dies Eingang nicht nur in die wissenschaftliche Diskussion gefunden (hierzu u.a. Flaxman/Goel/Rao, Public Opinion Quarterly Vol. 80 (2016), 298; Messingschlager/Holtz, in: Appel, Psychologie des Postfaktischen, S. 91 ff.; Schweiger, Der (des)informierte Bürger, S. $86 \mathrm{ff}$.), sondern wurde dadurch auch Grundlage rechtswissenschaftlicher insbesondere medienrechtlicher Überlegungen, siehe u.a. Mitsch, DVBl. 2019, 811 (812); Cornils, AfP 2018, 377 (381); Lischka, AfP 2018, 388 (390 f.); Paal/Hennemann, JZ 2017, 641; Drexl, ZUM 2017, 529 (531).

335 Für das Phänomen von Echokammern etwa Zollo u.a., PLoS ONE 12(7): e0121821, S. 3 f. Siehe auch Cornils, AfP 2018, 377 (381) und Liesem, UFITA 2019, 395 (406) jeweils m.w.N.

336 Schweiger, Der (des)informierte Bürger, S. 92.

337 Vgl. Messingschlager/Holtz, in: Appel, Die Psychologie des Postfaktischen, S. 99 f. Webster, Northwestern Law Review 104 (2010), 593 (603) hält das auch für den Grund, dass Informationen schnell und weitläufig verbreitet werden.

338 So etwa die zentralen Aussagen einer Studie von Mitarbeitern von Facebook Bakshy/Adamicl Messing, Science 348 (2015), S. 1130 mit Blick auf den News Feed. Allerdings blieb ihre Studie nicht unkritisiert, weil die Nutzerentscheidung eben auch maßgeblich von der Art und Weise der Präsentation der Inhalte selbst abhänge, so etwa Tufekci, How Facebook's Algorithm Suppresses Content Diversity und Jurgenson, Facebook: Fair and Balanced. Nach Sandvig, The Facebook „It's not our fault" Study, habe es den Anschein, dass das Unternehmen gerade die Aussage verhindern wollte, "that polarization is bad when you just have found that your own product increases it". Rau/Stier, ZfVP 2019, 399 (409) weisen jedenfalls daraufhin, dass es sich um eine interne und nicht replizierbare Studie handelt. Kritisch auch zu Aussagen von Verantwortlichen des Unternehmens, dass die Nutzer selbst entscheiden, was sie sehen wollen Brodnig, Hass im Netz, S. $201 \mathrm{f}$. Gleichwohl wird auch andernorts die Rolle der Nutzer deutlich stärker akzentuiert, vgl. etwa Garrett, Facebook's problem is more complicated than fake news, Scientific American, 2016; Messingschlager/Holtz, in: Appel, Psychologie des Postfaktischen, S. 91 (94 f., 99 f.). Zu anderen Intermediären Flaxman/Goel/Rao, Public Opinion Quarterly Vol. 80 (2016), 298 (312 ff.). Haim/Graefe/Brosius, Digital Journalism 2018, (339 ff.) halten das Szenario einer Filterblase in Bezug auf Google News für „overestimated“. Allgemeine Zurückhaltung bei der Bewertung der Auswirkungen sozialer Netzwerke wollen Holtz/ Kimmerle, in: Appel, Psychologie des Postfaktischen, S. 21 (28 f.) wahren. 
Echokammern haben, ${ }^{339}$ sondern die Nutzer selbst aufgrund der Verzerrungstendenzen dafür verantwortlich sind, so ändert dies nichts an der Tatsache, dass Meinungsroboter sich eben diese zunutze machen können. Wesentliche Hürde ist dann nur, dass die Inhalte auch in die Feeds gelangen, was aber durch die bereits aufgezeigten Strategien und die Verbindung mit den Nutzern selbst ohne Weiteres ermöglicht werden kann.

\section{Fazit}

Betrachtet man die vorgenannten Aspekte dann mögen sich Meinungsroboter in ihrer tatsächlichen Wirkkraft zwar nicht von anderen medialen Beeinflussungsversuchen unterscheiden. Gleichwohl bestehen erhebliche strukturelle Unterschiede in der Art und Weise, wie dieser Einfluss erzielt wird. Durch die Identitätstäuschung tritt eine neue Intransparenz und Informationsasymmetrie hinzu und ihre Reichweite unterscheidet sich strukturell von anderen Akteuren. Das folgt letztlich auch aus der Tatsache, dass ihnen netzwerkinterne Effekte hierbei helfen. Deshalb sind Meinungsroboter als neuartiges Phänomen politischer Propaganda und Agitation eben auch eigenständig zu beurteilen. Ein bloßer Verweis auf andersartige Einflussversuche reicht jedenfalls nicht aus, um die Unerheblichkeit von Meinungsrobotern zu begründen.

\section{VII. (Verfassungs-)Rechtliche Bedeutung der bisherigen Befunde?}

Was bedeuten die bisherigen Befunde aber nun für die rechtliche Bewertung des neuartigen Phänomens? Wegen der weitgehenden Kongruenz empirischer und verfassungsrechtlicher Vorstellungen des politischen Kommunikations- und Willensbildungsprozess geht mit jedem potentiell nachteiligen Einfluss auf die Diskursivität, Vielfalt, Transparenz und Offenheit - also die Grundfunktionen des Kommunikationsprozesses - eine auch (verfassungs-)rechtliche Implikation einher. Schon mit Blick auf den klassischen Rundfunk führten tatsächliche Entwicklungen im Medienbereich zu verfassungsrechtlichen Fragen, ${ }^{340}$ die die Länder zu beantworten hatten. Mit der Digitalisierung verlagert sich öffentliche Kommunikation und Meinungsbildung tendenziell in private Räume, über die der Staat keine oder nur eine begrenzte Kontrolle hat. Die jeweiligen Dienstan-

339 Genaue Aussagen lassen sich aber - insbesondere mit Blick auf Facebook als führendes soziales Netzwerk - nur schwer ausmachen, weil ein Zugriff auf die Daten zu Forschungszwecken kaum ermöglicht wird, vgl. zu diesem „Vorbehalt“ auch Lischka, AfP 2018, 388 (391).

340 Das gilt nicht nur hinsichtlich der Einführung des Rundfunks, hierzu BVerfGE 12, 205 (260) - 1. Rundfunkentscheidung, sondern auch für die Phase in denen durch technische Entwicklung die ursprüngliche Frequenzknappheit wegfiel und sich die Argumentation des BVerfG für die Rundfunkdogmatik wandelte, hierzu BVerfGE 119, 181 (214f.) - Rundfunkfinanzierungsstaatsvertrag. Ausführlicher zu dieser Rechtsprechung sogleich, 2. Teil, 1. Kapitel, A.I. 
bieter erlangen so nicht nur eine gesellschaftlich relevante Stellung, sondern können dadurch einen nicht unerheblichen Einfluss auf den Kommunikationsprozess ausüben. Mit diesem Strukturwandel der Kommunikationsräume geht aber ein Rollenwechsel des Staates - notwendiger Weise - einher. Er wird immer weniger Aggressor grundrechtlicher Freiheiten und des offenen und freien Kommunikationsprozesses. Vielmehr wird seine Rolle als Aufsichts- und Regulierungsbehörde zunehmend wichtiger. Das gilt aber nicht allein mit Blick auf die Anbieter digitaler Medien- und Intermediärdienste. Deutlich wird das eben auch mit Blick auf Meinungsroboter. Wenn private Akteure durch das Erstellen massenhafter Profile Einfluss auf die in den letzten Jahren exponentiell gestiegene Kommunikation in sozialen Netzwerken nehmen und insbesondere dadurch auffallen, dass sie Propaganda betreiben, desinformative Inhalte veröffentlichen, Diskussionen stören und so letztlich Einfluss auf die Informationsvermittlung, die Willensbildung und final politische Entscheidungen generieren wollen, dann führt das zumindest zu einem potentiellen Risiko für die kommunikationsgrundrechtlichen Freiheiten. Folgt aus diesem vorerst oberflächlichen Befund also eine staatliche, gar verfassungsrechtliche Verantwortung, kraft derer Nutzer vor dem risikobehafteten Verhalten Dritter zu schützen wären?

Das hierfür eine prinzipielle, rechtspolitische Notwendigkeit bestünde, kann durch eine etwas andere Sicht unterstrichen werden: Wenn im kommerziellen Wettbewerb Mitbewerber um Marktanteile konkurrieren, ein Mitbewerber aber unter Zuhilfenahme von Social Bots nicht nur die unternehmenseigenen Produkte bewirbt, sondern auch die Produkte der Mitbewerber kritisiert, schlechter und falsch bewertet ${ }^{341}$ und ihre Kommunikation in sozialen Netzwerken zu stören versucht, dann würde man kaum zögern, dieses Verhalten wegen Verstößen gegen das UWG als wettbewerbswidrig zu klassifizieren. ${ }^{342}$ Wenn das einfache Recht zur Sicherung eines freien und lauteren wirtschaftlichen Wettbewerbs hier dem Einsatz Grenzen zieht, wieso soll dann derselbe Einfluss auf den Meinungsmarkt ja möglicherweise gar auf die politische Willensbildung unproblematisch sein? Immerhin geht es nicht allein um potentielle Kaufentscheidungen, sondern um etwas viel Fundamentaleres: den Prozess der Meinungsbildung und damit dem schlichtweg konstituierenden Merkmal einer freiheitlich demokratischen Grundordnung ${ }^{343}$.

341 Anschaulich hierzu auch Weber, Roboterjournalismus, Chatbots \& Co., S. $90 \mathrm{f}$.

342 Hierzu Jülicher/Röttgen, InTeR 2018, 15 (16f.). Nichts anderes gilt jedenfalls für das sog. Stealth Marketing, also wenn man sich natürlicher Personen bedienen würde, ausführlicher hierzu Scheibel, (Weiter)Empfehlung, S. $127 \mathrm{ff}$. Speziell zu den wettbewerbsrechtlichen Vorgaben beim Einsatz von Chatbots, Köbrich/Frotzheim, WRP 2017, $1188 \mathrm{ff}$.

343 Vgl. BVerfGE 7, 198 (208) - Lüth. 


\section{Teil: Die Wandlung der Rolle des Staates im digitalen Zeitalter - Der Staat als Garant (manipulationsfreier) politischer Willensbildung?}

Auch wenn die prinzipielle Notwendigkeit einer staatlichen Intervention rechtspolitisch zumindest plausibel dargelegt werden kann, so folgt daraus freilich keine unmittelbare staatliche, ja gar verfassungsrechtlich determinierte Pflicht, diese Rolle auch wirksam auszufüllen und nunmehr Garant kommunikationsgrundrechtlicher Rechtsgüter in sozialen Netzwerken zu sein. Insoweit braucht es immerhin verfassungsrechtlicher Anknüpfungspunkte, die eine solche Rolle des Staates generell (1. Kapitel) und dann auch im Kontext der Meinungsroboter begründen können (2. Kapitel). Eine davon $\mathrm{zu}$ trennende Frage ist dann freilich, welcher Schutzbedarf sich daraus ergibt und inwieweit der Staat diesem unter Berücksichtigung anderweitiger verfassungsrechtlicher Anforderungen nachkommt oder nachkommen muss (3. Kapitel). Weil es sich aber bei dem digitalen Kommunikationsraum um einen dynamischen Regelungsbereich handelt, kann eine für alle Zeiten finale Regelung kaum denkbar sein, sodass auch ein Blick auf die zukünftige (Rechts-)Entwicklung gewagt werden darf (4. Kapitel). 


\section{Kapitel: Die Begründung einer staatlichen Verantwortung für die kommunikationsgrundrechtlichen Schutzgüter in digitalen Kommunikationsräumen}

Eine Verantwortung des Staates kann nur dort entstehen, wo ihm durch die Verfassung selbst ein entsprechender Auftrag respektive eine entsprechende Pflicht auferlegt wird. Kommt er dieser Verantwortung nicht nach, so „trifft [ihn] die Begründungslast, warum das so ist; ihn trifft die Argumentationslast dafür, ob und was dagegen getan werden muss; ihn trifft gegebenenfalls eine Handlungspflicht, um die Zustände wieder zu verbessern. "344 Blickt man in das Grundgesetz, so lassen sich durchaus verschiedene solcher Aufträge (explizit) entnehmen. Im grundrechtlichen Bereich gilt das etwa für Ausgestaltungsaufträge oder Einrichtungs- und Institutsgarantien. Sie sind notwendige Voraussetzung dafür, dass bestimmte grundrechtliche Freiheiten überhaupt in der Gesellschaft und im Verhältnis zu Dritten wahrgenommen werden können. Sie verpflichten den Gesetzgeber nicht nur einfach-rechtlich, die entsprechenden Institute vorzusehen, sondern sie auch so auszugestalten, dass sie Wirksamkeit erlangen. ${ }^{345}$ Das gilt nicht nur für die Vertragsfreiheit (Art. 2 Abs. 1 GG), die durch die vertragsrechtlichen Vorgaben gewährleistet wird ( $\mathbb{S} 104 \mathrm{ff}$. BGB) und durch allgemeine Vorkehrungen gegenüber Dritten Wirksamkeit entfaltet (etwa $\mathbb{S} \mathbb{S} 123,134,138$ BGB). ${ }^{346}$ Auch die Ehefreiheit (Art. 6 Abs.1, 1. Alt. GG) bedarf der Ausgestaltung und Absicherung. Das gilt nicht nur für die Eheschließungs- $(\mathbb{S} 1297 \mathrm{ff}$. BGB für die Eingehung, $\mathbb{S} \mathbb{S} 1564 \mathrm{ff}$. BGB für die Beendigung), sondern auch für die Ehegestaltungsfreiheit (insbesondere das eheliche Güterrecht, $\mathbb{S} \mathbb{S} 1363 \mathrm{ff}$. BGB). ${ }^{347} \mathrm{Am}$ deutlichsten kommt diese Verantwortung in Art. 14 Abs. 1 S. 2 GG zum Ausdruck: „Inhalt und Schranken [des Eigentums] werden durch die Gesetze bestimmt." Was also zum grundrechtlichen Eigentum gehört und wie weit dieses reicht, ist nicht - wie etwa der Lebensschutz oder die Menschenwürde - tatsächlich vorgegeben, sondern wird erst durch das einfache Recht bestimmt (etwa $\mathbb{\int} \int 903$ ff. BGB, UrhG etc. $)^{348}$. Daneben kommt bereits in Art. 1 Abs. 1 S. 2 GG und in Art. 6 Abs. 1 GG auch ein weiterer Aspekt zum Tragen: Ein Schutzauftrag für (bestimmte) grundrechtliche Freiheiten. Gemein sind diesen grundrechtlichen

344 Schimmele, Staatliche Verantwortung für diskursive Integrität, S. 149.

345 Siehe etwa Papier/Shirvani, in: Maunz/Dürig, Art. 14 Rn. 119; Möslein, in: Gsell u.a., Beck-online GK, $\$ 145$ BGB Rn. 23; Badura, in: Maunz/Dürig, Art. 6 Rn. 8 f., 70.

$346 \mathrm{Zu}$ dieser etwa BVerfGE 89, 214 (231f.) - Bürgschaftsverträge. Ausführlich zu dieser Verantwortung auch Möslein, in: Gsell u.a., Beck-online GK, $\mathbb{1} 145$, Rn. $23 \mathrm{ff}$.

347 Siehe hierzu auch Brosius-Gersdorf, in: Dreier, GG, Art. 6 Rn. $61 \mathrm{ff}$. zur Eheschließungsfreiheit und zu ihrer Ausgestaltung und Rn. 66 f. zur Ehegestaltungsfreiheit und ihrer Ausgestaltung.

348 Eine ausführliche Aufzählung mit Verweisen zur verfassungsgerichtlichen Judikatur findet sich etwa bei Axer, in: Epping/Hillgruber, BeckOK GG, Art. 14 Rn. $49 \mathrm{f}$. 
Aufträgen aber eines: Sie sollen im Ergebnis die grundrechtliche Freiheit absichern und schützen und das auch im Verhältnis zu (privaten) Dritten.

Auch andernorts finden sich spezifische Verantwortungen des Staates. Das gilt etwa für den Schutz der natürlichen Lebensgrundlage und der Tiere (Art. 20a GG). Und das Sozialstaatsprinzip (Art. 20 Abs. 1 GG) ist Grundlage für die staatliche Für- und Vorsorge, wozu insbesondere aber nicht ausschließlich ${ }^{349}$ der Bereich der Daseinsvorsorge zählt. Besondere Bereiche dieser Daseinsvorsorge werden explizit an späterer Stelle angesprochen: der Verkehr (Art. 87e GG) und die Post und Telekommunikation (Art. 87f GG). Insbesondere mit Blick auf letztere hat sich aber ein gewisser Wechsel in der Verantwortungsstruktur ergeben. Durch die Privatisierung der Deutschen Bundesbahn und der Deutschen Bundespost kann der Staat nicht mehr unmittelbar die Verantwortung dafür übernehmen, dass die Anforderungen der Daseinsvorsorge in diesen Bereichen tatsächlich erfüllt werden. Vielmehr beschränkt sich der tatsächliche Zugriff nun darauf, die entstandene private Macht zu kanalisieren und den Zugang zu den Dienstleistungen für alle zu gewährleisten (vgl. Art. 87e Abs. 4 S. 1, Art. 87f Abs. 1 GG). Insoweit hat er vor allem durch einfach-rechtliche Vorgaben (PostG, TKG) sicherzustellen, dass „flächendeckend angemessene und ausreichende Dienstleistungen“ bestehen. Insoweit wandelte sich die einstige Erfüllungsverantwortung in eine Gewährleistungsverantwortung für die im Wege der Liberalisierung erschaffenen Marktstrukturen. ${ }^{350}$

Was bedeutet all das für die hier gegenständliche Frage einer Verantwortung für den Kommunikations- und Willensbildungsprozess? Ein expliziter Auftrag hierfür lässt sich im Grundgesetz nicht in einer vergleichbaren Weise ausmachen. Im Gegenteil weisen die für ihn relevanten Grundrechte, insbesondere Art. 5 Abs. 1 S. 1, 2 GG, allein der Gesellschaft und ihren Kräften das Recht und insoweit die „Obliegenheit“ zu, den Kommunikationsprozess in eigener Verantwortung zu vollziehen. ${ }^{351}$ Das wird besonders in Art. 21 Abs. 1 S. 1 GG deutlich: „Die Parteien wirken bei der politischen Willensbildung des Volkes mit. [Hervorh. d. Aut.]“ Wegen seiner demokratischen Funktion macht dies auch nur Sinn, denn an dessen Ende steht immerhin die Legitimation staatlicher Gewalt. ${ }^{352}$

349 Ausführlicher zu den Teilgehalten etwa Wittreck, in: Dreier, GG, Art. 20 Rn. $27 \mathrm{ff}$.

$350 \mathrm{Zu}$ dieser Gewährleistungsverantwortung siehe etwa BVerfGE 108, 370 (392ff.) - Exklusivlizenz für Postdienstleistung. Ausführlicher zur Privatisierung staatlicher Aufgaben und der ihr korrespondierenden Gewährleistungsverantwortung etwa Kämmerer, Privatisierung, S. 474 ff. und Weiss, Privatisierung und Staatsaufgaben, S. $291 \mathrm{ff}$.

351 Ingold, in: Opelland, Propaganda als (neue) außen- und sicherheitspolitische Herausforderung, S. 81 (101). Vesting, JZ 2020, 975 (977) spricht von der „gesellschaftlichen Selbstorganisation“. Zur Konstitution des (sozialen) Kommunikationsprozesses durch die Grundrechte auch Schulze-Fielitz, in: Dreier, GG, Art. 5 Abs. 1-2 Rn. 42. Und das BVerfG, E 8, 104 (113) - Volksbefragung, begreift die öffentliche Meinungsbildung als „Erscheinung des gesellschaftlich-politischen und nicht des staatsorganschaftlichen Bereichs".

352 Vgl. BVerfGE 148, 11 (24) - Chancengleichheit politischer Parteien; 138, 102 (109) - Wahlkampfäußerungen von Regierungsmitgliedern. 
Gleichwohl war die öffentliche Kommunikation und Willensbildung stets auf die Bereitstellung öffentlicher Räume angewiesen, die originär vor allem durch den Staat bereitgestellt wurden (etwa öffentliche Straßen und Wege). Im digitalen Zeitalter verlieren diese Räume aber zunehmend an Bedeutung, und öffentliche Debatten verlagern sich in private, digitale Räume. ${ }^{353}$ Auch wenn damit die (faktische) Ausgangslage ähnlich ist wie im Bereich der Telekommunikation, so folgt allein hieraus keine eigenständige Gewährleistungsverantwortung des Staates. Anders als im Anwendungsbereich des Art. 87f GG bestand schon zuvor keine alleinige Verantwortung des Staates, entsprechende Räume zu gewährleisten. Schon durch private Print- und Rundfunkmedien wurde Öffentlichkeit erzeugt. Insoweit hat nicht der Staat Liberalisierungsmaßnahmen ergriffen, durch die originär staatliche Aufgaben für einen privaten Markt frei gegeben wurden. Vor allem technische Entwicklungen - die Erhöhung der Sendefrequenzen im Rundfunk oder die Entwicklung des Internets - führten zu einer Liberalisierung und einem Wandel von Öffentlichkeit und kommunikativen Räumen. Insofern geht es hier folglich nicht um eine „Privatisierung“, 354 sodass sich daraus keine gewandelte Verantwortung ergeben kann. Wenn der Staat auch in diesem Bereich gefordert sein soll - und insoweit von einer „Gewährleistungsverantwortung “ gesprochen wird $-{ }^{355}$ so muss diese Verantwortung verfassungsrechtlich anderweitig begründet werden.

Hierfür kommen potentiell zwei dogmatische Ansätze in Betracht: Erstens, hat die gesellschaftliche Primärverantwortung für den Kommunikationsprozess das BVerfG nicht davon abgehalten, frühzeitig im Bereich des Rundfunks eine staatliche Verantwortung dafür zu begründen, den Meinungsmarkt vor rundfunkspezifischen Gefahren zu schützen. Art. 5 Abs. 1 S. 2, 2. Var. GG sei hierfür ein spezieller Regelungsauftrag zu entnehmen (A.). ${ }^{356}$ Weil aber nicht nur der Rundfunk potentielle Risiken für den Meinungsmarkt zeitigt, sondern Risiken auch durch andere Kräfte insbesondere im digitalen Umfeld drohen, ließe sich - zweitens ganz generell darüber nachdenken, ob dem Staat nicht zumindest eine sekundäre Verantwortung dergestalt zukommt, die Wahrnehmung der kommunikativen Grundrechte gegen potentielle Bedrohungen abzusichern und so den gesellschaft-

353 Vgl. hierzu BVerfGE 128, 226 (251 ff.) - Fraport, wo das BVerfG in einem obiter dictum andeutete, auch private öffentlich Räume einer (starken) Bindung an die Versammlungsfreiheit unterwerfen zu wollen.

354 Ausführlicher zur Privatisierung und ihrer Erscheinungsformen etwa Weiss, Privatisierung und Staatsaufgaben, S. $28 \mathrm{ff}$.

355 Vgl. Fehling/Leymann, AfP 2020, 110 Rn. 6; Ingold, in: Oppelland, Propaganda als (neue) außenund sicherheitspolitische Herausforderung, S. 81 (100); Kube, in: Isensee/Kirchhof, HbStR IV, $\mathbb{S} 91$ Rn. 30; Kellner, Die Regulierung der Meinungsmacht von Internetintermediären, S. 119 oder Heilmann, MMR 2020, 162 (163).

356 Auf diese Kasuistik beziehen sich im Kontext der Meinungsroboter etwa Dankert/Dreyer, K\&R 2017, 73 (75). Allgemein zur Gewährleistungsverantwortung des Staates für Internetkommunikation etwa Kube, in: Isensee/Kirchhof, HbStR IV, $\mathbb{~} 91$ Rn. 30. 
lichen Kräften bei der Wahrnehmung ihrer „Obliegenheit“ Hilfe zu leisten. ${ }^{357}$ Insoweit könnte sich die nur vereinzelt ausdrücklich und spezifisch angesprochene grundrechtliche Schutzfunktion des Staates zu einer allgemeinen Verantwortung verdichten lassen: Einer grundrechtlichen Schutzverantwortung zugunsten der (Kommunikations-)Grundrechte (B.). ${ }^{358}$

\section{A. Der rundfunkrechtliche Regelungsauftrag des Gesetzgebers}

Schon früh hat das Bundesverfassungsgericht die Bedeutung des Rundfunks für die Meinungsbildung und damit für die Demokratie betont. ${ }^{359}$ Im Laufe der Jahre hat es zahlreiche Möglichkeiten erhalten, zu rundfunkrechtlichen Fragen Stellung zu nehmen, ${ }^{360}$ wobei eine Sonderdogmatik entstanden ist. Kern dieser Dogmatik ist ein spezifischer Handlungsauftrag, den Rundfunk und seine Wirkungen auf den Meinungsbildungsprozess zu regulieren.

\section{Entwicklung}

Den Weg der Entwicklung einer speziellen rundfunkrechtlichen Regelungs- und damit Schutzverantwortung ebnete die 1. Rundfunkentscheidung. ${ }^{361}$ Dort führte das BVerfG aus, dass der Rundfunk unabhängig vom Programmformat eminenter Faktor der öffentlichen Meinungsbildung sei. ${ }^{362}$ Gegenüber der Presse unterscheide sich der Rundfunk aber darin, dass wegen der Frequenzknappheit und der hohen Betriebskosten die Senderanzahl gering sei, wodurch keine Außen-Pluralität gewährleistet werden könne. Deshalb benötige es besonderer Vorkehrungen dafür, dass der Rundfunk „weder dem Staat noch einer gesellschaftlichen

357 Das ist letztlich auch der Gedanke, der dem verfassungsgerichtlichen Ansatz der Rundfunkdogmatik zugrunde liegt, siehe etwa BVerfGE 121, 30 (52) - Parteibeteiligung an Rundfunkunternehmen.

358 Den Bezug zu einer solchen staatlichen bzw. grundrechtlichen Schutzpflicht im Kontext der Meinungsroboter stellen demgegenüber Milker, ZUM 2017, 216 (220); ders., InTeR 2017, 199 (202f.); Löber/Roßnagel, MMR 2019, 493 (496); Klaas, MMR 2019, 84 (89) und Brings-Wiesen, JuWissBlog Nr. 93/2016, her. Und im Kontext der aufgezeigten Privatisierungsfragen weist auch Kämmerer, Privatisierung, S. 449 ff. auf die Bedeutung staatlicher Schutzpflichten als Gegenstand des „Privatisierungsfolgenrecht" hin.

359 Angefangen 1961 mit der ersten Rundfunkentscheidung BVerfGE, 12, 205 (259 ff.), wobei die Ausführungen hier noch recht oberflächlich waren. Wesentliche Grundlagen für die Dogmatik wurden dann 1967 in der zweiten Rundfunkentscheidung geschaffen, BVerfGE 31, 314 (325 ff.).

360 Ein umfassender Überblick über die Rundfunkentscheidungen des BVerfG bieten z.B. Holznagel, in: Spindler/Schuster, Elektronische Medien, Vor $\mathbb{1}$ RStV Rn. $22 \mathrm{ff} . ;$ Grabenwarter, in: Maunz/Dürig, GG, Art. 5 Abs. 1, 2 Rn. 539 ff. und Degenhart, in: BK-GG, Art. 5 Abs. 1, 2 Rn. 284 ff.

361 Dort sprach das BVerfG bereits von der „Besonderheit des Rundfunkwesens“, BVerfGE 12, 205 (260) - 1. Rundfunkentscheidung.

362 BVerfGE, ebd. 
Gruppe ausgeliefert wird. “363 Hierfür müssten die Rundfunkanstalten intern so organisiert sein, dass sie die Garantie dafür bieten, dass „alle in Betracht kommenden Kräfte [...] Einfluß haben und im Gesamtprogramm zu Wort kommen können " und dass der Inhalt selbst sachlich und ausgewogen ist. ${ }^{364} \mathrm{Um}$ dies gewährleisten zu können, müsse der Staat den Rundfunk entsprechend der Vorgaben gesetzlich regeln. ${ }^{365}$ Der Regelungsauftrag ${ }^{366}$ des Gesetzgebers für den Rundfunk war also geboren.

Dieser Auftrag beschränkte sich aber nicht nur auf den öffentlich-rechtlichen Rundfunk. Das BVerfG hatte schon damals klargestellt, dass auch ein privater Rundfunk existieren könne, für den es auch einer Organisation bedarf, die dem Aspekt der Pluralität hinreichend Rechnung trägt. Voraussetzung sei schon deshalb eine Staatsaufsicht. ${ }^{367}$ In der 3. Rundfunkentscheidung - es ging um die saarländische Regelung des privaten Rundfunks - sprach das BVerfG zum ersten Mal ausdrücklich von einer „dienenden (Rundfunk-)Freiheit“. ${ }^{368}$ Zugleich machte es Vorgaben für die Regelung des privaten Rundfunks: Der Gesetzgeber habe entsprechende Regelungen zu erlassen, die einerseits den Zugang und die Vergabe der Sendefrequenzen regeln ${ }^{369}$ andererseits aber auch die notwendige Pluralität absichern. ${ }^{370}$ Dabei habe er die Wahl zwischen einer binnen- oder einer außenpluralistischen Struktur. ${ }^{371}$ Bei Letzterem müsse aber durch das Gesamtangebot gewährleistet sein, dass das Angebot im Wesentlichen der bestehenden Meinungsvielfalt entspreche. ${ }^{372}$ In jedem Fall benötige es auch einer begrenzten Staatsaufsicht und einer vorherigen Überprüfung, ob die Veranstalter die Anforderungen erfüllen.

Hieran wurde weitestgehend auch in der 4. Rundfunkentscheidung festgehalten. Der terrestrisch verbreitete öffentlich-rechtliche Rundfunk sei für die Grundversorgung zuständig, weil private Anbieter dies nicht flächendeckend gewährleisten können. ${ }^{373}$ Gleiches gelte für die notwendige Pluralität, was auch an der Notwendigkeit der Werbefinanzierung des privaten Rundfunks liege. ${ }^{374}$ Deshalb bedürfe der private Rundfunk auch weiterhin einer Regulierung, die allerdings -

363 BVerfGE, a.a.O. (262) - 1. Rundfunkentscheidung; ebenso in E 90, 60 (87) - 8. Rundfunkentscheidung zur Gebührenausgestaltung. Vgl. auch zum Zugang zum Rundfunk für private Interessenten BVerfGE 73, 118 (182f.) - 4. Rundfunkentscheidung; 83, 238 (323) - 6. Rundfunkentscheidung.

364 BVerfGE 12, 205 (262) - 1. Rundfunkentscheidung; 31, 314 (326) - 2. Rundfunkentscheidung.

365 BVerfGE, 12, 205 (263) - 1. Rundfunkentscheidung.

366 Als solchen fast ausdrücklich bezeichnend dann BVerfGE 31, 314 (329) - 2. Rundfunkentscheidung: „Den Ländern ist von Verfassungs wegen aufgegeben, durch allgemeine Normen zu sichern, [...]“.

367 BVerfGE 12, 205 (262) - 1. Rundfunkentscheidung.

368 Seit BVerfGE, 57, 295. (320) - 3. Rundfunkentscheidung ständige Rechtsprechung.

369 Weil dies im Saarland aber nicht gewährleistet war, wurde die entsprechende Regelung für nichtig erklärt, BVerfGE 57, $295-3$. Rundfunkentscheidung.

370 Hierzu ausführlich BVerfGE 57, 295 (319 ff.) - 3. Rundfunkentscheidung.

371 BVerfGE, a.a.O (325) - 3. Rundfunkentscheidung.

372 BVerfGE, ebd.

373 BVerfGE 73, 118 (156f.) - 4. Rundfunkentscheidung.

374 BVerfGE, a.a.O. (155) - 4. Rundfunkentscheidung. 
wegen der gesicherten Grundversorgung - nicht gleich hohe Anforderungen erfüllen müsse. ${ }^{375}$ Die nachfolgenden Urteile ${ }^{376}$ verfestigten und präzisierten den beschrittenen Weg. ${ }^{377}$ Oftmals ging es dabei um die Absicherung des öffentlichen Rundfunks und seiner zentralen Bedeutung. ${ }^{378}$

Durch die zunehmende Digitalisierung wurde es allerdings möglich, dass das Angebot des privaten Rundfunks auf dem „Meinungsmarkt“ deutlich zunehmen konnte. Da das BVerfG zur Begründung seiner Dogmatik an die Frequenzknappheit angeknüpft hatte, ${ }^{379}$ fehlte ein wesentlicher Pfeiler für die Aufrechterhaltung der bisherigen Rechtsprechung. Allein der kommerzielle Einfluss als Gefahr der Meinungsvielfalt ${ }^{380}$ blieb erhalten. Schon in ihrem Sondervotum zur 2. Rundfunkentscheidung hatten die Richter Geiger, Rinck und Wand angedeutet, dass die Dogmatik mit der Frequenzknappheit stehen und fallen würde: ${ }^{381}$ Sobald eine Außen-Pluralität durch den Wettbewerb vergleichbar mit der im Pressewesen entstehe, gölten die strengen Vorgaben nicht mehr. ${ }^{382}$ Diesem Ansatz ist das BVerfG

375 BVerfGE, a.a.O. (158f.) - 4. Rundfunkentscheidung ausführlich zu den inhaltlichen Anforderungen solcher Regelungen BVerfGE, a.a.O. (159). Daran anknüpfend BVerfGE 83, 238 (316f.) - 6. Rundfunkentscheidung.

376 Eine detailliertere Darstellung findet sich u.a. bei Grabenwarter, in: Maunz/Dürig, GG, Art. 5 Abs. 1, $2 \mathrm{Rn} .540 \mathrm{ff}$

377 In der 5. Entscheidung, BVerfGE 74, 297, ging es um den verfassungswidrigen Ausschluss des öffentlich-rechtlichen Rundfunks von der Veranstaltung regionaler Sendungen und Programme. BVerfGE 97, 228 - Kurzberichterstattung, stellte klar, dass Art. 5 Abs. 1 S. 2 GG weitestgehend legitimiere, die Kurzberichterstattung von öffentlichen Veranstaltungen zuzulassen. Art. 12 Abs. 1 GG mache davon aber punktuell Ausnahmen, etwa in Hinblick auf die Unentgeltlichkeit, a.a.O. (262 f.), oder auf einzuhaltende Karenzzeiten, bspw. bei der Berichterstattung über Fußballspiele, a.a.O. (261). Durch BVerfGE 121, 30 (50 ff.) wurde das gesetzliche Verbot der Parteibeteiligung an Rundfunkunternehmen, durch die sie einen bestimmenden Einfluss auf die Programmgestaltung nehmen können, gebilligt.

378 So ging es u.a. um die Zulässigkeit des Engagements auch auf regionaler Ebene (in Konkurrenz mit dem privaten Rundfunk) BVerfGE 74, 297 (331ff.) - 5. Rundfunkentscheidung. Die Bestands- und Entwicklungsgarantie, die den Gesetzgeber bindet, wurde angedeutet in BVerfGE 74, 297 (350 f.) 5. Rundfunkentscheidung und ausdrücklich so benannt in BVerfGE 83, 238 (298) - 6. Rundfunkentscheidung. Um die Gewährleistung einer Finanzierung ging es jedenfalls thematisch bereits in BVerfGE 83, 238 (310 f.) - 6. Rundfunkentscheidung, wobei das Gericht konkrete Fragen explizit offenließ. Die Grenzen der Finanzierung werden dann näher in BVerfGE 87, 181 (199ff.) - 7. Rundfunkentscheidung, aufgezeigt. Zur Ausgestaltung der Gebühr und damit verbundenen Fragen BVerfGE 90, 60 - 8. Rundfunkentscheidung. Die Gebührenfinanzierung war dann auch wieder Gegenstand von BVerfGE 119, 181 - Rundfunkfinanzierungsstaatsvertrag. Zur Besetzung der Aufsichtsgremien als Gewährleistung der Binnenpluralität, BVerfGE 136, 9 - ZDF-Staatsvertrag. Und jüngst zur Legitimität des Rundfunkbeitrags BVerfGE 149, 222 - Rundfunkbeitrag.

379 So schon der Ansatz für die Unterscheidung zwischen Rundfunk und Presse in BVerfGE 12, 205 (261) - 1. Rundfunkentscheidung, und dann vor allem BVerfGE 31, 314 (334) - 2. Rundfunkentscheidung.

380 BVerfGE 31, 314 (334) - 2. Rundfunkentscheidung; 87, 181 (199) - 7. Rundfunkentscheidung.

381 So heißt es im Sondervotum der Richter, BVerfGE 31, 314 (338) - 2. Rundfunkentscheidung, „Das [gemeint ist das Verbot den Rundfunk dem Belieben Einzelner zu überlassen - Anm. d. Aut.] würde sich erst ändern, wenn allen interessierten Gruppen [...] eine Frequenz zugewiesen werden könnte $[\ldots]^{\text {“. }}$.

382 So führen sie weiter fort: „[...] und sich auf diese Weise ein Pluralismus der Meinungen und Anschauungen ähnlich wie im Bereich der Presse auch im Bereich von Rundfunk und Fernsehen herstellen ließe.“, BVerfGE, ebd. 
aber nicht gefolgt. In der Entscheidung zum Rundfunkfinanzierungsstaatsvertrag betonte es ausdrücklich, dass der Wegfall der Frequenzknappheit und das Entstehen eines Medienmarktes den Regelungsauftrag des Gesetzgebers nicht entbehrlich mache. ${ }^{383}$ Vielmehr resultiere der Regelungsauftrag aus der herausgehobenen Bedeutung des Rundfunks unter den Medien, die aus seiner Breitenwirkung, Aktualität und Suggestivkraft folge. ${ }^{384}$ Zudem bestünden neue Gefährdungen, denen zu begegnen sei. ${ }^{385}$ Insoweit blieb die rundfunkrechtliche Sonderdogmatik bis heute im Wesentlichen aufrecht erhalten. ${ }^{386}$

\section{Die verfassungsdogmatischen Aussagen des BVerfG}

Betrachtet man die Entscheidungen des BVerfG zur Rundfunkfreiheit, so kann man diese auf einige prägnante und wesentliche dogmatische Aussagen reduzieren: Die Rundfunkfreiheit gewährleistet zwar ein subjektiv-rechtliches Abwehrrecht für (potentielle) ${ }^{387}$ Rundfunkveranstalter. Dieses tritt aber weitestgehend hinter einer objektiv-rechtlichen Funktion zurück. ${ }^{388}$ Weil Rundfunk wesentliches Medium und Voraussetzung für den Kommunikationsprozess ist, müsse die Funktionsfähigkeit des Rundfunks insgesamt staatlich sichergestellt werden. Insoweit sieht das BVerfG mit dem Regelungsauftrag die Rundfunkfreiheit als ein normgeprägtes Grundrecht an, ${ }^{389}$ dass der gesetzlichen Ausgestaltung bedarf, ${ }^{390}$ wobei für den öffentlich-rechtlichen und den privaten Rundfunk nicht dieselben Anforderungen gelten. ${ }^{391} \mathrm{Zu}$ dieser Ausgestaltung sind die Länder verpflichtet, ${ }^{392}$ denen hierbei eine weite Einschätzungsprärogative zukommt. ${ }^{393}$ Maßnahmen zur Ausgestaltung stellen keine Eingriffe in die Rundfunkfreiheit dar, ${ }^{394}$ weshalb sie letztlich auch nicht an dem Schrankenvorbehalt des Art. 5 Abs. 2 GG zu messen sind. ${ }^{395}$ Weil aber auch ihnen belastende Wirkung zukommen kann, sind sie nur dann „verfassungsrechtlich nicht zu beanstanden, wenn sie geeignet sind, das

383 BVerfGE 119, 181 (214) - Rundfunkfinanzierungsstaatsvertrag.

384 BVerfGE 119, 181 (214f.) - Rundfunkfinanzierungsstaatsvertrag m.w.N.

385 Neben der zunehmenden Kommerzialisierung, zählt hierzu auch die zunehmende horizontale und vertikale Verflechtung auf den Medienmärkten, BVerfG, a.aO. (216 f.).

386 Vgl. zuletzt BVerfGE 149, 222 (260 ff.) - Rundfunkbeitrag und BVerfGE 136, 9 (28 ff.) - ZDFStaatsvertrag.

387 Hierzu BVerfGE 97, 298 (312 f.) - extra-radio; 121, 30 (55 f.) - Parteibeteiligung an Rundfunkunternehmen.

388 Vgl. Degenhart, in: BK-GG, Art. 5 Abs. 1, 2 Rn. 281 spricht von einer Überlagerung und von einem Zurückdrängen der individuell-abwehrrechtlichen Dimension Rn. 304.

389 So Beyerbach, in: Hornung/Müller-Terpitz, RechtsHb Social Media, 9 Rn. 7.

390 BVerfGE 97, 228 (266) - Kurzberichterstattung m.w.N. zur Judikatur.

391 BVerfGE 121, 30 (50) - Parteibeteiligung an Rundfunkunternehmen.

392 Grundlegend BVerfGE 12, 205 (223 ff.) - 1. Rundfunkentscheidung.

393 BVerfGE 121, 30 (50) - Parteibeteiligung an Rundfunkunternehmen, m.w.N. zur Judikatur.

394 Zur Abgrenzung von Ausgestaltungs- und Eingriffsgesetzen siehe etwa Grabenwarter, in: Maunz/ Dürig, GG, Art. 5 Abs. 1 Rn. 858.

395 So ausdrücklich BVerfGE 73, 118 (166) - 4. Rundfunkentscheidung. Siehe aber auch BVerfGE 97, 228 (267) - Kurzberichterstattung. 
Ziel der Rundfunkfreiheit zu fördern und die von Art. 5 Abs. 1 S. 2 GG geschützten Interessen angemessen berücksichtigen. “396

\section{Kritische Rezeption der Rechtsprechung}

Jedenfalls im Ergebnis wurde und wird dem BVerfG oftmals zugestimmt: Der Rundfunk ist regulierungsbedürftig. ${ }^{397} \mathrm{Im}$ Laufe der Jahre mehrten sich aber die Stimmen, die - auch angesichts des erstarkten Wettbewerbs im Rundfunkbereich - ${ }^{398}$ an der Rechtsprechung zweifelten. ${ }^{399}$ Mittlerweile dürfte die Rundfunkfreiheit Gegenstand einer der kontroversesten grundrechtsdogmatischen Diskussionen sein. ${ }^{400}$

Bis heute kann die Dogmatik des Bundesverfassungsgerichts nicht überzeugend erklären, warum allein der Rundfunkfreiheit eine besondere Regelungspflicht zu entnehmen ist. Zwar kommt dem Rundfunk eine wesentliche Bedeutung für die Meinungsbildung zu. Vor allem durch fehlenden Wettbewerb und fehlende Vielfalt entstehen damit besondere Risiken, denen aus Sicht eines auf Offenheit, Transparenz und Pluralität basierenden demokratischen Kommunikationsprozess zu begegnen ist. ${ }^{401}$ Insoweit wertet man das kollidierende Allgemeininteresse am Rundfunk zum ausschlaggebenden Schutzzweck der Norm mit der

396 BVerfGE 97, 228 (267) - Kurzberichterstattung.

397 So etwa Klein, Staat 20 (1981), 177 (184); Fink, DÖV 1992, 805 (812); vgl. Degenhart, in: BK-GG, Art. 5 Abs. 1, 2 Rn. 304, der zumindest die Notwendigkeit gesetzgeberischer Ordnungsbefugnisse erkennt. Thum, DÖV 2008, 653 (657) meint, dass die objektiv-rechtliche Erweiterung sinnvoll und notwendig ist, aber die abwehrrechtliche Funktion nicht überlagern dürfte.

398 So weist Degenhart, in: Merten/Papier, HbGR $\$ 105$ Rn. 18, darauf hin, dass die ,ursprüngliche und tragende Rechtfertigung, die rundfunkrechtliche Sondersituation entfallen ist. “ Ähnlich Cornils, Ausgestaltung der Grundrechte, S. 84. Siehe auch Gersdorf, Legitimation und Limitierung von Online Angeboten, S. 57 und Klein, Staat 20 (1981), 177 (183). Besonders deutlich mit Blick auf die neue, digitale Herausforderung einer „Content Curation“ etwa Vesting, JZ 2020, S. 975 ff.

399 So etwa der NdsStGH ZUM-RD 2006, 321 (326), unter Hinweis auf die europäischen und völkerrechtlichen Vorgaben, i.E. aber offenlassend. Ausdrücklich kritisch hingegen Degenhart, in: Merten/ Papier, HbGR IV, $\mathbb{\$} 105$, Rn. 17 ff.; ders., in: BK-GG, Art. 5 Abs. 1, 2 Rn. 304 ff.; Gersdorf, Legitimation und Limitierung von Online-Angeboten, S. 31 f., 50 ff.; Hain, in: Spindler/Schuster, Elektronische Medien, C. Rn. 122-125; ders., Rundfunkfreiheit und Rundfunkordnung, S. 83 ff.; ders., JZ 2008, 128 (130f.); Hartmann, JZ 2016, 18 (21 ff.); Hillgruber, in: Isensee/Kirchhof, HbStR IX, $\$ 200$ Rn. 71; Klein, Staat 20 (1981), 177 (189 ff.) m.w.N. in den Fn. 42-52; Kühling, in: Gersdorf/Paal, Informations- und Medienrecht, Art. 5 GG Rn. 80, 94 ff.; Stern, in: ders., Staatsrecht, Bd. VI/1, S. 1655 ff. scheint dies zwar auch kritisch zu sehen, aber wegen der gefestigten Rspr. spricht er von einer Irreversibilität. Zustimmend aber, Kube, in: Isensee/Kirchhof, HbStR, $\mathbb{S} 91 \mathrm{Rn} .13$; Schmidt, ZRP 1980, 132 (135 f.) und wohl auch Hoffmann-Riem, ZRP 1976, 291 (292 f.). Und speziell im Kontext digitaler Medien(intermediäre) auch Heidtke, Meinungsbildung und Medienintermediäre, S. $206 \mathrm{ff}$.

400 Hartl, Suchmaschinen und Meinungsmacht, S. 87, bezeichnet es als eines der „beständigsten Diskussionsfeldern im Bereich der Grundrechte".

401 Ohnehin haben sich auch die tatsächlichen Gegebenheiten gewandelt. Die ursprüngliche Sondersituation kann nicht mehr ausgemacht werden. Und die besondere Suggestivkraft konnte bisher jedenfalls ebenso wenig ausgemacht werden, sodass das gesamte argumentative Konstrukt mehr oder weniger im „luftleeren Raum“ schwebt. Ausführlicher hierzu etwa Pille, Meinungsmacht sozialer Netzwerke, S. 231 ff. und ebenfalls kritisch hierzu Cornils, Designing platform governance, S. 21. 
Folge auf, ${ }^{402}$ dass die individuell-abwehrrechtliche Konzeption der Grundrechte hier punktuell aufgegeben wird ${ }^{403}$ und dem Gesetzgeber wesentliche Entscheidungen für die Verwirklichung des Grundrechts vorbehalten bleiben, ${ }^{404}$ ohne dass die Norm hierfür etwas hergeben würde: ${ }^{405}$ Schon der Wortlaut enthält keinen Anhaltspunkt für eine solche Sichtweise. Berichterstattung kann höchstens - was das BVerfG $^{406}$ aber zu Recht ablehnte - als inhaltliches Kriterium verstanden werden. Eine Ausgestaltungspflicht kann hier aber ebenso wenig hineingelesen werden ${ }^{407}$ wie in das Merkmal der Gewährleistung, welches sich auf alle Medienfreiheiten bezieht.

Aus systematischer und teleologischer Sicht gilt Ähnliches, denn anders als in Art. 14 Abs. 1 S. 2 GG kommt ein Ausgestaltungsauftrag hier nicht zum Vorschein. ${ }^{408}$ Warum zudem gerade die Rundfunkfreiheit nicht mehr bloß ein subjektiv-rechtliches Abwehrrecht, sondern auch eine objektiv-rechtliche Regelungsverpflichtung enthalten soll, kann nicht plausibel erklärt werden. Dass den Grundrechten objektiv-rechtliche Funktionen zukommen, ist richtig. Das gilt etwa allgemein für die Kommunikationsrechte, bei denen die demokratiefördernde Funktion betont wird. ${ }^{409}$ Daraus wird aber auch woanders keine Schranke für die subjektiv-rechtliche Funktion gezogen. ${ }^{410}$ Im Gegenteil: Im Falle der Meinungsfreiheit wirkt sich dies gerade schutzintensivierend aus. ${ }^{411}$ Und mit Blick auf die

402 Vgl. Gersdorf, Legitimation und Limitierung von Online-Angeboten, S. 53; Bremer/Esser/Hoffmann, Der Rundfunkbegriff in der Verfassungs- u. Wirtschaftsordnung in Deutschland, S. 42.

403 Kritisch deshalb etwa Bremer/Esser/Hoffmann, Der Rundfunkbegriff in der Verfassungs- u. Wirtschaftsordnung in Deutschland, S. 41 f.; Degenhart, in: BK-GG, Art. 5 Abs. 1, 2 Rn. 304, 643; Klein, Staat 20 (1981), 177 (178 f.); Thum, DÖV 2008, 653 (657). Gersdorf, Legitimation und Limitierung von Online-Angeboten, S. 53 spricht bei der verfassungsrechtlichen Annahme von einem „scharfen Kontrast“ zur herkömmlichen Dogmatik und von einem „Restbereich personaler Subjektivität.“

404 Vgl. hierzu auch Thum, DÖV 2008, 653 (656), der vorrangig die unzulässige Wahrnehmung parlamentarischer Rechte durch das BVerfG durch das Aufstellen wesentlicher Kriterien bemängelt, nachrangig aber auch die Gestaltung des Grundrechts durch den Gesetzgeber.

405 Vgl. Thum, DÖV 2008, 653 (657).

406 BVerfGE 12, 205 (260)-1. Rundfunkentscheidung.

407 Kritisch zu dem Versuch hierauf gestützt die Sonderdogmatik herzuleiten auch Schmitt, in: Donath/ Bretthauer u.a., 59. ATÖR - Verfassungen, S. 203 (208f.).

408 Schmitt, a.a.O., (209); Pille, Meinungsmacht sozialer Netzwerke, S. 230. Allerdings bezeichnet Hain, in: Spindler/Schuster, Elektronische Medien, 1. Teil C. Rn. 123, das als banales Argument.

409 Zur Meinungsfreiheit BVerfGE 7, 198 (208) - Lüth; zur Informationsfreiheit BVerfGE 27, 71 (81) Leipziger Volkszeitung; zur Versammlungsfreiheit siehe etwa nur BVerfGE69, 315 (343 f.) - Brokdorf; zur Pressefreiheit BVerfGE 20, 162 (174) - Spiegel; und mit Blick auf Art. 5 Abs. 1 GG insgesamt BVerfGE 20, 56 (97) - Parteifinanzierung I. Auf diesen Aspekt ebenfalls hinweisend Degenhart, in: Merten/Papier, HbGR IV, $\$ 105$ Rn. 18; ders., in: BK-GG, Art. 5 Abs. 1, 2 Rn. 304; Gersdorf, Legitimation und Limitierung von Online-Angeboten, S. 31. Und in der Lüth-Entscheidung wird gar der gesamten grundrechtlichen Ordnung eine objektive Funktion als Wertordnung beigemessen, die in alle Rechtsgebiete ausstrahlt, BVerfGE 7, 198 (204 ff.) - Lüth.

410 Ähnlich auch Tief, Kommunikation auf Facebook, Twitter \& YouTube, S. 43.

411 Den Vergleich zur Meinungsfreiheit zieht auch Gersdorf, Legitimation und Limitierung von OnlineAngeboten, S. 31, 55. Kritisch zu dieser Unterscheidung auch Kull, in: Badura/Scholz, FS-Lerche, 1993, S. 663 (666). Und mit der Pressefreiheit vergleichend Schmitt, in: Donath/Bretthauer u.a., 59. ATÖR - Verfassungen, S. 203 (209). Thum, DÖV 2008, 653 (657) deutet an, dass ein objektiv-rechtliches Verständnis zulässig ist, wenn es die Wirkkraft verstärkt oder erweitert. Klein, Staat 20 (1981), 
Presse ergibt sich funktional kein Unterschied: Beide Medienfreiheiten haben eine wesentliche, unterstützende Funktion im Kommunikationsprozess. Die besondere Wirkmacht des Rundfunks und die damit einhergehenden Risiken für die Kommunikation können im Vergleich zur Presse zwar strengere Regelungen rechtfertigen und mögen den Rundfunk regelungsbedürftiger machen. Allein die Regelungsbedürftigkeit als tatsächlicher Moment ist aber kaum in der Lage, eine verfassungsrechtliche (inhaltlich präzise umrissene) Pflicht zu begründen. ${ }^{412}$ Vielmehr hat sich der Gesetzgeber trotz Regelungsbedürftigkeit an die grundrechtlichen Grenzen zu halten.

Auch eine völkerrechtsfreundliche Auslegung im Lichte von Art. 10 EMRK ${ }^{413}$ oder Art. 11 Abs. 2 EuGRC würde zu einem ausschließlich subjektiv-rechtlichen Verständnis führen. Zwar ist der Staat für den EGMR auch der „ultimate guarantor" für Pluralismus im Rundfunk ${ }^{414}$ und auch er erkennt objektiv-rechtliche Dimensionen der Meinungsfreiheit, speziell der Medienfreiheiten an ${ }^{415}$. Daraus folgt indes nicht die Überlagerung der subjektiv-rechtlichen Komponente. Maßnahmen zur Vielfaltssicherung werden vielmehr als Beschränkungen zum Schutze der Rechte anderer bzw. als notwendige Beschränkung für die demokratische Gesellschaft und nicht als Ausgestaltung angesehen, vgl. Art. 10 Abs. 2 EMRK. ${ }^{416}$

177 (186) sieht den Streit darin wurzelnd, ob Grundrechte entweder als subjektives Recht oder als objektiver Ordnungszusammenhang begriffen werden. „Je nachdem welcher Auffassung man folgt, haben die individualrechtlichen oder die objektivrechtlichen Elemente der Grundrechtsnormen prävalierenden oder dienenden Charakter; d.h. entweder verstärken die objektiven Komponenten der Grundrechte deren individualrechtlichen Kern oder umgekehrt: die Grundrechte [...] kommen nur im Rahmen ihnen objektivrechtlich vorgegebener Funktionszusammenhänge zum Tragen. " Dann kommen aber hinsichtlich Meinungsfreiheit und Pressefreiheit einerseits und Rundfunkfreiheit andererseits (scheinbar) unterschiedliche Ansätze zum Tragen. Schmidt, ZRP 1980, 133 (135) meint zwar, dass sich aus Art. 5 Abs. 1 S. 2 GG kein neues subjektives Recht ergibt, weil dies schon durch die Meinungsfreiheit ausreichend abgesichert sei. Das widerspricht aber der Stellung und auch der Formulierung vehement, so auch Klein, Staat 20 (1981), 177 (190 f.).

412 So auch Degenhart, in: BK-GG, Art. 5 Abs. 1, 2 Rn. 304.

413 Hierauf ebenfalls abstellend, Degenhart, in: Merten/Papier, HbGR IV, $\mathbb{} 105$ Rn. $19 \mathrm{ff}$ oder Tief, Kommunikation auf Facebook, Twitter \& YouTube, S. 44 f. Ausführlicher hierzu Gersdorf, Legitimation und Limitierung von Online-Angeboten, S. $59 \mathrm{ff}$.

414 EGMR, Urt. v. 24.11.993 - 13912/88 u.a. = EuGRZ 1994, 549 Rn. 34, 38 - Informationsverein Lentia u.a. v. Austria.

415 Siehe EGMR (GK), Urt. v. 23.09.1994 - 15890/89, Rn. 31 - Jersild, der betont, „that freedom of expression constitutes one of the essential foundations of a democratic society and that the safeguards to be afforded to the press are of particular importance. Whilst the press must not overstep the bounds set, (...) it is nevertheless incumbent on it to impart information and ideas of public interest. Not only does the press have the task of imparting such information and ideas: the public also has a right to receive them. Were it otherwise, the press would be unable to play its vital role of ,public watchdog'." Diese Grundsätze gelten, so der EGMR, auch für audio-visuelle Medien und damit für den Rundfunk.

416 Hierzu EGMR Urt. v. 24.11.993 - 13912/88 u.a. = EuGRZ 1994, 549 Rn. 34, 38 f. - Informationsverein Lentia u.a. v. Austria. Eine vergleichende Darstellung von BVerfG und EGMR findet sich bei Grote/Wenzel, in: Dörr/Grote/Marauhn, Konkordanzkommentar, Kap. 18 Rn. 22 f. und auch bei Thum, DÖV 2008, 653. Auf die völkerrechtliche, anders gelagerte Situation ebenfalls hinweisend Degenhart, in: Merten/Papier, HbGR IV, $\$ 105$ Rn. 20 und Hain, in: Spindler/Schuster, Elektronische Medien, Erster Teil C. Rn. 122. Deshalb wurde das staatliche Rundfunkmonopol in Österreich etwa 1994 mit folgender Begründung als ungerechtfertigter Eingriff eingestuft: „As a result of the technical 
Und letztlich soll auch noch auf die fehlende Praktikabilität in zwei Punkten ${ }^{417}$ hingewiesen werden: Es wird nicht nur eine Differenzierung zwischen Eingriff und bloßer Ausgestaltung notwendig. ${ }^{418}$ Darüber hinaus führt die Dogmatik auch zu einer breiten Diskussion über den Rundfunkbegriff, die vor allem durch die zunehmende Medienkonvergenz an Relevanz gewonnen hat. ${ }^{419}$

progress made over the last decades, justification for these restrictions [gemeint ist: das öffentlichrechtliche Monopol] can no longer today be found in considerations relating to the number of frequencies and channels available;" EGMR, Urt. v. 24.11.993 - 13912/88 u.a. = EuGRZ 1994, 549 Rn. 39 - Informationsverein Lentia u.a. v. Austria. Gleichwohl sehen Kaiser/Reiling, in: Unger/v. Ungern-Sternberg, Demokratie und künstliche Intelligenz, S. 85 (100), hier drin keinen Widerspruch.

417 Daneben speziell zu den Problemen, die sich jüngst in der Entscheidung des BVerfG zum Rundfunkbeitrag zeigten (BVerfGE 149, 222) und dadurch ergeben, dass das Konzept der gruppenorientierten Vielfalt auf digitale Intermediäre übertragen wird, auf die dieses aber nicht ohne Weiteres zutrifft, Vesting, JZ 2020, $975 \mathrm{ff}$.

418 Vgl. hierzu Hain, in: Spindler/Schuster, Elektronische Medien, Erster Teil C. Rn. 127. Kritisch deshalb etwa auch Pille, Meinungsmacht sozialer Netzwerke, S. $228 \mathrm{f}$.

419 Weil die Grenzen zwischen analoger Pressetätigkeit und digitaler Rundfunktätigkeit immer weiter im Internet verschwimmen, wird versucht, den Rundfunkbegriff zu modifizieren, oftmals um die negativen Regelungsfolgen, oder eine allzu weite Entfernung vom einfachrechtlichen Rundfunkbegriff zu vermeiden. Zentral lassen sich hierbei drei Strömungen ausfindig machen. Zwei davon versuchen den Rundfunkbegriff selbst auszulegen und sind durch ein enges bzw. weites Verständnis geprägt. Vertreter eines engen Verständnisses knüpfen dabei an das definitorische Merkmal der Darbietung an und laden dieses qualitativ auf. Teilweise wird eine publizistische bzw. meinungsbildende Relevanz (so Dörr, in: Dittmann/Fechner/Sander, Der Rundfunkbegriff im Wandel der Medien, S. 125; Ricker, AfP 1998, 437 [443]; Gersdorf, Rundfunkrecht, Rn. 91; ders., AfP 1995, 565 [569]), eine redaktionelle Aufbereitung (so etwa Holznagel, in: Hoeren/Sieber/derS. Multimedia-Recht, Teil 3 Rn. 63; ders., in: Spindler/Schuster, Elektronische Medien, $\mathbb{} 2$ RStV Rn.11; Jarass, AfP 1998, 133 [134f.]; SchulzeFielitz, in: Dreier, GG, Art. 5 Abs. 1, 2 Rn. 101; Schemmer, in: Epping/Hillgruber, Art. 5 Rn. 69; Grabenwarter, in: Maunz/Dürig, GG, Art. 5 Abs. 1, 2 Rn. 620; Ladeur, in: Paschke/Berlit/Meyer/Kröner, Medienrecht, 1. Teil 4. Abschnitt Rn. 75. In diese Richtung auch Papier/Schröder, epd Medien Nr. 60 [2010], 16 [18]; Dankert/Dreyer, K\&R 2017, 73 [74]), eine Vergleichbarkeit mit dem herkömmlichen Rundfunk, insbesondere mit den Kriterien der Aktualität, Suggestivkraft und Breitenwirkung (vgl. Beater, Medienrecht, Rn. 219. So wohl auch Klaes, ZUM 2009, 135 [141]), oder aber gar die Linearität des Angebots (so etwa Starck/Paulus, in: v. Mangoldt/Klein/Starck, GG, Art. 5 Rn. 252; Kube, in: Isensee/Kirchhof, HbStR, IV, $\mathbb{} 91$ Rn. 14.; Holznagel, AfP 2011, 532 [534 f.], der eine Abgrenzung zwischen Rundfunk und einer neu zu entwickelnden Internetdienstefreiheit ermöglichen will) gefordert. Vertreter eines weiten Rundfunkbegriffs knüpfen hingegen allein an den technischen Übertragungsweg an (so Paulus/Nölscher, ZUM 2017, 177 [178]; Schulz, in: Binder/Vesting, Rundfunkrecht, $\mathbb{} 2$ RStV Rn. 22; Hain, in: Spindler/Schuster, Elektronische Medien, C. Rn. 48. In diese Richtung auch Bethge, in: Sachs, GG, Art. 5 Rn. 90a f.; Grabenwarter, in: Maunz/Dürig, GG, Art. 5 Abs. 1, 2 Rn. 613 ff.; Wendt, in: v. Münch/Kunig, GG, Bd. 1, Art. 5 Rn. 57 f.; Stern, in: ders., Staatsrecht, Bd. VI/1, S. 1667 f., 1670 und speziell zum World Wide Web S. 1673 oder Ory, AfP 2011, 19). Eine davon zu unterscheidende Dritte Strömung versucht den Ausweg durch eine Weiterentwicklung der Medienfreiheiten selbst zu finden, entweder indem eine einheitliche Medienfreiheit (Kellner, Die Regulierung der Meinungsmacht von Internetintermediären, S. 110 ff.; in diese Richtung auch Kühling, in: Gersdorf/Paal, Informations- und Medienrecht, Art. 5 GG Rn. 76) oder zumindest eine eigenständige Internetfreiheit (Holznagel, AfP 2011, 532 [534]; ders./Schumacher, ZRP 2011, 74 [77]) geschaffen wird. Allgemein erweckt die Diskussion den Anschein, als würde man versuchen, die unliebsamen Rechtsfolgen der Rundfunkfreiheit bereits auf Tatbestandsseite zu umgehen. Dafür besteht aber selbst nach der verfassungsgerichtlichen Judikatur kein Grund. Das BVerfG hat betont, dass „[d]ie Ausgestaltung dieser [Rundfunk-]Ordnung [...] Aufgabe des Gesetzgebers [ist], der dabei einen weiten Spielraum, auch für Differenzierungen insbesondere nach der Regelungsart und Regelungsdichte, vorfindet [...].", so BVerfGE 121, 30 (50) - Parteibeteiligung an Rundfunkunternehmen. Eine solche Möglichkeit wird auch in der Literatur oftmals angenommen, so etwa Jarass, AfP 1998, 133 (133); Kresse/Heinze, AfP 1995, 574 (577f.); Castendyk/Böttcher, MMR 2008, 13 (15); Hain, in: Spindler/Schuster, Elektronische Medien, C. Rn. 48, 49; Dittmann, in: ders./Fechner/Sander, Rund- 
Einen Bruch der Dogmatik und dadurch begründete Schwierigkeiten ${ }^{420}$ könnte man anderweitig vermeiden: Der Rundfunk steht ebenso wie die Pressetätigkeit im Spannungsverhältnis zwischen individuellem Rechtsschutz und Allgemeininteresse, ${ }^{421}$ weil er in unmittelbarem Zusammenhang zur Informations- und Meinungsbildungsfreiheit der Rezipienten steht. ${ }^{422} \mathrm{Er}$ ist - insoweit ist dem BVerfG zuzustimmen - Forum und Faktor des Meinungsbildungsprozesses. Mit der Rundfunkfreiheit kollidieren dann aber gewichtige verfassungs- und insbesondere grundrechtlich geschützte Interessen, die - wie sonst üblich - auf Schrankenebene hätten berücksichtigt werden können, indem sie mögliche Begrenzungen rechtfertigen. ${ }^{423}$ So ließe sich dann auch der "dienenden Funktion“ des Rundfunks Rechnung tragen. Auf diesem Weg hätte der ursprüngliche Ansatz eines staatlichen Monopols wegen der bestehenden Frequenzknappheit auch begründet werden können: Das Allgemeininteresse an einer gesicherten pluralen Berichterstattung wiegt höher als die Freiheit des Einzelnen, Rundfunk zu veranstalten.

Legt man den Fokus in der Diskussion um die Regulierungsbedürftigkeit stärker auf die kollidierenden Interessen, so lässt sich damit auch ein Sonderweg vermeiden. Vielmehr stünde mit der aus der objektiv-rechtlichen Funktion der Grundrechte gewonnenen Schutzpflicht ${ }^{424}$ gerade ein Instrument zur Verfügung, welches für alle freiheitlichen Grundrechte eine staatliche Verantwortung begründen kann und ebenfalls im Einzelfall konkrete gesetzgeberischer Maßnahmen verlangt. ${ }^{425}$ Dass dieser gemeinsame Ansatz nicht von Anfang an verfolgt wurde, ist wohl dem Umstand geschuldet, dass die Sonderdogmatik des Rundfunks bereits entwickelt wurde, bevor überhaupt das BVerfG den Begriff der Schutzpflicht verwendet hat. Erst Mitte der 1970-er Jahre, also ein Jahrzehnt später, begann sich die Schutzpflichtendogmatik parallel zu der bereits näher ausdifferenzierten Rundfunkdogmatik zu entwickeln. Wenn Rundfunk aber „Faktor“ des Meinungsbildungsprozesses und damit wesentliche Voraussetzung für die Informations- und Meinungsbildungsfreiheit ist, dann könnte den Staat eine Schutz-

funkbegriff im Wandel, S. 19 (32 ff.) spricht diesbezüglich von einer Relevanztheorie. Jedenfalls andeutend Bullinger, in: Isensee/Kirchhof, HbStR VII, $\$ 163$ Rn. 150.

420 So auch Hain, in: Spindler/Schuster, Elektronische Medien, Erster Teil C. Rn. 130, mit Blick auf eine „konvergenztauglichen Fortentwicklung“.

421 Ähnlich Hain, Rundfunkfreiheit und Rundfunkordnung, S. 85 der die Interessen der Rezipienten an der Gestaltung der Rundfunktätigkeit anspricht.

422 Daraus ergibt sich letztlich der „dienende“ Charakter.

423 So auch Hain, in: Spindler/Schuster, Elektronische Medien, 1. Teil C. Rn. 126 hin; ders., JZ 2008, 128 (131); ders., Rundfunkfreiheit und Rundfunkordnung, S. 85. In diese Richtung auch Bremer/ Esser/Hoffmann, Rundfunk in der Verfassungs- und Wirtschaftsordnung in Deutschland, S. 42; Klein, Staat 20 (1981), 177 (184, 196); Hindelang, Freiheit und Kommunikation, S. 191. Vgl. auch Cornils, Ausgestaltung der Grundrechte, S. 72. Kritisch zu einem solchen Ansatz Fink, DÖV 1992, 805 (812).

424 Dazu ausführlich sogleich unter B.

425 Auf diese Schutzpflichtendimension weisen auch Schmitt, in: Donath/Bretthauer u.a., 59. ATÖR Verfassungen, S. 203 (220) und Pille, Meinungsmacht sozialer Netzwerke, S. 212 ff., maßgeblich hin. 
pflicht dahingehend treffen, diese Funktion abzusichern. ${ }^{426}$ Wie noch aufzuzeigen sein wird, besteht auch mit Blick auf den durch das BVerfG eingeräumten Entscheidungsspielraum eine nicht zu verkennende Parallele von Rundfunk- und Schutzpflichtendogmatik. ${ }^{427}$ Das BVerfG hat bislang den Absprung für die Überführung der Rundfunk- in die Schutzpflichtendogmatik verpasst, sodass Sterns Annahme einer Irreversibilität ${ }^{428}$ durchaus berechtigt ist. Gleichwohl ändert das nichts daran, dass - unabhängig von der Frage, ob es sich bei Meinungsrobotern überhaupt um Rundfunk handelt - mit den Schutzpflichten ein deutlich überzeugenderes Konstrukt zur Begründung einer staatlichen Verantwortung besteht als mit der Rundfunkdogmatik.

\section{B. Grundrechtliche Schutzpflichten des Staates}

Die Anerkennung grundrechtlicher Schutzpflichten ist deshalb vor Schwierigkeiten gestellt, weil Grundrechte primär als Abwehrrechte gegen den Staat konzipiert sind, um einen möglichst großen Raum individueller Freiheit zu gewährleisten. ${ }^{429}$ Zwar ist es seit jeher Aufgabe des Staates, nicht nur die Freiheit der Bürger zu achten, sondern auch ihre Sicherheit zu gewährleisten. ${ }^{430}$ Allerdings wurde der Sicherheitsaspekt meist nur als rechtfertigendes, kollidierendes Interesse freiheitsbeschränkender Maßnahmen verstanden.

Auch wenn deshalb Sicherheit und Freiheit oftmals in einem Spannungsverhältnis stehen, so kommt dem Aspekt der Sicherheit auch eine freiheitssichernde Bedeutung zu: ${ }^{431}$ Nicht allein vom Staat gehen Gefährdungen für die grundrechtliche Freiheit aus. Insbesondere dort, wo die Ausübung grundrechtlicher Freiheit im privaten (Rechts-)Raum erfolgt - sei es bei der Suche nach Arbeit, ${ }^{432}$ bei

426 Diese Schutzpflicht betont etwa auch Schmitt, in: Donath/Bretthauer u.a., Verfassungen, S. 203 (220) und will damit den Abschied von der dienenden Freiheit einleiten. In diese Richtung (wohl) auch Wendt, in: v. Münch/Kunig, Art. 5 Rn. 50.

427 Hartl, Suchmaschinen, Algorithmen und Meinungsmacht, S. $141 \mathrm{ff}$. zeigt diese Parallele durchaus auf, erkennt dabei jedoch auch einen entscheidenden Unterschied: Während die Handlungspflicht bei der Dogmatik der Schutzpflicht „je nach Dringlichkeit der Gefahr“ besteht, geht die „Dogmatik der dienenden Freiheit von einer ständigen Gefahrenlage aus.“ Daneben siehe auch Hindelang, Freiheit und Kommunikation, S. 203 f. Ausführlich zur Schutzpflicht und der Frage der Dringlichkeit der Gefahr, 2. Teil, 2. Kap. A.I.1 und Kap. 3 A.III.4.

428 So Stern, in: ders., Staatsrecht VI/1, S. 1657.

429 Vgl. BVerfGE 7, 198 (204) - Lüth; 50, 290 (370) - Mitbestimmung; Klein, NJW 1989, 1633 (1639). Hierzu auch unter Einbeziehung der Handlungspflichten Classen, Staatsrecht II, $\$ 4$ Rn. $24, \mathbb{} 6$ Rn. 33. Kritisch zu einer solchen Betonung der abwehrrechtlichen Konzeption, Weiss, Privatisierung und Staatsaufgaben, S. $150 \mathrm{f}$.

430 Hierzu Isensee, in: ders./Kirchhof, HbStR IX, $\mathbb{S} 191$ Rn. 181 ff.; siehe etwa auch BVerfGE 49, 24 (56) - Kontaktsperre-Gesetz.

431 Vgl. Isensee, in: ders./Kirchhof, HbStR IX, $\mathbb{1 9 1}$ Rn. 183.

432 Siehe hierzu BVerfGE 84, 133 (146) - Warteschleife. 
der Ausübung des religiösen Bekenntnisses im Arbeitsverhältnis, ${ }^{433}$ der Ausübung der Meinungsfreiheit in digitalen Kommunikationsräumen, ${ }^{434}$ der Ausübung der informationellen Selbstbestimmung im digitalen Zeitalter ${ }^{435}$ oder dem Übersenden von vertraulichen Informationen auf postalischem oder digitalem Wege ${ }^{436}$ gehen wesentliche Gefährdungen nicht von staatlicher Seite, sondern von privater Seite aus. ${ }^{437} \mathrm{Da}$ aber den Grundrechten (grundsätzlich) ${ }^{438}$ keine unmittelbare Wirkung gegenüber Privaten zukommt, ${ }^{439}$ wären die Grundrechte in ihrer Wirkung eingeschränkt ${ }^{440}$ und der Einzelne der Macht privater Dritter schutzlos ausgeliefert, ${ }^{441}$ sofern nicht der Gesetzgeber im Rahmen seiner Prärogative freiwillig einfach-rechtliche Schutzvorkehrungen trifft.

\section{Grundlagen grundrechtlicher Schutzpflichten}

Das grundlegende Bedürfnis einer Erweiterung der Grundrechte über bloße Abwehrrechte hinaus gerade mit Blick auf das Verhältnis Privater zueinander hat das BVerfG bereits in der Lüth-Entscheidung erkannt. Dort erstreckte es die Wirkung der Grundrechte auch auf private Rechtsbeziehungen. Denn Grundrechte seien nicht bloß subjektive Rechte, sondern stellten auch eine objektive Wertord-

433 Hierzu, allerdings aus europäischer Sicht, EuGH, Urt. v. 14.03.2017 - C-188/15 = EuZW 2017, 483 - Bougnaoui/Micropole SA und Urt. v. 14.03.2017 - C-157/15 = NJW 2017, 1087 - Achbita/G4S Secure Solutions NV.

434 Zur (mittelbaren Drittwirkung) der Meinungsfreiheit in sozialen Netzwerken und verschiedenen Fällen einer Löschung oder Sperrung von Inhalten bzw. Nutzerprofilen siehe u.a. OLG Hamm, Beschl. v. 15.09.2020 - 29 U 6/20 = GRUR-RS 2020, 25382; OLG Nürnberg, Urt. v. 04.08.2020 - 3 U 3641/19 = GRUR-RS 2020, 23456; OLG Dresden, Beschluss vom 07.04.2020 - 4 U 2805/19 =MMR 2020, 626; OLG München, Urt. v. 18.02.2020 - 18 U 3465/19 Pre = NJW-RR 2020, 1171 oder OLG Oldenburg, Urt. v. 01.07.2019 13 W 16/19 = MMR 2020, 41.

435 Auch hier aus europäischer Sicht etwa zur Übermittlung von Daten ins Ausland EuGH, Urt. v. 16.07.2020 - C-311/18 =NJW 2020, 2613 - Schrems II.

436 Denn zumindest mit der Privatisierung der Deutschen Post hat Art. 10 GG in seiner abwehrrechtlichen Wirkung an Bedeutung verloren und die Schutzdimension an Bedeutung gewonnen, hierzu Turner, in: Maunz/Dürig, GG, Art. 10 Rn. 76, 80; Ogorek, in: Epping/Hillgruber, BeckOK GG, Art. 10 Rn. 74. Insbesondere $\mathbb{} 39$ PostG ist nun Ausdruck der staatlichen Schutzverantwortung.

437 Ähnlich Hesse, in: FS Mahrenholz, S. 541 (544); Herdegen, in: Maunz/Dürig, GG, Art. 1 Abs. 3 Rn. 23.

438 Allein Art. 9 Abs. 3 S. 2 GG kann eine explizit angeordnete horizontale Wirkung entnommen werden.

439 Ursprünglich war die Frage einer unmittelbaren Drittwirkung streitig. So hatte insbesondere Nipperdey, DVBl. 1958, 445 (446 ff.) und auch das BAG eine unmittelbare Drittwirkung für bestimmte Grundrechte angenommen, für Art. 5 Abs. 1, Art. 3 Abs. 3 GG, BAGE 1, 185 (193 ff.); 24, 438 (441); für Art. 6 Abs. 1, 2 GG BAG NJW 1957, 1688 (1689). Zu einer unmittelbaren Drittwirkung siehe auch Böckenförde, Staat 29 (1990), 1 (11). Seit BVerfGE 7, 198 (205) - Lüth, ist aber allgemein anerkannt, dass die Grundrechte nur mittelbar zwischen Privaten wirken können. Aus der Literatur siehe nur Herdegen, in: Maunz/Dürig, GG, Art. 1 Abs. 3 Rn. 113; Hillgruber, in: Epping/ders., BeckOK GG, Art. 1 Rn. 72; Hermes, NJW 1990, 1764 f. Umfangreicher zur Frage der Drittwirkung der Grundrechte v. Münch/Kunig, in: dies., GG, Vorb. Art. 1-19 Rn. 15 ff.; Classen, AöR 122 (1997), $65 \mathrm{ff}$.

440 Hierzu Isensee, in: ders./Kirchhof, HbStR IX, $\$ 191$ Rn. 177.

441 Vgl. Klein, NJW 1989,1633; Isensee, in: ders./Kirchhof, HbStR IX, $\mathbb{S} 191$ Rn. 177. 
nung auf, die die Geltungskraft der Grundrechte verstärkt und für alle Bereiche des Rechts gilt. ${ }^{442}$ Von ihnen gingen Richtlinien und Impulse für den Staat aus. ${ }^{43}$ Deshalb hätten auch die Gerichte bei Anwendung und Auslegung des einfachen Rechts die Grundrechte im Verhältnis Privater zu berücksichtigen, was vor allem bei Generalklauseln, den „Einbruchsstellen“ der Grundrechte, relevant wird. ${ }^{44}$ Auch wenn dies durchaus plausibel und eine mittelbare Drittwirkung mittlerweile allgemein anerkannt ist, so führen die abstrakten Ausführungen des BVerfG letztlich zu einer Beachtung der Grundrechte in privaten Rechtsverhältnissen in zwei Fällen. In Abhängigkeit vom Ursprung der freiheitsbelastenden Wirkung kann entweder der Staat Urheber einer Freiheitsbeeinträchtigung sein oder aber der Private. In beiden Fällen sind Grundrechte zu beachten, wobei aber in der ersten Konstellation unmittelbar die Grundrechte als Abwehrrechte gefordert sind und in der zweiten die Gerichte die Grundrechte in einer Schutzfunktion zu beachten haben. ${ }^{445}$ Insofern liegt schon der objektiven Wertordnung eine gewisse Schutzfunktion zugrunde.

Deshalb ist es nicht verwunderlich, dass das BVerfG auf Basis dieser objektiven Wertordnung die schon für die Judikative enthaltene Schutzdimension auch auf die Staatsgewalt insgesamt erstreckte. Wesentliche Vorarbeiten hierfür wur-

442 BVerfGE, a.a.O (205) - Lüth.

443 BVerfGE, ebd.

444 BVerfGE, a.a.O (206).

445 So lag der Lüth-Entscheidung wegen des begehrten und von den Gerichten bewilligten Äußerungsverbotes eine abwehrrechtliche Konstellation zugrunde, so auch Canaris, Grundrechte und Privatrecht, S. 37; Alexy, Theorie der Grundrechte, S. 486 f. und Classen, Staatsrecht II, $\mathbb{3}$ Rn. 66. In anderen Konstellationen, etwa bei nachteiligen Wirkungen eines Vertrages (so etwa in BVerfGE 89, 214 Bürgschaftsverträge), bei einem Stadionverbot (so etwa in BVerfGE 148, 267), oder der Sperrung eines Netzwerkprofils (BVerfG NJW 2019, 1935 - III. Weg), ging es allein um eine Schutzwirkung der Grundrechte gegenüber diesem Dritten und damit um den eigentlichen Anwendungsfall der mittelbaren Drittwirkung der Grundrechte. Insoweit kommt also in der mittelbaren Drittwirkung bereits eine staatliche Schutzpflicht der Judikative zum Tragen, so letztlich auch Canaris, Grundrechte und Privatrecht, S. 38; Armbrüster, in: MüKo-BGB, $\$ 134$ Rn. 34. Siehe auch Alexy, Theorie der Grundrechte, S. 485. Diese Differenzierung ergibt sich letztlich mittelbar auch aus der Judikatur des BVerfG. Während bei Lüth und in ähnlich gelagerten Fällen (etwa BVerfGE 86, 1 - TITANIC; 97, 125 (144ff.) - Caroline von Monaco I) keine gesonderte Argumentation für die Berücksichtigung der Grundrechte - abseits der allgemeinen Ausführungen zur objektiven Wertordnung - erfolgte, begnügt sich das Gericht in anderen Fällen hiermit nicht. Vielmehr bedarf es eines besonderen Umstandes, warum die Grundrechte zu berücksichtigen sind. Bei der Vertragsautonomie war das etwa ein starkes Übergewicht einer Vertragspartei (BVerfGE 89, 214 (234ff.)). In der Stadionverbotsentscheidung hat das BVerfG für die Relevanz von Art. 3 Abs. 1 GG gerade die besondere Konstellation hervorgehoben, dass ein Privater „eine Veranstaltung aufgrund eigener Entscheidung einem großen Publikum ohne Ansehen der Person [...][zur Verfügung stellt] und [...] für die Betroffenen in erheblichem Umfang über die Teilnahme am gesellschaftlichen Leben entscheidet. Indem ein Privater eine solche Veranstaltung ins Werk setzt, erwächst ihm von Verfassungswegen auch eine besondere rechtliche Verantwortung.“, BVerfGE 148, 267 (283 f.). Im Beschluss zum Bierdosenflashmob, BVerfG NJW 2015, 2485 Rn. 5, knüpfte das BVerfG an das „öffentliche Forum“ aus BVerfGE 128, 226 (252 f.) - Fraport, an, um eine besondere Verpflichtung der Eigentümerin zu begründen. Und bei einem Ausschluss von Facebook hat das BVerfG jedenfalls auf die Bedeutung des Netzwerks und auf andere Kriterien hingewiesen, BVerfG, NJW 2019, 1935 Rn. 15 - III. Weg. In allen Fällen ging es aber um eine Schutzpflichten-Konstellation. 
den bereits im Hochschul-Urteil geleistet. Weil die Grundrechte eine objektive Wertordnung aufstellen, die maßgeblich für die gesamte Rechtsordnung ist und die Geltungskraft der Grundrechte verstärken soll, ist es nicht nur Aufgabe des Staates, staatliche Eingriffe zu unterlassen. ${ }^{446}$ „[S]ie schließt vielmehr das Einstehen des Staates [...] für die Idee einer freien Wissenschaft und seine Mitwirkung an ihrer Verwirklichung ein und verpflichtet ihn, sein Handeln positiv danach einzurichten, d.h. schützend und fördernd einer Aushöhlung dieser Freiheitsgarantie vorzubeugen. " 447 Auch wenn hier - ebenso wie in der Entscheidung zum numerus clausus $-{ }^{448}$ eher Leistungsansprüche gegen den Staat auf Bereitstellung notwendiger Mittel begründet wurden, so ging es im Ergebnis gleichwohl um einen Anspruch auf staatlichen Schutz.

Mit der ersten Entscheidung zum Schwangerschaftsabbruch erweiterte das BVerfG diesen Schutzaspekt unter Hinweis auf die objektive Wertordnung erstmals auch auf ein tripolares Verhältnis, in dem die Gefahren von einem Privaten (der Mutter) her drohten: Art. 2 Abs. 2 S. 1 GG enthält eine staatliche Schutzpflicht, die den Staat verpflichtet, „sich schützend und fördernd vor [das] Leben zu stellen, das heißt vor allem, es auch vor rechtswidrigen Eingriffen von Seiten anderer zu bewahren. An diesem Gebot haben sich die einzelnen Bereiche der Rechtsordnung, je nach ihrer besonderen Aufgabenstellung, auszurichten. “449 Diese allgemeine staatliche Schutzpflicht wurde daneben auch unter Hinweis auf das explizit normierte Schutzgebot in Art. 1 Abs. 1 S. 2 GG begründet. Die darauffolgenden Entscheidungen bestätigten diese Schutzpflicht zunächst nur hinsichtlich der Rechtsgüter des Art. 2 Abs. 2 S. 1 GG. ${ }^{450}$ Angesichts des zuvor vom BVerfG betonten Zusammenhangs mit der Menschenwürde - und damit mit dem explizit normierten Schutzgebot des Art. 1 Abs. 1 S. 2 GG - mag das auch verständlich sein. Im Verlauf verschob sich die Begründung aber immer mehr auf die dem Abwehrrecht zur Seite stehenden objektiv-rechtlichen Funktionen, ${ }^{451}$ und zunehmend hat das BVerfG auch für andere grundrechtliche Schutzgüter Schutzpflichten anerkannt, denen kein vergleichbar enger Bezug zur Menschenwürde zukommt. ${ }^{452}$ Insoweit ist der Verweis des BVerfG auf Art. 1 Abs. 1 S. 2 GG auch

\footnotetext{
446 BVerfGE 35, 79 (114) - Hochschul-Urteil.

447 BVerfGE, ebd.

448 BVerfGE 33, 303 (330 f.) - numerus clausus I.

449 BVerfGE 39, 1 (42) - Schwangerschaftsabbruch I.

450 BVerfGE 46, 160 (164) - Schleyer; 49, 89 (141f.) - Kalkar I; 53, 30 (57) - Mühlheim-Kärlich; 56, 54 (73) - Fluglärm.

451 Angefangen mit BVerfGE 49, 89 (141f.) - Kalkar I und dann fortgesetzt in BVerfGE 53, 30 (57) Mühlheim-Kärlich; 56, 54 (73) - Fluglärm; 121, 317 (356) - Rauchverbot; 142, 313 (337) Zwangsbehandlung. Lediglich in BVerfGE 88, 203 (251) - Schwangerschaftsabbruch II, wies das BVerfG erneut und explizit auf Art. 1 Abs. 1 S. 2 GG hin, was aber auch daran liegt, dass mit der Abtreibung gerade auch die Menschenwürdegarantie deutlicher tangiert war als in den anderen Fällen. Entsprechendes gilt auch für BVerfGE 115, 118 (152) - Luftsicherheitsgesetz.

452 Siehe - ohne Anspruch auf Vollständigkeit - zu Art. 2 Abs. 1 (Privatautonomie) BVerfGE 81, 242 (254 f.) - Handelsvertreter; 103, 89 (100 f.) - Unterhaltsverzichtsvertrag; 114, 1 (34) - Bestandsüber-
} 
nur als Hinweis auf die spezifische Nähe zur Menschenwürde im konkreten Fall zu deuten, ohne dabei aber die Schutzpflichten hieraus (abschließend) herleiten zu wollen.

Sicherheit ist also nicht mehr nur eine objektive Aufgabe des Staates in Ausformung der grundrechtlichen Schranken, ${ }^{453}$ sondern auch wesentliche Voraussetzung der Verwirklichung grundrechtlicher Freiheit selbst und in jedem Grundrecht angelegt, weshalb auch ein subjektiv-rechtlicher Anspruch auf staatlichen Schutz der Grundrechte besteht. ${ }^{454}$ Trotz anfänglicher Kritik ${ }^{455}$ und verschiedener anderer, teils ergänzender Begründungsansätze, ${ }^{456}$ besteht mittlerweile Einigkeit

tragung Lebensversicherung; 114, 73 (90) - Lebensversicherung mit Überschussbeteiligung; BVerfG (K), MMR 2007, 93; zu Art. 2 Abs. 1 GG i.V.m. Art. 1 Abs. 1 GG (allgemeines Persönlichkeitsrecht) BVerfGE 96, 56 (64) - Vaterschaftsauskunft; zur informationellen Selbstbestimmung BVerfG (K), MMR 2007, 93. Zu Art. 4 GG BVerfG (K), NVwZ 2001, 908; BVerfGE 125, 39 (78 ff.) - Adventssonntage Berlin; zu Art. 12 Abs. 1 BVerfGE 92, 26 (46) - Zweitregister; zuvor schon angedeutet in BVerfGE 84, 133 (146f.) - Warteschleife, BVerfGE 149, 126 Rn. 47 ff. - Teilzeit- und Befristungsgesetz m.w.N. auf die Judikatur; zu Art. 14 BVerfGE 114, 73 (90) - Lebensversicherungen mit Überschussbeteiligung; BVerfG, NJW 2018, 2312 Rn. 31 - Fliegerhorst Büchel. Und dort, wo bislang eine entsprechende Judikatur fehlt, ist dies eher einer fehlenden Möglichkeit denn einer fehlenden Bereitschaft geschuldet, so Gerbig, Grundrecht auf staatlichen Schutz, S. 55.

453 So etwa in BVerfGE 2, 118 (120) - Sicherungsverwahrung; 7, 377 (414f.) - Apotheken-Urteil; 9, 338 (346) - Hebammen; 23, 50 (57) - Nachtbackverbot I.

454 BVerfGE 125, 39 (78) - Adventssonntage Berlin: „Aus Grundrechten ist vielmehr auch eine Schutzpflicht des Staates für das geschützte Rechtsgut abzuleiten, deren Vernachlässigung von dem Betroffenen mit der Verfassungsbeschwerde geltend gemacht werden kann“, m.w.N. zur Judikatur. In der Literatur gibt es keine einheitliche Linie. Eine umfangreichere Darstellung dieses Problems findet sich bei Borowski, Grundrechte als Prinzipien, S. 373 ff. m.w.N.

455 Im Sondervotum der Richter Rupp-v. Brünneck und Simon wird die Schutzpflicht deshalb kritisch gesehen, weil die Grundrechte nicht mehr allein vor Eingriffen schützen, sondern als Grundlage für staatliche Eingriffe (durch den Gesetzgeber) dienen. Zudem wird auch die damit verbundene Verstärkung der Kompetenzen des BVerfG kritisiert, BVerfGE 39, 1 (73ff.) - Schwangerschaftsabbruch I. Zur Kritik insgesamt vgl. Isensee, in: ders./Kirchhof, HbStR IX, $\mathbb{1 9 1} \mathrm{Rn}$. $165 \mathrm{ff}$. mit entsprechenden weiteren Verweisen oder Calliess, in: Merten/Papier, HbG II, $\mathbb{} 44$ Rn. 8. Vor allem dem ersten Einwand erteilte das BVerfG in E 88, 203 (252f.) - Schwangerschaftsabbruch II eine Absage, wenn es das Verbot des Schwangerschaftsabbruchs als einzige Möglichkeit ansieht. Und dem zweiten Einwand begegnet das BVerfG durch einen weiten Spielraum des Gesetzgebers und einer zurückhaltenden Überprüfung seiner Entscheidungen, siehe hierzu nur BVerfGE 92, 26 (46) - Zweitregister.

456 Hierzu umfassend etwa Hermes, Grund und Grenzen staatlicher Schutzansprüche, S. 82ff.; Dietlein, Die Lehre von den grundrechtlichen Schutzpflichten, oder Unruh, Zur Dogmatik der grundrechtlichen Schutzpflichten, S.31ff. Überwiegend wird dem BVerfG in der Begründung über die objektive Wertordnung gefolgt (so u.a. Kahl, in: BK-GG, Bd. 1, Art. 1 Abs. 3 Rn. 177; Böckenförde, Staat 29 (1990), 1; vgl. Klein, NJW 1989, 1633 (1636); Dolderer, Objektive Grundrechtsgehalte, S. 196ff.; Robbers, Sicherheit als Menschenrecht, S. 186ff., unter Ergänzung durch einen vom Staat geschaffenen Vertrauenstatbestand; die Subjektivierung soll dann aufgrund einer sozialstaatliche Komponente erfolgen, ebd. S. 193f.; Hoffmann-Riem, JZ 2014, 53 (56); Stern, DÖV 2010, 241 (244) stützt sich auf Art. 1 Abs. 1 S. 2 GG und die Funktion als objektive Wertentscheidung) werden Schutzpflichten (ergänzend) auch aus dem Staatszweck der Gewährleistung von Sicherheit hergeleitet (grundlegend etwa Isensee, Grundrecht auf Sicherheit, S. 34ff.). Daneben wird - dogmatisch sauber - versucht, die Schutzpflicht aus Art. 1 Abs. 1 S. 2 GG zu gewinnen (so etwa Starck, in: ders., Praxis der Verfassungsauslegung, S. 70 ff.), wobei sich allerdings Probleme im Umfang ergeben, weil eine Begrenzung auf den jeweiligen in der Menschenwürde wurzelnden Kern der speziellen Grundrechte notwendig wäre (kritisch deshalb etwa Unruh, Zur Dogmatik der grundrechtlichen Schutzpflichten, S. 43f. oder Weiss, Privatisierung und Staatsaufgaben, S. 151ff.; ablehnend Robbers, Sicherheit als Menschenrecht, S. 188. Und Dietlein, Die Lehre von den grundrechtlichen Schutzpflichten, S. 66f. weist daraufhin, dass Art. 1 Abs. 1 S. 2 GG nur eine besondere Qualität enthält, weil dem Gesetzgeber - anders 
darüber, dass der Staat nicht nur eigene Eingriffe zu unterlassen hat, sondern ihn - jedenfalls hinsichtlich der freiheitsrechtlichen Rechtsgüter - ${ }^{457}$ auch Schutzpflichten treffen, ${ }^{458} \mathrm{kraft}$ derer er sich ,schützend und fördernd vor die [...] Rechtsgüter zu stellen [hat] “459. Im Verhältnis zu Privaten ist der Staat also

als bei den anderen Grundrechten - hier kein Ausgestaltungsspielraum im einfachen Recht verbleibt.) Teilweise wird die Schutzpflicht auch als Teil des Abwehrrechts verstanden und dies durch die staatliche Auferlegung einer Duldungspflicht des Betroffenen konstruiert: Wenn der Staat gefahrenbegründendes Verhalten Dritter erlaube, dann bürdet er den Betroffenen eine Duldungspflicht auf, kraft derer er selbst in die Grundrechte eingreift (vgl. etwa Murswiek, NVwZ 1986, 611; ders., Die staatliche Verantwortung für die Risiken der Technik, S. 89ff.; kritisch hierzu zu Recht etwa Unruh, Zur Dogmatik der grundrechtlichen Schutzpflichten, S. 46f.; Robbers, Sicherheit als Menschenrecht, S. $190 \mathrm{f}$. oder Dietlein, Die Lehre von den grundrechtlichen Schutzpflichten, S. 38ff. ). Und wieder andere sehen die Schutzpflicht als Kompensation" für die aus dem staatlichen Gewaltmonopol resultierende Friedenspflicht der Bürger an (siehe hierzu etwa Callies, JZ 2006, 321 (326); so im Ansatz etwa Dreier, in: ders., GG, Vorbem. Rn. 104; vgl. auch Klein, JuS 2006, 960; v. Kielmansegg, JuS 2009, 216 (217); kritisch zu diesem Begründungsansatz Klein, NJW 1989, 1633 (1636) und Weiss, Privatisierung und Staatsaufgaben, S. 162ff.). Insoweit findet sich wohl kein abschließender Anknüpfungspunkt und Begründungsansatz der Schutzpflichten. Vielmehr folgen diese einerseits aus der teilweisen Normierung im Verfassungstext, der Staatsaufgabe, Sicherheit - auch wegen des Gewaltmonopols herzustellen, dem Bedürfnis, die Grundrechte auch gegenüber Privaten zu schützen und damit aus objektiven Werten, die die Grundrechte aufstellen, so auch Ruffert, Vorrang der Verfassung und Eigenständigkeit des Privatrechts, S. 166 und Clausen, Das Verhältnis von Achtungs- und Schutzpflichten in Ausnahmesituationen, S. 61.

457 Bei gleichheitsrechtlichen Fragen wird dies indes schwieriger zu beurteilen sein, da kein Gleichbehandlungszwang zwischen Privaten besteht, siehe nur BVerfG NJW 2019, 3769 Rn. 6 - Hausverbot NPD-Funktionär. Allein in gewissen Konstellationen lässt sich hierüber nachdenken. Das BVerfG hat jüngst bei einem bundesweit geltenden Stadionverbot auch aus Art. 3 Abs. 1 GG Schutzfunktionen im Wege der mittelbaren Drittwirkung hergeleitet. Dies blieb aber auf Fälle beschränkt, in denen „einzelne Personen mittels des privatrechtlichen Hausrechts von Veranstaltungen ausgeschlossen werden, die von Privaten aufgrund eigener Entscheidung einem großen Publikum ohne Ansehen der Person geöffnet werden, und wenn der Ausschluss für die Betroffenen in erheblichem Umfang über die Teilhabe am gesellschaftlichen Leben entscheidet." Das war etwa dort nicht der Fall, wo es um die Beherbergung eines NPD-Funktionärs durch einen Hotelier ging, der ihm die Unterbringung verweigerte, hierzu BVerfG NJW 2019, 3769 - Hausverbot NPD-Funktionär. Denn hier handele es sich weder „um eine Veranstaltung, die in erheblichem Umfang über die Teilnahme am gesellschaftlichen Leben entscheidet, noch hat die Hotelbetreiberin eine Monopolstellung oder eine strukturelle Überlegenheit.“, ebd., Rn. 8. Daneben hält vor allem das AGG einen einfach-rechtlichen Diskriminierungsschutz bereit. Ausführlicher zur Frage einer Schutzpflicht für die Gleichheitsrechte etwa Gerbig, Grundrecht auf staatlichen Schutz, S. $57 \mathrm{ff}$.

458 Siehe etwa Böckenförde, Staat 29 (1990), 1 (12); Borowski, Grundrechte als Prinzipien, S. 369 f.; Dietlein, Die Lehre von den grundrechtlichen Schutzpflichten, S. 34 ff.; Gerbig, Grundrecht auf staatlichen Schutz, S. 33 ff., 83; Moritz, Staatliche Schutzpflichten gegenüber pflegebedürftigen Menschen, S. 96; Weiss, Privatisierung und Staatsaufgaben, S. 149; Calliess, JZ 2006, 321; Jaeckel, DVB1. 2011, 13 (14); Stern, DÖV 2010, 241 (244); Neubert, AöR 140 (2015), 267 (270 f.) m.w.N.; Canaris, AcP 184 (1984), 201 (225 f.); Hesse, in: FS-Mahrenholz, S. 541 (545); Classen, Staatsrecht II, $\$ 6$ Rn. 35; Epping, Grundrechte, Rn.124; Michael/Morlok, Grundrechte, Rn. 511. Zu alternativen Ansätzen einer Schutzpflicht und der Kritik hieran Callies, in: Merten/Papier, HbGR II, $\mathbb{\int} 44$ Rn. 12 ff. ders., JZ 2006, 321 (324). Umfassender auch Dietlein, Die Lehre von den grundrechtlichen Schutzpflichten, S. $35 \mathrm{ff}$. Aber nicht nur in Deutschland ist die Schutzfunktion der Grundrechte durch den Staat anerkannt. Auch der EGMR hat diese objektiv-rechtliche Deutung für die europäischen Menschenrechte übernommen, siehe nur EGMR, Urt. v. 16.03.2000 - 23144/93, Rn. 42 f. - Özgür Gündem/Türkei m.w.N. Und auch Art. 51 Abs. 1 S. 2 der EuGRC zielt mit der Verpflichtung, die Anwendung der Rechte zu fördern, auf Schutzpflichten der nach Satz 1 Gebundenen ab, hierzu Jarass, EuGRCh, Art. 51 Rn. 5.

459 Vgl. BVerfGE 39, 1, (1. LS, 42) - Schwangerschaftsabbruch I; 88, 203 (251) - Schwangerschaftsabbruch II zum Lebensrecht aus Art. 2 Abs. 2 GG. 
„Garant" ${ }^{460}$ für die Wahrnehmung grundrechtlicher Freiheiten. Kommt der Staat dieser „Garantenpflicht“ nicht nach, verletzt er selbst die Grundrechte. ${ }^{461}$ In jüngerer Vergangenheit gewinnt diese auch - bspw. hinsichtlich des eingeführten Masernimpfzwanges ${ }^{462}$, den Infektionsschutzmaßnahmen während der Covid-19Pandemie, ${ }^{463}$ Umweltschutzmaßnahmen ${ }^{464}$ oder allgemein angesichts des zu gewährenden grundrechtlichen Schutzniveaus wegen der zunehmenden Digitalisierung ${ }^{465}$ - zunehmend an Bedeutung. 466

\section{Voraussetzungen und Inhalt der Schutzpflichten}

Während die Voraussetzungen einer unzulässigen Beeinträchtigung der abwehrrechtlichen Dimension der Grundrechte mit der Trias aus Schutzbereich, Eingriff und Rechtfertigung feststehen, besteht ein solch dezidiertes Prüfprogramm für staatliche Schutzpflichten nicht. Am ehesten hat sich noch die - recht abstrakte - Differenzierung zwischen Tatbestand und Rechtsfolge etabliert. ${ }^{467}$ Insoweit trennt man also gedanklich zwischen zwei Fragen: Erstens, ob der Staat überhaupt - abstrakt - verpflichtet ist, Schutz zu gewähren und zweitens, was aus dieser Pflicht konkret folgt.

460 Kube, in: Isensee/Kirchhof, HbStR IV, $\mathbb{S} 91$ Rn. 30 sieht den Staat mit Blick auf die Internetkommunikation in der „Rolle des für die freiheitliche Kommunikationsordnung Gewährleistungsverantwortlichen“. Von Gewährleistungsverantwortung spricht auch Heilmann, MMR 2020, 162 (163).

461 Siehe nur BVerfGE 77, 170 (214) - Lagerung chemischer Waffen.

462 BT-Drs. 19/3452, jetzt in $\$ 20$ Abs. 8-13 InfSchG normiert. Hierzu siehe etwa Komp/Thrun, JA 2020, 195 ff.; Zuck, ZRP 2017, 118 (120) und vor allem auch BVerfG, NJW 2020, 1946 Rn. 15 - Masernschutzimpfung.

463 Zum Zusammenhang von Schutzpflicht und IfSchG etwa Kluckert, in: ders., Das neue Infektionsschutzrecht, $\$ 2$ Rn. $49 \mathrm{ff}$.

464 Hierzu etwa Voßkuble, NVwZ 2013, 1 (6 ff.) oder Bickenbach, JZ 2020, 168 (170 ff.).

465 Mit Blick auf die Ausnutzung von Sicherheitslücken von IT-Systemen durch staatliche Ermittlungsbehörden siehe etwa Derin/Golla, NJW 2019, 1111 (1114f.). Datenschutzrechtliche Fragen der Digitalisierung wurden (vorerst) auf europäischer Ebene mit dem Erlass der DS-GVO geklärt. Im Rahmen der 5G-Diskussion etwa lässt sich schon über das altbekannte Problem der Gefahren von Mobilfunkanlagen nachdenken, siehe hierzu bereits BVerfG NJW 2002, 1638 - Mobilfunkanlagen I; NVwZ 2007, 805 - Mobilfunkanlagen II, wo zwar die Grenzwerte nicht beanstandet wurden, weil aus wissenschaftlicher Sicht noch nicht verlässlich klar war, ob diese zum Schutz der Gesundheit ausreichend waren. Allerdings wurde deshalb die Beobachtungspflicht des Gesetzgebers betont. Zu den gesundheitlichen Risiken siehe u.a. Betzalel/Ishai/Feldman, Environmental Research 163 (2018), $208 \mathrm{ff}$. oder Di Ciaula, International Journal of Hygiene and Environmental Health 221 (2018), $367 \mathrm{ff}$. Und auch mit Blick auf autonomes Fahren stellen sich wesentliche Fragen, die Schutzpflichten berühren, etwa bei „Dilemmasituationen“, hierzu Steege, NZV 2019, 459 (463 f.).

466 So schon die Einschätzung von Hesse, in: FS-Mahrenholz, S. 541 (544); Gerbig, Grundrecht auf staatlichen Schutz, S. 15. In eine ähnliche Richtung geht auch Jarass, in: Badura/Dreier, FS-BVerfG, S. 35, der die Schutzpflichten als „größten Beitrag“ des BVerfG für die Grundrechtsdogmatik bezeichnet.

467 Statt vieler Isensee, in: ders./Kirchhof, HbStR IX, $\mathbb{1 9 1}$ Rn. 217. 


\section{Tatbestandliche Voraussetzungen}

Auf die erste Frage, wann Schutzpflichten überhaupt greifen, hat das Bundesverfassungsgericht bisher keine präzise Antwort gegeben; spezifische „Anspruchsvoraussetzungen“" wurden bislang nicht aufgestellt, weshalb eine Systematisierung schwerfällt. ${ }^{468}$ Notwendig wird zunächst vor allem die Abgrenzung des abwehrrechtlichen Anspruchs, staatliche Handlungen zu unterlassen, von dem positiven Anspruch, staatlichen Schutz gegenüber Dritten bereitzustellen. ${ }^{469}$ Soweit das Verhalten, gegen das Schutz begehrt wird, staatlichen Ursprungs bzw. dem Staat zurechenbar ist, ${ }^{470}$ aktiviert sich das Abwehrrecht. ${ }^{471}$ Droht die Gefahr von Seiten Dritter, ist der Schutzanspruch einschlägig.

Abseits klassischer Fälle der Gefahrenverursachung durch private Dritte kommen Schutzpflichten potentiell auch dort in Betracht, wo Gefahren durch Naturgewalten oder ausländische Staaten verursacht werden. ${ }^{472}$ Mit Blick auf natürliche Risiken kommt das jedenfalls dort in Betracht, wo es auch um von Menschen verursachte klimatische Folgen wie etwa beim Klimawandel geht. ${ }^{473}$ Und auch Gefahren, die von ausländischen Staaten drohen, können durchaus erfasst sein. ${ }^{474}$ Denn qualitativ besteht teilweise kein Unterschied zum Handeln der grundrechtsgebundenen inländischen Staatsgewalt oder zum Handeln privater Dritter. Im Gegenteil kann sich dies zu einer ganz erheblichen Gefährdung für

468 Dietlein, Grundrechtliche Schutzpflichten, S. 105 spricht insoweit von „systematischen Defiziten“.

469 Die dadurch entstehenden Abgrenzungsprobleme hat schon Dietlein, Grundrechtliche Schutzpflichten, S. 88 ff., besprochen und sollen hier nicht weiter behandelt werden. Eine solche Abgrenzungsschwierigkeit stellte sich etwa auch in BVerfG, NJW 2018, 2312 Rn. 28 ff., 44 f. - Fliegerhorst Büchel oder in BVerfGE 77, 170 (222) - Lagerung chemischer Waffen.

470 Nicht damit ist gemeint, dass der Staat durch ein fehlendes rechtliches Verbot der Handlung selbst Gefährder dadurch wird, dass er dem Betroffenen eine „Duldungspflicht“ aufbürdet. Zu dieser fehlerhaften Konstruktion der Schutzpflicht als Teil des Abwehrrechts siehe schon Fn. 456. Ausführlichere Kritik findet sich etwa bei Dietlein, Die Lehre von den grundrechtlichen Schutzpflichten, S. $37 \mathrm{ff}$.

471 BVerfG, Beschl. v. 04.09.2008 - 2 BvR 1720/03, Rn. 37 - juris. s. Vgl. hierzu auch BVerfGE 66,39 (57ff.) - Nachrüstung.

472 Insgesamt hierzu etwa Szczekalla, Grundrechtliche Schutzpflichten im deutschen und europäischen Recht, S. 276 ff. m.w.N.

$473 \mathrm{Zu}$ der Schutzpflicht hinsichtlich des Klimawandels siehe etwa Meyer, NJW 2020, 894 ff.; Buser, DVBl. 2020, 1389 (1391), der sich aber hier auch nur auf den menschengemachten Klimawandel bezieht und etwa Meteoriteneinschläge hiervon ausnehmen will. Zur Aktivierung allein bei „menschlicher Mitverursachung" und nicht bei rein natürlichen Risiken kommt auch Isensee, in: ders./Kirchhof, HbStR IX, $\mathbb{1} 191$ Rn. 243; Krings, Grund und Grenzen staatlicher Schutzansprüche, S. $217 \mathrm{ff}$. Bei Naturgewalten i.S.v. Naturkatastrophen ablehnend Dreier, in: ders., GG, Vorbem. Rn. 104. Wenn er sich aber darauf beruft, dass das private Handeln in derlei Konstellationen fehlt, ist das ein ZirkelschlusS. Es ist ja gerade fraglich, ob Schutzpflichten abschließend einen privaten Übergriff voraussetzen. Und aus Sicht des Schutzgutes macht es keinen Unterschied, ob etwa das Leben durch einen privaten oder durch Naturgewalten bedroht wird. Aufgrund einer schutzgutorientierten Sichtweise nimmt wohl Unruh, Zur Dogmatik der grundrechtlichen Schutzpflichten, S. 76, auch in derlei Fällen eine Schutzpflicht an. Und Dietlein, Die Lehre von den grundrechtlichen Schutzpflichten, S. 103, hält eine Differenzierung anhand der Gefahrenquelle für gänzlich unvereinbar. Weitere befürwortende Verweise finden sich etwa bei Krings, a.a.O., Fn. 223.

474 Umfangreiche Nachweise etwa bei Kleinlein/Rabenschlag, ZaöRV 2007, 1277 (1300) mit Fn. 136. 
die Bürger entwickeln. ${ }^{475}$ Das gilt etwa mit Blick auf die NSA-Affäre und den erheblichen Übergriff auf das informationelle Selbstbestimmungsrecht. ${ }^{476}$ Dass sich gegebenenfalls international politische Spannungen ergeben können, mag zwar gewichtig sein, allerdings ist das dann eher ein kollidierendes Interesse, welches auf Rechtsfolgenseite zu berücksichtigen wäre. ${ }^{477}$ Ohnehin können hier zumeist nur diplomatische Schritte unternommen werden. ${ }^{478}$ Das BVerfG hat Schutzpflichten zumindest bei einem gefahrenverursachenden Handeln eines ausländischen Staates, dass einen gewissen Inlandsbezug aufwies, ${ }^{479}$ angenommen, gewährte allerdings einen weiten politischen Spielraum. ${ }^{480}$

Aus inhaltlicher Sicht ist jedenfalls diskutabel, ob jedes risikobehaftete Handeln eines Dritten automatisch Schutzpflichten des Staates begründen kann. Denn im Ausgangspunkt macht auch ein Dritter von seinen grundrechtlich verbürgten Rechten Gebrauch. ${ }^{481}$ Die schwangere Frau, die sich entscheidet abzutreiben, übt ihr Selbstbestimmungsrecht, ${ }^{482}$ und der Betreiber eines angrenzenden, lärmintensiven Flughafens ${ }^{483}$ übt seine Eigentums- und Berufsfreiheit aus. Vergleichbares gilt für den Stadionbetreiber ${ }^{484}$ oder den Betreiber von sozialen Netz-

475 Dieses schutzgutorientierte Verständnis legt etwa auch Unruh, Zur Dogmatik der grundrechtlichen Schutzpflichten, S. 76, zugrunde.

476 Hier nimmt etwa Neubert, AöR 140 (2015), 267 ff. das Bestehen grundrechtlicher Schutzpflichten an. Ebenso Hoffmann-Riem, JZ 2014, 53 (56 f.).

477 So auch Dietlein, Zur Dogmatik der grundrechtlichen Schutzpflichten, S. 123. Nichts anderes galt letztlich auch in den Fällen, in denen Terroristen die Gefahrenverursacher waren und gewichtige politische bzw. strategische Belange einem Eingehen auf die erhobenen Forderungen im Wege standen, BVerfGE 46, 160 (164) - Schleyer.

478 Vgl. BVerfG NJW 2018, 2312 Rn. 45 f. - Fliegerhorst Büchel.

479 Das war nicht nur in BVerfG NJW 2018, 2312 Rn. 45 f. - Fliegerhorst Büchel, sondern auch in BVerfGE 77, 170 - Lagerung chemischer Waffen, und in BVerfGK 14, 192 (199) - Schloss Bensberg, der Fall. In den beiden ersten Fällen ging es jeweils um die Lagerung bzw. den Transport gefährlicher militärischer Waffen durch andere Staaten innerhalb des deutschen Staatsgebiets. Im Fall des Schlosses Bensberg um die Beanspruchung und verzögerte Räumung eines deutschen Grundstücks durch den belgischen Staat. Das OVG Münster, Urt. v. 19.03.2019 - 4 A 1361/15 = BeckRS 2019, 5666, hat die Schutzpflicht dann auch auf den Fall ausgedehnt, in dem ein auswärtiger Staat von dem im Hoheitsgebiet Deutschlands gelegenen Stützpunkt militärische Drohnen im Ausland betreibt. Das BVerwG hat das Urteil allerdings in der Revision geändert, BVerwG, Urt. v. 25.11.2020 - 6 C 7/19. Explizit sehen die Entscheidungen aber keine Beschränkungen auf allein inländische Sachverhalte vor. Insbesondere in BVerfG, Beschl. v. 04.09.2008 - 2 BvR 1720/03 = BeckRS 2010, 54358 Rn. 34 ist nur von einer „Pflicht [...] inländische Unternehmen vor Beeinträchtigungen durch fremde Staaten zu schützen“ die Rede, wobei Voraussetzung nur „die Schutzbedürftigkeit des Betroffenen“ sei. Allerdings sprach das BVerfG in BVerfGE 6, 290 (299) - Washingtoner Abkommen von der Schutzpflicht auch hinsichtlich im Ausland befindlicher Grundstücke. Für einen Inlandsbezug als Voraussetzung etwa Krings, Grund und Grenzen grundrechtlicher Schutzansprüche, S. 196, wobei aber auch schon die Staatsbürgerschaft ausreiche, ebd., S. 200 f. Gegenüber Drittstaaten ablehnend, Dreier, in: ders., GG, Vorbem. Rn. 104 und zur Kritik siehe auch Kleinlein/Rabenschlag, ZaöRV 2007, 1277 (1304).

480 Siehe nur BVerfG NJW 2018, 2312 Rn. 45 - Fliegerhorst Büchel, m.w.N.

481 Isensee, in: ders./Kirchhof, HbStR IX, $\mathbb{\$} 191 \mathrm{Rn} .220$ weist auf die Ambivalenz dieses Schutzeingriffes hin.

482 Vgl. BVerfGE 39, 1 (43 f.) - Schwangerschaftsabbruch I; 88, 203 - Schwangerschaftsabbruch II.

483 So etwa der Fall in BVerfGE 56, 54 - Fluglärm.

484 BVerfGE 148, 267 (281) - Stadionverbot. 
werken, der gestützt auf sein Hausrecht Dritte von der Nutzung ausschließt. ${ }^{485}$ Dort, wo die Ausübung der eigenen Freiheiten die Freiheitsrechte Dritter nicht oder nur unwesentlich berührt, wo es also um bloße Belästigungen oder Bagatellen geht, ist jedenfalls kein Raum für Schutzpflichten. ${ }^{486}$ Vielmehr kommt eine Schutzpflicht nur dort in Betracht, wo gegen den Willen des Betroffenen auf ein grundrechtliches Schutzgut eingewirkt wird. ${ }^{487}$ Insoweit handelt es sich also um eine „eingriffsäquivalente Lage“, die im Schrifttum mit dem Merkmal des „Übergriffs" beschrieben wird. ${ }^{488}$ Offensichtliche Fälle eines solchen Übergriffs liegen jedenfalls dann vor, wenn es bereits zu konkreten Störungen kommt, also Schäden bereits eingetreten sind. Aber auch schon bevor Schäden eintreten, greifen staatliche Schutzpflichten wegen ihres präventiven Schutzzwecks und wegen des ansonsten nur selten effektiv gewährleisteten Grundrechtsschutzes. ${ }^{489}$ Wie weit im Voraus staatliche Schutzpflichten bestehen, ist bisher aber noch nicht hinreichend geklärt.

\section{a. Einschränkungsversuche in der Literatur}

Die Diskussion um eine Restriktion des Tatbestandes wurde auch durch das BVerfG dadurch entfacht, dass es von einem "rechtswidrigen Eingriff“ sprach. ${ }^{490}$ Das führte im Schrifttum teilweise dazu, dass eine Überprüfung der Legitimität des Verhaltens bereits auf Tatbestandsebene gefordert wurde. ${ }^{491}$ Nur der mit der Verfassung unvereinbare Eingriff sei abzuwehren. ${ }^{492}$ Anderswo findet sich lediglich ein bloßer Verweis darauf, dass Schutzpflichten jedenfalls nicht durch „unerhebliche Gefahren“ begründet werden. ${ }^{493}$ Überwiegend wird allerdings eine

485 Hierzu vgl. BVerfG NJW 2019, 1935 - III. Weg. Eine ausführlichere Darstellung denkbarer Kollisionsfälle findet sich etwa bei Preu, JZ 1991, 265 (266).

486 Vgl. etwa BVerfGE 102, 347 (363) - Schockwerbung I, mit Blick auf „[e]in vom Elend der Welt unbeschwertes Gemüt des Bürgers“, BVerfGE 128, 226 (261) - Fraport, mit Blick auf sich aus „der Gruppenbezogenheit der Grundrechtsausübung [der Versammlungsfreiheit] ergeben[de] [Belästigungen]" oder BVerfG NJW 2011, 47, 1. Ls - Kritik an Abtreibungspraxis.

487 Isensee, in: ders./Kirchhof, HbStR IX, $\mathbb{1 9 1}$ Rn. 225; Gerbig, Grundrecht auf staatlichen Schutz, S. 60 .

488 Vgl. Callies, JZ 2006, 320 (326); Isensee, in: ders./Kirchhof, HbStR, XI, \$191 Rn. 225.

489 Vgl. Moritz, Staatliche Schutzpflichten gegenüber pflegebedürftigen Menschen, S. 110; Gerbig, Grundrecht auf staatlichen Schutz, S. 62.

490 So etwa bei BVerfGE 39, 1 (42) - Schwangerschaftsabbruch I; 46, 160 (164) - Schleyer; 53, 30 (57) Mühlheim-Kärlich.

491 Siehe hierzu etwa Isensee, in: ders./Kirchhof, HbStR XI, $\mathbb{1 9 1}$ Rn. 225 ff. Zumindest diese Richtung andeutend Hillgruber, in: Hoffmann/Knaup, Was heißt: In Würde sterben?, S. 115 (124). Zutreffende Kritik hiergegen findet sich aber schon bei Dietlein, Grundrechtliche Schutzpflichten, S. 106 oder auch bei Kämmerer, Privatisierung, S. 452.

492 Vgl. Isensee, in: ders./Kirchhof, HbStR IX, $\$ 191$ Rn. 227. Zu den Problemen des Begriffes und dem Rechtswidrigkeitsurteil siehe Hermes, Das Grundrecht auf Schutz von Leben und Gesundheit, S. 226 f. Ebenfalls kritisch Krings, Grund und Grenzen staatlicher Schutzansprüche, S. 233 und Kämmerer, Privatisierung, S. 452.

493 So etwa Schmidt-Aßmann, AöR 106 (1981), 205 (216). 
Parallele zum polizeirechtlichen Gefahrenbegriff gezogen. ${ }^{494}$ Demnach müsse eine Sachlage bestehen, die bei ungehindertem Verlauf mit hinreichender Wahrscheinlichkeit zu einem Schaden führt. ${ }^{495}$ Entscheidend für die Bestimmung der Gefahr sei das Produkt aus der Eintrittswahrscheinlichkeit und des Schadensausmaßes. ${ }^{496}$ Je größer der zu erwartende Schaden, desto geringer seien die Anforderungen an die Eintrittswahrscheinlichkeit. ${ }^{497}$ Damit wird vor allem versucht, die Risikovorsorge aus dem Anwendungsbereich herauszuhalten. So dürfe der Staat diese zwar verfolgen und sie stelle auch ein legitimes Ziel dar, sei aber nicht verfassungsrechtlich geboten. ${ }^{498}$ Dadurch könne der Schutzpflicht klare(re) Konturen verliehen werden, und man vermeide die Frage, ob und inwieweit sich im Bereich der Risikovorsorge subjektive Rechte begründen lassen. ${ }^{499}$

Anderswo wird dieser Ansatz scharf kritisiert. So sei es widersprüchlich, wenn teilweise dann doch auf wertende Elemente zurückgegriffen werde, um bestimmte (sozialadäquate) Gefahren wieder aus dem Anwendungsbereich herauszuhalten. ${ }^{500}$ Zudem sei der Gesetzgeber anders als die Exekutive kein Nothelfer, der im Einzelfall eingreife. ${ }^{501}$ Die zu erlassenden Schutzgesetze umfassen vielmehr verschiedene Szenarien, in denen auch kumulierte Risiken betrachtet werden müssen und bei denen es um eine (zeitlich) umfassendere Prognose geht. ${ }^{502}$ Gefahren mit bedenklichem Schadenausmaß entwickeln sich erst über Jahrzehnte hinweg. ${ }^{503}$ Eine Abgrenzung anhand des Gefährdungsniveaus berücksichtige dies nicht. Deshalb sei auch zwischen exekutiver Schutzpflicht, die durch den einfach-rechtli-

494 Neubert, AöR 140 (2015), 267 (272); Isensee, in: ders./Kirchhof, HbStR IX, \$191 Rn. 235; Hermes, Das Grundrecht auf Schutz von Leben und Gesundheit, S. 236; Murswiek, Die staatliche Verantwortung für die Risiken der Technik, S. 140 ff.; Epping, Grundrechte, Rn. 124; Schmidt am Busch, in: Kollmer/Klindt/Schucht, Arbeitsschutzgesetz, A. Rn. 29; Gärditz, in: Landmann/Rohmer, UmweltR, GG Art. 20a Rn. 86; Peine, in: Brauenschweigische Wissenschaftliche Gesellschaft, Jahrbuch 1999, S. 151 (153); Arndt/Droege, NVwZ 2003, 906 (908); Krings, Grund und Grenze staatlicher Schutzansprüche, S. 231; vgl. Hartl, Suchmaschinen, Algorithmen und Meinungsmacht, S. 144. Di Fabio, in: Maunz/Dürig, GG, Art. 2 Abs. 2 S. 1 Rn. 49 hält den Rückgriff jedenfalls für möglich.

495 Krings, Grund und Grenze staatlicher Schutzansprüche, S. 231. Zum polizeirechtlichen Gefahrenbegriff siehe etwa Kingreen/Poscher, Polizeirecht, $\mathbb{} 8$ Rn. 2. U.a. in $\mathbb{} 3$ Abs. 3 Nr. 1 SOG MV findet sich eine Legaldefinition des Gefahrenbegriffs.

496 Krings, ebd.; vgl. auch Hermes, Das Grundrecht auf Schutz von Leben und Gesundheit, S. 236 f. Ausführlicher zu dieser Produktformel Murswiek, Die staatliche Verantwortung für die Risiken der Technik, S. $165 \mathrm{ff}$.

497 Krings, ebd.; Murswiek, Die staatliche Verantwortung für die Risiken der Technik, S. 149 f.; Gärditz, in: Landmann/Rohmer, UmweltR, GG Art. 20a Rn. 86.

498 So Murswiek, Die staatliche Verantwortung für die Risiken der Technik, S. 144; Isensee, in: ders./ Kirchhof, HbStR IX, $\mathbb{1} 191$ Rn. 235. Vgl. auch Moritz, Schutzpflichten gegenüber pflegebedürftigen Menschen, S. 111; Dietlein, Die Lehre von den grundrechtlichen Schutzpflichten, S. 107.

499 Krings, Grund und Grenze staatlicher Schutzansprüche, S. 230.

500 So etwa Murswiek, Die staatliche Verantwortung für die Risiken der Technik, S. $141 \mathrm{f}$. Kritisch dazu Hermes, Das Grundrecht auf Schutz von Leben und Gesundheit, S. 238 f. und Dietlein, Die Lehre von den grundrechtlichen Schutzpflichten, S. 108.

501 Dietlein, Die Lehre von den grundrechtlichen Schutzpflichten, S. 108; Unruh, Zur Dogmatik der grundrechtlichen Schutzpflicht, S. 77.

502 Dietlein, ebd.; Unruh, a.a.O., S. 77.

503 Dietlein, ebd. 
chen Gefahrenbegriff oftmals vorgegeben ist, und der legislativen Gesetzgebungspflicht zu unterscheiden. ${ }^{504}$ Im letzteren Falle bestünde nämlich die eigentliche Problematik gar nicht darin, die Frage des Ob dezidiert zu klären. Wegen der eingeräumten Gestaltungsspielräume des Gesetzgebers werde das schutzpflichtenaktivierende Gefahrenniveau „erst dort relevant, wo sich die legislativen Gestaltungsspielräume [...] zu unabdingbaren Gesetzgebungspflichten ,auf Null' reduziert haben. "505 Insoweit seien die unterschiedlichen Gefahrenniveaus nicht auf Tatbestands-, sondern vielmehr auf Rechtsfolgenseite relevant. ${ }^{506}$ Diese Grenze sei aber spezifisch verfassungsrechtlich zu bestimmen. Der Gesetzgeber müsse in erster Linie Normen bereithalten, die die Exekutive dann zum Eingriff ermächtigt. Deshalb komme es für die Legislative weniger auf die Produktformel als vielmehr auf die Frage an, ob der Exekutive ein Eingriff bei Gefährdungen bereits ermöglicht werde. Insoweit sei auch die bestehende Rechtslage zu berücksichtigen. ${ }^{507}$

Moritz spricht sich zwar gegen eine Gefahrenschwelle aus, will aber auf Tatbestandsseite eine Abwägung im Einzelfall vornehmen, die „neben der Bedeutung des gefährdeten Rechtsguts, auch die Gefahrintensität, die möglichen Mittel sowie entgegenstehende Interessen “ berücksichtigt. ${ }^{508}$ Und anderswo werden überhaupt keine tatbestandlichen Restriktionen gefordert, sondern eine Differenzierung anhand des Gefährdungsniveaus allein auf Rechtsfolgenseite vorgenommen. ${ }^{509}$ So meint Gerbig, dass anhand einer am jeweiligen Schutzgut orientierten Wertung nur solche Sachverhalte zu identifizieren seien, „in denen die Freiheitsrechte einer zumindest vorstellbaren Bedrohung ausgesetzt sind. "510 Und auch nach Hermes können Gefahren, Risiken, Restrisiken oder das allgemeine Lebensrisiko Schutzpflichten auslösen. ${ }^{511}$ Ein verfassungsrechtliches Restrisiko gebe es folglich nicht.

504 Dielein, a.a.O., S. 109 ff. Ihm zustimmend etwa Clausen, Das Verhältnis von Achtungs- und Schutzpflicht in Ausnahmesituationen, S. $64 \mathrm{f}$.

505 Dietlein, Die Lehre von den grundrechtlichen Schutzpflichten, S. 112. In diese Richtung auch Hermes, Grundrecht auf Schutz von Leben und Gesundheit, S. 240.

506 Dietlein, ebd.

507 Ausführlich hierzu Dietlein, aa.O. S. $112 \mathrm{ff}$. In Anlehnung an ihn auch Clausen, Das Verhältnis von Achtungs- und Schutzpflicht in Ausnahmesituationen, S. 65.

508 Moritz, Schutzpflicht gegenüber pflegebedürftigen Menschen, S. 112.

509 So neben Gerbig, Grundrecht auf staatlichen Schutz, S. 62 f. und Hermes, Das Grundrecht auf Schutz von Leben und Gesundheit, S. 239 f. auch Heun, RW 2011, 376 (396). In diese Richtung auch Szczekalla, Grundrechtliche Schutzpflichten im deutschen und europäischen Recht, S. 304.

510 Gerbig, Grundrecht auf staatlichen Schutz, S. 64.

511 Hermes, Das Grundrecht auf Schutz von Leben und Gesundheit, S. 239 f.; Gerbig, Grundrecht auf staatlichen Schutz, S. 63. In diese Richtung tendenziell auch Heun, RW 2011, 376 (396). 


\section{b. Die Rechtsprechung des BVerfG:}

Das BVerfG hat sich nie ausdrücklich zu einer potentiellen Gefahrenschwelle als Voraussetzung der Schutzpflicht geäußert und damit auch nie dazu beigetragen, die von ihm selbst verursachten Ungewissheiten $\mathrm{zu}$ beseitigen. Soweit es zu Beginn selbst von unerheblichen Grundrechtsgefährdungen, ${ }^{512}$ sozialadäquaten Risiken ${ }^{513}$ oder rechtswidrigen Eingriffen ${ }^{514}$ gesprochen hat, war damit aber wohl nie eine Restriktion der Schutzpflicht gewollt. So hat das BVerfG etwa schon bloße Risiken und Realisierungstendenzen als erheblich eingestuft ${ }^{515}$ und ebenfalls als „Grundrechtseingriff“ bezeichnet. ${ }^{516}$ Und auch die Risikovorsorge hat es als „von der Schutzpflicht der staatlichen Organe umfasst“ angesehen. ${ }^{517}$ Die Sozialadäquanz eines Risikos wird allein bei der Frage relevant, ob ein absoluter Schutz notwendig ist oder ob nicht bestimmte Risiken geduldet und von allen getragen werden müssen (allgemeines Lebensrisiko), weil ansonsten etwa die Nutzung von Kernenergie ${ }^{518}$ oder die (kernphysikalische) Forschung 519 unmöglich wären. Insoweit ging es auch hier eher um eine Abwägung auf Rechtsfolgenseite. Und auch die Rechtswidrigkeit hat das BVerfG nie dezidiert geprüft. Vielmehr scheint es damit evidente Fälle zu kennzeichnen, wenn es heißt, dass Schutzpflichten „insbesondere“520 oder „vor allem“521 bei rechtswidrigen Eingriffen bestehen, was etwa bei Schwangerschaftsabbrüchen evident ist.

Eine Parallele zur polizeirechtlichen Gefahr kommt nicht nur in den vom BVerfG verwendeten Begriffen der Gefahr, ${ }^{522}$ Gefährdung $^{523}$ oder des Risikos ${ }^{524}$

512 So in BVerfGE 49, 89 (141) - Kalkar I; siehe auch BVerfG, NVwZ 2018, 1224 Rn. 43 - Fliegerhorst-Büchel.

513 Etwa in BVerfGE 49, 89 (143) - Kalkar I; BVerfG, NVwZ 2009, 171 (173) - Grafenrheinfeld; NvWZ 2009, 1489 Rn. 47 - Flughafen Leipzig/Halle; NVwZ 2010, 114 Rn. 23 - Schacht Konrad und NVwZ 2010, 702 Rn. 13 - CERN.

514 Siehe hierzu etwa BVerfGE 39, 1 (42) - Schwangerschaftsabbruch I; 46, 160 (164) - Schleyer; 53, 30 (57) - Müblheim-Kärlich.

515 BVerfG, NVwZ 2010, 702 Rn. 12 - CERN zur Realisierungstendenz und BVerfGE 49, 89 (141f.) Kalkar I zum Risiko. Auch andernorts spricht das BVerfG von der gebotenen „Risikovorsorge“, etwa in BVerfGE 53, 30 (58f.) - Mühlheim-Kärlich; 56, 54 (78) - Fluglärm.

516 Siehe zur (theoretisch herleitbaren) Gefährdung BVerfGE 49, 89 (141) - Kalkar I; 56, 54 (77, 78) Fluglärm; BVerfG, NVwZ 2010, 702 Rn. 12 - CERN. Zur Risikovorsorge BVerfGE 53, 30 (58f.) Mühlheim-Kärlich; 56, 54 (78) - Fluglärm.

517 BVerfG, NVwZ 2009,1489 Rn. 29 - Flughafen Leipzig/Halle m.w.N.

518 BVerfGE 49, 89 (142f.) - Kalkar I. Das Allgemeininteresse an dieser Nutzung betont das BVerfG auch in E 53, 30 (58) - Müblheim-Kärlich, allerdings nicht im Rahmen der Sozialadäquanz.

519 BVerfG, NVwZ 2010, 702 Rn. 13 - CERN.

520 BVerfGE 53, 30 (57) - Mühlheim-Kärlich.

521 BVerfGE 39, 1 (42) - Schwangerschaftsabbruch I.

522 Siehe etwa BVerfGE 88, 203 (252) - Schwangerschaftsabbruch II; 142, 313 Rn. 80 - Zwangsbehandlung; 149, 126 Rn. 41 - Teilzeit- und Befristungsgesetz; BVerfG, NVwZ, 2018, 1224 Rn. 45 - Fliegerhorst Büchel; NJW 2020, 1427 Rn. 14 - Coronabedingtes Verbot religiöser Zusammenkünfte.

523 BVerfGE 49, 89 (141) - Kalkar I.

524 In BVerfGE 56, 54 (78) - Fluglärm, betont das BVerfG gerade, dass auch die Risikovorsorge umfasst sein kann, allerdings bezieht es das wiederum auf „Grundrechtsgefährdungen“. In BVerfG, NVwZ 2010, 702 Rn. 12 - CERN spricht das BVerfG etwa von „Risikopotenzial“. 
zum Ausdruck. Teilweise finden sich auch deutliche Hinweise einer Produktformel. ${ }^{525}$ In der Entscheidung Kalkar I meinte das Gericht, dass grundrechtliche Schutzpflichten auch dort in Betracht kommen, wo gegenwärtig noch keine Grundrechtsverletzungen vorliegen. Schutzpflichten können auch zu Regelungen verpflichten, die die Gefahr von Grundrechtsverletzungen eindämmen. Ob, wann und mit welchem Inhalt eine solche Regelung verfassungsrechtlich geboten sei, hänge u.a. von einer Abwägung „der Art, der Nähe und dem Ausmaß möglicher Gefahren, der Art und dem Rang des verfassungsrechtlich geschützten Rechtsguts $[\ldots]^{\text {“ }}$ ab. ${ }^{526}$ Entsprechende Formulierungen finden sich auch in anderen Entscheidungen, in denen es um den Bereich der Risikovorsorge ging. ${ }^{527} \mathrm{Ob}$ das BVerfG aber diese Prüfung schon auf Tatbestandseite vornehmen will oder ob es vielmehr dazu tendiert, auch die Risikovorsorge als von der Schutzpflicht mit umfasst anzusehen und sich diese nur unter bestimmten Umständen zu einer konkreten Pflicht, Maßnahmen zur Risikovorsorge zu ergreifen, verdichtet, ist unklar. Deutlicher wird dieser Bezug in der Entscheidung zum Schwangerschaftsabbruch.528 So müsse „die Schutzverpflichtung des Staates [...] um so ernster genommen werden, je höher der Rang des in Frage stehenden Rechtsgutes innerhalb der Wertordnung des Grundgesetzes anzusetzen ist. “529 Ähnliche Stellen finden sich auch in weiteren Entscheidungen. ${ }^{530}$

In anderen Entscheidungen werden derartige Ausführungen hingegen eher auf Tatbestandsseite relevant. Allen voran die CERN-Entscheidung ist hierfür sinnbildlich. Unter Verweis auf kernphysikalische Überlegungen trug die Beschwerdeführerin vor, dass von einem Experiment des CERN potentielle Risiken für die gesamte Welt bestünden, weil Miniatur-Schwarze-Löcher entstehen könnten, welche dann die sie umgebende Materie absorbierten. Was wie ein Science-Fiction-Szenario klang, hat das BVerfG aber gerade nicht davon abgehalten, Schutzpflichten hier für einschlägig zu halten. Das BVerfG hat die hohe Bedeutung des Rechtsgutes (Art. 2 Abs. 2 S. 1 GG) betont und deshalb nur geringe Anforderungen an die Eintrittswahrscheinlichkeit gestellt.

„Eine nur theoretisch herleitbare Gefährdung von Leben oder Gesundheit kann ausnahmsweise als Grundrechtseingriff angesehen werden. Dabei gilt: Je größer das Risikopotenzial für Leben oder Gesundheit ist, desto niedriger liegt die Schwelle der Wahr-

525 Siehe etwa BVerfGE 49, 89 (141) - Kalkar I; 56, 54 (78) - Fluglärm.

526 BVerfGE 49, 89 (140) - Kalkar I.

527 Siehe nur BVerfG, NVwZ 2009, 1489 Rn. 29 - Flughafen Halle/Leipzig m.w.N.

528 Zur Kalkar-Entscheidung tendenziell a.A. Voland, NVwZ 2019, 114 (117). Szczekalla, Grundrechtliche Schutzpflichten im deutschen und europäischen Recht, S. 173 weist auf den Interpretationsspielraum hin: „Ob sich die genannten Äußerungen des BVerfG tatsächlich und zwingend auf die Prüfungsstufe ,Beeinträchtigung' (in einem weiten, Gefahr und Risiko einschließenden Sinne) beziehen oder ob es nicht vielmehr (schon) um Erwägungen zur Rechtfertigung ganz oder teilweise unterlassener Schutzmaßnahmen geht, ist unklar.".

529 BVerfGE 39, 1 (42) - Schwangerschaftsabbruch I.

530 Siehe etwa BVerfGE 49, 89 (142) - Kalkar I; BVerfG, NVwZ 2010, 702 Rn. 12 - CERN. 
scheinlichkeit für die Prognose eines Schadenseintritts, bei deren Überschreitung wirksame staatliche Schutzmaßnahmen geboten sind. Hinsichtlich schwerer Schäden an Leben oder Gesundheit einer Vielzahl von Grundrechtsträgern genügt prinzipiell bereits eine im Vorfeld erkannte Realisierungstendenz, um Schutzpflichten des Staates auszulösen [...]. “

Das legt durchaus den Schluss nahe, dass es sich dort zwar originär um den Bereich der Risikovorsorge handelt, sich das Risiko aber wegen eines gesenkten Wahrscheinlichkeitsmaßstabes bereits zu einer relevanten Gefahr verdichtet hat. ${ }^{531}$ Auch in der Entscheidung zum Fluglärm meint das BVerfG, „[d]aß auch eine auf Grundrechtsgefährdungen bezogene Risikovorsorge von der Schutzpflicht der staatlichen Organe umfaßt werden kann" ${ }^{532}$.

In wieder anderen Entscheidungen äußerte sich das BVerfG überhaupt nicht zu etwaigen Voraussetzungen. In der Entscheidung zur Zwangsbehandlung unterscheidet das BVerfG vielmehr zwischen allgemeiner und konkreter Schutzpflicht. Dies erweckt den Eindruck, dass allgemeine Schutzpflichten immer bestehen, konkrete hingegen nur in besonderen Fällen. ${ }^{533}$ Letztere ergaben sich immerhin aus der besonderen Hilfsbedürftigkeit der untergebrachten Personen und nach Abwägung der freiheitlichen mit den sicherheitsrechtlichen Belangen. ${ }^{534}$ Und auch in Entscheidungen zum Kündigungsschutz von Arbeitnehmern, ${ }^{535}$ zum Schutz der unterlegenen Vertragspartei, ${ }^{536}$ oder zur Sonntagsruhe ${ }^{537}$ wird auf eine Gefahrenprognose oder vergleichbare Aspekte nicht eingegangen, sondern von der Existenz von Schutzpflichten für einen bestimmten Bereich ausgegangen und im Rahmen einer Abwägung der kollidierenden Interessen dann bestimmte Regelung für (un-)vereinbar gehalten. ${ }^{538}$ Damit bewegt man sich aber auch hier eher auf Rechtsfolgenseite. Allerdings kann dies auch daran liegen, dass es hierbei um weitaus offensichtlichere Gefährdungslagen ging als etwa in den Fällen der Risikovorsorge.

Jüngst hat das BVerfG hinsichtlich des strafrechtlichen Verbots der geschäftsmäßigen Hilfe zur Selbsttötung ( $\$ 217$ StGB) formuliert, dass der Gesetzgeber verfassungsrechtlich „nicht gehindert [ist], aus generalpräventiven Gründen Handlungen, die lediglich generell geeignet sind, Rechtsgüter zu gefährden, unter

531 In diese Richtung Gärditz, in: Landmann/Rohmer, UmweltR, GG Art. 20a Rn. 86. Siehe auch Hermes, Das Grundrecht auf Schutz von Leben und Gesundheit, S. 237, 238.

532 BVerfGE 56, 54 (78) - Fluglärm.

533 So etwa auch Szczekalla, Grundrechtliche Schutzpflichten im deutschen und europäischen Recht, S. 172.

534 BVerfGE 142, 313 (338 ff.) - Zwangsbehandlung.

535 Siehe hierzu etwa BVerfGE 128, 157 (176f.) - Klinikprivatisierung, m.w.N. auf die verfassungsgerichtliche Judikatur. Hier wird nur darauf hingewiesen, dass der Staat der Schutzpflicht durch die Kündigungsschutzvorschriften entspricht. Kritisch zu dieser pauschalen Aussage und zur Entscheidung des BVerfG insgesamt Lindner, RdA 2005, 166 (167f.).

536 BVerfGE 114, 1 (42 f.) - Bestandsübertragung Lebensversicherung.

537 BVerfG, NVwZ 2010, 570 Rn. 150 ff. - Berliner Ladenöffnungsgesetz.

538 BVerfGE 114, 1 (43 ff.) - Bestandsübertragung Lebensversicherung; BVerfG, NVwZ 2010, 570 Rn. 162 ff. - Berliner Ladenöffnungsgesetz oder in BVerfGE 142, 313 (340 ff.) - Zwangsbehandlung. 
Umständen schon in einem frühen Stadium zu unterbinden [...]. Anderenfalls nähme man dem Gesetzgeber die Möglichkeit, Gefahren für hochrangige Rechtsgüter zu begegnen, die aufgrund fehlender gesicherter wissenschaftlicher oder empirischer Erkenntnisse nicht exakt einschätzbar sind. “539 Auch wenn das Gericht hier gerade die Prärogative des Gesetzgebers betont, folgt daraus nicht, dass eine Pflicht zum Schutz nicht besteht. Vielmehr bezieht sich dies auf die Auswahlmöglichkeiten im konkreten Fall und die Frage, ob das Strafrecht zum präventiven Lebensschutz eingesetzt werden darf.

Kurzum: Allein die Parallelen zur Produkt- und Je-desto-Formel können nicht zu dem Schluss führen, das BVerfG knüpfe ebenfalls an eine Gefahrenschwelle an. Vielmehr scheint es auch im Bereich der reinen Risikovorsorge Schutzpflichten anzunehmen und diesem Umstand dann auf Rechtsfolgenseite durch die Gewährung eines weitreichenderen Entscheidungsspielraums Rechnung zu tragen.

\section{c. Fazit: Gefahrenschwelle als Minimalkonsens}

Auch wenn im Einzelnen die Ansichten darüber auseinander gehen, ab welcher Gefährdungsschwelle Schutzpflichten eingreifen, so lässt sich jedenfalls ein Minimalkonsens ausmachen. Zumindest wenn eine nicht vom Staat verursachte Gefahr für grundrechtliche Schutzgüter besteht, ist der Staat in entsprechender Anwendung polizeirechtlicher Maßstäbe grundrechtlich zur Gewährung von Schutz verpflichtet. ${ }^{540}$

\section{Die Rechtsfolge - Inhalt und Ausgestaltung staatlicher Schutzpflichten}

Damit ist freilich noch nichts darüber gesagt, welche inhaltlichen Pflichten dies für den Staat zeitigt. Adressat der Schutzpflichten kann jedenfalls - in entsprechender Anwendung von Art. 1 Abs. 3 GG - die gesamte staatliche Gewalt sein. Primär und zuvörderst richten sie sich freilich an den Gesetzgeber, ${ }^{541}$ der ein dem Schutzbedarf gerecht werdendes Schutzniveau innerhalb der Rechtsordnung vorsehen muss, welches dann auf sekundärer Ebene durch Exekutive und Judikative verwirklicht werden kann. ${ }^{542}$

539 BVerfG, NJW 2020, 905 Rn. 271 - Recht auf Selbsttötung.

540 So etwa auch Neubert, AöR 140 (2015), 267 (272).

541 Holderer, Objektive Grundrechtsgehalte, S. 199; Stern, in: der, Staatsrecht III/1, S. 951. Vgl. BVerfGE 39, 1 (44) - Schwangerschaftsabbruch I; 88, 203 (252) - Schwangerschaftsabbruch II.

542 BVerfGE 142, 313 (337) - Zwangsbehandlung; im Ansatz auch BVerfGE 39, 1 (44) - Schwangerschaftsabbruch I. 


\section{a. Allgemeine Grenzen}

Weder auf abwehrrechtlicher Seite noch auf Seiten der Schutzpflichten ist staatliches Handeln unbegrenzt möglich. Faktische Grenzen ergeben sich schon aus der begrenzten territorialen Macht des Staates, sodass im Grundsatz rechtlicher und damit effektiver Schutz primär nur innerhalb des eigenen Herrschaftsbereichs gewährt werden kann. Im Ausland beschränkt sich der Schutz zumeist auf diplomatische Bemühungen. Weil es daneben aber durchaus Fälle geben kann, in denen staatliche Bemühungen ergebnislos bleiben oder von vornherein zum Scheitern verurteilt sind, ${ }^{543}$ steht die Schutzpflicht insoweit unter dem Vorbehalt der „Unmöglichkeit“. 544

Daneben ergeben sich schon aus der wechselseitigen Beziehung von Freiheit und Sicherheit verschiedene Grenzen. Da Sicherheitsaspekte zwangsläufig Freiheitsrechte Anderer begrenzen, ziehen die betroffenen Abwehrrechte und mit ihnen das Übermaßverbot Grenzen. ${ }^{545}$ Mit Blick auf Sicherheitsaspekte hat das BVerfG betont, dass die Reichweite der Schutzpflicht auch „mit Blick auf die [...] Schutzbedürftigkeit des zu schützenden Rechtsguts [...] zu bestimmen [sei]. "546 Denn die Grundrechte fördern und fordern autonomes Handeln der Bürger. ${ }^{547}$ Soweit den Betroffenen also Mittel zur eigenständigen Beseitigung des Risikos bzw. der Gefahr zur Verfügung stehen, bedarf es keines spezifischen, staatlichen Schutzes. ${ }^{548}$ Dieser Gedanke kommt nicht zuletzt schon in den polizeirechtlichen Subsidiaritätsklauseln zum Ausdruck. ${ }^{549}$ Zudem würde staatlicher Schutz hier

$543 \mathrm{Zu}$ denkbaren Fällen tatsächlicher Unmöglichkeit auch auf dem eigenen Herrschaftsgebiet siehe etwa Hermes, Grundrecht auf Schutz von Leben und Gesundheit, S. 244 f.

544 Vgl. Hermes, ebd.; Clausen, Das Verhältnis von Achtungs- und Schutzpflichten in Ausnahmesituationen, S. 157.

545 Ausführlicher auch zu diesem Spannungsverhältnis Hermes, a.a.O., S. $246 \mathrm{ff}$.

546 BVerfGE 85, 191 (213) - Nachtarbeitsverbot; 88, 203 (254) - Schwangerschaftsabbruch II; BVerfGK 14, 192 (199) - Schloss Bensberg; BVerfGE 142, 313 (341) - Zwangsbehandlung. Zur Schutzbedürftigkeit siehe auch Isensee, in: ders./Kirchhof, HbStR IX, $\mathbb{1 9 1}$ Rn. 219, $270 \mathrm{f}$. Kuch, DÖV 2019, 723 (724) beschreibt es so, „dass der staatsfreie Raum erst beginnen kann, wo die vordringlichen Schutzbedürfnisse des Bürgers enden.“

547 Vgl. hierzu Classen, Staatsrecht II, \$ 6 Rn. 33. In BVerfGE 142, 313 (341) - Zwangsbehandlung, stellt das BVerfG etwa darauf ab, dass die untergebrachten Personen „nicht zur Einsicht in die konkrete Notwendigkeit einer medizinischen Maßnahme fähig sind und darum Gefährdungen von Leib und Leben ausgeliefert wären, ohne in Freiheit selbst für den eigenen Schutz sorgen zu können. "Dieser Autonomiegedanke hat nicht nur Ausdruck in dem Leitbild eines mündigen und vernünftigen Bürgers der Aufklärung geprägt, sondern es ist auch das Idealbild, welches dem Grundgesetz zugrunde liegt, vgl. etwa BVerfGE 27, 71 (81f.) - Leipziger Volkszeitung; 124, 1 (21) - Nachwahl; oder jüngst BVerfG, Urt. v. 26.02.2020 - 2 BvR 2347/15 u.a., Rn. 274, 279 - Recht auf Sterben m.w.N. auf die verfassungsgerichtliche Judikatur. Die Autonomie als maßgebliches Leitbild betont das Gericht auch in BVerfG, NJW 2019, 3703 Rn. 127 - Hartz-IV Sanktionen, m.w.N.

548 Das ist letztlich auch bedeutsam für das Übermaßverbot, genauer gesagt für die Erforderlichkeit staatlicher Maßnahmen. Zur Selbsthilfemöglichkeit als Grenze ausführlicher Hermes, a.a.O., S. 245 f.

549 Isensee, in: ders./Kirchhof, HbStR IX, $\mathbb{1} 191$ Rn. 271. 
tendenziell Gefahr laufen, paternalistische Züge anzunehmen und die Autonomie des Einzelnen dadurch zu beeinträchtigen. ${ }^{550}$

Spezifische rechtliche Grenzen können sich daneben vor allem auch aus dem Unionsrecht ergeben. Wegen seines umfangreichen Anwendungsvorrangs auch vor dem nationalen Verfassungsrecht kann es den Handlungskorridor möglicher Schutzmaßnahmen begrenzen. Das gilt insbesondere dort, wo Sekundärrecht eine unionsweite (Voll-)Harmonisierung bewirkt ha, und insoweit kein oder nur ein geringer Spielraum für mitgliedstaatliche Abweichungen oder Alleingänge besteht. ${ }^{551}$

\section{b. Spezifische Vorgabe der Schutzpflichtendimension}

Die Dimension der grundrechtlichen Schutzpflichten gibt dem Staat indes ein bestimmtes Handlungsziel vor: einen effektiven Schutz grundrechtlicher Rechtsgüter. ${ }^{552}$

\section{aa. Ausgangspunkt Gestaltungsspielraum des Staates}

Das bedeutet freilich nicht, dass damit von vornherein spezifische Vorgaben bestehen oder gar ein optimaler Schutz gewährleistet werden muss. ${ }^{553}$ Allgemein kann sich die staatliche Gewalt stets auf gewisse Einschätzungs- und Beurteilungsspielräume berufen. Für die Exekutive und Judikative sind diese zwar deutlich geringer, weil sie schon an das einfache Recht gebunden sind und Spielräume auch zur Schutzgewährung durch das einfache Recht vorgegeben werden. ${ }^{54}$ Soweit hier ein „Schutz durch Eingriff“ gewährt werden soll, bedarf es wegen der Rechtfertigungslast des Staates immerhin einer (präzisen) gesetzlichen Grundlage. ${ }^{555}$ Für den Gesetzgeber folgt hingegen in erster Linie nur aus der Verfassung selbst eine Bindungswirkung. Insoweit stehen ihm deutlich weitere Gestaltungsspielräume zu, die ihm das BVerfG etwa bei der Beurteilung der Eignung oder Erforderlichkeit eines Gesetzes gewährt. ${ }^{556}$ Damit wird nicht zuletzt auch das

550 Hermes, a.a.O., S. 245, der hier etwa das Beispiel der Verbraucherhinweise auf Produkten zur Information über Gesundheitsrisiken nennt. Besonders deutlich wird das etwa in vertragsrechtlichen Fällen, in denen grundsätzlich die Vertragsautonomie hinreichend Schutz bietet, vgl. hierzu nur BVerfGE 89, 214 (231 f.) - Bürgschaftsverträge. In dem hier untersuchten Feld der Meinungsroboter wird das auch noch relevant, siehe hierzu unten, 3. Kapitel, B.II.1.

551 Das wird hier noch relevant werden, wenn es um Fragen der Vereinbarkeit spezifischer Maßnahmen mit der E-Commerce-Richtlinie geht, hierzu ausführlicher unten, 3. Kapitel, C.II.2.c.aa(III.) und mit Blick auf das Herkunftslandprinzip ebd, C.II.2.f.bb.

552 Vgl. Hermes, Grundrecht auf Schutz von Leben und Gesundheit, S. 261.

553 Krings, Grund und Grenzen grundrechtlicher Schutzansprüche, S. 260.

554 Siehe hierzu auch Weiss, Privatisierung und Staatsaufgaben, S. 179 f.

555 Vgl. hierzu etwa BVerfGE NJW 2020, 300 Rn. 86 - Recht auf Vergessen I.

556 Zum „Beurteilungs- und Prognosespielraum“ bei der Erforderlichkeit etwa BVerfG, NJW 2019, 3054 Rn. 66 - Mietpreisbremse; BVerfGE 116, 202 (225) - Tariftreueerklärung; 146, 71 (126) - Ta- 
verfassungsrechtliche Verhältnis zwischen primär verantwortlichem Gesetzgeber und nur sekundär verantwortlicher Verfassungsrechtsprechung geklärt und ein Übergriff der Judikative verhindert. ${ }^{557}$

Geht es um die Erfüllung grundrechtlicher Schutzpflichten, tritt die Notwendigkeit von Spielräumen noch deutlicher zu Tage. ${ }^{558}$ Denn während der negatorische Anspruch darauf gerichtet ist, dass eine konkrete schutzbereichsbeschränkende Maßnahme des Staates unterbleiben muss, ${ }^{559}$ ist die Schutzpflicht unbestimmt. ${ }^{560}$ Grundrechtliche Schutzgüter lassen sich in vielfältiger Weise schützen; ${ }^{561}$ eine konkrete Maßnahme kann also oftmals nicht ausgemacht werden. Hinzu kommt, dass die Schutzinteressen regelmäßig mit freiheitsrechtlichen Interessen kollidieren, sich also zwei Grundrechtsträger gegenüberstehen: Die Gewährung von Schutz führt dann zur Beschneidung der Freiheitsgrundrechte anderer. ${ }^{562}$ Notwendig wird also eine Abwägung und damit die Herstellung eines schonenden Ausgleichs. ${ }^{563}$ Das Ergebnis dieses Ausgleichs ist aber offen.

rifeinheitsgesetz. Zur Einschätzungsprärogative bei der Eignung etwa BVerfG NJW 2019, 3703 Rn. 166 - ALG-II-Sanktionen; BVerfGE 116, 202 (224) - Tariftreueerklärung. Daneben zum Einschätzungsspielraum bei der Auswahl von förderungsfähigen Zwecken (im Steuerrecht) etwa BVerfGE 138, 136 (182) - Erbschaftssteuer und zum Beurteilungsspielraum über die tatsächlichen Gegebenheiten als Grundlage einer Regelung BVerfG NJW 2017, 381 Rn. 62 - Kopftuchverbot in Kindertagesstätten oder BVerfGE 94, 114 (143f.) - sichere Herkunftsstaaten.

557 Andeutend Bethge, in: Maunz u.a., BVerfGG, $\mathbb{S} 90$ Rn. 27; deutlicher Walter, in: Maunz/Dürig, GG, Art. 93 Rn. 104 ff.

558 Siehe gerade mit Blick auf das Verhältnis von Gesetzgebung und Verfassungsgerichtsbarkeit etwa BVerfGE 56, 54 (819) - Fluglärm; BVerfG NJW 1998, 3264 (3265) - Waldsterben; NJW 2016, 1716 Rn. 19 - Pflegenotstand.

559 Krings, Grund und Grenzen grundrechtlicher Schutzansprüche, S. 262; Hesse, in: FS-Mahrenholz, S. $541(545)$.

560 BVerfGE 96, 56 (64) - Vaterschaftsauskunft; 115, 118 (160) - Luftsicherheitsgesetz; 142, 313 (337) - Zwangsbehandlung. Diesen Vergleich von abwehr- und schutzrechtlicher Seite ziehen neben Klein, NJW 1989, 1633 (1637) u.a. auch Unruh, Zur Dogmatik der grundrechtlichen Schutzpflichten, S. 79; Ruffert, Vorrang der Verfassung und Eigenständigkeit des Privatrechts, S. 167; Krings, Grund und Grenzen grundrechtlicher Schutzansprüche, S. 261; Calliess, in: FS-Starck, S. 201 (208) und Hesse, in: FS-Mahrenholz, S. 541 (545). Ähnlich auch Heun, RW 2011, 376 (398).

561 Siehe hierzu nur Krings, Grund und Grenzen grundrechtlicher Schutzansprüche, S. 263 f.

562 Das gilt etwa dann, wenn der Nasciturus durch strafrechtliche Verbote geschützt werden soll, die wiederrum die Mutter in ihrer Entscheidungsfreiheit einschränken (hierzu BVerfGE 39, 1 - Schwangerschaftsabbruch I und E 88, 203 - Schwangerschaftsabbruch II), wenn aus Gründen des Gesundheitsschutzes bestimmte Immissionswerte festgelegt und eingehalten werden müssen (hierzu etwa BVerfGE 56, 54 - Fluglärm; BVerfGE 79, 174 - Straßenverkehrslärm oder BVerfG, NJW 2002, 1638 - Mobilfunkanlagen) oder die Freiheit der Arbeitgeber durch Kündigungsschutzregeln zugunsten der Arbeitnehmer begrenzt wird (hierzu etwa BVerfGE 92, 26 (46) - Zweitregister; BVerfGE 149, 126 Rn. 47 ff. - Teilzeit- und Befristungsgesetz m.w.N).

563 Dies spielt auch in der verfassungsrechtlichen Judikatur eine entscheidende Rolle, siehe etwa schon BVerfGE 56, 54 (80) - Fluglärm. Umfangreiche Gedanken zu einer solchen Abwägung machte sich das BVerfG etwa in BVerfGE 142, 313 Rn. 78 ff. - Zwangsbehandlung, wo es um die Abwägung der Freiheit der Betreuten mit ihrem gesundheitlichen Schutz ging. In BVerfGE 96, 56 (64f.) - Vaterschaftsauskunft meint das BVerfG gar, dass die Abwägung zwischen Schutzrecht und Abwehrrecht, der maßgebliche Grund für den Gestaltungsspielraum des Gesetzgebers ist: „Gestaltungsspielräume bestehen vor allem dort, wo es um die Berücksichtigung widerstreitender Grundrechte geht. Das gilt auch dann, wenn - wie hier - der Schutz einer grundrechtlichen Position zwangsläufig die Beeinträchtigung des Grundrechts einer anderen Person zur Folge hat, weil die Abwägung in erster Linie 
Besonders deutlich wird dies dadurch, dass die Frage der Schutzintensität letztlich davon abhängt, wie bestimmte Gefahren, Risiken und potentielle Schäden bewertet werden. Dafür sind zwar wissenschaftliche Ergebnisse brauchbar, aber auch diese müssen nicht immer eindeutig sein. In Abhängigkeit von den gewählten Bewertungsgrundlagen kann sich das Ergebnis (erheblich) unterscheiden und zu anderen Maßnahmen führen. Denkbar sind neben Überwachungs- und Nachforschungsmaßnahmen als Risikovorsorge auch präventive Regelungen und Maßnahmen bis hin zur „schärfsten Waffe“564, der Repression mit Hilfe des Strafrechts. ${ }^{565}$ Insoweit ist die konkrete Auswahl der Maßnahmen nicht nur eine verfassungsrechtliche, sondern auch eine rechtspolitische ${ }^{566}$ Frage. Damit bewegt man sich aber auch hier auf der Verantwortungsebene des Gesetzgebers, weshalb ihm schon dem Grunde nach zur Beantwortung der „komplexen Frage“567 der Gewährung staatlichen Schutzes ein erheblicher Einschätzungs-, Gestaltungs- und Wertungsspielraum zugebilligt werden muss. ${ }^{568}$ Insoweit folgt aus den Schutzpflichten meist nur das Gebot, dass der Staat handeln muss. ${ }^{569}$ Nur selten wird ihm eine konkrete Handlungspflicht auferlegt. ${ }^{570}$ Freiheits- und schutzrechtliche Aspekte ebnen also lediglich einen „Handlungskorridor“, innerhalb dessen sich der Staat bewegen muss. ${ }^{571}$

den jeweils zuständigen staatlichen Organen zukommt.“ Ausführlicher zu dem „Konfliktschlichtungsauftrag“ des Gesetzgebers Ruffert, Vorrang der Verfassung und Eigenständigkeit des Privatrechts, S. $203 \mathrm{ff}$.

564 BVerfGE 39, 1 (45) - Schwangerschaftsabbruch I.

565 Vgl. hierzu BVerfGE 39, 1 (44 f.) - Schwangerschaftsabbruch I.

566 Besonders hinsichtlich des Nichtraucherschutzes in Gaststätten betont das Zuck, ZRP 2008, 165 (166).

567 So schon RiBVerfG Rupp-v. Brünneck, Simon, SV, BVerfGE 39, 1 (71) - Schwangerschafsabbruch I; zudem BVerfGE 56, 54 (81) - Fluglärm; BVerfG NJW 1987, 2287 - Aids-Bekämpfung; NJW 1996, 651 - Ozon; NJW 1998, 3264 (3265) - Waldsterben; NVwZ 2000, 309 (310) - Altlasten DDRUranbergbau; NJW 2016, 1716 Rn. 19 - Pflegenotstand. Hesse, in: FS-Mahrenholz, S. 541 (546) nennt es eine „komplexe Problematik“.

568 Vgl. BVerfGE 39, 1 (44) - Schwangerschaftsabbruch I. Später dann in eben dieser Formulierung etwa in BVerfGE 77, 170 (214 f.) - Lagerung chemischer Waffen; 85, 191 (212) - Nachtarbeitsverbot; 96, 56 (64) - Vaterschaftsauskunft; 142, 313 (337) - Zwangsbehandlung; BVerfG NJW 1996, 651 Ozon; NJW 1998, 3264 (3265) - Waldsterben; NVwZ 2000, 309 (310) - Altlasten DDR-Uranbergbau; NVwZ 2009, 1489 Rn. 30 - Flughafen Leipzig/Halle; NVwZ 2011, 991 Rn. 38 - Fluglärmschutzgesetz; NJW 2016, 1716 Rn. 19 - Pflegenotstand.

569 Heun, RW 2011, 376 (398). Vgl. BVerfGE 142, 313 (342) - Zwangsbehandlung.

570 BVerfGE 96, 56 (64) - Vaterschaftsauskunft; BVerfG, NJW 2016, 1716 Rn. 19 - Pflegenotstand. Gärditz, in: Landmann/Rohmer, UmweltR, GG Art. 20a Rn. 89. In der Judikatur des BVerfG war das selten der Fall, und kam vor allem dort in Betracht, wo es um das Recht auf Leben ging (so auch BVerfGE 46, 160 (164f.) - Schleyer). Vor allem hinsichtlich des Schwangerschaftsabbruchs meinte das BVerfG, dass das Strafrecht eingesetzt werden und der Schwangerschaftsabbruch als Unrecht gelten müsse, E 39, 1 (44) - Schwangerschaftsabbruch I; BVerfGE 88, 203 (252) - Schwangerschaftsabbruch II. Zum Luftsicherheitsgesetz hatte das BVerfG gemeint, dass der Abschuss entführter Flugzeuge die einzige Handlung sei. Wegen kollidierender Grundrechte der Insassen hatte es dies allerdings für verfassungswidrig gehalten, BVerfGE 115, 118 (159ff.) - Luftsicherheitsgesetz.

571 Calliess, JZ 2006, 320 (329); ders., in: FS-Starck, S. 201 (216). In der Sache ähnlich Ruffert, Vorrang der Verfassung und Eigenständigkeit der Privatrechtsordnung, S. 218; Mayer, Untermaß, Übermaß und Wesensgehaltsgarantie, S. 76; Borowski, Grundrechte als Prinzipien, S. $261 \mathrm{f}$. Illustrativ etwa Schimmele, Staatliche Verantwortung für diskursive Integrität, S. 117 f. In einem SV spricht RiBVerfG 


\section{bb. Untermaßverbot als Direktive der Effektivität}

Das bedeutet allerdings nicht, dass hierbei keinerlei Grenzen zu beachten wären. Auf abwehrrechtlicher Seite schränkt das Übermaßverbot die staatliche Gewalt ein. Es ist die Direktive für das grundrechtliche Unterlassungsgebot: Der Staat darf nur verhältnismäßige Eingriffe vornehmen. Sein Pendant auf der Seite der Schutzpflichten findet es im Untermaßverbot. ${ }^{572}$ Schutzpflichten sind keine Optimierungsgebote in dem Sinne, dass sie den Staat zu einem absoluten und damit optimalen Schutz verpflichten. ${ }^{573}$ Einen solchen kann es schon wegen der Kollision mit Freiheitsrechten regelmäßig nicht geben. ${ }^{574}$ Wegen der Forschungsfreiheit müssen bspw. gewisse Restrisiken von wissenschaftlichen Experimenten in Kauf genommen werden. ${ }^{575}$ Gleiches gilt auch bei der Erzeugung von Kernenergie ${ }^{576}$ und der Entsorgung des radioaktiven Materials. ${ }^{577}$ Demnach gewährleistet das Untermaßverbot nur einen Mindestschutz. ${ }^{578}$ Unterhalb dieser Schwelle darf sich der Staat nicht bewegen. Konkret bedeutet das, dass die getroffenen Vorkehrungen „für einen angemessenen und wirksamen Schutz ausreichend sein und zudem auf sorgfältigen Tatsachenermittlungen und vertretbaren Einschätzungen beruhen [müssen]. “579 Bewegt sich der Staat unterhalb des gebotenen Mindeststandards, verletzt er die Grundrechte, handelt also verfassungswidrig. Wo diese

Bryde davon, dass „der Gesetzgeber von Verfassungs wegen ohnehin schon zwischen Untermaßverbot [...] und Übermaßverbot [...] eingeklemmt ist.", BVerfGE 121, 317 (380) - Rauchverbot in Gaststätten.

572 Moritz, Staatliche Schutzpflichten gegenüber pflegebedürftigen Menschen, S. 113. Zum historischen Kontext des Untermaßverbotes siehe etwa Calliess, in: FS-Starck, S. 201 (202 ff.). In der verfassungsgerichtlichen Judikatur findet sich dies erstmals erwähnt in BVerfGE 88, 203 (254) - Schwangerschaftsabbruch II. Zustimmend im Schrifttum Moritz, Schutzpflicht gegenüber pflegebedürftigen Menschen, S.119; Bortnikov, Netzneutralität, S. 145 ff.; Krings, Grund und Grenze staatlicher Schutzansprüche, S. 262; Klein, JuS 2006, 960 (964); Sachs, in: ders., GG, Vor. Art. 1 Rn. 36; Callies, in: FS-Starck, S. 201 (211). Teilweise wird eine eigenständige Bedeutung verneint, weil im Dreiecksverhältnis Übermaß- und Untermaßverbot deckungsgleich seien, so etwa Hain, DVBl. 1993, 982 ff.; ähnlich auch Starck, JZ 1993, 816 (817).

573 So Krings, Grund und Grenzen grundrechtlicher Schutzansprüche, S. 262. Tendenziell anders Unruh, Zur Dogmatik der grundrechtlichen Schutzpflicht, S. 78, der von einem "größtmöglichen Schutz“ spricht. Zwar sprach auch das BVerfG in E 49, 89 (139) - Kalkar I von einer „bestmöglichen Gefahrenabwehr", worauf auch Unruh, aa.O, Fn. 227 verweist. Allerdings legte es hier den Willen des Gesetzgebers hinsichtlich des $\mathbb{\$} 7$ Abs. 2 AtomG aus und stellte nicht darauf ab, dass dies der Sinn der Schutzpflicht, sondern dass dies vielmehr die Vorgabe des Gesetzgebers an die Exekutive sei. So letztlich auch Krings, Grund und Grenzen grundrechtlicher Schutzansprüche, S. 260.

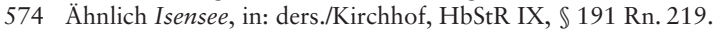

575 BVerfG NVwZ 2010, 702 Rn. 13 - CERN.

576 Vgl. BVerfGE 49, 89 (143) - Kalkar I.

577 BVerfG, NVwZ 2010, 114 Rn. 23 - Schacht Konrad.

578 Canaris, AcP 184 (1984), 201 (228); Sommermann, in: v. Mangoldt/Klein/Starck, GG, Art. 20 Rn. 319; Grzeszick, in: Maunz/Dürig, GG Art. 20 Rn. 126. Aus der verfassungsgerichtlichen Judikatur insbesondere BVerfGE 88, 203 (254 f.) - Schwangerschaftsabbruch II. 
Grenze exakt verläuft, hängt vom Einzelfall $a b^{580}$ und ist vor allem von der Art der drohenden Schäden, dem Umfang, der Bedeutung des Rechtsguts, der Nähe der Bedrohung und auch von den kollidierenden Interessen abhängig. ${ }^{581}$ Und ein einmal ergriffenes Schutzkonzept entbindet den Staat nicht davon, nachzuforschen, zu beobachten und gegebenenfalls nachzubessern, falls sich etwa die tatsächlichen Umstände ändern. ${ }^{582}$

\section{aa. Kontrolle durch das Bundesverfassungsgericht}

Ob Schutzpflichten bestehen und ob der Staat diese verletzt, hat regelmäßig das BVerfG zu entscheiden. Das führt aber deshalb zu Problemen, weil das Gericht mit der Wahl seiner Prüfungsdichte Einfluss auf den Gesetzgeber ausüben kann: ${ }^{83}$ Ein enger Maßstab führt dazu, dass das BVerfG nicht nur gesetzgeberische Entscheidungen überprüft, sondern dem Gesetzgeber auch die verfassungsrechtlichen Ergebnisse verbindlich vorgeben kann und somit in seinen Kompetenzbereich übergreift. ${ }^{584}$ Dieser Gefahr ist sich auch das BVerfG bewusst. ${ }^{585}$ So betont es den weiten Einschätzungs- bzw. Gestaltungsspielraum des Staates ${ }^{586}$ und nimmt damit die eigene gerichtliche Kontrolldichte zurück. Dabei weist die Judikatur allerdings unterschiedliche Maßstäbe auf: ${ }^{587}$ Insbesondere die erste Entscheidung zum Schwangerschaftsabbruch ist von einer eher strengen Kontrolle und von präzisen Vorgaben geprägt. Der Abbruch sei stets als Unrecht zu

580 So Canaris, AcP 184 (1984) 201, 228; Krings, Grund und Grenzen grundrechtlicher Schutzansprüche, S. 261; Schliesky u.a., Schutzpflichten und Drittwirkung im Internet, S. 50 f.; Stern, in: ders., Staatsrecht III/1, S. 953.

581 So etwa BVerfGE 49, 89 (142) - Kalkar I.

582 Siehe hierzu statt vieler BVerfGE 56, 54 (79ff.) - Fluglärm.

583 Ausführlicher zu dem Konflikt der gerichtlichen Kontrolle im Bereich der Schutzpflicht etwa Hesse, in: FS-Mahrenholz, S. $541 \mathrm{ff}$.

584 Diese Gefahr haben schon die RiBVerfG Rupp-v. Brünneck und Simon gesehen und zeigten in ihrem SV zum Schwangerschaftsabbruch grundlegende Probleme auf. Siehe hierzu BVerfGE 39, 1 (71 ff.) Schwangerschaftsabbruch I. Dort sprachen sie sich auch für eine zurückhaltende Position des BVerfG aus. Siehe auch Unruh, Zur Dogmatik der grundrechtlichen Schutzpflichten, S. 79 f.

585 Zu den wahrgenommenen Kompetenzkonflikten siehe u.a. BVerfGE 56, 54 (819) - Fluglärm; BVerfG NJW 1998, 3264 (3265) - Waldsterben; NJW 2016, 1716 Rn. 19 - Pflegenotstand.

586 In BVerfGE 39, 1 (42) - Schwangerschaftsabbruch I, heißt es etwa: „Die Schutzverpflichtung des Staates muß um so ernster genommen werden, je höher der Rang des in Frage stehenden Rechtsgutes innerhalb der Wertordnung des Grundgesetzes anzusetzen ist." In BVerfGE 88, 203 (254) - Schwangerschaftsabbruch II, heißt es: „Art und Umfang des Schutzes im Einzelnen zu bestimmen, ist Aufgabe des Gesetzgebers. Die Verfassung gibt den Schutz als Ziel vor, nicht aber seine Ausgestaltung im Einzelnen." Ähnliche Ausführungen finden sich auch bei BVerfGE 46, 160 (164) - Schleyer; 56, 54 (80 f.) - Fluglärm. In BVerfGE 77, 170 (214f.) - Lagerung chemischer Waffen, findet sich dann erstmals die Formulierung: „Dem Gesetzgeber wie der vollziehenden Gewalt kommt bei der Erfüllung dieser Schutzpflichten ein weiter Einschätzungs-, Wertungs- und Gestaltungsbereich zu, der auch Raum läßt, etwa konkurrierende öffentliche und private Interessen zu berücksichtigen. “ Diese wurde in den folgenden Entscheidungen dann stets - gebetsmühlenartig - wiederholt. Siehe hierzu BVerfGE 85, 191 (212) - Nachtarbeitsverbot; 79, 174 (202) - Straßenverkehrslärm; 92, 26 (46) - Zweitregister; 125, 39 (78) - Adventssonntage Berlin; 142, 313 (337) - Zwangsbehandlung.

587 Vgl. hierzu BVerfGE 50, 290 (333) - Mitbestimmungsgesetz. 
werten und müsse daher strafrechtlich verboten werden. Nur in besonderen Konstellationen dürfe man Ausnahmen schaffen, solange hierbei auch der Schutz des Ungeborenen gewährleistet bleibt. ${ }^{588}$ Und auch die ersten atomrechtlichen Entscheidungen sind nicht von Zurückhaltung geprägt. In Kalkar I überprüfte das BVerfG, ob der Gesetzgeber die Grenze praktischer Vernunft bei der Regelung eingehalten hatte. ${ }^{589}$ Eine Ausnahme bildet insoweit der Schleyer-Beschluss. Allerdings lag dies wohl auch an den besonderen Umständen des Falles, immerhin wurde hier ein spezifisches Handeln der Exekutive gefordert, um Schleyer aus der Gewalt der RAF-Terroristen zu befreien: Die Erfüllung der Forderungen der Geiselnehmer und damit u.a. die Freilassung von elf Inhaftierten. ${ }^{590}$

In der Entscheidung zum Fluglärm stellte das BVerfG dann hingegen auf einen Maßstab der Evidenz ab und nahm damit die eigenen Kontrollbefugnisse jedenfalls dort stark zurück, wo „nicht Rechtsgüter von höchster Bedeutung auf dem Spiel stehen “591. Das BVerfG könne - bei Nachbesserungspflichten erst dann eingreifen, „wenn evident ist, daß eine ursprünglich rechtmäßige Regelung wegen zwischenzeitlicher Änderung der Verhältnisse verfassungsrechtlich untragbar geworden ist, und wenn der Gesetzgeber gleichwohl weiterhin untätig geblieben ist oder offensichtlich fehlsame Nachbesserungsmaßnahmen getroffen hat. " 592 In folgenden Entscheidungen knüpfte man hieran an und betonte, dass das BVerfG nur eingreife, wenn der Schutz gänzlich ungeeignet oder völlig unzulänglich ist. ${ }^{593}$

Zwischenzeitlich brachte der Zweite Senat in der zweiten Entscheidung zum Schwangerschaftsabbruch das Untermaßverbot als Prüfungsmaßstab in die Diskussion und kontrollierte den Gesetzgeber dahingehend, ob er den erforderlichen Mindestschutz gewährleistet hatte. Allerdings führte der Senat sogleich aus, dass es sich hierbei um eine eingeschränkte Prüfung handelt, die danach fragt, ob die Entscheidung vertretbar sei. Dabei betonte er, dass Reichweite und der Umfang des gesetzgeberischen Spielraumes von verschiedenen Parametern abhingen. ${ }^{594}$

In den darauffolgenden Entscheidungen setzte sich hingegen wieder der Evidenzmaßstab durch. Demnach könne das BVerfG eine „Verletzung einer solchen Schutzpflicht nur feststellen, wenn Schutzvorkehrungen entweder überhaupt

588 BVerfGE 39, 1 (45 ff.) - Schwangerschaftsabbruch I. Kritisch hierzu etwa Denninger, in: FS-Mahrenholz, S. 561 (568f.).

589 BVerfGE 49, 89 (143) - Kalkar I. Daran anknüpfend dann BVerfGE 53, 30 (57 ff.) - Mühlheim-Kärlich.

590 BVerfGE 46, $160 \mathrm{ff}$. - Schleyer.

591 BVerfGE 56, 54 (81) - Fluglärm.

592 BVerfGE 56, 54 (81) - Fluglärm.

593 BVerfGE 77, 170 (214f.) - Lagerung chemischer Waffen; 79, 174 (202) - Straßenverkehrslärm; 85, 191 (212) - Nachtarbeitsverbot. Den Maßstab der „Evidenz“ findet man daneben aber auch in BVerfG NJW 1987, 2287 - AIDS-Bekämpfung; NJW 1998, 3264 (3265) - Waldsterben; NJW 2016, 1716 Rn. 19 - Pflegenotstand.

594 BVerfGE 88, 203 (254 ff.) - Schwangerschaftsabbruch II. 
nicht getroffen sind, wenn die getroffenen Regelungen und Maßnahmen offensichtlich ungeeignet oder völlig unzulänglich sind, das gebotene Schutzziel zu erreichen, oder wenn sie erheblich hinter dem Schutzziel zurückbleiben [...]. “595

Die Entscheidungen sind allerdings nicht zwingend als widersprüchlich zu begreifen. Im Ausgangspunkt dürfte sich die Evidenzkontrolle durchgesetzt haben. ${ }^{596}$ Evidenzkontrolle und Untermaßverbot greifen dabei ineinander: Ergreift der Staat keinen oder nur unzureichenden Schutz, so ist damit der verfassungsrechtlich vorgesehene Mindestschutz nicht eingehalten worden. Das Untermaßverbot wird dann evident verletzt. ${ }^{597} \mathrm{Ob}$ es dabei um eine allein prozessrechtliche Frage der Kontrollbefugnis geht, oder aber dies eine materiell-rechtliche Frage ist ${ }^{598}$ - Szczekalla spricht hier von einem Auseinanderfallen von Handlungs- und Kontrollnorm - ${ }^{599}$ ist mangels divergierender praktischer Konsequenzen hier zu vernachlässigen. ${ }^{600}$ In bestimmten Konstellationen kann es aber dazu kommen, dass sich der Spielraum des Gesetzgebers verengt und damit einhergehend die verfassungsgerichtliche Kontrolle intensiviert wird. Das wird insbesondere dort der Fall sein, wo es - wie in den Abtreibungsentscheidungen um erhebliche Gefährdungen bzw. Verletzungen elementarer Grundrechte geht. ${ }^{601}$

\section{cc. Operable Formel des Untermaßverbots}

Für die Praxis ist es allerdings notwendig, das Untermaßverbot operationalisierbar zu machen ${ }^{602}$ und dabei auch die Evidenzmaßstäbe des BVerfG einfließen

595 BVerfGE 142, 313 (337f.) - Zwangsbehandlung. Siehe zudem auch zuvor BVerfGE 92, 26 (46) Zweitregister; 125, 39 (78f.) - Adventssonntage Berlin; BVerfG NJW 2018, 2312 (2313) - Fliegerhorst Büchel m.w.N.

596 Aus der Literatur zustimmend etwa Krings, Grund und Grenzen staatlicher Schutzansprüche, S. 271; Gröpl, in: FS-Käfer, S. 95 (107); Hesse, in: FS-Mahrenholz, S. 541 (556f.). In diese Richtung auch Ruffert, Vorrang der Verfassung und Eigenständigkeit des Privatrechts, S. 215.

597 So lag der Fall etwa bei BVerfGE 125, 39 (84ff.) - Adventssonntage Berlin.

598 Diese Frage hat bisher auch das BVerfG nicht zufriedenstellend beantwortet, ist vielmehr selbst Verursacher dieses Problems, vgl. Denninger, in: FS-Mahrenholz, S. 561 (568).

599 Szczekalla, Grundrechtliche Schutzpflichten im deutschen und europäischen Recht, S. 353. Ähnlich auch Hesse, in: FS-Mahrenholz, S. 541 (557).

600 Zu dieser Diskussion siehe etwa Szczekalla, ebd., S. 353 ff.; Störring, Das Untermaßverbot in der Diskussion, S. $123 \mathrm{ff}$. jeweils m.w.N.

601 In diese Richtung das BVerfG, E 88, 203 (262f.) - Schwangerschaftsabbruch II: „Die im Beschluß des Senats vom 29. Oktober 1987 [...] enthaltenen Ausführungen zur Zulässigkeit einer Verfassungsbeschwerde gegen staatliches Unterlassen dürfen nicht dahin verstanden werden, als genügten der Erfüllung der Schutzpflicht des Staates gegenüber menschlichem Leben schon Maßnahmen, die nicht gänzlich ungeeignet oder völlig unzulänglich sind." Deutlich jüngst auch BVerfG, Urt. v. 26.02.2020 - 2 BvR 2347/15 u.a., Rn. 266 - Recht auf Sterben. Zu einem flexiblen Maßstab kommt auch Moritz, Schutzpflicht gegenüber pflegebedürftigen Menschen, S. 120. Vgl. auch Schmidt, in: Erfurter Kommentar, ArbR, 10. Einleitung Rn. 43; Bortnikov, Netzneutralität und Bedingungen kommunikativer Selbstbestimmung, S. 146 und Klein, JuS 2006, 960 (961).

602 Kritisch zur Entscheidung Schwangerschaftsabbruch II wegen fehlender praxistauglicher Konkretisierungen, Dietlein, ZG 1995, 75 (76f.). 
zu lassen. ${ }^{603}$ Überwiegend wird hierfür eine Anlehnung an das Übermaßverbot befürwortet. ${ }^{604}$ Eine solche Parallele ist allerdings nicht unkritisiert geblieben. ${ }^{605}$ Teilweise wird von einer Kongruenz von Übermaß- und Untermaßverbot ausgegangen: ${ }^{606}$ Innerhalb des Störungsdreiecks erlaube das Übermaßverbot ohnehin nur die mildesten und wirksamsten Mittel und entspreche daher i.E. dem Untermaßverbot. ${ }^{607}$ Das mag im Grundsatz durchaus richtig sein. ${ }^{608}$ Dort, wo allerdings kein Eingriff in Rechte Dritter erfolgt - etwa wenn man die Schutzpflicht auch gegenüber anderen Staaten oder Naturgewalten anerkennt - dann werden regelmäßig keine Grundrechte betroffen sein; ${ }^{609}$ dem Untermaßverbot kommt dann eine eigenständige, konstitutive Bedeutung zu. ${ }^{610}$ Zudem kann nicht von einer Ergebniskongruenz auf eine generelle Deckungsgleichheit geschlossen werden. Immerhin unterscheiden sich beide Institute im Ansatz und in der Funktion: im ersten Fall wird eine bestimmte Maßnahme abgewehrt, es entscheidet sich also, was der Staat tun darf, im zweiten soll Schutz gegen eine bestimmte (zukünftige) Handlung gewährt werden, es entscheidet sich also, was der Staat tun muss. ${ }^{611}$

Aber selbst wenn die Eigenständigkeit des Untermaßverbots angenommen wird, soll eine an das Übermaßverbot angelehnte Prüfung ausscheiden. ${ }^{612}$ Beim Übermaßverbot ginge es um eine konkrete Maßnahme, während beim Untermaßverbot der potentielle Spielraum offen für Wertungen sei. Und Dietlein meint gar, dass aus dem Untermaßverbot nichts anderes folge als aus dem Schutzgebot der Schutzpflicht. ${ }^{613}$ Allerdings ist die Frage eines Schutzgebotes von dessen In-

603 Ähnlich Bortnikov, Netzneutralität, S. 147.

604 In diese Richtung etwa Michael/Morlok, Grundrechte, Rn. 627 ff.; Bortnikov, Netzneutralität und Bedingungen kommunikativer Selbstbestimmung, S. 147 ff.; Hindelang, Freiheit und Kommunikation, S. 197 m.w.N.

605 Ausführlich zum Problem der Eigenständigkeit des Untermaßverbotes Szczekalla, Grundrechtliche Schutzpflichten im deutschen und europäischen Recht, S. 323 ff. m.w.N.

606 So vor allem Hain, DVBl. 1993, 982 ff.; ähnlich auch Starck, JZ 1993, 816 (817). In der Tendenz andeutend Stern, in: ders., StaatsR III/2, S. 813 f. Eine ausführliche und auch kritische Darstellung findet sich etwa auch bei Schimmele, Staatliche Verantwortung für diskursive Integrität, S. $117 \mathrm{ff}$. oder bei Borowski, Grundrechte als Prinzipien, $261 \mathrm{f}$.

607 So etwa Erichsen, Jura 1997, 85 (88); Hain, DVBl. 1993, 982 (983); Starck, JZ 1993, 816 (817).

608 Vgl. Klein, JuS 2006, 960 (963); ebenso Erichsen, Jura 1997, 85 (88).

609 Schimmele, Staatliche Verantwortung für diskursive Integrität, S. 119; Störring, Das Untermaßverbot in der Diskussion, S. 128; zu Naturgewalten Dietlein, ZG 1995, 131 (135).

610 In diese Richtung auch mit umfassenderer Kritik Krings, Grund und Grenzen staatlicher Schutzansprüche, S. 299 ff. Siehe auch Dietlein, ZG 1995, 131 (135 ff.).

611 Vgl. hierzu Klein, JuS 2006, 960 (962 f.) auch zu weiteren Divergenzen prozessualer Art; Isensee, in: ders./Kirchhof, HbStR IX, $\mathbb{\$} 191$ Rn. 304. Vertieft auch anhand der Entscheidung Schwangerschaftsabbruch II Dietlein, ZG 1995, 131 (136 ff.). Daneben siehe auch Krings, Grund und Grenzen grundrechtlicher Schutzansprüche, S. 300; Calliess, in: FS-Starck, S. 201 (211); Ruffert, Vorrang der Verfassung und Eigenständigkeit des Privatrechts, S. 216 f.; Störring, Das Untermaßverbot in der Diskussion, S. 127; Grzeszick, in: Maunz/Dürig, GG, Art. 20 Rn. 127.

612 In diese Richtung etwa Dietlein, ZG 1995, 131 (139). Jedenfalls kritisch zu einer Schematisierung Ruffert, Vorrang der Verfassung und Eigenständigkeit des Privatrechts, S. $222 \mathrm{f}$.

613 Dietlein, ZG 1995, 131 (140). Ähnlich auch Hain, DVBl. 1993, 982 (983). Kritisch hierzu Krings, Grund und Grenzen staatlicher Schutzansprüche, S. 301. 
halt zu trennen. Liegt eine Gefahrenlage vor, ist das Gebot aktiviert. Sein Inhalt liegt dann im Toleranzbereich zwischen Untermaß- und Übermaßverbot. ${ }^{614}$

Und auch wenn das Untermaßverbot ungenau ist, heißt das nicht, dass es nicht zumindest operationalisierbar gemacht werden kann und sollte. Ob das in Anlehnung an das Übermaßverbot erfolgen soll oder eigenständig ${ }^{615}$, ist jedenfalls für die Praxis unbedeutend und dürfte i.E. ohnehin keinen Unterschied machen. Da aber ansonsten kaum ein praxistauglicher Vorschlag gemacht wird, liegt es deutlich näher, sich auf diese Parallele einzulassen. ${ }^{616}$ Das führt gerade nicht zu einem bloßen „Wenn-Dann-Schema“. ${ }^{617}$ Vielmehr lassen sich die spezifischen Unklarheiten und Spielräume des Gesetzgebers hier angemessen berücksichtigen. Das gilt einerseits hinsichtlich der Auswahl des Schutzkonzeptes, andererseits aber vor allem auch hinsichtlich der Abwägung mit anderen Belangen. Hier ist dann letztlich auch Raum dafür, die freiheitsrechtliche Seite zu berücksichtigen, die die äußerste Grenze aufzeigt. In Anknüpfung an bereits vorhandene Darstellungen ${ }^{618}$ ergeben sich danach vier „Stufen“:

1. Zunächst gilt es zu klären, ob der Gesetzgeber bereits ein Schutzkonzept vorgesehen hat bzw. sonstwie zum Schutz tätig geworden ist. Ist dies nicht der Fall liegt ohnehin schon ein evidenter Verstoß gegen das Untermaßverbot vor, sofern dies nicht durch kollidierende Rechte und das Übermaßverbot bedingt ist.

2. Sodann ist zu klären, ob das Konzept zum Schutz auch geeignet ist, also dem Schutz in irgendeiner Weise dient. Besteht aus wissenschaftlicher Sicht noch keine Klarheit über Art und Ausmaß von Gefahren und werden hierzu unterschiedliche wissenschaftliche Meinungen vertreten, so ist es Aufgabe des Gesetzgebers, die Bewertung auf Grundlage vorsichtiger und vertretbarer Tatsachenermittlungen vorzunehmen, wobei er verpflichtet ist hierfür „Erkenntnisquellen auszuschöpfen und eine Risikoanalyse mit fachlicher Bewertung vorzunehmen.“ ${ }^{619}$ Sofern er hierbei eine Auswahl trifft, die nicht „feststehen-

614 In diese Richtung auch Callies, in: FS-Starck, S. 201 (212); Schimmele, Staatliche Verantwortung für diskursive Integrität, S. 119. Vgl. hierzu auch mit Unterscheidung zwischen obligatorischem und fakultativem Teil Isensee, in: ders./Kirchhof, HbStR IX, $\mathbb{1 9 1}$ Rn. 305.

615 So etwa befürwortend Moritz, Schutzpflicht gegenüber pflegebedürftigen Menschen, S. 120 m.w.N.

616 So i.E. jedenfalls Michael/Morlok, Grundrechte, Rn. 628 ff.; Bortnikov, Netzneutralität und Bedingungen kommunikativer Selbstbestimmung, S. $148 \mathrm{ff}$.; Hermes, Grund und Grenzen staatlicher Schutzansprüche, S. 253 f.; Calliess, JZ 2006, 321 (329); Krings, Grund und Grenzen staatlicher Schutzansprüche, S. 302 ff.; Lange, Grundrechtsbindung des Gesetzgebers, S. 404 f.

617 So Dietlein, ZG 1995, 131 (139).

618 Siehe hierzu u.a. Michael/Morlok, Grundrechte, Rn. 628 ff.; Bortnikov, Netzneutralität und Bedingungen kommunikativer Selbstbestimmung, S. $148 \mathrm{ff}$.; Hermes, Grund und Grenzen staatlicher Schutzansprüche, S. 253 f.; Krings, Grund und Grenzen grundrechtlicher Schutzansprüche, S. 302 ff.; Calliess, JZ 2006, 321 (329); ders., in: FS-Starck, S. 201 (215 f.); Krings, Grund und Grenzen staatlicher Schutzansprüche, S. 302 ff.; Mayer, Untermaß Übermaß und Wesensgehaltgarantie, S. $154 \mathrm{f}$. Ausführlicher hierzu Borowski, Grundrechte als Prinzipien, S. $266 \mathrm{ff}$.

619 BVerfG, NVwZ 2010, 702 Rn. 14 - CERN. 
de unvereinbare Tatsachen ignoriert “620, ist seine Entscheidung nicht angreifbar.

3. Das Schutzkonzept muss aber auch dasjenige sein, welches einerseits einen hinreichenden, effektiven Schutz bietet, andererseits aber am wenigsten stark in kollidierende Interessen eingreift. Solange keine Alternative denkbar ist, die offensichtlich bevorzugt werden müsste, handelt der Gesetzgeber in vertretbarer Wahrnehmung seines Spielraums.

4. Zuletzt muss das Schutzkonzept die Zweck-Mittel-Relation zwischen Schutz und kollidierenden (freiheitsrechtlichen) Belangen wahren. Auch hier besteht ein erheblicher Spielraum, der nur auf evidente Verstöße hin überprüft werden kann. Hierbei muss vor allem überlegt werden, ob etwaige Restrisiken, die bislang nicht geregelt sind, hingenommen werden müssen. Dies kann sich nur durch eine Abwägung ergeben, bei der Art und Ausmaß der Schäden, ihre Nähe und die Bedeutung des Rechtsgutes, die bestehenden Regelungen ${ }^{621}$ und auch die kollidierenden Grundrechtsbelange berücksichtigt werden. ${ }^{622}$

\section{c. Fazit: Untermaßverbot als rechtspraktische verfassungsrechtliche Grenze}

Was aus den jeweiligen Schutzpflichten konkret für den Staat - insbesondere den Gesetzgeber - folgt, ist in der Regel unbestimmt und hängt von verschiedenen Parametern ab. Dabei wird die Pflicht nicht nur durch den Vorbehalt des Unmöglichen oder durch rechtliche Grenzen außerhalb des Verfassungsrechts begrenzt. Wegen oftmals kollidierender Freiheitsrechte und dem zu berücksichtigenden Übermaßverbot bedarf es einer vorsichtigen und komplexen Abwägung von Freiheit und Sicherheit. Deshalb und weil mit der Schutzpflicht das Ziel, nicht aber der Weg dorthin vorgegeben ist, muss dem Gesetzgeber ein weiter Spielraum zukommen, der nur in wenigen Fällen auf eine bestimmte Maßnahme konkretisiert werden kann. Eine (justiziable) Verletzung der Schutzpflichten liegt nur dann vor, wenn der Gesetzgeber in evidenter Weise das Untermaßverbot verletzt, also Schutzmaßnahmen gar nicht ergreift oder erheblich hinter dem Mindestschutz zurückbleibt. Dies nachzuprüfen ist Aufgabe des Bundesverfassungsgerichts. Für die rechtspraktische Anwendung überzeugt es, in Anlehnung an das Übermaßverbot entsprechende Prüfungspunkte zu entwickeln. Hierbei gilt es einerseits den gesetzgeberischen Spielraum zu beachten, andererseits auch die abwehrrechtliche Seite kollidierender Grundrechte Dritter. Was final aus der Schutzpflicht folgt, hängt deshalb vom Einzelfall ab.

620 OVG Münster, Beschl. v. 05.05.2020 - 8 B 1153/20.N = BeckRS 2020, 7654 Rn. 25; Beschl. v. 19.05.2020 - 13 B 557/20.NE = BeckRS 2020, 9803 Rn. 47 m.w.N.

621 BVerfGE 49, 89 (142) - Kalkar I.

622 In diese Richtung Isensee, in: ders./Kirchhof, HbStR IX, $\mathbb{1 9 1}$ Rn. 297. 


\section{Kapitel: Aktivierung grundrechtlicher Schutzpflichten durch Meinungsroboter in digitalen Kommunikationsräumen?}

Vor diesem Hintergrund stellen sich auch mit Blick auf die Meinungsroboter und ihre oben beschriebenen Auswirkungen auf den Kommunikations- und Willensbildungsprozess (2. Teil, C.) zentrale Fragen, denen nachgegangen werden muss: Inwieweit begründet die Aktivität der Meinungsroboter eine staatliche Schutzpflicht (A.)? Und werden neben den Meinungsrobotern als „unmittelbare Störer“ auch die betreiberseitigen Verzerrungen relevant, die den Einsatz begünstigen können, sodass auch dies Schutzpflichten begründet (B.)?

\section{A. Schutzpflichten im Verhältnis (Bot-)Verwender-Staat-Nutzer}

Dass im politischen Kommunikations- und Willensbildungsprozess grundrechtliche Rechtsgüter relevant werden, sollte bereits hinreichend deutlich geworden sein (2. Teil. B.). Das bedeutet aber nicht zwangsläufig, dass Meinungsroboter diese in einer so erheblichen Weise tangieren, dass daraus auch eine staatliche Schutzpflicht für die betroffenen Rechtsgüter folgt. In den bislang vom Bundesverfassungsgericht entschiedenen Fällen konnte stets eine gewisse Gefahr ohne Weiteres ausgemacht werden. Das gilt nicht nur für den Schwangerschaftsabbruch $^{623}$, für die Bedrohung von durch Terroristen entführte Passagierflugzeuge $^{624}$ oder für das durch die friedliche Nutzung von Kernenergie ${ }^{625}$ gefährdete Lebensrecht, sondern etwa auch für die Gefahren für die (Vertrags-)Autonomie, die durch bestimmte Abhängigkeiten oder Machtgefälle entstehen. ${ }^{626}$ Im Falle der Meinungsroboter hingegen ist vieles unklar. Weder lässt sich zweifelsfrei feststellen, ob und in welchem Umfang Meinungsroboter aktiv sind, noch welche konkreten Einflüsse und Auswirkungen sie auf die Kommunikation und die Nutzer haben. Weil es damit - zumindest auf den ersten Blick - nur um ein Risiko geht, lässt sich hinterfragen, ob hieraus eine Schutzpflicht erwächst oder ob die Gewährung von Schutz nicht vielmehr der Prärogative des Gesetzgebers überlassen bleibt.

623 Hierzu BVerfGE 39, 1 ff. - Schwangerschaftsabbruch I; 88, 203 ff. - Schwangerschaftsabbruch II.

624 Siehe BVerfGE 115, 118 - Luftsicherheitsgesetz.

625 Hierzu statt vieler BVerfGE 49, 89 - Kalkar I.

626 Hierzu etwa BVerfGE 149, 126 Rn. 47 - Teilzeit- und Befristungsgesetz, m.w.N für den Schutz „strukturell unterlegene[r] Arbeitnehmerinnen und Arbeitnehmer“. Allgemein mit Blick auf die Vetragsautonomie etwa BVerfGE 81, 242 (254f.) - Handelsvertreter; 103, 89 (100f.) - Unterhaltsverzichtsvertrag; 114, 1 (34) - Bestandsübertragung Lebensversicherung; 114, 73 (90) - Lebensversicherung mit Überschussbeteiligung; BVerfG (K) MMR 2007, 93 - versicherungsvertragliche Obliegenheit zur Schweigepflichtentbindung. 
I. „Unschärfe“ im bisherigen Umgang mit der Schutzpflichten-Dogmatik im Kontext der Meinungsroboter

Schon die bisherige Diskussion im Kontext der Meinungsroboter ist von dem Bemühen geprägt, eine gewisse Relevanzschwelle verbindlich festlegen zu wollen, ab der Schutzpflichten bestehen sollen. Ausgangspunkt der Überlegungen ist meist die staatliche Verantwortung für die Aufrechterhaltung einer funktionierenden Kommunikationsordnung, ${ }^{627}$ wobei „[e]ine Handlungspflicht des Gesetzgebers [...] erst bei einer unmittelbaren Beeinträchtigung und ernsthaften Bedrohung ausgelöst [werde]. “628 Eine solche Lage soll nach divergierenden Auffassungen bei einer „existentiellen Gefährdung des normativen Vielfaltsbildes“ 629 oder dann vorliegen, wenn die Meinungsbildung unmöglich gemacht ${ }^{630}$ oder ein Diskussionskanal „vollständig lahmgelegt“ wird. ${ }^{631}$ Hiervon ausgehend werden spezifische Schutzmaßnahmen des Staates vor dem Einsatz der Meinungsroboter für (noch) nicht erforderlich gehalten. ${ }^{632}$ Allein Brings-Wiesen nimmt die Aktivierung von Schutzpflichten an, die eine zumindest vorsichtige Regulierung notwendig mache. ${ }^{633}$

Betrachtet man die Diskussion genauer, so lässt sich aber eine gewisse Unschärfe ausmachen. So wird nicht hinreichend deutlich, ob bereits die Aktivierung der Schutzpflicht unter den aufgezeigten Vorbehalten stehen soll und insoweit die Gefahrenschwelle konkretisiert wird, ${ }^{634}$ oder ob es sich vielmehr um eine Beschränkung der Rechtsfolge handelt. Dort, wo von einer „spezifischen staatlichen Schutzpflicht“635 bzw. „konkreten Schutzpflicht“636 die Rede ist, deutet

627 Im Kontext der Meinungsroboter Löber/Roßnagel, MMR 2019, 493 (496); Milker, ZUM 2017, 216 (220); Libertus, ZUM 2018, 20 (22); vgl. Klaas, MMR 2019, 84 (89). Allgemein mit Blick auf die Schutzgüter des Art. 5 Abs. 1 S. 1 GG etwa Grabenwarter, in: Maunz/Dürig, GG, Art. 5 Abs. 1 Rn. 108. Kühling, in: Gersdorf/Paal, Informations- und Medienrecht, Art. 5 GG Rn. 13 bezeichnet das als „Grundkonsens“, m.w.N. Ebenso Schimmele, Staatliche Verantwortung für diskursive Integrität, S. 202.

628 Löber/Roßnagel, MMR 2019, 493 (496). So auch Milker, ZUM 2017, 216 (220); ders., InTeR 2017, 199 (202f.). Auch hier allgemein mit Blick auf Art. 5 Abs. 1 S. 1 GG Grabenwarter, in: Maunz/ Dürig, GG, Art. 5 Abs. 1 Rn. 109.

629 Löber/Roßnagel, MMR 2019, 493 (496).

630 So etwa Steinbach, ZRP 2017, 101 (103), der damit Fälle „faktischer Knappheit“ erfasst wissen will. Siehe auch Milker, ZUM 2017, 216 (220).

631 Milker, ZUM 2017, 216 (220); ders., InTeR 2017, 199 (203). In diese Richtung auch Liesem, in: Litschka/Krainer, Der Mensch im Digitalen Zeitalter, S. 183 (194).

632 In diese Richtung etwa Löber/Roßnagel, MMR 2019, 493 (496); vgl. Milker, ZUM 2017, 216 (220 f.). Ausdrücklich - allerdings mit Blick auf eine konkrete Schutzpflicht - Liesem, in: Litschka/ Krainer, Der Mensch im digitalen Zeitalter, S. 183 (194). Offenlassend indes Klaas, MMR 2019, 54 (89).

633 Brings-Wiesen, JuWiss-Blog Nr. 93/2016. In diese Richtung auch Hostacna, AL 2018, 1 (4).

634 In diese Richtung wohl eher Löber/Roßnagel, MMR 2019, 493 (496), wenn es im Kontext der Handlungspflicht heißt, dass der Gesetzgeber den Meinungsbildungsprozess durch geeignete Maßnabmen schützen muss.

635 Milker, ZUM 2017, 216 (220).

636 Libertus, ZUM 2018, 20 (22); Milker, ZUM 2017, 216 (220). 
vieles darauf hin, dass die Schutzpflicht des Staates abstrakt für aktiviert gehalten wird und allein an der Notwendigkeit spezifischer regulativer Maßnahmen gezweifelt wird. ${ }^{637}$ Allein Klaas macht ausdrücklich deutlich, dass er zumindest Zweifel an der Aktivierung von Schutzpflichten hat, auch wenn er dies auf die Notwendigkeit konkreten Handelns bezieht. ${ }^{638}$

Soweit sich die vorgeschlagenen Eingrenzungen auf die Rechtsfolge beziehen und die Grenze für spezifische Handlungen markieren sollen, verkennt man aber, dass aus der Schutzpflicht immer ein spezifisches Handlungsziel folgt, nämlich Schutz vor den Risiken bzw. Gefahren zu gewähren. Eine davon zu trennende Frage ist dann, wie dieser Schutz auch mit Rücksicht auf kollidierende Belange verfassungskonform aussehen kann. Konkrete Handlungsanweisungen im Sinne einer ganz bestimmten Maßnahme werden also wegen der Prärogative des Gesetzgebers nur selten aus einer Schutzpflicht folgen. ${ }^{639}$ Insoweit wäre also das Bestehen einer Schutzpflicht von dem Bestehen einer Regelungspflicht zu trennen.

Soweit man die Begrenzung schon auf Tatbestandsseite fruchtbar macht, ${ }^{640}$ würde damit zwar dem Ansatz einer spezifischen Gefahrenschwelle entsprochen werden. Gleichwohl bestehen erhebliche Zweifel an den aufgezeigten Versuchen. Zum einen wird nicht begründet, warum die gewählte Schwelle dort verlaufen soll, wo sie gezogen wird. Wenn hier auf die grundlegende Bedeutung der Meinungsfreiheit hingewiesen wird, ${ }^{641}$ ist damit nicht viel gewonnen. Und zum anderen kann sich die Ernsthaftigkeit der Bedrohung auf das Schadensausmaß, die Eintrittswahrscheinlichkeit oder auf beides beziehen. Damit werden aber wesentlich höhere Anforderungen gestellt, als es die polizeirechtliche Gefahrenschwelle

637 Deutlich wird dieser Ansatz etwa bei Milker, ZUM 2017, 216 (220), der nicht nur von einer „,konkreten“ bzw. „spezifischen Schutzpflicht“ spricht, sondern hier auch mit dem allgemeinen Risiko eines nicht repräsentativen Internets argumentiert. Das entspricht folglich den „unentrinnbaren, sozialadäquaten Lasten“ die das BVerfG etwa bei der Nutzung von Kernenergie, BVerfGE 49, 89 (143) Kalkar I, oder risikobehafteter physikalischer Forschung, BVerfG, NVwZ 2010, 702 Rn. 13 CERN, betont hat. Deutlicher macht Milker das in InTeR 2017, 199 (203), wo er explizit den Gestaltungsspielraum anspricht und darauf hinweist, dass sich dieser nur bei einer ernsthaften Bedrohung verenge.

638 Klaas, MMR 2019, 84 (89).

639 BVerfGE 96, 56 (64) - Vaterschaftsauskunft; BVerfG, NJW 2016, 1716 Rn. 19 - Pflegenotstand. In der Judikatur des BVerfG war das selten der Fall. Hinsichtlich des Schwangerschaftsabbruchs meinte das BVerfG, dass das Strafrecht eingesetzt werden und der Schwangerschaftsabbruch als Unrecht gelten müsse, BVerfGE 39, 1 (44) - Schwangerschaftsabbruch I; 88, 203 (252) - Schwangerschaftsabbruch II. Zum Luftsicherheitsgesetz hatte das BVerfG gemeint, dass der Abschuss entführter Flugzeuge die einzig mögliche Handlung sei. Wegen kollidierender Grundrechte der Insassen hatte es dies allerdings für verfassungswidrig gehalten, BVerfGE 115, 118 (159 ff.) - Luftsicherheitsgesetz.

640 So klingt das etwa bei Löber/Roßnagel, MMR 2019, 493 (496), die nur von der „Handlungspflicht des Gesetzgebers“ sprechen und sodann meinen, wenn die genannte Schwelle erreicht ist, „muss der Gesetzgeber [...] durch geeignete Maßnahmen schützen“.

641 So insbesondere Grabenwarter, in: Maunz/Dürig, GG, Art. 5 Abs. 1 Rn. 109. 
vorsieht. ${ }^{642}$ Denn die elementare Bedeutung der betroffenen Rechtsgüter ${ }^{643}$ müsste eher zu einer Absenkung der Anforderungen führen. Andernorts wird gerade die Notwendigkeit präventiver Regelungen betont, um frühzeitig Fehlentwicklungen für den Kommunikationsprozess zu begegnen. ${ }^{644}$ Demnach scheint die Kategorie der „ernsthaften Bedrohung“ also klare Fälle zu umfassen, in denen vom Staat (regulative) Schutzmaßnahmen spätestens und zwingend zu ergreifen sind ${ }^{645}$ Sie kann aber nicht als abschließende Fallgruppe verstanden werden.

Möglicherweise sind die Versuche, eine pauschale Schwelle festlegen zu wollen, eher ergebnisorientiert zu erklären. Nicht jede Täuschung und auch nicht jede Beeinträchtigung ${ }^{646}$ der Äußerungsbereitschaft soll Regelungspflichten auslösen. Denn es wäre bedenklich, wenn der Staat schon bei geringfügigen Gefahren verpflichtet wäre, ein hohes, eingriffsintensives Schutzniveau zu gewähren, immerhin operiert er dann am offenen Herzen des demokratischen Prozesses. ${ }^{647}$ Das ist allerdings weder eine tatbestandliche Frage, noch schließt das eine Schutzverantwortung per se aus. Der Staat unterliegt immerhin Begrenzungen von zwei Seiten. Vor allem die abwehrrechtliche Seite der Meinungsfreiheit oder anderer kollidierender Grundrechte verhindert einen gesetzgeberischen Exzess. Den Prinzipien staatlicher Neutralität und der Staatsferne des Kommunikationsprozesses kommt also auf Rechtsfolgenseite entscheidende Bedeutung zu und verschmälert den „Handlungskorridor“. Allgemein zeigt sich an den Darstellungen in der Literatur die fehlende Systematik grundrechtlicher Schutzpflichten und mit ihr die fehlende Trennung zwischen tatbestandlichen Voraussetzungen und den eigentlichen Rechtsfolgen. ${ }^{648}$ Vieles spricht daher dafür, dass die genannten Schwellen eher angeben, ab wann konkrete Maßnahmen unbedingt notwendig

642 In der Tendenz geht das eher in die Richtung einer gegenwärtigen Gefahr (vgl. etwa $\$ 3$ Abs. 3 Nr. 3 SOG MV), womit qualitativ höhere Anforderungen gestellt werden. Vgl. hierzu auch BVerfGE 141, 220 Rn. 110, 111 - BKAG.

643 Siehe nur zur Meinungsfreiheit BVerfGE 7, 196 (208) - Lüth.

644 So insbesondere das BVerfG mit Blick auf Verzerrungen und Gefahren im herkömmlichen Rundfunk, BVerfGE 121, 30 (52) - Parteibeteiligung an Rundfunkunternehmen, m.w.N. aus der Judikatur. Aus dem Schrifttum etwa Brinkmann, UFITA 2019, 364 (377) und Fehling/Leymann, AfP 2020, 110 Rn. 41.

645 So jedenfalls ausdrücklich Milker, InTeR 2017, 199 (203).

646 Milker, ZUM 2017, 216 (220). Vgl. Schröder, DVBl. 2018, 465 (469).

647 Vgl. etwa Fehling/Leymann, AfP 2020,110 (115); Martini, Blackbox Algorithmus, S. 103, spricht hier von einer „Odysee zwischen Skylla und Charybdis: Auf der einen Seite läuft er [der Staat] Gefahr, die Funktionsbedingungen einer pluralistischen Demokratie zu unterspülen, wenn er soziale Netzwerke untätig treiben lässt. Auf der anderen Seite kann hektischer Aktionismus die von staatlichen Vorgaben freie Meinungsbildung des demokratischen Diskurses durch Einschüchterungseffekte staatlicher Wahrheitskontrolle gefährden." Vgl. auch Schimmele, Staatliche Verantwortung für diskursive Integrität, S. 266 die eine staatliche Pflicht zum Schutz vor fehlerhaften Informationen deshalb ablehnt, weil „dies faktisch zu einer staatlich gesteuerten Informationspolitik [führt].“

648 Denn wenn Schimmele, ebd., die Verantwortung des Nutzers für die kritische Rezeption von Informationen betont, dann werden damit der staatlichen Verantwortung unmittelbar verfassungs- bzw. grundrechtliche Grenzen gezogen. Diese Grenzziehung ist aber keine Frage des Tatbestandes und damit der Frage, $o b$ eine Verantwortung besteht, sondern begrenzt allein die Ausfüllung der Verantwortung (erheblich). 
sind und damit auch auf Rechtsfolgenseite gesetzgeberische Maßnahmen (zwingend) erfordern. ${ }^{649}$ Wenn also in der Literatur gemeint wird, dass der Staat keine Maßnahmen zum Schutz vor Meinungsrobotern ergreifen müsse, ${ }^{650}$ so heißt das nicht, dass eine Schutzverantwortung nicht besteht. Vielmehr können Aspekte der Neutralität und des freien Meinungsbildungsprozesses o.ä. hier der Wahrnehmung der Schutzverantwortung derart entgegenstehen, dass lediglich beobachtende oder informierende Maßnahmen - etwa im Wege der Öffentlichkeitsarbeit - zulässig sind. Insoweit sind die Begriffe „(konkrete) Schutzpflicht“ und „Regelungspflicht“ gerade nicht kongruent.

\section{Relevanter Übergriff auf grundrechtliche Schutzgüter?}

Besinnt man sich auf die allgemeinen tatbestandlichen Voraussetzungen für die Aktivierung einer Schutzpflicht zurück, so gilt es zunächst einmal zu ermitteln, ob ein für die Begründung der Schutzpflicht relevanter Übergriff in grundrechtliche Schutzgüter durch die Verwendung von Meinungsrobotern erfolgt. Dass es insoweit um eine klassische Dreieckskonstellation geht, die den Staat als Garanten fordert, liegt auf der Hand. Die hier relevanten Risiken rühren nicht von dem deutschen Staat, sondern von nicht grundrechtsgebundenen Dritten her. Soweit sie auch durch ausländische Staaten begründet wird, wie etwa im Falle der Internet Research Agency Russlands, ${ }^{651}$ besteht mit der Anknüpfung an den demokratischen Willensbildungsprozess und die im Inland eintretende Wirkung auch ein spezifischer Inlandsbezug. Insoweit ist allein zu untersuchen, ob Meinungsroboter auch in einer erheblichen Weise grundrechtliche Schutzgüter tangieren.

\section{1. Übergriff auf die Meinungsäußerungsfreiheit}

Die Meinungsfreiheit des Art. 5 Abs. 1 S. 1 GG wird dort relevant, wo der Einzelne unmittelbar oder faktisch daran gehindert wird, seine Meinung frei kundzutun. Sie umfasst vor allem das Recht, sich diskriminierungsfrei zu äußern und Gehör zu verschaffen ${ }^{652}$ und dabei auch über die Modalitäten der Äußerungen, insbesondere Ort und Zeit, zu entscheiden ${ }^{653}$. Insoweit ist also die Meinungsfrei-

649 So ausdrücklich etwa Milker, InTeR 2017, 199 (203).

650 In diese Richtung Steinbach, ZRP 2017, 101 (105), allerdings bezogen auf politische Wünsche, „den Einsatz von Social Bots in Wahlkampfzeiten zu verbieten.“; Milker, ZUM 2017, 216 (220 f.).

651 Siehe hierzu etwa Mueller, Report On The Investigation Into Russian Interference In The 2016 Presidential Election, Vol. I, S. 14 ff., und speziell zu den Botnetzwerken auf Twitter, ebd., S. $28 \mathrm{f}$.

652 Holzngael, in: Hoeren/Sieber/ders., Multimedia-Recht, Teil 3 Rn. 137, bezeichnet dies als Grundvoraussetzung. Wendt, in: v. Münch/Kunig, Art. 5 Rn.17, der auf die geistige Wirkung und die Möglichkeit „andere mit seiner Meinung werbend zu erreichen“ abstellt.

653 Statt vieler Grabenwarter, in: Maunz/Dürig, GG, Art. 5 Abs. 1 GG Rn. 82 ff., 91 ff. m.w.N. 
heit jedenfalls immer dann tangiert, wenn sich Personen gar nicht oder nur in begrenztem Umfang äußern können.

\section{a. Übergriff durch „psychische Zwangswirkung“?}

Anders als in Fällen, in denen mit von außen erzeugtem Druck oder Gewalt eine Äußerung verhindert werden soll (vgl. $\mathbb{S} \mathbb{S} 108$ Abs. 1, 240 Abs. 1 StGB) geht es im Falle deer Meinungsroboter allein um „psychische Zwangswirkungen“, die durch das wahrgenommene Meinungsklima des jeweiligen Kommunikationsumfeldes verursacht werden (Theorie der Schweigespirale) und damit eher um eine Selbst- denn Fremdzensur. ${ }^{654}$ Deshalb ist aber nicht der Charakter eines Übergriffs abzulehnen. Von der Wirkungsweise unterscheiden sich beide Methoden kaum. In beiden Fällen soll der Wille des Äußernden gebeugt werden. Zwar mögen Gewalt und Drohung illegitimer und unvereinbar mit den grundrechtlichen Wertvorstellungen sein. ${ }^{65}$ Gleichwohl sind in beiden Fällen wesentliche Wirkungsvoraussetzungen in der Außenwelt begründet, die dann maßgeblich die Willensbetätigung beeinflussen. Das ist insoweit vergleichbar mit den auf abwehrrechtlicher Seite bedeutsamen chilling effects. Denn wenn beispielsweise eine anonyme Äußerung erschwert wird, weil eine Datenspeicherung angeordnet würde, dann hat das auch erst einmal eine psychologische Wirkung dergestalt, dass aus Angst vor potentiellen Konsequenzen eine Meinungsäußerung unterbleibt. ${ }^{656}$ Gleichwohl führt das nicht dazu, dass man hier den Eingriffscharakter ablehnt. ${ }^{657}$ Auch wenn die Makroebene stetig auf die Mikroebene einwirkt und lediglich die Wirkungsintensität variiert, darf nicht außer Betracht bleiben, dass es nicht um „normale“ Einflüsse diskursiver Öffentlichkeit geht. Diese Wirkungen werden künstlich erzeugt: falsche Mehrheiten werden suggeriert und bestehende Heuristiken gezielt ausgenutzt, um die einschüchternde, selbst-zensierende Wirkung herbeizuführen. Insoweit ist der Vorgang der Selbst-Zensur zwar ein innerer. Die wesentlichen Grundbedingungen werden aber durch Dritte und damit extern gesetzt. Daran ändert auch die gedachte Anonymität im Internet nichts. Diese führt nicht unbedingt zu einer „Liberalisierung“ der Meinungsäußerung. Vielmehr legen Studien den Schluss nahe, dass die selbstzensierenden Effekte. wegen der

654 Zur Schweigespirale oben, 1. Teil B.III.3.a.

655 Mit Blick auf wirtschaftlichen Druck siehe etwa BVerfGE 25, 256 (264ff.) - Blinkfüer. Daneben siehe auch BVerfGE 62, 230 (244 f.) - Boykottaufruf; BVerfG NJW 1989, 381 (382) - Mietboykott.

$656 \mathrm{Zu}$ den chilling effects in derlei Konstellationen etwa Gräfe, PinG 2019, 5 (7).

657 Mit Blick auf Art.10 EMRK (hier die Pressefreiheit) EGMR, Urt. v. 27.03.1996 - 17488/90= BeckRS 2012, 18728 Rz. 39 - Goodwin v. UK. Vgl. auch Grabenwarter, in: Maunz/Dürig, GG, Art. 5 Abs. 1 Rn. 104. Kersten, JuS 2017, 193 (196), sieht die Vermeidung von chilling effects nicht nur als Frage der abwehrrechtlichen Dimension, sondern ebenfalls als „Ausdruck staatlicher Schutzpflichten, dass Bürgern [...] eine anonyme Meinungskundgabe ermöglicht wird, in denen beispielsweise individuelle oder soziale, rechtliche oder wirtschaftliche Repressalien für die freie Meinungsäußerung zu erwarten sind.“. 
Angst vor unkontrollierten und dadurch stärkeren Sanktionen in sozialen Netzwerken stärker sein können als in der realen Welt. ${ }^{658}$

\section{b. Überschreitung der Bagatellgrenze - Einsatz von Meinungsrobotern als bloße Belästigung?}

Insoweit könnte allein der Einwand vorgebracht werden, dass es sich hierbei nur um eine bloße Bagatelle, eine zumutbare Belästigung handelt, die hingenommen werden muss. ${ }^{659}$ Ausgangspunkt der Überlegung ist hierbei die dezentrale Kommunikationsstruktur innerhalb der sozialen Netzwerke: Anders als in herkömmlichen Meinungsforen gibt es keinen bestimmten Ort für themenspezifische Diskussionen. Vielmehr können sich diese situationsbedingt ergeben. Jeder Beitrag, jede Seite, jede Gruppe und jedes Hashtag können Diskussionsorte werden, ${ }^{660}$ sodass jeder Nutzer mehr als nur eine Möglichkeit hat, seine themenspezifische Meinung kundzugeben. Dort, wo Meinungsroboter nicht aktiv sind, haben sie auch keinen Einfluss auf die Äußerungsbereitschaft.

Das würde freilich voraussetzen, dass die Erheblichkeit einer Beeinträchtigung allein an quantitativen Maßstäben zu messen wäre. Im Schrifttum finden sich durchaus entsprechende Ansätze, mit denen jedenfalls im Ergebnis der (bislang) harmlose Charakter der Meinungsroboter begründet wird. So meint Milker etwa - auch wenn hierbei nicht der Tatbestand, sondern die Rechtsfolge begrenzt wird,$-{ }^{661}$ dass,$[e]$ ine konkrete Schutzpflicht [...] ausnahmsweise nur dann begründet [wird], wenn ein Diskussionskanal komplett lahmgelegt wird. “662 Und Steinbach meint etwa, dass sich die Unzulässigkeit aus Sicht der Meinungsbil-

658 Siehe hierzu neben Hampton u.a., Social Media and the ,Spiral of Silence', S. 4 auch Neubaum/ Krämer, Communication Research 45 (2018), 139 (157f.).

659 Isensee, in: ders./Kirchhof, HbStR IX, $\mathbb{1} 191 \mathrm{Rn} .239$. Vgl. hierzu etwa BVerfGE 102, 347 (364) Schockwerbung I, wo das BVerfG explizit meint, dass „[e]in vom Elend der Welt unbeschwertes Gemüt des Bürgers [...] kein Belang [ist], zu dessen Schutz der Staat Grundrechtspositionen einschränken darf. Anders kann es zu beurteilen sein, wenn ekelerregende, furchteinflößende oder jugendgefährdende Bilder gezeigt werden.“ Die Abgrenzung von gesundheitsgefährdender Umweltbelastung und hinnehmbarer Belästigung betont Voßkuble, NVwZ 2013, 1 (6).

660 So zu Twitter letztlich auch Shao u.a., IEEE Access Vol. 6 (2018), 75327 (75336).

661 Jedenfalls würde das der Differenzierung zwischen abstrakt bestehender Schutzverantwortung und konkreter Schutz- bzw. Regelungspflicht entsprechen. Vgl. zu der Differenzierung zwischen „allgemeiner" und „konkreter Schutzpflicht“ auch BVerfGE 142, 313 (338) - Zwangsbehandlung. Auch wenn der Ansatz eines vollständigen Lahmlegens plausibel klingen mag, so ergeben sich dann gerade wegen der dezentralen Kommunikationsstruktur Probleme bei der Anwendung. Was macht einen Diskussionskanal aus, und wann ist er im Sinne des Ansatzes von Milker vollständig lahmgelegt? Je nachdem, ob man einen einzelnen Beitrag oder ein einzelnes Hashtag, ein bestimmtes Angebot (etwa die Seite des ZDF) oder das gesamte Netzwerk als „Diskussionskanal“ begreift, variieren auch die Anforderungen an die Lahmlegung.

662 Milker, ZUM 2017, 216 (220); ders., InTeR 2017, 199 (203). In diese Richtung auch Liesem, in: Litschka/Krainer, Der Mensch im Digitalen Zeitalter, S. 183 (194) und vgl. auch Semizoğlu, in: Hetmank/Rechenberg, Kommunikation, Kreation und Innovation, S. 79 (95). 
dungsfreiheit erst dann ergebe, wenn es zu Fällen faktischer Knappheit, etwa einer limitierten Darstellung individueller Meinungen, käme. ${ }^{663}$

In der Judikatur finden sich auch Konstellationen, in denen das Gewicht von Beeinträchtigungen der Äußerungsmöglichkeiten mit quantitativen Überlegungen relativiert wurde. So hielt das VG Mainz den Ausschluss eines Nutzers von der Kommentarfunktion auf Seiten des ZDF wegen des wiederholten Publizierens von Hassrede für rechtmäßig. ${ }^{664}$ Zwar ging das VG schon davon aus, dass es sich bei den Äußerungen um Fomalbeleidigungen und Schmähkritik handelte. ${ }^{665}$ Aber auch unabhängig davon überwögen die kollidierenden Interessen, weil die Äußerungsmöglichkeit nicht insgesamt, sondern nur zu einem (unbedeutenden) Teil genommen wurde. ${ }^{666}$

„Zwar wird ihm hierdurch die Möglichkeit genommen, weiterhin an den Diskussionen auf den betroffenen Facebook-Profilen des Bekl. teilzunehmen und hier seine Meinungen zu äußern. Angesichts der großen Verbreitung von Facebook und der wachsenden Bedeutung virtueller öffentlicher Räume für die Ausübung der Meinungsfreiheit, ist diese einschränkende Wirkung für den Kl. auch nicht zu unterschätzen. Gleichwohl wird dem Kl. durch die Sperren nicht grds. die Möglichkeit genommen, seine Meinungen weiterhin öffentlich - auch auf einer Vielzahl von Plattformen im Internet - kundzutun. [...] Schließlich hat der Bekl. in der mündlichen Verhandlung auch klargestellt, dass es sich keineswegs um "lebenslange" Sperren handelt, der Bekl. - anders als Facebook technisch aber nicht die Möglichkeit habe, eine Sperre zugleich mit ihrer Vornahme zu befristen. " 667

Das LG Frankfurt a.M. urteilte unter Berufung auf das VG Mainz ähnlich. ${ }^{668}$ Der Kläger machte einen Anspruch aus den $\mathbb{S} \mathbb{S} 241$ Abs. 2, 1004 BGB gegen den Betreiber eines Netzwerkes gerichtet auf die Wiederherstellung eines gesperrten Kommentars ${ }^{669}$ geltend, der nach Ansicht des Betreibers gegen die Nutzungsbedingungen verstieß. Das LG gab dem Antrag nicht statt, weil ein Verfügungsanspruch gegen den Betreiber nicht bestünde. Zwar seien im Wege der mittelbaren Drittwirkung auch Grundrechte im Rahmen der Rücksichtnahmepflichten $(\mathbb{S} 241$ Abs. 2 BGB) zu berücksichtigen. Gleichwohl wurde die Sperrung nicht nur wegen der durch Hassrede beeinträchtigten Interessen des Betreibers, eine sachbezogene Diskussion und die freie Rede aller Nutzer zu gewährleisten, als rechtmäßig er-

663 Steinbach, ZRP 2017, 101 (103).

664 VG Mainz, MMR 2018, $556 \mathrm{ff}$.

665 VG Mainz, a.a.O. Rn. $122 \mathrm{ff}$.

666 VG Mainz, a.a.O. Rn. 139.

667 VG Mainz, a.a.O. Rn. 145.

668 LG Frankfurt a.M., MMR 2018, 770.

669 Unter einem Beitrag der „Welt“ zu einem Angriff auf Polizisten durch Asylbewerber veröffentlichte der Kläger: „Wasser marsch, Knüppel frei und dann eine Einheit Militärpolizisten! Dann ist schnell Ruhe! Und jeden ermittelten Gast Merkels ab in die Heimat schicken.“. 
achtet, sondern auch deshalb, weil der Umfang der Sperrung gering und daher die Löschung angemessen sei. ${ }^{670}$

„Demgegenüber geht es vorliegend darum, dass dem Ast. die Wiederholung seiner Äußerung allein auf der Plattform der Ag. untersagt wird. Es geht also gerade nicht darum, dass der Ast. sich überhaupt nicht derart äußern kann, dies steht ihm außerhalb der Plattform der Ag. unabhängig vom hiesigen Antrag frei [...]. Der Eingriff in die Rechte des Ast. ist daher insgesamt ggü. einer äußerungsrechtlichen Unterlassungsverfügung oder durch die genannten Äußerungsdelikte deutlich eingeschränkt. Hierbei verkennt die Kammer nicht, dass die Ag. [... ein] wesentlicher Marktplatz für Informationen darstellt und ein großes Interesse für den Ast. daran besteht, seine Meinung auf dieser konkreten Plattform äußern zu können. Der Eingriff in die Rechte des Ast. ist hier zweifelsohne erheblich, er enthält jedoch kein Gesamtverbot und ist damit jedenfalls im Vergleich dazu weniger schwerwiegend. "671

Auch der EGMR wies in der Rechtssache Delfi jedenfalls am Rande darauf hin, dass das Internet dem Einzelnen viele Möglichkeiten biete, sich Gehör zu verschaffen, weshalb die Verpflichtung eines Providers, Hassrede wirksam einzuschränken, „in keiner Weise mit einer, privaten Zensur' gleichgestellt werden [könne]. “672 Und letztlich findet sich das Argument der Quantität auch in der Literatur wieder. So weist etwa Lüdemann daraufhin, dass die Angewiesenheit auf das Netzwerk kein Argument dafür sei, die Regelungen der sozialen Netzwerke und ihre darauf gestützte Sperr- und Löschpraxis für unzulässig zu halten. Immerhin bestünden zahlreiche weitere Möglichkeiten, denselben Inhalt an anderer Stelle zu äußern. ${ }^{673}$

Generell ließe sich einem quantitativen Ansatz zwar schon entgegenhalten, dass die Bedeutung des Netzwerks für die Äußerung des Einzelnen und die Kommunikation insgesamt verkannt wird. ${ }^{674}$ Andere Dienste und internetbasierte Äußerungsmöglichkeiten haben immerhin nicht ansatzweise denselben Stellenwert und dieselbe Reichweite ${ }^{675}$ wie das streitgegenständliche Netzwerk, ${ }^{676}$ sodass allein die vom LG Frankfurt angesprochenen kollidierenden Interessen die

670 In einem ähnlich gelagerten Fall hat auch das LG Görlitz auf eine ähnliche Argumentation abgestellt. Die Entscheidung ist zwar nicht veröffentlicht, allerdings greift das OLG Dresden die Argumentation des LG auf. Zum Tatbestand siehe OLG Dresden, MMR 2018, 756 und zur Argumentation des LG ebd., Rn. 19.

671 LG Frankfurt, MMR 2018, 770 Rn. 43.

672 EGMR, Urt. v. 16.06.2015 - 64569/09 = NJW 2015, 2863 Rn. 157 - Delfi/Estland.

673 Lüdemann, MMR 2019, 279 (284).

674 So meint etwa Raue, JZ 2018, 961 (966), dass „die von den Plattformen angebotene Leistung nicht ohne Weiteres substituierbar [ist]." Und auch König, AcP 219 (2019), 611 (633), will zumindest die Bedeutung der Netzwerke für die Kommunikation bei der Interessenabwägung - allerdings im Falle einer Klauselkontrolle nach $\$ 307$ Abs. 1 BGB - berücksichtigt wissen.

675 Kritisch hierzu Lüdemann, MMR 2019, 279 (284), der darauf hinweist, dass aus Art. 5 Abs. 1 S. 1 GG kein Anspruch auf Reichweite folgt.

676 In diese Richtung etwa OLG Dresden, MMR 2018, 756 Rn. 19. Siehe hierzu auch BVerfG, NVwZ 2019, 959 Rn. 19 - III.-Weg. 
Rechtmäßigkeit begründen können. ${ }^{677}$ Abseits hiervon bestehen auf den ersten Blick durchaus Gemeinsamkeiten: Auch im Falle der Meinungsroboter werden Nutzer - faktisch - am Verfassen von Kommentaren gehindert. Diese faktische Sperre gilt nicht für das gesamte Netzwerk, sondern nur für betroffene Netzwerkknoten und Diskussionspunkte. Und wenn eine unmittelbare Beschränkung rechtmäßig ist, dann müsste das doch erst recht für lediglich mittelbar-psychologische Wirkungen gelten. Auf den zweiten Blick ergeben sich indes evidente Unterschiede: Der Nutzer setzt vorliegend keinen kausalen Beitrag, der Grundlage der Sperrung ist. Höher zu gewichtende, kollidierende Belange sind - anders als bei Nutzer- oder Inhaltssperren - nicht betroffen. Und auch die rein psychische Wirkung taugt nicht als Argument. Zum einen ist die Unterscheidung zwischen mittelbaren und unmittelbaren Zwangswirkungen oftmals theoretischer Natur, weil sie sich in ihrer tatsächlichen Wirkung ähneln. ${ }^{678}$ Zum anderen mag die faktische Sperrwirkung zwar allein von sozial-psychologischen Effekten abhängig sein, die von Person zu Person unterschiedlich stark sein können (Theorie der Schweigespirale) und die auch in der vor-digitalen Welt schon wirkten. Das verkennt allerdings, dass es ja nicht um eine unabdingbare Wirkung diskursiver Öffentlichkeit geht, sondern dass diese Wirkung im konkreten Einzelfall vielmehr künstlich erzeugt wird, gerade um eine diskursive Auseinandersetzung $\mathrm{zu}$ verhindern. Anlassbezogene betreiberseitige Sperrungen sollen hingegen diese ungestörte diskursive Öffentlichkeit schützen. Insoweit bestehen also gewichtige strukturelle Unterschiede.

Daneben lässt ein rein quantitativer Ansatz entscheidende Parameter unberücksichtigt. Wenn im Einzelfall nur wenige Diskussionspunkte betroffen sind, kann die Gesamtwirkung wesentlich gravierender sein als im umgekehrten Fall. Das mag zunächst verwundern, leuchtet aber angesichts des dezentralen Charakters der Netzwerke ein: Das individuelle Netzwerk eines jeden Nutzers unterscheidet sich und mit ihm die zentralen Netzwerkknoten und Diskussionspunkte. So ergeben sich etwa spezifische Punkte an denen überwiegend Personen mit derselben ideologischen Einstellung oder denselben politischen Ansichten aufeinandertreffen. Daneben bestehen aber auch neutralere Orte, die Schnittpunkte vieler individueller Netzwerke sind und die wegen der deutlich größeren Reichweite eine zentralere Bedeutung als andere Netzwerkknotenpunkte haben. Dass dies einen Unterschied machen kann, kann an den trending topics von Twitter verdeutlicht werden: Während des syrischen Bürgerkrieges ist etwa das Hashtag

677 Auf die Beeinträchtigungswirkung auf Diskussionen etwa hinweisend LG Frankfurt a.M., MMR 2018, 545 Rn. 13; ebd.,770 Rn. 14, (69, 70); LG Karlsruhe, Beschl. v. 26.11.2018 - 4 O 270/18 = BeckRS 2018, 42508 Rn. 30, 35; und im Anschluss hieran OLG Karlsruhe, NJW-RR 2019, 1006 Rn. 29. Nur im Ansatz VG München, MMR 2018, 418 Rn. 17, 24, 29 und VG Mainz, MMR 2018, 556 Rn. 88.

678 Das zeigt nicht zuletzt auch die oftmals nicht ergebnisrelevante Unterscheidung zwischen unmittelbaren und mittelbar-faktischen Eingriffen des Staates in grundrechtliche Schutzgüter. 
\#Syria für darauf bezogene Diskussionen kennzeichnend gewesen. ${ }^{679}$ In Mexiko wurde das Hashtag \#YaMeCanse zum Kennzeichen einer scharfen und breit geführten Kritik an staatlichen Behörden. ${ }^{680}$ In beiden Fällen sollen Bots versucht haben, die Diskussion und Informationsweitergabe zu stören, indem sie das Hashtag dekontextualisierten oder zum Verbreiten von Spam verwendeten. Dadurch wurde die Partizipation an der Diskussion und die Rezeption diskussionsspezifischer Inhalte erschwert. Im Falle von \#YaMeCanse führte das gar dazu, dass die Diskussion sukzessive zu \#YaMeCanse1, \#YaMeCanse2 usw. verlagert werden musste. Die Partizipation an der Diskussion ging zurück, wofür allerdings auch der lange Zeitraum (mit-)ursächlich gewesen sein kann. Auch wenn hier letztlich nur ein Schlagwort attackiert wurde, hat das eine potentiell bedeutsamere Wirkung. Von einer bloßen Belästigung lässt sich in solchen systematischen und gezielten Kampagnen kaum sprechen.

Der Einsatz von Meinungsrobotern lässt sich also nicht per se als bagatellhaft oder unbedeutend charakterisieren. Konkrete Auswirkungen variieren im Einzelfall. Entscheidend kommt es vor allem auf die Qualität einer Beeinträchtigung und damit eine wertende Betrachtung an. Werden zentrale Diskussionspunkte angegriffen und eine Diskussion gestört, so ist das am ehesten noch vergleichbar mit der Störung von Versammlungen im öffentlichen Raum durch Dritte. Auch hier wird nicht von einer Bagatelle gesprochen, sondern es werden Schutzpflichten bemüht. ${ }^{681}$ Insoweit handelt es sich auch beim Einsatz von Meinungsrobotern aus Sicht der Meinungsfreiheit um einen relevanten Übergriff.

\section{Veränderung der „kommunikativen Chancengleichbeit“}

Davon zu trennen ist allerdings die Frage, ob auch dann ein relevantes Drittverhalten vorliegt, wenn man sich äußert, die Äußerung aber in der Masse der Gegenbeiträge untergeht und so die Rezeptionswahrscheinlichkeit sinkt. Gemeint sind insbesondere Konstellationen, in denen Diskussionen dadurch gekapert werden, dass massenhaft Inhalte veröffentlicht oder Beiträge anderer Teilnehmer unterstützt werden, um eine gewisse Dominanz auszustrahlen. Dabei helfen sicherlich auch die Algorithmen, die Kommentare oder Inhalte an Diskussionspunkten nach verschiedenen Kriterien und nicht nur chronologisch anordnen. Wegen der suggerierten Nutzermehrheit durch Meinungsroboter können also andere „normale“ Beiträge unterdrückt oder weniger priorisiert angezeigt werden,

679 Hierzu Abokhodair/Yoo/McDonald, in: ACM, Proceedings of the $18^{\text {th }}$ ACM Conference on Computer Supported Cooperative Work \& Social Computing, S. 839 (849).

680 Suárez-Serrato u.a., in: Spiro/Ahn, SocInfo 2016: Social Informatics, S. $269 \mathrm{ff}$.

681 In diese Richtung etwa Stern/Sachs/Dietlein, in: Stern, Staatsrecht IV/1, S. 1274 f.; Schulze-Fielitz, in: Dreier, GG, Art. 8 Rn. 110, 113 jeweils m.w.N. Offenlassend aber BVerfGE 69, 315 (355) - Brokdorf. 
sodass ihnen auch mögliche Rezipienten keine oder zumindest weniger Beachtung schenken. Allerdings geht es hierbei nicht darum, einen grundrechtlichen „Anspruch“ auf tatsächliche Rezeption zu begründen. Dem stünde schon die negative Informationsfreiheit der anderen Nutzer im Wege. ${ }^{682}$ Vielmehr geht es um die Frage, inwieweit nicht auch die Kommunikationsfreiheiten ein gewisses Maß an chancengleicher Teilhabe gewährleisten, das staatlich abzusichern sein kann.

\section{a. Begründbarkeit einer Schutzpflicht zugunsten der „kommunikativen Chancengleichbeit"?}

Weit überwiegend wird das Prinzip „kommunikativer Chancengleichheit“ mit rundfunkrechtlichen Fragestellungen in Verbindung gebracht und hat dort seit jeher eine besondere Bedeutung, wenn es um Ausgestaltungsfragen von Meinungsvielfalt geht, ${ }^{683}$ etwa hinsichtlich der Netzneutralität oder der Chancengleichheit von Rundfunkveranstaltern. ${ }^{64}$ Insoweit ist kommunikative Chancengleichheit essentieller Bestandteil des rundfunkrechtlichen Regulierungsauftrags und wesentliche Direktive für den Rundfunkgesetzgeber. ${ }^{685}$ Das muss aber nicht zwingend den abschließenden Anwendungsbereich bilden. ${ }^{686}$ Denn öffentliche Kommunikation wurde ursprünglich im Wesentlichen medial vermittelt, weshalb es dort auch nur um die Chancengleichheit der Rundfunkunternehmen und die Chancengleichheit (von Gruppeninteressen) ${ }^{687}$ im Rundfunksektor gehen konnte. Mit der digitalen Entwicklung kann auch jeder Einzelne zur öffentlichen (Internet-)Kommunikati-

682 Cornils, ZUM 2019, 89 (93); ähnlich Gomille, GRUR 2017, 241 (245). Einen solchen Anspruch ebenfalls i.E. ablehnend etwa Grabenwarter, in: Maunz/Dürig, GG, Art. 5 Abs. 1 Rn. 81.

683 Meinungsvielfalt ist eines der zentralen Regelungsziele, die das BVerfG dem Rundfunkgesetzgeber vorgibt, siehe etwa BVerfGE 57, 295 (320, 321, 323) - 3. Rundfunkentscheidung; 73, 118 (153) - 4. Rundfunkentscheidung. Soweit es um die Herstellung einer Außenpluralität durch verschiedene Angebote geht, ist das durchaus für den Rundfunksektor überzeugend. Geht es hingegen um eine inhaltliche Meinungsvielfalt, so ist kommunikative Chancengleichheit die Vorbedingung, unter der sich diese erst i.E. tatsächlich einstellen kann. Zu diesem Zusammenhang siehe Hartl, Suchmaschinen, Algorithmen und Meinungsmacht, S. 36. In BVerfGE 73,118 (159) - 4. Rundfunkentscheidung, heißt es gar, dass der „Grundstandard gleichgewichtiger Vielfalt [...] die wesentlichen Voraussetzungen von Meinungsvielfalt [umfasst], die gegen konkrete und ernsthafte Gefährdungen zu schützen sind: die Möglichkeit für alle Meinungsrichtungen - auch diejenigen von Minderheiten -, im privaten Rundfunk zum Ausdruck zu gelangen [...]“ und in BVerfGE 25, 256 (265) - Blinkfüer, spricht das Gericht gar explizit von der „Gleichheit der Chancen beim Prozeß der Meinungsbildung“.

684 So etwa bei Gersdorf, AfP 2011, 209 (213f.); Bortnikov, K\&R 2015, 703 (704); Jahn, in: Spindler/ Schuster, Elektronische Medien, 2015, $\$ 52$ c RStV Rn. 13; Knothe/Nowosadtko, ZRP 2001, 33 f.; Stern/Sachs/Dietlein, in: Stern, Staatsrecht, $\mathbb{} 114$ S. 76; BT-Drs. 16/11570, S. 267.

685 Vgl. Schulz/Held/Kops, ZUM 2001, 621 (625).

686 So auch Bortnikov, Netzneutralität und Bedingungen kommunikativer Selbstbestimmung, S. 47, 50.

687 Vgl. BVerfGE 12, 205 (265 f.) - 1. Rundfunkentscheidung; deutlicher in BVerfGE 57, 295 (322) - 3. Rundfunkentscheidung; 73, 118 (153) - 4. Rundfunkentscheidung. Dieses gruppenfokussierte Verständnis ist im Bereich des Rundfunks pragmatisch begründet, weil so zumindest eine gewisse Identifizierung und Beteiligung unterschiedlicher Bevölkerungsgruppen möglich ist, Hindelang, Kommunikation und Freiheit, S. 159. Dieses Verständnis wird den Gegebenheiten im Internet aber gerade nicht mehr gerecht, ausführlicher hierzu Hindelang, a.a.O., S. 172 ff., insbesondere S. 176 ff. 
on beitragen, sodass ähnliche Probleme auch hier entstehen können. ${ }^{688}$ Zudem kennt das Grundgesetz mit der Wahlrechtsgleichheit in Art. 38 Abs. 1 S. 1, 4. Alt. GG für den zentralen demokratischen Akt der Wahl eine ganz besondere Ausprägung des Prinzips kommunikativer Chancengleichheit, dass nicht nur explizit normiert ist, sondern auch streng formal ${ }^{689}$ verstanden wird. Und auch mit Blick auf den politischen Wettbewerb der Parteien ist die Chancengleichheit (Art. 21 Abs. 1 i.V.m. Art. 3 Abs. 1 bzw. i.V.m. Art. 38 Abs.1, Var. 4 GG) wesentliche subjektiv-rechtliche Gewährleistung. ${ }^{690}$ Chancengleichheit ist somit ein Kernprinzip des demokratischen Staates. ${ }^{691}$

Wenn und weil sich aber die demokratische Meinungsbildung nicht allein in dem Wahlakt erschöpft, sondern auch ein stetiger Kommunikationsprozess entscheidend ist, ${ }^{692}$ muss die Chancengleichheit prinzipiell auch in diesem Bereich Geltung erhalten. ${ }^{63}$ Denn öffentliche Kommunikation kann nur dann die demokratische und integrative Funktion zukommen, wenn jeder grundsätzlich dieselbe Chance auf Teilhabe und Gehör erhält. ${ }^{694}$ Ansonsten würde gerade auch die minderheitsschützende Funktion ${ }^{695}$ partiell verkannt werden. Kommunikative Chan-

688 Vgl. hierzu neben Bock, Die Übertragbarkeit der Kommunikationsfreiheiten des Art. 5 GG auf das Internet, S. 259 f. auch Hindelang, Freiheit und Kommunikation, S. 71 f., 103, und 158 insbesondere zu dem daraus folgenden Wandel der Schutzfunktion. Das gilt etwa mit Blick auf die Debatte um die Netzneutralität und die Frage, inwieweit etwa Access-Provider bestimmte Signale und Inhalte privilegiert bzw. priorisiert weiterleiten dürfen.

689 BVerfGE 95, 408 (417) - Grundmandatsklausel, m.w.N. zur Judikatur. Ausführlich hierzu etwa Morlok, in: Dreier, GG, Art. 38 Rn. 103.

690 Siehe hierzu u.a. BVerfGE 138, 102 (110f.) - Wablkampfäußerungen von Regierungsmitgliedern; 140, 225 (227) - Äußerungsbefugnis Bundesministerin; 148, 11 (23ff.) - Chancengleichheit politischer Parteien und jüngst Urt. v. 09.06.2020 - 2 BvE 1/19= NJW 2020, 2096 Rn. 43 ff. - Seehofer.

691 Mit Blick auf die Chancengleichheit der Parteien ausdrücklich BVerfGE 120, 82 (104) - Sperrklausel Kommunalwahlen. Siehe daneben auch BVerfGE 138, 102 (109f.) - Wablkampfäußerungen von Regierungsmitgliedern.

692 Hierzu nur BVerfGE 8, 104 (112f.) - Volksbefragung; 20, 56 (98f.) - Parteifinanzierung I; 148, 11 (23) - Chancengleichheit politischer Parteien; und etwa Dreier, in: ders., GG, Art. 20 Rn. 77.

693 Das BVerfG spricht teilweise von einem Recht auf Teilhabe, E 12, 113 (115) - Schmid-Spiegel. Andernorts spricht es ausdrücklich davon, dass Voraussetzung ein „Willensbildungsprozeß [ist], an dem grundsätzlich alle wahlmündigen Bürger zu gleichen Rechten teilhaben können [...]“. Kube, in: Isensee/Kirchhof, HbStR IV, $\$ 91$ Rn. 27 entnimmt Art. 5 Abs. 1 S. 1 als „objektivrechtliche Gewährleistung " Aspekte einer Kommunikationsgerechtigkeit, wozu er insbesondere auch die kommunikative Chancengleichheit zählt. Zum Vermittlungsprozess durch Algorithmen von Medienintermediären etwa Kaiser/Reiling, in: Unger/v. Ungern-Sternberg, Demokratie und künstliche Intelligenz, S. 85 (96). Eine Möglichkeit der Ausweitung der „Netzneutralität“ sieht auch Bock, Übertragbarkeit der Kommunikationsgrundrechte des Art. 5 GG auf das Internet, S. $260 \mathrm{f}$.

694 Andernorts wird das eher als Offenheit des Kommunikationsprozess bezeichnet, so etwa bei Habel, Roboterjournalismus, S. 92; vgl. auch Dreier, in: ders., GG, Art. 20 Rn. 77; Heilmann, MMR 2020, 162 (163 f.). Diese Offenheit betont auch das BVerfG, wenn es um die Chancengleichheit der Parteien geht, so etwa in BVerfGE 148, 11 (24) - Chancengleich heit politischer Parteien.

$695 \mathrm{Zu}$ dieser etwa Stern/Sachs/Dietlein, in: Stern, Staatsrecht IV/2, $\$ 112$ S. 35. Vgl. auch Wendt, in: v. Münch/Kunig, Art. 5 Rn. 74. Kritisch deshalb zum Gruppenverständnis im klassischen Rundfunk Hindelang, Freiheit und Kommunikation, S. 175. Und auch das BVerfG betont, dass das demokratische Prinzip verlangt, dass auch die Minderheit zur Mehrheit werden kann, BVerfGE 138, 102 Rn. 28 - Wahlkampfäußerung von Regierungsmitgliedern; vgl. auch BVerfGE 123, 267 (340 ff.) Vertrag von Lissabon, was aber eine chancengleiche Teilhabe zwingend voraussetzt. 
cengleichheit ist also auch Ausdruck der auf demokratische Teilhabe ausgerichteten Kommunikationsfreiheiten des Art. 5 Abs. $1 \mathrm{GG}^{696}$ und Voraussetzung für die Offenheit des Kommunikationsprozesses und damit der Meinungsäußerungsund Informationsfreiheit. ${ }^{697}$ Deshalb folgt aus der kommunikativen Chancengleichheit nicht nur eine (Gewährleistungs-)Pflicht für den Staat, ${ }^{698}$ die Offenheit des Kommunikationsprozess zu gewährleisten, die sich etwa dort aktualisiert, wo es um Fragen des technischen Zugangs zur Online-Kommunikation geht. ${ }^{699}$ Daneben tritt gerade auch eine (Schutz-)Pflicht, bestimmten Verzerrungen, die durch Anbieter und Dritte hervorgerufen werden, zu begegnen. ${ }^{700}$ Damit wird nicht zuletzt gewährleistet, dass die grundrechtlich gesicherten kommunikativen Frei-

696 Mit Blick auf das Prinzip der Meinungsvielfalt spricht Habel, Roboterjournalismus, S. 84 f. von einer „akzessorischen Grundrechtsvoraussetzung“, welche „mit den Einzelgrundrechten eng verwoben [ist].“ Zur Netzneutralität vgl. Bock, Übertragbarkeit der Kommunikationsgrundrechte des Art. 5 GG auf das Internet, S. $260 \mathrm{f}$.

697 Kube, in: Isensee/Kirchhof, HbStR IV, $\mathbb{S} 91$ Rn. 27. Deshalb will Bock, Die Übertragbarkeit der Kommunikationsfreiheiten des Art. 5 GG auf das Internet, S. 260, die staatliche Schutzpflicht der „Netzneutralität" auch nicht als (alleinigen Bestandteil) der Medienfreiheiten verstanden wissen, sondern als „ein[en] allgemeine[n] Grundsatz jeglicher Kommunikation“.

698 Kube, in: Isensee/Kirchhof, HbStR IV, $\mathbb{\$} 91$ Rn. 27.

699 Das ist etwa dort problematisch, wo eine flächendeckende Versorgung mit Breitbandanschlüssen nicht sichergestellt ist und somit Zugangshürden für die Wahrnehmung digitaler Kommunikationsangebote bestehen, vgl. hierzu etwa Schütz, MMR 2018, 36 (38); Hindelang, Freiheit und Kommunikation, S. 107 f. und Kube, in: Isensee/Kirchhof, HbStR IV, $\mathbb{S} 91 \mathrm{Rn}$. 28. Insoweit entspricht das letztlich der Gewährleistungsverantwortung des Art. $87 \mathrm{f}$ Abs. 1 GG, zu dieser etwa Möstl, in: Maunz/Dürig, GG, Art. 87f Rn. 70 ff. Diese inhaltliche Kongruenz deutet etwa Hoffmann-Riem, JZ 2014, 53 (58) an, weist allerdings auf die unterschiedlichen Zielrichtungen hin: bei den Schutzpflichten geht es um Freiheits- und Persönlichkeitsschutz, bei Art. 87f GG um „Systemschutz“.

700 Vgl. Kube, a.a.O., Rn. 30; Schulze-Fielitz, in: Dreier, GG, Art. 5 Abs. 1-2 Rn. 42, und mit Blick auf die Informationsfreiheit und die dortige Kommunikationsgerechtigkeit Rn. 222. Siehe daneben auch Bock, Die Übertragbarkeit der Kommunikationsfreiheiten des Art. 5 GG auf das Internet, S. 258 ff.; Bortnikov, Netzneutralität und kommunikative Selbstbestimmung, S. 47 f.; Hindelang, Freiheit und Kommunikation, S. 196 f. entsagt sich der Formulierung der „kommunikativen Chancengleichheit“, sieht aber die Schutzpflicht des Staates dort, „wo [die kommunikative Autonomie des Einzelnen] droht, von den Möglichkeiten aktiver massenmedialer Betätigung im Rahmen der Informationsproduktions- und -distributionsprozesse durch andere Privatleute beeinträchtigt zu werden.", a.a.O., S. 197, siehe auch S. 204. Hierbei geht es um die Aspekte die das BVerfG in seiner Rundfunkjudikatur immer als Teil der Meinungsvielfalt versteht, also Vermeidung einer Meinungskonzentration u.ä. Aspekte. Diese Schutzverantwortung hat etwa Ausdruck im bisherigen Medienkonzentrationsrecht $(\mathbb{S} 26 \mathrm{ff}$. RStV) oder bei der inhaltlichen Ausgestaltung $(\mathbb{S} 25,52 \mathrm{~b}, 52 \mathrm{c}$ RStV) gefunden und aktualisiert sich auch in der Debatte um die von privaten Providern abhängige Netzneutralität. Zu Letzterer etwa Hindelang, Freiheit und Kommunikation, S. 111 f., der hier von einem „grundsätzlich diskriminierungsfreie[m] Senden von Informationen“ spricht. Ursprünglich ermächtigte $\$ 41$ a TKG die Bundesregierung, ,gegenüber Unternehmen, die Telekommunikationsnetze betreiben, die grundsätzlichen Anforderungen an eine diskriminierungsfreie Datenübermittlung und den diskriminierungsfreien Zugang zu Inhalten und Anwendungen festzulegen, um eine willkürliche Verschlechterung von Diensten und eine ungerechtfertigte Behinderung oder Verlangsamung des Datenverkehrs in den Netzen zu verhindern. “ Aspekte der Netzneutralität regelt mittlerweile EU-einheitlich die VO 2015/2120 über Maßnahmen zum Zugang zum offenen Internet [...] in der Union, ABl. 2015 L 310, $1 \mathrm{ff}$. Mit Blick auf soziale Netzwerke aktualisiert sich diese „Schutzpflichtendimension“ etwa im Vertragsrecht, wenn es um Zugangsansprüche zu den Netzwerken und die mittelbare Drittwirkung der Kommunikationsgrundrechte geht, vgl. hierzu etwa BVerfG, NJW 2019, 1935 ff. und Bock, Die Übertragbarkeit der Kommunikationsfreiheiten des Art. 5 GG auf das Internet, S. 257 f. 
heitsräume nicht durch eine faktische Begrenzung des Zutritts zu bzw. der Stellung in den digitalen Kommunikationsräumen beeinträchtigt wird. ${ }^{701}$

\section{b. Inhaltliche Reichweite des Schutzes kommunikativer Chancengleichheit}

Damit ist freilich noch nicht geklärt, welche inhaltliche Reichweite man dem Prinzip zuspricht. ${ }^{702}$ Anders als bei der Wahl lässt sich eine formale Chancengleichheit kaum garantieren, was schon an unterschiedlichen Positionen der einzelnen Kommunikationsteilnehmer liegt. ${ }^{703}$ Insoweit geht es zunächst vor allem um eine chancengleiche Teilhabemöglichkeit am Kommunikationsprozess und damit einhergehend die chancengleiche Möglichkeit der Rezeption durch andere, ${ }^{704}$ die sichergestellt sein muss. ${ }^{705}$ Denn eine unterschiedlich meinungsstarke Stellung der Teilnehmer ist jedenfalls dann gleichheitsrechtlich unproblematisch, wenn diese kommunikativ begründet ist, ${ }^{706}$ ihre Legitimation also aus der Funktionsweise des Meinungsmarkts selbst folgt. ${ }^{707}$ So kommt Inhalten von Influencern oder Medienunternehmen deshalb eine wesentlich stärkere Bedeutung als einer

701 Zur Notwendigkeit der staatlichen Steuerung etwa Schulze-Fielitz, in: Dreier, GG, Art. 5 Abs. 1-2 Rn. 42.

702 Auf die Frage des Verständnisses weist auch Cornils, ZUM 2019, 89 (93) hin.

703 Und auch das BVerfG unterscheidet hinsichtlich der Anforderungen, wenn es betont, dass „der Grundakt demokratischer Legitimation, die Wahl (...)“, „[i]n höchstem Maße der Integrität bedürftig ist“, BVerfGE 44, 125 (140) - Öffentlichkeitsarbeit, und wenig später der gleichberechtigten Teilhabe nur „grundsätzlichen“ Charakter zuspricht, ebd., S. 142.

704 Chancengleichheit in diesem Sinne heißt freilich auch, dass Einschränkungen möglich sind. Diese Einschränkungen ergeben sich in der Dreieckskonstellation vor allem aus kollidierenden Interessen der Betreiber digitaler Kommunikationsräume.

705 So auch Hindelang, Freiheit und Kommunikation, S. 170; Hartl, Suchmaschinen, Algorithmen und Meinungsmacht, S. 32; vgl. Holznagel, in: Hoeren/Sieber/Ders., Multimediarecht, Teil 3 C. Rn. 137; vgl. Knothe/Nowosadtko, ZRP 2001, 33 (34). Das BVerfG spricht hier von der grundsätzlich gleichberechtigten Teilhabe, BVerfGE 44, 125 (142) - Öffentlichkeitsarbeit und der „Gleichheit der Chancen beim Prozess der Meinungsbildung“, BVerfGE 25, 256 (265) - Blinkfüer. An anderer Stelle betont das BVerfG gerade, „dass [d] er Gesetzgeber [...] zwar nicht faktisch vorhandene, unterschiedliche Möglichkeiten der Einflußnahme auf diesen Prozeß auszugleichen“ braucht. Insoweit geht es also nicht um einen Anspruch auf Zurverfügungstellung wirksamer Instrumente zur Äußerung (einen solchen ablehnend Grabenwarter, in: Maunz/Dürig, Art. 5 Abs. 1 Rn. 111 m.w.N.). Sofern entsprechende Instrumente bestehen, kann ein Anspruch darauf entstehen, dass gewährleistet wird, dass diese chancengleich verwendet werden können. Dass damit nicht jede Form erfasst sein kann, ist schon angesichts der Schranken der Meinungsäußerungsfreiheit und kollidierender Interessen der Anbieter selbst offenkundig. Ein solcher Anspruch aktualisiert sich etwa in den Fällen der mittelbaren Drittwirkung, wenn es um Konten- oder Inhaltssperrungen geht, hierzu etwa BVerfG NJW 2019, 1935 Rn. 15 - III. Weg; LG Nürnberg-Fürth, MMR 2019, 541 oder OLG Karlsruhe, GRUR-RR 2019, 496.

706 Das ist nach Hartl, Suchmaschinen, Algorithmen und Meinungsmacht, S. 32 das entscheidende Kriterium für die kommunikative Chancengleichheit. Daneben siehe auch Knothe/Nowosadtko, ZRP 2001, 33; Habel, Roboterjournalismus, S. 92.

707 Eine gewisse Parallele findet sich bei Schulze-Fielitz, in: Dreier, GG, Art. 5 Rn. 4, wenn er meint, dass auf das „liberale Modell gesellschaftlicher Selbstregulierung auf dem ,Marktplatz der Meinungen'“ u.a. angesichts „vermachteter und konkurrierender Meinungsmärkte“ und „durch neue Medien“ nicht vertraut werden dürfe, sondern die Steuerung der Rezeptionschancengleichheit durch den Staat notwendig sei. 
Einzelmeinung zu, weil sie mit ihren Inhalten eine breitere Öffentlichkeit ansprechen und damit „überzeugender“" ${ }^{\text {708 }}$ sind als andere. Und auch die Bedeutung für den Kommunikationsprozess ist jedenfalls bei Medienunternehmen eine deutlich größere.

Indes anders zu beurteilen ist es, wenn eine im Kommunikationsprozess selbst angelegte Legitimationsgrundlage fehlt. ${ }^{709}$ Das hat schon das Bundesverfassungsgericht deutlich gemacht. In der Blinkfüer-Entscheidung ${ }^{710}$ ging es um einen Boykottaufruf des Presseverlages Axel Springer gegen die hamburgische Wochenzeitung, der an die lokalen Händler gerichtet war. Wegen des Abdrucks „der Ostzonenprogramme“ forderte man Händler auf, den Verkauf zu stoppen und drohte für den Fall, dass vom Verkauf nicht abgesehen wird, mit einer Beendigung des geschäftlichen Verhältnisses. Damit habe man aber nicht mehr von einem zulässigen Mittel im geistigen Meinungskampf Gebrauch gemacht. Entscheidend sei hier zwar nicht allein die wirtschaftliche Machtstellung des Verlagshauses, weil dem „Stärkeren nicht verwehrt [sei], einen geistigen Meinungskampf zu führen “, sondern die Tatsache, dass anstelle eines rein geistigen Arguments vorliegend die wirtschaftliche Stellung als maßgeblicher Überzeugungsfaktor genutzt wurde: ${ }^{711}$

„Die Ausübung wirtschaftlichen Druckes, der für den Betroffenen schwere Nachteile bewirkt und das Ziel verfolgt, die verfassungsrechtlich gewährleistete Verbreitung von Meinungen und Nachrichten zu verhindern, verletzt die Gleichheit der Chancen beim Prozeß der Meinungsbildung. Sie widerspricht auch dem Sinn und dem Wesen des Grundrechts der freien Meinungsäußerung, das den geistigen Kampf der Meinungen gewährleisten soll. " 712

Im Falle der Meinungsroboter wird zwar kein wirtschaftlicher Druck ausgeübt. Allerdings wird die Stärke des Argumentes ebenso wenig durch den geistigen Gehalt der Äußerung gewonnen, sondern durch das koordinierte Zusammenagieren der Nutzerkonten. Grundsätzlich besteht zwar für jeden Nutzer dieselbe Chance, sich mit anderen zusammen zu tun und etwa als Online-Protestbewegung den Einfluss zu steigern. Allerdings ist man insoweit auf die eigene Überzeugungskraft gegenüber anderen und damit auf die (geistige) Unterstützung durch andere angewiesen. ${ }^{713}$ Simplifiziert folgt das dem grundlegenden Prinzip „one man, one vote“. ${ }^{714}$ Bei Meinungsrobotern hingegen kann ein Einzelner ohne Zutun anderer

708 Vgl. Hindelang, Freiheit und Kommunikation, S. 41, 150. Illustrativ formuliert dies auch Heldt, CR 2018, 494 (497): „Auf dem Marktplatz der Ideen gewinnt derjenige, der seine Meinung am besten verkauft."

709 Für den Rundfunk werden diese insbesondere dort ausgemacht, wo die Verbreitungschancen nicht von inhaltlichen, sondern von wirtschaftlichen oder technischen Kriterien abhängen, vgl. Bortnykov, K\&R 2015, 703 (704).

710 BVerfGE 25, 256 (258) - Blinkfüer.

711 BVerfGE 25, 256 (264ff.) - Blinkfüer.

712 BVerfGE 25, 256 (265) - Blinkfüer.

713 Hierzu etwa Thieltges/Hegelich, ZfP 64 (2017), 493 (504).

714 Vgl. auch Steinbach u.a., Desinformation aufdecken und bekämpfen, S. 111. Schroeder, DVB1. 2018, 465 (472) schlägt vor, dieses Prinzip (hilfsweise) als Bestandteil der öffentlichen Ordnung im gefah- 
seine Überzeugungskraft selbst (suggestiv) steigern. ${ }^{715}$ Insoweit geht es also nicht um im kommunikativen Prozess gewonnene geistige Stärke. Hier gilt vielmehr das Prinzip „one man, many votes“. ${ }^{716}$ Dadurch werden aber nicht nur gezielt anderslautende Meinungen behindert. ${ }^{717}$ Es gelten auch nicht mehr für alle Teilnehmer dieselben Voraussetzungen für eine erfolgreiche Teilnahme. Die Chancenverteilung wird einseitig zugunsten des Verwenders verändert. ${ }^{718}$ Am ehesten lässt sich diese Situation noch mit Fragen der Netzneutralität vergleichen. Denn wenn technisch bedingt bestimmte Signale oder Daten priorisiert oder in anderer bevorzugter bzw. benachteiligter Weise weitergegeben werden, so ist das mit Blick auf die kommunikative Chancengleichheit schon seit jeher als problematisch und regulierungsbedürftig angesehen worden. ${ }^{719} \mathrm{Zwar}$ führt das vor allem zu Regulierungen bzw. Regulierungsvorschlägen für Netzbetreiber selbst, was hier eher dem Netzwerk entspräche. Anders als dort findet die eigentliche „Manipulation“ aber nicht beim Weiterleitungsprozess statt. ${ }^{720}$ Dass hier ggf. auch (legitime) Eigenrationalitäten der Netzwerkbetreiber - Stichwort Personalisierung und Filterblase relevant werden, ${ }^{721}$ ändert hieran nichts. Denn die auf dieser Eigenrationalität beruhenden Kriterien einer priorisierten Weiterleitung sind für alle Nutzer die gleichen. Die entscheidende Manipulation, die dann zu einer priorisierten Weiter-

renabwehrrechtlichen Sinne zu verstehen, um so staatliche Maßnahmen gegen die Betreiber zu ermöglichen.

715 Hierauf verweist wohl auch die Länderarbeitsgruppe „Social Bots“ in ihrem nicht veröffentlichten Bericht, vgl. die Antwort der Sächsischen Staatsregierung auf eine kleine Anfrage zu diesem Thema, Sächs-LT-Drs. 6/12131, S. 3 f.

716 Auf diesen Bruch mit dem Prinzip des „one man, one vote“ weisen auch Steinbach u.a., Desinformation aufdecken und bekämpfen, S. 111, hin.

717 Vgl. etwa Lamo/Calo, UCLA Law Review 66 (2019), 988 (999). Das zu verhindern, ist aber nach Holznagel, in: Hoeren/Sieber/Holznagel, Multimediarecht, Teil 3 C Rn. 137 zentrales Anliegen der kommunikativen Chancengleichheit.

718 So auch Fehling/Leymann, AfP 2020, 110 (118), die von einer „Verzerrung des chancengleichen Meinungswettbewerbs sprechen“. Vgl. hierzu auch Habel, Roboterjournalismus, S. 145 und Heilmann, Anonymität für User-generated Content?, S. 163. Aus ethischer Sicht kritisch hierzu Coleman, Journal of Media Ethics Vol. 33 (2018), 120 (125).

719 Siehe hierzu u.a. Holznagel, MMR 2011, 1 (2). Einfach-rechtlich wurde diese Einwirkung auf die kommunikative Chancengleichheit etwa in $\$ 52 \mathrm{c} R \mathrm{RtV}$ reguliert. Der MStV normiert nun für Medienintermediäre ebenfalls ein neutralitätswahrendes Diskriminierungsverbot in $\mathbb{9} 94 \mathrm{MStV}$. Ausführlich zu diesem noch später, 3. Teil, 3. Kap. C.II.2.b. Und auch im Übrigen legt der MStV einen gesteigerten Wert auf die Absicherung der kommunikativen Chancengleichheit etwa mit Blick auf die Auffindbarkeit ähnlicher Angebote $(\$ 83)$, den Zugang zu Medienplattformen und Benutzeroberflächen $(\mathbb{S} \mathbb{S} 82,83)$ oder deren Belegung $(\mathbb{S} 81)$.

720 Wohl gemerkt: Nur weil auch hier bei der Weiterleitung die kommunikative Chancengleichheit tangiert ist, bedeutet das freilich nicht, dass damit eine strikte Neutralitätsverpflichtung die Folge sein muss. Anders als bei Internetprovidern besteht hier nämlich ein erhebliches kommerzielles Interesse an der unterschiedlichen Gewichtung von Inhalten, die nicht nur allein in der Überzeugungskraft (hier wäre das etwa die Popularität eines Inhaltes) liegt. Das ist aber keine Frage, ob die kommunikative Chancengleichheit tangiert ist, sondern inwieweit hier die kollidierenden Interessen der Betreiber (und auch Nutzer) einer strengen Regulierung im Wege stehen. Zu diesem Ausgleich im Kontext des neuen Diskriminierungsverbotes aus $\$ 94$ Abs. 1, 2 MStV noch unten 3. Teil, 3. Kap. C.II.3.a.

721 Vgl. mit Blick auf Suchmaschinen Koreng, in: Stark/Dörr/Aufenanger, Die Googleisierung der Informationssuche, S. 245 (249). Auch dies kann aus Sicht der staatlichen Schutzverantwortung relevant sein, hierzu unten B. 
gabe führt, erfolgt zeitlich vorgelagert bei der Erstellung des weiterzuleitenden Inhalts, ${ }^{722}$ bzw. bei der Eingabe anderer Signale, die unmittelbar bedeutsam für die Relevanzbewertung des Inhalts ist. Im Ergebnis ist aber unerheblich, ob der Weiterleitungsprozess betreiberseitig eine Priorisierung bestimmter Inhalte vorsieht, oder ob dieser Weiterleitungsprozess durch manipulierte Meta(-Daten) der Inhalte korrumpiert wird.

\section{c. Bloße Bagatelle oder beachtlicher Übergriff?}

Auch mit Blick auf die kommunikative Chancengleichheit ließe sich prinzipiell überlegen, inwieweit der Einsatz von Meinungsroboter nicht als bloße Belästigung zu werten wäre. Aber wie auch schon mit Blick auf die Meinungsäußerungsfreiheit wäre es trügerisch, von der Möglichkeit, andernorts chancengleich seine Meinung äußern zu können, auf eine bloße Belästigung zu schließen. Auch hier kommt es weniger auf quantitative denn auf qualitative Maßstäbe an. Insoweit kann der Einsatz ebenfalls nicht als bloße Bagatelle qualifiziert werden. Das gilt umso mehr, als dass in vergleichbaren Konstellationen gerade die Debatte um Netzneutralität geführt wird. Insoweit handelt es sich auch aus Sicht der kommunikativen Chancengleichheit um einen beachtlichen Übergriff.

\section{3. Übergriff auf die Informations- und Meinungsbildungsfreiheit}

Die Informationsfreiheit des Art. 5 Abs. 1 S. 1, 2. Alt. GG schützt die Entscheidung, ob und wie sich der Einzelne informiert. Dabei ist die Wahl allein auf Quellen beschränkt, die geeignet sind, der Allgemeinheit Informationen zu verschaffen. ${ }^{723}$ Nicht nur herkömmliche Massenmedien unterfallen also dem Anwendungsbereich der Informationsfreiheit. Auch das Internet und die dort frei zugänglichen Dienstleistungen sind gerade darauf angelegt, Informationen an eine Vielzahl von Nutzern weiterzuleiten. Soziale Netzwerke bilden hiervon trotz der vorgenommenen Personalisierung - keine Ausnahme. ${ }^{724}$ Für den Schutz kommt es gerade nicht darauf an, dass jeder dieselben Informationen erhält, sondern vielmehr, dass der Quelle überhaupt eine Informationsfunktion im oben beschriebenen Sinne zukommt. ${ }^{725}$ Zentrales Informationsmedium in sozialen Netz-

722 Vgl. hierzu auch Hindelang, Freiheit und Kommunikation, S. 78 f. Das ist in einem sehr weiten Sinne etwa vergleichbar mit der Differenzierung zwischen normalen und Premium-Kunden, deren Signale priorisiert weitergeleitet werden, auch wenn dies im Weiterleitungsprozess selbst Berücksichtigung findet (hierzu Bortnikov, Netzneutralität, S. 108 ff., 118). Meinungsroboter machen sich selbst durch die gegenseitige Unterstützung zu solchen „Premium-Kunden“ bzw. die eingegebenen Daten zu „Premium-Inhalten".

723 Siehe nur BVerfGE 103, 44 (59) - Fernsehaufnahmen aus dem Gerichtssaal II m.w.N.

724 Grabenwarter, in: Maunz/Dürig, GG, Art. 5 Abs. 1, Abs. 2 Rn. 1008; Peukert, MMR 2018, 572 (575); vgl. Drexl, ZUM 2017, 529 (539).

725 Vgl. zu diesem weiten Verständnis, Grabenwarter, in: Maunz/Dürig, Art. 5 Abs. 1 Rn. 1004. 
werken ist vor allem der individuell zusammengestellte News Feed.$^{726}$ Daneben kann auch den trending topics - soweit vorhanden - wesentliche Bedeutung zukommen, ebenso einer individuellen Suche nach bestimmten Themen oder Begriffen über entsprechende Suchmasken. Trotz des individuellen Netzwerks, auf dem der News Feed beruht, ist es nicht zwingend notwendig, dass Meinungsroboter mit dem einzelnen Nutzer unmittelbar verbunden sind. Wo dies allerdings der Fall ist, kann ein Beitrag deutlich leichter in den jeweiligen News Feed gelangen. Daneben können sie aber auch mittelbar über gemeinsame Verbindungspunkte - gemeinsame Freunde, Interessen oder abonnierte Seiten - Einfluss auf den News Feed haben.

Bedeutsam hierfür wird der Rankingalgorithmus. ${ }^{727}$ Eine wesentliche Größe bei diesem ist die Relevanz der Inhalte für die Nutzergemeinschaft oder für Nutzer mit ähnlichem Nutzerprofil. ${ }^{728}$ Indem Meinungsroboter also ein gemeinschaftliches Interesse für einen Beitrag oder ein Thema suggerieren, können sie Einfluss auf die Weitergabe der Information nehmen, weil diese etwa im News Feed, in Kommentarspalten oder als trending topic priorisiert angezeigt werden. Wie stark dieser Einfluss tatsächlich ist, ist auch hier von verschiedenen Faktoren abhängig. Insbesondere dort, wo eine größere Schnittmenge zwischen den Bot- und den Nutzerinteressensprofilen besteht, ist die Wahrscheinlichkeit eines unmittelbaren oder mittelbaren Einflusses auf den News Feed und damit die Informationsvermittlung erhöht. ${ }^{729}$ Damit beeinflusst man aber bereits die Informationsgrundlage, aufgrund derer sich Nutzer potentiell eine Meinung bilden. Dadurch kann bereits bestimmt werden, ob die Grundstimmung zu einem bestimmten Thema eher positiv oder negativ ausfällt. ${ }^{730}$ Besonders prekär ist diese Situation etwa bei Falschinformationen zu aktuellen Themen oder in Krisenzeiten. ${ }^{731}$ Nutzer sind sich dieses Umstands ebenso wie dem des personalisierten Rankings oftmals nicht bewusst. ${ }^{732}$

726 Zur Bedeutung der Informationsbündelung durch algorithmisierte Feeds oder Streams etwa Schmidt u.a., Zur Relevanz von Online-Intermediären für die Meinungsbildung, S. $20 \mathrm{ff}$.

$727 \mathrm{Zu}$ diesem bereits oben, 2. Teil, A.II.2.

728 Vgl. Lischka, AfP 2018, 388 (390).

729 Zu derlei Einflüssen siehe etwa Thieltges/Hegelich, ZfP 64 (2017), 493 (497).

$730 \mathrm{Zu}$ der eher negativ konnotierten Informationsweitergabe während des katalanischen Referendums Stella/Ferrara/Domenico, PNAS Vol. 115 (2018), $12435 \mathrm{ff}$.

731 Auch während der Covid-19-Pandemie kursierten verschiedenen fehlerhafte Informationen über den Ausbruch der Krankheit, den Verlauf und weitere Aspekte der Pandemie im Internet. Auch hier konnte der Einsatz von (vermeintlichen) Meinungsrobotern beobachtet werden, hierzu etwa Ahmed u.a., J Med Internet Res 2020:22(5):e19458; Cinelli u.a., Scientific Reports 10 (2020), 16598; Ferrara, First Monday Vol. 25 (2020) Nr. 6; Gallotti u.a., Nature Human Behaviour 2020; Yang/TorresLugo/Menczer, Prevalence of Low-Credibility Information on Twitter During the Covid-19 Outbreak, 2020. Zur Verbreitung von Hate Speech auch durch Meinungsroboter während der Pandemie Uyheng/Carley, Journal of Computational Social Sciences 2020.

732 Kaiser/Reiling, in: Unger/v. Ungern-Sternberg, Demokratie und künstliche Intelligenz, S. 85 (103). Zur fehlenden „algorithmic awareness“ siehe etwa Schulz/Dankert, Die Macht der Informationsintermediäre, S. 42 f. mit Verweis auf eine durchgeführte Studie von Eslami/Rickman u.a. Siehe dane- 
Zwar ließe sich sagen, dass, solange nur die Informationsgrundlage selbst betroffen ist, keine Probleme auszumachen sind, weil unklar ist, ob und wie diese Informationen aufgenommen werden und die Informationsaufnahme primär ohnehin vom (unvorsichtigen) Auswahlverhalten der Nutzer selbst abhängig ist. ${ }^{733}$ Daran ist sicherlich richtig, dass die Informationen niemandem aufgezwungen werden. Verkannt wird aber, dass die Netzwerkbetreiber keine redaktionelle Vorprüfung vornehmen. Die Algorithmen sortieren gerade nicht nach sachlich-inhaltlichen, sondern primär nach ökonomisch-rationalen Kriterien. Es ist Aufgabe des Nutzers, relevante von irrelevanten Inhalten zu unterscheiden. ${ }^{734}$ Dabei ist er aber gerade anfällig für Verzerrungen, angefangen beim confirmation bias, über mögliche Beeinflussungen durch sensationelle oder emotionale Reize, bis hin zu den wesentlichen Kriterien zur Ermittlung der Relevanz. ${ }^{735}$ Diese nutzerseitigen Verzerrungen können aber durch den Einsatz von Meinungsrobotern gezielt ausgenutzt werden. ${ }^{736}$ Insoweit geht es hier also um die Lenkung der Aufmerksamkeit und die unbewusste Manipulation von Nutzern. Maßgebliche Wirkungsvoraussetzungen werden also nicht erst durch den Nutzer selbst, sondern schon vorher durch externe Faktoren geschaffen. Das ist gerade vergleichbar mit der algorithmischen Informationsweitergabe selbst ${ }^{737}$ und wird hier, wenn auch im anderen Gewand, wieder relevant. Insoweit ist zumindest ein Übergriff anzunehmen.

Informationsaufnahme und -Rezeption haben aber auch wesentliche Bedeutung für die Meinungsbildung. Betrachtet man Art. 5 Abs. 1 S. 1 GG, so findet sich zwar kein Hinweis darauf, dass auch der innere Prozess der Meinungsbildung geschützt ist. ${ }^{738}$ Informations- und Meinungsfreiheit gewähren prima vista nur einen „Input-Output-Schutz“. Damit wäre aber der zentrale geistige Vorgang vor von außen drohender Indoktrination ungeschützt. Wenn aber gerade die Meinungsbildung sowohl auf Mikro- als auch auf Makroebene das zentrale Anliegen der Kommunikationsfreiheiten ist, ${ }^{739}$ muss auch die eigentliche Meinungs-

ben auch die Darstellung und Verweise bei Schmidt u.a., Zur Relevanz von Online-Intermediären für die Meinungsbildung, S. 29.

733 Siehe hierzu bereits die Verweise in Fn. 338.

734 So letztlich auch BVerfGE 149, 222 (262) - Rundfunkbeitrag.

735 Dazu ausführlicher bereits oben, 2. Teil, B.III.3.b.

736 Dazu ausführlicher bereits oben, 2. Teil, C.III.

737 Siehe hierzu etwa Pille, Meinungsmacht sozialer Netzwerke, S. 322 ff.; Kellner, Die Regulierung der Meinungsmacht von Internetintermediären, S. 150 ff.; Heidtke, Meinungsbildung und Medienintermediäre, S. 124 ff. und zur Rolle der Intermediäre im Meinungsbildungsprozess und zu den negativen Effekten auf diesen, S. $130 \mathrm{ff}$.

738 Starck, in: v. Mangoldt/Klein/ders., GG, Art. 5 Rn. 37; Schmalenbach, NVwZ 2005, 1357.

739 Ähnlich Rossen-Stadtfeld, in: Binder/Vesting, Rundfunkrecht, $\mathbb{2 5}$ RStV Rn. 9; vgl. Degenhart, in: BK-GG, Art. 5 Abs. 1, 2 Rn. 57, der in der Meinungsbildung den funktionalen Schutzzweck erblickt; explizit Schmalenbach, NVwZ 2005, 1357, der auf einen ansonsten lückenhaften Schutz hinweist. Grundlegend zur Meinungsfreiheit BVerfGE 7, 198 (208) - Lüth; zur Rundfunkfreiheit etwa BVerfGE 57, 295 (319) - 3. Rundfunkentscheidung. 
bildung als von Art. 5 Abs. 1 S. 1 GG mitgeschützt angesehen werden, ${ }^{740}$ um einen lückenhaften Schutz zu verhindern. ${ }^{741}$ Auch hierbei handelt es sich freilich um einen inneren Prozess, auf den aber durch die aufgezeigten Manipulationen Einfluss genommen werden kann. Weil auch insoweit die „erschlichene Aufmerksamkeit" maßgeblich für die inneren Prozesse ist, handelt es sich auch hier um ein zu berücksichtigendes Drittverhalten. ${ }^{742}$

\section{4. Übergriff auf die Freiheit der Wablentscheidung}

Neben den klassischen kommunikationsgrundrechtlichen Schutzgütern könnte aber auch eine besondere Verantwortung für den entscheidenden Kommunikationsakt der Wahl aus Art. 38 Abs. 1 S. 1 Var. 3 GG folgen. Zwischen Kommunikationsprozess und Freiheit der Wahl besteht immerhin ein enger Sachzusammenhang, weil sie nicht nur die eigentliche Entscheidungsfreiheit vor unzulässigem Zwang oder sonstigen Beeinflussungen schützt, ${ }^{743}$ sondern auch gewährleisten will, dass der Wähler seine Entscheidung „in einem freien, offenen Proze[ss] der Meinungsbildung “ treffen kann. ${ }^{74}$ Insoweit ist auch der Kommunikationsprozess im - zeitlich begrenzten - Vorfeld der Wahl mitgeschützt. ${ }^{745}$ Probleme resultieren hier allerdings daraus, dass es sich bei den Wahlrechtsgrundsätzen um grundrechtsgleiche Rechte handelt und insoweit unklar ist, ob die grundrechtliche Schutzpflichtendogmatik ohne Weiteres übertragen werden kann. Denn einerseits hat das BVerfG die Wahlfreiheit in einer Wahlprüfungssache auch gegenüber Privaten ohne Rekurs auf eine Schutzpflicht zur Anwendung gebracht, ${ }^{746}$ andererseits hat es aus Art. 38 Abs. 1 S.1 GG eine Integrationsverantwortung hinsichtlich der verfassungsrechtlichen Grenzen für die unionsrechtliche Kompe-

740 So auch Grabenwarter, Maunz/Dürig, GG, Art. 5 Abs. 1, 2 Rn. 76; Gräfe, PinG 2019, 5 (8). I.E. auch aber einschränkend auf Tätigkeiten, die „,von außen bedrohbar sind“ Starck, in: v. Mangoldt/Klein/ ders., GG, Art. 5 Rn. 37; Schulze-Fielitz, in: Dreier, GG, Art. 5 Rn. 67; Schmidt-Jortzig, in: Isensee/ Kirchhof, HbStR VII, $\mathbb{S} 162$ Fn. 35; Kloepfer, Verfassungsrecht II, $\mathbb{S} 61 \mathrm{Rn} .29$, sieht es zumindest als objektiv-rechtlichen Gehalt an.

741 Ähnlich Schmalenbach, NVwZ 2005, 1357; Schmidt-Jortzig, in: Isensee/Kirchhof, HbStR VII, $\$ 162$ Fn. 35.

742 Freilich gilt das auch für herkömmliche Medien, denen ähnliche Beeinflussungswirkungen zukommen. Dass sich Meinungsroboter hiervon aber unterscheiden, wurde bereits herausgearbeitet, 2. Teil, C.VI. Und zudem ist die Legitimität des Handelns keine Frage des Übergriffs. Auch die Presse unterliegt etwa mit den Sorgfaltspflichten (etwa $\$ 5$ PresseG MV) oder dem Gegendarstellungsanspruch (etwa $\mathbb{1} 10$ PresseG MV) spezifischen Vorgaben, die bestimmten Risiken entgegenwirken sollen. Inwieweit entsprechende regulative Absicherungen notwendig sind und wie intensiv diese sein müssen, ist aber eine Frage der Abwägung kollidierender Belange.

743 BVerfGE 7, 63 (69) - Listenwahl; 15, 165 (166) - Briefwahl; 47, 253 (282) - Bezirksvertretung; 66, 369 (380) - Wablbeeinflussung durch Unternehmer; 124, 1 (24) - Nachwabl Dresden.

744 BVerfGE 44, 125 (139) - Öffentlichkeitsarbeit der Bundesregierung; 66, 369 (380) - Wablbeeinflussung durch Unternehmer; 124, 1 (24) - Nachwahl Dresden.

745 Trute, in: v. Münch/Kunig, GG, Art. 38 Rn. 43, der den Schutz durch einen funktionalen Zusammenhang mit der Wahl begrenzen will.

746 BVerfGE 66, 369 (380) - Wählerbeeinflussung durch Unternehmer. 
tenzübertragung hergeleitet, die den Schutzpflichten nicht unähnlich sei. ${ }^{747}$ Insoweit ergibt sich kein klares Bild.

$\mathrm{Zu}$ berücksichtigen ist aber einerseits, dass die Wahlprüfungsbeschwerde nicht dem Schutz individueller Rechte dient, sondern die Integrität der Wahlen bewahren will. ${ }^{748}$ In der oben genannten Entscheidung rekurriert das BVerfG zudem im Wesentlichen auf die einfach-rechtliche Norm der Wählernötigung ( $\$ 108$ StGB) und damit nur mittelbar auf Art. 38 Abs. 1 S. 1 GG. ${ }^{749}$ Und bei der Integrationsverantwortung geht es auch nicht primär um die in Art. 38 Abs. 1 S. 1 geschützten Wahlrechtsgrundsätze, sondern um den Schutz des Wesenskerns ${ }^{750}$ des demokratischen Wahlrechts vor einer inhaltlichen Entleerung. Art. 38 Abs. 1 S. 1 GG ist notwendig, um ein justitiables, subjektives Recht zu begründen $;^{751}$ der inhaltliche Schwerpunkt der Verantwortung liegt aber eher beim Demokratieprinzip. ${ }^{752}$ Ebenso wenig steht der Charakter eines grundrechtsgleichen Rechts im Weg. Denn zum einen sind Grundrechte und grundrechtsgleiche Rechte vergleichbar ausgestaltet, insbesondere justiziable subjektive Rechte. ${ }^{753}$ Und mit Blick auf die Wahlgrundsätze, insbesondere die Wahlfreiheit, gilt es zu berücksichtigen, dass anders als in den Fällen des Statusrechts (Art. 33 GG) oder der justiziellen Rechte (Art. 101, 103, 104 GG) ${ }^{754}$ nicht allein aus der staatlichen Sphäre Bedrohungen resultieren. Gerade die Wahlfreiheit ist auch durch Private angreifbar, sodass

747 Siehe hierzu BVerfGE 142, 123 Rn. 166 - OMT-Programm; 151, 202 Rn. 142 - Europäische Bankenunion; BVerfG, Urt. v. 05.05.2020 - 2 BvR 859/15 u.a., Rn. 114 - EZB Staatsanleihenkaufprogramm. Diese Verantwortung verpflichtet die Verfassungsorgane, sich schützend und fördernd vor die durch Art. 38 Abs. 1 S. 2 i.V.m. Art. 20 Abs. 2 S. 1 GG geschützten Rechtspositionen des Einzelnen zu stellen, wo dieser nicht selbst für ihre Integrität sorgen kann (...).“, so in BVerfGE 142, 123 Rn. 166 - OMT-Programm; 151, 202 Rn. 142 - Europäische Bankenunion.

748 BVerfGE 66, 369 (378) - Wählerbeeinflussung durch Unternehmer, m.w.N.

749 BVerfGE 66, 369 (380 ff.) - Wählerbeeinflussung durch Unternehmer. Das ist insoweit konsequent, als dass die Frage der Unzulässigkeit wegen der grundsätzlich gewollten Beeinflussung im Kommunikationsprozess eine Abwägung zwischen kollidierenden Gütern voraussetzt, deren Ergebnis dann letztlich $\$ 108$ StGB ist, hierzu in Ansätzen Müller, in: v. Mangoldt/Klein/Starck, GG, Art. 38 Rn. $140 \mathrm{f}$.

750 Schon in der Maastricht-Entscheidung, BVerfGE 89, 155, wird von den „unverzichtbaren Mindestanforderungen " gesprochen, ebd., S. 172. Siehe hierzu aber auch die nachfolgenden Urteile BVerfGE 142, 123 Rn. 126 - OMT-Programm; 151, 202 Rn. 92 - Europäische Bankenunion; BVerfG, Urt. v. 05.05.2020 - 2 BvR 859/15 u.a., Rn. 104 - EZB Staatsanleihenkaufprogramm.

751 Vgl. hierzu die erste Entscheidung des BVerfG, E 89, 155 (171f.) - Maastricht; und dann nachfolgend BVerfGE 123, 267 (340 ff.) - Lissabon; 129, 124 (167f.) - Euro-Rettungsschirm; 151, 202 Rn. 91 f. - Europäische Bankenunion; BVerfG, Urt. v. 05.05.2020 - 2 BvR 859/15 u.a., Rn. 98 ff. EZB Staatsanleihenkaufprogramm.

752 Bereits in BVerfGE 89, 155 (172) - Maastricht, wird hier der unveränderliche Kern des Art. 20 Abs. 1, 2 i.V.m. Art. 79 Abs. 3 GG betont. Siehe hierzu auch die späteren Entscheidungen BVerfGE 123, 267 (341 ff.) - Lissabon; 129, 124 (177 f.) - Euro-Rettungsschirm; 142, 123 Rn. 127 ff. - OMTProgramm; 151, 202 Rn. 115 ff. - Europäische Bankenunion.

753 Vgl. Dreier, in: ders., GG, Vorbem. Art. 1 GG Rn. 64, der sie als „materielle Grundrechte“ bezeichnet.

754 Der Begriff des Gerichts bzw. Richters erfasst hier nur staatliche Institutionen, was schon der Zusammenhang mit Art. 92 GG zeigt. Vgl. zu Art. 101 Abs. 1 GG Classen, in: v. Mangoldt/Klein/Starck, GG, Art. 101 Rn. 11; zu Art. 103 GG Nolte/Aust, in: v. Mangoldt/Klein/Starck, GG, Art. 103 Rn. 16. 
auch hier die Notwendigkeit eines staatlichen Schutzes besteht. ${ }^{755}$ Und das BVerfG hat nicht nur die Bedeutung der Grundsätze betont, ${ }^{756}$ sondern auch den Bezug zur Menschenwürde. ${ }^{757}$ Wenn aber die Freiheit der Wahl ein so elementarer verfassungsrechtlicher Wert ist, der auch durch Dritte beeinträchtigt werden kann, wäre es nur konsequent, dass sein Schutz auch Staatsaufgabe ist. ${ }^{758}$

Hiervon zu trennen ist allerdings die Frage, ob die Anerkennung einer Schutzpflicht für die hier behandelte Fragestellung einen wesentlichen Mehrwert bietet. Soweit nur der offene und plurale Kommunikationsprozess als Voraussetzung einer freien Wahl Anknüpfungspunkt für die Schutzverantwortung des Staates ist, ist die Schutzverantwortung hier kongruent mit der für die kommunikationsgrundrechtlichen Schutzgüter. Art. 38 Abs. 1 S. 1 GG kommt also keine eigenständige, sondern nur eine verstärkende Wirkung zu. Anders als in Fällen der unmittelbaren Beeinflussung der Wahlentscheidung durch Nötigungshandlungen (vgl. $\$ 108$ StGB) besteht hier also kein originärer Ansatz für eine Schutzpflicht.

\section{Fazit: Übergriff auf die kommunikationsgrundrechtlichen Güter}

Meinungsroboter können auf allen Stufen des Kommunikationsprozesses relevant werden und berühren schon deshalb die hierfür relevanten grundrechtlichen Schutzgüter. Das beginnt bei der Informationsvermittlung durch die Steigerung gewisser Relevanzmerkmale und setzt sich fort bei der Störung von Diskursen und dem Verringern mehrheitskonträrer Äußerungen. Dadurch wird nicht zuletzt die kommunikative Chancengleichheit zulasten anderer Diskursteilnehmer verzerrt und der individuelle Meinungsbildungsprozess beeinflusst. Auch wenn es sich hierbei um interne Verarbeitungsprozesse der Nutzer handelt, werden die für eine Entscheidung maßgeblichen Parameter extern erzeugt und so die Aufmerksamkeit der Nutzer für bestimmte Inhalte erschlichen. Dadurch kann letztlich auch der interne Meinungsbildungsprozess mittelbar beeinflusst werden. Weil dieser Informations-, Meinungsbildungs- und Meinungsäußerungsprozess

755 Wenn man, wie teilweise in der Literatur vertreten (s.o. Fn. 455) das Gewaltmonopol als Grundlage der Schutzpflichten sieht gilt das also erst recht. Denn insoweit ist der Einzelne auf staatliche Hilfe angewiesen, um sich bestimmten Beeinflussungen (etwa Nötigungen) erwehren zu können.

756 Siehe nur BVerfGE 99, 1 (13) - Bayerische Kommunalwablen.

757 Siehe hierzu etwa BVerfGE 123, 267 (341) - Lissabon.

758 Beiläufig hat das etwa auch das BVerfG betont. So heißt es in BVerfGE 59, 119 (127) - Briefwahl, dass der Gesetzgeber „,auch künftig für eine bestmögliche Sicherung und Gewährleistung der Wahlrechtsgrundsätze zu sorgen [habe].“ Er müsse getroffene Regelungen angesichts neuer Entwicklungen überprüfen. „Treten dabei Mißbräuche zutage, die geeignet sein können, die Freiheit der Wahl [...] mehr als unumgänglich zu gefährden, so erwächst daraus die verfassungsrechtliche Pflicht, die ursprüngliche Regelung im Wege der Nachbesserung zu ergänzen oder zu ändern. "Und auch in der Literatur werden Schutzpflichten zugunsten der Wahlgrundsätze des Art. 38 Abs. 1 S. 1 GG ohne nähere Begründung angenommen, so etwa Stern/Sachs/Dietlein, in: Stern, Staatsrecht IV/2, $\$ 115$ S. 196 mit Blick auf die Allgemeinheit der Wahl; zum Grundsatz der geheimen Wahl Klein, in: Maunz/ Dürig, GG, Art. 38 Rn. 110 und explizit zur Wahlfreiheit etwa Sachs, Grundrechte, $\mathbb{} 33$ Rn. 23. 
notwendige und wesentliche Voraussetzung für die freie Wahlentscheidung der Nutzer ist, besteht auch ein wesentlicher Zusammenhang mit dem Grundsatz der Freiheit der Wahl. Das führt zwar nicht per se zu einem Übergriff in dieses Schutzgut. Jedenfalls ist es aber als verstärkendes Element mit zu berücksichtigen.

\section{Fakultative Risikovorsorge oder obligatorische Gefahrenabwehr?}

Auch wenn ein Übergriff in grundrechtliche Schutzgüter vorliegt, muss das nicht zwingend zu einer staatlichen Verantwortung für Meinungsroboter führen. Vielmehr hängt das maßgeblich davon ab, welches Aktivierungsniveau man für eine staatliche Schutzpflicht für erforderlich hält. Bedeutung erlangt hier die Diskussion um eine Gefahrenschwelle ${ }^{759}$. Denn wie schon ausführlicher aufgezeigt (1. Teil C.IV), ist einerseits unklar, ob Meinungsroboter überhaupt in einem relevanten Umfang aktiv sind und andererseits, welche genauen Auswirkungen von ihnen tatsächlich ausgehen. Wegen dieser Unsicherheiten bewegt man sich prima vista im Bereich der Risikovorsorge, der oftmals allein der Prärogative des Gesetzgebers zugeschrieben wird. ${ }^{760}$

Was auf den ersten Blick simpel und plausibel erscheint, wird dadurch erschwert, dass die bereits aufgezeigte Produktformel aus Schadenshöhe und Eintrittswahrscheinlichkeit die Gefahrenschwelle beweglich macht. Bei größeren zu erwartenden Schäden für bedeutendere Rechtsgüter ist eine deutlich geringere Eintrittswahrscheinlichkeit ausreichend als bei geringerer Schadensintensität. Insoweit sind „Gefahren [...] Risiken ab einer bestimmten Größe. “761 Das führte in der Judikatur des Bundesverfassungsgerichts dazu, dass hinsichtlich des Lebensrechts schon „theoretisch herleitbare Risiken“ und damit eine ganz entfernte Wahrscheinlichkeit ausreichte, um Schutzpflichten zu aktivieren. ${ }^{762}$ Im ersten Urteil zum Schwangerschaftsabbruch hatte das Gericht gar gemeint, dass die Schutzpflicht umso ernster genommen werden müsse, je höher der Rang des in Frage stehenden Rechts innerhalb der Wertordnung ist. ${ }^{763}$ Dies wurde teils kritisch rezipiert, weil damit suggeriert werde, dass den unterschiedlichen

\footnotetext{
759 Hierzu oben, A.I.1.

$760 \mathrm{Zu}$ diesem Gleichlauf von Risikovorsorge und gesetzgeberischer Prärogative hinsichtlich der Frage des „Ob“ einer Schutzgewährung allgemein Murswiek, Die staatliche Verantwortung für die Risiken der Technik, S. 144; Isensee, in: ders./Kirchhof, HbStR IX, $\mathbb{S} 191$ Rn. 235. Vgl. auch Moritz, Schutzpflichten gegenüber pflegebedürftigen Menschen, S. 111; Dietlein, Die Lehre von den grundrechtlichen Schutzpflichten, S. 107. In diese Richtung im Kontext der Intermediärsregulierung hinsichtlich einer Vielfaltssicherung etwa Cornils, AfP 2018, 377 (383) und Liesem, ZUM 2020, 377 (378 f.).

761 Krings, Grund und Grenzen staatlicher Schutzansprüche, S. 228.

762 So etwa in BVerfG, NVwZ 2010, 702 Rn. 12 f. - CERN.

763 BVerfGE 39, 1 (42) - Schwangerschaftsabbruch I. In BVerfGE 121, 317 (353) - Rauchverbot in Gaststätten, wird u.a. auch mit dem „hohen Gewicht“ des Lebensrechts „in der Wertordnung des Grundgesetzes“ argumentiert.
} 
Rechtsgütern ein unterschiedlicher Wert zukomme, dem Grundrechtskatalog also eine Werthierarchie entnommen werden könne. ${ }^{764}$ Um eine solche Wertordnung dürfte es indes gar nicht gehen. Vielmehr wird damit berücksichtigt, dass bei gewissen (existenziellen) Schutzgütern die Anforderungen wegen der damit einhergehenden Risiken geringer ausfallen müssen als bei anderen, ${ }^{765}$ weil sich ihre $R e$ stituitionsfähigkeit unterscheidet. ${ }^{766}$ Denn die Frage, ob ein Zustand ex post behebbar ist, hat unmittelbaren Einfluss auf die Frage, ob man Schutzmaßnahmen früher ergreifen muss, um nicht oder nur schwer korrigierbare Fehlentwicklungen $\mathrm{zu}$ vermeiden. ${ }^{767}$ Das erklärt letztlich auch, warum beim Lebensgrundrecht ein deutlich geringerer Maßstab angelegt wird. ${ }^{768}$ Für die Produktformel sind also drei wesentliche Faktoren entscheidend: die Bedeutung des Rechtsguts, die Bedrohungs- bzw. Schadensintensität und die Eintrittswahrscheinlichkeit. Eine höhere Bewertung potentieller Schäden und der Bedeutung des Rechtsguts rechtfertigt also eine Verringerung des Wahrscheinlichkeitsgrades des Schadeneintritts. ${ }^{769}$

\section{Bedeutung der betroffenen Grundrechtsgüter}

Anders als das Lebensrecht haben die betroffenen Kommunikationsfreiheiten keine physisch-existenzielle Bedeutung. ${ }^{770}$ Wenn dort die Wahrscheinlichkeitsanforderungen auf ein Minimum abgesenkt werden, ${ }^{771}$ ist das durchaus konsequent. ${ }^{772}$ Allerdings ist die Meinungsäußerungsfreiheit für die politisch-gesell-

$764 \mathrm{Zu}$ diesem Problem etwa Ruffert, Vorrang der Verfassung und Eigenständigkeit des Privatrechts, S. 194; Dietlein, Die Lehre von den grundrechtlichen Schutzpflichten, S. 86.

765 In diese Richtung hinsichtlich des Lebensgrundrechts jedenfalls Hermes, Das Grundrecht auf Schutz von Leben und Gesundheit, S. 237. Vgl. hierzu eben auch BVerfGE 121, 317 (352 f.) - Rauchverbot in Gaststätten.

766 Vgl. Klein, NJW 1989, 1633 (1638).

767 Zwar nicht zur Schutzpflicht, allerdings mahnt das BVerfG dies in Entscheidungen zur Rundfunkfreiheit deutlich an. „Bei dieser Sachlage würde es dem verfassungsrechtlichen Gebot, die Freiheit des Rundfunks zu gewährleisten, nicht gerecht werden, wenn nur staatliche Eingriffe ausgeschlossen würden und der Rundfunk dem freien Spiel der Kräfte überlassen würde; dies um so weniger, als einmal eingetretene Feblentwicklungen - wenn überhaupt - nur bedingt und nur unter erheblichen Schwierigkeiten rückgängig gemacht werden könnten. [Hervorh. d. Autors]“, BVerfGE 57, 295 (323) - 3. Rundfunkentscheidung. Ähnlich auch BVerfGE 73, 118 (160) - 4. Rundfunkentscheidung.

768 Ähnlich Murswiek, Die staatliche Verantwortung für die Risiken der Technik, S. 179; Klein, NJW 1989, 1633 (1638).

769 Murswiek, Die staatliche Verantwortung für die Risiken der Technik, S. 179 hat allerdings darauf hingewiesen, dass sich hieraus allein eine grobe Richtschnur ergebe, weil der Verfassung „nur wenige Kriterien für die Beurteilung des Schadenumfangs zur Verfügung stellt“. Insbesondere immaterielle Schäden können nur schwer quantifiziert werden, ebd. S. 175.

770 „Das menschliche Leben stellt, wie nicht näher begründet werden muß, innerhalb der grundgesetzlichen Ordnung einen Höchstwert dar; es ist die vitale Basis der Menschenwürde und die Voraussetzung aller anderen Grundrechte. “, BVerfGE 39, 1 (42) - Schwangerschaftsabbruch I. Für die Schutzpflicht des ungeborenen Lebens hat das BVerfG gemeint: „Ihre Erfüllung ist eine Grundbedingung geordneten Zusammenlebens im Staat“, BVerfGE 88, 203 (252) - Schwangerschaftsabbruch II.

771 Statt vieler und besonders instruktiv BVerfG NVwZ 2010, 702 Rn. 12 - CERN, wo bereits theoretisch herleitbare Gefahren ausreichend waren.

772 So i.E. jedenfalls Murswiek, Die staatliche Verantwortung für die Risiken der Technik, S. 179. 
schaftliche Sphäre elementar; sie ist der Kern der Kommunikationsverfassung ${ }^{773}$ und von fundamentaler Bedeutung für den „Ausdruck der menschlichen Per-

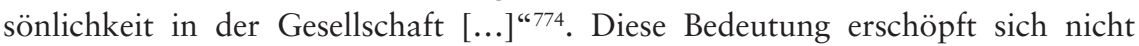
ausschließlich in dieser subjektiv-rechtlichen Wirkung. Vielmehr sind auch die gesellschaftlichen und demokratischen Zielsetzungen zu berücksichtigen. ${ }^{775}$ Denn der Einzelne ist in ein Gesamtkonstrukt eines Meinungsbildungsprozesses und einer diskursiven Öffentlichkeit eingebettet. Individuelle Meinungsbildung und -äußerung ist Voraussetzung für die kollektive und umgekehrt. ${ }^{776}$ Deshalb kann der Meinungsfreiheit auch eine demokratiefördernde, objektiv-rechtliche Funktion nicht abgesprochen werden. ${ }^{777}$ Die Meinungsfreiheit ist „schlechthin konstituierend für die freiheitlich-demokratische Grundordnung “778 und der daraus entstehende Prozess der öffentlichen Meinungsbildung das „Lebenselement“ ${ }^{\text {“779 }}$ einer Demokratie. ${ }^{780}$ Und dieser Diskurs wiederum ermöglicht es erst, seine Meinung selbst weiterzubilden, zu überprüfen und ihr sodann im Wahlakt Ausdruck zu verleihen. ${ }^{781}$

Dem steht die Informations- und Meinungsbildungsfreiheit in nichts nach. Sie hat eine wesentliche Funktion für den Meinungsbildungsprozess und damit für die Demokratie. ${ }^{782}$ Informationen sind notwendige Bedingung der Meinungsbildung nicht nur als Voraussetzung, um im Kommunikationsprozess partizipieren und politisch verantwortungsvoll handeln zu können, sondern sie sind auch für die soziale Stellung entscheidend. ${ }^{783}$ Und ohne das Recht, Informationen frei empfangen zu können, verlöre auch die Meinungsäußerungsfreiheit an Wirk-

773 Die amerikanische Verfassung macht es gerade vor, dass zur Gewährleistung eines umfangreichen Kommunikationsschutzes allein die Meinungsfreiheit ( $1^{\text {st }}$ Amendment) ausreicht. Ähnliches gilt mit Blick auf Art. 10 Abs. 1 S. 1, 2 EMRK oder für Art. 11 Abs. 1 EuGRC. Der EGMR bezeichnet sie als „essential foundation of a democratic society“, EGMR, Urt. v. 07.12.1976 - 5493/72, Rz. 49 - Handyside/Vereinigtes Königreich. Insoweit merkt Dörr, in: Merten/Papier, HbGR IV, $\mathbb{} 103$ Rn. 13, zutreffend an, dass es sich bei den einzelnen Kommunikationsgrundrechten „lediglich um die Konkretisierung eines einzigen Grundrechts handelt" und die Meinungsfreiheit den Ausgangspunkt [bildet]“.

774 BVerfGE 7, 198 (208) - Lüth. Zum Aspekt der Persönlichkeitsentfaltung etwa auch BVerfGE 82, 272 (282) - Zwangsdemokrat Strauß, und Jestaedt, in: Merten/Papier, HbGR IV, $\mathbb{S} 102$ Rn. 7.

$775 \mathrm{Zu}$ dieser doppelten Schutzrichtung schon BVerfGE 7, 198 (208) - Lüth. Deutlicher etwa in BVerfGE 82, 272 (282) - Zwangsdemokrat Strauß. Siehe auch Schulze-Fielitz, in: Dreier, GG, Art. 5 Abs. 1-2 Rn. 40.

776 Jestaedt, in: Merten/Papier, HbGR IV, $\$ 102$ Rn. 19 formuliert es anschaulich als „die Wechselbezüglichkeit von individueller und öffentlicher Meinungsbildung, [...] die Notwendigkeit eines öffentlichen Kommunikationsprozesses als Stimulans, Katalysator und Resonanzboden individueller Meinungsbildung und Meinungsäußerung [...]“.

777 So schon BVerfGE 7, 198 (208) - Lüth. Ausführlicher zu dieser Funktion und ihrer (dogmatischen) Bedeutung etwa Jestaedt, in: Merten/Papier, HbGR IV, $\mathbb{1} 102 \mathrm{Rn} .13 \mathrm{ff}$.

778 So die vom BVerfG seit BVerfGE 7, 198 (208) - Lüth verwendete Formulierung.

779 So BVerfGE 7, 198 (208) - Lüth.

780 Vgl. BVerfGE 5, 85 (135) - KPD-Verbot; 7, 198 (208) - Lüth; 20, 56 (98) - Parteifinanzierung I.

781 Böckenförde, in: Isensee/Kirchhof, HStR II, \$24 Rn. 68; ähnlich Holznagel, NordÖR 2011, 205 (206).

782 BVerfGE 27, 71 (81) - Leipziger Volkszeitung.

783 BVerfGE 27, 71 (81) - Leipziger Volkszeitung. 
kraft. $^{784}$ Zudem sorgt eine plurale Informationsaufnahme für Toleranz und Akzeptanz anderer Meinungen und führt so zur Beseitigung von Vorurteilen. ${ }^{785}$ Die Informationsfreiheit ergänzt somit die Meinungs- und Medienfreiheiten aus der Empfängerperspektive. ${ }^{786}$ Ohne ihre Gewährleistung wäre der demokratische Kommunikationsprozess unvollständig. ${ }^{787}$

Die Relevanz der kommunikativen Freiheiten in ihrer Gesamtheit findet nicht zuletzt auch ihren Ausdruck darin, dass Voraussetzung der freien Wahlausübung gerade der offene, plurale und freie Kommunikationsprozess ist.

\section{Potentielles Schadensausmaß}

Hinsichtlich des konkreten Schadensausmaßes lässt sich schon auf die oben skizzierten Wirkungszusammenhänge verweisen. Auch hier wird es also nicht um existentielle Bedrohungslagen gehen, wie in Fällen des Lebensrechts. Gleichwohl sind verschiedene gewichtige Faktoren zu berücksichtigen, die den Meinungsrobotern eine erhöhte Relevanz zukommen lassen (können).

\section{a. Allgemeine Bedeutung der Netzwerke als digitale Informations- und Kommunikationsräume}

Generell ist die Bedeutung der sozialen Netzwerke und die Netzwerkkommunikation für die Meinungsäußerung und für die Informationsaufnahme zu berücksichtigen. ${ }^{788}$ Die Bedeutung für die Meinungsäußerung folgt dabei vor allem aus der Reichweite, die in der analogen Welt keine Entsprechung findet. Inhalte und Äußerung manifestieren sich, bleiben für alle sichtbar erhalten und abrufbar, sodass sie dauerhaft und über territoriale Grenzen hinweg wirken können. Zudem kann unmittelbar auch mit gesellschaftlichen und politischen Akteuren interagiert werden. Auch diese Interaktivität sucht man in der analogen Welt vergebens. Dort ist man etwa auf die in den Medien zur Verfügung gestellten Leserbriefsparten angewiesen und damit auch von einem redaktionellen Filter abhängig. Und allgemein sind Äußerungen in der analogen Welt für Personen, denen gegenüber sie nicht kundgegeben werden, in der Regel nicht wahrnehmbar. Diese Reichweite führt letztlich dazu, dass einer Äußerungsmöglichkeit in

\footnotetext{
784 Dörr, in: Merten/Papier, HbGR IV, $\mathbb{S} 103$ Rn. 13. Stern, in: ders., Staatsrecht IV/1, $\mathbb{S} 108$, S. 1387 bezeichnet sie deshalb zu Recht als "Zwillingsgrundrechte“ und Grabenwarter, in: Maunz/Dürig, GG, Art. 5 Abs. 1 Rn. 985 als „Komplementärstück zur Meinungsfreiheit“.

785 BVerfGE 27, 71 (82) - Leipziger Volkszeitung.

786 BVerfGE 90, 27 (31) - Parabolantenne I.

787 BVerfGE 90, 27 (31) - Parabolantenne I; vgl. BVerfGE 27, 71 (81) - Leipziger Volkszeitung.

788 Das betont auch Milker, InTeR 2017, 199 (203).
} 
sozialen Netzwerken eine deutlich höhere Bedeutung zukommt. ${ }^{789}$ Das gilt umso mehr, je relevanter auch die Nutzung der Plattformen selbst wird.

Entsprechendes gilt auch mit Blick auf die Informationsvermittlung. Soziale Netzwerke fungieren als Medienintermediäre. Sie erleichtern den Zugriff auf Informationen erheblich einerseits dadurch, dass in ihnen die verschiedenen medialen Subangebote sowie politische und wirtschaftliche Informationen zusammenlaufen und durch die Intermediäre selbst und nur nach den potentiellen Interessen des Nutzers vorgefiltert werden und andererseits dadurch, dass der Zugriff auf die Informationen barrierefrei und vor allem - jedenfalls aus Sicht der Nutzer - „kostenfrei“ möglich ist. Freilich ist dies dahingehend zu relativieren, dass die sozialen Netzwerke keine Monopolstellung bei der Informationsvermittlung innehaben, sondern abseits hiervon ein plurales Medienangebot zur Verfügung steht, welches ebenso viele Informationen bereithält ${ }^{790}$ und welches aktuell jedenfalls noch deutlich umfangreicher genutzt wird. ${ }^{791}$ Allerdings wäre es trügerisch deshalb bereits jegliches Bedrohungspotential abzusprechen. Je nach individuellem Nutzungs- und Medienkonsumverhalten variiert die Bedeutung der Netzwerke und damit auch die potentielle Beeinträchtigung durch entsprechende Manipulationen in ihnen. Besonders bedeutsam werden Manipulationen für Nutzer, die überwiegend über soziale Netzwerke kommunizieren und Informationen aufnehmen, ${ }^{792}$ etwa weil sie den herkömmlichen Medien weniger Vertrauen entgegenbringen. Das wird - aktuell - zwar nur ein Bruchteil der Nutzer sein. Aus demokratischer Sicht gibt es aber gerade keine zu vernachlässigende Größe, wenn es um die Partizipation am Meinungsbildungsprozess geht.

\section{b. Meinungsäußerungsfreiheit}

Zwar wird die Äußerungsmöglichkeit anders als bei Kontensperren nicht generell genommen. Von der Intensität her kann das aber eine vergleichbare Ausschluss-

789 Diese zentrale Bedeutung wird auch andernorts betont, etwa wenn es um die Frage der Zulässigkeit von Äußerungen und damit um Fragen der mittelbaren Drittwirkung geht. Das OLG Dresden, ZUMRD 2019, 2 (6), und Urt. v. 16.06.2020 - 4 U 2890/19 = GRUR-RS 2020, 12291 Rn. 18 spricht von einer „Quasi-Monopolstellung“. Das OLG München, ZUM 2019, 269 (270) spricht hier von einem „öffentlichen Marktplatz“; ebenso LG Bamberg, MMR 2019, 56 Rn. 51. Das OLG Nürnberg, Urt. v. 04.08.2020 - 3 U 3641/19 = GRUR-RS 2020, 23456 Rn. 72 betont hingegen nur die „Bedeutung des [...] betriebenen Netzwerkes [...], die ihm aufgrund seiner erheblichen Reichweite zukommt“. und andernorts wird vor allem die marktbeherrschende Stellung betont und daraus eine starke mittelbare Bindung an die Meinungsfreiheit angenommen, so etwa OLG Hamm, Beschl. v. 15.09.2020 29 U 6/20 = GRUR-RS 2020, 25382 Rn. 109.

790 Hierauf ebenfalls hinweisend Milker, ZUM 2017, 216 (220). Allgemein zur Bedeutung des Konsumverhaltens im Kontext der Filterblasendiskussion etwa Schweiger, Der (des)informierte Bürger, S. $91 \mathrm{f}$.

791 Siehe hierzu Reuters Institute, Digital News Report 2020, S. 70.

792 Nach dem Reuters Institute, Digital News Report 2020, S. 11, haben etwa 39 \% der Befragten zu Beginn der Corona-Krise (auch) soziale Medien als Nachrichtenquelle verwendet. Und 2019 haben insgesamt $10 \%$ soziale Medien als Hauptnachrichtenquelle angegeben, hierzu Hölig/Hasebrink, Reuters Institute Digital News Report 2019 - Ergebnisse für Deutschland, S. 21. 
wirkung vor allem dann haben, wenn der Ort der Diskussion zentral war. Insoweit kommt es allein und entscheidend auf die qualitative Wirkung an. Hierbei ist auch zu berücksichtigen, dass etwaige zensierende Effekte durch Mehrheiten tendenziell stärker in den Netzwerken sein können als in der realen Welt, was vor allem auch daran liegt, dass potentielle Folgen nicht überschaut und ihnen vor allem nur begrenzt begegnet werden kann. ${ }^{793}$ Welche tatsächlichen Auswirkungen dies hat, ist einzelfallabhängig und kann nicht pauschal beantwortet werden. Dass hier ein strafrechtlich relevantes Niveau noch nicht erreicht ist, weil keine taugliche Nötigungshandlung oder keine relevante Ausübung von Druck vorliegt, ${ }^{794}$ ist jedenfalls unbeachtlich. Strafrechtliche Maßnahmen sind die ultima ratio ${ }^{795}$ und insoweit nicht maßgeblich für die Bewertung des Schadensausmaßes. ${ }^{796}$ Insofern mag das allein auf Rechtsfolgenseite Berücksichtigung finden. Nicht zu verkennen ist aber, dass i.E. auch mithilfe der Meinungsroboter dieselbe faktische Wirkung hergestellt werden kann wie durch die strafrechtlich pönalisierten Nötigungshandlungen.

\section{c. Kommunikative Chancengleichbeit}

In der Debatte um „Netzneutralität“ wird gerade wegen des Einflusses auf die Chancengleichheit eine staatliche Regulierung für notwendig gehalten. ${ }^{797}$ Das gilt nicht zuletzt auch mit Blick auf die Wirkung der Algorithmen von Intermediären. ${ }^{798}$ Wenn aber nicht vom Algorithmus die unmittelbare Gefahr eines diskriminierenden Einflusses auf die Chancengleichheit ausgeht, sondern die Manipulation u.a. von statistischen Daten maßgeblich für eine privilegierte Weitergabe von Informationen ist, wäre es nicht plausibel, dies hier für unbeachtlich zu halten. Denn objektiv betrachtet ist es gleichgültig, ob der Algorithmus und da-

793 Vgl. Cornils, AfP 2018, 377 (380 ff.) und Ingold, MMR 2020, 82 (84), die bestehende Ungewissheiten im Umgang mit potentiellen Gefahren der Online-Kommunikation aufzeigen.

$794 \mathrm{Zu}$ den wahlrechtsspezifischen Delikten, auch zur Wählernötigung, siehe etwa $v$. Ungern-Sternberg, in: Unger/dies., Demokratie und künstliche Intelligenz, S. 3 (15f.). Ausführlicher hierzu noch unten, 3. Teil, C.I.4.a.

795 Vgl. BVerfGE 39, 1 (45) - Schwangerschaftsabbruch I. Ausführlicher zu einem ultima-ratio-Prinzip Jahn/Brodowski, ZStW 129 (2017), 363 (366 ff.).

796 Vgl. Schulze-Fielitz, in: Dreier, GG, Art. 5 Abs. 1 Rn. 222.

797 Mit Blick auf die Neutralität der Intermediäre selbst siehe etwa Bund-Länder-Kommission, Bericht zur Medienkonvergenz, S. 32 f.; zu Suchmaschinen siehe etwa Dörr/Natt, ZUM 2014, 829 (843). Umfassend zum Aspekt der Netzneutralität Bortnikov, Netzneutralität und Bedingungen kommunikativer Selbstbestimmung. Das eigentliche Problem der Netzneutralität der Access-Provider war ursprünglich sogar in $\$ 41$ a TKG durch Verordnungsermächtigung der Bundesregierung geregelt, was sich jetzt in Art. 3 der VO 2015/2120, ABl. 2015 L 310/1 wiederfindet.

798 Jedenfalls kritisch mit Blick auf Aspekte der Netzneutralität BVerfGE 149, 222 Rn. 79 - Rundfunkbeitrag; die Regulierungsbedürftigkeit vor diesem Hintergrund zeigt auch Heilmann, MMR 2020, 162 (163) auf, wobei sie - zutreffend - betont, dass anders als in der Telekommunikationsdebatte wegen der Funktion der Suchmaschinen keine absolute Neutralität möglich ist. So auch und kritisch zur Parallele der Netzneutralität Hartl, Suchmaschinen, Algorithmen und Meinungsmacht, S. $197 \mathrm{f}$. Deshalb spricht er eher von Suchmaschinenneutralität, S. $198 \mathrm{ff}$. 
mit das Bewertungsverfahren von Inhalten und ihren Metadaten eine priorisierte Weitergabe verursacht,oder ob diese aufgrund manipulierter, für die Bewertung relevanter (Meta-)Daten ${ }^{799}$ selbst erfolgt. In beiden Fällen ist das Resultat ähnlich: ein verzerrtes Bild aus dem eigenen Netzwerk. Kern der Neutralitätsdebatte ist aber gerade, dass eine technisch bedingte Priorisierung bzw. Benachteiligung verhindert werden soll.

\section{d. Informations-und Meinungsbildungsfreibeit}

Insbesondere für die oben bereits angesprochenen Nutzer, die überwiegend über soziale Netzwerke kommunizieren und Informationen aufnehmen, ${ }^{800}$ etwa weil sie den herkömmlichen Medien weniger Vertrauen entgegenbringen, können Meinungsroboter bedeutsam werden. Das wird - aktuell - zwar nur ein Bruchteil der Nutzer sein. Aus demokratischer Sicht gibt es aber gerade keine zu vernachlässigende Größe, wenn es um die Partizipation am Meinungsbildungsprozess geht. Besonders mit Blick auf diese Nutzergruppe haben Meinungsroboter aber dann auch eine wesentlich größere Bedeutung. Dies resultiert einerseits aus einer potentiell schon bestehenden Informationsdisbalance infolge personalisierter, algorithmischer Informationsweitergabe und homogeneren Teilnetzwerken. Meinungsroboter mit einem entsprechenden Nutzerprofil können also gezielten Einfluss auf die Informationsweitergabe an „Gleichgesinnte“ haben. ${ }^{801}$ Entscheidend wird hierbei freilich auch das individuelle Nutzerverhalten. ${ }^{802}$ Denn der Einfluss wird potentiell dadurch größer, dass entsprechende Profile eine direkte Verbindung miteinander eingehen. Gerade im Bereich der hier angesprochenen Nutzergruppe besteht aber die Gewohnheit einer deutlicheren sozialen Isolation im Vergleich zu anderen Nutzergruppen. ${ }^{803}$ Gelingt es Meinungsrobotern also in diese Kreise zu gelangen und „social capital“804 aufzubauen, dann wird auch der Einfluss größer sein. ${ }^{805}$

799 Gemeint sind hiermit etwa die Relevanz des Inhalts für Nutzergemeinschaft und statistische Daten wie Likes, Reaktionen oder Anzahl der Kommentar etc., also solche Informationen, die für die Bewertung des Inhalts relevant werden.

800 Gräfe, PinG 2019, 5 (9) sieht im Kontext des sog. Microtargetings ebenfalls, dass diese Nutzergruppe „eines stärkeren Schutzes“ bedarf.

801 Strategisch zielt das in gewisser Weise auf ein Microtargeting ab, indem unterschiedlichen Nutzergruppen unterschiedliche Informationen angezeigt werden, um zielgruppenorientiert bestimmte Wirkungen etwa von Wahlwerbung o.ä. zu erzeugen. Siehe ausführlicher zum Microtargeting Klaas, MMR 2019, 84 (89) und Künast, ZRP 2019, 62 (63).

802 Hierzu illustrativ Thieltges/Hegelich, ZfP 64 (2017), 493 (504 ff.).

803 Allgemein zur Tendenz der homophilen Kommunikationsnetzwerke etwa Schweiger, Der (des)informierte Bürger, S. 89 f. Mit Blick auf die Netzwerkstruktur von Impfgegnern siehe etwa Yuan/ Schuchard/Crooks, Social Media + Society 2019, 1 (6, 8, 9).

$804 \mathrm{Zu}$ diesem etwa Coleman, Journal of Media Ethics Vol. 33 (2018), 120 (123).

$805 \mathrm{Zu}$ diesem Social Capital als Wirkungsvoraussetzung etwa Murthy u.a., IJoC 10 (2016), $4952 \mathrm{ff}$. Siehe zum Einfluss in diesen geschlossenen Netzwerken auch Hagen u.a., Social Science Computer Review, 1 (13 ff.) mit Blick auf politisch rechte Netzwerke. 
Spezifische Probleme nicht nur in Bezug auf diese Nutzergruppen ergeben sich dann, wenn „alternative Fakten“ verbreitet und deren Glaubwürdigkeit durch entsprechende Interaktionen suggeriert wird. Diverse Erfolgsgeschichten von Falschinformationen zeigen, dass diese nicht allein in einem kleinen Kreis Gehör finden, sondern ihnen eine größere Breitenwirkung zukommt. ${ }^{806}$ Zwar sind desinformative Inhalte kein neues Phänomen der virtuellen Welt. Allerdings verstärken soziale Netzwerke die nachteiligen Wirkungen erheblich, ${ }^{807}$ was nicht nur an der Geschwindigkeit der Kommunikation liegt. Dass es sich hierbei um von der Mehrheit abweichende Fakten handelt, ist dabei nicht entscheidend, sondern ihr fehlender sachlicher Kern. Das gilt nicht nur deshalb, weil durch sie Verwirrung gestiftet ${ }^{808}$ oder ein Klima der Verunsicherung geschaffen werden kann, indem unklar wird, welche Informationen richtig und welche falsch sind. ${ }^{809}$ Diese Verunsicherung mag zu Zweifeln an einer Mehrheitsmeinung oder rational-faktenbasierten Informationen führen, was wiederum Einfluss auf die weitere Informationsaufnahme und -verarbeitung haben kann. ${ }^{810}$ Wenn aufgrund unzutreffender Informationen eine Meinung gebildet wird, entfernt man sich von einer sachlichen Diskussion und damit vom Lebenselement der Demokratie. ${ }^{811}$ Eine kritische Überprüfung der eigenen, auf Grundlage ganz anderer Informationen gebildeten Meinung ist so deutlich schwieriger. Gefördert wird dadurch vor allem die Entstehung von extremen, regierungs- oder staatsfeindlichen Positionen, die teils auch verschwörungstheoretischen Charakter haben, ${ }^{812}$ was nicht minder problematisch ist. Solche Informationen kursierten zwar schon in der analogen Welt ${ }^{813}$ und können auch in der virtuellen Welt erfolgreich ohne Zutun von Meinungsrobotern verbreitet werden. Meinungsroboter helfen aber gerade, diese Informationen für einen größeren Kreis der Nutzer sichtbar zu machen

806 Und zwar auch bei Nutzern, die weniger stark die sozialen Medien nutzen, vgl. hierzu Vosoughi/Roy/ Aral, Science Vol. 359 (2018), 1146 (1149).

807 von der Weth/Vachery/Kankanhalli, in: Proceedings of the International Conference on Multimedia \& Expo Workshops (ICMEW), S. 1.

808 Hierzu etwa Coleman, Journal of Media Ethics Vol. 33 (2018), 120 (126).

809 Hegelich, Die Politische Meinung, Nr. 543 (2017), 32 (36).

810 Hegelich, ebd.

811 Das ist letztlich auch die wesentliche Ratio, die sich hinter dem vom BVerfG vertretenen Ausschluss bewusst unwahrer Tatsachenbehauptungen vom Schutz des Art. 5 Abs. 1 S. 1 GG verbirgt, vgl. BVerfGE 12, 113 (130) - Schmid-Spiegel; 54, 208 (219) - Böll.

812 Neben der Reichsbürgerbewegung, die die Existenz der Bundesrepublik anzweifeln, zählt hierzu u.a. auch die Leugnung des menschengemachten Klimawandels, zahlreiche Verschwörungstheorien hinsichtlich der Covid-19 Pandemie, insbesondere dass der Staat versuche, individuelle Freiheiten massiv und dauerhaft zu beschränken. In der Studie von Hagen u.a., Social Science Computer Review, $1 \mathrm{ff}$. konnte etwa nachgewiesen werden, dass (wahrscheinlich) automatisierte Nutzerprofile vorwiegend dem rechten Spektrum zugeordnet werden konnten (S. 10). Zu einer Verbreitung ,various alt-right narratives [...] which are pushing divisive political ideology” während der Covid-19-Pandemie kommt auch Ferrara, First Monday Vol. 25 (2020) Nr. 6.

813 Hill, DÖV 2020, 205 (209f.); Appel/Doser, in: Appel, Die Psychologie des Postfaktischen, S. 9 (11f.) mit einigen Beispielen. Hierauf ebenfalls, wenn auch in einem anderen Zusammenhang, hinweisend Pfengler, GreifRecht 2020, 1 (10). 
und mit ihrer Hilfe weiterzuverbreiten. ${ }^{814}$ Insoweit bleibt dann der betroffene Nutzerkreis gerade nicht auf eine Minderheit beschränkt. Das gilt umso mehr, wenn man die zunehmende Bedeutung sozialer Netzwerke in den vergangenen Jahren berücksichtigt. Mit zunehmender Nutzung und Verweildauer steigt auch die Wahrscheinlichkeit, in Kontakt mit entsprechenden Manipulationen zu kommen. ${ }^{815}$ Und letztlich ist auch allgemein im Blick zu behalten, dass qualitätssichernde Vorfilter wie in herkömmlichen Massenmedien online nicht in demselben Umfang existieren, weshalb der Nutzer die Bewertung und Trennung von relevanten Inhalten selbst übernehmen muss, dabei aber besonders anfällig für Verzerrungen und Manipulationen ist. ${ }^{816}$ Ein bloßer Verweis auf das Bestehen alternativer, qualitätssichernder Informationsmöglichkeiten greift deshalb insgesamt zu kurz, um das Gefahrenpotential zu negieren. ${ }^{817}$

\section{e. Wechselseitige Verstärkungen der Effekte und das übergeordnete Schutzgut der Kommunikation}

Nicht aus dem Blick zu verlieren ist bei der Diskussion auch, dass die Teilfreiheiten des Art. 5 Abs. 1 S. 1 GG in einem größeren Zusammenhang zu betrachten sind. Sie sichern mit den subjektiven Gewährleistungen auch den objektiven Kommunikationsprozesses als solchen $a b .{ }^{818}$ Insoweit sind die Teilfreiheiten und die beeinträchtigenden Auswirkungen nicht nur isoliert, sondern eben auch in ihrer kumulierten Wirkungsweise zu betrachten. Kumuliert wirken die Meinungsroboter aber aus Sicht des Kommunikationsprozesses an den entscheidenden Stellen. Nicht nur die Weitergabe der Informationen, sondern gerade auch die diskursive Begegnung der Nutzer wird beeinträchtigt. ${ }^{819}$ Die diskursive Öffentlichkeit ist aber - unabhängig davon, dass soziale Netzwerke eher verschiede-

814 Zum Zusammenhang zwischen Falschinformationen bzw. „low-credibility-information“ und dem Einsatz von Meinungsrobotern vgl. etwa Yang/Torres-Lugo/Menczer, Prevalence of Low-Credibility Information on Twitter During the COVID-19 Outbreak, 2020, S. 2 f. und Gallotti u.a., Nature Human Behaviour 2020, im Kontext der Covid-19-Pandemie. Hill, DÖV 2020, 205 (210) spricht von einer „neuen Dimension“, die Fake News auch durch den Einsatz von Bots angenommen habe.

815 Wohl auch deshalb mahnen Kaiser/Reiling, in: Unger/v. Ungern-Sternberg, Demokratie und künstliche Intelligenz, S. 85 (96) zu einer „vorausschauenden Regulierung“ mit Blick auf die Meinungsvielfalt in sozialen Netzwerken.

816 Allgemeiner zu diesen Verzerrungen bereits ausführlich oben, 2. Teil, B.III.3.b. Das BVerfG sagt das zwar nicht ausdrücklich, allerdings leitet es auch dadurch die noch immer bedeutende Rolle des öffentlich-rechtlichen Rundfunks in einer veränderten Medienwelt her, BVerfGE 149, 222 Rn. 80 Rundfunkbeitrag.

817 In diese Richtung ebenfalls und von Bedrohung sprechend Kaiser/Reiling, in: Unger/v. Ungern-Sternberg, Demokratie und künstliche Intelligenz, S. 85 (96). Mit Blick auf die Bedrohungsszenarien der Fragmentierung durch Intermediäre ähnlich Brinkmann, UFITA 2019, 364 (370 f.).

818 Vgl. zu diesem Zusammenhang von Informationsfreiheit, Meinungsfreiheit und Meinungsbildungsprozess statt vieler BVerfGE 74, 297 (323) - 5. Rundfunkentscheidung.

819 Dies stellt für Kaiser/Reiling, in: Unger/v. Ungern-Sternberg, S. 85 (95) aber im Kontext der Filterblasenproblematik eine (besondere) Gefahr dar. 
ne Teilöffentlichkeiten als eine gesamte Öffentlichkeit aufweisen $-{ }^{820}$ entscheidende Voraussetzung des Kommunikationsprozesses. Das setzt nicht nur voraus, dass Räume der Begegnung bestehen, sondern dass gerade alle Meinungen und Informationen in gleichberechtigter Weise Zugang zu ihnen erhalten können. ${ }^{821}$ Meinungsroboter versuchen aber insbesondere dies zu verhindern.

Berücksichtigt man also auch die kumulierten (Wechsel-)Wirkungen, bedeutet das in den Kategorien der Schutzpflichtendogmatik gesprochen, dass das potentielle Schadensausmaß damit steigt. Im Falle der Meinungsroboter wird das u.a. dort besonders deutlich, wo unliebsame Meinungen unterdrückt und zeitgleich fehlerhafte Informationen verbreitet werden. Potentiell kritische Stimmen werden gar nicht laut oder zumindest nicht mehr im gleichen Umfang wahrgenommen, wohingegen abweichende Fakten zur wesentlichen Grundlage der Diskussion werden können. In Abhängigkeit vom Thema, dem Nutzerkreis und anderen situationsspezifischen Faktoren kann das unterschiedliche und nicht abschließend abschätzbare Auswirkungen haben: Besonders in empfänglichen Nutzerkreisen kann das zu einem noch größeren Vertrauensverlust in die herkömmlichen Medien, die Politik, das Staatswesen und dadurch zu einer anhaltenden und weiteren Radikalisierung führen. Und auch die notwendige Begegnung unterschiedlicher Meinungen zur Findung der Wahrheit durch den kommunikativen Prozess wird so, wenn auch u.U. nur marginal, verhindert. ${ }^{822}$ Das gilt umso mehr dann, wenn desinformative Inhalte verbreitet werden, eine Begegnung also schon aufgrund ganz unterschiedlicher Tatsachen erfolgt. ${ }^{823}$ Eine gemeinsame Lösung oder einen Kompromiss zu finden, ist so unter erheblich erschwerten Bedingungen und nur begrenzt möglich. ${ }^{824}$ In der ohnehin gespaltenen Internetöffentlichkeit, in der schon jetzt aggressive und politisch polare Kommunikation zu beobachten ist, ${ }^{825}$ kann das zusätzlich den Spaltungsprozess und die Entgrenzung der Netzwerköf-

820 Vgl. hierzu nur Brinkmann, UFITA 2019, 364 (375 f.); Gräfe, PinG 2019, 5 (6); Vesting, in: Unger/v. Ungern-Sternberg, Demokratie und künstliche Intelligenz, S. 33 (45).

821 Zur Offenheit als zentrale Voraussetzung der „Öffentlichkeit“ siehe bereits oben, 1. Teil, B.I.

822 Vgl. Keller/Klinger, Political Communication Vol. 36 (2019), 171 (174).

823 Ähnlich, allerdings im Kontext der Filterblasen und Echokammern, Kaiser/Reiling, in: Unger/v. Ungern-Sternberg, Demokratie und künstliche Intelligenz, S. 85 (95 f.).

824 Keller/Klinger, Political Communication Vol. 36 (2019), 171 (174). Vgl. hierzu Schaal, in: BedfordStrohm/Höhne/Zeyher-Quattlender, Digitaler Strukturwandel der Öffentlichkeit, S. 121 (127): „Twitterbots [...] sind nicht darauf programmiert, dem zwanglosen Zwang des besseren Arguments zu folgen - das Gegenteil ist der Fall [...]. Da sie im Gegensatz zu Menschen in Kommunikationen frustrationstolerant sind, besteht die Gefahr, dass die Ergebnisse deliberativer Prozesse in der demokratischen Öffentlichkeit in Zukunft eine systematische Schlagseite zugunsten von Twitterbots besitzen werden. Durch die Verzerrung der informationellen Basis des Prozesses der Präferenzgenese kann das deliberative Ideal demokratischer Politik insgesamt untergraben werden, da die Ergebnisse deliberativer Verfahren nicht mehr die Vermutung höherer Rationalität für sich beanspruchen können. Vor diesem Hintergrund steht die normative Integrität öffentlicher Diskurse unter Vorbehalt und die Idee der räsonierenden Öffentlichkeit wird selbst in Frage gestellt. " Und ergänzend, a.a.O., S. 128 f.

Brinkmann, UFITA 2019, 364 (368f.). 
fentlichkeit beflügeln. ${ }^{826} \mathrm{Zu}$ berücksichtigen ist zudem, dass die Wirkungen weder auf den Einzelnen noch auf die Online-Umgebung allein beschränkt bleiben. Wegen der Orientierungsfunktion digitaler Öffentlichkeiten und des digitalen Meinungsklimas für die Kommunikation in der analogen Welt ${ }^{827}$ können die Wirkungen weiterreichen. ${ }^{828}$

\section{f. Zwischenfazit}

Deshalb ist weder eine pauschale Verharmlosung noch eine maßlose Übertreibung angezeigt. ${ }^{829}$ Quintessenz bleibt vielmehr, dass die tatsächlichen Auswirkungen der Meinungsroboter gerade die Grundsätze des von Art. 5 Abs. 1 S. 1 GG geschützten offenen und gleichberechtigten Kommunikationsprozess berühren und mit der interaktiven und auf der Nutzergemeinschaft beruhenden Kommunikationsstruktur der sozialen Netzwerke jedenfalls die Grundlage für einen erfolgreichen Einfluss der Meinungsroboter gelegt ist. Den Meinungsrobotern wohnt damit jedenfalls ein nicht unerhebliches Schadenspotential ${ }^{830}$ inne, das mit Blick auf die kommunikative Chancengleichheit und die Informationsweitergabe tendenziell vergleichbar mit Aspekten der Netzneutralität sein kann. Und aus Sicht der Meinungsäußerungsfreiheit ergibt sich im Ergebnis kein Unterschied zu den bereits strafrechtlich normierten Verboten der Willensbeeinflussung. Alles in allem spricht also vieles für ein erhöhtes potentielles Schadensausmaß, dem zwar nicht - wie schon mehrfach betont - Allgemeingültigkeit zukommen kann, sondern das letztlich vielmehr von den konkret-individuellen Umständen abhängig

826 Vgl. Steinmaurer, in: Litschka/Krainer, Der Mensch im digitalen Zeitalter, S. 31 (36) der von einer „Erosion und Vergiftung öffentlicher Diskursqualitäten“ spricht.

827 Vgl. Thieltges/Hegelich, ZfP 64 (2017), 493 (507f.). Maßgeblich ist insoweit auch die von den Netzwerkthemen und Diskussionen ausgehende Agenda-Setting-Funktion. Daneben werden Sie aber etwa auch für statistische Erhebungen oder sonstige Analysen relevant, hierzu Muble/Ackland/Graham, ZParl 2018, 618 (638). Schaal, in: Bedford-Strohm/Höhne/Zeyher-Quattlender, Digitaler Strukturwandel der Öffentlichkeit, S. 121 (128) bezeichnet die öffentliche Meinung als Seismograph und zeigt gleichzeitig auch die Probleme auf, die dadurch entstehen, ebd., S. $127 \mathrm{f}$.

$828 \mathrm{Zu}$ der Möglichkeit dieses Einflusses etwa Oberer/Erkollar/Stein, in: Stumpf, Digitalisierung und Kommunikation, S. 311 (316) und Muble/Ackland/Graham, ZParl 2018, 618 (638). Dankert, in: Hoffmann-Riem, Big Data, S. 157 (164) bezeichnet das als die „schwerwiegendere Gefahr“. In diese Richtung auch Weber, Roboterjournalismus, ChatBots und Co, S. 86.

829 Krüper, in: Unger/v. Ungern-Sternberg, Demokratie und künstliche Intelligenz, S. 67 (69) spricht hier von einer doppelten Gefahr: „Weder darf sie sich zum Diener von Horrorszenarien einer Tyrannin der Algorithmen machen, noch sollte sie sich in digitalpolitischer Ignoranz üben. " Und mit Blick auf das Filterblasennarrativ betonen Kaiser/Reiling, in: Unger/v. Ungern-Sternberg, Demokratie und künstliche Intelligenz, S. 85 (90) die Notwendigkeit einer „differenzierte[n] Bewertung, die weder in ein ,Filterblasen-Lamento“ verfällt, noch die Gefahr einer Filterblase [...] kleinzureden versucht.“ Diese beiden Extrema zeigt auch Hegelich, Die Politische Meinung, Nr. 543 (2017), 32 auf. Ebenfalls vor (politischen) Fehlschlüssen im Kontext digitalisierter Öffentlichkeiten warnend Drerup, in: Binder/ders., Demokratieerziehung und Bildung digitaler Öffentlichkeiten, S. 29 (37).

830 Steinmaurer, in: Litschka/Krainer, Der Mensch im digitalen Zeitalter, S. 31 (36) spricht mit Blick auf Fake News und Social Bots von einem hohen Gefährdungspotential. 
wäre. ${ }^{831}$ Letzteres ist aber für die Frage einer Schutzpflicht des Staates insgesamt unbedeutend. ${ }^{832}$

\section{Die Eintrittswahrscheinlichkeit - empirische Unsicherheiten und Rationalitätsanforderungen an die Schutzpflicht}

Entscheidend für die verfassungsrechtliche Betrachtung werden die bereits aufgezeigten Unsicherheiten im Umgang mit Meinungsrobotern. Denn wenn nicht hinreichend klar ist, ob erstens Meinungsroboter überhaupt in einem bedeutenden Umfang aktiv sind und zweitens, welche genauen Auswirkungen sie haben, dann ist der Eintritt der aufgezeigten Schäden nicht hinreichend belegbar. ${ }^{833}$ Prima facie würde das also zu einer eher geringen Eintrittswahrscheinlichkeit führen. Das muss vor dem Hintergrund der Je-desto-Formel zwar nicht Schutzpflichten ausschließen. Es verlangt aber einen höheren Begründungsaufwand. Betrachtet man vor diesem Hintergrund die rundfunkrechtlichen Prämissen, die das BVerfG dem Regelungsauftrag des Gesetzgebers zugrunde legt, so fällt insbesondere eines in diesem Zusammenhang auf: Das Gericht betont stets den notwendigen, präventiven Charakter der „Handlungspflicht“: ${ }^{834}$ Erst einmal eingetretene Fehlentwicklungen im Kommunikations- und Meinungsbildungsprozess lassen sich wenn überhaupt - nur sehr schwer rückgängig machen. ${ }^{835}$ Anders gewendet wird die Eintrittswahrscheinlichkeit von Schäden im Bereich des Kommunikationsprozesses regelmäßig nicht hinreichend abschätzbar sein, was aber einer präventiven Regelungspflicht nicht entgegensteht. Abzuwarten, bis eine Schädigung eintritt oder sich konkret abzeichnet, kann mit ganz erheblichen Nachteilen verbunden sein, weshalb bereits eine „abstrakt-generelle Gefahr“836 der Beeinträchtigung ausreicht. ${ }^{837}$

$831 \mathrm{Zu}$ diesen Faktoren siehe bereits oben, 2. Teil, C.V.

832 Immerhin geht es hier um die Frage einer Schutzpflicht, der durch abstrakt-generelle Regelung und damit anders als im Polizeirecht nicht durch Verfügung im konkreten Einzelfall begegnet werden soll.

$833 \mathrm{Zu}$ den Schwierigkeiten der Konkretisierung einer Eintrittswahrscheinlichkeit siehe auch Thieltges/ Hegelich, ZfP 64 (2017), 493 (509).

834 In diese Richtung etwa Heitdke, Meinungsbildung und Medienintermediärsregulierung, S. 209 ff., der aber auch nur von einer Obliegenheit ausgeht.

835 Darauf stets hinweisend das BVerfG, E 57, 295 (323) - 3. Rundfunkentscheidung; 73, 118 (160) - 4. Rundfunkentscheidung; 119, 181 (217) - Rundfunkfinanzierungsstaatsvertrag. So auch in der generellen Diskussion um eine Intermediärsregulierung und unter Bezugnahme auf diese Rechtsprechung etwa Bund-Länder-Kommission, Bericht zur Medienkonvergenz, 2016, S. 32. In diese Richtung auch Brinkmann, UFITA 2019, 364 (377); Fehling/Leymann, AfP 2020, 110 Rn. 41 und Paal, MMR 2018, 567 (568).

836 Vgl. Heidtke, Meinungsbildung und Medienintermediäre, S. 210; Dörr/Natt, ZUM 2014, 829 (841).

837 Mit Blick auf soziale Netzwerke Fehling/Leymann, AfP 2020, 110 Rn. 41. Mit Blick auf Suchmaschinen Dörr/Natt, ZUM 2014, 829 (841). Vgl. auch zu einem eher präventiv-vorsorgenden Ansatz, allerdings mit Blick auf Nanotechnologie, Softwareentwicklungen und Algorithmen, Martini, BlackBox Algorithmus, S. 121. Hartl, Suchmaschinen, Algorithmen und Meinungsmacht, S. $141 \mathrm{f}$. meint sogar, dass wegen der Funktion der „dienenden Rundfunkfreiheit“ eine ständige Gefahrenlage bestehe. In- 
Eine Übertragung dieses „Vorsorgegedankens“ auf die neuen digitalen Probleme wird allerdings durchaus kritisch bewertet. So meint etwa Cornils, dass die Risikovorsorge zwar regulative Eingriffe rechtfertige, aber hieraus keineswegs eine Regelungspflicht gewonnen werden könne. ${ }^{838}$ Ansonsten bestünde etwa die Gefahr, dass das auf den klassischen Rundfunk bezogene Diktum der Meinungsvielfalt auf alle Medien erstreckt wird, ,wenn und soweit eine Akkumulation von Meinungsmacht durch diese [...] vorstellbar ist. “839 Insoweit „verbiet[e] sich die Annahme, [...] Maßnahmen [- etwa zur Entflechtung oder Aufmerksamkeitslenkung -] könnten nicht nur erlaubt, sondern auch noch verfassungsrechtlich obligatorisch sein. “840 Daher sei nicht von einer Pflicht, sondern einer gesetzgeberischen Prärogative auszugehen. ${ }^{841}$ Und Ingold weist daraufhin, dass im Bereich der neuen Medien und der Meinungsroboter eine doppelte Ungewissheit bestünde, einerseits in Hinblick auf die Eignung der zu wählenden Instrumente im „kommunikativen Wirkungsgefüge“ und andererseits hinsichtlich der Grundannahmen über die gesellschaftlichen Wirkungszusammenhänge der zu regulierenden Phänomene. ${ }^{842}$ Zudem seien die Kommunikationstechniken keine „Hochrisikotechnologieanlagen “ ${ }^{843}$ bei denen entsprechend geringere Anforderungen gestellt wurden. ${ }^{844}$ Ohnehin müssten im digitalen Raum ganz andere Konzepte gefunden werden, weil es hier gerade nicht mehr um eine Angebotsvielfalt ginge. ${ }^{845}$

Prinzipiell ist der Kritik durchaus zuzustimmen. Die neuen Herausforderungen divergieren ganz erheblich von denen, die der herkömmliche Rundfunk mit sich gebracht hat. Dass altbekannte Regelungsmodelle nicht ohne Weiteres als Status quo für den gesamten Medienbereich Geltung beanspruchen können, folgt schon aus den ganz verschiedenartigen Gefahrenlagen. ${ }^{846} \mathrm{Im}$ Internet ist nicht

soweit sieht er einen Unterschied im Regel-Ausnahme-Verhältnis im. Vergleich zu dem bei der Schutzpflicht. Hier sei der Veruicht auf schützende Handlung die Regel.

838 Cornils, AfP 2018, 377 (382). Ebenso Liesem, UFITA 2019, 395 (409).

839 Cornils, ebd.

840 Cornils, a.a.O., S. 383. In diese Richtung auch Ingold, MMR 2020, 82 (85).

841 Cornils, a.a.O., S. 384; so letztlich auch Liesem, UFITA 2019, 395 (409).

842 Ingold, MMR 2020, 82 (84). Zumindest mit Blick auf das kommunikative Wirkungsgefüge auch Cornils, AfP 2018, 377 (383).

843 Ingold, MMR 2020, 82 (84).

844 So etwa bei BVerfG, NVwZ 2010, 702 - CERN.

845 Ingold, ebd.

846 Nichts anderes hat auch das BVerfG betont, wenn es formulierte, dass „die Ausgestaltung dieser Ordnung [- gemeint ist die plurale Rundfunkordnung nach den Vorgaben des BVerfG -] Aufgabe des Gesetzgebers [ist], der dabei einen weiten Spielraum, auch für Differenzierungen insbesondere nach der Regelungsart und Regelungsdichte, vorfindet [...]“, BVerfGE 121, 30 (50) - Parteibeteiligung an Rundfunkunternehmen. Vgl. daneben BVerfGE 73, 118 (202) - 4. Rundfunkentscheidung, in der das BVerfG es für unproblematisch ansah, dass ausländische Programme nicht der in $\$ 15$ LRG normierten Ausgewogenheit verpflichtet waren. In BVerfGE 83, 238 (302) - 5. Rundfunkentscheidung, stellte es klar, dass für neue Medien wegen der noch geringen Bedeutung, vorerst keine Grundversorgung notwendig war. 
mehr die generelle, sondern die individuelle Vielfalt das Problem, ${ }^{847}$ sodass zwangsläufig ganz andere Regelungsansätze notwendig werden. Das bedeutet aber nicht, dass der Kerngedanke der Aufrechterhaltung des offenen und pluralen Kommunikationsprozesses damit nicht mehr einschlägig ist. Auch hier führt die rundfunkrechtliche Sonderdogmatik mit ihren vergleichsweise strikten Regelungs(ziel)vorgaben zu einer starken Verunsicherung im Umgang mit neuen digitalen Phänomenen. Durch die Übertragung althergebrachter Regelungsziele und -Vorgaben befürchtet man eine zu starke Regulierung aller meinungsrelevanten Dienste. Hier zeigen sich erneut die Vorteile der Dogmatik grundrechtlicher Schutzpflichten: $O b$ potentielle Fehlentwicklungen wegen ihrer Irreversibilität eine Pflicht des Gesetzgebers auslösen, ist von der Frage des Inhalts einer solchen Pflicht klar zu trennen. ${ }^{848}$ Eindeutige, obligatorische Maßnahmen sind im Bereich der „Risikovorsorge“ schon wegen der kollidierenden Interessen nur schwer zu begründen. Hier bleibt es eher bei der Vorgabe eines aus dem Schutzbedarf erwachsenden Schutzziels. Die Auswahl der konkreten Schutzinstrumente obliegt regelmäßig und ausschließlich dem Staat respektive dem Gesetzgeber. ${ }^{849}$ Denn die von Ingold angesprochenen empirischen Wissenslücken über die Auswirkungen bestimmter Phänomene ebenso wie über die Auswirkungen noch zu ergreifender Instrumente sind auch abwehrrechtlich problematisch und im Rahmen der Verhältnismäßigkeit durchaus zu berücksichtigen (dazu noch später, 3. Teil C.II.3). Aus Sicht der Kommunikationsgrundrechte ist die Staatsferne des Kommunikationsprozesses zu beachten, und durch den Staat selbst begründete, schadhafte Verzerrungen sind zu vermeiden. ${ }^{850}$ Das kann dazu führen, dass regulative Maßnahmen nicht ergriffen werden können, sondern anderweitig Schutz zu gewähren ist.

847 Liesem, AfP 2020, 277 (278) spricht insoweit nicht mehr von der Angebots-, sondern von der Rezeptionsvielfalt. Ingold, MMR 2020, 82 (85) verweist darauf, dass es eher um individuelle Autonomie denn um objektive Anforderungen der Repräsentativität o.ä. geht.

848 Müller-Terpitz, in: Kühling/Zimmer, Neue Gemeinwohlherausforderungen, S. 177 (194) scheint dies etwa zu sehen, wenn er einerseits meint, dass eine Aufgabe zur Gefahrenvorsorge besteht, andererseits aber meint, dass die Länder zwar keine Regelungen angesichts der abstrakten Gefahr treffen müssen, dies aber dürfen. Indes verkannt wird diese Differenzierung auch von Schimmele, Staatliche Verantwortung für diskursive Integrität, S. 204, wenn sie einerseits erkennt, dass der algorithmenbasierten Informationsvermittlung eine Verzerrungstendenz immanent ist, sie aber darin keine „Auflehnung gegen eine funktionierende Kommunikationsordnung, die staatliche Schutzpflichten auslöst“ sieht, weil wirtschaftliche Zielsetzungen auch bei analogen Medien vorzufinden sind. Das steht nicht der prinzipiellen Verpflichtung im Wege, sondern es sind vielmehr Aspekte, die dann auf Rechtsfolgenseite berücksichtigt werden können, weil Art. 3 Abs. 1 GG und Art. 12 Abs. 1 GG insoweit Grenzen ziehen könnten.

849 So Liesem, UFITA 2019, 395 (409); dies., AfP 2020, 277 (279); dies., in: Litschka/Krainer, Der Mensch im digitalen Zeitalter, S. 183 (194).

850 Vgl. zu dieser Gefahr im Intermediärsbereich etwa Liesem, AfP 2020, 277 Rn. 19 ff. mit Blick auf die Vorgabe einer positiven Diskriminierung durch den Staat. Vgl. zu diesem Problem auch Fehling/ Leymann, AfP 2020, 110 Rn. 26; Ingold, MMR 2020, 82 (84), und in Bezug auf Propaganda und Desinformation ders., in: Opelland, Propaganda als (neue) außen- und sicherheitspolitische Herausforderung, S. 85 (101). 
Auch hier stehen die empirischen Defizite nicht per se einer Schutzpflicht im Wege. Schon die Debatte um die Rundfunkregulierung ist - nach Entfallen der Frequenzknappheit - mit der besonderen Suggestivkraft ${ }^{851}$ recht stark von Annahmen geprägt, die so nie wirklich belegt werden konnten und zunehmend in Zweifel gezogen werden ${ }^{852}$. Ohnehin handelt es sich bei der Meinungsbildung um einen komplexen Prozess, bei dem einzelne Wirkungsfaktoren nur schwer bestimmt werden können. ${ }^{853}$ Letzteres wird auch noch dadurch erschwert, dass man es mittlerweile mit einer wesentlich komplexeren und vielfältigeren Mediennutzung zu tun hat, sodass ohnehin keine absolute Gewissheit eintreten kann, ob und wie bestimmte Phänomene online und offline Wirkung entfalten. ${ }^{854}$ Dass insbesondere die Kommunikations- und Medienwissenschaften deshalb (berechtigte) Zurückhaltung bei der Bewertung bestimmter Phänomene und ihrer Rückführung auf digitale Prozesse wahren, ist vor diesem Hintergrund nur verständlich. ${ }^{855}$ Für eine medienrechtliche Regulierung hat das aber keine zwingende Konsequenz. ${ }^{856}$ Hier geht es ja gerade um die Verhinderung eines (zukünftigen) Gefahreneintritts für den demokratischen Kommunikationsprozess, dessen endgültiger Beweis nicht abgewartet werden sollte und der ohnehin kaum in dieser Weise zu erbringen wäre. ${ }^{857}$ Diese Unsicherheit ist nicht nur der Medienregulie-

851 BVerfGE 90, 60 (87) - 8. Rundfunkentscheidung; 119, 181 (214f.) - Rundfunkfinanzierungsstaatsvertrag.

852 Hierauf ebenfalls, wenn auch in anderem Kontext, hinweisend Pille, Meinungsmacht sozialer Netzwerke, S. 166 f., 233 f. Ebenfalls kritisch Cornils, Designing platform governance, S. 21; ders., AfP 2018, 377 (380 f.).

853 Schon das BVerfG sprach in der analogen Zeit davon, dass „[z]wischen den Faktoren und Medien des komplexen Prozesses der Meinungs- und Willensbildung [...] mannigfache Beziehungen, Abhängigkeiten und Einflußnahmen [wirken]“, BVerfGE 20, 56 (99) - Parteifinanzierung I.

854 Besonders deutlich formuliert das auch Hegelich, Die Politische Meinung, Nr. 543 (2017), 32 (35): „[...] [E]ines wird die Wissenschaft in absehbarer Zeit nicht zustande bringen: den unumstößlichen Nachweis, dass diese Formen der Manipulation einen politischen Effekt haben. Denn diese Gretchenfrage entzieht sich dem sozialwissenschaftlichen Zugriff. Es lässt sich nie mit hundertprozentiger Sicherheit sagen, wie sich dieselben Menschen ohne den Kontakt mit den sozialen Medien in genau der gleichen Situation verhalten hätten." Siehe daneben auch Brinkmann, UFITA 2019, 364 (376), und Mitsch, DVBl. 2019, 811 (816f.) begründet mit dieser vielschichtigeren Nutzung der Medien gerade die Unzulänglichkeit des bestehenden medienrechtlichen Rahmens.

855 Brinkmann, UFITA 2019, 364 (376).

856 So letztlich mit Blick auf die allgemeinen Probleme der Medienintermediäre ganz ausdrücklich Brinkmann, UFITA 2019, 364 (377, 379), der zudem - und, wie noch in dem vorliegenden Fall aufzuzeigen sein wird, zu Recht - betont, dass gerade im Bereich der Telemedien bislang kaum ein regulatorischer Zugriff erfolgt ist, was deshalb gerade die Notwendigkeit eines solchen nochmals deutlicher aufzeigt (a.a.O., S. 383). Auch das spricht hier dafür, dass es keine rein fakultative Aufgabe sein kann, (endlich) vorsorgend tätig zu werden. Nach Brinkmann, a.a.O., S. 383 „könnte sich [die Untätigkeit] als fahrlässiges Experiment an den realen Verhältnissen erweisen “ und „,[d]er medienrechtliche Auftrag [könne] sich [nicht] hinter fehlende[n] gesellschaftswissenschaftliche[n] Beweisführungen [...] zurückziehen.".

857 Vgl. hierzu auch Brinkmann, UFITA 2019, 364 (377, 383). Denn selbst wenn nachgewiesen werden würde, dass Meinungsroboter mit Nutzern interagieren und so einen potentiellen Einfluss haben, bleibt immer noch unklar, inwieweit dies auch realiter das Meinungsbild des jeweiligen Nutzers beeinflusst. Speziell mit Blick auf die Wirkungszusammenhänge von Meinungsrobotern Kind u.a., Social Bots, S. 57. Anders jedenfalls mit Blick auf die netzwerkinternen Wirkungen Muble/Ackland/ Graham, ZParl 2018, 618 (638). 
rung immanent, ${ }^{858}$ sondern ohnehin ein Kernaspekt jeder staatlichen Risikovorsorge und Gefahrenabwehr. ${ }^{859}$

Die Bedeutung der empirischen Defizite für die Begründung einer Schutzpflicht im Falle der Meinungsroboter sind daneben aber auch aus anderen Gründen zu einem gewissen Grad zu relativieren: Studien, die selbst entsprechende Profile verwendet haben, konnten immerhin aufzeigen, dass die Implementierung der Meinungsroboter in den sozialen Netzwerken möglich ist und zumindest ein gewisser Einfluss durchaus bestehen kann. ${ }^{80}$ Insoweit bleibt also allein ungewiss, in welcher Anzahl Meinungsroboter insgesamt aktiv sind und welchen Einfluss sie tatsächlich haben. Weil dies aber auch von verschiedenen konkret-individuellen Faktoren abhängt (hierzu oben, 2. Teil, C.V.), ist eine konkrete Aussage ohnehin nur schwer möglich. Gleichwohl zeigt sich insbesondere im Erfolg desinformativer Inhalte ${ }^{861}$ deutlich, dass der Nutzer in der Online-Welt, in der er selbständig „die Verarbeitung und die massenmediale Bewertung übernehmen [muss], die herkömmlich durch den Filter professioneller Selektionen und durch verantwortliches journalistisches Handeln erfolgt[e]“862, unvorsichtig agiert und in erheblicher Weise manipulierbar sein kann. ${ }^{863}$ Diese nutzerseitigen kognitiven Verzerrungstendenzen lassen sich auch - anders als etwa betreiberseitig geförderte Filterblaseneffekte $-{ }^{864}$ nachweisen. Entsprechendes gilt auch für potentielle

$858 \mathrm{Zu}$ der Notwendigkeit und dem Wesen des Medienrechts auf unklare aber sich abzeichnende Risiken zu reagieren etwa Brinkmann, UFITA 2019, 364 (377).

859 Auch die Rasterfahndung war etwa darauf angelegt Personen ausfindig zu machen, die in einem späteren Schritt erst zu möglichen Gefährdern werden. Aus Sicht des Rechtsgüterschutzes wurde dies auch nicht beanstandet, sondern allein wegen der damit einhergehenden unverhältnismäßigen Eingriffe in das Recht auf informationelle Selbstbestimmung der Betroffenen, hierzu BVerfGE 115, 320 (345 ff.) - Rasterfahndung II. Siehe zudem auch BVerfGE 109, 133 (158) - Langfristige Sicherungsverwahrung.

860 So etwa Boshmaf u.a., Computer Networks 57 (2013), 556 ff.; Grimme u.a., Big Data Vol. 5 (2017), 279 ff. Murthy u.a., IJoC 10 (2016), $4952 \mathrm{ff}$.; Freitas u.a., Social Network Analysis and Mining (2016), 6:23, S. 22 ff.; Zhang u.a., in: 2013 IEEE Conference on Communications and Network Security (CNS), S. $46 \mathrm{ff}$. mit Blick auf den "digital influence" allerdings aus kommerzieller und nicht politischer Sicht. Und zu einem speziellen Literaturnetzwerk Aiello u.a., in: Proceedings of the $6^{\text {th }}$ International AAAI Conference on Weblogs and Social Media, S. $10 \mathrm{ff}$.

$861 \mathrm{Zu}$ dieser Entwicklung auf der „unteren Ebene der vernetzten digitalen Kommunikation“, Brinkmann, UFITA 2019, 364 (372). Besonders relevant wurden letztere Inhalte auch während der Covid-19-Pandemie, siehe hierzu etwa Ahmed u.a., J Med Internet Res 2020;22(5):e19458; Cinelli u.a., Scientific Reports 10 (2020), 16598; Ferrara, First Monday Vol. 25 (2020) Nr. 6 und Gallotti u.a., Nature Human Behaviour 2020; Yang/Torres Lugo/Menczer, Prevalence of Low-Credibility Information on Twitter During the COVID-19 Outbreak, 2020.

862 BVerfG 149, 222 Rn. 80 - Rundfunkbeitrag.

863 Vgl. hierzu mit Blick auf FakeNews und einer mangelnden Medienkompetenz Kalwey, in: Möller, Was tun gegen Fake News und Hate Speech?, S. 139; Schweiger, Der (des)informierte Bürger, S. 109 und Thieltges/Hegelich, ZfP 64 (2017), 493 (498).

864 Auf diese Ungewissheiten und die mittlerweile anderslautenden empirischen Ergebnisse weist Cornils, AfP 2018, 377 (381); ders., ZUM 2019,89 (95), hin. Zumindest zurückhaltend gegenüber Regulierungsvorschlägen ohne empirische Basis Kaiser/Reiling, in: Unger/v. Ungern-Sternberg, Demokratie und künstliche Intelligenz, S. 85 (89) mit Fn. 20. Gleichwohl sehen sie hier eine den Handlungsbedarf auslösende Bedrohung, S. 96. Allerdings gilt es auch hierbei zu bedenken, dass letztlich aus dem Status quo die fehlende Regulierungsbedürftigkeit abgeleitet wird, obgleich unbestritten sein dürfte, dass 
Auswirkungen der Schweigespirale nicht nur allgemein. Ihre Wirkung lässt sich teilweise stärker -in der Online-Kommunikation belegen. ${ }^{865}$ Obgleich gewisse Zweifel an den in der bisherigen Empirie angewandten Methoden bei der Erkennung der Bots (hierzu oben 2. Teil, C.IV.) angebracht sind, so konnten zumindest entsprechende Automatisierungstendenzen und Phänomene weltweit beobachtet werden. ${ }^{866}$ Die Netzwerkstruktur, die auf nutzerfreundliche und einfache Interaktion und auf der Auswertung dieser Interaktionssignale beruht, bietet ein ideales Umfeld für Meinungsroboter. Sie sind besonders vorbildliche und interaktive Nutzer. ${ }^{867}$ Und viele der aufgezeigten Einsatzstrategien lassen sich einfach erstellen. Insoweit sind also wesentliche Voraussetzungen für einen tatsächlich erfolgreichen Einsatz geschaffen, sodass sich die aufgezeigten Schäden durchaus plausi-

die Intermediäre jedenfalls die Möglichkeit und die Macht hätten, die Informationsvermittlung im Sinne dieser Narrative zu steuern (siehe hierzu etwa Schmidt u.a., Zur Relevanz von Online-Intermediären für die Meinungsbildung, S.20; Heidtke, Meinungsbildung und Medienintermediäre, S. 124 ff.; und zu Suchmaschinen Hartl, Suchmaschinen, Algorithmen und Meinungsmacht, S. 38ff). Insoweit kann sehr wohl ein in die Zukunft gerichtetes Bedürfnis bestehen, einen potentiellen Missbrauch - gerade angesichts monopolartiger Marktstrukturen - zu verhindern, so etwa auch MüllerTerpitz, in: Kühling/Zimmer, Neue Gemeinwohlherausforderungen, S. 177 (193), der hier zudem von „einer verfassungsrechtlichen Aufgabe einer Gefahrenvorsorge" spricht, ebd., S. 194. Ausführlicher zu den potentiellen Risiken hier Pille, Meinungsmacht sozialer Netzwerke, S. 322 ff., der hieraus gerade eine besondere Gefahrgeneigtheit ableitet (S.345), die zu einer präventiven Regulierung führen muss (S. $346 \mathrm{ff}$.), mit Blick auf die potentielle Möglichkeit eines Eingriffs in die Informationsvermittlung, (S. $352 \mathrm{ff}$.). Aber auch hier wird betont, dass wegen der theoretischen Plausibilität das Phänomen „ernst zu nehmen [ist]“, was gerade auch deshalb der Fall sei, weil „eine empirisch fundierte Aussage über den gegenwärtigen Impetus seiner Algorithmen derzeit nur von Facebook selbst getroffen werden kann, weil eine für die Wissenschaft zugängliche Datenbasis fehlt." Hartl, Suchmaschinen, Algorithmen und Meinungsmacht, S. $70 \mathrm{f}$.

865 Insbesondere mit Blick auf den Einsatz der Meinungsroboter - wenn auch nur aus theoretischer Sicht - Ross u.a., European Journal of Information Systems 2019, S. 394 ff. Allgemeiner zur Schweigespirale in sozialen Netzwerken Neubaum/Krämer, Communication Research 45 (2018), 139 (158ff.) und Hampton u.a., Social Media and the ,Spiral of Silence', S. 8. Insoweit geht es gerade nicht allein um bloße Vermutungen, die nicht erfasst sein sollen, so etwa Krings, Grund und Grenzen grundrechtlicher Schutzansprüche, S. 231.

866 Siehe etwa zu Venezuela Forelle u.a., Political Bots and the Manipulation of Public Opinion in Venezuela, 2017; zu Japan Schäfer/Evert/Heinrich, Big Data Vol. 5 (2017) Nr. 5, zu den USA Bessil Ferrara, First Monday Vol. 21 Nr. 11; Howard u.a., Junk News and Bots during the US Election, 2017; zu Mexiko Suárez-Serrato u.a., in: Spiro/Ahn, SocInfo 2016: Social Informatics, S. 269 ff., und Treré, Trípodos, Número 39 (2016), 35 (39ff.); zum Iran Thieltges u.a., Effects of Social Bots in the Iran-Debate on Twitter, 2018; zu Brasilien vgl. Department of public policy analysis, Bots and Brazil's Electoral legal system, Policy Paper 3, 2019; zum katalanischen Referendum Stella/Ferraral Domenico, PNAS Vol. 115 (2018), 12435 ff.; zum Ukrainekonflikt Hegelich/Janetzko, in: Proceedings of the Tenth International AAAI Conference on Web and Social Media (ICWSM 2016), S. 579 ff. Weitere Nachweise auch bei Woolley, First Monday Vol. 21(2016). Zu Deutschland in Ansätzen Brachten u.a., Strategies and Influence of Social Bots in a 2017 German state election, 2017; Grimme u.a., PropStop Bericht Bundestagswahl 2017; Institute for Strategic Dialogue, Make Germany Great Again, S. 18 f.; Pfaffenberger/Adrian/Heinrich, in: Holtz-Bacha (Hrsg.), Die (Massen-)Medien im Wahlkampf, S. 97 (99). Und. Im Kontext der Covid-19 Pandemie u.a. Ahmed u.a., J Med Internet Res 2020:22(5):e19458; Cinelli u.a., Scientific Reports 10 (2020), 16598; Ferrara, First Monday Vol. 25 (2020) Nr.6; Gallotti u.a., Nature Human Behaviour 2020; Yang/Torres-Lugo/Menczer, Prevalence of Low-Credibility Information on Twitter During the COVID-19 Outbreak, 2020 und Uyheng/Carley, Journal of Computational Social Sciences 2020.

867 Kaerlein, in: Hug/Pallaver, Talk with the Bots, S. 17 (24f.). 
bel darlegen lassen können ${ }^{868}$ und keine Anhaltspunkte dafür bestehen, dass gerade Deutschland ${ }^{869}$ auf Dauer verschont bleiben sollte. Deshalb warnen auch Empiriker davor, dass entsprechende Szenarien zukünftig gerade nicht auszuschließen sind. ${ }^{870}$ Die grundlegende Bedeutung der kumulativ betroffenen Gehalte der Kommunikationsfreiheiten würde die Herabsetzung der Wahrscheinlichkeitsanforderungen rechtfertigen. Insoweit geht es nicht mehr ausschließlich um abstrakte Risikopotentiale, die in ferner Zukunft in „konkrete Gefahren umschlagen könnten" ${ }^{871}$. Man bewegt sich also nicht mehr allein in einem Bereich bloßer Risikovorsorge. Die betroffenen Grundrechte erlauben es, die Anforderungen an die Eintrittswahrscheinlichkeit in einer Weise herabzusetzen, dass sich die theoretisch herleitbaren und rational nachvollziehbaren Risikopotentiale zu einer - wenn auch sehr abstrakten - Gefahr verdichten. ${ }^{872}$

868 Brinkmann UFITA 2019, 364 (383), scheint es zu genügen, wenn sich Fehlentwicklungen im Bereich der Medien „abzeichnen“. Ein bestimmter Nachweis bestimmter Strategien von Meinungsrobotern konnten unter anderem Hagen u.a., Social Science Computer Review, $1 \mathrm{ff}$. führen.

869 Und selbst hier wurden entsprechende Phänomene bereits entdeckt, auch wenn das Ausmaß (teilweise) gering war, siehe bereits die Verweise in Fn. 866.

870 Vgl. Thieltges/Hegelich, ZfP 64 (2017), 493 (509f.), die differenziert die Risiken beurteilen. Mit Blick auf netzwerkintern-individuelle Risiken sprechen sie von einer „hohen Eintrittswahrscheinlichkeit“ und „multiplen Risiken“, bei netzwerkintern-sozialen Risiken sei hingegen die Eintrittswahrscheinlichkeit eher geringer (S. 510). Entsprechendes gilt für netzwerkextern-individuelle bzw. -soziale Risiken (S. 510 f.). Zukünftig und insgesamt gehen sie aber von einer Erhöhung der Eintrittswahrscheinlichkeit aus (S. 511). Siehe auch Kind u.a., Social Bots, S. 40. Steinmaurer, in: Litschka/Krainer, Der Mensch im digitalen Zeitalter, S. 31 (36) spricht schon jetzt - allerdings im Kontext von Fake News und Social Bots - von einem „hohe[n] Gefährdungspotenzial für demokratische Kommunikationsprozesse." Kaerlein, in: Hug/Pallaver, Talk with the Bots, S. 17 (22 f.) meint, dass sich etwaige Befürchtungen nicht „, $[\mathrm{g}] \mathrm{anz}$ von der Hand zu weisen sind [...].“ Ross u.a., European Journal of Information Systems, 394 (408) bezeichnen sie im Kontext der Schweigespirale als „plausible mechanism of manipulation“ und meinen, „that their existence can considerably undermine democratic processes[...]. “ Und auch die Enquete-Kommission Künstliche Intelligenz plädiert jedenfalls dafür, „dass die Situation - auch wenn stichhaltige Belege für Social Bots bislang fehlen - ernst genommen werden sollte“, BT.-Drs. 19 /2700, S. 481.

871 So etwa Heidtke, Meinungsbildung und Medienintermediäre, S. 210, der im Kontext der Intermediäre „(noch) nicht von einer Pflicht zur Ausgestaltung“, sondern von einer „Obliegenheit zur Risikovorsorge" ausgeht. Brinkmann, UFITA 2019, 364 (383) weist aber zutreffend daraufhin, dass die Untätigkeit ansonsten schnell zu einem „fahrlässigen Verzicht“ werden kann.

872 Davon ging scheinbar auch die Länderarbeitsgruppe „Social Bots“ in ihrem nicht veröffentlichten Bericht aus, vgl. Sächs-LT-Drs. 6/12131, S. 4. Blickt man auf die Literatur zur Medienregulierung digitaler Phänomene finden sich durchaus vergleichbare Stimmen. Hinsichtlich der Intermediärsregulierung spricht Müller-Terpitz, in: Kühling/Zimmer, Neue Gemeinwohlherausforderungen, S. 177 (194) nicht nur von einer verfassungsrechtlichen Aufgabe zur Gefahrenvorsorge, sondern gerade auch von einer abstrakten Gefahr. Mit Blick auf Suchmaschinen sehen das etwa auch Dörr/Natt, ZUM 2014, 829 (841) ähnlich: „Das Schutzgut der Meinungsfreiheit ist für eine demokratische Gesellschaft von solcher Bedeutung, dass allein die abstrakt-generelle Möglichkeit der Einflussnahme ausreicht, um regulierend tätig zu werden. [...] [D]en Gesetzgeber [trifft] eine Gewährleistungspflicht“. Mit Blick auf soziale Netzwerke, allerdings im Kontext der Ausgestaltungsdoktrin des Rundfunks, meinen Kaiser/ Reiling, in: Unger/v. Ungern-Sternberg, Demokratie und künstliche Intelligenz, S. 85 (102): „Von Verfassungs wegen zu fordern ist demnach eine Anpassung des Rundfunkstaatsvertrags an die moderne Gefährdungslage, die sich durch die sozialen Medien insgesamt und durch den personalisierten Algorithmus im Besonderen ergibt." Sie sprechen hier zudem - trotz vielfältiger Mediennutzung - von einer Bedrohung, S. 96. Mit Blick auf potentielles Microtargeting und Webtracking meint Gräfe, PinG 2019, 5 (11) „[...] [F]ür eine Regulierung [ließe sich] argumentieren, dass zum Schutz der De- 


\section{Obligatorische Risikovorsorge und Gefahrenabwehr?}

Generell wird deutlich, dass die für die Begründung einer prinzipiellen Handlungspflicht gewählte Unterscheidung zwischen Risikovorsorge und Gefahrenabwehr erhebliche Probleme aufwirft, weil eine präzise Abgrenzung kaum möglich ist. Die Grenze zwischen bloßem Risiko- und damit „Obliegenheit zur Risikovorsorge“ "873 - und zumindest abstrakter Gefahr - und damit obligatorische Gefahrenabwehr - kann nicht klar gezogen werden. Gerade in Fällen empirischer Wissensdefizite wird das offensichtlich. Potentielle Risiken lassen sich kaum handhabbar bewerten und einem Gefahrenmodell zuordnen. Ein Risiko kann sich bereits zur Gefahr verdichten oder nur ein Risiko bleiben. Das erklärt vielleicht auch, warum das BVerfG nie ausdrücklich zu diesem Abgrenzungsproblem Stellung bezogen hat. Oftmals ging es ohnehin um offensichtliche Fälle rechtswidriger Eingriffe. ${ }^{874}$ Auch würde damit attestiert werden, dass die Gefahrenschwelle des Polizeirechts kongruent mit der grundrechtlichen Schutzpflicht des Staates verläuft. ${ }^{875}$ Mit anderen Worten wäre die tatbestandliche Gefahrenschwelle nicht mehr selbst Ergebnis der Wahrnehmung der gesetzgeberischen Schutzpflicht, sondern die notwendige und zentrale Voraussetzung für das Eingreifen eben dieser. ${ }^{876}$ Gerade mit Blick auf empirische Unsicherheiten führte

mokratie Schutzpflichten des Staates aus den Kommunikationsgrundrechten bestehen.“ Explizit zu den Meinungsrobotern geht auch Brings-Wiesen, JuWiss-Blog Nr. 93/2016, davon aus, dass Schutzpflichten jedenfalls in einem Maße aktiviert sind, die einen „vorsichtig regulierenden Eingriff“ notwendig machen. Tendenziell mit Blick auf die Schutzpflichten, allerdings ohne dies ausdrücklich klarzustellen, Schmitt, in: Donath u.a., Verfassungen - 59. ATÖR, S. 203 (220 f.). Hartl, Suchmaschinen, Algorithmen und Meinungsmacht, S. 70 f., spricht sich jedenfalls wegen der Plausibilität von Personalisierungsmaßnahmen auf die Informationsvermittlung dafür aus, dies „ernst zu nehmen“. Anderswo wird die (medienrechtliche) „Risikovorsorge“ als „Obliegenheit“ des Gesetzgebers (Heidtke, Meinungsbildung und Medienintermediäre, S. 201) bzw. als verfassungsrechtlich nicht geboten angesehen (Cornils, AfP 2018, 377 [384]; Liesem, ZUM 2020, 377 [378 f.]). Hierbei geht es aber eher um die Frage, ob bestehende Vorgaben zur Vielfaltssicherung zwingend auf Intermediäre und das Internet zu übertragen sind.

873 Vgl. Heidtke, Meinungsbildung und Medienintermediäre, S. 210. Ähnlich auch Cornils, AfP 2018, 377 (384); Liesem, ZUM 2020, 377 (378 f.). I.E. jedenfalls ebenso Murswiek, Die staatliche Verantwortung für die Risiken der Technik, S. 144.

874 So etwa mit dem Schwangerschaftsabbruch und dem Beenden des menschlichen Lebens (BVerfGE 39, 1 [42] - Schwangerschaftsabbruch I und E 88, 203 [251] - Schwangerschaftsabbruch II), bei der Bedrohung des Lebens durch Geiselnahme (E 46, 160 [164] - Schleyer), den Gefahren der friedlichen Nutzung von Atomenergie (S. nur E 53, 30 [57] - Mühlheim-Kärlich) oder bei terroristischen Angriffen (E 115, 118 [152] - Luftsicherheitsgesetz). In all diesen Entscheidungen findet sich gerade der Rekurs - gekennzeichnet durch die konkreten Seitenangaben - auf den „rechtswidrigen Angriff“.

875 In diese Richtung etwa Hartl, Suchmaschinen, Algorithmen und Meinungsmacht, S. 144.

876 Vgl. hierzu etwa Gerbig, Grundrecht auf staatlichen Schutz, S. 63, die deshalb durch Zurücknahme der Anforderungen eine „eigenständige Bedeutung“ herstellen will. Siehe auch Dietlein, Grundrechtliche Schutzpflichten, S. 108 mit Blick auf die exekutiven Schutzpflichten. Er betont hier, dass die grundrechtliche[n] Schutzpflichten zur ,kleinen Münze' des Polizeirechts [verkommen]. "Krings, Grund und Grenzen staatlicher Schutzansprüche, S. 229, sieht das aber nicht als „prinzipiell problematisch“ an, „sondern aus verfassungsrechtlicher Sicht erwünscht und notwendig.“ Dabei schließt er aber von der einfach-rechtlichen Schwelle als Ausdruck der Transformation des Gesetzesvorbehaltes auf die eingeschränkte Verantwortung des Staates. Insoweit scheint er Regelungspflicht und Schutz- 
das aber zu Problemen: Wenn unklar ist, ob Risiken überhaupt bestehen, so könnte der Gesetzgeber zwar freiwillig tätig werden, eine Pflicht, für Aufklärung zu sorgen, besteht aber gerade nicht. Das führte aber zu absurden Ergebnissen: Solange der Staat auf die unzureichenden Forschungsergebnisse verweisen kann, kann er sich aus der Verantwortung ziehen, obwohl es gerade entscheidend wäre, dass zumindest dafür Sorge getragen wird, entsprechende Unsicherheiten $\mathrm{zu}$ beseitigen. ${ }^{877}$ Er wäre also nicht mal verpflichtet - um im polizeirechtlichen Jargon zu bleiben - Maßnahmen der Gefahrenerforschung vorzunehmen. ${ }^{878}$

Plausibler wäre es, die Unsicherheiten vor allem auf Rechtsfolgenseite zu berücksichtigen: ${ }^{879}$ Die Auswahl staatlicher Maßnahmen kann wegen dieser Ungewissheit weitreichend sein. Von Unterstützung empirischer Forschungsprojekte durch Bereitstellen finanzieller Mittel im Haushaltsplan und Vergabe durch die zuständigen Stellen oder die Beauftragung mit der Durchführung entsprechender Studien bis hin zu vorsichtig regulierenden Eingriffen ist vieles denkbar. ${ }^{800}$ Insoweit kommt dem Gesetzgeber ein weitreichender Spielraum zu, der ausfüllungsbedürftig und nicht vorgezeichnet ist. Das ändert aber nichts an der bestehenden Verantwortung des Staates. Wenn hiergegen eingewendet wird, dass damit das Verhältnis von Freiheit und Schutz ganz strukturell verändert wird, weil jede Freiheitsausübung potentiell risikobehaftet und deshalb i.E. rechtfertigungsbedürftig wird, ${ }^{881}$ so ist dem in dieser Verallgemeinerung nicht zuzustimmen. Es mag richtig sein, dass nicht jedes Verhalten Schutzpflichten auslöst, sondern ein gewisser Bagatellvorbehalt besteht. ${ }^{882}$ Freiheit und Schutz sind aber schon

pflicht kongruent zu verstehen. Plausibler wäre es, wenn man die flexible Gefahrenschwelle heranzöge, um zu bestimmen, ab wann konkrete regulative Maßnahmen notwendig werden und damit eine Regelungspflicht besteht.

877 Zur Gefahr, dass sich der Staat seiner Verantwortung entzieht Hermes, Grundrecht auf Schutz von Leben und Gesundheit, S.233, und mit Blick auf eine Pflicht selbst für Aufklärung zu sorgen ebd., S. 234.

878 Aus polizeirechtlicher Sicht wäre das aber notwendige Voraussetzung, um einen weitreichenderen Spielraum für die Sicherheitsbehörden zu schaffen. Mit der Frage des Vorliegens einer Gefahr entscheidet sich also, inwieweit danach tatsächlicher Schutz zu gewähren wäre. Zum Gefahrenerforschungseingriff siehe etwa Weiß, NVwZ 1997, 737 f.; Pewestorf, in: ders./Söllner/Tölle, Polizei- und Ordnungsrecht, $\mathbb{} 1$ Rn. 17.

879 So auch Hermes, Grundrechte auf Schutz von Leben und Gesundheit, S. 234, 240; Gerbig, Grundrecht auf staatlichen Schutz, S. 63 f.; Siehe auch Meyer, NJW 2020, 894.

$880 \mathrm{Zu}$ einer solchen allgemeinen Abstufung der Pflichtigkeit gelangt auch Gerbig, Grundrecht auf staatlichen Schutz, S. 63. Siehe hierzu auch Martini, BlackBox Algorithmus, S. 121: „Fördert eine Risikoanalyse hinreichende Anhaltspunkte für eine Schadensneigung zu Tage, knüpft sich daran das prozedurale Gebot, weitere Erkundungen und Analysen zu betreiben, um die Gefahren näher zu erforschen und zu ermitteln. Die Risikoprognose mündet in die Abwägung, ob die Reaktion zwischen Eintrittswahrscheinlichkeit und Schadenshöhe gesellschaftlich noch hinnehmbar oder bereits unzumutbar ist. Der Pflichtige muss dann mit Blick auf die diagnostizierte Risikohöhe alle technologischen Maßnahmen ergreifen, die zur Verfügung stehen, um einen Schaden zu vermeiden - auch wenn ein solcher nicht unmittelbar droht".

881 In diese Richtung etwa Murswiek, Die staatliche Verantwortung für die Risiken der Technik, S. 143; Dietlein, Grundrechtliche Schutzpflichten, S. 105.

882 Hierzu bereits oben, A.I.1 mit Fn. 486. 
immer kollidierende Aspekte gewesen, die in einen Ausgleich zueinander zu bringen sind. Eine generelle Risikotragungspflicht besteht also nicht per se, ${ }^{883}$ sondern ergibt sich erst im Wege dieses Ausgleichs, ${ }^{884}$ der zuvörderst Aufgabe des Gesetzgebers ist. ${ }^{85}$ Mit steigendem Risiko, fehlenden Schutzvorkehrungen und dem Vorliegen weiterer Aspekte wie der Irreversibilität von Schäden steigen die Anforderungen an das zu erreichende Schutzniveau; ${ }^{886}$ der Schutzgedanke kann dann die Freiheit überwiegen. Konkret führt das also dazu, dass sich der Handlungs- und Gestaltungsspielraum mit zunehmendem Risiko immer weiter verengt und mit Eintritt in eine relevante Gefahr auch regulative Maßnahmen (zwingend) notwendig werden können. ${ }^{887}$ Das bedeutet indes nicht, dass vorher keinerlei Verantwortung besteht. ${ }^{88}$ Nur wird der Staat sich hier eher auf die Rolle eines

883 So aber Murswiek, ebd.

884 Vgl. Gerbig, Grundrecht auf staatlichen Schutz, S. 63. Das BVerfG hat die Sozialadäquanz und das allgemeine Lebensrisiko in seinen Entscheidungen auch nicht als Ausschluss der Schutzpflichten begriffen, sondern erst unter Berücksichtigung der Forschungsfreiheit (BVerfG NVwZ 2010, Rn. 14 CERN) oder den Interessen an der Nutzung der Kernenergie (vgl. BVerfGE 49, 89 [144] - Kalkar I) betont. Insoweit ist es gerade verfehlt, wenn Krings, Grund und Grenzen staatlicher Schutzansprüche, S. 230 meint, dass der Gefahrenbegriff als Korrektiv und als „Zuordnungs- und Ausgleichskriterium zwischen Freiheit und staatlichem Schutzhandeln [Hervorh. d. Aut.] “ dient. Damit betont er gerade doch die Rechtsfolge des Interessensausgleichs. Insoweit unterscheidet sich der Gefahrenbegriff für exekutives Handeln entscheidend. Er ist Maßgabe für das rechtmäßige freiheitseinschränkende Handeln der Polizei- und Ordnungsbehörden, weil die Schwelle unterhalb der Gefahr meist keine Rechtfertigung hierfür bietet. Dies wird ja auch hier nur dann anders beurteilt, wenn unklar ist, ob überhaupt eine Gefahr besteht. Dem begegnet man dann aber mit einer Einschränkung auf Rechtsfolgenseite, nur sog. Gefahrenerforschungseingriffe vornehmen zu können. Zum Gefahrenverdacht und den Gefahrenerforschungseingriffen etwa Wei $\beta$, NVwZ 1997, 737 f. oder Pewestorf, in: ders./Söllner/ Tölle, Polizei- und Ordnungsrecht, $\mathbb{\$} 1 \mathrm{Rn} .17$. Die Gefahrenerforschung gehört also eher zum Bereich der Risikovorsorge.

885 Vgl. hierzu BVerfGE 96, 56 (64f.) - Vaterschaftsauskunft. Ausführlicher zu dem „Konfliktschlichtungsauftrag“ des Gesetzgebers Ruffert, Vorrang der Verfassung und Eigenständigkeit des Privatrechts, S. $203 \mathrm{ff}$. Auch daraus resultiert der umfangreiche Spielraum, den das BVerfG betont, etwa in BVerfGE 39, 1 (44) - Schwangerschaftsabbruch I; 77, 170 (214f.) - Lagerung chemischer Waffen; 85, 191 (212) - Nachtarbeitsverbot; 96, 56 (64) - Vaterschaftsauskunft; 142, 313 (337) - Zwangsbehandlung; BVerfG NJW 1996, 651 - Ozon; NJW 1998, 3264 (3265) - Waldsterben; NVwZ 2000, 309 (310) - Altlasten DDR-Uranbergbau; NVwZ 2009, 1489 Rn. 30 - Flughafen Leipzig/Halle; NVwZ 2011, 991 Rn. 38 - Fluglärmschutzgesetz; NJW 2016, 1716 Rn. 19 - Pflegenotstand.

886 Nichts anders liegt letztlich auch der Konzeption von präventivem Verbot mit Erlaubnisvorbehalt und repressivem Verbot mit Genehmigungsvorbehalt zugrunde. Im ersten Fall handelt es sich um weniger risikobehaftete Tätigkeiten, bei der im Einzelfall aber kollidierende Interessen gewichtig sein können, was eine staatliche Kontrolle zumindest erforderlich macht. Im zweiten Fall ist die Ausübung der Freiheitsrechte mit ganz erheblichen Risiken verbunden; die kollidierenden Interessen legitimieren ein grundsätzliches Verbot, von dem nur im Einzelfall eine Ausnahme zugelassen werden kann.

887 Das entspricht letztlich auch den verfassungsgerichtlichen Ansätzen, wenn es betont: „Ob, wann und mit welchem Inhalt sich eine solche Ausgestaltung von Verfassungs wegen gebietet, hängt von der Art, der Nähe und dem Ausmaß möglicher Gefahren, der Art und dem Rang des verfassungsrechtlich geschützten Rechtsguts sowie von den schon vorhandenen Regelungen ab“, BVerfGE 49, 89 (142) Kalkar I. Auch im Falle des Schwangerschaftsabbruchs wird das deutlich: „Die Schutzverpflichtung des Staates muß um so ernster genommen werden, je höher der Rang des in Frage stehenden Rechtsgutes innerhalb der Wertordnung des Grundgesetzes anzusetzen ist.“, BVerfGE 39, 1 (42) Schwangerschaftsabbruch I.

888 So im Wesentlichen auch Dietlein, Grundrechtliche Schutzpflichten, S. 108; Gerbig, Grundrecht auf staatlichen Schutz, S. 63 f. 
Überwachers bzw. Beobachters zurückziehen dürfen. ${ }^{889}$ Gerade in Bereichen, in denen wissenschaftlich vieles ungeklärt ist, kommt das also in Betracht, weil eingriffsintensive Maßnahmen kaum verhältnismäßig sein werden. ${ }^{890}$

Ein letztes Argument hiergegen mag zwar sein, dass das Fehlen einer bestimmten qualitativen Schwelle die Justiziabilität einer Verletzung der Schutzpflichten und damit der Grundrechte erleichtert. Denn wenn ein subjektives Recht auf staatlichen Schutz der potentiell bedrohten Grundrechtsträger besteht, ${ }^{891}$ wäre eine Verletzung der Grundrechte möglich und insoweit eine Verfassungsbeschwerde durchaus zulässig. Aber mit dem weiten Spielraum des Gesetzgebers geht auch eine deutliche Zurücknahme der gerichtlichen Kontrolldichte ${ }^{892}$ und damit auch erhöhte Substantiierungsanforderungen einher. ${ }^{893}$ Die Gefahr einer ausufernden Justiziabilität der Schutzpflicht besteht daher im Ergebnis gerade nicht.

Insgesamt sprechen also keine gewichtigen Gründe für eine Restriktion des Tatbestandes der Schutzpflicht. Auch der Bereich der Risikovorsorge ist tatbestandlich erfasst. ${ }^{894}$ Er führt allerdings zu einem größeren Spielraum auf Rechtsfolgenseite. Eine Regelungspflicht ergibt sich also nicht zwingend; die Begriffe Schutz- und Regelungspflicht sind gerade nicht kongruent. ${ }^{895}$

889 Vgl. Unruh, Zur Dogmatik der grundrechtlichen Schutzpflichten, S. $77 \mathrm{f}$

890 Vgl. hierzu etwa BVerfG NVwZ 2010, 702 Rn. 14 - CERN und auch BVerfGE 141, 220 Rn. 113 $B K A G$ : Dort, wo „nur relativ diffuse Anhaltspunkte für mögliche Gefahren bestehen, [d]ie Tatsachenlage [...] durch eine hohe Ambivalenz der Bedeutung einzelner Beobachtungen gekennzeichnet [ist, und] [d]ie Geschehnisse [...] in harmlosen Zusammenhängen verbleiben, aber auch den Beginn eines Vorgangs bilden [können], der in eine Gefahr mündet“, bestünde keine genügende Grundlage für eingriffsintensive Maßnahmen.

891 BVerfGE 125, 39 (78) - Adventssonntage Berlin: „Aus Grundrechten ist vielmehr auch eine Schutzpflicht des Staates für das geschützte Rechtsgut abzuleiten, deren Vernachlässigung von dem Betroffenen mit der Verfassungsbeschwerde geltend gemacht werden kann“, m.w.N. zur Judikatur. In der Literatur gibt es keine einheitliche Linie. Teilweise wurde ein subjektives Recht gänzlich abgelehnt, so etwa Steinberg, NJW 1984, 457 (461), teilweise bejaht, aber unter einen Evidenzvorbehalt gestellt, oder vollständig anerkannt, so Klein, NJW 1989, 1633 (1637); Isensee, in: ders./Kirchhof, HbStR IX, $\$ 191$ Rn. 194; Alexy, Theorie der Grundrechte, S. 414 f.; in diese Richtung auch Gerbig, Grundrecht auf staatlichen Schutz, S. 19; Calliess, in: Merten/Papier, HbGR II, $\mathbb{4} 4$ Rn. 24; Classen, Staatsrecht II, $\mathbb{} 6$ Rn. 13 ff.; Jarass, in: Badura/Dreier, FS-BVerfG, S. 35 (48f.); Stern, DÖV 2010, 241 (248) bejaht ein subjektives Recht, aber nur auf ein Tätigwerden, nicht auf eine bestimmte gesetzliche Maßnahme. Eine umfangreichere Darstellung dieses Problems findet sich bei Borowski, Grundrechte als Prinzipien, S. $373 \mathrm{ff}$.

892 Dazu oben, 1. Kapitel, B.II.aa.

893 Vgl. BVerfGE NVwZ 2010, 702 Rn. 15f. - CERN. Hierzu auch Iben, GreifRecht 2020, 36 (41) und Meyer, NJW 2020, 894 (899f.).

894 So spricht Richterin Haas in ihrem Sondervotum zur Rasterfahndung, BVerfGE 115, 320 (380) gerade auch von der gesetzgeberischen „Pflicht zur Risikovorsorge“. So auch mit Blick auf die CoronaPandemie, und damit allerdings wieder in Bezug auf das elementare Grundrecht des Art. 2 Abs. 2 GG, Schwarz, JA 2020, 321 (326). Daneben siehe auch Dietlein, Grundrechtliche Schutzpflichten, S. 107 ff.; Gerbig, Grundrecht auf staatlichen Schutz, S. 63 f.; Unruh, Zur Dogmatik der grundrechtlichen Schutzpflicht, S. $77 \mathrm{f}$.

895 Dieser Eindruck entsteht aber bei Krings, Grund und Grenzen staatlicher Schutzansprüche, S. 229 f. 
IV. Fazit: Schutzpflicht wegen einer zumindest abstrakten Gefährlichkeit

Durch den Einsatz von Meinungsrobotern bestehen vielseitige potentielle Risiken für die kommunikationsgrundrechtlichen Schutzgüter, deren Wirkung sich gegenseitig verstärken kann. In der Theorie konnten nicht nur wesentliche Wirkungen bereits aufgezeigt und herausgearbeitet werden, gerade auch die netzwerk- und nutzerseitigen Grundlagen für einen erfolgreichen Einsatz sind geschaffen. Allein die Frage nach den tatsächlichen Auswirkungen kann bislang nicht zufriedenstellend beantwortet werden, was aber auch daran liegt, dass es sich um einen komplexen Prozess handelt, bei dem einem einzelnen Faktor kein numerischer Einflusswert zugeschrieben werden kann. Vielmehr hängt dieser stark von individuellen Umständen ab. Wegen der grundlegenden Bedeutung der kommunikationsgrundrechtlichen Schutzgüter (in toto) ist es allerdings ausreichend, dass diese grundlegenden Gefahrenpotentiale aufgezeigt und plausibel dargelegt werden können. In Bezug auf diese „abstrakte Gefährlichkeit“ ist eine staatliche Schutzpflicht aktiviert. ${ }^{896}$ Davon ist strikt die Frage zu trennen, ob das den Staat zugleich auch zu konkreten (regulativen) Maßnahmen verpflichtet. Letzteres kann ohnehin nicht allein mit Blick auf die schutzrechtliche Dimension beantwortet werden. Vielmehr können sich hier - auch wegen der Ungewissheiten - anderweitig verfassungsrechtliche Grenzen ergeben, die nur einen vorsichtigen (regulierenden) Eingriff zulassen. ${ }^{897}$ Das gilt - nach der hier vertretenen Ansicht - selbst dann, wenn man tendenziell die Risiken nicht als zu einer Gefahr verdichtet bewertet und sie als noch im Rahmen der Risikovorsorge befindlich einordnet. Auch sie ist Bestandteil der Schutzpflicht, nur ergibt sich ein weitaus größerer Spielraum für den Gesetzgeber.

\section{B. Schutzpflichten im Verhältnis Staat-Nutzer-Netzwerkbetreiber}

Daneben können aber auch im Dreiecksverhältnis zwischen Netzwerkbetreiber, Nutzer und Staat Schutzpflichten aktiviert sein. Hierfür sind zwei Ansatzpunkte denkbar: die allgemeine Tätigkeit als Intermediär, die erst dafür sorgt, dass entsprechende Inhalte Nutzer erreichen, und die Bereitstellung einer für Manipulationen anfälligen bzw. eine Automatisierungsprozesse begünstigenden Infrastruktur.

896 In diese Richtung letztlich auch Brings-Wiesen, JuWiss-Blog Nr. 93/2016. Wohl auch Milker, ZUM 2017, 216 (220), wenn er lediglich davon spricht, dass keine konkreten Schutzpflichten eingreifen. Vgl. auch Hartl, a.a.O., S. 142 f. der bei neuen Entwicklungen in der Kommunikationsordnung per se von der Aktivierung ausgeht.

897 Hierauf weist letztlich auch Brinkmann, UFITA 2019, 364 (383) hin. 
Die Algorithmen der Betreiber sind primär dafür verantwortlich, welche Informationen überhaupt wahrgenommen werden. ${ }^{898}$ Dass den Algorithmen dabei bestimmte Verzerrungstendenzen und damit Risiken für den Meinungsbildungsprozess immanent sein oder ihnen gar bewusst implementiert werden können, ist vergleichsweise unbestritten. ${ }^{899}$ Wenn der Algorithmus aber manipulierte Inhalte priorisiert weiterleitet, so leistet auch der Betreiber einen (wesentlichen) kausalen Beitrag dafür, dass entsprechende Inhalte überhaupt die intendierte Wirkung entfalten können. Zudem sind die Nutzerprofile und der auf sie zurückgreifende Algorithmus auch dafür verantwortlich, dass eine segregierte Informationsvermittlung erfolgen kann. Durch Personalisierungsmaßnahmen ermittelt der Algorithmus einen Kreis potentiell interessierter Rezipienten. Insofern ist auch der Algorithmus dafür verantwortlich, wenn durch Meinungsroboter verursachte Trends Nutzer erreichen und so überhaupt erst Wirkung entfalten können. Zwar lässt sich nicht sicher feststellen, ob betreiberseitige Verzerrungen tatsächlich bestehen oder ob für eine einseitige Informiertheit nicht vielmehr die Nutzer selbst verantwortlich sind, ${ }^{900}$ und daher kein Unterschied zum einseitigen Informationskonsum in der analogen Welt besteht. ${ }^{901}$ Insoweit lässt sich trefflich darüber streiten, ob bereits eine abstrakte Gefahr vorliegt oder doch nur ein gewisses Risiko. Darauf kommt es - wie soeben aufgezeigt - aber nicht an. In jedem Fall ist eine staatliche Verantwortung - und nicht nur die staatliche Prärogative 902 zumindest im Bereich der Risikovorsorge begründet. ${ }^{903}$ Davon ist erneut die

898 Lischka, AfP 2018, 388 (391) vergleicht die Wirkung der Algorithmen letztlich mit den Auswahlentscheidungen durch die redaktionelle Auswahl bei herkömmlichen Medien. Illustrativ im Kontext der Meinungsroboter Oehmer/Pedrazzi, UFITA 2020, 7 (15).

899 Siehe hierzu u.a. Bauer, WRP 2020, $171 \mathrm{f} . ;$ Martini, Blackbox Algorithmus, S. 99 ff.; Holznagel, NordÖR 2011, 205 (209ff.); Schwartmann/Hermann/Mühlenbeck, MMR 2019, 498 ff.; Paall Heidtke, ZUM 2020, 230 (231); Paal/Hennemann, ZRP 2017, 76f.; Schmid/Braam/Mischke, MMR 2020, 19 f.; Paal, Intermediäre: Regulierung und Vielfaltssicherung, S. 10 ff.; ders., ZRP 2015, 34 ff.; ders., MMR 2018, 567 ff.; Drexl, ZUM 2017, 529 ff.; Heidtke, Meinungsbildung und Medienintermediäre, S. 275.

900 Kritisch zu den Narrativen der Filterblase und Echokammer und den daraus teilweise folgenden Forderungen nach Regulierung deshalb u.a. Cornils, AfP 2018, 377 (381ff.); Liesem, ZUM 2020, 377 (378f.); Ingold, MMR 2020, 82 (83 ff.). Mit Blick auf die Rolle der Nutzer siehe daneben vor allem auch die Verweise in Fn. 338.

901 In diese Richtung etwa Pfengler, GreifRecht 2020, 1 (10) und Gräfe, PinG 2019, 5 (10), der das als „ein Problem demokratischer Kultur" bezeichnet.

902 Insbesondere Heidtke, Meinungsbildung und Medienintermediäre, S. 210 spricht hier (noch) von einer bloßen „Obliegenheit“. Unklarer bei Cornils, AfP 2018, 377 (383), der zwar einerseits die Risikoprärogative betont, andererseits dies aber auch als Argument dafür nimmt, dass gerade keine spezifischen Regelungen oder Vorkehrungen geboten sind.

903 So etwa auch Paal, MMR 2018, 567 (569) mit Blick auf Intermediäre. Und mit Blick auf Suchmaschinen - wenn auch unter dem Blickwinkel des Regelungsauftrages - etwa Dörr/Natt, ZUM 2014, 829 (841), die hier von einer abstrakt-generellen Gefahr für den objektiv-rechtliche Dimension der Meinungsfreiheit und einer Gewährleistungspflicht sprechen. 
Frage, ob hieraus auch spezifische Regulierungspflichten bereits folgen können, zu trennen. ${ }^{904}$

Wenn es demgegenüber um die Bereitstellung einer manipulierbaren Infrastruktur geht - etwa mit Blick auf manipulierbare algorithmische Prozesse, ${ }^{905}$ die faktische Duldung automatisierter Prozesse oder die prinzipielle Bereitstellung entsprechender Instrumente hierfür,$-{ }^{906}$ ist die Begründung schwieriger. Hierbei handelt es sich immerhin um eine wertneutrale und aus Sicht einer partizipativen Öffentlichkeit zu begrüßenden Handlung. ${ }^{907}$ Risiken entstehen erst dann, wenn eine manipulative Absicht des jeweiligen Nutzers hinzutritt. ${ }^{908}$ Dass die Interaktionsstrukturen ideale Bedingungen für Meinungsroboter bieten und dass deshalb darüber nachzudenken ist, ob den Betreibern nicht bestimmte Vorgaben gemacht werden können, die zum Schutz vor den Meinungsrobotern angezeigt sind und entsprechende Manipulationen verhindern sollen, ist im Kern zutreffend. ${ }^{909}$ Weder aus der Bereitstellung noch aus der grundsätzlichen Duldung automatisierter Prozesse ergeben sich indes spezifische Gefahren für grundrechtliche Güter, die eine Schutzpflicht auslösen. ${ }^{910}$ Anknüpfungspunkt bleibt insoweit allein die Tätigkeit bei der Informationsvermittlung und damit die spezifischen Gefahren, die einerseits durch Personalisierungsprozesse und andererseits durch die algorithmenbasierte Orientierung an statistischen Daten bei der Informationsweiter-

904 Insbesondere die durchaus berechtigten unternehmerischen Interessen an einer personalisierten Weiterleitung sind als abwehrrechtliche Grenze zu berücksichtigen, so auch Hindelang, Freiheit und Kommunikation, S. 205. Und auch die Nutzer haben ein Interesse an einer gewissen Vorselektion, was letztlich auch dem Vorgehen herkömmlicher Medien entspricht. Und zuletzt bestehen auch spezifische Gefahren, wenn der Staat regulierend eingreift, weil er selbst Verzerrungen schaffen kann, was mit Blick auf die Kommunikationsgrundrechte des Art. 5 Abs. 1 GG nicht weniger problematisch wäre, so hinsichtlich strenger Neutralitätsvorgaben für Medienintermediäre etwa Liesem, AfP 2020, 277 (281); siehe auch Cornils, ZUM 2019, 89 (103).

905 Im Kontext von sog. Trollen etwa Lee/Kerr, First Monday Vol. 25 (2020) Nr. 6.

906 Vgl. Oehmer/Pedrazzi, UFITA 2020, 7 (9).

907 Vgl. hierzu BGH GRUR 2013, 751 - Autocomplete, der einerseits das Interesse der Nutzer betonte (Rn. 22) und zeitgleich explizit meinte, dass die Entwicklung und Verwendung der entsprechenden Software als Ergebnis der Ausübung grundrechtlicher Freiheiten nicht vorwerfbar sei (Rn. 26). Daneben vgl. auch OLG Braunschweig, GRUR-RR 2014, 385 (389).

908 Hegelich, Die Politische Meinung 543 (2017), S. 32 (34) bringt das ganz treffend auf den Punkt: „Es ist also nicht die Technik, die die Probleme verursacht, sondern es sind die Interessen, denen diese Technik dienstbar gemacht wird. " Im Kontext der strafrechtlichen Verantwortlichkeit für Beleidigungen weist auch Reinbacher, Neue Kriminalpolitik 2020, 186 (189) auf den „Missbrauch“ des Nutzers, durch den erst die Ehrverletzung erfolgt. Dieser Gedanke liegt letztlich auch den Entscheidungen des BGH zugrunde, wenn es um die Störerhaftung von Intermediären geht. Im Falle von Google betonte der BGH etwa, dass allein das Entwickeln der Software nicht vorzuwerfen sei, weil es hierbei um eine geschützte Tätigkeit ginge, die zudem nicht auf Rechtsverletzungen ausgerichtet sei. „Nur durch das Hinzutreten eines bestimmten Nutzerverhaltens können ehrverletzende Begriffsverbindungen entstehen.", BGH GRUR 2013, 751 Rn. 26 - Autocomplete.

909 Kaerlein, in: Hug/Pallaver, S. 17 (19) begreift deshalb Bots nur als „Symptom der Funktionsweise des Social Webs“. Sie seien besonders soziale Bewohner der Plattformen, S. $24 \mathrm{f}$.

910 Vgl. hierzu mit Blick auf eine Garantenstellung der Anbieter für Beleidigungen Reinlicher, Neue Kriminalpolitik 2020, 186 (189). 
leitung, ${ }^{911}$ wo der Fokus „stärker auf Steuerungsmechanismen der Aufmerksamkeitsökonomie [liegt] “912, entstehen. ${ }^{913}$

911 Vgl. hierzu Lischka, AfP 2018, 388 (390).

912 So Martini, Blackbox Algorithmus, S. 100.

913 Spezifisch zu sozialen Netzwerken etwa Pille, Meinungsmacht sozialer Netzwerke, S. 322 ff. Zu den Gefährdungen durch Medienintermediären Heidtke, Meinungsbildung und Medienintermediäre, S. $131 \mathrm{ff}$. Andernorts wird die Frage eher unter dem Gesichtspunkt des Gestaltungsauftrags des Gesetzgebers für den Rundfunk diskutiert und die entsprechenden Algorithmen als Rundfunk im Sinne des Art. 5 Abs. 1 S. 2 Var. 3 GG angesehen, siehe hierzu u.a. Kaiser/Reiling, in: Unger/v. UngernSternberg, Demokratie und künstliche Intelligenz, S. 85 (97 ff.). 


\section{Kapitel: Rechtsfolgen der Schutzpflicht - Schutzbedarf und Handlungsoptionen des Staates}

Auch wenn der Staat die Verantwortung trägt, vor potentiellen Gefahren der Meinungsroboter zu schützen, so folgt daraus im Ausgangspunkt nicht zwingend eine konkrete Handlungsanweisung oder gar eine Legitimation eingriffsintensiver Maßnahmen ${ }^{914}$. Was konkret aus ihr folgt, ist maßgeblich von den zwei grundrechtlichen Polen und dem dadurch vorgezeichneten Handlungskorridor abhängig: die Vorgaben des Übermaßverbotes auf der einen (A.) und den speziellen Vorgaben der Schutzpflichten (B.), insbesondere des Untermaßverbotes (C.), auf der anderen Seite.

\section{A. Das Übermaßverbot und kollidierende Grundrechte als relative Grenze der Schutzverantwortung}

Die Schutzverantwortung des Staates wird nach oben durch die kollidierenden Grundrechte und damit durch abwehrrechtliche Aspekte relativ begrenzt. Relativ deshalb, weil auch die Freiheitsgrundrechte nicht absolute Geltung beanspruchen, sondern insbesondere durch Gesetze, die dem grundrechtlichen Schutz dienen und damit die verfassungsimmanenten Schranken konkretisieren, eingeschränkt werden können. Insoweit genießt weder die Freiheitssphäre noch die Schutzsphäre per se Vorrang. Der Grenzverlauf hängt somit vom Einzelfall ab und ergibt sich erst durch die Berücksichtigung des Übermaßverbotes und innerhalb dessen zumeist erst im Rahmen einer umfangreichen Abwägung der kollidierenden Belange (zu eben dieser unten, C.II.3).915 Der Schutzverantwortung des Staates beim Einsatz von Meinungsrobotern stehen dabei potentiell drei Freiheitssphären gegenüber: die der Verwender, die der Netzwerkbetreiber und auch die der Nutzer selbst, die durch staatliche Intervention auch abwehrrechtlich tangiert sein kann.

\section{Der grundrechtliche Schutz der Meinungsroboter unter der Meinungsfreiheit}

Dass die Betätigung der Verwender zumindest den (subsidiären) Schutz der allgemeinen Handlungsfreiheit des Art. 2 Abs. 1 GG genießt, dürfte unter Berücksichtigung der Elfes-Entscheidung kaum streitig sein. ${ }^{916}$ Mit der Meinungsfreiheit des

914 Vgl. Hartl, Suchmaschinen, Algorithmen, Meinungsmacht, S. 143.

915 Vgl. hierzu etwa BVerfGE 115, 320 (358 f.) - Rasterfahndung II.

916 BVerfGE 6, 32 (36 ff.) - ElfeS. Zu der Diskussion um die Bedeutung der „Entfaltung der Persönlichkeit“ im Schutzbereich des Art. 2 Abs. 1 GG und dem - zutreffenden - Verständnis als Auffanggrund- 
Art. 5 Abs. 1 S. 1, 1. Var. 2 GG könnte aber ein spezifisches Freiheitsrecht einschlägig sein. Zwangsläufig müssen hierbei aber Bedenken geweckt werden, wenn die Meinungsroboter einerseits darauf gerichtet sind, wesentliche Voraussetzungen eines offenen Kommunikationsprozesses zu stören, die Verwender andererseits aber einen für diesen zentralen grundrechtlichen Schutz genießen sollen. Schützt die Meinungsfreiheit also auch den Urheber der Meinungsroboter und die von ihnen „produzierten“ Inhalte, sodass potentielle staatliche Eingriffe den hohen Rechtfertigungsanforderungen des Art. 5 Abs. 2 GG genügen müssen?

\section{Automatisierte Äußerungen als geschützte Meinungsäußerung?}

Im Ausgangspunkt lässt sich schon überlegen, ob für automatisiert und teilautonom veröffentlichten Content überhaupt die Meinungsfreiheit einschlägig sein kann. ${ }^{917}$ Das ist vor allem eine Zurechnungsfrage: Der jeweilige Verwender von Meinungsrobotern mag zwar Grundrechtsträger der Meinungsfreiheit sein. ${ }^{918}$ Art. 5 Abs. 1 S. 1, 1. HS. GG gewährleistet aber nur, seine Meinung zu äußern. Während Hufen eine solche Zurechnung im Falle der Meinungsroboter ablehnt, ${ }^{919}$ lassen sich - vor allem US-amerikanisch geprägte - Überlegungen ausfindig machen, die eine Extension der Meinungsfreiheit nicht nur auf Algorithmen selbst, ${ }^{920}$ sondern aus funktionalen Argumenten auch auf die „Speech“ künstlich intelligenter Systeme annehmen wollen. ${ }^{921}$ Für das US-amerikanische Verfas-

recht, siehe statt vieler Di Fabio, in: Maunz/Dürig, GG, Art. 2 Abs. 1 Rn. 12 ff. und Dreier, in: ders., GG, Art. 2 Rn. 26 f.

917 Diese Frage wirft auch Mehrens, in: Steinbrecher/Rager, Wenn Maschinen Meinung machen, S. 20 (33) auf.

918 So auch Semizoğlu, in: Hetmank/Rechenberg, Kommunikation, Kreation und Innovation, S. 79 (84). Ähnlich auch Milker, InTeR 2017, 199 (201). Im Kontext der US-amerikanischen Debatte im Lichte der Freedom of Speech siehe etwa Hines, Houston Law Review 57 (2019), 405 (418).

919 Hufen, Staatsrecht II, $\mathbb{2} 25$ Rn. 52.

920 Vgl. hierzu die US-amerikanische Rspr. in Search King, Inc. v. Google Tech. Inc., Nr. CIV-02-1457M, S. 9 - (PageRank-Algorithmus erfasst vom First Amendement); Bernstein v. United States Department of State, 922 F. Supp. 1426 (1996) (1436) - (Computercodes als geschützte „Speech“). Insgesamt ist die Frage aber - angesichts der damit verbundenen, teilweise als nicht tragbar bezeichneten, Extension - nicht unumstritten. Ausführlicher zur Problematik u.a. Kerr, Washington and Lee Law Review, Vol. 57 (2000), 1287 (1290 ff.); Benjamin, Algorithms and Speech, Pennsylvania Law Review 161 (2013), 1445 (1447f.) m.w.N. in Fn. 5-7. Das Unternehmen Apple beruft sich auf den ersten Verfassungszusatz der Verfassung der Vereinigten Staaten von Amerika von 1791, der „Freedom of Speech“, da es vom FBI dazu gezwungen wird eine bestimmte Version des Betriebssystems iOS bereit zu stellen, um so dem FBI den ungeschützten Zugriff auf das Mobiltelefon des Attentäters von San Bernadino zu ermöglichen. Apple sieht darin ein Verstoß gegen die (negative) Freiheit der Rede, weil ihnen diese "Aussage“ vordiktiert wird und sie der eigenen Haltung widerspricht, dem Kunden eine absolut sichere Nutzung ihrer Geräte zu ermöglichen. Dabei sei die Programmiersprache gleich zu behandeln mit den anderen Sprachen, da auch durch sie Befehle, Meinungen und Aussagen wiedergegeben werden können. Hierzu ausführlicher Cohn, Code is Free Speech, Time Magazine und Khamooshi, Breaking Down Apple's iPhone Fight with the U.S. Government, New York Times.

921 In diese Richtung etwa der BGH hinsichtlich automatisiert generierter Suchvorschläge BGH, GRUR 2013, 751 Rn. 22 - Autocomplete, allerdings ohne hierauf näher einzugehen. Siehe auch zu sog. Journalismusrobotern Habel, Roboterjournalismus, S. 133 ff. Daneben auch Massaro/Norton, Nw. 
sungsrecht mag dies aufgrund des eher sachlich ausgerichteten Schutzes des ersten Verfassungszusatzes überzeugend sein. ${ }^{922}$ Gilt dies aber auch für die Zurechnung automataisierter Äußerungs- und Interaktionsprozesse nach dem deutschen Verständnis? Oder führt die Zwischenschaltung eines Programms hier zu einer „Zäsur“, durch die die Zurechnung unterbrochen wird?

\section{a. Zurechnung aufgrund der Verantwortungs- und Herrschaftssphäre als einfach-rechtlicher Grundsatz}

Zurechnungsprobleme und Verantwortungsfragen beim Einsatz von Automatisierungsprozessen sind der Rechtsordnung mit zunehmender Digitalisierung und Technologisierung nicht mehr fremd. Auch wenn diese Entwicklung nicht zwingend Bedeutung für die Auslegung der ranghöheren Grundrechte haben mag, so wäre es doch widersprüchlich, wenn einerseits (nachteilige) Folgen von Automatisierungsprozessen einer Person zugerechnet werden, eine grundrechtliche Zurechnung aber nicht erfolgt, vor allem dann, wenn Begründungsansätze und Interessenlagen vergleichbar wären.

Einfach-rechtliche Zurechnungs- und Verantwortungsfragen werden vor allem anhand der Kriterien der Beherrschbarkeit und der Risikosphäre der potentiell Verantwortlichen beantwortet. Auf eine einfache Regel heruntergebrochen gilt: Derjenige, der sich Vorteile eines automatisierten Systems zu eigen macht, muss auch die daraus resultierenden Nachteile tragen. ${ }^{923}$ So wird der Einsatz automatisierter Willenserklärungen im E-Commerce wie die Erklärung eines Boten behandelt. ${ }^{924}$ Dem Verwender wird die jeweilige Erklärung also als eigene, quasi antizipierte Willenserklärung zugerechnet, ${ }^{925}$ weil er die Kriterien, wann eine entsprechende Erklärung automatisiert abgegeben werden soll, abstrakt festgelegt hat. ${ }^{926}$ Und dort, wo die Abgabe (teil-)autonom erfolgt, wird jedenfalls im Ergebnis auch eine Zurechnung angenommen, weil dem Verwender als Verantwortli-

U. L. Rev. 110, 1169 ff.; Massaro/Norton/Kaminski, Minn. Lev 101 (2016), 2481 ff., die damit argumentieren, dass der Ansatz für den grundrechtlichen Schutz - auch nach der Rechtsprechung des US Supreme Court - eher inhaltlich ausgerichtet ist und eher auf den Wert für die Zuhörer abstellt. Aus deutscher Sicht siehe etwa auch Lewke, InTeR 2017, 207 (210 f.) und - wenn auch i.E. ablehnend Krupar, in: Taeger, Recht 4.0, S. 275 (281f.).

922 Vgl. hierzu Massaro/Norton/Kaminski, Minnes. L. Rev. 101 (2017), 2481 (2486 ff.).

923 Vgl. BGH GRUR 2007, 890 Rn. 36; BGH, GRUR 2015, 1129 Rn. 42. Dieses Prinzip kommt letztlich u.a. in $\mathbb{S} \mathbb{S} 31,166$ Abs. 1, 2, 278 S. 1, 831 Abs. 1 S. 1 BGB zum Ausdruck.

924 Rhiem, ITRB 2014, 113f; vgl. BGH NJW 2005, 976 (977) der $\mathbb{} 120$ BGB hier (entsprechend) anwendet.

925 So schon Brehm, FS-Niederländer, S. 234; und - trotz ursprünglich anderer Ansichten, z.B. Clemens NJW 1985, 1998 (2001) - mittlerweile auch allgemein anerkannt, vgl. Spindler, in ders./Schuster, Recht elektronischer Medien, Vorbem. $\mathbb{S} 116$ ff. BGB Rn. 5; Glossner, in: Leupold/dies., AnwHb ITRecht, Teil 2 Rn. 15; vgl. hierzu auch Specht/Herold, MMR 2018, 40 (41).

926 Glossner, in: Leupold/dies., AnwHb IT-Recht, Teil 2 Rn. 15; Paulus/Matzke, ZfPW 2018, 431 (440 ff.). 
chem das von ihm geschaffene Risiko zuzurechnen ist. ${ }^{927}$ Streitig ist allein, ob es sich um einen Unterfall der automatisierten Erklärung handelt.

Probleme ergeben sich auch im Bereich der zivilrechtlichen, verschuldensabhängigen Haftung. Bei der vertraglichen Haftung ist $\$ 278 \mathrm{BGB}$ nicht direkt anwendbar. Allein eine analoge Anwendung käme in Betracht, sofern man eine „funktionale Verschuldensäquivalenz“ ausreichen ließe. ${ }^{928}$ Die h.M. sieht dafür jedoch keinen Raum, ${ }^{929}$ sondern löst dies über allgemeine Sorgfaltspflichtanforderungen. ${ }^{930}$ Vergleichbares gilt im deliktischen Bereich. Hier kommt eine Haftung des Verwenders nach $\mathbb{\int} 823$ Abs. 1 S. 1 BGB dort in Betracht, wo er Verkehrssicherungs- oder Überwachungspflichten verletzt. ${ }^{931}$ Dem liegt der „allgemeine Rechtsgrundsatz [zugrunde] [...], dass jeder, der in seinem Verantwortungsbereich eine Gefahrenquelle schafft oder andauern lässt, die ihm zumutbaren Maßnahmen und Vorkehrungen treffen muss, die zur Abwendung der daraus Dritten drohenden Gefahren notwendig sind. “932

Im Falle von automatisiert erstellten Suchvorschlägen hat der BGH Google gar als mittelbaren Störer i.S.d. $\$ 1004$ BGB angesehen. ${ }^{933}$ Nach dem BGH ist eine Äußerung, bei der ein Algorithmus dem Suchenden bestimmte Suchvorschläge unterbreitet, der diese anhand verschiedenster Informationen ermittelt, ${ }^{934}$ dem Verwender zurechenbar. ${ }^{935}$ Begründet hat dies der BGH damit, dass Google selbst durch Auswertung des Nutzerverhaltens die Suchvorschläge generiert und für

927 Hierauf hinweisend Paulus/Matzke, ZfPW 2018, 431 (445); vgl. hierzu auch Heuer-James/Chibanguza/Stücker, BB 2018, 2818 (2821, 2822), die darauf hinweisen, dass ansonsten rechtsfreie Räume entstehen, in denen sich der Verwender von den (Haftungs-)Folgen freizeichnen könne (S. 2822).

928 Zech, ZfPW 2019, 198 (211f.), weil die Situation beim Einsatz eines Erfüllungsgehilfen und eines diesen ersetzenden Automatisierungsprozesses vergleichbar bleibe.

929 So u.a. Schaub, JZ 2017, 342 (343); Günther/Böglmüller, BB 2017, 53 (55); Caspers, in: Staudinger, \278 Rn. 5; Grundmann, in: MüKo BGB, Bd. 2, \$278 Rn. 46; Medicus/Lorenz, Schuldrecht AT, Rn. 386.

930 Grundmann, in: MüKo BGB, Bd. 2, \278 Rn. 46; vgl. Müller-Hengstenberg/Kirn, MMR 2014, 307 (311).

931 Schaub, JZ 2017, 342 (344); Zech, ZfPW 2019, 198 (206 f.) und zu diesen Verkehrssicherungspflichten, a.a.O., S. 210 f. Speziell zu den Problemen bei anpassungsfähigen Systemen Etzkorn, MMR 2020, $360 \mathrm{ff}$. De lege ferenda könnte auch ein eigener Gefährdungshaftungstatbestand geschaffen oder eine versicherungsrechtliche Lösung eingeführt werden. Diese Möglichkeiten andeutend Schaub, JZ 2017, 342 (345 f.). Zur Lösung durch ein Haftpflichtversicherungssystem für autonome Systeme, sodass zumindest für Geschädigte eine Kompensation sichergestellt wäre, etwa Günther/Böglmüller, BB 2017, 53 (55). Und zum Ansatz einer Gefährdungshaftung siehe Borges, NJW 2018, 977 (982 f.) und zur Weiterentwicklung des Produkthaftungsrechts Seehafer/Kohler, EuZW 2020, $213 \mathrm{ff}$.

932 So BGH GRUR 2007, 890 Rn. 36. Siehe auch BGH, GRUR 2015, 1129 Rn. 42.

933 Ausreichend ist nämlich, dass man „in irgendeiner Weise willentlich und adäquat kausal zur Beeinträchtigung des Rechtsguts beiträgt.“, BGH GRUR 2012, 311 Rn. 21 - Blog-Eintrag; a.a.O., 751 (753) - RSS-Feed; NJW 2018, 2324 Rn. 31; vgl. auch BGH GRUR 2013, 751 Rn. 24 - Autocomplete.

934 BGH, GRUR 2013, 751 Rn. 28 f. - Autocomplete; ausführlicher begründet das der BGH in NJW 2018, 2324 Rn. 34 - Prüfpflichten eines Suchmaschinenbetreibers, wo die elementare Bedeutung von Suchmaschinen betont wird. Kritisch zur Autocomplete Entscheidung etwa Spindler/Volkmann, in: Spindler/Schuster, Elektronische Medien, $\$ 1004$ BGB Rn. 16.

935 So BGH, GRUR 2013, 751 Rn. 17 - Autocomplete. 
Nutzer zum Abruf unmittelbar bereit hält. ${ }^{936}$ Insoweit stellt er im Wesentlichen auch auf die Risiko- und Herrschaftssphäre des Unternehmens ab. Die Haftung wurde lediglich unter dem Gesichtspunkt der Zumutbarkeit eingeschränkt. ${ }^{937}$

Im Strafrecht ${ }^{938}$ reicht nach der Kausalitätslehre bei vorsätzlichen Erfolgsdelikten ${ }^{939}$ schon die Inbetriebnahme eines autonomen Systems aus, um eine „Verantwortung“ zu begründen, sofern sie conditio sine qua non für den tatbestandlichen Erfolg ist. ${ }^{940}$ Eine Zäsur im Sinne eines Dazwischentretens durch das autonome Programm wird regelmäßig nicht erfolgen. Die maßgebliche Kontrolle und Befehlsgewalt über das System oder die Maschine hat der Verwender. Denn die genauen Umstände und Ausführungsarten der Handlungen werden dem System vorgegeben. Auch wenn derjenige die (Tat-)Handlung selbst nicht ausführt, fungiert das System als „Tatwerkzeug“941 im konkreten Moment. Weil aber zumindest der bedingte Vorsatz regelmäßig fehlen wird, dürfte es überwiegend eher um Fahrlässigkeitsdelikte gehen. Hierbei kommt allerdings dann wieder der Risikosphärengedanke zum Tragen, der spezifische Anforderungen an die Sorgfalt des Verwenders stellt. ${ }^{942}$ Auch speziell im Bereich der Äußerungsdelikte hält der Verwender durch die konkreten Anweisungen an das Programm die Herrschaft darüber in den Händen, unter welchen Umständen welcher Inhalt an welchem Ort veröffentlicht werden soll. ${ }^{943}$ In gewisser Weise ist das also sogar vergleichbar zu der Lage bei einer automatisierten Willenserklärung. Auch hier handelt es sich faktisch um eine antizipierte Äußerung.

\section{b. Bedeutung für die Frage einer grundrechtlichen Zurechnung der Äußerungen}

Wenn aber im einfachen Recht (nachteilige) Folgen an den Einsatz automatisierter Systeme geknüpft werden und insbesondere bei automatisierten Äußerungen eine Zurechnung vorgenommen wird, bestünde ein Wertungswiderspruch, wenn man dies im grundrechtlichen Bereich anders sähe. ${ }^{944}$ Denn das Botprogramm

936 BGH, ebd.

937 BGH GRUR 2012, 311 Rn. 22 ff. - Blog-Eintrag; NJW 2018, 2324 Rn. 36 - Prüfpflichten eines Suchmaschinenbetreibers.

938 Umfassend zu intelligenten Agenten und dem Strafrecht Gleß/Weigend, ZStW 2014, 561.

939 Bei Fahrlässigkeitsdelikten stellt sich die Frage nicht in gleichem Maße, denn Anknüpfungspunkt ist hier ein Sorgfaltswidrigkeitsverstoß. Wenn zwischen ihm und dem Erfolg ein Pflichtwidrigkeitszusammenhang besteht, führt das schon zur Strafbarkeit. Hierbei könnte lediglich zwischen sozialadäquaten und sozialinadäquaten Verstößen zu unterscheiden sein, so Markwalder/Simmler, AJP 2017, 171 (175 ff.) aus schweizerischer Perspektive.

940 Vgl. hierzu Markwalder/Simmler, AJP 2017, 171 (179); Gleß/Weigend, ZStW 2014, 561 (579f.).

941 So für Social Bots Volkmann, MMR 2018, 58 (60). Vgl. hierzu auch Markwalder/Simmler, AJP 2017, 171 (179).

942 Vgl. zum autonomen Fahren etwa Sander/Hollering, NStZ 2017, 193 (200 f.) oder Schuster, DAR 2019, 6 (8). Zu diesen Pflichten vgl. auch Bilski/Schmid, NJOZ 2019, 657 (660 f.).

943 Umfassend im Rahmen von $\$ 130$ StGB Volkmann, MMR 2018, 58 (60 ff.).

944 So aber scheinbar Hufen, Staatsrecht II, $\$ 25$ Rn. 52: „Noch schwieriger dürfte es sein, die für den demokratischen Diskurs nicht ungefährlich polarisierende Wirkung von [...] ,Likes ‘ und ,Dislikes“ 
und die von ihm getroffenen Entscheidungen sind auch hier maßgebliches Resultat menschlichen Denkens und Handelns ${ }^{945}$ und die Kommunikate damit unmittelbarer Ausdruck der Ansichten des jeweiligen Grundrechtsträgers. ${ }^{946}$ Der Urheber formuliert in abstrakten Anweisungen die zu veröffentlichenden Inhalte und die Umstände, unter denen diese publiziert werden; ${ }^{947}$ sie beinhalten - wie bei der automatisierten Willenserklärung - eine „antizipierte“ Äußerung ${ }^{948}$. Das Programm ist auch hier an die Vorgaben gebunden. ${ }^{949}$ Die wesentliche Kontrolle über die Vorgänge verbleibt beim Verwender ${ }^{950}$, selbst wenn er zum Zeitpunkt der Äußerung nicht anwesend ist und von der finalen Äußerung nur eine ungefähre Vorstellung hat. ${ }^{951}$

Daneben lassen sich aber auch aus der Meinungsfreiheit selbst gewonnene Argumente vortragen, die eine Zurechnung legitimieren: Die Meinungsfreiheit ist technikneutral; ${ }^{952}$ ihr Schutz greift unabhängig von der Äußerungsmodalität, sodass auch neuartige Kommunikationswege erfasst sind. ${ }^{953}$ Der Äußernde kann frei entscheiden, wann er seine Meinung wie und vor allem wo kundgibt, um die von ihm intendierte Wirkung zu erreichen. ${ }^{954}$ Entscheidet sich dieser, automa-

mit verfassungskonformen Mitteln zu bekämpfen. Diese fallen, jedenfalls wenn sie wahrheitsgemäß und von Personen und nicht von Robotern stammen, in den Schutzbereich der Meinungsfreiheit [Hervorhebung d Verf.]“.

945 Herberger, NJW 2018, 2825 (2827) bezeichnet Algorithmen als „Gebilde des menschlichen Geistes“.

946 Lewke, InTeR 2017, 207 (211). Ähnlich für Suchmaschinenergebnisse Milstein/Lippold, NVwZ 2013, 182 (185).

947 Lewke, InTeR 2017, 207 (210 f.); Milker, InTeR 2017, 199 (201); Semizoğlu, in: Hetmank/Rechenberg, Kommunikation, Kreation und Innovation, S. 79 (87). Aus US-amerikanischer Sicht etwa $\mathrm{Hi}$ nes, Houston Law Review 57 (2019), 405 (419).

948 So auch Hostacna, AL 2018, 1 (4); Lewke, InTeR 2017, 207 (211); Milker, ZUM 2017, 216 (217); Liesem, in: Litschka/Krainer, Der Mensch im digitalen Zeitalter, S. 183 (189) und Semizoğlu, in: Hetmank/Rechenberg, Kommunikation, Kreation und Innovation, S. 79 (87). Siehe auch Krüper, in: Unger/v. Ungern-Sternberg, Demokratie und künstliche Intelligenz, S. 67 (74), der von einem ,generalisierten Willen “ spricht. Starck/Paulus, in: v. Mangoldt/Klein/Starck, GG, Art. 5 Rn. 87, gehen etwa bei den algorithmisch erstellten Suchmaschinenergebnissen wegen der ,entsprechenden Programmierung " von einem Werturteil aus, m.w.N. Nicht überzeugend ist deshalb auch der Einwand, dass kein manuelles Erstellen und kein willentlicher Handlungsentschluss im Zeitpunkt der Äußerung vorliegen, vgl. etwa Stadler, Haftung für Informationen im Internet, S. 293 hinsichtlich des Setzens von Links durch Suchmaschinen.

949 Nach Lewke, InTeR 2017, 207 (211) steuert der Computer lediglich seine Rechnungsleistung bei.

950 Für Suchmaschinen maßgeblich auf die Kontroll- und Eingriffsmöglichkeiten des Urhebers abstellend Milstein/Lippold, NVwZ 2013, 182 (185) und mit Blick auf den News Feed Pille, Meinungsmacht sozialer Netzwerke, S. 178.

951 Vgl. Lewke, InTeR 2017, 207 (211). Deshalb ist die Bezeichnung als „Sprachrohr“ von Steinbach, ZRP 2017, 101 (102) durchaus zutreffend.

952 Steinbach, ZRP 2017, 101 (102); ebenso Dankert/Dreyer, K\&R 2017, 73 (74); vgl. auch Milker, InTeR 2017, 199 (201). Semizoğlu, in: Hetmank/Rechenberg, Kommunikation, Kreation und Innovation, S. 79 (88) nennt das „entwicklungsoffen“ und Grabenwarter, in: Maunz/Dürig, GG, Art. 5 Rn. 93 bezeichnet es als Medienneutralität.

953 So meint etwa Semizoğlu, in: Hetmank/Rechenberg, Kommunikation, Kreation und Innovation, S. 79 (87), dass eine Einengung nicht mehr „zeitgerecht“ und die Meinungsfreiheit entwicklungsoffen sei, S. 88. Mit Blick auf die US-amerikanische Freedom of Speech etwa Lamo/Calo, UCLA Law Review 66 (2019), 988 (1004).

954 Vgl. BVerfGE 93, 266 (289) - Soldaten sind Mörder; Grabenwarter, in: Maunz/Dürig, GG, Art. 5 Rn. 84; Schemmer, in: Epping/Hillgruber, Beck-OK GG, Art. 5 Rn. 9. 
tisiert Äußerungen zu verschicken, die durch die programmatischen Vorgaben seinem Willen entsprechen, ist das also zunächst seine Entscheidung. ${ }^{955}$ Es dürfte folglich keinen Unterschied machen, ob der Äußernde selbst fortwährend seine Äußerung niederschreibt und sodann kundgibt oder dies aus Effizienzgründen einem Programm überlässt. ${ }^{956}$ Die zeitliche Zäsur zwischen Herstellung des Programms und Verbreitung der Äußerung findet sich in vergleichbarer Weise etwa auch bei Flugblättern zwischen Herstellung und eigentlicher Verbreitung. ${ }^{957}$ Sähe man dies anders, käme man in Zeiten fortschreitender Technologisierung zu unlösbaren Problemen. Automatisierungsprozesse sind in Zeiten des Internets notwendig, um auf die sich sekündlich ändernde Umgebung angemessen reagieren zu können. ${ }^{958}$ Sie können zudem gerade auch diskursfördernde Wirkung entfalten $^{959}$ und darüber hinaus für die Ausübung anderer grundrechtlich gewährleisteter Freiheiten Bedeutung erlangen. Auch deshalb wäre es aus grundrechtlicher Perspektive unplausibel, hier eine technisch bedingte Grenze festlegen zu wollen. ${ }^{960}$

Das muss selbst dann gelten, wenn das Botprogramm vorgegebene Inhalte zur Herstellung einer gewissen Varianz umformuliert oder sonstige Änderungen vornimmt, solange die Kernaussage und die intendierte Wirkung unverändert bleiben. Auch hier handelt das System nämlich nicht völlig autonom. ${ }^{961}$ Problematischer wird die Grenzziehung, wenn künstliche Intelligenz durch DeepLearning und neuronale Netze hinzutritt, ${ }^{962}$ sich der Algorithmus also selbstständig weiterentwickelt und von den ursprünglichen Anweisungen des Urhebers „loslöst“. 963 Soweit ersichtlich, scheint dies allerdings noch ein eher zukunftsori-

955 So in Bezug auf Journalismusroboter letztlich auch Habel, Roboterjournalismus, S. 139.

$956 \mathrm{Zu}$ diesem Aspekt der Effizienz als allgemeiner Charakter von Bots siehe etwa Hines, Houston Law Review 57 (2019), 405 (410).

957 Auch Schröder, DVBl. 2018, 465 (467) zieht hier Parallelen zu bereits bekannten Fällen, in denen Formulierung und Äußerung auseinanderfallen. Und auch Haeg, JPE 2017, 85 (92) sieht in der zeitlichen Diskrepanz zwischen Formulierung und Äußerung keinen Grund, um Bots als aus ethischer Perspektive „unzulässig“ anzusehen.

958 Vgl. in Bezug auf automatisierte Suchmaschinen Stadler, Haftung für Informationen im Internet, S. 199.

959 Auf diese Funktionalität weisen auch Semizoğlu, in: Hetmank/Rechenberg, Kommunikation, Kreation und Innovation, S. 79 (88) und Lewke, InTeR 2017, 207 (211), hin.

960 Pille, Meinungsmacht sozialer Netzwerke, S. 179, macht jedenfalls mit Blick auf den News Feed zur Voraussetzung, dass der Output manuell ausgewählt oder zumindest der Algorithmus „derart engmaschig programmiert [wird], dass nur ganz konkrete Inhalte ausgewählt werden“. Dabei geht es aber eher nicht um die Frage der technischen Modalitäten, sondern darum festzulegen, ab wann eigentlich überhaupt eine Meinung im eigentlichen Sinne vorliegt, wann man sich also die weitergegebenen Inhalte zu eigen macht.

961 Vgl. hierzu Habel, Roboterjournalismus, S. 137 f.

962 Zum Deep-Learning und den neuronalen Netzen siehe etwa Krug, beck.digitax 2020, 74 ff.

963 Aber selbst der dann bestehende Lernprozess ist das Ergebnis menschlicher Vorgaben. Dieser bewegt sich also auch in dem durch die Algorithmen vorgegebenen Lernrahmen, sodass auch hier einiges dafür spräche, eine Zurechnung vorzunehmen, vgl. Schröder, DVBl. 2018, 465 (467). Eine Zurechnung scheint Lewke, InTeR 2017, 207 (211) auch zu befürworten. 
entiertes Problem zu sein. ${ }^{964}$ Eine Grenze wäre dann nach Habel erreicht, wenn ex post die Auswahl und die konkrete Formulierung eines Kommunikats nicht mehr nachvollzogen werden kann. ${ }^{965}$ Das ist jedenfalls insoweit überzeugend, als dass der Anknüpfungspunkt gerade der Schutz des Individuums und damit die kognitive Leistung des Einzelnen ist und nicht allein die objektive Funktion eines Kommunikats. ${ }^{966}$ In jedem Fall ist der Umstand einer automatisierten Äußerung aber nicht per se in der Lage, eine grundrechtliche Zurechnung des Outputs und damit die Anwendbarkeit der Meinungsfreiheit auszuschließen. ${ }^{967}$

964 So auch Lewke, InTeR 2017, 207 (208); ähnlich Oebmer/Pedrazzi, UFITA 2020, 7 (10). Dann würde sich letztlich die Frage stellen, warum die Kontrolle beim Grundrechtsträger verbleiben muss. Algorithmen, die selbständig erlernen unter welchen Bedingungen sie ein vorher bestimmtes Verhalten vollziehen müssen, können ebenso förderlich für die Ausübung der grundrechtlich gewährleisteten Freiheiten sein und erleichtern sogar ihre Ausübung (siehe hierzu eindrucksvoll Müller, Selbstlernende Algorithmen, Computerwoche). Aus Sicht der US-amerikanischen Diskussion ist das letztlich ja auch der Ansatz, um den Schutz der Freedom of Speech zu begründen, vgl. die Verweise in Fn. (921) und auch Lewke, InTeR 2017, 207 (211) argumentiert mit der Funktionalität des Grundrechts. Spätestens dann käme man u.U. zu Wertungswidersprüchen, wenn zu hohe Anforderungen an die Kontrolle gestellt würden. Denn auch in diesen Fällen lägen menschliche Entscheidungen und Denkleistungen dem Algorithmus zugrunde und der Urheber kann jederzeit steuernd eingreifen. So meint Schröder, DVBl. 2018, 465 (466), dass „de constitutione ferenda“ diskutabel wäre, ob mit künstlicher Intelligenz ausgestatteten Robotern die Grundrechtsfähigkeit zukommen kann. In den USA ist man hier schon bei dieser Diskussion angekommen, siehe etwa Massaro/Norton, NWU Law Review Vol. 110, S. 1169-1194. Zumindest aus privatrechtlicher Sicht hinterfragt Teubner, AcP 218 (2018), 155, den Status autonomer Softwareagenten. Angesichts zunehmender Automatisierung und Robotisierung und der Entwicklung hin zu einer künstlichen Intelligenz hat der Rechtsausschuss des EU-Parlaments der Kommission 2017 anlässlich seines Berichts (A8-0005/2017) eine Empfehlung zu zivilrechtlichen Regelungen im Bereich der Robotik unterbreitet. In ihm wird darauf hingewiesen, dass jedes Reglement die Frage der Rechtsnatur von „Robotern“ aufwirft und deshalb eine neue „Kategorie mit speziellen Merkmalen und Implikationen geschaffen werden sollte“. Hierfür böte sich (u.a.) ein spezieller rechtlicher Status als „elektronische Person“ - insbesondere für haftungsrechtliche Fragen - sowie die Anwendung einer „elektronischen Persönlichkeit“ an, Empfehlung 2015/2103(INL), S. 8, $21 \mathrm{f}$. Zur Einführung einer Rechtspersönlichkeit für Computersysteme auch Kluge/Müller, InTeR 2017, 24 (29); Heuer-James/Chibanguza/Stücker, BB 2018, 2818 (2821).

965 So jedenfalls mit Blick auf Journalismusroboter Habel, Roboterjournalismus, S. 139.

966 So unter Rekurs auf den aus der Menschenwürde resultierenden Schutz des Individuums, Habel, Roboterjournalismus, S. 140.

967 I.E. auch eine Zurechnung bei Bots annehmend: Brings-Wiesen, JuWiss-Blog Nr. 93/2016; Dankert/ Dreyer, K\&R 2017, 73 (74); Hostacna, AL 2018, 1 (4); Jürlicher/Röttgen, InTeR 2018, 15 (16); dies., in: Taeger, Recht 4.0, S. 230; Milker InTeR 2017, 199 (201); ders., ZUM 2017, 216 (217f.); Lang/Wilms, Staatsrecht II, Rn. 631; Lewke, InTeR 2017, 207 (211); Oebmer/Pedrazzi, UFITA 2020, 7 (17); Steinbach, ZRP 2017, 101 (102); Semizoğlu, in: Hetmank/Rechenberg, Kommunikation, Kreation und Innovation, S. 79 (88). Hinsichtlich Journalismusrobotern Habel, Roboterjournalismus, S. 143. Mit Blick auf algorithmische Suchmaschinenergebnisse nehmen auch Starck/Paulus, in: v. Mangoldt/Klein/Starck, GG, Art. 5 Rn. 86 oder Milstein/Lippold, NVwZ 2013182 (185) eine Zurechnung vor. Auch der BGH scheint diese vornehmen zu wollen, wenn er in der Autocomplete-Entscheidung auch die Meinungsfreiheit der beklagten Firma Google ins Feld führte, BGH, GRUR 2013, 751 Rn. 22. Allerdings kam er hierauf nicht zurück, sondern sprach dann bei den Suchvorschlägen von einer „durch Art. 2, 14 GG geschützte[n] wirtschaftliche[n] Tätigkeit“. Anders hingegen Hufen, Staatsrecht II, $\mathbb{} 25 \mathrm{Rn} .52$. 


\section{Sachlicher Schutz der Inhalte}

In sachlicher Hinsicht werden also auch automatisierte Meinungen im Sinne von Art. 5 Abs. 1 S. 1, 1. HS. GG geschützt. Der Begriff der Meinung ist dabei weit zu verstehen. ${ }^{968}$ Zunächst erfasst er die eigentliche Meinung als subjektives Werturteil und damit Äußerungen im Rahmen einer geistigen Auseinandersetzung, die durch Elemente der Stellungnahme, des Dafürhaltens und des Meinens geprägt sind. ${ }^{969}$ Meinungen zeichnen sich also durch die subjektive Beziehung des Äußernden zum geäußerten Inhalt aus. ${ }^{970}$ Daneben werden aber auch Tatsachenbehauptungen erfasst, obgleich der Wortlaut diese nicht vorsieht, ${ }^{971}$ und ihnen bei näherer Betrachtung die zuvor genannten subjektiven Elemente fehlen ${ }^{972}$. Sie sind durch „eine objektive Beziehung [...] [zur] Wirklichkeit geprägt und der Überprüfung mit Mitteln des Beweises zugänglich [...]. “973 Ihr Schutz leitet sich aus einer derivativen Funktion für die Meinungsbildung ab, ${ }^{974}$ sodass sie nur dann geschützt werden, sofern sie Grundlage und Voraussetzung für die Bildung einer Meinung sein können. ${ }^{975}$

Die Trennung zwischen den Äußerungsarten hat vor allem praktische Folgen, wenn es um desinformative Inhalte geht. Während es für den Schutz einer Meinung nicht darauf ankommt, ob sie emotional oder rational, begründet oder grundlos, wertvoll oder wertlos ist, ${ }^{976}$ werden nach der ständigen Judikatur des Bundesverfassungsgerichts die im Zeitpunkt der Äußerung erwiesen oder bewusst unwahren Tatsachenbehauptungen gar nicht erst vom Schutzbereich er-

968 So BVerfGE 61, 1 (9) - CSU NPD Europas; 71, 162 (179) - Werbeverbot für Ärzte; BVerfG (K), NJW 1991, 1529 - Zahnärztestreik.

969 Vgl. BVerfGE 61, 1 (8) - CSU NPD Europas; 71, 162 (179) - Werbeverbot für Ärzte; 85, 1 (14) Bayer-Aktionäre; 90, 241 (247) - Auschwitzlüge.

970 BVerfGE 33, 1 (14) - Strafgefangene; 90, 241 (247) - Auschwitzlüge. Aus jüngerer Zeit BVerfG (K), NJW 2016, 2643 Rn. 11 - ACAB; BVerfG, Beschl. v. 26.02.2015 - 1 BvR 1036/14, Rn. 11 - FCK CPS.

971 Schemmer, in: Epping/Hillgruber, Beck-OK GG, Art. 5 Rn. 6.

972 BVerfGE 61, 1 (8) - CSU NPD Europas; 85, 1 (15) - Bayer-Aktionäre; Degenhart, in: BK-GG, Art. 5 Abs. 1, 2 Rn. 112; Schmidt-Jortzig, HbStR VII, $\$ 162$ Rn. 21.

973 Siehe hierzu nur BVerfG, Beschl. v. 17.03.2017 - 1 BvR 3085/15 Rn. 13 m.w.N. auf die verfassungsgerichtliche Rechtsprechung.

974 Hierzu etwa Degenhart, BK-GG, Art. 5 Abs. 1, 2 Rn. 112; Schulze-Fielitz, Dreier GG, Art. 5 Abs. 1, 2 Rn. 63.

975 BVerfGE 7, 198 (210) - Lüth; 61, 1 (8) - Meinungsäußerung im Wablkampf; 90, 241 (247) - Auschwitzlüge. Ob diese Abgrenzung überhaupt Sinn macht, ist zweifelhaft. Ziel ist es, dass solche Äußerungen aus dem Schutzbereich fallen, die irrelevant für die Meinungsbildung sind. Bedenkt man aber, dass jede Tatsachenmitteilung in einem wertenden Kontext stehen kann, scheint diese Einschränkung kaum sinnvoll zu sein, das Herausfallen aus dem Schutzbereich bleibt die (sehr) seltene Ausnahme, vgl. Schulze-Fielitz, Dreier GG, Art. 5 Abs. 1, 2 Rn. 64. Ausführlichere Kritik an dieser Rspr. findet sich etwa bei Jestaedt, in: Merten/Papier, HbGR IV, $\$ 102$ Rn. $37 \mathrm{f}$.

976 BVerfGE 61, 1 (9) - Meinungsäußerung im Wablkampf; 90, 241 (247) - Auschwitzlüge; 124, 300 (320) - Wunsiedel. 
fasst. ${ }^{977}$ Dem wird u.a. ${ }^{978}$ entgegengehalten dass bereits aufgrund der schwierigen Trennung zwischen Meinung und Tatsachenbehauptung derlei Einschränkungen abzulehnen seien ${ }^{979}$. Unwahre Tatsachenbehauptungen können allerdings zu keinem Zeitpunkt einen Mehrwert für die Meinungsbildung bieten. ${ }^{90}$ Die Meinungsfreiheit soll einen sinnvollen, sachlichen und ergiebigen Diskurs fördern. Diesem Zweck sind Lügen abträglich, können ihn sogar gefährden ${ }^{981}$ und sind deshalb nicht schützenswert. ${ }^{982}$. Die Abgrenzungsschwierigkeit wird dadurch relativiert, dass bei einer untrennbaren Verbindung von Meinung und Tatsache von einer einheitlichen Meinungsäußerung auszugehen ist. ${ }^{983}$

Für die Abgrenzung zwischen Meinung und Tatsachenbehauptung kommt es weder auf die subjektive Absicht des Äußernden noch auf das subjektive Verständnis des von der Äußerung Betroffenen an. ${ }^{984}$ Vielmehr ist der Äußerung der Sinn beizumessen, den ihr ein unvoreingenommener und verständiger Empfänger geben würde. ${ }^{955}$ Dem Urheber der Äußerung darf aber kein Inhalt untergeschoben werden, den er ihr erkennbar nicht beilegen wollte. Im Zweifel wird zumeist von einer Meinungsäußerung auszugehen sein.

977 St. Rspr., BVerfGE 54, 208 (219); 61, 1 (8) - Meinungsäußerung im Wahlkampf; 85, 1 (15) - BayerAktionäre; 90, 241 (247) - Auschwitzlüge; zustimmend u.a. Odendahl, in: Schmidt-Bleibtreu, GG, Art. 5 Rn. 4; Schmalenbach, JA 2005, 749 (750); Huster, NJW 1996, 487 (487). Aus der Linie der ansonsten einheitlichen Entscheidungen des BVerfG fällt die unklare Passage in der Stolpe-Entscheidung, BVerfGE 114, 339 (352) - mehrdeutige Äußerungen. Dort heißt es: „Grundsätzlich tritt die Meinungsfreiheit daher bei Tatsachenbehauptungen, die bewusst unwahr oder erwiesenermaßen falsch sind, hinter das Persönlichkeitsrecht zurück“. Dies legt eine Lösung auf Rechtfertigungsebene nahe (so Degenhart, BK-GG, Art. 5 Abs. 1, 2 Rn. 118), würde aber der st. Rspr. widersprechen. Da in der Entscheidung auf BVerfGE 85, 1 (17) - Bayer-Aktionäre verwiesen wurde, ist vom BVerfG die Fundstelle wohl eher unsauber wiedergegeben worden. Dort heißt es nämlich: „Enthält die Meinungsäußerung erwiesen falsche oder bewußt unwahre Tatsachenbehauptungen, so wird regelmäßig das Grundrecht der Meinungsfreiheit hinter dem [...] geschützten Recht zurücktreten. " Hier wird also nur Rekurs genommen auf das, was regelmäßig bei der Vermengung von überwiegender Meinung und bewusst unwahrer Tatsachenbehauptung gilt: der Schutz durch die Meinungsfreiheit mit der Folge einer umfassenden Abwägung auf Rechtfertigungsebene. Kritisch hierzu Steinbach, ZRP 2017, 101 (103).

978 Umfassender hierzu u.a. Herzog, in: Maunz/Dürig, GG, EL 30, Stand Dez. 1992, Art. 5 Rn. 50 ff. und Jestaedt, in: Merten/Papier, HbGR IV, $\mathbb{} 102$ Rn. 36 ff., jeweils mit weiteren Nachweisen. Siehe insbesondere auch im Kontext von „Fake News“ Steinbach, JZ 2017, 653 (656 ff.).

979 Schulze-Fielitz, in: Dreier, GG, Art. 5 Abs. 1, 2 Rn. 65. Kritisch wegen des Subjektbezugs reiner Tatsachenbehauptungen auch Jestaedt, in: Merten/Papier, HbGR IV, $\mathbb{1 0 2}$ Rn. 37.

980 Hierzu BVerfGE 54, 208 (219) - Böll; 61, 1 (8) - Meinungsäußerung im Wahlkampf; 90, 241 (247) Auschwitzlüge. Vgl. hierzu auch BVerfGE 12, 113 (130) - Schmidt-Spiegel.

981 Huster, NJW 1996, 487 (487).

982 Siehe nur BVerfGE 54, 208 (219) - Böll; 61, 1 (8) - Meinungsäußerung im Wablkampf; 85, 1 (15) Bayer-Aktionäre.

983 BVerfGE 61, 1 (9) - Meinungsäußerung im Wablkampf; 85, 1 (15) - Bayer-Aktionäre. Vgl. auch BVerfGE 93, 266 (296) - Soldaten sind Mörder.

984 BVerfGE 93, 266 (295) - Soldaten sind Mörder; 114, 339 (348) - mehrdeutige Äußerungen.

985 BVerfGE 93, 266 (295) - Soldaten sind Mörder; 107, 275 (281) - Schockwerbung II; 114, 339 (348) - mehrdeutige Äußerungen. Ebenso BGHZ 95, 212 (215) - Wehrmachtsoffizier; 132, 13 (19) Luftsicherheitsgesetz. Kritisch hierzu Jestaedt, in: Merten/Papier, HbGR IV, $\$ 102$ Rn. 92 f., der sich vielmehr für einen subjektiven Auslegungsansatz ausspricht. 
Lässt sich über die Richtigkeit von Tatsachenangaben streiten, so sind derartige Äußerungen nicht vom Schutz ausgenommen. ${ }^{986}$ Vielmehr treffen den Äußernden Wahrheits- und Sorgfaltspflichten. ${ }^{987}$ Verstöße gegen diese Pflichten können, insbesondere in Kollisionsfällen mit anderen grundrechtlichen Interessen, zu Einschränkungen des Schutzes führen. ${ }^{988}$ So müssen i.d.R. sorgfaltswidrig unwahre Tatsachenbehauptungen - Behauptungen, deren objektive Unwahrheit sich später herausstellt - zurücktreten, während für wahre Tatsachenbehauptungen für gewöhnlich der Vorrang der freien Rede gilt. ${ }^{989}$

Im Falle von Hate Speech handelt es sich meist um personenbezogene Meinungen oder verbundene Aussagen, sodass hier regelmäßig Persönlichkeitsrechte Dritter beeinträchtigt werden. Grundsätzlich ist auch hier der Schutzbereich für noch so diffamierende Äußerungen eröffnet. ${ }^{990}$ Eine Grenze ist dort erreicht, wo nur reine Beleidigungen geäußert werden (sog. Formalbeleidigungen). ${ }^{991}$ Im Übrigen ergeben sich die Grenzen allein durch kollidierende Rechte, insbesondere durch das allgemeine Persönlichkeitsrecht Betroffener. Einfach-rechtlich schlägt sich das in den $\mathbb{S} \mathbb{S} 130,185,186,187$ StGB nieder. Im Falle von Schmähkritik tritt die Meinungsfreiheit regelmäßig hinter den Schutz der persönlichen Ehre zurück. ${ }^{992}$ Die Schwelle hierfür liegt hoch. Sie ist erst dann anzunehmen, wenn der Äußerung kein irgendwie gearteter sachlicher Kern mehr zu entnehmen ist, sondern die Diffamierung im Vordergrund steht. ${ }^{993}$

Einfachere Bots sind lediglich darauf programmiert, bestimmten politischen Parteien oder Personen zu folgen. Zwar handelt es sich hierbei um non-verbale Kommunikation. Der Betätigung des „Gefällt-mir“-Knopfs kann aber derselbe

986 Vgl. BVerfGE 61, 1 (8) - Meinungsäußerung im Wablkampf.

987 Siehe zu diesen Wahrheitspflichten etwa die Ausführungen in BVerfG, NJW 2016, 3360 Rn. 20 Doping in der DDR. Vgl. auch BVerfGE 90, 241 (247 f.) - Auschwitzlïge; Grabenwarter, in: Maunz/ Dürig, GG, Art. 5 Abs. 1 Rn. 49; Schmalenbach, JA 2005, 749 (750). Einfach-rechtlich kommen diese Pflichten etwa in $\$ 186$ StGB im Tatbestandsmerkmal „nicht erweislich wahr“ zum Ausdruck.

988 BVerfGE 61, 1 (8) - Meinungsäußerung im Wahlkampf, dort heißt es: „,soweit unrichtige Tatsachenbehauptungen nicht schon von vorneherein [aus dem] Schutzbereich [fallen], sind sie Einschränkungen [...] leichter zugänglich als das Äußern einer Meinung." Siehe hierzu auch BVerfGE 99, 185 (197) - Scientology.

989 Degenhart, BK-GG, Art. 5 Abs. 1, 2 Rn. 453. Das BVerfG urteilte: „Für die Verbreitung unwahrer Tatsachenbehauptungen gibt es in der Regel keinen rechtfertigenden Grund“, BVerfGE 114, 339 (352) - mehrdeutige Äußerungen. Siehe hierzu außerdem nur $\mathbb{} 186 \mathrm{StGB}$, der dies auch einfach-gesetzlich zum Ausdruck bringt.

990 So ausdrücklich BVerfGE 33, 1 (15) - Strafgefangene; 93, 266 (289) - Soldaten sind Mörder. Siehe aber auch BVerfGE 61, 1 (8) - Meinungsäußerung im Wablkampf.

991 Hierzu Grabenwarter, in: Maunz/Dürig, GG, Art. 5 Abs. 1 Rn. 62. Zum fehlenden grundrechtlichen Schutz in diesen Fällen BVerfGE 60, 234 (242) - Kredithaie; 82, 43 (51) - Strauß-Transparent. In der jüngeren Judikatur klingt das tendenziell anders, wenn Formalbeleidigung und Schmähkritik zusammen erwähnt werden und davon die Rede ist, dass „die Meinungsfreiheit regelmäßig hinter den Ehrenschutz zurück[tritt].“, vgl. BVerfG, NJW 2019, 2600 Rn. 18 - Hexenprozess. So auch Schemmer, in: Epping/Hillgruber, BeckOK GG, Art. 5 Rn. 4.

992 Statt vieler BVerfG NJW 2019, 2600 Rn. 18 - Hexenprozess, m.w.N. auf die verfassungsgerichtliche Judikatur.

993 BVerfG, NJW 2003, 3760 - politische Polemik; NJW 2009, 3016 (3017) jeweils m.w.N. 
Erklärungsgehalt wie einer verbalen Zustimmung entnommen werden, sodass damit gerade auch ein subjektives Werturteil verbunden ist. Das gilt auch deshalb, weil der jeweilige Nutzer stets mit dem so markierten Beitrag in Verbindung gebracht und aus den markierten Inhalten auf eine bestimmte politische oder ideologische Grundhaltung geschlossen werden kann. Deshalb ist auch dieser non-verbale Vorgang eine von Art. 5 Abs. 1 S. 1 GG geschützte Meinungsäußerung. ${ }^{994}$ Durch das Folgen oder Abonnieren anderer Nutzer, gibt man hingegen nur zu erkennen, dass man über Aktivitäten des jeweiligen Nutzers informiert bleiben will. Über die genaue Einstellung des Folgenden wird dadurch nicht zwingend etwas bekannt, ebenso wenig über seine subjektive Bewertung der Inhalte. Im Zweifel ließe sich höchstens qua Gewohnheit davon ausgehen, dass nur solchen Profilseiten gefolgt werden wird, an denen man auch interessiert ist. Als sichere Erkenntnis kann dies aber nicht gelten, sodass es vom Einzelfall abhängig sein wird, ob eine Meinungsäußerung vorliegt.

\section{Restriktion des Schutzbereichs im Falle von Meinungsrobotern?}

Bis hierhin würden die Verwender also den Schutz der Meinungsfreiheit genießen. Nur in geringem Umfang sind schädliche Inhalte - etwa Desinformation vom Schutzbereich ausgenommen. Insoweit ist zumindest diskutabel - die einschlägige Literatur macht das vor ${ }^{995}$, ob der Schutzbereich im Falle der Meinungsroboter $\mathrm{zu}$ restringieren und den Verwendern der Schutz zu verwehren wäre.

\section{a. Allgemeines zur Konzeption von Schutzbereichsausnahmen}

Schon Art. 8 Abs. 1 GG stellt grundrechtsspezifische Beschränkungen des Schutzes dadurch auf, dass er nur „friedliche Versammlungen ohne Waffen“ als geschützt ansieht. Anderweitige Schutzbereichsausnahmen sieht das Grundgesetz selbst zwar nicht vor. Gleichwohl versucht die Rechtsprechung, grundrechtliche Schutzbereiche zu restringieren, um unerwünschte Handlungsweisen den freiheitsrechtlichen Schutz zu versagen. ${ }^{996}$ Das gilt nicht nur mit Blick auf die Mei-

994 I.E. ebenso Fublrott/Oltmanns, NZA 2016, 785 (786f.); Schimmele, Staatliche Verantwortung für diskursive Integrität, S. 103. Im Einzelfall können allerdings Umstände eine andere Wertung zulassen, bspw. wenn das Drücken des „Gefällt-mir“-Knopfs die Voraussetzung für die Teilnahme an einem Gewinnspiel ist, hierzu LG Hamburg, Urt. v. 10.01.2013 - 327 O 438/11, Rn. 22 ff.

995 So etwa Brings-Wiesen, JuWiss-Blog Nr. 93/2016; Hostacna, AL 2017, 1 (4); Löber/Roßnagel, MMR 2019, 493 (495) oder Schröder, DVBl. 2018, 465 (467).

996 Und allgemein wird lebhaft darüber diskutiert, ob man grundrechtliche Schutzbereiche nicht von Anfang an auf einen bestimmten Gewährleistungsgehalt reduzieren müsste. Während das Schrankenmodell den grundrechtlichen Schutz weit versteht und Begrenzungen erst im Rahmen der Abwägung vornehmen will (in diese Richtung Kahl, Der Staat 43, 167 (184ff.) m.w.N. in Fn. 16; ders., AöR 131, 579 (617); Classen, Staatsrecht II, $\$ 5$ Rn. 8; Hufen, NJW 1999, 1504 (1508); Höfling, Offene 
nungsfreiheit und die Herleitung des derivativen Schutzes der Tatsachenbehauptungen und dem dadurch bezweckten Ausschluss erwiesen bzw. bewusst unwahrer Tatsachenbehauptungen. Daneben genießen Werbeaussagen nur dann den Schutz der Meinungsfreiheit, wenn sie einen wertenden, meinungsbildenden Inhalt aufweisen. ${ }^{997}$ Und auch der Ausschluss unrichtiger Zitate aus dem Schutzbereich ist von dem Bemühen gekennzeichnet, dies mit dem Telos der Meinungsfreiheit zu begründen. ${ }^{998}$ Daneben hat das BVerfG im Falle des Sprayers von Zürich den Schutz der Kunstfreiheit aus Art. 5 Abs. 3 GG dort abgelehnt, wo es um „die eigenmächtige Inanspruchnahme oder Beeinträchtigung fremden Eigentums zum Zwecke der künstlerischen Entfaltung “ ging. ${ }^{999}$ Mit der Sampling-Entscheidung hat das BVerfG diese Auffassung aber aufgegeben und eine Lösung im Rahmen der Abwägung präferiert. ${ }^{1000}$ Auch bei der Berufsfreiheit aus Art. 12 Abs. 1

Grundrechtsinterpretation, S. 175 ff; Sachs, in: ders., GG, Vor Art. 1 Rn. 77a; wohl auch Jarass, in: ders./Pieroth, GG, Vorb. Vor Art. 1 Rn. 21), versucht die Tatbestandslösung schon auf Ebene des Schutzbereiches Beschränkungen vorzunehmen und den Schutz auf bestimmte Verhaltensweisen zu beschränken. Während der Sachbereich nur das einschlägige Thema umfasse, gebe der Gewährleistungsbereich der Grundrechte die konkrete Weite des Schutzes vor. Eben dieser Gewährleistungsbereich soll dann restriktiv gehandhabt werden, in diese Richtung u.a. Böckenförde, Der Staat 42, 165 (174 ff.); Rusteberg, Grundrechtlicher Gewährleistungsbereich; Hillgruber, in: Isensee/Kirchhof, HbStR, Bd. IX, $\mathbb{2} 200$ Rn. 32; Hesse, Grundzüge Verfassungsrecht, Rn. 300, 428. Problematisch an der Schrankenlösung ist freilich, dass eine gewisse Einzelfallkasuistik entsteht, was die Vorhersehbarkeit und damit die Rechtssicherheit beeinträchtigt, kritisch deshalb etwa Leisner, NJW 1997, 636 (638f.) Andererseits sorgt dies aber gerade für eine Flexibilität und dafür, dass im Einzelfall sachgerechte Ergebnisse erzielt werden können, vgl. etwa Hillgruber, in: Isensee/Kirchhof, HbStR, Bd. IX, $\mathbb{2} 200$ Rn. 32. Die Rspr. des BVerfG ist ebenfalls nicht einheitlich. Vereinzelt kann eine gewisse Tendenz zu tatbestandlichen Restriktionen ausgemacht werden. Insbesondere der Sprayer von Zürich Beschluss (NJW 1984, 1293 [1294]) sowie die Entscheidungen Glykol (BVerfGE, 105, 252 [265]) und Osho (BVerfGE 105, 279 [294f.]) weisen eher in Richtung des GewährleistungsmodellS. In der Entscheidung zum Maastricht Vertrag differenziert das BVerfG sogar expressis verbis zwischen „Sachbereich“ und „Gewährleistungsinhalt“, BVerfGE 89, 155 (172). Überwiegend versucht allerdings auch das BVerfG eine Lösung im Wege der Abwägung zu finden. 2016 hat das BVerfGE auch die Auffassung im Falle des Sprayers von Zürich revidiert, BVerfGE 142, 74 (96ff.) - Sampling. Eine ausführlichere Darstellung der Problematik findet sich u.a. bei Rusteberg, Grundrechtliche Gewährleistungsinhalte; Cornils, Ausgestaltung der Grundrechte, S. 40 ff.; Hillgruber, in: Isensee/Kirchhof, HbStR, Bd. IX, $\mathbb{2} 200 \mathrm{Rn}$. $24 \mathrm{ff}$. Siehe auch Camilo de Oliveira, Abwägung in der Grundrechtsdogmatik, S. $173 \mathrm{ff}$. umfassend und kritisch zu der voranschreitenden Lösung grundrechtlicher Spannungen im Rahmen der Abwägung und S. 232 zu alternativen Modellen sowie S. 281 zu einer alternativen Grundrechtsdogmatik.

997 BVerfGE 71, 162 (175) - Werbeverbot für Ärzte. In BVerfGE 40, 371 (382) - Werbefahrten, hatte das BVerfG ebenfalls Art. 5 Abs. 1 GG erwähnt aber, „da es in dem zur Entscheidung stehenden Fall nur um die Wirtschaftswerbung geht“, hierzu nichts weiter ausgeführt. Ein solcher Charakter kann bspw. dann angenommen werden, wenn die Aussagen „wirtschaftliche, politische, soziale und kulturelle Probleme zum Gegenstand haben“, so BVerfGE 102, 347 (362) - Schockwerbung I. Siehe hierzu auch BVerfGE, 107, 275 (282 f., 285 f.) - Schockwerbung II.

998 Hierzu heißt es etwa „Weder die öffentliche Meinungsbildung noch die demokratische Kontrolle können [...] unter dem Erfordernis leiden, richtig zitieren zu müssen. “, BVerfGE 54, 208 (220) Böll.

999 BVerfG (K), NJW 1984, 1293 (1294) - Sprayer von Zürich; zustimmend Hoffmann, NJW 1985, 237 (243).

1000 BVerfGE 142, 74 (Rn. 84, 89 f., 92 ff.) - Sampling; mit dieser Tendenz - wenn auch hinsichtlich des Persönlichkeitsrechts - schon zuvor BVerfGE 119, 1 (23) - Esra. Insgesamt ist die Entscheidung im Falle des Sprayers von Zürich auf erhebliche Kritik in der Literatur gestoßen, die eine Lösung im 
GG gab es seit jeher Ambitionen, den Schutzbereich einzuengen. So haben BVerwG und BVerfG den Schutz zunächst auf gesetzlich oder behördlich erlaubte Tätigkeiten beschränkt. ${ }^{1001}$ Inzwischen sollen „unerlaubte Tätigkeiten“ nur solche sein, die „schon nach ihrem Wesen als verboten anzusehen sind, weil sie aufgrund ihrer Sozial- und Gemeinschaftsschädlichkeit schlechthin nicht am Schutz [...] teilhaben können “. ${ }^{1002}$ Auch hier war die Judikatur bemüht, die Restriktion unabhängig von der Überzeugungskraft - teleologisch zu begründen. Und auch im Übrigen stützt sich das Streben nach Restriktionen grundrechtlicher Schutzbereiche maßgeblich auf teleologische Erwägungen, ${ }^{1003}$ was letztlich selbst für die ausdrücklich normierte Begrenzung der Versammlungsfreiheit gilt. ${ }^{1004}$ Insoweit

Rahmen der Abwägung bevorzugte, siehe hierzu nur Wittreck, in: Dreier, GG, Art. 5 III (Kunst) Rn. 49; $v$. Arnauld, in: Isensee/Kirchhof, HStR VII, $\mathbb{S} 167$ Rn. 44.

1001 BVerwGE 2, 110 (111) - Kasinospiel; 4, 294 (296) - Lotterie; 87, 37 (40) - Glykol; so auch BVerfGE 7, 377 (397) - Apotheken-Urteil: ,[Der Begriff ,Beruf'] umfaßt [...] auch die vom Einzelnen frei gewählten untypischen (erlaubten) Betätigungen [...].“; BVerfGE 13, 97 (106) - Handwerksordnung; 14, 19 (22) - Warenautomat; 32, 311 (317) - Steinmetz-Wettbewerb; 48, 376 (388) - Tierversuche; 81, 70 (85) - Mietwagenunternehmer; 112, 197 (213) - Spielbankgesetz Baden-Württemberg. Und auch das OVG Münster, NJW 1986, 2783 zu glykolhaltigem Wein, und der BayVGH, NJW 1987, 727 zu Schwangerschaftsabbrüchen durch Ärzte, haben noch auf die Maßgeblichkeit einfach-rechtlicher Verbote abgestellt. Anders aber BVerwGE 22, 286 (288f.) - Astrologie, wo auf die Sozialschädlichkeit abgestellt wird. Diese „kann, aber muß nicht [darin begründet sein] [...], daß eine Tätigkeit verboten oder strafbar ist“. Deshalb könnte man auch die vorherige Rechtsprechung in dieser restriktiveren Weise verstehen. Entsprechende Bedenken finden sich etwa auch bei Breuer, in: Isensee/Kirchhof, HStR VIII, $\$ 170$ Rn. 68.

1002 So BVerfGE 115, 276 (301) - Sportwetten; 117, 126 (137) - Hufbeschlaggesetz; andeutend aber offenlassend in BVerwGE 97, 12 (22) - Buchmacher; zuvor bereits BVerwGE 22, 286 (288 f.) - Astrologie. Zu Recht ist die ursprüngliche Rechtsprechung auf Kritik gestoßen. Denn dann würde der grundrechtliche Schutz für den Gesetzgeber disponibel. Zur Kritik siehe etwa Berg, GewArch 1977, 249; Scholz, in: Maunz/Dürig, GG, Art. 12 Rn. 35; umfangreicher bei Breuer, in: Isensee/Kirchhof, HStR VIII, $\mathbb{1 7 0}$ Rn. 68. Und auch die Restriktion auf „nur“ sozial- und gemeinschaftsschädliche Tätigkeiten sieht sich dem Einwand ausgesetzt, dass der Schutz dann maßgeblich - wie bei der Prostitution oder beim Handel mit Marihuana - von der momentanen gesellschaftlichen Anschauung abhängt, wodurch es zu Rechtsunsicherheiten kommen kann, Kämmerer, in: v. Münch/Kunig, GG, Art. 12 Rn. 17; vgl. Breuer, in: Isensee/Kirchhof, HStR VIII, S. 118 Fn. 268. Legalisiert der Gesetzgeber ein Rauschgift, wie es momentan für Marihuana gefordert wird, so endet damit dann auch die Gemeinschaftsschädlichkeit, so Kämmerer, in v. Münch/Kunig, GG, Art. 12 Rn. 17. Und mit Blick auf die Prostitution ist, trotz ursprünglich anderer Auffassung (Berufsqualität i.S.d. Art. 12 Abs. 1 GG in einem obiter dictum ablehnend BVerwGE 22, 286 [289]; ähnlich BVerwGE 84, 314 [320]; offenlassend hingegen OVG Lüneburg, NordÖR 2003, 26 (27); in der Tendenz für die Anerkennung des Schutzes VG Berlin, NJW 2001, 983 [989] jeweils m.w.N.), jedenfalls durch das Prost $\mathrm{G}$ die gesetzgeberische Entscheidung dafür gefallen, den Prostituierten rechtswirksame Forderungen zuzugestehen und die Tätigkeit zu legalisieren. Anders hingegen Kurz, GewArch 2002, 142 (145).

1003 Die mittels Drucks geäußerte oder vermittelte Meinung wird, da sie nicht mehr auf den geistigen Meinungskampf abzielt, nicht von Art. 5 Abs. 1 S. 1 geschützt, vgl. Grabenwarter, in: Maunz/Dürig, Art. 5 Rn. 67. Die Restriktion des verfolgten Zwecks von Versammlungen - dem BVerfG nach beschränkt auf die Teilhabe an der öffentlichen Meinungsbildung durch Kundgebungen (BVerfGE 104, 92 [104] - Sitzblockade III) - begrenzt den Schutzbereich der Versammlungsfreiheit aus Art 8 Abs. 1 GG aufgrund des Bezugs zum Prozess der öffentlichen Meinungsbildung, BVerfGE a.a.O.

1004 Dieser Ausschluss soll klarstellen, dass die Versammlung als Instrument der geistigen Auseinandersetzung und Einflussnahme auf die politische Willensbildung dienen soll, sodass gerade der Einsatz von Gewalt - zumal ein staatliches Gewaltmonopol beseht - diesem Zweck diametral gegenüber- 
lassen sich Begrenzungsversuche also noch am ehesten mit einer teleologischen Reduktion ${ }^{1005}$ vergleichen.

\section{b. Übertragung auf politische Bots}

Ansatzpunkt einer Schutzbereichsbegrenzung der Meinungsfreiheit kann folglich auch hier nur die Vereinbarkeit der Meinungsroboter mit dem Schutzzweck des für die Demokratie unerlässlichen Prozesses einer sachlich-geistigen Auseinandersetzung sein. Zweifel an dieser Kompatibilität begründen vor allem zwei Charakteristika: die Identitätstäuschung und die vorgetäuschte Meinungsmacht. ${ }^{1006}$

\section{aa. Identitätstäuschung}

Durch die falschen Nutzerkonten täuschen die Verwender zahlreiche falsche Identitäten vor, denen konkrete Äußerungen zugeordnet werden können. Sie erschaffen dadurch also Teile des virtuellen Kommunikationsraumes, ohne dass dies von anderen Nutzern ohne Weiteres bemerkt werden kann. Dass aber die Kopplung einer Äußerung an einen konkreten Urheber für die Rezeption bedeutsam ist, hat das Bundesverfassungsgericht bereits hinsichtlich anonymer Äußerungen deutlich gemacht. Ihnen fehle „häufig dasjenige Maß an Authentizität und Glaubhaftigkeit, welches ihnen erst den gewünschten Einfluss verleiht oder Reaktionen hervorruft“. ${ }^{1007}$ Und dies lässt sich auch der Entscheidung des BVerfG über den Schutzbereichsausschluss unrichtiger Zitate entnehmen, wenn dort mit dem Interesse der öffentlichen Meinungsbildung an der Information argumentiert wird. ${ }^{1008}$ Anders als bei anonymen Äußerungen fehlt eine entsprechende Warnfunktion bei Meinungsrobotern. Ähnlich wie bei den unrichtigen Zitaten ${ }^{1009}$ wird hier vielmehr eine Äußerung einer fiktiven Person zugeordnet, die für die Nutzer echt zu sein scheint, sodass jedenfalls konkludent mit ihnen die Aussage einhergeht, sie seien reale Personen. ${ }^{1010}$ Dabei handelt es sich aber ebenso um eine Lüge, wie auch im Falle einer unwahren Tatsachenbehauptung. Geht man aber davon aus, dass bewusst oder ersichtlich unwahre Tatsachenbe-

steht, Vgl. BVerfGE 69, 315 (359f.); ähnlich Benda, in: BK-GG, Art. 8 Rn. 37; andeutend Michael/ Morlok, Grundrechte, Rn. 271, 275.

1005 So bezeichnet auch Schröder, DVBl. 2018, 465 (466) die Herangehensweise.

1006 Diese Differenzierung findet sich auch in der einschlägigen Literatur, siehe etwa bei Andersen, HRN 2017, 9 (10 ff.) oder Brings-Wiesen, JuWiss-Blog Nr. 93/2016.

1007 BVerfGE 97, 391 (398) - Missbrauchsbezichtigung.

1008 BVerfGE 54, 208 (220) - unrichtiges Zitat.

1009 Der maßgebliche Unterschied besteht hier darin, dass das BVerfG in seiner Entscheidung E 54, 208 (219f.) - unrichtiges Zitat, maßgeblich auch auf das betroffene allgemeine Persönlichkeitsrecht abgestellt hat, welches hier freilich gar nicht erst einschlägig sein wird.

1010 Milker, InTeR 2017, 199 (201). 
hauptungen keinen Gewinn für die Meinungsbildung bringen, liegt der Verdacht nahe, dass das entsprechend auch für die Täuschung über die Identität gilt. ${ }^{1011}$

\section{(I.) Identitätstäuschung als Bestandteil der Modalitätsfreiheit}

Was im Ausgangspunkt plausibel klingt, wird aber mit Blick auf die Modalitätsfreiheit zu einem Problem. Denn die Wahl der Äußerungsmodalitäten hat unmittelbar Bedeutung für die Wirkung, die eine Meinung erzeugen soll und obliegt schon deshalb allein dem Grundrechtsträger. ${ }^{1012}$ Genau genommen ermöglichen es Meinungsroboter sogar erst, dass eine gewisse Öffentlichkeit in sozialen Netzwerken hergestellt wird, ${ }^{1013}$ eine Meinung also überhaupt eine bestimmte Wirkung entfalten kann. Im Entferntesten kann man sie noch mit Flugblättern vergleichen: ${ }^{1014}$ Verteilt eine Person Flugblätter, maximiert sie nicht nur die Zahl ihrer Äußerungen, sondern auch die Rezeptionswahrscheinlichkeit. Eine vergleichbare Möglichkeit besteht in sozialen Netzwerken hingegen nicht. Der Einzelne ist hier vielmehr darauf angewiesen, dass er durch den Beitrag die Filter der Netzwerke durchdringt und andere Personen damit auch tatsächlich erreicht. $^{1015}$

Freilich tritt hier anders als im Falle der Flugblätter die Identitätstäuschung hinzu. Darin wird aber oftmals kein Widerspruch gesehen, weil mit anonymen und pseudonymen Äußerungen identitätsbezogene Täuschungen einher gingen, diese aber gerade von der Meinungsfreiheit geschützt werden. ${ }^{1016}$ Dem ist zuzugeben, dass keine Pflicht besteht, durch die Verwendung eines (richtigen) Namens Rückschlüsse auf den Urheber zu ermöglichen. ${ }^{1017}$ Vielmehr werden auch anonyme und pseudonyme Äußerungen vom Schutz erfasst, ${ }^{1018}$ weil nur so eine Mei-

1011 Brings-Wiesen, JuWiss-Blog Nr. 93/2016. In diese Richtung auch Andersen, HRN 2017, 9 (10f.); Hostacna, AL 2018, 1 (4).

1012 Allgemein hierzu BVerfG NJW 2003, 1109 (1110) - Informationsweitergabe an Presse, m.w.N aus der verfassungsgerichtliche Judikatur. Auf diese Freiheit in der Debatte um den Schutz der Meinungsroboter verweisend Steinbach, ZRP 2017, 101 (102, 103) und Semizoğlu, in: Hetmank/ Rechenberg, Kommunikation, Kreation und Innovation, S. 79 (90).

1013 Ähnlich Paal/Hennemann, ZRP 2017, 76 (77).

1014 Haeg, JPE 2017, 85 (89) bezeichnet sie als „new communicational tool“ und Bock, Übertragbarkeit der Kommunikationsfreiheiten, S. 195 als „Meinungsmultiplikator“.

1015 Insoweit geht es hier um die bereits oben angesprochenen Netzwerkeffekte, die durch die Nutzergemeinschaft und das Prinzip der sozialen Bewährtheit maßgeblich hervorgerufen werden, hierzu bereits oben 2. Teil, B.III.3.a.

1016 So etwa Steinbach, ZRP 2017, 101 (102 f.); Zumkeller-Quast, JuWiss-Blog Nr. 2/2017; Schroeder, DVBl. 2017, 465 (467 f.); Milker, InTeR 2017, 199 (201f.).

1017 BGH, NJW 2009, 2888 (2892); ähnlich Steinbach, ZRP 2017, 101 (103); Zumkeller-Quast, JuWiss-Blog Nr. 2/2017.

1018 So die wohl h.M.: Trotz Pseudonym lehnte das BVerfG jedenfalls nicht den Schutzbereich ab, BVerfG NJW 2012, 3712 ff. Daneben auch BGH NJW 2009, 2888 (2892); OLG Hamm ZD 2011, 179; Grabenwarter, in: Maunz/Dürig, GG, Art. 5 Rn. 86; Kühling, in: Gersdorf/Paal, Informationsund Medienrecht, Art. 5 GG Rn. 33; Kersten, JuS 2017, 193 (196); Dankert/Dreyer, K\&R 2017, 73 (75); Heckmann, NJW 2012, 2631 (2632); Zumkeller-Quast, JuWiss-Blog Nr. 2/2017. Und selbst 
nung ohne Angst vor Repressalien frei geäußert werden kann. ${ }^{1019}$ Die ansonsten abschreckende Wirkung und damit einhergehende Selbstzensur ${ }^{1020}$ würde insbesondere Minderheitsmeinungen schwächen. ${ }^{1021}$

Allerdings bestehen strukturelle Unterschiede, die einer Gleichsetzung der Meinungsroboter mit anonymen Äußerungen im Wege stehen: Zwar geht auch mit Pseudonymen eine Täuschungswirkung einher. ${ }^{1022}$ Während bei anonymen Äußerungen keinerlei Rückschlüsse auf den Äußernden möglich sind, verschleiert ein Pseudonym regelmäßig die Identität des Urhebers. Im Ausnahmefall kann dies sogar dazu führen, dass eine neue Identität entsteht. Gleiches gilt zwar - in potentiell größerem Ausmaß - ebenfalls für Meinungsroboter. ${ }^{1023}$ Daraus einen Gleichlauf des Schutzes der Meinungsroboter zu deduzieren, überzeugt indes nicht. Pseudonyme sollen - ebenso wie Anonymität - die eigene Identität schützen (vgl. hierzu $\mathbb{3}$ Abs. 6a BDSG a.F., Art. 4 Nr. 5 DSGVO). Sie stehen damit im engen sachlichen Zusammenhang mit der informationellen Selbstbestimmung (Art. 2 Abs. 1 GG i.V.m. Art. 1 Abs. 1 GG) ${ }^{1024}$ und haben zudem eine die Meinungsfreiheit fördernde Wirkung ${ }^{1025}$. Die Täuschungswirkung ist also notwendige Begleiterscheinung, aber nicht Hauptzweck des Pseudonyms. Bei den Meinungsrobotern hingegen geht es weniger um den Schutz der Identität des Urhebers; der Zweck eines Pseudonyms ist hier vielmehr notwendige Begleiterscheinung. ${ }^{1026}$ Hauptsächlich soll aber durch ein falsches Profile eine andere menschliche Identität suggeriert werden. Die eigene Identität tritt also in den Hintergrund und muss dies letztlich auch tun, um maximalen Erfolg bei der Einflussnahme haben zu können. Insofern geht es primär um die Teilhabe am öffentlichen Dis-

diejenigen, die anonyme Äußerungen kritischer sehen, nehmen zumindest entsprechende Begrenzungen erst im Rahmen der Abwägung vor, statt vieler siehe nur Benreuther, AfP 2011, 218.

1019 BGH NJW 2009, 2888 (2892); OLG Hamm ZD 2011, 179; Kersten, JuS 2017, 193 (196). Vgl. hierzu auch Steinbach, JZ 2017, 653 (661) hinsichtlich des damals geplanten Auskunftsanspruchs des $₫ 14$ Abs. 2 TMG.

1020 So bezeichnet es der BGH, NJW 2009, 2888 (2892).

1021 Aufgrund der Schweigespirale (oben 2. Teil, B.III.3.a) besteht bereits das Phänomen, dass (radikale) Minderheitsmeinungen oftmals nicht kundgegeben werden. Der Schutz einer anonymen Äußerung würde also dem entgegenwirken. Nimmt man das BVerfG wörtlich - der Schutz von Meinungen besteht auch bei gefährlichen, wertlosen oder irrationalen Meinungen - ist dieser Schutz dann aber gerade zwingend, um ein „faktisches Mehrheitsdiktat“ zu vermeiden.

1022 So Steinbach, ZRP 2017, 101 (103) und Schröder, DVBl. 2018, 465 (467). I.E. auch ZumkellerQuast, JuWiss-Blog Nr. 2/2017. Milker, InTeR 2017, 199 (202) spricht von einer strukturellen Vergleichbarkeit.

1023 Jedenfalls andeutend auch Steinbach, ZRP 2017, 101 (103).

1024 Im Ansatz auch Milker, ZUM 2017, 216 (218); deutlicher ders., InTeR 2017, 199 (202); Spindler/ Nink, Recht der elektronischen Medien, $\mathbb{1 3}$ TMG Rn. 21; Knopp, DuD 2015, 527; Caspar, ZRP 2017, 233 (233, 235); ähnlich Roßnagel/Scholz, MMR 2000, 721 (722).

1025 Zutreffend deshalb BGH NJW 2009, 2888 (2892) - spickmich.de. Daneben siehe Milker, ZUM 2017, 216 (218); vgl. auch Caspar, ZRP 2015, 233 (235). Auch der Europarat erkennt diesen Zusammenhang, Empf. „On the Protection of human rights with regard to social networking services v. 04.04.2012, CM/Rec(2012)4 unter I. 3.

1026 In diese Richtung auch Semizoğlu, in: Hetmank/Rechenberg, Kommunikation, Kreation und Innovation, S. 79 (91). 
kurs mittels Täuschung und weniger um den Schutz vor Selbstzensur, den Art. 5 Abs. 1 S. 1 GG gewährleisten will. ${ }^{1027}$ Insoweit unterscheiden sich Meinungsroboter also schon strukturell erheblich von den geschützten Modalitäten. ${ }^{1028}$

Ähnliches gilt auch für die Täuschungswirkung: Während Pseudonyme in sozialen Netzwerken erkennbar sein können, gilt das für Meinungsroboter gerade nicht. Gerieren sie sich als reale Person, indem das Profil mit scheinbar echten personenbezogenen Daten gefüllt und so eine falsche Identität kreiert wurde, so fehlt die maßgebliche "Warnfunktion“, die eine anonyme oder pseudonyme Äußerung für den Rezipienten hat. Dieser ist sich der maschinellen Äußerung und der möglichen Manipulation nicht bewusst. Hierdurch wird der Wirkungsgrad der Meinung aber vergrößert. ${ }^{1029}$ Anders als Steinbach ${ }^{1030}$ es nahelegt, muss gerade deshalb auch die Vervielfältigung als Strukturelement berücksichtigt werden. Hier zeigt sich noch deutlicher, dass die Meinungsroboter mit der geschützten Pseudonymität kaum gleichgesetzt werden können ${ }^{1031}$, da es hierbei doch fast ausschließlich um die Erschaffung eines „Kommunikationsnetzwerkes“ unter Ausnutzung der strukturellen Besonderheiten sozialer Medien und Netzwerke geht. Diese Unterschiede würden folglich eine differenzierte Behandlung der Meinungsroboter durchaus rechtfertigen.

\section{(II.) Berücksichtigung der Rechtsprechung bei unrichtigen Zitaten}

Der Ausschluss wird daneben auch mit der verfassungsgerichtlichen Judikatur zu unrichtigen Zitaten begründet, ${ }^{1032}$ wo ebenfalls die Parallel zu den unwahren Tat-

1027 Siehe hierzu BGH NJW 2009, 2888 (2892) - spickmich.de mit Verweis auf Ballhausen/Roggenkamp, K\&R 2008, 403 (406). Hierauf weist letztlich auch $v$. Ungern-Sternberg, in: Unger/dies., Demokratie und künstliche Intelligenz, S. 3 (18f.); auch Andersen, HRN 2017, 9 (11) sieht diesen strukturellen Unterschied.

1028 So im Ergebnis auch Semizoğlu, in: Hetmank/Rechenberg, Kommunikation, Kreation und Innovation, S. 79 (91). Vgl. auch v. Ungern-Sternberg, in: Unger/dies., Demokratie und künstliche Intelligenz, S. 3 (18f.), die hier zwar trotz der soeben aufgezeigten Unterschiede von einer ,anonymen Modalität“ spricht, aber sodann zu dem Schluss kommt, dass diese nicht „gleichermaßen schützenswert ist“, ebd., S. 19. Vgl. auch Andresen, HRN 2017, 9 (11). Und auch mit Blick auf die US-amerikanische Freedom of Speech des First Amendement gibt Stricke, Vanderbilt Journal of Entertainment \& Technology Law Vol. 22 (2020), 839 (889), zu bedenken: „Anonymity can increase speech by reprisal $[\ldots]$. But the speaker's alleged right to pose online as another individual seems harder to defend in a context as dire as an election".

1029 Vgl. hierzu BVerfGE 97, 391 (402) - Missbrauchsbezichtigung, das im dort zu entscheidenden Fall davon ausging, dass die mit der Aussage (des sexuellen Missbrauchs und der Nicht-Nennung des eigenen Namen in diesem Zusammenhang) verbundene „Wirkung [...] auf die Öffentlichkeit [...] verringert [wird], weil die mit der Namensnennung regelmäßig verbundene Glaubhaftigkeit und Authentizität der Schilderung auszubleiben droht." Das bedeutet aber - argumentum e contrario dass diese Authentizität bei einer menschlich wirkenden Identität in der Regel gegeben ist.

1030 Steinbach, ZRP 2017, 101 (103).

1031 In diese Richtung Andersen, HRN 2017, 9 (11). Milker, InTeR 2017, 199 (202) meint, dass Bots über die pseudonyme Nutzung wesentlich hinausgehen.

1032 So etwa $v$. Ungern-Sternberg, in: Unger/dies., Demokratie und künstliche Intelligenz, S. 3 (18). 
sachenbehauptungen gezogen wird. ${ }^{1033}$ Auf den ersten Blick mag das überzeugen, denn auch mit einem unrichtigen Zitat versucht der Zitierende Aufmerksamkeit durch die Verbindung einer Aussage mit einer (angesehenen) Person zu erhalten. Bei Meinungsrobotern liegt der Fall aber tendenziell anders. Hier existiert bereits die Person des Äußernden nicht. Insoweit mag zwar eine unwahre Modalität vorliegen. Nicht übersehen werden darf aber zum einen, dass im Falle unrichtiger Zitate auch der Äußerung selbst kein Wert beigemessen wird. Im vorliegenden Fall ist die Äußerung aber an sich geschützt, sofern sie sich in den aufgezeigten Grenzen bewegt. Also würde allein eine unwahre Modalität den an sich berechtigten Schutz verhindern. ${ }^{1034}$ Und zum anderen darf nicht übersehen werden, dass das BVerfG die Wahrheits- bzw. Kennzeichnungspflicht bei interpretierenden Wiedergaben von Zitaten vor allem auch mit den kollidierenden Grundrechten des Zitierten begründet. ${ }^{1035}$ Hier aber ist das Persönlichkeitsrecht eines anderen durch die Äußerung gerade nicht tangiert, sofern fiktive Nutzer erstellt werden. Vielmehr bleibt allein die Täuschungswirkung der Anknüpfungspunkt.

\section{(III.) Widerspruch zum Schutzzweck?}

Widerspricht aber allein die Identitätstäuschung dem Schutzzweck in einer Weise, die eine Zurücknahme des Schutzes rechtfertigt? Dass der Begriff der Meinungsroboter negativ konnotiert ist, ist auch der Tatsache geschuldet, dass überwiegend nur die negativen Auswüchse dieses Phänomens berücksichtigt werden. ${ }^{1036}$ Nur vereinzelt werden auch andere Anwendungsbereiche aufgezeigt. ${ }^{1037}$ Das ist zwar verständlich, weil aus wissenschaftlicher Sicht dort ein größeres Interesse besteht, wo auch ein höheres Gefahrenpotential auszumachen ist. Und in der Tat liegt in solchen Fällen der Schluss nahe, es ginge nicht mehr um eine sachliche Auseinandersetzung und um „die Stärke des Arguments“1038. Gleichwohl führt diese einseitige Diskussion womöglich zu einem Trugschluss, denn die

1033 BVerfGE 54, 208 (219 f.) - Böll, wobei auch darauf hingewiesen wird, dass durch die damit einhergehende Wahrheitspflicht für Zitate keine unangemessene und zu strenge Wahrheitspflicht an die Zitierenden gestellt wird.

1034 Kritisch deshalb zur Schutzbereichsausnahme grundsätzlich auch Semizoğlu, in: Hetmank/Rechenberg, Kommunikation, Kreation und Innovation, S. 79 (90).

1035 BVerfGE 54, 208 (220 ff.) - Böll.

1036 So etwa nur Thieltges/Hegelich, ZfP 2017, 493; Steinbach, ZRP 2017, 101 und Milker, ZUM 2017, 216 in Bezug auf die Auswirkungen auf den Wahlkampf, Volkmann, MMR 2018, 58 aus strafrechtlicher Sicht oder Janal/Isikay, GRUR-Prax 2018, 393 für einen kommerziellen Einsatz. Selbst diese Arbeit bildet davon ja keine Ausnahme. Dieses Vorgehen ist ja auch nur verständlich, weil die „guten Einsatzarten“ von weniger politischer Relevanz sind. Diese Einseitigkeit in der Diskussion sieht auch Siri, in: Akademie der Wissenschaften zu Göttingen, Digitalisierung, Privatheit und öffentlicher Raum, S. 95 (102).

1037 So etwa von Velásquez/Yazdani/Suárez-Serrato, SocialBots Supporting Human Rights, mit Blick auf eine unterstützende Rolle im Kommunikationsprozess oder Graham/Ackland, in: Gehl/Bakardjieva, Socialbots and their friends, S. $187 \mathrm{ff}$.

1038 Brings-Wiesen, JuWiss-Blog Nr. 93/2016. 
eigentlich problematischen Fälle - seien es persönlichkeitsverletzende, hetzerische oder desinformative Inhalte - fallen entweder schon aus dem Schutzbereich der Meinungsfreiheit heraus oder würden oftmals hinter den kollidierenden Rechten zurücktreten. Im Übrigen handelt es sich aber um Inhalte, die auf eine sachliche Auseinandersetzung gerichtet sind und als solche den Schutz der Meinungsfreiheit ohne Weiteres genießen. ${ }^{1039}$ Insoweit würde man also die Modalität einer Äußerung höher bewerten als den eigentlichen Inhalt selbst, obwohl dieser tendenziell entscheidender für den Schutzzweck sein kann. ${ }^{1040}$ Diese Aufwertung der Modalität gegenüber dem Inhalt vernachlässigt aber, dass es durchaus Einsatzmöglichkeiten gibt, die dem Schutzzweck der Meinungsfreiheit gerade dienlich sind. Besonders ${ }^{1041}$ zwei Studien können legen dies nahe: eine Studie aus Mexiko $^{1042}$ und eine jüngere Studie zur Covid-19-Kommunikation. ${ }^{1043}$ In einer mexikanischen Region kam es am 22.05.2015 während einer Razzia zu einem Schusswechsel zwischen vermeintlichen Kartellmitgliedern und der Polizei. Dabei starben 42 Zivilisten. ${ }^{1044}$ Wie ein Bericht der Comision Nacional de Derechos Humanos $^{1045}$ (Nationale Kommission für Menschenrechte) später herausfand, wurden 22 von ihnen willkürlich hingerichtet. Unter dem Hashtag „\#Tanhuato“ sollen Bots versucht haben, die Informationsdiffusion zu beeinflussen und einen Trend zu erschaffen, um die Aufmerksamkeit auf den Bericht und das staatliche Fehlverhalten zu lenken. ${ }^{1046}$ Auch während der Covid-19-Pandemie wurden ähnliche Phänomene hinsichtlich kritischer Äußerungen zum Vorgehen Chinas in Hongkong beobachtet, die ansonsten ignoriert oder zensiert worden wären. ${ }^{1047}$

Auch in derlei Fällen ${ }^{1048}$ wird über die Identität getäuscht, ohne dass aber das Ziel der Meinungsfreiheit, einen Austausch der Ansichten und eine bereichernde öffentliche Kommunikation zu ermöglichen, in einer gleichen Weise gefährdet werden würde. Das verdeutlicht, dass durch die Identitätstäuschung einmal mehr

1039 In diese Richtung auch Krüper, in: Unger/v. Ungern-Sternberg, Demokratie und künstliche Intelligenz, S. 67 (79).

1040 Grundsätzlich dieser Ansicht auch Semizoğlu, in: Hetmank/Rechenberg, Kommunikation, Kreation und Innovation, S. 79 (90). So auch Lang/Wilms, Staatsrecht II, Rn. 631.

1041 Siehe auch die Verweise in Fn. (1037). Und schon Boshmaf u.a., Computer Networks 57 (2013), 556 (559), haben hierauf hingewiesen, m.w.N.

1042 Velásquez/Yazdani/Suárez-Serrato, SocialBots Supporting Human Rights, S. 13.

1043 Darauf deuten etwa erste Ergebnisse von Ferrara, What Types of COVID-19 Conspiracies are Populated by Twitter Bots?, arXiv, S. 20, hin.

1044 Siehe hierzu Castillo, 43 killed in Mexican shootout, CNN.

1045 Comisión Nacional de los Derechos Humanos, Recomendación Nr. 4VG /2016.

1046 Über die Streaming-API von Twitter wurden die Account-Ids gefiltert und mit Hilfe des Dienstes BotorNot überprüft. Von den knapp 9.700 Usern wurden 1800 als Bots eingestuft.

1047 Ferrara, First Monday Vol. 25 (2020) Nr. 6.

1048 Daneben siehe auch zu möglichen positiven Einsätzen Brachten u.a., Social Bots in Social Media Crisis Communication oder Coleman, Journal of Media Ethics Vol. 33 (2018), 120 (126). Hagen u.a., Social Science Computer Review, 1 (2) gehen sogar - allerdings bezogen auf die webbasierten Botprogramme insgesamt - davon aus, dass Webbots gewöhnlich sind „and typically benign“. 
das Paradigma des technischen Fortschritts zu Tage tritt: ${ }^{1049}$ Grundsätzlich bietet er fortschrittliche und positive Einsatzmöglichkeiten, in den falschen Händen ist er aber auch dem Missbrauch zugänglich. Wegen dieser negativen Auswirkungen gleich ein gesamtes Feld ohne Differenzierungen (weitestgehend) vom grundrechtlichen Schutz auszunehmen, wird dem nicht gerecht, ${ }^{1050}$ denn „the role and landscape of socialbots is more complex than simple binary categorizations. "1051

Zwar geht auch Semizoğlu grundsätzlich von dem Schutz der Meinungsfreiheit für Meinungsroboter aus, allerdings will er eine Ausnahme zulassen: Im US-amerikanischen Präsidentschaftswahlkampf sollen bewusst Nutzerkonten mit spanischen Namen erstellt worden sein, um die lateinamerikanische Bevölkerung $\mathrm{zu}$ beeinflussen und so die schlechten Umfragewerte für den konservativen Kandidaten Donald J. Trump zu verbessern. Hierzu sollen entsprechend positive Beiträge veröffentlicht worden sein, um dem negativen Meinungsklima zu begegnen. ${ }^{1052}$ Nach Semizoğlu soll den Modalitäten in derlei Fällen „eine ähnlich gewichtige Relevanz wie [dem] Inhalt [zukommen]." ${ }^{1053}$ Aber auch er folgert die Unzulässigkeit eher aus dem zu missbilligendem Einsatzzweck. Denn denkbar kann die konkrete Gestaltung von Profilen etwa auch dort werden, wo bestimmte andere Nutzergruppen angesprochen werden sollen. So könnte man versuchen, Verschwörungstheoretiker - sei es im Rahmen der jüngsten Covid-19Pandemie oder in sonstigen Fällen - gezielt mit richtigen Fakten zu versorgen, indem man Profile mit vergleichbaren Interessen u.ä. erstellt, um entsprechende Netzwerke zu „infiltrieren“. Wenn aber entsprechende staatskritische, eklatant falsche und für Verunsicherung sorgende Informationen in ihrer Wirkungsweise gestört werden sollen, ist das aus Sicht des Kommunikationsprozesses weniger problematisch. Insofern mögen eher die besonderen Umstände des Falles - es ging um eine ethnische Differenzierung während des Wahlkampfes - Anlass für

1049 Ähnlich Ferrara u.a.., Communications of the ACM 59 (7), 96 (97); Milker, ZUM 2017, 216 (218) spricht davon, dass der Algorithmus (der Bots) „wie jede andere technische Entwicklung [...] auch freiheitseinschränkend für Dritte eingesetzt werden“ kann. Dies klingt zumindest auch bei Haeg, JPE 2017, 85 (88) an: „The Problems with malicious polibots (...) aren't problems or features connected to the polibot technology per se, but rather the specific (...) aims they have".

1050 Mit Blick auf die US-amerikanische Diskussion ähnlich, Lamo/Calo, UCLA Law Review 66 (2019), 988 (1024): „, But there seems to be no basis by which to lump all bot speech together as a category. To do so, especially at this early stage of its development, would prejudge bot speech and perhaps enable or even encourage the censorship of that speech. " Diese grundsätzlichen Bedenken finden wohl auch die Stütze bei Dankert/Dreyer, K\&R 2017, 73 (74) oder Steinbach, ZRP 2017, 101 (102), wenn sie von einem technikneutralen Schutz durch Art. 5 Abs. 1 S. 1 GG sprechen. Immerhin wird auch an anderer Stelle dafür plädiert, sachlich korrekt zu differenzieren, um den unterschiedlichen Erscheinungsformen gerecht zu werden, so bspw. Ballhausen/Roggenkamp, K\&R 2008, 403 (405) hinsichtlich Bewertungsplattformen. Und auch hinsichtlich der Bots wird teilweise eine Kategorisierung in gut oder schlecht vorgenommen, so etwa Ferrara u.a., Communications of the ACM Vol. 59, 96 f. oder Haeg, JPE 2017, 85 (87).

1051 So Velásquez/Yazdani/Suárez-Serrato, SocialBots Supporting Human Rights, S. 13.

$1052 \mathrm{Zu}$ diesem konkreten Fall siehe auch Woolley/Howard, Bots Unite to Automate the Presidential Election.

1053 Semizoğlu, in: Hetmank/Rechenberg, Kommunikation, Kreation und Innovation, S. 79 (90). 
eine besonders kritische Bewertung gewesen sein. Die grundlegenden Fragen stellen sich aber in jedem Fall, in dem gezielt bestimmte Nutzergruppen adressiert werden sollen.

\section{bb. Meinungsmacht - quantitative Legitimität}

Eng mit der Identitätstäuschung verwoben ist auch das zweite Charakteristikum, das eine Diskussion um eine Begrenzung des Schutzbereiches dominiert: die Meinungsmacht bzw. die quantitative Legitimität. ${ }^{1054}$ Im Kern geht es hierbei um folgende Überlegung: Durch den Einsatz einer Vielzahl von Bots erlangt die Öffentlichkeit nicht nur Kenntnis von einer bestimmten Meinung. Vielmehr wird dadurch der Anschein erweckt, sie habe einen bestimmten Rückhalt in der Gesellschaft. Andresen sieht darin eine direkte Manipulation des Meinungsmarktes. ${ }^{1055}$ Tatsächlich lässt sich diese potentielle Manipulation auch sozialpsychologisch unter Rekurs auf die Theorie der Schweigespirale begründen. ${ }^{1056}$ In diesen Fällen geht es nicht mehr um die Stärke des Arguments und damit um eine geistige Auseinandersetzung, sondern vielmehr um eine subtile, quantitative Täuschung und eine Manipulation der Mehrheitsverhältnisse, ${ }^{1057}$ die letztlich auch die kommunikative Chancengleichheit einseitig verändert. ${ }^{1058}$ Gleichwohl argumentiert Zumkeller-Quast, dass solch verzerrende Darstellungen „schon mindestens solange möglich [seien], wie es schriftliche Meinungsäußerung gibt. “1059 Bei Printmedien kann die verzerrende Darstellung aber auf eine medienpolitische Einstellung des Presseverlages zurückgeführt werden, von dem der Leser grundsätzlich auch Kenntnis erlangen kann. ${ }^{1060}$ Und zudem entspricht die Darstellung letztlich den redaktionellen Leitlinien, die ohnehin nur eine Auswahl des Weltgeschehens erfassen. ${ }^{1061}$ Meinungsroboter geben zwar auch eine persönliche Meinung wieder, allerdings entsteht hier ja gerade der Eindruck, dass nicht ein bestimmter Verlag oder eine bestimmte Person dieser Ansicht ist, sondern dass

1054 Hierauf berufen sich etwa Löber/Roßnagel, MMR 2019, 493 (495) und insbesondere Andresen, HRN 2017, 9 (11f.). Zur quantitativen Legitimität insbesondere Brings-Wiesen, JuWiss-Blog Nr. 93/2016. Und Schröder, DVBl. 2017, 465 (468) bezeichnet dies als „Verzerrung des Meinungsmarktes".

1055 Andresen, ebd.

1056 So letztlich auch die Argumentation von Löber/Roßnagel, MMR 2019, 493 (495). Zur Schweigespirale bereits oben, 2. Teil, B.III.3.a. und mit Blick auf die Auswirkung für die Meinungsfreiheit anderer Nutzer oben, A.II.1.

1057 So auch Löber/Roßnagel, a.a.O.

1058 Dazu ausführlicher oben 3. Teil, 2. Kap. A.II.2.b. Auch Andresen, HRN 2017, 9 (12) argumentiert hiermit, wenn er ausführt, dass „,[d]er Einzelmeinung übriger Personen [...] als Konsequenz faktisch ein geringeres Gewicht schon deshalb [zukomme], weil diese einer vermeintlich schon gefestigten Gegenmeinung gegenüber steht."

1059 Zumkeller-Quast, JuWiss-Blog Nr. 2/2017.

1060 Vgl. Paal/Hennemann, JZ 2017, 641 (642).

1061 Paal/Hennemann, a.a.O. 
ein erheblicher Teil der Gesellschaft eine Ansicht teilt. ${ }^{1062}$ Anders als bei Medien, die bewusst gewählt werden, beeinflussen Meinungsroboter Rezipienten auf einer wesentlich subtileren Ebene. ${ }^{1063}$

Entscheidender ist, dass die Veränderung der quantitativen Legitimität zwangsläufige Begleiterscheinung der Meinungsroboter ist und zwar unabhängig vom konkreten Einsatzziel. Denn auch in der mexikanischen Fallstudie wird die Relevanz eines Inhaltes fiktiv gesteigert. Aber auch dort ist der verbreitete Inhalt (besonders) schutzwürdig, weil er zentrale Informationen zu einem öffentlichen Anliegen enthält und damit Grundlage des Kommunikationsprozesses ist. Insoweit würde (erneut) allein die Modalität den inhaltlichen Schutz verdrängen. Das scheint aber nicht angezeigt, denn auch ansonsten prävaliert der Inhalt die Umstände einer Äußerung. Das zeigt sich nicht nur daran, dass die Rechtsprechung die Kategorie der „unwahren Tatsachenbehauptungen“ restriktiv handhabt. Es muss zum einen um Fälle gehen, wo keine zulässige Meinung mit der Aussage verbunden ist und zum anderen muss die faktische Grundlage evident fehlen. Nur dann kann kein positiver Beitrag zum Kommunikationsprozess geleistet werden. Und im Kontext öffentlicher Debatten reicht der sachliche Kern einer Aussage aus, um diffamierende Elemente zu verdrängen und eine Vermutung zugunsten der freien Rede zu begründen. ${ }^{1064}$ Im Falle von Bots verlässt man diesen zurückhaltenden, vorsichtigen Ansatz aber, wenn ganz ohne Differenzierung das gesamte Phänomen vom grundrechtlichen Schutz ausgeschlossen werden soll. Das lässt nicht nur die notwendige Vorsicht vermissen, sondern ist in dieser Pauschalität abzulehnen. ${ }^{1065}$

\section{cc. Manipulative Zielsetzung}

Ob tatsächlich erhebliche Spannungen mit dem Schutzzweck der Meinungsfreiheit bestehen, ist allein von der Intention des Verwenders abhängig. Nur wenn er in boshafter Absicht agiert, kann die Kumulation von Identitätstäuschung und Meinungsmacht negative Auswirkungen für den Kommunikationsprozess zeitigen. Entscheidend wäre für eine Schutzbereichsausnahme - wenn überhaupt - also nur die Differenzierung zwischen kommunikationsfördernder und -behindernder Zielsetzung. ${ }^{1066}$ Unabhängig von der Frage, ob eine solche Differenzierung im

1062 Diesen strukturellen Unterschied erkennt auch Schröder, DVBl. 2018, 465 (468); zu den Unterschieden auch Gasser/Kraatz, VerfBlog, 2017/1/16.

1063 Zu den Unterschieden bereits ausführlich oben, 2. Teil, C.VI.

1064 Siehe etwa BVerfG NJW 2020, 2631 Rn. 16, 22 - Beleidigung von Politikern; NJW 2020, 2636 Rn. 15, 19 - Beleidigung einer Amtsperson, jeweils m.w.N.

1065 I.E. letztlich auch Milker, InTeR 2017, 199 (202); ausführlicher zu den Verzerrungen des Meinungsmarktes Schröder, DVBl. 2017, 465 (468f.). Anders indes Andersen, HRN 2017, 9 (12).

1066 Diese Abgrenzungsmöglichkeit erkennt jedenfalls auch Schröder, DVBl. 2018, 465 (468), obgleich er sie mangels Praktikabilität - ohne weitere Gedanken hierzu - ablehnt. Haeg, JPE 2017, 87 differenziert zwischen malicious und saintly; Coleman, Journal of Media Ethics, Vol. 33 (2018), 120, 
grundrechtlichen Bereich überhaupt angezeigt ist, ${ }^{1067}$ führt das aber vor allem zu Beurteilungs- und Bewertungsproblemen. Was ist der Maßstab für eine manipulative Absicht? Wenn Minderheitsmeinungen mannigfaltig veröffentlicht werden, heißt das nicht zwingend, dass damit ein Missbrauch des Kommunikationsprozesses gewollt ist. Bestes Beispiel hierfür sind die bereits erwähnten mexikanischen Bots. Offensichtlicher hingegen mag die Beurteilung in den Fällen sein, in denen Diskussionen von Bots durch aggressive Kommentare oder Falschinformationen gestört werden sollen. ${ }^{1068} \mathrm{Ab}$ wann aber die Schwelle zur manipulativen Zielsetzung überschritten wird, ist unklar. Probleme ergeben sich hier vor allem deshalb, weil zunächst einmal der Nachweis einer Automatisierung erbracht und sodann die konkrete Zielsetzung aus den Inhalten ermittelt werden müsste. Das wird zwangsläufig zu erheblichen Beweis- und Beurteilungsschwierigkeiten führen. ${ }^{1069}$

\section{(I.) „Prima-facie-Beweis“ einer manipulativen Absicht?}

Am einfachsten wäre es daher, prima facie von einer manipulativen Absicht auszugehen. Da aber mit einem Anscheinsbeweis die Beweisführung durch die Berücksichtigung der allgemeinen Lebenserfahrung erleichtert werden soll, ${ }^{1070}$ müsste gerade ein solcher Erfahrungswert hier vorliegen. Voraussetzung wäre insoweit, dass zumindest mit einer hohen Wahrscheinlichkeit ${ }^{1071}$ eine boshafte Intention vorliegt. Ausgehend von der Dominanz boshafter Bots in wissenschaft-

zwischen civilly und uncivilly. Und Stieglitz u.a., Categorisation of Social Bots, S. 4, unterscheiden zwischen „benign“ and „malicious“.

1067 Im Kontext von personenbezogenen Äußerungen und auf Abwägungsebene etwa kritisch Lenski, Personenbezogene Massenkommunikation als verfassungsrechtliches Problem, S. 235 f. Und allgemein ablehnend wohl auch Schimmele, Staatliche Verantwortung für diskursive Integrität, S. 122.

1068 So lag etwa der Fall beim \#YaMeCanse in Mexiko, siehe hierzu Suárez-Serrato u.a., in: Spiro/Ahn, SocInfo 2016: Social Informatics, S. 269 ff. Haeg, JPE 2017, 85 (87) spricht in diesen Fällen ebenfalls von „malicious polibots“.

1069 Sinnbildlich hierfür ist die Aussage von Thieltges u.a., Effects of Social Bots on the Iran-Debate on Twitter, S. 3: „[...] the fraudulent intent could only be assumed, because the specific intention of the users or the 'backers' is unknown".

1070 Entwickelt wurde dieser im Zivilrecht bei schwer nachvollziehbaren Handlungsabläufen, in denen eine Vielzahl von Faktoren eine Rolle spielten. Ursprünglich betraf dies Schiffskollisionen, bei denen vor allem natürliche Faktoren wie Wind, Wellengang und Meeresströmungen die maßgeblich zu berücksichtigenden Faktoren waren. In derlei Fällen schien es unbillig, die „Beweisnot“ (so Diederichsen, VersR 1966, 211 [217]) einer Partei aufzubürden, wenn nach allgemeiner Erfahrung objektive Anhaltspunkte einen bestimmten Schluss nahegelegt haben, hierzu Stück, JuS 1996, 153. Im Zivilrecht wird etwa prima facie von der Ursächlichkeit einer Verletzung von Verkehrssicherungspflichten für die entstandene Rechtsgutverletzung im Rahmen von $₫ 823$ Abs. 1 BGB ausgegangen, siehe hierzu u.a. RGZ 134, 237 (240); BGH, v. 06.11.1973 - VI ZR 76/72, Rn. 10 ff. - juris. Eine umfangreiche Darstellung zivilrechtlicher Fallgruppen findet sich bei Prütting, in: MüKo ZPO, $\$ 286$ Rn. 67-79. Er ist deshalb „von größter Bedeutung“, ders., a.a.O., Rn. 48.

1071 Hierzu Prütting, in: MüKo ZPO, Bd.1, $\$ 286$ Rn. 56-60. 
lichen und medialen Beiträgen ${ }^{1072}$ wäre dies auf den ersten Blick anzunehmen. Auf den zweiten Blick ist diese Dominanz aber trügerisch, weil sie auch dem erhöhten Interesse der Wissenschaft geschuldet sein kann. ${ }^{1073}$ Hinzu kommt, dass es sich bei den Bots um ein neues Phänomen handelt, welches noch nicht abschließend bewertet wurde und sich ständig weiterentwickelt. Anders als bei Erfahrungssätzen zu Auffahrunfällen ${ }^{1074}$ oder Schiffskollisionen ${ }^{1075}$, bei denen letztlich auch unveränderliche Naturgesetze relevant werden, können sich so Neuerungen ergeben, die ein Anscheinsbeweis nicht hinreichend berücksichtigt. Und letztlich macht nicht allein der Einsatz von Bots die manipulative Absicht aus. Vielmehr kommt es entscheidend auch auf die Intention der Verwender an. Insofern werden die Handlungsanweisungen und die konkreten Inhalte und Umstände des Einsatzes wesentlich deutlicher zeigen, welche Ziele verfolgt werden. In dem Einsatz mag zwar ein Indiz für eine bösartige Absicht des Verwenders gesehen werden, ein entsprechender Erfahrungssatz lässt sich dem aber nicht ohne Weiteres entnehmen. Schon deshalb dürfte ein Anscheinsbeweis ausscheiden. ${ }^{1076}$

Aber auch aus grundrechtlicher Sicht ist dies abzulehnen. So ist anerkannt, dass die fehlerhafte Einordnung einer Aussage als Schmähkritik regelmäßig zu einer eigenständigen Verletzung des Grundrechts der Meinungsfreiheit führt, ${ }^{1077}$ weil bereits auf der „Deutungsebene Vorentscheidungen über die Zulässigkeit oder Unzulässigkeit von Äußerungen fallen [...]." ${ }^{1078}$ Gleiches muss dann aber auch bei falscher Bewertung der Absicht oder zu hohen Anforderungen an die Entkräftung des Anscheinsbeweises gelten. Denn wenn eine Verletzung der Meinungsfreiheit bei der fehlerhaften Einordnung einer Äußerung als Schmähkritik vorliegt - also in Fällen, in denen der Schutzbereich zwar eröffnet ist, die Mei-

1072 Siehe hierzu nur die einschlägigen rechtswissenschaftlichen Abhandlungen, die sich mit dem Thema beschäftigen, so bspw. Steinbach, ZRP 2017, $101 \mathrm{ff} . ;$ Volkmann, MMR 2018, $58 \mathrm{ff}$.; Wolf, WRP 2019, 440 ff. Und auch die hierzu durchgeführten Studien erfolgten eher zu den negativen Seiten des Boteinsatzes, so etwa nur Forelle u.a., Political Bots Venezuela, 2016; Suárez-Serrato u.a., in: Spiro/ Ahn, SocInfo 2016: Social Informatics, S. 269ff; Howard/Woolley/Calo, Journal of Technology \& Politics Vol. 15, $81 \mathrm{ff}$.

1073 Auf diese Einseitigkeit weisen auch Haeg, JPE 2017, 85 (87) und Cresci, Communications of the ACM Vol. 63 (2020), 72 (73), hin. Und auch Siri, in: Akademie der Wissenschaften zu Göttingen, Digitalisierung, Privatheit und öffentlicher Raum, S. 95 (102) zeigt hier gewisse Unterschiede im Umgang mit politischen und unpolitischen Bots auf. Kritisch zum Schluss „social bots = social bad“, Coleman, Journal of Media Ethics Vol. 33 (2018), 120 (122).

1074 Hierzu nur BGH NJW 2017, 1177 Rn. 9 m.w.N. auf die höchstrichterliche Judikatur.

1075 BGH NJW-RR 1989, 474 (475).

1076 Kokott, Beweislastverteilung und Prognoseentscheidung, S. 101, weist ebenfalls hinsichtlich der grundrechtlichen Beweislastverteilung daraufhin, dass diese „nicht durch abstrakte Regelungen wie [...] Prima-facie-Beweis, Beweisnot, Beweislastumkehrung etc.“ gelöst werden könne, sondern vielmehr auch hierbei die Grundrechte zu beachten sind. Anders aber Klein/Bethge, in: Maunz u.a., BVerfGG, $\mathbb{3} 30$ Rn. 10.1, die sich - wenn auch vage - für die Möglichkeit eines Anscheinsbeweises aussprechen.

1077 Siehe hierzu u.a. BVerfGE 93, 266 (295) - Soldaten sind Mörder; BVerfG, NJW 2019, 600 Rn. 19 Hexenprozess m.w.N.

1078 BVerfGE 93, 266 (295) - Soldaten sind Mörder. 
nungsfreiheit aber hinter das allgemeine Persönlichkeitsrecht zurücktritt ${ }^{1079}$-, dann muss das erst recht gelten, wenn der Schutz vollständig versagt wird. ${ }^{1080}$ Ansonsten käme es auch hier „im Ergebnis zur Unterdrückung einer zulässigen Äußerung. “1081

\section{(II.) Materielle Beweislast des Grundrechtsträgers?}

Trägt der Grundrechtsträger dann aber wenigstens die materielle Beweislast? Eine Orientierungshilfe könnten insoweit die Grundrechte sein, für deren Schutzbereich persönliche Motive oder Umstände maßgeblich sind, also zum einen die Religions- und Gewissensfreiheit (Art. 4 GG) und zum anderen das Asylgrundrecht (Art. 16a GG). Hier trägt der Grundrechtsträger die Darlegungs- und Beweislast für die geistig-religiöse Motivation seines Handelns bzw. für seinen Status als politisch Verfolgter, wobei allerdings das erforderliche Beweismaß gesenkt wird. ${ }^{1082}$ Im Falle von Gewissenskonflikten (Art. 4 Abs. 2, 3 GG) obliegt es dem Grundrechtsträger, objektivierbare Anhaltspunkte glaubhaft zu machen, aus denen mit einer gewissen Sicherheit auf das Vorliegen einer Gewissensnot geschlossen werden kann. ${ }^{1083}$ Im Falle der Religionsfreiheit muss er „hinreichend substantiiert [darlegen], dass sich das Verhalten tatsächlich nach geistigem Gehalt und äußerer Erscheinung in plausibler Weise dem Schutzbereich des Art. 4 GG zuordnen lässt, also tatsächlich einer als religiös anzusehenden Motivation [entspringt]. “1084

1079 Siehe hierzu nur BVerfG NJW 1999, 2262 (2263); BVerfGE 61, 1 (12) - Meinungsäußerung im Wahlkampf; 93, 266 (294) - Soldaten sind Mörder und aus neuerer Zeit BVerfG NJW, 1460 Rn. 14 - Obergauleiter der SA-Horden; BVerfG, NJW 2019, 719 Rn. 11 - Äußerung in kommunalpolitischer Auseinandersetzung, und BVerfG, Beschl. v. 14.06.2019 - 1 BvR 2433/17 - Hexenprozess.

1080 Das BVerfG formuliert: „Urteile, die den Sinn der umstrittenen Äußerung erkennbar verfehlen und darauf ihre Würdigung stützen, verstoßen gegen das Grundrecht auf Meinungsfreiheit“, BVerfGE 93, 266 (295) - Soldaten sind Mörder.

1081 So BVerfGE 93, 266 (295) - Soldaten sind Mörder.

1082 Zur Beweis- und Darlegungslast bei Art. 16a GG u.a. BVerwG NVwZ 1985, 658 (660). Zum Herabsenken des Beweismaßes heißt es dort: „Die Beweisschwierigkeiten des Flüchtlings bestehen häufig - im Fehlen der üblichen Beweismittel. In der Regel können unmittelbare Beweise im Verfolgerland nicht erhoben werden. Mit Rücksicht darauf kommt dem persönlichen Vorbringen des Kl. und dessen Würdigung gesteigerte Bedeutung zu. Zur Asylanerkennung kann schon allein der Tatsachenvortrag des Asylsuchenden führen, sofern seine Behauptungen unter Berücksichtigung aller sonstigen Umstände in dem Sinne, glaubhaft (Hervorh. d. Autor) sind, daß sich das Tatsachengericht von ihrer Wahrheit überzeugen kann.“

1083 Vgl. hierzu etwa BVerwG, NJW 1973, 576 (578) zum ärztlichen Notfalldienst; NVwZ 1994, 578 (579) zu einem religiös bedingten Konflikt und BeckRS 2018, 19416 Rn. 14 zur Kriegsdienstverweigerung.

1084 BVerfGE 138, 296 (329) - Kopftuchverbot; ebenso BVerfG NJW 2017, 461 (467) - Stilleschutz Karfreitag; andeutend BVerfG, NJW 2017, 381 (383) - Kopftuchverbot Kindergarten; a.a.O., 2333 (2335) - Kopftuchverbot Hessen. Zustimmend, Mückl, in: BK-GG, Art. 4 Rn. 89; de Wall/Muckel, Kirchenrecht, $\mathbb{1} 10$ Rn. 2. Das BVerwG, E 94, 82 (87) spricht expressis verbis von einer Darlegungslast. 
Da es auch im Falle der Meinungsroboter um innere, geistige Voraussetzungen geht, lassen sich jedenfalls gewisse Parallelen nicht von der Hand weisen. Eine analoge Anwendung muss aber dennoch ausscheiden, weil schon die Interessenlage vollkommen unterschiedlich ist. Zum einen ist die Herangehensweise bei Art. 4 GG dem Umstand geschuldet, dass der Staat den Beweis gar nicht führen kann, ohne selbst eine Bewertung von Gewissensentscheidungen oder religiöser Motive vorzunehmen, was ihm aber gerade verwehrt sein soll; ${ }^{1085}$ allein der Grundrechtsträger bzw. die jeweilige religiöse Gemeinschaft befindet darüber, welche Inhalte und Gebote ihren Glauben und ihre Religion prägen. ${ }^{1086}$ Es kommt also bereits bei der Interpretation des Schutzgehaltes maßgeblich auf das Selbstverständnis an. Zum anderen lassen sich Gewissensentscheidungen nicht als zutreffend oder unzutreffend nachweisen. ${ }^{1087}$ Überdehnt man daher in diesen Fällen die Beweisanforderungen an den Grundrechtsträger, so kommt es zu einer Entleerung des Grundrechts. Andererseits führten geringe Anforderungen zu einer Ausdehnung bis hin zu einem „uneingeschränkten Verweigerungsrecht“. ${ }^{1088}$ Insoweit ist der Rekurs auf das Glaubhaft-Machen ein Kompromiss, der diese Problemlage entschärft. Und während es bei Art. 4 GG um die Frage geht, ob der Schutzbereich der Religions- und Gewissensfreiheit überhaupt eröffnet ist, ${ }^{1089}$ steht dies für die Äußerungen der Bots prinzipiell fest und es soll nur eine Rückausnahme gemacht werden. Deshalb käme nur ein eigenständiger Ansatz für die materielle Beweislast bei Art. 5 Abs. 1 S. 1 GG in Betracht.

\section{(1.) Negative Grundregel der Beweislast}

Das gestaltet sich aber im grundrechtlichen Bereich deutlich schwieriger ${ }^{1090}$ als bspw. im Zivilprozess, wo die objektive Beweislast die beweisbelastete Partei trifft und im Falle eines non liquet vom Nichtvorliegen der streitigen Tatsache

1085 Muckel, in: Berliner Kommentar GG, Art. 4 Rn. 39. Der Staat soll gerade nicht „theologische Dispute (...) schlichten“, so Mückl, in: BK-GG, Art. 4 Rn. 89. So letztlich auch das BVerfG, dass darauf hinweist, dass der Staat andernfalls die „nach dem Grundgesetz gewährte Eigenständigkeit und [...] Selbständigkeit [...] [verletzt]“, E 24, 236 (248) - Aktion Rumpelkammer.

1086 Vgl. hierzu in Ansätzen BVerfGE 41, 29 (49) - Simultanschule. Deutlicher wird dies u.a. in BVerfGE 108, 282 (298 f.) - Kopftuch; 138, 296 (329) - Kopftuchverbot in NRW.

1087 Ähnlich Jarass/Pieroth, GG, Art. 4 Rn. 46, die darauf verweisen, dass die Schwierigkeit auch daraus resultiert, dass es - anders als bei der Religionsfreiheit - keine geteilte Gewissensauffassung gibt, an der man sich orientieren kann.

1088 Zu diesem Zwiespalt Herzog, in: Maunz/Dürig, GG, Art. 4 Rn. 161. Ähnlich auch die Zusammenfassung von Kokott, Beweislastverteilung, S. 435.

1089 „Da das Grundrecht selbst nur unter dieser einschränkenden Voraussetzung gewährleistet ist,“ bürdet das BVerwG NJW 1959, 1792 (1793) explizit dem Grundrechtsträger die Beweislast auf.

1090 Kokott hat sich in ihrer Habilitationsschrift diesem Problemkreis ausführlich angenommen, dies., Beweislastverteilung bei Grund- und Menschenrechten, 1993. 
ausgegangen wird (sog. negative Grundregel). ${ }^{1091}$ Hinsichtlich der Motivation des Urhebers besteht aber das erste Problem bereits darin, dass es sich hierbei nur um eine im Wege der teleologischen Auslegung gewonnenen Grenze handelt, nicht aber um ein festes Tatbestandsmerkmal wie die „politische Verfolgung “ in Art. 16a GG oder die „Gewissensentscheidung“ bei Art. 4 GG. Und selbst wenn man diese Grundregel dann trotzdem anwendet, kommt es zu der von Herzog angesprochenen Entleerung der Grundrechte ${ }^{1092}$. Denn in Zweifelsfällen wären die speziellen Grundrechte dann nie einschlägig.

\section{(2.) Normentheorie}

Im Verwaltungsprozess wird, sofern das materielle Recht keine Aussagen trifft, an die Normentheorie angeknüpft. ${ }^{1093}$ Danach trägt derjenige die Beweislast, dem die fraglichen Umstände bzw. das fragliche Merkmal einer Norm zugutekommen würde. ${ }^{1094}$ Die Günstigkeit ist dabei „durch Auslegung der materiell-rechtlichen Norm zu ermitteln“, insbesondere auch unter Berücksichtigung einschlägiger Grundrechte ${ }^{1095}$, wobei danach zu fragen ist, „welche Verteilungsanordnung die in ihr enthaltene ungeschriebene Beweislastnorm trifft. “1096 Beruft sich der Grundrechtsträger auf seine Glaubensfreiheit, so muss er jedenfalls belegen, warum sie einschlägig ist, um den Schutz beanspruchen zu können. Er beruft sich auf ein ihm günstiges Abwehrrecht gegenüber dem Staat. Im Falle der Meinungsroboter würde der Schutz der Meinungsfreiheit dem Grunde nach aber eingreifen,

1091 Hierzu OLG Düsseldorf, ZUM 2005, 825 (833); Saenger, in: ders., ZPO, \$286 Rn. 34, 53. Nagler, Beweislast im Öffentlichen Recht, S. 108 ff., 462, will diese Grundregel auch im Öffentlichen Recht zur Anwendung bringen.

1092 Herzog, in: Maunz/Dürig, GG, Art. 4 Rn. 161.

1093 Siehe hierzu BVerwG, LKV 2008, 171 (176); NVwZ 2008, 1371 (1375); Schmidt-Aßmann, in: Maunz/Dürig, GG, Art. 19 Abs. 4 Rn.228; Vgl. Engel/Pfau, in: Mann/Sennekamp/Uechtritz, VwVfG $\$ 24$ Rn. 60, die von einem Grundsatz sprechen. Ausführlicher zur Normentheorie im Öffentlichen Recht Dawin, in: Schoch/Schneider/Bier, VwGO, $\$ 108$ Rn. 90 ff. Freilich gibt es zahlreiche Ansätze zur Beweislastverteilung in grundrechtlichen Konstellationen. Grabitz hat beispielsweise zum Grundsatz der Verhältnismäßigkeit ein System von Vermutungs- bzw. Beweislastregeln entwickelt. Grundsätze wie in dubio pro auctoritate oder in dubio pro libertate werden zwar stets genannt, wegen der unzureichenden Differenzierung aber zu Recht abgelehnt und kommen deshalb ebenfalls nicht in Betracht, so etwa Schmidt-Aßmann, in: Maunz/Dürig, GG, Art. 19 Abs. 4 Rn. 227 m.w.N.

1094 BVerwGE 80, 290 (296f.) m.w.N.; 131, 171 (Rn. 41); Benda/Klein, Verfassungsprozessrecht, Rn. 320; Lenz/Hansel, BVerfGG, $\$ 26$ Rn. 12; siehe auch Schenke, in: BK-GG, Art. 19 Abs. 4 Rn. 142; Ibler, in: Friauf/Höfling, GG, Art. 19 Abs. 4 Rn. 251; Lechner/Zuck, BVerfGG, $\mathbb{} 26$ Rn. 6 und Schmidt-Aßmann, in: Maunz/Dürig, GG, Art. 19 Abs. 4 Rn. 228.

1095 Schmidt-Aßmann, in: Maunz/Dürig, GG, Art. 19 Abs. 4 Rn. 228. Das BVerwG hat beispielsweise hinsichtlich der Rechtmäßigkeit von Tatsachen im Verfassungsschutzbericht - und damit in einem grundrechtssensiblen Bereich - dem Verfassungsschutz die objektive Beweislast aufgebürdet, BVerwGE 131, 171 (Rn. 41 f.). Im Falle der Kriegsdienstverweigerung hat es gerade auch auf diesen Grundsatz hingewiesen, aber ihn nicht angewendet, da Art. 4 Abs. 3 GG keine Ausnahmeregel gegenüber einer allgemeingültigen Wehrpflicht beinhalte, BVerwG NJW 1959, 1792 (1793).

1096 BVerwGE 131, 171 (Rn. 41). 
der Grundrechtssauschluss wäre daher eine für den Grundrechtsträger ungünstige Tatsache. Weil sich freiheitsbeschränkende Maßnahmen dann nicht mehr am strengen Maßstab des Art. 5 Abs. 2 GG messen lassen müssen, wäre dies eine für den Staat günstige Tatsache. ${ }^{1097}$ Der Einwand einer manipulativen Absicht gleicht dann in der Wirkung einer rechtsvernichtenden Einwendung, weshalb der Staat beweisbelastet sein müsste. Das entspräche auch der Rechtfertigungslast: Nicht der Grundrechtsträger muss sich für die Ausübung seiner Grundrechte rechtfertigen, sondern der Staat für die Einschränkung eben dieser. ${ }^{1098}$

\section{(3.) Grundrechtsimmanente Beurteilung der Beweislastverteilung}

Kokott hat sich bei der Inanspruchnahme von Grundrechten gegen abstrakte Regeln ausgesprochen. ${ }^{1099}$ Vielmehr müsse man die konkrete Beweislastverteilung im Einzelfall aus dem einschlägigen Grundrecht, insbesondere aus der ihm immanenten Wertung gewinnen. ${ }^{1100}$ Hieraus werden sich aber kaum Unterschiede ergeben. Begreift man die Meinungsfreiheit nicht nur als individualschützendes Freiheitsrecht, sondern weist man ihr auch eine objektiv-rechtliche, demokratische Funktion - und damit elementare Bedeutung ${ }^{1101}$ - zu, trägt zwingend der Staat die objektive Beweislast. Die demokratische Bedeutung von Kommunikation führt zu einer Vermutung zugunsten der freien Rede. ${ }^{1102}$ Diese Vermutung würde aber konterkariert, wenn man in Fällen eines non liquet dem Grundrechtsträger die Beweislast aufbürdet, sodass der Grundrechtsschutz regelmäßig keine Wirkung entfaltet. Das ist durchaus vergleichbar mit den Fällen, in denen der Wahrheitsgehalt von Tatsachen nicht feststeht. Auch hier trägt nicht der Grundrechtsträger die Beweislast für den Wahrheitsgehalt der Tatsache. Vielmehr bleibt der

1097 Vgl. Pieroth/Hartmann, DVBl. 2002, 421 (424), die bei einer Anwendung von Art. 2 Abs. 1 GG anstelle von Art. 12 Abs. 1 GG davon sprechen, dass „dem Staat die Rechtfertigung leichter [fällt]“.

1098 Vgl. BVerfG NJW 2007, 2167 Rn. 21 und im Anschluss daran BVerwGE 131, 171 (Rn. 41).

1099 Siehe hierzu Kokott, Beweislastverteilung, S. 71-88.

1100 Kokott, Beweislastverteilung, S. 113-119. Das scheint deshalb plausibel, weil so die Grundrechte nicht nur materiell-rechtliche, sondern gerade auch verfahrensrechtliche Bedeutung erlangen würden. Anderswo wird gerade auch die verfahrensrechtliche Absicherung als Aspekt der Grundrechtsverwirklichung auch aus dem individuellen Grundrecht und nicht etwa aus Art. 19 Abs. 4 GG hergeleitet (Dolde, NVwZ 1982, 65; Starck, in: v. Mangoldt/Klein/ders., GG, Art. 1 Rn. 199 ff.; Schmitz, in: Stelkens/Bonk/Sachs, VwVfG, $\mathbb{S} 1$ Rn. 45 ff. Zum fehlenden Aussagegehalt von Art. 19 Abs. 4 zur Beweislastverteilung etwa Schmidt-Aßmann, in: Maunz/Dürig, GG, Art. 19 Abs. 4 Rn. 227; Niehaus, Beweismaß und Beweislast, S. 448 f.; Kokott, Beweislastverteilung, S. 99 f.). Die eigentliche Beweislastverteilung bei einem non liquet ist aber für einen effektiven (prozessualen) Grundrechtsschutz von zentraler Bedeutung (andeutend Kokott, Beweislastverteilung, S. 102, und Schmidt-Aßmann, in: Merten/Papier, HbGR II, $\mathbb{} 45 \mathrm{Rn}$. 47). Zudem lassen sich so auch für die unterschiedlichsten Konstellationen sachgerechte Ergebnisse herstellen, obgleich der Rechtssicherheit damit kein Gefallen getan wird. Kritisch zu dem Ansatz von Kokott etwa Dawin, in: Schoch/Schneider/Bier, VwGO, $\mathbb{} 108$ Rn. 98 ff.

1101 Grundlegend hierzu BVerfGE 7, 198 (207f.) - Lüth.

1102 BVerfGE 7, 198 (212) - Lüth; 30, 336 (354) - jugendgefährdende Schriften; 85, 1 (16) - Bayer-Aktionäre; 124, 300 (324) - Wunsiedel. 
Schutz aufrechterhalten. ${ }^{1103}$ Erst auf Ebene der Rechtfertigung erfolgen mögliche Beschränkungen, so etwa durch den das Persönlichkeitsrecht Dritter schützende $\$ 186$ StGB. Wenn aber bei nur derivativ geschützten Inhalten ${ }^{1104}$ (Tatsachenbehauptungen) der grundrechtliche Schutz aufrechterhalten bleibt, muss dies auch für alle Inhalte von Meinungsrobotern gelten. Denn Anknüpfungspunkt ist hier nur die Modalität, nicht aber der Inhalt.

\section{(4.) Fazit: keine materielle Beweislast des Grundrechtsträgers}

Wenn auch im Ausgangspunkt eine Parallele zu Art. 4 GG naheliegt, zeigt sich bei näherer Betrachtung, dass man es mit unterschiedlichen Ausgangslagen zu tun hat, die einer analogen Anwendung der Grundsätze im Wege stehen. Vielmehr müsste ein eigener Ansatz gefunden werden. Nach den bisher geltenden Beweislastverteilungsregeln würde aber niemals der Grundrechtsträger die Beweislast tragen, weil es sich bei der manipulativen Absicht um einen Einwand gegen den grundsätzlich bestehenden grundrechtlichen Schutz handelt. Die Zuweisung des non liquet stünde zudem in unmittelbaren Widerspruch mit der grundsätzlich weit verstandenen Vermutung des Vorranges der freien Rede. Ausnahmsweise bewahrheitet sich hier also das Prinzip in dubio pro libertate.

\section{Fazit: Schutz der Meinungsroboter durch die Meinungsfreibeit}

So sehr die moralischen, ethischen, rechtlichen und politischen Bedenken, die der Einsatz von Meinungsrobotern auslöst, auch Anlass zu Kritik geben mögen, eine Schutzbereichsausnahme können sie nicht rechtfertigen. ${ }^{1105}$ Weder die technische Seite der Äußerungen noch die möglichen Täuschungen über die Identität oder die quantitative Legitimität können einen Ausschluss überzeugend und widerspruchsfrei begründen. Andernfalls käme man zu Problemen bei fortschreitender Digitalisierung und Technologisierung, wenn man den Grundrechtsschutz nicht technikneutral begreift. Zudem verbietet sich die pauschale Verurteilung der immanenten Täuschungswirkung. Das liegt aber nicht an dieser selbst, sondern vielmehr an dem prävalierenden Schutz der Inhalte. Erhebliche Konflikte mit dem Schutzzweck der Meinungsfreiheit ergeben sich ohnehin nur dort, wo der Einsatz von einer manipulativen Zielsetzung getragen ist. Überzeugender wäre es also, diese zum Gegenstand einer Differenzierung zu machen. Allerdings

1103 Zur Erinnerung: die einschlägige Grenze wird erst bei bewusst oder erwiesen unwahren Tatsachen gezogen.

1104 Jestaedt, in: Merten/Papier, HbGR IV, $\$ 102$ Rn. 35.

1105 So im Ergebnis auch Steinbach, ZRP 2017, 101 (102 f.); Milker ZUM 2017, 216 (220); derS. InTeR 2017, 199 (201f.); Zumkeller-Quast, JuWiss-Blog Nr. 2/2017; Dankert/Dreyer, K\&R 2017, 73 (75). Differenzierender Semizoğlu, in: Hetmank/Rechenberg, Kommunikation, Kreation und Innovation, S. 79 (90), Kritik hierzu aber bereits oben A.I.3.b.aa(III.). 
stellen sich dann schwierige Bewertungs- und Beurteilungsfragen, die nicht zufriedenstellend innerhalb des Schutzbereiches beantwortet werden können. Die Ablehnung einer Schutzbereichsausnahme bedeutet auch nicht, dass Meinungsroboter grenzenlos aktiv sein können und sollten. Grenzen können dann aber nicht schon wegen des fehlenden spezifischen Schutzes der Meinungsroboter und des daraus folgenden Überwiegens der Schutzinteressen (leichter) gerechtfertigt werden. Vielmehr bedarf es dann einer präziseren und vorsichtigeren Abwägung zwischen den kollidierenden Interessen. ${ }^{1106}$

\section{Grundrechtlicher Schutz der Interessen der Netzwerkbetreiber}

Bei einer Regulierung können aber auch die Interessen der Netzwerkbetreiber tangiert sein. Das gilt vor allem dann, wenn sie selbst - etwa wegen ihrer Algorithmen, der Nutzeroberfläche oder aus anderen Überlegungen - Adressat einer Regelung sind. In erster Linie wird es hierbei vor allem um die Berufsfreiheit aus Art. 12 Abs. 1 GG gehen. Auch wenn überwiegend die Stammsitze innerhalb des europäischen Binnenmarkts im EU-Ausland liegen, so bedeutet das jedenfalls nicht den Ausschluss vom Anwendungsbereich des Art. 19 Abs. 3 GG ${ }^{1107}$ und damit vom grundrechtlichen Schutz. Schon wegen des Diskriminierungsverbotes aus Art. 18 AEUV ist jedenfalls im Ergebnis auch im EU-Ausland ansässigen (juristischen) Personen derselbe grundrechtliche Schutz zu gewähren. Allein die dogmatische Herleitung dieses Ergebnisses ist umstritten. ${ }^{1108}$ Eine notwendige zumindest objektiv berufsregelnde Tendenz ${ }^{1109}$ kommt Maßnahmen hier auch regelmäßig zu, etwa dann, wenn unmittelbar Regeln für die Diensterbringung aufgestellt werden. Meist wird es hierbei aber nur um Berufsausübungsregelungen gehen, die vergleichsweise leicht zu rechtfertigen sind, weil hierfür bereits „vernünftige Überlegungen des Gemeinwohls“ ausreichen, sofern die Regelung zur Verfolgung dieser Ziele auch verhältnismäßig ist. ${ }^{1110}$ Dabei steigen die Anforde-

1106 Vgl. Schimmele, Staatliche Verantwortung für diskursive Integrität, S. $122 \mathrm{f}$.

1107 Grundlegend hierfür BVerfG 129, 78 (92ff.) - Anwendungserweiterung.

1108 Dieser Schutz kann einerseits durch eine Extension des Deutschenbegriffs im Lichte des Art. 18 AEUV gewährt werden (in diese Richtung wohl Breuer, in: Isensee/Kirchhof, HbStR VIII, $\mathbb{1 7 0}$ Rn. 43, wenn er von der „supranationalen Erweiterung des Trägerkreises“ spricht), andererseits aber auch dadurch, dass der Anwendungsvorrang des Diskriminierungsverbotes eine „europarechtskonforme“ Anwendung notwendig macht, sodass nach Art. 12 GG i.V.m. Art. 18 AEUV jedenfalls EU-Ausländer geschützt werden, in diese Richtung etwa Ruffert, in: Epping/Hillgruber, BeckOK GG, Art. 12 Rn. 37. Wegen des eindeutigen Wortlauts des Art. 12 Abs. 1 GG wird anderswo der Schutz über Art. 2 Abs. 1 GG gewährt und die jeweils spezifischen Schrankenregelungen übertragen, um eine Diskriminierung im Ergebnis zu vermeiden, so etwa das BVerfG, NJW 2016, 1436 Rn. 10ff. - Preisbindung Arzneimitte; siehe auch Starck, in: v. Mangoldt/Klein/ders., GG, Art. 1 Rn. 208 und Manssen, a.a.O., Art. 12 Rn. 267 Dreier, in: ders., GG, Vorbem. Rn. 116 jeweils m.w.N.

1109 Siehe hierzu statt vieler BVerfGE 129, 208 (266f.) - TKÜ-Neuregelung m.w.N.

1110 Grundlegend zu den Rechtfertigungsanforderungen für die Berufsausübung BVerfGE 7, 377 (405) Apotheken-Urteil. 
rungen an die Verhältnismäßigkeit allerdings auch mit der Eingriffsintensität, insbesondere wenn es um spezifische Regelungen für die Nutzeroberfläche oder den Algorithmus geht. Denn beide sind letztlich für den wirtschaftlichen Erfolg der Netzwerkbetreiber ursächlich. Die auf einfachen Interaktionen beruhende Oberfläche führt zu deutlich mehr Interaktionen der Nutzer und zu einer einfachen Handhabung des Netzwerks selbst. ${ }^{1111}$ Und diese Interaktionen sind letztlich maßgebliche Größe für die auf Personalisierung angelegten Prozesse und damit auch für die Vermarktung entsprechender Werbeflächen. ${ }^{1112}$ Daneben sorgt der Algorithmus letztlich für die Weitergabe der personalisierten Informationen und versucht dadurch Nutzer möglichst lange im Netzwerk „gefangen“ zu halten. ${ }^{113}$ Auch dies hat letztlich mittelbaren Einfluss auf die Vermarktung der Werbeplätze und damit wesentliche Bedeutung für den wirtschaftlichen Erfolg. Die privaten Unternehmen investieren viel Zeit und Aufwand in die (Fort-)Entwicklung und Optimierung ihrer Dienste. Auch im Wettbewerb hat der jeweilige Algorithmus eine wesentliche Bedeutung, was sich letztlich auch aus den jeweiligen QuasiMonopolen entsprechender Anbieter ableiten lässt. ${ }^{1114}$ Aus all diesen Gründen wird ersichtlich, warum die Algorithmen auch gegenüber Nutzern weitestgehend geheim gehalten und nur oberflächliche und abstrakte Informationen geteilt werden. ${ }^{1115}$ Dieses Geschäfts- und Betriebsgeheimnis genießt daher einen besonderen grundrechtlichen Schutz, ${ }^{1116}$ sodass an den Algorithmus anknüpfende Regelungen einen eingriffsintensiven Charakter haben können. ${ }^{1117}$ Bei alldem ist zudem zu berücksichtigen, dass aus Sicht des Übermaßverbots Differenzierungen angezeigt

1111 Hierzu Lischka, AfP 2018, 388 (389). Zur grundrechtlichen Bedeutung der Anzahl der Nutzer und der Attraktivität der Dienste vgl. OLG Nürnberg, Urteil vom 04.08.2020 - 3 U 3641/19= GRUR-RS 2020, 23456 Rn. 63.

1112 Zum Zusammenhang von personalisierungsmaßnahmen und Refinanzierung etwa Heldt, CR 2018, 494 (495); Schneiders, Das ist doch Diskriminierung, S. 3 m.w.N. Ausführlicher zur Finanzierung der Netzwerke und Intermediäre etwa Tief, Kommunikation auf Facebook, Twitter \&YouTube, S. 30 f. Daneben siehe auch BKartA, Beschl. v. 06.02.2019 - B6-22/16, BeckRS 2019, 4895 Rn. $220 \mathrm{f}$.

1113 Vgl. Kaiser/Reiling, in: Unger/v. Ungern-Sternberg, Demokratie und künstliche Intelligenz, S. 85 (88).

1114 Auf dem Gebiet der Suchmaschinen ist Google mit seinem Pagerank-Algorithmus weit abgeschlagen der Marktführer. Im Bereich der sozialen Netzwerke dominiert Facebook weitestgehend den Markt u.a. mit den Diensten Facebook und Instagram, zur Marktbeherrschung siehe etwa BKartA, Beschl. v. 06.02.2019 - B6-22/16 = BeckRS 2019, 4895 Rn. 374 ff.

1115 Ähnlich auch Drexl, ZUM 2017, 529 (531).

1116 Martini, Blackbox Algorithmus, S. 38; Schulz/Dankert, Informatik Spektrum 2017, 352 (354); explizit (auch) zu Art. 12 Abs. 1 GG Werner, NJOZ 2019, 1041 (1043) zum Page-Rank-Algorithmus von Google, allerdings mit Blick auf Art. 14 Abs. 1 GG, Heilmann, MMR 2020, 162 (165); vgl. auch Paal/Hennemann, ZRP 2017, 76 (78); Zwar nicht explizit auf den grundrechtlichen Schutz stellte der BGH bei der Tätigkeit der Schufa ab, gleichwohl sprach er von dem besonders schutzwürdigen Interesse an der Geheimhaltung der Scoreformel, die sie nicht offenlegen müsste, BGH, NJW 2014, 1235 Rn. 15.

1117 Mit Blick auf Art. 14 Abs. 1 GG Heilmann, MMR 2020, 162 (165); hinsichtlich einer Offenlegungspflicht Paal/Hennemann, ZRP 2017, 76 (78); dies., JZ 2017, 641 (652); Hoffmann-Riem, AöR 142 (2017), 1 (32). 
sein können, soweit ansonsten unzumutbare Belastungen entstehen. So können für marktstärkere Netzwerkunternehmen durchaus strengere Anforderungen in Betracht kommen als für kleinere Anbieter. ${ }^{1118}$ Auch gleicheitsrechtlich ist das ohne Weiteres möglich.

Weil der Algorithmus aber auch Ergebnis einer geistigen Leistung ist, aus eigenen Mitteln selbst geschaffen wurde und exklusiv dem jeweiligen Unternehmen zugeordnet wird, ${ }^{1119}$ kommt daneben auch ein Schutz als Eigentum im Sinne des Art. 14 Abs. 1 GG in Betracht. ${ }^{1120}$ Dieser kann sich insbesondere neben Art. 12 Abs. 1 GG aktualisieren, wenn - aus Gründen des Allgemeinwohls - die Offenbarung der Funktionsweise der Algorithmen verlangt oder i.Ü. inhaltliche Anforderungen an sie (Neutralität, Must-Carry o.ä.) gestellt und insoweit Inhalts- und Schrankenbestimmungen getroffen werden.

Und letztlich lässt sich auch darüber diskutieren, ob die Leistung der Intermediäre insgesamt nicht auch als Rundfunk i.S.d. Art. 5 Abs. 1 S. 2, 1. Var. GG einzuordnen wäre. ${ }^{1121}$ Auch hier steht Art. 18 AEUV einer Diskriminierung wegen des Niederlassungsortes im Wege, sodass auch im EU-Ausland ansässige Unternehmen sich nach Art. 19 Abs. 3 GG auf den Schutz berufen können. ${ }^{1122}$ Und richtiger Weise sind auch die Leistungen der digitalen Medienintermediäre als Rundfunk anzusehen. ${ }^{1123}$ Die Zusammenfassung der Medienfreiheiten ${ }^{1124}$ oder die Erfindung einer neuen Internetfreiheit ${ }^{1125}$ bedarf es hierfür nicht. Angesichts der Technikneutralität des grundrechtlichen Schutzes sind nicht nur lineare Dien-

1118 Denn was für den einen Anbieter lediglich eine Berufsausübungsschranke darstellt, kann für den anderen zu einer faktischen Berufswahlschranke werden, wenn nach Art und Umfang solche Pflichten statuiert werden, dass sie einen erheblichen und nicht zumutbaren Aufwand für kleinere Unternehmen bedeuten. Zu diesen fließenden Übergängen zwischen Berufsausübungs- und Berufswahlregelung siehe etwa Scholz, in: Maunz/Dürig, GG, Art. 12 Rn.342; Manssen, in: v. Mangoldt/Klein/ Starck, GG, Art. 12 Rn. 141.

1119 Vgl. zur einfach-rechtlichen Zuordnung, auch als Geschäftsgeheimnis, Martini, Blackbox Algorithmus, S. 33 ff. Und speziell zum auch urheberrechtlichen Schutz statt vieler Dreier, in: ders./Schulze, UrhG, $\mathbb{6} 69$ a Rn. 22.

1120 Heilmann, MMR 2020, 162 (165). Siehe auch BGH, GRUR 2013, 751 Rn. 26 - Autocomplete, zu Suchmaschine von Google.

1121 Anzweifelnd etwa OLG Nürnberg GRUR-RS 2020, 23456 Rn. 65.

1122 Ausführlich hierzu BVerfGE 129, 78 (94 ff.) - Anwendungserweiterung.

1123 So auch Paal, MMR 2018, 567 (568); Heilmann, MMR 2020, 162 (165). Ausführlich zur Einordnung von Suchmaschinen als Rundfunk etwa Hartl, Suchmaschinen, Algorithmen und Meinungsmacht, S. 103 ff. Kritisch etwa OLG Nürnberg, GRUR-RS 2020, 23456 Rn. 65.

1124 So etwa Kellner, Regelung der Meinungsmacht, S. 110 ff.; in diese Richtung auch Kühling, in: Gersdorf/Paal, Informations- und Medienrecht, Art. 5 GG Rn. 76.

1125 So etwa Holznagel, AfP 2011, 532 (534); ders./Schumacher, ZRP 2011, 74 (77). Hierfür besteht nur dann Bedarf, wenn der grundrechtliche Schutz erhebliche Lücken aufweist, die zu schließen wären. So lag der Fall etwa bei dem Recht auf Vertraulichkeit und Integrität informationstechnischer Systeme. Für die besonderen Gefahrenlagen gewährte Art. 2 Abs. 1 GG keinen ausreichenden Schutz, was schon an den vergleichsweise niedrigen Rechtfertigungsanforderungen lag. Vgl. hierzu BVerfGE 120, 274 (303 ff.) - Online-Durchsuchungen. 
ste erfasst. ${ }^{1126}$ Und ebenso wenig lassen sich aus dem Begriff der Darbietung inhaltliche Kriterien entwickeln. Eine redaktionelle Aufbereitung kann nur taugliches Abgrenzungskriterium zwischen Medienfreiheiten und Meinungsfreiheit sein. ${ }^{1127}$ Und soweit eine publizistische oder meinungsbildende Relevanz gefordert wird, ist unklar welche qualitative Schwelle überschritten sein muss. Ohnehin hat das BVerfG ja betont, dass nicht nur klassischen Nachrichtensendungen die Rundfunkqualität zukommt, sondern auch unterhaltenden Beiträgen. Mit all diesen Ansätzen versucht man eher den befürchteten, negativen Konsequenzen der Sonderdogmatik des BVerfG zu entkommen. ${ }^{1128}$ Dass diese Sonderdogmatik ohnehin nicht überzeugt und aufgegeben werden sollte, wurde bereits ausführlicher begründet. ${ }^{1129}$ Aber selbst das BVerfG hält eine Differenzierung zwischen verschiedenen Rundfunkangeboten auf Rechtsfolgenseite für notwendig. ${ }^{1130}$ Einziges sinnvolles Merkmal bleibt neben der Verbreitung an die Allgemeinheit (Massenkommunikation) allein der elektronische Übertragungsweg. ${ }^{1131}$ Ohnehin wird der grundrechtliche Schutz nur dort relevant, wo es um die Gestaltung des „Programms“ geht. Bei Intermediären betrifft das aber vor allem - mangels eigener Inhalte - Fälle, wo Vorgaben für die algorithmenbasierte Verbreitung der fremden Inhalte, also für ihre „redaktionelle Vorfilterung“ gemacht werden. Das wäre etwa dort der Fall, wo die Benachteiligung bzw. Bevorzugung bestimmter Inhalte untersagt oder qualitative Anforderungen an die Algorithmen gestellt werden würden. Das entspräche also der „Programm- bzw. Tendenzfreiheit“ klassischer Medienunternehmen. ${ }^{1132}$

1126 So aber Starck/Paulus, in: v. Mangoldt/Klein/Starck, GG, Art. 5 Rn. 252; Kube, in: Isensee/Kirchhof, HbStR IV, $\$ 91$ Rn. 14; Holznagel, AfP 2011, 532 (534 f.), der so eine Abgrenzung zwischen Rundfunk und einer neu zu entwickelnden Internetdienstefreiheit ermöglichen will.

1127 So auch Jarass, AfP 1998, 133 (135). Denn sowohl Presse- als auch Rundfunkfreiheit sind durch redaktionelle Leistungen gekennzeichnet.

1128 Vgl. etwa Grabenwarter, in: Maunz/Dürig, GG, Art. 5 Abs. 1, 2 Rn. 606: „ein weiterer, dynamischer Rundfunkbegriff hingegen führt dazu, dass andere mediale Erscheinungsformen, die rundfunktypische ,Suggestivkraft, Aktualität und Breitenwirkung' aufweisen, ebenfalls von Verfassungs wegen reguliert werden müssten bzw. könnten“.

1129 Oben 3. Teil, A.III.

1130 BVerfGE 121, 30 (50) - Parteibeteiligung an Rundfunkunternehmen. Vgl. daneben BVerfGE 73, 118 (202) - 4. Rundfunkentscheidung in der das BVerfG es für unproblematisch ansah, dass ausländische Programme nicht der in $\$ 15$ LRG normierten Ausgewogenheit verpflichtet waren. In BVerfGE 83, 238 (302) - 5. Rundfunkentscheidung, stellte es klar, dass für neue Medien wegen der noch geringen Bedeutung, vorerst keine Grundversorgung notwendig war. Eine solche Differenzierungsmöglichkeit wird in der Literatur oftmals betont, so etwa Jarass, AfP 1998, 133 (133); Kressel Heinze, AfP 1995, 574 (577f.); Castendyk/Böttcher, MMR 2008, 13 (15); Hain, in: Spindler/ Schuster, Elektronische Medien, C. Rn. 48, 49; Dittmann, in: ders./Fechner/Sander, Rundfunkbegriff im Wandel, S. 19 (32 ff.) spricht diesbezüglich von einer Relevanztheorie. Jedenfalls andeutend Bullinger, in: Isensee/Kirchhof, HbStR VII, $\$ 163$ Rn. 150.

1131 So auch Hain, in: Spindler/Schuster, Elektronische Medien, Erster Teil C. Rn. 48; Papier/Schröder, epd Medien Nr. 60/2010, 16 (22); Wendt, in: v. Münch/Kunig, GG Art. 5 Rn. 58; wohl auch Schemmer, in: Epping/Hillgruber, BeckOK GG, Art. 5 Rn. 66 ff.

1132 Vgl. Hartl, Suchmaschinen, Algorithmen und Meinungsmacht, S. 199 f., 219; Liesem, UFITA 2019, 395 (410). Zur Tendenzfreiheit des Rundfunks etwa C. Wagner, AfP 2018, 281 (282) oder Grabenwarter, in: Maunz/Dürig, GG, Art. 5 Art. 1, 2 Rn. 746, der diese als Synonym für die Programmau- 


\section{Kommunikationsfreiheiten der Nutzer}

Neben den Interessen der Verwender und der Betreiber werden die Nutzerinteressen aber nicht nur als schutzrechtlicher Aspekt relevant. Zugleich bilden sie auch aus abwehrrechtlicher Perspektive eine wesentliche Schranke staatlichen Handelns. Dort, wo Inhalte gelöscht, gesperrt oder gekennzeichnet werden, nimmt man Einfluss auf die Meinungsfreiheit des jeweiligen Urhebers. Im Falle der Meinungsroboter wurde aber schon die empirische Forschung dafür kritisiert, dass falsch-positive Ergebnisse bei der Überprüfung von Netzwerkprofilen produziert wurden. Beabsichtigt der Staat also, dass auffällige Konten gesperrt, gelöscht oder gekennzeichnet werden, dann besteht damit auch immer das Risiko, dass entsprechende Regelungen in ihrer praktischen Anwendung originären und damit erlaubten Content berühren. Und aus Sicht der Rezipienten werden ihnen im Falle von Kennzeichnungen bestimmte Informationen de facto aufgedrängt und im Falle von Löschungen oder Sperrungen Informationen entzogen, die unter normalen Umständen zugänglich gewesen wären, sodass zumindest auch die Informationsfreiheit potentieller Rezipienten tangiert sein kann. ${ }^{1133}$

Eine solche staatliche Intervention will daneben auch das Gebot der Staatsfreiheit bzw. Staatsferne verhindern. Zwar ist dieses vor allem im Demokratieprinzip verankert und folgt nur mittelbar daraus, dass der Gesellschaft die Aufgabe zukommt, einen politischen Willen zu bilden. ${ }^{1134}$ Wegen der engen Verbindung zu den Kommunikationsgrundrechten kommt diesem aber mittelbar Bedeutung zu. Dabei geht es freilich nicht um ein umfängliches Verbot staatlicher Intervention und Aktion, denn schon durch die Öffentlichkeitsarbeit kommt es zu einer berechtigten Teilnahme an diesem Prozess. ${ }^{1135}$ Vielmehr geht es um das Verbot einer parteiergreifenden Intervention und somit um ein Sachlichkeits- und Neutralitätsgebot. ${ }^{1136}$ Wegen der Bedeutung der Medien für die Meinungsbildung kommt der Staatsferne im Medienbereich eine entscheidende Bedeutung zu. Dies wird insbesondere dadurch verwirklicht, dass die Aufsicht und Kontrolle der Einhaltung von gesetzlichen Vorgaben im Bereich der Presse durch Einrichtungen

tonomie begreift; und mit Blick auf Intermediäre etwa Kellner, Die Regulierung der Meinungsmacht von Internetintermediären, S. 147.

1133 Die Informationsfreiheit des Art. 11 EuGRCh bemüht etwa auch regelmäßig der EuGH, wenn es um die Auferlegung von Pflichten geht, die zur Sperrung oder Löschung von Inhalten führen, siehe etwa EuGH, Urt. v. 24.11.2011 - C-70/10 = MMR 2012, 174 Rn.52- Scarlet Extended SA/ SABAM; Urt. v. 16.02.2012 - C-360/10 = MMR 2012, 334 Rn. 50 - SABAM/Netlog.

1134 Hierzu siehe etwa BVerwG, NVwZ 2018, 433 Rn. 28 f. - Licht aus.

1135 Hierzu etwa nur BVerfGE NJW 2020, 2096 Rn. 49 ff. - Seehofer. Aus grundrechtlicher Sicht zur Öffentlichkeitsarbeit etwa BVerfGE 105, 252 (268f.) - Glykol; a.a.O., 279 (301ff.) - Osho und BVerwG, NVwZ 2018, 433 Rn. 18 - Licht aus.

1136 Hierzu Ostermann, Transparenz und öffentlicher Meinungsbildungsprozess, S. $354 \mathrm{ff}$. Zum Sachlichkeitsgebot siehe etwa BVerfGE 105, 252 (265 ff.) - Glykol. Hierzu auch BVerwG, NVwZ 2018, 433 Rn. 26 ff. - Licht aus, und zur Abgrenzung zum Neutralitätsgebot Rn. 23 ff. 
der freiwilligen Selbstkontrolle ${ }^{1137}$ und im Rundfunkbereich durch die staatsfern organisierten Landesmedienanstalten erfolgt.

B. (Spezifische) Grenzen der grundrechtlichen Schutzpflicht im Falle der Meinungsroboter

Neben den abwehrrechtlichen Grenzen sind aber die schon oben (3. Teil, 1. Kapitel A.I.2) angesprochenen spezifischen Grenzen der staatlichen Schutzpflicht zu berücksichtigen. Dies gilt nicht allein in Hinblick auf das einzuhaltende Untermaßverbot als Maßstab für den zu gewährenden Mindestschutz (III.). Auch mit Blick auf die territoriale Entgrenzung des Internets stellen sich Fragen zu den Grenzen des tatsächlich und rechtlich Möglichen (I.). Und letztlich gilt es auch präzise herauszuarbeiten, worin eigentlich der genaue Schutzbedarf der betroffenen Nutzer und ihrer grundrechtlichen Rechtsgüter besteht (II.). Immerhin ist der Staat nicht verpflichtet, über diesen Schutzbedarf hinaus zu gehen.

\section{Vorbehalt der Möglichkeit staatlichen Schutzes}

Die staatliche Schutzpflicht steht unter dem Vorbehalt des Möglichen. Dabei können neben rechtlichen Grenzen auch faktische bestehen, wegen derer der Staat keinen oder nur einen eingeschränkten Schutz bereithalten kann bzw. darf. Abseits der abwehrrechtlichen Implikationen kommen hier vor allem Kompetenzgrenzen nicht nur auf nationaler Ebene, sondern gerade auch im Verhältnis zur Europäischen Union in Betracht. Und aus faktischer Sicht ergeben sich im digitalen Raum auch ganz andere Grenzen als in der realen Welt.

\section{Dichotome Gesetzgebungszuständigkeiten - Adressat der Schutzpflichten}

Geht es um die Zuweisung der Zuständigkeit im föderalen Bundesstaat, so können potentiell aufgrund der dichotomen Kompetenzstruktur zwei Adressaten verpflichtet sein: Bund und Länder. Besonders veranschaulicht wird das im Bereich der (digitalen) Medienregulierung durch das Telemediengesetz des Bundes (TMG) und den Rundfunk- bzw. Medienstaatsvertrag der Länder, die koexistieren und partielle Schnittmengen aufweisen können. ${ }^{1138}$ Insoweit ist die Reichweite der Schutzpflicht nicht zuletzt auch von der Reichweite der jeweiligen Gesetz-

1137 Vor allem durch den Deutschen Presserat erfolgt diese Selbstregulierung.

1138 Das wird etwa mit Blick auf den Begriff des Telemediums deutlich oder mit Blick auf die Kennzeich-

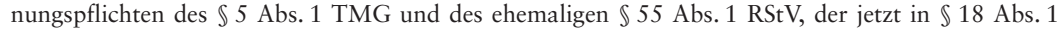
$\mathrm{MStV}$ zu finden ist. 
gebungskompetenz abhängig. Im Bereich des Medienrechts ${ }^{1139}$ ist der Bund neben der Infrastruktur und der Signalübertragung (Art. 73 Abs. 1 Nr. 7 GG) ${ }^{1140}$ vor allem für die Regulierung der wirtschaftlichen Aspekte der Medien zuständig (Art. 72 Abs. 2, 74 Abs. 1 Nr.11 GG). So wurde neben dem TMG auch das NetzDG kompetenziell auf diesen Titel gestützt. ${ }^{1141}$ Daneben käme etwa der Kompetenztitel des Strafrechts (Art. 74 Abs. 1 Nr.1, 2. Var. GG) dort in Betracht, wo es um eine repressive Pönalisierung des Einsatzes von Meinungsrobotern oder konkreter Inhalte ginge. Demgegenüber leitet sich die Kompetenz der Länder (Art. 70 Abs. 1 GG) aus ihrer Kulturhoheit ab. ${ }^{142}$ Sie sind für die publizistischen, kommunikationsbezogenen Regelungen und damit vor allem für die inhaltlichen Vorgaben im Medienbereich zuständig. ${ }^{1143}$ Freilich können hierfür auch Regelungen notwendig werden, die strafrechtlichen oder wirtschaftsrechtlichen Charakter haben, aber mit dem „kompetenzbegründenden Schwerpunkt“"1144 der Regelungen eng verbunden sind. ${ }^{1145}$ Das gilt etwa für die im zuvor im RStV bislang vorgesehenen Ordnungswidrigkeiten oder die Vorgaben für Werbung und Teleshopping im Rundfunk. ${ }^{1146}$ Wenn es also um die Regulierung der Meinungsmacht und der potentiellen Einflüsse der Meinungsroboter auf den Kommunikationsprozess geht, so ist dies Sache der Länder.

Diese duale Zuständigkeitsverteilung führt nicht selten zu Abgrenzungsschwierigkeiten. ${ }^{1147}$ Oft lässt sich nämlich nicht vermeiden, dass eine Regelung sowohl einen inhaltlichen, kommunikationsbezogenen als auch einen wirtschaftsrechtlichen Charakter aufweist. ${ }^{1148}$ So wurde dem Bund bei Erlass des NetzDG vorgeworfen, er überdehne in verfassungsrechtlich unzulässiger Weise seinen eigenen Kompetenzbereich zulasten der Länder. ${ }^{1149}$ Teilweise verliert das aber dadurch an Brisanz, dass inhaltlich deckungsgleiche Regelungen vorzufinden sind (so etwa im Falle von $\mathbb{S}$ Abs. 1 TMG und $\mathbb{S} 5$ Abs. 1 RStV bzw. $\mathbb{} 18$

1139 Grundlegend zu der Kompetenzabgrenzung zwischen Bund und Ländern im Bereich der Rundfunkregulierung BVerfGE 12, 205 (225 ff.) - 1. Rundfunkentscheidung.

1140 Vgl. hierzu BVerfG, ebd.

1141 Zum NetzDG siehe BT-Drs. 18/12356, S. 13; zum TMG siehe BT-Drs. 16/3078 S. 12.

1142 Grundlegend für das Rundfunkrecht BVerfGE 12, 205 (229) - 1. Rundfunkentscheidung. Siehe auch Martini, in: Gersdorf/Paal, Informations- und Medienrecht, $\mathbb{1}$ RStV Rn. 4; Vesting, in: Binder/ders., Rundfunkrecht, $\mathbb{\int} 1$ RStV Rn. 10; Uble, in: Maunz/Dürig, GG, Art. 70 Rn. 106.

1143 Hierzu auch Ohr, in: Schwartmann, PraxisHb Medienrecht, Kap. 11 Rn. 13.

1144 Schede/Schuldt, NVwZ 2019, 1572 (1574).

1145 Vgl. Vesting, in: Binder/ders., Rundfunkrecht, $\mathbb{} 1$ RStV Rn. 13.

1146 Zur Werberegulierung Vesting, in: Binder/ders., Rundfunkrecht, $\mathbb{S} 1 \mathrm{RStV}$ Rn. 13.

1147 So auch Martini, in: Gersdorf/Paal, Informations- und Medienrecht, $\mathbb{1}$ RStV Rn. 6.

1148 Vesting, in: Binder/ders., Rundfunkrecht, $\mathbb{S} 1 \mathrm{RStV}$ Rn. 12 weist darauf hin, dass die ursprüngliche inhaltliche Regulierung des öffentlich-rechtlichen Rundfunks mit Hinzutreten des privaten Rundfunks eher wettbewerbsrechtlich ausgerichtet ist und die Länderkompetenz hier nur wegen einer „sehr weit gehende[n] Relativierung der wirtschaftsrechtlichen Kompetenz des Bundes“ möglich ist, die aber „nicht mehr so ohne Weiteres plausibel gemacht werden kann“.

1149 Ausführlich zur Kompetenzwidrigkeit etwa Gersdorf, MMR 2017, 439 (440 ff.). Daneben u.a. auch Kalscheuer/Hornung, NVwZ 2017, 1721 (1724f.). 
Abs. 1 MStV). ${ }^{1150}$ Und auch im Bereich des Medienkonzentrationsrechts lassen sich gewisse Parallelen zum allgemeinen Kartellrecht nicht von der Hand weisen. ${ }^{151} \mathrm{Im}$ Streitfalle, wenn beide Regulierungskompetenzen berührt sind, entscheiden regelmäßig materielle Kriterien, insbesondere der eigentliche Regelungsschwerpunkt, ${ }^{1152}$ über die Zuständigkeit. ${ }^{1153}$ Und nach dem Prinzip der Bundestreue sind Bund und Länder bei der Wahrnehmung der Kompetenzen zu gegenseitiger Rücksichtnahme verpflichtet. ${ }^{1154}$

\section{Unionskompetenzen, sekundärrechtliche Medienregulierung und Herkunftslandprinzip}

Eine vergleichbar dichotome Kompetenzverteilung findet sich auch im Verhältnis der Mitgliedstaaten zur EU wieder, ${ }^{1155}$ die auch hier maßgeblich aus dem Doppelcharakter der Medien einerseits als Kulturgut, andererseits als Wirtschaftsgut resultiert. ${ }^{1156}$ Die Verträge sehen keinen eigenständigen Bereich der Medienpolitik vor; der Union fehlt also eine entsprechende, von den Mitgliedstaaten übertragene Kompetenz im Bereich der Medien ${ }^{1157}$ und wegen des Prinzips der begrenzten Einzelermächtigung (Art. 5 Abs. 1, 2 EUV) kann eine solche auch nicht begründet werden. ${ }^{1158}$ Primär liegt die Medienregulierung daher im Verantwortungsund Kompetenzbereich der Mitgliedstaaten. Für inhaltliche, pluralismussichernde Aspekte folgt dies auch hier aus ihrer „Kulturhoheit“. ${ }^{1159}$ Dass sich der unio-

$1150 \mathrm{Zu}$ dieser Kongruenz siehe auch noch später, C.I.3.c.

1151 So auch Martini, in: Gersdorf/Paal, Informations- und Medienrecht, $\mathbb{\$} 1 \mathrm{RStV}$ Rn. 6.3. Siehe hierzu auch Vesting, in: Binder/ders., Rundfunkrecht, $\mathbb{1}$ RStV Rn. $15 \mathrm{ff}$.

1152 Vgl. Martini, in: Gersdorf/Paal, Informations- und Medienrecht, $\mathbb{1}$ RStV Rn. 5; Uhle, in: Maunz/ Dürig, GG, Art. 74 Rn. 12.

1153 Ausführlicher hierzu etwa Erbguth/Schubert, in: Sachs, GG, Art. 30 Rn. 20 ff.

1154 Vgl. hierzu etwa BVerfGE 12, 205 (241f.) - 1. Rundfunkentscheidung oder E 61, 149 (205) Amtshaftung.

1155 Siehe zur vertikalen Kompetenzabgrenzung im Medienbereich etwa Knodel, Medien und Europa, S. $193 \mathrm{ff}$.

1156 Auf diesen Doppelcharakter weisen auch Burggraf/Gerlach/Wiesner, Media Perspektiven 2018, 49, Zagouras, Pluralismus in Europa, S. 57 f. und Schwarze, ZUM 2000, 779 (780) hin. Ausführlicher hierzu etwa Knodel, Medien und Europa, S. 32 ff. Seidel, NVwZ 1991, 120 (122) spricht insoweit vom „Rundfunk als Ware“ und vom „Rundfunk als öffentliche Aufgabe und Sache der Allgemeinheit unter nationaler Kontrolle“.

1157 Burggraf/Gerlach/Wiesner, Media Perspektiven 2018, 496; Schladebach/Simantiras, EuR 2011, 784; Ferreau, in: Spindler/Schuster, Elektronische Medien, Erster Teil B. Rn. 2; Oppermann/Classen/ Nettesheim, Europarecht, $\mathbb{} 34$ Rn. 50; Knödel, Medien und Europa, S. 114. So auch zur damaligen EG Seidler, NVwZ 1991, 120 (124); Schwarze, ZUM 2000, 779 (781); Hain, AfP 2007, 527 (531f.).

1158 Ferreau, in: Spindler/Schuster, Elektronische Medien, Erster Teil B. Rn. 2. Ukrow, in: Bröhmer, Europa und die Welt, S. 197 (231) überlegt, ob nicht realiter - gestützt auf die Schutzpflichten der einschlägigen grund- bzw. menschenrechtlichen Gewährleistungen - eine Veränderung des Kompetenzgefüges angesichts international agierender Unternehmen notwendig wäre.

1159 Vgl. Schladebach/Simantiras, EuR 2011, 784 (785); vgl. auch EuGH, Urt. v. 13.12.2007 Rs. C-250/06 = EuZW 2008, 86 Rn. 41 - United Pan-Europe Communications Belgium SA u.a./ Belgien, m.w.N.; EuGH, Urt. v. 25.07.1991 - Rs. C-288/89 = GRUR Int 1991, 223 Rn. 23 - Sticht- 
nale Kompetenzbereich aber im Ergebnis doch auf die nationale Medienregulierung auswirkt, ist neben einer unterstützenden Rolle im Bereich der Kultur ${ }^{1160}$ vor allem auch der Zuordnung der Medientätigkeiten zum Gewährleistungsbereich der Dienstleistungsfreiheit ${ }^{1161}$ geschuldet. Denn von national divergierenden Regelungen bei grenzüberschreitenden Sachverhalten gehen potentielle Gefahren für die Binnenmarktziele der Union aus. Dieser binnenmarktrechtliche Bezug begründet die funktionale, wirtschaftsrechtliche (Harmonisierungs-)Kompetenz der Union (Art. 59, Art. 62 i.V.m Art.53, Art. 114 AEUV). ${ }^{1162}$ Auch im Verhältnis zur EU setzt sich damit das schwierige Abgrenzungsproblem zwischen wirtschaftsrechtlichen und kulturpolitischen Fragestellungen der Medienregulierung fort, ${ }^{1163}$ was auch noch zu Problemen führen wird. ${ }^{1164}$

Inhaltlich beeinflusst vor allem das harmonisierende europäische Sekundärrecht die Medienregulierung in Deutschland. Besonders die E-Commerce-Richtlinie 2000/31/EG (ECRL) ${ }^{1165}$ und die durch sie harmonisierte Haftungsprivilegierung von Hostprovidern (Art. 14 Abs. 1 und Art. 15 Abs. 1 ECRL) wird im Kontext von Regulierungsfragen neuer digitaler - und nicht überwiegend audio-visueller - ${ }^{1166}$ Medien bedeutsam, insbesondere wenn es um inhaltliche Überwa-

ing Collectieve Antennevoorziening Gouda/Commissariaat voor de Media. Zur pluralismussichernden und inhaltsbezogenen Ausrichtung der mitgliedstaatlichen Kompetenz siehe Ferreau, ZUM 2017, 632 (638) und die Ausführungen des BVerfG in E 92, 203 (241) - EG-Fernsehrichtlinie, das gerade die Notwendigkeit einer wirtschaftsorientierten Harmonisierung des Binnenmarktes sah und diese auch deshalb akzeptierte, weil „[der] Kernbereich der Landeskompetenz zur Regelung der organisatorischen sowie politisch-kulturellen Materien des Rundfunkwesens nicht [berührt sei].“.

1160 Hier hat die Union gem. Art. 6 S. 1, S. 2 lit. c) AEUV eine Unterstützungs-, Koordinierungs- und Ergänzungsfunktion, die durch Art. 167 AEUV näher präzisiert wird. Aber hierbei geht es nur um vergleichsweise kleine Aspekte des Medienrechts, vgl. hierzu Oster/Wagner, in: Dauses/Ludwigs, Hb EU-WirtschaftsR, E. V. Rn. 7; Ferreau, ZUM 2017,632 (638).

1161 Für Betreiber sozialer Netzwerke etwa Liesching, MMR 2018, 26 (29). Mit Blick auf VideoSharing-Plattformen etwa Ukrow/Ress, in: Grabitz/Hilf/Nettesheim, Recht der EU, Art. 167 AEUV Rn. 214.

1162 Vesting, in: Binder/ders., Rundfunkrecht, $\mathbb{1}$ RStV Rn. 25 f. Vgl. zur binnenmarktrechtlichen Zielsetzung Hain, AfP 2007, 527 (531 f., 534), allerdings noch zum EGV, und Roßnagel/Kleist/Scheuer, Die Reform der Regulierung elektronischer Medien in der EU, S. 34, 40.

1163 Vgl. Vesting, in: Binder/Ders., Rundfunkrecht, $\mathbb{S} 1$ RStV Rn. 25b und Knodel, Medien und Europa, S. 150. Burggraf/Gerlach/Wiesner, Media Perspektiven 2018, 496 sprechen hier von einem „nicht immer konfliktfreie[n] Spannungsfeld mitgliedstaatlicher und gemeinschaftlicher Politik“. Ausführlicher zu der Kompetenzabgrenzung etwa Knodel, Medien und Europa, S. 103 ff., und speziell im Kontext der Vielfaltssicherung - allerdings noch zum EGV - Zagouras, Pluralismus in Europa, S. $54 \mathrm{ff}$.

1164 Hierbei geht es vor allem um die Vereinbarkeit bestimmter Regelungen des Medienstaatsvertrages für Medienintermediäre mit dem Sekundär- und Primärrecht. Siehe hierzu noch unten C.II.2.c.aa(III.) und C.II.2.f.

1165 Richtlinie 2000/31/EG des Europäischen Parlaments und des Rates vom 8. Juni 2000 über bestimmte rechtliche Aspekte der Dienste der Informationsgesellschaft, insbesondere des elektronischen Geschäftsverkehrs, im Binnenmarkt („Richtlinie über den elektronischen Geschäftsverkehr“), ABl. 2000 L 178, S. 1 ff.

1166 Hier wird nämlich die Richtlinie 2018/1808 des Europäischen Parlaments und des Rates vom 14. November 2018 zur Änderung der Richtlinie 2010/13 zur Koordinierung bestimmter Rechtsund Verwaltungsvorschriften der Mitgliedstaaten über die Bereitstellung audiovisueller Medien- 
chungs- und Prüfpflichten für Intermediäre geht. ${ }^{1167}$ Daneben wird auch das Herkunftslandprinzip aus Art. 3 Abs. 1 ECRL relevant. Es verhindert, dass nationales Sachrecht auf alle elektronischen Dienste angewendet wird, die einen Inlandsbezug haben, weil damit erhebliche Hindernisse für den Binnenmarkt einhergehen würden. ${ }^{1168}$ Deutsches Recht findet vielmehr nur auf Dienste Anwendung, die in Deutschland niedergelassen sind, also von hieraus ihre Geschäftstätigkeit tatsächlich ausüben (Art. 3 Abs. 1 i.V.m. Art 2 lit c) und Erwgr. 19 ECRL bzw. $\mathbb{S} 3$ Abs. 1, $\mathbb{S}$ a Abs. 1 S. 2 TMG), auch wenn die Dienste in anderen EU-Staaten angeboten werden (vgl. $\mathbb{} 3$ Abs. 1 TMG). Für audiovisuelle Medien, die sowohl der AVMD-RL 2018/1808 als auch der ECRL unterfallen, enthalten $\mathbb{2} 2 \mathrm{a}$ Abs. 2, 3 TMG abweichende Regelungen. Erfasst ist aber nur deutsches Recht, dass die Fragen des koordinierten Bereichs betrifft (Art. 3 Abs. 1 i.V.m. Art. 2 lit.h) ECRL) und nicht von dem Anwendungsbereich der Richtlinie ausgenommen ist. Bei grenzüberschreitenden, internationalen Fragen entscheidet zudem das deutsche Kollisionsrecht über das anwendbare Sachrecht, das dann gegebenenfalls selbst zu modifizieren ist, sofern damit Beschränkungen des Geschäftsverkehrs einher gehen. ${ }^{1169}$ Die ECRL trifft gerade keine Aussagen hierüber (vgl. Art. 1 Abs. 4 ECRL). ${ }^{1170}$

\section{Faktische Grenzen staatlicher Regulierung}

Wendet man sich den tatsächlichen Gegebenheiten und damit faktischen Grenzen einer Regulierung zu, so stößt man zunächst auf Konflikte mit dem Territorialitätsprinzip der staatlichen Gewalt. Dem Internet und seinen Diensten sind territoriale Grenzen fremd. Jeder Nutzer kann (potentiell) jeden Dienst des Internets in Anspruch nehmen und somit über Grenzen hinweg mit anderen Personen in Kontakt treten. Das führt vorliegend zu drei zentralen Problemen: Erstens müssen potentielle Urheber von Meinungsrobotern nicht vom Inland aus agieren, sondern können auch aus dem Ausland den Meinungsbildungsprozess beeinflussen, sodass sie im Einzelfall als (Regelungs-)Adressaten nur schwer greifbar sein könnten. ${ }^{1171}$ Zweitens sind auch die Anbieter selbst nur selten mit

dienste (Richtlinie über audiovisuelle Mediendienste) im Hinblick auf sich verändernde Marktgegebenheiten (ABl. 2018 L 303, S. 69 ff.) bedeutsam.

1167 Speziell zu dem Problem des Art. 15 der Richtlinie siehe noch unten im Zusammenhang mit $\mathbb{S} 93$ Abs. 4 MStV, C.II.2.c.aa(III.).

1168 Vgl. Erwgr. 5 ECRL.

1169 Ausführlicher zu der Frage des anwendbaren Kollisionsrecht und zu den daraus folgenden Problemen etwa Weller, in: Gersdorf/Paal, Informations- und Medienrecht, $\mathbb{} 3$ TMG Rn. $7 \mathrm{ff}$. und Nordmeier, in: Spindler/Schuster, Elektronische Medien, $\mathbb{3}$ TMG Rn. $9 \mathrm{ff}$. Grundlegend zu dieser Sichtweise EuGH, Urt. v. 25.10.2011 - Rs. C-509/09 u. C-161/10 = NJW 2012, 137 Rn. 53 ff. - eDateAdvertising. Und zum Beschränkungsverbot auch BGH, NJW 2012, 2197 Rn. 30.

1170 Grundlegend hierzu EuGH, Urt. v. 25.10.2011 - Rs. C-509/09 u. C-161/10 = NJW 2012, 137 Rn. 60 ff. $-e$ Date-Advertising.

1171 Vgl. Redeker, IT-Recht, D. Rn. 1291. 
einem Sitz im Inland vertreten und damit für die staatliche Gewalt greifbar. Und drittens führt das bei konkreten Inhalten nicht selten zu Problemen, weil sie aus Sicht der inländischen Rechtsordnung rechtswidrig, mit Blick auf andere Rechtsordnungen aber rechtmäßig sein können. ${ }^{1172}$ Deshalb lässt sich an der Wirksamkeit nationaler Regeln für einen internationalen Kommunikationsraum jedenfalls zweifeln. ${ }^{1173}$

Abseits dessen bringen aber auch andere internetspezifische Umstände ihre Probleme mit sich. Riesige Datenmengen gilt es im Zweifel zu analysieren, wenn entsprechende Manipulationen ausfindig gemacht werden sollen. Und wenn es um die Verantwortung der Urheber geht, so verbergen sich diese hinter der Maske der Anonymität, was Ermittlungen nicht selten erschwert. ${ }^{1174}$ Als greifbarer Adressat bleibt folglich oftmals nur der Netzwerkbetreiber. Und letztlich ist auch die (empirische) Überprüfbarkeit begrenzt, weil Netzwerkbetreiber keinen Einblick hinter die Kulissen gewähren und black boxes bleiben. Das ist insoweit problematisch, als dass eine vernünftige Regulierung zumindest eine gewisse Kenntnis über den Regulierungsgegenstand, insbesondere seine Funktionsweise, voraussetzt. ${ }^{1175}$

\section{Die Schutzbedürftigkeit der Nutzer als Grundprämisse und Grenze staatlichen Schutzes}

Geht es um den Willensbildungsprozess und potentielle Verzerrungen, so wird vor allem auch die Schutzbedürftigkeit der Nutzer relevant. Denn die potentiellen Gefahren für die Willensbildung der Nutzer resultieren nicht allein - anders als etwa in den typischen Konstellationen des Gesundheitsschutzes - von Seiten Dritter. Vielmehr werden auch autonome, unvorsichtige Entscheidungen der Nutzer conditio sine qua non für die oben beschriebenen Einflüsse der Meinungsroboter. ${ }^{1176}$ Sind Nutzer also überhaupt schutzbedürftig? Und wenn

1172 Das gilt etwa mit Blick auf die äußerungsrechtlichen Vorgaben Deutschlands und der Vereinigten Staaten. Letztere haben ein weitaus liberaleres Verständnis von Meinungsfreiheit als Deutschland. Darauf auch hinweisend Schmitz/Berndt, The German Act on Improving Law Enforcement on Social Networks: A Blunt Sword?, S. 11. Mit Blick auf die urheberrechtliche Diskussion um Uploadfilter auch Ingold, in: Unger/v. Ungern-Sternberg, Demokratie und künstliche Intelligenz, S. 183 (195).

1173 So weist Classen, in: Pirson/Rüfner/Germann/Muckel, HbStaatskirchenR, $\$ 38$ Rn. 15 auf die „[w]egen der Internationalität der elektronischen Verbreitung [...] nur begrenzt[en] Wirkung “ hin. Und auch Pille, Meinungsmacht sozialer Netzwerke, S. 279 meint, „dass [das] nationalstaatliche Recht faktisch nur noch in Grenzen funktionstüchtig ist.“. Ausführlicher zur „Entgrenzung des Internets" und den Vollzugsdefiziten, ders., ebd., S. 282 ff. In Bezug auf die hier relevanten Social Bots Dankert/Dreyer, K\&R 2017, 73 (78).

1174 Vgl. Redeker, IT-Recht, D. Rn. 1291. Mit Bezug zu den Meinungsrobotern etwa Hagen u.a., Social Science Computer Review, 1 (4).

1175 Vgl. Lischka, AfP 2018, 388 (391); Hartl, Suchmaschinen, Algorithmen und Meinungsmacht, S. 231.

1176 Allgemein zu den bestehenden internen Verzerrungen im Meinungsbildungsprozess oben, 2. Teil B.III.3.b und zur Ausnutzung dieser durch die Meinungsroboter, 2. Teil, C.III. 
ja, worin besteht die Schutzbedürftigkeit? Diese Fragen müssen in Relation zum betroffenen „Schutzgut“ beantwortet werden. Insoweit gilt es zwischen der Meinungsäußerungs-, Informations- und Meinungsbildungsfreiheit (1.), der kommunikativen Chancengleichheit (2.) und der kollektiven Sphäre (3.) zu unterscheiden.

\section{Schutzbedarf der Meinungsäußerungs-, Informations- und Meinungsbildungsfreibeit}

Besonders deutlich zeigt sich die Bedeutung individueller Verzerrungstendenzen im Falle der Meinungsäußerungs-, Informations- und Meinungsbildungsfreiheit. Denn wenn Nutzer bestimmten Angeboten eher vertrauen, einseitig Information aufnehmen, Informationen nicht kritisch rezipieren (Informations- und Meinungsbildungsfreiheit) oder sich von einer gefühlten Mehrheit einschüchtern lassen (Meinungsäußerungsfreiheit), so ist vor allem auch die eigene „Verantwortungssphäre“ betroffen. Weil die Nutzer hierbei aber gerade auch Gebrauch von ihrer grundrechtlich geschützten Autonomie ${ }^{1177}$ machen, läuft der Staat Gefahr, nicht mehr allein vor externen Gefahren, sondern auch vor eigenen, unvorsichtigen und unvernünftigen Entscheidungen zu schützen. Das bedeutet freilich nicht, dass man damit zwangsläufig in einen Konflikt mit der grundrechtlichen Autonomie geraten muss und der Staat den Nutzer bevormunden würde (a.). ${ }^{1178}$ Gleichwohl muss genau überlegt werden, worin der Schutzbedarf besteht, um diese Gefahr zu minimieren (b., c.).

\section{a. Kein prinzipieller Widerspruch zur grundrechtlichen Autonomie}

Wenn Nutzer auch vor eigenen unvernünftigen Entscheidungen geschützt werden, so kann staatlicher Schutz paternalistische Züge annehmen und Gefahr laufen, in Widerspruch mit dem grundrechtlichen Idealbild der Autonomie zu geraten. ${ }^{1179}$ Allerdings ist „Paternalismus [...] nicht gleich Paternalismus“. ${ }^{1180}$ Bereits die verfassungsgerichtliche Judikatur zeigt, dass eine gewisse Differenzierung angezeigt ist. ${ }^{1181}$ Diese ergibt sich bereits aus dem Handlungsziel: In Fällen, in denen eine Gefahr aus Gründen des Drittschutzes unterbunden wird und da-

1177 Ähnlich Martini, Blackbox Algorithmus, S. 103. Auf diese bewussten Entscheidungen weist auch Drexl, ZUM 2017, 529 (535) hin.

1178 Vgl. zu dem Problem auch im Kontext der diskursiven Integrität Schimmele, Staatliche Verantwortung für diskursive Integrität, S. $110 \mathrm{ff}$.

1179 So bezeichnen etwa von der Weth/Vachery/Kankanhalli, in: Proceedings of the International Conference on Multimedia \& Expo, S. 1, ein staatliches Verbot desinformativer Inhalte als „hard paternalism".

1180 Volkmann, Darf der Staat seine Bürger erziehen?, S. 45.

1181 Ausführlich zum Problem des staatlichen Schutzes vor sich selbst etwa Kolbe, Freiheitsschutz vor staatlicher Gesundheitssteuerung, S. $118 \mathrm{ff}$. 
durch auch die Gefahren für den Gefahrverursacher (zwangsläufig) erfasst werden, hat das BVerfG das Problem einer Bevormundung gar nicht aufgeworfen. ${ }^{1182}$ Ging es hingegen um die Abwendungen von Gefahren, die jemand allein für sich selbst setzt, ${ }^{1183}$ hat es stets betont, dass sich die individuelle Entscheidung „nicht an einem Maßstab objektiver Vernünftigkeit“ auszurichten habe und dem Staat keine „Vernunfthoheit“ zukomme, kraft derer er den Willen des Grundrechtsträgers außer Kraft setzen dürfe. ${ }^{184}$ Die Grundrechte gewährten vielmehr auch das Recht, unvernünftige Entscheidungen zu treffen, sofern überhaupt ein freier, autonomer Wille gebildet werden könne. ${ }^{1185}$ Das ist insoweit zutreffend, als dass schon der zentrale Wert der Menschenwürde eine autonome Lebensgestaltung gewährleistet ${ }^{1186}$ und ohnehin keine objektiven Kriterien für die Bewertung einer Handlung als vernünftig oder unvernünftig bestehen. ${ }^{187}$ Hier wird dem Staat also gerade eine Grenze durch den Autonomiegedanken der Grundrechte gesetzt.

Insoweit ist nicht nur zwischen indirektem und direktem, sondern bei letzterem auch zwischen hartem und weichem Paternalismus zu unterscheiden. Geht es um die Abwehr von primär eigenständig gesetzten Gefahren, handelt der

1182 Das gilt etwa im Fall des Rauchverbots in Gaststätten und Diskotheken das vor allem mit dem Schutz der Gesundheit der Nichtraucher begründet wurde. Zwangsläufig unterbindet das aber auch den schädlichen Konsum der Tabakprodukte durch die Raucher selbst. BVerfGE 121, 317 ff. Rauchverbot in Gaststätten. Lediglich RiBVerfG Masing hat die Probleme angesprochen und in seinem SV in der „Radikallösung eines absoluten gaststättenrechtlichen Rauchverbots (...) ein Weg edukatorischer Bevormundung “ gesehen, a.a.O (388). Problematischer könnte das auch bei der EUweiten Pflicht fotografischer Warnhinweise vor den Langzeitschäden des Rauchens sein, weil damit letztlich bezweckt wird, (junge) Personen vom Rauchen abzuhalten. Das BVerfG hat aber textliche Warnhinweise als legitim eingestuft, ohne dabei auf dieses Problem einzugehen, BVerfGE 95, 173 (185 f.) - Warnhinweise für Tabakerzengnisse. Zwar mag hier argumentiert werden, dass es um eine Beschränkung eines Dritten, der Hersteller, ging. Gleichwohl sieht etwa Birnbacher, in: v. Hirsch/ Neumann/Seelmann, Paternalismus im Strafrecht, S. 11 (16) auch in derlei Konstellationen ein paternalistisches Staatshandeln.

1183 So etwa im Falle von Zwangsbehandlungen BVerfGE 128, 282 (303) - Zwangsbehandlung im Maßregeelvollzug; 142, 313 (339) - Zwangsbehandlung; oder beim Suizid BVerfG, Urt. v. 26.02.2020 - 2 BvR 2347/15 u.a.- Recht auf Sterben. Zum aufgedrängten Schutz vor sich selbst ausführlicher auch mit Blick auf die Schutzpflichtendogmatik Kuch, DÖV 2019, $723 \mathrm{ff}$.

1184 Hierzu BVerfG, Urt. v. 26.02.2020 - 2 BvR 2347/15 u.a, Rn. 208 ff. - Recht auf Sterben. Zuvor wurde etwa betont, dass „die öffentliche Gewalt jedem in den Arm fallen [dürfe], der sich selbst zu töten anhebt" (Di Fabio, in: Maunz/Dürig, GG, Art. 2 Abs. 2 Rn. 47) und ein entsprechendes „Selbsttötungsrecht“ abgelehnt (D. Lorenz, JZ 2009, 57 (60); Kunig, in: v. Münch/ders., GG, Art. 1 Rn. 36; Starck, in: v. Mangoldt/Klein/ders., GG, Art. 2 Rn. 192.) oder nur unter der Voraussetzung gewährt, dass die Lebenserhaltung nicht zu Konflikten mit der Menschenwürde führt (Di Fabio, ebd.). Und das BVerwG, NJW 2017, 2215 Rn. 24 hatte ein Recht auf Sterben nur für schwer oder unheilbar kranke Menschen ausdrücklich anerkannt. Zu der schon vor dem Urteil weitgehend verbreiteten Befürwortung eines solchen Rechts - wobei allein die dogmatischen Begründungen variierten - statt vieler Neumann, in: Kindhäuser/ders./Paeffgen, StGB, Vorbem. $\$ 211$ Rn. 43 m.w.N.

1185 Vgl. zuletzt BVerfG, Urt. v. 26.02.2020 - 2 BvR 2347/15, Rn. 239 ff., 279 ff. - Recht auf Sterben, m.w.N.

1186 Siehe etwa BVerfG NJW 2019, 3703 Rn. 127 - Hartz-IV Sanktionen. Vgl. hierzu Duttge, ZStW 2017, 448.

1187 Volkmann, Darf der Staat seine Bürger erziehen?, S. 45 spricht von einer „Alleinzuständigkeit des Einzelnen und (...) einem Vorrang der eigenen Entscheidung “. Aus der Rspr. etwa BVerfG NJW 2019, 3703 Rn. 27 - Hartz-IV Sanktionen. 
Staat direkt paternalistisch, während die zwangsläufig mit dem primär intendierten Schutz Dritter verbundene Abwehr dieser Gefahren indirekt paternalistisch ist. ${ }^{1188}$ Schon deshalb sind indirekt paternalistische Eingriffe zulässig. Während beim harten, direkten Paternalismus unmittelbar mit Verboten oder vergleichbaren Instrumenten gearbeitet wird, ${ }^{1189}$ soll weicher, direkter Paternalismus eher eine unterstützende und die Autonomie des Einzelnen bewahrende Funktion insbesondere dort einnehmen, wo „Defizite“ bestehen. ${ }^{1190}$ In diese Kategorie lassen sich etwa die Entscheidungen zur Zwangsbehandlung einordnen. ${ }^{1191}$ Dieser Ansatz ist schon deshalb richtig, weil durchaus Konstellationen denkbar sind, in denen normatives Leitbild eines mündigen Bürgers und Realität voneinander abweichen. ${ }^{1192}$ Dann geht es aber gerade darum, die Voraussetzungen für eine

1188 Zu dieser Differenzierung ausführlicher etwa Kirste, JZ 2011, 805 ff. Allgemein zum indirekten Paternalismus. Duttge, ZStW 2017, 448 (449f.); Gutmann, NJW 1999, 3387 (3388). Mit Blick auf \$29 BtMG Oğlakcıoğlu, in: MüKo StGB, Bd. 6, Vorbem. \$29 BtMG Rn. 17. Honer, DÖV 2019, 940 (946) spricht hier von einem „Sozialbezug“. Das gilt auch in anderen Fällen, etwa wenn es um die Helmpflicht im Straßenverkehr geht, wenn dies mit dem Schutz vor Traumatisierung anderer Verkehrsteilnehmer oder mit der Last für die Sozialversicherungen begründet wird., vgl. hierzu etwa VGH Mannheim BeckRS 2017, 124386 Rn. 34 und zentral zu der Begründung der Legitimität BVerwG NJW 2019, 3466 Rn. 22. Zum Argument der Lasten für die Allgemeinheit bzw. das Sozialsystem siehe BVerfGE 59, 275 (279) - Helmpflicht. Ob diese Begründung des BVerwG allerdings überzeugend ist, soll dahin gestellt bleiben (kritisch zum Argument etwa Volkmann, Darf der Staat seine Bürger erziehen?, S. 21 mit Fn. 39 und $v$. Hirsch, in: ders./Neumann/Seelmann, Paternalismus im Strafrecht, S. 57 (66f.)). Aus jüngerer Zeit gehören etwa die Masernimpfpflicht ebenso wie die Schutzmaßnahmen während der Covid-19-Pandemie oder die geplante, aber nicht beschlossene Widerspruchslösung bei der Organspende hierzu. Zentrales Anliegen der Impfpflicht ist die Ausbreitung des Masernvirus zu verhindern und damit Risikogruppen und solche Personen zu schützen, die wegen einer medizinischen Kontraindikation nicht selbst geimpft werden, vgl. hierzu schon die Gesetzesbegründung BT-Drs. 19/ 13452 und BVerfG, Beschl. v. 11.05.2020 - 1 BvR 469/20, 1 BvR 470/20, Rn. 15. Zuck, ZRP 2017, 118 (121) betont aber auch hier die Autonomie des Einzelnen und hält eine solche Pflicht i.E. für unzulässig. Bei den Kontaktbeschränkungen und -Verboten ging es auch darum die Verbreitung des Corona-Virus zu verlangsamen und so die Risikogruppen zu schützen bzw. das Gesundheitssystem nicht zu überlasten. Und bei der Widerspruchslösung ging es um sog. staatliches Nudging, also darum den Bürger auf den richtigen bzw. vernünftigen und solidarischen Pfad zu „stupsen“, hierzu ausführlicher Honer, DÖV 2019, 940 ff.

$1189 \mathrm{Zu}$ diesem harten Paternalismus siehe etwa Kolbe, Freiheitsschutz vor staatlicher Gesundheitssteuerung, S. 119 f.; siehe auch $v$. Hirsch, in: ders./Neumann/Seelmann, Paternalismus im Strafrecht, S. 57 ff. Speziell mit Blick auf das Strafrecht.

1190 Vgl. hierzu Kuch, DÖV 2019, 723 (732), der die Selbstbestimmung als „Grund und Grenze“ der staatlichen Schutzpflicht bezeichnet. Ausführlicher hierzu Kolbe, ebd., S. $126 \mathrm{ff}$. Vgl. auch Duttge, ZStW 129 (2017), 448 (449). Von liberalem Paternalismus hier sprechend Weber/Schäfer, Staat 56 (2017), 561 (576); Volkmann, Darf der Staat seine Bürger erziehen?, S. 44. Das gilt etwa in Fällen fehlender Einsichtsfähigkeit oder Selbstbestimmtheit, also dort wo bereits die Fähigkeit zur Bildung eines freien Willens fehlt, vgl. BVerfG, Urt. v. 26.02.2020 - 2 BvR 2347/15 u.a., Rn. 241 - Recht auf Sterben m.w.N.

1191 Denn Maßgabe des BVerfG, E 142, 313 - Zwangsbehandlung, war dort, dass die Betreuten nicht nur spezifisch hilfsbedürftig sind (Rn. 73), sondern dass sie oftmals nicht in der Lage sind, einen freien Willen über Behandlungen zu bilden (Rn. 75 f.). Daraus rechtfertigt sich der Dispens eines entgegenstehenden natürlichen Willens in besonderen Fällen einer „schwerwiegenden Gesundheitsbeeinträchtigung “ (Rn. $78 \mathrm{ff}$.).

1192 Vgl. Honer, DÖV 2019, 940 (945); Duttge, ZStW 129 (2017), 448 (451f.). Volkmann, in: Unger/ v. Ungern-Sternberg, Demokratie und künstliche Intelligenz, S. 51 (61) spricht diesbezüglich von einer Kontrafaktizität zwischen ,gehaltvoller Vorstellung von Autonomie mit den Befunden empirischer Demokratiebeobachtung “. 
autonome Entscheidungsfindung immer wieder $\mathrm{zu}$ überprüfen und bei Bedarf entsprechend nachzujustieren, um so das normative Leitbild am Leben zu halten, ${ }^{1193}$ ohne dass damit aber dieses konterkariert wird ${ }^{1194}$ oder a priori der Einwand des Paternalismus erhoben werden kann. ${ }^{195}$ Vielmehr ist es gerade Aufgabe des Gesetzgebers hier unterstützend tätig zu werden. ${ }^{1196}$ Harter, direkter Paternalismus hingegen ist unzulässig, weil hier gerade die autonome Entscheidung des Einzelnen missachtet und sich über sie hinweg gesetzt wird. Der Staat geriert sich als „Vernunftpolizei“. Solche Eingriffe stehen aber in eklatantem Widerspruch zur Konzeption des grundrechtlichen Menschenbildes, weshalb sie nicht zu rechtfertigen sind. ${ }^{1197}$ Probleme ergeben sich dadurch, dass die unterschiedlichen Kategorien nur schwer voneinander unterschieden werden können,

1193 Volkmann, in: Unger/v. Unger-Sternberg, Demokratie und künstliche Intelligenz, S. 51 (65) meint, dass sich aus diesem Widerspruch „kein Einwand gegen das normative Programm, sondern umgekehrt ein Argument, sich gerade mit dieser Entwicklung kritisch auseinanderzusetzen " erwächst.

1194 Vgl. Honer, DÖV 2019, 940 (945); Duttge, ZStW 129 (2017), 448 (451f.).

1195 In der Tendenz ist das etwa in der Debatte um staatliches Nudging der Fall, vgl. nur Hufen, JuS 2020,193 (197). Und allgemein wird dieser in der Debatte oftmals wegen der damit einhergehenden Freiheitsbeschränkung erhoben, vgl. zu diesem Einwand Eidenmüller, JZ 2011, 814 (815).

1196 Im Falle der geschäftsmäßigen Suizidförderung hat dies letztlich auch das BVerfG angedeutet, Urt. v. 26.02.2020 - 2 BvR 2347/15 u.a., Rn. 242, 246, 274 ff. - Recht auf Sterben. Das ist letztlich auch der Ansatz des liberalen Paternalismus, um Rationalitätsdefiziten zu begegnen, siehe hierzu Eidenmüller, JZ 2011, 814 (817f.). Und bezogen auf die Diskrepanz von normativem Leitbild und empirischer Wirklichkeit der Demokratie meint Volkmann, in: Unger/v. Unger-Sternberg, Demokratie und künstliche Intelligenz, S. 51 (65), „[d]ie Verfassung (...) zeigt Richtungen an, in denen mögliche Lösungen gesucht werden können, gibt aber in der Regel nicht schon die Lösung vor. "In jüngerer Zeit ist hier vor allem die Frage virulent geworden, ob nicht Ergebnisse aus der Verhaltensökonomik Berücksichtigung finden müssen, weil das normative Ideal und die empirische Wirklichkeit nicht kongruent sind und deshalb im Wege eines „libertären Paternalismus“ angeglichen werden können bzw. müssen, in diese Richtung etwa Duttge, ZStW 129 (2017), 448 (451f.). Siehe zu libertärem bzw. liberalem Paternalismus auch Eidenmüller, JZ 2011, 814 ff. Zurückhaltend indes hinsichtlich moralischer Vorstellungen der Diskursqualitität, wo die Verantwortung vor allem auch beim Einzelnen und der Zivilgesellschaft liegt, was aber staatliche Intervention nicht unmöglich mache, Schimmele, Staatliche Verantwortung für diskursive Integrität, S. $112 \mathrm{f}$.

1197 Vgl. Kuch, DÖV 2019, 723 (726); Volkmann, Darf der Staat seine Bürger erziehen?, S. 45; nur mit Blick auf strafrechtliche Interventionen $v$. Hirsch, in: ders./Neumann/Seelmann, Paternalismus im Strafrecht, S. 57 (61 ff.). Mit Blick auf etwaige Mitwirkungspflichten für Arbeitslose hat dies auch das BVerfG deutlich gesagt: „Das [gemeint ist die Menschenwürde und die damit garantierte Autonomie] schließt Mitwirkungspflichten aus, die auf eine staatliche Bevormundung oder Versuche der „Besserung“ gerichtet sind.“, BVerfG NJW 2019, 3703 Rn. 127 - Hartz-IV-Sanktionen. Ein Beispiel hierfür ist etwa, dass der Suizid selbst nicht strafbar ist, die Tötung auf Verlangen aber schon (\$216 StGB). Und auch das Verbot geschäftsmäßiger Förderung des Suizids ist jedenfalls deshalb und insoweit verfassungswidrig, als dass es dem Einzelnen faktisch die Möglichkeit nimmt, selbstbestimmt aus dem Leben durch entsprechende Tätigkeiten Dritter zu scheiden, vgl. hierzu BVerfG, Urt. v. 26.02.2020 - 2 BvR 2347/15 u.a. - Recht auf Sterben, Rn. 215 ff. zum faktischen Eingriff in das Selbstbestimmungsrecht und Rn. $222 \mathrm{ff}$. zur (fehlenden) Rechtfertigung. Zu den hier relevanten Aspekten des Paternalismus bzw. der Freiheit des Einzelnen siehe insbesondere Rn. $273 \mathrm{ff}$. Letztlich zu ähnlichen grundrechtlichen Fragen im Rahmen von $\mathbb{} 216$ StGB etwa $v$. Hirsch/Neumann, in: dies./Seelmann, Paternalismus im Strafrecht, S. 71 ff. und S. 99 ff. Nach Birnbacher, in: v. Hirsch/ Neumann/Seelmann, Paternalismus im Strafrecht, S. 11 (12), ist auch nur der harte direkte Paternalismus als Paternalismus im eigentlichen Sinne zu begreifen. Nach ihm könne aber auch dieser ethisch gerechtfertigt werden. Beim indirekten Paternalismus bestünden nur „strukturell mehr Möglichkeiten“ hierfür, Birnbacher, ebd., (18). In diese Richtung auch Kleinig, in: v. Hirsch/Neumann/ Seelmann, Paternalismus im Strafrecht, S. 145 ff. 
die Übergänge tendenziell fließend sind und meist zumindest auch Drittinteressen verfolgt werden, sodass nur selten ein echter Fall des illegitimen, harten Paternalismus vorliegen wird. ${ }^{1198}$

\section{b. Schutzbedarf der Meinungsäußerungsfreibeit}

Blickt man unter diesen Vorzeichen auf die Meinungsäußerungsfreiheit, so mag zwar die Entscheidung über die eigentliche Äußerungshandlung allein beim Äußernden liegen. Der vorgelagerte interne und kognitive Entscheidungsprozess, ob, wann und wie die eigene Meinung kundgegeben wird, basiert aber maßgeblich auch auf der Auswertung externer Faktoren, die dem Einfluss des Nutzers entzogen sind. Verändern sich diese Faktoren, hat das auch Einfluss auf die endgültige Entscheidung über die Äußerung und ihre Modalitäten. So wird man zu einer anonymen Äußerung eher dort tendieren, wo Repressalien für die Äußerung oder anderen Konsequenzen erwartet werden. ${ }^{1199}$ Im vorliegenden Kontext wird das deshalb relevant, weil letztlich der äußere Schein der Mehrheitsmeinung eine ähnliche Wirkung haben kann. ${ }^{1200}$ Auch wenn das grundsätzlich ein normaler und deshalb unbedenklicher Effekt interpersonaler Kommunikation sein mag, wird er hier künstlich und gezielt zur Unterdrückung von Meinungen erzeugt und ist damit gerade nicht mehr normale Begleiterscheinung diskursiver Kommunikation. Hinsichtlich dieser „digitalen Zwangswirkung “1201 besteht also ein konkreter Schutzbedarf, wobei dieser darauf gerichtet ist, eine autonome Entscheidung des Nutzers befreit von äußeren (künstlichen) Zwangswirkungen zu treffen. Er hat so lediglich einen unterstützenden Charakter, ${ }^{1202}$ sodass es hier allein um indirekte und schwach paternalistische Schutzmaßnahmen gehen wird.

Daneben besteht kein spezifischer Schutzbedarf für die Meinungsäußerung bei der Wahl, denn im Zeitpunkt der Stimmabgabe ist die Einflussmöglichkeit begrenzt und die eigentliche Wahlentscheidung ist bereits zuvor gefällt. Insoweit

1198 Vgl. Duttge, ZStW 129 (2017), 448 (450). Im Falle der Hartz-IV-Sanktionen meinte das BVerfG etwa, dass das Ziel die Selbstentfaltung zu fördern illegitim, die Mitwirkung zur Überwindung der eigenen Hilfsbedürftigkeit zu stärken aber zulässig sei, BVerfG NJW 2019, 3703 Rn. 127. Volkmann, Darf der Staat seine Bürger erziehen?, S. 45 fässt das prägnant so zusammen: „Paternalismus [...] ist nicht gleich Paternalismus. " Mit Blick auf $\$ 217$ StGB - der vom BVerfG für verfassungswidrig erklärt wurde (Urt. v. 26.02.2020 - 2 BvR 2347/15 u.a. - Recht auf Sterben) - weist etwa Duttge, a.a.O., (457f.) daraufhin, dass das Verbot geschäftsmäßiger Suizidförderung für manche Personen eine hart paternalistische Wirkung hat, weil sie ihrer autonomen Entscheidung nicht nachgehen können. Das war letztlich auch ein entscheidender Aspekt für das BVerfG, aa.O. Rn. 266.

1199 Zur Bedeutung der Anonymität im Kontext von sozialem Konformitätsdruck siehe etwa Beater, NJ 2019, 365 (366).

1200 Hierzu bereits oben 2. Teil B.III.3.a.

1201 Ähnlich Milker, InTeR 2017, 199 (203).

1202 Vgl. hierzu auch BVerfG, Urt. v. 26.02.2020 - 2 BvR 2347/15 u.a., Rn. 243 - Recht auf Sterben, Voraussetzung einer „freien Suizidentscheidung“ sei demnach auch, „, dass der Betroffene keinen unzulässigen Einflussnahmen oder Druck ausgesetzt ist.“ Vgl. zur Einwilligungsfähigkeit bei medizinischen Heileingriffen auch BVerfGE 128, 282 (301) - Zwangsbehandlung im Maßregelvollzug. 
resultiert das Schutzbedürfnis hier eher aus den zuvor bestehenden Einflüssen auf die Informationsverarbeitung und Meinungsbildung.

\section{c. Schutzbedarf der Informationsfreiheit bzw. Meinungsbildung}

Soweit es um den internen Prozess der Meinungsbildung geht, besteht kein Schutzbedarf, weil hierauf nicht eingewirkt werden kann. ${ }^{1203}$ Entscheidend ist vielmehr der vorgelagerte Prozess der Informationsaufnahme selbst. Auch hier resultiert der Schutzbedarf nicht abschließend aus der Gefahrenverursachung durch Dritte, die die Art und Weise der Informationsaufnahme und -verarbeitung gesendeter Inhalte beeinflussen. ${ }^{1204}$ Denn dieser Prozess wird maßgeblich von autonomen und bewussten Entscheidungen der Rezipienten gesteuert. Die tatsächliche Informiertheit ist also Ergebnis autonomer Entscheidungen. ${ }^{1205}$ Das gleiche gilt dort, wo auf statistische Daten und damit auf Heuristiken für die Verifizierung von Inhalten vertraut wird, und auch dort, wo eine kritische Rezeption etwa von desinformativen Inhalten nicht erfolgt. Auch hier besteht also die Gefahr, dass staatlicher Schutz paternalistische Züge annehmen kann. Diese Gefahr besteht mit Blick auf drei zentrale Wirkungsmechanismen der Bots: die Täuschungswirkung, die Verbreitung desinformativer Inhalte und hinsichtlich einer einseitigen Informationsrezeption. ${ }^{1206}$

\section{aa. Täuschungswirkung}

Eine Täuschungswirkung besteht bei Meinungsrobotern einerseits hinsichtlich der Identität des Urhebers andererseits hinsichtlich der „quantitativen Legitimität“. Die Gefahr geht hier einerseits vom Verwender aus, weil er die Täuschungswirkung anlegt, andererseits vom Rezipienten, weil er sich täuschen lässt. Gleichwohl könne nach Steinbach von den Nutzern erwartet werden, „sich im durch Pseudonymität und Anonymität geprägten Internet nicht auf die scheinbare Echtheit eines Nutzeraccounts [...] [zu] verlassen. " 1207 Und Milker meint gar, dass der

1203 Vgl. Starck/Paulus, in: v. Mangoldt/Klein/Starck, GG, Art. 5 Rn. 99.

1204 Deshalb bestehen spezifische Probleme, wenn es um die personalisierte Vermittlung von Informationen durch Intermediäre geht. Dass hier ein spezifischer Schutzbedarf besteht, der in der Vorbeugung von Meinungsvielfalt und anderen Aspekten besteht, ist schon mit Blick auf den Rundfunk seit langer Zeit anerkannt. Ausführlicher zu den Problemen und dem Regelungsbedarf, der aus den algorithmisierten Tätigkeiten der Intermediäre resultiert u.a. Pille, Meinungsmacht sozialer Netzwerke; Hartl, Suchmaschinen Algorithmen und Meinungsmacht; Heidtke, Meinungsbildung und Medienintermediäre; und Kellner, Die Regulierung der Meinungsmacht von Internetintermediären.

1205 Vgl. Drexl, ZUM 2017, 529 (535) zur Rationalität von einseitiger oder alternativer Informationsaufnahme.

1206 Alle drei aktivieren immerhin rationale und autonom gesetzte Denkmuster auf Seiten der Rezipienten.

1207 Steinbach, ZRP 2017, 101 (103). Hierauf weist auch Küper, in: Unger/Ungern-Sternberg, Demokratie und künstliche Intelligenz, S. 67 (74) hin, allerdings geht es hier um die Frage einer Schutzbe- 
Bürger nicht zwingend vor Identitätstäuschungen geschützt werden müsse, weil diese zum „allgemeinen Lebensrisiko“ gehören. ${ }^{1208}$ Ein Schutzbedarf wird hier folglich nicht angenommen, sondern vielmehr auf die Mündigkeit und Vernunft der Nutzer vertraut.

\section{(I.) Vergleichbare, einfach-rechtliche Konstellationen}

Auch wenn das im Ausgangspunkt plausibel erscheinen mag, so wird das dadurch konterkariert, dass das einfache Recht für andere Täuschungssituationen und Informationsasymmetrien einen spezifischen Schutz durch Transparenz- und Informationspflichten gewährt, obwohl man den Verbraucher hier auch ohne Weiteres auf seine Mündigkeit ${ }^{1209}$ verweisen könnte. Auch hier folgt die Schutzgewährung daraus, dass sich insbesondere das Verbraucherrecht stark an den verhaltensökonomischen Erkenntnissen orientiert, ${ }^{1210}$ ihm also nicht mehr das Leitbild des homo oeconomicus, ${ }^{1211}$ sondern eines aufzuklärenden, schutzbedürftigen Verbrauchers zugrunde liegt. ${ }^{1212}$ Entscheidend mag daneben auch sein, dass der freie Wettbewerb allein niemals Gewähr dafür bieten kann, dass alle zentralen und entscheidungsrelevanten Informationen verfügbar sind, wodurch eine Asymmetrie zwischen den Marktteilnehmern entstehen kann, die allein durch staatliche Maßnahmen reduziert werden kann. ${ }^{1213}$

So sieht das Wettbewerbsrecht etwa einen Schutz gegen besondere, kommerziell motivierte Täuschungen vor, um den Entscheidungsprozess des Verbrauchers abzusichern und Gewähr dafür zu tragen, dass er auf Grundlage sachlich richtiger Informationen eine rationale und autonome Entscheidung treffen kann. ${ }^{1214}$

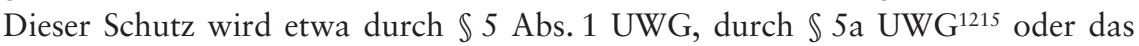
„Per-se-Verbot“ des Anhangs Nr. $11^{1216}$ verwirklicht. Maßstab für die Bestim-

reichsausnahme von Art. 5 Abs. 1 S. 1 GG. Gerade der confirmation bias ist Folge des Bedürfnisses einstellungskonforme Inhalte zu konsumieren und so innere Dissonanzen zu vermeiden.

1208 Milker, ZUM 2017, 216 (220).

1209 Im einschlägigen Schrifttum wird letztlich auch immer die Mündigkeit als Argument dafür herangezogen, dass konkrete Maßnahmen die Überschreitung einer hoch gesetzten Schwelle erfordern. Siehe hierzu etwa Steinbach, ZRP 2017, 101 (103): „Eine Grenze wäre dort zu ziehen, wo eine Meinungsbildung durch die Marginalisierung individueller Meinungsbeiträge schlichtweg unmöglich gemacht wird. (...) Auch ist es Bürgern zumutbar, den Inhalt einer Aussage kritisch zu hinterfragen und auf ihren Wahrheitsgehalt zu prüfen und sich im durch Pseudonymität und Anonymität geprägten Internet nicht auf die scheinbare Echtheit eines Nutzeraccounts zu verlassen. “.

1210 Siehe hierzu etwa Engel/Stark, ZEuP 2015, 32 (38ff.).

1211 Zum homo oeconomicus bereits ausführlich oben, 1. Teil B.III.2. Kritisch zu diesem als Maßgabe für die rechtliche Sphäre, Alexander, in: Gsell u.a, Beck Großkommentar BGB, $\$ 13$ Rn. 370.1 m.w.N.

1212 Vgl. Tamm/Tonner, Verbraucherrecht, $\mathbb{1} 1$ Rn. 25, 27. Allgemein zur Kritik am homo oeconomicus, siehe etwa Weber/Schäfer, Staat 56 (2017), 561 (564 ff.).

1213 Vgl. hierzu BVerfGE 105, 252 (266f.) - Glykol.

1214 Vgl. Nordemann, in: Götting/ders., UWG, $\mathbb{S} 5$ Rn. 0.1.

1215 Nordemann, in: Götting/ders., UWG, $\mathbb{S} 5$ a Rn. 1.

1216 Alexander, in: MüKo Lauterkeitsrecht, Bd. 1, $\$ 3$ Abs. 3 Nr. 11 UWG Rn. 5. 
mung einer irreführenden Handlung ist hierbei der durchschnittlich informierte, situationsadäquat aufmerksame und verständige Verbraucher ${ }^{1217}$ (vgl. auch $\mathbb{} 3$ Abs. 4 UWG).

Auch im Medienrecht findet sich mit den Kennzeichnungspflichten ein ähnlicher Ansatz. $\int 6$ TMG verpflichtet etwa zur Kennzeichnung eines kommerziellen Zwecks. Und $\mathbb{S} 8$ bzw. $\mathbb{S} 18$ Abs. $1 \mathrm{MStV}$ verpflichtet zur Kennzeichnung kommerzieller Inhalte. Auch hier geht es zumindest darum, das Informationsinteresse des Verbrauchers zu schützen, damit er eine zutreffende Einschätzung gesendeter oder angezeigter kommerzieller Inhalte vornehmen und insoweit nicht getäuscht werden kann. ${ }^{1218}$ Denn gerade dort, wo der Eindruck entsteht, dass die Aussage von einem Dritten und nicht vom Werbenden selbst stammt, wird Aussagen eine andere Bedeutung beigemessen. ${ }^{1219}$ In jüngerer Zeit ist dies besonders mit Blick auf das sog. Influencer-Marketing problematisch geworden, weil der Verbraucher in sozialen Netzwerken nicht immer mit kommerziellen Inhalten rechnet, ${ }^{1220}$ sich vielmehr private und kommerzielle Zwecke und Interessen vermengen und die Abgrenzung dadurch schwierig wird. ${ }^{122} \mathrm{Ob}$ entsprechende Informationen er-

1217 Andeutend EuGH, Urt. v. 06.07.1995 - Rs. C-470/93 = NJW 1995, 3234 Rn. 24 - Mars; ausdrücklich dann im Urt. v. 16. 7. 1998 - Rs. C-210-96 = NJW 1998, 3183 Rn. 31 - Gut Springenheide; Urt. v. 28.01.1999 -C-303/97 = GRUR Int. 1999, 345 Rn. 36 - Sektkellerei Kessler; Urt. v. 04.04.2000 - C-465/98 = GRUR Int. 2000, 756 Rn. 20 - D'arbo Naturrein. Grundlegend in der nationalen Judikatur für dieses Verständnis BGH, GRUR 200, 619 (621) - Orient-Teppich; GRUR 2002, 715 (716) - Scanner-Werbung. Kritisch zu diesem mit Blick auf ein Behavioral Microtargeting Ebers, MMR 2018, 423 (424 f.).

1218 Primär mag $\mathbb{1} 18 \mathrm{MStV}$ zwar verhindern, dass ein unzulässiger Einfluss infolge der Abhängigkeit von Werbeunternehmen auf das Rundfunkprogramm entsteht, vgl. Döpkens, in: Spindler/Schuster, Elektronische Medien, $\$ 7$ RStV Rn. 4. Letztlich führt das aber auch für Rezipienten zu einer klaren Trennung von Werbung und Programm und damit dem Schutz vor Täuschungen, Holznagel, in: Hoeren/Sieber/ders., Multimedia-Recht, Teil 3 Rn. 157 m.w.N. $\mathbb{5} 58$ Abs. 1 RStV erweitert diese Wirkung auf Telemedien, vgl. Fiedler, in: Gersdorf/Paal, Informations- und Medienrecht, $\mathbb{\$} 58 \mathrm{RStV}$ Rn. 2, 5. Zu 6 TMG siehe etwa Micklitz/Schirmbacher, in: Spindler/Schuster, Elektronische Medien, $\$ 6$ TMG Rn. 7; Pries, in: Gersdorf/Paal, Informations- und Medienrecht, $\$ 6$ TMG Rn. 2, 4.

1219 Fiedler, in: Gersdorf/Paal, Informations- und Medienrecht, $\mathbb{5} 58 \mathrm{RStV}$ Rn. 2, zurückgehend auf BGH NJW 1995, 3177 (3179). So auch mit Blick auf $\$ 3$ Abs. 3 NR. 11 UWG Alexander, in: MüKo Lauterkeitsrecht, Bd. 1, $\$ 3$ Abs. 3 Nr. 11 UWG Rn. 6.

1220 Ausführlicher hierzu Fries, Influencer-Marketing, S. 163ff; Mallick/Weller, WRP 2018, 155 (159).

1221 Zur Frage der Abgrenzung zwischen kommerziellen und privaten Zwecken siehe etwa LG Itzehoe, MMR 2019, 186 Rn. 27 ff.; LG München I, MMR 2019, 544 ff., das u.a. mit der Verifikation (blauer Haken) die Erkennbarkeit des kommerziellen Zwecks begründet (anders aber Köhler, in: ders./Bornkamp/Feddersen, UWG, $\mathbb{S} 5$ a Rn. 7.80a); KG Berlin, MMR 2019, 175 Rn. 18, keine generelle Vermutung einer kommerziellen Kommunikation. Sinnbildlich für das Problem der Unterscheidbarkeit etwa Pfeifer, GRUR 2018, 2118 (1220): „[...] Jeder Tourist, der T-Shirts mit Werbeaufdruck trägt, gerät potenziell mit seinem Instagram-Foto in den Verdacht des Werbenden. Aber auch ein Blogger oder Journalist, der Produkte bespricht, vorstellt, kritisiert und dadurch zumindest bekannt macht, wird verdächtig. Lauterkeitsrechtlich geht es um die Frage, ab wann Kennzeichnungspflichten bestehen, medienrechtlich auch um die Frage, ob aufgrund des erlangten Meinungseinflusses dem Protagonisten Grenzen für Produktplatzierungen auch deswegen gesetzt werden müssen, weil es um den normativ erwünschten Schutz seiner Funktion als unabhängiger Meinungsbildner geht." Ausführlich zum Influencer-Marketing aus rechtlicher Perspektive u.a. Fries, Influencer-Marketing; Suwelack, MMR 2017, $661 \mathrm{ff}$.; Ahrens, GRUR 2018, $1211 \mathrm{ff}$. und Pfeifer, ebd., $1218 \mathrm{ff}$ 
kennbar sind, wird auch hier aus Sicht eines durchschnittlichen Verbraucher beantwortet. ${ }^{1222}$

Und aus Sicht des vertraglichen Verbraucherschutzrechts ergibt sich ein konkreter Schutzbedarf einerseits aus bestimmten Überraschungsmomenten ${ }^{1223}$ und andererseits aus dem bei Fernabsatzverträgen bzw. außerhalb von Geschäftsräumen geschlossenen Verträgen vorhandenen Informationsdefiziten, ${ }^{1224}$ denen die Informationspflichten der $\mathbb{S} \mathbb{S} 312 \mathrm{a}$ Abs. 1, 2, 312d BGB Rechnung tragen. Insoweit geht es auch hier darum, die Grundlage einer informierten und rationalen Entscheidung zu garantieren. ${ }^{1225}$ Auch hier wird für die Frage der klaren und verständlichen Information letztlich das Verbraucherleitbild relevant. ${ }^{1226}$

Trotz des Leitbildes eines mündigen Verbrauchers sieht also das einfache Recht durchaus Schutzmechanismen vor, die in unterschiedlichen Konstellationen eine rationale und autonome Entscheidungsfindung absichern ${ }^{1227}$ und den durch Dritte verursachten (täuschungsbedingten) Informationsasymmetrien begegnen sollen. Damit knüpft man also an einen Zeitpunkt vor der eigentlichen autonomen Entscheidungsfindung der Verbraucher an. Durch die Informationspflichten wird gerade sichergestellt, dass die für die Entscheidung wesentlichen Informationen auch verfügbar sind. Eine autonome Entscheidung wird so unterstützt, sodass es nur um schwache Formen eines indirekten Paternalismus geht. $^{1228}$

1222 Zu den rundfunkrechtlichen Vorschriften etwa BVerwG ZUM 2016, 194 Rn. 24; Fiedler, in: Gersdorf/Paal, Informations- und Medienrecht, $\$ 58$ RStV Rn. 7; Goldbeck, in: Paschke/Berlit/Meyer/ Kröner, Medienrecht, 26. Abschnitt Rn. 89. Zu $\$ 6$ TMG siehe nur Pries, in: Gersdorf/Paal, Informations- und Medienrecht, $\$ 6$ TMG Rn. 6.

1223 Siehe hierzu bereits Erwggr. 21 der RL 2011/ 83/EU, ABl. L 304, S. 64 (66) aber auch Wendehorst, MüKo-BGB, Bd. 2, $\$ 312$ d Rn. 2.

1224 So Müller-Peltzer/Franck, in: Taeger, Recht 4.0, S. 241 (245); Wendehorst, in: MüKo-BGB, Bd. 2, \312a Rn. 6 bzw. $\$ 312$ d Rn. 2.

1225 Vgl. Alexander, in: Beck Großkommentar BGB, $\mathbb{1 3}$ BGB Rn. 58 f.; Honer, DÖV 2019, 940 (942). Wendehorst, in: MüKo BGB, Bd. 2, $\$ 312$ a Rn. 6 spricht von der Ermöglichung ,eine[r] zutreffende[n] Einschätzung der mit dem Vertrag verbundenen Vorteile und Nachteile sowie eine überlegte Entscheidung hinsichtlich des Vertragsschlusses [...]“.

1226 Vgl. Alexander, in: Beck Großkommentar BGB, $\$ 13$ BGB Rn. 378 ff.

1227 Für das Wettbewerbsrecht siehe grundlegend Beater, Verbraucherschutz und Schutzzweckdenken im Wettbewerbsrecht, 2000. Daneben siehe etwa auch Alexander, in: MüKo Lauterkeitsrecht, Bd. $1, \mathbb{3}$ Abs. 3 Nr. 11 UWG Rn. 5; Sosnitza, in: Ohly/ders., $\$ 5$ UWG Rn. 89. Für die Kennzeichnungspflichten des TMG bzw. RStV vgl. etwa Pries, in: Gersdorf/Paal, Informations- und Medienrecht, $\$ 6$ TMG Rn. 2, 4; Fiedler, in: Gersdorf/Paal, Informations- und Medienrecht, $\$ 58 \mathrm{RStV}$ Rn. 2, 5. Zur Informationspflicht des $\$ 312$ a BGB Wendehorst, in: MüKo BGB, Bd. 2, $\mathbb{3} 312$ a Rn. 6; zu der des $\$ 312$ d BGB ders., a.a.O., $\$ 312$ d Rn. 2. Allgemein zur Bedeutung von vorenthaltenen Informationen für die Autonomie etwa Kirsten, JZ 2011, 805 (806).

1228 Anders wohl Kirsten, JZ 2011, 805 (807), der in der Reihung von Informationen überhaupt keinen Paternalismus erkennt. Engel/Stark, ZEuP 2015, 32 (42 ff.) sehen darin ebenfalls einen liberalen Paternalismus, äußern sich aber auch kritisch zu einer allzu unbedachten Ausweitung europäischen Verbraucherschutzrechte, sondern plädieren vielmehr für ein situationspezifischen Schutz. 
(II.) Vergleichbarkeit und Konsequenzen für die Täuschungswirkung von Meinungsrobotern

Was bedeutet das aber für die Täuschungswirkung der Meinungsroboter? Wenn es auch hier darum geht, was von mündigen Nutzern erwartet werden kann, so ist es nur überzeugend, wenn systematisch-kohärent auch hier auf den durchschnittlichen Nutzer abgestellt und der Schutzbedarf in Abhängigkeit von diesem Maßstab entwickelt wird. Damit orientiert man sich jedenfalls an der Realität und macht nicht ein übergeordnetes, kaum existentes Ideal zum Vorbild für $\mathrm{zu}$ erwartende Verhaltensmuster. Und dadurch ermöglicht man auch einen wesentlich engeren Bezug zum Autonomiegedanken, ohne dass dabei allerdings überspannte Anforderungen gestellt werden, etwa indem auf die Sicht und Informiertheit von Digital Natives abgestellt wird, die im Umgang mit den Netzwerken bewandert sind. Insoweit findet man einen Kompromiss zwischen ihnen und dem anderen Extrem eines unvorsichtigen, flüchtigen Nutzers. ${ }^{122}$

Damit ist aber noch nichts darüber gesagt, was dieses Leitbild über den Schutzbedarf hinsichtlich der Täuschungswirkung aussagt. Zwar ist dem durchschnittlichen Nutzer die grundsätzliche Möglichkeit der Pseudo- und Anonymität bewusst, sodass der Ausgangspunkt bei Steinbach durchaus zutreffend ist. ${ }^{1230}$ Gleichwohl wird die durchschnittliche Erwartungshaltung von Nutzern regelmäßig dahin gehen, dass Anonymisierungen oder Pseudonymisierungen der Nutzer aus Gründen des Daten- oder Persönlichkeitsschutzes vorgenommen werden und nicht um Manipulationen zu ermöglichen. ${ }^{1231}$ Wenn also die Pseudonymisierung auffällt, muss das nicht zwingend zu dem Schluss einer falschen Identität oder einer manipulativen Absicht führen. Das liegt sicherlich auch daran, dass der herkömmliche Kontakt mit unter Pseudonymen auftretenden Nutzern auch im eigenen Netzwerk erfolgt, die Nutzer aber oftmals einander mit bürgerlichem Namen bekannt sein dürften. Aber selbst wenn dies nicht der Fall ist, so ergibt sich dadurch ein Unterschied, dass hier falsche Identitäten realitätsgetreu erstellt werden, sodass es sich ohnehin nicht um einen - erkennbaren - Fall von Pseudonymisierung handelt, der dem Schutz des Äußernden vor nachteiligen Folgen dienen soll. ${ }^{1232}$

1229 Letzterer war der ursprüngliche Ansatz des BGH in wettbewerbsrechtlichen Fällen, etwa bei BGH, WRP 1990, 235 (236); aa.O., 819 (820). Diese beiden Extrema finden sich auch mit Blick auf die allgemeine "Kluft" in Hinblick auf die Probleme der Netzwerkkommunikation wieder, so etwa bei Brinkmann, UFITA 2019, 364 (374), der hier auch von einem „Digital Divide“ (a.a.O., S. 375) spricht.

1230 Auch der BGH sieht die Anonymität als dem Internet wesensimmanent an, BGH MMR 2009, 608 Rn. 38 - spickmich.de.

1231 Vgl. Quandt, EJoC 27 (2012), 7 (15f.).

1232 Hierzu schon ausführlicher oben, A.I.3.b.aa(I.). So auch Semizoğlu, in Hetmank/Rechenberg, Kommunikation, Kreation und Innovation, S. 79 (91) und v. Ungern-Sternberg, in: Unger/dis., Demokratie und künstliche Intelligenz, S. 3 (18f.). 
Diese Erwartungshaltung und das daraus folgende Schutzbedürfnis eines durchschnittlichen Nutzers kann auch durch einen Vergleich mit dem InfluencerMarketing verdeutlicht werden. Influencer verwenden ein normales Nutzerkonto, um mit anderen Nutzern auch zu Marketingzwecken in Kontakt zu treten. Von anderen Nutzerkonten sind sie (objektiv) nur durch die Reichweite zu unterscheiden. ${ }^{1233}$ Während in normalen kommerziellen Settings der kommerzielle Zweck erkennbar und eine entsprechende Kennzeichnung meist entbehrlich ist, weil die Nutzer dies erkennen und dadurch Inhalte kritischer rezipieren können, ${ }^{1234}$ ist dies beim Influencer-Marketing oftmals anders. Weil soziale Netzwerke in erster Linie sozial ausgerichtet sind, wird der durchschnittliche Nutzer regelmäßig nicht von einer kommerziellen Einflussnahme ausgehen, wenn es um originäre Inhalte anderer Nutzer geht, ${ }^{1235}$ sodass dann auch keine kritischere Rezeption erfolgt. ${ }^{1236} \mathrm{Im}$ Grundsatz gilt: was nicht als kommerziell gekennzeichnet oder deutlich erkennbar ist, wird als private Meinung angesehen. ${ }^{1237}$ Das führt dann oftmals zu Verstößen gegen $\mathbb{\int} 5$ a Abs. 6 UWG. ${ }^{1238}$

1233 Das LG München I, MMR 2019, 544 Rn. 41 ff. betont die Verifikation von Profilen und eben die deutlich größere Reichweite, um daraus letztlich den offensichtlichen, kommerziellen Zweck abzuleiten.

1234 Im Kontext von $\$$ 5a Abs. 6 UWG BGH, GRUR 2013, 644 Rn. 16 m.w.N. zu redaktionellen Inhalten. KG Berlin, MMR 2019, 175 Rn. 32, 34; LG München I, MMR 2019, 544 Rn. 41 ff. und OLG Celle, MMR 2017, 769 Rn. 14 f. bezogen auf soziale Netzwerke. Zu $\$ 6$ TMG siehe etwa Micklitz/ Schirmbacher, in: Spindler/Schuster, Elektronische Medien, $\$ 6$ TMG Rn. 27. Generell dürfte das etwa bei der Präsenz von Unternehmen in sozialen Netzwerken der Fall sein, vgl. Fuch/Hahn, in: Jahnke, Influencer-Marketing, S. 161 (165). Zum Einsatz von Chat-Bots machen Müller-Peltzer/ Franck, in: Taeger, Recht 4.0, S. 241 (245 f.), dies von dem Interesse des Nutzers abhängig. Wenn es i.E. keinen Unterschied macht, ob ein Bot oder ein Mensch mit dem Nutzer kommuniziert, so sei eine diesbezügliche transparente Information nicht erforderlich.

1235 Fries, Influencer-Marketing, S. 163; im Ansatz auch Scherer, WRP 2019, 277. In diese Richtung mit Blick auf $\$ 5$ Abs. 6 UWG im Falle von nicht oder nur unzureichend gekennzeichnet kommerziellen Beiträgen sog. Influencer, OLG Celle, MMR 2017, 769 Rn. 14 ff.; LG Hagen, MMR 2018, 106 Rn. 23; LG Berlin, MMR 2018, 543 Rn. 22. Deutlicher LG Karlsruhe, GRUR-RR 2019, 328 Rn. 47, das in der bewussten Vermengung privater und werblicher Inhalte eine gezielte Reduzierung der Erkennbarkeit von Werbung sieht. Allgemein anders hingegen LG München I, MMR 2019, 544 Rn. 46 ff. Bruhn, in: Gloy/Loschelder/Danckwerts, WettbewerbsR, $\mathbb{5} 59$ a Rn. 31, 32 betont jedenfalls die schwierige Trennung zwischen Privat und kommerziell. Mit Bezug zum Influencer-Marketing betont Gerecke, GRUR 2018, 153 (156), dass gerade private Kommunikation suggeriert werden soll.

1236 Vgl. zu redaktionellen Inhalten BGH, GRUR 2013, 644 Rn. 16 m.w.N.; bezogen auf soziale Netzwerke KG Berlin, MMR 2019, 175 Rn. 32, 34. Im Bereich des Influencer Marketing liegt das an der vermittelten Authentizität der Profile, die zu einer Einordnung als neutraler Dritter bei den Nutzern führt, Mallick/Weller, WRP 2018, 1289 (1292); Scherer, WRP 2019, 277 (281) und Holznagel/Hartmann, in: Hoeren/Sieber/Holznagel, Multimedia-Recht, Teil 3 Rn. 212.

1237 Fries, Influencer-Marketing, S. 163. Vgl. zu diesem in der Regel privaten Charakter LG Itzehoe, MMR 2019, 186 Rn. 30; OLG Celle MMR 2017, 769 Rn. 17 f.; LG Berlin, MMR 2018, 543 Rn. 24. Vgl. Schmid/Braam/Mischke, MMR 2020, 19 (23) die als persönlich getarnte politische Werbung einen (potentiell) höheren Einfluss auf die Meinungsbildung nachsagen. Vgl. hierzu auch Pille, Meinungsmacht sozialer Netzwerke, S. $307 \mathrm{ff}$.

1238 Siehe hierzu u.a. OLG Celle, MMR 2017, 769 ff.; KG Berlin, MMR 2018, 98 ff.; LG Itzehoe, MMR 2019, 186 ff.; LG Hamburg, MMR 2020, $130 \mathrm{ff}$. Das ist auch andernorts die grundlegende Annahme, vgl. etwa Alexander, in: MüKo Lauterkeitsrecht, $\$ 3$ Abs. 3 Nr. 11 UWG Rn. 6. 
Aber auch in der hiesigen Konstellation - das ist ja letztlich die Grundannahme der potentiellen Bedrohung durch Meinungsroboter - wird der durchschnittliche Nutzer von sozialen Interaktionen von Privaten ausgehen, ${ }^{1239}$ sodass auch hier oftmals keine kritische Rezeption erfolgt. ${ }^{1240}$ Das gilt besonders in Konstellationen, in denen die Aufmerksamkeit eher den Inhalten und weniger den Urhebern gilt, was vor allem dort der Fall sein wird, wo mehrere, einander unbekannte Nutzer zusammentreffen. Nicht nur wird selten eine kritische Identitätsüberprüfung vorgenommen - ohnehin bestehen Zweifel daran, inwieweit das überhaupt angesichts der begrenzten Aufmerksamkeit und Zeit realitätsnah und zumutbar wäre -, sondern es fehlen regelmäßig auch Warnhinweise, die sich aus einem ganz bestimmten Setting ergeben können. Anders als bei „Nutzern des öffentlichen Lebens“, bei denen ein Verifikationshinweis die Gewähr für ihre Authentizität bietet ${ }^{1241}$ und die Interessenverfolgung dadurch auch deutlicher zutage tritt, ${ }^{1242}$ fehlt ein solcher für herkömmliche Nutzerkonten. Etwas anderes mag (wohl) in Konstellationen gelten, in denen eine direktere Interaktion mit anderen Nutzern stattfindet, etwa bei Freundschafts- oder Abonnement-Anfragen. Hier dürfte die durchschnittliche Aufmerksamkeit höher sein, weil man dem anderen Zugriff auf persönliche Informationen und das eigene individuelle Netzwerk ermöglicht. ${ }^{1243}$

Wenn es daneben um statistische Daten - und damit um Fragen der „quantitativen Legitimität“ - geht, wird die Aufdeckung noch schwieriger, weil die Nutzer nur das aggregierte Endergebnis angezeigt bekommen. Eine Verifizierung ist faktisch unmöglich, weil jeder bewertende Nutzer überprüft werden müsste. Gerade deshalb kommt der sozialen Bewährtheit und dem Vertrauen in die „Schwarmintelligenz" Bedeutung zu. Vergleichbar ist dieses Vertrauen durchaus mit dem Vertrauen in die sachliche und neutrale Arbeit der Medien, auf dem letztlich auch dort die Wirkungsmöglichkeit beruht.

Ähnliches gilt für die Leitfunktion des Meinungsbildes von Diskussionen, Abstimmungen o.ä. Auch hier wird es faktisch unmöglich sein, einzelne Beiträge

1239 Milker, ZUM 2017, 216 (218); Brachten u.a., Social Bots in Social Media Crisis Communication, S. 3. Das dem durchaus so ist, haben etwa Edwards u.a., Computers in Human Behavior 33 (2014), 372 ff. aufgezeigt. Vgl. auch Cheng/Luo/Yu, Physica A 2020, 124163, S. 2.

1240 So mit Blick auf als persönliche Meinung getarnte politische Werbung Schmid/Braam/Mischke, MMR 2020, 19 (23). Mit Blick auf Bots vgl. Drexl, ZUM 2017, 529 (534). Vgl. allgemein zur Kommunikation in sozialen Netzwerken Pille, Meinungsmacht sozialer Netzwerke, S. 308.

1241 Sowohl bei Facebook als auch bei Twitter erfolgt diese Verifikation durch einen blauen Haken hinter dem Namen des jeweiligen Nutzerkontos.

1242 Vgl. hierzu Das LG München I, MMR 2019, 544 Rn. $41 \mathrm{ff}$.

1243 Allerdings kann auch hier die Glaubwürdigkeit des Profils unmittelbar Einfluss auf den Umfang einer Überprüfung durch Nutzer haben. Je eher ein Bot schon mit anderen Personen aus dem Netzwerk verbunden, desto höher ist die Wahrscheinlichkeit dafür, dass auch andere Nutzer Freundschaftsanfragen etwa akzeptieren, vgl. hierzu Boshmaf u.a., Computer Networks 57 (2013), 556 (565). Allgemein zur auch hier fehlenden Vorsicht Abulaish/Fazil, IEEE Technology and Society Magazine 2020, 52 (58). 
dahingehend zu überprüfen, ob hinter ihnen ein echter Nutzer steht. Zwar lässt sich aus einer bestimmten Aufmachung bzw. bestimmten Profilangaben und -Inhalten auf die politische Gesinnung rückschließen. Das wird aber nur bedingt weiterhelfen, wenn es um die Frage geht, was die Mehrheit der Nutzer denkt. Dass das Internet zwar nicht zwingend repräsentativ ist, ${ }^{1244}$ mag Nutzern noch bekannt sein. Gleichwohl ändert dies nichts an der Orientierungswirkung entsprechender Meinungsbilder. Immerhin orientieren sich auch klassische Medien oder Umfragen durchaus an den Stimmungsbildern in den Netzwerken. Selbst bei einer erhöhten Aufmerksamkeit der Nutzer besteht also kaum die Möglichkeit, eine entsprechende Manipulation offen zu legen.

\section{(III.) Fazit: Insbesondere Förderung von Transparenz und Medienkompetenz}

Auch vorliegend besteht mit Blick auf die Identitätstäuschung und die Täuschung über die quantitative Legitimität letztlich ein ähnlicher Schutzbedarf, wie in den bereits regulierten Konstellationen im kommerziellen Bereich. Er resultiert auch hier aus kognitiven Verzerrungen und Verhaltens- bzw. Denkanomalien, die letztlich auf fehlende Transparenz zurückzuführen sind. Letztere ist aber Voraussetzung dafür, eine autonome und sachliche Entscheidung auch in digitalen Räumen treffen zu können. ${ }^{1245}$ Insoweit besteht auch kein allzu großes Spannungsverhältnis zur Nutzerautonomie; diese gilt es vielmehr durch relevante Informationen zu unterstützen. Wie auch in anderen einfach-rechtlichen Konstellationen geht es hier also primär um die Förderung von Transparenz. ${ }^{1246}$

Weil aber der Umgang mit Informationen erlernt werden muss - das gilt sowohl für herkömmliche wie für die neuen digitalen Medien - kann bereits dieser auf fehlerhaften Annahmen beruhen. Gerade die aufgezeigten Heuristiken und Denk- bzw. Verhaltensmuster sind letztlich Resultat eines fehlerhaften Verständnisses der Bedeutung bestimmter Informationen in sozialen Netzen. Wo vorher

1244 Besonders betont das etwa Milker, ZUM 2017, 216 (220) und sieht als endgültigen Ausweg hier nur eine absolute Klarnamenpflicht an.

1245 Mancherorts wird deshalb - wenn auch nicht in Konstellationen, in denen es (ausschließlich) um Meinungsroboter - geht von der digitalen Dimension der Grundrechte gesprochen, vgl. etwa Graf $v$. Westphalen, IWRZ 2019, 61 (63), m.w.N. Mit Blick auf ein kommerzielles Microtargeting spricht etwa Ebers, MMR 2018, 423 (424) von „einer Erosion der Privatautonomie“. Allgemein zur Bedeutung von Informationen für „Marktteilnehmer“ siehe auch BVerfGE 105, 252 (266 f.) - Glykol.

1246 Das wird im Schrifttum oftmals ähnlich bewertet, wenn spezifische Forderungen nach Kennzeichnungspflichten erhoben werden, so etwa Klaas, MMR 2019, 84 (90); Löber/Roßnagel, MMR 2019, 493 (497); Drexl, ZUM 2017, 529 (543). Heldt, CR 2018, 494 (497 formuliert es so, dass, „[u]m die freiheitliche Voraussetzung zu erfüllen, [...] die wichtigsten Informationen für alle Teilnehmer einsehbar sein [müssen].“ Siehe hierzu auch der von Heldt, ebd., zitierte Art. 3 Abs. 1 der VO 1169/2011 betreffend die Information der Verbraucher über Lebensmittel: „Die Bereitstellung von Informationen über Lebensmittel dient [...] Interessen der Verbraucher, indem Endverbrauchern eine Grundlage für eine fundierte Wahl [...] unter besonderer Berücksichtigung von gesundheitlichen, wirtschaftlichen, umweltbezogenen, sozialen und ethischen Gesichtspunkten geboten wird. [Hervorh. d. Autors]". 
redaktionelle und vor allem professionelle Vorfilter existierten, ist dies nun die Aufgabe der Nutzer. ${ }^{1247}$ Das setzt aber voraus, dass sie die Instrumente besitzen, um dieser Aufgabe realiter nachkommen zu können. Eine kritische Rezeption setzt neben den transparenten Informationen daher auch die Fähigkeit voraus, diese zutreffend einordnen, bewerten oder interpretieren zu können. Insoweit geht es vor allem um eine grundlegende „Medienkompetenz“, die es zu erlernen gilt. Dort, wo diese nicht oder nur schwach vorhanden ist, besteht jedenfalls Bedarf, diese gegebenenfalls mit staatlicher Hilfe zu erlangen.

\section{bb. Desinformative Inhalte}

Betrachtet man die individuelle Wirkungssphäre von Desinformation, so kann es hier nicht darum gehen, eine Aufnahme allein sachlich-richtiger Informationen zu gewährleisten oder sonst wie bestimmte sachlich richtige Informationen jemandem faktisch aufzuzwingen. ${ }^{1248}$ Denn die eigentliche Gefahr resultiert nicht allein aus der Information selbst, sondern ist vielmehr in erster Linie im Rezeptionsverhalten der Nutzer zu sehen. Immerhin lässt sich nachweisen, dass desinformative Inhalte deutlich mehr Nutzer ansprechen und von deutlich mehr Nutzern geteilt werden, als herkömmliche Nachrichten. ${ }^{1249}$ Dies wird u.a. darauf zurückgeführt, dass Desinformation bereits vorhandene Denkmuster aktivieren und meist auch auf einer emotionalen Ebene wirken. ${ }^{1250}$ Bedeutung erlangen insoweit also vor allem die aufgezeigten Verzerrungstendenzen bei der Informationsaufnahme und -verarbeitung, insbesondere der Rückgriff auf Heuristiken, kraft derer bestimmten Inhalten mehr Aufmerksamkeit zukommt (etwa soziale Bewährtheit), und solchen Verzerrungen, die zu einer bevorzugten Wahrnehmung populistischer Inhalte führt, weil sie der bereits vorhandenen Grundeinstellung entsprechen (etwa confirmation bias). Dadurch werden nicht zuletzt auch Spannungen emotionaler oder rationaler Art vermieden (sog. Theorie der kognitiven Dissonanz) ${ }^{1251}$. Und auch das allgemeine (oberflächliche) Konsumverhalten als Ausdruck einer Kosten-Nutzen-Erwägung oder der Verzicht von klassischen Medien infolge von Vertrauenskrisen oder durch das Gefühl der Vernachlässigung partikularer Interessen begünstigt die Beeinflussbarkeit für entsprechende Inhalte. Diese „Anomalien“ sind aber vorhersehbar und können durch Dritte ausge-

1247 BVerfGE 149, 222 Rn. 80 - Rundfunkbeitrag.

1248 Mahnend etwa Milker, ZUM 2017, 216 (220).

1249 Hierzu Vosoughi/Roy/Arai, Science 359 (2018), S. $1146 \mathrm{ff}$.

1250 In diese Richtung etwa Voshougi/Roy/Arai, a.a.O., (1149f.), weil Desinformation neuartiger als richtige Information ist und insoweit u.a. einen höheren Überraschungseffekt besitzt. Zum Einfluss des confirmation bias etwa Steinebach u.a., Desinformation aufdecken und wirksam bekämpfen, S. 80 und zum Einfluss vom Negativity Bias und Emotionen vgl. S. 81.

1251 Zu dieser siehe etwa Bedford-Strohm, in: ders./Höhne/Zeyer-Quattlender, Digitaler Strukturwandel der Öffentlichkeit, S. 201 (215). 
nutzt werden. ${ }^{1252}$ Und mithilfe der Unterstützung fiktiver Nutzer kann gerade auch die Glaubwürdigkeit und Relevanz der Information gesteigert werden. Vor diesem Hintergrund besteht auch hier Anlass dafür, zumindest „Hilfe zur Selbsthilfe“ ${ }^{1253}$ zu gewähren, um so zu ermöglichen, dass die nutzerseitig vorhandenen Schwächen und Anfälligkeiten eigenständig behoben werden können. Das setzt aber voraus, dass Nutzer auf eben diese aufmerksam gemacht werden. Das kann durch zusätzliche transparenzerhöhende Informationen erfolgen ${ }^{1254}$ oder alternativ bzw. kumulativ durch die begleitete Entwicklung und Förderung einer Medienkompetenz der Nutzer. ${ }^{1255}$

\section{cc. Einseitige Informationsrezeption}

Geht es hingegen um den dritten und letzten Aspekt einer einseitigen Informationsrezeption, so werden auch hier unterschiedliche Faktoren relevant. Betrachtet man den Weitergabeprozess, so kommt zunächst den Algorithmen selbst Bedeutung zu, weil sie darüber entscheiden, welche Informationen den Nutzer überhaupt und priorisiert erreichen sollen. Zwar ließe sich argumentieren, dass dem Nutzer selbst die wesentliche Entscheidung zukommt, welche Inhalte er konkret wahrnimmt. Insoweit könnte auch ein „neutraler“ Algorithmus, eine einseitige Informationsrezeption nicht verhindern, weil seit jeher die Tendenz zu einem eher einseitigeren Inhalts- und Medienkonsum besteht. ${ }^{1256}$

Das wird aber mit Blick auf die algorithmenbasierte Weitergabe dadurch in den Hintergrund gerückt, dass hier die Weitergabe in den meisten Fällen von dem bisherigen Nutzerverhalten und den bisherigen Nutzerinteressen abhängig ist. Bei herkömmlichen Medien obliegt dem Einzelnen die bewusste Entscheidung über die Auswahl des Mediums aus einem vielfältigen Angebot. Im Internet finden (notwendige) Vorselektierungen aber bereits statt, ohne dass der Nutzer

1252 Vgl. mit Blick auf das kommerzielle sog. Behavioral Microtargeting Ebers, MMR 2018, 423 (424). Besonders sog. Clickbaiting zeigt das Potential dahinter. Hier hinter versteckt sich die Strategie, Nutzer durch kurze Informationen neugierig zu machen, um sie dazu zu bewegen, einen bestimmten Link oder Inhalt zu klicken, um mehr Informationen zu erhalten. Zum Clickbaiting aus persönlichkeitsrechtlicher Sicht siehe etwa OLG Köln, GRUR-RR 2019, 396ff, insbeS. Rn. 29.

1253 So Wiebe, GewArch 2016, 456 (457) in Bezug auf unternehmerische Informationspflichten.

1254 Vgl. hierzu auch Drexl, ZUM 2017, 529 (542) der dies im Zusammenhang mit einer Regulierung der Algorithmen der Intermediäre überlegt.

1255 Allgemein mit Blick auf Meinungsroboter etwa Milker, InTeR 2017, 199 (207); Graber/Lindemann, in: Sachs-Hombach/Zywietz, Fake News Hashtags \& Social Bots, S. 51 (65); Kind u.a., Social Bots, S. 65 und Rückert, in: Albrecht u.a., Strafrecht und Politik, S. 167 (184); Fehling/Leymann, AfP 2020, 110 (118); Oberer/Erkollar/Stein, in: Stumpf, Digitalisierung und Kommunikation, S. 311 (323); Dankert, in: Hoffmann-Riem, Big Data, S. 157 (164). Und mit Blick auf Desinformation etwa Kalwey, in: Möller, Was tun gegen Fake News und Hate Speech?, S. 139 ff.; Schweiger, Der (des)informierte Bürger, S. 190; Steinebach u.a., Desinformation aufdecken und bekämpfen, S. 206 und Varol/Uluturk, First Monday Vol. 23. Nr. 5 (2018).

1256 Darauf u.a. hinweisend Pfengler, GreifRecht 2020, 1 (10). Und Kaiser/Reiling, in: Unger/v. UngernSternberg, Demokratie und künstliche Intelligenz, S. 85 (89) bezeichnen Filterblasen online und offline als „etwas gesellschaftlich Normales“. 
diese Selektion bewusst nachvollzieht oder steuert und ohne dass der Nutzer um diese technischen ${ }^{1257}$ oder um die internen Verzerrungen weiß. ${ }^{1258}$ Ihnen müsste dafür zumindest offenbart werden, welche Verhaltensweisen zu einer bestimmten priorisierten Weitergabe führen, um diese gegebenenfalls ändern zu können.

Daneben ist der persönliche Informationszuschnitt nur begrenzt nutzerorientiert. Anbieter verfolgen hiermit kommerzielle Interessen, weil sie sich über die Aufenthaltsdauer, die Präzision des persönlichen Zuschnitts und die darin angezeigten Werbeinhalte refinanzieren. ${ }^{1259}$ Und letztlich trifft zwar der Nutzer die Entscheidung, welchen konkreten Inhalt er vertieft anschaut. Das setzt aber zumindest voraus, dass der entsprechende Inhalt ihm auch vorher angezeigt wird. Insoweit geht es hier neben erneuten Transparenzaspekten vor allem um grundlegende Fragen der Meinungspluralität, die klassischerweise die rundfunkrechtliche Debatte seit Anbeginn dominiert haben. ${ }^{1260}$ Voraussetzung einer autonomen Entscheidung ist immerhin, dass plurale Informationen einer Entscheidung zugrunde gelegt werden können. Ob sie tatsächlich zugrunde gelegt werden, ist insoweit irrelevant und Ergebnis einer autonomen Entscheidung. Mithin besteht mit Blick auf die Informationsweitergabe vor allem Bedarf hinsichtlich der Transparenz und (rezeptiven) Pluralität. ${ }^{261}$

Theoretisch ließe sich auch mit Blick auf die Anomalien fragen, inwieweit hier Schutzbedarf besteht. Weil es aber um interne Prozesse der Informationsverarbeitung geht, beschreitet man hier einen nicht nur aus praktischer Sicht schwer

1257 Zur fehlenden „algorithmic awareness“ vgl. Schulz/Dankert, Die Macht der informationsintermediäre, S. 42 f., mit Verweis auf eine durchgeführte Studie von Eslami/Rickman u.a., oder Kaiser/ Reiling, in: Unger/v. Ungern-Sternberg, Demokratie und künstliche Intelligenz, S. 85 (103) Siehe daneben auch die Darstellung und Verweise bei Schmidt u.a., Zur Relevanz von Online-Intermediären für die Meinungsbildung, S. 29.

1258 Immerhin kommt hier dem unterbewusst agierenden Denksystem I maßgebliche Bedeutung zu. Hierzu bereits oben, 2. Teil, B.III.3.b.

1259 Vgl. Kaiser/Reiling, in: Unger/v. Ungern-Sternberg, Demokratie und künstliche Intelligenz, S. 85 (88). Dieses Problem hat auch das BVerfG angemahnt, E 149, 222 Rn. 79 - Rundfunkbeitrag.

1260 Zum klassischen Rundfunk siehe etwa das SV von den Richtern Geiger, Rinck und Wand BVerfGE 31, 314 (338) - 2. Rundfunkentscheidung. Daneben aber auch die Senatsentscheidungen in BVerfGE 73, 118 (152 ff.) - 4. Rundfunkentscheidung; 74, 297 (323 ff.) - 5. Rundfunkentscheidung; 83, 238 (295) - 6. Rundfunkentscheidung. Ausführlich zur Frage von Meinungsmacht und -pluralität in sozialen Netzwerken und dem Erfordernis einer Regulierung etwa Pille, Meinungsmacht sozialer Netzwerke; Heidtke, Meinungsbildung und Medienintermediäre; oder Kellner, Die Regulierung der Meinungsmacht von Internetintermediären. Zur ähnlichen Frage bei Suchmaschinen Hartl, Suchmaschinen, Algorithmen und Meinungsmacht. Zur aktuellen Diskussion der Vielfaltssicherung bei Internetintermediären, siehe etwa Paal, MMR 2018, 567 ff.; Heilmann, MMR 2020, $162 \mathrm{ff}$.

1261 Vgl. hierzu im Ergebnis auch die Ausführungen im Schrifttum zu Internetintermediären und den Regulierungsoptionen bzw. -Empfehlungen Paal, MMR 2018, 567 (569); Heilmann, MMR 2020, 162 (164f.); Schwartmann/Hermann/Mühlenbeck, MMR 2019, 498 (499ff.). Zur Transparenz etwa auch Kaiser/Reiling, in: Unger/v. Ungern-Sternberg, Demokratie und künstliche Intelligenz, S. 85 (103). Speziell zum Aspekt der Vielfaltssicherung Kreile, ZUM 2017, 268 ff., mit Blick auf Suchmaschinen; Paal/Hennemann, ZRP 2017, 76 (77 ff.); Dörr/Natt, ZUM 2014, 29 (844ff.). 
gangbaren Pfad. Auch die Autonomie der Nutzer steht hier im Weg. ${ }^{1262}$ Eine Einwirkung auf die inneren Vorgänge ist nur schwer möglich. Vielmehr wird es auch hier darauf ankommen, die Grundsteine dafür zu legen, dass der Einzelne über das Wissen verfügt, um dem Idealbild entsprechend mit Informationen umgehen zu können. Das setzt freilich auch hier eine gewisse Medienkompetenz voraus. ${ }^{1263}$ Denn im digitalen Umfeld insbesondere bei Intermediären kann nicht mehr allein auf unabhängige und dem Sachlichkeitsgebot unterworfenen Redaktionen vertraut werden, sondern hier ist eine deutlich größere geistige Leistung der Nutzer erforderlich, Informationen nicht nur aufzunehmen und zu verarbeiten, sondern diese auch in einen Kontext einzubinden, sie zu bewerten und dabei auch kritisch zu hinterfragen. Das Vertrauen auf die Nutzergemeinschaft ist folglich nur Mittel zum Zweck und substituiert in gewisser Weise das Vertrauen in die Auswahl durch Redaktionen. Dieser neue Umgang mit Informationen muss aber erstmal erlernt werden.

\section{d. Fazit: Transparenz, Medienkompetenz als wesentliche Schutzmaximen}

Betrachtet man die Wirkungsmechanismen der Meinungsroboter, so ergeben sich verschiedenartige Schutzbedürfnisse. Aus Sicht der Meinungsäußerungsfreiheit geht es hier vor allem um den Schutz vor den potentiellen Zwangswirkungen. Mit Blick auf die Informationsfreiheit geht es zumindest darum, der bestehenden Informationsasymmetrie, die vergleichbar mit kommerziellen Konstellationen ist, $\mathrm{zu}$ begegnen. Insoweit geht es hier nicht darum, dem Nutzer die Mündigkeit abzusprechen, sondern vielmehr die Autonomie durch Beseitigung dieser Asymmetrien zu stärken, was letztlich auf Transparenzerfordernissen in den verschiedenen Konstellationen hinausläuft. Daneben besteht aber auch ein Bedürfnis,

1262 In diese Richtung wohl Cornils ZUM 2019, 89 (95) wenn er meint, dass „[1]enkende Eingriffe in die Nutzergewohnheiten der Netzkommunikation durch Verbote oder subtilere Anreize (wieder weg von dem "schnelles Denken begünstigenden Design " der WWW-Intermediäre und wieder zurück zum Modus bewusster Aufmerksamkeitsanstrengung etwa bei der Rezeption klassischer Medienberichterstattung) [...][kaum] grundrechtskonform [...] vorstellbar [erscheinen].“.

1263 Diese Medienkompetenz wird auch weit überwiegend im Kontext der Meinungsroboter betont, so etwa Dankert, in: Hoffmann-Riem, Big Data, S. 157 (164); Fehling/Leymann, AfP 2020, 110 (118); Graber/Lindemann, in: Sachs-Hombach/Zywietz, Fake News Hashtags \& Social Bots, S. 51 (65); Kind u.a., Social Bots, S. 65 und Rückert, in: Albrecht u.a., Strafrecht und Politik, S. 167 (184); Milker, InTeR 2017, 199 (207); Oberer/Erkollar/Stein, in: Stumpf, Digitalisierung und Kommunikation, S. 311 (323). Mit Blick auf die allgemeinen „Transparenz- und Orientierungsdefizite“ etwa Brinkmann, UFITA 2019, S. 364 (384). Im Kontext von Fake News etwa Kalwey, in: Möller, Was tun gegen Fake News und Hate Speech?, S. 139 ff.; Schweiger, Der (des)informierte Bürger, S. 190 und Steinebach u.a., Desinformation aufdecken und bekämpfen, S. 206. Mit Blick auf die Rechtswahrung und die Sensibilisierung vor Rechtsverletzungen etwa Bock, Übertragbarkeit der Kommunikationsfreiheiten auf das Internet, S. $284 \mathrm{f}$. und Rößner, in: Hill/Kugelmann/Martini, Digitalisierung in Recht, Politik und Verwaltung, S. 55 (63). Allgemeiner auch Drexl, ZUM 2017, 529 (543); Wardle/Derakhshan, Information Disorder, S. 70 f. oder vgl. Auch Steinmaurer, in: Litschka/Krainer, Der Mensch im digitalen Zeitalter, S. 31 (36). 
Meinungspluralität zu gewährleisten und letztlich muss auch eine grundlegende Medienkompetenz der Nutzer geschaffen und gestärkt werden. ${ }^{1264}$

\section{Schutzbedarf der kommunikativen Chancengleichheit}

Die „kommunikative Chancengleichheit“ ist schon vom Anwendungsbereich allein auf die Gefährdung der chancengleichen Teilhabe am Kommunikationsprozess gerichtet, sodass der Schutzbedarf hier allein auf die Beseitigung entsprechender Benachteiligungen oder Bevorzugungen gerichtet sein kann. Im Falle der Meinungsroboter würde das vor allem bedeuten, dass Schutz vor der technischen Verzerrung der Weitergabe- und damit der Rezeptionswahrscheinlichkeit gewährt werden müsste, so wie es letztlich auch in der Debatte um die Netzneutralität zum Ausdruck gekommen ist. ${ }^{1265}$

\section{Kumulation der Wirkungsweisen und Schutzbedarf des Kommunikationsprozesses in toto}

Und zuletzt besteht nicht nur ein Schutzbedarf für die individuelle Seite. Wenn die individuelle Sphäre Grundlage und Voraussetzung für die kollektive Sphäre der Willensbildung ist, so lässt sich auch der individuelle Schutzbedarf zu einem kollektiven potenzieren. ${ }^{1266}$ Weil und wenn eine diskursive Öffentlichkeit notwendig für die Willensbildung und das Entstehen einer öffentlichen Meinung ist, wird diese wesentliche Voraussetzung jedenfalls dann gestört, wenn eine ungleiche Verteilung der Rezeptionschancen begründet wird und Teilnehmer von Äußerungen abgehalten werden, wodurch nicht mehr alle Ideen in den digitalen Meinungsmarkt vordringen können. Dass der tatsächliche Eintritt solcher Szenarien wegen des vielfältigen Informationsmarkts eher unwahrscheinlich sein mag, ${ }^{1267}$ hat für die Frage nach einem grundsätzlich bestehenden Schutzbedarf keinerlei Bedeutung. Insoweit ist auch für die kollektive Sphäre die bereits aufgezeigte Transparenz und die Medienkompetenz der Nutzer bedeutsam. Noch prekärer wird die Lage dann, wenn desinformative Inhalte kursieren und so eine widersprüchliche Informationslage geschaffen wird, um entweder Unsicher-

1264 Siehe hierzu die umfangreichen Verweise in Fn. 1263.

1265 Zu dieser Diskussion siehe umfassend etwa Bortnikov, Netzneutralität und Bedingungen kommunikativer Selbstbestimmung.

1266 Vgl. zu diesem Zusammenhang auch Cornils, ZUM 2019, 89 (95). Diesen Zusammenhang stellt auch Brinkmann, UFITA 2019, 364 (384) her allerdings bezogen auf die Transparenz- und Orientierungsdefiziten auf individueller und die verstärkende Wirkung von Fragmentierung auf „überindividueller Ebene".

1267 Auf das bestehende plurale Angebot am Informationsmarkt weist etwa Milker, ZUM 2017, 216 (220) hin. 
heit hervorzurufen ${ }^{1268}$ oder um gegenüberstehende Meinungslager auseinanderzutreiben. Denn wenn der demokratische Diskurs auf falschen Informationen fußt, wird eine sachlich-geistige Auseinandersetzung zumindest erschwert. Von sachlicher Wahrheits- oder Kompromissfindung kann dann nicht mehr die Rede sein. ${ }^{1269}$

Dass Schutz vor bestimmten Inhalten auch aufgrund dieser kollektiven Sphäre notwendig sein kann, zeigt nicht nur der Tatbestand der Volksverhetzung, $\mathbb{\$} 130$ Abs. 1 StGB. ${ }^{1270}$ Auch die Rechtsprechung des BVerfG ist erkennbar davon geprägt, wenn es Lügen aus dem Schutzbereich der Meinungsfreiheit heraushalten will und dies mit dem verfehlten Zweck für den Meinungsbildungsprozess begründet. Nicht nur in den klassischen Fällen der Lüge, sondern auch in anderen nicht erfassten Fällen desinformativer Inhalte wird dieser Zweck eher gefährdet denn gefördert. Dies kann zu einer Disruption der informativen Grundlage des eigentlichen Meinungsbildungsprozesses führen. Insoweit ist eine solch ablehnende Haltung gegenüber bestimmten Inhalten auch Ausdruck der wehrhaften Demokratie, ${ }^{1271}$ die zwar einerseits Meinungsvielfalt und auch kritische Äußerungen zulässt, andererseits aber eben solchen Inhalten kritisch gegenübersteht, die selbst die wesentlichen Grundvoraussetzungen der Demokratie negieren. ${ }^{1272}$

Weil aber die Transparenzerfordernisse und die Verbesserung der Medienkompetenz nur individuell wirken und es insoweit nicht auszuschließen ist, dass konkrete Inhalte - auch unabhängig von Meinungsrobotern - ${ }^{1273}$ weiterhin entsprechend nachteilige Wirkungen entfalten, besteht weiterhin ein eigenes kollektives Schutzbedürfnis, dem man (regulativ) begegnen kann. Neben der Verpflichtung der Betreiber im Kampf gegen solche Inhalte könnte auch eine strafrechtliche Pönalisierung in Betracht kommen. ${ }^{1274}$

1268 Besonders deutlich wurde das etwa während der Covid-19-Pandemie und zahlreichen Desinformationen, die im Netz kursierten, siehe hierzu u.a. Cinelli u.a., Scientific Reports 10 (2020), 16598; Ferrara, First Monday Vol. 25 Nr. (2020).

1269 Vgl. Keller/Klinger, Political Communication Vol. 36 (2019), 171 (174). Vgl. hierzu Schaal, in: Bedford-Strohm/Höhne/Zeyher-Quattlender, Digitaler Strukturwandel der Öffentlichkeit, S. 121 (127).

1270 Einen ähnlichen Vergleich zieht auch Mafi-Gudarzi, ZRP 2019, 65 (68), wenn es um Überlegungen einer Pönalisierung von Desinformation de lege ferenda geht.

1271 Ähnlich auch Mafi-Gudarzi, ZRP 2019, 65 (67).

1272 Verfassungsrechtlich sind hier besonders die Artt. 21 Abs. 2, 20 Abs. 4, 18, 9 Abs. 2 GG zu nennen. Einfach-rechtlich sehen insbesondere Versammlungsgesetze entsprechende Klauseln vor, etwa $\mathbb{} 15$ Abs. VersG. Und auch $\mathbb{1 3 0 A b s .} 4$ StGB ist eine einfach-rechtliche Antwort der wehrhaften Demokratie, vgl. hierzu Berg, in: Dannecker, FS-Otto, 2007, S. 1065 (1075 ff.).

1273 So etwa Mafi-Gudarzi, ZRP 2019, 65 (68).

1274 Hierauf wird noch zurückzukommen sein, siehe dazu unten 3. Teil, 4. Kap. G. 
C. Untermaßverbot - Erfüllung und Konkretisierung der staatlichen Schutzpflicht?

Der Schutzbedarf hat jedenfalls die notwendigen Ansatzpunkte eines solchen Schutzkonzeptes aufgezeigt: Meinungsäußerung, Informationsvermittlung, Meinungsbildung und die kollektive Seite des Kommunikationsprozesses. Inwieweit aber nicht nur abstrakter, sondern auch konkreter Bedarf einer Regulierung besteht, wird maßgeblich durch das Untermaßverbot vorgegeben. Hierbei erlangen vor allem auch die bisherigen staatlichen Regelungen und sonstigen Maßnahmen Bedeutung, ${ }^{1275}$ die im Folgenden näher untersucht werden sollen. Dabei ist die Darstellung zweigeteilt, weil sich im Laufe der Untersuchung die relevanten normativen Gegebenheiten geändert haben. In einem ersten Teil liegt der Fokus auf den bisher ergriffenen Maßnahmen vor der Einigung der Länder auf einen Medienstaatsvertrag im Dezember 2019 (I.). Der Medienstaatsvertrag soll den bisherigen Rundfunkstaatsvertrag ablösen und diesen an die digitalen Veränderungen anpassen. Hierbei gerieten nicht nur die Medienintermediäre selbst in das Visier der Regulierer, was mittelbar Bedeutung für die Meinungsroboter haben kann. Vielmehr finden sich hier auch Vorgaben, die das Feld der Meinungsroboter unmittelbar betreffen. Auf diese Veränderungen wird erst danach eingegangen (II.).

I. Das bisherige Schutzkonzept des Staates

Eine Analyse des bisherigen Schutzkonzeptes des Staates vor automatisierten Manipulationen durch Meinungsroboter kann nicht ohne eine gewisse Systematisierung ergriffener Maßnahmen und regulativer Ansätze auskommen. Dabei können zunächst allgemeine politische Bemühungen im Kontext der Meinungsroboter aufgezeigt werden (1.). Erst danach stellt sich die Frage, inwieweit das bestehende Recht bereits allgemeine rechtliche Vorgaben für den Einsatz bereithält (2.) und inwieweit auch spezielle Schutzmechanismen für die Informationsund Meinungsfreiheit der Nutzer bestehen (3., 4.), die hier zumindest eine gewisse Wirkung entfalten. Denn nur selten wird der Gesetzgeber vollkommen untätig geblieben sein, die grundrechtlichen Schutzgüter vor privaten Übergriffen zu schützen.

1275 So letztlich schon BVerfGE 49, 89 (142) - Kalkar I. 
1. Beobachtung, Überwachung und Erkenntnissicherung durch den (Bundes-)Gesetzgeber

Der Bundestag - genauer gesagt sein Ausschuss für Bildung, Forschung und Technikfolgenabschätzung - hatte bereits im Februar 2017 zu einem Fachgespräch eingeladen, in dem das Feld der Meinungsroboter beleuchtet wurde. Daneben wurde das Büro für Technikfolgenabschätzung beauftragt, eine entsprechende (Vor-)Studie durchzuführen. Resultat sowohl des Fachgesprächs als auch der Studie war, dass zwar auch hier die Unsicherheiten bei der Beurteilung der Meinungsroboter betont und insbesondere darauf verwiesen wurde, dass die Forschung mehr Daten bräuchte. Allerdings wurde den Meinungsrobotern ein grundsätzlich hohes Risikopotential zugeschrieben, welches in ganz unterschiedlichen Bereichen Wirkung entfalten könne. Mit Blick auf die politische Wirkungssphäre kommt die Studie zu dem Ergebnis:

„Social Bots tragen zur Veränderung der politischen Debattenkultur im Internet bei und können durch die massenweise Verbreitung von (Falsch-) Nachrichten zu einer Desinformation und "Klimavergiftung "im öffentlichen Diskurs führen. Social Bots bergen das Potenzial, das Vertrauen in die Demokratie zu unterlaufen."

Damit entsprechen die Studienergebnisse auch den überwiegend, wenn auch vorsichtigen Bewertungen durch andere Forscher. ${ }^{1276}$ Entscheidend ist zudem, dass die Studie deutlich hervorhebt, dass es zurzeit (2017) noch an technischen Möglichkeiten zur Enttarnung fehle. Entscheidend sei deshalb gerade auch, dass nicht nur die Kompetenz der Nutzer im Umgang mit den neuen Medien und den dortigen Herausforderungen gestärkt werden müsse, sondern dass weitergehende Forschung notwendig sei, was aber an nur eingeschränkten Datenzugängen oftmals scheitere.

\section{Allgemeine rechtliche Vorgaben für den Einsatz von Meinungsrobotern:}

Allgemeine rechtliche Vorgaben für den Einsatz ergeben sich primär im vertraglichen Verhältnis zwischen den Verwendern der Meinungsroboter und den Netzwerkbetreibern. Daneben könnten potentielle Grenzen aber auch im gesetzlichen Schuld-, dem Urheber- und dem Strafrecht bestehen.

1276 Siehe hierzu bereits die Verweise in Fn. 870. 


\section{a. Vertragsrechtliche Vorgaben}

Unabhängig von der genauen vertragstypologischen Einordnung des Nutzungsverhältnisses $^{1277}$ wird der konkrete Inhalt des Vertrages maßgeblich durch die vom Netzwerkbetreiber gestellten Geschäftsbedingungen und die sie ergänzenden Regularien ${ }^{1278}$ konkretisiert. In diesen finden sich auch Regeln für den Einsatz von Meinungsrobotern. In inhaltlicher Hinsicht verbietet Facebook etwa, dass Fake News, Hassrede und andere beleidigende Inhalte geteilt werden. Mit Blick auf die Profildaten enthalten AGB, Gemeinschaftsstandards und die Namensrichtlinie Bestimmungen, wonach keine falschen Namen und Identitäten verwendet werden dürfen, sondern vielmehr der bürgerliche Name verwendet werden muss (Klarnamenzwang). ${ }^{1279}$ Zudem ist der automatisierte Zugriff auf Netzwerkdaten verboten, sofern dies nicht vorab von den Betreibern genehmigt wurde. ${ }^{1280}$ Und auch Twitter hat zumindest Regeln zum Identitätsbetrug aufgestellt ${ }^{1281}$ und untersagt daneben „eine massenweise oder aggressiv ausgeführte Aktivität, die versucht, Twitter oder die Erfahrung der Nutzer auf Twitter zu manipulieren oder zu stören, um den Datenverkehr oder die Aufmerksamkeit für andere Accounts, Produkte, Dienste oder Initiativen zu erhöhen. “1282 Und auch zum Schutz der Integrität von Wahlen bestehen Regularien des Netzwerks. Unter diesen Umständen wären Bots zumindest aus vertragsrechtlicher Sicht nicht nur wegen möglicher Inhalte (Desinformationskampagnen, Hate Speech) verboten, sondern auch weil personenbezogene Daten fingiert (Verstoß gegen die Klarnamenpflicht), multiple Konten eines Nutzers registriert werden und der

1277 Neben Redeker, in: Hoeren/Sieber/Holznagel, Multimedia-Recht, Teil 12 Rn. 423 f., siehe auch Bräutigam/Sonnleithner, in: Hornung/Müller-Terpitz, Rechtshb Social Media, 3 Rn. $18 \mathrm{ff}$. Hennemann/Moritz, ZUM 2017. 544 (548); Bräutigam, MMR 2012, 635 (638 ff.); Schmidt, Social Media, S. 106. Nach dem OLG München NJW 2018, 3115 Rn. 18 und NJW 2018, 3120 Rn. 18 dürfte es sich mangels Entgeltlichkeit bei dem Nutzungsvertrag bei Facebook um einen Vertrag eigener Art handeln. Das LG Frankfurt, MMR 2018, 545 Rn. 9, hat sich nur sporadisch dazu geäußert. Nach ihm handele „es sich um einen schuldrechtlichen Vertrag mit miet-, werk- und dienstvertraglichen Elementen“. Ebenso KG Berlin, ZD 2017, 386 Rn. 56. Der BGH hat zuletzt die Rechtsnatur des Vertrages mangels Erheblichkeit offengelassen, BGH, NJW 2018, 3178 Rn. 19.

1278 So hat Facebook einige ergänzende und themenspezifische Richtlinien durch In-Bezugnahme in den AGBs mit zum Vertragsinhalt gemacht. Hierzu gehören bspw. die spezifischen Bedingungen für Facebookseiten (https://www.facebook.com/policies/pages_groups_events/\#) oder die Datenrichtlinie (https://www.facebook.com/about/privacy/). Auch Twitter verwendet AGB und verweist in diesen auf weitere Regularien, wie die Datenschutzrichtlinie (https://twitter.com/de/privacy) oder die allgemeinen Twitter-Regeln (https://help.twitter.com/de/rules-and-policies/twitter-rules), (Stand aller: 30.05.2021).

1279 Siehe hierzu Nr. 1, 2 der Inhalte zur Registrierung und Kontosicherheit in der Erklärung der Rechte und Pflichten von Facebook, https://www.facebook.com/legal/terms?ref=pf, (Stand: 30.05.2021).

1280 Nr. 3 UAbs. 2 Nr. 3. der Facebook-Nutzungsbedingungen, https:/www.facebook.com/legal/terms?re $\mathrm{f}=\mathrm{pf}$, (Stand: 30.05.2021).

1281 Sog. Impersonation policy, https://help.twitter.com/en/rules-and-policies/twitter-impersonation-policy, (Stand: 30.05.2021.

1282 So die Regel zum Identitätsbetrug, https://help.twitter.com/de/rules-and-policies/twitter-rules, (Stand: 30.05.2021). 
Zugriff auf die Netzwerke automatisch, unautorisiert und mit dem Ziel erfolgt, Aufmerksamkeit anderer Nutzer auf gewisse Inhalte zu lenken.

\section{aa. Wirksamkeit der Nutzungsbedingungen}

Sofern also die Nutzungsbedingungen und die sie ergänzenden Regelwerke wirksamer Vertragsbestandteil geworden sind, ${ }^{1283}$ wären Meinungsroboter schon aus vertragsrechtlicher Sicht unzulässig, soweit die Klauseln nicht selbst gegen zwingendes Recht verstoßen also insbesondere einer Klauselkontrolle standhalten. Für die Bewertung müsste aber überhaupt das deutsche Recht anwendbar sein.

\section{(I.) Anwendbarkeit des deutschen Vertragsrechts?}

Weil regelmäßig nicht die nationale, sondern im europäischen Raum die irische Niederlassung Vertragspartnerin wird, ${ }^{1284}$ es sich also um einen grenzüberschreitenden vertraglichen Sachverhalt handelt, bestimmt die Rom- I-Verordnung das anwendbare nationale Recht. ${ }^{1285}$ Facebook macht in seinen Nutzungsbedingungen jedenfalls mit Wirkung gegenüber Verbrauchern von dem Grundsatz der freien Rechtswahl (Art. 3 Abs. 1 S. 1 Rom-I-VO) Gebrauch. ${ }^{1286}$ Als Verbraucher in diesem Sinne dürften (grundsätzlich) die Nutzer der Profile gelten. Denn während diese der privaten Selbstdarstellung dienen, sollen die Seiten eine öffentlichkeitswirksame Darstellung vor allem kommerzieller Zwecke ermöglichen. ${ }^{1287}$ Das entspräche auch der nach Art. 6 Abs. 1 Rom-I-VO erforderlichen Differenzierung. Nicht nur lässt sich so der subjektive Vertragszweck der Nutzer bestimmen, dieser wird auch objektiv für den Vertragspartner erkennbar. ${ }^{1288}$ Das wird

1283 Einen allgemeinen Überblick hierzu bieten Schwartmann/Ohr, in: Schwartmann, PraxisHb, 11 Rn. 34 ff. sowie Bräutigam/v. Sonnleithner, in: Hornung/Müller-Terpitz, Rechtshb Social Media, Kap. 3.3.

1284 Siehe hierzu das Impressum von Facebook, https://www.facebook.com/legal/terms? ref=pf. Die nationale Tochtergesellschaft, Facebook Germany GmbH mit Sitz in Hamburg, ist nur für das Akquirieren von Werbeanzeigen für den Hauptsitz verantwortlich und unterstützt diesen beim nationalen Marketing, so etwa die Ausführungen vom VG Hamburg, BeckRS 2016, 43614 und vom VG Schleswig BeckRS 2013, 46930.

Siehe zu Twitter AGB Nr. 6, abrufbar unter https://twitter.com/de/tos, (Stand: 30.05.2021).

1285 Verordnung v. 17. Juni 2008, Nr. 593, ABl. L 177/6.

1286 In den Nutzungsbedingungen heißt es unter Ziffer 4.4: „Wenn du ein Verbraucher bist und deinen ständigen Wohnsitz in einem Mitgliedstaat der Europäischen Union hast, gelten die Gesetze dieses Mitgliedstaats für jeglichen Anspruch, Klagegegenstand oder Streitfall, den du uns gegenüber hast und der sich aus diesen Nutzungsbedingungen oder aus den Facebook-Produkten oder im Zusammenhang damit ergibt („Anspruch“). Du kannst deinen Anspruch vor jedwedem Gericht in diesem Mitgliedsstaat klären lassen, das für den Anspruch zuständig ist.“

1287 Vgl hierzu https:/www.facebook.com/business/help/2148431558717386? id=939256796236247, (Stand: 30.05.2021).

1288 Zur Maßgeblichkeit des subjektiven Vertragszwecks soweit er objektiv erkennbar ist siehe Rühl, in: Gsell u.a., Grosskommentar, Art. 6 Rom-I-VO, Rn. 66; Magnus, in: Staudinger, Art. 6 Rom-I-VO Rn. 46; Leible, in: Hüßtege/Mansel, BGB, Art. 6 Rom-I-VO Rn. 21. 
auch für Meinungsroboter gelten, selbst dann, wenn sie gegen Aufwandsentschädigung bzw. Entgelt betrieben werden. Dieser Charakter ist für den Betreiber ohnehin nicht erkennbar. Und zudem spricht vieles dafür, dass die Verbrauchereigenschaft hier extensiver und ggf. vergleichbar mit dem der RL 2011/83/EU ${ }^{1289}$ verstanden werden sollte ${ }^{1290}$. So könnten zumindest die (zwingenden) materiellrechtlichen Verbraucherschutzvorschriften zur Anwendung kommen (Art.6 Abs. 2 Rom-I-VO). ${ }^{1291}$ Anders als bei Art. 13 EuGVÜ geht es hier ja nicht bloß um die Frage des Gerichtsstandes. ${ }^{1292}$ Sofern der kommerzielle Zweck nicht deutlich überwiegt, wäre also von der Verbrauchereigenschaft auszugehen. ${ }^{1293}$ Deshalb dürfte auch hinsichtlich der Verträge bei Twitter, wo keine Rechtswahl erfolgt ist, deutsches Recht gem. Art. 6 Abs. 1 Rom-I-VO regelmäßig Anwendung finden, soweit es um simulierte Nutzerkonten geht. Denn Twitter richtet seinen Dienst gerade auch für Nutzer innerhalb Deutschlands aus (Art. 6 Abs. 1 lit. b) Rom-I-VO). Entsprechendes gölte dann auch für andere Anbieter aus dem europäischen Ausland.

1289 ABl. 2011 L 304/64.

1290 Jedenfalls für ein deutlich weiteres Verständnis als der EuGH Magnus, in: Staudinger, Art. 6 Rom-IVO Rn. 47; in diese Richtung auch Leible, in: Hüßtege/Mansel, BGB, Art. 6 Rom-I-VO Rn. 24. Weller/Nordmeier, in: Spindler/Schuster, Elektronische Medien Art. 6 Rom-I-VO Rn. 8 weisen zumindest auf die Verbraucherrechte-RL und die Auslegungsmöglichkeit hin. Martiny, in: MüKo, BGB, Bd. 13, Art. 6 Rom-I-VO Rn. 15 hält die Anknüpfung an die prozessrechtlichen Vorgaben des EuGH jedenfalls für zweifelhaft. Der gängigen Praxis, den materiell-rechtlichen Verbraucherbegriff ebenfalls eng auszulegen - siehe hierzu die rechtsvergleichende Darstellung von Gottschalk, RiW 2006, 576 (577) -, ist durch die RL 2011/83/EU gerade eine Absage erteilt worden. Rühl, in: Gsell u.a., Grosskommentar, Art. 6 Rom-I-VO Rn. 73 hält, eher die Nähe zu Art. 17 Abs. 1 Brüssel-Ia-VO für ausschlaggebend. Bei Erlass der VO habe der Verordnungsgeber in den Erwägungsgründen eine solche Klarstellung in Kenntnis der RL 2011/83/EU gerade nicht getroffen.

1291 In diese Richtung auch Magnus, in: Staudinger, Art. 6 Rom-I-VO Rn. 47; Leible, in: Hüßtege/ Mansel, BGB, Art. 6 Rom-I-VO Rn. 24. Gottschalk, RiW 2006, 576 (577) weist darauf hin, dass es in erster Linie um die Frage geht, ob die besondere Erfahrung eines Geschäftsmannes vorliegt, die einem Schutz entgegenstehen würde. Den Aspekt des Verbraucherschutzes betont der EuGH besonders in seinem Urt. v. 25.01.2018 - C-498/16 = NJW 2018, 1003 Rn. 40 - Schrems, allerdings ging es hier um Art. 15 Brüssel-I-VO.

1292 Denn hier hat der EuGH stets ein enges Verständnis betont. In der Rechtssache Gruber (EuGH, Urt. v. 20.01.2005 - Rs. C-464/01 = Slg. I-00439, Rn. 39 ff. - Gruber/BayWa AG; siehe zuvor aber auch EuGH, Urt. v. 03.07.1997 - Rs. C-269/95, Slg. I-03767, Rn. 16 - Benincasa) entschied der Gerichtshof, dass ein gemischter Vertrag nur dann die Verbrauchereigenschaft begründe, wenn der beruflich-gewerbliche Zweck „derart nebensächlich ist, dass er im Gesamtzusammenhang (...) nur eine ganz untergeordnete Rolle spielt $(\ldots)^{\text {“. }}$

1293 In diesem Sinne Magnus, in: Staudinger, Art. 6 Rom-I-VO Rn. 47; Leible, in: Hüßtege/Mansel, BGB, Art. 6 Rom-I-VO Rn. 24. Siehe hierzu auch EuGH, Urt. v. 25.01.2018 - Rs. C-498/16 = NJW 2018, 1003 Rn. 36 ff. - Schrems, wo der EuGH betont, dass allein die Verbindung eines Nutzerkontos mit einer Seite nicht per se zur Ablehnung der Verbrauchereigenschaft führt. Hierbei ging es zwar um Art. 15 Brüssel-I-VO, dieser gleicht aber dem Wortlaut von Art. 6 Abs. 1 Rom-I-VO. 
(II.) Transparenzerfordernis des $\mathbb{3} 305$ Abs. 2 BGB

Gestützt auf das Transparenzerfordernis des $\mathbb{} 305$ Abs. 2 BGB wurde teilweise Kritik laut, die zwar nicht die Verlinkungspraxis der Betreiber betraf, ${ }^{1294}$ sondern vielmehr den erheblichen Umfang der Regelwerke. Denn neben den eigentlichen Nutzungsbedingungen werden auch zahlreiche ergänzenden Regelwerke - dazu gehören Communitystandards, Datenschutz und Cookie-Richtlinien u.v.m. Vertragsbestandteil. Nach vereinzelter Ansicht setze $\$ 305$ Abs. 2 Nr. 2 BGB aber voraus, dass die AGB einen im Verhältnis zum Vertrag vertretbaren Umfang haben. ${ }^{1295}$ Es sei dem Nutzer nicht zuzumuten, sich durch die zahlreichen Regelungen durchzuklicken, zumal hiervon ohnehin regelmäßig abgesehen wird, weshalb die AGB nicht wirksam einbezogen worden seien. ${ }^{1296}$ Angesichts der umfangreichen und länderübergreifenden rechtlichen Verpflichtungen ${ }^{1297}$ in Online-Netzwerken, muss man aber zumindest anerkennen, dass der Umfang der AGB schon deshalb deutlich größer sein wird als bei anderen Vertragstypen. ${ }^{1298}$ Und allgemein ist allein der Umfang kein tauglicher Anknüpfungspunkt für die Beurteilung einer wirksamen Einbindung. ${ }^{1299}$ Schon der Tatbestand gibt für eine solche Sichtweise nichts her. Mit der „Zumutbarkeit“ ist allein die barrierefreie Möglichkeit der Kenntnisnahme gemeint, denn nach $\mathbb{3} 305$ Abs. 2 Nr. 2 BGB liegt es in der Verantwortung des Verbrauchers, von der Möglichkeit der Kenntnisnahme auch tatsächlich Gebrauch zu machen. ${ }^{1300}$ Daran ändert auch der empirische Befund, dass viele die AGB nicht wahrnehmen, nichts. ${ }^{1301}$ Von den Netzwerkbetreibern ist mit Blick auf die formale Transparenz insbesondere eine klare Strukturierung und einfache Textgestaltung ${ }^{1302}$ zu fordern. Dem kommen sie aber durch die

1294 Die AGB werden bei Vertragsschluss nicht unmittelbar angezeigt, sondern nur verlinkt. Erst wenn dieser Link angeklickt wird, öffnet sich ein Fenster, in dem eine druckbare Version der AGB zu finden ist. Zu der wirksamen Einbindung in derlei Fällen siehe BGH NJW 2006, 2976 (2977); Schuster, in: Spindler/ders., Elektronische Medien, \$305 BGB Rn. 44 und Berberich, MMR 2010, 736 (737).

1295 Grüneberg, in: Palandt, $\mathbb{3} 305$ Rn. 37; Schlosser, in: Staudinger, BGB, $\mathbb{3} 305$ Rn. 140; kritisch dazu Schmidt, NJW 2011, 1633 (1635).

1296 So u.a. v. Westphalen, VuR 2017, 323 (325); Bräutigam/Sonnleithner, in: Hornung/Müller-Terpitz, 3 Rn. 48 sehen das zumindest als diskutabel an.

1297 Neben vertragsrechtlichen Fragen stellen sich hier u.a. auch datenschutz- und urheberrechtliche Fragen, die es zu klären gilt. Insbesondere die Vorgaben der DS-GVO setzen wiederum hohe Anforderungen an die Transparenz, sodass sich der Umfang letztlich auch anders erklären lässt.

1298 Vgl. mit Blick auf die äußerungsrechtlichen Fragen der Zulässigkeit bestimmter durch AGB verbotener Äußerungen Friede, NJW 2020, 1697.

1299 Kritisch hierzu Schmidt, NJW 2011, 1633 (1635); als mittlerweile unproblematisch ansehend Basedow, in: MüKo BGB, Bd. 2, $\$ 305$ Rn. 75; ebenso schon Löhning, NJW 1997, 1688 (1689).

1300 Denn den Verwender trifft insoweit nur die Obliegenheit diese Möglichkeit bereit zu halten, vgl. Lehmann-Richter, in: Beck Großkommentar, \$305 BGB Rn. 219.

1301 Im Rahmen der datenschutzrechtlichen Einwilligung wird dieses Argument bspw. angeführt, um zu belegen, dass die Einwilligung weder freiwillig noch informiert erfolgt, vgl. hierzu etwa Rothmann, in: Roßnagel/Friedewald/Hansen, Fortentwicklung des Datenschutzes, S. $59 \mathrm{ff}$.

1302 Das OLG Dresden, NJW 2018, 3111 (3113) verweist hinsichtlich des in den Gemeinschaftsstandards erläuterten Begriffs der „Hassrede“ hierauf. 
thematische Sortierung und die textliche Gestaltung nach. Das Problem einer Informationsflut ist durchaus existent. Ein zu harscher Umgang führte aber dazu, dass die AGB auf ein Minimum verkürzt werden, was wiederum zu erheblichen Verständniseinbußen führen kann. Und soweit es wegen des Umfangs um Fragen des Verständnisses geht, ist die inhaltliche Transparenz allein bei $\mathbb{S} 307$ Abs. 1 S. 2 BGB zu verorten. ${ }^{1303}$ Insgesamt sind die AGB also trotz des Umfanges wirksam eingebracht. ${ }^{1304}$

(III.) Inhaltskontrolle der AGBs - Unwirksamkeit der Regelungen?

Davon zu trennen ist die Wirksamkeit der konkreten Inhalte, deren Kontrolle sich überwiegend auf den Maßstab des $\mathbb{3} 307$ Abs. 1, 2 BGB beschränken wird. Die Vorgaben der AGB zu den veröffentlichungsfähigen Inhalten ${ }^{1305}$ werfen zumindest dann keine Bedenken auf, wenn sie unter Beachtung der Rechte der betroffenen Nutzer angewendet werden. ${ }^{1306}$ Insbesondere hinsichtlich der Löschung von Inhalten wird dabei die Meinungsfreiheit der Nutzer zu berücksichtigen sein. ${ }^{1307}$ Etwas anderes kann in Hinblick auf die Klarnamenpflicht gelten. So wurde darin ein Verstoß gegen $\mathbb{1 3}$ Abs. 6 TMG gesehen, weil auch im Verhältnis zu anderen Nutzern der bürgerliche Name verwendet werden muss. ${ }^{1308}$ Allerdings wird nach In-Kraft-Treten derr DS-GVO $\$ 13$ Abs. 6 TMG jedenfalls im Anwendungsbereich der DS-GVO für nicht anwendbar gehalten. ${ }^{1309}$ Mangels

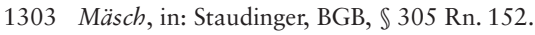

1304 So ausdrücklich, ohne dies zu problematisieren, Schuster, in: Spindler/ders., Elektronische Medien, \305 BGB Rn. 44; Berberich, MMR 2010, 736 (737); Solmecke/Dam, MMR 2012, 71 f. So wohl auch das OLG Dresden, NJW 2018, 3111 (3112f.), wenn es nur eine Inhaltskontrolle vornimmt und zugleich in der Weiterverlinkung auf die zusätzlichen Gemeinschaftsstandards kein Verstoß gegen das Tranzparenzverbot des $₫ 307$ Abs. 1 BGB sieht. Differenzierend zwischen der Möglichkeit, die AGB nur online zu lesen und der Möglichkeit, diese zusätzlich ohne weitere Hürden auszudrucken Kollmann, in: Dauner-Lieb/Langen, BGB, $\$ 305$ Rn. 77.

1305 Auf andere Klauseln, die problematisch sein können, soll hier nicht eingegangen werden. Eine umfangreiche Darstellung hierzu findet sich u.a. bei Bräutigam/Sonnleithner, in: Hornung/Müller-Terpitz, 3 Rn. 54 ff. oder bei Schwartmann/Ohr, in: Schwartmann, PraxisHb, 11 Rn. 34 ff.

1306 Zu den Anwendungsproblemen siehe etwa OLG Stuttgart, NJW-RR 2019, 35 Rn. 27; OLG Dresden, NJW 2018, 3111 Rn. 12; OLG München NJW 2018, 3115 Rn. 25 ff. Siehe hierzu auch LG Frankfurt MMR 2018, 545 und die Anmerkung von Müller-Riemenschneider, a.a.O., 54 und auch Lüdemann, MMR 2019, $279 \mathrm{ff}$.

1307 Hierzu LG Frankfurt MMR 2018, 545; OLG Karlsruhe NJW 2018, 3110; OLG Dresden NJW 2018, 3111; OLG München NJW 2018, 3115; NJW 2018, 3119.

1308 So Caspar, ZRP 2015, 233 (234); deutlich Hullen/Roggenkamp, in: Plath, DS-GVO/BDSG, $\mathbb{} 13$ TMG Rn. 42; Roggenkamp/Krieg, in: Schmidt/Taddicken, Hb. soziale Medien, S. 360; Nink, in: Spindler/Schuster, Elektronische Medien, $\mathbb{1} 13$ TMG Rn. 22; in diese Richtung scheint auch Stadler, ZD 2011, 57 (58 f.) zu tendieren. Das LG Berlin, MMR 2018, 328 Rn. 62 ff. lässt diese Frage offen, sieht aber die Selbstverpflichtung wegen Verstoßes gegen $\$ \mathbb{S} 4,4 \mathrm{a}$ BDSG a.F. als unwirksam an. Krit. hierzu aber Konrad, K\&R 2018, 275. Und Ziebarth, ZD 2013, 375 (377), sieht in der Klarnamenpflicht eine überraschende Klausel i.S.v. $\$ 305$ c Abs. 1 BGB. Ausführlicher zur Klarnamenpflicht u.a. Caspar, ZRP 2015, 233 und bei Kluge, K\&R 2017, 230 (231 ff.).

1309 Zwar trifft die DS-GVO unmittelbare Vorgaben für das Datenschutzrecht, dem letztlich auch die $\mathbb{S} \int 11 \mathrm{ff}$. TMG unterfallen. Allerdings geht die DS-GVO auf die DatenschutzRL 1995/46 zurück, die 
entsprechender Vorgaben innerhalb der DS-GVO kann das gleiche Ergebnis höchstens durch allgemeine Grundsätze oder durch die Rechtfertigungstatbestände $^{1310}$ herbeigeführt werden, immerhin kennt auch die DS-GVO den Grundsatz der Datenminimierung, Art. 5 Abs. 1 lit.c). ${ }^{1311}$ Hullen/Roggenkamp gehen daher davon aus, dass auch unter der DS-GVO weiterhin „eine Nutzung [...] zumindest pseudonym möglich sein muss, wenn dies ,zumutbar “ ist. “1312 Allerdings geht es hier ohnehin nur um das Verhältnis zu anderen Nutzern und nicht um das interne Verhältnis zum Betreiber, ${ }^{1313}$ denn hier erlaubt schon Art. 6 Abs. 1 lit. b) DS-GVO die Verarbeitung und auch $\$ 13$ Abs. 6 TMG stand dem nicht im Weg. ${ }^{1314}$ Selbst wenn die Klarnamenpflicht unwirksam wäre, bliebe der Vertrag und die AGB i.Ü. davon unberührt (\$306 Abs. 1 BGB). Insbesondere der Ausschluss automatischer Zugriffe und das Verbot des mehrfachen Registrierens bleiben bestehen und führen bei Verwendung von Bots zu einem Verstoß gegen die vertraglichen Regelungen. Und auch wenn die geposteten Inhalte nicht den Community-Standards entsprechen, kann ein Vertragsverstoß vorliegen.

\section{bb. Vertragliche Sanktionen bei Vertragsverstößen}

Bei Verstößen behalten sich die Netzwerkbetreiber regelmäßig die Löschung oder Sperrung von Inhalten oder Nutzerkonten vor. ${ }^{1315}$ Angesichts der Bedeutung der sozialen Netzwerke muss auch dieses Recht jedenfalls so ausgeübt werden, dass die Rechte der Nutzer berücksichtigt werden. ${ }^{1316}$ Wegen der weitreichenden nachteiligen Folgen auch für die Betreiber selbst kommt bei unautorisiertem Einsatz der Meinungsroboter eine sofortige Löschung durchaus in Betracht, weil da-

Vorgaben des TMG auf die immer noch wirksame ePrivacy-RL 2002/58/EG. Gleichwohl wird überwiegend davon ausgegangen, dass $\$ 13$ Abs. 6 TMG, von der DS-GVO verdrängt wird, sofern die Anwendungsbereiche kongruent sind; so etwa Conrad/Hausen, in: Auer-Reinsdorff/Conrad, Hb ITund Datenschutzrecht, $\mathbb{S} 36$ Rn. 21; Jandt, ZD 2018, 405 (407); Hullen/Roggenkamp, in: Plath, DSGVO/BDSG, $\$ 13$ TMG Rn. 3; Nebel, K\&R 2019, 148 (149). In diese Richtung auch Kluge, K\&R 2017, 230 (236). Eine genaue Übersicht findet sich hierzu etwa bei Conrad/Hausen, a.a.O., $\mathbb{3 6}$ Rn. 19 ff. Siehe aber jüngst hierzu auch OLG München, Urt. v. 08.12.2020 - 18 U 2822/19 Pre = BeckRS 2020, 34203Rn. 42 ff. und zur unionsrechtskonformen Auslegung Rn. 53 ff.

1310 So Nebel, K\&R 2019, 148 (152).

1311 Nebel, K\&R 2019, 148 (149f.) geht aber davon aus, dass sich hieraus nichts eindeutig ableiten lässt, weil der Verantwortliche nach seinen Vorstellungen den Verarbeitungszweck (frei) bestimmen kann.

1312 Hullen/Roggenkamp, in: Plath, DS-GVO/BDSG, $\mathbb{1 3}$ TMG Rn. 43a. Kluge K\&R 2017, 230 (236) deutet zumindest an, dass ein solches Verständnis der DS-GVO entnommen werden kann. Nebel, K\&R 2019, 148 (152) spricht sich i.E. auch gegen eine Klarnamenpflicht aus, leitet das aber aus dem Rechtfertigungstatbestand des Art. 6 Abs. 1 lit. f DS-GVO her. Anders indes jüngst OLG München, Urt. v. 08.12.2020 - 18 U 2822/19 Pre = BeckRS 2020, 34203Rn. $53 \mathrm{ff}$.

1313 So bereits zu $\$ 13$ Abs. 6 TMG andeutend OLG Hamburg, ZUM 2009, 417 (420); und zu $\$ 4$ Abs. 6 TDDSG OLG Düsseldorf, MMR 2008, 618 (620).

1314 Müller-Broich, TMG, $\mathbb{1 3}$ Rn. 10.

1315 Vgl. Ohr, in: Schwartmann, Praxishb Medienrecht, Kap. 11 Rn. 40.

1316 Hierzu etwa LG Frankfurt a.M. ZUM-RD 2018, 626 ff., in Ansätze auch OLG Karlsruhe, GRURRS 2018, 50856 und OLG München, NJW-RR 2020, 1171 Rn. 62 ff. 
rin regelmäßig ein „wichtiger Grund“ i.S.v. $\mathbb{S} 314$ Abs. 1 S. 1 BGB liegen wird. ${ }^{1317}$ Weil der Verwender regelmäßig auch schuldhaft i.S.d. $\$ 276$ Abs. 1 BGB handeln dürfte, kommt ein Schadensersatzhaftung nach $\mathbb{} 280$ Abs. 1 S. 1 BGB in Betracht. Der erlittene Schaden wird hier vor allem in der unrechtmäßigen Beanspruchung der Serverkapazitäten zu sehen sein, sodass es auch hier um die Beseitigung der Inhalte gehen wird ( $\$ 249$ Abs. 1 BGB). Daneben kann ein finanzieller Ausgleich jedenfalls dann in Betracht kommen, wenn auch der Unternehmenswert beeinflusst wurde, wobei aber die Rückführbarkeit von Wertveränderungen auf den Bot-Einsatz kaum möglich sein dürfte.

\section{b. Gesetzliches Schuldrecht-(Quasi-)negatorischer Unterlassungs-und Beseitigungsanspruch, SS 1004 Abs. 1, 823 Abs. 1 BGB}

Sofern deutsches Recht auch mit Blick auf Eigentumsbeeinträchtigungen einschlägig ist, ${ }^{1318}$ besteht mit dem negatorischen Unterlassungs- und Beseitigungsanspruch nach $\mathbb{S} 1004$ Abs. 1 BGB die Möglichkeit, Beeinträchtigungen absoluter Rechte und Rechtsgüter zu beseitigen ( $\$ 1004$ Abs. 1 S. 1 BGB, ggf. analog) und für die Zukunft zu verhindern ( $\mathbb{S} 1004$ Abs. 1 S. 2 BGB, ggf. analog). Als Anknüpfungspunkt hierfür kommt neben dem Sacheigentum i.S.d. $\mathbb{9 0 3}$ BGB auch ein „virtuelles Hausrecht“1319 der Netzwerkbetreiber in Betracht. Zumindest an

$1317 \mathrm{Zu}$ einer solchen Kündigung etwa bei Verstößen gegen inhaltliche Vorgaben OLG Dresden, GRURRS 2020, 1229; LG Frankenthal, GRUR-RS 2020, 23730 Rn. 28 ff.

1318 Bei Facebook ist dies im Verhältnis zu Verbrauchern ohne Weiteres der Fall. Bei anderen Anbietern, die keine Rechtswahl getroffen haben, richtet sich dies hingegen nach den kollisionsrechtlichen Bestimmungen der Rom-II-VO bzw. des Art. 40 EGBGB.

1319 Ein solches Recht ist in der Judikatur durchaus anerkannt. Das LG Bonn, MMR 2000, 109 (110), meinte, dass es wegen des virtuellen Hausrechts nahe läge, „die Regelungen über das Eigentum entsprechend anzuwenden.“ Zustimmend Ladeur, MMR 2001, 787 (788) Das OLG Köln, MMR 2001, 52. Das BSG, MMR 2013, 675 (676) nahm ein solches Recht des Betreibers an, damit „einer denkbaren Haftung als (...) Betreiber einer Internetplattform“ vorgebeugt werden kann. Das OLG Dresden, NJW 2018, 3111 (3114), kam bei der Inhaltskontrolle der Nutzungsbedingungen von Facebook nach $\$ 307$ Abs. 1 S. 1 BGB hierauf zu sprechen. Streitgegenständlich war der Ausschluss eines Nutzers vom Netzwerk aufgrund eines veröffentlichen Inhalts, der gegen den Gemeinschaftsstandard der Hassrede verstieß. Insoweit kam es auf eine Abwägung der kollidierenden Rechte und Interessen des Nutzers und des Anbieters an. Für Letzteren stritt sein virtuelles Hausrecht. Zur Begründung führte das OLG zwei Gedanken an: Erstens stünde den Betreibern regelmäßig das Eigentumsrecht aus $\$ 903$ BGB an der Hardware, auf denen die Inhalte der Nutzer gespeichert werden, zu. Nach „\$S 903 S. 1 Alt. 2, 1004 BGB kann daher der Betreiber jeden anderen von der Nutzung der Hardware durch das Speichern von Inhalten auf dieser abhalten." Im Falle nur gemieteter Hardware griffe jedenfalls der possessorische Besitzschutzanspruch aus $\mathbb{S} 8662$ Abs. 1 S. 1,858 Abs. 1 BGB. Zweitens haftet der Betreiber als Intermediär für die Beiträge seiner Nutzer und kann insbesondere auf Unterlassung in Anspruch genommen werden, sodass er ein Recht brauche, haftungsbegründende Beiträge zu löschen. Ein virtuelles Hausrecht des Eigentümers der Hardware bejahen auch das LG Ulm, NJW-RR 2015, 1167 f., das LG Mosbach, Beschl. v. 01.06.2018 - 1 O 108/18 - BeckRS 2018, 20323 Rn. 17 und auch das OLG München, NJW 2018, 3115 Rn. 28, wie auch das LG Leipzig, Endurteil v. 12.07.2019 - 08 O 2491/18 = GRUR-RS 2018, 38785 Rn. 48. Ein solches scheint auch das LG Offenburg, Urt. v. 26.09.2018 - 2 O 310/18, Rn. 50, anzunehmen., wenngleich es seine Relevanz bei vertraglichen Verhältnissen als niedrig einzuschätzen scheint, a.a.O., Rn. 51. I.E. - u.a. - ebenfalls anerkennend Conraths/Krüger, MMR 2016, 310 (311f.); 
den Datenträgern der Server werden Eigentumsrechte der Betreiber bestehen. ${ }^{1320}$ Veröffentlicht ein Nutzer Inhalte oder betätigt er sich durch andere Interaktionen in den Netzwerken, werden die Inhalte und Daten auf diesen gespeichert. Der Nutzer nimmt damit Einfluss auf das Eigentum der Netzwerkbetreiber. ${ }^{1321}$ Erfolgt der Zugriff widerrechtlich - d.h. außerhalb des vertragsrechtlichen Rahmens -, besteht keine Duldungspflicht der Betreiber i.S.v. $\$ 1004$ Abs. 2 BGB. Der Störer ist dann zur Beseitigung und ggf. Unterlassung verpflichtet.

Sofern entsprechende Eigentumsrechte der Netzwerkbetreiber bestehen, kommt einem „virtuellen Hausrecht“ daneben keine eigenständige Bedeutung zu. ${ }^{1322}$ Einzig für Betreiber von Seiten, die keine eigenen Server betreiben und daher eine Sperrung oder Löschung von Inhalten nicht schon auf ihr Eigentumsrecht aus $\$ 903$ BGB stützen können, kann das virtuelle Hausrecht zu einem absoluten Recht verhelfen. ${ }^{1323}$ Das wird bei sozialen Netzwerken aber nur selten der Fall sein.

\section{c. Urheberrecht:}

Der Einsatz von Meinungsrobotern kann daneben auch urheberrechtlich relevant sein, was insbesondere Ansprüche auf Beseitigung und Unterlassung einer Rechtsverletzung nach $\mathbb{} 97$ Abs. 1 UrhG nach sich ziehen würde. Wird der Schutz vor Verletzungen im deutschen Territorium begehrt, ist das deutsche Urheberrecht jedenfalls einschlägig (lex loci protectionis). ${ }^{1324}$ Bei Streitigkeiten mit Bezug zu Mitgliedstaaten der EU regelt dies Art. 8 Rom-II-VO. Weil aber unklar ist, ob und wenn ja welche Daten beim Einsatz von Meinungsrobotern ausgetauscht und verwendet werden und welche netzwerkeigenen Strukturen hierfür in Anspruch genommen werden, lassen sich die urheberrechtlichen Spannungsfelder nur skizzieren: Mit Blick auf die netzwerkeigenen Daten der Betreiber geht etwa Libertus ohne Weiteres davon aus, dass die Installation von Social Bots eine Vervielfältigungshandlung i.S.d. \16 UrhG darstellt. ${ }^{1325}$ Dabei nimmt er aller-

Maume, MMR 2007, 620. Ausführlich mit dem virtuellen Hausrecht beschäftigen sich u.a. Schmidl, K\&R 2006, 563 und Maume, MMR 2007, 620 ff. Kritisch zu diesem Recht etwa Raff, in: MüKo BGB, Bd. 8, $\$ 1004$ Rn. 33.

1320 Vgl. Determann, ZD 2018, 503 (505). Denga, NJW 2018, 1371, spricht wohl deshalb den dort gespeicherten Daten im Falle der Vernichtung des Trägermediums nur einen „akzessorischen Integritätsschutz“ zu. Siehe hierzu auch Spindler, JZ 2016, 805 (812).

1321 So die maßgebliche Begründung des OLG Dresden, NJW 2018, 3111 (3114); siehe hierzu auch Elsaß/Labusga/Tichy, CR 2017, 234 (236).

1322 Vgl. Conraths/Krüger, MMR 2016, 310 (310, 311).

1323 Hier käme dann eine analoge Anwendung der $\$ \mathbb{S} 903,1004$ BGB dann in Betracht, wenn ein klar definierbarer Raum vorliegt, der durch tatsächliche Einwirkungsmöglichkeiten beherrscht werden kann, hierzu Maume, MMR 2007, 620 (624); Schmidl, K\&R 2006, 563 (564); Schwenke, K\&R 2012, 305 (306). Jedenfalls i.E. auch bei nur bestehendem Besitz anerkennend Conraths/Krüger, MMR 2016, 310 (311f.).

1324 BGH, ZUM 2003, 225 (226) - Felsberg; LG Hamburg, ZUM 2016, 887 (889).

1325 Libertus, ZUM 2018, 20 (21). 
dings Bezug auf zwei Entscheidungen zu Bots, die in der Abwesenheit des Nutzers Charaktere in Online-Spielen steuerten (sog. Cheat-Bots). Das OLG Dresden sah in solchen Bots eine Vervielfältigungshandlung, ${ }^{1326}$ denn zum Programmieren und Ausführen der Bot-Programme musste auf die eigentlichen Spieldaten zugegriffen werden, die dadurch in den Arbeitsspeicher der PCs geladen wurden. ${ }^{1327}$ Eine Beeinträchtigung des Rechts ergab sich aufgrund der Zweckübertragungslehre ${ }^{1328}$, weil nur eine private Nutzung vertraglich gestattet war, die Bots aber gewerblich betrieben wurden.

Eine Übertragung dieser Rechtsprechung kann vor allem in den Fällen erfolgen, in denen netzwerkinterne Daten Eingang in das Botprogramm finden. Inwieweit dies beim normalen Browser-Zugriff der Fall sein kann, also die zwischen dem Anwender-PC und den Servern von Facebook ausgetauschten Daten für den Aufbau der Webseite für die Botprogramme relevant sind, ist unklar. ${ }^{1329}$ Komplexe Botprogramme werten aber vor allem die Netzwerkdaten mit Hilfe der API aus, um situationsspezifisch reagieren zu können. ${ }^{1330}$ Das gilt etwa dort, wo auf bestimmte Schlagwörter reagiert werden soll. Hierfür werden Netzwerkdaten über die API bereitgestellt, die zumindest in die Arbeitsspeicher der PCs gelangen. Weil die Daten aus verschiedenen Inhalten bestehen, kann in ihnen eine

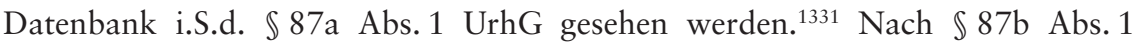
UrhG besitzen aber die Hersteller, also die Netzwerkbetreiber, die ausschließlichen Nutzungsrechte an diesen Datenbanken, sodass eine und für den Einsatz der Bots wiederholte und systematische Vervielfältigung dieser Daten auf dem Anwender-PC dieses Recht jedenfalls dann verletzen kann, wenn die berechtigten Interessen der Netzwerkbetreiber unzumutbar beeinträchtigt werden. Weil aber der manipulative Einsatz den Betrieb auch für andere Nutzer stört und so nicht nur die „Integrität“, sondern auch die Attraktivität der Dienste bedroht, und schon deshalb vertragsrechtlich untersagt ist, wäre dies regelmäßig der Fall. ${ }^{1332}$ Entsprechendes gilt letztlich auch nach $\$ 55$ a UrhG für den Fall, dass die übermittelten Daten als Datenbankwerk ( $\mathbb{4} 4$ Abs. 2 UrhG) zu qualifizieren wären. ${ }^{133}$

Daneben kann auch die Verwendung von unternehmenseigenen Applikationen entgegen der Nutzungsbedingungen urheberrechtlich relevant sein. Vorausset-

1326 OLG Dresden, MMR 2015, 402 (403); siehe auch Jülicher/Röttgen, InTeR 2017, 15 (17).

1327 Ausführlicher dazu das OLG Dresden, a.a.O., (403).

1328 Zu dieser statt vieler Schulze, in: Dreier/ders., UrhG, $\mathbb{} 31$ Rn. 110 f. m.w.N.

1329 Ein herkömmlicher Abruf geschützter Bild-, Audio- oder Videodateien ( $\$ 2$ Abs. 1 Nr. 2., 5, 6 UrhG) ist unschädlich, sofern diese nur im Zwischenspeicher als normale Begleiterscheinung der Kommunikation zwischen Browser und Server gespeichert werden, $\$ 44$ a UrhG. Grundlegend hierzu EuGH, Urt. v. 05.06.2014 - Rs. C-360/13 = EuZW 2014, 637, Public Relations Consultants Association Ltd./Newspaper Licensing Agency Ltd u.a.

1330 Ausführlicher hierzu mit Blick auf die Twitter-Streams Wolf, WRP 2019, 440 (443 ff.).

1331 Wolf, a.a.O., (444).

1332 Wolf, ebd.

1333 Wolf, a.a.O., (445). 
zung wäre dann nicht nur, dass sie als Computerprogramme nach $\mathbb{S} \mathbb{S} 1,2$ Abs. 1 Nr. 1 Var. 4, 69a Abs. 1 UrhG geschützt sind, sondern auch dass sie zum Einsatz der Bots Verwendung finden und hierfür nicht nur genutzt, sondern auf dem jeweiligen Endgerät installiert, also vervielfältigt werden $(\mathbb{S} 16$ Abs. 1 UrhG). Sehen die Nutzungsbedingungen für Zugriffe bestimmte Restriktionen, etwa hinsichtlich der Modalitäten vor, die die Verwendung von Bots ausschließen, handelt man außerhalb des eingeräumten Nutzungsrechts und beeinträchtigt urheberrechtlich geschützte Interessen. ${ }^{1334}$ Entsprechendes dürfte auch für Fälle gelten, in denen über die API auf die Netzwerke zugegriffen wird, sofern sie als Computerprogramme i.S.d. $\int 69$ a Abs. 1 UrhG geschützt sind. ${ }^{1335}$ In diesen Fällen bestünde folglich ein Anspruch aus $\mathbb{S} 97$ Abs. 1 S. 1 UrhG, den das Unternehmen als Ausübungsberechtigter ( $\$ 69$ b Abs. 1 UrhG) geltend machen kann.

\section{d. Strafrecht}

Auch das Strafrecht könnte den Einsatz der Roboter bereits regulieren. Deutsches Strafrecht ist jedenfalls anwendbar, wenn der tatbestandliche Erfolg im Inland eintritt oder der Täter im Inland gehandelt hat, $\mathbb{S} 33,9$ Abs. 1, Var. 3 StGB. ${ }^{1336}$ Bei Taten, wo dies nicht der Fall ist, ist zumindest über $\mathbb{\int} 7$ Abs. 2 Nr. 1 StGB bei einem deutschen Täter deutsches Strafrecht anwendbar.

aa. Das Verändern statistischer Daten als tatbestandliche Handlung der $\mathbb{\int} \int 303 a, b$ StGB?

Die $\int \mathbb{S} 303 a$, b StGB könnten die Plattformbetreiber insbesondere dort vor automatisierten Zugriffen schützen, wo Bots Einfluss auf die Auswertung der statistischen Daten haben, auf die die Algorithmen der Netzwerke reagieren.

\section{(I.) Datenveränderung, \303a StGB}

Darin könnte zunächst einmal eine Datenveränderung im Sinne von $\mathbb{3} 303 \mathrm{a}$ StGB zu sehen sein. Voraussetzung wäre, dass Daten inhaltlich so umgestaltet werden,

1334 So auch Libertus, ZUM 2018, 20 (22), auch wenn dort nicht deutlich wird, dass sein Bezugspunkt nicht jegliche Nutzung, sondern nur die Nutzung der Apps ist.

1335 Umfangreicher zu der Programmqualität von APIs, Grützmacher, in: Wandtke/Bullinger, UrhR, \$69a UrhG Rn. 31 m.w.N.

1336 Bei abstrakten Gefährdungsdelikten wie der Volksverhetzung soll diese Norm aber nach der StA Hamburg nicht anwendbar sein, weil dies in Zeiten des Internets zu einer Allzuständigkeit des deutschen Strafrechts selbst in Fällen führt, in denen Äußerungen im Ausland getätigt wurden, dort aber straffrei sind, siehe hierzu StA Hamburg, Entscheidung v. 08.03.2016 - 7101 AR 57/16 = BeckRS 2016, 8783. Siehe hierzu auch BGH, NStZ 2015, 81 Rn. 8. 
dass sie einen anderen Informations- oder Aussagegehalt erhalten. ${ }^{1337}$ Die Netzwerke basieren maßgeblich auf einem Ranking der relevanten Inhalte, welches verschiedene Informationen und Parameter, wie die Anzahl der Likes und Reposts einzelner Inhalte und damit Daten i.S.v. $\$ 202$ a Abs. 2 StGB, ${ }^{1338}$ berücksichtigt. Indem Bots auf die Relevanz bestimmter Themen, Beiträge oder auch Hashtags Einfluss nehmen, werden Teile der dem Ranking zugrundeliegenden akkumulierten Daten verändert. Insoweit kann eine Datenveränderung durchaus vorliegen. ${ }^{1339}$ Auch das zusätzliche, einschränkende Tatbestandsmerkmal der Rechtswidrigkeit ${ }^{1340}$ steht einer Strafbarkeit nicht entgegen, weil den Verwendern gerade keine eigentümerähnliche Befugnis hinsichtlich der Daten zukommt. ${ }^{1341}$ Neben dem Verwender kann auch der Urheber der Bots strafbar sein, denn nach $\mathbb{\$} 303 \mathrm{a}$ Abs. 3 StGB gilt $\$ 202$ c StGB für Vorbereitungshandlungen entsprechend und gem. $\ 202$ c Abs. 1 Nr. 2 StGB ist eine solche gerade die Herstellung von „Computerprogrammen, deren Zweck die Begehung einer solchen Tat ist“.

\section{(II.) Computersabotage, $\mathbb{} 303$ b Abs. 1, 2 StGB}

Zugleich ist das Rankingverfahren auch eine Datenverarbeitung i.S.d. $\mathbb{S} 303 \mathrm{~b}$ StGB ${ }^{1342}$, sodass auch eine Computersabotage in Betracht käme. Voraussetzung hierfür ist, dass sie von wesentlicher Bedeutung für einen anderen ist. Auch wenn Zaczyk die Formulierung als uferlos bezeichnet, ${ }^{1343}$ geht es hierbei letztlich um den Ausschluss von Bagatellfällen ${ }^{1344}$. Das Rankingverfahren aber ist eines der

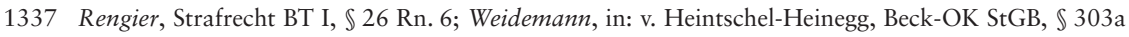
Rn. 14.

1338 Davon geht jedenfalls auch Libertus, ZUM 2018, 20 (23), aus. Zur Weite des Datenbegriffs siehe u.a. Graf, in: MüKo-StGB, Bd. 5, $\$ 302$ a Rn. 12.

1339 Hierzu auch Libertus, ZUM 2018, 20 (23). Insgesamt dürfte das davon abhängen, welchen Einfluss die veränderten Informationen haben, also ob durch das mehrfache Liken oder Teilen eines Beitrages letztlich der Rankingwert für eine Vielzahl von Nutzern verändert wird. Das aber kann nicht ohne Weiteres festgestellt werden. Auch die offiziellen Informationen von Facebook zu ihrem Ranking Verfahren helfen nicht weiter. Bei Twitter ist das wesentlich leichter zu beantworten, da es hier immer Trending Hashtags gibt, deren Beurteilung maßgeblich auch von der Anzahl der Posts abhängt, die das Hashtag enthalten.

1340 So u.a. Weidemann, in: v. Heintschel-Heinegg, Beck-OK StGB, $\mathbb{3} 303$ a Rn. 5, 6; Rengier, Strafrecht BT I $₫ 26$ Rn. 7; Heger, in: Lackner/Kühl, StGB, $\$ 303 a$ Rn. 4; Gercke, in: Spindler/Schuster, Elektronische Medien, $\mathbb{S} 303$ a StGB Rn. 8; ein solches Verständnis legt auch BT-DrS. 10/5058, S. 34 nahe.

1341 Vgl. OLG Nürnberg, ZD 2013, 282 (283); Weidemann, in: v. Heintschel-Heinegg, Beck-OK StGB, \303a Rn. 5; hierfür sprechen auch die Ausführungen in BT-Drs. 10/5058, S. 34.

1342 So letztlich auch Libertus, ZUM 2018, 20 (23).

1343 Zaczyk, in: Kindhäuser/Neumann/Paeffgen, StGB, $\mathbb{3} 303 \mathrm{~b}$ Rn. 5.

1344 So die Gesetzesbegründung, BT-Drs. 16/3656, S. 13. Der zugrundeliegende Rahmenbeschluss 2005/222/JI des Rates schließt die Strafbarkeit auch dann aus, „wenn [ein] leichter Fall vorliegt.“ Krit. hierzu Zaczyk, a.a.O., Rn. 1, 5, der die Norm wegen Verstoßes gegen Art. 103 Abs. 2 GG für verfassungswidrig hält. Auch Roos/Schumacher, MMR 2014, 377 (379) weisen auf die mit dem Begriff verbundene Unklarheit hin. 
zentralen Mechanismen für die personalisierte Darstellung der Inhalte ${ }^{1345}$ und damit für den wirtschaftlichen Erfolg und das Nutzererlebnis entscheidend. Ein Bagatellfall liegt somit fern. Vielmehr kommt sogar der Qualifikationstatbestand aus $\mathbb{S} 303$ b Abs. 2 StGB in Betracht. ${ }^{1346}$ Weitere Voraussetzung ist aber, dass die Datenverarbeitung auch erheblich gestört werden muss. ${ }^{1347}$ Für die Störung reicht es zwar aus, dass der reibungslose Ablauf beeinträchtigt wird. ${ }^{1348}$ Anders als bspw. bei DDoS-Angriffen ${ }^{1349}$, bei denen der Dienst nur eingeschränkt zugänglich ist, führt eine Manipulation der zugrundeliegenden Daten - zumal sich diese nur auf die Täuschung über die Beliebtheit bzw. Relevanz eines Inhalts beschränkt nicht dazu, dass das gesamte Rankingverfahren unbrauchbar wird. Der Datenverarbeitungsvorgang an sich bleibt also unberührt. Er wird nur systematisch ausgenutzt, indem relevante Daten auf den Servern gespeichert werden, die sodann Eingang in den Verarbeitungsvorgang finden. Eine erhebliche Störung bzw. eine - sowie es das das Übereinkommen des Europarates über Computerkriminalität vom 23. November 2001 in Art. 3 und der Rahmenbeschluss des Rates 2005/222/JI in Art. 3 formulieren - „schwere Behinderung oder Störung“ liegt nicht vor. ${ }^{1350}$

Mit der Änderung des $\$ 303 b$ StGB hat sich aber auch seine Schutzrichtung geändert. ${ }^{1351}$ Nun soll „das Interesse der Betreiber und Nutzer von Datenverarbeitungen allgemein an deren ordnungsgemäßer Funktionsweise“ geschützt werden. ${ }^{1352}$ Legt man das Merkmal der erheblichen Störung in diesem Lichte aus, kann auch ein fehlerhaftes Ergebnis die Strafbarkeit begründen. Das gilt etwa, wenn die Rankingverfahren beliebte Themen der Nutzergemeinschaft oder die

1345 Der Gesetzgeber hat bei Privatpersonen gerade auf die zentrale Bedeutung für die Lebensgestaltung abstellen wollen, s. hierzu BT-Drs. 16/3656, S. 13.

1346 So nahm das LG Düsseldorf, MMR 2011, 624 (625), bei einem DDoS-Angriff auf Pferdewettanbieter, (scheinbar) an, dass die wesentliche Bedeutung jedenfalls dann zu bejahen ist, wenn die „Datenverarbeitung (...) für die betroffenen Firmen von einigem Wert [sind]“.

1347 Urteile, an denen man sich ggf. orientieren könnte, existieren bislang nicht, was die Frage nach der Bedeutung nicht gerade einfacher macht. Das LG Düsseldorf, MMR 2011, 624 hat $\$ 303$ b StGB nur oberflächlich angesprochen. Der BGH, NStZ 2017, 470, hat sich in seinem Beschluss auch nur mit der Frage auseinandergesetzt, ob $\$ 303 \mathrm{~b}$ StGB auch Datenverarbeitungen, die zu rechtswidrigen Zwecken eingesetzt werden, schützt.

1348 Weidemann, in: v. Heintschel-Heinegg, Beck-OK StGB, $\mathbb{3} 303$ b Rn. 15 m.w.N.

1349 Distributed Denial of Service Angriffe: Hierbei werden einzelne Dienste oder Server durch zahlreiche Zugriffe so überlastet, dass sie zusammenbrechen und ein Zugriff nicht mehr oder nur eingeschränkt möglich ist. Der Straftatbestand wurde auch wegen des Aufkommens derartiger Angriffe geschaffen. 2001 etwa war Lufthansa Opfer eines solchen Angriffs. Da $\$ 303$ b Abs. 1 Nr. 2 StGB zu dieser Zeit noch nicht existierte, war die Strafbarkeit nur nach $\$ 240$ Abs. 1 StGB zu beurteilen, hierzu AG Frankfurt MMR 2005, 863 und das Revisionsverfahren vor dem OLG Frankfurt, MMR 2006, 547. Hierzu aus Sicht des \$303b StGB etwa Hoffmanns, ZIS 2012, 409 (411f.).

1350 Die Änderung des $\$ 303$ b StGB diente immerhin der Umsetzung der beiden Akte, so BT-Drs. 16/ 3656, S. 12.

1351 So BT-Drs. 16/3656, S. 13.

1352 BT-Drs. 16/3656, S. 13. Zaczyk, in: Kindhäuser/Neuman/Paeffgen, StGB, \303b Rn. 1, sieht wegen dieses diffusen Interesses Spannungen mit Art. 103 Abs. 2 GG, die für ihn die Verfassungswidrigkeit begründen. 
persönliche Relevanz von Inhalten bewerten sollen. Voraussetzung wäre aber, dass die Veränderung des Ergebnisses auch erheblich ist. Das dürfte - um eine Ausweitung der Strafbarkeit zu verhindern - nur dann in Betracht kommen, wenn der Einsatz von Meinungsrobotern vergleichbare Wirkungen hat wie andere technische Funktionsbeeinträchtigungen wie etwa bei DDoS-Angriffen. Dort, wo die Datenverarbeitung maßgeblich allein auf Aktivitätsindikatoren der Nutzer beruht, die dann durch Meinungsroboter massenhaft simuliert werden, kommt das eher in Betracht als dort, wo dem Verarbeitungsvorgang vielfältigere Parameter zugrunde liegen und deshalb nur ein (Teil-)Aspekt betroffen ist. Das Rechenergebnis muss dann erheblich gestört sein, sofern alle anderen Parameter unberührt geblieben sind. Insoweit kann sich ein Unterschied etwa zwischen den Trending-Topics ${ }^{1353}$ und der algorithmenbasierten Weitergabe der Inhalte im News Feed ergeben. Die Strafbarkeit bleibt aber eine Frage des Einzelfalles. Als taugliche Tathandlung kommen die des $\mathbb{S} 303 \mathrm{a}$ Abs. 1 StGB $(\mathbb{S} 303 \mathrm{~b}$ Abs. 1 Nr. 1 StGB) oder die Dateneingabe oder -Übermittlung nach Nr. 2 in Betracht. Im letzten Falle müsste dann noch das eingrenzende subjektive Merkmal der Nachteilszufügungsabsicht erfüllt sein.

\section{bb. Grenzen für Beitragsinhalte}

Besonders wenn es um Hate Speech oder auch Desinformation ${ }^{1354}$ geht, bestehen mit den $\mathbb{S} \mathbb{S} 130,185 \mathrm{ff}$. StGB, $145 \mathrm{~d}$ StGB auch inhaltliche Grenzen. Für $\mathbb{S} \mathbb{S} 185 \mathrm{ff}$. StGB müsste dafür ein ehrverletzender Inhalt kundgegeben werden, wobei insbesondere die Grenzziehung zwischen Ehrverletzung und zulässiger Äußerungen Schwierigkeiten bereiten wird. ${ }^{1355}$ Entscheidend wird dann eine Abwägung zwischen Persönlichkeitsrecht und Meinungsfreiheit. ${ }^{1356}$ Die $\mathbb{S} \mathbb{1 8 6 - 1 8 7}$ StGB sind nur bei Tatsachenbehauptungen einschlägig und differenzieren nach dem Wahrheitsgrad und der Kenntnis des Täters hiervon. ${ }^{1357}$

\footnotetext{
1353 Dies etwa bejahend Libertus, ZUM 2018, 20 (23f.), der daraufhin weist, dass die Manipulation im Extremfall zu einem vollkommen unbrauchbaren Ergebnis führen könne.

1354 Ausführlicher zur Strafbarkeit der Verbreitung von Fake News Hoven, ZStW 129 (2017), 718.

1355 Etwa bei Kollektivbeleidigungen, siehe hierzu BVerfGE 93, 266 (301ff.) - Soldaten sind Mörder; BVerfG, NJW 2015, 2222 Rn. 16 f. - FCK CPS; NJW 2016, 2643 Rn. 16 f. - ACAB.

1356 Ob dies über $\mathbb{1} 193$ StGB erfolgt (so Zaczyk, in: Kindhäuser/Neumann/Paeffgen, StGB, $\mathbb{1 9 3}$ Rn. 6 ; BVerfGE 12, 113 (125) - Schmid-Spiegel; 42, 143 (152) - Deutschland-Magazin bezeichnet $\$ 193$ als „eine besondere Ausprägung des Grundrechts der Meinungsfreiheit“, jeweils unter Bezugnahme auf BGHSt 12, 287 (293).) oder direkt dem Art. 5 Abs. 1 S. 1 GG zu entnehmen ist (Joecks/Pegel/ Regge, in: MüKo StGB, Bd. 4, $\$ 193$ Rn. 3; Lencker/Eisele, in: Schönke/Schröder, StGB, $\mathbb{} 193$ Rn. 1), kann dahingestellt bleiben.

1357 Die Meinungsfreiheit wird hier kaum relevant, denn bei $\mathbb{1} 187$ StGB besteht ohnehin kein Schutz unter Art. 5 Abs. 1 S. 1GG. Und auch bei $\$ 186$ StGB besteht höchstens ein abgeschwächter Schutz. Der Tatbestand trägt den Sorgfaltspflichten Rechnung, die für die derivativ geschützten Tatsachenbehauptungen entwickelt wurden, hierzu bereits oben A.I.2.
} 
Bei qualifizierten Äußerungen ${ }^{1358}$ die sich auf eine anhand bestimmter Merkmale unterscheidbare Personengruppen beziehen, diese herabwürdigen und geeignet sind den öffentlichen Frieden zu stören, kommt zusätzlich ${ }^{1359}$ der Tatbestand der Volksverhetzung nach $\mathbb{\int} 130 \mathrm{StGB}^{1360}$ in Betracht. Insbesondere bei der aggressiven Verunglimpfung von Flüchtlingen ${ }^{1361}$, religiösen Gruppen ${ }^{1362}$ oder anderer in Nr. 1 aufgeführter Bevölkerungsgruppen kann der Tatbestand einschlägig sein. Probleme ergeben sich hier, wenn fremde Inhalte repostet werden. ${ }^{1363}$ Allein der volksverhetzende Charakter der ursprünglichen Äußerungen reicht für eine eigenständige Strafbarkeit nicht aus. ${ }^{1364}$ Nach Ansicht des BGH ist die Wiedergabe fremder Äußerungen „nur dann tatbestandsmäßig, wenn sich der Täter die Äußerung ausdrücklich oder konkludent derart zu eigen macht, dass er selbst beschimpft oder verächtlich macht." ${ }^{1365} \mathrm{Ob}$ dies der Fall ist, ist eine Frage der Auslegung, bei der alle Umstände einer Gesamtwürdigung zuzuführen sind. ${ }^{1366}$ Für Meinungsroboter stellt Volkmann auf den Kanon der Schlagwörter ab, die ihnen bei der Auswahl an die Hand gegeben werden. ${ }^{1367}$ Freilich können die kalibrierten Schlagwörter maßgeblich einen Schluss auf die Intention des Verwenders zulassen. Zielt er nur auf degradierende und beleidigende Schlagwörter ab, geht es nicht um eine (kritische) Rezeption. Vielmehr spricht dann einiges dafür, dass er selbst die Wirkung der bereits tatbestandsmäßigen Äußerungen intensivieren, mit den Worten des BGH also selbst beschimpfen will. Nicht ganz ersichtlich ist aber, warum nicht auch die jeweiligen Begleitumstände mit einzubeziehen sind. Werden tatbestandsmäßige Äußerungen in rechten Gruppen oder als Kommentar

1358 Allgemein zu den tatbestandsmäßigen, qualifizierten Äußerungen im Falle der Volksverhetzung: BGHSt 40, 97 (102); 46, 212 (217); BGH, Urt. v. 20.09.2011 - 4 StR 129/11, Rn. 18 zu \130 Abs. 1 Nr. 1 StGB; zu Nr. 2 BGHSt 40, 212 (216); BGH, Urt. v. 03.04.2008 - 3 StR 394/07, Rn. 17 (Beschimpfen) und BGH NStZ-RR 2006, 305 (306); BayOLG NJW 1995, 145 (Verächtlich machen). Zur Böswilligkeit OLG Stuttgart NStZ 2010, 453 (454); BayObLG NJW 1995, 145; BGH NJW 1964,1481 (1483). Zum Angriff auf die Menschenwürde BGH NJW 1994, 1421; vgl auch schon zuvor BGH NStZ 1981, 258; bestätigt durch BVerfG NJW 2001,61 (63) - Bezeichnung als Jude.

1359 In gruppenbezogenen Äußerungen kann nämlich zugleich auch eine Kollektivbeleidigung zu sehen sein, die auch die Ehre des Gruppenangehörigen verletzt. Hierzu etwa BVerfG, NJW 2015, 2222 Rn. 16 f. - FCK CPS; K\&R 2016, 494 Rn. 16 f. - ACAB; NJW 2016, 2643 Rn. 16 f. - ACAB.

1360 Ausführlich hierzu beim Einsatz von Social Bots Volkmann, MMR 2018, 58.

1361 Hierzu Galetzka/Krätschmer, MMR 2016, 518 (519f.). Explizit hinsichtlich Asylbewerber BGH NStZ 2007, 216 (Rn. 5); hierzu auch OLG Frankfurt NStZ-RR 2000, 368; BayOLG NJW 1995, 145 einschränkend in Bezug auf Asylbewerber, die keinen Anspruch auf Asyl haben und kollektiv als „Asylbetrüger“ bezeichnet wurden; OLG Karlsruhe BeckRS 1995, 8221 Rn. 33.

1362 Vgl. hierzu OLG Stuttgart, Urt. v. 19.05.2011 - 1 Ss 175/11, Rn. 8 - juris.

1363 Ausführlich zum Problem der Strafbarkeit am Beispiel der Weiterverbreitung von Hate Speech in sozialen Medien Reinbacher, JZ 2020, $558 \mathrm{ff}$.

1364 Siehe nur BGH NStZ 2015, 512 (513); 2018, 589 (590).

1365 BGH NStZ 2018, 589 (590).

1366 BGH NStZ 2015, 512 (513); Hörnle, NStZ 2002, 113 (117). Nach Reinbacher, JZ 2020, 558 (559) ist das Teilen nicht ausreichend, sondern erst die Ergänzung durch eigene Kommentare o.ä. soll die Strafbarkeit begründen.

1367 Volkmann, MMR 2018, 58 (62). 
unter ebenfalls beleidigenden Inhalten Dritter veröffentlicht, besteht vielmehr Grund zur Annahme, dass der Verwender selbst beschimpfen will. Zudem dürfte letzteres auch im Einzelfall leichter zu beweisen sein als die tatsächlich im Quellcode verankerten Schlagwörter. Deshalb scheint eine Kombination aus mehreren Faktoren eine sicherere Bestimmung zu ermöglichen und sie wird auch eher der Betrachtung der Gesamtumstände gerecht, die der BGH fordert.

\section{e. Spezialfall: völkerrechtliches Interventionsverbot}

In der Vergangenheit konnte eine Beeinflussung von inländischen Meinungsbildungsprozessen durch ausländische Kräfte beobachtet werden. Oftmals sind die Versuche auf die Internet Research Agency Russlands rückführbar gewesen. Auch wenn hierbei nicht unbedingt Meinungsroboter, sondern Trolle verwendet wurden, so besteht doch eine Gemeinsamkeit in beiden Fällen: ein möglicher Verstoß gegen das völkerrechtliche Interventionsverbot. Seine Grundlage findet das gewohnheitsrechtlich anerkannte Verbot in der souveränen Gleichheit der Staaten untereinander (Art. 2 Abs. 1 UN-Charta). ${ }^{1368}$ Es verbietet die Einmischung in die inneren Angelegenheiten anderer Staaten mit Zwangsmitteln, die geeignet sind, den Willen des anderen Staates zu beugen, sofern dies nicht mehr als sozialadäquat angesehen werden kann. ${ }^{1369}$ Aber auch Propaganda soll jedenfalls dann hierunter fallen, wenn sie „den Effekt hat, die Autorität eines anderen Staates zu untergraben und ihn auf diese Weise zu destabilisieren [...], [d]enn in diesen Fällen handelt es sich nicht um den legitimen Ausdruck politischer Meinung, sondern um die Ausübung (wenngleich versteckten und indirekten) Zwanges, die eine substantielle Souveränitätsverletzung des anderen Staates bewirkt. " 1370

Unabhängig von der Frage ob und unter welchen qualitativen Anforderungen der Einsatz von Meinungsrobotern durch ausländische Staaten ein Verstoß gegen das Interventionsverbot sein kann, ${ }^{1371}$ dem dann diplomatisch oder etwa vor dem IGH begegnet werden könnte, ${ }^{1372}$ wird es rein faktisch oftmals schwierig sein, den zielgerichteten und absichtlichen Einsatz spezifischer Meinungsroboter auf einen ausländischen Staat zurückzuführen, sodass im Zweifel ein privater Beein-

1368 Thielbörger, in: Oppelland, Propaganda als (neue) außen- und sicherheitspolitische Herausforderung, S. 63 (74).

1369 Grundlegend zum Interventionsverbot IGH, Military and Paramilitary Activities in and against Nicaragua (Nicaragua v. United States of America), Judgment of 27 June 1986, ICJ Reports 1986, S. 14 Rn. 202 ff. Daneben siehe etwa Geiger, Grundgesetz und Völkerrecht, $\$ 62$ II.

1370 Thielbörger, in: Opelland, Propaganda als (neue) außen- und sicherheitspolitische Herausforderung, S. 63 (74).

1371 Bei Fake-News etwa bejahend Baade, in: v. Kielmansegg/Krieger/Sohm, Die Wiederkehr der Landesund Bündnisverteidigung, S. 161 (166ff.); und für bestimmte propagandistische Inhalte auch Thielbörger, in: Opelland, Propaganda als (neue) außen- und sicherheitspolitische Herausforderung, S. $63(75)$.

1372 Freilich nur, wenn die Zuständigkeitsvoraussetzungen der Artt. 34 ff. IGH-Statut erfüllt sind. 
flussungsversuch vorliegt. ${ }^{1373}$ Und selbst wenn dies gelingt, werden diplomatische und völkerrechtliche Maßnahmen - so denn sie überhaupt ergriffen werden - regelmäßig nur eine kompensatorische Wirkung haben. Der bereits ausgeübte Einfluss kann damit kaum rückgängig gemacht oder verhindert werden.

\section{f. Fazit: Betreiber als (ineffektive) Garanten konkreten Schutzes}

Allgemeine Grenzen für den Einsatz von Meinungsrobotern ergeben sich primär aus privatautonom geschlossenen Vereinbarungen, die die Betreiber selbst aufgestellt haben, oder aus anderen Vorschriften, die die betrieblichen Interessen der Betreiber schützen. Dieser Schutz wird vor allem durch das Privat-, Urheberund Strafrecht verfolgt. Vor allem dort, wo ein vertragswidriger automatisierter Zugriff auf die Netzwerke erfolgt, greift dieser Schutz ein. Daneben werden vor allem inhaltliche Regelungen relevant, die die konkrete Art der Verwendung begrenzen. Auch diese sind nicht nur in den Nutzungsbedingungen enthalten, sondern ergeben sich daneben auch aus dem strafrechtlichen Äußerungsrecht. Obgleich damit ein mittelbares Schutzkonzept für die Nutzer besteht, gilt es zu berücksichtigen, dass die Durchsetzung allein den Netzwerkbetreibern obliegt. Ein staatlicher Zugriff beschränkt sich auf eine sekundäre Ebene, etwa wenn es um die Beurteilung der Rechtmäßigkeit von Sperrungen geht oder die Bestrafung der Verwender der Bots durch die Zivil- und Strafgerichte. In der Vergangenheit fehlte es aber - ebenso wie in anderen Fällen $-{ }^{1374}$ gerade an der Bereitschaft der Betreiber, eine wirksame Durchsetzung zu gewährleisten. ${ }^{1375}$ Das mag auch daran liegen, dass eine erhöhte Nutzerzahl durchaus positive Auswirkungen auf die wirtschaftliche Stellung der Unternehmen haben kann. Das erklärt immerhin auch, warum erst dann Bewegung in die Sache kommt, wenn der öffentliche Druck auf die Betreiber wächst. Insoweit dürfen Bedenken an der Effektivität des allgemeinen Schutzkonzeptes durchaus geäußert werden.

\section{Spezifische Schutzmechanismen für die Informationsfreiheit}

Neben diesen allgemeinen Regelungen könnten auch bereits Vorgaben bestehen, die der Sicherung der Informationsfreiheit der Nutzer dienen. Relevant werden

1373 Ausführlicher zur Zurechnungsfrage im Lichte von Cyberangriffen Diggelmann/Hadorn, in: Schubel u.a., Jahrbuch für Vergleichende Staats- und Rechtswissenschaften - 2016/2017, S. 256 (269).

1374 Facebook war beispielsweise lange Zeit zurückhaltend, wenn es um die Veröffentlichung von FakeNews auch durch Politiker ging. Das Unternehmen sah lange Zeit davon ab hier zu intervenieren. Erst als wirtschaftlicher Druck dadurch aufkam, dass zahlreiche finanzstarke Werbepartner vorübergehend von der weiteren Kooperation absahen, wurde das Unternehmen aktiv. Hierzu https://w ww.tagesschau.de/wirtschaft/facebook-werbeboykott-101.html, (Stand: 30.05.2021).

1375 Speziell mit Blick auf Fake Accounts bei Facebook etwa Silverman/Mac/Dixit, „I Have Blood on MY Hands": A Whistleblower says Facebook Ignored Global Politcal Manipulation, BuzzFeed News. 
insoweit nicht nur Versuche einer Medienkompetenzvermittlung und bestehende Soft-law-Ansätze, sondern auch medien- und datenschutzrechtliche Vorgaben.

\section{a. Soft-law Regularien}

Soft-law-Regelungen gehen zwar nicht vom Staat aus, sondern beruhen zumeist auf einem selbstregulativen Ansatz. Gleichwohl werden Sie aus Sicht der Schutzpflicht deshalb relevant, weil bei einer wirksamen Selbstregulierung bereits die Notwendigkeit eines staatlichen Schutzes bezweifelt werden kann.

aa. Maßnahmen der Betreiber

Zuvörderst haben die Netzwerkbetreiber aus eigenem intrinsischen Motiven Vorkehrungen getroffen, um eine illegale Verwendung ihrer Dienste zu verhindern. Das Facebook Immune System überwacht etwa jede Aktivität innerhalb des Netzwerkes, um Unregelmäßigkeiten und dadurch auch Anzeichen einer Automatisierung ausfindig zu machen. ${ }^{1376}$ Und auch Twitter überwacht die Datenströme. ${ }^{1377}$ Beide Netzwerkbetreiber suspendieren auffällige Accounts oder löschen entsprechende Profile. Unklar ist dabei allerdings, auf welchen Parametern die Erkennung im Detail beruht. Dass diese nicht öffentlich gemacht werden, entspricht freilich berechtigten Interessen der Betreiber. Dennoch lässt sich - bezogen auf Twitter - zumindest eine gewisse prozentuale Kongruenz zwischen den vorgenommenen Suspendierungen und den Ergebnissen anderer Erkennungssoftware aufzeigen. ${ }^{1378}$

Aber nicht nur mit Blick auf die Nutzer selbst finden Kontrollen statt. Seitdem die Kritik an den sozialen Netzwerken insbesondere nach 2016 zugenommen hat, engagieren sich die Betreiber auch selbst aktiver bei der inhaltlichen Kontrolle, um insbesondere Desinformation frühzeitig zu erkennen. Facebook arbeitet etwa mit unabhängigen Factcheckern zusammen, die Inhalte weltweit auf ihre Glaubwürdigkeit hin überprüfen. Voraussetzung ist allerdings, dass Nutzer entsprechende Inhalte melden, die dann einer Kontrolle unterzogen werden, oder dass Factchecker selbst proaktiv nach entsprechenden Inhalten suchen. Wird ein Inhalt als Falschmeldung eingestuft, so wird dafür Sorge getragen, dass dieser weiter unten im Feed erscheint und so seine Sichtbarkeit faktisch abnimmt. Zudem wird dem Inhalt ein entsprechender Warnhinweis beigefügt und sicher-

1376 Ausführlicher zum System etwa He u.a., Security and Communication Networks 9 (2016), 2157 (2160).

1377 Vgl. Chavoshi/Hamooni/Mueen, in: Proceedings of the $16^{\text {th }}$ International Conference on Data Mining, S. 817 (820); Silva/Proksch, American Political Science Review 2020, 1 (2).

1378 Siehe hierzu Chavoshi/Hamoon/Mueen, ebd. Die Übereinstimmung mit den Ergebnissen des Botometers betrug knapp 37\%, bei DeBot nur 21,06\% - im Jahr 2015. 
gestellt, dass Nutzer, die den Inhalt konsumieren oder weiterleiten wollen, auf zusätzliche, ergänzende Informationen aufmerksam gemacht werden. ${ }^{1379}$ Bei wiederholten Verstößen von einzelnen Nutzern werden weitere Maßnahmen ergriffen, wobei sichergestellt ist, dass der Urheber eines Inhalts zumindest Einspruch gegen eine Entscheidung der Faktenprüfer einlegen kann. ${ }^{1380}$

Auch Twitter geht seit jüngstem aktiver gegen desinformative Inhalte vor, setzt dabei aber nicht auf die Zusammenarbeit mit unabhängigen Prüfern, sondern geht eigenständig gegen Inhalte vor, die entweder technisch manipuliert wurden (bspw. DeepFakes), in irreführender Weise verbreitet (etwa durch Veränderungen des originären Inhaltes) oder schädliche Auswirkungen haben können (etwa Einschüchterungseffekte, Gewaltaufrufe o.ä). Je nach Ausfall der Prüfung variiert die Reaktion von Kennzeichnung bis Löschung. ${ }^{1381}$ Das führte etwa auch dazu, dass ein Post von Donald Trump zur Briefwahl im Mai 2020 entsprechend gekennzeichnet worden ist. ${ }^{1382}$

Das Problem, was hierbei zutage tritt, ist ein Allgemeines, wenn es um Kontrollmechanismen und die Überprüfung von Inhalten geht: die potentielle Begrenzung der Meinungsfreiheit der Nutzer durch gezieltes Vorgehen, insbesondere aber durch eine fehlerhafte Einordnung als falscher Inhalt und die damit bewirkte „Überregulierung“. Besonders schwierig wird das etwa bei Inhalten, wo die Grenzen zwischen zulässiger Meinung, Verunglimpfung oder Satire verschwimmen oder wo es um eine sehr kontrovers geführte Debatte geht. ${ }^{1383}$ Zudem sind den Bemühungen schon faktische Grenzen gesetzt, die aus der begrenzten Arbeitskraft der Faktchecker und der Unmenge an potentiellen Inhalten resultieren. ${ }^{1384}$ Und letztlich können Informationen bereits ihre Wirkung entfalten, bevor sie überhaupt entdeckt und überprüft werden. ${ }^{1385}$

\section{bb. EU-Verhaltenskodex}

Aufgrund der zunehmenden Bedeutung der sozialen Netzwerke und der Debatte um die Einflussnahme auf die Willensbildung entschloss sich die europäische Kommission bereits 2018, einen umfangreichen Aktionsplan gegen desinformati-

$1379 \mathrm{Zu}$ etwaigen lauterkeitsrechtlichen Problemen durch die Verlinkung anderer Angebote siehe etwa Peukert, WRP 2020, $391 \mathrm{ff}$.

1380 Siehe hierzu die betreiberseitigen Informationen, abrufbar unter https://www.facebook.com/busines s/help/182222309230722, (Stand 30.05.2021) und unter https://about.fb.com/news/2018/06/hard -questions-fact-checking/, (Stand 30.05.2021).

1381 Siehe auch hierzu die unternehmenseigenen Informationen, abrufbar unter https://help.twitter.com/ de/rules-and-policies/manipulated-media, (Stand 30.05.2021).

1382 Hierzu etwa Klein, Wie Soziale Netzwerke gegen Fake News kämpfen.

1383 Das sehen freilich auch die Unternehmen selbst, so etwa ausdrücklich auch Facebook, abrufbar unter https://about.fb.com/news/2018/06/hard-questions-fact-checking/, (Stand 30.05.2021).

1384 Vgl. hierzu Shao u.a., IEEE Access Vol. 6 (2018), 75327 (75338).

1385 Auch hierzu Shao u.a., IEEE Access Vol. 6 (2018), 75327 (75330f.). 
ve Inhalte zu verabschieden. ${ }^{1386}$ Ein wesentlicher Bestandteil dieses Aktionsplanes ist ein Verhaltenskodex zur Bekämpfung von Desinformation im Internet, der bereits 2018 von den großen Unternehmen - neben Google auch Facebook und Twitter - unterzeichnet wurde. ${ }^{1387}$

\section{(I.) Allgemeines}

Nach der Präambel wird Desinformation definiert „als , nachweislich falsche oder irreführende Informationen', die insgesamt, mit dem Ziel des wirtschaftlichen Gewinns oder der vorsätzlichen Täuschung der Öffentlichkeit konzipiert, vorgelegt und verbreitet werden' und ,öffentlichen Schaden anrichten können', worunter ,Bedrohungen für die demokratischen politischen Prozesse und die politische Entscheidungsfindung sowie für öffentliche Güter wie den Schutz der Gesundheit der EU-Bürgerinnen und -Bürger, der Umwelt und der Sicherheit zu verstehen“ sind.“ Inhaltlich sieht der Kodex vier zentrale Verpflichtungen vor: Umgang mit politischer Werbung, Stärkung der Integrität der Dienste, der Position der Verbraucher und der Forschungsgemeinschaft. In diesem Zusammenhang sind besonders die konkreten Verpflichtungen des Abschnitts II.C. und II.D. hervorzuheben. Hiernach sind die Unterzeichner verpflichtet, „in ihren Diensten klare Richtlinien in Bezug auf die Identität und den Missbrauch automatisierter Bots festzulegen und diese in der EU durchzusetzen“ (Nr. 5) und zudem „Richtlinien dafür festzulegen, was eine unzulässige Verwendung automatisierter Systeme darstellt, und diese Richtlinien auf der Plattform öffentlich verfügbar und für die Nutzer in der EU zugänglich zu machen“ (Nr.6). Zugleich sehen die Verpflichtungen des Abschnitts II.D. vor, die Position der Verbraucher dadurch zu stärken, dass sie fundierte Entscheidungen treffen können sollen, wenn sie auf möglicherweise falsche Nachrichten stoßen (Nr. 7) und zudem andere Maßnahmen, die die Meinungsbildung auf Grundlage von zuverlässigen Informationen ermöglichen und stärken sollen (Nr. 8-9). So soll letztlich auch die Medienkompetenz gestärkt werden (Nr. 10).

\section{(II.) Würdigung und spezifische Probleme}

Auch wenn der Verhaltenskodex ein wichtiges Signal war, verbinden sich mit ihm Probleme. ${ }^{1388}$ Das beginnt schon damit, dass der Verhaltenskodex ein Instrument der freiwilligen Selbstregulierung ist. Die EU-Kommission kann also die Einhal-

\footnotetext{
1386 Aktionsplan gegen Desinformation, JOIN(2018) 36 final, v. 05.12.2018, abrufbar unter https://eeas .europa.eu/sites/eeas/files/aktionsplan_gegen_desinformation.pdf, (Stand 30.05.2021).

1387 Siehe Mitteilung der Kommission JOIN(2018) 36 final, S. 2.

1388 Eine ausführliche Bewertung hat die European Regulators Group for Audio-visual Media Services (ERGA), Report on Disinformation, 2020, vorgenommen.
} 
tung der Verpflichtungen nicht kontrollieren. Vielmehr obliegt dies den Unterzeichnern selbst. Dieser Kontrolle kommen sie u.a. durch einen zu veröffentlichenden jährlichen und öffentlich zugänglichen Bericht (siehe hierzu Abschnitt III des Kodex) nach, der zwar einer „objektiven externen“ aber selbst zu wählenden Stelle zur Prüfung vorzulegen ist (ebd. Nr. 20). Die Kommission kann nur im Rahmen der Zusammenarbeit (ebd. Nr. 21) auf die weitere Entwicklung einwirken. Es fehlen also unabhängige Überwachungs- und Kontrollmechanismen, ${ }^{1389}$ die aber auch aus Sicht der europäischen Grundrechte u.U. notwendig wären. ${ }^{1390}$

Und auch das regulative Konzept des Kodex ist jedenfalls angreifbar. So sieht er schon keine genauen Vorgaben über die Inhalte der Berichte vor, sodass die Berichte selbst zwar die Ergebnisse, insbesondere die „Outputindikatoren “, 1391 der Tätigkeiten veröffentlichen, aber daraus nicht ersichtlich wird, welche Daten hierfür begutachtet wurden und vor allem wie sich die Ergebnisse auf die einzelnen Mitgliedstaaten verteilen. ${ }^{1392}$ Insoweit können den Berichten kaum spezifische Informationen entnommen werden, um validieren zu können, wie und inwieweit die Unternehmen die vorgesehenen Verpflichtungen erfüllen oder Fortschritte erzielt haben. ${ }^{1393}$ Und auch der Zugang für die Forschung wird nur sporadisch gewährt, sodass eine unabhängige Kontrolle erschwert wird. ${ }^{1394}$

Mit Blick auf Meinungsroboter enthalten zwar die Erwägungsgründe Hinweise auf eine mögliche Kennzeichnungspflicht, ${ }^{1395}$ die Verpflichtungen kommen hierauf aber nicht zurück, sondern sehen nur vor, dass Richtlinien für die Definition und den Umgang von Bots vorgesehen werden müssen. Diese finden sich aber schon seit langem in den Nutzungsbedingungen. Insoweit enthält der Kodex keine „Innovationen“. ${ }^{1396}$ Entsprechende Anpassungen sind aber durchaus not-

1389 Kritisch deshalb auch Mafi-Gudarzi, ZRP 2019, 65 (66).

1390 Zu diesem Aspekt etwa Kuczerawy, in: Terzis u.a., Disinformation and Digital Media as a Challenge for Democracy, S. $291 \mathrm{ff}$.

1391 Vgl. EU-Kommission, EuZW 2019, 884.

1392 Vgl. EU-Kommission, Erklärung Nr. 19/6166, EuZW 2019, 884. Speziell zu Abschnitt C. des Kodexes und damit zu den Meinungsrobotern ERGA, Report on Disinformation, S. $24 \mathrm{f}$.

1393 Auch die ERGA kommt in ihrem Report on Disinformation v. 04.05.2020, S. 3 zu dem Ergebnis: „there is a need for greater transparency about how the signatories are implementing the Code".

1394 Vgl. EU-Kommission, Erklärung Nr. 19/6166, EuZW 2019, 884. Das ist auch ein wesentlicher Kritikpunkt der ERGA, Report on Disfinformation, S. 43. Auch die Kommission selbst weist auf das Problem angesichts der Desinformationen während der Covid-19-Pandemie nochmals hin, JOIN(2020) 8 final, S. 9. Und auch die Gesetzesbegründung zum NetzDG weist auf die fehlende Aussagekraft der Informationen der Betreiber hin, allerdings mit Blick auf die Löschung bzw. Sperrung von Inhalten, BT.-Drs. 18/12356, S. 11.

1395 In Abschnitt II.C. heißt es „Im Einklang mit der Mitteilung der Europäischen Kommission erkennen die Unterzeichner die Bedeutung der, Verstärkung der Bemühungen zur Schließung von Scheinkonten und [des] Nachweis[es] ihrer Wirksamkeit‘ sowie die Bedeutung der Einführung ,klare[r] Kennzeichnungsregeln und -systeme für Bots und [der] Gewährleistung, dass ihre Tätigkeiten nicht mit menschlicher Interaktion verwechselt werden könne’ an.“.

1396 Das wird auch dadurch sichtbar, dass die in dem Anhang aufgeführten Maßnahmen letztlich nur die bereits bestehenden Maßnahmen der unterschiedlichen Betreiber aufgelistet werden, auf die aber gerade in Abschnitt II.C Nr. 5 verwiesen wird. Kritisch deshalb auch Mafi-Gudarzi, ZRP 2019, 65 (66). 
wendig, können aber ebenfalls von der EU-Kommission nur begleitet werden. ${ }^{1397}$ Die Unterzeichner müssen sich auf die Ergreifung von Folgemaßnahmen einigen und diese beschließen. ${ }^{1398}$ Und ganz allgemein hat etwa die European Regulators Group for Audiovisual Media Services (ERGA) - zu Recht - darauf hingewiesen, dass der Kodex zwar zu begrüßen, er aber viel zu unpräzise und allgemein sei, was sicherlich auch daran liegt, dass er für ganz unterschiedliche Unterzeichner und damit Dienste gilt. ${ }^{1399}$ Insgesamt bestehen also starke Zweifel nicht nur an der Verbindlichkeit, sondern vor allem auch an der Wirksamkeit der getroffenen Regelungen. ${ }^{1400}$

\section{b. Medienkompetenzvermittlung}

Wesentliche Voraussetzung der Wirkung von Meinungsrobotern ist eine gewisse Leichtfertigkeit im Umgang mit und eine unreflektierte Rezeption der Inhalte innerhalb sozialer Netzwerke. Notwendig ist also ein grundlegendes Verständnis der Funktionsweise und der technischen Besonderheiten sozialer Netzwerke und dort vorzufindender Phänomene (Fake News, Social Bots, Filterblasen etc.), ebenso wie die Bewertung und kritische Rezeption von Inhalten als Ausprägungen einer umfangreichen „digitalen Medienkompetenz“1401 der Nutzer. ${ }^{1402}$

1397 Siehe dazu Abschnitt IV des Kodex. Die ERGA sieht deshalb die Notwendigkeit von „specific monitoring activities (...) and (...) enforcement tools to ensure the compliance to the rules.", Erga, Report on Disinformation, S. 3 .

1398 In Abschnitt IV des Kodex heißt es dazu: „Sie werden die Fortführung des Kodex erörtern und gegebenenfalls Folgemaßnahmen besprechen und vorschlagen.“.

1399 ERGA, Report on Disinformation, S. 42, 43. Kritisch auch Steinebach u.a., Desinformation aufdecken und bekämpfen, S. 179 .

1400 Ganz drastisch formuliert es das Sounding Board der Expertengruppe EURACTIV, Final opinion on the so-called Code of Practice, unter 3.: „[T] he ,Code of practice“ as presented by the working group contains no common approach, no clear and meaningful commitments, no measurable objectives or KPIs, hence no possibility to monitor process, and no compliance or enforcement tool: it is by no means self-regulation, and therefore the Platforms, despite their efforts, have not delivered a Code of Practice.".

1401 Auch wenn der Begriff der Medienkompetenz in diesem Zusammenhang zu eng sein mag (hierzu Kalwey, in: Moll, Was tun gegen Fake News?, S. 139 (140 ff.)), wird er vorliegend in einem weiten Sinne verstanden. Es geht um die Kompetenz im Umgang mit digitalen Diensten, ihren Inhalten und dem Verständnis der technischen, gesellschaftlichen und individuellen Einflüsse. Insoweit erfasst die hier vorgeschlagene „Medienkompetenz“ auch zwangsläufig Fragen der Nachrichtenkompetenz, der Medienpädagogik und Medienbildung. Zu diesen einzelnen Aspekten siehe neben Dengeln, Medienpädagogik 2018, 11 ff. auch Kalwey, ebd.

1402 Zur Notwendigkeit eben dieser Kompetenz mit Blick auf Meinungsroboter etwa Milker, InTeR 2017, 199 (207); Graber/Lindemann, in: Sachs-Hombach/Zywietz, Fake News Hashtags \& Social Bots, S. 51 (65); Kind u.a., Social Bots, S. 65 und Rückert, in: Albrecht u.a., Strafrecht und Politik, S. 167 (184); Fehling/Leymann, AfP 2020, 110 (118); Oberer/Erkollar/Stein, in: Stumpf, Digitalisierung und Kommunikation, S. 311 (323); Dankert, in: Hoffmann-Riem, Big Data, S. 157 (164). Mit Blick auf die allgemeinen „Transparenz- und Orientierungsdefizite“ etwa Brinkmann, UFITA 2019, S. 364 (384). Im Kontext von Fake News etwa Kalwey, in: Möller, Was tun gegen Fake News und Hate Speech?, S. 139 ff.; Schweiger, Der (des)informierte Bürger, S. 190 und Steinebach u.a., Desinformation aufdecken und bekämpfen, S. 206. Mit Blick auf die Rechtswahrung und die Sensibilisierung vor Rechtsverletzungen etwa Bock, Übertragbarkeit der Kommunikationsfreiheiten auf das In- 
aa. Bildung der Medienkompetenz im schulischen Bereich

Die Bildung einer Medienkompetenz ist vor allem ein erzieherisches Anliegen, welches auf staatlicher Seite primär im Bereich der allgemeinen schulischen Erziehung und Bildung als Bestandteil der Schulaufsicht des Staates (Art. 7 Abs. 1 GG) verfolgt wird. ${ }^{1403}$ Schon der Digitalpakt Schule 2019-2024 ${ }^{1404}$ ebenso wie die gemeinsame Strategie der Kultusministerkonferenz (KMK) aus dem Jahr 2016 - „Bildung in der digitalen Welt“ - haben dieses Bedürfnis erkannt. ${ }^{1405}$ Tatsächliche Auswirkungen für den schulisch-inhaltlichen Bereich hat aber nur die KMK-Strategie. ${ }^{1406}$ Diese setzt bei der Umsetzung nicht auf ein eigenes Schulfach, sondern auf die Integration in alle Fächer und zwar schulformübergreifend. ${ }^{1407}$ Die bereits aufgezeigten Problembereichen (Filterblase, Echokammer, Fake News, Social Bots) werden aber nicht unmittelbar adressiert. ${ }^{1408}$ Konkretisierte Vorgaben finden sich beispielsweise ${ }^{1409}$ im Rahmenlehrplan „Digitale Kompetenzen“ des Landes Mecklenburg-Vorpommern, der die erforderlichen Kompetenzen näher beschreibt und Umsetzungsbeispiele für unterschiedliche Fächer aufzeigt. ${ }^{1410}$ So wird etwa Deutsch als Leitfach dafür bestimmt, dass unterschiedliche Online-Inhalte analysiert, interpretiert und kritisch bewertet werden sollen. Das

ternet, S. 284 f. und Rößner, in: Hill/Kugelmann/Martini, Digitalisierung in Recht, Politik und Verwaltung, S. 55 (63). Allgemeiner auch Drexl, ZUM 2017, 529 (543); Wardle/Derakhshan, Information Disorder, S. 70 f. oder Steinmaurer, in: Litschka/Krainer, Der Mensch im digitalen Zeitalter, S. $31(36)$.

$1403 \mathrm{Zu}$ diesem eigenständigen Bildungs- und Erziehungsauftrag siehe u.a. Jestaedt, in: Isensee/Kirchhof, HbStR VI, $\$ 156$ Rn. 1, 43 f. und in Abgrenzung zur Elternverantwortung Rn. 46. Aus der Judikatur des BVerfG zu beidem etwa E 34, 165 (182) - Förderstufe und E 93, 1 (21) - Kruzifix.

1404 Verwaltungsvereinbarung DigitalPakt Schule 2019-2024, S. 2.

1405 KMK, Bildung in der digitalen Welt - Strategie der Kultusministerkonferenz, S. 11.

1406 Der Digitalpakt wurde oftmals wegen der Fokussierung auf das Lernen mit Medien kritisch bewertet, so etwa Kalwey, in: Möller, Was tun gegen Fake News und Hate Speech?, S. 139 (143). In der Tat beantwortet der Digitalpakt vor allem finanzielle Fragen infrastruktureller Anforderungen des digitalen Lernens. Inhaltliche Fragen der Planung und Organisation der Lehrinhalte ist als Teil der Schulaufsicht aber allein den Ländern vorbehalten. Insoweit ist vor allem die KMK-Strategie bedeutsam, an die die Rahmenlehrpläne der Länder angepasst werden, vgl. nur $\ 9$ Abs. 2 S. 2, 3. Var. SchulG MV. Angesichts des Digitalpaktes kam es auch zu einer Änderung des GG. Nach Art. 104c Abs. 1 GG können entsprechende Bundesinvestitionen in den eigentlich allein den Ländern zugewiesenen Bereich erfolgen.

$1407 \mathrm{Zu}$ Recht wird nämlich betont, dass ansonsten die Medienkompetenz zu einem „Privileg höherer Bildungsschichten wird“, so Bock, Die Übertragbarkeit der Kommunikationsfreiheiten des Artikel 5 GG auf das Internet, S. 286.

1408 Siehe dazu die Kompetenzbereiche die in der Strategie, S. 16 ff. näher abgesteckt wurden. So wird etwa mit Blick auf den Datenschutz vor allem von der Datensicherheit und dem Schutz vor Datenmissbrauch gesprochen (4.2.1) oder allgemein von Risiken und Gefahren in digitalen Umgebungen gesprochen (4.1.1).

1409 Von einer umfangreichen Auswertung der länderspezifischen Regelungen wurde schon wegen des Umfanges und der Besonderheiten abgesehen. Im Kontext der Meinungsroboter finden sich Ausführungen zu dem Ansatz von Hamburg etwa in HH-Drs. S21/18626, S. 2 f.

1410 Der Rahmenplan „Digitale Kompetenzen“ ist abrufbar unter https://www.bildung-mv.de/export/sit es/bildungsserver/downloads/unterricht/rahmenplaene_allgemeinbildende_schulen/fachuebergreifen d/Finalfassung-Rahmenplan-digitale-Kompetenzen.pdf, (Stand: 30.03.2021). 
Fach Informatik und Medienbildung wird als Leitfach für das Erkennen und Formulieren von Algorithmen bestimmt. ${ }^{1411}$ Zeitgleich wird aber auch aufgezeigt, wie auch in anderen Fächern ein thematischer Zugriff erfolgen kann. ${ }^{1412}$ Für das Fach Sozialkunde wird exemplarisch vorgeschlagen, „die Folgen des Einsatzes von social bots für Wahlen und Meinungsbildung ein[zu]schätzen “. ${ }^{1413}$

Auch wenn die jeweiligen Rahmenpläne verbindlich sind ( $\mathbb{S} 8$ Abs. 2 S. 2 SchulG MV), so obliegt es aber nicht nur den einzelnen Schulen diese umzusetzen. Vor allem ist es auch von der jeweiligen Lehrkraft abhängig, wie, in welchem Umfang und in welcher Qualität entsprechende Inhalte thematisch mit einbezogen werden. ${ }^{1414}$ Insoweit ist die Unterrichtsgestaltung also auch von der Kompetenz der Lehrkraft abhängig, entsprechende Lerninhalte zu vermitteln. ${ }^{1415}$ Gerade bei älteren Lehrkräften mag die Medienkompetenz ebenso fehlen ${ }^{1416}$ wie bei den Schülern. ${ }^{1417}$ Insofern ist es notwendig, dass auch die Lehrkräfte für die entsprechenden Themen sensibilisiert und geschult werden. Dieses Problem hat auch die KMK gesehen und adressiert. ${ }^{1418}$ Bemerkenswert ist insoweit die Bemühung des Landes Niedersachsen, eine gewisse Vereinheitlichung dadurch zu erreichen, dass Unterrichtsmaterialien bereitgestellt werden, die sich den Themen „Fake News“ und „Social Bots“ widmen und die in den Sekundarbereichen I

1411 A.a.O., S. 34.

1412 Mit Blick auf das Fach Informatik und Medienbildung heißt es dort etwa: „Suchergebnisse in Bezug auf Relevanz bewerten, Authentizität und Vertrauenswürdigkeit der Quelle abschätzen (Urheber, Seitenfinanzierung), Wahrheitsgehalt einer Information untersuchen, kommerzielle Interessen erkennen“. Im Fach Philosophie wird beispielsweise das Thema vorgeschlagen „Der gläserne Mensch? Das Verhältnis von Mensch und Maschine problematisieren“. Und mit Blick auf den Sozialkundeunterricht findet sich auch der Vorschlag, ,verschiedene Internetquellen zu einem bestimmten aktuellen Ereignis im Hinblick auf ihre Güte kritisch hinterfragen (Fake News, „Lügen mit Statistik“). Hierzu a.a.O., S. 5 ff.

1413 Rahmenplan Digitale Kompetenzen, S. 36.

1414 Dies betont auch Kalwey, in: Möller, Was tun gegen Fake News und Hate Speech?, S. 139 (146). Zwar sind Lehrkräfte an die Rahmenpläne gebunden, $\mathbb{1 0 0}$ Abs. 2 S. 2 SchulG MV. Und der Rahmenplan Digitale Kompetenzen des Landes MV weist auch stets ein Leitfach aus, das verbindlich die „Basiskompetenzen“ vermitteln soll, ebd., S. 1. Diese können zwar auch in den fachspezifischen Fachplänen aufgenommen werden - der Rahmenplan Digitale Kompetenzen schlägt insoweit Möglichkeiten vor -, allerdings ist das bislang noch nicht immer erfolgt. So soll im Fach Sozialkunde etwa auch der Einfluss von Social Bots (Rahmenplan Digitale Kompetenz, S. 36) oder Fragen der Algorithmen, insbes. Transparenz und Filterblase, thematisiert werden (a.a.O. S. 34). In den fachspezifischen Rahmenplänen findet man hierzu aber noch nichts. Insoweit bleibt es also „der eigene[n] pädagogische[n] Verantwortung “ der Lehrkräfte überlassen, wie umfangreich entsprechende Themen behandelt werden $(\mathbb{S} 100$ Abs. 2 S. 1 SchulG MV). Zu den faktischen Unterschieden zwischen Lehrkräften, die zu einer weniger starken Thematisierung führen, vgl. Institut für Demoskopie Allensbach, Die Vermittlung von Nachrichtenkompetenz in der Schule, S. $93 \mathrm{ff}$.

1415 Hierzu Kalwey, in: Möller, Was tun gegen Fake News und Hate Speech?, S. 139 (145).

1416 Allgemein zu den erheblichen Lücken auf Seiten der Lehrkräfte etwa Institut für Demoskopie Allensbach, Die Vermittlung von Nachrichtenkompetenz in der Schule, S. 66 ff.

1417 So auch Graber/Lindemann, in: Sachs-Hombach/Zywietz, Fake News Hashtags \& Social Bots, S. 51 (65); Schweiger, Der (des)informierte Bürger, S. 190. Zu der Kluft innerhalb der Lehrerschaft Kalwey, in: Möller, Was tun gegen Fake News und Hate Speech?, S. 139 (145).

1418 KMK, Bildung in der digitalen Welt - Strategie der Kultusministerkonferenz, S. $24 \mathrm{ff}$. Kritisch allerdings Dengel, Medienpädagogik 2018, 11 (24) mit Blick auf die aktuelle Ausbildung der Lehrkräfte. 
und II in den Unterricht integriert werden sollen. ${ }^{1419}$ Diese sollen Schülern u.a. vermitteln, wie man die Glaubwürdigkeit von Inhalten beurteilt und so Fehlbzw. Desinformation erkennt. Dabei geht es nicht nur um textliche, sondern auch um bildliche Inhalte (Übung 1-5). ${ }^{1420}$ Zeitgleich werden in einer weiteren Einheit zentrale Begriffe der Filterblase, Echokammer und Social Bots nahe gebracht und u.a. versucht, auch im Rahmen von Selbstversuchen für die Problematik zu sensibilisieren (Übung 7). Freilich bleiben die Unterrichtseinheiten insbesondere hinsichtlich des technischen Hintergrundes relativ oberflächlich, was auch hier dem fehlenden Spezialwissen der Lehrkräfte, die dies letztlich vermitteln müssen, geschuldet sein mag. Entscheidend ist aber, dass von den Unterrichtseinheiten eine Sensibilisierungswirkung ausgehen kann und die Schüler auch im Selbstversuch den Problemen in der digitalen Welt nachspüren können.

\section{bb. Medienkompetenz älterer Nutzerschichten}

Durch die Anpassung der schulischen Bildung an die veränderten Umstände erreicht man aber nur einen Bruchteil der Internetnutzer und zudem nur solche, die als „Digital Natives“ ohnehin mit den Kommunikationstechniken aufwach$\operatorname{sen}^{1421}$. Ältere Nutzerschichten werden nicht oder - wenn überhaupt - nur mittelbar durch die Schülerinnen und Schüler erreicht. Bislang ist es zentrale Aufgabe der Medien, bestimmte gesellschaftsrelevante Phänomene in der digitalen Welt $\mathrm{zu}$ thematisieren und so „aufzuklären“.. ${ }^{1422}$ Insbesondere öffentlich-rechtliche Rundfunkanbieter sind diesbezüglich bemüht, indem sie Hintergründe liefern, FakeNews begegnen und dabei auch sehr aktiv in sozialen Medien agieren. ${ }^{1423}$ Weil aber spezifische Verzerrungen auf Konsumentenseite bestehen, ist nicht sichergestellt, dass flächendeckend eine solche Sensibilisierung auch erfolgt. Das

1419 Niedersächsisches Kultusministerium, Fake News und Social Bots im digitalen Zeitalter, abrufbar unter https://www.nibis.de/uploads/1chaplin/files/FakeNews_SekI.pdf, (Stand: 30.05.2021). Damit begegnet man auch dem Problem, dass viele Lehrbücher und Unterrichtsmaterialien sich vorwiegend mit herkömmlichen Medien befassen und digitale Angebote außen vor lassen, kritisch deshalb Kalwey, in: Möller, Was tun gegen Fake News und Hate Speech?, S. 139 (144).

1420 Die ergänzenden Bildmaterialien, umfassen etwa auch einen Facebookbeitrag, der die bereits angesprochenen (desinformative) Verbindung von einem Merkel-Selfie mit einem Flüchtling und die Beteiligung desselben bei einem Terroranschlag herstellt.

1421 Wobei das zwar die technische Kompetenz erhöht, aber nicht den Umgang mit den Medieninhalten, Schweiger, Der (des)informierte Bürger, S. 108 f., 111; Schmid/Stock/Walter, in: Sachs-Hombach/ Zywietz, Fake News Hashtags \& Social Bots, S. 69 (86).

1422 Vgl. zu der medialen Verantwortung im Umgang mit Meinungsrobotern (allerdings bezogen auf den Umgang mit den Datenbeständen aus sozialen Netzwerken) Dankert, in: Hoffmann-Riem, Big Data, S. 157 (164f.).

1423 Vgl. Schmid/Stock/Walter, in: Sachs-Hombach/Zywietz, Fake News Hashtags \& Social Bots, S. 69 (86). Brinkmann, UFITA 2019, 364 (384) weist allerdings kritisch daraufhin, dass die eigentliche Bildung einer Medienkompetenz als Teil der Medienpädagogik nicht Aufgabe der Medien ist und deshalb in einem entsprechenden Umfang nicht erwartet werden kann. Nach $\$ 30$ Abs. $2 \mathrm{MStV}$ sollen insbesondere die Telemedienangebote „die technische und inhaltliche Medienkompetenz aller Generationen und von Minderheiten gefördert werden.". 
versuchen die Betreiber durch den mittlerweile mehr oder minder freiwilligen Kampf gegen Fake-News, die Kooperation mit unabhängigen Fact-Checkern oder etwa durch die Gewährleistung entsprechender transparenter Hintergrundinformationen zu den Facebook-Seiten zu kompensieren. Allerdings sind das keine verbindlichen Vorgaben, die flächendeckend und vor allem kontrolliert umgesetzt werden. ${ }^{1424}$

cc. Fazit:

Prinzipiell sind mit der infrastrukturellen Versorgung der Schulen (Digitalpakt) und der „feste[n] Verankerung der Medienbildung in der Bildungspolitik “1425 (KMK-Strategie und Rahmenpläne) jedenfalls zwei grundlegende Anforderungen für die Herausbildung einer Medienkompetenz prinzipiell abgesichert. Insgesamt bleibt dennoch abzuwarten, wie die Vorgaben im Lehralltag umgesetzt werden und ob daraus tatsächlich eine stärkere Medienkompetenz erwächst. ${ }^{1426}$ Wesentlich schwieriger ist hingegen, auch ältere Nutzerschichten zu erreichen. Öffentlich-rechtliche Medien versuchen gewisse Gegenmaßnahmen zu ergreifen und auch die Betreiber engagieren sich nach zunehmendem, öffentlichem Druck deutlich stärker. Insgesamt ist aber nicht sichergestellt, dass die Informationen alle Nutzer erreichen, und von den unterschiedlichen Nutzerschichten auch in gleicher Weise verstanden werden können. Insbesondere bei älteren Nutzerschichten kann so eine Schere mit Blick auf die unterschiedlich stark ausgeprägte Medienkompetenz entstehen.

\section{c. Transparenzvorgaben des Medienrechts, insbesondere $\iint 5 \mathrm{ff}$. TMG, $55 \mathrm{RStV}$}

Anders als die Soft-law Ansätze statuiert insbesondere das Medienrecht verbindliche Pflichten für Anbieter von Telemediendiensten. Dabei geht es unter anderem auch darum, den Umgang mit ihnen verbraucher- und nutzerfreundlicher, insbesondere transparenter zu gestalten. ${ }^{1427}$ Wegen der bereits angesprochenen dichotomen Kompetenzverteilung finden sich entsprechende Regelungen auf

1424 Probleme ergaben sich mit Blick auf die Fact-checking-Methoden etwa aus wettbewerbsrechtlicher Sicht, hierzu OLG Karlsruhe, ZUM-RD 2020, $446 \mathrm{ff}$.

1425 Kalwey, in: Möller, Was tun gegen Fake News?, S. 139 (142).

1426 Insoweit kritisch wegen des Aufwandes und des „ohnehin vollen Lehrplans der Schulen“ Schmid/ Stock/Walter, in: Sachs-Hombach/Zywietz, Fake News Hashtags \& Social Bots, S. 69 (88). Wegen der spezifischen Besonderheiten in den Schulsystemen der Länder (etwa Wahlpflichtfächer in NRW) ist dies genau zu beobachten, um vor allem eine landes- und schulformübergreifende, flächendeckende Ausbildung der Medienkompetenz zu gewährleisten. Zu den schon zuvor bestehenden Unterschieden etwa Kalwey, in: Möller, Was tun gegen Fake News und Hate Speech?, S. 139 (145 f.). Und wegen des ungewissen Ergebnisses kritisch von der Weth/Vachery/Kankanhalli, in: Proceedings of the International Conference on Multimedia \& Expo Workshops, S. 1.

1427 Siehe hierzu u.a. die Gesetzesbegründung zu \$6 TDG, BR Drs. 966/96, S. 23. 
Landesebene in dem seit 2020 den RStV ersetzenden Medienstaatsvertrag (MStV) bzw. auf Bundesebene im TMG. ${ }^{1428}$ Mit Blick auf Meinungsroboter könnten vor allem die allgemeinen Kennzeichnungspflichten des $\mathbb{5}$ Abs. 1 TMG bzw. des $\mathbb{5} 5$ Abs. $1 \mathrm{RStV}$ - dessen normativer Gehalt durch $\mathbb{1 8}$ Abs. $1 \mathrm{MStV}$ unverändert übernommen wurde - relevant sein. ${ }^{1429}$

\section{aa. Kennzeichnungspflicht nach $\mathbb{S}$ Abs. $1 \mathrm{TMG}$}

Die Kennzeichnungspflicht des $\mathbb{5}$ Abs. 1 TMG verpflichtet Anbieter von Telemedien, die in Deutschland niedergelassen sind und von hieraus ihre Geschäftstätigkeit tatsächlich ausüben ( $\mathbb{2} 2 \mathrm{a}$ Abs. $1 \mathrm{~S} .2 \mathrm{TMG}$ ) oder unter den Voraussetzungen des $\mathbb{S} 2 \mathrm{a}$ Abs. 2 oder 3 TMG und das auch dann, wenn die Dienste in anderen EU-Staaten angeboten werden ( $\mathbb{3}$ Abs. 1 TMG). Während soziale Netzwerke selbst als Telemedium zu qualifizieren sind, ${ }^{1430}$ ist dies für die Nutzer- und damit Botprofile zumindest diskutabel. Das TMG unterscheidet schließlich zwischen Anbietern ( $\mathbb{2}$ Nr. 1 TMG) und Nutzern ( $\mathbb{2}$ Nr. 3 TMG). Hierbei handelt es sich aber nicht um Antonyme. Halten Nutzer eigene Telemedien bereit, können sie auch Dienstanbieter sein. ${ }^{1431}$ Entscheidend ist allein, ob eine objektiv erkennbare kommunikationsbezogene Eigenständigkeit besteht, also ob - aus der Sicht eines durchschnittlichen Nutzers ${ }^{1432}$ - ein eigenständiges Kommunikationsund Informationsangebot geschaffen wird. ${ }^{1433}$ Entscheidend hierfür ist die tatsächliche Kontrolle und damit die Herrschaft über das Medium. ${ }^{1434}$ Kommerziel-

1428 Insbesondere mit dem Erlass des RStV und des TMG haben sich Länder und Bund auf eine Vereinheitlichung der Regelung „neuer Medien“ geeinigt. Vorher wurden mit den Mediendiensten (MStV) und den Telediensten (TDG) unterschiedliche Begriffe verwendet. Dies führte teilweise dazu, dass die Abgrenzung des Anwendungsbereiches recht schwierig war. Vgl. hierzu Roßnagel, NVwZ 2007, 743 (743 f.), der die Politik als „rückschrittlich“ und die Unterscheidung als wenig überzeugend bezeichnet.

1429 Dort, wo Social Bots kommerzielle Interessen verfolgen, kommt auch die besondere Kennzeichnungspflicht nach \6 TMG in Betracht, hierzu etwa Dankert/Dreyer, K\&R 2017, 73 (75).

1430 Altenhain, in: MüKo StGB, Bd. 6, $\mathbb{1}$ TMG Rn. 26; Karg/Fahl, K\&R 2011, 453 (456); Hullen/ Roggenkamp, in: Plath, BDSG/DS-GVO, $\mathbb{1} 11$ TMG Rn.6; vgl. auch EuGH, Urt. v. 16.02.2012 Rs. C-360/10 = EuZW 2012, 261, Rn. 27 - SABAM/Netlog NV zur Anwendbarkeit von Art 14 der E-Commerce RL.

1431 Martini, in: Gersdorf/Paal, Informations- und Medienrecht, $\$ 2$ TMG Rn. 18; Rockstroh, MMR 2013, 627 (629); ausführlicher Heilmann, User-generated Content, S. 232 ff., der richtiger Weise auch daraufhin weist, dass in Zeiten des Web 2.0 die nutzereigenen Inhalte gerade die Dichotomie der Begriffe an ihre Grenzen gebracht hat, a.a.O., S. 24, 229. Anders indes B. Lorenz, VuR 2008, 321 (322 f.); ders., VuR 2014, 83 (84).

1432 Vgl. LG Regensburg, MMR 2013, 246 (248).

1433 Vgl. OLG Frankfurt, Urt. v. 06.03.2007 - 6 U 115/06, Rn. 30 - juris; OLG Düsseldorf, GRUR-RR 2013, 433 (435) LG Stuttgart ZUM 2014, 988 (990); Rockstroh, MMR 2013, 627 (628f.); Janall Isikay, GRUR-Prax 2018, 393 (394); vgl. Ricke, in: Spindler/Schuster, Elektronische Medien, $\$ 2$ TMG Rn. 3; Martini, in: Gersdorf/Paal, Informations- und Medienrecht, $\$ 2$ TMG Rn. 7a.

1434 Ricke, in: Spindler/Schuster, Elektronische Medien, $\$ 2$ TMG Rn. 3; Müller-Broich, TMG $\$ 2$ Rn. 1. Auch das OLG Frankfurt, a.a.O., Rn. 29 stellt in seiner Begründung auf die Gestaltungsmöglichkeiten $\mathrm{ab}$. 
le Seiten wurden regelmäßig als eigenständige Telemedien eingestuft. ${ }^{1435}$ Aber auch herkömmliche Nutzerprofile lassen sich - in gewissen Grenzen - vom jeweiligen Nutzer formal und inhaltlich frei gestalten. Der Nutzer ist Administrator seines Profils und besitzt auch die Möglichkeit, fremde Inhalte von seiner Seite zu löschen oder zumindest zu verbergen. Auch ein durchschnittlicher Nutzer weiß, dass die jeweilige Seite unabhängig vom Netzwerk durch den Nutzer verwaltet wird, ${ }^{1436}$ sodass auch Nutzer Anbieter sein können. ${ }^{1437}$ Sofern versucht wird, den Begriff dahingehend einzuschränken, dass rein privat genutzte Profile nicht erfasst werden, ${ }^{1438}$ findet das weder in $\$ 2 \mathrm{Nr}$. 1 TMG noch in $\mathbb{} 1 \mathrm{Abs} .1 \mathrm{TMG}$ eine Stütze. Auch der Gesetzesbegründung ist eine solche Einschränkung nicht zu entnehmen. Und letztlich spricht auch die Formulierung in $\mathbb{5} 5 \mathrm{Abs} .1 \mathrm{RStV}-$ immerhin ist die Anbietereigenschaft hier keine andere ${ }^{1439}$ - dagegen, weil die Norm ansonsten rein deklaratorischer Natur wäre.

Entscheidend für die Abgrenzung ist das Merkmal „in der Regel gegen Entgelt angeboten“. Denn geschäftsmäßig sind Angebote, die nachhaltig, d.h. für eine gewisse Dauer angeboten werden. ${ }^{1440}$ Woitke wollte den Begriff ursprünglich te-

1435 Allgemein zu kommerziell genutzten Seiten OLG Düsseldorf, MMR 2008682 (683); für Händlerseiten bei eBay LG Stuttgart, ZUM 2014, 988 (990); zu Facebook-Accounts LG Aschaffenburg MMR 2012, 38; LG Regensburg, MMR 2013, 246 und Rockstroh, MMR 2013, 627 (629). Siehe auch Micklitz/Schirmbacher, in: Spindler/Schuster, Elektronische Medien, $\$ 5$ TMG Rn. 7.

1436 Solmecke, in: Hoeren/Sieber/Holznagel, Multimedia-Recht, Teil 21.1 Rn. 85.

1437 Von dieser ausdrücklich ausgehend Lange, ZJS 2013, 141 f.; Dankert/Dreyer, K\&R 2017, 73 (76); Solmecke, in: Hoeren/Sieber/Holznagel, Teil 21.1 Rn. 85; Beyerbach, in: Hornung/Müller-Terpitz, RechtsHb Social Media, Kapitel 9 Rn.69; Brock/Schmitmann, in: Schwartmann, PraxisHb, 10 Rn. 54. So i.E. auch Rauschofer, MMR-aktuell 2010, 302790; und wohl auch Pießkalla, ZUM 2014, 368 (369), wenn er nur die Geschäftsmäßigkeit bei $\$ 5$ TMG verneint, ebenso Föhlisch, in: Hoeren/Sieber/Holznagel, Multimedia-Recht, Teil 13.4 Rn. 53. So wohl auch OLG Düsseldorf, ZUM 2014, 587 (588), wenn es auf eine Anbieterkennzeichnungspflicht von Nutzern näher eingeht. Daneben auch Richter, MMR 2014, 517; andeutend Stadler, ZD 2011, 57 (58); mangels Entscheidungserheblichkeit offenlassend, LG Freiburg MMR 2014, 118 (120). Differenzierender Ricke, in: Spindler/Schuster, Elektronische Medien, $\mathbb{S} 2$ Rn. 2 der die Anbietereigenschaft nur dann ablehnt, wenn ausschließlich persönliche Zwecke verfolgt werden. Ebenso Föhlisch, in: Hoeren/Sieber/ Holznagel, Multimedia-Recht, 13.4 Rn. 53. Nur im Falle von Facebook bejahend, Roggenkamp/ Krieg, in: Schmidt/Taddicken, Hb soziale Medien, S. 347.

1438 Schunicht, Informationelle Selbstbestimmung in sozialen Netzwerken, S. 179; Ricke, in: Spindler/ Schuster, Elektronische Medien, $\mathbb{2}$ TMG Rn. 2; Föhlisch, in: Hoeren/Sieber/Holznagel, Multimedia-Recht, 13.4 Rn. 53.

1439 Vgl. Krieg, jurisPR-ITR 13/2011 Anm. 3. Die Vereinheitlichung war immerhin das Ziel der damaligen Neuregelung des RStV und des TMG, BT-Drs. 16/3078, S. 11.

1440 LG Stendal, Urt. v. 24.02.2010 - 21 O 242/09; Ott, in: Gersdorf/Paal, Informations- und Medien-

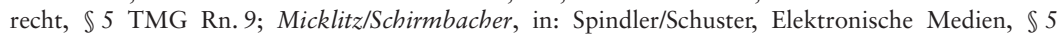
TMG Rn. 8; Hoeren, NJW 2007, 801 (803); Kitz, ZUM 2007, 368 (371); so schon zu \$6 TDG Brunst, MMR 2004,8 (9 f.); Stickelbrock, GRUR 2004, 111 (112); Beyerbach, in: Hornung/MüllerTerpitz, RechtsHb Social Media, Kapitel 9 Rn.91. Grundlage dieses Verständnisses ist eine Orientierung an $\$ 6$ TDG, an die hierfür gegebene Gesetzesbegründung (BR Drs. 966/96, S. 23.) und an $\$ 3$ Nr. 5 TKG a.F. bzw. $\$ 3$ Nr. 10 TKG n.F. (sieh nur Ott, in: Gersdorf/Paal, Informations- und Medienrecht, $\mathbb{5} 5$ TMG Rn. 9). Sofern der Begriff teleologisch reduziert werden sollte, um private Anbieter aus dem Anwendungsbereich auszunehmen (so Woitke, NJW 2003, 871 (872); im Ansatz auch Stickelbrock, GRUR 2004, 111 (112f.)) besteht nun mehr wegen des zusätzlichen Merkmals der „Entgeltlichkeit“, hierfür kein Bedarf. 
leologisch reduzieren, um private Anbieter aus dem Anwendungsbereich auszunehmen. ${ }^{1441}$ Wegen des zusätzlichen Merkmals der „Entgeltlichkeit“ besteht hierfür indes kein Bedürfnis mehr, sodass jede Art von Telemedium - sofern es nicht nur einmalig oder gelegentlich angeboten wird $-^{1442}$ geschäftsmäßig ist. Was aber unter der Wendung „in der Regel gegen Entgelt“ verstanden werden kann, ist nicht gerade offensichtlich. ${ }^{1443}$ Folgt man stringent dem Wortlaut, wäre $\mathbb{S} 5$ Abs. 1 TMG eine inhaltsleere Floskel. Die überwiegende Anzahl von Web- und Profilseiten sind frei zugänglich und werden gerade nicht gegen Entgelt angeboten. ${ }^{1444}$ Nach dem nationalen Gesetzgeber soll es davon abhängen, ob die Onlinepräsenz ohne jeglichen wirtschaftlichen Hintergedanken betrieben wird. ${ }^{1445}$ Dies sei der Fall, wenn sie als „Einstiegsmedium“ dient, durch das einem Kunden im Ergebnis eine entgeltliche Leistung angeboten wird. ${ }^{1446}$ So werden beispielsweise auch werbefinanzierte Inhalte erfasst. ${ }^{1447}$ Für Bots könnte also zumindest eine Kennzeichnungspflicht nach $\mathbb{S}$ Abs. 1 TMG bestehen, wenn sie wirtschaftlich orientierte Handlungen vornehmen. ${ }^{1448}$ Bei politischen Bots hingegen werden weder gegenüber Nutzern noch durch die Inhalte wirtschaftliche Zwecke verfolgt, sodass eine wirtschaftliche Relevanz gar nicht vorliegt. Erst die Tatsache, dass die Betreiber ein Entgelt erhalten, begründet ein wirtschaftliches Handlungsmotiv.

1441 Woitke, NJW 2003, 871 (872); im Ansatz auch Stickelbrock, GRUR 2004, 111 (112 f.).

1442 Ähnlich B. Lorenz, VuR 2014, 83 (85). Vgl. hierzu auch BT-Drs. 13/ 7385, S. 21.

1443 Eine ausführliche Auslegung des Begriffes findet sich bei Heilmann, Anonymität für User-Generated Content, S. $275 \mathrm{ff}$.

1444 Ablehnend auch B. Lorenz, VuR 2010, 323 (324); Held, in: Paschke/Berlit/Meyer/Kröner, Medienrecht, 69. Abschn. Rn. 31; Ott, in: Gersdorf/Paal, Informations- und Medienrecht, $\$ 5$ TMG Rn. 11. A.A. Hartmann, in: Wandtke/Ohst, PraxisHb MedienR, Kapitel $1 \rrbracket 5$ Rn. 180, nach dem es gerade darauf ankommen soll, „ob der konkrete Dienst vergütungspflichtig ist oder typischerweise wäre.".

1445 BT-Drs. 16/3078, S. 14; darauf verweisend Kitz, ZUM 2007, 368 (371). Rockstroh, MMR 2013, 627 (629 f.) legt das Merkmal im Lichte des Art. 57 AEUV und der hierzu ergangenen Rechtsprechung des EuGHs aus, kommt aber dadurch zu keinem anderen Ergebnis.

1446 Micklitz/Schirmbacher, in: Spindler/Schuster, Elektronische Medien, $\$ 5$ TMG Rn. 11; Ott, in: Gersdorf/Paal, Informations- und Medienrecht, $\mathbb{} 5$ TMG Rn. 11; in diese Richtung auch Brock/Schmittmann, in: Schwartmann, Praxishandbuch Medien, IT- und UrhR, 10 Rn. 63; Beyerbach, in: Hornung/Müller-Terpitz, RechtsHb Social Media, Kapitel 9 Rn. 93; Held, in: Paschke/Berlit/Meyer/ Kröner, Medienrecht, 69. Abschnitt Rn. 31; Haug, NJW 2015, 661; so auch LG Regensburg, MMR 2013, 246 (248). Ähnlich, mit umfassender Herleitung über Art. 57 AEUV, Rockstroh, MMR 2013, 627 (629f.) nach dem der Hintergrund einer wirtschaftlichen Tätigkeit ausreicht, so letztlich auch die Gesetzesbegründung BT-DrS. 16/3078, S. 14. Deutlich enger will Heilmann den Begriff verstehen, siehe hierzu nur das Fazit von dems., Anonymität für User-generated Content, S. 316, Hiernach bestünde nie eine Kennzeichnungspflicht für Bots, gleich welcher Art, weil das Telemedium selbst kein entgeltpflichtiges Angebot beinhaltet und auch keine Kontrahierungsmöglichkeit bietet. So letztlich auch, wenn man Hartmann, in: Wandtke/Ohst, PraxisHb MedienR, Kapitel 1 \5 Rn. 181 folgt; in diese Richtung auch Fischer, Medienrecht und Medienmärkte, S. 66, der nur wirtschaftliche Leistungen, die über Telemedien angeboten werden, als erfasst ansieht.

1447 Kitz, ZUM 2007, 368 (371); Ott, MMR 2007, 354 (355); ausführlicher und differenzierend hierzu

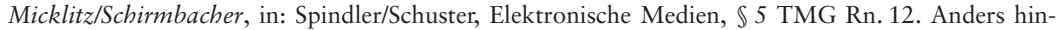
gegen Hartmann, in: Wandtke/Ohst, PraxisHb MedienR, Kapitel 1 \$ Rn. 181.

1448 Dankert/Dreyer, K\&R 2017, 73 (76); andeutend Müller-Peltzer/Franck, in: Taeger, Recht 4.0, S. 247. 
Das aber reicht nicht aus, um eine Kennzeichnungspflicht zu begründen. Maßgeblich ist nicht der konkrete Dienst, sondern die Frage, ob vergleichbare Dienste für gewöhnlich gegen Entgelt angeboten werden. ${ }^{1449}$ Entscheidend hierfür ist die objektive Sicht eines durchschnittlichen Nutzers. ${ }^{1450}$ Da es sich aber um gewöhnliche, nicht kommerzielle Nutzerprofile handelt, mit Hilfe derer kommuniziert wird, ist dies gerade nicht der Fall. ${ }^{1451}$ Für wirtschaftsbezogene Auftritte gibt es bei Facebook spezielle Seiten, die hierfür angelegt werden können und mit herkömmlichen Profilen nicht identisch sind. Und auch bei Twitter dürfte ein privater Account nicht mit den dort vertretenen kommerziell orientierten Presse- und Kurznachrichtendiensten vergleichbar sein, die die Nutzer oftmals über Links zum eigenen Online-Auftritt führen und als verifizierte Accounts gelistet sind. Eine Impressumspflicht nach $\mathbb{S}$ Abs. 1 TMG liegt somit für Botprofile fern.

Etwas anderes könnte sich nur dann ergeben, wenn man dem Ansatz von Lange folgt und wegen des Datenaustausches zwischen Netzwerkbetreiber und Nutzer eine Entgeltlichkeit annehmen würde. ${ }^{1452}$ Zwar kann auch die Leistung gegenüber einem Dritten die „Entgeltlichkeit“ begründen. ${ }^{1453}$ Für gewöhnlich geht es aber in den Konstellationen der Werbefinanzierung um eine Leistung, die dem jeweiligen Inhaber des Profils zufließt, während die Daten hier als „vertragliche Gegenleistung "1454 abfließen. Auch der Verweis von Lange auf die Einbindung der Bürgschaftsverträge in $\mathbb{} 312$ Abs. 1 BGB, ${ }^{1455}$ hilft nicht weiter. Denn dort ist schon die Perspektive eine andere, weil nicht Dritten Informationen zur Hand gegeben werden sollen, sondern der vertragsschließende Verbraucher um seiner selbst willen umfassend geschützt sein soll. ${ }^{1456}$ Entscheidend ist hier zudem neben kompetenziellen Aspekten auch die grundrechtliche Perspektive: Sofern auch private Auftritte in diesem weiten Sinne als entgeltlich angesehen würden, verlässt man damit den Bereich des Kompetenztitels „Recht der Wirtschaft“ des

1449 Beyerbach, in: Hornung/Müller-Terpitz, RechtsHb Social Media, Kap. 9 Rn. 92; Ott, in: Gersdorf/ Paal, Informations- und Medienrecht, $\$ 5$ TMG Rn. 11.

1450 Beyerbach, ebd. Lediglich von objektiven Maßstäben sprechend, Micklitz/Schirmbacher, in: Spindler/Schuster, Elektronische Medien, $\mathbb{5}$ TMG Rn. 20.

1451 Vgl. hierzu Beyerbach, in: Hornung/Müller-Terpitz, RechtsHb Social Media, Kap. 9 Rn. 93 , 95.

1452 Lange, ZJS 2013, 141 (144). Vgl. auch Ernst, in: Paal/Pauly, DS-GVO/BDSG, Art. 2 DS-GVO Rn. 19, der dies im Rahmen von Art. 2 Abs. 2 lit. C DS-GVO so sieht.

1453 Sonst ließe sich ja die Entgeltlichkeit von Medien nicht begründen, die werbe- und damit durch Dritte finanziert sind. Siehe hierzu die Verweise in Fn. 1447.

1454 Hierzu ausführlicher Bräutigam/v. Sonnleithner, in: Hornung/Müller-Terpitz, RechtsHb Social Media, Kap. 3 Rn. 18; Bräutigam, MMR 2012, 635 (636); vgl. auch Wendehorst, in: MüKo-BGB, Bd. 2, $\$ 312$ BGB Rn.38f. Siehe hierzu auch Metzger, AcP 2016, $818 \mathrm{ff}$. Die EU-Kommission hat in Art. 3 Abs. 1 eines Richtlinienvorschlags, $\operatorname{KOM}(2015) 634$ final, das Modell Daten als Entgelt vorgeschlagen, allerdings wurde das recht kritisch bewertet, u.a. auch weil es zu Spannungen mit der DS-GVO käme, hierzu etwa $v$. Westphalen/ Wendehorst, BB 2016, 2179 ff.; Specht, JZ 2017, $763 \mathrm{ff}$.

1455 Hierzu BGH, NJW 1998, 2356. Jüngst hat der BGH, NJW 2020, 3649, aber auch diesen Ansatz für Bürgschaftsverträge aufgegeben.

1456 Erwägungsgrund 3 und Art. 1 der RL 2011/83/EU, ABl. L 304, S. 64, 72. Zu diesem Schutzgedanken vgl. auch EUGH, Urt. v. 17.03.1998 - Rs. C-45/96 = NJW 1998, 1295 Rn. 19 - Dietzinger. 
Bundes (Art. 74 Abs. 1 Nr. 11 GG) ${ }^{1457}$ und begibt sich eher auf das Gebiet einer allgemeinen Medienregulierung und damit in den Kompetenzbereich der Länder. Entsprechendes gilt auch für das Verhältnis der EU zu den Mitgliedstaaten, denn die Richtlinie erfasst nur den elektronischen Geschäftsverkehr und beruft sich deshalb auf die Art. 47 Abs. 2, 55 und 95 EGV. ${ }^{1458}$ Und mit Blick auf die Grundrechte wäre zu bedenken, dass damit ein faktischer Klarnamenzwang im Internet bestünde, was nicht nur mit Blick auf die informationelle Selbstbestimmung, sondern auch mit Blick auf die Meinungsfreiheit problematisch wäre. ${ }^{1459}$ Das zeigt nicht zuletzt auch die Ausnahme des $\mathbb{} 18$ Abs. $1 \mathrm{MStV}$ für ausschließlich private Zwecke. ${ }^{1460}$ Eine extensive Auslegung auch auf private Nutzerprofile nach dem Vorbild von Lange kann daher nicht überzeugen. ${ }^{1461}$

\section{bb. Kennzeichnungspflicht des $\$ 55$ Abs. $1 \mathrm{RStV}$}

Mit Blick auf $\mathbb{S} 55$ Abs. 1 RStV - dessen normativer Gehalt durch $\mathbb{} 18$ Abs. 1 MStV (unverändert) übernommen wurde - bestünde eine Kennzeichnungspflicht, sofern das jeweilige Nutzerprofil nicht ausschließlich persönlichen oder familiären Zwecken dient. Wann aber ist das der Fall? Die Darstellung zum Telemediengesetz hat jedenfalls gezeigt, dass eine binäre Differenzierung zwischen Nutzer und Anbieter von Telemedien ${ }^{1462}$ nicht in Betracht kommt. Systematisch liegt es durchaus nahe, die Abgrenzung anhand des schon bei $\mathbb{S}$ Abs. 1 TMG relevant gewordenen Merkmals des wirtschaftlichen Hintergedankens vorzunehmen. ${ }^{1463}$ Aber nur weil kein wirtschaftlicher Zweck verfolgt wird, verfolgt das jeweilige Angebot nicht per se ausschließlich persönliche Zwecke. ${ }^{1464}$ Das Kriterium einer Zugangsbeschränkung ${ }^{1465}$ mag schon eher einen Anhaltspunkt für die persönliche Nutzung geben. Gleichwohl kommt es nach $\mathbb{5} 5$ Abs. $1 \mathrm{MStV}$ auf die (subjektiven) Zwecke des Nutzers und nicht auf die potentiellen Adressaten an. ${ }^{1466}$ Wie

1457 Hierzu und zur Begründung des Bedürfnisses einer bundeseinheitlichen Regelung i.S.d. Art. 72 Abs. 2 GG BT-Drs. 16/3078, S. 12.Und zudem findet sich hier der Hinweis, dass sich „,[d]iese Anforderungen (...) vor allem an Dienste, die im Zusammenhang mit einer Wirtschaftstätigkeit angeboten werden", richten.

1458 Hierzu die allgemeinen Ausführungen der RL 2000/31/EG. Und zudem werden in Erwägungsgrund 18 ausdrücklich nur Dienste erfasst, „(...) soweit es sich überhaupt um eine wirtschaftliche Tätigkeit handelt (...)“.

$1459 \mathrm{Zu}$ den grundrechtlichen Implikationen der Informationspflichten ausführlich Heilmann, Anonymität für User-generated Content, S. $81 \mathrm{ff}$.

1460 Vgl. Heilmann, Anonymität für User-generated Content, S. 268.

1461 Kritisch hierzu auch Beyerbach, in: Hornung/Müller-Terpitz, RechtsHb Socal Media, Kap. 9 Rn. 93.

1462 So etwa B. Lorenz, VuR 2014, 83 (84) m.w.N.

1463 So Weiner/Schmelz, K\&R 2006, 453 (458).

1464 I.E. vgl. hierzu Hartmann, in: Wandtke/Ohst, Medienrecht Bd. V, Kap. 1 Rn. 171.

1465 Rockstroh, MMR 2013, 627 (630). Ähnliche Kriterien fanden sich schon zuvor, wenn es um die Kennzeichnungspflichten von Webseitenbetreibern ging, siehe hierzu etwa Ott, MMR 2007, 354 (356).

1466 So etwa LG Köln, Urt. v. 28.12.2010 - 28 O 402/10, Rn. 46 - juris. 
das LG Köln zu Recht betont hat, ist den Internetangeboten die Offenheit wesensimmanent. ${ }^{1467}$ Und vielen privaten Angeboten fehlt ohnehin oftmals (unbewusst) eine Zugangsbeschränkung. Auch aus teleologischer Sicht bestehen gegen einen solchen Ansatz Bedenken. $\mathbb{S} 5$ Abs. $1 \mathrm{RStV}$ will einen Ausgleich zwischen informationeller Selbstbestimmung und Sicherung der Meinungsfreiheit ${ }^{1468}$ auf Seiten des Anbieters und dem Interesse Dritter an der Transparenz des Meinungsbildungsprozesses ${ }^{1469}$ und einer wirksamen Rechtsverfolgung im Streitfalle ${ }^{1470}$ schaffen. Sowohl ein zu restriktives als auch ein zu extensives Verständnis beeinträchtigen die Belange einer Seite. ${ }^{1471}$ Nur dort, wo die entgegenstehenden Interessen Dritter überwiegen, kommt eine Kennzeichnungspflicht in Betracht. Schon deshalb kann nicht allein eine (zufällig) vorhandene Zugangsbeschränkung entscheidend sein. ${ }^{1472}$ Werden nur private Inhalte veröffentlicht, interessiert das regelmäßig nur Personen des näheren Umkreises, denen die Identität ohnehin bekannt ist. ${ }^{1473}$ Private Inhalte können aber auch politische Beiträge sein ${ }^{1474}$, weil man ansonsten durch die Kennzeichnungspflicht chilling effects heraufbeschwören würde ${ }^{1475}$. Erst mit steigender Reichweite und Bedeutung eines Nutzers nimmt auch das Drittinteresse an einer Identifizierung und damit Kennzeichnung zu. ${ }^{1476}$ Und je eher Alternativen für eine Identifizierung zur Verfügung stehen - so besteht mit $\mathbb{} 14$ Abs. 3 TMG gegenüber dem Host-Provider die Möglichkeit, Auskunft über die Identität von Nutzern im Falle einer Rechtsverletzung zu verlangen und gerichtlich geltend $\mathrm{zu}$ machen $-{ }^{1477}$ desto eher kann auch auf eine

1467 LG Köln, ebd.

1468 Held, in: Binder/Vesting, Rundfunkrecht, $\mathbb{} 55$ RStV Rn. 10 f. Der baden-württembergische Gesetzgeber weist auf mögliche chilling effects hin, LT B-W Drs. 14/558, S. 39; ebenso Dankert/Dreyer, K\&R 2017, 73 (77).

1469 Hierauf stellt jedenfalls die Begründung der Vorgängernorm im Mediendienstestaatsvertrag, auf die bei Neuregelung durch das 9. RÄStV maßgeblich Bezug genommen wurde, ab. Die Begründung im MDStV ist abgedruckt bei Held, in: Binder/Vesting, Rundfunkrecht, $\mathbb{\int} 55$ RStV Rn. 7.

1470 Beyerbach, in: Hornung/Müller-Terpitz, RechtsHb Social Media, Kap. 9 Rn. 64 erwähnt beide Aspekte.

1471 Ein extensives Verständnis führt dazu, dass nicht mehr anonym oder pseudonym (informationelle Selbstbestimmung) und damit ohne Angst vor Repressalien kommuniziert werden kann (Meinungsfreiheit). Ein restriktives Verständnis führt zur Einschränkung der Transparenz und der erschwerten Rechtsverfolgung im Streitfall.

1472 Kritisch hierzu auch Hartmann, in: Wandtke/Ohst, Medienrecht Bd. V, Kap. 1 Rn. 176. Held, in: Binder/Vesting, Rundfunkrecht, $\mathbb{S} 55$ RStV Rn. 27a; ders., in: Paschke/Berlit/Meyer/Kröner, Medienrecht, 69. Abschnitt Rn. 54, greift deshalb auch auf inhaltliche Aspekte zurück.

1473 Hierzu LT B-W Drs. 14/558, S. 39, da „[in] diesen Fällen (...) entweder durch die persönliche Bekanntschaft oder aber über den Plattformanbieter sichergestellt [ist], dass die schutzwürdigen Belange der Beteiligten gewahrt werden können.

1474 So jedenfalls die Gesetzesbegründung, u.a. nachzulesen bei LT B-W Drs. 14/558, S. 38. Hierauf hat sich auch das LG Köln, Urt. v. 28.12.2010 - 28 O 402/10, Rn. 46 berufen.

1475 Ähnlich auch der baden-württembergische Gesetzgeber, LT B-W Drs. 14/558, S. 39. Dankert/Dreyer, K\&R 2017, 73 (77) weisen hierauf ebenfalls hin.

1476 Heilmann, Anonymität für User-generated Content?, S. 334; Dankert/Dreyer, K\&R 2017, 73 (77).

1477 Ebenfalls hierauf hinweisend Schunicht, Informationelle Selbstbestimmung in sozialen Netzwerken, S. 252. Wegen des ursprünglichen Fehlens einer solchen Vorschrift hat Lent, in: Gersdorf/Paal, In- 
Kennzeichnung verzichtet werden. ${ }^{1478}$ Bezweifelt werden kann allein, ob der so bestehende Schutz einem Impressum gleich kommt. ${ }^{1479}$ Hartmann gibt aber zu recht zu bedenken, dass „Verletzer, die Vorkehrungen dagegen treffen (..) ermittelt werden zu können, (...) sich kaum von einer Impressumspflicht beeindrucken lassen." 1480

Keines der genannten Kriterien kann also für sich überzeugen. Vielmehr sind alle Kriterien als wechselseitige Korrektive im Einzelfall zu berücksichtigen. ${ }^{1481}$ Existiert eine Zugangsbeschränkung, kann ohne Weiteres ein persönlicher Zweck angenommen werden. Ist dies hingegen nicht der Fall, führt das nicht automatisch zur Kennzeichnungspflicht. Entscheidend sind dann die Umstände des Einzelfalles, insbesondere aber die Inhalte, der damit verfolgte Zweck und der Kreis der Adressaten. ${ }^{1482}$ Auch die Möglichkeit, die Identifizierbarkeit anderweitig sicherzustellen, kann berücksichtigt werden. ${ }^{1483}$ Im Regelfall dürfte ein herkömmliches Nutzerprofil wegen der persönlichen Inhalte von der Kennzeichnungspflicht aber befreit sein. ${ }^{1484}$

Was bedeutet das für Botprofile? Nur weil Meinungsroboter sich normale Nutzerprofile zu eigen machen, heißt das nicht, dass für sie grundsätzlich eben-

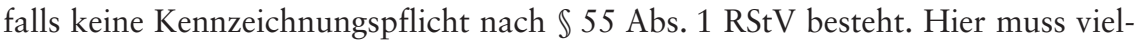
mehr differenziert werden: Automatisiert man sein eigenes Profil, dann hat das jedenfalls keinerlei Auswirkungen auf die Kennzeichnungspflicht. Ein strukturel-

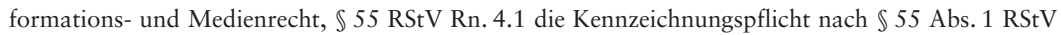
als nicht angemessene Lösung zwischen den kollidierenden Interessen angesehen.

1478 So maßgeblich das LG Köln, Urt. v. 28.12.2010 - 28 O 402/10, Rn. 47.

1479 Allgemein, ohne auf die Grundlagen einzugehen, Richter, MMR 2014, 517 (519), der verlangt, dass nicht nur E-Mail-Adressen gespeichert werden, die vorher nicht auf ihre Authentizität überprüft werden. Für gängige soziale Netzwerke bedeutet das, dass ihr Schutzniveau nicht an $\mathbb{} 55$ Abs. 1 RStV herankommen würde. Für das LG Köln scheint es schon auszureichen, wenn der Betreiber moderierend agiert und auf Grundlage der Nutzungsbedingungen den Zugriff auf Inhalte und Blogs verhindern kann, Urt. v. v. 28.12.2010 - 28 O 402/10, Rn. 47.

1480 Hartmann, in: Wandtke/Ohst, Medienrecht Bd. IV, Kap. 1 Rn. 176.

1481 Beyerbach, in: Hornung/Müller-Terpitz, RechtsHb Social Media, Kap. 9 Rn. 70; umfassend hierzu Heilmann, Anonymität für User-generated Content, S. $317 \mathrm{ff}$.

1482 So i.E. auch Heilmann, Anonymität für User-generated Content?, S. 342.

1483 Das war ein maßgebliches Argument des LG Köln, Urt. v. 28.12.2010 - 28 O 402/10, Rn. 46.

1484 So Beyerbach, in: Hornung/Müller-Terpitz, RechtsHb Social Media, Kap. 9 Rn. 70; Schunicht, Informationelle Selbstbestimmung in sozialen Netzwerken, S. 252; i.E. auch allerdings mit anderer Begründung Hartmann, in: Wandtke/Ohst, IT-Recht, Kap. 1 Rn.176. Lent, in: Gersdorf/Paal, Informations- und Medienrecht, $\mathbb{} 55 \mathrm{RStV}$ Rn. 4; Held, in: Binder/Vesting, Rundfunkrecht, $\mathbb{} 55 \mathrm{RStV}$ Rn. 27a; in diese Richtung tendiert wohl auch das LG Köln, Urt. v. 28.12.2010 - 28 O 402/10, Rn. 46 f. Differenzierend Roggenkamp/Krieg, in: Schmidt/Taddicken, Hb Soziale Medien, S. 349; ebenso Stadler, ZD 2011, 57 (58f.). Eine Kennzeichnungspflicht i.d.R. annehmend Richter, MMR 2014, 517 (519f.) und Rockstroh, MMR 2013, 627 (630). Letztendlich wird das Ergebnis maßgeblich von der Frage abhängen, ob das informationelle Selbstbestimmungsrecht hinter Drittinteressen - insbesondere einer wirksamen Rechtsverfolgung - zurücktreten sollte. Erachtet man diese Drittinteressen als immer und überall gefährdet (in diese Richtung Richter, ebd.) liegt eine Kennzeichnungspflicht nahe. Allerdings verkennt man dann womöglich die spezifische Gefährdungslage, in der sich der Kennzeichnungspflichtige befindet, wenn seine Daten dauerhaft im Internet gespeichert werden, hierzu Hartmann, in: Wandtke/Ohst, Medienrecht Bd. IV, Kap. 1 Rn. 176. 
ler Wandel des Profils findet nicht statt, sofern dadurch nicht die Reichweite erheblich erhöht oder Inhalte hinzutreten, die den privaten Charakter verändern. ${ }^{1485}$ Anders liegt es nur dann, wenn der Verwender aufgrund eines Auftrages tätig wird oder wenn Bots systematisch und (wahlkampf-)strategisch eingesetzt werden. Im ersten Fall handelt es sich schon nicht mehr um ausschließlich persönliche Zwecke, weil der Verwender nicht aus eigenem Antrieb tätig wird. Er agiert zumindest auch für einen anderen. Im zweiten Fall geht es nicht mehr ausschließlich um die persönliche Teilhabe an Diskussionen. Vielmehr wird agiert, um eine möglichst große Teilhabe an der öffentlichen Kommunikation und damit Reichweite $\mathrm{zu}$ erzielen. Dadurch tritt ein noch verfolgter ideell-persönliche Zweck in den Hintergrund. Von einem ausschließlich persönlichen Zweck kann dann nicht die Rede sein. ${ }^{1486}$ In diesen Fällen müsste also ein Impressum bereitgehalten werden, ${ }^{1487}$ sodass Name und Anschrift des Inhabers leicht erkennbar, unmittelbar erreichbar und ständig verfügbar sind.

\section{cc. Durchsetzung der Kennzeichnungspflicht}

Nach $\mathbb{5} 59$ Abs. 2 und Abs. 3 S. 1, 2 RStV kann die zuständige Aufsichtsbehörde bei Verstößen gegen die Kennzeichnungspflicht des $\mathbb{} 55$ Abs. 1 RStV einschreiten. Sie kann dann die zur Beseitigung des Verstoßes erforderlichen Maßnahmen treffen, was insbesondere - allerdings als ultima ratio - die Angebotsuntersagung und Sperrung nach $\mathbb{S} 59$ Abs. 3 S. 2 RStV beinhaltet. Derlei Maßnahmen können nicht nur gegenüber dem eigentlichen Verantwortlichen, sondern auch gegenüber den Netzwerkbetreibern getroffen werden, wenn sich Maßnahmen gegen Ersteren als nicht durchführbar oder nicht erfolgreich erweisen ( $\mathbb{S} 59$ Abs. 4 S. 1 RStV). Allerdings darf bezweifelt werden, dass dies im Falle der Meinungsroboter erfolgt, weil die Behörde von einem Verstoß gegen $\mathbb{} 55$ Abs. 1 RStV erst einmal Kenntnis erlangen muss. Das setzt aber voraus, dass die Behörde auch den Umstand einer Automatisierung oder zumindest die Reichweite des automatisierten Profils erkennen kann. Angesicht der Identitätskreierung wird ersteres aber häufig schwerfallen. Und letzteres scheitert an einem fehlenden behördlichen Zugriff auf die Netzwerke. Insoweit dürfte der behördlichen Ermächtigung nicht nur im Falle der Meinungsroboter, sondern in sozialen Netzwerken allgemein eine geringe Bedeutung zukommen.

1485 Dankert, in: Hofmann-Riem, Big Data - Regulative Herausforderungen, S. 157 (161) sieht in dem Einsatz von (mehreren) SocialBots jedenfalls ein starkes Indiz für einen nicht ausschließlich privaten Zweck.

1486 So Dankert/Dreyer, K\&R 2017, 73 (77).

1487 So i.E. auch Dankert/Dreyer, K\&R 2017, 73 (77) und auch - allerdings ohne nähere Begründung Klaas, MMR 2019, 84 (89). 
dd. Fazit: Lückenhafte Transparenzvorgaben des Medienrechts

Die bestehenden Kennzeichnungspflichten erfassen Meinungsroboter ungenügend. ${ }^{1488}$ Sofern $\mathbb{S} 55$ Abs. $1 \mathrm{RStV}$ und behördliche Kontrollrechte einschlägig sind, werden besonders zwei Schwachstellen sichtbar: Zum einen sind die Bots als solche für die außenstehende Behörde kaum zu erkennen, was erhebliche Auswirkungen auf die Durchsetzungsfähigkeit hat. Zum anderen wirkt die Kennzeichnungspflicht, wenn man davon ausgeht, dass sie befolgt wird, ${ }^{1489}$ nur mit Blick auf den eigentlichen Verwender transparenzerhöhend. Die Profile können so zwar einer Person zugeordnet werden. Im normalen Diskurs zwischen den Nutzern hat das aber keine Auswirkung, weil die Automatisierung weiterhin unentdeckt bleibt. ${ }^{1490}$ Zudem kann ein vorhandenes (falsches) Impressum die Glaubwürdigkeit noch weiter steigern.

\section{d. Datenschutzrechtliche Vorgaben}

Mittelbar werden auch die Netzwerkbetreiber und ihre Rankingalgorithmen für die Informations- und Meinungsbildungsfreiheit relevant, weil diese conditio sine qua non für eine privilegierte und auch segregierte Informationsdiffusion sind. ${ }^{1491}$ Da diese aber maßgeblich auf Personalisierungsprozessen beruhen, können auch datenschutzrechtliche Implikationen relevant werden. Die hiermit verbundenen Fragen werden seit dem 25.05.2018 - zumindest bei privaten Verarbeitern - europaweit durch die DS-GVO beantwortet, die innerhalb ihres Anwendungsbereichs die nationalstaatlichen Regelungen, allen voran das BDSG und das TMG, verdrängt. ${ }^{1492}$ Auch wenn in der Praxis unklar sein mag, ob die entsprechenden Verarbeitungsschritte im Binnenmarkt oder im EU-Ausland erfolgen, haben die Betreiber jedenfalls die DSGVO zu beachten, sofern die Verarbeitung einen unionalen Bezug aufweist (vgl. Art. 3 Abs. 2 DS-GVO), was angesichts der Ausrichtung auf den europäischen Binnenmarkt stets der Fall sein wird.

1488 So letztlich auch Löber/Roßnagel, MMR 2019, 493 (494 f.).

1489 Schon das ist aber nicht überwiegend wahrscheinlich, weil die Risiken für die Verwender überschaubar sind. Im schlimmsten Fall werden die entdeckten Profile gesperrt. Das hindert sie aber nicht daran, neue Profile zu registrieren. Es läuft also auf ein Katz-und-Maus-Spiel hinaus.

1490 So auch Löber/Roßnagel, MMR 2019, 493 (495); Dankert, in: Hofmann-Riem, Big Data - Regulative Herausforderungen, S. 157 (161 f.).

$1491 \mathrm{Zu}$ den Personalisierungsprozessen siehe bereits oben, 1. Teil A.II.1 bzw. A.II.2. Und zur mittelbaren Bedeutung dieser netzwerkinternen Prozesse oben, 1. Teil C.VI.3.

1492 Vgl. nur im Ansatz Erwägungsgrund 10, 16 der DS-GVO 2016/679, ABl. L 119. Weil es sich aber um eine Hybrid zwischen Verordnung und Richtlinie handelt, so etwa Bilz, Margin of Appreciation, S. 240 m.w.N., werden daneben auch ganz besonders national-rechtliche Vorgaben relevant. Das gilt etwa, wenn es in Art. 6 Abs. 1 lit.c) um die „rechtlichen Verpflichtungen“ geht, oder etwa hinsichtlich der Öffnungsklausel des Art. 85 oder des Art. 91 DS-GVO. Zu diesen und den dadurch entstehenden mitgliedstaatlichen Regelungsspielräumen, etwa Bilz, ebd., S. $240 \mathrm{ff}$. 
Die DS-GVO ist aber kein geeignetes Instrument, um Fragen der Informationsdiffusion, der Meinungsvielfalt oder andere Fragen des Medienrechts in der digitalen Welt vernünftig beantworten zu können. ${ }^{1493}$ Das liegt nicht nur an dem ganz anderen Regelungszweck, sondern auch daran, dass sie einer Verarbeitung personenbezogener Daten der Nutzer zum Zwecke der Personalisierung der Dienste (und damit auch der Informationsweiterleitung) nicht dem Grunde, sondern nur dem Umfang nach im Weg steht. Art. 6 Abs. 1 DS-GVO erlaubt grundsätzlich die Erhebung entsprechender Daten, sofern und soweit eine rechtliche Grundlage einschlägig ist. In Betracht kommt neben der Einwilligung (Art. 6 Abs. 1 lit. a DS-GVO) auch die Erforderlichkeit oder die berechtigten Interessen der Betreiber, ${ }^{1494}$ wobei sich jeweils spezifische Probleme ergeben: Eine Einwilligung der Nutzer kann nicht nur an der fehlenden Freiwilligkeit (Art. 4 Abs. 1 Nr. 11 DS-GVO) scheitern. Denn die starke wirtschaftliche Machtstellung ${ }^{1495}$ einzelner Anbieter und die auch soziale Abhängigkeit der Nutzer infolge von Lockin-Effekten u.ä. ${ }^{1496}$ führt de facto dazu, dass man sich den Diensten kaum entziehen kann, ohne sich auch in eine gewisse soziale Isolation zu begeben. Zudem ist den Nutzern eine Disposition über die Art und den Umfang erhobener gar nicht oder nur in einem nicht nennenswerten Umfang möglich, sodass er entweder einwilligen oder dem Dienst fernbleiben muss. ${ }^{1497}$ Im Lichte des Erwägungsgrundes 43 könnte deshalb schon keine freiwillig erteilte Einwilligung vorliegen. ${ }^{1498}$ Daneben ist zumindest diskutabel, in wie weit eine informierte Einwilligung angesichts

1493 So etwa auch Kaiser/Reiling, in: Unger/v. Ungern-Sternberg, Demokratie und künstliche Intelligenz, S. 85 (101).

1494 Hierzu auch v. Ungern-Sternberg, in: Unger/Dies., Demokratie und künstliche Intelligenz, S. 3 (22 ff.).

1495 Vgl. hierzu etwa Ernst, in: Paal/Pauly, DS-GVO/BDSG, Art. 4 DS-GVO Rn. 76; ders., ZD 2017, 110 (112), der formuliert: „Wer als Anbieter mit einem signifikanten Marktanteil (wie (...) Anbieter großer Social-Media-Dienste) als Bedingung für die Inanspruchnahme der eigenen Leistungen die Zustimmung zu einer umfassenden Verwendung personenbezogener Daten zwingend verlangt, kann nicht davon ausgehen, dass diese Einwilligung tatsächlich ,freely given' ist. " In diese Richtung auch Gierschmann, ZD 2016, 51 (54). Umfangreich zu dieser Machtstellung im Falle von Facebook BKartA, Beschluss vom 06.02.2019 - B6-22/16 = BeckRS 2019, 4895 Rn. 163 ff. und zur Frage der Freiwilligkeit der Einwilligung Rn. 639 ff. Auf die fehlende Alternative stellt auch Golland, MMR 2018, 130 (131), ab. Nocun, in: Roßnagel/Friedewald/Hansen, Fortentwicklung des Datenschutzes, S. 39 (40) bezeichnet Facebook als einen der wichtigsten Gatekeeper und als de-facto Standard für soziale Netzwerke. Anders indes Klement, in: Simitis/Hornung/Spiecker gen. Döhmann, Datenschutzrecht, Art. 7 DS-GVO Rn. 63 und Kipker/Voskamp, DuD 2012, 737 (739).

1496 Siehe hierzu etwa Noyb, Beschwerde gegen Facebook nach Art. 77 Abs. 1 DS-GVO, S. 11.

1497 Zur Relevanz für die Freiwilligkeit siehe etwa BKartA, Beschluss vom 06.02.2019 - B6-22/16 = BeckRS 2019, 4895 Rn. 645.

1498 So BKartA, Beschluss vom 06.02.2019 - B6-22/16 = BeckRS 2019, 4895 Rn. 645 f.; Knebel, Drittwirkung der Grundrechte gegenüber Privaten, S. 197; Jørgensen/Desai, NJHR 35 (2017), 106; so auch die Begründung von Noyb, Beschwerde an die österreichische Datenschutzbehörde, S. 11. In diese Richtung wohl auch Ernst, in: Paal/Pauly, DS-GVO/BDSG, Art. 7 DS-GVO Rn. 76; ders., ZD 2017, 110 (112); Pomana, BB 2018, 965 (972 f.); Golland, MMR 2018, 130 (130 f.); jedenfalls in Betracht ziehend Kamp/Rost, DuD 2013, 80 (82). A.A. Kipker/Voskamp, DuD 2012, 737 (738f.); wohl auch Kampert, Datenschutz in sozialen Online-Netzwerken, S.93; Klement, in: Simitis/ Hornung/Spiecker gen. Döhmann, Datenschutzrecht, Art. 7 DS-GVO Rn. 63. 
der umfangreichen Datenrichtlinien und ihrer teils nur recht vagen Aussagen erfolgt. ${ }^{1499}$ Und auch das Kopplungsverbot des Art. 7 Abs. 4 DS-GVO könnte einer Einwilligung entgegenstehen, weil der Zugang zum Netzwerk von der Datenpreisgabe abhängig gemacht wird. Das hängt aber zum einen davon ab, wie man die die „Erforderlichkeit“ versteht, und zum anderen davon, ob Art. 7 Abs. 4 DSGVO ein absolutes oder doch nur ein relatives Kopplungsverbot enthält. ${ }^{1500}$

In welchem Umfang Betreiber ${ }^{1501}$ dann gestützt auf Art. 6 Abs. 1 lit. b) DSGVO Daten erheben können, hängt von dem Tatbestandsmerkmal der Erforderlichkeit ab. Klar ist insoweit nur, dass allein die Leistungsbeschreibungen der Betreiber nicht maßgeblich für die datenschutzrechtliche Bewertung sein können, weil online wesentlich mehr Daten anfallen als in herkömmlichen analogen Vertragsbeziehungen. Wenn hier alle Daten ${ }^{1502}$ als erforderlich für die personalisierte Erbringung der Dienste angesehen würden, würden die hohen Anforderungen der Einwilligung umgangen ${ }^{1503}$ und auch das Prinzip der Datensparsamkeit ad absurdum geführt. Der datenschutzrechtliche Schutz wäre so zur Disposition des geschickten Verwenders von AGB gestellt. ${ }^{1504}$ Unklar ist bislang allein, wie - me-

1499 Greve, in: Sydow, DS-GVO, Art. 12 Rn. 13 sieht zumindest ein Spannungsfeld. Zu empirischen Fragen, der rechtlichen Bedeutung und Verbesserungen etwa Gerpott, MMR 2020, $739 \mathrm{ff}$. Ebenfalls zur Empirie Rothmann, in: Roßnagel/Friedewald/Hansen, Fortentwicklung Datenschutz, S. $59 \mathrm{ff}$. Bezugnehmend auf eine österreichische Studie; hierzu auch ders./Buchner, DuD 2018, 342 (344ff.). Diese Informiertheit anzweifelnd B. Wagner, ZD-Aktuell 2018, 06103. Kritisch hinsichtlich der Floskel "We use the information we collect from all of our services to provide, maintain, protect and improve them, to develop new ones [...]”, die gewisse Ähnlichkeit zur Datenschutzrichtlinie von Facebook hat, Golba, Annuals of the Administration and Law 17 (2), 253 (258). Kritisch auch Noncun, in: Roßnagel/Friedewald/Hansen, Fortentwicklung des Datenschutzes, S. 45; ebenfalls mit Blick auf Art. 5 Abs 1 lit. a) DS-GVO BKartA, Beschl. v. 06.02.2019 - B6-22/16 = BeckRS 2019, 4895 Rn. 791 f. Und zur Verarbeitung der durch die business tools erlangten Daten Rn. 863. Letztlich geht es hier darum einen Spagat zwischen umfangreicher Information auf der einen und dem Verhindern einer Informationsflut auf der anderen Seite zu schaffen (vgl. Kampert, Datenschutz in sozialen Online-Netzwerken, S. 90 f.). Angesichts dessen werden in letzter Zeit zusätzlich zu den Datenschutzerklärungen sog. „One-pager“ verlangt, die die essentiellen Informationen kurz und bündig zusammenfassen (hierzu Kuntz, ZD-Aktuell 2015, 04909; Hennemann, ZUM 2017, 544 (551).). Ob das aber zwingend bedeutet, dass die gängige Praxis datenschutzrechtlich unzulässig ist, ist unklar.

1500 In die Richtung eines absoluten Kopplungsverbotes tendierend Remmertz, GRUR-Prax 2018, 254 (255); anders hingegen Artikel-29-Datenschutzgruppe, WP 259 rev. 01, S. 10; Veil, NJW 2018, 3337 (3340).

1501 Betrachtet man einschlägige Regelwerke, bspw. von Facebook, so fällt auf, dass ein Groß der Daten nicht auf Grundlage einer Einwilligung erhoben wird. Vielmehr beruft man sich überwiegend auf die Erforderlichkeit zur Vertragserfüllung. Siehe hierzu bspw. die ergänzenden Erklärungen von Facebook, abrufbar unter https://www.facebook.com/about/privacy/legal_bases (Stand: 30.05.2021). Darauf weist auch das BKartA, Beschl. v. 06.02.2019 - B6-22/16 = BeckRS 2019, 4895 Rn. 641 f., hin. Das überrascht auch deswegen, weil sich die einschlägige Literatur zumeist auf die Einwilligung stürzt und die Frage der Erforderlichkeit für die Vertragserfüllung gar nicht oder nur unzureichend aufgeworfen und beantwortet wird, siehe hierzu nur Söbbing, InTeR 2018, 182 (184).

1502 Ausführlicher zu den Datenquellen BKartA, Beschl. v. 06.02.2019 - B6-22/16 = BeckRS 2019, 4895 Rn. $483 \mathrm{ff}$.

1503 Schantz, in: Simitis/Hornung/Spiecker gen. Döhmann, Datenschutzrecht, Art. 6 DSGVO Rn. 33.

1504 So etwa auch $v$. Ungern-Sternberg, in: Unger/Dies., Demokratie und künstliche Intelligenz, S. 3 (25). Wenn Facebook sein Angebot als personalisiertes Erlebnis und personalisierten Dienst beschreibt, 


\section{thodisch überzeugend - eine Restriktion des Tatbestandes erfolgen kann. ${ }^{1505}$ In jedem Fall ist aber die umfangreiche Nutzung sämtlicher auch netzwerkexterner,}

werden hierfür potentiell alle personenbezogenen Informationen notwendig. Eine bestimmte Grenze lässt sich so nur schwer ziehen, sodass Art. 6 Abs. 1 lit. b DS-GVO immer einschlägig sein könnte, vgl. zu personalisierten e-Commerce-Diensten Krohm/Müller-Peltzer, ZD 2017, 551 (554). Damit wird aber die Einwilligung mit ihren hohen Rechtmäßigkeitsanforderungen unterlaufen (so Buchner/Petri, in: Buchner/Kühling, DS-GVO/BDSG, Art. 6 DS-GVO Rn. 6 Wendehorst/Graf v. Westphalen, NJW 2016, 3745 [3747], weshalb sich die Schutzrichtung im Vergleich zum Vertragsrecht hier ändert: es geht nicht mehr um den Schutz vor einschränkenden, sondern vor ausufernden Leistungsbeschreibungen, [3746f.]) und wesentliche Ziele der DS-GVO können nicht erreicht werden. Deshalb bezeichnet Hennemann, ZUM 2017, 544 (546) Art. 6 Abs. 1 lit b) DS-GVO wohl auch als „trojanisches Pferd“. Kritisch hierzu auch Nocun, in: Roßnagel/Friedewald/Hansen, Fortentwicklung des Datenschutzes, S. 43.

1505 Das BKartA, Beschl. v. 06.02.2019 - B6-22/16 = BeckRS 2019, 4895 Rn. 672 stellt folgende Voraussetzung: „Die Datenverarbeitung ist danach nur dann für die Vertragserfüllung erforderlich, wenn bei vernünftiger Betrachtung das Angewiesensein des Unternehmens auf das konkret in Frage stehende Mittel zu bejahen und ein Verzicht auf die Daten nicht sinnvoll oder unzumutbar ist. Dabei gilt die Bestimmung gerade nicht für Situationen, in denen die Verarbeitung für die Vertragserfüllung nicht wirklich notwendig ist, sondern der Vertragsinhalt und die Datenverarbeitung der betroffenen Person von dem für die Verarbeitung Verantwortlichen einseitig auferlegt wird. “ In der Literatur wird versucht, Art. 6 Abs. 1 lit. b DS-GVO teleologisch zu reduzieren, wenn die erhobenen Daten auch zu kommerziellen Zwecken vom Verantwortlichen genutzt werden (Wendehorst/Graf $v$. Westphalen, NJW 2016, 3745 (3747); in diese Richtung auch Buchner/Petri, in: Buchner/Kühling, DS-GVO/BDSG, Art. 6 DS-GVO Rn. 40; wohl auch Hennemann, ZUM 2017, 544 (547); jedenfalls i.E. für den Ausschluss der Rechtsgrundlage bei kommerzieller Verwendung Albrecht/Jotzo, Datenschutzrecht der EU, Teil 3 Rn. 44). Damit würde man aber in systematisch fragwürdiger Weise nach den Interessen des Datenverarbeitenden differenzieren. Andernorts sollen unbillige Ergebnisse über eine zivilrechtliche Vertrags- und Klauselkontrolle korrigiert werden (Engeler, ZD 2018, 55 (58); so auch - als alternativer Ansatz bis zur endgültigen Klärung durch den EuGH -Wendehorst/Graf $v$. Westphalen, NJW 2016, 3745 [3748f.]; in diese Richtung auch Schantz, in: Simitis/Hornung/ Spiecker gen. Döhmann, Datenschutzrecht, Art. 6 DS-GVO Rn. 27 f.; Indenhuck/Britz, BB 2019, 1091 [1093]). Gangbar wäre es aber auch zu fragen, was den Wesenskern des Vertrages ausmacht, welche Daten also für den Sinn des Vertrages notwendig sind (so Buchner/Petri, in: Buchner/ Kühling, DS-GVO/BDSG, Art. 6 DS-GVO Rn. 38 f.; in diese Richtung auch BGH, ZD 2018, 477 Rn. 71; ähnlich Gierschmann, MMR 2018, 7 [8]; vgl. hierzu auch die Darstellungen bei B. Wagner, ZD-Aktuell 2018, 06103 und Engeler, ZD 2018, 55 [57f.]. Das EDPB formuliert in seinen Guidelines 2/2019, Rn. 25: „Article 6 (1) (b) will not cover processing which is useful but not objectively necessary for performing the contractual service [...].") Die Einwilligung würde so nicht durch das „trojanische Pferd“ (so Hennemann, ZUM 2017, 544 [546]) der Vertragserfüllung unterlaufen. Das EDPB, Guidelines 2/2019, Rn. 17, meint explizit: „(...) in some circumstances it may be more appropriate to rely on freely given consent under Article 6 (1) (a). "Dabei verlässt man auch nicht den Wortlaut der Norm, weil es dann immer noch im Kern auf den konkreten Vertrag ankommt, nur entzöge man die Dispositionsbefugnis über die Frage der Erforderlichkeit den Datenverarbeitern. Das EDPB, Guidlines 2/2019, Rn. 30, bekräftigt dies, wenn es schreibt: „A contract cannot artificially expand the categories of personal data or types of processing operation that the controller needs to carry out for the performance of the contract within the meaning of Article 6 (1) (b)." Worin aber soll der Wesenskern eines Nutzungsvertrages bei sozialen Netzwerken bestehen? In der Nutzung? In der Abstimmung des Angebots an die jeweiligen Nutzerbedürfnisse? Kann man nicht sogar die Daten als vertragliche Hauptleistungspflicht anerkennen (siehe nur Bräutigam, MMR 2012, 635), sodass stets von der Erforderlichkeit auszugehen wäre? Nimmt man den Restriktionsversuch ernst, wäre es plausibel, wenn an die Grundfunktionen angeknüpft wird, also die Kommunikation und Interaktion mit Bekannten und Freunden (vgl. Buchner, DuD 2016, 155 (158); A $\beta$ mus, in: Jandt/Steidle, Datenschutz, B. III. Rn. 225). Die bedarfsgerechte, also personalisierte, Erbringung der Leistungen ist insoweit nützlich, aber nicht erforderlich (vgl. Buchner/Petri, in: Buchner/Kühling, DS-GVO/BDSG, Art. 6 Rn. 42; EDPB, Guideline 2/2019, Rn. 54 und die Beispiele 7 und 8). Jedenfalls eine Erhebung über die Netzwerkdaten hinaus, wäre also nicht mehr durch Art. 6 Abs. 1 lit. b DS-GVO legitimiert, in diese Richtung Buchner, DuD 2016, 155 (158). 
personenbezogener Daten nicht erfasst, ${ }^{1506}$ sondern lediglich ein eng begrenzter Ausschnitt der - allein netzwerkinternen - Daten.

Weil die Verarbeitung der Daten zwar der Refinanzierung der Dienste dient, haben die Betreiber ein berechtigtes Interesse an der Verarbeitung (Art. 6 Abs. 1 lit. f) DS-GVO). Allerdings werden auch hier dort Grenzen gezogen, wo „die Interessen oder Grundrechte und Grundfreiheiten der betroffenen Person, die den Schutz personenbezogener Daten erfordern, überwiegen (...).“ Insoweit kann freilich auch hierunter keine sachlich unbegrenzte Verarbeitung netzwerkinterner und -externer Daten erlaubt sein. ${ }^{1507}$

Und letztlich können selbst sensible Daten (Art. 9 Abs. 1 DS-GVO) verarbeitet werden. Mit Blick etwa auf die „politische Meinung“ gehören hier nicht nur direkte Angaben über die politische Zugehörigkeit zu, sondern auch solche Daten, aus denen sich mittelbar die politische Meinung deduzieren lässt. ${ }^{1508}$ Den Betreibern wird die Verarbeitung solcher Daten aber nicht unmöglich gemacht. Sie ist vor allem dann erlaubt, wenn der Betroffene die Information bewusst ins Internet stellt und diese ohne wesentliche Hürden einer Vielzahl von Personen zugänglich sind (Art. 9 Abs. 2 lit e) DS-GVO) ${ }^{1509}$.

Auch aus datenschutzrechtlicher Sicht sind also Personalisierungsprozesse legitimer Bestandteil - immerhin haben auch Nutzer einen Mehrwert davon ${ }^{1510}$ der Netzkultur. Lediglich der Umfang ist hier wohl durch die DS-GVO deutlich weiter beschränkt, als es die Praxis vermuten lässt. Das dürfte zwar einen Einfluss auf die Personalisierungsmaßnahmen haben. Gleichwohl werden zumindest die freiwillig angegebenen Daten und die geteilten Inhalte noch erfasst sein, sodass sich hieraus wesentliche Datenbestände ergeben, die eine selektierte und personalisierte Informationsdiffusion ermöglichen. ${ }^{1511}$ Die DS-GVO reagiert hierauf selbst mit entsprechenden, die Transparenz erhöhenden Maßnahmen, insbesondere mit den Informationspflichten und Auskunftsrechten der Art.13ff. DSGVO.

1506 Ausführlich hierzu BKartA, Beschl. v. 06.02.2019 - B6-22/16 = BeckRS 2019, 4895 Rn. $671 \mathrm{ff}$.

1507 Hierzu umfassender BKartA, Beschl. v. 06.02.2019 - B6-22/16 = BeckRS 2019, 4895 Rn. 727 ff.

1508 So letztlich auch der BKartA, Beschl. v. 06.02.2019 - B6-22/16 = BeckRS 2019, 4895 Rn. 586. Ähnlich Albers/Veit, in: Wolff/Brink, Datenschutzrecht, Art. 9 DS-GVO Rn. 19; Schneider, ZD 2017, 303 (304). Ausführlicher Petri in: Simitis/Hornung/Spiecker gen. Döhmann, Datenschutzrecht, Art. 9 DSGVO Rn. $11 \mathrm{ff}$

1509 Albers/Veit, in: Wolff/Brink, Datenschutzrecht, Art. 9 DS-GVO Rn. 65 f.; Petri, in: Simitis/Hornung/ Spiecker gen. Döhmann, Datenschutzrecht, Art. 9 DS-GVO Rn. 57 f. Explizit zu sozialen Netzwerken Petri, in: Simitis/Hornung/Spiecker gen. Döhmann, Datenschutzrecht, Art. 9 DS-GVO Rn. 58.

1510 Schafft/Ruoff, CR 2006, 499 (500).

1511 Das zeigt ja schon die Prediction-API „Apply Magic Sauce“, die „nur“ mit netzwerkinternen Datenbeständen arbeitet, den Post, den Likes, den Kommentaren und den Profilinformationen und damit meist über Daten die für die Bereitstellung der Dienste erforderlich sind. Denn die Inhalte müssen ja gespeichert werden, um den Dienst erfüllen und sie letztlich anderen Nutzern anzeigen zu können. Siehe hierzu https://applymagicsauce.com/about-us, (Stand 30.05.2021.). 


\section{e. Fazit: Primär selbstregulatorischer Schutz durch Betreiber}

Der Schutz der Informationsfreiheit wird in erster Linie auf einer selbstregulatorischen Ebene gewährleistet. Allerdings bleibt es hier meist nur bei einem Gewährleistungsversuch. Weder die Nutzungsbedingungen noch der gemeinsame Verhaltenskodex der EU-Kommission bieten hinreichend Gewähr dafür, dass Meinungsroboter tatsächlich effektiv bekämpft werden. Dafür mangelt es an spezifischen Vorgaben und aufsichtsrechtlichen Befugnissen. Verbindliche, die Transparenz erhöhende Regeln und aufsichtsrechtliche Befugnisse bestehen zwar im Medienrecht. Aber selbst wenn diese Regeln einschlägig sind, so bieten sie nur eine allgemeine Antwort auf das Bedürfnis, den Anbieter eines Telemediums identifizieren zu können. Im Falle der Meinungsroboter führt das aber gerade nicht zu der Aufdeckung des eigentlichen Charakters. Dem Hauptanliegen der Transparenz wird also mit Blick auf die Informationsfreiheit nicht hinreichend Rechnung getragen. Datenschutzrechtliche Erwägungen werden zwar auch mittelbar relevant, soweit sie von Bedeutung für die algorithmenbasierte Weitergabe der Informationen sind. Allerdings steht die personalisierte Informationsweitergabe nicht unter einem generellen, sondern allein unter dem Vorbehalt, dass nur ein bestimmter (geringer) Datenbestand hierfür verwendet wird. Mit den Informationspflichten (Art.13f. DS-GVO) wird zwar Transparenz mit Blick auf die Zwecke der Verarbeitung geschaffen. Inwieweit dabei aber auch Transparenz über den algorithmisierten Weiterleitungsprozess geschaffen wird, ist zweifelhaft und hängt vor allem von den jeweiligen Datenschutzerklärungen ab. Den Schutzbedürfnissen der Informationsfreiheit wird so kaum Rechnung getragen.

\section{Spezifische Schutzmechanismen für die Meinungsäußerungsfreiheit}

Betrachtet man etwaige Schutzvorkehrungen für die Meinungsäußerungsfreiheit, so kann Anknüpfungspunkt hierfür - zur Erinnerung - allein die Gewährleistung der Freiheit der Willensbetätigung vor einem externen Einfluss sein. ${ }^{1512}$ Insoweit können nicht nur speziell für die Willensbetätigung im politisch-demokratischen Kontext Schutzmechanismen bestehen, sondern auch ganz generell für die freie Willensbetätigung.

\section{a. Einfach-rechtlicher Schutz vor Beeinflussung der Willensbetätigung}

Einfach-rechtlich spiegelt sich diese Unterscheidung vor allem in der Unterscheidung zwischen dem generellen Schutz der Willensbetätigung durch den „Grundtatbestand“ der Nötigung $(\mathbb{S} 240$ StGB) und dem Schutz der freien Willensbetäti-

1512 Zum Schutzbedarf oben, B.II.1.b. 
gung als Voraussetzung des zentralen politischen Akts der Wahl ( $\mathbb{S} 108 \mathrm{StGB})$ wider. Diese strafrechtliche Schutznorm wird durch das wahlrechtliche Verbot bestimmter Einflussnahmen am Wahltag in $\$ 32$ Abs. 1 BWG ergänzt.

\section{aa. Schutz der allgemeinen freien Willensbetätigung, $\mathbb{S} 240 \mathrm{StGB}$}

\240 StGB schützt aber nur vor Nötigungen. Es muss entweder mit einem empfindlichen Übel gedroht oder Gewalt angewendet werden. Bei Meinungsrobotern ist es durchaus denkbar, dass anderen Nutzern etwaige Übel in Aussicht gestellt werden, worauf die jeweiligen Bots Einfluss zu haben scheinen. Abhängig ist das aber von den konkreten Kommunikaten. Entscheidender wäre die Frage, ob durch die Suggestion einer gesellschaftlichen Mehrheit Gewalt im Sinne der Norm angewendet wird. Bereits in anderen Fällen wurde aber deutlich gemacht, dass allein ein psychisch vermittelter Zwang keine Gewalt darstellt, weil man damit die Grenze des Wortlauts in verfassungswidriger Weise überschreitet (Art. 103 Abs. 2 GG). ${ }^{1513}$ Demnach ist das zentrale Kriterium für den Begriff der Gewalt eine doppelte Körperlichkeit: einerseits eine körperliche Kraftentfaltung des Täters andererseits eine körperliche Zwangswirkung beim Opfer.

In den hier relevanten Fällen werden aber allein Mehrheiten suggeriert und damit die normalen Zwangswirkungen von Diskursen und Gruppendynamiken ausgenutzt. Dies führt zwar zu dem Zwang, die eigene Meinung nicht kundgeben $\mathrm{zu}$ wollen - insoweit bestünde also eine willensbeugende Wirkung -, allerdings basiert diese Zwangswirkung eher auf der geistigen, denn auf einer körperlichen Wirkung. Insoweit ergibt sich gerade kein Unterschied zwischen der physischen Anwesenheit von Personen etwa im Rahmen einer Versammlung, die zwar Einschüchterungseffekte auslösen kann, aber als solche zulässig ist, ${ }^{1514}$ und der Anwesenheit anderer Nutzer und ihrer Meinungen als „manifestierte physische Präsenz“ innerhalb der Netzwerke. In der Konsequenz müssten ansonsten immer Mehrheitsmeinungen wegen der psychologischen Effekte als Gewalt angesehen werden. ${ }^{1515}$ Dass das nicht gewollt sein kann, liegt auf der Hand. Erst wenn bestimmte Begleitumstände hinzutreten, kann auch die „physische Präsenz“ in eine taugliche Nötigungshandlung „umschlagen“..1516

1513 Siehe hierzu nur BVerfG 92, 1 (14ff.) - Sitzblockade I.

1514 BVerfGE 104, 92 (101 f.) - Sitzblockade III m.w.N.

1515 Zwar ergibt sich ein Unterschied durch die künstliche Erzeugung der Effekte, was jedenfalls das Eingreifen einer Schutzpflicht rechtfertigen kann. Das kann aber hier nicht maßgebliches Differenzierungskriterium für die Beurteilung der strafrechtlichen Relevanz bestimmter psychologischer Wirkungen sein. Unterschiede im Ergebnis ergeben sich dann erst durch die konkrete Art der Verwendung, die Inhalte und durch ähnliche Faktoren.

1516 Etwa dann, wenn es nicht mehr um die psychische Wirkungskraft geht, sondern die Anwesenheit zur Bereitung von Hindernissen genutzt wird und so Dritte, die halten müssen, als Werkzeug zur Errichtung eines Hindernisses benutzt werden (sog. Zweite-Reihe-Rechtsprechung, zurückgehend auf BGHSt 41, 182). Dieser Ansatz wurde vom BVerfG, NJW 2011, 3020 ff., gebilligt. 
bb. Schutz der politischen Willensbetätigung im Rahmen von Wahlen

Die $\mathbb{S} \int 108 \mathrm{ff}$. StGB sichern daneben die freie, wahlspezifische Betätigung des politischen Willens ab. Dabei schützt $\$ 108$ Abs. 1 StGB den Wahlberechtigten vor unzulässiger Einwirkung auf seine Entscheidungsfreiheit der Wahlrechtsausübung und weist Parallelen zum Nötigungstatbestand auf. Auch hier setzt der Tatbestand Gewalt oder vergleichbare Nötigungshandlungen voraus, sodass es auch hier schon regelmäßig an einer tauglichen Tathandlung fehlen wird. Und selbst wenn diese vorläge, wird dies wohl nur in Ausnahmefällen eine tatsächliche Wahlhinderung oder eine Nötigung zu einer spezifischen Wahlrechtsausübung zur Folge haben. Wenn überhaupt kommt also nur eine Versuchsstrafbarkeit nach Abs. 2 in Betracht.

\108a Abs. 1 StGB pönalisiert Täuschungen, die zu Irrtümern über den konkreten Inhalt der abgegebenen Wahlentscheidung führen bzw. die dazu führen, dass die Wahlentscheidung ungültig wird. Täuschungen bei der Willensbildung und dadurch bedingte Motivirrtümer werden gerade nicht erfasst. Sofern also Meinungsroboter nur politische Inhalte teilen und ihre Relevanz steigern - also höchstens Einfluss auf die vorgelagerte Willensbildung haben - und keine Desinformation über Modalitäten der Wahl verbreiten, ist $\mathbb{1 0 8 a}$ Abs. 1 nicht einschlägig. Und mangels „Stimmenkaufs“ scheidet auch die Wählerbestechung gem. $\$ 108$ b Abs. 1 StGB aus.

cc. Schutz der politischen Meinungsäußerung durch das Wahlrecht

Der strafrechtliche, wahlrechtsspezifische Schutz wird durch $\mathbb{} 32$ Abs. 1 BWahlG - und die entsprechenden landesrechtlichen Normen - ${ }^{1517}$ ergänzt. Verboten ist danach jegliche Beeinflussung während der Wahlzeit im und am Wahlgebäude. Eine Nötigung muss also gerade nicht vorliegen, vielmehr reicht die abstrakte Gefährlichkeit entsprechender Handlungen aus.

Freilich können die Bots bis zur Wahlhandlung in sozialen Netzwerken und damit innerhalb der maßgeblichen Wahlzeit ( $\$ 47$ Abs. 1 BWO) aktiv Propaganda betreiben. Diese müsste aber auch in bzw. am Wahlgebäude vorgenommen werden. Folgt man dem Wortlaut, wäre dies nicht per se abzulehnen. Durch tragbare elektronische Endgeräte kann die Wirkung auch noch bis zum Wahlakt selbst und damit innerhalb des von $\mathbb{3} 32$ Abs. 1 BWahlG geschützten Bereichs

1517 Siehe hierzu $\$ 35$ Abs. 1 LWG BaWü; Art. 20 Abs. 1 GLKrWG bzw. Art. 12 Abs. 1 LWG Bay; $\mathbb{2 8}$

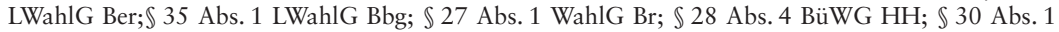

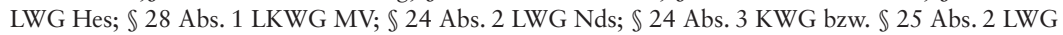
NRW; $\mathbb{} 18$ Abs. 1 LWG Rhpf; $\mathbb{2} 29$ Abs. 1 LWG Sl; $\mathbb{3} 30$ Abs. 1 LWG S; $\mathbb{3} 30$ Abs. 1 LWG SA; $\mathbb{3} 38$ Abs. 2 WahlG SH; $\mathbb{} 33$ Abs. 1 LWG Th. 
Wirkung entfalten. ${ }^{1518}$ Allerdings kann dies deshalb anders zu bewerten sein, weil man freiwillig auf sozialen Netzwerken aktiv und dort von Einflussnahmen unterschiedlichster Art betroffen ist und nicht wie in den klassischen Fällen ${ }^{1519}$ durch Dritte derart beeinträchtigt wird, dass man sich dem nur durch das Fernbleiben von der Wahl entziehen kann. $\$ 32$ Abs. 1 BWahlG dient gerade der spezifischen Gefahrenlage, die dadurch entsteht, dass der Wähler darauf angewiesen ist, am Wahltag das Wahllokal aufzusuchen. ${ }^{1520}$ Weitestgehend wird dieses subjektiv-rechtliche Verständnis durch Wahlprüfungssachen ${ }^{1521}$ und ministeriale Hinweise $^{1522}$ ebenso wie durch die Gesetzgebungsmaterialien ${ }^{1523}$ bestätigt. Soweit in der Judikatur (auch) auf die Integrität der Wahl als objektiv-rechtlicher Schutzzweck abgestellt wird, ${ }^{1524}$ steht das dem hier aufgezeigten Verständnis der Norm nicht im Wege. Denn - wie schon der Zusammenhang von Mikro und MakroEbene zeigt - wird die Integrität des Wahlvorgangs vor allem durch die Gewährleistung der Freiheit der Wähler gesichert. Entscheidet sich also der Wähler selbst dazu, politische Inhalte wahrzunehmen, sei es in sozialen Netzwerken oder sei es dadurch, dass er noch kurz vor Stimmenabgabe die Fragen des Wahl-O-Mat beantwortet, so besteht zwar ein potentieller Einfluss auf die Stimmabgabe. Dieser ist aber nicht von externen Faktoren abhängig. Deshalb ist nach der hier vertretenen Ansicht $\mathbb{3} 2$ Abs. 1, 1. Alt BWahlG - und mit ihm die landesrechtlichen Pendants - teleologisch zu reduzieren und damit vorliegend nicht einschlägig.

\section{b. Spezifischer verfassungsrechtlicher Schutz der Wabläußerung vor Einflussnahme}

Abseits einfach-rechtlicher Vorschriften bestehen auch verfassungsrechtliche Schutzmechanismen, insbesondere mit Blick auf die Äußerung im Rahmen der Wahl und dem hiermit einhergehenden Kommunikationsprozess. Konkret wird es hierbei um die für eine Wahlprüfung relevanten Fälle eines Wahlfehlers gehen, unabhängig davon, ob diese bei der Wahlvorbereitung, der Wahlhandlung oder

1518 So sieht Hablen, in: Schreiber, BWahlG, $\mathbb{S} 32$ Rn. 3, bspw. auch Propaganda, die mittels SMS- oder Twitter-Nachrichten verbreitet wird, als vom Verbot des $\$ 32$ Abs. 1 BWahlG erfasst an.

1519 Siehe hierzu u.a. VGH Kassel NVwZ 1991, 702 ff.; OVG Lüneburg, NVwZ 1994, 589.

1520 Vgl. VGH Kassel NVwZ 1991, 702 f., dass maßgeblich auf die Beeinflussung des Wählers abstellt; deutlicher VGH Kassel, NVwZ-RR 1993, 116 (119). Das OVG Lüneburg, NVwZ 1994, 589 (590), weist zumindest auch auf den Wahlgang des Bürgers hin.

1521 BT-Drs. 14/1560, Anlage 84, S. 227 wo es heißt, dass „[d]as Verbot der Wählerbeeinflussung in $\$ 32$ Abs. 1 BWahlG (...) der Gewährleistung der freien Ausübung der Wahl (...) “ dient.

1522 In der offiziellen Mitteilung des Innenministeriums des Landes Mecklenburg-Vorpommerns, Vorbereitung und Durchführung der Bundestagswahl am 27. September 2009, Az. II 210-115.2.2-1, Rn. 12.1 und ebenso in der Anweisung, Vorbereitung und Durchführung der Euopaparlaments- und Kommunalwahlen am 7. Juni 2009, Az. II 210-115.4.2-2, Rn. 12.1 heißt es: „Entscheidend ist, dass allen Wahlberechtigten ihr politisches Grundrecht zu wählen ungehindert gewährleistet [wird].“

1523 BT-Drs. 10/2834, S. 25.

1524 In diese Richtung etwa BVerwG NVwZ 1991, 681 (682). Auch das OVG Lüneburg, NVwZ 1994, 589 (590) weist hierauf zusätzlich hin. 
bei Feststellung des Ergebnisses erfolgen. ${ }^{1525}$ Wahlfehler kommen vorliegend insbesondere dann in Betracht, wenn staatliche Akteure, insbesondere Regierungsmitglieder, in den Kommunikationsprozess eingreifen (aa.), aber auch wenn Meinungsroboter durch private Akteure (bb.) oder durch Parteien (cc.) eingesetzt werden.

\section{aa. Einsatz durch staatliche Akteure:}

Staatliche Akteure haben eine besondere Zurückhaltung im politischen Kommunikationsprozess zu wahren. Sie trifft eine strikte Neutralitätspflicht, ${ }^{1526}$ die nicht nur der Chancengleichheit im politischen Wettbewerb (Art. 21 Abs. 1 i.V.m. Art. 38 Abs. 1. S. 1 GG) dient ${ }^{1527}$ und aus der Freiheit der Wahl und des Meinungsbildungsprozess selbst (Art. 28 Abs. 1 S. 2 GG bzw. Art. 38 Abs. 1 S. 1 GG) folgt, ${ }^{1528}$ sondern Ausdruck des Demokratieprinzip selbst ist. ${ }^{1529}$ Einseitige Parteinahmen und zielgerichtete Eingriffe in den politischen Wettbewerb sind hiernach unzulässig. ${ }^{1530}$ Wegen der Zurechnung der automatisierten Kommunikate gilt diese Neutralitätspflicht folglich auch für den Einsatz von Meinungsrobotern.

Nicht nur wegen einer grundsätzlich zulässigen Öffentlichkeitsarbeit stellen sich in der Praxis schwierige Abgrenzungsfragen. ${ }^{1531}$ Weil staatliche Amtsträger nicht nur als solche auftreten können, sondern zugleich auch Parteipolitiker und Privatpersonen sind - als solche aber nicht der Neutralitätspflicht unterliegen 1532 ergeben sich auch deshalb Abgrenzungsprobleme. Nach der Rechtsprechung des BVerfG ist von einem staatlichen Handeln auszugehen, wenn ein Rückgriff

1525 Brocker, in: Epping/Hillgruber, BeckOK GG, Art. 41 Rn. 3; Klein, in: Maunz/Dürig, GG, Art. 41 Rn. 103.

1526 Grundlegend BVerfGE 44, 125 (138ff.) - Öffentlichkeitsarbeit. Ob aus der Rspr. des BVerfG eine allgemeine Neutralitätspflicht für staatliche Akteure im gesamten Meinungskampf gefolgert werden kann, bezweifelt Payandeh, Staat 55, 519 (525 ff.) jedenfalls stark. Hierfür spricht jedenfalls auch BVerfGE 136, 323 - Äußerungsbefugnis d. Bundespräsidenten, da hier für den Bundespräsidenten ein dogmatisch eigenständiger Ansatz entwickelt wurde. Wegen der Intergrationsfunktion und der Parteiferne des Bundespräsidenten, habe er einen Ermessensspielraum hinsichtlich seiner Äußerungen und Wortwahl. Eine Grenze ist erst bei einer willkürlichen Parteiergreifung erreicht. Insbesondere für Regierungsmitglieder gilt indes ein strikter Maßstab, hierzu neben BVerfGE 138, 102 Rn. 31 ff. - Schwesig; 148, 11 Rn. 44 ff. - Chancengleichheit politischer Parteien; und zuvor schon die einstweilige Anordnung in BVerfGE 140, 225 ff. - Rote Karte und jüngst etwa BVerfG NJW 2020, 2096 ff. - Seehofer.

1527 Statt vieler siehe nur die jüngste Entscheidung, BVerfG, NJW 2020, 2096 Rn. 43 ff. - Seehofer, m.w.N.

1528 Vgl. BVerfGE 103, 111 (130) - Wahlprüfung Hessen; NJW 2020, 2096 Rn. 44 - Seehofer, m.w.N. Aus der verwaltungsgerichtlichen Judikatur etwa BVerwG, NVwZ 2003, 983 (985).

1529 Grundlegend BVerfGE 44, 125 (138ff.) - Öffentlichkeitsarbeit.

1530 Statt vieler BVerfG, NJW 2020, 2096 Rn. 51 - Seehofer, m.w.N.

1531 Insoweit geht es um die Abgrenzung von zulässiger Informations- und Öffentlichkeitsarbeit zur unzulässigen Einflussnahme, vgl. BVerfG NJW 2020, 2096 Rn. 49 ff. - Seehofer. Hierbei kommt insbesondere dem Gebot der Sachlichkeit Bedeutung zu, BVerfG, a.a.O. Rn. 52 m.w.N.

1532 BVerfG, NJW 2020, 2096 Rn. 54 f. - Seehofer m.w.N. 
auf die mit dem Regierungsamt eröffneten Mittel und Möglichkeiten erfolgt, ${ }^{1533}$ was dann der Fall ist, wenn die Autorität des Amtes oder die damit verbundenen Ressourcen in spezifischer Weise in Anspruch genommen werden. ${ }^{1534}$ Auch wenn unklar sein mag, ab wann diese Amtsautorität in sozialen Netzwerken Verwendung findet, ${ }^{1535}$ hat das für den Einsatz von Meinungsrobotern keinerlei Bedeutung. Steinbach weist zutreffend darauf hin, dass das bisherige Kriterium der Wahrnehmung amtsspezifischer Autorität hier gerade nicht mehr anwendbar ist, weil diese wegen der Identitätsverschleierung nicht nach außen dringt. ${ }^{1536} \mathrm{Maß-}$ gebliches Kriterium wäre also nur die Inanspruchnahme staatlicher, amtsbezogener Ressourcen beim Betrieb oder bei der Unterhaltung entsprechender Profile oder des Botprogramms selbst. Dafür müsste dann die technische Infrastruktur des Staates - etwa Server- oder Rechenkapazitäten - genutzt oder an Mitarbeiter Aufgaben der Überwachung und Betreuung delegiert werden. ${ }^{1537}$ Nicht ausreichend wäre es, wenn die Bots nur auf den offiziellen Webseiten Inhalte veröffentlichen, beispielsweise bei Facebook auf der Seite der Ministerien, ohne aber sonstige amtsspezifische Ressourcen zu verwenden. ${ }^{1538}$ Insoweit bestünde gerade kein Unterschied zu anderen Bürgern, die Inhalte veröffentlichen, oder anderen Akteuren, die Meinungsroboter verwenden. Insgesamt dürfte daher der Einsatz durch staatliche Akteure nicht die Neutralitätspflicht aktivieren.

\section{bb. Einsatz durch private Akteure}

Wahrscheinlicher ist es ohnehin, dass Beeinflussungsversuche von privaten Dritten ausgehen. Aber auch diese können die Integrität der Willensbildung selbst beeinträchtigen. Allen voran der Grundsatz der Wahlfreiheit wird bei Beeinflussungsversuchen durch Private relevant. ${ }^{1539}$ Er gewährleistet, dass der Wähler sein Wahlrecht ohne Zwang oder sonstige unzulässige Beeinflussung ausüben kann, ${ }^{1540}$ um so seine Entscheidung ,in einem freien, offenen Proze[ss] der Meinungsbildung“ treffen zu können ${ }^{1541}$. Dabei ist allerdings zu berücksichtigen, dass keine strenge Neutralitätspflicht eingreift, sondern im Gegenteil auch

1533 BVerfG, NJW 2020, 2096 Rn. 56 m.w.N.

1534 BVerfG, ebd. m.w.N. Kritisch zur Rspr. Madjarov, GreifRecht 2015, 59 (61 f.).

1535 Harding, NJW 2019, 1910 (1914), nimmt dies dann an, wenn im Impressum ein Hinweis auf die amtliche Tätigkeit gegeben wird.

1536 Steinbach, ZRP 2017, 101 (104).

1537 Vgl. hierzu auch v. Ungern-Sternberg, in: Unger/dies., Demokratie und künstliche Intelligenz, S. 5 (15f.).

1538 Anders liegt der Fall, wenn auf der Internetseite des Ministeriums eine Pressemitteilung veröffentlicht wird, vgl. hierzu BVerfG, NJW 2018, 928 (Rn. 67 ff.) - Wanka.

1539 So etwa bei beeinflussenden Äußerungen von Arbeitgebern und Gewerkschaften, BVerfGE 66, 369.

1540 BVerfGE 7, 63 (69) - Listenwahl; 15, 165 (166) - Briefwabl; 47, 253 (282) - Bezirksvertretung; 66, 369 (380) - Wablbeeinflussung durch Unternehmer; 124, 1 (24) - Nachwahl Dresden.

1541 BVerfGE 44, 125 (139) - Öffentlichkeitsarbeit der Bundesregierung; 66, 369 (380) - Wahlbeeinflussung durch Unternehmer; 124, 1 (24) - Nachwabl Dresden. 
auf Seiten der Einflussnehmenden Grundrechte zu berücksichtigen sind. ${ }^{1542}$ Das macht die Bestimmung verfassungs- bzw. wahlrechtlicher Grenzen der Zulässigkeit von Äußerungen schwierig; grundsätzlich ist hier - wie auch allgemein bei Äußerungen - von einem Vorrang der freien Rede auszugehen. ${ }^{1543}$ Nach der verfassungsrechtlichen Judikatur sind Äußerungen nur dann unzulässig und als Wahlfehler einzuordnen,

„[...] wenn private Dritte, einschließlich Parteien [...], mit Mitteln des Zwangs oder Drucks die Wablentscheidung beeinflusst haben oder wenn in ähnlich schwerwiegender Art und Weise auf die Wählerwillensbildung eingewirkt worden ist, ohne dass eine hinreichende Möglichkeit zur Abwehr - zum Beispiel mit Hilfe der Gerichte oder der Polizei oder des Ausgleichs, etwa mit den Mitteln des Wahlwettbewerbs, bestanden hätte. "1544

Jedenfalls die erste Fallgruppe der Nötigung scheidet im Kontext der Meinungsroboter aus. Denn hierfür orientiert sich das Gericht am Tatbestand des $\mathbb{} 108$ StGB. ${ }^{1545}$ Insoweit kommt allein die zweite Fallgruppe einer ähnlich schwerwiegenden Einwirkung in Betracht. Auch wenn Volkmann die Formel zu Recht als unpraktikabel bezeichnet, ${ }^{1546}$ so kann ein Wahlfehler nur angenommen werden, wenn eine gewisse Vergleichbarkeit zu den Verbotstatbeständen bestünde. ${ }^{1547}$

\section{(I.) Vergleichbarkeit der Charakteristika der Meinungsroboter}

Diese Vergleichbarkeit nimmt von Ungern-Sternberg im Falle der Meinungsroboter wegen der gezielten und massenhaften Täuschung von Nutzern an. ${ }^{1548} \mathrm{Maß}$ geblich seien hierfür vor allem die Identitätstäuschungen. Sie können weder durchschaut werden, noch lässt sich Abhilfe etwa durch Löschungsansprüche oder die Richtigstellung der Täuschungen durch Dritte schaffen. Und selbst wenn nachträglich gelöscht würde, war der Einsatz dennoch lange Zeit möglich. ${ }^{1549}$ So-

1542 Vgl. Steinbach, ZRP 2017, 101 (104); Klein, in: Maunz/Dürig, GG; Art. 41 Rn. 120. Das Sächsische OVG, Urt. v. 15.01.2013 - 4 A 462/12, Rn. 25 - juris weist allgemein daraufhin, „[d]ass private Dritte für die Wahl von Parteien und Kandidaten werben und zu ihren Gunsten beeinflussen wollen, (...) Bestandteil der in einer Demokratie geführten Wahlkämpfe [ist].“.

1543 Nach dem Sächsischen OVG, Urt. v. 15.01.2013 - 4 A 462/12, Rn. 25, seien „Einwirkungen privater Dritter auf den Wähler grundsätzlich hinzunehmen“ und nach dem BVerwG NVwZ 2003, 983 (985) ist auch die „Verbreitung von Täuschungen und Lügen, (...) nicht zu beanstanden, [auch] wenn sie sittlich zu missbilligen sind. “ ̈̈hnlich auch BVerwG, Urt. v. - 8 B 24/12, Rn. 12 - juris; OVG Lüneburg, Urt. v. 29.01.2009 - 10 LA 316/08, Rn. 11 - juris; VG Stade, Urt. v. 20.03.2013 1 A 1517/11, Rn. 65- juris. Vgl. auch BVerfGE 103, 111 (128) - Wahlprüfung Hessen.

1544 BVerfGE 103, 111 (132f.) - Wahlprüfung Hessen; 122, 304 (315) - negatives Stimmengewicht; 124, 1 (20f.) - Nachwahl Dresden; zustimmend LVerfG Bbg, Beschl. v. 18.09.2015 - VfGBbg 18/15, Rn. 24 - juris.

1545 Siehe hierzu nur BVerfGE 66, 369 (380) - Wablbeeinflussung durch Unternehmer; 103, 111 (132) Wahlprüfung Hessen. Zur fehlenden Tatbestandsmäßigkeit siehe bereits zuvor, C.I.4.a.bb.

1546 Volkmann, in: Unger/v. Ungern-Sternberg, Demokratie und künstliche Intelligenz, S. 51 (65 f.) kritisiert diese Formel, weil sich hierunter nicht sinnvoll subsumieren lasse.

1547 So $v$. Ungern-Sternberg, in: Unger/dies., Demokratie und künstliche Intelligenz, S. 3 (21).

1548 v. Ungern-Sternberg, in: Unger/dies., Demokratie und künstliche Intelligenz, S. 3 (21).

$1549 v$. Ungern-Sternberg, a.a.O., S. $21 \mathrm{f}$. 
weit es um die faktischen Aussagen geht, ist von Ungern-Sternberg ohne Weiteres zuzustimmen. Gerade die inhärenten Täuschungswirkungen (Identitätstäuschung und Täuschung über die quantitative Legitimität) sind als solche für die Nutzer nicht erkennbar und jedenfalls nach der bislang aufgezeigten Rechtslage kann ihnen deshalb auch nicht begegnet werden. ${ }^{1550}$ Vor allem auch die Täuschungsqualität unterscheidet sich deutlich von anderen netzwerk- oder internetimmanenten Täuschungen. ${ }^{1551}$ Gleichwohl können allein die Charakteristika der Bots nicht die Vergleichbarkeit zu den $\mathbb{S} \mathbb{S} 108,108$ a StGB begründen. $\mathbb{S} 108$ StGB pönalisiert immerhin die Nötigung als unmittelbare unterdrückende Einwirkung auf die Willensentschließung des Wählers. \108a StGB erfasst zwar Täuschungen, allerdings auch nur solche, die unmittelbar zu einem Irrtum über den Inhalt der Wahlerklärung führen oder zu einem Irrtum, infolgedessen der Wähler seinen Willen nicht (wirksam) kundgeben kann. Die Täuschungswirkungen führen aber gerade nicht zu einer unmittelbaren Einwirkung auf den Willen des Wählers dergestalt, dass er seine Wahlentscheidung nicht mehr frei treffen und wirksam kundgeben kann. Vielmehr werden dadurch nur die Glaubwürdigkeit und Reichweite der fiktiven Nutzer und ihrer Inhalte erhöht. Die Frage, ob und wie man die Äußerungen wahrnimmt, ist allein vom Rezipienten und den Umständen abhängig. ${ }^{1552}$ Die Entscheidung, die aus der einseitigen Informationsaufnahme oder der Orientierung an statistischen Daten zur Beurteilung der Glaubwürdigkeit und Relevanz folgt, ist damit nicht weniger frei als die Entscheidung ohne entsprechende Einflüsse. Sie ist lediglich „fehlerhaft“ zustande gekommen. Alle Manipulationen der Entscheidungsgründe sind für die strafrechtlichen Tatbestände irrelevant und stellen nur unerhebliche Motivirrtümer dar. ${ }^{1553}$ Auch in anderen von der Rechtsprechung entschiedenen Fällen wurde nicht an die Umstände, sondern an das konkrete Verhalten angeknüpft. ${ }^{1554}$ Und zudem wäre dann auch hier der positive Einsatz erfasst, wenn etwa Informationen transparent gemacht werden, die sonst keine Aufmerksamkeit erhalten hätten. ${ }^{155}$

Auch der digitale Zwang reicht hierfür nicht aus. Weder ist dieser nötigungsäquivalent ${ }^{1556}$ noch dauert die Zwangswirkung - anders als etwa im Falle des nicht strafbaren Einflusses von Unternehmern auf die Belegschaft - nicht bis zur eigentlichen Wahlhandlung fort; die psychische Wirkung beschränkt sich auf den

$1550 \mathrm{Zu}$ den hierfür relevanten Kennzeichnungspflichten oben, C.I.3.c.

1551 Hierzu bereits oben 2. Teil, C.VI.1.

1552 Hierauf im Kontext von Desinformation hinweisend Schimmele, Staatliche Verantwortung für diskursive Integrität, S. 194.

1553 Vgl. Kargl, in: Kindhäuser/Neumann/Paeffgen, StGB, $\mathbb{1 0 8 a}$ Rn. 2.

1554 Siehe etwa VGH B-W 1 S 581/19, Rn. 30 f. - juris, zu falschen personenbezogenen Tatsachen, die i.E. aber nicht als unzulässig bewertet wurden; vgl. zur Verletzung des amtlichen Wahrheitsgebots BVerwG NVwZ 2003, 983 (985).

1555 Insoweit greifen hier also auch die Bedenken, die schon gegen die Annahme einer Schutzbereichsausnahme von Art. 5 Abs. 1 S. 1 GG vorgetragen wurden. Hierzu oben, A.I.3.

1556 Dazu bereits oben, C.I.4.a.aa. 
digitalen Diskurs und die Wahrnehmung der Meinungsäußerungsmöglichkeit. Der eigentliche Entscheidungsprozess bleibt unberührt und die Entscheidung selbst wird nicht in einem vergleichbaren Sinne erzwungen.

\section{(II.) Kumulation von Äußerungsinhalten und Verbreitungsform bei Desinformation?}

Etwas anderes könnte sich nur dann ergeben, wenn man zusätzlich noch die konkreten Inhalte berücksichtigt. Besondere Bedeutung erlangt das bei Desinformationskampagnen. Ausgangspunkt ist hierbei die Überlegung, dass die Freiheit der Wahl auch das Recht verbürgt, „sich (...) über Ziele und Verhalten der Wahlbewerber frei von Manipulationen [zu] informieren "1557 und seine Entscheidung „in einem freien, offenen Proze[ss] der Meinungsbildung “ treffen zu können ${ }^{1558}$. Das OVG Münster - wie auch zuvor das BVerwG ${ }^{1559}$ - kam deshalb zu dem Entschluss, dass auch Des- oder Fehlinformation eine Beeinflussung der Entscheidungsfreiheit darstellen kann, ${ }^{1560}$ denn „,[die] Wahrheit ist auch im Wahlkampf als Rahmenbedingung sozialer Kommunikation unentbehrlich."1561 Allerdings ging es hierbei um die Vorenthaltung von richtigen, wahlrechtsrelevanten Information durch Amtsträger. ${ }^{1562}$ In derlei Fällen besteht nämlich keine Möglichkeit, dass die Vorenthaltung auf anderem Wege abgewehrt werden kann. ${ }^{1563}$

Geht es aber um private Fehlinformation, ist der angelegte Maßstab deutlich liberaler. ${ }^{1564}$ Ganz ausdrücklich formulierte es das VG Freiburg, dass eine unzulässige Beeinflussung gänzlich ablehnt, „wenn der Wähler die Unrichtigkeit oder Fragwürdigkeit einer Behauptung ohne allzu große Anstrengung erkennen konnte“1565. Auch das BVerfG wies daraufhin, dass man schon früher bei privaten „Täuschungen und Lügen“ keinen Grund sah, diese zu beanstanden, selbst wenn

1557 BVerwG, NVwZ 2003, 983 (984 f.); OVG Münster, Urt. v. 15.12.2011 - 15 A 876/11, Rn. 69; ähnlich VG Freiburg, Urt. v. 22.03.2006 - 1 K 1844/05 -, Rn. 67.

1558 BVerfGE 44, 125 (139) - Öffentlichkeitsarbeit der Bundesregierung; 66, 369 (380) - Wablbeeinflussung durch Unternehmer; 124, 1 (24) - Nachwabl Dresden.

1559 BVerwG, NVwZ 2003, 983 (985).

1560 OVG Münster, Urt. v. 15.12.2011 - 15 A 876/11, Rn. 69.

1561 BVerwG, NVwZ 2003, 983 (985). Hain, K\&R 2014, 492 (493) betont: „Voraussetzungen für das Gelingen individueller Selbstbestimmung bilden nun nicht zuletzt die Wahrheit und Repräsentativität der dem Individuum (massen-)kommunikativ übermittelten Daten.".

1562 In OVG Münster, Urt. v. 15.12.2011 - 15 A 876/11, hatten die beklagten ehemaligen Oberbürgermeister nicht wahrheitsgemäße Aussagen zu der aktuellen Haushaltslage getätigt. Auch bei BVerwG NVwZ 2003, 983 ging es um die Vorenthaltung von amtlichen Informationen. Vgl. hierzu auch VG Neustadt (Weinstraße), Urt. v. 25.03.2013 - 3 K 857/12.NW - juris. Ausführlicher zu staatlicher Fehl- und Desinformation etwa Ingold, in: Oppelland, Propaganda als (neue) außen und sicherheitspolitische Herausforderung, S. 81 (93 ff.).

1563 BVerwG, NVwZ 2003, 983 (985); OVG Münster, Urt. v. 15.12.2011 - 15 A 876/11, Rn. 69; VG Neustadt (Weinstraße), Urt. v. 25.03.2013 - 3 K 857/12.NW Rn. 44 - juris.

1564 Wegen dieses Maßstabes wird auch eine Beeinträchtigung der Wahlfreiheit durch Meinungsroboter abgelehnt, so etwa Steinbach, ZRP 2017, 101 (104); Milker, ZUM 2017, 216 (220f.).

1565 VG Freiburg, Urt. v. 22.03.2006 - 1 K 1844/05 -, Rn. 67, juris. 
sie sittlich zu missbilligen waren. ${ }^{1566}$ Und selbst in der einschlägigen Literatur wird überwiegend unter dem Stichwort der Medienkompetenz an die Vernunft der Rezipienten appelliert. ${ }^{1567}$ Grundsätzlich ist dagegen nichts einzuwenden, denn private Fehlinformationen oder Täuschungen führen primär auch nur zu Motivirrtümern und wirken sich nicht unmittelbar auf die Wahlentscheidung selbst aus. Der eigentliche geistige Entscheidungsprozess bleibt vor unmittelbarer Einwirkung ebenso wie die finale Entscheidung unberührt. Gleichwohl ist schon der Judikatur zu amtlicher Wahlwerbung die Idee immanent, dass bestimmte Informationen, ihre Vorenthaltung oder eine Täuschung über sie relevanter ist als in anderen Fällen. Bestimmte Informationen sind nämlich schon per se dazu geeignet, den Wählerwillen positiv oder negativ zu beeinflussen und schlagen insoweit auf die Wahlentscheidung durch. Auch wenn die Vorenthaltung von Informationen im Ausgangspunkt deshalb bereits anders zu bewerten ist als die Vermittlung falscher Informationen, weil im letzteren Fall eine Aufdeckung und Abwehr durch Medien, andere Informationsquellen und den Meinungskampf möglich ist, ${ }^{1568}$ ergeben sich dennoch neue Herausforderungen insbesondere dort, wo die Informationsaufnahme in sozialen Netzwerken erfolgt. Filterblasen-Phänomene, einseitige Informationsaufnahme und die durch kognitive Verzerrungen gewonnene Aufmerksamkeit führen ohnehin schon zu einem starken Erfolg von Desinformation. ${ }^{1569}$ Meinungsroboter können dies durch zusätzliche Manipulationen erheblich verstärken. Besonders dann, wenn die konkreten Inhalte eine besondere wahlkampfpolitische Bedeutung haben - so wie etwa 2015 während der Flüchtlingsdebatte oder aktuell während der Covid-19-Pandemie - können sie in ihrer Gesamtheit dieselbe faktische Bedeutung für die finale Wahlentscheidung haben wie vorenthaltene, amtliche Informationen im Wahlkampf. Wegen der dezentralen Kommunikationsstruktur ist zudem nicht sichergestellt, dass eine Löschung generell ${ }^{1570}$ oder zumindest eine Richtigstellung einer Äußerung so erfolgt, dass diese auch die betroffenen Nutzer erreicht. ${ }^{1571}$ Der schon von Schimmele ${ }^{1572}$ herausgearbeitete Unterschied besteht hier aber darin, dass es nicht um „externe Eingriffe“ in die Wahlfreiheit geht, sondern vor allem interne Ver-

1566 BVerfGE 103, 111 (128) - Wahlprüfung Hessen. In Bezug auf die Meinungsroboter so ausdrücklich Milker, ZUM 2017, 216 (220).

1567 So etwa Milker, ZUM 2017, 216 (220).

1568 So letztlich auch Schimmele, Staatliche Verantwortung für diskursive Integrität, S. 194; Milker, ZUM 2017, 216 (219).

1569 Zur Verbreitung von Fake News in sozialen Netzwerken siehe Vosoughi/Roy/Aral, Science 359, 1146.

1570 Das NetzDG sieht in $\ 3$ Abs. 1 ein obligatorisches Beschwerdemanagment für rechtswidrige Inhalte vor. Die Betreiber von sozialen Netzwerken ( $\mathbb{1}$ Abs. 1 S. 1 ) haben sicher zu stellen, dass es ein solches Verfahren gibt und dass etwaige Beschwerden über rechtswidrige Inhalte unverzüglich überprüft werden. Sind sie begründet, so hat der Betreiber den Inhalt unverzüglich zu löschen oder den Zugang zu ihm zu sperren ( $\$ 3$ Abs. 2 Nr. 3 ).

1571 Auch hierzu Vosoughi/Roy/Aral, Science 359, 1146.

1572 Vgl. Schimmele, Staatliche Verantwortung für diskursive Integrität, S. 194. 
zerrungen der Nutzer relevant werden, die immer schon bestanden. ${ }^{1573}$ Allein die Qualität ist in sozialen Netzwerken eine andere, was auch daran liegt, dass keine professionellen Filter bestehen und es insoweit auf die bislang nur rudimentär bis schlecht ausgeprägte Medienkompetenz der Nutzer ankommt. ${ }^{1574}$ Dass daher gewisse Spannungen mit den Kommunikationsgrundsätzen bestehen und daraus ein gewisser Schutzbedarf der Nutzer erwächst, bedeutet aber nicht, dass damit auch eine ähnlich schwerwiegende Einwirkung angenommen werden kann. Denn die Wahlfehler dienen - anders als die Schutzpflichten - nicht dem Schutz subjektiver Rechte, sondern dem Schutz der Integrität des demokratischen Wahlprozesses. ${ }^{1575}$ Dieser Prozess muss aber an dem Leitbild eines vernünftigen, wohlinformierten Bürgers ausgerichtet sein, um nicht selbst in Widerspruch mit dem Demokratieprinzip zu geraten. ${ }^{1576}$ Insoweit ist gedanklich zwischen dem durch bestimmte Gefahren begründete Schutzbedarf des Einzelnen bzw. des Kommunikationsprozesses als Voraussetzung der Wahlentscheidung und dem Schutz der demokratisch-kollektiven Wahlentscheidung selbst zu differenzieren.

Das bedeutet freilich nicht, dass (durch Meinungsroboter) verbreitete Desinformation nicht auch die Qualität eines Wahlfehlers erreichen kann. Während sich bei der Ausübung unzulässigen Drucks ohne Weiteres auf eine gewisse Beeinflussungseignung schließen lässt, ist das bei einem bestimmten desinformativen Inhalt, dessen Reichweite mithilfe von Meinungsrobotern erhöht wird, nicht der Fall. Hier bedarf es vielmehr besonderer Anforderungen in quantitativer und qualitativer Hinsicht. ${ }^{1577}$ Gerade der offene und plurale Kommunikationsprozess muss dann aber insgesamt beeinträchtigt sein, was etwa dann anzunehmen sein könnte, wenn die Informationsvermittlung primär durch soziale Netzwerke erfolgt, massive Manipulationen durch (von Meinungsrobotern) verbreitete desin-

1573 Denn die in $\mathbb{} 108$ StGB normierten Drucksituationen gefährden die Wahrnehmung des zuvor frei gebildeten Willens, während die Verzerrungstendenzen lediglich beeinflussen, welche Inhalte wahrgenommen werden, ohne dabei aber per se das Ergebnis zu prädeterminieren.

1574 Auf die veränderte Rolle der Rezipienten in sozialen Netzwerken und die Schwierigkeiten weist auch das BVerfG, E 149, 222 Rn. 80 - Rundfunkgebühr, hin.

1575 So jedenfalls zum Sinn der Wahlprüfung und der Überprüfung auf Wahlfehler BVerfGE 66, 369 (378) - Wablbeeinflussung durch Unternehmer m.w.N. Vgl. zur fehlenden Kongruenz von Wahlfreiheitschutz durch die Wahlanfechtung und der freiheitssichernden Funktion der Wahlfreiheit als solcher BVerwG, NVwZ 2003, 983 (985).

1576 In diese Richtung etwa auch Milker, ZUM 2017, 219 (220), wenn er die Medienkompetenz der Bürger betont. Das BVerfG spricht hier von der „Idee der freien Selbstbestimmung aller [Kursiv. d. Aut.]“, BVerfGE 44, 125 (142) - Öffentlichkeitsarbeit der Bundesregierung. Zu diesem Leitbild vgl. auch Di Fabio, in: Görres-Gesellschaft, Staatslexikon, Demokratie I. 3.

1577 Es ist durchaus denkbar, dass $v$. Ungern-Sternberg, in: Unger/dies., Demokratie und künstliche Intelligenz, S. 3 (21 f.) auch nur in solchen Fällen und nicht generell einen Wahlfehler annehmen will, wenn sie (einschränkend) schreibt, dass „sich besonders schwerwiegende Formen des Einsatzes [...] als unerlaubte Wahlhandlung einordnen [lassen]. "Vgl. hierzu in Ansätzen Klaas, MMR 2019, 84 (89), der eine solche Qualitätsschwelle fordert, um von einer Beeinträchtigung u.a. der Chancengleichheit ausgehen zu können. Hohe Anforderungen stellen daneben auch Schimmele, Staatliche Verantwortung für diskursive Integrität, S. 194 und Milker, ZUM 2017, 216 (220). Sie verläuft bei beiden dort, wo die Kommunikation unmöglich gemacht wird. 
formative Inhalte erfolgen und diese Darstellungen von herkömmlichen Medien nicht mehr, nur unzureichend oder ineffektiv korrigiert werden können. Bislang ist das jedenfalls noch nicht der Fall.

\section{cc. Besonderheiten beim Einsatz durch Parteien}

Gilt Selbiges auch für den Einsatz durch Parteien? Zwar ist das Wesen politischer Parteien von einer Zwitterstellung geprägt: Einerseits sind sie staatsnaher Akteur, ${ }^{1578}$ andererseits private Vereinigung. Im Kontext der Wählerbeeinflussung prävaliert aber der private Charakter; Parteien unterliegen - was ohnehin dem Parteienwettbewerb zuwiderlaufen würde - keiner strikten Neutralitätspflicht. Insoweit gelten eigentlich dieselben Voraussetzungen wie für private Akteure. ${ }^{1579}$ Gleichwohl ergeben sich hier spezifische Fragen, wenn Social Bots benutzt werden, um Wähler zu beeinflussen.

\section{(I.) Bedeutung und Funktion der Parteien}

Bedenken resultieren hierbei vor allem aus der verfassungsrechtlichen Stellung der Parteien: Sie sind Kernbestandteil einer Demokratie. ${ }^{1580}$ Art. 21 Abs. 1 S. 1 GG weist den Parteien deshalb gerade einen verfassungsrechtlichen Auftrag ${ }^{1581}$ zur Willensbildung des Volkes in politischen Angelegenheiten zu. ${ }^{1582}$ Auch wenn Absatz 1 S. 1 klarstellt, dass ihnen keine Monopolstellung zukommt („mitwirken“), ${ }^{1583}$ so sind doch die Parteien maßgeblicher Mittler zwischen Staat und Bürger. ${ }^{1584}$ Durch sie kann der Bürger Einfluss auf die Entscheidungen oberster

1578 Das BVerfG spricht hier davon, dass sie „in den Bereich der institutionalisierten Staatlichkeit hineinwirken, ohne diesem selbst anzugehören (...).“, NJW 2018, 928 Rn. 41 - Wanka, m.w.N.

1579 Auch für Parteien dürfte hinsichtlich der Wahlrechtsfreiheit nichts anderes gelten als für Private. Selbst (bewusst) falsche Wahlversprechen dürfen abgegeben werden, weil sie ein legales, wenn auch sittlich zu missbilligendes Instrument sind, vgl. BVerfGE 103, 111 (128) - Wahlprüfung Hessen.

1580 Grimm, in: Benda/Maihofer/Vogel, Hb VerfR, $\mathbb{S} 14$ Rn. 2, spricht davon, dass „der Schritt zur Massendemokratie [...] Parteien unentbehrlich macht“. Im Kern ebenso Schmitz, Grundrechtskollisionen, 1995, S. 31 f. Siehe auch BVerfGE 85, 264 (284) - Parteifinanzierung II; BVerfGE 138, 102 (109) - Äußerungen von Regierungsmitgliedern; BVerfG NJW 2018, 928 (929) - Wanka. Instruktiv zur Bedeutung der Parteien für die Demokratie auch aus kritischer Sicht Towfigh, Das Parteien-Paradox, S. $77 \mathrm{ff}$.

1581 Klein, in: Maunz/Dürig, GG, Art. 21 Rn. 150 bezeichnet es schlicht als verfassungsrechtliche Aufgabe. Damit haben die Parteien nicht nur ein verfassungsrechtlich gewährleistetes Recht, sondern dem korrespondiert auch eine Pflicht, der sie nachkommen müssen, hierzu Klein, a.a.O., $162 \mathrm{ff}$.

1582 Aber auch ohne eine derartige ausdrückliche Aufgabenzuweisung wären die Parteien notwendige „Folgeerscheinung der parlamentarisch organisierten Mitsprache der Gesellschaft an staatlichen Entscheidungen“, Grimm, in: Benda/Maihofer/Vogel, Hb VerfR, $\$ 14$ Rn. 3.

1583 BVerfG, E 85, 264 (284) - Parteienfinanzierung II.

1584 So ausdrücklich BVerfG NJW 2018, 928 Rn. 41 - Wanka. Siehe auch BVerfGE 85, 264 (284) Parteienfinanzierung II. 
Staatsorgane nehmen. ${ }^{1585}$ Die nicht handlungsfähige, heterogene Volksmasse wird durch die Parteien erst strukturiert und zusammengeführt. ${ }^{1586}$ Ohne die Parteien bestünde folglich keine Möglichkeit, wirksam auf das staatliche Geschehen Einfluss zu nehmen. ${ }^{1587}$ Diese wesensmäßige, demokratische Funktion erschöpft sich nicht allein in der Vorbereitung und Durchführung von Wahlen. Vielmehr ist die Willensbildung ein stetiger Prozess ${ }^{1588}$, muss also durch einen andauernden Austausch von Informationen zwischen Bürger und Staat gewährleistet werden (vgl. $\mathbb{1}$ Abs. 2 PartG). ${ }^{1589}$ So sorgen die Parteien dafür, dass wandelnde Ideen und Ansichten staatliche Entscheidungen auch unabhängig von Wahlen mit beeinflussen. Die Parteien existieren also nicht um ihrer selbst willen. Ihre Funktion leitet sich vielmehr aus der Notwendigkeit einer Strukturierung und Effektuierung des demokratischen Willensbildungsprozess ab. ${ }^{1590}$

\section{(II.) Verfassungsunmittelbare Grenzen des Einsatzes?}

Im Ausgangspunkt ist es freilich Sache der Parteien, wie sie ihren Mitwirkungsauftrag erfüllen und welche Medien sie hierfür verwenden. ${ }^{1591} \mathrm{Im}$ herkömmlichen Sinne geht es hierbei aber um offene Wahlwerbung innerhalb des politischen (Parteien-)Wettbewerbs und nicht um einen verdeckten manipulativen Einsatz in digitalen Diskursräumen. Deshalb wird gerade versucht aus der derivativen Funktion der Parteien spezifische verfassungsrechtliche Bedenken gegen den Einsatz von Meinungsrobotern zu artikulieren ${ }^{1592}$ und insoweit verfassungsunmittelbare, demokratiespezifische Grenzen aufzuzeigen. So meinen Gasser und Kraatz, dass „[d]er Einsatz [...] von Verfassungs wegen untersagt [sei]“, ${ }^{1593}$ weil die Parteien ihre Stellung als Mittler verlassen und in einer dem Demokratieprinzip widersprechenden Weise die Willensflussrichtung umkehren: Es werde nur noch suggeriert, dass diese von unten (Volk) nach oben (Staat) erfolge, denn

1585 BVerfGE 20, 56 (99) - Parteifinanzierung I; BVerfGE 44, 125 (140) - Öffentlichkeitsarbeit der Bundesregierung.

1586 So letztlich auch das BVerfG, NJW 2018, 928 Rn. 41 - Wanka, m.w.N. auf die verfassungsgerichtliche Rechtsprechung, siehe insbesondere auch BVerfGE 85, 264 (285) - Parteifinanzierung II. Daneben siehe etwa auch Grimm, in: Benda/Maihofer/Vogel, Hb VerfR, $\$ 14$ Rn. 13.

1587 BVerfG NJW 2018, 928 Rn. 41 - Wanka.

1588 So schon das BVerfG, E 20, 56 (98) - Parteienfinanzierung; vgl. auch BVerfGE 44, 125 (139f.) Öffentlichkeitsarbeit.

1589 Nach dem BVerfG vollziehen sich die „Willensbildung des Volkes und [die] Willensbildung in den Staatsorganen [...] in vielfältiger und tagtäglicher Wechselwirkung. Politisches Programm und Verhalten der Staatsorgane wirken unablässig auf die Willensbildung des Volkes ein und sind selbst Gegenstand der Meinungsbildung des Volkes“, E 44, 125 (139 f.).

1590 Vgl. BVerfGE 121, 30 (54) - Parteibeteiligung an Rundfunkunternehmen, m.w.N.

1591 BVerfGE 148, 11 Rn. 43 - Chancengleichheit politischer Parteien m.w.N.

1592 Anders indes Milker, ZUM 2017, 216 (220), der den Einsatz für ethisch zu missbilligen, aber grundsätzlich für (rechtlich) unbedenklich hält. In diese Richtung auch Steinbach, ZRP 2017, 101 (105).

1593 Gasser/Kraatz, VerfBlog, 2017/1/16. 
die verbreiteten Meinungen haben ihren Ausgangspunkt in der Parteizentrale. „Der Rückkopplungsprozess zwischen Partei und Bevölkerung und schließlich auch mit dem Staat wird dadurch zumindest marginalisiert.“ Klaas ${ }^{1594}$ meint, „der Handlungsspielraum politischer Parteien [sei] im Willensbildungsprozess durch den Sinn und Zweck, eine demokratische Grundverfassung zu fördern und zu gewährleisten, begrenzt. “ Daher seien sie gehindert, die Wähler durch die Täuschung über die gesellschaftliche Stärke zu beeinflussen, weil damit die informative Grundlage verfälscht werde, und sie dürften nicht den chancengleichen Wettbewerb aller am Kommunikationsprozess Beteiligter beeinträchtigen. Notwendig sei allerdings das Überschreiten einer gewissen "Qualitätsschwelle“, die anhand verschiedenster Parameter zu bestimmen sei. Daneben wird aber auch auf die - schon im Rundfunk - bestehenden demokratischen Transparenzinteressen hinsichtlich der Parteiaktivitäten hingewiesen. ${ }^{1595}$

Auch wenn das von Gasser und Kraatz vorgebrachte Argument der demokratischen Flussrichtung besonders prägnant ist, so führt dies zunächst nur zu der oben aufgezeigten Neutralitätspflicht staatlicher Akteure. Selbst hier sind - in gewissen Grenzen - Wechselbeziehungen durchaus legitim, etwa wenn es um amtliche Informationen und Öffentlichkeitsarbeit geht. ${ }^{1596}$ Diese Wechselbeziehung ist aber bei Parteien deutlich stärker ausgeprägt, weil sie nicht im herkömmlichen Sinne staatlich sind und weil ganz regelmäßig bestimmte politische Vorschläge ihren Ursprung in der „Parteizentrale“ haben. ${ }^{1597}$ Die Mitwirkung an der Willensbildung kann auch durch die Hingabe bestimmter Vorschläge oder Ansichten denkbar sein. ${ }^{1598}$ Entscheidend ist nur, dass ein kritischer Kontrollprozess durch das Volk selbst erfolgt und Inhalte dann übernommen werden, bevor sie Eingang in staatliche Entscheidungen finden. Der Einsatz von Meinungsrobotern widerspricht aber dieser Kontrolle, weil künstlich die Überzeugungskraft - zumindest im virtuellen Raum - in einem Maß gesteigert werden kann, wodurch die Aufnahme und Verarbeitung der Informationen erleichtert wird, ohne dass dabei wegen der Identitätstäuschung die wesentlichen Informationen über die Herkunft der Meinung mitgeteilt werden. Insoweit wird versucht, die Hürde einer kritischen Rezeption gezielt zu umgehen. Bei einer solchen gezielten Meinungssteue-

1594 Klaas, MMR 2019, 84 (88).

1595 So etwa - wenn auch in anderem Kontext - v. Ungern-Sternberg, in: Unger/dies., Demokratie und künstliche Intelligenz, S. 3 (19). Und auch Klaas, MMR 2019, 84 (88) zieht zumindest die Parallele zur rundfunkrechtlichen Rechtsprechung.

1596 Allgemein zu den Wechselwirkungen etwa BVerfGE 121, 60 (55) - Parteibeteiligung an Rundfunkunternehmen, m.w.N. Speziell zur Öffentlichkeitsarbeit siehe statt vieler BVerfG NJW 2020, 2096 Rn. 49 ff. - Seehofer, m.w.N.

1597 Vgl. hierzu Ipsen, in: Sachs, GG, Art. 21 Rn. 26; BVerfGE 44, 25 (140) - Öffentlichkeitsarbeit der Bundesregierung; 138, 102 (111) - Wahlkampfäußerungen von Regierungsmitgliedern.

1598 In BVerfGE 121, 30 (57) - Parteibeteiligung an Rundfunkunternehmen, heißt es dazu: "Sie [die Parteien] sammeln die auf politische Macht und deren Ausübung gerichteten Meinungen, Interessen und Bestrebungen, gleichen sie in sich aus, formen sie und versuchen, ihnen auch im Bereich der staatlichen Willensbildung Geltung zu verschaffen. [Hervorh. d. Autors]“. 
rung aktualisiert sich auch das Risiko, welches aus der staatsnahen Stellung der Parteien resultiert. ${ }^{1599}$ Eine Parallele hierzu findet sich in dem durch die verfassungsgerichtliche Judikatur bestätigten Verbot einer bestimmenden Beteiligung an Rundfunkunternehmen. ${ }^{1600}$ Medien sind wesentliche Informationsgeber und haben Einfluss auf die Art der Rezeption und damit auf die Steuerung des Kommunikationsprozesses. ${ }^{1601}$ Wenn also staatsnahe Akteure Einfluss auf Medienunternehmen gewinnen, folgt daraus auch eine entsprechende Manipulationsgefahr. ${ }^{1602}$ Das BVerfG hat zum Schutz der kommunikativen Chancengleichheit ein Verbot einer bestimmenden Beteiligung der Parteien für verfassungsmäßig und aus Gründen der Transparenz eine darunter liegende Beteiligung zwar nicht für verboten, aber für offenlegungsbedürftig gehalten. ${ }^{1603}$ Auch dort ging es also um das Risiko der Veränderung der kommunikativen Chancengleichheit und um das Risiko einer verdeckten meinungssteuernden Betätigung der Parteien. ${ }^{1604}$

Das sowohl mit Blick auf den demokratischen Kontrollprozess als auch mit Blick auf den verdeckten Einfluss zur Geltung kommende Transparenzbedürfnis unterscheidet sich auf den ersten Blick zwar nicht von dem allgemeinen. ${ }^{1605}$ Allerdings handeln Parteien anders als Private gerade nicht um ihrer selbst willen. ${ }^{1606}$ Besonders die demokratische, im Öffentlichkeitsprinzip niedergelegte Transparenz staatlichen Handelns wird durch das Grundgesetz selbst explizit auch auf die wirtschaftliche Betätigung der Parteien erstreckt (Art. 21 Abs. 1 S. 4 GG). ${ }^{1607}$ Damit soll verhindert werden, dass ein nicht unerheblicher, verdeckter Einfluss auf Parteien ausgeübt wird. ${ }^{1608}$ Aber auch abseits hiervon folgt aus der besonderen, einzigartigen staatsnahen Stellung der Parteien ein Öffentlichkeits- respektive Transparenzbedürfnis, welches Art. 21 Abs. 1 GG immanent ist. ${ }^{1609}$ Wenn schon der wirtschaftliche Einfluss von außen verfassungsrechtlich zwingend transparent gemacht werden muss, um final eine mittelbar-verdeckte Beeinflus-

1599 Zu dieser Stellung ausführlicher BVerfGE 121, 30 (54 f.) - Parteibeteiligung an Rundfunkunternehmen. Im Falle staatlicher Akteure fordern etwa Dankert/Dreyer, K\&R 2017, 73 (76) und Milker, JA 2017, 647 (654) die Transparenz hinsichtlich des Urhebers der Bots bzw. der Kommunikationsinhalte.

1600 Hierzu BVerfGE 121, 30 (60 ff.) - Parteibeteiligung an Rundfunkunternehmen. Diese Parallele zieht auch Klaas, MMR 2019, 84 (88).

1601 Allgemein hierzu bereits oben, 2. Teil, B.III.

1602 So letztlich die Begründung des BVerfG, E 121, 30 (61 f.) - Parteibeteiligung an Rundfunkunternehmen.

1603 Zum Verbot beherrschender Beteiligungen BVerfGE, a.a.O., (60 f.) und zur Offenlegung anderer Beteiligungen (67).

1604 Diesen Vergleich zieht auch Klaas, MMR 2019, 84 (88).

$1605 \mathrm{Zu}$ diesem allgemeinen Transparenzbedürfnis bei Meinungsrobotern siehe oben, B.II.1.c.aa.

1606 Vgl. zu diesem Unterschied auch BVerfGE 20, 56 (107) - Parteifinanzierung I, mit Blick auf die staatliche Förderung privater Vereinigungen und Parteien.

1607 Zu diesem Zusammenhang etwa Shirvani, NVwZ 2017, 1321 (1322).

1608 Siehe hierzu nur BVerfGE 85, 264 (319) - Parteienfinanzierung II; 111, 54 (83) - fehlerhafte Rechnungslegung.

1609 Hierzu Klein, in: Maunz/Dürig, GG, Art. 21 Rn. 316 f.; Streinz, in: v. Mangoldt/Klein/Starck, GG, Art. 21 Rn. 176; allgemein zum Transparenzbedürfnis Boehme-Neßler, NVwZ 2017, 528 f. 
sung des Meinungsbildungsprozesses zu verhindern, ${ }^{1610}$ und auch im Rundfunk jedenfalls Transparenzvorgaben notwendig werden, dann muss das noch deutlich stärker dort gelten, wo sie unmittelbar und verdeckt die digitalen Diskursräume beeinflussen. ${ }^{1611}$ Denn hier aktiviert sich nicht nur in besonderer Weise das Risiko der Staatsnähe, sondern es widerspricht auch der wesentlichen Konzeption der Parteien. Je gravierender dabei der Einsatz ist, also je eher sich die zuvor abstrakte Gefahr für die kommunikative Chancengleichheit und den Kommunikationsprozess konkretisiert, desto stärker wird das grundlegende Transparenzbedürfnis ${ }^{1612}$ und es kann sich mit Erreichen der von Klaas angesprochenen „Qualitätsschwelle“ von einem einfach-rechtliche Transparenzpflichten rechtfertigenden, verfassungsimmanenten Interesse ${ }^{1613} \mathrm{zu}$ einer verfassungsunmittelbaren Grenze des Einsatzes verdichten. Maßgeblich werden hierfür vor allem die tatsächliche Reichweite, der Umfang und die Art des Einsatzes. ${ }^{1614}$ Bisherige Beeinflussungen konnten diese Verdichtung deshalb nicht herbeiführen, weil es einerseits um neutrale wirtschaftsbezogene Handlungen ging (Rundfunkbeteiligung) ${ }^{1615}$, die Gefahr von externer Seite aus begründet wurde (Parteispenden) oder bei herkömmlicher Agitation im Wahlkampf gerade die Verbindung einer Aussage zur Partei entscheidend war, sodass hier Transparenz schon aus intrinsischen Motiven hergestellt wurde.

\section{c. Fazit: Kein spezifischer Schutz der Meinungsäußerungsfreiheit}

Weder einfach-rechtlich noch verfassungsrechtlich bestehen gegen den Einsatz von Meinungsrobotern durch private Akteure Grenzen, die dem Schutz der Meinungsäußerungsfreiheit sowohl im Kommunikationsprozess allgemein als auch speziell mit Blick auf die Wahl selbst dienen. Einfach-rechtlich sind die vorgesehenen Grenzen einer Nötigungshandlung allein durch den Einsatz nicht erreicht und können nur durch spezifische Inhalte begründet werden. Verfassungsrechtlich folgt das letztlich daraus, dass Meinungsroboter - anders als die bereits nor-

1610 BVerfGE 20, 56 (106) - Parteifinanzierung I.

1611 Für das BVerfG führte dieser Aspekt immerhin dazu, die Beteiligung an Rundfunkunternehmen jedenfalls nicht unbegrenzt zuzulassen, weil sonst eine staatsähnliche Indoktrination des Kommunikationsprozesses droht, hierzu BVerfGE 121, 30 (53 ff.) - Parteibeteiligung an Rundfunkunternehmen.

1612 Eine solche Abstufung nimmt letztlich das BVerfG auch hinsichtlich des Rundfunks vor, wo dies von Verbot bis zur Offenlegung reicht, BVerfGE 121, 30 (60 ff.) - Parteibeteiligung an Rundfunkunternehmen. Eine solche Unterscheidung nach der "Schwere“ liegt aber auch der Abstufung der Transparenzpflicht im Falle der Parteispenden zugrunde, vgl. BVerfGE 24, 300 (356) - Parteifinanzierung I; 85, 264 (321) - Parteifinanzierung II.

1613 Vgl. zu diesem grundsätzlichen Interesse $v$. Ungern-Sternberg, in: Unger/dies., Demokratie und künstliche Intelligenz, S. 3 (19) speziell im Kontext der Meinungsroboter und allgemeiner etwa Boehme-Neßler, NVwZ 2017, $528 \mathrm{f}$.

1614 Klaas, MMR 2019, 84 (88).

1615 Vgl. zu der Legitimität der Beteiligung BVerfGE 121, 30 (60) - Parteibeteiligung an Rundfunkunternehmen. 
mierten und strafbaren Handlungen - nicht die freie Wahlentscheidung der Wähler unterdrücken wollen, sondern viel früher ansetzen. Der Willensbildungsprozess basiert auf falschen oder verfälschten Informationen; der Willensbildungsprozess bleibt aber weiterhin frei. ${ }^{1616}$

Speziell staatliche Akteure unterliegen zwar einer verfassungsrechtlichen Neutralitätspflicht. Folgt man aber der bisherigen verfassungsgerichtlichen Judikatur, so begründet dies regelmäßig nicht die Unzulässigkeit des Einsatzes von Meinungsrobotern, weil der staatliche Autoritätscharakter hier nicht nach außen dringt und insoweit keine amtliche Beeinflussung vorliegt. Etwas anderes kann dort gelten, wo Parteien Meinungsroboter einsetzen. Sie haben immerhin einen verfassungsrechtlichen Auftrag zu erfüllen, durch den sie sich nicht um ihrer selbst willen, sondern als notwendige Institution im demokratischen Staat betätigen. Insofern haben sie eine unterstützende Funktion. Der Einsatz der Meinungsroboter konfligiert hiermit aber deshalb, weil es nicht mehr um eine Unterstützung des Kommunikationsprozesses, sondern um die Ausnutzung dessen für die eigene Parteilinie geht. Dieser „Guerilla-Wahlkampf“ steht nicht nur im Widerspruch zu demokratischen Grundprinzipien, sondern tangiert auch ganz erhebliche, demokratische Transparenzbedürfnisse, die sich zu einer verfassungsunmittelbaren Grenze verdichten können.

\section{Bewertung des bisherigen Schutzkonzeptes im Lichte des Untermaßverbots}

Das bislang geltende Recht weist kaum Schutzvorkehrungen auf, welche den spezifischen Charakteristika und Einflüssen der Meinungsrobotern begegnen und so den aufgezeigten Schutzbedürfnissen im Ansatz gerecht werden können. Eine wirksame Antwort auf Meinungsroboter überlässt der Staat vor allem den Netzwerkbetreibern und ihrer Selbstregulierung, ohne dass dabei eine wirksame staatliche Kontrolle gewährleistet wäre. Das gilt nicht nur mit Blick auf die vertragliche Beziehung zwischen Netzwerkbetreiber und Nutzer. Selbst dort, wo das einfache Recht Anknüpfungspunkte für eine rechtliche Lösung bereithält, weist es die Befugnis zur Geltendmachung den Betreibern zu. Das gilt insbesondere hinsichtlich der straf- und urheber-rechtlichen Normen, die bei der Aktivität von Meinungsrobotern eingreifen können. Insoweit bleibt ein staatlicher Zugriff wenn überhaupt - allein auf die sekundäre Schlichtungssphäre im Streitfalle begrenzt. Und selbst der europäische Verhaltenskodex stellt zwar verbindliche Verpflichtungen auf, überlässt aber die Durch- und Umsetzung ebenfalls allein den Netzwerkbetreibern. Dabei knüpft er - jedenfalls mit Blick auf die Meinungsroboter - an den Status quo an und trifft keinerlei innovative Vorkehrungen.

1616 Das zeigt nochmals deutlich die Notwendigkeit eines Schutzes für den vorgelagerten Meinungsbildungsprozess vor den Einflüssen der Meinungsroboter. 
Aber auch mit Blick auf die spezifischen Schutzbedürfnisse der Informationsund Meinungsäußerungsfreiheit besteht kein überzeugendes Schutzkonzept. Die Kennzeichnungspflicht des $\mathbb{S} 55$ Abs. $1 \mathrm{RStV}$ - jetzt $\mathbb{} 18$ Abs. $1 \mathrm{MStV}-$ mag zwar einschlägig sein, bietet aber gerade keinen Schutz gegen die Täuschungswirkung. Und selbst wenn ihr nicht nachgekommen wird, so besteht - wegen des objektiven Scheins privater Kommunikation - schon keine faktische Grundlage für staatliche Nachforschungsmaßnahmen, für die ohnehin ein direkter Zugang zu den Netzwerkdaten und eine konkrete Ermächtigung fehlt. Auch gegen das besondere Problem desinformativer Inhalte ist - soweit jedenfalls kein persönlichkeitsverletzender oder volksverhetzender Charakter ausgemacht werden kann $(\mathbb{S} 185 \mathrm{ff}$ StGB bzw. $\mathbb{1} 130 \mathrm{StGB})$ - kein straf- oder äußerungsrechtlicher Schutz vorgesehen. Das gilt noch stärker für die kollektive Ebene des Kommunikationsprozesses. Sofern daneben auch die Personalisierungsprozesse der Netzwerkunternehmen einen kausalen Beitrag für die Beeinflussung bestimmter Nutzergruppen leisten können, wird dieser Einfluss durch die Vorgaben der DS-GVO jedenfalls nicht gänzlich unterbunden. Allein der Umfang der Datenerhebung wird auf das (rechtlich) erforderliche bzw. angemessene Maß reduziert. Mit Blick auf die Meinungsäußerungsfreiheit sieht jedenfalls das einfache Recht nur in exzessiven Ausnahmefällen Grenzen des Einsatzes vor. Das gilt dann, wenn die konkreten Inhalte der Meinungsroboter eine willensbeugende Wirkung, sowohl mit Blick auf die allgemeine ( $\mathbb{S} 240$ Abs. 1 StGB) als auch die wahlspezifische Willensbetätigung ( $\mathbb{S} 108$ StGB), haben. Aus verfassungsrechtlicher Sicht bestehen nur gegen einen parteigesteuerten Einsatz Bedenken, sofern dieser eine gewisse exzessive Form annimmt. Einzig die Vermittlung einer Medienkompetenz versuchen die Länder im schulischen Bereich sicher zu stellen, wobei hier aber erst die Grundbausteine gelegt sind und noch völlig unklar ist, ob dies auch gewinnbringend sein wird.

Zusammengenommen bietet der Staat selbst also keine hinreichende Gewähr dafür, dass den Einflüssen von Meinungsrobotern in sozialen Netzwerken wirksam begegnet wird. Bislang wird weder den besonderen Transparenzerfordernissen noch den aus kommunikativer Sicht besonderen Gefahrenlagen für die Meinungsäußerungsfreiheit und dem (kollektiven) Kommunikationsprozess Rechnung getragen. Nur in krassen Ausnahmefällen bestehen Möglichkeiten, dem Einsatz von Meinungsrobotern zu begegnen. Diese Schutzmaßnahmen sind jedoch allesamt repressiv ausgestaltet, stehen einem prinzipiellen Einsatz also nicht im Wege. Vielmehr setzen sie voraus, dass Meinungsroboter in erheblicher beeinträchtigender Weise zuvor tätig geworden sind. Wegen der grundsätzlichen Möglichkeit privater Intervention, kann man zwar nicht davon ausgehen, dass der Staat Schutzvorkehrungen überhaupt nicht bereithält oder dass diese völlig ungeeignet wären, sodass man zumindest über die ersten beiden Stufen des Untermaßverbotes hinwegkäme. Anders sieht das aber mit Blick auf die zwei 
weiteren Stufen der Effektivität und Angemessenheit aus. Der Staat vertraute lange Zeit auf eine Regulierung durch den (Meinungs-)Markt selbst. Auch wenn dies aus Sicht des Übermaßverbots sicherlich ein verständlicher Ansatz ist, ${ }^{1617}$ setzt dies aber voraus, dass vom Markt selbst eine gewisse regulierende Wirkung (bspw. Wettbewerb) ausgeht, was schon angesichts oligopoler Strukturen zweifelhaft ist. ${ }^{1618}$ Faktisch würde das auch voraussetzen, dass die Interessen von Betreibern, Nutzern und demokratischer Gesellschaft jedenfalls so kongruent sind, dass hinreichend Gewähr für eine wirksame Regulierung besteht. ${ }^{1619}$ Dass die Netzwerkbetreiber selbst an dem störungs- und manipulationsfreien Betrieb ihrer Dienste ein nicht unerhebliches Interesse haben, ${ }^{1620}$ ist zwar richtig. Ob allein dieses Interesse ausreicht, um die Wirksamkeit der Maßnahmen - auch aus Sicht des Gesetzgebers - anzunehmen, darf indes bezweifelt werden. ${ }^{1621}$ Schon die Klarnamenpflicht selbst wird kaum durchgesetzt und auch anderen betreiberseitigen Regelungen wurde erst nach Erlass von gesetzgeberischen Maßnahmen oder erst durch öffentlichen, wirtschaftlichen oder politischen Druck mehr Beachtung geschenkt. ${ }^{1622}$ Vor dem Netzwerkdurchsetzungsgesetz soll etwa nur unzureichend von der Sperr- bzw. Löschmöglichkeit von Inhalten Gebrauch gemacht worden

1617 Zu diesem Vorrang der Regulierung durch den Markt etwa Milker, InTeR 2017, 199 (203). Demgegenüber sieht Brinkmann, UFITA 2019, 364 (373), den „Markt der Meinungen“ als „eine unzulängliche Projektion des ökonomischen Regulationsmodells" an.

1618 So auch Schneiders, Das ist doch Diskriminierung, S. 10. Umfangreicher zur Problematik einer reinen Selbstregulierung des Marktes im Bereich der Medien etwa Brinkmann, UFITA 2019, 364 (379 ff.).

1619 Das dies nicht immer der Fall ist wurde schon mehrfach aufgezeigt. Das beginnt bereits bei der Ausrichtung an Relevanz und nicht an journalistisch-redaktionellen Kriterien und endet nicht erst bei den in erster Linie ökonomischen Interessen der Betreiber, wodurch Handlungsbedarf erst dann besteht, wenn dies die ökonomische Rationalität gebietet.

1620 Darauf hinweisend etwa Dreyer/Schulz, Stellungnahme zum 2. MStV, 2019, S. 16; im Kontext der Intermediärsregulierung auch Drexl, ZUM 2017, 529 (542).

1621 Allgemein kritisch mit Blick auf eine Selbstregulierung Bock, Die Übertragbarkeit der Kommunikationsfreiheiten des Artikel 5 GG auf das Internet, S. 283, 304. König, AcP 219 (2019), 611 (637) meint gar, dass kein originäres Eigeninteresse daran bestehe Inhalte zu sperren oder Nutzer zu vergraulen; dies mache „betriebswirtschaftlich in der Regel keinen Sinn“. Speziell mit Blick auf Social Bots Bock, a.a.O., S. 196 und speziell zu Fake Accounts bei Facebook siehe Silverman/Mac/Dixit, I Have Blood on My Hands": A Whistleblower Says Facebook Ignored Global Political Manipulation, BuzzFeed News. Und Gorwa/Guilbeault, Policy \& Internet Vol. 12 (2020), 225 (242) weisen ganz allgemein daraufhin, dass die Betreiberinteressen und die öffentlichen Interessen oftmals nicht kongruent verlaufen.

1622 Erst der Werbeboykott durch namhafte Werbepartner führte etwa bei Facebook zum Umdenken hinsichtlich des Umgangs mit Fake News, die auch von Politikern verbreitet wurden, siehe hierzu Tagesschau, Kritik und Werbeboykott - Facebook reagiert, abrufbar unter https://www.tagesscha u.de/wirtschaft/facebook-werbeboykott-101.html, (Stand: 30.05.2021). Und zur Untätigkeit des Unternehmens im Umgang mit Fake-Accounts und Bots siehe insbesondere Silverman/Mac/Pranav, „I Have Blood on My Hands“: A Whistleblower Says Facebook Ignored Global Political Manipulation, BuzzFeed NewS. Insgesamt kritisch zu selbstregulierenden Ansätzen auch Hines, Houston Law Review 57 (2019), 405 (434) der darauf hinweist, dass eine (effektive) Regulierung erst dann erfolgen wird „until it is directly profitable or until their ubiquity is an existential threat - a time that may never come for some of the larger tech firms." 
sein. ${ }^{1623}$ Und erst durch die US-Präsidenschaftswahlen wuchs das Interesse an datenschutzrechtlichen Fragen - Stichwort: Cambridge-Analytica ${ }^{1624}$ - und dem Einsatz von Meinungsrobotern in sozialen Netzwerken. Ein solcher Druck kann aber im Falle der Meinungsroboter nur entstehen, wenn bestimmte kritische Phänomene auch für die Öffentlichkeit einsehbar sind. Anders als bei der Untätigkeit oder der fehlenden Effektivität im Kampf gegen Desinformation sind aber die konkreten Auswirkungen von automatisierten Fake-Accounts nicht öffentlich sichtbar. Und die freiwilligen, aber wenig detaillierten Berichte begründen eine solche (wirksame) Kontrolle bislang auch nicht. Auch deshalb stellt sich hier bereits die Frage, ob man privaten Betreibern tatsächlich eher vertrauen kann als dem Staat. Auch das BVerfG hat immerhin die Notwendigkeit von transparenten Informationen für die Marktteilnehmer zur Gewährleistung der Funktionsfähigkeit des Wettbewerbs und damit des Marktes betont und dabei klargestellt, dass diese Transparenz nicht allein marktseitig begründet werden kann. ${ }^{1625}$ Und allgemein lässt sich zumindest kritisch fragen, ob die Regelungen aus Sicht der Betreiber tatsächlich unmittelbar den Nutzern zugutekommen oder nicht vielmehr primär der Steigerung der wirtschaftlichen Wertschöpfung dienen ${ }^{1626}$ und schon deshalb eine geringere Motivation für eine effektive Durchsetzung besteht, weil mit den falschen Nutzerprofilen die Zahl der Nutzer und mit Meinungsrobotern die Aktivität in den Netzwerken ansteigt. ${ }^{1627}$ Die sporadischen Informationen, die die Netzwerkbetreiber herausgeben, lassen kaum Schlüsse auf die genaue Praxis im Umgang mit falschen Nutzerprofilen erkennen. ${ }^{1628}$ Eine Kompensation durch den Zugang zu den Netzwerkdaten für Forschungszwecke findet nur begrenzt statt. Insgesamt kann deshalb nicht nur die Wirksamkeit bezweifelt werden, vielmehr zeigt sich dadurch gerade die Notwendigkeit eines - wenn auch nur vorsichtigen $-{ }^{1629}$ Zugriffs durch den Staat. ${ }^{1630}$

1623 Vgl. hierzu die Begründung des Regierungsentwurfes BT-Drs. 18/12356, S. 1 f., der auf entsprechende Ergebnisse einer Studie verweist. Bei Facebook sei demnach nur in 39\% und bei Twitter nur in $1 \%$ der Fälle ein Inhalt tatsächlich gelöscht worden.

1624 Ausführlich hierzu etwa Cadwalladr/Graham-Harrison, Revealed: 50 million Facebook profiles harvested for Cambridge Analytica in major data breach, The Guardian.

1625 Siehe hierzu BVerfGE 105, 252 (266f.) - Glykol.

1626 Kritisch deshalb auch Bock, Die Übertragbarkeit der Kommunikationsfreiheiten des Artikel 5 GG auf das Internet, S. 197.

1627 Darauf ebenfalls hinweisend Bock, ebd.; Keller/Klinger, Political Communication 36 (2019), 171 (178). Siehe auch Abulaish/Fazil, IEEE Technology and Society Magazine 2020, 52 (59).

1628 Das wurde auch schon in dem Gesetzesentwurf zum NetzDG kritisiert, hierzu BT.-Drs. 18/12356, S. 11.

1629 In diese Richtung nämlich Brings-Wiesen, JuWiss-Blog Nr. 93/2016.

1630 Vgl. hierzu auch Bock, Die Übertragbarkeit der Kommunikationsfreiheiten des Artikel 5 GG auf das Internet, S. 283, 304. Anders indes Cornils/Liesem, Stellungnahme zum Diskussionsentwurf eines Medienstaatsvertrages der Rundfunkkommission der Länder, S. 21. Gräfe, PinG 2019, 5 (11) meint - wenn auch in anderem Kontext - ganz generell, dass es „Aufgabe des Staates und nicht der sozialen Netzwerke [ist], die Kommunikationsfreiheiten zu schützen.“. 
Das hätte aber - vor allem bei unveränderter Rechtslage - nicht zwingend eine Verletzung der Schutzpflicht begründen müssen. Denn verfassungsrechtlich lässt sich nur schwer ein verpflichtender, zeitlicher Horizont für die Gewährung des Mindestschutzes ausmachen. Das liegt freilich auch daran, dass es mit Blick auf das Übermaßverbot einer präzisen Abwägung etwaiger Schutzkonzepte mit den kollidierenden Belangen bedarf. Das wirft ganz besonders dort Schwierigkeiten auf, wo es nicht nur um ein bi- oder tripolares, sondern ein mehrpoliges Verhältnis geht. Dort steigen nicht nur allgemein die Anforderungen für die Rechtfertigung, weil nicht mehr nur der „Störer“ tangiert ist. Eine angemessene Abwägung setzt hier auch notwendigerweise eine gewisse faktische Grundlage voraus, aufgrund derer Risiken eingeschätzt werden müssen. ${ }^{1631}$ Weil es sich aber um ein vergleichsweise junges Phänomen handelt, dessen Wirkungszusammenhänge und Risiken sich erst allmählich herauskristallisiert haben, setzt eine gesetzgeberische Antwort notwendiger Weise eine gewisse Nachforschung und Evaluation sowie die Bewertung, Gewichtung und Beantwortung daraus resultierender rechtlicher Fragen voraus. Schon deshalb ist dem Gesetzgeber auch ein zeitlicher Spielraum zu gewähren. Dieser folgt hier letztlich auch aus der schon aufgezeigten Komplexität nicht nur der grundrechtlichen Fragen. Gerade auch kompetenzielle Fragen zwischen Bund und Ländern aber auch im Verhältnis zur Europäischen Union bedürfen einer zeitaufwendigen Analyse.

Berücksichtigt man allerdings, dass der Bundestagsausschuss für Bildung, Forschung und Technikfolgenabschätzung bereits im Januar $2017 \mathrm{zu}$ einem öffentlichen Fachgespräch eingeladen hat, und eine entsprechend (Vor-)Studie von Oktober 2016 bis April 2017 vom Büro für Technikfolgenabschätzung durchgeführt wurde, und dass auch weitere wissenschaftliche Publikationen in vergleichbarer Weise das Risiko- und Gefahrenpotential aus Sicht des Kommunikationsprozesses aufzeigen, ${ }^{1632}$ so überrascht es zumindest, dass es bis auf konkretere Forderungen $^{1633}$ über einen langen Zeitraum - knapp 4 Jahre - nur bei Planungen

1631 Insoweit könnte hier also auch ein Schutz ohne Eingriff denkbar sein. Das ähnelt also in gewisser Weise der Situation im Polizeirecht, in dem lediglich ein Gefahrenverdacht vorliegt. Dann können die Behörden zwar Gefahrenermittlung gestützt auf die Generalklausel betreiben, aber dabei keine rechtsbeschränkenden Verfügungen gegen den „Verdachtsstörer“ erlassen oder ihn sonst wie in Anspruch nehmen. Zu der Lage bei der Schutzpflicht siehe etwa Krings, Grund und Grenzen grundrechtlicher Schutzansprüche, S. $264 \mathrm{f}$. Zum Gefahrenverdacht im Polizeirecht statt vieler Mühl/ Fischer, in: Möstl/Bäuerle, BeckOK POR Hessen, $\mathbb{1}$ HSOG Rn. $70 \mathrm{ff}$.

1632 Dazu zählen u.a. Suárez-Serrato u.a., in: Spiro/Ahn, SocInfo 2016: Social Informatics, S. 269ff; Thieltges u.a., Effects of Social Bots in the Iran-Debate on Twitter; Ferrara u.a., Communications of the ACM Vol. 59 (2018), 96 ff.; Thieltges/Hegelich, ZfP 64 (2017), (503 ff.). Speziell zum Einfluss in Diskussionen etwa Hagen u.a., Social Science Computer Review, 1 ff., insbesondere die Zusammenfassung, S. 18 f.; zum Einfluss auf die Informationsvermittlung etwa Stella/Ferrara/Domenico, PNAS Vol. 115 (2018), 12435 ff.; zur Verbreitung von Fake-News Shao u.a., Nature Communications (2018)9:4787.

1633 So etwa der Antrag der Fraktion Bündnis 90/Die Grünen u.a., BT.-Drs. 18/11856, S. 2. 
blieb. ${ }^{1634}$ Die wesentlichen überwiegend kritischen Auffassungen zur Einschätzung der Risikopotentiale haben sich nicht deutlich verändert und waren jedenfalls grundlegend schon vor dem Jahr 2016 bekannt. ${ }^{1635}$ Wäre der Status-quo unverändert geblieben, hätte sich die Frage der Verfassungswidrigkeit unweigerlich stellen müssen. Angesichts des Medienstaatsvertrages, der am 7. November 2020 in Kraft getreten ist und der nicht nur die Medienintermediäre einer medienrechtlichen Regelung unterworfen hat, sondern auch Vorkehrungen für den Einsatz von Meinungsrobotern in sozialen Netzwerken vorsieht, könnte diese Lage aber entschärft worden sein.

\section{Die Neuregelung des RStV durch den MStV}

Mit dem „Staatsvertrag zur Modernisierung der Medienordnung in Deutschland“1636 haben die Länder neue Regelungen für die bislang unregulierten Netzwerke und andere digitale Anbieter mit meinungsbildender Relevanz eingeführt; der veraltete Rundfunkstaatsvertrag ist dadurch aufgehoben worden (Art. 2 des Entwurfes). ${ }^{1637}$ Nach Änderung der Richtlinie für audio-visuelle Medien (RL 2010/13/EU) ${ }^{1638}$ durch die Richtlinie 2018/1808 ${ }^{1639}$ wurde eine Anpassung für Video-Sharing-Dienste ohnehin bis zum 19. September 2020 notwendig (Art. 2 der RL). Im Zuge der Umsetzung wurde eine allgemeine und zeitgerechtere Überarbeitung des bestehenden Regelungsregimes befürwortet, um dieses an die im Zuge der Digitalisierung veränderte Medienlandschaft anzupassen ${ }^{1640}$. Vorliegend kann und soll freilich nicht auf alle Einzelheiten des Staatsvertrages eingegangen werden. ${ }^{1641}$ Zentral wird es hier vielmehr um zwei Aspekte gehen:

1634 So erachtet etwa Steinmaurer, in: Litschka/Krainer, Der Mensch im digitalen Zeitalter, S. 31 (36) „Maßnahmen [für] notwendig, die einen substanziellen Beitrag zur Absicherung einer „digital resilienten Demokratie“ leisten. “ Anders indes Liesem, in: Litschka/Krainer, Der Mensch im digitalen Zeitalter, S. 183 (194).

1635 Das Potential der Verbreitung von Desinformation und der Manipulation der öffentlichen Meinung erkennen bereits Boshmaf u.a., Computer Networks, 53 (2013), 556 (557).

1636 So die offizielle Bezeichnung, siehe nur BayLT-Drs. 18/7640, S. 1.

1637 BayLT-Drs. /7640, S. 69.

1638 Richtlinie 2010/13/EU des Europäischen Parlaments und des Rates vom 10. März 2010 zur Koordinierung bestimmter Rechts- und Verwaltungsvorschriften der Mitgliedstaaten über die Bereitstellung audiovisueller Mediendienste (Richtlinie über audiovisuelle Mediendienste), ABl. 2010 L 95, S. $1 \mathrm{ff}$.

1639 Richtlinie (EU) 2018/1808 des Europäischen Parlaments und des Rates vom 14. November 2018 zur Änderung der Richtlinie 2010/13/EU zur Koordinierung bestimmter Rechts- und Verwaltungsvorschriften der Mitgliedstaaten über die Bereitstellung audiovisueller Mediendienste (Richtlinie über audiovisuelle Mediendienste) im Hinblick auf sich verändernde Marktgegebenheiten, ABl. 2018 L 303, S. 69 ff. Zu den vorgesehenen Neuerungen der Richtlinie etwa Gundel, ZUM $2019,131 \mathrm{ff}$.

1640 Ladeur/Gostomzyk, K\&R 2018, 686 (687).

1641 Für einen Überblick über die Neuregulierung etwa Ory, ZUM 2019, 139 ff.; d'Orville, ZUM 2019, $104 \mathrm{ff}$. Gesondert zu Medienplattformen und Benutzeroberflächen etwa Thomale, ZUM 2019, $122 \mathrm{ff}$. Und zu Medienintermediären etwa Paal/Heidtke, ZUM 2020, 230 ff.; Müller-Terpitz, in: Kühling/Zimmer, Neue Gemeinwohlherausforderungen, S. 177 (183 ff.); ders., in: Eifert/Gostom- 
Einerseits spezifische Vorschriften für Anbieter von Meinungsrobotern $(\mathbb{S} 18$ Abs. $3 \mathrm{MStV}$ ) und andererseits die spezifischen Regelungen für sog. Medienintermediäre ( $\mathbb{S} 91-96 \mathrm{MStV})$.

\section{Spezifische Kennzeichnungspflicht für Meinungsroboter}

In $\mathbb{S} 18 \mathrm{MStV}$ finden sich weitestgehend die Transparenzregelungen wieder, die zuvor in $\mathbb{S} 55 \mathrm{RStV}$ normiert waren. Diese werden durch einen Absatz 3 erweitert, der eine Antwort auf das Phänomen der Meinungsroboter darstellen und die Transparenz schaffen soll, die durch die bisherigen Informationspflichten nicht gewährt wurde. Anbieter von Telemedien, deren Inhalte automatisiert durch ein Computerprogramm erstellt wurden, müssen diesen Umstand kennzeichnen, sofern das hierfür verwendete Nutzerkonto den Anschein eines echten Nutzers erwecken kann. Damit geht Deutschland einen ähnlichen Weg wie schon zuvor der Staat Kalifornien. ${ }^{1642}$ Bedeutsamkeit erlangt die Regelung damit in zweierlei Richtung: Einerseits bestätigt sie das oben genannte Ergebnis, dass auch Nutzerkonten als Telemedien anzusehen sind. Andererseits wird die bislang bestehende und bereits oben beschriebene Regelungslücke der bisherigen Transparenzregelungen (C.I.3.c.) geschlossen.

Erfasst werden aber nur Automatisierungsprozesse, denen eine (menschliche) Identitätstäuschung immanent ist. Andere automatisierte Kommunikation, etwa durch kommerzielle Facebook-Seiten, unterliegen also keinen weiteren Einschränkungen als zuvor mit den $\mathbb{S} \mathbb{S} 5,6$ TMG. Automatisierte Meldungen auf diesen Seiten sind also immer noch zulässig, ohne dass eine Kennzeichnung erfolgen muss. Durch $\mathbb{S} 18$ Abs. 3 S. 3 MStV wird zudem klargestellt, dass die Art der Information und ihre (redaktionelle) Aufbereitung irrelevant ist. Sowohl das Teilen von fremden Inhalten als auch das Veröffentlichen eigener Inhalte ist kennzeichnungspflichtig. Die Kennzeichnungspflicht trifft allerdings nach $\mathbb{1}$ Abs. 7 MStV nur Anbieter, die nach den Regelungen des TMG ( $\mathbb{S} 2$ Abs. 2 Nr. 2, 2a Abs. 1 TMG) als in der Bundesrepublik niedergelassen anzusehen sind.

zyk, Netzwerkrecht, S. 45 (56 ff.); kritisch hierzu etwa Kottmann, ZUM 2019, 119 ff. Ausführlich zu den Medienintermediärsregelungen Kellner, Die Regulierung der Meinungsmacht von Internetintermediären, S. $283 \mathrm{ff}$. zur Transparenzregelung, S.288f. zur Diskriminierungsvorschrift und Heidtke, Meinungsbildung und Medienintermediäre, S. $319 \mathrm{ff}$.

1642 Im September 2018 wurde in Kalifornien der sog. „Bolstering Online Transparency Act“ (California Senate Bill 1001) beschlossen, wonach bestimmte Arten von Bots als solche gekennzeichnet werden müssen. Nach Sec. 17491 (a) des California Business and Profession Code ist es „unlawful for any person to use a bot to communicate or interact with another person in California online, with the intent to mislead the other person about its artificial identity for the purpose of knowingly deceiving the person about the content of the communication in order to [...] influence a vote in an election. A person using a bot shall not be liable under this section if the person discloses that it is a bot.“ Nach Sec. 17491 (b) muss die Kennzeichnung „clear, conspicuous, and reasonably designed to inform persons with whom the bot communicates or interacts that it is a bot.". 
Durch die Kennzeichnung muss der Umstand der Automatisierung erkennbar sein, was nach $\mathbb{S} 18$ Abs. 3 S. 2 einen gut lesbaren Hinweis verlangt, der dem jeweiligen Inhalt bei- oder vorangestellt wird. ${ }^{1643}$ Wie dieser Hinweis konkret auszusehen hat, beantwortet die Norm nicht. Wegen der Parallele zu den anderen Kennzeichnungsvorschriften ${ }^{1644}$ müsste ein solcher Hinweis so erfolgen, dass ein durchschnittlicher Nutzer die Information ohne Weiteres erkennen kann. Das dürfte etwa durch einen Zusatz hinter dem jeweiligen Profilnamen gewährleistet werden. Insoweit könnte sich eine Parallele zu den vom LG München ${ }^{1645}$ angesprochenen blauen Häkchen als Kennzeichnung der Verifikation von Profilen (Abb. 1) anbieten. Konkret könnte statt des Hakens etwa ein entsprechendes Symbol angezeigt werden, dass bei längerem Verweilen des Cursors kurze, prägnante Hinweise auf die Bedeutung gibt und durch einen Link auf ergänzende, ausführlicher Hinweise verweist. Auch dies ist bei dem „blauen Haken“ ähnlich (Abb. 2).

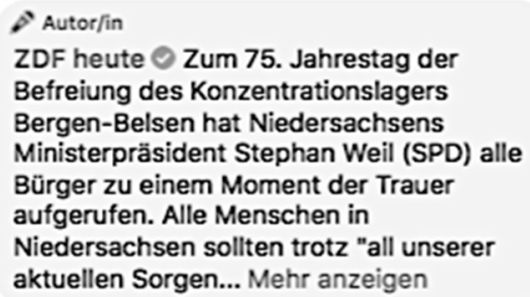

Abb. 1: Verifikation der ZDF heute Seite bei Facebook mittels „blauem Haken“ in der Kommentarspalte

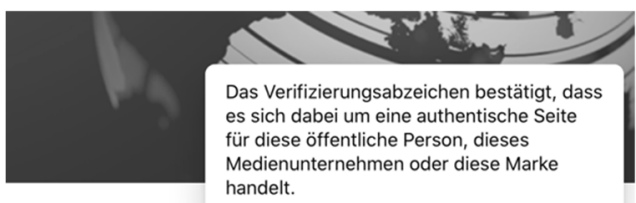

\section{ZDF heute}

@ZDFheute.Rundfunk- und

Medienproduktionsfirma

Abb. 2: Hintergrundinformationen zum Verifikationshinweis

1643 Damit folgt man den Ansätzen der Kennzeichnung beim Influencer-Marketing aufgrund des $₫ 5 \mathrm{a}$ Abs. 6 UWG, OLG Celle, NJW-RR 208, 42 Rn. 11 f. Und zu der Kennzeichnung im Lebensmittelrecht siehe etwa Heldt, CR 2018, 494 (498).

1644 Siehe hierzu bereits oben, B.II.1.c.aa.

1645 Das LG München, MMR 2019, 544 Rn. 43 ff. begründete damit nämlich, dass der kommerzielle Zweck der Kommunikation für Nutzer ersichtlich sei, sodass kein Verstoß gegen $\$ 5$ a Abs. 6 UWG vorlag. 
Auch wenn der Tatbestand auf den ersten Blick keine spezifischen Probleme aufwerfen mag, so wurde bereits während der Synopse wegen der konkreten Formulierung der Norm Kritik laut, auf die hier kurz eingegangen werden soll.

\section{a. Telemediendienstanbieter}

So wurde vereinzelt gemeint, dass unklar sei, wer als Telemediendienstanbieter eigentlich verpflichtet wird. ${ }^{1646}$ Allerdings ist vergleichsweise offensichtlich, dass durch $\mathbb{S} 18$ Abs. 3 MStV allein die Urheber und damit die hinter den Nutzerkonten stehenden Personen verpflichtet werden und nicht die Netzwerkbetreiber.

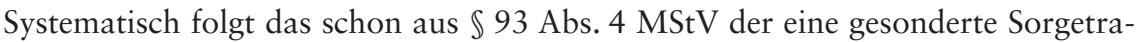
gungspflicht der Netzwerkbetreiber enthält, die ansonsten leerliefe. ${ }^{1647}$ Teleologisch ist aber auch zu berücksichtigen, dass die bestehende Regelungslücke für automatisiert erstellte Inhalte geschlossen werden soll. Da die Netzwerkbetreiber aber für die konkreten Inhalt ohnehin nicht verantwortlich sind, sondern nur die Plattform zur Verfügung stellen, kann nur der Verwender Adressat der Regelung sein.

\section{b. Definition eines Meinungsroboters}

Daneben wurde auch die gesetzgeberische „Definition“ der Meinungsroboter kritisiert, weil die Umschreibung des Regelungsgegenstandes zu weit sei. ${ }^{1648}$ Dem Wortlaut nach beschränkt sich die Norm nämlich nicht nur auf den problematischen Bereich der Meinungsroboter.

\section{aa. Begrenzung auf eine doppelte Täuschungswirkung?}

Diese zeichnen sich immerhin durch eine doppelte Täuschungswirkung aus: falsche Identität und Vorspiegelung menschlichen Verhaltens. ${ }^{1649}$ Die Norm erfasst aber auch Fälle, in denen ein real existierender Nutzer entsprechende Automatisierungen an seinem eigenen Profil vornimmt, beispielsweise wenn Personen des öffentlichen Lebens (Politiker oder Influencer) zur Vereinfachung bestimmter Aufgaben auf automatisierte Prozesse zurückgreifen, etwa um Antworten auf

1646 So etwa Dreyer/Schulz, Schriftliche Stellungnahme zum zweiten Diskussionsentwurf eines Medienstaatsvertrages der Länder vom Juli 2019, S. 16 zu dem wortgleichen damaligen $\$ 55$ Abs. 3 MStVE.

1647 Diese hat aber auch der zweite Entwurf schon in $\$ 53 \mathrm{~d}$ Abs. $4 \mathrm{MStV}$ enthalten. Insoweit ist nicht recht verständlich, warum Dreyer/Schulz, ebd. von einem unklaren Begriffsverständnis ausgehen.

1648 So etwa Verband der Internetwirtschaft, Stellungnahme zum MStV, LT-NRW Stellungnahme 17/2784, S. 5. Anders indes Löber/Roßnagel, MMR 2019, 493 (498).

1649 So auch Facebook, Positionspapier zum Diskussionsentwurf zu den Bereichen Rundfunkbegriff, Plattformregulierung und Intermediäre „Medienstaatsvertrag“, 2018, S. 8. Siehe auch Lent, in: Gersdorf/Paal, Informations- und Medienrecht, $\mathbb{1} 18$ MStV Rn. 20. 
Nutzerkommentare zu verfassen. Auch hier würde der Norm zufolge eine Kennzeichnungspflicht bestehen. ${ }^{1650}$ Dass eine solche Extension nicht gewollt ist, mag schon die Gesetzesbegründung nahelegen, wenn dort explizit auf Social Bots und auf ihren verdeckten Einfluss auf den Kommunikationsprozess hingewiesen wird. ${ }^{1651}$ Entscheidend mag aber vor allem sein, dass die Automatisierung grundrechtlich von der Meinungsäußerungsfreiheit geschützt ist. ${ }^{1652}$ Zwar kann die Meinungsäußerungsfreiheit wegen der Äußerungsmodalität hinter anderen Interessen zurücktreten. Dabei geht es aber - wie auch hinsichtlich anonymer Äußerungen $-{ }^{1653}$ um Konstellationen, indem mit der Automatisierung und der Identitätstäuschung kein sinnvoller, ${ }^{1654}$ sondern ein manipulativer Zweck verfolgt wird, der zu Reibungen mit den Grundsätzen des Kommunikationsprozesses führt. ${ }^{1655}$ Dies wärre bei Meinungsrobotern und einer doppelten Täuschungswirkung wegen des spezifische Transparenzinteresses ohne Weiteres anzunehmen. Automatisieren echte User ihre Profile, fehlt dieses Interesse indes, ${ }^{1656}$ denn hier ergibt sich kein wesentlicher Unterschied zwischen Automatisierung und Beauftragung einer anderen Person, die stellvertretend für den Profilinhaber entsprechende Aufgaben wahrnimmt. ${ }^{1657}$ Auch hier ist nach außen nicht erkennbar, dass nicht der eigentliche Profilinhaber handelt. Das ist aber auch nicht notwendig, weil die jeweilige Äußerung dem Inhaber des Profils ohne Weiteres zugerechnet werden kann. In derlei Fällen besteht der eigentliche Schutzbedarf also nicht in der Offenbarung der Betreuung des Profils durch Dritte, sondern in der Offenbarung der Identität des Äußernden. ${ }^{1658}$ Dort, wo eine Automatisierung bzw. eine Betreuung der Prä-

1650 Das kalifornische Recht ist dahingehend etwas restriktiver. Ein Bot liegt nach Sec. 17490 (a) des California Business und Professions Code vor, wenn alle oder „substantially all“ Interaktionen automatisiert und damit nicht "the result of a person “ sind. Dadurch werden zwar der sogleich aufgezeigte, aber nicht überzeugende weite Anwendungsbereich vermieden, aber neue Unklarheiten dadurch geschaffen, dass nicht hinreichend klar ist, wann die Interaktionen als „substantially all“ $\mathrm{zu}$ qualifizieren sind, hierzu und umfassend zu den Fragen, die die Norm auch im Übrigen aufwirft, Stricke, Vanderbilt Journal of Entertainment \& Technology Law Vol. 22 (2020), 839 ff.

1651 BayLT-Drs. 18/7640, S. 90; NiedersLT-Drs. 18/6414, S. 98.

1652 Lamo/Calo, UCLA Law Review 66 (2019), 988 (1009ff.) diskutieren dies im Kontext der Freedom of Speech auch unter dem Aspekt der „compelled speech“, weil der Betreiber zur Offenbarung gezwungen wird, wobei sie auch hier die Bedeutung der Informationen für Dritte herausarbeiten (S. $1010 \mathrm{ff}$.$) .$

1653 Hierzu etwa Benreuther, AfP 2011, $18 \mathrm{ff}$.

1654 Zwar nicht zu Meinungsrobotern, doch weisen etwa Franck/Pelzer, in: Taeger, Recht 4.0, S. 241 (244ff.) daraufhin, dass ein gesondertes Informationsbedürfnis bei Chatbots für Verbraucher und aus verbraucherschutzrechtlicher Sicht nicht bestünde.

1655 Ausführlich hierzu, wenn auch mit Blick auf die Meinungsfreiheit, bereits oben, A.I.3.b.

1656 Nach den Transparenzerwartungen der Nutzer differenzierend Lent, in: Gersdorf/Paal, Informations- und Medienrecht, $\mathbb{1} 18 \mathrm{MStV}$ Rn. 20, der zudem etwa den Gebrauch von Assistenzsoftware, die nur eine zeitliche Voreinstellung für den Versand von Inhalten ermöglicht, nicht erfasst wissen will, Rn. 19.

1657 Diesen Vergleich zieht etwa auch Coleman, Journal of media ethics Vol. 33 (2018), 120 (121) und hält diese Form des Einsatzes nicht „necessarily ethically transgressive“. Ebenso, allerdings mit Blick auf den Einsatz im E-Commerce, Raue/v. Ungern-Sternberg, ZRP 2020, 49 (52).

1658 Mit Blick auf die kalifornische Regelung weist Stricke, Vanderbilt Journal of Entertainment \& Technology Law Vol. 22 (2020), 839 (845), daraufhin, dass das Gesetz nur die durch die Äußerung 
senz notwendig wird, dürfte aber regelmäßig entweder die Kennzeichnungspflicht des $\mathbb{S} 5$ Abs. 1 TMG oder zumindest die des $\mathbb{} 18$ Abs. 1 MStV (ehem. \55 Abs. $1 \mathrm{RStV}$ ) ausgelöst werden, weil das Telemedium regelmäßig nicht mehr „ausschließlich persönlichen Zwecken“ dienen wird. Und blickt man etwa auf das Beispiel der Influencer, dient die Automatisierung gerade dem berufsorientierten Einsatz, sodass auch der grundrechtliche Schutz von Art. 12 Abs. 1 GG zu berücksichtigen wäre. ${ }^{1659}$ Hier mag aber auch ein erhebliches Interesse daran bestehen, dass dieser Umstand nicht nach außen hin bekanntgegeben werden muss, weil man ansonsten einen Vertrauens- oder Imageschaden riskiert. Auf diesem Vertrauen beruht immerhin der berufliche Erfolg. ${ }^{1660}$ Insoweit spricht vieles für eine Restriktion des Anwendungsbereichs von $\mathbb{S} 18$ Abs. $3 \mathrm{MStV}$ auf Fälle einer doppelten Täuschungswirkung und damit auf die eigentlichen Meinungsroboter. ${ }^{1661}$

von Bots begründeten „most social concern“ erfasst. Insoweit liegt dem also auch dort eine gewisse „Abwägung“ der kollidierenden Interessen zugrunde.

1659 Denn das Profil ist dann das wesentliche „Asset“ und Automatisierungsprozessen kommt hier dann eine gewisse Hilfsfunktion zu. Diese Hilfsfunktion ist etwa der Anknüpfungspunkt für die von Lent, in: Gersdorf/Paal, Informations- und Medienrecht, $\mathbb{} 18 \mathrm{MStV}$ Rn. 19 vorgenommene verfassungskonforme Reduktion der Norm bei Assistenzsoftware.

1660 Zu diesem Zusammenhang etwa Lettmann, GRUR 2018, 1206 (1208f.); Holznagel/Hartmann, in: Hoeren/Sieber/Holznagel, Multimedia-Recht, Teil 3 Rn. 212.

1661 In diese Richtung auch Dreyer/Schulz, Stellungnahme zum zweiten Diskussionsentwurf eines Medienstaatsvertrages, 2019, S. 16 f.; Liesem, K\&R 2019, 687 (691), die „eine funktionsorientierte Differenzierung " für notwendig hält, ohne dabei allerdings zu erklären, was damit genau gemeint ist. Jedenfalls auch kritisch zu einer Ausweitung der Kennzeichnungspflicht auf alle „Fälle einer Verwechslungsgefahr" Raue/v. Ungern-Sternberg, ZRP 2020, 49 (52). Holznagel, MMR 2018, 18 (22) meint - allerdings unabhängig von einer konkreten Regelung -, dass es „, [f]ür den Einsatz bestimmter Arten [...] Transparenzvorgaben geben [sollte]. " Lent, in: Gersdorf/Paal, Informations- und Medienrecht, $\mathbb{S} 18 \mathrm{MStV}$ Rn. 20, will es von der Transparenzerwartung der Nutzer im Einzelfall und damit von „Kommunikationsgegenstand und Nutzungskontext" abhängig machen. Anders indes Löber/Roßnagel, MMR 2019, 493 (497); Tendenziell in diese Richtung wohl auch Fechner, Medienrecht, Kap. 10 Rn. 211, wenn er meint, dass dem Nutzer ermöglicht werden soll, „zu unterscheiden, ob er es in einem sozialen Netzwerk mit einer natürlichen Person oder lediglich mit einer computergenerierten Mitteilung zu tun hat." Sollte sich - was der Gesetzesbegründung nicht zu entnehmen ist - auch an dem kalifornischen Vorschlag orientiert worden sein, so ist auch hier darauf hinzuweisen, dass der California Business and Professions Code in Sec. 17491 (a) gerade eine Begrenzung auf bestimmte Täuschungszwecke vorsieht. Neben kommerziellen Zwecken ist vor allem die Beeinflussung einer Wahlentscheidung erfasst (,,with the intent to $[\ldots]$ influence a vote in an election“). Und hier wird explizit nicht nur eine bloße Einschränkung auf soziale Netzwerke vorgesehen, vielmehr gilt die Kennzeichungspflicht hier im gesamten Online-Kommunikationsraum, S. Sec. 17490 (b). Für eine Begrenzung auf die eigentlichen Meinungsroboter spricht auch ein Hinweis in der Antwort auf eine kleine Anfrage der Staatsregierung Sachsens (Sächs DrS. 6/12131), wo unter Hinweis auf die Länderarbeitsgruppe „Social Bots“ auf die Frage was unter Social Bots zu verstehen sei, ausgeführt wird: „Die Arbeitsgruppe [...] hat, ,Social Bots“ $[\ldots]$ als Computerprogramme eingeordnet, die automatisiert in sozialen Medien kommunizieren und dabei vortäuschen, eine menschliche Identität zu haben. Ihr verdeckter Einsatz ziele darauf ab, menschliche Äußerungen zu fingieren.“, ebd. S. 3. Ähnliche Ausführungen finden sich ebenfalls im Antrag des Bündnis 90 /Die Grünen u.a., BT-DrS. 18/11856, S. 2. 
bb. Begrenzung auf allein meinungsbildungsrelevante Inhalte?

Daneben kann sich eine Notwendigkeit der Restriktion auch mit Blick auf die Verteilung der Kompetenzen zwischen Bund und Ländern ergeben. Zwar differenziert auch schon die allgemeine Kennzeichnungspflicht ( $\mathbb{S} 18$ Abs. $1 \mathrm{MStV}$ ) nicht zwischen kommerziell und nicht kommerziellen bzw. meinungsbildungsrelevanten Inhalten. Das ist aber deshalb zu vernachlässigen, weil das vom Bund erlassene TMG ohnehin eine entsprechende Regelung enthält ( $\$ 5$ Abs. 1 TMG) und zudem lässt sich das „Alles-oder-Nichts-Prinzip“ gut damit begründen, dass eine sinnvolle und praktikable Trennung bei gemischten Angeboten im Falle der Impressumspflicht kaum möglich ist. Hier könnte eine solche Differenzierung aber deshalb notwendig werden, weil eine Kennzeichnung bei rein kommerziellen Inhalten auch eine verbraucherschützende und wettbewerbsregulierende Funktion erfüllen würde, wodurch der wirtschaftsrechtliche Charakter der Regelung überwiegen würde. ${ }^{1662}$ Auf Bundesebene hat man aber sowohl mit dem TMG als auch mit dem UWG ${ }^{1663}$ abschließende Regelungen geschaffen, die keine Abweichungsmöglichkeiten der Länder vorsehen. ${ }^{1664}$ Ein Beispiel verdeutlicht das Problem: Wenn automatisiert in einem Netzwerk negative Bewertungen auf Unternehmensseiten veröffentlicht werden, dann besteht kein oder nur ein marginaler Zusammenhang zum Regelungszweck des MStV. Vielmehr wäre hier gerade das UWG einschlägig. ${ }^{1665}$ Die Rechtsfolge des MStV (Kennzeichnung) ist aber eine andere als die des UWG (Beseitigung, Unterlassung usw.). ${ }^{1666}$

Auch wenn die Kompetenzen pauschal an den Kriterien wirtschaftlich-kommerziell und inhaltlich-meinungsbildungsrelevant abgegrenzt werden können, so gilt das für die verschiedenen Inhalte gerade nicht. Ein Inhalt kann zuvörderst kommerziell bezweckt sein, aber zugleich eine meinungsbildende Relevanz auf-

1662 Noch deutlicher wird das durch den im Bundesstaat Kalifornien erlassen Bot Disclosure Act, der entsprechende Kennzeichnungspflichten in den Sec. 17490 ff. des California Business and Profession Codes eingefügt hat und vor allem auch explizit den kommerziellen Einsatz erfasst, hierzu siehe Sec. 17491 (a). Und auch dort stellen sich scheinbar Fragen mit Blick auf das föderale System, andeutend etwa Hines, Houston Law Review 57 (2019), 405 (414).

1663 Rauelv. Ungern-Sternberg, ZRP 2020, 49 (52) weisen auf den (ausreichenden) „Irreführungsschutz des UWG“ in kommerziellen Szenarien.

1664 In der SächsDrs. 6/12131, S. 2 wird gar explizit gefordert, dass der „Bundesgesetzgeber Akteuren mit Sitz in Deutschland und Drittstaaten, die Social Bots einsetzen, durch eine Ergänzung des Telemediengesetzes eine Pflicht zur Kennzeichnung von geschäftsmäßigen Beiträgen, die von Social Bots erstellt und versandt werden, auferlegen [sollte]. [Hervorh. d. Aut.] “ Allerdings spricht das nicht zwingend für eine Reduktion der medienstaatsvertraglichen Norm, sondern kann auch nur als sinnvolle Ergänzung u.a. zur Vermeidung von Missverständnissen gemeint sein. Denn wenig später wird gerade auch die Einführung der allgemeinen Norm ( $\$ 18 \mathrm{MStV})$ befürwortet, ebd. S. $2 \mathrm{f}$.

$1665 \mathrm{Zu}$ lauterkeitsrechtlichen Grenzen von Social Bots siehe in Ansätzen etwa Röttgen/Jülicher, in: Taeger, Recht 4.0, S. 227 (230f.); Dankert/Dreyer, K\&R 2017, 73 (77).

1666 Allerdings führt das nicht zu einem Widerspruch i.E., denn insoweit wäre nach dem UWG ja dennoch die unerlaubte Handlung zu Unterlassen ( $\$ 8$ Abs. 1 UWG). Insoweit wird die reine Kennzeichnung freilich „,verdrängt“. 
weisen. ${ }^{1667}$ Insoweit würden sich hier praktische Abgrenzungsschwierigkeiten ergeben. Zudem muss vorsichtig zwischen dem Hauptzweck und den unvermeidbaren Begleiterscheinungen der Regelung differenziert werden. Die Kennzeichnungspflicht zielt in erster Linie auf die Sensibilisierung der Nutzer und vor allem auf die Aufdeckung automatisierter Tätigkeiten in digitalen Kommunikationsräumen ab. Damit liegt der Hauptzweck aber gerade nicht auf den verbraucherschutz- oder wettbewerbsrechtlichen Aspekten des Einsatzes; sowohl im Verhältnis B2C als auch im Verhältnis B2B bleibt es vielmehr bei den bisherigen bundesrechtlichen Vorgaben. ${ }^{1668}$ Nicht zuletzt wird ein zu harscher Übergriff in die Bundeskompetenz gerade auch dadurch vermieden, dass erstens - anders als im kalifornischen Recht ${ }^{1669}$ - von vornherein nur bei einer Identitätstäuschung die Kennzeichnungspflicht greift und zweitens bestimmte - auch kommerziell motivierte - Automatisierungsprozesse aus dem Anwendungsbereich herausgenommen werden. Insoweit bedarf es gerade keiner Reduktion im Lichte der Kompetenzverteilung allein auf meinungsbildungsrelevante Inhalte.

\section{c. Soziales Netzwerk}

Und auch der Begriff des sozialen Netzwerks ist in seinen Konturen jedenfalls unscharf, was auch daran liegt, dass er - anders als die zentralen Begriffe in $\mathbb{} 2$ MStV - nicht legaldefiniert wird. Auch wenn begriffstechnisch (hierzu bereits oben 2. Teil, A.I) klar sein dürfte, dass es um Dienste geht, die maßgeblich auf sozialen Strukturen zwischen den Nutzern basieren und Interaktionen zwischen ihnen zulassen, so sind damit aber keine inhaltlichen Grenzen gezogen. Denn auch bei Diensten, bei denen die soziale Komponente allein spezifischen Zwecken dient, kann in einem weiten Sinne von einem sozialen Netzwerk gesprochen werden. Das gilt etwa für die beruflich ausgerichteten Dienste XING oder LinkedIn. Ein solch weites Verständnis des Begriffs konfligiert aber ebenfalls mit der Kompetenzverteilung, weil dann der Schwerpunkt eher auf der kommerziellen Regulierung und damit auf dem Recht der Wirtschaft (Art. 74 Abs. 1 Nr. 11 GG) und weniger auf der aus der Kulturpolitik der Länder folgenden inhaltlichen Medienregulierung liegt. ${ }^{1670}$ Deshalb überrascht es, dass nicht mal die Gesetzesbegründung Hinweise enthält, was konkret unter dem Begriff zu verstehen ist.

\footnotetext{
1667 Bestes Beispiel sind etwa die Werbebemühungen von Benetton, die das BVerfG letztlich unter den Schutzbereich auch der Meinungsfreiheit subsumierte, hierzu BVerfGE 102, 347 (362 ff.) -Schockwerbung I; 107, 275 (280 ff.) - Schockwerbung II. Und im Rundfunkbereich hat das BVerfG dies letztlich seit jeher betont, wenn es die Rundfunkfreiheit nicht allein auf politische Sendungen bezogen hat, etwa in BVerfGE 31, 314 (325 f.) - 2. Rundfunkentscheidung.

1668 Vgl. hierzu auch Heilmann, Anonymität für User-generated Content, S. 71, der deshalb auch nicht \55 RStV als Marktverhaltensregelung im Sinne von $\ 4$ Nr. 11 UWG begreifen will.

1669 Siehe auch hierzu erneut California Business and Professional Code Sec. $17490 \mathrm{f}$.

1670 Dazu schon oben, B.I.1.
} 
Einen überzeugenden Ausweg kann aber die Anlehnung an die in $\mathbb{S} 1$ Abs. 1 S. 1 NetzDG enthaltene Legaldefinition bieten. Einerseits würde man damit - wie schon bei der parallelen und abgestimmten Normierung von TMG und RStV - ${ }^{1671}$ kongruente Begriffe verwenden und so die Rechtsklarheit und -Einheit stärken. Andererseits wurde mit dem NetzDG mittelbar auch der Kommunikationsprozess geschützt, ${ }^{1672}$ sodass sich auch in sachlicher Hinsicht zumindest Schnittmengen ergeben. Nach $\mathbb{S} 1$ Abs. 1 NetzDG meint der Begriff „Telemediendiensteanbieter“ Anbieter, die mit Gewinnerzielungsabsicht Plattformen im Internet betreiben, die dazu bestimmt sind, dass Nutzer beliebige Inhalte mit anderen Nutzern teilen oder der Öffentlichkeit zugänglich machen." Entscheidende Bedeutung kommt mit Blick auf das oben beschriebene Problem dem Merkmal der „beliebigen Inhalte“ zu. Denn schon nach der Gesetzesbegründung des NetzDG ging es darum, Plattformen, die zwar eine soziale Interaktion aufweisen, aber spezifisch themenbezogenen Zwecken dienen, nicht zu erfassen. ${ }^{1673}$ Exemplarisch wurden hierbei gerade die berufsorientierten Netzwerke genannt. ${ }^{1674}$ Zwar kann es auch dort vereinzelt dazu kommen, das meinungsbildungsrelevante Inhalte geteilt werden, entscheidend ist aber die allgemeine Ausrichtung der Plattform. ${ }^{1675}$ Beim NetzDG resultiert die Unterscheidung aus der Anzahl der potentiellen Rechtsverstöße, die sich bei spezifischen themenbezogenen Plattformen nicht in demselben Umfang ergeben werden. ${ }^{1676}$ Diese Überlegungen dürften hier analoge Anwendung finden, weil entsprechende Plattformen keine oder eine deutlich geringere Bedeutung für den Kommunikationsprozess haben. ${ }^{1677}$ Insoweit bietet sich also auch hier eine Orientierung am eigentlichen Zweck des jeweiligen Angebots an. Ist dieser vor allem kommerziell ausgerichtet oder handelt es sich um spezialisierte Angebote (bspw. Themenforen), die dem eigentlichen Zweck politischer Meinungsbildung nicht oder nur in einem unerheblichen Umfang dienen, so sind sie nicht als soziales Netzwerk im Sinne der Norm anzusehen. ${ }^{1678}$ Und auch für Dienste, bei denen sich das soziale Element überwiegend auf eine Oneto-One-Konversation erstreckt, dürfte das Vorliegen eines sozialen Netzwerks bezweifelt werden. Denn hier wird - wie auch schon bei $\$ 55$ Abs. 1 RStV ${ }^{1679}$ kein

1671 Hierzu statt vieler Roßnagel, NVwZ 2007, $743 \mathrm{f}$.

1672 Der Gesetzesentwurf BT-DrS. 18/12356, spricht hier insoweit von der „Debattenkultur“ (S. 1, 11) oder von einer „massiven Veränderung des gesellschaftlichen Diskurses im Netz“ (S. 11). Und mit Blick auf die Legaldefinition soll zudem gerade sichergestellt werden, „dass die Berichtspflicht nur die Betreiber großer sozialer Netzwerke mit Meinungsmacht [...] trifft". (S. $11 \mathrm{f}$.).

1673 BT.-Drs. 18/12356, S. 19.

1674 BT.-Drs. 18/12356, S. 19.

1675 BT.-Drs.18/12356, ebd.

1676 Hoven/Gersdorf, in: Gersdorf/Paal, Informations- und Medienrecht, $\$ 1$ NetzDG Rn. 16.

1677 Das jedenfalls ist auch der Ansatz, der zu der Einschränkung des Anwendungsbereichs des MStV auf Medienintermediäre führt, vgl. hierzu $\mathbb{} 91$ Abs. 2 Nr. 2, 3 MStV und die Gesetzesbegründung hierzu, BayLT.-Drs. 18/7640, S. 108 oder NiedersächsLT-Drs. 18/6414, S. 115.

1678 Vgl. zum NetzDG BT.-Drs. 18/12356, S. 19.

1679 Dazu siehe bereits oben, C.I.3.c.bb. 
schutzwürdiges Interesse an einer Kennzeichnung bestehen, immerhin wird die gegenüberstehende Person meist bekannt sein. Das findet auch eine Stütze in $\mathbb{} 1$ Abs. 1 S. 1 NetzDG: Weder werden Inhalte mit Nutzern geteilt noch der Öffentlichkeit zugänglich gemacht.

\section{d. Fazit: Meinungsroboter als zentraler Regulierungsgegenstand}

Die Formulierung des $\mathbb{S} 18$ Abs. 3 des $\mathrm{MStV}$ geht weit über den eigentlich diskutierten Anwendungsfall sog. Meinungsroboter hinaus. Dabei ist durchaus diskutabel, ob man damit verfassungsrechtliche, insbesondere grundrechtliche und kompetenzielle Grenzen, überschreitet. Allerdings ließe sich die Norm auch teleologisch und in verfassungskonformer Weise reduzieren, sodass die Probleme eher auf die Ebene der Rechtsanwendung verlagert werden. Für den hier vorliegenden Untersuchungsgegenstand ist dies aber letztlich gleichgültig. Die Meinungsroboter sind immerhin das gesetzgeberische Leitbild für die neu eingeführte Kennzeichnungspflicht.

\section{Vorgaben für Medienintermediäre, $\int \mathbb{S} 91-96 \mathrm{MStV}$}

Neben den Vorgaben für die Verwender von Meinungsrobotern enthält der MStV auch spezielle Vorgaben für Medienintermediäre, die einerseits mittelbare Bedeutung erlangen, wenn es um allgemeine Vorgaben den Prozess der Informationsvermittlung betreffend geht (b.) und andererseits unmittelbar relevant werden, soweit die Betreiber sozialer Netzwerke auch für Meinungsroboter eine gewisse Sorgfaltspflicht zu erfüllen haben (c.).

\section{a. Begriff des Medienintermediärs}

Als Medienintermediär gilt gem. $\$ 2$ Abs. 2 Nr. 16 MStV ein „Telemedium, das auch journalistisch-redaktionelle Angebote Dritter aggregiert, selektiert und allgemein zugänglich präsentiert, ohne diese zu einem Gesamtangebot zusammenzufassen“. Wenn auch hier Unklarheiten bei einzelnen Diensten bestehen mögen, ${ }^{1680}$ so erfolgt eine solche Aggregation und Selektion jedenfalls auch mithilfe der Algorithmen sozialer Netzwerke. Der im Rahmen der Onlinebeteiligung gemachte Vorschlag enthielt eine entsprechende Auflistung, die diese explizit auf-

1680 Der Digitale Gesellschaft e.V., Stellungnahme zum Entwurf des 23. Rundfunkänderungsstaatsvertrages „Medienstaatsvertrag“, 2018, S. 6, weist etwa daraufhin, dass unklar sei, ob auch Dienste wie Wikipedia hierunter fallen, weil die Inhalte von Usern verfasst werden - also als redaktionell gestaltet gelten könnten - und durch Wikipedia letztlich bereitgestellt werden. 
führte ${ }^{1681}$ und in der Gesetzesbegründung werden sie ebenfalls als „Regelfall“ eingestuft. ${ }^{1682}$ Ähnlich wie beim NetzDG ( $\mathbb{1}$ Abs. 2 NetzDG) stellt $\mathbb{9} 91$ Abs. 2 MStV allerdings die Verpflichtungen unter einen Bagatellvorbehalt ( Nr. 1), was weiterhin Innovationen erlaubt, ${ }^{1683}$ und womit die Regulierung gerade von einer gewissen (vermuteten) Meinungsbildungsrelevanz des jeweiligen Dienstes abhängt. ${ }^{1684}$ Zeitgleich wird durch den Ausschluss kommerzieller Dienste sichergestellt, dass hier nur der originäre, medienrechtlich relevante Bereich erfasst wird $\left(\right.$ Nr. 2) ${ }^{1685}$ und dass nicht regulierungsbedürftige Privatkommunikation nicht den Verpflichtungen unterfällt (Nr. 3).

\section{b. Allgemeine Regulierungsvorgaben für Medienintermediäre}

Inhaltlich zielt die Regulierung mit $\mathbb{S} 93 \mathrm{MStV}$ auf die Verbesserung der Transparenz für die Nutzer ab und ordnet insbesondere an, dass bestimmte für die Informationsvermittlung notwendige Hintergrundinformationen für Nutzer „leicht wahrnehmbar, unmittelbar erreichbar und ständig verfügbar zu halten [sind]“ ( $\$ 93$ Abs. $1 \mathrm{MStV}) .{ }^{1686}$ Neben den Kriterien, die für die Veröffentlichung entscheidend sind (Abs. 1 Nr. 1), gehören auch die zentralen Kriterien, die den Algorithmen zugrunde liegen, hierzu (Nr. 2). Was aber als zentrales Kriterium anzusehen ist, verschweigt die Norm. ${ }^{1687}$ Die noch von der Bund-Länder-Kommission $^{1688}$ geforderten Regelbeispiele wurden nicht normiert. ${ }^{1689}$ Insoweit liegt die Entscheidung hierüber zunächst in der Hand der Betreiber. Allerdings müssen sich die Angaben und ihr Umfang an dem Ziel der Transparenzvorschrift orien-

1681 Siehe hierzu Diskussionsentwurf für einen „Medienstaatsvertrag“, $\mathbb{2}$ Abs. 2 Nr. 13b, abrufbar unter https:/www.rlp.de/fileadmin/rlp-stk/pdf-Dateien/Medienpolitik/MStV-E_Synopse_2019-07_Onl ine_.pdf, (Stand: 30.05.2021).

1682 NiedersächsLT-Drs. 18/6414, S. 94; BayLT-Drs. 18/7640, S. 85.

1683 So etwa Bund-Länder-Kommission, Bericht zur Medienkonvergenz, 2016, S. 36. Und zum NetzDG siehe BT-Drs. 18/12356, S. 19, auch wenn hier eher auf den mit den normierten Pflichten einhergehenden Aufwand abgestellt wurde. Gleichwohl wird a.a.O., S. 12 explizit auch auf „Start-up-Unternehmen" hingewiesen.

1684 Kritisch zur gewählten Anzahl der „unique user“ etwa Ory, ZUM 2019, 139 (152).

1685 So auch der Hinweis in der Gesetzesbegründung, siehe nur BayLT-Drs. 18/7640, S. 108.

$1686 \mathrm{Zu}$ den inhaltlichen Anforderungen ausführlicher etwa Schwartmann/Hermann/Mühlenbeck, Transparenz von Medienintermediären, S. $118 \mathrm{ff}$.

1687 Auch die Gesetzesbegründung hilft nicht weiter. Allein mit Blick auf Nummer 2 sind zwei Beispiele genannt: „der Standort des Nutzers oder dessen Sprache“, NiedersächsLT-Drs. 18/6414, S. 115; BayLT-Drs. 18/7640, S. 108. Deshalb aber auch allgemein kritisch zur Norm Ladeur/Gostomzyk, K\&R 2018, 686 (690f.); Cornils, ZUM 2019, 89 (102). Zur ursprünglich vorgeschlagenen Regelung des damaligen $₫ 53$ d Abs. 1 Nr. 2 MStV Kellner, Die Regulierung der Meinungsmacht von Internetintermediären, S. 28.

1688 Bund-Länder-Kommission, Bericht zur Medienkonvergenz, 2016, S. 36.

1689 Das hat die P2B-Verordnung 2019/1150, ABl. 2019 L 186, S. 57 ff. besser gelöst. Sie gibt in Art. 5 Abs. 5 lit. a) -c) zumindest gewisse Leitlinien vor, an denen sich die Plattformen bei der Veröffentlichung von transparenten Informationen zu orientieren haben. 
tieren. ${ }^{1690}$ Hier kann es aber - das zeigt nicht nur der Wortlaut, sondern auch ein systematischer Vergleich innerhalb des $\mathrm{MStV}^{1691}$ und mit anderen Transparenzvorschriften ${ }^{1692}$ - allein um die Stärkung der Nutzerautonomie gehen, sodass sich die Informationen jedenfalls an diesem Ziel zu orientieren haben. ${ }^{1693}$ Dass die Umsetzung im Einzelnen schwierig ist, weil nicht hinreichend klar ist, welcher Umfang angezeigt ist, in welcher Tiefe Informationen notwendig sind und wie diese darzustellen sind, ${ }^{1694}$ ändert an der prinzipiellen Verpflichtung nichts. Mit \$96 MStV besteht jedenfalls die Möglichkeit, die abstrakten Vorgaben etwa durch Best-Practice-Beispiele zu konkretisieren. ${ }^{1695}$ Nehmen die Netzwerkbetreiber Änderungen an den Kriterien vor, müssen diese nach $\$ 93$ Abs. 3 MStV unverzüglich und in derselben Weise kenntlich gemacht werden. Teilweise wurde kritisch darauf hingewiesen, dass die Algorithmen stetig Änderungen unterliegen. ${ }^{1696}$ Allerdings setzt die Norm ja nicht voraus, dass per se jede Änderung offenbart wird, sondern nur wenn und soweit sie die zentralen Kriterien betreffen.

Neben diesen Transparenzanforderungen sieht $\mathbb{S} 94$ Abs. 1 MStV ein Diskriminierungsverbot für redaktionell-journalistische Angebote ${ }^{1697}$ vor. Damit be-

1690 Vgl. Dogruel u.a., Media Perspektiven, 139 (142 f.). Vgl. auch Zimmer, in: Gersdorf/Paal, Informations- und Medienrecht, $\mathbb{S} 93 \mathrm{MStV} \mathrm{Rn}$. 14. Und erste Überlegungen zu den formellen und inhaltlichen Anforderungen finden sich a.a.O., Rn. $5 \mathrm{ff}$.

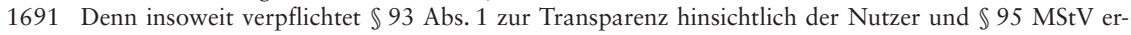
weitert diese durch entsprechende Berichtspflichten auch auf das zuständige Kontrollgremium (ZAK). Durch die Berichtspflicht könnten also entsprechend präzisere Informationen verlangt werden.

1692 Schon Heldt, CR 2018, 494 (498) zieht die Parallele zu den lebensmittelrechtlichen Transparenzpflichten. Auch dort heißt es in Art. 12 Abs. 1 der VO 608/2004, dass die verpflichtenden Informationen „verfügbar“ und „leicht zugänglich“ sein müssen und in Art. 15 heißt es, dass die Informationen „in einer für die Verbraucher [...] leicht verständlichen Sprache abzufassen [sind]. “ Siehe daneben aber auch Art. 5 Abs. der VO 2019/1150 zur Förderung von Fairness und Transparenz für gewerbliche Nutzer von Online- Vermittlungsdiensten, ABl. L 186, S. 57, der eine ähnliche Formulierung aufweist und die Position der kommerziellen Nutzer stärken soll, hierzu auch Erwggr. 18.

1693 So auch Laude, JuWiss-Blog Nr. 126/2020.

1694 Siehe hierzu etwa Laude, JuWiss-Blog Nr. 127/2020. Teilweise wurden hier Bestimmtheitsbedenken geäußert und gefordert, dass schon der Gesetzgeber eine gewisse Differenzierung vornimmt, in diese Richtung etwa Liesem, AfP 2020, 277Rn. 26, 27. Zu berücksichtigen ist aber, dass die Anforderungen an die Bestimmtheit vom Regelungsgegenstand und Bereich abhängen (hierzu mit zahlreichen Nachweisen auf die verfassungsgerichtliche Judikatur etwa Grzeszick, in: Maunz/Dürig, GG, Art. 20 Rn. 58 ff.). Insoweit ist hier einerseits zu berücksichtigen, dass ganz unterschiedliche Angebote erfasst sind, die eine unterschiedliche Akzentuierung notwendig machen, sodass man dem in der Breite - auch für zukünftige Anbieter - nicht gesetzlich gerecht werden kann. Andererseits ist auch zu berücksichtigen, dass hierfür eine gewisse Kenntnis der unterschiedlichen Dienste notwendig wäre. Ein staatlicher Einblick wird aber erst durch die Ermittlungsbefugnisse $(\mathbb{\$} \$ 95,56 \mathrm{MStV})$ ermöglicht, sodass eine Konkretisierung nur in Abhängigkeit entsprechender Kenntnisse erfolgen kann. Insoweit ist es durchaus sinnvoll, den Betreibern zunächst die Umsetzung zu überlassen und diese danach zu konkretisieren (\$96 MStV).

1695 Ausführlicher zu $\$ 96 \mathrm{MStV}$ noch unten, C.II.2.d. Zur Notwendigkeit einer Konkretisierung gerade durch derartige Beispiele, siehe etwa Dogruel u.a., Media Perspektiven 2020, 139 (143).

1696 So allen voran Facebook Germany, Positionspapier zum Diskussionsentwurf zu den Bereichen Rundfunkbegriff, Plattformregulierung und Intermediäre „Medienstaatsvertrag“, 2018, S. 6.

1697 Hierzu mit Blick auf das Diskriminierungsverbot (auch kritisch) etwa Kellner, Die Regulierung der Meinungsmacht von Internetintermediären, S. 289. 
dient man sich der Begrifflichkeit, die auch zuvor im RStV verwendet wurde. ${ }^{1698}$ Als redaktionell-journalistisch versteht man hierbei nur solche Angebote, die eine gewisse Periodizität aufweisen, publizistisch ausgerichtet ${ }^{1699}$ und universell, aktuell und faktenbasiert sind (journalistisch), sofern dabei eine Inhaltsauswahl und -bearbeitung sowie eine gewisse Vereinheitlichung stattfindet und eine gewisse organisatorische Verfestigung vorhanden ist, die Kontinuität gewährleistet (redaktionell). ${ }^{1700}$ Die Abgrenzung kann im Einzelfall schwierig sein. ${ }^{1701}$ Während kommerzielle Kommunikation nicht erfasst sind, ${ }^{1702}$ könnten persönliche Blogs oder ähnliche Angebote hierunter fallen. ${ }^{1703}$ Entscheidend wird insoweit die publizistische Ausgestaltung ${ }^{1704}$ des Angebots und der Grad der redaktionellen Arbeit ${ }^{1705}$, um den angebotsprägenden Charakter zu ermitteln. ${ }^{1706}$

Eine Diskriminierung solcher Inhalte kommt in zwei Fällen in Betracht: einerseits, wenn von den zu veröffentlichenden Kriterien systematisch abgewichen wird ( $\mathbb{S} 94$ Abs. 2, 1. Alt. MStV) und andererseits, wenn die Kriterien selbst eine mittelbare oder unmittelbare systematische unbillige Behinderung bestimmter Inhalte darstellen. Insoweit wird also verhindert, dass durch die transparenten Kriterien selbst eine neue Diskriminierung geschaffen wird. In beiden Fällen soll verhindert werden, dass bestimmte Angebote allein wegen ihrer inhaltlichen oder

1698 Siehe etwa $\mathbb{S} 54$ Abs. 2, 55 Abs. 2, 56 Abs. 1, 57 RStV. Dieser Begriff wird auch einheitlich verwendet, „da Rechte und Pflichten der Anbieter solcher Dienste in einem ausgewogenen Verhältnis stehen. Dies würde aufgebrochen werden, wenn einzelne Rechte oder Pflichten isoliert einzelnen Ange-

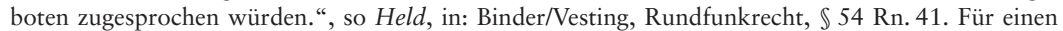
einheitlichen Begriff auch Lent, in: Gersdorf/Paal, Informations- und Medienrecht, $\mathbb{S} 54$ RStV Rn. 5.

1699 Das bedeutet nicht nur, dass sie an die Öffentlichkeit gerichtet sind, sondern auch dass sie eine publizistische Relevanz besitzen und damit mit der herkömmlichen Presse vergleichbar sind, vgl. Weyhe, in: Paschke/Berlit/Meyer/Kröner, Medienrecht, 37. Abschnitt Rn. 192.

1700 Siehe hierzu etwa Lent, in: Gersdorf/Paal, Informations- und Medienrecht, $\$ 54$ RStV Rn. 5; Held, in: Binder/Vesting, Rundfunkrecht, $\mathbb{5} 54 \mathrm{Rn} .48$. Aus der Rspr. etwa OVG Berlin-Brandenburg, LKV 2015, 180 (181); VGH Baden-Württemberg, ZUM-RD 2014, 396 (399); OLG Bremen, NJW 2011, 1611 (1612), VG Schwerin, ZUM-RD 2015, 353 (355).

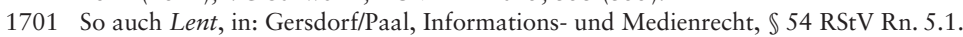

1702 So etwa OVG Bautzen, MMR 2017, 716 Rn. 9; OVG Berlin Brandenburg, LKV 2015, 180 (181).

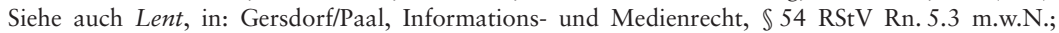
Held, in: Binder/Vesting, Rundfunkrecht, $\$ 54$ Rn. 59.

1703 Vgl. Held, in: Binder/Vesting, Rundfunkrecht, $\mathbb{\int} 54$ Rn. 39; Weyhe, in: Paschke/Berlit/Meyer/Kröner, Medienrecht, 39. Abschnitt Rn. 193. Eine Auflistung verschiedener Angebote die als redaktionelljournalistisch einzuordnen sind, findet sich etwa bei Lent, in: Gersdorf/Paal, Informations- und Medienrecht, $\mathbb{S} 54 \mathrm{RStV}$ Rn. $5.1 \mathrm{ff}$.

1704 Den fehlenden meinungsbildenden Bezug betont etwa der BGH, NJW 2015, 3377 Rn. 38 . Ebenso das OVG Schleswig-Holstein, ZUM-RD 2017, 696 (698) zum presserechtlichen Auskunftsanspruch, oder das OVG Bautzen, MMR 2017, 716 Rn. 8. Und der BayVGH, ZUM-RD 2017, 349 (350) betont gerade die meinungsbildende Zielsetzung des Dienstes als wesentliches Kriterium. Ebenso Held, in: Binder/Vesting, Rundfunkrecht, $\mathbb{} 54$ Rn. 51 und 58 a mit Blick auf Twitterprofile.

1705 Hieran scheiterte es etwa in der Entscheidung des OVG Greifswald, NordÖR 2013, 258 (259), weil eine Vorauswahl gar nicht getroffen wurde. Dies betont auch Held, in: Binder/Vesting, Rundfunkrecht, $\mathbb{S} 54 \mathrm{Rn} .39$.

1706 Vgl. Lent, in: Gersdorf/Paal, Informations- und Medienrecht, $\mathbb{5 4}$ RStV Rn. 5.3; VGH Mannheim (1. Senat), Urteil vom 09.05.2017 - 1 S 1530/16 = BeckRS 2017, 112670, Rn. 63, 64, 67f. Siehe wenn auch zu $\mathbb{4} 41$ Abs. 1 BDSG a.F. BGH, NJW 2009, 2888 Rn. 21. 
politischen Ausrichtung oder aufgrund anderer Kriterien benachteiligt werden. ${ }^{1707}$ Eine Diskriminierung kann aber sachlich gerechtfertigt werden, insbesondere wenn rechtswidrige Inhalte betroffen sind. ${ }^{1708}$ Und eine unbillige systematische Behinderung liegt auch erst dann vor, wenn die Interessen der Betreiber gegenüber den Interessen der Betroffenen zurückzutreten haben. ${ }^{1709}$ Deshalb können insbesondere Personalisierungsprozesse, die mittelbar $\mathrm{zu}$ einer systematischen Bevorzugung von Inhalten führen, durchaus billig im Sinne der Vorschrift sein. ${ }^{1710}$

Das Diskriminierungsverbot ist auch prozessual ausgestaltet und gibt betroffenen Anbietern von Inhalten die Möglichkeit, Verstöße bei der zuständigen Landesmedienanstalt geltend zu machen ( $\mathbb{S} 93$ Abs. 3 S. 1 MStV). In offensichtlichen Fällen kann die Landesmedienanstalt auch von Amts wegen tätig werden. ${ }^{1711}$

\section{c. Sorgetragungspflicht für Betreiber sozialer Netzwerke nach $\int 93$ Abs. 4 MStV}

Besonders hervorzuheben ist, dass die bereits genannte Kennzeichnungspflicht des $\mathbb{S} 18$ Abs. $3 \mathrm{MStV}$ in $\mathbb{S} 93$ Abs. $4 \mathrm{MStV}$ auf die Betreiber sozialer Netzwerke - sofern sie als Medienintermediäre gelten - erweitert wird. Damit wird gerade ein anderer Weg eingeschlagen als etwa durch den kalifornischen Gesetzgeber. ${ }^{1712}$ Sie selbst trifft hiernach die Pflicht, für eine Kennzeichnung entsprechender Inhalte Sorge zu tragen.

\section{aa. Die Bedeutung der Sorgetragungspflicht des $\mathbb{} 93$ Abs. $4 \mathrm{MStV}$}

Allerdings wird - anders als bei $\mathbb{S} 18$ Abs. $3 \mathrm{MStV}$ - nicht erkennbar, durch welche Maßnahmen die Betreiber dieser Pflicht nachkommen können. ${ }^{1713}$ Dass dies

1707 Vgl hierzu NiedersächsLT-Drs. 18/6414, S. 116; BayLT-Drs. 18/7640, S. 109.

1708 NiedersächsLT-Drs. 18/6414, S. 117; BayLT-Drs. 18/7640, S. 110.

1709 NiedersächsLT-Drs. 18/6414, S. 117; BayLT-Drs. 18/7640, S. 110.

1710 Vgl. Schneiders, Das ist doch Diskriminierung, S. 6.Ausdrücklich so Zimmer/Liebermann, in: Gersdorf/Paal, Informations- und Medienrecht, $\mathbb{\$} 94 \mathrm{MStV}$ Rn. 8. Die Gesetzesbegründung weist immerhin auf die „nach Artikel 12 des Grundgesetzes [geschützte] unternehmerische Freiheit“ hin, NiedersächsLT-Drs. S. 18/6414, S. 116; BayLT-Drs. S. 18/7640, S. 109.

1711 Nach den vorherigen Diskussionsentwürfe konnten nur betroffene Inhalteanbieter einen Verstoß geltend machen, $\mathbb{S} 53$ e Abs. $3 \mathrm{MStV}$ in der Fassung vom Juli 2019.

1712 Zwar wurden ähnliche Verpflichtungen auch dort diskutiert. Sie fanden allerdings keinen Eingang in das endgültige Gesetz (hierzu Stricke, Vanderbilt Journal of Entertainment\&Technology Law Vol. 22 (2020), 839 (843)). Sec. 17492 (c) des California Business and Professions Code sieht nämlich ausdrücklich vor, dass durch die neu eingefügten Regeln den Dienstanbietern von online Plattformen keine Pflichten auferlegt werden. Hier bleibt es allein bei einem möglichen Auskunftsersuchen an die Betreiber, ausführlich hierzu Stricke, ebd., S. 844, 860 ff. Eine entsprechende Ausweitung der Verantwortlichkeit der Netzwerkbetreiber fordert aber Hines, Houston Law Review 57 (2019), 405 (434).

1713 So auch Laude, JuWiss-Blog Nr. 127/2020 v. 02.11.2020. Vergleichsweise abstrakt bleiben auch die Forderungen von Hines, ebd.: „a future bot law must not only go after users, but must also go after social networks for not taking steps to prevent the behavior themselves." Der von US-Senatorin 
durchaus gewollt und sinnvoll ist, wird noch aufzuzeigen sein, vermag aber nicht über die Tatsache hinwegzutäuschen, dass - gerade auch mit Blick auf den Bußgeldtatbestand des $\mathbb{S} 115$ Abs. 1 S. 1 Nr. 42 MStV - die Unklarheit erhebliche Probleme mit sich bringt. Das führt zwar nicht per se zu ihrer Unbestimmtheit, denn die Bestimmtheitsanforderungen sind immer auch abhängig vom konkreten Regelungsbereich und den damit verbundenen Schwierigkeiten einer Regulierung. Solange sich die Norm mithilfe allgemeiner Auslegungscanones näher präzisieren lässt, kann die Norm auch den Bestimmtheitsanforderungen genügen, zumal - wie noch aufzuzeigen wird - hier gerade Gründe dafür bestehen, dass die weite Formulierung gewählt wurde. Besonders auch verfassungs- und unionsrechtliche Implikationen sind hierbei zu berücksichtigen.

\section{(I.) National-rechtliche Auslegungscanones}

\section{(1.) Wortlaut „Sorge tragen“}

Geht man vom Wortlaut der Norm aus, so kann jedenfalls keine „Sicherstellung“ verlangt werden. Sorge trägt schon derjenige, der sich nur um die Erreichung eines Ziels bemüht. ${ }^{1714}$ Insoweit ist jedenfalls die untere Grenze des "Sorge-Tragens“ klar definierbar: der Betreiber darf nicht untätig bleiben. Die Sorgetragungspflicht betrifft aber letztlich zwei potentielle Verantwortungssphären: den Dienst als eigenes Handeln und die Tätigkeiten der Nutzer und damit fremdes Handeln. Im ersten Fall würde das "Sorge tragen“ tendenziell darauf abzielen, die Nutzer selbst in die Lage zu versetzen, die Kennzeichnung vornehmen zu können. In den Worten von Löber und Roßnagel geht es darum, die „technischen Strukturen bereitzustellen“1715. Geht es indes um fremdes Handeln, insbesondere um eine Verantwortung in Fällen, in denen eine Kennzeichnung (absichtlich) unterbleibt, bleibt unklar, was unter „Sorge tragen“ zu verstehen ist. Müssen die Betreiber gewährleisten, dass weit überwiegend die Kennzeichnungspflicht eingehalten wird? ${ }^{1716}$ Muss der Netzwerkbetreiber hierfür selbst kennzeichnen oder die Nutzerkonten selbst überprüfen? Die potentielle Reich-

Feinstein 2018 eingebrachte US Senate Bill 3127 (sog. „Bot Disclosure and Accountability Act 2018“) sieht demgegenüber konkretere Vorgaben vor (S. 8 des Gesetzesvorschlags): Einerseits „a process by which the social media provider will take reasonable preventative and corrective action to mitigate efforts by a user to use an automated software program or process intended to impersonate or replicate human activity online without disclosure as required under paragraph (1), which may include suspension or any other action authorized by the Commission “ und andererseits „a process by which the social media provider will remove posts, images, or any other online activity of a user or profile making use of an automated software program or process intended to impersonate or replicate human activity online that is not in compliance with the policy under paragraph (1) ".

1714 So auch Löber/Roßnagel, MMR 2019, 493 (498).

1715 Löber/Roßnagel, MMR 2019, 493 (498).

1716 Dies ablehnend Laude, JuWiss-Blog Nr. 127/2020. 
weite reicht hier von einem Identitätsnachweis bei Registrierung, ${ }^{1717}$ der Einrichtung eines Beschwerdemanagements vergleichbar mit dem des NetzDG bis hin zu einer Überprüfung der Netzwerkdaten, durch die auffällige Nutzerkonten ausgemacht werden und diese dann betreiberseitig zu kennzeichnen ${ }^{1718}$ oder gar zu sperren $^{1719}$. Der Wortlaut hilft bezüglich dieser zweiten Sphäre nicht weiter.

\section{(2.) Systematik}

Ein systematischer Vergleich $\mathrm{zu}$ anderen Regelungstatbeständen fällt deshalb schwer, weil sich andere Kennzeichnungspflichten ausschließlich an den Anbieter des Telemediums richten. Allerdings wurde auch im Bereich des Influencer-Marketings auf der Plattform Instagram bereits das Problem ersichtlich, dass eine nur unzureichende Gestaltungsmöglichkeit zu Problemen bei der Kennzeichnung von kommerziellen Inhalten führen kann. Dort war lange Zeit unklar, wie eine ordnungsgemäße Kennzeichnung erfolgen muss, weil man darauf beschränkt war, durch entsprechende Bildbeschreibung - etwa durch Hashtags (\#ad, \#sponsoredby etc.) - den kommerziellen Charakter aufzuzeigen, ${ }^{1720}$ was $\mathrm{zu}$ uneinheitlichen Praktiken führte. ${ }^{1721}$ Auch hier war man also darauf angewiesen, dass der Betreiber zur Vereinheitlichung eine entsprechende Bezeichnung einführt. ${ }^{1722}$ Dieses Problem umgeht man also, wenn $\mathbb{S} 93$ Abs. $4 \mathrm{MStV}$ die Betreiber zum Sorge-Tragen verpflichtet. Insoweit geht es also zunächst um die Gewährleistung der technischen Voraussetzungen.

Berücksichtigt man zudem bisherige Haftungstatbestände, so trifft die Anbieter der Netzwerke bislang keine eigene Verantwortung, die Inhalte der Nutzer entsprechend zu kennzeichnen. Primär ist allein der jeweilige Profilinhaber verantwortlich. Dritte sind nur dann verantwortlich, wenn ihnen dies zugerechnet werden kann, etwa beim beauftragenden Unternehmen unter den Voraussetzungen des $\mathbb{\int} 8$ Abs. 2 UWG. ${ }^{1723}$ Eine Haftung der Betreiber der Plattformen käme al-

1717 Milker, InTeR 2017, 199 (205, 206).

1718 In diese Richtung Löber/Roßnagel, MMR 2019, 493 (498).

1719 Auch wenn die Regelung auf das Sorge tragen einer Kennzeichnung abzielt, so kann eine Sperrung auffälliger Nutzerkonten letztlich eine disziplinierende Wirkung auf andere Nutzer entfalten, der Kennzeichnungspflicht nachzukommen. Insoweit fiele auch eine solche Maßnahme in den Rege-

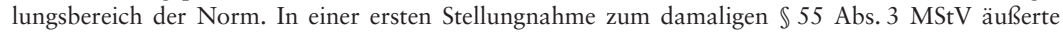
Facebook dies ausdrücklich und lehnte eine Kennzeichnungsverantwortung deutlich ab: „Eine Kennzeichnung solcher Konten wird es auf Facebook somit nicht geben. Vielmehr werden wir alle gefälschten Konten auf Facebook entfernen, ganz gleich, ob diese von Menschen oder Bots gesteuert werden. “, siehe hierzu Facebook, Positionspapier zum Diskussionsentwurf, 2018, S. 10 bzw. Facebook, Positionspapier zum Diskussionsentwurf, 2019, S. 7.

1720 Zur richtigen Kennzeichnung siehe etwa Gerecke, GRUR 2018, 153 (158).

1721 Siehe u.a. Jaworski/Kraetzig, GRUR-Prax 2020, 302 (304) die das auch auf die Rechtsunsicherheit zurückführen, oder Köhler, in: ders./Bornkamm/Feddersen, UWG, $\mathbb{S} 5 \mathrm{a}$ Rn. 7.80a.

1722 Vgl. Micklitz/Schirmbacher, in: Spindler/Schuster, Elektronische Medien, $\$ 6$ TMG Rn. 49.

1723 Gerecke, GRUR 2018, 153 (159); Micklitz/Schirmbacher, in: Spindler/Schuster, Elektronische Medien, $\mathbb{6} 6$ TMG Rn. 52. 
so höchstens dann in Betracht, wenn sie Kenntnis von rechtswidrigen Tätigkeiten erhalten ( $\mathbb{S} \mathbb{S}$ Abs. 2, 10 Abs. 1 TMG). Vor diesem Hintergrund kann aber $\mathbb{S} 93$ Abs. 4 MStV gerade deshalb erlassen worden sein, um die Verantwortung für Verstöße auszudehnen und entsprechende Lücken, wie Sie sich an anderer Stelle offenbart haben, zu vermeiden. Mit Blick auf den Tatbestand der Ordnungswidrigkeit und der angedrohten Geldstrafe von bis zu 500.000 Euro ( $\mathbb{S} 117$ Abs. 1 S. 2 Nr. 42, Abs. 2, 1. Var. MStV) erscheint es auch nicht plausibel, dass nur die Pflicht zur Bereitstellung der Kennzeichnungsinstrumente besteht. Vielmehr deutet das auf eine darüberhinausgehende eigene Verantwortlichkeit hin. Im Lichte der allgemeinen Haftungsgrundsätze käme deshalb vor allem die Einrichtung eines Beschwerdemanagements als verbindliche Vorgabe in Betracht, um Haftungsfolgen überhaupt auslösen zu können.

\section{(3.) Normgenese}

Eine Kennzeichnungspflicht war schon seit längerem nicht nur auf Landesebene im Gespräch. ${ }^{1724} \mathrm{Zu}$ Beginn wurde dabei eher die Verantwortung der Verwender betont. ${ }^{1725}$ Abgesichert werden sollte das nur durch eine Berichtspflicht und einen Meldeweg, durch den die Betreiber auf auffällige Konten aufmerksam gemacht werden sollten. ${ }^{1726}$ Allerdings wurde schon frühzeitig im Gesetzgebungsprozess die nun im MStV vorgesehene Regelung gefordert. ${ }^{1727}$ In der Gesetzesbegründung wird explizit darauf hingewiesen, dass erstens die „konkrete Art und Weise der Pflichterfüllung (...) bewusst nicht festgelegt wird“1728, und zweitens, „gerade nicht die Einführung einer Pflicht der Anbieter von Medienintermediären zur eigenen Verfolgung und Sanktionierung von Verstößen gegen $\mathbb{1} 18$ Abs. 3 [bezweckt wird]. “1729 Danach wären also auf den ersten Blick alle Maßnahmen denkbar, die nicht eine proaktive Tätigkeit der Netzwerkbetreiber erfordern. Liest man aber genauer, dann gibt es zwei Anknüpfungspunkte, wie eine proaktive Pflicht dennoch begründet werden kann. Einerseits soll die eigene Verfolgung und Sanktionierung ausgeschlossen sein. Das öffnet aber den Spielraum dafür, dass etwa anerkannte Einrichtungen der Selbstkontrolle und damit externe Stel-

1724 Auf Bundesebene siehe etwa den Antrag der Fraktion Bündnis 90/Die Grünen, BT-Drs. S. 18/11856, S. 2 .

1725 So wird etwa in einem Antrag der Grünen im Bundestag gefordert, dass die Betreiber ihre Nutzer verpflichten müssten, Social Bots zu kennzeichnen, BT-Drs. 18/11856, S. 3. Nur von einer bußgeldbewehrten Kennzeichnungspflicht redend BRat-Drs. 519/18, S.2, 3. Beide Möglichkeiten in Betracht ziehend Milker, InTeR 2017, 199 (205 f.).

1726 BT-Drs. 18/11856, S. 3. Deutlicher eine Berichtspflicht fordernd - im Rahmen der Debatte um die Weiterentwicklung des NetzDG - BT-Drs. 19/5950, S. 6.

1727 Damals noch als $\mathbb{5} 5 \mathrm{~d}$ Abs. $4 \mathrm{MStV}$, siehe hierzu Rundfunkkommission der Länder, Diskussionsentwurf für einen Medienstaatsvertrag, 2019.

1728 Siehe u.a. BayLT-Drs.18/7640 S. 109; NiedersächsLT-Drs. 18/6414, S. 116.

1729 BayLT-Drs. 18/7640 S. 109; NiedersächsLT-Drs. S. 18/6414, 116. 
len eine solche Überprüfung übernehmen. ${ }^{1730}$ Andererseits schließt der Wortlaut nur die Verfolgung und Sanktionierung aus. Damit knüpft man aber an eine Phase an, in der der (mögliche) Rechtsverstoß schon bekannt geworden ist. Die Gesetzesbegründung schließt gerade nicht die Überwachung des Netzwerks zur vorgelagerten (präventiven) Ermittlung von Rechtsverstößen aus. Wenn in derlei Fällen eine eigenständige Kennzeichnung vorgenommen wird, geht davon auch keine sanktionierende Wirkung aus. Vielmehr wird der rechtlich vorgesehene Normalzustand hergestellt.

\section{(4.) Telos der Norm}

Der Regelungszweck der Norm ist vielseitig, ohne dass aber alle Facetten Eingang in die Gesetzesbegründung gefunden hätten. Zunächst - und wohl auch primär - geht es um die Schließung von Lücken, die durch eine fehlende Bereitschaft zur Kennzeichnung entstehen und damit um die Effektuierung der Kennzeichnungspflicht. ${ }^{1731}$ Zweitens geht es auch um eine gewisse Vereinheitlichung, um Transparenz auch tatsächlich erreichen zu können. Drittens begegnet der Gesetzgeber so seiner eigenen Unkenntnis von den technischen Hintergründen der Netzwerke und der Bots. Weil der Entwurf letztlich eine Vielzahl von Intermediären betreffen soll, deren Geschäftsmodelle divergieren und vor allem nicht en détail bekannt sind, scheint es sinnvoller eine allgemeine Verpflichtung zu statuieren, und die Konkretisierung vorerst ${ }^{1732}$ den Netzwerkbetreibern zu überlassen. ${ }^{1733}$ Das führt letztlich auch zu einem vierten Aspekt: die Betreiber verfügen nicht nur über das erforderliche Wissen und die nötigen Zugriffe, um eine entsprechende Pflicht umzusetzen. ${ }^{1734}$ Sie können auch viel eher darüber entscheiden, wie man die Pflicht am besten innerhalb des Dienstes umsetzen kann. ${ }^{1735}$ Denn die bisherigen Versuche durch entsprechende Software Bots zu identifizieren, basieren nur auf den Datenbeständen, zu denen die Netzwerkbetreiber externen Forschern Zugang gewähren. Dieser Aspekt ist vor allem - fünftens - für die

1730 Dass entspricht in gewisser Weise der Zusammenarbeit bspw. von Facebook mit externen Faktenprüfern, dazu oben, C.I.3.a.aa. Das sieht letztlich auch das NetzDG - wenn auch in anderem Kontext $(\$ 3$ Abs. 2 Nr. 3 lit. b) NetzDG) vor.

1731 So auch Fehling/Leymann, AfP 2020, 110 Rn. 37. In diese Richtung auch Schröder, DVBl. 2018, 465 (470 f.). Gerade wegen dieser Effektuierung durch die Verantwortlichkeit der Netzwerkbetreiber wird das Fehlen entsprechender Pflichten im kalifornischen Recht kritisch bewertet. Im ursprünglichen Entwurf war noch ein Notice-and-Takedown-Verfahren vorgesehen. Hierzu J.F. Weaver, Journal of Robotic, Artificial Intelligence \& Law 2018, 431 (435).

1732 Mit $\$ 96 \mathrm{MStV}$ besteht immerhin eine Konkretisierungsbefugnis der Landesmedienanstalten.

1733 Vgl. BayLT-Drs. 18/7640 S. 109; NiedersächsLT-Drs. 18/6414, S. 116. Auf diese notwendigen Unterscheidungen zwischen den Betreibern weist etwa auch Liesem, AfP 2020, 277 Rn. 23 hin.

1734 Dies betonen etwa auch Simon Hegelich zit. nach Grandjean, ZUM 2017, 565 (570); Laude, JuWiss-Blog Nr. 127/2020; Schröder, DVBl. 2018, 465 (471) und J.F. Weaver, Journal of Robotics, Artificial Intelligence \& Law Vol. 1 (2018), 431 (435).

1735 Vgl. Löber/Roßnagel, MMR 2019, 493 (498). Einen solchen „technischen Spielraum“ fordert auch Milker, InTeR 2017, 199 (206). 
freiheitsrechtliche Sphäre der Anbieter entscheidend. Man bewegt sich hier immerhin im Bereich der Berufsausübungsfreiheit, Art. 12 Abs. 1 GG $^{1736}$ und der Dienstleistungsfreiheit des Art. 56 ff. AEUV ${ }^{1737}$.

(5.) Zwischenfazit: Infrastrukturelle Verantwortung und regulierte Selbstregulierung im Kampf gegen Meinungsroboter

Eine Auslegung führt also zunächst nur zu dem Ergebnis, dass $\mathbb{9} 93 \mathrm{Abs.} 4 \mathrm{MStV}$ nicht nur die infrastrukturellen Voraussetzungen schaffen, sondern gerade bestehende Schutzlücken durch eine eigene, bei Verstößen bußgeldbewehrte Verantwortlichkeit der Betreiber schließen will. Dabei ist aber ein (zwingend) notwendiger Ermessensspielraum der Netzwerkbetreiber vorgesehen, der einerseits aus der fehlenden technischen Kenntnis des Gesetzgebers, andererseits aus den freiheitsrechtlichen Garantien der Netzwerkbetreiber folgt. Insoweit verfolgt die Norm also den Ansatz einer „regulierten Selbstregulierung“, die ein Spektrum von einer Identitätskontrolle über die Einführung eines Notice-and-Takedown-Sytsems, ${ }^{1738}$ bis hin zu einer eigenständigen Kennzeichnung ${ }^{1739}$ beinhalten kann. Und auch die gängige Löschpraxis von auffälligen, falschen Profilen einiger Anbieter könnte so noch - als mittelbarer Zwang zur Kennzeichnung - den Anforderungen der Norm gerecht werden. ${ }^{1740}$

(II.) Verfassungs- bzw. grundrechtliche Implikationen

Berücksichtigt man hier allerdings den verfassungsrechtlichen Hintergrund, so fragt sich, ob die Sorgetragungspflicht nicht auf konkrete Maßnahmen reduziert werden kann bzw. muss. Entscheidend kommt es insoweit auf einen sachgerechten Ausgleich der kollidierenden grundrechtlichen Belange an.

(1.) Interessen der Betreiber, insbes. Art. 12 Abs. 1 GG

Auf Betreiberseite wird die bereits angesprochene berufliche Freiheit relevant. ${ }^{1741}$ Insoweit ist allerdings nicht die Schaffung der infrastrukturellen Voraussetzungen

1736 Auf diese grundrechtliche Sphäre ebenfalls hinweisend Milker, InTeR 2017, 199 (206).

1737 Hierauf wird noch ausführlicher zurückzukommen sein, unten, C.II.2.f.cc.

1738 Das wurde etwa in der US-amerikanischen Debatte durchaus vorgeschlagen, vgl. Stricke, Vanderbilt Journal of Entertainment\&Technology Law Vol. 22 (2020), 839 (843); J.F. Weaver, Journal of Robotics, Artificial Intelligence \& Law 2018, 431 (435). In diese Richtung auch Dankert, in: Hoffmann-Riem, Big Data, S. 157 (164).

1739 Hier drin sehen Schwartmann/Hermann/Mühlenbeck, Transparenz von Medienintermediären, S. 136 scheinbar den maßgeblichen Regelungsgehalt der Norm.

1740 Gefordert werden kann das wohl aber nicht, denn damit fordert man gerade eine „Sanktionierung “ durch die Netzwerkbetreiber.

1741 S. o., A.II. 
ein Problem. Hiermit ist ein vergleichsweise wenig intensiver Eingriff in die beruflichen Interessen der Betreiber verbunden. Vielmehr geht es um die Frage, was von den Betreibern darüber hinaus verlangt werden kann. Dass zur Wahrung der Interessen ein gewisser Spielraum bezweckt und auch notwendig ist, wurde bereits erwähnt. Dieser Spielraum darf aber weder dazu führen, dass Unzumutbares, also Unverhältnismäßiges verlangt wird, noch das (offensichtlich) ineffektive Maßnahmen ergriffen werden. Mit Blick auf die Zumutbarkeit gilt es allerdings zweierlei zu berücksichtigen: Zum einen wird teilweise eine Automatisierung betreiberseitig ermöglicht, gefördert oder zumindest geduldet. ${ }^{1742}$ Dann aber trifft zumindest den Netzwerkbetreiber eine gewisse Verantwortung für solche Phänomene, ${ }^{1743}$ derer er sich nicht durch Hinweis der bloßen Anbietereigenschaft entziehen kann. Vielmehr bewegt man sich aus Sicht der zu schützenden Interessen in einem ähnlichen Bereich wie beim Zweckveranlasser, ${ }^{1744}$ bei ingerentem Verhalten oder bei Gefahrenlagen, die Verkehrssicherungspflichten auslösen. ${ }^{174}$ Dieser Überlegung liegt letztlich „der allgemeine Rechtsgrundsatz [zugrunde][...], dass jeder, der in seinem Verantwortungsbereich eine Gefahrenquelle schafft oder andauern lässt, die ihm zumutbaren Maßnahmen und Vorkehrungen treffen muss, die zur Abwendung der daraus Dritten drohenden Gefahren notwendig sind. "1746 Das kann eine durchaus stärkere Inpflichtnahme rechtfertigen.

Zum anderen ist auch der konkrete Dienst und seine Stellung im Meinungsbildungsprozess bzw. seine Meinungsbildungsrelevanz zu berücksichtigen. ${ }^{1747}$ Denn

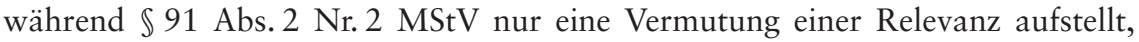

1742 So auch Oehmer/Pedrazzi, UFITA 2020, 7 (9, 15). Insbesondere für Twitter gilt dies, dessen Dienst offen für die Anbindung von Eingabeprogrammen o.ä. ist und eine Automatisierung gerade zulässt.

1743 Diese Verantwortung betonen etwa auch Oehmer/Pedrazzi, UFITA 2020, 7 (18).

1744 Diese Parallele zieht etwa W. Beck, DVBl. 2020, 253 (255).

1745 Vergleichbare Fälle finden sich etwa im Wettbewerbsrecht, wenn es um wettbewerbsrechtliche Verkehrspflichten geht, die auf die Verhinderung von Verletzungen wettbewerbsrechtlicher Interessen gerichtet sind. Auch diese stehen unter dem Vorbehalt des Möglichen und Zumutbaren. Hierzu etwa mit Blick auf eBay, wo mehrfach jugendgefährdende Medien angeboten wurden, BGH, GRUR 2007, 890 Rn. 36. Vgl. auch W. Beck, DVBl. 2020, 253 (256).

1746 So BGH GRUR 2007, 890 Rn. 36. Siehe auch BGH, GRUR 2015, 1129 Rn. 42. Pille, Meinungsmacht sozialer Netzwerke, S. 181 meint hier, dass „sich aus der kommunikativen Verantwortung jener Angebote auch kommunikationsbezogene Verpflichtungen ableiten [lassen], die ggf. durch eine Regulierung zu erzwingen wären.“ W. Beck, DVBl. 2020, 253 (255) sieht jedenfalls eine ,aus überlegener Organisationsmacht resultierende Verantwortung“, die sich aus der polizeirechtlichen Figur des Zweckveranlassers ergebe.

1747 Diesen Ansatzpunkt sieht auch W. Beck, DVBl. 2020, 253 (257). Vgl. hierzu auch Paal, MMR 2018, 567 (568), der bei den Rahmenbedingungen für eine Medienintermediärsregelung auch auf „Bindungen für Medienintermediäre auf Grund deren herausgehobener Bedeutung für den Meinungsbildungsprozess" und sodann auf das obiter dictum des BVerfG in der Fraport-Entscheidung abstellt, was im Wesentlichen aussagt, dass die Grundrechtsbindung Privater einer staatlichen nahe oder gar gleich kommen kann, hinsichtlich der Kommunikationsgrundrechte insbesondere dann, „wenn private Unternehmen die Bereitstellung schon der Rahmenbedingungen öffentlicher Kommunikation selbst übernehmen [...]“, BVerfGE 128, 226 (249). Im Kontext der Diskussion um Filtertechnologien bei Intermediären vgl. zudem auch Müller-Terpitz, ZUM 2020, 365 (372). Vgl. für die Bedeutung dieser Relevanz für die rundfunkrechtliche Regulierung auch Kaiser/Reiling, in: Unger/v. Ungern-Sternberg, Demokratie und künstliche Intelligenz, S. 85 (105f.). 
kann es im Rahmen der Verantwortung einen Unterschied machen, ob ein Dienst gerade so die Grenze überschreitet oder zentraler Anbieter auf dem Markt ist. ${ }^{1748}$ Ähnliche Gedanken werden etwa auch in der Diskussion um eine mittelbare Drittwirkung der Grundrechte gegenüber den Betreibern ${ }^{1749}$ relevant. Hier stellt man zur Begründung einer (staatsähnlichen) ${ }^{1750}$ Wirkung der Grundrechte im Vertragsverhältnis auf die Markt- bzw. Entscheidungsmacht ${ }^{1751}$, die Eröffnung kommunikativer Räume ${ }^{1752}$, die „,hervorgehobenen Wirkmacht für den öffentlichen Diskurs“, ${ }^{1753}$ eine staatsgleiche Stellung ${ }^{1754}$ bzw. die faktische Übernahme staatlicher Aufgaben ${ }^{1755}$ oder auf eine Kombination von Kriterien ${ }^{1756}$ ab. ${ }^{1757}$

1748 Auch der Bagatellvorbehalt in $\$ 1$ Abs. 2 NetzDG wurde maßgeblich mit diesen (grundrechtlichen) Erwägungen begründet. In der Gesetzesbegründung heißt es hierzu: „Der Grund für eine solche Regelung liegt darin, dass kleinere soziale Netzwerke von aufwendigen Prüfpflichten befreit werden sollen. Die umfassenden gesetzlichen Anforderungen können nur von sozialen Netzwerken mit entsprechenden Ressourcen und Kapazitäten bewältigt werden. “, BT-Drs. 18/12356, S. 19.

1749 Ausführlich hierzu etwa Knebel, Die Drittwirkung der Grundrechte und -freiheiten gegenüber Privaten, S. $47 \mathrm{ff} ., 174 \mathrm{ff}$.

1750 Vgl. Knebel, Die Drittwirkung der Grundrechte und -freiheiten gegenüber Privaten, S. 67, $107 \mathrm{ff}$.

1751 Aus der fachgerichtlichen Judikatur etwa OLG Dresden, ZUM-RD 2019, 2 (6) das von einer "Quasi-Monopolstellung “ spricht. Der Mächtigkeit widmet sich etwa Knebel, Die Drittwirkung der Grundrechte und -freiheiten gegenüber Privaten, S. $111 \mathrm{ff}$. umfassend und begründet damit eine auch staatsgleiche Bindung, S. 109, 301. Kritisch zu einer Differenzierung zwischen marktmächtigen und anderen Unternehmen mit Blick auf die Drittwirkungsproblematik bei Kommunikationsstandards Lüdemann, MMR 2019, 279 (283f.). In anderen vertragsrechtlichen Konstellationen wurde ebenfalls auf die strukturelle Unterlegenheit eines Vertragspartners abgestellt, siehe etwa BVerfGE 89, 214 (232) - Bürgschaftsverträge.

1752 So etwa BVerfGE 128, 226 (251) - Fraport; kritisch zu einer Übertragung auf soziale Netzwerke aber Lüdemann, MMR 2019, 279 (280). Das OLG Dresden, stellt hier auch den Bezug zur FraportEntscheidung her, und zwar nicht nur für Facebook, ZUM-RD 2019, 2 (6) und Urt. v. 16.06.2020 4 U 2890/19 = GRUR-RS 2020, 12291 Rn. 18, sondern auch für Twitter, MMR 2020, 626 Rn. 14. Das OLG München, ZUM 2019, 269 (270) spricht hier von einem „öffentlichen Marktplatz“; ebenso LG Bamberg, MMR 2019, 56 Rn. 51.

1753 LG Leipzig, Endurteil v. 12.07.2019 - 08 O 2491/18 = GRUR-RS 2018, 38785 Rn. 50.

1754 Heidtke, Meinungsbildung und Medienintermediäre, S. 212. Vgl. hierzu Lüdemann, MMR 2019, 279 (280). Mit Blick auf die Stadionverbotsentscheidung des BVerfG bezeichnet Smets, NVwZ 2019, 34 (36) den DFB als „Teilbereichsstaat“.

1755 Das betont etwa das BVerfG in seinem obiter dictum, E 128, 226 (252) - Fraport: „Wenn heute die Kommunikationsfunktion der öffentlichen Straßen, Wege und Plätze zunehmend durch weitere Foren wie Einkaufszentren, Ladenpassagen oder sonstige Begegnungsstätten ergänzt wird, kann die Versammlungsfreiheit für die Verkehrsflächen solcher Einrichtungen nicht ausgenommen werden, soweit [...] Private im Wege der mittelbaren Drittwirkung in Anspruch genommen werden können. Das LG Leipzig, Endurteil v. 12.07.2019 - 08 O 2491/18, GRUR-RS 2019, 38785 Rn. 47 hat etwa von einer „mit der traditionellen Pflichten- oder Garantenstellung des Staates vergleichbaren Lage“ gesprochen. Und hieran knüpft auch das OLG Dresden, Urt. v. 16.06.2020 - 4 U 2890/19= GRUR-RS 2020, 12291 Rn. 18 an. Aus der Literatur vgl. etwa Jobst, NJW 2020, 11 (15); vgl. auch Specht-Riemenschneider/Riemenschneider, in: Tamm/Tonner/Brönneke, Verbraucherrecht, $\$ 4 \mathrm{~b}$ Rn. 51.

1756 So im Ansatz etwa die Überlegung des BVerfG (K), NJW 2019, 1935 - Accountsperrung in sozialem Netzwerk, auch wenn es dies i.E. offenlässt. In BVerfGE 148, 267 Rn. 41 - Stadionverbot, stellt das Gericht auf die Eröffnung für den allgemeinen Verkehr und die Bedeutung der Veranstaltung ab. Vgl. hierzu auch LG Bamberg, MMR 2019, 56, dass nicht nur auf den öffentlichen Marktplatz abstellt (Rn. 51), sondern daneben auch die Quasi-Monopolstellung (Rn. 54) und die zentrale Stellung des Dienstes (Rn. 55) betont. Jobst, NJW 2020, 11 (15), führt die Öffnung für ein unbegrenztes Publikum, die Begründung eines „öffentlichen Forums“, dessen „überragende Bedeutung“, eine strukturelle Überlegenheit und die Privatisierung von Kommunikationsräumen an. Und Raue, JZ 
Auch wenn es hier nicht um die Frage einer mittelbaren Drittwirkung geht, so können diese Kriterien gleichwohl Berücksichtigung finden. Aus ihnen folgt nämlich ein gestuftes Regelungsprinzip, dass mit steigender Bedeutung und Macht des Dienstes stärkere Anforderungen stellt. Das kommt vor allem für Facebook und Twitter in Betracht. Dem liegt auch die Überlegung zugrunde, dass die Dienstanbieter von der zentralen Stellung und dem Abhängigkeitsverhältnis der Nutzer in Folge der Marktmacht, den Lock-in-Effekten ${ }^{1758}$ und der Bedeutung des Dienstes im Kommunikationsprozess wesentlich profitieren, ${ }^{1759}$ und eine solche Wirkung u.a. durch entsprechendes Contentmanagement intendiert ist, um die Nutzer möglichst lange innerhalb des Dienstes zu fangen. ${ }^{1760}$ Das führt zu einer starken Marktmacht weniger Dienste. ${ }^{1761}$ Insbesondere der Facebook-Konzern dominiert den Markt. Twitter hat daneben zwar eine (deutlich) geringere Nutzerzahl, der Dienst ist aber - anders als Facebook - auch für nicht registrierte Nutzer zugänglich und bietet mit dem auf Kurznachrichten basierten Dienst ein spezielles Angebot, welches sich von Facebook unterscheidet. ${ }^{1762}$ Wenn aber erhebliche Profite aus dieser Stellung folgen, und diese (gesellschaftliche) Stellung gerade intendiert ist, so muss damit auch eine größere (gesellschaftliche) Verantwortung einhergehen als etwa für kleinere Angebote, spezifische themenbezogene Dienste oder Meinungsforen. ${ }^{1763}$ Denn auch die finanziellen und organisatorischen Belastungen, die mit einer Verpflichtung einher gehen, nehmen mit zuneh-

2018, 961 (965 ff.) knüpft an die Überlegenheit, die Eröffnung eines Verkehrs und das geschaffene öffentliche Forum an. Ebenso v. Ungern-Sternberg, in: Unger/dies., Demokratie und künstliche Intelligenz, S. 3 (27), die zusätzlich auch auf die in der Stadionverbotsentscheidung relevant gewordene Teilnahme am gesellschaftlichen Leben hinweist. Und Pille, Meinungsmacht sozialer Netzwerke, S. 375 meint, dass eine ,intensive Grundrechtsbindung zumindest dann gerechtfertigt ist, wenn Private ,öffentliche Kommunikationsräume beherrschen' und als kommunikationsbezogene Monopolisten im Internet agieren.", m.w.N.

1757 Diese Varianz der Faktoren betonte auch zuletzt das BVerfGE, 148, 267 Rn. 33 - Stadionverbot.

1758 Ausführlich zu diesen etwa Knebel, Die Drittwirkung der Grundrechte und -freiheiten gegenüber Privaten, S. $155 \mathrm{ff}$.

1759 In Ansätzen mit Blick auf die Drittwirkungsfrage ähnlich Raue, JZ 2018, 961 (965 f.).

1760 Vgl. Oehmer/Pedrazzi, UFITA 2020, 7 (10); zur Verweildauer als ökonomisches Ziel auch Kaiser/ Reiling, in: Unger/v. Ungern-Sternberg, Demokratie und künstliche. Intelligenz, S. 85 (88).

1761 Hierzu etwa BKartA, Beschl. v. 06.02.2019 - B6-22/16 = BeckRS 2019, 4895 Rn. 175.

1762 Denn Facebook ist in erster Linie auf soziale Interaktion aus, Hegelich zit. nach Grandjean ZUM 2017, 565 (571) spricht hier davon, „[dass] [d] as ganze Netzwerk [...] für private Kommunikation und für Wohlfühlen ausgelegt [sei], wo man nicht richtig über die Dinge nachdenken müsse, sondern schnelle Reaktionen erzielen wolle.“

1763 Diese Überlegung findet sich etwa auch in anderem Zusammenhang bei Jobst, NJW 2020, 11 (15 f.). Krüger, in: Unger/v. Ungern-Sternberg, Demokratie und künstliche Intelligenz, S. 67 (83) weist hier lediglich, aber immerhin daraufhin, dass den Netzwerkbetreiber eine „Gewährleistungsverantwortung für den netzgebundenen Diskurs übertragen werden müsste“. Die gesellschaftliche Verantwortung der Betreiber betonen etwa auch Steinebach u.a., Desinformation aufdecken und bekämpfen, S. 203, wenn es um die Tätigkeit der Betreiber im Kampf um Desinformation geht und Steinmaurer, in: Litschka/Krainer, Der Mensch im digitalen Zeitalter, S. 31 (36 f.). Vgl. hierzu auch LG Leipzig, Urt. v. 12.07.2019 - 08 O 2491/18 = GRUR-RS 2018, 38785 Rn. 50, betont, dass aus der Wirkmacht für den öffentlichen Diskurs auch eine stärkere Bindung an grundrechtliche Maßstäbe folge. 
mender Marktmacht ab. ${ }^{1764}$ Deshalb ist auch gleichheitsrechtlich eine solche Differenzierung problemlos möglich.

Zudem handelt es sich hierbei lediglich um Berufsausübungsschranken, also wenig eingriffsintensive Maßnahmen, die bereits durch überwiegende wichtige Allgemeinwohlinteressen gerechtfertigt werden können. Gerade mit Blick auf die Abwägung ist zu berücksichtigen, dass die Verpflichtung nur in einer bestimmten Konstellation gilt und nicht - wie etwa bei Upload-Filtern - ganz verschiedene Rechtsverletzungen von einer Vielzahl von Rechtsinhabern erfasst werden. Dabei begrenzen $\mathbb{S} \mathbb{S} 18$ Abs. 3, 1 Abs. $7 \mathrm{MStV}$ die Sorgetragungspflicht auch nur auf einen Bruchteil der problematischen Fälle, nämlich auf Telemedien, die die Voraussetzungen von $\mathbb{\$} 18$ Abs. $3 \mathrm{MStV}$ erfüllen und nach den Vorschriften des Telemediengesetzes in Deutschland niedergelassen sind ( $\mathbb{1} 1 \mathrm{Abs} .7 \mathrm{MStV})$. Durch die Orientierung an den jeweils verwendeten IP- oder Profildaten wäre eine solche Eingrenzung auch technisch ohne Weiteres möglich. Auch in Kombination mit anderen Verpflichtungen des NetzDG bestehen keine Bedenken, weil das NetzDG materiell-rechtlich keine neuen Löschpflichten aufbürdet, sondern nur verfahrensrechtliche Vorgaben für alle Anbieter trifft. Daneben lassen sich die Aufgaben durchaus auf primärer Ebene automatisiert durchführen ${ }^{1765}$ und erfordern nur eine sekundäre Überprüfung durch Personal. Das notwendige technische Know-how haben die Unternehmen jedenfalls, sodass die Beeinträchtigung auch dadurch abgemildert werden kann. Zudem ist zu berücksichtigen, dass die großen Netzwerkbetreiber schon jetzt automatisiert die Netzwerkdaten überprüfen lassen, um bestimmte Manipulationen frühzeitig zu erkennen und zu verhindern. Paradebeispiel ist das Facebook Immune System ${ }^{1766}$.

Betrachtet man unter diesen Prämissen den möglichen Bereich der Maßnahmen, so muss man einerseits attestieren, dass ein bloßes Beschwerdemanagementverfahren der aufgezeigten Verantwortung kaum gerecht wird. Entscheidender könnten proaktive Kontrollmaßnahmen sein. Insbesondere mit Blick auf die Stellung der zentralen Anbieter legitimiert das nicht nur bloße Identitätskontrollen oder Zugangskontrollen zur API, ${ }^{1767}$ sondern auch darüberhinausgehende proaktive Überwachungspflichten. Denkbar ist hierfür auch ein Modell regulierter Selbstregulierung in dem Sinne, wie es auch schon das NetzDG ${ }^{1768}$ vorsieht: Die eigentlichen Kontrollaufgaben werden an eine unabhängige, externe, aber

1764 Vgl. hierzu bereits die Gesetzesbegründung zu $₫ 1$ Abs. 2 NetzDG, BT.-Drs. 18/12356, S. 19.

1765 Entsprechende Erkennungssoftware wird seit einigen Jahren erprobt und in der Forschung finden sich ebenfalls verschiedene Ansätze, wie man „malicious Bots“ erkennen kann. Einen Überblick gewährt etwa Cresci, Communication of the ACM Vol. 63 (2020), 72 (76 ff.).

1766 Ausführlicher zu diesem etwa Stein/Chen/Mangla, Facebook Immune System, 2011. Siehe daneben auch die Informationen des Unternehmens zum Umgang mit Fake Accounts, abrufbar unter https:// about.fb.com/news/2019/05/fake-accounts/, (Stand: 30.05.2021).

1767 So der Vorschlag etwa von Milker, InTeR 2017, 199 (206).

1768 Vgl. hierzu $\$ 3$ Abs. 2 Nr. 3 lit. b) und $\$ 3$ Abs. 3 NetzDG. 
anerkannte Stelle abgegeben. Das entspricht in einer gewissen Weise auch dem Ansatz, den die Betreiber bei der Bekämpfung von desinformativen Inhalten verfolgen, wenn sie mit unabhängigen Faktenprüfern zusammenarbeiten. Dann beschränkt sich die Sorgetragungspflicht darauf, den Zugang von Dritten auf die Netzwerkdaten zu ermöglichen und ihre Arbeit zu kontrollieren.

Hinsichtlich der Identitätskontrolle besteht zwar auch ein betreiberseitiges Interesse an einer noch genaueren Zuordnung der Nutzungsdaten, ${ }^{1769}$ allerdings darf nicht übersehen werden, dass es Gründe dafür gibt, dass die Unternehmen bisher von einer wirksamen Durchsetzung der Klarnamenpflicht abgesehen haben und an anderer Stelle gerade anonyme Zugangsmöglichkeiten schaffen ${ }^{1770}$. Denn in Zeiten zunehmenden datenschutzrechtlichen Bewusstseins und dem Willen nach „anonymer“ Nutzung könnte das zu einer erheblichen Abschreckungswirkung führen, die die Dienste weniger attraktiv machen. ${ }^{171}$ Das gilt gerade dann, wenn etwa Ausweispapiere vorgelegt oder auf anderem Wege die Identität nachgewiesen werden muss. Vergleichbares kann aber auch mit Blick auf die API gelten. Insbesondere Twitter will ja den Dienst möglichst barrierefrei anbieten. Zugangshürden bei der Registrierung können sich also auch auf die Inanspruchnahme des Dienstes auswirken. ${ }^{1772}$

Das gilt zwar auch für proaktive Überwachungs- oder Kontrollpflichten und den mit ihnen einhergehenden finanziellen, personellen, technischen und organisatorischen Aufwand. Diese laufen aber im Hintergrund und sind - ebenso wie die Personalisierungsprozesse - nicht für Nutzer sichtbar ab. Insoweit geht damit keine vergleichbare „Abschreckungswirkung“ einher. Betreiberseitig lassen sich diese Aufgaben primär - etwa mit entsprechender Erkennungssoftware automatisiert bewältigen. Zur Gewährleistung richtiger Ergebnisse wäre eine händische Nachkontrolle notwendig. Angesichts der riesigen Datenmengen wäre das auch praktisch gar nicht anders zu bewerkstelligen. Gewisse automatisierte Methoden zur Erkennung (nicht) natürlichen Verhaltens von Profilen liegen den Netzwerkalgorithmen ja auch im positiven Sinne zugrunde. Die ökonomisch orientierte Selbstregulierung wird insofern nur um eine gesellschaftliche Sphäre ergänzt. Die Konkretisierung ist ja auch hier den Betreibern überlassen, sodass der notwendige Spielraum besteht, um dienstspezifisch entsprechende Lösungen zu entwickeln.

1769 Dieses berechtigte Interesse sieht etwa Heberlein, Datenschutz im Social Web, S. 164 f. Zu der besseren Vermarktung solcher Daten siehe etwa Caspar, ZRP 2015, 233 (234f.).

1770 So ermöglicht etwa Facebook die Nutzung in China über eine Seite im Darkweb, bei der sichergestellt ist, dass die Verbindungsdaten nicht auf einen einzelnen Nutzer zurückverfolgt werden können.

1771 Zur grundrechtlichen Bedeutung der Anzahl der Nutzer und die Attraktivität der Dienste vgl. OLG Nürnberg, Urteil vom 04.08.2020 - 3 U 3641/19 = GRUR-RS 20202, 23456 Rn. 63.

1772 So letztlich u.a. auch Gumz/Kar, in: Weber, ÖFIT-Trendschau, Trendthema 44: Social Bots. Mit Blick auf sog. Captchas als implementierter Turingtest um Bots zu erkennen Hitaj u.a., Capture the Bot, S. 2. 


\section{(2.) Schutzbedarf der Kommunikationsfreiheiten der Nutzer}

Aus Sicht des oben bereits aufgezeigten Schutzbedarfs bestehen vor allem Bedenken gegen ein reines Beschwerdeverfahren. Wenn die Grundprämisse darin besteht, dass Meinungsroboter als solche regelmäßig nicht für Nutzer erkennbar sind, ${ }^{1773}$ und deshalb der Gesetzgeber Transparenz schaffen will, so liefe ein solches isoliertes Verfahren der Intention zuwider. Das heißt freilich nicht, dass Netzwerkbetreiber nicht auch ein Beschwerdeverfahren vorsehen können. Dies allein erfüllt aber - anders als bei konkreten Inhalten (vgl. $\mathbb{3}$ Abs. 1 NetzDG) nicht den erforderlichen Schutzbedarf und kann damit also nicht mal den Mindestmaßstab für die Sorgetragungspflicht bilden. Insoweit wären also zumindest aktive Kontrollen des Registrierungsverfahrens oder des Zugriffs (über die API) als absoluter Mindeststandard erforderlich. Allerdings ist unklar, inwieweit solche Maßnahmen einerseits hinreichend effektiv und manipulationssicher sind, ${ }^{1774}$ aber andererseits nicht erhebliche Auswirkungen auf die Benutzerfreundlichkeit haben. ${ }^{1775}$ Speziell mit Blick auf die API führt das auch zu Problemen, weil aus Transparenzgründen dann zwischen automatisierten und nicht-automatisierten Zugriffen differenziert werden müsste. Das liegt einerseits daran, dass das einfach-rechtlich geschützte Transparenzbedürfnis nur in den Fällen der Meinungsroboter besteht. ${ }^{1776}$ Und andererseits würde man dem Transparenzbedürfnis schaden, wenn jeder Zugriff über die API entweder immer als automatisiert oder immer nur als „API-Zugriff“ gekennzeichnet würde. ${ }^{1777}$ In letzterem Fall kommt erschwerend hinzu, dass die Angabe eines solchen Ursprungs oft weniger verständlich für den Durchschnittsnutzer sein wird als die Angabe, dass es sich um einen automatisiert erstellten Inhalt handelt. Daneben hilft eine Kontrolle der API in solchen Fällen nicht weiter, wo Bots über (simulierte) HTTP-Requests agieren, also gar nicht die Programmier-Schnittstellen verwenden. Orientiert man sich zudem etwa an der bislang erfolgten eigenständigen Überprüfung von FakeAccounts bei Facebook, so umfasst auch diese nicht nur die primäre Ebene der Registrierung. Weil hierdurch nur offensichtliche Fälle identifiziert werden kön-

1773 So schon Boshmaf u.a., Computer Networks 57 (2013), 556 (557); Edwards u.a., Computers in Human Behavior 33 (2014), 372 ff. Siehe daneben auch Kaerlein, in Hug/Pallaver, Talk with the Bots, S. 17 (21).

1774 Kritisch etwa Simon Hegelich auf der Tagung des Instituts für Urheber- und Medienrecht am 28.04.2017, zit nach Grandjean, ZUM 2017, 565 (571).

1775 Das mahnt etwa Milker, InTeR 2017, 199 (204) mit Blick auf sog. Captchas an.

1776 Insoweit zeigt sich hier erneut die fehlende Differenzierung die auch schon tatbestandlich $\mathbb{} 18$ Abs. 3 MStV zugrunde liegt: dem eigentlichen Anwendungsbereich der Meinungsroboter (doppelte Täuschung) und dem Zugriff echter Nutzer über die API. Dazu siehe bereits oben, C.II.1.b.

1777 Auf diese bereits bestehende Herangehensweise bei Twitter, die Methode zu kennzeichnen, mit der Beiträge ins Netzwerk gelangt sind, weist etwa Riedel, Wir brauchen eine faktenbasierte Digitalund Medienpolitik, medium.com, hin. 
nen, wird auch eine sekundäre Kontrolle des Nutzerverhaltens notwendig. ${ }^{1778}$ Insoweit wäre für den Schutzbedarf auch eine über die Registrierung oder den Zugang zum Netzwerk hinausgehende proaktive Kontrolle notwendig.

\section{(3.) Weitere kollidierende grundrechtliche Interessen}

Mit präventiven Identitätskontrollen verbinden sich zwangsläufig datenschutzrechtliche und damit die informationelle Selbstbestimmung (Art. 2 Abs. 1 i.V.m Art. 1 Abs. 1 GG) betreffende Fragen. Die DS-GVO erlaubt allerdings die Erhebung der Identität des jeweiligen Vertragspartners schon jetzt (Art. 6 Abs. 1 lit. b) DSGVO) und auch aufgrund national-rechtlicher Vorgaben (Art. 6 Abs. 1 lit.c) DS-GVO). Sofern sich die Erhebung der Identitätsdaten nur auf das interne Vertragsverhältnis beschränkt und nicht auch auf das Außenverhältnis zu anderen Nutzern, wäre dies auch grundsätzlich zulässig. ${ }^{1779} \mathrm{Ob}$ dies angesichts der Marktmacht und der Bedeutung bestimmter Anbieter für die Kommunikation sinnvoll und von den Ländern rechtspraktisch gewollt wäre, darf bezweifelt werden. ${ }^{1780}$ Denn damit schafft man tendenziell neue Missbrauchsgefahren im Umgang mit personenbezogenen Daten. ${ }^{1781}$ Zwar könnte der Zugang über die API immer eine automatische Kennzeichnung auslösen. Die schon zuvor aufgezeigte fehlende Differenzierung führt aber dann zu Konflikten mit den berechtigten -

1778 Siehe hierzu die Informationen des Unternehmens, abrufbar unter https://about.fb.com/news/201 9/05/fake-accounts/, (Stand 30.05.2021). Hiernach werden bei der Registrierung unterschiedliche Signale erfasst, die implizieren, dass zusammenhängend mehrere Profile registriert werden sollen (etwa Zugriff über bestimmte IP-Adressen geben, die sodann blockiert werden) oder wenn auffällige E-Mail-Adressen verwendet oder andere Signale beobachtet werden, die zuvor bei Fake-Accounts häufig zu beobachten waren. Aber nicht alle Fake-Accounts werden schon bei der Registrierung erkannt und gesperrt. Sofern sie die Registrierung erfolgreich meistern, müssen sie in ihrem Nutzungsverhalten Auffälligkeiten zeigen. Insoweit entspricht das letztlich auch dem Ansatz für Meinungsroboter.

1779 Das ist letztlich auch die Argumentation der Länder Niedersachsen und Mecklenburg-Vorpommern in ihrer Gesetzesinitiative betreffend die Indentifizierungspflicht von sozialen Netzwerken, BT-Drs. $70 / 20$, S. 8 .

1780 Denn damit würde die ohnehin bestehende Möglichkeit der personenbezogenen Speicherung von Daten nur noch stärker ermöglicht. Und zudem ist zweifelhaft, ob der Klarname aus wirtschaftlicher Sicht notwendig wäre, denn bereits jetzt lassen sich über wenige Nutzungsdaten recht eindeutige Schlüsse auf den jeweiligen Nutzer ziehen. Für die wirtschaftliche Verwertung der Daten im Wege des Ad-Placements dürfte der Klarname also keine gesteigerte Bedeutung haben, sodass hier womöglich die datenschutzrechtlichen Interessen höher zu gewichten sein können. Simon Hegelich hält eine Klarnamenpflicht etwa für „gefährlich“ zit. nach Grandjean, ZUM 2017, 565 (571). Hier stellt sich die Frage, ob nicht auch ein Recht auf anonyme bzw. pseudonyme Nutzung im Verhältnis zu den Netzwerkbetreibern in Betracht kommt, hierzu etwa Schunicht, Informationelle Selbstbestimmung in sozialen Netzwerken, S. $263 \mathrm{ff}$. Ausschlaggebend wären hier letztlich nur Aspekte der Rechts- und Strafverfolgung und damit wieder kollidierende Interessen Dritter, siehe hierzu Kersten, JuS 2017, 193 (195 f.); ausführlicher hierzu noch unten, 4. Kapitel, E.

1781 Kritisch etwa Laufer, Der Identifizierungszwang für Gamer hat das Zeug zum Desaster, netzpolitik.org. Sofern etwa die Identifizierung über ein entsprechendes amtliches Ausweisdokument verlangt wird, werden mit dem Bild auch biometrische und damit besonders sensible Daten (Art. 9 DSGVO) erfasst. 
und grundrechtlich geschützten - Interessen derjenigen Nutzer, die die API für die Verwaltung des eigenen Profils nutzen. Ein hiermit kollidierendes Offenbarungsinteresse der Öffentlichkeit an diesem Umstand besteht nicht, ${ }^{1782}$ sodass auch gleichheitsrechtliche Fragen auftreten würden. Eine irgendwie geartete proaktive Kontrolle wäre also vermutlich in jedem Fall notwendig. ${ }^{1783}$ Damit gehen zwar potentielle Gefahren für die Meinungsäußerungsfreiheit der Nutzer einher, etwa wenn eine fälschliche Einordnung und Kennzeichnung als Meinungsroboter erfolgt. Dem kann aber durch verfahrensrechtliche Anforderungen durchaus begegnet werden. ${ }^{1784}$ Entscheidender mag auch hier sein, dass eine Überwachung neue Risiken für die Datenschutzinteressen der betroffenen Nutzer mit sich bringen. ${ }^{1785}$ Weil es hierbei aber nur um die Auswertung ohnehin zwangsläufig mit der Nutzung verbundener Daten geht, die zudem teilweise ohnehin manuell für alle einsehbar sind, wird also keine vollkommen neue Gefahr geschaffen. Auch hier ginge es dann darum, sicherzustellen, dass die Daten nur zu dem gesetzlich vorgesehenen Zweck und nicht länger als notwendig verarbeitet werden. ${ }^{1786}$

\section{(4.) Zwischenfazit: Zugangskontrolle als Mindestschutz, präventive Überwachung als zumutbare Anforderung möglich}

Berücksichtigt man die kollidierenden (grundrechtlichen) Interessen, so wäre eine präventive Kontrolle bei Registrierung und die Kontrolle des Zugangs über die zur Verfügung gestellte API das zu fordernde Mindestmaß. Dabei wäre jedenfalls rechtlich auch eine Identitätskontrolle ohne Weiteres möglich. Wegen potentieller Abschreckungswirkungen kann das aber vor allem von kleineren Anbietern kaum verlangt werden, um nicht noch weitere Wettbewerbsnachteile zu begründen. Speziell für die gesellschaftlich weitaus relevanteren Anbieter Twitter und Facebook kann es aber bei diesem Mindestmaß nicht bleiben. Auch mit Rücksicht auf die geschützten betrieblichen Interessen ist es ihnen zumutbar, darüber hinaus auch die Netzwerkdaten selbst in einer Weise zu überwachen, die die Identifizierung auffälliger Nutzerprofile ermöglicht, um so gerade ihrer gesteigerten gesellschaftlichen Verantwortung nachzukommen. Bei der konkreten Umsetzung verbleibt ihnen ein ausreichender Spielraum.

1782 Etwas anderes gölte freilich, wenn man wie Löber/Roßnagel, MMR 493 (498) annähme, dass jegliche automatisierte Kommunikation erfasst ist. Dann würde der Ansatz über die API ausreichen. Das dem aber nicht so ist, wurde bereits aufgezeigt, S. oben C.II.1.b.

1783 In diese Richtung etwa auch Löber/Roßnagel, MMR 2019, 493 (496), wenn Sie darauf hinweisen, dass „er [- der Plattformanbieter - ] die dynamische Entwicklung der Bot-Technik beobachten und seine Erkennungs- und Kennzeichnungsmechanismen immer wieder dieser Entwicklung anpassen [müsse].”.

1784 Hierauf wird noch zurückzukommen sein, unten C.II.3.b.bb(II.)(3.).

1785 Vgl. Conrad/Hausen, in: Auer-Reinsdorff/Conrad, Hb IT- u. Datenschutzrecht, $\$ 36$ Rn. 67.

1786 Siehe hierzu noch ausführlicher unten, C.II.2.c.aa(IV.). 
(III.) Auslegung im Lichte der E-Commerce-RL

\93 Abs. 4 MStV kann allerdings auch nicht unabhängig von europäischen Vorgaben betrachtet werden. Nimmt man soziale Netzwerke als Host-Provider ${ }^{1787}$ für das Verhalten von Nutzern der Dienste in Anspruch, so sind vor allem die Haftungsprivilegien der Artt. 14 Abs. 1, 15 Abs. 1 ECRL zu berücksichtigen. Hiernach sind Hostprovider solange nicht für fremde Inhalte oder fremdes Verhalten verantwortlich, wie sie keine Kenntnis hiervon haben oder haben müssten (Art. 14 Abs. 1 ECRL). Abgesichert wird dies durch Art. 15 Abs. 1 ECRL, der den Mitgliedsstaaten verbietet, Providern allgemeine proaktive Überwachungspflichten bzw. Nachforschungspflichten hinsichtlich der fremden Inhalte aufzuerlegen. ${ }^{1788}$ Auch wenn zur Zeit zwei Verfahren beim EuGH anhängig sind, in denen geklärt werden soll, inwieweit eine gewisse Content-Moderation der Betreiber dazu führt, dass sie ihre neutrale Rolle als Provider und damit die Haftungsprivilegien verlassen, ${ }^{1789}$ soll jedenfalls mit Blick auf die Meinungsroboter davon

1787 Siehe neben EuGH, Urt. v. 16.02.2012, Rs. C-360/10 = MMR 2012, 334 Rn. 27 - Sabam/Netlog auch die jüngste Entscheidung Urt. v. 03.10.2019 - C-18/18 = GRUR 2019, 1208 Rn. 22 - Glawischnig-Pisczeck.

1788 Probleme mit Art. 15 Abs. 1 der RL 2000/31/EG sehen auch Schulz/Dreyer, Stellungnahme zum Diskussionsentwurf eines Medienstaatsvertrag, S. 17.

1789 Hierzu die erste Vorlagefrage des OGH Österreichs in der Rs. C-500/19 - Puls 4 TV/YouTube, ABl. der EU 2019/C-295/13. Ähnliches gilt auch für die Vorlage des BGH, NJOZ 2019, 25ff, dort Vorlagefrage 2, Rs. C-682/18 - LC/ YouTube, ABl. 2019 C 82/2. Zum Zusammenhang zwischen (inhalts)neutraler Diensterbringung und Haftungsprivilegierung Erwägungsgrund 42 der RL 2000/31, ABl. 2000 L 178/6. EuGH, Urt. v. 23.03.2010 - Rs. C-236/08 u.a. = GRUR 2010, 455 Rn. 114 - Google France; Urt. v. 12.07.2011 - C-324/09 = MMR 2011, 596 Rn. 113 - L’Oréal SA. In die Richtung eines solchen Rollenwechsels bei aktiver Moderation etwa Cobbe/Singh, European Journal of Law and Technology Vol. 10 Nr. 3 (2019), S. 13. Eine solche Einordnung für Facebook deutlich ablehnend LG Würzburg, ZUM 2017, 437 (446) unter Berufung darauf, dass die Inhalte nicht „,...] in irgendeiner Weise verändert, insbesondere redaktionell aufbereitet, würden. Nur dann, wenn aber aktiv auf den Inhalt der von den Nutzern eingestellten Informationen eingewirkt würde und insoweit Veränderungen vorgenommen werden würden, würde dies zum Verlust der Haftungsprivilegierung führen. “In diese Richtung dürfte auch die Rspr. des BGH hindeuten, der etwa hinsichtlich von Hotelbewertungsportalen (GRUR 2015, 1129 Rn. 35) meint, dass „eine über die Aussonderung gegen die Nutzungsbedingungen verstoßender Beiträge hinausgehende inhaltliche Einflussnahme nicht erfolgt", m.w.N. Insoweit kommt es darauf an, ob der Anbieter nicht allgemein, sondern mit Blick auf den konkreten rechtsverletzenden Inhalt eine aktive Rolle eingenommen hat. Nur insoweit wird dann das Haftungsprivileg zurückgenommen, so ausdrücklich EuGH, Urt. v. 12.07.2011 - Rs. C-324/09 = MMR 2011, 596 Rn. 116 - L’Oréal SA. Bei der Hilfestellung oder eigenständigen Schaltung von Anzeigen, die unmittelbar auf rechtswidrige Inhalte verweisen, ist dies etwa der Fall, EuGH, Urt. v. 12.07.2011 - Rs. C-324/09 = MMR 2011, 596 Rn. 113 ff.L'Oréal SA; BGH GRUR 2013, 1229 Rn. 48 - Kinderhochstühle II; GRUR 2015, 485 Rn. 56 Kinderhochstühle III. Der italienische Corte di cassazione geht in seinem Urteil v. 19.03.2019 - Sez. 1 n. 7708/2019, 4.3 - RTI spa v. Yahoo! Inc. einen anderen Weg und gibt gewisse Indizien vor, die eine aktive Rolle begründen sollen: das Filtern, Selektieren, Indizieren, Organisieren, Katalogisieren, die Aggregation, Bewertung, Verwendung, Änderung oder Förderung der Inhalte [...] etwa durch die Einführung einer Verhaltensbewertungstechnik der Benutzer, um ihre Loyalität zu erhöhen [...]“. Wegen der Unklarheiten in diesem Bereich wird auch über eine Nach- bzw. Neujustierung de lege ferenda nachgedacht, In diese Richtung etwa Cobbe/Singh, ebd., S. 13 ff.; Stalla-Bourdillon/ Thorburn, The scandal of intermediary, S. $18 \mathrm{ff}$ und Buitem/de Streel/Peitz, Rethinking Liability Rules for Online Hosting Platforms, S. $15 \mathrm{ff}$. 
ausgegangen werden, dass die sozialen Netzwerke ihre Dienste „neutral“ erbringen und ihnen die Privilegien weiterhin zugutekommen. Insoweit müssten also auch die in Betracht kommenden Maßnahmen in dem oben bereits genannten Spektrum von Identitätskontrolle bis eigenständiger bzw. beauftragter Überwachung und Kennzeichnung mit den Privilegierungen vereinbar sein.

(1.) Unproblematische Fälle: Identitätskontrollen, Beschwerdemanagement, Zugangskontrollen

Jedenfalls einer Identitätskontrolle ${ }^{1790}$ steht die Richtlinie nicht im Weg. Sie ist vollkommen unabhängig von den weitergeleiteten Informationen möglich, sodass der Anwendungsbereich der Artt. 14 Abs. 1, 15 Abs. 1 gar nicht betroffen wäre. Auch ein Beschwerdeverfahren und

-Management ${ }^{1791}$ würde in erster Linie nur dafür sorgen, dass der Netzwerkbetreiber von konkreten Auffälligkeiten überhaupt erst einmal in Kenntnis gesetzt und damit die Haftungsschwelle des Art.14 Abs. 1 überhaupt erst erreicht wird. ${ }^{1792}$ Die Möglichkeit einer entsprechenden Verfahrensregulierung in Bezug auf rechtswidrige Inhalte (ab Kenntniserlangung) sieht Art. 14 Abs. 3 ohnehin schon vor. Auch würde man letztlich keine allgemeine Überwachung anordnen, sondern in einem spezifischen Einzelfall tätig werden, was von Erwägungsgrund 47 sogar ausdrücklich erlaubt wäre. ${ }^{1793}$ Und auch wenn es um eine Kontrolle der API o.ä. ginge, bestehen keine Bedenken, ${ }^{1794}$ weil es schon nicht um eine allgemeine inhaltliche Überprüfung geht.

\section{(2.) Proaktive Überwachung der Datenströme und eigenständige Kennzeichnung}

Eine proaktive Pflicht, Datenströme zu überwachen, um zunächst auffällige Konten ausfindig zu machen und ggf. eigenständig zu kennzeichnen - also der eigentlich interessante und effektivste Anwendungsfall des $\$ 93$ Abs. $4 \mathrm{MStV}$-, wirft dann schon eher Probleme auf. Das gilt aber nur dort, wo der Netzwerkbetreiber selbst etwaige Überwachungsaufgaben übernimmt. Umgehen ließe sich das dadurch, dass ihre „Sorgetragungspflicht“ - vergleichbar mit dem freiwilligen Vorgehen gegen Fake-News - sich darauf beschränkt, Dritten effektiven Zugang zu

1790 So etwa vorgeschlagen von Milker, InTeR 2017, 199 (206). Vgl hierzu auch EuGH, Urt. v. 15.09.2016 - Rs. C-484/14 - EuZW 2016, 821 Rn. 90 ff. - Mc Fadden/Sony Music.

1791 Besonders hinsichtlich des NetzDG wurde der Konflikt mit Art. 15 Abs. 1 der RL 2000/31/EG diskutiert, aber die Norm für mit Art. 15 der Richtlinie vereinbar gehalten, so etwa Bock, Die Übertragbarkeit der Kommunikationsfreiheiten des Art. 5 GG auf das Internet, S. 301.

1792 Vgl. Drexl, ZUM 2017, 529 (539).

1793 Vgl. hierzu EuGH, Urt. v. 03.10.2019 - Rs. C-18/18 = MMR 2019, 798 Rn. 35 - Glawischnig-Piesczek.

1794 So auch Milker, InTeR 2017, 199 (206). 
den Netzwerkdaten zu gewähren, die dann eigenständig die Kontrolle übernehmen. Insoweit beschränkt sich die Verantwortung der Betreiber nicht mehr auf eine proaktive Maßnahme, sondern lediglich auf den Zugang und eine Überwachung der Tätigkeit beauftragter Dritter. Dort, wo eine solche Delegation nicht stattfindet, entstehen die eigentlich interessanten Spannungsfelder mit Art. 15 Abs. 1 RL. ${ }^{1795}$

\section{(a.) Überzeugende Einwände gegen eine Kollision mit Art. 15 Abs. 1 ECRL?}

Gleichwohl werden im Schrifttum drei Begründungsansätze dafür vorgebracht, dass dies mit den Vorgaben der ECRL dennoch vereinbar ist: Erstens ginge es hier nicht um Inhalte, sondern um die Modalitäten des Inhalts, sodass Art. 15 Abs. 1 ECRL gar nicht betroffen sei. ${ }^{1796}$ Zweitens sei der Einsatz von Meinungsrobotern - jedenfalls nicht gegenüber Dritten - rechtswidrig und damit keine rechtswidrige Tätigkeit i.S.v. Art. 15 Abs. 1 ECRL. Und drittens wäre keine deliktsrechtliche Verantwortlichkeit der Betreiber die Folge. ${ }^{1797}$

Was zunächst plausibel klingen mag, wird bei genauerer Betrachtung schon dem Normtext nicht gerecht und auch die bisherige Rechtsprechung des EuGH deutet eher daraufhin, dass man sich mit einer solchen proaktiven Pflicht gerade im Anwendungsbereich des Art. 15 Abs. 1 ECRL bewegt: Auch wenn es primär um die Modalität der Äußerung geht, so muss der Netzwerkbetreiber diese ausfindig machen. Er muss also aktiv nach bestimmten Auffälligkeiten in den Netzwerkdaten - etwa nach Ähnlichkeiten in den Inhalten, sprachlichen Auffälligkeiten, zeitlichen Abständen und anderen Parametern - suchen, sodass hier die Grenze zwischen bloßer Modalität und Inhalt verschwimmen. ${ }^{1798}$ Hinzu kommt, dass die Richtlinie ganz allgemein von Information spricht, sodass damit nicht nur die eigentlichen Inhalte, sondern alle nutzerseitig eingebrachten Informationen gemeint sind. ${ }^{1799}$ Daneben hat der EuGH in der Rechtssache SABAM u. Net$\log$ entschieden, dass ein generelles Filtersystem, welches „die Ermittlung, systematische Prüfung und Verarbeitung der Informationen in Bezug auf die auf dem sozialen Netzwerk von dessen Nutzern geschaffenen Profile bedeute[t][...]“1800 und präventiv, zeitlich unbegrenzt und auf eigene Kosten erfolgt, ${ }^{1801}$ nicht mit

\footnotetext{
1795 Dreyer/Schulz, Schriftliche Stellungnahme zum zweiten Diskussionsentwurf eines Medienstaatsvertrages der Länder vom Juli 2019, S. 16 allerdings noch mit Blick auf $\mathbb{5} 55$ Abs. 3 des MStV aus dem Juli 2019, der der jetzigen Regelung i $₫ 18$ Abs. 3 MStV entspricht.

1796 In diese Richtung allerdings eher mit Blick auf Identitätskontrollen, Milker, InTeR 2017, 199 (206); allgemeiner Löber/Roßnagel, MMR 2019, 493 (495).

1797 So Löber/Roßnagel, MMR 2019, 493 (495).

1798 Vgl. hierzu Schlussantrag von GA Szpunar, SA v. 04.06.2019 - Rs.18/18, Rn.26, der meint, dass die Identifikation von Inhalten die Überwachung oder Filterung voraussetzt.

1799 In diese Richtung auch GA Szpunar, SA v. 04.06.2019 - Rs. 18/18, Rn. 48.

1800 EuGH, Urt. v. 16. 02. 2012 - Rs. C-360/10 = GRUR 2012, 382 Rn. 49 - SABAM u. Netlog.

1801 EuGH, ebd., Rn. 26 - SABAM u. Netlog.
} 
Art. 15 Abs. 1 der RL vereinbar ist. Ein vergleichbarer Filter müsste aber auch in dem hiesigen Fall vorgehalten werden. ${ }^{1802}$ Insoweit könnte nur entscheidend sein, dass der inhaltliche Bezug marginal ist, während er im Fall von Netlog überwog. ${ }^{1803}$

Zwar ist der Einsatz von Meinungsrobotern nicht rechtswidrig. Allerdings gilt das nur unter der Bedingung, dass entweder keine Täuschungswirkung mit ihnen einher geht oder eine Kennzeichnung dieser Wirkung erfolgt ist, $(\mathbb{S} \mathbb{S} 18$ Abs. 3, 117 Abs. 1 S. 2 Nr. 2 MStV). Und auch wenn für den Netzwerkbetreiber keine deliktsrechtliche Haftung begründet wird, so sieht $\$ 117$ Abs. 1 S. 2 Nr. $42 \mathrm{MStV}$ doch ein „täterschaftliches Haftungsregime“ vor. Verlangt man also eine Überwachung, so führte das gerade dazu, dass der Betreiber aktiv nach Umständen suchen muss, die auf ein rechtswidriges Unterlassen hindeuten. Die Verantwortlichkeit im Sinne der Art. 12-15 der Richtlinie hat aber keinen rein zivilrechtlichen Bezug, ${ }^{1804}$ sondern erfasst auch die straf- und ordnungswidrigkeitsrechtliche Verantwortlichkeit. ${ }^{1805}$ Insoweit deutet eigentlich vieles auf die Unvereinbarkeit einer proaktiven Pflicht hin. ${ }^{1806}$

\section{(b.) Richtlinienkonforme Ausnahme im Falle der Meinungsroboter?}

Jedenfalls unter Berücksichtigung der bisher vorgetragenen Argumente im Schrifttum stünde also ein solches Verständnis von $\mathbb{S} 93$ Abs. 4 MStV mit Art. 15 Abs. 1 ECRL in einem eklatanten Widerspruch. Eine Ausnahme käme nur dann

1802 Hierauf weist auch Kuczerawy, The Proposed Regulation on Preventing the Dissemination of Terrorist Content Online, S. 14 hinsichtlich Art. 6 der geplanten Verordnung zur Verhinderung terroristischer Online-Inhalte in der Fassung des Kommissionsvorschlags KOM(2018) 640 endg. hin. Ebenso zu Art. 17 der Urheberrechtsrichtlinie Frosio/Mendis, in: Frosio, The Oxford Handbook of Online Intermediary Liability, Ch. 28 S. 19.

1803 Deshalb sieht etwa die Richtlinie über das Urheberrecht und die verwandten Schutzrechte im digitalen Binnenmarkt und zur Änderung der Richtlinien 96/9/EG und 2001/29/EG (RL 2019/790) sieht in Art. 17 Abs. 3 eine Ausnahme der Haftungsprivilegierung des Art. 14 Abs. 1 der RL 2000/31/EG vor, sodass bei sog. Uploadfilter ein solcher Konflikt zukünftig jedenfalls nicht mehr besteht. Ausführlicher zu den Problemen dieser Regelung etwa Gerpott, MMR 2019, $420 \mathrm{ff}$.

1804 So aber Löber/Roßnagel, MMR 2019, 493 (495) mit dem Hinweis auf die deliktsrechtliche Haftung. Das suggerieren jedenfalls auch die bisherigen Entscheidungen, in denen es um persönlichkeitsrechtliche Unterlassungs- und Beseitigungsansprüche, so etwa im jüngsten Urteil des EuGH, v. 03.10.2019 - Rs. C-18/18 = MMR 2019, 798 - Glawischnig-Piesczek, oder um Urheberrechtsverletzungen ging, so etwa in EuGH, Urt. v. 16. 02. 2012 - Rs. C-360/10 = GRUR 2012, 382 Rn. 49 SABAM $u$. Netlog.

1805 Schon die Kommission hat im Verfahren die strafrechtliche Verantwortlichkeit mit angesprochen, $\operatorname{KOM}(1998)$ 568, Erwgg. 16, ABl. 1999 C 30/4. Und so letztlich auch Oermann, in: HofmannRiem, Big Data - Regulative Herausforderungen, S. 145 (150); vgl. Spindler, NJW 2002, 921 (924); Richter, ZD-Aktuell 2017, 05623; Valerius, in: v. Heintschel-Heinegg, BeckOK StGB, \185 Rn. 44 ff.; BT-Drs. 14/6098, S. 23. Das ergibt sich letztlich auch aus der Zielsetzung, die Verantwortlichkeit der Dienstanbieter abschließend zu harmonisieren, weil gerade solche Vorschriften erhebliche wettbewerbsbegrenzende Wirkung entfalten können, siehe Erwägungsgrund 40 der Richtlinie 2000/31/EG, ABl. 2000 L 178/6.

1806 Das etwa andeutend Dreyer/Schulz, Stellungnahme zum zweiten Diskussionsentwurf eines Medienstaatsvertrages, S. 16. 
in Betracht, wenn die Richtlinie andernorts einen Anknüpfungspunkt für eine Ausnahme bereithielte oder im Wege der Auslegung eine solche Ausnahme in die RL hineingelesen werden könnte. Hierfür bestehen drei potentielle Anknüpfungspunkte: Erstens ist nur eine allgemeine aber keine spezifische Verpflichtung untersagt (aa.). Zweitens stammt die E-Commerce-RL aus dem Jahr 2000. Seitdem haben sich aber die zugrundeliegenden technischen Sachverhalte deutlich geändert, sodass das zugrunde liegende (grundrechtliche) Konzept nicht mehr auf alle Bereiche Anwendung finden könnte (bb.). Und drittens enthält Art. 1 Abs. 6 der RL eine Öffnungsklausel für Mitgliedstaaten im Bereich des Medienpluralismus (cc.).

(aa.) $\mathbb{9} 93$ Abs. $4 \mathrm{MStV}$ als „spezifischer Fall“?

Art. 15 Abs. 1 ECRL i.V.m Erwägungsgrund 47 verbietet nur allgemeine Verpflichtungen. In spezifischen Fällen bleibt eine proaktive Überwachungspflicht möglich. ${ }^{1807}$ Handelt es sich also bei $\mathbb{S} 93$ Abs. $4 \mathrm{MStV}$ um einen spezifischen Fall? Für gerichtliche Anordnungen hatte der EuGH jüngst in der Rechtssache Glawischnig-Piesczek ${ }^{1808}$ Kriterien hierfür zu entwickeln: Ein österreichisches Gericht hatte Facebook verpflichtet, bestimmte persönlichkeitsverletzende Inhalte eines Nutzers sowie alle wort- und sinngleichen Inhalte anderer Nutzer zu entfernen. Generalanwalt Szpunar wies in seinem Schlussantrag darauf hin, dass es im Falle gerichtlicher Anordnungen für die Frage nach einem spezifischen Fall auf die Zielgerichtetheit ankomme, die sich gesamtheitlich aus der Dauer, der konkret benannten Rechtsverletzung, dem Gegenstand und dem Urheber ergebe. ${ }^{1809}$ Diese Kriterien hat der EuGH seiner Entscheidung zu Grunde gelegt. In der Anordnung sah er einen spezifischen Fall, weil die Rechtsverletzung und der konkrete Inhalt der Anordnung so deutlich waren, dass der Betreiber keine autonome Überprüfung vornehmen müsse, sondern vielmehr durch automatisierte Verfahren dies bewerkstelligen könne. ${ }^{1810}$ Im Gegensatz hierzu hat der Gerichtshof allgemeine Pflichten etwa dann angenommen, wenn „sämtliche Daten jedes Einzelnen [...] Kunden aktiv zu überwachen [wären], um jeder künftigen Verletzung von Rechten des geistigen Eigentums vorzubeugen“"1811. Anbieter müssen gerade nicht „aktiv alle Angaben eines jeden seiner Kunden [...] überwachen, um jeder

1807 Ob Löber/Roßnagel, MMR 2018, 493 (496) hierauf hinweisen, wenn sie meinen, dass es um „einen nach allgemeinen Kriterien abgrenzbaren Sachverhalt" geht, ist unklar.

1808 EuGH, v. 03.10.2019- Rs. C-18/18 = MMR 2019, 798 ff. - Glawischnig-Piesczek.

1809 Schlussantrag v. 04.06.2019 - Rs. C-18/18 - Glawischnig-Piesczek.

1810 EuGH, v. 03.10.2019- Rs.C-18/18 = MMR 2019, 798 Rn. 46, 53 - Glawischnig-Piesczek.

1811 EuGH, Urt. v. 24.11.2011 - Rs. C-70/10 = MMR 2012, 174 Rn. 40 - Scarlet/SABAM. 
künftigen Verletzung von Rechten des geistigen Eigentums über die Seite dieses Anbieters vorzubeugen [Hervorh. d. Aut.]“. ${ }^{1812}$

Auch in nationalen Verfahren geht es meist um bestimmte gerichtliche Anordnungen spezifische Rechtsverletzungen des Klägers betreffend, denen abgeholfen und die zukünftig vermieden werden sollen. Dem BGH kam es diesbezüglich auch weniger auf die Anzahl der Inhalte als vielmehr auf die konkrete Rechtsverletzung an, die es zu verhindern gilt. ${ }^{1813}$ Insoweit hat er keine allgemeine Pflicht in der Überprüfung von Inhalten dort angenommen, wo es um das Verhindern des Uploads von ein und demselben urheberrechtlich geschützten Werk auch durch andere Nutzer ging. ${ }^{1814}$ Andernorts wird besonders eine zeitliche oder sachliche Begrenzung gefordert, weil ansonsten mittelbar eine allgemeine Pflicht eingeführt werden könne. ${ }^{1815}$ Demnach läge ein spezifischer Fall also auch dann vor, wenn es um eine bestimmte konkrete Rechtsverletzung geht, die hinreichend klar umrissen ist. Entscheidend ist dabei nicht, dass sie durch eine Vielzahl von Nutzern begangen werden kann, sofern allerdings keine zeitlich unbefristete Pflicht hieraus erwächst.

Mit derlei Ansätzen kann ein spezifischer Charakter von $\mathbb{9} 93$ Abs. 4 MStV aber nicht begründet werden. Auch wenn es bei der Überwachung der fehlenden Kennzeichnung um eine bestimmte und hinreichend umrissene Rechtsverletzung geht, so ist die Ermittlung eines Verstoßes weitestgehend den Betreibern überlassen. Kriterien für die Überwachung wie in der Rechtssache Glawischnig-Piesczek werden gerade nicht gegeben, sodass die Anbieter erst einmal eine nicht unerhebliche geistige Arbeit erbringen müssen, um die Kriterien für einen Rechtsverstoß zu bestimmen und danach auf Grundlage dieser ein eigenständiges Urteil zu fällen. Dadurch verlässt der Betreiber aber seine neutrale Rolle. ${ }^{1816}$ Entscheidend ist hier zudem, dass es um eine abstrakt-generelle Regelung geht, die sich grundsätzlich an alle Anbieter sozialer Netzwerke richtet, sofern nicht die Bagatellgrenze des $\mathbb{\int} 91$ Abs. $2 \mathrm{MStV}$ unterschritten ist. Abstrakt-generelle Regelung und spezifi-

1812 EuGH, Urt. v. 12.07.2011 - Rs. C-324/09 = MMR 2011, Rn. 139 - L'Oréal/Ebay. Dies bezog er in der Rechtssache SABAM/Netlog auch auf den Betreiber eines sozialen Netzwerks, dem durch ein nationales Gericht angeordnet wurde, ein Filtersystem bereitzuhalten, dass präventiv, zeitlich unbegrenzt und auf alle Nutzer bezogen Inhalte nach Urheberrechtsverletzungen durchsucht und diese blockiert, EuGH, Urt. v.16.02.2012 - Rs. C-360/10 = GRUR 2012, 382 Rn. 49 - SABAM u. Net$\log$.

1813 So auch Holznagel, ZUM 2018, 350 (351 f.).

1814 Siehe etwa BGH, ZUM 2013, 288 Rn. 32 - Alone in the Dark. Auch im Schrifttum wird eine Überwachung aller Inhalte für zulässig gehalten, sofern es um eine bestimmte Rechtsverletzung geht, Holznagel, ZUM 2018, 350 (355); Spindler, NJW 2019, 3274 (3275). Mit Bezug zu Urheberrechtsverletzungen meint Nordemann, GRUR 2011, 977 (980), dass ein spezifischer Fall dann vorliege, wenn es um ein und dasselbe Werk oder wenn es um denselben „Wiederholungstäter" und um „Werke der gleichen Kategorie“ gehe.

1815 Siehe etwa Kastl, GRUR 2016, 671 (676). Das zeitliche Element betont letztlich auch GA Szpunar, Schlussantrag v. 04.06.2019 - Rs. C-18/18, Rn. 47, 49.

1816 Hierauf weist etwa auch GA Szpunar in seinem Schlussantrag v. 04.06.2019 - RS. C-18/18 Rn. 73 mit Blick auf die Erkennung sinngleicher Inhalte anderer Nutzer hin. 
scher Einzelfall lassen sich so nur schwer miteinander vereinbaren, zumal hier potentiell alle Netzwerkdaten und -konten überwacht werden müssten und das für einen unbegrenzten Zeitraum. ${ }^{1817}$

(bb.) Bereichsausnahme des Haftungsprivilegs im Falle von $\mathbb{9} 93$ Abs. 4 MStV?

Ursprünglich wollte die E-Commerce-Richtlinie den Herausforderungen einer zunehmend digitalisierten Wirtschaft gerecht werden. Dienste der Informationsgesellschaft wurden als Treibkraft für Innovation und wirtschaftlichen Wachstum ausgemacht, ${ }^{1818}$ weshalb zentrale rechtliche Hemmnisse in den Rechtsordnungen der Mitgliedstaaten beseitigt und harmonisiert werden sollten, ${ }^{1819}$ wozu insbesondere die Verantwortlichkeit der Informationsdienste gehörte. ${ }^{1820}$ Bei Erlass der Richtlinie im Jahr 1999 waren Begriffe wie „Web 2.0“ oder „User-generatedContent" aber nicht mal bekannt; ${ }^{1821}$ entsprechendes gilt für die gewachsene Bedeutung der Dienste für sozial-demokratische Prozesse und damit auch die Risiken, die Dienste hierfür begründen können. ${ }^{1822}$ Mit zunehmender Relevanz der Dienste für demokratische Prozesse führt das zu erheblichen Konflikten, ${ }^{1823}$ was gerade bei $\mathbb{\$} 93$ Abs. $4 \mathrm{MStV}$ deutlich wird.

Ein potentieller Ausweg könnte hier im Wege einer (teleologischen) Reduktion vor allem unter Berücksichtigung der maßgeblichen grundrechtlichen Erwägungen der Haftungsprivilegien (vgl. Erwgr. 41) gefunden werden. Im Spannungsfeld von unternehmerischer Freiheit der Betreiber (Art. 16 EuGrCh), ${ }^{1824}$ Informationsund Meinungsfreiheit der Nutzer (Art. 11 EuGrCh) und geschützten Rechtsinter-

1817 Das hält aber GA Szpunar, Schlussantrag v. 04.06.2019 - Rs. C-18/18 - Glawischnig-Piesczek, für „schwerlich mit der Vorstellung von einer auf spezifische Fälle im Sinne des 47. Erwägungsgrundes der Richtlinie 2000/31 ausgerichteten Verpflichtung vereinbar." Vgl. hierzu auch Kuczerawy, The proposed Regulation on preventing the dissemination of terrorist content online, S. 14.

1818 Erwägungsgrund 2 der Richtlinie 2000/31/EG, ABl. 1999 L 178/1.

1819 Erwägungsgrund $3 \mathrm{ff}$.

1820 Erwägungsgrund 40.

1821 Auf diesen Aspekt weisen auch Cole/Etteldorf/Ullrich, Europäischer Rechtsrahmen und die Rechtsdurchsetzung bei der grenzüberschreitenden Verbreitung von Online-Inhalten, S. 6, hin, um damit auf die nur unzureichende Differenzierung der Dienste in der E-Commerce-RL hinzuweisen. In diese Richtung auch Policy Department for Citizens' Rights and Constitutional Affairs, Disinformation and propaganda - impact on the functioning of the rule of law in the EU and its Member States, S. 81. Siehe auch Nölscher, ZUM 2020, 301 (309), der hierauf in der Diskussion um die Vereinbarkeit des NetzDG mit der E-Commerce-RL eingeht.

1822 Mit Blick auf die Gatekeeper-Funktion betonen etwa Cole/Etteldorf/Ullrich, Cross-Border Dissemination of Online Content, S. $200 \mathrm{f}$. diesen Wandel.

1823 Von Hanfeld, Im digitalen Sandkasten, Faz.net, wird sie gar als „demokratiegefährdend“ betitelt. Eifert, in: ders./Gostomzyk, Netzwerkrecht, S. 9 (24) weißt hierauf und auf die Bedürftigkeit einer Überarbeitung hin.

1824 Im Kontext von Art. 15 Abs. 1 der E-Commerce-RL siehe EuGH, Urt. v. 24.11.2011 - Rs. C-70/10 = MMR 2012, 174 Rn. 46 ff. - Scarlet $/ S A B A M$; Urt. v.16.02.2012- C-360/10 = GRUR 2012, 382 Rn. 44 - SABAM/Netlog. Siehe auch GA Szpunar, Schlussantrag v. 04.06.2019 - Rs. C-18/18, Rn. 63 - Glawischnig-Piesczek. 
essen - etwa Urheber- (Art. 17 EuGrCh) ${ }^{1825}$ oder Persönlichkeitsrechte (Art. 7 Eu$\mathrm{GrCh})^{1826}$ - galt es einen gerechten Ausgleich zu finden, der hier nur kurz skizziert werden soll: Die Anbieter haben keine Kontrolle über die konkreten Inhalte oder die Verwendung ihrer Dienste durch die Nutzer, sodass eine breite Verantwortlichkeit für das Nutzerverhalten die wirtschaftliche Tätigkeit erheblich beeinträchtigen würde. Das gilt nicht nur hinsichtlich der rechtlichen und finanziellen Belastung, die eine Haftung der Anbieter bereits mit der Veröffentlichung von Inhalten unweigerlich zur Folge hätte. Auch der Aufwand, um allen potentiellen Rechtsverletzungen durch Nutzer präventiv zu begegnen oder proaktiv ausfindig zu machen und sodann abzustellen, würde den Rahmen des Zumutbaren sprengen. ${ }^{1827}$ Ein automatisiertes System kann allein kaum die Aufgaben bewerkstelligen, ohne dabei - vor allem in Graubereichen, in denen das Ergebnis stark vom Einzelfall abhängig ist $-{ }^{1828}$ fehleranfällig zu sein. ${ }^{1829}$ Insbesondere diese Fehleranfälligkeit aber auch allgemein die betreiberseitige Kontrolle und Löschung von Inhalten führt daneben nicht nur zu einer Beeinträchtigung der Äußerungsfreiheit der Nutzer. Betroffene Inhalte können auch nicht mehr wahrgenommen werden, sodass auch die Informationsfreiheit tangiert wird. ${ }^{1830}$ Besonders im Falle nach-

1825 Siehe hierzu im Kontext des Art. 15 Abs. 1 der E-Commerce-RL etwa EuGH, Urt. v. 24.11.2011 C-70/10 = MMR 2012, 174 Rn. 43 ff. - Scarlet $/ S A B A M$; EuGH, Urt. v.16.02.2012- C-360/10 = GRUR 2012, 382 Rn. 41 ff. - SABAM u. Netlog. Daneben auch EuGH, Urt. v. 29.01. 2008 C-275/06 = GRUR 2008, 241 Rn. 62 - Promusicae/Telefónica m.w.N.

1826 Zwar stellt der EuGH in seinem Urt. v. 03.10.2019- Rs. C-18/18 = MMR 2019, 798 Rn. 36 f., 41, 44 - Glawischnig-Piesczek, nicht auch die Charta ab, gleichwohl betont es die Bedeutung der Verteilung von Inhalten in sozialen Netzwerken für eine Intensivierung der (Persönlichkeits-)Rechtsverletzung. Siehe auch GA Szpunar, Schlussantrag v. 04.06.2019 - Rs. C-18/18, Rn. 64 - GlawischnigPiesczek.

1827 So EuGH, Urt. v. 24.11.2011 - Rs. C-70/10 = MMR 2012, 17446 ff. - Scarlet/SABAM; Urt. v.16.02.2012- Rs. C-360/10 = GRUR 2012, 382 Rn. 46 - SABAM u. Netlog. In seinem Urt. v. 03.10.2019- Rs. C-18/18 = MMR 2019, 798 - Glawischnig-Piesczek, war letztlich auch der erforderliche Aufwand maßgeblich für die endgültige Entscheidung des EuGH, Rn. 44 ff. Auf den Aufwand berufen sich auch die deutschen Gerichte im Bereich der Störerhaftung, wenn es um die Frage von zumutbaren Prüfpflichten der Dienstanbieter geht, siehe hierzu u.a. BGH, GRUR 2013, 370 Rn. 28 - Alone in the Dark; GRUR 2013, 1229 Rn. 47 - Kinderhochstüble im Internet I; LG Würzburg, ZUM 2017, 437 (447). Zum personellen Aufwand einer notwendigen händischen Nachkontrolle vgl. BGH, GRUR 2015, 1129 Rn. 37 - Hotelbewertungsportal; KOM(2018) 640 final, S. 10.

1828 Das gilt etwa für die Frage der Rechtswidrigkeit einer Äußerung, wo Satire und Ironie Probleme mit sich bringen und wo auch die Grenze zwischen persönlichkeitsverletzender und berechtigter Meinungsäußerung fließend ist. Deshalb betont der EuGH in seinem Urt. v. 03.10.2019- Rs. C-18/18 = MMR 2019, 798 - Glawischnig-Piesczek, auch, dass Facebook hier keine autonome Entscheidung treffen muss, vielmehr die Rechtswidrigkeit maßgeblich durch das nationale Gericht schon vorgegeben wird, a.a.O Rn. 45 f., 53. Ähnliches gilt im Falle von Urheberrechtsverletzungen, wo unklar sein kann, ob etwaige Ausnahme- oder Genehmigungstatbestände eingreifen, hierzu im Kontext von Art. 15 Abs. 1 e-Commerce-RL GA Szpunar, Schlussantrag v. 04.06.2019 - Rs. C-18/18, Rn. 63 Glawischnig-Piesczek.

1829 Vgl. hierzu Heldt, EuR 2020, 238 (243 f., 245); Kuczerawy, The proposed Regulation on preventing the dissemination of terrorist content online, S. 14.

1830 Vgl. EuGH, Urt. v. 24.11.2011 - Rs. C-70/10 = MMR 2012, 174 Rn. 52 - Scarlet/SABAM; Urt. v.16.02.2012- C-360/10 = GRUR 2012, 382 Rn. 50 - SABAM u. Netlog. Vgl. auch Kuczerawy, The proposed Regulation on preventing the dissemination of terrorist content online, S. $14 \mathrm{f}$. 
teiliger (ökonomischer) Folgen, bestünde die Gefahr eines „Overblockings“.. ${ }^{1831}$ Zusätzlich hat der EuGH hier aber auch auf den Schutz personenbezogener Daten hingewiesen, der bei einer proaktiven Verpflichtung beeinträchtigt werden kann. ${ }^{1832}$ Auch wenn durch die Erbringung der Dienste eine nicht unerhebliche Gefährdung der Rechtsgüter von Personen droht, auch weil sich Inhalte schnell und weit verbreiten lassen, ${ }^{183}$ begegnen dem die Haftungsprivilegien dadurch, dass Betroffene ihre Rechte gegenüber den Betreibern geltend machen können, sie dadurch in Kenntnis eines rechtswidrigen Inhaltes setzen und so die Haftung nach den allgemeinen Regeln begründen können. Dabei kann die Verantwortung sich dann auch nicht nur auf den konkreten Inhalt, sondern auf inhaltsgleiche Daten beziehen, sofern dies den Betreibern zumutbar ist. ${ }^{1834}$ Insoweit kann das aus Artt. 14 Abs.1, 15 Abs. 1 ECRL resultierende Notice-and-take-down-Verfahren „in vielen Fällen als angemessenes Werkzeug zum Ausgleich der Rechte und Interessen aller Beteiligten funktionieren [...]. “1835

Im Kontext der Meinungsroboter sind aber verschiedene Aspekte zu berücksichtigen, die von dem soeben skizzierten grundrechtlichen Gleichgewicht (erheblich) abweichen. Das beginnt schon bei dem Inhalt der Pflicht. Auch wenn ein spezifischer Fall fernliegen mag, so bleibt die Pflicht dennoch auf einen konkreten Anwendungsfall beschränkt: eine Automatisierung menschlich wirkender Nutzerprofile. Insoweit geht es also gar nicht darum, allen erdenklichen Rechtsverletzungen durch Nutzer betreiberseitig vorzubeugen. Auch die bedeutsamen Haftungsrisiken bestehen hier nicht. Der Einsatz von Meinungsrobotern führt nicht zu schadensersatz- oder sonstigen Ansprüchen finanzieller Art von Seiten der Rechteinhaber. Zwar wird mit $\$ 117$ Abs. 1 S. 2 Nr. 42 MStV eine strafrechtliche Haftung begründet, sie gilt aber nicht inhaltsbezogen, sondern sichert nur $\mathrm{ab}$, dass die Betreiber überhaupt und nicht in völlig ineffektiver Weise tätig werden. ${ }^{1836}$ Insoweit wäre also der eigentliche Anwendungsbereich von Art. 14 Abs. 1 der Richtlinie nicht tangiert und damit auch nicht die sichernde Funktion

1831 Vgl. hierzu die Diskussion beim NetzDG etwa bei Liesching, MMR 2018, 26 (27f.) oder bei Schiff, MMR 2018, 366 (369f.).

1832 EuGH, Urt. v. 24.11.2011 - Rs. C-70/10 = MMR 2012, 174 Rn. 50 f. - Scarlet/SABAM mit Blick auf potentiell zu speichernde IP-Adressen der Nutzer. Mit Blick auf die Profildaten der Nutzer in sozialen Netzwerken Urt. v. 16.02.2012 - Rs. C-360/10 = GRUR 2012, 382 Rn. 50 - SABAM $u$. Netlog.

1833 Vgl. hierzu auch EGMR, Urt. v. 16.06.2015 - 64569/09 = GRURInt 2016, 81 Rn. 110 - Delfil Estland. Und das war auch die maßgebliche Begründung des NetzDG, vgl. BT-Drs. 18/12356, S. 19.

1834 Mit Blick auf persönlichkeitsverletzende Inhalte EuGH, Urt. v. 03.10.2019- Rs. C-18/18 = MMR 2019, 798 Rn. 38 ff. - Glawischnig-Piesczek. Mit Blick auf urheberechtsverletzende Inhalte etwa BGH, GRUR 2013, 370 Rn. 32 - Alone in the Dark.

1835 EGMR, Urt. v. 02.02.2016 - 22947/13 = NJW 2017, 2091 Rn.91- Magyar Tartalomszolgáltatók Egyesülete u. Index.hu Zrt/Ungarn. Vgl. auch EGMR, Urt. v. 16.06.2015 - 64569/09 = GRURInt 2016, 81 Rn. 159 - Delfi/Estland.

1836 Hier drin besteht einer der wesentlichen Unterschiede zum „Haftungsregime“ des NetzDG, welches ein deutlich engeres Korsett in $\$ 4$ Abs. 1 schnürt. 
von Art. 15 Abs. 1 der Richtlinie ${ }^{1837}$. Zudem ist hier zu berücksichtigen, dass die Norm den Anbietern den notwendigen Freiraum belässt, eigenständig maßgebliche Entscheidungen organisatorischer Art zu treffen. Insoweit ist das Ziel, nicht aber der Weg dorthin vorgegeben, ${ }^{1838}$ sodass auch der unternehmerischen Freiheit Rechnung getragen wird.

Betrachtet man die Rolle der Netzwerkbetreiber, so ergeben sich ebenfalls Unterschiede: Sie ermöglichen die Automatisierung von Nutzerkonten durch die Vorhaltung von Programmierschnittstellen und eine nur unzureichende Kontrolle ihrer eigenen Standards. Bei Twitter gilt das noch deutlich stärker, denn das Netzwerk erlaubt - unter Einhaltung entsprechender Vorgaben - die Automatisierung von Profilen. Insoweit erschöpft sich der Beitrag nicht nur in der „sozialadäquaten " 1839 Bereitstellung der Kommunikationsinfrastruktur, sondern es wird gerade auch die Gefährdungslage mitverursacht. ${ }^{1840}$ Entsprechendes gilt auch für die Nutzeroberfläche, deren Design den erfolgreichen Einsatz von Meinungsrobotern begünstigt. ${ }^{1841}$ Insoweit geht es nicht nur um die Verantwortlichkeit für das (Fremd-)Verhalten der Nutzer, sondern auch um die Verantwortung für die gesellschaftlich-demokratische Wirkung der eigenen Dienste.

Anders als bei inhaltlich ausgerichteten Pflichten ist ein Einfluss auf die Meinungsäußerung nur hinsichtlich der Modalität gewollt. Die Äußerung als solche bleibt nach dem gesetzgeberischen Leitbild zulässig. Das wiederum führt auch nicht dazu, dass anderen Nutzern Informationen vorenthalten werden. Vielmehr wird eine sachgerechte Einordnung der Äußerung so erst ermöglicht. Das macht auch den wesentlichen Unterschied zu bisherigen Fallkonstellationen aus. ${ }^{1842}$ Fehler bei der Erkennung schlagen also nicht so erheblich ins Gewicht wie bei einer vollständigen Löschung oder Sperrung der Inhalte.

Entscheidend ist hier auch, dass - abseits des verfolgten Schutzes - kein Bezug zu kollidierenden Rechten besteht, aus denen eine Inanspruchnahme der Betreiber durch Dritte folgen könnte. ${ }^{1843}$ Eine Mitteilung würde - nach bisheriger Rechtslage - keine rechtliche Verpflichtung der Betreiber begründen, entsprechende Inhalte zu kennzeichnen oder zu entfernen, sodass das bisherige Rege-

1837 Vgl. hierzu Marly, in: Grabitz/Hilf, Recht der EU, A. 4. Art. 15 Rn. 3.

1838 Hierin unterscheidet sich die vorliegende Situation aber von den national-gerichtlichen Anordnungen, die der EuGH zu beurteilen hatte.

1839 So in Bezug auf den Telos Fitzner, GRURInt 2012, 109 (113).

1840 Das gleiche Problem stellt sich etwa hinsichtlich der Cambridge-Analytica-Affäre und Facebook, hierzu Cadwalladr/Graham-Harrison, Revealed: 50 million Facebook profiles harvested for Cambridge Analytica in major data breach, The Guardian. Hier soll das Unternehmen ja auch von der Sicherheitslücke gewusst haben, aber nichts gegen die Extraktion der Daten zahlreicher Nutzer unternommen und so - durch Unterlassen - auch einen wesentlichen Kausalbeitrag geleistet haben.

1841 Hierzu ausführlicher Kaerlein, in: Hug/Pallaver, Talk with the bots, S. 17 (23 ff.).

1842 Denn in den Fällen vor dem EuGH ging es immer um Anordnungen, die eine endgültige Entfernung der Inhalte zur Folge haben sollte, siehe hierzu vor allem EuGH, Urt. v.16.02.2012- Rs. C-360/10 = GRUR 2012, 382 Rn. 48 ff. - SABAM/Netlog.

1843 Ähnlich auch Schröder, DVBl. 2018, 465 (472). 
lungssystem der Art. 14 Abs. 1, 15 Abs. 1 ECRL in Hinblick auf die potentiellen Auswirkungen von Meinungsrobotern ohnehin ins Leere liefe. Die Einführung eines Managementsystem würde zwar die nachteiligen Folgen für die Anbieter abmildern. Das wird aber der Konstellation hier nicht gerecht, denn während die Rechtswidrigkeit von Inhalten ohne Weiteres ersichtlich sein oder zumindest ernste Zweifel hervorrufen und durch Rechtsinhaber nachvollzogen werden kann, ist der Inhalt bei Meinungsrobotern nicht per se problematisch und dort, wo der Inhalt dies ist, besteht mit dem Notice-and-Takedown-Verfahren des NetzDG jedenfalls ein ausreichender, dem Grunde nach ${ }^{1844}$ richtlinienkonformer Schutz. Die Täuschungswirkung tritt hier aber oftmals nicht offen zu Tage, sondern kann erst durch eine Analyse der Profil-, Nutzungs- und Metadaten aufgedeckt werden. Ein umfangreicher Einblick in diese ist aber den Betreibern vorbehalten. Während also bei rechtswidrigen Inhalten durchaus von einem Kompromiss zwischen den kollidierenden Interessen gesprochen werden kann, laufen wesentliche Erwägungen der Norm im Fall der Meinungsroboter ins Leere.

Prinzipiell scheint auch beim unionalen Gesetzgeber die Kenntnis gereift, dass der liberale Ansatz der Haftungsprivilegierungen nicht in jeder Konstellation sinnvoll ist. ${ }^{1845}$ Das zeigt nicht nur der angesprochene Verhaltenskodex (oben C.I.3.a.bb). Auch die Kommissionsempfehlung 2018/334 sieht in Kapitel II, Rn. 18 vor, dass „Hostingdiensteanbieter [...] aufgefordert werden [sollten], in Bezug auf illegale Inhalte, soweit angemessen, besondere proaktive, verhältnismäßige Maßnahmen zu ergreifen“, was auch den „Einsatz von Systemen zur automatischen Erkennung illegaler Inhalte umfassen [...][kann].“ Auf die Haftungsprivilegierungen der E-Commerce-RL soll dies aber ausweislich des Kapitel I Nr. 3 keinen Einfluss haben. Diese Empfehlung konkretisiert sich etwa im Kommissionsvorschlag für eine Verordnung zur Verhinderung der Verbreitung terroristischer Online-Inhalte aus dem Jahr 2018. ${ }^{1846}$ So sollen die Betreiber nach Art. 6 Abs. 1 „gegebenenfalls proaktive Maßnahmen [ergreifen], um ihre Dienste vor der Verbreitung terroristischer Inhalte zu schützen. “1847 Die Haftungsprivile-

1844 Ausführlicher zu den eigentlichen Kernproblemen aus unionsrechtlicher Sicht Spindler, ZUM 2017, $473 \mathrm{ff}$.

1845 Kuczerawy, The Proposed Regulation on Preventing the Dissemination of Terrorist Content Online, S. 5 sieht die geplante Verordnung als „major shift in the approach towards the obligations of online hosting services“ an. Vgl. hierzu auch die Darstellungen von Riis/Schwemer, Journal of Internet Law Vol 22 (2019), 1 (12 ff.). Allgemein die zunehmende Inpflichtnahme von Intermediären betont auch Eifert, in: ders./Gostomzyk, Netzwerkrecht, S. 9 (13f.), wobei er auch darauf verweist, dass die Haftungsprivilegierung eine thematische Auseinandersetzung über eine „angemessenen und inhaltlichen Kontrolle der Inhalte durch die Intermediäre“, S. 11. Frosio/Mendis, in: Frosio, Oxford Handbook of Intermediary liability, Ch. 28 S. 4 sprechen hier sogar von einem „global shift“. Cole/ Etteldorf/Ullrich, Cross-Border Dissemination of Online Content, S. 201 sprechen allgemein von einer „tendency away from the traditional liability framework towards responsibility“ und stellen hier Vorschläge eines „Duty of Care“-Ansatzes vor, ebd, S. $202 \mathrm{ff}$.

$1846 \operatorname{KOM}(2018) 640$ final.

1847 Im weiteren Verfahren wurde proaktiv allerdings durch spezifisch ersetzt, sodass unklar ist, ob damit nur Fälle in einem konkreten Einzelfall gemeint sind (vgl. Erwägungsgrund. 47 RL 2000/31/ 
gierung des Art. 14 Abs. 1 der RL 2000/31/EG soll aber unberührt bleiben. ${ }^{1848}$ Entscheidend für eine Aufrechterhaltung des Haftungssystems mag sein, dass der Betreiber zu wichtigen gesellschaftlichen Zwecken aktiv wird, ${ }^{1849}$ man auf ihn bei der Bekämpfung angewiesen ist und eine allein reaktive Kontrolle die (demokratiegefährdende) Wirkung solcher Inhalte unverändert lassen würde, sodass ihm eine aktive Rolle nicht zum Nachteil gereicht werden soll. ${ }^{1850}$ Anders ist die Situation bei der Harmonisierung des Urheberrechts und die Diskussion um einen sog. Value Gap. ${ }^{1851}$ In Art. 17 Abs. 1 der „Richtlinie 2019/790 über das Urheberrecht und die verwandten Schutzrechte im digitalen Binnenmarkt (...) “1852 wird eine Haftung der Betreiber für Urheberrechtsverletzungen der Nutzer begründet. Art. 14 Abs. 1 der E-Commerce-RL findet keine Anwendung (Art. 17 Abs. 3). Hier wird der Betreiber nicht als "guter Samariter“ in Anspruch genommen, ${ }^{1853}$ sondern als (angeblicher) wirtschaftlicher Profiteur urheberrechtsverletzender Handlungen. Art. 17 Abs. 4 macht hiervon Einschränkungen, wenn der Anbieter drei kumulative Anforderungen zur Vermeidung der Verletzungen erfüllt. ${ }^{1854}$ Insoweit dreht sich das Regel-Ausnahme-Verhältnis des Art. 14 Abs. 1 der

EG), oder ob darüber hinaus auch weiterhin eine proaktive Überwachung zumindest hinsichtlich terroristischer Inhalte - und damit nicht allgemein für alle Rechtsverletzungen - erfolgen soll. Vgl. hierzu auch Kuczerawy, The Proposed Regulation on Preventing the Dissemination of Terrorist Content Online, S. 13 f. zu den - mittlerweile gestrichenen - Art. 6 Abs. 3, 4 des Kommissionsvorschlags.

1848 Erwägungsgrund 5 des Kommissionsvorschlags. In der durch das Parlament beschlossenen geänderten Fassung kommt dies noch deutlicher zum Vorschein. Hiernach heißt es in Art. 6 Abs. 1: „Unbeschadet der Richtlinie (EU) 2018/1808 und der Richtlinie 2000/31/EG können die Hostingdiensteanbieter spezifische Maßnahmen ergreifen, um ihre Dienste vor der öffentlichen Verbreitung terroristischer Inhalte zu schützen.", Entschließung des Parlamentes v. 17.04.2019, P8_TAPROV(2019)0421, Abänderung 85.

1849 Vgl. hierzu Erwägungsgrund 19, KOM(2018) 640 endg.: „Angesichts der besonders schwerwiegenden Risiken, die mit der Verbreitung terroristischer Inhalte verbunden sind,[...].“

1850 So etwa auch Kuczerawy, The proposed Regulation on preventing the dissemination of terrorist content online, S. 5. Allgemein wird darüber diskutiert, ob für solche Zwecke den Haftungsvorschriften nicht eine sog "Good Samaritan Clause“ nach amerikanischem Vorbild beigefügt werden soll, siehe hierzu etwa European Parliament Research Service, Reform of the EU liability regime for online intermediaries, S. 17 f. Ingold, in: Unger/v. Ungern-Sternberg, Demokratie und künstliche Intelligenz, S. 183 (196) sieht deshalb im Fall von Kinderpornographie und den besagten terroristischen Inhalten durchaus die Möglichkeit, „,eine entsprechende Indienstnahme verhältnismäßig [...] [auszugestalten] “. Das ist letztlich auch der Ansatz der Kommission für den geplanten Digital Service Act. Siehe hierzu Art. 6 des Entwurfes COM(2020) 825 final.

1851 Vgl. hierzu auch die amtliche Begründung in Erwggr. 62 der RL 2019/790, ABl. 2019, L 130/92. Näher und vor allem kritisch zum „Value Gap“ etwa Suwelack, MMR 2018, 582 (585); Wimmers/ Barudi, GRUR 2017, 327 ff. und Nolte, ZUM 2017, 304 (308 ff.).

1852 ABl. 2019, L 130/92.

1853 Vgl. hierzu Kuczerawy, The EU Commission on voluntary monitoring: Good Samaritan 2.0 or Good Samaritan 0.5?.

1854 Ausführlicher hierzu etwa Hofmann, ZUM 2019, 617 (620ff.); Pravemann, GRUR 2019, 783 (785 ff.) Vor allem die Voraussetzung der branchenüblichen Sorgfalt in Art. 17 Abs. 4 lit. b) der RL führte zu der Diskussion um sog. Uploadfilter, vgl. Gielen/Tiessen, EuZW 2019, 639 (644); Wandtke, NJW 2019, 1841 (1846). 
RL 2000/31/EG im Urheberrecht um. Gleichwohl sollen auch hier dem Betreiber keine allgemeinen Prüfpflichten auferlegt werden (Art. 17 Abs. 8). ${ }^{1855}$

Was folgt also hieraus für $\mathbb{S} 93$ Abs. $4 \mathrm{MStV}$ ? Gewisse Gemeinsamkeiten mit der Verpflichtung der Betreiber durch die geplante Verordnung können jedenfalls nicht von der Hand gewiesen werden. Auch hier ist die Inanspruchnahme maßgeblich dem Umstand geschuldet, dass eine Regulierung erheblich an Wirksamkeit einbüßt, wenn Betreiber nur rudimentär im Einzelfall verpflichtet werden können. In beiden Fällen geht es aber - anders als im Urheberrecht - nicht darum, die Betreiber als Verantwortliche in die Pflicht zu nehmen. Vielmehr sieht man die Netzwerkbetreiber als „gute Samariter“ an, die nicht aus Angst vor haftungsrechtlichen Folgen wirksame Maßnahmen unterlassen sollen. ${ }^{1856} \mathrm{Im}$ Medienstaatsvertrag soll das u.a. durch den Ordnungswidrigkeitentatbestand abgesichert werden, in der geplanten Verordnung durch spezifische Vorgaben der Mitgliedstaaten und eine Berichtspflicht (Art. 6 Abs. 4). ${ }^{1857}$ Insoweit ist man auch auf EU-Ebene darauf bedacht, sachgerechte Lösungen für eine (zeitgemäßere) Verantwortung der Betreiber zu finden, ohne dass das Damoklesschwert einer Haftung über ihnen schwebt. Während aber hinsichtlich terroristischer Inhalte eine (strafrechtliche) Haftung grundsätzlich in Betracht käme, die grundrechtlichen Erwägungen der Haftungsprivilegien hier also durchaus greifen, ist dies bei der Sorgetragungspflicht des $\mathbb{S} 93$ Abs. $4 \mathrm{MStV}$ gerade anders: Eine Haftung besteht nicht und insoweit auch nicht die Gefahr einer Umgehung des Haftungssystems, ${ }^{1858}$ sodass es nicht zwingend einer Bereichsausnahme wie im Falle terroristischer Inhalte bedürfte. Dass damit gleichwohl ein gewisser Konflikt mit den Zielen der Richtlinie besteht, kann nicht von der Hand gewiesen werden. Insoweit ist es zweifelhaft, ob allein derartige (teleologische) Überlegungen ausreichen können, um eine Ausnahme zu begründen. Denn mit Rücksicht auf die Wirksamkeit der Richtlinie besteht jedenfalls die Gefahr, dass mitgliedstaatliche Allein-

1855 Das führt aber zu Widersprüchen, weil Art. 17 Abs. 4 lit. b) eine irgendwie geartete, technische Vorabprüfung verlangt, die angesichts der zahlreichen Inhalte einen generellen Charakter annehmen kann, Wandtke, NJW 2019, 1841 (1846). Zwar müssen die Rechteinhaber den Dienstanbieter entsprechende Informationen zur Verfügung stellen, sodass es um es um spezifische Inhalte und damit um einen spezifische, vorbeugende Prüfpflicht gehen könnte, in diese Richtung etwa Spindler, GRUR 2020, 253 (256); Pravemann GRUR 2019, 783 (787). Wegen der Massen an potentiellen Informationen der Rechtsinhaber und der Masse der gleichwohl zu überprüfenden Inhalte, ist die Grenze zu einer allgemeinen Überwachungspflicht aber noch weiter verschwommen, als ohnehin schon. Allgemein zu diesem Problem Kuczerawy, The proposed Regulation on preventing the dissemination of terrorist content online, S. 14. Speziell zum Urheberrecht, Gielen/Tiessen, EuZW 2019, 639 (644); Wandtke/Hauck, ZUM 2019, 627 (629); Spindler CR 2019, 277 (287); Frosio/ Mendis, in: Frosio, The Oxford Handbook of Online Intermediary Liability, Ch. 28 S. 18 f.

1856 Dieses Problem sieht auch die Kommission, $\operatorname{KOM}(2016) 288$ endg., S. 10. Das US-amerikanische Recht löst das durch eine "Good-samaritan-protection“ in 47 U.S. Code $\$ 230$ (c). Kritisch zu dieser Klausel als Vorbild einer europäischen Lösung etwa Kuczerawy, The EU Commission on voluntary monitoring: Good Samaritan 2.0 or Good Samaritan 0.5?.

1857 Das gilt auch für die zurzeit gültige Version des Europäischen Parlamentes, siehe hierzu ST 8663 2019 INIT, Abänderung 88.

1858 Soweit Löber/Roßnagel, MMR 2019, 493 (495) also hierauf hinweisen, ist ihnen zuzustimmen. 
gänge dann wieder zu einem „Flickenteppich“ der Verantwortlichkeiten führen. Insoweit dürfte es auch unwahrscheinlich sein, dass der EuGH mit dieser Begründung die Konformität der Sorgetragungspflicht mit dem Unionsrecht bejahen würde. Vielmehr erwächst auch hieraus das Bedürfnis einer zeitgerechteren Regulierung.

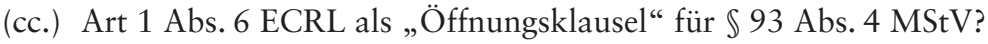

Entscheidender dürfte indes die bereits angesprochene Kompetenzverteilung zwischen Mitgliedstaaten und Europäischer Union (oben B.I.2) sein. Denn - wie bereits im Verhältnis von Bund und Ländern thematisiert (B.I.1) - steht bei den Normen des MStV die originär medienrechtliche, demokratieschützende Komponente der Regulierung im Vordergrund. Damit bewegt man sich aber primär im Bereich der mitgliedstaatlichen Kompetenz. Dem grundlegenden Spannungsverhältnis zwischen originärer Medienregulierung als Kompetenz der Mitgliedstaaten und binnenmarktorientierter Wirtschaftsregulierung der Union trägt Art. 1 Abs. 6 ECRL Rechnung. Hiernach bleiben einzelstaatliche Maßnahmen, „die unter Wahrung des Gemeinschaftsrechts der Förderung der kulturellen und sprachlichen Vielfalt und dem Schutz des Pluralismus dienen, [...] von dieser Richtlinie unberührt." Nach dem Wortlaut wären also jedenfalls die Meinungsvielfalt sichernde Regelungen zulässig. Berücksichtigt man allerdings hierbei auch Erwägungsgrund 63, ${ }^{1859}$ wären auch Regelungen erfasst, „die die Mitgliedstaaten im Einklang mit dem Gemeinschaftsrecht erlassen könnten, um soziale, kulturelle und demokratische Ziele unter Berücksichtigung ihrer sprachlichen Vielfalt, der nationalen und regionalen Besonderheiten sowie ihres Kulturerbes zu erreichen [...]. “ ${ }^{1860} \mathrm{Ob}$ allerdings - worauf der Wortlaut hindeutet - es hier nur um demokratiefördernde Maßnahmen gehen soll, die gerade mit den genannten Kriterien (sprachliche Vielfalt, nationale oder regionale Besonderheiten oder Kulturerbe) begründet werden können, ist zweifelhaft. Ginge man davon aus, so ließe sich überlegen, ob die präventive Medienregulierung in Deutschland nicht eine „nationale Besonderheit“ wäre. Das BVerfG hatte jedenfalls in seiner Lissabon-Entscheidung zumindest Teilbereiche des Medienrechts als unbedingt den Mitgliedstaaten verbleibende Materie deklariert, ${ }^{1861}$ wobei allerdings der genaue Umfang dieses Teilbereichs unklar bleibt. ${ }^{1862}$

1859 Allgemein zur Auslegung des verfügenden Teils im Lichte der Begründung etwa EuGH MMR 2012, 45 Rn. 54 ff. m.w.N.

1860 RL 2000/31/EG, ABl. 1999 L 178/8.

1861 BVerfGE 123, 267 (358, 363) - Vertrag von Lissabon.

1862 Auf diese Unklarheit weist etwa auch Classen, JZ 2009, 881 (887f.) hin. Kritisch insbesondere mit Blick auf die Integrationsoffenheit des GG und auf die Entstehung einer europäische Öffentlichkeit Ukrow, in: Bröhmer, Europa und die Welt, S. 197 (208) m.w.N. In der Entscheidung zur EG-Fernsehrichtlinie hat das BVerfG zwar die wirtschaftsorientierte Harmonisierung bestimmter Aspekte 
Ein solcher Umweg ist vorliegend aber womöglich gar nicht erforderlich. (Meinungs-)Pluralismus ist seither prägender Aspekt der (deutschen) Medienregulierung, ${ }^{1863}$ und auf dieses Regelungsziel weist auch die Gesetzesbegründung des MStV hin. ${ }^{1864}$ Das heißt freilich weder, dass jede Norm des MStV automatisch dem Meinungspluralismus dient, noch dass dieser Charakter dort zu verneinen ist, wo dieser nicht unmittelbar ersichtlich ist. Gerade letzteres muss hinsichtlich der Intermediäre berücksichtigt werden. Sie und damit auch die erforderlichen Regelungskonzepte unterscheiden sich strukturell erheblich vom herkömmlichen, für die eigenen Inhalte redaktionell verantwortlichen Rundfunk. ${ }^{1865}$ Während es bei redaktionell verantwortlichen (Rundfunk-)Medien um die Absicherung einer Angebotsvielfalt - etwa durch die Gewährleistung von Binnenoder Außen-Pluralität - geht, ${ }^{1866}$ besteht diese im Internet generell. ${ }^{1867}$ Die Frage ist bei Intermediären also nur, welcher Ausschnitt davon wahrnehmbar gemacht wird und dann auch von den Nutzern wahrgenommen wird. Insoweit geht es auch hier um pluralismussichernde Aspekte, ${ }^{1868}$ allerdings im Sinne einer Rezeptionsvielfalt. ${ }^{1869}$ Der Medienstaatsvertrag setzt hierfür neben der Stärkung der Nutzerautonomie (Transparenz) auch auf die Stärkung der Verantwortung der Dienste mit Blick auf die gesellschaftliche Sphäre (Diskriminierungsverbot). ${ }^{1870}$

Um diese Rezeptionsvielfalt geht es aber letztlich auch im Falle der Meinungsroboter. Auch hier resultiert die Gefahr aus einer verzerrten Wahrnehmung der Onlineumgebung infolge ihres intransparenten Einflusses. Primär soll das durch die transparenzerhöhende Kennzeichnung und die damit verbundene Stärkung

des Rundfunks anerkannt, gleichwohl betonte es dort auch, dass „[der] Kernbereich der Landeskompetenz zur Regelung der organisatorischen sowie politisch-kulturellen Materien des Rundfunkwesens nicht [berührt sei] “, BVerfGE 92, 203 (241). Insoweit ist der Medienstaatsvertrag aber gerade Ausdruck dieser Kompetenz und insbesondere die vorwiegend demokratieschützenden Aspekte der eigentliche Kernbereich der Rundfunkregulierung. Grundlegend zur Kompetenz der Länder und dem Rundfunkrecht BVerfGE 12, 205 (225 ff.) - 1. Rundfunkentscheidung.

1863 Vgl. etwa BVerfGE 31, 314 (326) - 2. Rundfunkentscheidung; 57, 295 (320, 322 ff.) - 3. Rundfunkentscheidung; 73, 118 (152f.) - 4. Rundfunkentscheidung; 74, 297 (324) - 5. Rundfunkentscheidung; 90,60 (88) - 8. Rundfunkentscheidung und jüngst etwa BVerfGE 149, 222 (259) Rundfunkbeitrag.

1864 Siehe nur BayLT-Drs. 18/7640 S. 78, 79.

1865 Vgl. hierzu Ingold, MMR 2020, 82 (84 f.), der hier anmerkt, dass man sich mit dem individual-gezogenen Regulierungsansatz von dem eigentlich ursprünglichen Sinn der Medienregulierung entfernt. Bedenkt man aber, dass die neuen Angebote gerade individuell zugeschnitten sind, ist hier drin wohl eher der spezifische Regulierungsansatz zu erkennen, der sich von dem beim herkömmlichen Rundfunk eben unterscheidet. Vgl. hierzu auch Liesem, K\&R 2019, 687 (692), die hier zwar das Ziel der Meinungsvielfalt mit Blick auf das Transparenzgebot betont, aber diesbezüglich auch Bedenken äußert.

1866 Zu dieser Angebots- bzw. publizistischen Vielfalt vgl. BVerfGE 73, 118 (156) - 4. Rundfunkentscheidung; 74, 297 (328, 331 f.) - 5. Rundfunkentscheidung; 83, 238 (296f.) -6. Rundfunkentscheidung und jüngst etwa BVerfGE 149, 220 (260, 261) - Rundfunkbeitrag.

1867 So auch Liesem, AfP 2020, 277 Rn. 3.

1868 BVerfGE 149, 220 (262) - Rundfunkbeitrag.

1869 Zu dieser Differenzierung siehe Liesem, AfP 2020, 277 (280).

1870 Ganz ausdrücklich als Aspekte der Pluralismussicherung ansehend Enquete-Kommission Künstliche Intelligenz, BT.-Drs. 19/23700, S. 486. 
der Nutzerautonomie gewährleistet werden ( $\mathbb{S} 18 \mathrm{Abs.} 3 \mathrm{MStV})$. So können suggerierte Mehrheitsmeinungen enttarnt, manipulierte Trends aufgedeckt und wesentliche hilfreiche Hintergrundinformationen für die Aufnahme von Inhalten gegeben werden, um dem Nutzer final eine realitätsgerechtere und potentiell vielfältigere Informationsgrundlage geben zu können. ${ }^{1871}$ Man begegnet durch die speziellen Vorgaben also der „Meinungsmacht“ von Meinungsrobotern. ${ }^{1872} \mathrm{Um}$ aber der - anders als bei den Intermediären selbst - bestehenden Gefahr zu begegnen, dass dieser Pflicht nicht nachgekommen wird, sollen Betreiber sozialer Netzwerke hierfür eine „Hilfsfunktion“ übernehmen ( $\mathbb{9} 93$ Abs. $4 \mathrm{MStV}$ ). Insoweit schlägt das Regelungsziel des $\mathbb{S} 18$ Abs. $3 \mathrm{MStV}$ unweigerlich auf $\mathbb{\$} 93$ Abs. 4 MStV durch. Auch hier geht es im Ergebnis um Aspekte einer pluralismussichernden Medienregulierung. ${ }^{1873}$ Damit bewegt man sich aber nicht nur in dem Bereich, der den Mitgliedstaaten vorbehalten bleibt, sondern auch innerhalb des Anwendungsbereichs von Art. 1 Abs. 6 ECRL, mit der Folge, dass $\mathbb{\$} 93$ Abs. $4 \mathrm{MStV}$ von der E-Commerce-RL gerade „unberührt bleibt“. ${ }^{1874}$ \$ 93 Abs. 4 und damit auch eine proaktive Verpflichtung der Betreiber ist - jedenfalls der Sache nach $-{ }^{1875}$ unionsrechtlich zulässig. ${ }^{1876}$

\section{(c.) Fazit: Vereinbarkeit mit der E-Commerce-RL}

Auch wenn $\int 93$ Abs. $4 \mathrm{MStV}$ in dem hier aufgezeigten Verständnis zu einer vergleichbaren technischen Verpflichtung führt, wie sie der EuGH mit Blick auf inhaltliche Überwachungssysteme für unzulässig hält und auch vieles dafür spricht, dass eine abstrakt-generelle Verpflichtung in diesem Sinne eine allgemeine Überwachungspflicht statuiert, so zeigt eine nähere Betrachtung doch, dass die besonderen Umstände im Falle der Meinungsroboter die Vereinbarkeit mit den europäischen Vorgaben begründen können. Auch wenn hierbei aufgezeigt werden kann,

1871 Vgl. hierzu bereits die Mitteilung der EU-Kommission im Notifizierungsverfahren des Medienstaatsvertrages TRIS(2020) 00328 unter Nr. 9. Vgl. auch die Begründung des MStV etwa BayLTDrs. 18/7640, S. 90.

1872 Vgl. hierzu bereits dieselbe Mitteilung der EU-Kommission unter Nr. 8. Vgl. auch die Begründung des MStV etwa BayLT-Drs. 18/7640, S. 90.

1873 Vgl. Paal/Heidtke, ZUM 2020, 230 (234) zum Diskriminierungsverbot und zum Transparenzgebot, aber auch zu der die Social Bots betreffende Transparenzpflicht. Zumindest beim Transparenzgeund Diskriminierungsverbot ( $\$ 93$ Abs. 1 bzw. $\$ 94$ Abs. $1 \mathrm{MStV})$ spiegelt das auch der Wortlaut wider.

1874 Tendenziell kritisch zur Diskrepanz zwischen individueller Autonomie und dem eigentlichen öffentlichen Anliegen von Vielfalt und Repräsentativität Ingold, MMR 2020, 82 (85).

1875 Art. 1 Abs. 6 der E-Commerce-Richtlinie sieht daneben aber auch vor, dass die Regelung in Einklang mit dem Gemeinschaftsrecht stehen muss. Hierauf wird noch zurück zu kommen sein, unten 3. Kapitel C.II.2.f.

1876 Allgemein mit Blick auf die Intermediärsregulierung des MStV Müller-Terpitz, in: Kühling/Zimmer, Neue Gemeinwohlherausforderungen - Konsequenzen für Wettbewerbsrecht und Regulierung, S. 177 (186) mit Fn. 31.

Die Probleme, die ansonsten entstehen würden, illustriert Hanfeld unter der Überschrift „Killt die EU die Medienpolitik“, FAZ v. 24.04.2020. 
dass die grundlegenden Überlegungen, die zur Haftungsprivilegierung von Hostprovidern führten, im Falle der Meinungsroboter nicht ohne Weiteres übertragbar sind, so kann damit allein nur schwer ein nationalstaatlicher Alleingang begründet werden. Schon auf unionaler Ebene wird versucht eher bereichsspezifisch aber eben EU-einheitlich eine Lösung für spezifische Probleme zu finden, um so letztlich auch die Wirksamkeit der E-Commerce-RL nicht zu gefährden. Entscheidender ist hier vielmehr die durch Art. 1 Abs. 6 der RL bewirkte Öffnung für mitgliedstaatliche Maßnahmen zur Pluralismussicherung, die sich nicht an den (materiell-rechtlichen) ${ }^{1877}$ Vorgaben der Richtlinie messen lassen müssen. Gerade $\mathbb{9} 93$ Abs. $4 \mathrm{MStV}$ dient als spezifisches Instrument zur Gewährleistung einer unverfälschten Informationsaufnahme und damit der Rezeptionsvielfalt. Die Einführung einer proaktiven Pflicht der Betreiber als Ausprägung einer Sorgetragungspflicht des $\$ 93 \mathrm{Abs.} 4 \mathrm{MStV}$ ist mit der E-Commerce-RL so sehr wohl vereinbar.

Damit wird nicht verkannt, dass dennoch ein grundlegendes Bedürfnis einer EU-einheitlichen Regelung besteht, um Risiken für den Binnenmarkt zu minimieren. Für die Union bietet sich durch die deutsche Regelung aber gerade eine nicht zu unterschätzende Möglichkeit: Einerseits zeigt sich hier das Problem, dass die E-Commerce-Richtlinie selbst nicht mehr zeitgemäß ist, insbesondere neuere Konstellationen nicht sachgerecht erfasst, wodurch erhebliche rechtliche Fragen offenbleiben. ${ }^{1878}$ Andererseits kann sie auch einen wichtigen Impuls für europäische Regulierungsvorhaben insbesondere mit Blick auf Art. 114 AEUV geben. ${ }^{1879}$ Denn mit ihm besteht das Werkzeug, divergierende nationale Vorgaben zu vereinheitlichen, wofür nur dort praktischer Bedarf besteht, wo dies auch realiter der Fall ist. Insoweit könnte $\mathbb{\$} 93$ Abs. $4 \mathrm{MStV}$ einer europäischen Lösung Antrieb leisten. ${ }^{1880}$

\section{(IV.) Vereinbarkeit einer proaktiven Pflicht mit der DS-GVO?}

Eine proaktive Überwachungspflicht der Netzwerkbetreiber müsste zuletzt auch datenschutzrechtlich zulässig sein. Die DS-GVO steht dem nicht prinzipiell im Weg. Art. 6 Abs. 1 lit c) DS-GVO erlaubt immerhin die Verarbeitung, wenn und

1877 Verfahrensrechtlich könnte aber gleichwohl das Herkunftslandprinzip des Art. 3 E-CommerceRL zu berücksichtigen sein. Hierzu ausführlich unten, C.II.2.f.bb.

1878 In diese Richtung auch Eifert, in: ders./Gostomzyk, Netzwerkrecht S. 9 (24), der dies mit Blick auf das NetzDG zwar nicht als „rechtsstaatliches Ideal“, aber u.U. als „akzeptable politische Provokation “ verstehen will, um „durch einen kalkulierten Verstoß eine Dynamik [zu] befördern“.

1879 So bezeichnet Cornils, ZRP 2020, 60 (62) den MStV etwa als „rechtliche Pionierarbeit, die in Europa kein vergleichbares Beispiel hat und daher auch derzeit großes Interesse in den Mitgliedstaaten und auf EU-Ebene findet.".

1880 Liesem, ZUM 2020, 377 (382) spricht insgesamt beim Medienstaatsvertrag von einer „Pionierleistung“ einem „medienpolitischem Signal“, das als „Blaupause für eine unionsweite Regulierung“ dienen kann. 
soweit sie zur „Erfüllung einer rechtlichen Verpflichtung erforderlich“ ist. Insoweit ist es zwar grundsätzlich möglich, dass Mitgliedstaaten entsprechende Verpflichtungen vorsehen. Diese Rechtsgrundlagen müssten dann aber auch mit den betroffenen Grundrechten vereinbar sein, insbesondere nur soweit wie erforderlich das Datenschutzrecht (Art. 2 Abs. 1 i.V.m. Art. 1 Abs. 1 GG bzw. 8 EUGrCh) der betroffenen Nutzer einschränken. Denn automatisierte Filtertechnologien stellen eine potentielle Gefahr für den Datenschutz dar, weil damit auch eine Beobachtung des jeweiligen Nutzerverhaltens einhergeht. ${ }^{1881}$ Hierbei gilt es zu berücksichtigen, dass zum einen nur eine Verarbeitung netzwerkinterner Daten notwendig ist. ${ }^{1882}$ Diese fallen aber zwangsläufig bei der Nutzung an und werden allerdings in wenig durchsichtiger Weise - ohnehin schon jetzt von den Netzwerkbetreibern verarbeitet. Auch wenn dies nicht in dem vorgesehenen Umfang und zu den vorgesehenen Zwecken rechtmäßig ist, so wird durch eine proaktive Pflicht der Zweck dieser „Überwachung“ ebenso wie beim Facebook-ImmuneSystem klar vorgegeben: Es geht nicht um die Beseitigung von Inhalten oder die Beobachtung der Nutzer zu Zwecken der Profilbildung und Monetarisierung, sondern um die Erkennung von automatisierten Zugriffen, dem nicht ein wirtschaftliches, sondern ein öffentliches Interesse zugrunde liegt. Zum anderen handelt es sich oftmals ohnehin nur um solche Daten, die - allerdings deutlich mühsamer - auch manuell nachvollzogen werden können, weil diese für andere Nutzer der Netzwerke (bewusst) öffentlich gemacht worden und damit sichtbar sind. Das gilt etwa für die Zeitpunkte eines Uploads, die Inhalte, die Freunde und Follower, persönliche Interessen, abonnierte Seiten, Inhalte, Kommentare usw. Diese bewusste Veröffentlichung rechtfertigt nach Art. 9 Abs. 2 lit. e) DS-GVO aber sogar die Erhebung besonders sensibler Daten. Und potentiell kann dafür Sorge getragen werden, dass Daten nach einer gewissen (kurzen) Zeit wieder gelöscht werden, soweit etwa unauffällige Nutzerinteraktionen betroffen sind. Insoweit ist weniger die rechtliche Grundlage als die Durchsetzung und Kontrolle der Einhaltung der datenschutzrechtlichen Standards entscheidend. Die DS-GVO steht also einer auch proaktiven Pflicht nicht per se im Wege.

(V.) Fazit: präventive Kontrolle als Mindestmaß, proaktive Überwachung als Verantwortung meinungsrelevanter Betreiber

Für die Auslegung der Sorgetragungspflicht ergibt sich damit letztlich folgendes: \$93 Abs. 4 MStV normiert zweierlei Pflichten. Infrastrukturell müssen die Anbieter überhaupt die Möglichkeit dafür schaffen, dass entsprechende Inhalte ge-

1881 Vgl. Conrad/Hausen, in: Auer-Reinsdorff/Conrad, Hb IT- u. Datenschutzrecht, \$36 Rn. 67.

$1882 \mathrm{Zu}$ den verschiedenen Ansätzen einer Erkennung und die dafür notwendigen Daten siehe etwa Cresci, Communications of the ACM 2020, $72 \mathrm{ff}$. 
kennzeichnet werden können. Daneben ist aber - nicht nur wegen der lückenhaften Pflicht des $\mathbb{S} 18$ Abs. $3 \mathrm{MStV}$ - ein Vorgehen der Betreiber erforderlich. Der Inhalt dieser betreiberseitigen Vorkehrungen variiert hierbei vor allem in Abhängigkeit von der Bedeutung und „Meinungsbildungsrelevanz“, um so auch dem Kriterium der Zumutbarkeit Rechnung zu tragen. Als Mindestschutzmaßnahme muss dabei zumindest eine gewisse präventive Kontrolle des Registrierungsprozess oder des Zugriffs über die API erfolgen. Von meinungsmächtigen Anbietern kann hingegen mehr gefordert werden: eine proaktive Überwachung der Datenströme, um Automatisierungsprozesse herauszufiltern und entsprechende menschlich wirkende Nutzerprofile im Sinne des $\mathbb{} 18$ Abs. $3 \mathrm{MStV}$ eigenständig zu kennzeichnen.

bb. Verhältnis von $\mathbb{\int} 93$ Abs. $4 \mathrm{MStV}$ zu den Regelungen der Netzwerkbetreiber

Betrachtet man die bestehenden Regelungen etwa von Facebook (hierzu oben C.I.2.aI.3.a.aa), so werden falsche und damit auch mögliche automatisierte Profile nicht gekennzeichnet, sondern gelöscht. Eine entsprechende Praxis ist auch bei Twitter zu beobachten. ${ }^{1883}$ Das kollidiert zwar mit den gesetzgeberischen Vorgaben, die den Einsatz für grundsätzlich zulässig erachten und lediglich eine Kennzeichnung bezwecken. Allerdings ist der Norm keine Leitbildfunktion dergestalt zu entnehmen, dass davon abweichende, strengere vertragliche Vorgaben der Betreiber damit unvereinbar wären. ${ }^{1884}$ Denn die gesetzliche Regelung schafft nur einen Mindeststandard der Transparenz. ${ }^{1885}$ Ein solches Verständnis wäre zudem nur schwer mit der Berufsfreiheit der Betreiber (Art. 12 Abs. 1 GG) in Einklang zu bringen, denen die Entscheidung über die konkrete Ausrichtung und den Betrieb obliegt. Davon umfasst sind gerade auch die Modalitäten für die Nutzung. Das ist schon deshalb legitim, weil etwa das Konzept von Facebook mehr auf der sozialen, menschlichen Interaktion und Vernetzung beruht, als etwa bei Twitter. $\mathbb{S} 93$ Abs. $4 \mathrm{MStV}$ steht also schärferen Regelungen der Anbieter gerade nicht im Weg. Besondere Probleme ergeben sich nur dann, wenn im Einzel-

1883 Vgl. statt vieler Chavoshi/Hamooni/Mueen, in: Proceedings of the 16th International Conference on Data Mining, S. 817 (820).

1884 Ein solcher Anknüpfungspunkt bestünde etwa durch $\$ 307$ Abs. 1 S. 1, Abs. 2 Nr. 1 BGB, wonach Bestimmungen in AGB im Zweifel als unangemessene Benachteiligung anzusehen, also unwirksam sind, wenn sie „mit wesentlichen Grundgedanken der gesetzlichen Regelung, von der abgewichen wird, nicht zu vereinbaren [sind]. "Eine Leitbildfunktion kann auch Normen außerhalb des BGB zukommen, sofern diese für die vertragliche Beziehung bedeutsam sind, Wurmnest, in: MüKo-BGB, Bd. 2, $₫ 307$ Rn. 70 f.

1885 Vergleichbares gilt etwa auch mit Blick auf das NetzDG. Die Löschpflichten regeln das rechtliche Minimum. Vertraglich können die Netzwerkbetreiber durchaus auch strengere Vorschriften vorsehen. Eine andere Frage ist dann, ob dies im Einzelfall auch - etwa wegen einer starken mittelbaren Drittwirkung - zulässig wäre. Siehe hierzu insgesamt Friehe, NJW 2020, 1697 (1698). Diesen Mindeststandard im Falle des NetzDG betont etwa das LG Leipzig, Endurteil v. 12.07.2019 - 08 O 2491/18 = GRUR-RS 2018, 38785 Rn. 50 m.w.N. 
fall eine Löschung fälschlich erfolgt. Dann bestünde - ähnlich wie im Falle einer fälschlichen Löschung von Inhalten - ein Anspruch auf Wiederherstellung des Profils ( $\mathbb{S} \int 280$ Abs. 1, 249 Abs. 1 BGB). ${ }^{1886}$

\section{d. Ergänzende Satzungsbefugnis der Medienanstalten, $\int 96 \mathrm{MStV}$}

Kritik an den Vorgaben für Intermediäre wurde u.a. auch deshalb laut, weil ihre Pflichten vergleichsweise vage formuliert worden sind. Zwar lässt sich im Wege der Auslegung - das zeigen schon die ergänzenden Überlegungen zu $\$ 93$ Abs. 4 MStV - eine gewisse inhaltliche Schärfe durchaus gewinnen, gleichwohl begründet das eine gewisse Rechtsunsicherheit auch hinsichtlich der Umsetzung. Daraus folgt indes nicht ein verfassungsrechtlich problematischer Grad der Unbestimmtheit. ${ }^{1887}$ Der Grad der rechtsstaatlich gebotenen Bestimmtheit einer Norm hängt von „der Eigenart der zu ordnenden Lebenssachverhalte [ab] [...]. Die Betroffenen müssen die Rechtslage erkennen und ihr Verhalten danach ausrichten können. [...] Dabei sind die Anforderungen an den Grad der Klarheit und Bestimmtheit umso strenger, je intensiver der Grundrechtseingriff ist, den eine Norm rechtfertigen soll." ${ }^{1888}$ Aber nicht nur handelt es sich mit Blick auf die Betreiber um vergleichsweise niedrigschwellige Eingriffe, die die Diensterbringung und ihre berechtigten Interessen nicht erheblich beeinträchtigen, ${ }^{1889}$ sondern es ist auch zu berücksichtigen, dass angesichts der Bandbreite zu regulierender Intermediäre von Suchmaschinen über Newsaggregatoren bis hin zu sozialen Netzwerken ganz unterschiedliche Dienste und Funktionsweisen geregelt werden, sodass die Regelungen schon deshalb abstrakter bleiben müssen. ${ }^{1890}$ Insoweit gewährt man den Betreibern einen Gestaltungs- und Umsetzungsspielraum. Maßgeblich daneben ist auch, dass der Medienstaatsvertrag selbst dies durch eine sekundäre Norm-

1886 Zur Löschung von Inhalten siehe etwa LG Leipzig, Endurteil v. 12.07.2019 - 08 O 2491/18= GRUR-RS 2019, 38785, Rn. 18; OLG München, GRUR-RR 2020, 174 Rn. 60. Der Restore-Anspruch wird im Falle von inhaltsbedingten Kontensperrungen daneben auch auf die $\mathbb{S} \$ 241$ Abs. 2 , 1004 BGB gestützt, so etwa LG Karlsruhe, Beschluss vom 12.06.2018 - 11 O 54/18 = BeckRS 2018, 20324 Rn. 7; LG Frankfurt a.M., MMR 2018, 545 Rn. 8. Zumindest im letzten Fall ging es aber um Äußerungen, die auch Rechte Dritter tangierten, so dass die ursprüngliche Löschung im Rahmen der Störerhaftung $(\$ 1004$ BGB) erfolgte. Andernorts wird nur auf den Nutzungsvertrag i.V.m. $\$ 241$ Abs. 2 BGB hingewiesen, so etwa OLG Oldenburg, ZUM-RD 2020, 367 (368); OLG Dresden, MMR 2018, 756 Rn. 8; OLG München, NJW 2018, 3115 (3116), wobei $\$ 241$ Abs. 2 gerade keine Anspruchsgrundlage bereithält. Entsprechendes gilt für den Nutzungsvertrag. In einer unberechtigten Löschung ist vielmehr eine Verletzung der Haupt- oder Nebenleistungspflichten diese „normiert“ $\$ 241$ Abs. 2 BGB -, sodass die Verletzung nach $\mathbb{S} 280$ Abs. 1, 241 BGB restituiert werden muss, was hier der Wiederherstellung entspräche.

1887 Liesem, AfP 2020, 277 Rn. 24 deutet dies jedenfalls an, wenn sie die Frage aufwirft, ob die „so wichtige Konkretisierungsaufgabe nicht in den Händen des Normgebers hätte verbleiben sollen “.

1888 Statt vieler BVerfGE 149, 293 Rn. 77 - Fixierung von Patienten, mit zahlreichen weiteren Nachweisen.

1889 Ausführlicher hierzu noch später, C.II.3.b.bb(II.)(1.).

1890 Zumindest im Ansatz ähnlich Heidtke, Meinungsbildung und Medienintermediäre, S.276f. Auf diese Divergenzen weist auch. Liesem, AfP 2020, 277 Rn. 23, hin. 
konkretisierung „entschärft“. ${ }^{1891}$ Nach $\mathbb{9} 96$ MStV können die Landesmedienanstalten durch Satzungen oder Richtlinien Einzelheiten der Intermediärspflichten konkretisieren. Nach $\mathbb{\int} 96$ S. $2 \mathrm{MStV}$ ist dabei die jeweilige Orientierungsfunktion für die jeweiligen Nutzerkreise zu berücksichtigen. Die Landesmedienanstalten haben also „dem positiven Nutzen dieser Dienste für die Informationsgewinnung durch die Bürgerinnen und Bürger Rechnung [zu tragen] "1892 und damit eine dienstspezifische Konkretisierung vorzunehmen. Inhaltliche Grenzen der Normsetzungsbefugnis der Landesmedienanstalten ergeben sich insoweit allein aus rechtsstaatlichen und grundrechtlichen Erwägungen. Vor allem die Wesentlichkeitstheorie kann hier Grenzen aufzeigen, wenn die Medienanstalten in wesentliche, grundrechtssensible Bereiche eingreifen. ${ }^{1893}$ Das wird aber wohl eher die Ausnahme bleiben, sofern man die Konkretisierung der Normen nicht über Gebühr ausdehnt. So ließe sich in Wahrnehmung der „Konkretisierungsbefugnis“ etwa die im Wege der Auslegung gewonnene Konkretisierung des $\mathbb{S} 93$ Abs. 4 MStV auch durch Satzung für große soziale Netzwerke verbindlich und unzweideutig festlegen. Und mit Blick auf die allgemeinen Transparenzvorgaben des \93 Abs. 1 MStV könnten so die „zentralen Kriterien“ dienstspezifisch näher festgelegt werden.

\section{e. Kontrollbefugnisse und Durchsetzungsmöglichkeiten des Staates}

Der Medienstaatsvertrag sieht aber nicht nur einfache strafbewehrte Pflichten der jeweiligen Dienstanbieter vor. Er kleidet diese Pflichten auch in ein Korsett aus kontroll- bzw. aufsichtsrechtlichen Befugnissen der Landesmedienanstalten ein.

\section{aa. Ordnungswidrigkeitentatbestände}

Verstöße gegen die Kennzeichnungspflicht ( $\$ 115$ Abs. 1 S. 2 Nr. 2 MStV) ebenso wie gegen die Sorgetragungs- ( $\$ 115$ Abs. 1 S. 2 Nr. 45 MStV) und übrigen Intermediärspflichten ( $\mathbb{S} 115$ Abs. 1 S. 2 Nr. 41-47) sind unabhängig von der individuellen Vorwerfbarkeit ordnungswidrig ( $\$ 115$ Abs. 1 S. 2 Nr. 2, Nr. 42 MStV) und können mit einer Geldstrafe von bis zu 500.000 Euro (Abs. 2, 1. Var.) durch die zuständige Landesmedienanstalt ( $\$ 36$ Abs. 1 Nr. 1 OWiG, $\mathbb{S} 108 \mathrm{MStV}$ ) bestraft werden. Besonderes Augenmerk soll hier auf $\mathbb{} 115$ Abs. 1 S. 2 Nr. 45 MStV liegen. Hiernach handelt ordnungswidrig, wer „entgegen $\mathbb{S} 93$ Abs. 4 als Anbieter

1891 Für Laude, JuWiss-Blog Nr. 127/2020, ist dieses Regelungskonzept angesichts empirischer Ungewissheiten „alternativlos“. Ausführlicher zu der Befugnis etwa Liebermann, in: Gersdorf/Paal, Informations- und Medienrecht, $\mathbb{\$} 96 \mathrm{MStV}$ Rn. $1 \mathrm{ff}$.

1892 So die Begründung des Staatsvertrags, BayLT-Drs. 18/7640, S. 110.

1893 So Ladeur/Gostomzyk, K\&R 2018, 686 (692). 
eines Medienintermediärs nicht dafür Sorge trägt, dass Telemedien im Sinne von \18 Abs. 3 gekennzeichnet werden“. Pönalisiert wird also die abstrakte Gefährlichkeit des Normungehorsams ${ }^{1894}$ bzw. des Unterlassens entsprechender Schutzvorkehrungen. Wegen der relativen Unbestimmtheit der Pflicht selbst ist allerdings fraglich, ab wann der Tatbestand eingreift. ${ }^{1895}$ Anknüpfend an das bereits oben Gesagte bietet sich hier erneut eine Differenzierung an. Hinsichtlich der infrastrukturellen Voraussetzungen besteht eine absolute Pflicht. Hält ein Betreiber diese nicht vor, so handelt er ordnungswidrig.

Die relative Pflicht, durch eigene Maßnahmen die Einhaltung der Kennzeichnungspflicht zu gewährleisten, wird jedenfalls dann verletzt, wenn Betreiber vollständig untätig bleiben. Darüber hinaus hat Milker vorgeschlagen, den Tatbestand ähnlich zu handhaben wie die Störerhaftung. Danach sollen „Sanktionen bei Verstößen erst eintreten, wenn der Netzwerkbetreiber nicht alles Zumutbare zur Bot-Erkennung getan hat [...]." ${ }^{1896}$ Das ist insoweit überzeugend, als dass damit nicht nur eine sachgerechte Differenzierung in Abhängigkeit von den nach \$93 Abs. 4 MStV auferlegten Rechtspflichten des jeweiligen Dienstanbieters möglich ist. Es wird damit auch eine Parallele zur strafrechtlichen Dogmatik der Unterlassungsdelikte ${ }^{1897}$ oder den zivilrechtlichen Verkehrssicherungspflichten ${ }^{1898}$ gezogen, mit denen die Sorgetragungspflicht jedenfalls in einem weiten Sinne vergleichbar ist. ${ }^{1899}$ Probleme könnten sich hier nur aus dem Bestimmtheitsgebot des Art. 103 Abs. 2 GG $^{1900}$ ergeben, weil so nicht hinreichend erkennbar ist, wo die ordnungsrechtliche Schwelle exakt verläuft. Sie ergibt sich erst unter Berücksichtigung der auch grundrechtlichen Belange und der Bedeutung des jeweiligen Dienstes. Das ist aber dem Strafrecht nicht fremd. Insbesondere die Beleidigungstatbestände sind schließlich ebenfalls davon geprägt, dass zwar ein abstraktes Verbot aufgestellt wird. Ob eine Äußerung aber strafbar ist, ist auch hier im Einzelfall und unter Berücksichtigung grundrechtlicher Aspekte zu klären. Anders als dort geht es hier aber „nur“ um eine bußgeldbewehrte Ordnungswidrigkeit, also eine wenig intensive Strafandrohung, sodass ohnehin kein allzu strenger

1894 So allgemein in Bezug auf Ordnungswidrigkeiten $v$. Heintschel-Heinegg, in: ders., BeckOK StGB, Lexikon des Strafrechts, Deliktstypen und ihre spezifischen Eigenheiten Rn. 32.

1895 So auch Löber/Roßnagel, MMR. 2019, 493 (498).

1896 Milker, InTeR 2017, 199 (206). Nolte, ZUM 2017, 552 (560) hat einen Zumutbarkeitsvorbehalt auch beim NetzDG gefordert. In diese Richtung auch Gersdorf, MMR 2017, 439 (446).

1897 Siehe etwa Heger, in: Lackner/Kühl, StGB, $\mathbb{1 3}$ Rn. 5; Gaede, in: Kindhäuser/Neumann/Paeffgen, StGB, $\mathbb{1 3}$ Rn. 17 f.; Heuchemer, in: v. Heintschel-Heinegg, BeckOK StGB, $\$ 13$ Rn. 91 oder Freund, in: MüKo-StGB, Bd. 1, $\mathbb{S} 13$ Rn. 201, der keinen gesonderten Aspekt der Zumutbarkeit anerkennt, sondern dies vielmehr bei der Verhaltensnorm selbst berücksichtigt.

1898 Hierzu etwa BGH, GRUR 2007, 890 Rn. 36; GRUR 2015, 1129 Rn. 42.

1899 Siehe hierzu bereits oben, C.II.2.c.aa(II.)(1.).

1900 Dieser findet auch auf Ordnungswidrigkeiten Anwendung, siehe hierzu statt vieler Remmert, in: Maunz/Dürig, GG, Art. 103 Abs. 2 Rn. 56 m.w.N. 
Maßstab anzulegen sein dürfte. ${ }^{1901}$ Immerhin ist auch eine Konkretisierung der Sorgetragungspflicht durch $\mathbb{\int} 96 \mathrm{MStV}$ zukünftig ohne Weiteres möglich ist. ${ }^{1902}$

\section{bb. Aufsichtsrechtliche Befugnisse und Maßnahmen der Landesmedienanstalten}

Damit aber ordnungsrechtlich überhaupt auf Grundlage der Tatbestände des \115 Abs. 1 S. $2 \mathrm{MStV}$ vorgegangen werden kann, bedarf es aufsichtsrechtlicher Befugnisse, die eine (effektive) Kontrolle der Intermediäre und Netzwerkbetreiber sicherstellen. Der Medienstaatsvertrag kommt dem dadurch nach, dass der für die Überwachung der Intermediäre zuständigen Kommission für Zulassung und Aufsicht (ZAK) (\$ 107 Abs. 1 Nr. $10 \mathrm{MStV}$ ) nicht nur - mit Ausnahme des Diskriminierungsverbots - ein uneingeschränkter Amtsermittlungsgrundsatz auferlegt wird, ${ }^{1903}$ sondern dafür auch wesentliche Kontrollinstrumente bereitstellt. Nach $\mathbb{S} 95$ MStV kann sie die Vorlage erforderlicher Unterlagen verlangen. Dies wird durch die entsprechend anwendbaren Auskunftsrechte und Ermittlungsbe-

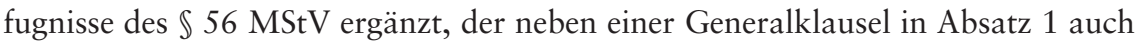
spezielle grundrechtssensible Eingriffsbefugnisse in Abs. 4 (Betretungs- und Einsichtsrecht) und Abs. 6 (Durchsuchung) vorsieht.

Kommt die ZAK zu dem Ergebnis, dass gegen rechtliche Pflichten verstoßen wurde, so kann sie aufsichtsrechtliche Anordnungen auf Grundlage der Generalklausel des $\mathbb{S} 109$ Abs. 1 S. 1 MStV treffen. Maßnahmen, die beispielhaft in Satz 2 aufgezählt werden, sind neben der Beanstandung auch die Untersagung oder Sperrung, wobei freilich das Gebot der Verhältnismäßigkeit zu beachten ist (vgl. $\mathbb{S} 109$ Abs. 2 S. 1 MStV). Im Falle von Telemedienanbietern, die einer Verpflichtung nach $\mathbb{S} 18$ Abs. $3 \mathrm{MStV}$ nicht nachkommen, bestünde die (subsidiäre) Möglichkeit, den Netzwerkbetreibern nach $\mathbb{1} 109$ Abs. $3 \mathrm{MStV}$ in die Pflicht zu nehmen. Ohne Zugang zu den Netzwerkdaten dürfte diese Befugnis aber ins Leere laufen (vgl. hierzu oben C.I.3.c.cc.)

Entscheidend für die Wirksamkeit einer Kontrolle ist alleine die Frage, welche Unterlagen und in welchem Umfang diese verlangt werden können. Denn für die Kontrolle der Einhaltung der Transparenz- und Diskriminierungsvorgaben bedarf es u.U. eines umfassenden Einblickes in die Struktur der Anbieter und insbesondere in die Funktionsweise der Netzwerkbetreiber, als es etwa die transparen-

1901 Vgl. BVerfGE 126, 170 (195) - Präzisierungsgebot Untreuetatbestand und zuvor auch BVerfGE 75, 329 (342) - Verwaltungsakzessorietät im Umweltstrafrecht: „[D]er Gesetzgeber [muss] die Strafbarkeitsvoraussetzungen umso genauer festlegen und präziser bestimmen [...], je schwerer die von ihm angedrohte Strafe ist."

1902 Vgl. zur Zulässigkeit einer solchen Konkretisierung durch Satzungen etwa BVerfGE 32,346 (362 f.) - Strafbestimmungen in Gemeindesatzungen .

1903 Denn in $\$ 94$ Abs. 3 S. 2 kann die Landesmedienanstalt hier nur in offensichtlichen Fällen tätig werden. Eine solche Einschränkung hat man für die Transparenzpflichten des $\$ 93$ gerade nicht vorgesehen. Hierauf hinweisend auch die Gesetzesbegründung, s. nur BayLT-Drs. 18/7640, S. 109. 
ten Informationen den Nutzern ermöglichen sollen. ${ }^{1904}$ Denn nur so kann sichergestellt werden, dass tatsächlich die zentralen Kriterien offenbart wurden und keine systematischen Benachteiligungen vorliegen. ${ }^{1905}$ Entsprechendes gilt auch mit Blick auf die Sorgetragungspflicht des $\mathbb{9} 93$ Abs. 4 MStV. ${ }^{1906}$ Die vorzulegenden Unterlagen müssen Einblicke in die konkret ergriffenen Maßnahmen, ihre Umsetzung und ihre Wirksamkeit ermöglichen und so eine effektive Kontrolle ermöglichen. Dass so umfangreiche Pflichten durchaus mit den besonders zu schützenden wirtschaftlichen und geheimhaltungsbedürftigen Interessen der Betreiber kollidieren, wird ohne Weiteres ersichtlich. ${ }^{1907}$ Allerdings ist eine solch weitreichende Transparenz der Unternehmen wichtig, um die bisher bestehenden Probleme eines rein selbstreguliativen Ansatzes zu beheben und eine effektive staatliche Kontrolle zu ermöglichen. ${ }^{1908}$ Prozessual werden die kollidierenden Interessen immerhin durch $\mathbb{S} 58 \mathrm{MStV}$ dadurch abgesichert, dass den Medienanstalten eine besondere Geheimhaltungspflicht auferlegt wird. ${ }^{1909}$

\section{f. Vereinbarkeit mit den europäischen Vorgaben im Übrigen}

Abseits der konkreten europarechtlichen Probleme des $\mathbb{\$} 93$ Abs. 4 stellt sich aber auch im Übrigen die Frage, inwieweit die Regelungen für Medienintermediäre mit dem Unionsrecht vereinbar sind. Hierbei wird es nicht nur um die Vereinbarkeit mit der Platform-to-business-Verordnung 2019/1150 ${ }^{1910}$ (P2B-VO) oder mit den Grundfreiheiten gehen. Speziell im Kontext der Meinungsroboter könnte auch das durch $\mathbb{S} 1$ Abs. $8 \mathrm{MStV}$ eingeführte Marktortprinzip mit den Vorgaben der E-Commerce-Richtlinie brechen.

1904 Dass die Informationen die nach $\mathbb{S} 93$ Abs. 1-3 MStV für eine wirksame Aufsicht nicht ausreichen und insoweit deutliche mehr Informationen verlangt werden müssen, erkennt auch Heidtke, Meinungsbildung und Medienintermediäre, S. 348.

1905 Deshalb fordert etwa Laude, JuWiss-Blog Nr. 127/2020 v. 02.11 .2020 eine Ergänzung um verschärfte Transparenzpflichten im Verhältnis der Betreiber zu den Landesmedienanstalten.

1906 Die fehlende Nachvollziehbarkeit bisher veröffentlichter Angaben zur Löschpraxis auffälliger Accounts bei Twitter betonen etwa Silva/Proksch, American Political Science Review 2020, 1 (2).

1907 Hierauf ebenfalls hinweisend Ladeur/Gostomzyk, K\&R 2018, 686 (692); Schwartmann/Hermann/ Mühlenbeck, Transparenz bei Medienintermediären, S. 152.

1908 Zu diesen Schwächen siehe insbesondere und zusammenfasend oben, C.I.5. Auf die effektive Kontrolle weist etwa auch die Enquete-Kommission, BT.-Drs. 19/23700, S. 485, hin.

1909 Schon die Begründung des $\mathbb{\$} 95$ und auch der Normtext des $\mathbb{S} 58 \mathrm{MStV}$ deuten jedenfalls auf ein solches Verständnis hin, wenn hier explizit von Betriebs- und Geschäftsgeheimnissen gesprochen wird. Siehe hierzu auch Schwartmann/Hermann/Müblenbeck, Transparenz bei Medienintermediären, S. 152.

1910 Verordnung (EU) 2019/1150 des Europäischen Parlaments und des Rates vom 20. Juni 2019 zur Förderung von Fairness und Transparenz für gewerbliche Nutzer von Online- Vermittlungsdiensten, AB1. 2019 L186/57. 
aa. Vereinbarkeit mit der P2B-Verordnung

Bereits die P2B-VO sieht Transparenzverpflichtungen für Anbieter von Vermittlungsdiensten i.S.v. Art. 2 Nr. 2 vor, wozu auch ausweislich des Erwägungsgrunds 11 soziale Netzwerke gehören können. Maßgeblich ist nämlich nicht - wie auch bei $\mathbb{5}$ Abs. 1 TMG -, dass die Transaktion zwischen gewerblichen Nutzern und den Verbrauchern auf der Plattform abgeschlossen wird; allein das In-KontaktTreten kann ausreichen (vgl. Art. 2 Nr. 2 lit. b).

Ohne zu ausführlich auf den Regelungsbereich einzugehen, ${ }^{1911}$ lässt sich durchaus eine inhaltliche Kongruenz der Verordnungsregelungen und des Medienstaatsvertrags mit Blick auf die allgemeinen Transparenz- und Antidiskriminierungsvorgaben ausmachen. Denn redaktionell journalistische Inhalte im Sinne der $\mathbb{S} \int 93,94 \mathrm{MStV}^{1912}$ stammen auch von gewerblichen Nutzern - etwa Medienunternehmen o.ä. - und führen nicht selten zu der Weiterleitung auf die eigenen externen Angebote, wo etwa direkte Transaktionen mit Verbrauchern - etwa in Form von Abonnements - erfolgen können. ${ }^{1913}$ Hinsichtlich der Transparenzvorgaben ergibt sich allerdings kein nennenswerter Unterschied. Auch nach Art. 5 Abs. 1, bzw. Abs. 2 P2B-VO sind die für das Ranking relevanten Hauptparameter zu veröffentlichen. Dazu gehören auch nach Absatz 5 spezifische Erläuterungen der Funktionsweise der Rankingalgorithmen. Daneben bestehen besondere Informationspflichten hinsichtlich einer wirtschaftlichen Kooperation mit den Plattformen, die für das Ranking relevant sein können (Art. 5 Abs. 3).

Die Antidiskriminierungsvorschrift des Art. 7 der P2B-VO unterscheidet sich von $\int 94$ Abs. 2, 3 allerdings dahingehend, dass hiernach nur transparenzerhöhende Maßnahmen verpflichtend sind, was Erläuterungen darüber beinhaltet, ob und wie eine differenzierte Behandlung von Angeboten überhaupt stattfindet (Abs. 2) bzw. welchen Einfluss kommerzielle Eigeninteressen der Plattformen durch eigene Angebote oder durch Kooperationen mit Dritten haben (Abs. 1). Soweit eine differenzierte Behandlung aufgrund von Maßnahmen der Plattformen erfolgt, sind diese nach Abs. 3 ebenfalls transparent zu machen, wobei bestimmte Regelbeispiele in lit. a)-d) aufgezählt werden. Schon wegen der eigenen, grundrechtlich geschützten kommerziellen Interessen der Plattformbetreiber hat

1911 Hierzu etwa Busch, GRUR 2019, 788 ff.; Voigt/Reuter, MMR 2019, 783 ff.

1912 Dazu näher oben, C.II.2.b.

1913 Kritisch deshalb etwa Facebook, Positionspapier zum Diskussionsentwurf für einen „Medienstaatsvertrag“, 2019, S. 3; Bitkom, Medienstaatsvertrag - Überarbeiteter Vorschlag der Rundfunkkommission, 2019, S. 9 sieht einen drohenden Konflikt mit den europäischen Vorgaben; ebenso Google, Stellungnahme zum Diskussionsentwurf (Juli 2019) der Rundfunkkommission der Länder für einen „Medienstaatsvertrag”, S. 2. 
die P2B-VO also - anders als der MStV - kein Diskriminierungsverbot normiert. ${ }^{1914}$

Bevor man aber allzu schnell europarechtliche Bedenken äußert, ${ }^{1915}$ sollte folgendes berücksichtigt werden: Einerseits gilt die P2B-VO nur im Verhältnis zwischen Plattform und kommerziellen Nutzern (Art. 1 Abs. 1) und tangiert nur mittelbar Nutzer, ${ }^{1916}$ wohingegen der MStV vor allem nutzer- bzw. gemeinwohlorientiert ausgestaltet ist. Andererseits verfolgen MStV und P2B-VO divergierende Ziele. Beide wollen zwar Transparenz und Fairness gewährleisten, dies allerdings in verschiedenen Bereichen: Meinungsbildungsprozess einerseits, wirtschaftlicher Wettbewerb andererseits. ${ }^{1917}$ Der nationale Gesetzgeber bewegt sich damit aber innerhalb der ihm belassenen Kompetenz, was gerade der Regelungsbereich der journalistisch-redaktionellen Inhalte des $\mathbb{S} 94$ Abs. 1 MStV zeigt. Die Überschneidung ist hier wieder allein dem Doppelcharakter der Medien geschuldet. Insoweit obliegt es aber auch hier den Mitgliedstaaten, die Folgen entsprechender Praktiken im Umgang mit journalistisch-redaktionellen Inhalten für den Kommunikationsprozess abzuwägen und sich über die allein wirtschaftlich ausgerichteten europäischen Vorgaben partiell hinwegzusetzen. Wegen der meinungsbildenden Relevanz entsprechender Dienstleister liegen hierfür auch überzeugende Gründe vor. Auch von den Ländern wurde das grundsätzliche Problem der Kongruenz gesehen, aber durch $\mathbb{\$} 91$ Abs. 2 Nr. 2 MStV und durch die Reduzierung des $\mathbb{S} 94$ Abs. 1, $2 \mathrm{MStV}$ auf journalistisch-redaktionelle Inhalte weitestgehend entschärft. Rein kommerzielle Inhalte fallen demnach auch weiterhin allein unter den Regelungsbereich der P2B-VO, deren Effektivität zudem nicht geschmälert wird. Insbesondere die Diskriminierungsvorgaben des $\mathbb{\$} 94$ Abs. 1, 2 MStV berühren die Informationspflichten nicht, sondern ergänzen diese im nationalstaatlichen Kompetenzbereich zur Sicherung von Meinungspluralismus. Daher spricht vieles dafür, dass die $\mathbb{S} \mathbb{S} 93,94 \mathrm{MStV}$ auch mit der P2B-VO vereinbar sind.

1914 Vielmehr ergeben sich hier Diskriminierungsverbote weiterhin allein aus wettbewerbsrechtlichen

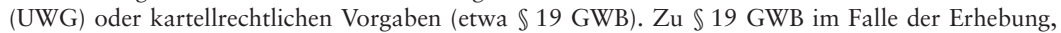
Zusammenführung und Verwendung von Nutzerdaten durch den Facebook-Konzern ausführlich etwa BKartA, Beschl. V. 06.02.2019 - B6-22/16 = BeckRS 2019, 4895 Rn. 163 ff.

1915 Kritisch etwa Facebook, Positionspapier zum Diskussionsentwurf für einen „Medienstaatsvertrag“, 2019, S. 3; Bitkom, Medienstaatsvertrag - Überarbeiteter Vorschlag der Rundfunkkommission, 2019, S. 9, sieht einen drohenden Konflikt mit den europäischen Vorgaben; ebenso Google, Stellungnahme zum Diskussionsentwurf (Juli 2019) der Rundfunkkommission der Länder für einen „Medienstaatsvertrag”, S. 2. Liesem, AfP 2020, 377 Rn. 36 sieht zumindest das „Damoklesschwert eines möglichen verdrängenden Anwendungsvorrangs“.

1916 So auch schon Erwägungsgrund 2 S. 3, Erwgr. 3 S. 2. Insoweit können also eigene Dienste systematisch präferiert werden, solange dies für die kommerziellen Nutzer hinreichend deutlich erkennbar ist. Ein Verbot ergibt sich also auch hier nur mit Blick auf kartellrechtliche bzw. wettbewerbsrechtliche Vorschriften, nämlich bei Missbrauch einer marktbeherrschenden Stellung (etwa i.S.v. Art. 102 AEUV), so wie es etwa bei Google Shopping der Fall war, siehe hierzu die Zusammenfassung des Beschlusses der Kommission v. 27.07.2017 im Fall AT.39740, ABl. 2018 C 9/11 - Google Search (Shopping).

1917 Auf diesen Unterschied weist etwa auch Schwartmann/Hermann/Mühlenbeck, Transparenz bei Medienintermediären, S. 95 hin. 
bb. Marktortprinzip d. $\mathbb{1} 1$ Abs. $8 \mathrm{MStV}$ - Verstoß gegen das Herkunftslandprinzip d. Art. 3 ECRL?

Mit $\mathbb{S}$ Abs. $8 \mathrm{MStV}$ wird die Regelungskompetenz auch über das eigene Staatsgebiet hinaus auf das europäische Ausland jedenfalls insoweit erstreckt, wie u.a. im Ausland ansässige Medienintermediäre ihre Dienste auch im Inland anbieten (sog. Marktortprinzip). Daher sind auch die Dienste von Facebook, Google und Twitter regulativ erfasst, obwohl sie in Irland niedergelassen sind. Damit begibt man sich in einen offenen Widerspruch mit dem Herkunftslandprinzip des Art. 3 Abs. 1, 2 der RL 2000/31/EG. ${ }^{1918}$ Nach ihm dürfen Mitgliedstaaten den „freien Verkehr von Diensten der Informationsgesellschaft aus einem anderen Mitgliedstaat nicht aus Gründen einschränken, die in den koordinierten Bereich fallen.“ Hierzu gehören aber gerade solche Maßnahmen, die „die Ausübung der Tätigkeit eines Dienstes der Informationsgesellschaft“ berühren (vgl. Art. 2 lit. h) i) zweiter Spiegelstrich RL 2000/31/EG). Mit anderen Worten weist die Richtlinie die Regulierungskompetenz für Intermediäre und die hier relevanten sozialen Netzwerken ${ }^{1919}$ dem Staat zu, in dem sie ihre Niederlassung haben (Art. 3 Abs. 1 RL 2000/31/EG), ${ }^{1920}$ Im Falle der drei angesprochenen Dienste wäre also allein Irland zuständig. ${ }^{1921}$ Schon die entsprechende Regelung des $\mathbb{} 1$ Abs. 1 NetzDG wurde deshalb für mit dem Herkunftslandprinzip unvereinbar gehalten. ${ }^{1922}$

1918 Insbesondere Facebook hat sich deshalb auf die Unionsrechtswidrigkeit im Beteiligungsverfahren berufen, Facebook, Positionspapier zum Diskussionsentwurf für einen Medienstaatsvertrag, 2019, S. 3. Aber auch im Notifizierungsverfahren der EU-Kommission hat sich etwa DigitalEurope, Comment on the draft Inter-State Treaty on modernising the media regulation (MStV) in Germany, S. 2, ähnlich geäußert; ebenso der Breitbandverband ANGA, Statement by The Broadband Association ANGA (Germany), S. 1. Kritisch zu $\$ 53$ c MStV-E (2018) auch Ladeur/Gostomzyk, K\&R 2018, 686 (690). Siehe auch Schröder, DVBl. 2018, 465 (471).

1919 So zu Facebook EuGH, Urt. v. 03.10.2019- Rs. C-18/18 = MMR 2019, 798 Rn. 22 - GlawischnigPiesczek. Zuvor auch schon zu Netlog EuGH, Urt. v.16.02.2012- Rs. C-360/10 = GRUR 2012, 382 Rn. 27 - SABAM/Netlog.

1920 Siehe ergänzend auch Erwägungsgrund 19 zur Bestimmung des Ortes der Niederlassung und 22 zum Herkunftslandprinzip, ABl. 2000 L 178/4. Ausführlicher zur Geltungswirkung des Herkunftslandprinzip etwa Nordmeier, in: Spindler/Schuster, Elektronische Medien, Elfter Teil $\mathbb{} 3 \mathrm{TMG}$ Rn. $9 \mathrm{ff}$.

1921 Dass sie Zweigstellen in anderen Mitgliederstaaten gegründet haben, die u.a. Hilfestellung für die Vermarktung der Werbeflächen geben (siehe etwa die Feststellungen des VG Hamburg, ZD 2016, 43 oder des OVG Hamburg, ZD 2018, 230 hinsichtlich Facebook), ist ausweislich Erwägungsgrund 19 S. 3 unbeachtlich.

1922 Hierzu Claussen, Medialaws 2018, 110 (128f.); Liesching, MMR 2018, 26 (29); ders., NetzDG, Dörr, Die regulatorische Relevanz der Organisation massenhafter Individualkommunikation, S. 25 f.; ausführlicher Spindler, ZUM 2017, 473 (475 ff.), ders., Legal Expertise concerning the notified German Act to Improve Enforcement of the Law in Social Networks, S. 6 ff.; Hain/Ferreau/ Brings-Wiesen, K\&R 2017, 433 ff. Zumindest i.E. für europarechtswidrig haltend Nolte, ZUM 2017, 552 (561); Adelberg, Rechtspflichten und -Grenzen der Betreiber sozialer Netzwerke, S. 113 f. m.w.N. Daneben hat sich auch die Fachabteilung Europa des Bundestages der überwiegenden Meinung in der Literatur angeschlossen, Ausarbeitung der Unterabteilung Europa des Bundestages, PE 6 - 3000 - 36/17, S. 6 ff. Anders indes etwa Nölscher, ZUM 2020, 301 (307ff.). 
Zwar ist in Art. 3 Abs. 4 eine Ausnahme vom Herkunftslandprinzip normiert. Allerdings steht nicht nur der Wortlaut (bestimmter Dienst) einer Verpflichtung aller in $\mathbb{\int} 1$ Abs. $8 \mathrm{MStV}$ genannten Dienstanbieter im Weg. Vielmehr lässt sich auch der abstrakt-generelle Charakter der unterschiedlichen Verpflichtungen kaum mit dem Begriff der Maßnahmen in Einklang bringen, ${ }^{1923}$ weil die in Art. 3 Abs. 4 lit. b) RL 200/31/EG vorgesehene Kooperation mit dem Sitzstaat ansonsten kaum Sinn ergäbe. ${ }^{1924}$ Wie schon bei Erlass des NetzDG ${ }^{1925}$ spricht vieles gegen eine Ausnahme für die gesamten medienrechtlichen Maßnahmen. ${ }^{1926}$

Ein Ausweg könnte auch hier dann nur über Art. 1 Abs. 6 RL 2000/31/EG zu finden sein: Denn während das NetzDG unmittelbar Anforderungen an die Sorgfaltspflichten der Dienstanbieter im Umgang mit rechtswidrigen und potentiell haftungsauslösenden Inhalten stellt, sich also innerhalb des Regelungsbereichs der Artt. 12-14 der E-Commerce-RL und damit im koordinierten Bereich bewegt, ${ }^{1927}$ hat der MStV eine völlig andere inhaltliche Ausrichtung, die von Art. 1 Abs. 6 der E-Commerce-RL gerade den Mitgliedstaaten kompetenziell zugewiesen ist. ${ }^{1928}$ Auch die Länder sind davon ausgegangen, dass das eingeführte Marktortprinzip „in Ermangelung entsprechender europäischer Regelungen und aufgrund der fehlenden Regelungskompetenz der Europäischen Union [...]“ zulässig ist. ${ }^{1929}$

Dem tritt die Europäische Kommission allerdings entgegen. Sie genehmigte zwar den Entwurf, wies dabei aber auch deutlich darauf hin, dass sie grundlegende Bedenken gegen eine rein national-rechtliche Lösung hat. ${ }^{1930}$ In einem noch

1923 Vgl. hierzu bereits die Verweise in Fn. 1922. Mit Blick auf den Begriff der Maßnahme muss allerdings berücksichtigt werden, dass GA Villalón in seinem Schlussantrag v. 29.03.2011 Rs. C-509/09, 161/10, Rn. 80 meinte, dass auch andere Staaten zum Schutz der genannten Güter „Ausnahmen entweder über materiell-rechtliche Maßnahmen oder sogar über Vorschriften vorsehen [können], mit denen das anwendbare Recht korrigiert wird“.

1924 Vgl. Nölscher, ZUM 2020, 301 (307f.). Umfangreich zur Auslegung der Ausnahmen etwa Liesching, MMR-Beil. 2020, 3 (8f.).

1925 Ausführlich zu diesem Problem etwa die Ausarbeitung der Unterabteilung Europa des Bundestages, PE 6 - 3000 - 36/17 oder Spindler, ZUM 2017, 473 (475 ff.), ders., Legal Expertise concerning the notified German Act to Improve Enforcement of the Law in Social Networks, S. $6 \mathrm{ff}$.

1926 Mit Blick auf den Medienstaatsvertrag etwa Liesching, Das Herkunftslandprinzip der E-CommerceRichtlinie, S. 84 f. speziell mit Blick auf die Inpflichtnahme von Intermediären im Kontext der Meinungsroboter Schröder, DVBl. 2018, 465 (471). Tendenziell anders aber, auch mit Blick auf einen vorrangig zu berücksichtigenden angemessenen Grundrechtsschutz, Nölscher, ZUM 2020, 301 (309).

1927 So auch Dörr, Die regulatorische Relevanz der Organisation massenhafter Individualkommunikation, S. 25.

1928 Dazu ausführlicher bereits oben A.I.1.a.aa(I.)(1.)(b.)(cc.). Auch in der Gesetzesbegründung berufen sich die Länder gerade darauf, dass „[d]ie Verankerung des Marktortprinzips ist auch in Ermangelung entsprechender europäischer Regelungen und aufgrund der feblenden Regelungskompetenz der Europäischen Union notwendig [sei] [...].“ (Hervorh. d. Aut.), NiedrsächsLT-Drs. 18/6414, S. 89; BayLT-Drs. 18/7640, S. 80.

1929 Siehe hierzu BayLT-Drs. 18/7640, S. 80, NiedersächsLT.-Drs., S. 89.

1930 Vgl. hierzu etwa die Pressemitteilung v. 28.04.2020, abrufbar unter https://ec.europa.eu/germany/ne ws/20200428-medienstaatsvertrag_de, (Stand 30.05.2021). 
nicht veröffentlichten Dokument begegnet die Kommission sogar explizit dem Argument, dass Art. 1 Abs. 6 der E-Commerce-RL eine Abweichung vom Herkunftslandprinzip legitimiere:
„Die deutschen Behörden machen geltend, dass der notifizierte Entwurf unter Artikel 1 Absatz 6 [...] falle, wonach, Maßnahmen auf gemeinschaftlicher Ebene oder einzelstaatli- cher Ebene, die unter der Wahrung des Gemeinschaftsrechts [...] dem Schutz des Pluralis- mus dienen, unberührt bleiben'. Um sich auf eine solche Bestimmung zu berufen, müssen die Maßnabmen tatsächlich und objektiv dem Schutz des Medienpluralismus dienen und in einem angemessenen Verhältnis den Zielen der Maßnahme stehen. [...] Darüber hinaus müssen Mitgliedstaaten gemäß Artikel 1 Absatz 6, auch wenn die Richtlinie [...] die Maßnahmen der Mitgliedstaaten zur Förderung des Pluralismus nicht berührt, beim Er- lass solcher Maßnahmen das weitere EU-Recht beachten, zu dem auch die Bestimmungen der Richtlinie [...] gehören. Daher werden mit Artikel 1 Absatz 6 die Bestimmungen der Richtlinien (im Gegensatz zu Artikel 1 Absatz 5) nicht ausgeschlossen, sondern wird vielmehr die Bedeutung hervorgehoben, die die EU dem Schutz des Pluralismus als einem Faktor beimisst, den die Mitgliedstaaten bei der Regulierung der Erbringung von Diensten der Informationsgesellschaft berücksichtigen mögen [...]. " 1931

Aus allein binnenmarktrechtlichen Gründen ist dieses Verständnis durchaus nachvollziehbar, dient das Herkunftslandprinzip doch gerade einer klaren Zuständigkeitsverteilung, dem Abbau von ansonsten bestehenden Hemmnissen durch kumulativ zu beachtende und divergierende nationale Regeln, ${ }^{1932}$ und ist deshalb zur Verwirklichung des Binnenmarktes essentiell. ${ }^{1933}$ Gleichwohl ist die Begründung der Kommission angreifbar. Wenn mitgliedsstaatliche Regelungen gerade von der Richtlinie unberührt bleiben sollen, müsste das - auch aufgrund der systematischen Stellung - ${ }^{1934}$ in letzter Konsequenz für die gesamten Vorgaben der Richtlinie gelten. Zudem ist der Hinweis auf die vorausgesetzte Vereinbarkeit mit dem Unionsrecht deshalb zirkulär, weil gerade zu klären ist, ob darunter auch das Herkunftslandprinzip fällt oder ob das vor allem als Verweis auf die Grundfreiheiten gemeint ist, was angesichts vergleichbarer Formulierungen andernorts ${ }^{1935}$ naheliegender wäre. Dass ursprünglich der Rundfunk und

1931 So der Wortlaut der bisher unveröffentlichten Mitteilung der Kommission v. 27.04.2020 - MSG 303 IND - 2020/0026/D), zitiert nach Liesching, Das Herkunftslandprinzip der E-CommerceRichtlinie, S. 79.

1932 Vgl. in Ansätzen Spindler, RabelsZ 2002, 633 (638f.). Daneben auch Marly, in: Grabitz/Hilf, Recht der EU, A 4. Art. 3 Rn. $11 \mathrm{f}$.

1933 Siehe etwa Schulz/Grothe, Caution, Loose Cornerstone: The Country of Origin Principle under Pressure; Müller Broich, TMG, $\mathbb{S} 3$ Rn. 1. Kritisch allerdings mit Blick auf die nachteiligen Folgen für den Binnenmarkt hinsichtlich unterschiedlicher Regelungen der Staaten der Niederlassungen Nölscher, ZUM 2020, 301 (308).

1934 Artikel 1 Abs. 6 ECRL steht immerhin unter der Überschrift „Zielsetzung und Anwendungsbereich".

1935 Vgl. etwa die Hinweise des EuGH bei der Ausübung der mitgliedstaatlichen Kompetenz im Bereich der direkten Steuern, wo stets eine Prüfung anhand grundfreiheitlicher Maßstäbe folgte. Siehe u.a. EuGH, Urt. v. 25.02. 2010 - Rs. C-337/08 = EuZW 2010, 512 Rn. 16 ff. - X Holding B V/Staatssecretaris van Financiën, mit Blick auf die Niederlassungsfreiheit; EuGH, Urt. v. 17.01.2008 Rs. C-152/05 = EuZW 2008, 127 Rn. 16 ff - Europäische Kommission/Bundesrepublik Deutsch- 
damit der eigentliche Anwendungsbereich der Medienregulierung ohnehin nicht der ECRL unterfiel, ${ }^{1936}$ ist jedenfalls mit Blick auf die Regulierung der digitalen Medien kaum ein überzeugendes Argument. ${ }^{1937}$

Der Verweis auf Art. 1 Abs. 5 ECRL ist zwar gewichtig, denn auch das Glücksspielrecht (Art. 1 Abs. 5 lit. d) dritter Spiegelstrich ECRL) ist eine originär mitgliedsstaatliche Materie, aber unterfällt explizit nicht dem Anwendungsbereich der Richtlinie. Allerdings mag das auch dem Umstand geschuldet sein, dass im Glücksspielrecht gerade wegen der gravierenden mitgliedstaatlichen Unterschiede ${ }^{1938}$ eine einheitliche Harmonisierung kaum in Betracht kommt und in den übrigen Fällen des Art. 1 Abs. 5 lit. d) ECRL nur ein geringer Überschneidungsgrad mit den inhaltlichen Vorgaben der Richtlinie besteht. Bei digitalen, meinungsrelevanten Medien wäre zudem ein genereller Ausschluss des Anwendungsbereichs wegen ihres Doppelcharakters (Kultur- und Wirtschaftsgut) nicht möglich, ohne dass damit eine erhebliche Verschmälerung des Anwendungsbereichs einher geht. Art. 1 Abs. 6 ECRL kann vor diesem Hintergrund gerade dazu dienen, einen „Kompromiss“ dergestalt zu finden, dass die ECRL auch hier jedenfalls solange anwendbar ist, wie sie nicht einer Medienvielfaltsregulierung durch die Mitgliedstaaten im Wege steht. Dass würde zumindest auch erklären, warum die im ursprüngliche Kommissionsentwurf nicht enthaltene ${ }^{1939}$ Kulturklause $^{1940}$ durch den Gemeinsamen Standpunkt des Rates eingefügt ${ }^{1941}$ und zeitgleich die zunächst in Art. 22 enthaltenen Ausnahmen vom Anwendungsbereich in Art. 1

land, mit Blick auf die Freizügigkeit (Art. 18 EGV), Arbeitnehmerfreizügigkeit (Art. 39 EGV) und Niederlassungsfreiheit (Art. 43 EGV); EuGH, Urteil vom 12. 7. 2005 - Rs. C-403/03 = EuZW 2005 Rn. $19 \mathrm{ff}$. - Egon Schempp/Finanzamt München V, mit Blick auf das Diskriminierungsverbot (Art. 12 EGV) und die Freizügigkeit (Art. 18 EGV). Vgl. daneben auch die wortlautgleiche Verwendung in anderen Vorschriften, etwa in Art. 1 Abs. 6 RL 2006/123/EG, ABl. 2006 L, S. 36.; oder die Erwägungsgründe der RL 90/385/EWG („,...] Diese harmonisierten Vorschriften berühren daher nicht die Möglichkeit der Mitgliedstaaten, die oben genannten Maßnahmen unter Wahrung des Gemeinschaftsrechts durchzuführen.").

1936 So etwa Kramer, Das sekundärrechtliche Herkunftslandprinzip, S. 60.

1937 Gerade angesichts der zunehmenden tatsächlichen Veränderungen im Bereich digitaler Medien gerät das Herkunftslandprinzip und die E-Commerce-RL insgesamt zunehmend unter Druck, vgl. Schmitz/Berndt, The German Act on Improving Law Enforcement on Social Networks: A Blunt Sword?, S. 23. Deshalb sieht Nölscher, ZUM 2020, 301 (309) die Möglichkeit, Ausnahmen vom Herkunftslandprinzip vorzusehen.

1938 Auch der EuGH betont hier die „sittlichen, religiösen oder kulturellen Besonderheiten“ und gewährt (deshalb) einen weitgehenden Spielraum, EuGH, Urt. v. 24.03.1994 - Rs. C-275/92 = NJW 1994, 2013 Rn. 60 ff. - Gerhart Schindler; Urt. v. 08.09.2010 - C-316/07 u.a. = NVwZ 2010,1409 Rn. 76 f. - Markus Stoß u.a. Zudem finden sich unterschiedliche Regelungsmodelle von teilweiser Beschränkung über Verbote bis zu offenen Märkten, vgl. Brüning, NVwZ 2013, 23 (24), der insoweit von einem nicht existenten europäischen Markt ausgeht, S. 26.

$1939 \operatorname{KOM}(1998) 586$ endg., ABl. 1999 C 30, S. 4 ff.

1940 So Marly, in: Grabitz/Hilf, Recht der EU, Art. 1 E-Commerce-RL Rn. 4.

1941 Gemeinsamer Standpunkt des Rates 22/2000, ABl. 2000 C 128, 32 ff. 
Abs. 5 der Richtlinie übernommen wurden. ${ }^{1942}$ Insoweit scheint es dem Rat gerade auch um den Erhalt nationaler Kompetenzen gegangen zu sein.

Gerade diese Kompetenz wird aber durch das Verständnis der Kommission (erheblich) bedroht, weil damit das Herkunftslandprinzip zu einer Kompetenzausübungsschranke im Bereich digitaler Medien wird. Denn der koordinierte Bereich und damit das diesen absichernde Herkunftslandprinzip reichen weit über die eigentlichen materiellen Regelungsgehalte der Richtlinie hinaus, ${ }^{1943}$ ohne dass insgesamt eine Harmonisierung sichergestellt wäre. Vielmehr obliegt es dem Staat der Niederlassung, entsprechende ergänzende materielle Regelungen vorzusehen. ${ }^{1944}$ Schon deshalb kann das Herkunftslandprinzip Hemmnisse durch dennoch divergierende nationale Regeln nicht verhindern. ${ }^{1945}$ Für Video-SharingDienste und klassischen Rundfunk hat das die AVMD-RL inzwischen besser gelöst. Nicht nur das Herkunftslandprinzip in Art. 2 Abs. 1 AVMD-RL ist deutlich präziser. ${ }^{1946}$ Auch finden sich wesentlich umfangreichere Harmonisierungen inhaltlicher Art. ${ }^{1947}$ Diese fehlen aber mit Blick auf Medienintermediäre, soweit es nicht um audiovisuelle Inhalte geht (AVMD-RL) oder die Beziehung zwischen Plattform und kommerziellem Nutzer tangiert ist (P2B-VO). ${ }^{1948}$ Das führt aber dazu, dass die Mitgliedstaaten das nach nationalem, verfassungsrechtlichem Verständnis vorgesehene Schutzniveau hinsichtlich digitaler Medien gar nicht eigenständig gewährleisten können, ${ }^{1949}$ sondern vielmehr davon abhängig sind, dass

1942 Vgl. hierzu den ursprüngliche (KOM(1998) 586 endg., ABl. 1999 C 30, S. 4 ff.) und überarbeiteten Kommissionsentwurf (KOM(1999) 427 endg., ABl. 2000 C 248 E, S. 69 ff.) mit dem gemeinsamen Standpunkt des Rates 22/2000, ABl. 2000 C 128, 32 ff. In der ursprünglichen Fassung fand sich in Art. 22 des Kommissionsvorschlags auch keine Ausnahme für das Glücksspielrecht. Auch diese Ausnahme wurde erst durch den Gemeinsamen Standpunkt des Rates aufgenommen.

1943 Zu dieser Ausweitung etwa Spindler, RabelsZ 66 (2002), 633 (638 f.).

1944 Kritisch mit Blick auf den Harmonisierungsbedarf etwa Weller, in: Gersdorf/Paal, Informationsund Medienrecht, 3 TMG Rn. 1; Spindler, RabelsZ 66 (2002), 633 (638) der von einer erheblichen Verschärfung des Wettbewerbs der Rechtsordnungen spricht.

1945 So etwa zum NetzDG Nölscher, ZUM 2020, 301 (308f.), der deshalb meint, dass das Herkunftslandprinzip „zur Erreichung seines originären Zwecks nur bedingt geeignet [sei].“, S. 309. Das ist letztlich auch der Grund, warum die Notwendigkeit einer Neujustierung im Wege eines „Digital Service Act" gesehen wird, vgl. etwa den Inception Impact Assessment der EU-Kommission v. 04.06.2020, ARES(2020)2877686, S. 3 f.

1946 Ähnlich schon mit Blick auf die Fernsehrichtlinie Spindler, RabelsZ 66 (2002), 633 (639f.).

1947 Zurückhaltend euphorisch zu den Änderungen etwa Nölscher, ZUM 2020, 301 (308, 309).

1948 So auch Ory, ZUM 2019, 139 (145).

1949 In diese Richtung auch Nölscher, ZUM 2020, 301 (309) der zudem auf die nur sporadische Harmonisierung von Video-Sharing-Dienste hinweist. Kellner, Die Regulierung der Meinungsmacht von Internetintermediären, S. 278 meint, dass „[e]ine unionsweit einheitliche Regulierung [...] vor dem Hintergrund der starken Einbeziehung nationaler grundrechtlicher Wertungen rechtspolitisch nicht angezeigt [ist].“. 
der Sitzstaat entsprechende Regelungen ${ }^{1950}$ trifft. ${ }^{1951}$ Insofern sprechen gewichtige Gründe für ein Verständnis des Art. 1 Abs. 6 E-Commerce-RL als „Öffnungsklausel“" und gegen das Verständnis der Kommission, das eher an die Querschnittsklausel des Art. 167 Abs. 4 AEUV erinnert. ${ }^{1952}$

Das bedeutet freilich nicht, dass entsprechende grenzüberschreitende Aspekte hier nicht zu harmonisieren wären. Eine europäische Lösung durch Rechtsangleichung wird hier auf lange Sicht sinnvoll und notwendig werden. ${ }^{1953}$ Voraussetzung hierfür ist aber gerade, dass von nationalstaatlichen Regelungen Hemmnisse für den Binnenmarkt ausgehen. Solange diese keine entsprechenden vielfaltssichernden Regelungen erlassen haben, hält sich der Bedarf einer Harmonisierung durch Sekundärrecht also ohnehin in Grenzen. Insoweit kann der deutsche Vorstoß einer europäischen Lösung Antrieb leisten. ${ }^{1954}$

1950 Pille, Meinungsmacht sozialer Netzwerke, S. 144 f. zeigt aber auf, dass es mitgliedstaatlich ganz unterschiedliche regulative Ansätze gibt, die nicht kongruent verlaufen. Und wegen der unterschiedlichen Erfahrungen und Vorstellungen wird eine einheitliche Regulierung auch „[f]aktisch [...] scheitern".

1951 Nölscher, ZUM 2020, 301 (309) weist darauf hin, dass die neuartigen Gefahrenlagen anders bewertet und nicht gleich ernst genommen werden könnten. Und Kellner, Die Regulierung der Meinungsmacht von Internetintermediären, S. 278 weist auf die „[grundsätzliche] Diskrepanz der Regulierungsbereitschaft der Mitgliedsstaaten“ hin. Das entspräche aber dann gerade auch der Situation, die beim Glücksspielrecht vorzufinden ist, wo der EuGH den nationalen Spielraum wegen unterschiedlicher Ansichten gerade betont, siehe nur EuGH, Urt. v. 03.06.2010 - Rs. C-203/08 = MMR 2010, 850 Rn. 27 ff. - Betfair/Minister van Justitie.

1952 Ausdrücklich nimmt Heidtke, Meinungsbildung und Medienintermediäre, S. 166, 351, die Vereinbarkeit an. In diese Richtung auch Müller-Terpitz, in: Kühling/Zimmer, Neue Gemeinwohlherausforderungen - Konsequenzen für Wettbewerbsrecht und Regulierung, S. 177 (189) mit Fn. 42; Ders., in: Eifert/Gostomzyk, Netzwerkrecht, S. 45 (50); Hönig d'Orville, ZUM 2019, 104 (107). Und - den pluralismussichernden Charakter der nationalen Norm vorausgesetzt - spricht sich auch Pille, Meinungsmacht sozialer Netzwerke, S. 148 f. gegen die Anwendung des Herkunftslandprinzip im Anwendungsbereich des Art. 1 Abs. 6 E-Commerce-RL aus. Unklar ist die Formulierung bei Ferreau, in: Spindler/Schuster, Elektronische Medien, Erster Teil B. 142, der mit Blick auf Art. 1 Abs. 6 ECRL nur auf EU-ausländische Anbieter eingeht.

1953 So bereits andeutend Spindler, RabelsZ 66 (2002), 633 (708f.); nach Kellner, Die Regulierung der Meinungsmacht von Internetintermediären, S. 278, sei diese wünschenswert. Mit Blick auf eine Effektuierung der Kennzeichnungspflicht des MStV Löber/Roßnagel, MMR 2019, 493 (495). Vesting, in: Binder/ders., Rundfunkrecht, $\mathbb{S} 1$ RStV Rn. 25b meint, dass ein Bedeutungszuwachs der Unionskompetenzen hier auf lange Sicht unvermeidbar seien und dies auch zu Recht geschehe. Ausführlicher zu den Vorteilen auch Cornils, Designing platform governance, S. $77 \mathrm{ff}$. Mit Blick auf Maßnahmen gegen Bots Fehling/Leymann, AfP 2020, 110 Rn. 36. Auf europäischer Ebene ist gerade eine Anpassung der E-Commerce-Richtlinie als Teil eines „Digital Service Act“ im Gespräch, der u.a. auch die Haftungsvorschriften anpassen bzw. verändern soll, siehe hierzu etwa den Inception Impact Assessment der EU-Kommission v. 04.06.2020, ARES(2020)2877686, S. 4 f.

1954 Deshalb betont etwa Eifert, in: ders./Gostomzyk, Netzwerkrecht, S. 9 (43), dass zwar europarechtliche Bedenken gegen das NetzDG bestehen, aber diese auch zum „Treiber einer europäischen Entwicklung werden [kann]“. Liesem, ZUM 2020, 377 (382) spricht insgesamt beim Medienstaatsvertrag von einer „Pionierleistung“ einem „medienpolitischen Signal“, das als „Blaupause für eine unionsweite Regulierung“ dienen kann. 
cc. Vereinbarkeit mit der Dienstleistungsfreiheit

Zuletzt müssten die getroffenen Regelungen auch das Unionsrecht und damit die Grundfreiheiten wahren. Maßgeblich ist vor allem die Dienstleistungsfreiheit des Art. 56 AEUV, wobei aber $\mathbb{S} 18$ Abs. $3 \mathrm{MStV}$ weniger Probleme aufwirft, weil die Kennzeichnungspflicht ohnehin nur für im Inland niedergelassene Anbieter gilt und sie insoweit nur gegenüber ausländischen Anbietern schlechter gestellt sind (Inländerdiskriminierung). ${ }^{1955}$ Problematischer erscheinen die $\mathbb{S}$ 93, $94 \mathrm{MStV}$. Zwar treffen die Regelungen alle Anbieter sozialer Netzwerke unabhängig von ihrer Ansässigkeit. Tatsächlich - und das zeigt gerade auch $\mathbb{} 1$ Abs. $8 \mathrm{MStV}-$ geht es aber zurzeit überwiegend um die Regulierung der im europäischen Ausland ansässigen Netzwerkbetreiber. Auch wenn inländische Anbieter existieren mögen, so werden sie selten die vorgesehene Bagatellgrenze von einer Million monatlich aktiven Nutzern ( $\$ 91$ Abs. 2 Nr. $1 \mathrm{MStV}$ ) erreichen. ${ }^{1956}$ Insoweit wirkt sich die Regulierung der Länder in erster Linie auf die Tätigkeit im EU-Ausland ansässiger Medienintermediäre aus. Durch die deutlich strengere Regelung als im Sitzland ist die Regelung also nicht nur geeignet die Tätigkeit zu beeinträchtigen, ${ }^{1957}$ sie erschwert diese gerade. Weil es sich hierbei aber nur um eine mittelbare Diskriminierung handelt, können Belange des Allgemeinwohls im Sinne der Cassis-de-Dijon-Doktrin die Beschränkung rechtfertigen, was im Bereich der Medienregulierung nach der Rechtsprechung des EuGH gerade durch den Aspekt des Medienpluralismus möglich ist. ${ }^{1958}$

Entscheidende Schranke ist hier letztlich das Prinzip der Verhältnismäßigkeit. Bereits die Ausführungen zum Schutzbedarf zeigen jedenfalls, dass eine solche Regelung durchaus geeignet wäre, den strukturellen Verzerrungen und der „Meinungsmacht“ der Meinungsroboter zu begegnen. Hinsichtlich der Erforderlichkeit räumt der EuGH den Mitgliedstaaten ein „weites Ermessen“ ein. ${ }^{1959}$ Zwar steht dies unter dem Vorbehalt des effet utile unionsrechtlicher Vorgaben. ${ }^{1960}$ Wie aber aufgezeigt, beeinträchtigt $\$ 93$ Abs. $4 \mathrm{MStV}$ nicht die Anwendung der ECRL oder der P2B-VO, und für die meinungsrelevanten Aspekte der $\mathbb{S} \mathbb{S} 93,94$

1955 Zur Irrelevanz dieser Diskriminierung aus Sicht des Unionsrechts statt vieler Müller-Graff, in: Streinz, EUV/AEUV, Art. 56 AEUV Rn. 71.

1956 Vgl. zu den deutschsprachigen Angeboten und deren Marktanteilen BKartA, Beschl. v. - B6-22/16 Rn. 346, $390 \mathrm{ff}$.

1957 Zu dieser Formel EuGH, Urt. v. 13. 12. 2007 - Rs. C-250/06 = EuZW 2008, 86 Rn. 29 - United Pan-Europe Communications Belgium SA u.a./Belgien, m.w.N.

1958 Vgl. etwa EuGH, Urt. v. 13. 12. 2007 - Rs. C-250/06 = EuZW 2008, 86 Rn. 41 - United Pan-Europe Communications Belgium SA u.a./Belgien, m.w.N. ging es etwa um eine belgische Regelung, die Kabelnetzanbieter verpflichtete bestimmte Inhalte $\mathrm{zu}$ senden; EuGH, Urt. v. 25.07.1991 Rs. C-288/89 = GRUR Int 1991, 223 Rn. 23 - Stichting Collectieve Antennevoorziening Goudal Commissariaat voor de Media.

1959 EuGH, Urt. v. 13. 12. 2007 - Rs. C-250/06 = EuZW 2008, 86 Rn. 44 - United Pan-Europe Communications Belgium SA u.a./Belgien.

1960 EuGH, ebd., Rn. 45. 
MStV findet sich bislang keine sekundärrechtliche Harmonisierung. Entscheidend wird es also auf eine Abwägung kollidierender Belange ankommen. Mit Blick auf $\mathbb{S} 93$ Abs. $4 \mathrm{MStV}$ sind bereits zahlreiche Aspekte aufgezeigt worden, die hier entsprechend zu berücksichtigen sind. ${ }^{1961}$ Im Übrigen ist auch das Funktionieren des demokratischen Meinungsbildungsprozesses aus europäischer Sicht elementar. ${ }^{1962}$ Während zur Sicherung bislang nur herkömmliche Medien reguliert wurden, verschiebt sich der Fokus - zu Recht - zunehmend auf digitale Angebote. Der MStV sieht hierfür in den $\mathbb{S} \mathbb{S} 93-94 \mathrm{MStV}$ nur Maßnahmen geringer Eingriffsintensität vor, die in anderem Kontext auch auf europäischer Ebene bereits normiert worden sind. ${ }^{1963}$ Allein $\$ 94$ Abs. 2 MStV kann eine etwas umfangreichere Anpassung der Algorithmen verlangen, wenn diesen eine gewollte und systematische Verzerrung der Darstellung bestimmter Inhalte zugrunde liegt. Aber selbst wenn man unterstellt, dass dies bislang der Fall war, so lässt sich das ohne Weiteres mit der zentralen Stellung entsprechender Anbieter im Kommunikationsprozess rechtfertigen. An diese zentrale Stellung wird auch im Übrigen auf europäischer Ebene angeknüpft und die Verantwortlichkeit der Anbieter im Vergleich zu den Regeln der E-Commerce-RL deutlich verschärft. ${ }^{1964}$ Der MStV weicht davon nicht wesentlich ab. Hierbei gilt es auch zu berücksichtigen, dass wegen des engen Bezugs zu demokratischen Grundvoraussetzungen - Informiertheit, kommunikative Chancengleichheit, Pluralismus etc. - hier auch ein deutlich gewichtigeres Ziel verfolgt wird, als dies etwa bei der P2B-VO mit ihrer rein wettbewerbs- und damit wirtschaftspolitischen Ausrichtung der Fall ist. Zusammengenommen dürften also auch keine durchgreifenden Bedenken an der Vereinbarkeit mit der Dienstleistungsfreiheit bestehen.

\section{g. Fazit: Zulässiges, tranzparenzerhöhendes Schutzkonzept der Länder}

Die Regelungen für Medienintermediäre wollen vor allem die Transparenz erhöhen, indem Informationen zur Funktionsweise der Rankingalgorithmen bereitgehalten werden müssen $(\mathbb{S} 93 \mathrm{MStV})$. Ein an die Transparenzpflicht der Intermediäre anknüpfendes Diskriminierungsverbot ( $\mathbb{S} 94$ Abs. 1, $2 \mathrm{MStV}$ ) ergänzt die Regelungen und verhindert zumindest einen systematischen, benachteiligenden Einfluss der Betreiber auf die Auswahl und Anzeige der Inhalte. Besonders her-

1961 Das gilt vor allem mit Blick auf die grundrechtlichen Ausführungen unter C.II.2.c.aa(II.).

1962 So betont etwa der EuGH regelmäßig auch die Bedeutung des Pluralismus für die Meinungsfreiheit, siehe etwa EuGH, Urt. v. 13. 12. 2007 - Rs. C-250/06 = EuZW 2008, 86 Rn. 41 - United Pan-Europe Communications Belgium SA u.a./Belgien.

1963 Das gilt sekundärrechtlich vor allem mit Blick auf die P2B-Verordnung der EU (hierzu bereits oben C.II.2.f.aa) aber auch mit Blick auf den Verhaltenskodex zur Bekämpfung von Desinformation (hierzu oben C.I.3.a.bb(I.)) in den alle drei Aspekte aufgenommen worden sind.

1964 Das gilt vor allem mit Blick auf die bereits erwähnte UrheberrechtsRL und die geplante VO zur Bekämpfung von terroristischen Online-Inhalten. Zu diesen Veränderungen bereits oben, A.I.1.a.aa(I.) (1.)(b.)(bb.). 
vorzuheben ist die gesteigerte Verantwortlichkeit der Betreiber sozialer Netzwerke für die Meinungsroboter. Die Kennzeichnungspflicht des $\mathbb{9} 93$ Abs. 4 ergänzt das Schutzkonzept in den Fällen, in denen der Pflicht des $\mathbb{S} 18$ Abs. $3 \mathrm{MStV}$ nicht nachgekommen wird. Dabei kann von ihnen - auch in Übereinstimmung mit dem Unionsrecht - durchaus auch die Überwachung der Netzwerkdaten und der Betrieb einer proaktiv agierenden Erkennungssoftware gefordert werden. Dass das neue Regelungssystem auch über die territorialen Grenzen hinaus vor allem für die in Irland ansässigen „Big Player“ Facebook und Twitter konzipiert ist, verursacht nur auf den ersten Blick Spannungen mit dem Unionsrecht. Die P2BVerordnung wird in ihrem wirtschaftsrechtlichen Ziel im Wesentlichen nicht berührt und der MStV orientiert sich - bis auf das Diskriminierungsverbot - weitestgehend an ihren Vorgaben, sodass es nicht zu eklatanten Widersprüchen kommt. Auch das durch $\mathbb{\int} 1$ Abs. $8 \mathrm{MStV}$ eingeführte Marktortprinzip ist i.E. mit dem Herkunftslandprinzip deshalb vereinbar, weil Art. 1 Abs. 6 ECRL eine umfangreiche „Öffnungsklausel“ für die hier erfolgte mitgliedstaatliche, medienrechtliche Pluralismussicherung vorsieht. Und die materiell-rechtlichen Vorgaben sind auch mit der Dienstleistungsfreiheit ohne Weiteres in Einklang zu bringen.

\section{Bewertung des Schutzkonzeptes anhand des Untermaßverbots}

Was bedeutet das aber nun mit Blick auf den staatlichen Schutzauftrag und den zu erfüllenden „Mindestschutz“? Kommen zumindest die Länder mit dem MStV nun der Schutzverantwortung in einer überzeugenderen und vor allem mit dem Untermaßverbot kompatiblen Art und Weise nach oder bestehen (evidente) Schutzlücken, sodass das Schutzkonzept auch weiterhin verfassungsrechtlich angreifbar bleibt? Und berücksichtigen sie dabei auch hinreichend die abwehrrechtlichen Implikationen?

Um einen Verstoß auf erster Stufe wird es schon deshalb nicht gehen, weil bereits zuvor ein - wenn auch sehr rudimentäres und wenig spezifisches - Schutzkonzept bestanden hat und insoweit nur dessen Wirksamkeit Zweifel hervorgerufen hat (dazu bereits oben,C.I.5.). Ebenso wenig wird es um einen Verstoß auf zweiter Stufe gehen. Vergleicht man die geänderten Regelungen mit den bisherigen, so kommt der Gesetzgeber in spezifischer Weise seinem Schutzauftrag nach und das nicht nur allgemein hinsichtlich der Medienintermediäre, sondern gerade auch mit Blick auf Meinungsroboter. ${ }^{1965}$ Dabei verfolgen die Regelungen insgesamt einen überzeugenden Ansatz: die Herstellung von Transparenz als wesentliche Voraussetzung einer informierten autonomen Entscheidung der Nutzer. ${ }^{1966}$

1965 Positiv bewertet auch Heidtke, Meinungsbildung und Medienintermediäre, S. $331 \mathrm{f}$. , die Vorgaben für Medienintermediäre insgesamt.

1966 Vgl. Liesem, K\&R 2019, 687 (691); dies., AfP 2020, 277 Rn. 25 Dogruel u.a., Media Perspektiven 2020, 139 (142); Brinkmann, UFITA2019, 364 (384f.). 
Das entspricht weitestgehend dem bereits aufgezeigten Schutzbedarf der Nutzer nicht nur mit Blick auf die allgemeine Informationsverarbeitung, die auch durch Medienintermediäre beeinflusst wird ( $\mathbb{S} 93$ Abs.1-3 MStV, zum Schutzbedarf oben B.II.1.c.cc), sondern gerade auch spezifisch mit Blick auf die doppelte Täuschung bei Meinungsrobotern $(\mathbb{S} 18$ Abs. 3 MStV, zum Schutzbedarf oben B.II.1.c.aa.). Auf Seiten der Intermediäre wird das noch durch das Diskriminierungsverbot abgesichert. Damit begegnen die Länder den aufgezeigten Gefahren, die speziell für die Willensbildung der Nutzer drohen und die durch die Empirie jedenfalls theoretisch aufgezeigt werden konnten. Die Narrative der Filterblase und Echokammer sind nicht erst seit wenigen Jahren bekannt. Die Grundannahmen einer Fragmentierung der Öffentlichkeit (Filterblase) und einer bloßen Wiederspiegelung der eigenen Ansichten in der Informationsvermittlung (Echokammer) haben sich seitdem nicht wesentlich geändert. Dass jüngst die Narrative bzw. die Kausalität betreiberseitiger Personalisierung relativiert wurden, ist aber nicht missachtet worden, denn das grundlegende Potential einer solchen intransparenten Beeinflussung bleibt unabhängig davon bestehen, ob Intermediäre bereits transparente Informationen bereithalten, oder spezifische Diskriminierungen bislang nicht ersichtlich waren. ${ }^{1967}$

Entsprechendes gilt auch mit Blick auf die Meinungsroboter. Denn auch hier stagniert die empirische Forschung aus den nur verständlichen und bereits aufgezeigten Gründen (2. Teil, C.IV) zu den tatsächlichen Auswirkungen. Wesentliche Grundannahmen bleiben unverändert und ein (erhebliches) Risikopotential wird ihnen jedenfalls weit überwiegend beigemessen. ${ }^{1968}$ Die bisherigen Regelungen (hierzu oben C.I.) erfassen die Meinungsroboter aber nur als „Eindringlinge“ in die IT-Systeme der Netzwerkanbieter und blenden dabei die gesamte gesellschaftliche und individuelle Wirkungsebene aus. ${ }^{1969}$ Durch die Kennzeichnungspflicht hingegen nimmt man den Einsatzstrategien, den potentiell schädlichen Inhalten sowie den einschüchternden Wirkungen und anderen potentiellen Einflüssen eine wesentliche Wirkungsvoraussetzung. ${ }^{1970}$ Durch $\mathbb{9} 93$ Abs. $4 \mathrm{MStV}$ wird nicht nur die Voraussetzung für eine Kennzeichnung geschaffen, sondern diese - unter dem Vorbehalt der Zumutbarkeit jedenfalls bei großen Anbietern - in Fällen abgesichert, in denen eine Kennzeichnung nicht erfolgt.

Ein effektiver Schutz wird also nicht mehr allein auf einer selbstregulierten Ebene gewährt, sondern vielmehr stehen jetzt auch klassische aufsichtsrechtliche Befugnisse bereit, um die Durchsetzung der Verpflichtung zu kontrollieren und

1967 Hierauf weist etwa auch Müller-Terpitz, in: Kühling/Zimmer, Neue Gemeinwohlherausforderungen, S. 177 (193), hin.

1968 Siehe hierzu bereits die Verweise in Fn. 870.

1969 Denn im Kontext von kommerziellen Entscheidungen sieht jedenfalls das UWG über seine Generalklauseln die Möglichkeit vor, auch den kommerziell motivierten Einsatz von Social Bots zu erfassen, hierzu etwa Dankert/Dreyer, K\&R 2017, 73 (77).

1970 In diese Richtung auch Liesem, K\&R 2019, 687 (691). 
deren Einhaltung auch mithilfe des Ordnungsrechtes ( $\mathbb{S} 115$ Abs. 1 S. 2 Nr. $42-47$ $\mathrm{MStV}$ ) sicher stellen zu können. Insofern wird man gerade hier der gewandelten Stellung des Staates gerecht: Auch hier wird gewährleistet, dass der Staat seine Rolle als Garant für die grundrechtlichen Schutzgüter und den demokratischen Kommunikationsprozess wahrnehmen kann. Insoweit sieht der MStV also ein geeignetes Konzept vor, um den unterschiedlichen Schutzbedürfnissen beim Einsatz von Meinungsrobotern Rechnung zu tragen, ohne dabei wissenschaftliche Erkenntnisse völlig aus dem Blick zu verlieren. Eine hiervon zu trennende Frage ist, ob - auch angesichts der unklaren empirischen Ausgangslage - das Konzept auch die Anforderungen der dritten und vierten Stufe des Untermaßverbots gerecht wird: die Effektivität unter Berücksichtigung der abwehrrechtlichen „Erforderlichkeit“ (3. Stufe) und der praktischen Konkordanz zwischen grundrechtlichen Schutzbedürfnissen und grundrechtlichen Eingriffsverboten (4. Stufe).

\section{a. Bewertung der allgemeinen Intermediärsvorgaben}

$\mathrm{Ob}$ die allgemeinen Intermediärsregelungen den verfassungsrechtlichen Anforderungen genügen, soll schon deshalb in der gebotenen Kürze analysiert werden, weil sie nur mittelbar im Falle der Meinungsroboter relevant werden. Ausführliche Analysen mit Blick auf die Meinungsmacht der Intermediäre selbst finden sich bereits andernorts. ${ }^{1971}$

aa. Vereinbarkeit mit den Grundsätzen „Effektivität“ und „Erforderlichkeit“ (3. Stufe)?

Auch wenn die Transparenzregelung recht vage bleibt und die P2B-VO das mit ihren „Zielvorgaben“ (Art. 5 Abs. 5) besser gelöst hat, ${ }^{1972}$ so ist es mit Rücksicht auf die unternehmerischen Interessen und die unterschiedliche Ausrichtung und Funktionsweis der Selektionsalgorithmen ${ }^{1973}$ durchaus überzeugend, die primäre Auswahl den Netzwerkbetreibern zu überlassen und auf sekundärer Ebene allenfalls die Kohärenz der Informationen zu überprüfen ( $\mathbb{S} 95 \mathrm{MStV})$ und Vorgaben $\mathrm{zu}$ konkretisieren ( $\mathbb{S} 96 \mathrm{MStV})$. Analog zur P2B-VO könnten dann spezifische Zielvorgaben aufgestellt werden. ${ }^{1974}$ Aber auch so gehen mit den Transparenzvor-

1971 So etwa bei Heidtke, Meinungsbildung und Medienintermediäre, S. $319 \mathrm{ff}$. oder Schwartmann/ Hermann/Müblenbeck, Transparenz von Medienintermediären.

1972 Diese Informationen betreffen die Frage, welchen Einfluss die Eigenschaften der Waren/Dienstleistungen haben (Art. 5 Abs. 5 lit.a), die Relevanz dieser Merkmale für die Verbraucher (lit.b)) und speziell für Suchmaschinen die Gestaltungsmerkmale der jeweiligen Webseite (llit c)).

1973 Deshalb eher kritisch gegenüber der Regelung Ladeur/Gostomzyk, K\&R 2018, 686 (690). Zu dieser Differenzierung Hartung, „Damit der Nutzer nicht zum Spielball der Interessen der Anbieter wird“, Medienpolitik.net.

1974 Hierauf weist auch Liesem, AfP 2020, 277 (282) hin. Der Bericht der AG Intermediäre sah ebenfalls präzisere Vorgaben vor. Das betraf nicht nur Angaben zur Bevorzugung eigener Inhalte, son- 
gaben indirekte Sensibilisierungseffekte einher, die die Netzwerkbetreiber zu einem Durchdenken der Funktionsweise ihrer Algorithmen anhalten. ${ }^{1975}$ Und indem Betreiber die zentralen Kriterien in einer „verständlichen Sprache“ offenlegen müssen, kann das Verständnis der Nutzer für die technischen Bedingungen begründet und geschärft und so die Nutzerautonomie gestärkt werden. ${ }^{1976} \mathrm{Ob}$ man der Regelung eine bloße „Alibi-Bedeutung“ beimessen sollte, ${ }^{1977}$ darf deshalb bezweifelt werden. ${ }^{1978}$ Probleme können freilich daraus resultieren, dass die Nutzer letztendlich keine Möglichkeit haben, von einer informierten Entscheidung Gebrauch zu machen, weil es keine Alternative gibt, auf die die Nutzer ausweichen können, weshalb es meist bei einem „Take it or leave it" bleibt. ${ }^{1979}$ Entscheidend zu weit gingen indes Vorschläge, die Funktionsweise der Algorithmen en détail offenzulegen und so eine vollständige Transparenz herzustellen, ${ }^{1980}$ weil sie dann nur in quantitativer aber nicht in qualitativer Hinsicht gewährleistet wird, was Nutzer überfordern würde, sodass die Informationsasymmetrie weiterhin besteht. ${ }^{1981}$ Zudem missachtet das unternehmerische und besonders geschütz-

dern auch „die Kenntlichmachung, ob Inhalte aus weltanschaulicher, religiöser oder politischer Motivation heraus bevorzugt, nachgeordnet oder überhaupt nicht dargestellt werden. “, Bund-LänderKommission, Bericht zur Medienkonvergenz, 2016, S. 35. Zudem wurde empfohlen, gesetzliche Regelbeispiele einzuführen, S. 36.

1975 Droguel u.a., Media Perspektiven 2020, 139 (143). So auch Thomas Fuchs in seinem Interview mit Hartung, „Damit der Nutzer nicht zum Spielball der Interessen der Anbieter wird“, Medienpolitik.net. Diesen Sensibilisierungseffekt zeigt auch Heidtke, Meinungsbildung und Medienintermediäre, S. 340 .

1976 Ähnlich auch Fehling/Leymann, AfP 2020, 110 (116); Heldt, CR 2018, 494 (499); Kaiser/Reiling, in: Unger/v. Ungern-Sternberg, Demokratie und künstliche Intelligenz, S. 85 (104); Paal, MMR 2018, 567 (571); Schwartmann/Hermann/Müblenbeck, MMR 2019, 498 (500). Und hierauf stellt letztlich auch die Gesetzesbegründung selbst ab: „Ausgehend vom Ziel der Vorschrift, der Sicherung der Meinungsvielfalt, beabsichtigen die Vorgaben, dem durchschnittlichen Nutzer eines Medienintermediärs die wesentlichen Grundzüge der technischen Vorgänge, die zu bestimmten Ergebnissen führen, zu erläutern. “, BayLT-Drs. 18/7640, S. 108. Kritisch mit Blick auf Suchmaschinen und einer nur abstrakten Transparenz, aber dennoch befürwortend Hartl, Suchmaschinen, Algorithmen und Meinungsmacht, S. 228 ff. Ausführlich zu den Aspekten der Transparenzvorgaben für die Nutzer etwa Schwartmann/Hermann/Mühlenbeck, Transparenz von Medienintermediären, S. $80 \mathrm{ff}$. Demgegenüber sehen Ladeur/Gostomzyk, K\&R 2018, 686 (690f.) die Vorschrift als „praktisch nicht anwendbar" an.

1977 So etwa Cornils, ZUM 2019, 89 (102).

1978 Für Bedeutsam erachten die Transparenzvorschrift etwa Fuchs und Schwartmann im Interview mit Hartung, „Damit der Nutzer nicht zum Spielball der Interessen der Anbieter wird“, Medienpolitik.net. Und Schomberg/Reubert, ZD-Aktuell 2019, 06877 bezeichnen sie - trotz der Bedenken als „ersten Schritt in die richtige Richtung“.

1979 Vgl. Droguel u.a., Media Perspektiven 2020, 139 (144). Kritisch deshalb auch Kaiser/Reiling, in: Unger/v. Ungern-Sternberg, Demokratie und künstliche Intelligenz, S. 85 (104).

1980 So wohl die ganz herrschende Ansicht u.a. Cornils, ZUM 2019, 89 (101); Fehling/Leymann, AfP 2020, 110 Rn. 29; Heidtke, Meinungsbildung und Medienintermediäre, S. 339 f.; Heilmann, MMR 2020, 162 (165); Kellner, Die Regulierung der Meinungsmacht von Internetintermediären, S. 282; Liesem, AfP 2020, 277 Rn. 26; Paal, MMR 2018, 567, 57.

1981 So letztlich auch Heidtke, Meinungsbildung und Medienintermediäre, S. 339; Kaiser/Reiling, in: Unger/v. Ungern-Sternberg, Demokratie und künstliche Intelligenz, S. 85 (104) und Fehling/ Leymann, AfP 2020, 110 (116). 
te Interessen angesichts der geheimhaltungsbedürftigen Algorithmen, ${ }^{1982}$ was auch deshalb problematisch ist, weil damit auch Kriminellen ein wesentlich detaillierterer Einblick in die Funktionsweise der Algorithmen gewährt wird, wodurch die Funktionsweise der Algorithmen noch besser ausgenutzt werden kann. ${ }^{1983}$

Auch das Diskriminierungsverbot bleibt zurückhaltend, wenn es eine „systematische“ Benachteiligung verlangt. ${ }^{1984}$ Die ersten Diskussionsentwürfe sahen damals noch in $\mathbb{S} 53$ e Abs. $1,2 \mathrm{MStV}$ - vor, dass eine Diskriminierung schon dann vorliegt, wenn bewusst und zielgerichtet abgewichen wird. ${ }^{1985}$ Aber schon wegen des bestehenden, auch nutzerseitigen Interesses an einer differenzierten Darstellung bestimmter Inhalte, ist es zu begrüßen, dass der Gesetzgeber sich allein auf die systematische Benachteiligung von Inhalten festgelegt hat. ${ }^{1986}$ Damit wurde auch etwaigen Bedenken ${ }^{1987}$ an der Bestimmtheit der Vorgängerversionen begegnet. Und mit der zweiten Variante einer unmittelbaren oder mittelbaren systematischen Behinderung wird jedenfalls sichergestellt, dass die Netzwerkbetreiber die Diskriminierungsverbote nicht ohne Weiteres umgehen können. ${ }^{1988}$ Probleme ergeben sich hier dadurch, dass die Transparenzerfordernisse nur die zentralen Kriterien offenlegen sollen und so von außen nicht ohne Weiteres erkennbar ist, ob eine systematische Benachteiligung überhaupt vorliegt. Deshalb wurden Bedenken gegen die Operationalisierbarkeit vorgebracht, ${ }^{1989}$ was unmittelbare Auswirkungen auf die Effektivität der Regelungen haben würde. Das ver-

1982 Vgl. Ladeur/Gostomzyk, K\&R 2018, 686 (690), Liesem, K\&R 2019, 687 (61 mit Fn. 47); Pille, Meinungsmacht sozialer Netzwerke, S. 362; Cornils, ZUM 2019, 89 (101); Kellner, Die Regulierung der Meinungsmacht von Internetintermediären, S. 282; Heidtke, a.a.O., S. 339 f. m.w.N. Darauf berufen sich letztlich auch die Länder in der Gesetzesbegründung für die Transparenzvorschrift, vgl. nur BayLT-DrS. 18/7640, S. 109.

1983 So auch Heidtke, Meinungsbildung und Medienintermediäre, S. 339; Kellner, Die Regulierung der Meinungsmacht von Internetintermediären, S. 282. Mit Blick auf kriminelle Interventionen Ladeur/ Gostomzyk, K\&R 2018, 686 (690). Mit Blick auf die Manipulationsgefahr Liesem, K\&R 2019, 687 (61 mit Fn. 47); Ingold, in: Unger/v. Ungern-Sternberg, Demokratie und künstliche Intelligenz, S. 183 (210); Cornils, ZUM 2019, 89 (101). Pille, Meinungsmacht sozialer Netzwerke, S. 363 ist aber der Ansicht, dass diese Gefahr grundsätzlich hinzunehmen sei, wenn man dem Informationsbedürfnis der Nutzer gerecht werden will.

1984 Kritischer allerdings hierzu und eine kartellrechtliche Regulierung bevorzugend Kaiser/Reiling, in: Unger/v. Ungern-Sternberg, Demokratie und künstliche Intelligenz, S. 85 (108).

1985 Der entsprechende Diskussionsentwurf ist abrufbar unter https:/www.rlp.de/fileadmin/rlp-stk/pdf -Dateien/Medienpolitik/ MStV_Synopse_2019-07_Online_.pdf (Stand: 30.05.2021).

1986 Ähnlich auch Fehling/Leymann, AfP 2020, 110 (116).

1987 Vgl. Heidtke, Meinungsbildung und Medienintermediäre, S. 344; Ladeur/Gostomzyk, K\&R 2018, 686 (691).

1988 Allerdings bleibt immer noch die Möglichkeit, dass konkrete Inhalte bewusst oder fälschlich in einer Art bewertet werden, die nach den transparenten Kriterien eine nachteilige Weiterleitung rechtfertigt. Insoweit besteht das Problem dann weder in einer Abweichung von den Kriterien noch in den Kriterien selbst, hierzu Droguel u.a., Media Perspektiven 2020, 139 (144). Zur Wandlung des Tatbestandes siehe etwa Liesem, UFITA 2019, 395 (418).

1989 Siehe hierzu etwa Cornils, ZRP 2020, 60 (62), aber auch Droguel u.a., Media Perspektiven 2020, 139 (144f.) und allgemein zu dem Aspekt der Operationalisierbarkeit Heidtke, Meinungsbildung und Meinungsintermediäre, S. 347 ff. Andeutend auch Liesem, AfP 2020, 277 (282). 
mag aber nichts daran zu ändern, dass in jedem Fall offensichtliche Fälle so ausfindig gemacht oder verhindert werden können. ${ }^{1990}$ Zudem besteht jedenfalls die Möglichkeit, dass die Landesmedienanstalten im Rahmen ihrer Ermittlungsbefugnisse $(\mathbb{S} 95 \mathrm{MStV})$ ergänzende Informationen verlangen, um gemeldete Verstöße nicht nur oberflächlich zu prüfen. ${ }^{1991}$ Und durch eine Konkretisierung der Transparenzpflichten über $\mathbb{\$} 96 \mathrm{MStV}$ ließe sich auch das Diskriminierungsverbot operationalisierbarer machen. Insoweit ist es eine Frage der Rechtsfortentwicklung, eine Effektuierung der Durchsetzung zu ermöglichen. ${ }^{1992}$ Der Erstzugriff des Gesetzgebers ist auch hier verständlich und vertretbar, ${ }^{1993}$ zumal angesichts der unklaren empirischen Lage um bestehende Verzerrungstendenzen im Sinne der Filterblasen- oder Echo-Kammer-Metaphorik aktuell effektivere Regelungen kaum gefordert werden können. ${ }^{1994}$ Insoweit wird die Diskussion um das vorzugswürdigste Regelungsmodell vor allem die Weiterentwicklung und Ergänzung der nun mehr bestehenden Regelungen prägen. ${ }^{1995}$ Mit $\mathbb{9} 96 \mathrm{MStV}$ besteht

1990 Das hängt letztlich auch nicht zuletzt davon ab, welchen Umfang man den Transparenzpflichten beimisst. So wird mit einer Transparenz ex post, also hinsichtlich der konkreten Anordnung und die relevanten Faktoren hierfür, auch ein besser zu kontrollierendes Diskriminierungsverbot geschaffen, vgl. Schwartmann/Hermann/Müblenbeck, Transparenz bei Medienintermediären, S. $137 \mathrm{f}$.

1991 Das hiergegen Bedenken bestehen, weil damit gerade eine detaillierte Offenlegung erfolgt, die aber in erheblichem Maße die betrieblichen Interessen der Anbieter tangieren, mag zwar richtig sein. Allerdings wird durch $\mathbb{5} 58 \mathrm{MStV}$ ja zumindest dem besonderen Geheimhaltungsbedürfnis verfahrensrechtlich Rechnung getragen.

1992 Ähnlich Heidtke, Meinungsbildung und Medienintermediäre, S. 349. Ähnlich Liesem, K\&R 2019, 687 (692) mit Blick auf die staatliche Kontrollarchitektur. Zu diesem Prozess der Weiterentwicklung und konkreten Regulierungsvorschlägen ausführlicher etwa Heidtke, Meinungsbildung und Medienintermediäre, S. 355 ff. Ohnehin wird darauf hingewiesen, dass ,die Verantwortung nicht zu sehr auf die Nutzer [geschoben wird], weil auch die gut informierten und kritischen unter ihnen, letztendlich keine wirksame Durchsetzungsmacht haben“. Deshalb müssen sie vielmehr „durch ,gute Regulierung geschützt werden [...““, Droguel u.a., Media Perspektiven 2020, 139 (144). Hannah Klein, Leiterin des Media Policy Lab, meint etwa, „dass das Diskriminierungsverfahren ein Einstieg in eine [...] Struktur der Begründungspflicht für Intermediäre sein könnte.“, zit. nach Droguel u.a., Media perspektiven 2020, 139 (145). Inwieweit die Medienanstalten selbst personell Änderungen vornehmen müssen, um das Verständnis über den neuen Bereich ihrer Aufsicht zu schärfen (so etwa auch Droguel u.a., Media Perspektiven 2020, 139 (145); Schwartmann/Hermann/Mühlenbeck, Transparenz von Medienintermediären, S. 150 f.; Enquete-Kommission Künstliche Intelligenz, BT.Drs. 19/23700, S. 486), ist eine davon zu trennende Frage.

1993 Droguel u.a., Media Perspektiven 2020, 139 (147) bezeichnen die Transparenzanforderungen als einen „wichtigen Schritt“ für die Gewährleistung der Meinungsvielfalt „angesichts der neuen digitalen Herausforderungen“. Schmid/Braam/Mischke, MMR 2020, 19 (21) bezeichnen das Diskriminierungsverbot als „zwingend erforderlich“. Im Ausgangspunkt auch begrüßend Dörr, WRP 2021, $168(172)$

1994 Deshalb bezeichnet es Cornils, ZUM 2019, 89 (100) auch „als gar nicht einmal so unkluge Zurückhaltung".

1995 (Intensivere) Regelungsmodelle sollen etwa konkrete Vorgaben für die zu präsentierenden Inhalte machen. So schlagen etwa Schwartmann/Hermann/Müblenbeck, MMR 2019, 498 ff. ein Zwei-Säulen Modell zur Sicherung der Meinungsvielfalt vor. Das Angebot auf der ersten Säule spiegelt die individuelle Vielfalt wider und ist von den Personalisierungstendenzen der Betreiber geprägt. Diese werden um eine zweite Säule der „Pluralen Vielfalt“ ergänzt, wodurch eine „gesetzlich vorzugebende positive Ordnung“ abgebildet werden. Die Inhalte aus Säule 1 und Säule 2 werden dann im Nutzerinterface zusammengeführt. Kaiser/Reiling, in: Unger/v. Ungern-Sternberg, Demokratie und künstliche Intelligenz, S. 85 (106) deuten die Möglichkeit von technischen Lösungen an, etwa einem 
zumindest ein Hebel, um die noch sehr abstrakten Vorgaben angebotsspezifisch zu konkretisieren. ${ }^{1996} \mathrm{Ob}$ den Regelungen insgesamt lediglich eine Symbolkraft zukommt, bleibt daher abzuwarten. ${ }^{1997}$ Die Länder gehen aber einen vertretbaren Weg. ${ }^{1998}$

\section{bb. Angemessener Ausgleich zwischen Schutzbedürfnissen und Abwehrrechten (4. Stufe)}

Hinsichtlich des angemessenen Ausgleichs kann auf vieles zuvor Genannte verwiesen werden. Die allgemeine Intermediärsregulierung hat sich jedenfalls den spezifischen Gefahren der digitalen Medienintermediäre behutsam genähert, ${ }^{1999}$ indem ein Kompromiss zwischen zumindest grobmaschiger Kenntnis der Funktionsweise der Algorithmen ${ }^{2000}$ und den unternehmerischen Interessen geschaffen wird. Damit wird zumindest der Schleier, hinter dem die „Black Boxes“ agieren, ${ }^{2001}$ teilweise gelüftet, ohne dass damit erhebliche Beeinträchtigungen der unternehmerischen Freiheit der Betreiber einhergehen. ${ }^{2002}$ Und zudem werden keine neuen (Missbrauchs-)Gefahren durch den Staat begründet. Angesichts der unterschiedlichen Angebote macht es durchaus Sinn, die genaue Frage darüber, was zentrale Kriterien sind, auch gesetzgeberisch zunächst offen zu lassen und

„opposing viewpoint button“ oder eines „serendipity buttons“, m.w.N. Dieser Vorschlag findet sich auch bei Sunstein, \#republic, S. $231 \mathrm{f}$. Und zum Ansatz einer Must-Carry-Regelung und denkbaren unterschiedlichen Umsetzungen siehe etwa Mitsch, DVBl. 2019, 811 (817f.). Daneben soll Transparenz nicht in einem allgemeinen Sinne hergestellt werden, sondern mit Bezug auf den einzelnen konkret angezeigten Inhalt, In diese Richtung unter Bezugnahme auf lebensmittelrechtliche Vorgaben Heldt, CR 2018, 494 (498). Und Schwartmann/Hermann/Müblenbeck, Transparenz bei Medienintermediären, S. 25 f., 125 ff., sehen dies schon als vom Regelungsgegenstand der Transparenzpflicht umfasst an. Damit wäre aber auch ein deutlicher Mehraufwand für die Netzwerkbetreiber verbunden, wenn hinsichtlich jedes einzelnen Inhaltes und seiner Position im Ranking maßgebliche Informationen transparent gemacht werden müssten. Und teilweise wird gar die Forderung nach einem öffentlich-rechtlichen Angebot laut, vgl. Kaiser/Reiling, in: Unger/v. Ungern-Sternberg, Demokratie und künstliche Intelligenz, S. 85 (109). Woanders wird etwa die Stärkung der Nutzerentscheidung betont, der auswählen können sollte, zu welchem Grad er Personalisierung zulassen will, vgl. etwa Droguel u.a., Media Perspektiven 2020, 139 (144). Ausführlich zu zukünftigen Entwicklungen auch Heidtke, Meinungsbildung und Medienintermediäre, S. 355 ff.

1996 Vgl. Schwartmann/Hermann/Müblenbeck, Transparenz von Medienintermediären, S. 149.

1997 So auch Liesem, UFITA 2019, 395 (417) zum Diskussionsentwurf 2019.

1998 Mit Blick auf den Aspekt der Meinungsvielfalt halten Schwartmann/Hermann/Mühlenbeck, Transparenz bei Medienintermediären, S. 155 f., die Vorgaben allerdings für nicht hinreichend.

1999 Liesem, K\&R 2019, 687 (691). Cornils, ZUM 2019, 89 (100) bezeichnet es „als gar nicht einmal so unkluge Zurückhaltung“.

2000 Kellner, Die Regulierung der Meinungsmacht von Internetintermediären, S. 283. Ähnlich auch Fehling/Leymann, AfP 2020, 110 (116).

$2001 \mathrm{Zu}$ der oftmals fehlenden Kenntnis einer algorithmenbasierten Kuratierung von Inhalten vgl. Schulz/Dankert, Die Macht der Informationsintermediäre, S. $42 \mathrm{f}$. mit Verweis auf eine durchgeführte Studie von Eslami/Rickman u.a. Siehe daneben auch die Darstellung und Verweise bei Schmidt u.a., Zur Relevanz von Online-Intermediären für die Meinungsbildung, S. 29.

2002 Kellner, Die Regulierung der Meinungsmacht von Internetintermediären, S. 282. 
erst auf sekundärer Ebene über entsprechende Satzungen und Richtlinien zu konkretisieren. ${ }^{2003}$

Das Diskriminierungsverbot bemüht sich daneben keine allzu strengen Vorgaben, sondern vielmehr die „Diskriminierung“ von sachlichen Gründen abhängig zu machen. Zwar begrenzt man damit auch die „Programmfreiheit“ der Betreiber, hinsichtlich der Auswahl weitergeleiteter Inhalte. ${ }^{2004}$ Hierbei ist aber einerseits zu berücksichtigen, dass sich ihre Dienste primär auf die Weitergabe fremder Inhalte beschränken und sie sich insoweit von herkömmlichem Rundfunk unterscheiden. Und andererseits verlangt das Diskriminierungsverbot zunächst vor allem, dass dies transparent gemacht wird ( $\mathbb{S} 93$ Abs. $1 \mathrm{MStV}),{ }^{2005}$ und erlaubt dies i.Ü., sofern das sachlich gerechtfertigt werden kann. Insoweit erfasst man nur offensichtliche Fälle, in denen die kommunikative Chancengleichheit schon im Ausgangspunkt nicht gewahrt wird. ${ }^{2006}$ Das ist schon deshalb konsequent, weil eine gewisse Selektion und damit Verkürzung der Informationsflut auch aus Sicht der Nutzer wünschenswert und essentieller Bestandteil der Online-Angebote ist. ${ }^{2007}$ Und zeitgleich erkennt man damit auch das grundlegende kommerzielle Interesse der Anbieter an, Dienste nutzerorientiert anzubieten und dies über die Vermarktung von (personalisierten) Werbeflächen zu refinanzieren. ${ }^{2008}$ Die Tendenz einer homophilen Informationsvermittlung ist zudem nicht nur online, sondern ebenso offline zu bemerken. Nur resultiert sie offline aus einer bewussten Entscheidung für bestimmte Medien, die zumindest eine gewisse Gewähr für die Einhaltung redaktionell-journalistischer Sorgfalt bieten. Online wird durch die personalisierte Kuratierung, aber eine Gefahr einer externen Verzerrung geschaffen. ${ }^{2009}$ Dieser Besonderheit wird man durch das an die Transparenzvorgaben anknüpfende Diskriminierungsverbot durchaus gerecht. Insoweit versucht man jedenfalls auch der Informationsasymmetrie zwischen Nutzern und Betreibern zu begegnen und so die Autonomie der Nutzer zu stärken ${ }^{2010}$ und

2003 Liesem, K\&R 2019, 687 (691) weist daraufhin, dass die normative Vorgabe bestimmter zentraler Kriterien zu einer Beeinträchtigung der Rechte der Netzwerkbetreiber führt. Diesen Umsetzungsspielraum befürwortet etwa auch Heldt, CR 2018, 494 (500). Vgl. auch Müller-Terpitz, in: Kühling/Zimmer, Neue Gemeinwohlherausforderungen, S. 177 (192).

2004 So auch Liesem, UFITA 2019, 395 (410).

2005 Vgl. zu diesem Aspekt Kellner, Die Regulierung der Meinungsmacht von Internetintermediären, S. 153.

2006 Denn im Ausgangspunkt gilt für jeden Inhalt, dass die Weitergabe- und Rezeptionswahrscheinlichkeit maßgeblich auch von den Berechnungen anhand der den Algorithmen vorgegebenen Kriterien abhängt. Soweit aber von den Kriterien abgewichen wird, verändert man (negativ) eben diese Wahrscheinlichkeit. Vgl. hierzu auch Liesem, UFITA 2019, 395 (409).

2007 Statt vieler Liesem, UFITA 2019, 395 (410).

2008 Webster, Northwestern Law Review 104 (2010), 593 (605, 606); Heidtke, Meinungsbildung und Medienintermediäre, S. 341. Ähnlich auch Cornils, ZUM 2019, 89 (93); Fehling/Leymann, AfP 2020, 110 (116).

2009 Vgl. Webster, Northwestern Law Review 104 (2010), 593 (605). Hierzu auch Hartl, Suchmaschinen, Algorithmen und Meinungsmacht, S. 71; Heilmann, MMR 2020, 162 (164 f.); Paal, MMR 2018, 567 (570 f.); Liesem, UFITA 2019, 395 (404, 405 f.).

2010 So auch Liesem, K\&R 2019, 687 (691). Ähnlich auch Heilmann, MMR 2020, 162 (165). 
nicht etwa durch „Must-Carry-Regelungen“2011 oder vergleichbare Instrumente den Nutzern Inhalte staatlich zu oktroyieren. ${ }^{2012}$ Angesichts der (noch) unklaren empirischen Lage, des im Wesentlichen auf herkömmliche journalistische Medien ausgerichteten Medienkonsums und den selbstregulativen (vorsichtigen) Bemühungen der Netzwerkbetreiber besteht zudem auch bislang kein Grund, eingriffsintensivere Maßnahmen zu ergreifen. ${ }^{2013}$ Denn immerhin besteht die Gefahr, dass damit von staatlicher Seite auch neue Fehlentwicklungen und Gefahren im Kommunikationsprozess begründet werden. ${ }^{2014}$ Deshalb wahren die allgemeinen Vorgaben für Medienintermediäre (momentan) jedenfalls die Relation zwischen dem verfolgten Schutzzweck und den damit kollidierenden Interessen der Netzwerkbetreiber. ${ }^{2015}$

\section{b. Bewertung des Schutzkonzepts gegen Einflüsse der Meinungsroboter}

Genügt daneben auch das Konzept zum Schutz vor den Risiken der Meinungsroboter den verfassungsrechtlichen Anforderungen?

2011 Zu möglichen Ansätzen solcher Regelungen siehe etwa Mitsch, DVBl. 2019, 811 (817f.).

2012 Denn dadurch zwingt der Staat mittelbar bestimmte Inhalte den Nutzern auf. Und außerdem ist dann zweifelhaft welche Inhalte weiterzugeben sind und ob etwa das „Privileg“ des öffentlich-rechtlichen Rundfunks auch in diesem Sinne Wirkung entfalten soll, kritisch hierzu mit Blick auf eine Must-be-found-Regelung bei Suchmaschinen etwa Hartl, Suchmaschinen, Algorithmen und Meinungsmacht, S. 219 ff. Kritisch mit Blick auf die kommunikative Chancengleichheit Liesem, AfP 2020, 277 (281) und allgemeiner dies., UFITA 2019, 395 (409).

2013 So mit Blick insbesondere auf die bereits selbst ergriffenen Maßnahmen der Netzwerkbetreiber Kaiser/Reiling, in: Unger/v. Ungern-Sternberg, Demokratie und künstliche Intelligenz, S. 85 (107). Mit Blick auf eine geforderte, strengere Neutralitätspflicht Fehling/Leymann, AfP 2020, 110 (116) und insgesamt zur Regulierung, S. 118. Anders Schwartmann/Hermann/Mühlenbeck, Transparenz bei Medienintermediären, S. 155 die die ergriffenen Maßnahmen aus Sicht der Meinungsvielfalt für nicht hinreichend erachten.

2014 So auch Cornils, ZUM 2019, 89 (103). Das gilt insbesondere mit Blick auf etwaige Must-carry-Regelungen oder sonstige Privilegierungen, weil damit wiederrum die kommunikative Chancengleichheit tangiert wäre und bestimmte (öffentlich-rechtliche) Angebote zulasten anderer medialer Angebote bevorzugt werden würden, kritisch hierzu etwa Liesem, AfP 2020, 277 (281) oder Hartl, Suchmaschinen, Algorithmen und Meinungsmacht, S. $219 \mathrm{ff}$.

2015 So letztlich auch Liesem, K\&R 2019, 687 (691); dies., AfP 2020, 277 (283). In diese Richtung auch v. Ungern-Sternberg, in: Unger/dies., Demokratie und künstliche Intelligenz, S. 3 (31). Mit Blick auf Transparenzvorgaben i.E. auch Heldt, CR 2018, 494 (499); wohl auch Heilmann, MMR 2020, 162 8165). Zu beidem auch Müller-Terpitz, in: Kühling/Zimmer, Neue Gemeinwohlherausforderungen, S. 177 (192 ff.), der in beiden Fällen auf die wegen der offen formulierten Normen mögliche grundrechtskonforme Anwendung hinweist. Eine Transparenzpflicht jedenfalls befürwortend Kaiser/ Reiling, in: Unger/v. Ungern-Sternberg, Demokratie und künstliche Intelligenz, S. 85 (110), aber kritisch im Falle von Facebook zu einem Diskriminierungsverbot, S. 108. 


\section{aa. Ausgleich zwischen „Effektivität“ und „Erforderlichkeit“ (3. Stufe)}

Aus Effektivitätsgründen überzeugt auch hier der Transparenzansatz. ${ }^{2016}$ Die Aufdeckung des wesentlichen Charakters entsprechender Äußerungen bzw. Profile ist prinzipiell dazu geeignet, unreflektierte heuristische Denkprozesse zu unterbinden und wesentliche Informationen für die Einordnung und Bewertung der Profile, ihres Verhaltens und veröffentlichter Inhalte bereitzustellen - jedenfalls sofern man nicht die Informationen ignoriert oder ihnen keinerlei Gewicht beimisst. Man ermöglicht so eine unverfälschte und damit freie Rezeption und zugleich geht davon eine Sensibilisierungswirkung für potentielle Manipulationsgefahren aus. ${ }^{2017}$ Auch wenn $\mathbb{1} 18$ Abs. 3 MStV wegen der fehlenden Kennzeichnungsbereitschaft ${ }^{2018}$ in seiner eigenständigen Wirksamkeit beschränkt sein mag, ${ }^{2019}$ so erlangt er doch auch dadurch an Bedeutung, dass er maßgebliche Richtschnur für die Sorgetragungspflicht der Netzwerkbetreiber ist und insoweit eine sekundäre Bedeutung erlangt.

Ursprünglich waren auch bundes- und hier vor allem strafrechtliche Ansätze in der Diskussion, gleichwohl ist zu begrüßen, dass diese Ansätze nicht weiter verfolgt wurden. ${ }^{2020}$ Das gilt zunächst für die Forderung eines digitalen Hausfriedensbruchs, der jede „unbefugte Nutzung informationstechnischer Systeme erfassen sollte, ${ }^{2021}$ und damit - wie das US-amerikanische Pendant $-^{2022} \mathrm{zu}$ einem „Vertragsstrafrecht“ führen kann, weil jeder Verstoß gegen Nutzungsbedingungen potentiell strafbar wäre. ${ }^{2023}$ Aber auch ein strafrechtliches Verbot des Einsat-

2016 So auch - allerdings eher aus ethischen und nicht aus rechtlichen Gründen - Liesem, in: Litschka/ Krainer, Der Mensch im digitalen Zeitalter, S. 183 (194).

2017 Liesem, in: Litschka/Krainer, Der Mensch im digitalen Zeitalter, S. 183 (194); Cornils/dies., Stellungnahme zum Diskussionsentwurf eines Medienstaatsvertrages, S. 21. In diese Richtung auch Müller-Terpitz, in: Kühling/Zimmer, Neue Gemeinwohlherausforderungen, S. 177 (191).

2018 In diese Richtung etwa Cornils, ZRP 2020, 60 (62). Vgl. auch Daniel/Cappiello/Ben, IEEE Internet Computing online 2019, 40 (45).

2019 Kritisch insoweit etwa Verband der Internetwirtschaft, Stellungnahme zum MStV, LT-NRW Stellungnahme 17/2784, S. 5. v. Ungern-Sternberg, in: Unger/dies., Demokratie und künstliche Intelligenz, S. 3 (17) weist in diesem Zusammenhang darauf hin, ,dass die Suche nach dem normativ Richtigen oder Wünschenswerten nicht von Vornherein diejenigen Regelungsoptionen ausschließen darf, die - mutmaßlich - ohnehin nicht befolgt würden oder leicht zu umgehen seien. “.

2020 Kritisch zu einem generellen Verbot etwa Schröder, DVBl. 2018, 465 (470). Anders etwa der Vorschlag im US-Senate Bill 3127, 115 ${ }^{\text {th }}$ Congress aus dem Jahr 2018 (sog. Bot Disclosure and Accountability Act) unter Sec. 325 (S. 11 des Vorschlags).

2021 Hierzu BR-Drs. 338/16.

2022 Sog. Computer Fraud and Abuse Act, 18 U.S.C. $\mathbb{\$} 1030$ (A) (2) (c). Hierzu und insbesondere auch zu der Frage, ob Verstöße gegen Nutzungsbedingungen erfasst sind Constant, Tulane Journal of Technology \& IP Vol. 16 (2013), S. 231 ff. Diese Frage ist zurzeit auch beim US Supreme Court in der Rechtssache Van Buren v. United States, Nr. 19-783 anhängig.

2023 Kritisch wegen der Reichweite des Tatbestandes zu Recht auch Marvany, ZRP 2016, 221 (223) und Bär, in: Wabnitz/Janovsky/Schmitt, Handbuch Wirtschafts- und Steuerstrafrecht, Kap. 15 Rn. 100h. Zudem lässt sich auch hier - jedenfalls sofern man die oben aufgezeigten strafrechtlichen Implikationen (wie hier vertreten) zugrunde legt - an der Notwendigkeit und einer bestehenden Schutzlücke zweifeln, ebenso Marvany, a.a.O. (222 f.) und Gercke, ZUM 2019, 798 (801). 
zes selbst würde nicht nur die schon mehrfach angesprochene Differenzierung zwischen den Einsatzarten vermissen lassen. ${ }^{2024}$ Zudem würden damit auch die Inhalte selbst - wenn auch nur mittelbar - verhindert werden. Angesichts der noch immer bestehenden Ungewissheit würde also das eingriffsintensivste Mittel gewählt. ${ }^{2025}$ Entscheidender Schwachpunkt eines solchen bundesrechtlichen Ansatzes ist zudem die fehlende Möglichkeit einer unionsrechtskonformen Inanspruchnahme der Netzwerkbetreiber. Das Privileg aus Art. 1 Abs. 6 ECRL steht dem Bundesgesetzgeber im innerdeutschen föderalen Kompetenzgeflecht gerade nicht zu. ${ }^{2026}$ Insoweit müsste er dann - wie auch beim NetzDG i.Ü. - auf ein Beschwerdemanagement zurückgreifen. Dass dies dem Problem der Meinungsroboter kaum gerecht wird, wurde schon mehrfach thematisiert. ${ }^{2027}$

Gleichwohl lässt auch der MStV möglicherweise entscheidende Fragen offen, die an der Effektivität erhebliche Zweifel wecken können. Das gilt nicht nur mit Blick auf die fehlende Berücksichtigung akkumulierter Netzwerkdaten (I.), sondern auch hinsichtlich einer fehlenden präventiven Wirkung (II.), einer möglicherweise fehlerhaften Anreizwirkung (III.) und dem begrenzten Anwendungsbereich selbst (IV.)

\section{(I.) Fehlende Berücksichtigung akkumulierter Netzwerkdaten}

Während der MStV zwar die Transparenz für die Nutzer in direkten Interaktionsszenarien stärkt, gilt das nicht dort, wo es um die Präsentation akkumulierter statistischer Daten geht (Likes, Abonnenten-Zahlen u.ä. Parameter), ${ }^{2028}$ die aber entscheidende Orientierungspunkte sind. ${ }^{2029}$ Entsprechendes gilt auch für die Frage, inwieweit der Algorithmus diese akkumulierten Daten für das Ranking der Inhalte verwendet. Hier bleibt die Entscheidung allein den Netzwerkbetreibern überlassen, die entsprechende Nutzerprofile gesondert anzeigen, nicht berücksichtigen oder ganz allgemein löschen müssten. Ein solches Vorgehen wäre zwar betreiberseitig ohne Weiteres möglich, kann aber auf Grundlage der bisherigen Regeln des MStV nicht gefordert werden. Insoweit besteht die potentielle Gefahr, dass zumindest Teilwirkungen der Meinungsroboter immer noch bestehen bleiben könnten, sofern die Netzwerkbetreiber nicht von den vertraglichen Sperrund Löschrechten (hierzu oben C.I.2.a) Gebrauch machen oder anderweitig

2024 So auch Wolf, WRP 2019, 440 (442 f.).

2025 Ein solches Verbot lehnen deshalb auch Fehling/Leymann, AfP 2020, 110 Rn. 37; Milker, InTeR 2017, 199 (205) und Schröder, DVBl. 2018, 465 (472) ab.

2026 Grundlegend zur Kompetenzverteilung BVerfGE 12, 205 - 1. Rundfunkentscheidung.

2027 Siehe hierzu u.a. oben, C.II.2.c.aa(II.)(1.).

2028 Auch Lamo/Calo, UCLA Law Review 66 (2019), 988 (1017) weisen darauf hin, dass derartige Einflüsse eine Transparenzregelung deshalb „underinclusive“ machen können.

2029 Kaerlein, in: Hug/Pallaver, Talk with the Bots, S. 17 (23) macht diese Struktur der Netzwerke auch als eigentliches Problem der Bots aus frei unter dem Slogan „Don't blame the bots, blame the platforms! [...]". 
Vorkehrungen treffen. Die Informationsasymmetrie, der durch die Regelungen begegnet werden soll, bleibt also partiell aufrechterhalten. ${ }^{2030}$ Eine Erweiterung der Verpflichtung würde aber auch die Netzwerkbetreiber noch stärker in der Darstellung ihres Angebotes beschneiden, weil nicht nur im Hintergrund ablaufende proaktive Überwachungsaktivitäten auferlegt werden, sondern auch konkrete Vorgaben für das Userinterface und die Darstellung akkumulierter Daten gegeben werden müssten. Die jeweilige Oberfläche und die auf diesen Daten beruhende Netzwerkstruktur ist nicht nur wesentlicher Bestandteil der nutzerfreundlichen Netzwerkoberfläche, sondern gerade auch für den wirtschaftlichen Erfolg mitverantwortlich, ${ }^{2031}$ sodass jedenfalls die Erweiterung auf die akkumulierten Daten nicht offensichtlich angezeigt ist. Zudem dürfte hier auch das betreiberseitige Interesse durchschlagen, gekennzeichnete Profile in solchen statistischen Aussagen unberücksichtigt zu lassen. ${ }^{2032}$ Und zuletzt besteht mit der Satzungs- und Richtlinienbefugnis der Landesmedienanstalten die Möglichkeit, die Anforderungen gerade auch mit Blick auf diese Situationen zu konkretisieren.

\section{(II.) Fehlende präventive Wirkung?}

Daneben lässt sich kritisch darauf hinweisen, dass auf den Einsatz von Meinungsrobotern oftmals nur ex post reagiert wird. ${ }^{2033}$ Denn mit der Erkennung automatisierten Verhaltens ist eine zeitliche Verzögerung verbunden, die die Wirkung - in einem begrenzten Umfang - zunächst ermöglicht. Und zudem wird den Nutzern die Bürde auferlegt, die transparenten Informationen zu verstehen und daraus die richtigen Schlüsse zu ziehen. ${ }^{2034}$ Wie erfolgreich das ist, hängt nicht nur von dem Design einer entsprechenden Kennzeichnung selbst ab, sondern auch davon, inwieweit dieses Design erklärt und eingeordnet werden kann. Hier bedarf es ggf. spezifischer Angebote, die diese Fähigkeit vermitteln. Allerdings kann eine präventive Wirkung nur dadurch erzielt werden, dass Zugriffsrechte auf die API erheblich beschränkt werden oder eine Registrierung fortan gewisse Erfordernisse wie etwa eine Identitätsprüfung erfüllen müsste. Dadurch greift man aber auch wesentlich stärker in die unternehmerische Freiheit ein, über Design und Zugangsfragen selbstständig zu entscheiden. Die API sind essentiell für die Anknüpfung externer Dienste und auch deshalb zumindest unter Art. 12

2030 Schon oben wurde aufgezeigt, dass in derlei unübersichtlichen Situationen eine tiefergehende Überprüfung des akkumulierten Ergebnisses nur selten erfolgen wird und auch wenig erfolgversprechend sein wird, B.II.1.c.aa(II.).

2031 Vgl. Oehmer/Pedrazzi, UFITA 2020, 7 (30).

2032 Das Hauptproblem liegt ja hier eher auf der primären Ebene, Betreiber überhaupt zu wirksamen Erkennungsmaßnahmen zu verpflichten, aus denen dann weitere konkrete Maßnahmen (freiwillig) hervorgehen können. Das wird aber mit $\$ 93$ Abs. 4 MStV ermöglicht.

2033 Vgl. Löber/Roßnagel, MMR 2019, 493 (498).

2034 Vgl. Hines, Houston Law Review 57 (2019), 405 (415) m.w.N. 
Abs. 1 GG besonders geschützt. ${ }^{2035}$ Eine Identifizierungspflicht - wie etwa die teilweise bereits geforderte Klarnamenpflicht in sozialen Netzwerken $-{ }^{2036}$ kann nicht nur zu Einschüchterungseffekten hinsichtlich der Meinungsäußerungsfreiheit führen, sondern dürfte regelmäßig auch Nutzer von der weiteren Inanspruchnahme betroffener Dienste abhalten. ${ }^{2037}$ Und eine entscheidende Schwachstelle haben diese Ansätze auch: Anders als bei einer Kennzeichnung erlangen die Nutzer keine Kenntnis von dem Phänomen und werden auch nicht hierfür sensibilisiert.

Tendenziell mag diese Wirkung zwar auch dadurch beseitigt werden, dass Betreiber auffällige Konten sperren oder löschen. Auch dann ist aber nicht sichergestellt, dass eine Sensibilisierungswirkung nach außen tritt, denn nach $\$ 96 \mathrm{MStV}$ besteht allein gegenüber der Landesmedienanstalt eine mittelbare Berichtspflicht. Die notwendige Transparenz in derlei Fällen ließe sich dann nur durch eine Berichtspflicht, die in Anlehnung u.a. ${ }^{2038}$ an $\mathbb{2}$ Abs. 1 NetzDG Betreiber zur Veröffentlichung eines Berichts über ihre Sorgetragungsmaßnahmen verpflichtet, der leicht erkennbar und unmittelbar erreichbar bereitzuhalten wäre. ${ }^{2039}$ Das ist aber dennoch kein durchschlagendes Effektivitätsproblem. Zum einen wird diese Lage durch die Selbstregulierung der Netzwerkbetreiber denn durch die gesetzliche Pflicht verursacht. Zum anderen führt das lediglich dazu, dass bei der Evaluation der bestehenden Regelungen und zukünftigen Änderungen dieses Problem mitbedacht werden sollte.

(III.) Faktische Wirksamkeitshemmung durch ökonomisch-rationale Herangehensweise der Betreiber?

Schon beim NetzDG bestand ein Dilemma, weil sich private Betreiber weder die Duldung von Hass-Postings oder anderen Rechtsverletzungen vorwerfen lassen noch als privater Zensor angesehen werden wollen. ${ }^{2040}$ Hier beziehen sich die Überlegungen zwar eher auf abwehrrechtliche Probleme einer überschießenden Sperrung von rechtmäßigen Inhalten. ${ }^{2041}$ Gleichwohl kann beim MStV u.U. der

2035 Hierauf ebenfalls hinweisend Milker, InTeR 2017, 199 (206).

2036 So mit Blick allerdings auf Hass-Kriminalität und im Kontext des NetzDG BR-Drs. 70/20. Vgl. Libertus, ZUM 2018, 20 (22). Das wurde auch auf der Tagung des Instituts für Urheber- und Medienrecht am 28.04.2017 diskutiert, vgl. Grandjean, ZUM 2017, 565 (572).

2037 Kritisch hierzu im Kontext der Meinungsroboter Schröder, DVBl. 2018, 465 (472).

2038 Neben dem NetzDG setzt auch schon der Verhaltenskodex im Kampf um Desinformation (oben, C.I.3.a.bb) auf eine solche Transparenz. Zu den Problemen, ebd. Und auch die geplante „Verordnung zur Verhinderung der Verbreitung von terroristischen Online-Inhalten " sieht in Artikel 18 eine entsprechende Berichterstattungspflicht vor, allerdings geht es auch in diesem Kontext eher um eine mitgliedstaatliche Kontrolle der Anbieter bei Durchführung von proaktiven bzw. spezifischen Maßnahmen, siehe hierzu Art. 18 in der aktuellen Version im Verfahren 2018/0331 (COD).

$2039 \mathrm{Zu}$ einer solchen Berichtspflicht ausführlicher noch unten, 4. Kapitel, A.

2040 Eifert, NJW 2017, 1450 (1452).

2041 Eifert, ebd. 
gegenteilige Effekt ausgemacht werden. Denn während zumindest die „geduldeten " Inhalte nach außen erkennbar bleiben und insoweit jedenfalls öffentlicher Druck bestehen kann, der Betreiber zu einer strengeren Aufsicht veranlasst, sind die automatisierten Profile nicht ohne Weiteres erkennbar. Die hier begründete Dilemmasituation zwischen überschießender Sperrung ${ }^{2042}$ bzw. Kennzeichnung und dem „Laissez-faire“ kann sich also schon deshalb einseitig zugunsten letzterer und damit zulasten der Effektivität verschieben. Das kann vor allem auch dadurch gefördert werden, dass die Anzahl der aktiven Nutzer immerhin auch den Wert eines sozialen Netzwerks mitbestimmt. ${ }^{2043}$ Insoweit kollidiert hier das intrinsische Interesse an der Entfernung von Fake-Accounts mit denen an einer möglichst stabilen Nutzerzahl. Der ordnungsrechtliche Tatbestand steht hier nicht wirklich absichernd im Wege, weil er eben nur eingreift, wenn Betreiber völlig untätig bleiben oder zumutbare Vorkehrungen nicht treffen. Gleichwohl resultiert daraus nicht per se ein Konflikt mit der Effektivität, weil eine gewisse Vorsicht bereits wegen kollidierender Interessen bei fehlerhaften Einordnungen durch Erkennungssoftware geboten ist. Und zudem ist es gerade Aufgabe der ZKA im Rahmen ihrer aufsichtsrechtlichen Befugnisse die Betreiber zu kontrollieren und ggf. spezifische Vorgaben zu machen.

(IV.) Faktische Wirksamkeitshemmung durch das Herkunftslandprinzip des $\mathbb{} 1$ Abs. $7 \mathrm{MStV}$ ?

Ein letztes Problem könnte in $\mathbb{} 1$ Abs. 7 MStV selbst zu erblicken sein, denn das Herkunftslandprinzip führt dazu, dass $\mathbb{} 18$ Abs. $3 \mathrm{MStV}$ sich nur auf Telemedienanbieter mit Niederlassung im Inland bezieht, was sich dann auch bei der Sorgetragungspflicht der Betreiber fortsetzt. Insoweit sind nur Meinungsroboter erfasst, die von Deutschland aus betrieben werden. Auf den ersten Blick ist das jedenfalls überzeugend, wenn man davon ausgeht, dass solche Telemedien schon wegen ihrer vermutlich überwiegend deutschsprachigen Ausrichtung bedeutsamer für den Willensbildungsprozess sind. Rein faktisch ist es aber durchaus möglich, dass auch Meinungsroboter aus dem Ausland agieren und einen entsprechenden Einfluss im Inland ausüben. ${ }^{2044}$ Zumindest mit Blick auf entsprechende Angebote aus Drittstaaten wäre eine Erstreckung vergleichsweise unproblema-

2042 Dazu ausführlicher noch sogleich unter C.II.3.b.bb(II.)(3.).

2043 Im Falle des NetzDG tritt Nolte, ZUM 2017, 552 (556), auch dem Einwand des wirtschaftlichen Interesses an Äußerungen im Graubereich deshalb entgegen, weil „ein einzelner Inhalt keinen nennenswerten wirtschaftlichen Wert [hat].“ In diese Richtung auch Jobst, NJW 2020, 11 (14). Vgl. hierzu auch König, AcP 219 (2019), 611 (637). Und zum Wert der Nutzer siehe etwa Keller/Klinger, Political Communication 36 (2019), 171. (178) oder Bock, Die Übertragbarkeit der Kommunikationsfreiheiten des Artikel 5 GG auf das Internet, S. 197.

2044 Vgl. hierzu auch Bock, die Übertragbarkeit der Kommunikationsfreiheiten des Art. 5 GG auf das Internet, S. 297. Kritisch deshalb mit Blick auf die Möglichkeit einer Erstreckung des rechtlichen Rahmens auf im Ausland ansässige Anbieter auch Milker, ZUM 2017, 216 (221). 
tisch ${ }^{2045}$ und vielleicht sogar ratsam gewesen angesichts vielfältiger Einflussbemühungen der russischen Internet Research Agency in politisch brisanten Entscheidungen. ${ }^{2046}$ Die Lösung hätte hier ein punktuelles Marktortprinzip sein können. Ähnlich wie bei $\mathbb{S} 1$ Abs. $8 \mathrm{MStV}$ wäre es dann entscheidend, ob der ausländische Dienst für die Nutzung in Deutschland bestimmt ist. Maßgeblich wäre insoweit eine Gesamtschau, wobei vor allem der sprachlichen Ausrichtung Bedeutung zukäme.

Allerdings ist nicht per se erwiesen, dass das Fehlen eines punktuellen Marktortprinzips den Schutz lückenhaft macht. ${ }^{2047}$ Ohnehin könnte die Notwendigkeit eines solchen auch rein faktisch zu relativieren sein: Die Betreiber könnten im Verhältnis zu deutschen Nutzern - die Kennzeichnungspflicht (freiwillig) ausdehnen, weil automatisierte Software ohnehin den gesamten Datenstrom überwachen muss. Dadurch würden Betreiber auch eigenständig das Vertrauen in die Integrität der Dienste steigern. ${ }^{2048}$ Zudem ist die Suche nach dem tatsächlichen Ursprung der Inhalte aufwendiger als die Orientierung an den konkreten Profiloder Seiteninformationen. Geht man aber grundsätzlich davon aus, dass Meinungsroboter den Eindruck eines echten und damit in unserem Falle deutschen Nutzers erwecken wollen, werden auch entsprechende Profilinformationen eher einen inländischen Ursprung nahelegen, sodass rein faktisch nicht nur tatsächlich im Inland ansässige Nutzer erfasst sein werden.

Das steht dann letztlich auch in einem unmittelbaren Zusammenhang mit der abwehrrechtlichen Perspektive. Unabhängig von der Frage, ob ein punktuelles Martkortprinzip nicht auch über Art. 1 Abs. 6 E-Commerce-RL europarechtskonform eingeführt werden könnte, führt es zu intensiveren Beeinträchtigungen der unternehmerischen Freiheit. Denn über die Erkennung automatisierter Profile hinaus, müssten dann auch solche Profile ausfindig gemacht werden, die im Ausland betrieben werden, aber für die Nutzung im Inland bestimmt sind. Damit drohen aber auch neue Ungenauigkeiten abseits der ohnehin schon schwierigen Abgrenzung von automatisierten und originären Inhalten. Insoweit ist es mit Blick auf die unternehmerische Freiheit der Betreiber aber auch mit Blick auf kollidierende Interessen fälschlich betroffener Nutzer verständlich, dass eine solche Ausweitung unterblieben ist.

2045 Vgl. Ferreau, in: Spindler/Schuster, Elektronische Medien, Erster Teil B. Rn. 142.

2046 Zur US-amerikanischen Präsidentschaftswahl siehe vor allem Mueller, Report on the Investigation into Russian Interference in the 2016 Presidential Election. Eine entsprechende (nicht-öffentliche) Empfehlung hat wohl auch die Länderarbeitsgruppe „Social Bots“ unterbreitet, vgl. SächsLT-Drs. 6/12131, S. 2 .

2047 Vgl. hierzu auch mit Blick auf eine rein nationale Ausweispflicht etwa Bock, Die Übertragbarkeit der Kommunikationsfreiheiten des Art. 5 GG auf das Internet, S. 297.

2048 Das ist etwa eines der Kernanliegen des Facebook-Immune-System mit Blick auf Fake Accounts, vgl. Stein/Chen/Mangla, Facebook Immune System, S. 4. 
(V.) Zwischenergebnis:

Die $\mathbb{S} \int 18$ Abs. 3, 93 Abs. 4 MStV gewähren auch im Kontext der Meinungsroboter einen Mindestschutz durch Transparenz. Trotz bestehender Schutzlücken, die der MStV nicht selbst beantwortet, ist das Schutzkonzept nicht offensichtlich ineffektiv. Die weitere Umsetzung durch die Netzwerkbetreiber mag zwar zunächst allein auf freiwilliger Basis von statten gehen müssen. Gleichwohl besteht immer auch die Möglichkeit, die abstrakten Vorgaben an die Betreiber stärker zu konkretisieren und so auch hinsichtlich des Interfaces spezifischere Vorgaben zu geben $(\mathbb{S} 96 \mathrm{MStV})$. Insofern bleibt es aber abzuwarten und eben zu kontrollieren, welche Maßnahmen Betreiber ergreifen. Auch das fehlende Marktortprinzip führt nicht per se zu einer erheblich verschlechterten Wirksamkeit. Gleichwohl wird die Schutzlücke auf lange Sicht nur durch eine unionsweit einheitliche Regelung geschlossen werden können.

bb. Angemessener Ausgleich zwischen grundrechtlichen Schutzbedürfnissen und grundrechtlichen Abwehrrechten (4. Stufe)

Die Beantwortung der Frage, ob die Regelungen einen angemessenen Ausgleich zwischen den kollidierenden Interessen schaffen, wirft bei $\mathbb{} 18$ Abs. $3 \mathrm{MStV}$ nicht solche Schwierigkeiten auf, wie sie bei der Sorgetragungspflicht der Betreiber bestehen, was schon daran liegt, dass ein vor allem bipolares Verhältnis (Boturheber - Nutzer) sich dort in ein mehrpoliges Verhältnis wandelt und insoweit auch anderweitige grundrechtliche Interessen zu berücksichtigen sind.

\section{(I.) Kennzeichnungspflicht des $\mathbb{1 8}$ Abs. $3 \mathrm{MStV}$}

Die Kennzeichnungspflicht selbst schafft einen angemessenen Ausgleich zwischen dem aus der Informationsasymmetrie stammenden Transparenzinteresse und insbesondere der Meinungsfreiheit der Urheber. ${ }^{2049}$ Das liegt maßgeblich auch daran, dass die beschränkende Wirkung für letztere zu relativieren ist. Zum einen deshalb, weil der Meinungsfreiheit nicht derselbe Wert beizumessen ist wie in normalen Äußerungskonstellationen. Schon in der Diskussion um den Schutz anonymer Äußerung, die durch das spickmich-Urteil des BGH ausgelöst wurde, ${ }^{2050}$ wird argumentiert, dass anonyme Äußerungen nur dort einen gleichwertigen

2049 So i.E. auch Steinebach u.a., Desinformation aufdecken und bekämpfen, S. 185.

2050 Dort hatte der BGH betont, dass Art. 5 Abs. 1 S. 1 GG sehr wohl auch anonyme Äußerungen erfasse. Ein von offenen Äußerungen abweichendes Gewicht wurde ihnen nicht zugesprochen, sodass u.a. deswegen im Ergebnis die Löschungs- und Beseitigungsansprüche der klagenden Lehrerin, die eine Persönlichkeitsverletzung geltend machte, nach Ansicht des Senates nicht bestanden haben, BGH MMR 2009, 608 Rn. 36ff, insbes. Rn. 38. 
Schutz genießen, wo entweder die Anonymität gerade zwingende Voraussetzung dafür ist, dass Aussagen überhaupt getätigt werden, ${ }^{2051}$ oder wo keine „objektiven Belange“ der anonymen Äußerung gegenüber stehen. ${ }^{2052}$ Im Übrigen bestünde hingegen kein gleichwertiges Schutzbedürfnis, weil der Äußernde bei anonymer Äußerung zu keinem Zeitpunkt persönlich für die Meinung eintreten und am Meinungsaustausch partizipieren wolle. ${ }^{2053}$ Vielmehr hafte der Äußerung „die Vermutung an, mit der Deckmantelmeinung fehlende Aufrichtigkeit verbergen zu wollen.“ 2054 Auch bestehe ein geringes öffentliches Interesse an anonymen Äußerungen. ${ }^{2055}$ Und letztlich entstehe durch die Anonymität eine erhebliche Missbrauchsgefahr. ${ }^{2056}$ Unabhängig davon, ob ein solcher Ansatz mit Blick auf anonyme Äußerungen richtig ist oder ob es nicht ohnehin eine Frage der Abwägung aller Umstände des Einzelfalles ist, ${ }^{2057}$ inwieweit auch eine anonyme Äußerung den Vorrang der freien Rede genießt, ${ }^{2058}$ liegt der Fall vorliegend ohnehin anders. Die Meinungsroboter dienen gerade nicht demselben Zweck wie andere, mit einer gewissen Täuschungswirkung verbundenen Äußerungsmodalitäten. ${ }^{2059}$ Es geht also nicht um Fälle einer „Neoliberalisierung“ von Kommunikationsräumen angesichts der Risiken, die mit der Namensnennung verbunden sind. Vielmehr geht es in erster Linie um das Erschleichen von Reichweite und Aufmerksamkeit.

2051 So etwa Benreuther, AfP 2011, 218 (219). Grabenwarter, in: Maunz/Dürig, GG, Art. 5 Abs. 1 Rn. 86 sieht das jedenfalls als den unproblematischen Fall einer zulässigen Äußerung an, lässt aber offen, ob anonyme Kommunikation allgemein geschützt ist. Kritisch aber etwa Brodowski, JR 2013, 511 (515).

2052 So und hierzu ausführlicher Benreuther, AfP 2011, 218 (219 f.).

2053 Kritisch hierzu Kersten, JuS 2017, 193 (196); anders auch OLG Hamm ZD 2011, 179 f., dass die Anonymität iRd. Abwägung nicht weiter thematisierte.

2054 Benreuther, AfP 2011, 218 (218, 219).

2055 Das BVerfG scheint in diese Richtung zu tendieren, wenn es ausführt, dass „die mit der Namensnennung regelmäßig verbundene Glaubhaftigkeit und Authentizität der Schilderung auszubleiben droht“, wenn man nicht mit seinem Namen für seine Meinung einsteht, BVerfGE 97, 391 (402) Missbrauchsbezichtigung.

2056 Kübling, NJW 2015, 447 (448).

2057 So eher Brodowski, JR 2013, 511 (515) und Beater, NJ 2019, 365 (371). Kritisch auch Schwadner, ZRP 2019, 207 (208 f.), und, allerdings ohne nähere Begründung, Kersten, JuS 2017, 193 (196).

2058 Denn während bei Bewertungsportalen - wie in der spickmich-Entscheidung - mit entsprechenden Inhalten von Nutzern nicht interagiert werden kann, also dem Betroffenen keine Möglichkeit zum kommunikativen Gegenschlag offensteht, ist dies bei sozialen Netzwerken anders. Insoweit ist die Frage, ob eine Möglichkeit zur Gegenwehr besteht eine Frage von vielen. Und im Falle von spickmich.de muss letztlich auch berücksichtigt werden, dass zumindest bei schlechter Bewertung der Lehrkräfte und bei Verwendung des Klarnamens die Möglichkeit von chilling effects durch das Autoritätsverhältnis im Schulbetrieb bestehen kann (so letztlich auch der BGH MMR 2009, 608 Rn. 38 - spickmich.de). Dort wo die Anonymität ohnehin nur zum Instrument für persönlichkeitsverletzende Äußerungen genutzt wird, dürfte schon der bloße Inhalt nicht den Vorrang genießen. Und das Argument der Missbrauchsgefahr überzeugt jedenfalls insoweit nicht, als dass erstens - seit dem 01.10.2017 - zumindest über $\mathbb{S} \mathbb{S} 14$ Abs. 2, 3 TMG eine mittelbare Identifizierung möglich ist, und zweitens die Verunglimpfung nicht mehr allein ein Phänomen anonymer Kommunikation ist, sondern mittlerweile allgemeines Problem der Netzwerkkommunikation, statt vieler Caspar, ZRP 2015, 233 (235).

2059 Ausführlicher hierzu oben A.I.3.b.aa(I.). 
Damit geht es aber nicht allein um den geistigen Wettbewerb, bei dem das bessere Argument gewinnt.

Zum anderen adressiert der Gesetzgeber genau diesen Aspekt, wenn er lediglich die Offenlegung des technischen Vorgangs verlangt. Insoweit trägt er dem Umstand Rechnung, dass auf sekundärer inhaltlicher Ebene jedenfalls zulässige Äußerungen erfolgen können, die den grundrechtlichen Schutz verdienen. Er beschränkt also nicht den generellen Einsatz von Meinungsrobotern, ${ }^{2060}$ sodass auch eine von der Meinungsfreiheit geschützte automatisierte Äußerung weiterhin möglich bleibt. ${ }^{2061}$ Die Meinung und damit der vermittelte Inhalt als solcher bleibt hiervon vollkommen unberührt. ${ }^{2062}$ Und dort, wo auch der Inhalt problematisch ist, gelten ohnehin die bereits bestehenden allgemeinen Schranken. Allein im internen Vertragsverhältnis zwischen den Betreibern und den Verwendern können sich hiervon abweichende Vorgaben ergeben. ${ }^{2063}$

Sofern daneben abwehrrechtliche Probleme durch die vergleichsweise breite Definition der Meinungsroboter verursacht werden, lässt sich dies durch eine auf das eigentliche regulative Problem der Meinungsroboter beschränkte Auslegung vermeiden. ${ }^{2064}$

Aus inhaltlicher Sicht unterscheidet die Norm - anders als sein kalifornisches Pendant - zwar nicht nach der Absicht des Urhebers und erfasst damit auch demokratiefreundliche bzw. -fördernde Einsätze. Potentielle Beweisprobleme sollen im kalifornischen Recht gegebenenfalls dadurch vermieden werden, dass schon gewisse Indizien die Vermutung der Täuschungsabsicht nahelegen sollen. ${ }^{2065}$ Das hätte auch ein gangbarer Weg für eine deutsche Regelung sein können. Anders als in der Debatte um die grundrechtliche „Beweislast“ bei der Begründung der

2060 Kritisch hierzu, insbesondere zu einem strafrechtlichen Verbot Milker, InteR 2017, 199 (205); Schröder, DVBl. 2018, 465 (477).

2061 Sofern die Betreiber privatautonom darüber hinaus gehen und Nutzerprofile sperren oder löschen, ist das jedenfalls kein grundrechtliches Problem im Verhältnis Staat-Nutzer. Vielmehr entscheiden dies die Betreiber privatautonom.

2062 Lamo/Calo, UCLA Law Review (1009); Stricke, Vanderbilt Journal of Entertainment \& Technology Law (889). Insoweit handelt es sich gerade um eine inhaltsneutrale Regelung, die von Art. 5 Abs. 2 GG durch die „allgemeinen Gesetze“ gefordert wird. Das rein faktisch gegebenenfalls mehr „rechte“ als etwa „linke“ Bots reguliert werden, ist eine (unbeachtliche) Folge, die den neutralen Charakter nicht negieren kann. Denn rein faktisch wird auch der Tatbestand der Volksverhetzung eher rassistische und damit rechtsradikale Ansichten erfassen, ohne dass das aber problematisch wäre.

2063 Zu Erinnerung: Während Facebook den Einsatz von Meinungsrobotern gänzlich verbietet, erlaubt Twitter den Einsatz, soweit der Charakter der Meinungsroboter als solcher auch kenntlich gemacht wird. Siehe hierzu oben C.I.2.a.

2064 Dazu schon ausführlicher oben, C.II.1.b.aa.

2065 Hierzu Stricke, Vanderbilt Journal of Entertainment \& Technology Law 22 (2020), 839 (856f.). Aber Hines, Houston Law Review 57 (2019), 405 (433) spricht sich - auch angesichts der Beweisprobleme einer entsprechenden politisch motivierten Täuschungsabsicht - dafür aus den „political prong“ der Regelung zu streichen und eher den kommerziellen Teil auf die Refinanzierung durch Werbung auszuweiten, um so Verwender davon abzuhalten von falschen politischen Inhalten zu profitieren. 
Schutzbereichsausnahme (hierzu oben A.I.3.b.cc) würde das einfache Recht dann nicht den prinzipiellen Schutz der Meinungsfreiheit in Frage stellen, sondern kollidierende Transparenzinteressen im Zweifel den Vorrang einräumen. ${ }^{2066}$ Eine solche Differenzierung geht zudem auch deshalb fehl, weil das Transparenzbedürfnis infolge der Informationsasymmetrie in beiden Fällen identisch ist. Auch gutartige Meinungsroboter machen sich die geschilderten Verzerrungstendenzen zunutze, sodass sich mit Blick auf die Wirkungsweise kein Unterschied ergibt. ${ }^{2067}$ Darin besteht letztlich auch der Unterschied zum kalifornischen Recht: Dort wird sämtliche automatisierte Kommunikation online von der Kennzeichnungspflicht erfasst. Jeder automatisierte Online-Account (California Business and Professions Code Sec. 17940 [a]) im World Wide Web (ebd. Sec. 17490 [b]) ist zu kennzeichnen, sofern er die Anforderungen von Sec. 17491 (a) erfüllt. Schon wegen dieser erheblichen Ausweitung auf den gesamten Online-Bereich bedarf es des restriktivierenden Merkmals der Täuschungsabsicht hinsichtlich besonders besorgniserregender Einsatzzwecke ${ }^{2068}$, um eine überschießende Regelungswirkung und Beschränkung technischer Innovation einerseits, aber auch zulässiger „Speech“ im Sinne des First Amendements andererseits, ${ }^{2069}$ zu verhindern. ${ }^{2070}$

Freilich hätte zur Abmilderung des Eingriffs der deutschen Regelung überlegt werden können, eine zeitliche Beschränkung der Norm etwa auf Krisensituationen oder Wahlkampfzeiten vorzusehen. ${ }^{2071}$ Allerdings ginge das schon deshalb fehl, weil der Willensprozess stetig verläuft ${ }^{2072}$ und außerhalb vom Wahlkampf gerade nicht ruht. ${ }^{2073}$ Und ohnehin wäre es schwer, abstrakt-generell einen ent-

2066 Damit wird dann also der Ausweg auf Rechtsfolgenseite und nicht schon im Schutzbereich gesucht.

2067 Vgl. hierzu Klaas, in: Möller, Was tun gegen Fake News und Hate Speech?, S. 47 (54), der auch bei dem positiven Einsatz Fragen der „ethischen Vertretbarkeit“ ausmacht, weil auch hier eine Beeinflussung der freien Meinung der Nutzer unter falschen Vorwänden [stattfindet].“.

2068 Stricke, Vanderbilt Journal of Entertainment \& Technology Law 22 (2020), 839, S. 845 spricht von den „most social concerns“.

2069 Hierzu Hines, Houston Law Review 57 (2019), 405 (431f.) und auch J.F. Weaver, Journal of Robotics, Artificial Intelligence \& Law 2018, 431 (433), der darauf hinweist, dass die ursprüngliche Fassung eine solche Restriktion nicht vorsah und insoweit nicht „narrowly tailored“ sei, weshalb es einer gerichtlichen Prüfung am Maßstab der Freedom of Speech nicht standgehalten hätte.

2070 Und auch an der vorgenommenen Restriktion des kalifornischen Gesetzgebers bestehen noch verfassungsrechtliche Zweifel, weil möglicherweise auch andere Gebiete reguliert werden, die einen besonderen Schutz genießen. Lamo/Calo, UCLA Law Review 66 (2019), 988 (1016), weisen hier etwa auf kreative Einsatzzwecke von Bots hin, die gerade die Illusion eines Nutzers erzeugen sollen.

2071 So wie etwa das französische LOI n 2018-1202 du 22 décembre 2018 relative à la lutte contre la manipulation de l'information, Art. L. 163-1 mit Blick auf Transparenzpflichten von Anbietern und Art. L. 163-2 mit Blick auf die Bekämpfung von desinformativen Inhalten. Der Zeitraum ist in beiden Fällen auf die drei Monate vor dem Monat der Wahl und bis zur Stimmabgabe festgelegt. $\mathrm{Zu}$ einer solchen Überlegung etwa Oehmer/Pedrazzi, UFITA 2020, 7 (24).

2072 BVerfGE 8, 51 (68) - 1. Parteispenden-Urteil; 20, 56 (98 f.) - Parteifinanzierung I.

2073 So auch Stricke, Vanderbilt Journal of Entertainment \& Technology Law 22 (2020), 839 (858). 
sprechenden Zeitraum ${ }^{2074}$ oder Kriterien für eine solche Krisensituation festzulegen. $\$ 18$ Abs. $3 \mathrm{MStV}$ stellt also einen sachgerechten Ausgleich her.

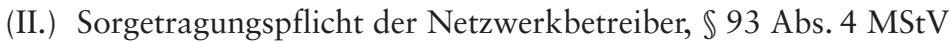

Schwieriger gestaltet sich die Frage, ob es auch aus verfassungsrechtlicher Sicht geboten bzw. vertretbar ist, dass auch die Betreiber in Anspruch genommen werden. Wesentliche grundrechtliche Aspekte wurden jedenfalls zur Konkretisierung der Sorgetragungspflicht bereits aufgezeigt. Nicht geklärt wurde allerdings, ob die Verpflichtung der Netzwerkbetreiber auch in Relation zum Schutzbedarf angemessen wäre.

\section{(1.) Beeinträchtigung der unternehmerischen Freiheit, Art. 12 Abs. 1 GG}

In erster Linie führen die Pflichten zu Beeinträchtigungen der unternehmerischen Freiheit der Betreiber (Art. 12 GG). Dabei wird ihren Interessen allerdings in zweierlei Hinsicht Rechnung getragen: Einerseits durch einen entsprechenden Umsetzungsspielraum, ${ }^{2075}$ andererseits durch eine abgestufte Intensität der Pflichten in Abhängigkeit von der Bedeutung und der Reichweite des Netzwerkes. ${ }^{2076}$ Besonders von großen, marktbeherrschenden Anbietern kann demnach mehr, insbesondere proaktive Maßnahmen, verlangt werden. ${ }^{2077}$ Dieses Erfordernis tritt zu der ohnehin bestehenden Bagatellschwelle des $\$ 91$ Abs. 2 Nr. 1 MStV hinzu.

Zwar hat der EuGH in anderen Konstellationen - meist ging es um Urheberrechtsverletzungen - eine proaktive und automatisierte Überprüfung mit Blick auf die unternehmerische Freiheit (Art.16 EUGrCh) für unverhältnismäßig gehalten, u.a. weil damit eine „[qualifizierte] Beeinträchtigung der unternehmerischen Freiheit des Hosting-Anbieters [...] [einher geht, indem er verpflichtet wäre], ein kompliziertes, kostspieliges, auf Dauer angelegtes und allein auf seine Kosten betriebenes Informatiksystem einzurichten. “2078 Und jüngst hat er dies noch einmal implizit bestätigt, indem er darauf hinwies, dass eine „übermäßige Verpflichtung des Hosting-Anbieters“ jedenfalls dort nicht vorliegt, wo er mithilfe automatisierter Techniken vorgehen kann und wo keine autonome Entschei-

2074 Denn hier geht es ja nicht allein um den Schutz der freien Stimmabgabe, wie es bei den einschlägigen Regelungen der Wahlgesetze der Fall ist, siehe etwa $\$ 35$ Abs. 1 BWG und zu diesem oben, C.I.4.a.cc.

2075 Löber/Roßnagel, MMR 2019, 493 (497).

2076 Vgl. hierzu Müller-Terpitz, ZUM 2020, 365 (372); W. Beck, DVBl. 2020, 253 (257).

2077 Auch das BVerfG betont immerhin in BVerfGE 148, 267 (284) - Stadionverbot, dass aus einer bestimmten tatsächlichen Stellung eine erhöhte rechtliche Verantwortung folgen kann.

2078 EuGH, Urt. v. 24.11.2011 - Rs. C-70/10 = MMR 2012, 174 Rn. 48 - Scarlet Extended SA/Société belge des auteurs, compositeurs et éditeurs SCRL (SABAM); Urt. v. 16.02.2012 - C-360/10 = GRUR 2012, 382 Rn. 46 - SABAM/Netlog. 
dung notwendig wird. ${ }^{2079}$ Angesichts der ohnehin freiwillig ergriffenen Maßnahmen zur automatischen Erkennung bestimmter Manipulationen - Stichwort Facebook Immune System - und den Fortschritten bei der automatisierten Erkennung von bestimmten Mustern, ist fraglich, ob diese Argumentation hier überzeugt. $^{2080}$ Die umfassenden, wenn auch freiwilligen Kontrollen legen jedenfalls nahe, dass es um keine allzu großen finanziellen Hürden geht. Ohnehin werden den Betreibern keine generellen inhaltlichen Überwachungspflichten, sondern allein auf Meinungsroboter begrenzte Pflichten auferlegt. ${ }^{2081}$ Zwar entbehrt das nicht sekundäre, händische Kontrollen durch geschultes Personal. Zu berücksichtigen ist hier anders als in den Fällen, die der EuGH zu entscheiden hatte, dass ein anderes Regelungsmodell ohne Inpflichtnahme der Betreiber zum Scheitern verurteilt ist. ${ }^{2082}$ Ein staatlicher direkter Zugriff auf die Netzwerkdaten geht auch mit intensiveren Eingriffen - nicht nur - in die unternehmerische Freiheit einher. Das und die nur unzureichend durchsetzbare Pflicht des $\mathbb{1 8}$ Abs. 3 MStV machen rein praktisch eine entsprechende Verantwortung der Betreiber notwendig. ${ }^{2083}$ Daran ändern auch die bereits vertraglich vorgesehenen Vorgaben der Netzwerkbetreiber ${ }^{2084}$ - aus bereits genannten Gründen - nichts. Insofern spiegelt sich in der Sorgetragungspflicht eine normierte Spezifizierung der allgemeinen Verantwortung der Betreiber für die eigenen (manipulierbaren) Dienste und ihre gesellschaftlichen Auswirkungen wider. ${ }^{2085}$

Und außerdem folgen aus der Pflicht zu proaktiver Überwachung und Kennzeichnung keine Verantwortung für übersehene Konten oder gar rechtswidrige Inhalte. Denn der Tatbestand des $\mathbb{} 115$ Abs. 1 S. 2 Nr. 42 MStV knüpft allein an die Sorgetragungspflicht und damit an die Frage an, ob der Betreiber überhaupt tätig geworden ist. Im Übrigen gilt für Inhalte weiterhin das Notice-and-Takedown-Prinzip der E-Commerce-RL und des NetzDG.

2079 EuGH, Urt. v. 03.10.2019- Rs. C-18/18 = MMR 2019, 798 Rn. 46 - Glawischnig-Piesczek/Facebook Ireland Ltd.

2080 Löber/Roßnagel, MMR 2019, 493 (497) meinen, dass das Verfahren technisch-organisatorisch umsetzbar und finanziell zumutbar sei.

2081 Dadurch kann aber auch der Erfüllungsaufwand wesentlich geringer sein als etwa im Falle einer umfangreichen Filter- und Löschpflicht von rechtswidrigen Inhalten, die nicht nur äußerungsrechtlicher, sondern auch urheberrechtlicher Art sein können und damit das Spektrum - auch mit Blick auf mögliche Ausnahmen - wesentlich größer ist.

2082 In diese Richtung etwa auch J.F. Weaver, Journal of Robotics, Artificial Intelligence \& Law Vol. 1 (2018), $431(435,436)$.

2083 So auch Fehling/Leymann, AfP 2020, 110 (118). Aus amerikanischer Perspektive wegen einer fehlenden vergleichbaren Pflicht im kalifornischen „Bot Diclosure Act“ (California's Busines and Professions Code Sec. 17940 ff.) gerade kritisch J.F. Weaver, Journal of Robotics, Artificial Intelligence \& Law 2018, 431 (435 f.).

2084 Hierauf zwar hinweisend, allerdings ohne „Verhältnismäßigkeitsbedenken“ Cornils, AfP 2018, 377 (385). Dreyer/Schulz, Stellungnahme zum 2. MStV, S. 16; Liesem, K\&R 2019, 687 (691), halten die Regelung deshalb nicht als „zwingend notwendig“.

2085 Vgl. zu dieser Verantwortung auch W. Beck, DVBl. 2020, 253 (255, 256, 259). 
Anders als in den Fällen des EuGH knüpft die Regelung auch unmittelbar an die kommunikative Verantwortung der Netzwerke ${ }^{2086}$ an und damit an eine Pflicht, die aus dem Betrieb des Dienstes folgt und nicht an eine Pflicht für Inhalte der Nutzer. Besonders deutlich wird das etwa im Falle von Twitter, das durch weitreichende Zugriffe über die API gerade auch die Automatisierung von Interaktionen grundsätzlich ermöglichen will. Aus technischer Sicht wird den Netzwerken auch keine unlösbare und damit unzumutbare Aufgabe gestellt. Zwar besteht ggf. ein Interesse der Betreiber daran, entsprechende Kennzeichnungen nur für deutsche Nutzer anzuzeigen und nicht auch für andere Nutzer. Allerdings können entsprechende HTTP-Requests der Nutzerbrowser, ob auf lokalen oder auf mobilen Geräten, auf eine bestimmte IP-Adresse zurückverfolgt werden. In Abhängigkeit vom Standort der IP-Adresse können dann für die Kennzeichnung erforderliche zusätzliche Daten innerhalb der für den Aufbau und die Darstellung der jeweiligen Seite relevanten HTML-Datei mitgesendet werden. Und im Übrigen ließ sich eine solche Darstellung auch in Abhängigkeit von den jeweiligen Profilinformationen, sofern dort der Wohnort oder das Herkunftsland angegeben ist, verwirklichen. Mit Blick auf die unternehmerische Freiheit wirft die Norm damit keine grundlegenden Bedenken auf.

\section{(2.) Privatisierung staatlicher Kontrollverantwortung?}

Schon das Netzwerkdurchsetzungsgesetz wurde deshalb kritisiert, weil es originär staatliche Rechtsdurchsetzungsaufgaben privatisiere. Ein solcher Einwand wird auch hier laut, weil der Staat sich der Betreiber bedient, um letztlich seiner staatlichen Verantwortung nachzukommen. ${ }^{2087}$ Erfolgt also mit der Sorgetragungspflicht eine (verfassungsrechtlich unzulässige) Privatisierung originär staatlicher Kontrollaufgaben?

Der Einwand einer Privatisierung suggeriert, dass der Staat vollständig in Teilbereichen seine Hoheitsgewalt und Verantwortung auf private Akteure überträgt, so wie es etwa klassischerweise im Bereich der Daseinsvorsorge in den 1990er Jahren erfolgte (Deutsche Post AG, Deutsche Telekom AG). ${ }^{2088}$ Aber schon beim NetzDG ist bereits fraglich, ob man angesichts der bestehenden finalen staatlichen Entscheidungshoheit für Löschungen vor den Zivilgerichten von einer „Privatisierung der Rechtsdurchsetzung“ sprechen kann, ${ }^{2089}$ oder ob

\footnotetext{
2086 Darauf beruft sich auch schon der Bundesgesetzgeber bei Erlass des NetzDG, vgl. nur BT 19/18792, S. 17.

2087 Vgl. etwa Dankert, in: Hoffmann-Riem, Big Data, S. 157 (163).

2088 Zur Privatisierung von Staatsaufgaben und damit einhergehende Fragen ausführlich etwa Kämmerer, Privatisierung.

2089 Vgl. MMR-Aktuell 2017, 387965; Müller-Franken, AfP 2018, 1 (5). Zu Recht kritisch hierzu Kalbhenn/Hemmert-Halswick, MMR 2020, 518 (521); Sabl/Bielzer, ZRP 2020, 2 (3f.); deutlich auch Eifert, NJW 2017, 1450 (1451) und Schiff, MMR 2018, 366 (368).
} 
es nicht vielmehr im Kern um die Frage geht, inwieweit der Staat privaten Unternehmen entsprechende Pflichten auferlegen darf, die sie ökonomisch rational erfüllen werden, was zu einem freiheitsgefährdenden Overblocking führen kann. ${ }^{2090}$ Soweit dies seine Parallele auch im MStV findet, wird hierauf noch sogleich einzugehen sein (cc.).

Mit Blick auf die Sorgetragungspflicht des MStV lässt sich freilich auch kritisieren, dass hier die Betreiber eine staatliche Schutzverantwortung final wahrnehmen müssen. Anders als beim NetzDG ist die Sorgetragungspflicht aber in ein deutlich engeres aufsichtsrechtliches Korsett gekleidet. Der Staat entzieht sich hier also nicht seiner Verantwortung, sondern delegiert lediglich die primäre Verantwortung an die Betreiber und sichert diese sekundär ab. ${ }^{2091}$ Anders ließe sich das wohl auch nicht bewerkstelligen, ohne dass der Staat einen umfangreichen Zugriff auf die Netzwerkdaten erlangt, was seinerseits neue grundrechtliche Spannungen hervorrufen würde. Insoweit geht es vielmehr darum, eine ohnehin bestehende „Macht“ der Betreiber und ergriffene Maßnahmen zu den notwendigen Schutzzwecken zu kanalisieren. ${ }^{2092}$ Von einer unzulässigen Privatisierung staatlicher Aufgaben kann folglich (auch) im Falle des $\$ 93$ Abs. 4 MStV nicht die Rede sein.

\section{(3.) Die Meinungsfreiheit betroffener Nutzer bei falsch-positiven} Kennzeichnungen bzw. Sperrungen

Verfassungsrechtliche Bedenken wirft die Norm aber mit Blick auf die Meinungsfreiheit der Nutzer auf. ${ }^{2093}$ Denn fordert man eine proaktive Überwachung und eigenständige Kennzeichnung auffälliger Konten durch die Netzwerkbetreiber, führt das letztlich dazu, dass ihre Glaubwürdigkeit und damit final ihre Rezeptionswahrscheinlichkeit sinkt. ${ }^{2094}$ Vergleichbares gilt auch in den Fällen, in denen keine Kennzeichnung, sondern gleich eine Sperrung oder Löschung der Profile er-

2090 So eher die Akzentuierung von Dankert, in: Hoffmann-Riem, Big Data, S. 157 (163). Hierauf auch maßgeblich hinweisend Lüdemann, in: Eifert/Gostomzyk, Netzwerkrecht, S. 153 (156ff.). Allerdings knüpft der Bußgeldtatbestand des $\mathbb{} 4$ Abs. 1 Nr. 2 NetzDG nach den Bußgeldleitlinien v. 22.03.2018, S. 7 f. an ein „systemisches Versagen“ an, sodass also allein eine fehlerhafte Bewertung eines Inhaltes nicht per se zu den empfindlichen Bußgeldern führt. Insoweit kann zumindest bezweifelt werden, ob die Anreizstruktur tatsächlich so gewichtig ist, dass in Zweifelsfällen stets eine Löschung vorgenommen wird, ähnlich auch Schiff, MMR 2018, 366 (369). Kritisch hierzu auch Löber/Roßnagel, MMR 2019,71 (73); Fehling/Leymann, AfP 2020, 110 Rn. 18 f. Und die jüngst vorgeschlagenen Änderungen, insbesondere in Hinblick auf ein Gegendarstellungsverfahren und die Ergänzung aufsichtsrechtlicher Befugnisse (siehe hierzu $\mathbb{3} 3$ b, $\mathbb{4} 4 \mathrm{a}$ NetzDG-E, BT-Drs. 19/18792, S. 9,13) sichert noch mal mehr die Meinungsfreiheit betroffener Nutzer ab.

2091 Insoweit gleicht das der etwa von Ibler, in: Maunz/Dürig, GG, Art. 86 Rn. 118 beschriebenen „funktionalen (Erfüllungs-)Privatisierung“".

2092 Vgl. zum NetzDG Eifert, NJW 2017, 1450 (1451); Müller-Franken, AfP 2018, 1 (6).

2093 So auch Löber/Roßnagel, MMR 2019, 493 (497).

2094 Zu Meinungsrobotern Schröder, DVBl. 2018, 465 (472). Vgl. zu anonymen Äußerungen BVerfGE 97, 391 (398) - Missbrauchsbezichtigung. 
folgt. Auch wenn das mit Blick auf originäre Meinungsroboter unproblematisch ist, ${ }^{2095}$ führt das hier deshalb zu Bedenken, weil bei jedem automatisierten und menschlichen Verfahren auch falsch-positive Ergebnisse vorkommen. ${ }^{2096}$ M.a.W. könnten auch normale Nutzer fälschlich als Meinungsroboter eingestuft werden. Konsequenz wäre dann, dass die Betreiber die Inhalte als automatisiert kennzeichnen oder gar den Account sperren oder löschen. Im ersten Fall wird der Äußerung fälschlich eine Modalität attestiert, die unmittelbar für die Wahrnehmung durch Dritte bedeutsam ist und so den „objektiven Wert“ der Äußerung schmälern kann. Im zweiten Fall wird dem Nutzer die Kommunikationsmöglichkeit innerhalb des Netzwerks vollständig entzogen. Wären hierfür also nicht verfahrensrechtliche Absicherungen notwendig, um die Rechte betroffener Nutzer zu wahren?2097

\section{(a.) Vergleichbare Problemlagen}

Das Fehlen entsprechender verfahrensrechtlicher Vorkehrungen ist nicht nur ein Problem von $\mathbb{\int} 93$ Abs. $4 \mathrm{MStV}^{2098}$ Auch der kalifornische Bot Disclosure Act wurde wegen der Gefährdung anonymer Äußerungen kritisiert. Denn wenn staatliche Ermittlungsverfahren wegen eines vermeintlichen Verstoßes eingeleitet werden, dann müsste sich der jeweilige Nutzer zu erkennen geben; ${ }^{2099}$ verfahrensrechtliche Vorkehrungen, die das verhindern, bestehen nicht oder sind kaum denkbar. ${ }^{2100}$ Und auch die Ursprungsfassung des NetzDG hat organisatorische Pflichten für Netzwerkbetreiber Inhalte eingeführt, ohne dabei sicherzustellen, dass der betroffene Nutzer im (Lösch-)Verfahren beteiligt wird. ${ }^{2101}$ Auch hier wird die Meinungsfreiheit in verfahrensrechtlicher Hinsicht nicht hinreichend geschützt. ${ }^{2102}$ Nach $\mathbb{S}$ NetzDG sind der Beschwerdeführer und der betroffene Nutzer nur über die Entscheidung unverzüglich zu informieren und die Entschei-

2095 Dazu schon zuvor, C.II.3.b.bb(I.).

2096 Allgemein zu Filterprozessen etwa Müller-Terpitz, ZUM 2020, 365 (373). Hinsichtlich des Botometers etwa Keller/Klinger, Political Communication Vol. 26 (2019), 171 (186); Varol u.a., in: Proceedings of the $11^{\text {th }}$ International AAAI Conference on Web and Social Media, S. 280 (284). Ähnlich auch Thieltges u.a., Effects of Social Bots on the Iran-Debate on Twitter, S. 3.

2097 Vgl. hierzu im Kontext falsch-positiver Ergebnisse von Filtern auch Leistner, ZUM 2012, 722 (729f.).

2098 Neben den hier angesprochenen Fällen weist Oermann, in: Hofmann-Riem, Big Data - Regulative Herausforderungen, S. 145 (154) hierauf auch im Kontext der gemeinsamen Terrorpropagandadatenbank hin.

2099 So etwa Lamo/Calo, UCLA Law Review 66 (2019), 988 (1009. 1022 ff.).

2100 Lamo/Calo, a.a.O., (1023).

2101 Kritisch deshalb auch Fechter/Arnold, in: Frenz, Handbuch Industrie 4.0, S. 133 (150); Hoven/ Gersdorf, in: Gersdorf/Paal, Informations- u. Medienrecht, $\mathbb{1}$ NetzDG Rn. 6 oder Müller-Franken, AfP 2018, 1 (10). Sabl/Bielzer, ZRP 2020, 2 (4) wollen eine solche Möglichkeit jedenfalls de lege ferenda einführen.

2102 Diese Bedenken teilen u.a. auch Kalscheuer/Hornung, NVwZ 2017, 1721 (1724); Gersdorf, MMR 2017, 439 (446). 
dung ist ihnen gegenüber zu begründen. Das ist deshalb besonders prekär, weil das NetzDG nach verbreiteter Ansicht ein Overblocking durch die Organisationspflichten $(\mathbb{S}$ NetzDG) und Bußgeldtatbestände (insbesondere $\mathbb{S} 4$ Abs. 1 Nr. 2 NetzDG) fördert, indem aus ökonomisch-rationalen Gründen - einerseits um ein hohes Bußgeld zu vermeiden und andererseits um durch Standardisierung den Aufwand möglichst begrenzt zu halten - im Zweifel auch rechtmäßige Inhalte gelöscht werden. ${ }^{2103}$

\section{(b.) Vertragsrechtliches Problem oder grundrechtliche Relevanz?}

Nun geht von $\mathbb{S} 93$ Abs. 4 MStV freilich keine originär staatliche Beeinträchtigung aus, vielmehr bewirken private Akteure die nachteiligen Folgen. ${ }^{2104}$ Insoweit könnte dann die Lösung - so wie in den üblichen Fällen von Accountsperrungen oder Inhaltslöschungen auch $-^{2105}$ allein auf vertraglicher Ebene zu suchen sein. Denn wenn das Profil nicht als automatisiert bzw. als Fake-Account zu klassifizieren wäre, entspräche es den vertraglichen Anforderungen und wäre damit zulässig. Dann bestünde ein vertraglicher Anspruch aus den $\mathbb{S} \mathbb{S} 280$ Abs. 1, 249 Abs. 1 BGB gerichtet auf die Rückgängigmachung einer Kennzeichnung bzw. Wiederherstellung oder Entsperrung des Profils, ${ }^{2106}$ der gerichtlich ohne Weiteres geltend gemacht werden kann. Auch wenn das zumindest mit Blick auf die privatautonome Entscheidung einer Sperrung überzeugend klingt, so begründet im Falle des $\mathbb{S} 93$ Abs. 4 MStV doch der Staat die besondere Gefahrenlage durch Auferlegung von Überwachungspflichten. ${ }^{2107}$ Die Betreiber agieren nicht mehr (allein) im Rahmen ihrer Privatautonomie, sondern (auch) in Erfüllung einer durch den Staat begründeten und ordnungsrechtlich abgesicherten Pflicht. ${ }^{2108}$ In

2103 Zum Aspekt der Standardisierung etwa Eifert, NJW 2017, 1450 (1452). Mit Blick auf die Bußgelder etwa Papier, NJW 2017, 3025 (3030); Nolte, ZUM 2017, 552 (555 f.). Zu beidem Lüdemann, in: Eifert/Gostomzyk, Netzwerkrecht, S. 153 (156 ff.).

2104 Vgl. Lamo/Calo, UCLA Law Review 66 (2019), 988 (1025).

2105 Zur Löschung von Inhalten siehe etwa LG Leipzig, Endurteil v. 12.07.2019 - 08 O 2491/18= GRUR-RS 2019, 38785, Rn. 18; OLG München, GRUR-RR 2020, 174 Rn. 60. Der Restore-Anspruch wird im Falle von inhaltsbedingten Kontensperrungen daneben auch auf die $\mathbb{\$} \$ 241$ Abs. 2 , 1004 BGB gestützt, so etwa LG Karlsruhe, Beschluss vom 12.06.2018 - 11 O 54/18 = BeckRS 2018, 20324 Rn. 7; LG Frankfurt a.M., MMR 2018, 545 Rn. 8. Zumindest im letzten Fall ging es aber um Äußerungen, die auch Rechte Dritter tangierten, so dass die ursprüngliche Löschung im Rahmen der Störerhaftung $(\$ 1004$ BGB) erfolgte. Andernorts wird nur auf den Nutzungsvertrag i.V.m. $\$ 241$ Abs. 2 BGB hingewiesen, so etwa OLG Oldenburg, ZUM-RD 2020, 367 (368); OLG Dresden, MMR 2018, 756 Rn. 8; OLG München, NJW 2018, 3115 (3116), wobei $\$ 241$ Abs. 2 gerade keine Anspruchsgrundlage bereit hält (so auch Jobst, NJW 2020, 11 (15)). Entsprechendes gilt für den Nutzungsvertrag.

2106 Auf einen vertraglichen Anspruch verweisen etwa auch Steinebach u.a., Desinformation aufdecken und bekämpfen, S. 185 .

2107 Vgl. Lüdemann, in: Eifert/Gostomzyk, Netzwerkrecht, S. 153 (158).

2108 Lamo/Calo, UCLA Law Review 66 (2019), 988 (1025). Ebenfalls mit Blick auf die Meinungsroboter und einer Kennzeichnungspflicht, Schröder, DVBl. 2018, 465 (472). Im Kontext des NetzDG wurde das auch mit Blick auf exzessive Löschungen (sog. Overblocking) virulent. Auch dort sah 
ähnlichen Konstellationen hatte aber der EuGH grundrechtliche Bedenken auch wegen der Meinungs- und Informationsfreiheit geäußert. ${ }^{2109}$ Und mit dem Umweg über die vertraglichen Rechte und Pflichten belastet man den jeweiligen Nutzer mit der gerichtlichen Geltendmachung seiner Rechte. ${ }^{2110}$ Mit anderen Worten greift der Staat mit der Auferlegung der Sorgetragungspflicht mittelbarfaktisch in die Meinungsfreiheit fälschlich gekennzeichneter Nutzer ein, sodass das Fehlen einer verfahrensrechtlichen Absicherung auch ein grundrechtliches Problem ist. ${ }^{2111}$ Daran ändert sich auch nichts dadurch, dass der ordnungsrechtliche Tatbestand ( $\$ 115$ Abs. 1 S. 2 Nr. 45 MStV) nur dann eingreift, wenn zumutbare Vorkehrungen überhaupt nicht getroffen wurden und damit ähnlich wie $\mathbb{} 4$ Abs. 1 Nr. 2 NetzDG ein „systemisches Versagen“2112 verlangen. Zwar mag hier ein deutlich größerer wirtschaftlicher Zwang bestehen, möglichst vorsichtig zu agieren, weil nicht „nur“ Inhalte der Nutzer betroffen sind, sondern die Accounts als solche. Diese sind aber auch bedeutsam für die wirtschaftliche Bedeutung der Netzwerke, sodass starke intrinsische Motive bestehen, einer exzessiven Kennzeichnung oder gar Löschung vorzubeugen. ${ }^{2113}$ Gleichwohl ändert das nichts an dem grundsätzlich nicht vermeidbaren Problem der Fehlentscheidungen. Insoweit mag die Gefahr eines „Overblocking“ hier geringer, aber eben nicht auf Null reduziert sein.

man vor allem den Staat in der Pflicht entsprechende Gegenmaßnahmen zu ergreifen, weil er die Anreizstrukturen für ein solches Overblocking geschaffen hatte, hierzu Schiff, MMR 2018, 366 (369f.), der von einem Eingriff in die Meinungsfreiheit spricht; Fechner/Arnhold, in: Frenz, Handbuch Industrie 4.0, S. 133 (150). Siehe auch Müller-Franken, AfP 2018, 1 (11f.). Lüdemann, in: Eifert/Gostomzyk, netzwerkrecht, S. 153 (164) meint, dass sich „ein staatlich initiiertes Verhalten [...] verfassungsrechtlich nun einmal kategorisch von einem Verhalten Privater unterscheidet." Sabl/Bielzer, ZRP 2020, 2 (4) halten es jedenfalls für sinnvoll verpflichtende Vorgaben für ein plattforminternes Anhörungsverfahren festzulegen. Der Änderungsentwurf zum NetzDG sieht deshalb auch nur hinsichtlich der wegen des NetzDG vorgenommenen Löschungen und nicht auch in Fällen, in denen sich die Betreiber selbst zu einer Löschung freiwillig entscheiden, ein entsprechendes Gegenvorstellungsverfahren für betroffene Nutzer vor, BT-Drs. 19/18792, S. 17, 48.

2109 EuGH, Urt. v. 24.11.2011 - Rs. C-70/10 = MMR 2012, 174 Rn. 52 - Scarlet Extended SA/Société belge des auteurs, compositeurs et éditeurs SCRL (SABAM); Urt. v. 16.02.2012 - C-360/10 = GRUR 2012, 382 Rn. 50 - SABAM/Netlog.

2110 Zum NetzDG deshalb zumindest kritisch Müller-Franken, AfP 2018, 1 (13); Nolte, ZUM 2017, 552 (558f.).

2111 In diese Richtung auch Schröder, DVBl. 2018, 465 (472). Vgl. hierzu im Kontext des NetzDG Schiff, MMR 2018, 366 (367f.); Kalscheuer/Hornung, NVwZ 2017, 1721 (1723f.). Müller-Terpitz, ZUM 2020, 365 (371) geht etwa bei einer präventiven Filterpflicht von einer verfassungsrechtlich unzulässigen Zensur aus, weil diese dem Staat zuzurechnen sei. Mit Blick auf die ganz ähnliche Lage beim NetzDG statt vieler Lüdemann, in: Eifert/Gostomzyk, Netzwerkrecht, S. 153 (164).

2112 Siehe hierzu die Bußgeldleitlinien v. 22.03.2018, S. 7 f.

2113 Zu dieser betriebswirtschaftlichen Eigenrationalität vgl. König, AcP 219 (2019), 611 (637). 
(c.) Verfahrensrechtliche Absicherung als vom Übermaßverbot geforderter Schutz?

Aus Sicht des Übermaßverbotes ist dieser Eingriff zwar logische Folge der Abwehr spezifischer Gefahren. Gleichwohl könnte dieser Eingriff schon deshalb nicht erforderlich sein, weil man die grundrechtlichen Interessen der Nutzer verfahrensrechtlich hätte absichern können bzw. müssen. Die prinzipielle Notwendigkeit einer solchen Absicherung hat die Judikatur immerhin auch in rein privatrechtlichen Konstellationen betont. Das gilt etwa mit Blick auf die vollumfängliche Sachaufklärung bei potentiell persönlichkeitsverletzenden Äußerungen. Hier fordert der BGH auch die Anhörung des jeweiligen Urhebers der Äußerung durch den Betreiber. ${ }^{2114}$ Und das BVerfG hat mit Blick auf bundesweit verhängte Stadionverbote ebenfalls (grundsätzlich) eine vorherige Anhörung des Betroffenen gefordert. ${ }^{2115}$ Das muss dann aber erst recht dort gelten, wo der Staat durch die Statuierung entsprechender Pflichten mittelbar Gefahren für Grundrechte der Nutzer schafft. ${ }^{2116}$

Eine davon zu trennende Frage ist, ob eine solche verfahrensrechtliche Absicherung nicht der Effektivität der Regelung im Wege stünde. ${ }^{2117}$ Denn während bei reinen Inhalten die Darlegung für die Bewertung einer Äußerung notwendigen Umstände durchaus hilfreich sein kann, führt allein die bloße Darlegung der Menschlichkeit eines Nutzerprofils kaum weiter. Vielmehr würde das Risiko geschaffen, dass die Kennzeichnungspflicht ( $\mathbb{S} 18$ Abs. 3) bzw. die Sorgetragungspflicht ( $\mathbb{S} 93$ Abs. $4 \mathrm{MStV}$ ) durch die Hintertür umgangen wird. Insoweit müssten spezifische (technische) Hürden geschaffen werden, die regelmäßig nur von echten Nutzern umgangen werden können, wofür insbesondere Captchas in der Vergangenheit eingesetzt wurden. ${ }^{2118}$ Bei Captchas handelt es sich um kleinere Rätsel, etwa ein verzerrter oder visuell veränderter Text bzw. Textzeichen-Zahlen-Kombinationen, die zur Verifikation gelöst werden müssen. ${ }^{2119}$ Allerdings können auch Bots durchaus in der Lage sein, entsprechende Rätsel ohne Weiteres zu lösen; und theoretisch besteht auch die Möglichkeit, dass solche Rätsel von echten Menschen gelöst werden, um sodann den automatisierten Zugriff wieder

2114 BGH, MMR 2012, 124 Rn. 25 ff. - Blog-Eintrag; MMR 2016, 418 LS. 2 und Rn. 24 - Ärztebewertung III. Hierauf im Falle des NetzDG ebenfalls hinweisend Hoven/Gersdorf, in: Gersdorf/Paal, Informations- u. Medienrecht, $\mathbb{} 1$ NetzDG Rn. 6; Koreng, GRUR-Prax 2017, 203 (205).

2115 BVerfGE 148, 267 (285 f.) - Stadionverbot.

2116 Mit Blick auf das NetzDG etwa Müller-Franken, AfP 2018, 1 (13); Schiff, MMR 2018, 366 (368).

2117 Andeutend Schröder, DVBl. 2018, 465 (470).

2118 Auf diese Captchas im Kontext der Meinungsroboter ebenfalls hinweisend Milker, InTeR 2017, 199 (204); Schröder, DVBl. 2018, 465 (472); Gummz/Kar, in: Weber, ÖFIT-Trendschau, Trendthema 44: Social Bots, und Daniel/Cappiello/Ben, IEEE Internet computing online 2019, 40 (45). 
zu ermöglichen. ${ }^{2120}$ Wegen derlei Effektivitätsbedenken ${ }^{2121}$ ist es nicht unvertretbar von der Erforderlichkeit einer Sorgetragungspflicht ohne verfahrensrechtliche Anforderungen auszugehen.

Mit Blick auf die herzustellende praktische Konkordanz überzeugt es aber kaum, dass weder die Regelungen eine verfahrensrechtliche Absicherung explizit vorsehen, noch dass die Länder bei Erlass - jedenfalls ist dies nicht aus der Gesetzesbegründung ersichtlich - sich zumindest Gedanken über dieses Problem gemacht hätten. ${ }^{2122}$ Insoweit wäre auch die Frage, ob eine solche Absicherung überhaupt vorgesehen ist, von der Frage, wie diese konkret aussehen kann, zu trennen. Dass hier nicht auf die Verfahren bei inhaltlichen Löschungen oder Stadionverboten zurückgegriffen werden kann, liegt schon in der Besonderheit der Meinungsroboter begründet.

\section{(d.) Verfassungswidrigkeit des MStV?}

Im Ausgangspunkt gebietet also die abwehrrechtliche Dimension der Grundrechte, dass der Staat verfahrensrechtliche Schutzvorkehrungen vorsieht, um solch nachteilige Folgen für die Nutzer und ein „Overblocking“ seitens der Netzwerkbetreiber zu verhindern. ${ }^{2123} \mathrm{Ob}$ das den MStV aber schon deshalb verfassungswidrig macht, ist zumindest diskutabel. Entscheidend hierfür ist nicht, dass die tatsächliche Belastung der Meinungsfreiheit von dem Umfang falsch-positiver Ergebnisse abhängt. ${ }^{2124}$ Denn bestehende Erkennungssoftware ist nie fehlerfrei und kann dies angesichts der fortschreitenden Simulation menschlichen Verhaltens auch zukünftig nicht werden. Allerdings sieht der MStV eine Nachbesserungsmöglichkeit durch die Satzungs- und Richtlinienbefugnis der Landesmedienanstalten $(\mathbb{S} 96 \mathrm{MStV})$ vor. Sofern diese Kompetenz nur mit Blick auf die Konkretisierung der sie betreffenden Verpflichtungen des dritten Unterabschnitts „Medienintermediäre“ gilt, ließe sich argumentieren, dass verfahrensrechtliche Anforderungen Bestandteil der Sorgetragungspflicht im weiteren Sinne sind und damit \93 Abs. 4 MStV zugeordnet werden können. Damit fänden aber ganz spezifische Vorgaben, die keinerlei Eingang in den MStV oder in die Begründung gefunden haben - über die sich der Gesetzgeber möglicherweise also gar nicht bewusst war -, über die Satzungsbefugnis der Landesmedienanstalten Eingang in den Me-

2120 Auf diese Unsicherheiten ebenfalls hinweisend Schröder, DVBl. 2018, 465 (472).

2121 Zur Ineffektivität von Captchas siehe etwa Boshmaf u.a., Computer Networks 57 (2013), 556 (559). Zu Verbesserungsmöglichkeiten siehe etwa Hitaj u.a., Capture the Bot.

2122 I.E. ebenfalls Schröder, DVBl. 2018, 465 (472). Allgemein mit Blick auf Filtertechnologien und der Notwendigkeit einer verfahrensrechtlichen Absicherung etwa Müller-Terpitz, ZUM 2020, 365 (373). Zur Verbesserung von Captchas auch zur effektiven Bekämpfung von Meinungsroboter siehe etwa Hitaj u.a., Capture the Bot.

2123 Allgemein im Rahmen von Filtertechnologie Müller-Terpitz, ZUM 2020, 365 (373). In diese Richtung zum NetzDG tendenziell auch Müller-Franken, AfP 2018, 1 (13).

2124 Vgl. Leistner, ZUM 2012, 722 (730). 
dienstaatsvertrag. Aus Sicht der Meinungsfreiheit und potentiell betroffener Nutzer handelt es sich hierbei aber um eine wesentliche Frage der Grundrechtsausübung, sodass viel dafürspricht, dass es Sache des Gesetzgebers wäre, entsprechende verfahrensrechtliche Anforderungen zumindest in einer $\mathbb{\int} 93 \mathrm{Abs} .4 \mathrm{MStV}$ entsprechenden, abstrakten Weise vorzugeben. ${ }^{2125}$

Die Kompetenz der Landesmedienanstalten könnte aber etwa die Einführung einer Übergangsfrist umfassen, in der von einer strengen Durchsetzung vorerst abgesehen wird. Insoweit könnte vorübergehend allein das aufgezeigte Mindestmaß verpflichtend sein und die strengere proaktive Überwachungspflicht vorerst zeitlich begrenzt aufgeschoben werden. Dadurch könnte gewährleistet werden, dass die Betreiber nicht nur genügend Zeit haben, die Vorgaben umzusetzen, sondern dass sie dabei auch vernünftige und sachgerechte Maßnahmen auch in Abstimmung mit der Forschungsgemeinschaft treffen und so in einen Diskurs über die möglichen Ansätze einer möglichst fehlerfreien Erkennungssoftware eintreten und diesen fortführen. Von der drohenden Sorgetragungspflicht und der Ordnungswidrigkeit ( $\mathbb{S} 115$ Abs. 1 S. 2 Nr. $45 \mathrm{MStV}$ ) ginge jedenfalls ein entsprechender Druck aus, entsprechende Lösungen finden zu müssen.

Gleichwohl bleibt auch hier eine verfahrensrechtliche Absicherung zwingend notwendig, um der Meinungsfreiheit fälschlich betroffener Nutzer Rechnung zu tragen. Solange diese fehlt, wird die Sorgetragungspflicht des $\mathbb{9} 93$ Abs. $4 \mathrm{MStV}$ die Meinungsfreiheit der Nutzer verletzen und deshalb verfassungswidrig sein.

\section{(e.) Zukünftige Verfahrensrechtliche Ausgestaltung}

In jedem Fall wäre also eine Nachbesserung notwendig, die eine konkrete Ausgestaltung im MStV notwendig macht. Dabei bedarf es nicht nur wegen des erneuten Eingriffs in die unternehmerische Freiheit, sondern auch wegen der Komplexität potentieller Lösungsansätze, eines gewissen Umsetzungsspielraumes, um auch hier den unterschiedlichen Angeboten gerecht werden zu können. ${ }^{2126}$ Wie könnte also eine Lösung aussehen?

Hierfür lohnt ggf. ein Blick auf vergleichbare Probleme und ihre Lösung. Weil auch im NetzDG verfahrensrechtliche Absicherungen bislang fehlten, sieht der Änderungsentwurf des NetzDG in $\mathbb{3}$ b NetzDG-E ein Gegenvorstellungsverfahren vor. ${ }^{2127}$ Hiernach hat „,[d]er Anbieter eines sozialen Netzwerks [...] ein wirksames und transparentes Verfahren [...] [vorzuhalten], mit dem sowohl der Be-

2125 So liegt der Fall letztlich auch beim NetzDG, vgl. $\$ 3$ b NetzDG-E, BT-Drs. 19/18792, wobei hier freilich eine Konkretisierungsbefugnis der Aufsichtsbehörde fehlte und insoweit der Gesetzgeber selbst tätig werden musste. Mit Blick auf den MStV vgl. hierzu Liebermann, in: Gersdorf/Paal, Informations- und Medienrecht, $\mathbb{} 96 \mathrm{MStV}$ Rn. 6.

2126 So auch Löber/Roßnagel, MMR 2019493 (497).

2127 In der Gesetzesbegründung wird explizit auf das Overblocking hingewiesen, und auf die Notwendigkeit dafür Sorge zu tragen, dass die Nutzerrechte in diesem Kontext abgesichert werden, BT-Drs. 
schwerdeführer als auch der Nutzer, für den der beanstandete Inhalt gespeichert wurde, eine Überprüfung einer [...] getroffenen Entscheidung [...] herbeiführen kann. Der Überprüfung bedarf es nur, wenn der Beschwerdeführer oder der Nutzer, für den der beanstandete Inhalt gespeichert wurde, unter Angabe von Gründen einen Antrag auf Überprüfung innerhalb von zwei Wochen nach der Information über die ursprüngliche Entscheidung stellt (Gegenvorstellung). Der Anbieter des sozialen Netzwerks muss zu diesem Zweck ein leicht erkennbares Verfahren zur Verfügung stellen, dass eine einfache elektronische Kontaktaufnahme und eine unmittelbare Kommunikation mit ihm ermöglicht. [...].“ Der Verhaltenskodex der EU sieht zumindest auch Verpflichtungen zur Wahrung der Grundrechte der Nutzer vor. Und auch die geplante Verordnung zur Verhinderung der Verbreitung terroristischer Inhalte enthält verpflichtende Absicherungen. So ist schon bei eigenständig ergriffenen Maßnahmen nach Art. 6 „den Grundrechten der Nutzer sowie der grundlegenden Bedeutung der Meinungsund Informationsfreiheit in einer offenen und demokratischen Gesellschaft Rechnung zu tragen" 2128 , was in dem Kommissionsentwurf durch eine Berichtspflicht in Art. 6 Abs. 2 abgesichert werden sollte, die aber das Europäische Parlament in der ersten Lesung gestrichen hat. ${ }^{2129}$ Und im Falle der Verwendung automatisierter Werkzeuge haben die Anbieter gem. Art. 9 Abs. 1 Schutzvorkehrungen für die Meinungs- und Informationsfreiheit zu treffen, was nach Abs. 2 insbesondere eine menschliche Aufsicht und Überprüfung erfordert. Zudem - und das ist das hier eigentlich interessante - soll nicht nur Art. 9a die Mitgliedstaaten zur Schaffung wirksamer Rechtsbehelfe verpflichten, sondern Art.10 erweitert dies auch auf die Anbieter, die ein Beschwerdeverfahren bereithalten müssen.

Dass ein vergleichbares „Gegenvorstellungsverfahren“ wenig Sinn ergibt, weil es hier nicht um Inhalte, sondern um menschlich wirkende Kommunikation geht, wurde bereits angesprochen. Allerdings wäre es angezeigt, zumindest eine der Sorgetragungspflicht entsprechende „abstrakte Schutzvorkehrungspflicht“ einzuführen, die $\mathbb{S} 93$ Abs. $4 \mathrm{MStV}$ beigefügt wird, was - in Anlehnung an Art. 1 Abs. 1 lit. b) und Art. 10 Abs. 1 der VO - wie folgt aussehen könnte:

$\int 93 \mathrm{MSt} V$

(4) Anbieter von Medienintermediären, die soziale Netzwerke anbieten, haben dafür Sorge zu tragen, dass Telemedien im Sinne von $\$ 18$ Abs. 3 gekennzeichnet werden. Sie ha-

19/18792, S. 17. Kritisch zu dem Änderungsentwurf etwa Spiegel/Heymann, K\&R 2020, 344 (347f.).

2128 So in der ursprünglichen Version der $\operatorname{KOM}(2018) 640$ final, die durch das Europäische Parlament leicht abgewandelt aber im Kern beibehalten wurde (siehe hierzu zuletzt das Dossier des Rats der EU 8663/19).

2129 Legislative Entschließung des Europäischen Parlaments vom 17. April 2019 zu dem Vorschlag für eine Verordnung des Europäischen Parlaments und des Rates zur Verhinderung der Verbreitung terroristischer Online-Inhalte, P8_TA-PROV(2019)0421. 
ben auch für geeignete und wirksame Vorkehrungen zum Schutz der rechtlichen Interessen fälschlich betroffener Telemediendienstanbieter Sorge zu tragen.

Dadurch würde man auch mit Blick auf die Schutzvorkehrungen eine abgestufte Regelung vorsehen, die mit der abgestuften Sorgetragungspflicht korrespondiert. Je eher eine proaktive Tätigkeit gefordert ist, desto eher werden auch geeignete und wirksame ${ }^{2130}$ Schutzvorkehrungen notwendig. Dadurch würde man auch den (sinnvollen) Ansatz einer regulierten Selbstregulierung fortschreiben und so auch der unternehmerischen Freiheit Rechnung tragen. ${ }^{2131}$ Zudem verbieten sich konkrete Vorgaben hier aber auch schon aus demselben Grund, wie bei der Sorgetragungspflicht: geeignete Schutzvorkehrungen für Nutzer - wie etwa die oben angesprochenen Captchas - sind schon jetzt nicht unüberwindbar. Feste Vorgaben würden also Gefahr laufen, dass der Gesetzgeber hinter der technischen Wirklichkeit zurückbleibt oder den Entwicklungen nicht in derselben Geschwindigkeit folgen kann. Schon deshalb macht es durchaus Sinn, dass den Betreibern und ihrem technischen Sachverstand die Umsetzungskompetenz zukommt. ${ }^{2132}$ Mit den Kontrollrechten und der Satzungsbefugnis der Landesmedienanstalten ( $\mathbb{S} 96$ $\mathrm{MStV}$ ) kann immer noch lenkend und regulierend interveniert werden. Insgesamt würde der Staat dadurch zumindest seiner primären Verantwortung, mittelbarfaktische Eingriffe zu vermeiden, gerecht werden. Auf sekundärer Ebene ist es ihm zudem durch die entsprechenden aufsichtsrechtlichen Befugnisse (etwa $\mathbb{S}$ 95, $56 \mathrm{MStV}$ ) möglich, die Einhaltung der Vorgaben wirksam zu kontrollieren und seine Verantwortung also auch hinsichtlich des Normvollzugs wahrzunehmen.

Auf Seiten der Betreiber könnte ein abgestuftes Schutzkonzept eine konforme Umsetzung sein. Die Schutzvorkehrungen greifen auf verschiedenen Ebenen ein, einerseits beim Registrierungsprozess, sofern etwa Zugriffe von bereits bekannten und kritischen IP-Adressen erfolgen. Primär wird es aber darum gehen, mithilfe automatisierter und algorithmisierter Verfahren die Datenströme zu überwachen, um bestimmte, auf Bots hinweisende Auffälligkeiten ausfindig zu machen. ${ }^{2133}$ Hierfür werden ganz unterschiedliche Ansätze verfolgt, die wegen

2130 In anderen Fällen wird explizit gefordert, dass eine umgehende Überprüfung eingehender Beschwerden erfolgen muss (etwa $\$ 3$ a Abs. 2 Nr. 3 NetzDG-E, BT-Drs. 19/18792, S. 10, ,unverzüglich einer Überprüfung[...]; Art. 10 Abs. 2 der geplanten VO zur Verhinderung der Verbreitung terroristischer Online-Inhalte sieht im KOM-Entwurf vor, dass umgehend jede eingehende Beschwerde überprüft wird und der Inhalt unverzüglich reaktiviert wird, wenn dessen Entfernung oder Sperrung nicht gerechtfertigt war. Und in Art. 9 Abs. 2 sollen Schutzvorkehrungen bei proaktiven Maßnahmen besonders die Aufsicht und Überprüfung durch Menschen beinhalten, COM(2018) 640 final).

2131 Als geeignete Schutzvorkehrung kämen neben technischen Lösungen vor allem eine händische Nachkontrolle in Betracht. Dies scheint die Enquete-Kommission Künstliche Intelligenz gar als notwendige Voraussetzung bei automatisierten Verfahren anzusehen, BT.-Drs. 19/23700, S. 492, 497.

2132 Allgemein betonen Kaiser/Reiling, in: Unger/v. Ungern-Sternberg, Demokratie und künstliche Intelligenz, S. 85 (110), die Grenze „der Schnelligkeit der technischen Entwicklung“.

2133 Nach der normativen Pflicht beträfe das allerdings nur Aufrufe bzw. Interaktionen, die aus Deutschland also mit einer in Deutschland registrierten IP-Adresse vorgenommen werden, sofern 
der Vielfältigkeit nur abstrakt aufgezeigt werden können: ${ }^{2134}$ Einige Verfahren wie der Botometer - betrachten nur die individuellen Accounts und dazu gehörige Daten (u.a. Follower-Followee-Ratio, Sentiment, Tweets-per-Day-Ratio, Reaktionsgeschwindigkeit usf.). ${ }^{2135}$ Andere Ansätze ziehen ergänzend oder in erster Linie inhaltliche Aspekte heran. ${ }^{2136}$ Teilweise wird auch eher eine breitere Netzwerkanalyse vorgenommen, die vor allem die Interaktionen der Profile untereinander im Blick hat, um so kollektive Phänomene - etwa Botnetzwerke ausfindig zu machen. ${ }^{2137}$ Und wieder andere setzen auf eine „adversarial machine-learning bot detection“. Hierbei dienen nicht nur (hochentwickelte) Bots und legitime Nutzerprofile als Trainingsdaten, auf Grundlage derer ein sog. Discriminator organische von anorganischen Daten bzw. authentisches von nicht authentischem Verhalten unterscheiden soll. ${ }^{2138}$ Ein sog. Generator entwickelt zudem fortlaufend weitere von den Trainingsdaten abweichende Beispiele. Dadurch wird der Discriminator auch über aktuelle Beispiele hinaus „trainiert“. Unabhängig von der konkreten Erkennungssoftware - insoweit ist es auch Aufgabe der Empirie den Weg zu einem sinnvollen Ansatz zu ebnen - ${ }^{2139}$ dürfte das Ziel jedenfalls darin bestehen, eine gewisse „Automatisierungswahrscheinlichkeit“ eines jeden Nutzerprofils zu berechnen. Je nach Wahrscheinlichkeitsgrad könnte sodann das weitere Vorgehen variieren:2140 Bei geringeren Wahrscheinlichkeiten könnten entsprechende Konten ohne Weiteres von einer weiteren Kontrolle ausgenommen werden. Bei höheren Wahrscheinlichkeiten böte es sich an, entsprechende Profile zu markieren und in einen Speicher aufzunehmen, der eine weitergehende und präzisere Kontrolle des Profils ermöglicht. Daneben könnten auch besondere Verifikationsmechanismen in solchen Fällen eingreifen, sodass entsprechende Profile ihre Menschlichkeit nachweisen müssten, ehe sie wieder Zugang

nicht die Profilinformationen selbst - etwa durch den angegebenen Wohnort o.ä. - den Schluss eines in Deutschland niedergelassenen Nutzers nahelegen (vgl. $\$ 1$ Abs. $7 \mathrm{MStV}$ ).

2134 Ein Überblick hierüber und über die Entwicklung der Erkennungsmethoden bietet etwa Cresci, Communication of the ACM Vol. 63 (2020), 72 (76 ff.).

$2135 \mathrm{Zu}$ diesem etwa Varol u.a., in: Proceedings of the Eleventh International AAAI Conference on Web and Social Media, S. $280 \mathrm{ff}$. Ähnliche Ansätz finden sich etwa bei Ferrara, First Monday Vol. 22 Nr. 8 (2017); Thieltges u.a., Effects of Social Bots in the Iran-Debate on Twitter, 2018 oder Uyheng/ Carley, Journal of Computational Social Sciences 2020, S. 7.

2136 In diese Richtung etwa Sumaila/Wakamiya/Aramaki, DBSJ Journal Vol. 18 (2020), 1ff; Schäfer/ Evert/Heinrich, Big Data Vol. 5 (2017), 294 (300).

$2137 \mathrm{Zu}$ diesen gruppenbezogenen, kollektiven Erkennungsansätzen siehe ebenfalls Cresci, Communication of the ACM Vol. 63 (2020), 72 (78 f.).

2138 Ausführlicher hierzu Cresci, a.a.O., (80 f.).

2139 Cresci, ebd. spricht sich vor allem für den zuletzt aufgezeigten Ansatz aus. Ross u.a., European Journal of Information Systems, 394 (408) meinen, dass eine Kombination von „natural language processing, social network analysis and other fields[...]" notwendig ist, um ein effektives System zu entwickeln. Und Grimme u.a., Big Data Vol. 5 (2017), 279 (291 meinen, dass nur inhalts- und profilbasierte Informationen Grundlage einer Erkennung sein können.

2140 Damit würde man auch den Ungewissheiten begegnen, ab welcher Automatisierungswahrscheinlichkeit eigentlich ein „Meinungsroboter" vorliegt, zu diesen Problemen etwa Oehmer/Pedrazzi, UFITA 2020, 7 (11). 
zum Netzwerk erhalten. ${ }^{2141}$ Inwieweit das aber per se zu einer Verifizierung führen sollte, hängt von den Verifizierungsmethoden ab. ${ }^{2142}$ Insoweit kann vor allem auch eine händische Nachkontrolle ratsam sein. Nur in offensichtlicheren Fällen und damit bei sehr hohen Wahrscheinlichkeiten dürfte eine umgehende direkte Kennzeichnung der Profile durch die Betreiber angezeigt sein. Auch hier wäre dann aber ein Verifikationsmechanismus bereit zu halten, damit in falsch-positiven Fällen eine Rückgängigmachung in Betracht kommt.

(4.) Zwischenfazit: Abgestuftes Schutzkonzept als Kompromiss zwischen Schutz- und Abwehrrechten

Auch wenn die Pflicht des $\mathbb{S} 93$ Abs. $4 \mathrm{MStV}$ der Betreiber allein mit Blick auf die unternehmerischen Interessen kaum angreifbar ist, sondern sich vielmehr im Rahmen der Prärogative der Länder bei Ausgestaltung ihrer grundrechtlichen Schutzpflicht bewegt, bestehen verfassungsrechtliche Bedenken in den Fällen, in denen durch die Sorgetragungspflicht neue Gefahren für die Meinungsfreiheit der Nutzer geschaffen werden, die fälschlicher Weise als Meinungsroboter identifiziert und daraufhin gekennzeichnet werden. Insoweit wäre es auf lange Sicht notwendig, dass die Länder hier - ähnlich wie es der Bund nun auch beim NetzDG vorhat - nachbessern und zumindest die Sorgetragungspflicht um abstrakte Vorgaben ergänzen würden. Auch hierbei gölte dann, dass mit zunehmender Meinungsbildungsrelevanz und daraus erwachsender gesellschaftlicher Verantwortung auch zunehmende Sicherungsvorkehrungen sowohl vor den Meinungsrobotern als auch vor negativen Auswirkungen für die Meinungsfreiheit zu treffen wären. Eine denkbare Option wäre ein abgestuftes Verfahren in Abhängigkeit von der „Automatisierungswahrscheinlichkeit“ oder anderen entsprechenden Parametern. Dieses kann neben einer engmaschigen Überwachung auffälliger Accounts auch bestimmte Verifikationsmethoden und in offensichtlicheren Fällen zu einer Kennzeichnung durch die Betreiber führen. Dabei muss freilich sichergestellt sein, dass echte Nutzer die (effektive) Möglichkeit haben, sich fälschlich ergriffener Maßnahmen zu erwehren.

2141 In diese Richtung auch mit Verweis auf Captchas Oehmer/Pedrazzi, UFITA 2020, 7 (23).

2142 Zu den Problemen bei Captchas siehe bereits oben, C.II.3.b.bb(II.)(3.)(c.). Identitätsüberprüfungen anhand von Ausweisdokumenten o.ä. könnten demgegenüber „sicherer" sein. Twitter hat etwa auf die Verifikation mittels Mobiltelefon-Nachrichten gesetzt. Erfolgte eine Verifikation nicht innerhalb einer festgelegten Frist, wurden entsprechende Profile endgültig gelöscht, Silva/Proksch, American Political Science Review 2020, 1 (2). 


\section{Bilanz des Medienstaatsvertrages}

Die Regelungen des MStV sind keine „Wunderwaffe“, um den herausgearbeiteten Schutzbedürfnissen zu begegnen. ${ }^{2143}$ Allerdings ging es auch nie darum jeglichen Einflüssen, die Auswirkung auf den Meinungsbildungsprozess der Nutzer haben können, durch eine engmaschige, eingriffsintensive Regulierung entgegenzutreten. Vielmehr vertrauen die Länder grundsätzlich weiterhin auf die primär gesellschaftliche und vor allem auch nutzerautonome Problemlösung und adressiert nur spezifische Probleme im Wege einer regulierten Selbstregulierung. ${ }^{2144}$ Angesichts vieler Ungewissheiten wahrt man damit jedenfalls auch die berechtigten Interessen der Netzwerkbetreiber und verhindert, selbst Verursacher noch viel gravierender Verzerrungen zu werden. ${ }^{2145}$ Die Länder adressieren auf Seiten der Intermediäre nur bestehende Risikopotentiale, die aus einer Informationsasymmetrie oder einem potentiellen Einfluss auf die priorisierte Weiterleitung von Inhalten resultieren. Mit Blick auf Erstere orientieren sich die Länder an den seit langem erhobenen Transparenzforderungen. ${ }^{2146}$ Das mag zwar eine „Pionierleistung “2147 deshalb sein, weil damit der erste Mitgliedstaat der EU einen spezifischen medienrechtlichen Vorstoß in die digitale Welt der Intermediäre wagt. Allerdings setzt schon die P2B-VO im kommerziellen Bereich auf vergleichbare Transparenzanforderungen. ${ }^{2148}$ Eine wirkliche Neuerung, die das spezifische Risikopotential adressiert, ist insoweit das Diskriminierungsverbot.

Fortschrittlicher mag dann wohl eher der Versuch der Regulierung potentiell problematischer, automatisierter Nutzerprofile sein. Das gilt aber auch hier nicht unbedingt für die gesetzlich angeordnete Transparenz, durch die der Einfluss von Meinungsrobotern insoweit neutralisiert werden kann, wie er auf einer Täuschungswirkung beruht. Auch der Staat Kalifornien hat mit dem Bot Disclosure Act bereits ähnliche Maßnahmen ergriffen. Wie auch schon beim NetzDG kommt vor allem der normierten Verantwortlichkeit der großen Netzwerkbetreiber zu, die eine Absicherung der Kennzeichnungspflicht auch durch

2143 Hinsichtlich des kalifornischen „Bot Disclosure Act“ meint etwa Hines, Houston Law Review 57 (2019), 405 (434). „While SB 1001 may not totally solve the problem of unregulated bots, it is necessary for government to at least start and act, if only to build public confidence that the issue is not out of control“.

2144 Vgl. zum Konzept regulierte Selbstregulierung Blane, ZUM 2005, 30 (38).

2145 Das gilt etwa mit Blick auf die diskutierten Vorschläge einer auch inhaltsspezifischen Regulierung der Medienintermediäre, sei es im Rahmen einer Must-Carry-Regelung oder einer zweiten informativen und von der Personalisierung unabhängigen Informationssäule. Kritisch zu derartigen positiven Diskriminierungen Liesem, AfP 2020, 377 Rn. 19 ff. oder Ingold, MMR 2020, 82 (85).

2146 Liesem, AfP 2020, 377 Rn. 25 bezeichnet dies als „conditio sine qua non einer wirksamen Regulierung“. In diese Richtung ebenfalls Enquete-Kommission Künstliche Intelligenz, BT.-Drs. 19/23700, S. 486. Kritisch aus demokratietheoretischer Sicht zu dieser fehlenden Transparenz etwa Drerup, in: Binder/ders., Demokratieerziehung und Bildung digitaler Öffentlichkeit, S. 29 (37).

2147 So betitelnd Liesem, AfP 2020, 377.

2148 Gleichwohl bezeichnen Fehling/Leymann, AfP 2020, 110 Rn. 29 die Transparenzpflicht als ,innovativer und zielführender" als das Diskriminierungsverbot. 
eine proaktive Überwachung gewährleisten müssen. Die betrieblichen Interessen der Betreiber stehen dem jedenfalls nicht im Weg. Probleme ergeben sich hier lediglich aus der kollidierenden Meinungsfreiheit Dritter, die potentiell die Verfassungswidrigkeit begründen können. Derlei Gefahren lassen sich aber durch (Fein-)Justierungen ohne Weiteres minimieren.

Zusammen genommen sorgt der Medienstaatsvertrag dafür, dass wesentliche Voraussetzungen einer autonomen, reflektierten und vielfaltsorientierten Rezeption von Inhalten rechtsverbindlich gewährleistet werden. ${ }^{2149}$ Das führt - trotz bestehender Schwächen der Regelungen - zu einem vertretbaren und jedenfalls überzeugenden Mindestschutz der grundrechtlich zu schützenden Interessen der Nutzer im Hinblick auf die Beeinflussung durch Meinungsroboter. ${ }^{2150}$ Nicht nur wird die Täuschungswirkung der Meinungsroboter adressiert und aufgedeckt, sondern es wird auch ein Verständnis für betreiberseitig hervorgerufene Verzerrungen bei der Informationsweitergabe - die eben auch für den Einfluss der Meinungsroboter kausal werden - ermöglicht.

Gleichwohl lässt der MStV mit Blick auf die Meinungsroboter ${ }^{2151}$ auch weiterhin gewisse Fragen unbeantwortet. So gewährleistet der Staatsvertrag zwar mit den Transparenzpflichten, dass notwendige Informationen für die Nutzer verpflichtend bereitstehen. Allerdings ist damit nicht sichergestellt, dass diese Informationen auch tatsächlich in einer Weise verstanden werden, dass den Nutzern deren Bedeutung, der Einfluss und die technischen Zusammenhänge in einer Weise bewusst werden, die nachhaltig ihr Rezeptions- und Nutzungsverhalten verändern kann. Man adressiert bei dem „problem of many hands“2152 lediglich die staatliche Seite und die der Betreiber. Bedenkt man aber, dass vor allem auch die individuellen, unterbewussten Entscheidungen maßgeblich für eine tatsächlich vielfältige Informiertheit sind, dann geht es - ebenso wie mit Blick auf die Transparenz - auch weiterhin um die Gewährleistung einer gewissen „digitalen Medienkompetenz“ der Nutzer. Neben diesen faktischen Schwächen bleibt die Frage unbeantwortet, inwieweit nicht nur die eigentlichen Inhalte automatisierter Profile als solche gekennzeichnet werden müssen, sondern auch bei akkumulierten Daten dafür Sorge zu tragen ist, dass diese ohne den Einfluss von Meinungsrobotern zustande kommen. Ohnehin ist der räumliche und sachliche Anwendungsbereich mit der Fokussierung auf rein inländische Anbieter

2149 Ähnlich auch Fehling/Leymann, AfP 2020, 110 Rn. 29.

2150 Kritisch allerdings jedenfalls mit Blick auf die allgemeinen Intermediärsvorgaben Cornils, AfP 2018, 377 (385).

2151 Abseits der Meinungsrobotern ist vor allem das fehlende Medienkonzentrationsrecht für die digitalen Angebote zu nennen (kritisch hierzu etwa Enquete-Kommission Künstliche Intelligenz, BT.-Drs. 19/23700, S. 486). Insoweit bleibt es (vorerst) bei dem Primat des Wettbewerbsrechts als potentielle Grenzen für die Marktmacht bestimmter Angebote.

2152 So bezeichnen Oehmer/Pedrazzi, UFITA 2020, 7 (9, 15), das aus den unterschiedlichen Verantwortungssphären resultierende Problem. 
zumindest begrenzt. Und auch ist der Medienstaatsvertrag kein probates Mittel, um besonders - auch unabhängig von Meinungsrobotern - das Problem der Verbreitung desinformativer Inhalte zu adressieren.

III. Fazit: Prinzipiell wirksame Konkretisierung der Schutzverantwortung durch die Länder

Dass sich der durch soziale Netzwerke geschaffene digitale und partizipative Meinungsmarkt selbst regulieren kann, ist angesichts der nicht immer kongruenten Interessen der Nutzer und der Betreiber und der bislang oligopolen Struktur eher eine „unzulängliche Projektion eines ökonomischen Regulationsmodells“2153 denn eine Gewissheit, die staatliche Regulierung entbehrlich macht. Es ist vor allem eine Frage des Vertrauens, ob Anbieter wirksamen Schutz bereithalten oder dies nicht vielmehr der Staat in spezifischer Weise zu erbringen hat. Das bisherige Recht vertraute lange Zeit in vielen Bereichen und so auch hinsichtlich der Meinungsroboter ausschließlich der Selbstregulierung der Betreiber und vernachlässigte damit eine auch staatliche Verantwortung für diesen Bereich. ${ }^{2154}$ Angesichts der zunehmenden Relevanz der digitalen Medien und der fehlenden wirksamen Kontrollmöglichkeit der Betreiber kann dieses Konzept nicht mehr überzeugen. Der Medienstaatsvertrag schlägt insoweit eine Brücke zwischen einerseits rechtsverbindlichen Vorgaben, ihrer wirksamen staatlichen Kontrolle und andererseits behutsamen regulativen Vorgehens. Insoweit konkretisieren die Länder die bestehende Schutzverantwortung für Meinungsroboter jedenfalls in einer nicht offensichtlich fehlerhaften Weise, die zumindest aus Sicht der grundrechtlichen Schutzpflicht und des Untermaßverbotes (vorerst) nicht angreifbar ist.

2153 Brinkmann, UFITA 2019, 364 (373).

2154 Ähnlich mit Blick auf Meinungsroboter Oehmer/Pedrazzi, UFITA 2020, 7 (9). 


\section{Kapitel: Ausblick auf die zukünftige (Rechts-)Entwicklung}

Auch wenn zurzeit keine zusätzlichen Maßnahmen (verfassungsrechtlich) gefordert werden können, so endet die rechtliche, wissenschaftliche und politische Diskussion damit noch lange nicht. Für den Gesetzgeber folgt dies nicht zuletzt daraus, dass sowohl Unter- als auch Übermaßverbot eine weitere Überwachung der getroffenen Regelungen erfordern. Aus Sicht des Untermaßverbotes geht es dabei um die Frage einer potentiellen Nachbesserungspflicht für den Fall, dass die getroffenen Regelungen nur einen lückenhaften oder unzureichenden Schutz gewähren. ${ }^{2155}$ Aus Sicht des Übermaßverbots folgt dies daraus, dass - sollte sich herausstellen, dass die Risiko- und Gefahrenpotentiale von Meinungsrobotern (offensichtlich) fehlerhaft eingeschätzt worden sind - die entsprechenden Verpflichtungen jedenfalls der Netzwerkbetreiber zurückzunehmen wären. ${ }^{2156}$ Insoweit gilt es, die weitere (technische) Entwicklung zu beobachten und auch kritisch zu begleiten. Denn nicht zuletzt bleibt der Kampf gegen Meinungsroboter ein Katz-und-Maus-Spiel. ${ }^{2157}$ Daher soll abschließend die weitere (rechtliche) Entwicklung ${ }^{2158}$ und potentielle Maßnahmen, die die aufgezeigten Schwächen des $\mathrm{MStV}$ adressieren oder die Regelungen sinnvoll ergänzen könnten, skizziert und bewertet werden. Das betrifft etwa die Frage, wie der durch die $\mathbb{\$} \$ 18$ Abs. 3, 93 Abs. $4 \mathrm{MStV}$ bezweckte Sensibilisierungseffekt dort erreicht werden kann, wo keine Kennzeichnung erfolgt, sondern auffällige Profile unmittelbar gelöscht werden. In Betracht käme hier eine öffentliche Berichtspflicht der Anbieter (A.). Daneben kann man auch über das Design der Nutzeroberflächen und Algorithmen einen großen Einfluss darauf nehmen, ob und inwieweit bestimmte replizierbare Strategien erfolgreich umgesetzt werden können (B.). Bislang wurde auch die empirische Forschung kaum ins Blickfeld regulatorischer Bemühungen genommen. ${ }^{2159}$ Um zukunftsgerichtet eine faktenbasierte Politik zu ermöglichen, bedarf es hierfür nicht nur entsprechender finanzieller Förderung. Es ist vielmehr auch darüber nachzudenken, ob es nicht verpflichtender Forschungszugänge zu den sozialen Netzwerkdaten bedarf (D.). Um auch eine strengere rechtliche Handhabung über Einsätze durch Parteien oder ausländische Akteure zu ermöglichen, könnten spezifische Verbote notwendig werden (E.). „Dauerbrenner“ bleiben da-

2155 Ausführlicher und grundlegend zu dieser aus der grundrechtlichen Schutzpflicht folgende Nachbesserungspflicht etwa BVerfGE 49, 89 (130 f.) - Kalkar I; 56, 54 (78 ff.) - Fluglärm.

2156 Vgl. BVerfGE 50, 290 (335 f.) - Mitbestimmungsgesetz; 110, 141 (157 f.) - Kampfhunde m.w.N.

2157 Freitas u.a., Social Network Analysis and Mining (2016), 6:23, S. 14; Oehmer/Pedrazzi, UFITA 2020, 7 (29).

2158 Eine ausführliche Darstellung und Bewertung der zukünftigen, vor allem inhaltlichen Regulierung von Medienintermediäre findet sich u.a. bei Heidtke, Meinungsbildung und Medienintermediäre, S. 355 ff. Vorschläge einer Regulierung der Meinungsroboter finden sich etwa auch bei Oehmer/ Pedrazzi, UFITA 2020, 7 (16 ff.).

2159 Wegen der noch nicht hinreichend klaren empirischen Lage etwa kritisch zu den Vorgaben des MStV Riedel, Wir brauchen eine faktenbasierte Medienpolitik. 
neben ein (mittelbarer) Klarnamenzwang in sozialen Netzwerken (D.) und die Stärkung der Medienkompetenz der Nutzer (E.). Und letztlich steht nicht nur eine Antwort im Kampf gegen desinformative Inhalte weiterhin aus (F.). Vor allem auf europäischer Ebene besteht - auch wegen der jüngsten mitgliedstaatlichen Maßnahmen - Handlungsbedarf (G.).

\section{A. Berichtspflichten als Maßnahme der Sensibilisierung und Stärkung der Nutzerautonomie}

Erfolgt statt einer Kennzeichnung eine unmittelbare Löschung auffälliger Profile, kann der durch die Kennzeichnung bezweckte Sensibilisierungseffekt ${ }^{2160}$ gegenüber den Nutzern nicht eintreten und auch nicht durch die Befugnisse der Landesmedienanstalten mittelbar herbeigeführt werden. Eine Kontrolle ist nur im internen Verhältnis zwischen Netzwerkbetreiber und zuständiger Landesmedienanstalt möglich $(\mathbb{S} \mathbb{S} 95,56 \mathrm{MStV})$. Vorgelegte Berichte und Informationen sind nicht öffentlich und wegen der Geheimhaltungspflicht ( $\$ 58 \mathrm{MStV})$ können sie ohne Erlaubnis nicht öffentlich gemacht werden. Dass Berichtspflichten nicht nur ein wichtiges Kontrollinstrument sind, ${ }^{2161}$ sondern auch für die Allgemeinheit Transparenz herstellen und Vertrauen schaffen, ist nicht unbekannt. Neben dem NetzDG (\$ 2) setzt auch der EU-Verhaltenskodex (oben 3. Kapitel, C.I.3.a.bb.) und die geplante Verordnung zur Bekämpfung von terroristischen Online-Inhalten jedenfalls in der Fassung des Kommissionsvorschlags ${ }^{2162}$ auf solche Berichtspflichten (Art. 6 Abs. 2).

Durch spezifische inhaltliche Vorgaben ließe sich im Falle der Meinungsroboter gewährleisten, dass die Berichte auch sensibilisierende und ggf. auch edukative Zwecke erfüllen. Ob dies noch als Bestandteil der Sorgetragungspflicht des \93 Abs. $4 \mathrm{MStV}$ verstanden werden und über $\mathbb{\$} 95 \mathrm{MStV}$ verwirklicht werden könnte, kann jedenfalls deshalb kritisch hinterfragt werden, weil damit ein ganz neues Konzept verfolgt wird, welches in anderen Gesetzgebungsakten eine explizite gesetzliche Grundlage gefunden hat. De lege ferenda könnte eine entsprechende Berichtspflicht in Anlehnung an $\$ 2$ Abs. 2 NetzDG-E allgemeine zur Veröffentlichung von Informationen u.a. darüber verpflichten, welche Maßnahmen der Betreiber ergriffen hat, wie viele Sperrungen respektive Löschungen vorgenommen worden sind, ob bestimmte Merkmale der Profile hierfür ausschlaggebend waren und insbesondere auch, ob gewisse inhaltliche Auffälligkeiten bestanden. Daneben könnten Grundzüge einer vorgenommenen automatisierten Er-

2160 Vgl. hierzu Liesem, K\&R 2019, 687 (691).

2161 So etwa nur Eifert, NJW 2017, 1450 (1453).

$2162 \operatorname{KOM}(2018) 0640$. 
kennung entsprechender Profile hilfreich sein. Formal müssten zumindest gewisse Vorgaben für die Auffindbarkeit gemacht werden, um - so wie bei den Informationspflichten insgesamt - sicher zu stellen, dass diese Berichte faktisch überhaupt einsehbar sind und von ihnen Gebrauch gemacht werden kann. ${ }^{2163}$

\section{B. Veränderungen der Nutzeroberfläche oder des Algorithmus}

Schon Kaerlein hat darauf hingewiesen, dass der potentielle Erfolg von Meinungsrobotern nicht losgelöst von der Nutzeroberfläche betrachtet werden kann. ${ }^{2164}$ Denn sie ist darauf angelegt, möglichst viele Interaktionen der Nutzer auszulösen, u.a. um die später kommerzialisierten Nutzerprofile mit Daten anzureichern. Das führt letztlich nicht nur dazu, dass die Aktivitätsdaten (Likes etc.) Hauptaugenmerk der Nutzer sind, ${ }^{2165}$ sondern auch dazu, dass der aktivste Nutzer belohnt wird. ${ }^{2166}$ Diese Leistungskriterien, sind aber für Bots wie für normale Nutzer gleichbedeutend. „Je mehr [also] die Internetkultur eine Template-Kultur mit standardisierten Schnittstellen geworden ist, umso einfacher ist die Simulation von Agilität und Lebhaftigkeit [...]. “2167 Insoweit ist das nicht nur ein Problem der Meinungsroboter. Auch zusammenagierende Nutzergruppen oder sog. Trolle - Fake-Accounts die durch reale Personen gesteuert werden - können sich diese netzwerkinternen Effekte zu eigen machen, um manuell entsprechende Verzerrungen auszulösen. ${ }^{2168}$ Vermeiden ließe sich das u.U. dadurch, dass groß angelegte Veränderungen an der Nutzeroberfläche vorgenommen werden:2169

2163 Der Kommissionsentwurf des Digital Service Acts, COM(2020) 825 final, sieht in Art. 31 Abs. 2 lit b) eine Berichtspflicht durchaus vor.

2164 Hierzu Kaerlein, in: Hug/Pallaver, Talk with the Bots, S. 17 (23 ff.) der dies ganz sinnbildlich formuliert: Don't blame the bots, blame the platforms!". In diese Richtung auch Leistert, in: Seyfert/ Roberge, Algorithmuskulturen, S. 215 (218 f.); Oehmer/Pedrazzi, UFITA 2020, 7 (9). Zum Zusammenhang von automatischen, impulsiven Reaktionen der Nutzer und der Bedeutung für die Erbringung der Dienste siehe auch Lischka, AfP 2018, 388 (389, 390).

2165 Kaerlein, in: Hug/Pallaver, Talk with the Bots, S. 17 (23 f.). Mit Blick auf die Informationsaufnahme bzw. Vermittlung Lischka, AfP 2018, 388 (389f.). Siehe auch Thieltges/Hegelich, ZfP 64 (2017), 493 (498).

2166 Mit Blick auf die priorisierte Anzeige von Kommentaren etwa Brodnig, Hass im Netz, S. 199f; ebenso Thieltges/Hegelich, ZfP 64 (2017), 493 (496).

2167 Leistert, in: Seyfert/Roberge, Algorithmuskulturen, S. 215 (221).

2168 Deshalb etwa kritisch zu den Vorgaben der $\mathbb{S} \int 18$ Abs. 3, 93 Abs. 4 MStV Riedel, Wir brauchen eine faktenbasierte Digital- und Medienpolitik, medium.com. Ausführlicher zu den Trollen und ihren Einflüssen innerhalb sozialer Netzwerke Lee/Kerr, First Monday Vol. 25 (2020) Nr. 6.

2169 Besonders Hegelich hat auf der Tagung „Der Code als Gatekeeper“ des Instituts für urheber- und Medienrecht am 28.04.2017 darauf hingewiesen, dass „man über Designfragen großen Einfluss darauf nehmen [könne], wie die Nutzer diese Plattform in Zukunft nutzten“, zit. nach Grandjean, ZUM 2017, 565 (571). Zur Änderung der Informationsvermittlung durch Algorithmen vgl. Lisch$k a$, AfP 2018, 388 (391). Und hinsichtlich der Förderung eines respektvollen Umgangs in sozialen Netzwerken, Brodnig, Hass im Netz, S. 200. Im Kontext der Meinungsroboter schlagen Oehmer/ Pedrzzi, UFITA 2020, 7 (26) etwa eine Limitierung der Aktivitäten pro Zeiteinheit vor. 
Interaktionszahlen könnten nicht mehr öffentlich einsehbar sein, ${ }^{2170}$ neben der Äußerung positiver Gefühle könnten auch andere Reaktionen zugelassen werden, es könnten spezielle Bewertungsmechanismen für die Glaubwürdigkeit eingeführt werden, die nur verifizierten Nutzern oder autorisierten Dritten zugänglich sind oder auch Anpassungen des Algorithmus vorgenommen werden, um „natürlich“ entstandene Trends zu erkennen. ${ }^{2171}$

Die Nutzeroberfläche ist aber neben dem Algorithmus wesentlicher Faktor für den wirtschaftlichen Erfolg der Netzwerkbetreiber. ${ }^{2172}$ Und aus Sicht der Nutzer ist zweifelhaft, ob durch das Verbergen allein etwas gewonnen wäre. Auch wenn die Heuristiken nicht nur im Kontext der Meinungsroboter problematisch sind, so geben Sie den Nutzern zumindest eine gewisse Hilfe an die Hand. ${ }^{2173}$ Insoweit wäre es ggf. ratsamer, abseits von Likes weitere Reaktionsmöglichkeiten zu ermöglichen. Das hat Facebook mit seinen Reaction-Emojis seit 2016 bereits versucht. ${ }^{2174}$ Damit wird aber auch die Profilbildung weiter intensiviert, weil sie einen noch genaueren Aufschluss über Interessen und Gefühle der Nutzer ermöglichen. Schon deshalb und wegen vieler Ungewissheiten, wie sich ein Eingriff auf die Nutzeroberfläche oder die Algorithmen letztlich auswirkt und welche Kriterien eigentlich sinnvoll dafür sind, entsprechende Ziele zu erreichen, sollte dies vorerst der Selbstregulierung überlassen bleiben. ${ }^{2175}$ Aufgabe der Netzwerkbetreiber wird es auch zukünftig sein, entsprechendes unnatürliches Verhalten innerhalb der Netzwerke ausfindig zu machen und frühzeitig zu unterbinden.

2170 In der Vergangenheit hat insbesondere Facebook bereits Testläufe unternommen, bei denen die Likes als sichtbare Datenquelle ausgeblendet wurden. Offiziell sollte damit die Jagd nach Anerkennung verhindert werden. Hierzu etwa Breithut, Facebook blendet Likes aus, Spiegel.

2171 Inwieweit hier eine gewisse Analogie zu den Versuchen von Suchmaschinenanbietern, suchmaschinenoptimierte Inhalte ausfindig zu machen, die die für das Ranking relevanten Faktoren künstlich in unzulässiger Weise erhöhen, denkbar ist, ist unklar. Zu derlei Bemühungen siehe etwa den Bericht von Google, abrufbar unter https://support.google.com/webmasters/answer/9044175, (Stand: 30.05.2021).

2172 Vgl. Oehmer/Pedrazzi, UFITA 2020, 7 (30).

2173 Zur hilfreichen ökologisch rationalen Bedeutung von Heuristiken siehe etwa Hill, DÖV 2020, 205 (215).

2174 Hierzu etwa auch Brodnig, Hass im Netz, S. $200 \mathrm{f}$.

2175 (Grund-)rechtliche Bedenken äußert etwa Cornils ZUM 2019, 89 (5) mit Blick auf die Nutzergewohnheit und lenkende Eingriffe des Staates in das Design der Intermediäre. Zumindest kritisch unter Hinweis auf die schwierige Regulierung aus technischer Sicht im Kontext von Fake-News Drexl, ZUM 2017, 529 (542). Kritisch mit Blick auf Vorgaben zur Gestaltung von Suchalgorithmen, Hartl, Suchmaschinen, Algorithmen und Meinungsmacht, S. 199, und mit Blick auf inhaltliche Vorgaben für die Verteilung der Inhalte Kaiser/Reiling, in: Unger/v. Ungern-Sternberg, Demokratie und künstliche Intelligenz, S. 85 (107) eine staatliche Vorgabe angesichts selbstregulativer Maßnahmen für zu „eingriffsintensiv“ und damit unverhältnismäßig. Hierauf setzt letztlich auch die Kommission in ihrem Entwurf des Digital Service Acts, COM(2020) 825 final in Art. 26, 27 die „sehr große Anbieter" (Art. 25 Abs. 1 ) dazu verpflichten Risiken zu ermitteln (At. 26 Abs. 1) und sodann zu mindern (Art. 27 Abs. 1 S. 1), wobei ihnen ein weiter Entscheidungsspielraum zusteht. 


\section{Spezielles Einsatzverbot für bestimmte Urheber?}

Auch wenn bereits aus verfassungsrechtlicher Sicht ein Verbot für Parteien besteht, Meinungsroboter einzusetzen, so ist dieses bislang - unterhalb einer Gefahrenschwelle - nicht rechtlich „vollstreckbar“. Insoweit könnte der Gesetzgeber einfach-rechtliche Verbotsnormen im Parteiengesetz schaffen, ${ }^{2176}$ die etwa zur Rückforderung der staatlichen Finanzierung oder zu Strafzahlungen führen können, ähnlich wie dies auch bei anderen Verstößen gegen die Transparenzvorgaben des Parteienrechts gilt (vgl. $\mathbb{S} \mathbb{S} 31$ a Abs. 1, 31b, 31c PartG). Ferreau schlägt zudem vor, dass in Ergänzung des $\$ 93$ Abs. $4 \mathrm{MStV}$ die Betreiber zur Überwachung verpflichtet werden. ${ }^{2177}$ Dasselbe soll auch mit Blick auf den Einsatz durch ausländische Staaten gelten. ${ }^{2178}$ Auch wenn damit der Schwäche der vor allem kompensatorischen Wirkung von Verstößen gegen das Interventionsverbot - so denn es verletzt wäre - begegnet würde (dazu oben, C.I.2.e), so bleibt das Problem der Staatenverantwortlichkeit und der Zurechnung. Nichts anderes gilt letztlich auch mit Blick auf die Parteien. Denn nur weil Meinungsroboter parteinahe Inhalte verbreiten, heißt das noch lange nicht, dass die Partei auch dafür als eigentlicher Urheber verantwortlich ist. ${ }^{2179}$ Insoweit könnten entsprechende Verbote und Überwachungspflichten schon rein faktisch leerlaufen. Und im Verhältnis zu ausländischen Staaten dürfte es auch nur bei diplomatischen Bemühungen bleiben, den jeweiligen Staat zum Unterlassen zu bewegen. Insoweit folgt hieraus keine nennenswerte Neuerung zum bereits bestehenden völkerrechtlichen Interventionsverbot.

\section{Forschungszugang zu sozialen Netzwerken}

Auch wenn Berichtspflichten die öffentliche Transparenz stärken, lassen sie den notwendigen detaillierten Einblick, der als Grundlage einerseits für eine medienwissenschaftliche Aufbereitung andererseits für eine faktenbasierte Regulierung bestimmter Phänomene unabdingbar ist, vermissen. ${ }^{2180}$ Das gilt insbesondere dort, wo sich bestimmte Forschungsfragen nicht durch das Beobachten und Scraping bestimmter Inhalte oder durch Simulationen klären lassen, ${ }^{2181}$ sondern

\footnotetext{
2176 In diese Richtung auch Ferreau, in: Möller/Hamaleers/ders., Typen von Desinformation und Misinformation, S. 44 (71).

2177 Ferreau, ebd.

2178 Ferreau, a.a.O., S. 74 f.

2179 Die Unmöglichkeit der Identifikation der Urheber betonen etwa Silva/Proksch, American Political Science Review 2020, 1 (2).

2180 Ausloos/Leerssen/Thije, Operationalizing Research Access in Platform Governance, S. $21 \mathrm{f}$. Siehe auch Enquete-Kommission Künstliche Intelligenz, BT.-Drs. 19/23700, S. 485.

2181 Das wäre etwa durch Crawling mit Blick auf Desinformation denkbar, hierzu ausführlicher Steinbach u.a., Desinformation aufdecken und bekämpfen, S. $113 \mathrm{ff}$. Allgemein zu den Vorteilen der API
} 
eine umfangreichere Auswertung netzwerkinterner Daten notwendig wird. Eine umfangreiche wissenschaftliche Auseinandersetzung wird aber durch einen eingeschränkten oder nicht vorhandenen Zugang zu den API der Netzwerke ${ }^{2182}$ bzw. zu den erforderlichen Netzwerkdaten erschwert, sodass gewisse Annahmen gar nicht überprüft werden können. ${ }^{2183}$ Während Twitter einen relativ liberalen Umgang pflegt und einen Zugriff auf das Netzwerk und die öffentlich gemachten Daten der Nutzer grundsätzlich gewährt, ${ }^{2184}$ ist dies bei Facebook anders. Wer in welchem Umfang und zu welchen Zwecken auf die Daten zugreifen darf, entscheidet das Unternehmen nach eigenen Maßstäben. ${ }^{2185}$ Auch wenn das im Ausgangspunkt verständlich ist, weil man Forschern immerhin auch (weite) Einblicke in die Funktionsweise des Dienstes und in die Netzwerkdaten gewährt, so führt das deshalb zu Problemen, weil die Betreiber willkürlich über die Zugriffsberechtigungen entscheiden können und so nur bestimmten Forschungsprojekten Zugang gewährt wird, die keine Gefahr für die wirtschaftlich orientierten Plattformen darstellen. ${ }^{2186}$ Dadurch besteht letztlich auch die Gefahr, dass „the resulting research are unlikely to deliver true accountability. “2187 Deshalb wurde der Ruf nach einem (verpflichtenden) Forschungszugang laut, ${ }^{2188}$ dem die bishe-

im Vergleich zu anderen Methoden Halavais, Information, Communication \& Society 22 (2019), 1567 (1573).

2182 Etwas ausführlicher zu diesen API und den gewährten Zugriffen u.a. Rat für Sozial- und Wirtschaftsdaten, Big Data in den Sozial-, Verhaltens- und Wirtschaftswissenschaften ", S. 9 ff.

2183 Lischka, AfP 2018, 388 (391). Ausloos/Leerssen/Thije, Operationalizing Research Access in Platform Governance, S. 9 bezeichnen die Betreiber als „de facto gatekeepers of research and reporting agendas".

2184 Hierzu etwa die Informationen über die Twitter-API unter https://help.twitter.com/de/rules-and-poli cies/twitter-api, (Stand 30.03.2021). Darauf hinweisend, wobei auch nicht ganz ohne Kritik, Ausloos/Leerssen/Thije, Operationalizing Research Access in Platform Governance, S. 17. Siehe hierzu auch Rat für Sozial- und Wirtschaftsdaten, Big Data in den Sozial-, Verhaltens- und Wirtschaftswissenschaften“, S. 11. Ausführlich zum API-Zugang bei Twitter etwa Pfaffenberger, Twitter als Basis wissenschaftlicher Studien, S. $41 \mathrm{ff}$.

2185 Hierzu auch Steinebach u.a., Desinformation aufdecken und wirksam bekämpfen, S. 117.

2186 Kritisch mit Blick auf die bisherige Praxis etwa auch Lischka, AfP 2018, 388 (390). Zu der vehementen Weigerung von Facebook gewisse Daten zuzulassen vgl. etwa Bröckling, Facebook behindert Forschung zu „Fake News“, Netzpolitik.org. Und auf Gefahren für eine unabhängige Forschung durch bspw. gewisse verpflichtende Abnahmeprozesse weisen Ausloos/Leerssen/Thije, Operationalizing Research Access in Platform Governance, S. 10 hin. Zur potentiellen Diskriminierung anhand von Eigeninteressen siehe dies., a.a.O., S. 21. Zum Verhältnis der Netzwerkbetreiber und kritischer Untersuchungen Bruns, Information, Communication \& Society 2019, $1544 \mathrm{ff}$. Allgemein weist der Rat für Sozial- und Wirtschaftsdaten in seinem Gutachten „Big Data in den Sozial-, Verhaltens- und Wirtschaftswissenschaften“, S. 8 daraufhin, dass „[e]in Datenzugang [...] häufig nur gewährt [wird], wenn das Unternehmen von dem spezifischen Forschungsprojekt profitiert.“

2187 Ausloos/Leerssen/Thije, Operationalizing Research Access in Platform Governance, S. 21, die hier zudem zu Recht von einem „chilling effect“ für die Forschungsarbeit sprechen. Kritisch mit Blick auf die ergriffenen Initiativen zum Kampf gegen Desinformation etwa Wardle/Derakhshan, Information Disorder, S. 62.

2188 So etwa Lischka, AfP 2018, 388 (391); Lischka/Stöcker, Digitale Öffentlichkeit, S. 49 mit Blick auf die Plattformökonomie und die Informationsvermittlung. Im Kontext des $₫ 93 \mathrm{MStV}$ Zimmer, in: Gersdorf/Paal, Informations- und Medienrecht, $\mathbb{\$} 93$ MStV Rn. 22. Mit Blick auf die Bekämpfung von Hasskriminalität etwa die Antonio Amadeu Stiftung, Stellungnahme zum erweiterten Entwurf 
rigen Vorkehrungen im Verhaltenskodex der $\mathrm{EU}^{2189}$ schon mangels unmittelbarer Durchsetzungsfähigkeit ${ }^{2190}$ seitens der Forschergruppen nicht gerecht werden können.

\section{Grundrechtliche Implikationen}

Ein staatlich verordneter Zugriff auf die Netzwerkdaten führt zu (neuen) Konflikten mit den unternehmerischen Interessen der Betreiber und den Datenschutzinteressen der Nutzer. ${ }^{2191}$ Schon mit Blick auf potentiell kritische Forschungsergebnisse besteht ein wirtschaftliches und durch Art. 12 Abs. 1 GG grundrechtlich geschütztes Interesse der Betreiber, eigenständig und nach selbst festgelegten Kriterien Forschungspartner auszuwählen. Dabei steigt das Auswahl- und Geheimhaltungsinteresse in Abhängigkeit von dem Umfang und dem Ziel des ersuchten Forschungszugriffs. So besteht ein substantielles Interesse nicht nur dort, wo es um den Einfluss der Algorithmen - den bedeutsamsten Unternehmenswert - für die Informationsvermittlung geht. Auch dort, wo es um die Anzahl von FakeUsern, Bots und ihren Einfluss innerhalb des Netzwerkes geht, stehen erhebliche wirtschaftliche Interessen einer weiten Offenbarung gegenüber. Soweit es um die Algorithmen oder die APIs geht, insbesondere wenn ein verpflichtender API-Zugang eingeführt wird, kommt daneben auch der Schutz der Eigentumsfreiheit (Art. 14 Abs. 1 GG) in Betracht. Weil ein Forschungszugang letztlich Weitergabe von Nutzerdaten und zu ihrer Verarbeitung durch Dritte führt, wird daneben Recht der Nutzer auf informationelle Selbstbestimmung tangiert (Art. 2 Abs. 1 i.V.m. Art. 1 Abs. 1 GG).

All das steht aber dem Vorhaben nicht per se im Weg. Denn angesichts der bestehenden Forschungslücken und dem nicht diskriminierungsfreien Zugang zu den Netzwerkdaten besteht ein grundlegendes Bedürfnis, die Forschungsarbeit nicht nur im Kontext der Meinungsroboter zu ermöglichen. Wegen des engen Zusammenhangs zwischen empirischer Medienforschung und medienpolitischen Erwägungen würde so vor allem die rechtliche Diskussion „versachlicht“ werden

des NetzDG. Neudert im Ausschuss für Digitale Agenda, Ausschussdrucksache 19(23)046, S. 5. Siehe daneben auch BT-Drs. 19/237000, S. 481.

2189 Der Verhaltenskodex der EU sieht in der Verpflichtung Nr. 12 vor, dass die Unterzeichner „unabhängige Anstrengungen “, die Desinformation verfolgen und die Auswirkungen untersuchen wollen, unterstützen. Nach Nr. 13 sollen die Unterzeichner seriöse Forschung nicht be- oder verhindern, sondern fördern (Nr. 14).

2190 Kritisch zu den selbstregulativen Versuchen etwa Ausloos/Leerssen/Thije, Operationalizing Research Access in Platform Governance, S. 10.

2191 Ob hierbei auch europäische grundrechtliche Gewährleistungen berücksichtigt werden müssen, weil es um die „Durchführung von Unionsrecht“ geht (Art. 51 Abs. 1 EUGrCh), oder - weil es um eine kompetenzielle Zuordnung geht - dies nicht der Fall ist (vgl. Jarass, Charta der Grundrechte der EU, Art. 51 Rn. 20a), ist zweifelhaft. Im Wesentlichen dürfte der Schutz aber weitestgehend kongruent verlaufen, statt vieler BVerfG, NJW 2020, 300 Rn. 55ff - Recht auf Vergessen I. 
können. ${ }^{2192}$ Und zudem fördert man dann auch eine (kritische) Überprüfung der Einhaltung der durch den Medienstaatsvertrag eingeführten Verpflichtungen.

Zudem werden die unternehmerischen Interessen vor allem dort bedeutsam, wo ein Einblick in die internen, geheimhaltungsbedürftigen Betriebsabläufe und damit die Algorithmen erfolgt. Dort, wo es etwa um das Nutzerverhalten, Nutzerdaten, oder das Endprodukt und seine gesellschaftlichen Auswirkungen geht, ist dieses spezifische und besondere Geheimhaltungsbedürfnis kaum betroffen. Vielmehr besteht ein Interesse lediglich daran, dass potentielle Risiken der netzwerkbasierten Kommunikation nicht nachgewiesen und öffentlich bekannt werden können. Eine vergleichbare Interessenlage besteht auch auf Seiten von Produktanbietern in der analogen Welt. Anders als in der digitalen Welt werden Forscher allerdings rein faktisch nicht davon abgehalten, Produkte zu erwerben und etwaige schädliche Auswirkungen nachzuweisen. Und teilweise werden gar spezifische Pflichten normiert, um die gesellschaftliche Verantwortung für Produkte dadurch zu stärken, dass zusätzliche Daten öffentlich gemacht werden. ${ }^{2193}$ Insoweit ergibt sich - jedenfalls solange nicht betriebliche Geheimnisse tangiert sind - kein plausibler Grund, warum sich die sozialen Netzwerke und sonstige Intermediäre gerade dieser gesellschaftlichen und wissenschaftlichen Kontrolle und dem Diskurs entziehen können sollen. Im Vergleich zu analogen Produkten kommt ihnen immerhin auch eine deutlich größere gesellschaftliche Bedeutung zu, die durch die Betreiber selbst ökonomisiert wird. Dieses ökonomische Interesse ist aber nicht tangiert, denn die alleinige Kommerzialisierung der Daten durch die Betreiber zur Refinanzierung ihrer Dienste bleibt unberührt.

Auch wenn man die API als (intellektuelles) Eigentum ansieht ${ }^{2194}$ und dem Schutzbereich von Art. 14 Abs. 1 GG unterwirft, führt zu keinem anderen Ergebnis. Denn ein Forschungszugriff wäre lediglich als Inhalts- und Schrankenbestimmung einzuordnen, und angesichts des erheblichen gesellschaftlichen Interesses an der Überprüfung und Erforschung der „black boxes“ führt das zu einer stärkeren Allgemeinwohlbindung. Nicht zuletzt zeigt schon $\$ 60$ c Abs. 1, 2 UrhG, dass ein besonderes Bedürfnis an der wissenschaftlichen Verwendung geistigen Eigentums bestehen kann. $\$ 60 \mathrm{~h}$ UrhG sieht hier immerhin eine Kompensation der Rechtsinhaber vor. Ein entsprechendes Kompensationsmodell für die Inanspruchnahme der Dienste der Betreiber ließe sich auch im Kontext des For-

2192 Lischka, AfP 2018, 388 (391); siehe auch Hartl, Suchmaschinen, Algorithmen und Meinungsmacht, S. 231. Vgl. zur Notwendigkeit eines Zugangs aus aufsichtsrechtlicher Sicht auch Enquete-Kommission Künstliche Intelligenz, BT.-Drs. 19/23700, S. 485. Im Bereich der Telekommunikation sieht $\$ 125$ TKG gerade eine wissenschaftliche Begleitung der Bundesnetzagentur vor.

2193 Vgl. hierzu Ausloos/Leerssen/Thije, Operationalizing Research Access in Platform Governance, S. 24 mit Fn. 88. Und zum öffentlichen Transparenzbedürfnis etwa im Kontext von $\$ 16$ a GenTG siehe Ostermann, Transparenz und öffentlicher Meinungsbildungsprozess, S. $475 \mathrm{ff}$.

2194 Hierzu u.a. Czychowski/Siesmayer, in: Kilian/Heussen, Computerrechts-Handbuch, 20.4 Rn. 36; Dreier, in: ders./Schulze, UrhG, $\mathbb{\int} 69$ a Rn. 23. 
schungszugriffs ohne Weiteres normieren. Daneben sieht $\$ 49$ Abs. 2 TKG etwa einen Zugangsanspruch zu der API gegenüber Rechteinhabern vor, um die für den Betrieb notwendigen Informationen zu erhalten und kompensiert das auch nur durch eine angemessene Vergütung. ${ }^{2195}$ Insoweit geht man auch hier vom Vorrang des Allgemeininteresses an der Kenntnis entsprechender Informationen aus.

Und zuletzt bietet gerade auch kritische Forschung die Möglichkeit, Transparenz zu schaffen und so auch das Vertrauen in die Integrität der Dienste längerfristig zu erhöhen. ${ }^{2196}$ Darin besteht also auch eine Chance für die Netzwerkbetreiber. Sofern damit ein zusätzlicher, erheblicher Aufwand verbunden ist, wäre auch ein kompensatorischer Ausgleich durchaus vorstellbar. Freilich werden durch Forschungszugriffe neue Gefahren des Missbrauchs geschaffen. Diesen kann man aber durch entsprechende inhaltliche oder verfahrenstechnische Anforderungen an Forschungsprojekte begegnen. Zudem lassen sich auch die Zugriffe auf das Netzwerk in Abhängigkeit von den Forschungszwecken technisch beschränken.

Diese Beschränkung wäre zudem schon deshalb erforderlich, weil regelmäßig auch die Verarbeitung von Nutzerdaten erforderlich sein wird. ${ }^{2197}$ So setzt die Erkennung von Meinungsrobotern die Analyse und Verarbeitung personenbezogener Daten ebenso voraus wie die Erforschung des Einflusses auf die Nutzer und die Nutzergemeinschaft. ${ }^{2198} \mathrm{Da}$ an der Verarbeitung auch ein wesentliches politisches und gesellschaftliches Interesse - und zwar weltweit - besteht, spricht auch vieles dafür, dass die Forschungsinteressen jedenfalls dann überwiegen, wenn ein hinreichender verfahrensrechtlicher und technischer Schutz der Daten sichergestellt ist. Das zeigt nicht zuletzt schon die einfach-rechtliche Ausgestaltung des Datenschutzes im Kontext wissenschaftlicher Verarbeitungszwecke, ${ }^{2199}$ die zahlreichen Privilegierungen vorsieht. Neben der entfallenden Zweckbindung (Art. 5 Abs. 1 lit. b), Art. 89 Abs. 1 DS-GVO) kann auch eine entsprechende Information der Betroffenen über die Drittverarbeitung entfallen (Art. 14 Abs. 5 lit.b) DSGVO), sofern entsprechende Schutzvorkehrungen getroffen werden. ${ }^{2200}$ Und auch anderweitige Rechte der Betroffenen (Art. 15, 16, 18, 21 DS-GVO) können gem.

2195 Ausführlicher zu $\ 49$ Abs. TKG etwa Janik, in: Geppert/Schütz, TKG, $\$ 49$ Rn. 12-23.

2196 Lischka, AfP 2018, 388 (391).

2197 So auch BT-Drs. 19/23700, S. 481.

2198 Etwa wenn es darum geht, individuelle Netzwerke zu analysieren und mögliche Bezugspunkte von Meinungsrobotern und normalen Nutzern ausfindig zu machen oder um die Interaktionen zwischen ihnen nachzuvollziehen.

2199 Mit Blick auf die Vorgaben der DSGVO zu der Verbreitung von Daten zu Forschungszwecken umfassend Kotsios u.a., An Analysis of the Consequences of the General Data Protection Regulation on Social Network Research. Daneben aber auch Steinebach u.a., Desinformation aufdecken und bekämpfen, S. $118 \mathrm{ff}$.

2200 Beispielhaft zu diesen etwa Kotsios u.a., An Analysis of the Consequences of the General Data Protection Regulation on Social Network Research, S. $14 \mathrm{f}$. Und zu den Problemen hinsichtlich einer tatsächlichen Informierung siehe dies., a.a.O., S. 24 f. 
Art. 85 Abs. 2 DS-GVO i.V.m. $\$ 27$ Abs. 2 S. 1 BDSG insoweit unberücksichtigt bleiben, wie sie die Verwirklichung der Forschungszwecke zumindest ernsthaft beeinträchtigen und die Beschränkung für die Zwecke erforderlich ist. Daten dürfen zudem gem. Art. 5 Abs. 1 lit. e), 2. HS. DS-GVO auch länger gespeichert werden; eine unmittelbare Löschpflicht nach Beendigung der Forschung besteht nicht. Und nach $\mathbb{} 27$ Abs. 1 BDSG, Art. 9 Abs. 2 lit. j) DS-GVO können auch besonders geschützte Daten abseits einer Einwilligung verarbeitet werden, sofern Schutzvorkehrungen i.S.v. $\$ 22$ Abs. 2 S. 2 BDSG getroffen werden. ${ }^{2201}$ Voraussetzung ist, dass entsprechende Nutzerdaten für die Forschung erforderlich sind und die Forschungsinteressen erheblich überwiegen. Ist dies der Fall, ist auch die Datenverarbeitung nicht besonders geschützter Daten gem. Art. 6 Abs. 1 lit.f) DSGVO zulässig. Insoweit ist eine unbegrenzte Verarbeitung auch hier nicht möglich. Vielmehr müsste der Forschungszweck zuvor so hinreichend präzisiert werden, dass anhand dessen auch eine Begrenzung der Nutzerdaten auf das hierfür erforderliche Maß möglich ist. ${ }^{2202}$ Das entspräche nicht zuletzt auch dem Grundsatz der Datenminimierung (Art. 5 Abs. 1 lit.c) DS-GVO). Im Übrigen wären auch die anderen Grundsätze der Verarbeitung (Art. 5 DS-GVO) zu berücksichtigen. Dazu gehört insbesondere der Schutz der Betroffenen, dem etwa durch eine Pseudo- oder Anonymisierung ihrer Daten im Einzelfall entsprochen werden kann (vgl. Art. 5 Abs. 1 lit. e) DS-GVO). Dort, wo es um die Analyse von Inhalten $^{2203}$ oder der Informationsdiffusion geht, also die individuellen Nutzerdaten weniger relevant sind, käme das etwa in Betracht. Im Einzelfall kann es - um entsprechend weitreichendere Daten zu erhalten - auch ratsam sein, eine freiwillige Bereitstellung von Nutzerdaten zu ermöglichen und Daten so auf Grundlage einer Einwilligung zu verarbeiten. Dann muss nur dafür Sorge getragen werden, dass diese Einwilligung auch informiert erfolgt (Art. 4 Nr. 11 DS-GVO).

\section{Perspektive für eine (gesetzliche) Ausgestaltung}

Insoweit ist die Verwirklichung eines Zugriffs vor allem eine Frage einer verfassungs- und datenschutzkonformen Ausgestaltung, die die aufgezeigten Interessen hinreichend berücksichtigt und in einen angemessenen Ausgleich bringt. Mit Blick auf die durch Behörden gespeicherten Informationen sieht das Informationsfreiheitsgesetz entsprechende Ansprüche und verfahrensrechtliche Ausgestaltungen bereits vor. ${ }^{2204}$ Hieran könnte sich orientiert werden. Forschungsprojekten

2201 Bedeutsam ist das etwa dort, wo Inhalte verarbeitet werden oder sonstige Informationen, aus denen besonders geschützte Daten hervorgehen. Hierzu Kotsios u.a., a.a.O., S. $16 \mathrm{f}$.

2202 Das gilt aber auch im Falle des Art. 6 Abs. 1 lit. e). Auch hiernach muss die Verarbeitung für die Wahrnehmung einer Aufgabe, die im öffentlichen Interesse liegt, erforderlich sein.

2203 Vgl. Dietrich/Gersin/Herweg, in: Frindte/Dietrich, Muslime, Flüchtlinge und Pegida, S. 235 (248).

2204 Durch das Informationsfreiheitsgesetz wird in $\$ 1$ Abs. 1 ein entsprechender Anspruch normiert. Dem Anspruch können bestimmte in $\$ 3$ normierte öffentliche Belange oder der Schutz behördlicher 
könnte dafür ein Anspruch gewährt werden, Zugang zu den erforderlichen Netzwerk- und Nutzungsdaten vor allem über (zu schaffende) API zu erhalten. Dieser Anspruch müsste verfahrensrechtlich an gewisse Voraussetzungen geknüpft werden. Um zu verhindern, dass illegitime Projekte - so wie etwa im Fall von Cambridge Analytica - Zugriff auf die Netzwerkdaten erhalten und diese missbrauchen, bedarf es vor allem einer vorherigen Verifizierung der Seriosität des jeweiligen Projekts. Insoweit wäre also ein an $\$ 7$ IFG angelehntes Verfahren vorzusehen. Projekten könnte dann ein individueller Zugangscode gewährt werden. Dieser Schutz müsste aber nicht nur primär im Rahmen einer Verifikation erfolgen, sondern daneben auch sekundär einerseits mit Blick auf den inhaltlichen und zeitlichen Umfang des gewährten Zugriffs und andererseits mit Blick auf zu ergreifende Vorkehrungen zum Schutz der Daten. Forschungsanfragen müssten also vorher so präzise formuliert werden, dass eine gewisse inhaltliche Begrenzung des Zugriffs möglich wäre. Dadurch würde gewährleistet, dass nur ein begrenzter Einblick in die Dienste gewährt werden muss und dass ggf. auch nur die anonymisierte bzw. pseudonymisierte Weitergabe von Nutzerdaten erfolgt. ${ }^{2205}$ Und zudem müsste schon vor dem Datenzugriff hinreichend klar sein, wie die Sicherheit der (sensiblen) Daten gewährleistet wird (vgl. Art. 25 Abs. 1, 2 DS-GVO). ${ }^{2206}$ Insoweit ist also ein umfangreicher Forschungsplan erforderlich. Praktisch ließe sich der Zugriff dann auch in Abhängigkeit der Zugriffscodes ausgestalten. Ihnen kann nicht nur ein zeitliches Verfallsdatum zugewiesen werden, sondern auch unterschiedliche und für das Forschungsvorhaben nur erforderliche Berechtigungen erteilt werden.

Abgesehen von den verfahrenstechnischen Anforderungen ${ }^{2207}$ müsste auch ganz grundlegend überlegt werden, wer für eine entsprechende Verifikation und Überprüfung des Forschungszwecks, der Überprüfung etwaiger Schutzvorkehrungen und der erforderlichen Daten zuständig sein sollte. Freilich kann dies schon auf vertraglicher Ebene zwischen Betreiber und Forschern ermöglicht werden, indem etwa ein „Kontrahierungszwang“ eingeführt wird, sofern gewisse

Entscheidungsprozesse $(\mathbb{4} 4)$ im Wege stehen. Über einen Antrag entscheidet gem. $\$ 7$ die über die Informationen verfügende Behörde. Hierbei wird auch überprüft, inwieweit personenbezogene Daten weitergegeben werden. Nach $\mathbb{S} 5$ Abs. 1 . S. 1 erfolgt dies grundsätzlich nur, ,soweit das Informationsinteresse des Antragstellers das schutzwürdige Interesse des Dritten am Ausschluss des Informationszugangs überwiegt oder der Dritte eingewilligt hat." Gegen eine ablehnende Entscheidung ist der Rechtsweg eröffnet, $\mathbb{\$} 9$ Abs. 4. Wird der Antrag genehmigt, werden gem. $\mathbb{1} 10$ Gebühren und Auslagen erhoben.

2205 Soweit es aber um die Analyse von Nutzerverhalten geht, müssen die Nutzer voneinander zumindest unterschieden werden können, weil etwa Verbreiter und oder andere Korrelationen ausfindig gemacht werden müssen. Vgl. hierzu Steinebach u.a., Desinformation aufdecken und bekämpfen, S. $119 \mathrm{f}$.

2206 Sobald der Zugriff erfolgt, haben die Projekte aber ebenfalls ihre Verpflichtungen einzuhalten, wozu u.a. die Protokollierung der Verarbeitung (Art. 30 DS-GVO) gehört.

2207 Einen Überblick und Vorschlag über „best practices“ für die Regulierung von Dateizugriffen findet sich etwa bei Ausloos/Leersen/Thije, Operationalizing Research Access, S. 3 f. 
Anforderungen erfüllt werden. Um generell zu verhindern, dass es zu neuen betreiberseitigen Verzerrungen hierbei kommt, böte es sich ggf. an, ein staatliches Verfahren zu schaffen. ${ }^{2208}$ So könnte etwa den Landesmedienanstalten als staatsferne Stellen ${ }^{2209}$ ein entsprechendes Mandat erteilt werden. Das würde auch gewährleisten, dass bei der für Intermediäre zuständigen Regulierungsbehörde zentrale Informationen der Betreiber $(\mathbb{S} 95 \mathrm{MStV})$ und Informationen über aktuelle Forschungsvorhaben zusammenlaufen und insoweit auch die regulierenden Stellen erreichen. ${ }^{2210}$ Daneben ließe sich auch überlegen, diese Aufgaben an eine unabhängige, anerkannte Stelle der Selbstregulierung zu delegieren. ${ }^{2211}$ Über die Anerkennung könnte dann die Landesmedienanstalt - ähnlich wie bei $\mathbb{\int} 19 \mathrm{JMStV}-$ entscheiden. In jedem Fall erscheint es plausibel, wie im Falle des IFG entstehende Kosten hierfür anteilig auf die Forschungsprojekte als „Verursacher“ umzulegen. Sie müssten aber so angesetzt werden, dass keine „chilling effects“ von ihnen ausgehen. ${ }^{2212}$

\section{Spezieller Zugriff zur Implementation von Meinungsrobotern?}

Gerade im Kontext von Meinungsrobotern wäre es aber u.U. durchaus sinnvoll, dass Forschern auch ein Zugriff ermöglicht wird, der es erlaubt, Meinungsroboter zu implementieren. Damit umgeht man die Erkennungsprobleme und kann unmittelbar ihre Auswirkungen beobachten. Bislang würde ein entsprechender Einsatz - ohne Genehmigung der Betreiber - gegen die Nutzungsbedingungen verstoßen. ${ }^{2213}$ Auch hier stellen sich freilich Fragen um die rechtliche Zulässigkeit, immerhin wird auch hier die Integrität des Dienstes selbst berührt. Diese Fragen lassen sich aber mit entsprechenden Sicherheitsvorkehrungen beantworten. Probleme bereiten hier eher die ethischen Fragen, die mit einem entsprechenden Zugriff vor allem im Verhältnis zu den Nutzern auftauchen und zwingend

2208 Ein solches Verfahren deutet der Kommissionsentwurf des Digital Service Acts, COM(2020) 825 final, in Art. 31 Abs. 2, 4 vor, wenn das Verlangen von dem staatlichen Koordinators für digitale Dienste ergehen muss (Abs. 2) und die Forscher die Anforderungen des Abs. 4 erfüllen müssen.

2209 Für die Notwendigkeit eben solcher Ausloos/Leerssen/Thije, Operationalizing Research Access in Platform Governance, S. $83 \mathrm{f}$.

2210 Weil aber die Landesmedienanstalten in der Regel keine datenschutzrechtlichen Fragen zu klären haben, müsste dafür Sorge getragen werden, dass eine Sensibilisierung für die datenschutzrechtlichen Aspekte erreicht wird. Ob das durch geeignetes Personal und die Schaffung eines besonderen Gremiums erfolgen soll, oder ob das doch dafür spräche, eine andere Stelle damit zu beauftragen, soll hier aber nicht weiter geklärt werden.

2211 Insoweit könnte man sich etwa an den Vorgaben des $\$ 3$ Abs. 6 NetzDG bzw. $\$ 19$ JMStV orientieren.

2212 Hier kann vor allem dem Staat die Aufgabe zukommen, durch finanzielle Unterstützung von Forschungsprojekten den Zugang zu den Netzwerken zu gewähren.

2213 Zu diesen Bedingungen oben, 3. Teil, 3. Kapitel, C.I.2.a. 
zu klären sind. ${ }^{2214}$ Denn Nutzer dürften den eigentlich automatisierten Charakter nicht erfahren, ${ }^{2215}$ wenn die Integrität der Forschungsergebnisse gewährleistet werden soll. ${ }^{2216}$ Problematisch wäre hierbei insbesondere die Simulation der bedenklichen Strategien. ${ }^{2217}$ Inwieweit dem allein durch eine nachträgliche Information über die Eigenschaft und die konkreten Interaktionen mit den Meinungsrobotern abgeholfen werden kann, ist diskutabel. Mit Hilfe der Netzwerkbetreiber könnten auch spezielle Subnetzwerke geschaffen werden, zu denen sich Nutzer freiwillig und ohne Kenntnis des konkreten Forschungsziels registrieren können, in denen abseits der Meinungsroboter aber dieselben Funktionen und Inhalte zur Verfügung stehen. ${ }^{2218}$ Ein potentielles Problem eines solchen Ansatzes bleibt aber, dass die Nutzerstruktur dann womöglich nicht repräsentativ ist. Insoweit gilt es hier vor allem gewisse Leitlinien festzulegen, ob und unter welchen Bedingungen entsprechende Forschung möglich sein soll. Das aber ist keine Frage, die der Gesetzgeber allein beantworten kann. Fest steht insoweit nur, dass erstens ein grundlegendes Bedürfnis für eine entsprechende Forschung besteht und zweitens allein die Nutzungsbedingungen der Netzwerkbetreiber Forschungsvorhaben nicht per se im Weg stehen sollten. 2219

2214 Diese ethischen Fragen deuten u.a. auch schon Krafft/Macy/Pentland, in: Proceedings of the 2017 ACM Conference on Computer Supported Cooperative Work and Social Computing, S. 183 (185 ff.) und Lou/Flammini/Menczer, Information Pollution, S. 2, an.

2215 In dem Versuch von Shirado/Christakis, Nature Vol. 545 (2017), $370 \mathrm{ff}$. hat man (wohl) deshalb die Testpersonen auch nicht über den Einsatz von Bots informiert. Ziel war es zu untersuchen, ob und wie Bots die menschliche Koordination in sozialen Netzwerken (positiv) beeinflussen können. Wegen der fehlenden Information bei Durchführung ihrer Studie äußern Murthy u.a., IJoC 10 (2016), 4952 (4954), ethische Bedenken.

2216 Die Kennzeichnungspflicht dürfte - auch in verfassungskonformer Auslegung im Lichte des Art. 5 Abs. 3 GG - hierfür jedenfalls nicht gelten.

2217 Insoweit geht es nicht um bloße Buchempfehlungen wie bei Aiello u.a., in: Proceedings of the $6^{\text {th }}$ International AAAI Conference on Weblogs and Social Media, S. 10 ff., die in ihrer Folge vergleichsweise unproblematisch sind. Diese Diskussion hat auch schon ein Experiment von Facebook ausgelöst, bei dem der News Feed von Nutzern manipuliert wurde, um zu untersuchen, ob und inwieweit die Stimmung von Nutzern beeinflusst wird, hierzu etwa Meyer, Nature 511 (2014), 265.

2218 Damit würde jedenfalls die datenschutzrechtlichen Fragen entschärft, weil dann zumindest eine freiwillige und informierte Einwilligung als Rechtsgrundlage von Datenverabreitungsvorgängen in Betracht kommt. Allerdings ist fraglich, ob ein grundlegendes Vertrauen der Nutzer besteht, diesen Zugriff zu erlauben. Halvais, Information, Communication \& Society 29 (2019), 1567 (1577) bezeichnet das fehlende Vertrauen als „[o]ne of the greatest barriers“.

2219 Diese Tendenz lässt sich zumindest auch im Urteil Sandvig et al. v. United States, Civil Action Nr. 16-1368 (JDB), US District Court for the District of Columbia erkennen, in dem der von den Angeklagten - akademische Forscher - begangene Verstoß gegen die Nutzungsbedingungen der Webseiteninhaber nicht als Verstoß gegen den Computer Fraud Act 18 U.S.C. \$1030 gewertet wurde. 


\section{E. Klarnamenpflicht für soziale Netzwerke}

Schon mehrfach wurde auch die Möglichkeit der Einführung einer Klarnamenpflicht angesprochen. Auch wenn diese Forderung mit Blick auf Meinungsroboter vorerst nicht durchgreifen kann, ${ }^{2220}$ so bekommt sie mittelbar dadurch Bedeutung, dass auch mit Blick auf den (gefühlten) Anstieg von Hasskriminalität und anderen Rechtsverletzungen in sozialen Netzwerken die Forderung nach einer besseren Identifizierbarkeit der pseudonym oder anonym agierenden Nutzer erhoben wird. ${ }^{2221}$ So hat der Bundesrat zum Zwecke der Bekämpfung von Hasskriminalität im Februar 2020 eine Gesetzesinitiative auf den Weg gebracht, wonach ein neuer $\mathbb{3}$ a NetzDG eingeführt werden soll, der die Betreiber verpflichtet, Nutzer bei der Registrierung zu identifizieren (Abs. 1 S.1) und dabei neben dem Namen und dem Geburtsdatum auch die Anschrift des Nutzers zu erfassen (Abs. 1 S. 2).2222 Nach Abs. 2 soll die Identifizierung natürlicher Personen nur anhand von qualifizierten Identitätsnachweisen erfolgen (Lichtbildausweis, elektronischer Identitätsnachweis u.ä.). Mit dem konkreten Vorschlag verfolgt man eine relative Klarnamenpflicht, die die offensichtlichen verfassungsrechtlichen Probleme einer absoluten Klarnamenpflicht ${ }^{2223}$ dadurch umgeht, dass kein „digitales Vermummungsverbot “2224 eingeführt wird, sondern eine Identifizierung nur im vertraglichen (Innen-)verhältnis zum Netzwerkbetreiber erfolgt. Nach dem Vorbild des $\$ 13$ Abs. 6 TMG bleibt eine anonyme oder pseudonyme Nutzung möglich. So könnte nicht nur eine psychische Hürde vor bestimmten rechtswidrigen und moralisch verwerflichen Handlungen geschaffen werden. Mittelbar könnte

2220 Siehe hierzu bereits oben, 3. Teil, 3. Kapitel, C.II.2.c.aa(II.)(3.) und C.II.3.b.aa(II.). Ein konkretes Bedürfnis kann sich allerdings dann ergeben, wenn entweder die Erkennung von Meinungsrobotern in ihrer Wirkung kaum bedeutsam ist oder wenn sie den zukünftigen technischen Entwicklungen irgendwann nicht mehr gerecht werden kann. Immerhin läuft dies auf ein Katz-und-Maus-Spiel hinaus, bei dem die Betreiber zumeist nur auf Entwicklungen reagieren können.

2221 Hierzu etwa BR-Drs. 70/20. Auch in Österreich wurde ein vergleichbarer Gesetzesentwurf diskutiert, hierzu ZD-Aktuell 2019, 06589. Ausführlich zu einer Ausweispflicht etwa Bock, Die Übertragbarkeit der Kommunikationsfreiheiten des Artikel 5 GG auf das Internet, S. 289ff; zur Klarnamenpflicht ausführlich etwa Griess, Klarnamenspflicht im Internet.

2222 BR-Drs.70/20. Ein solches Modell ebenfalls aufzeigend Bock, Die Übertragbarkeit der Kommunikationsfreiheiten des Art. 5 GG auf das Internet, S. 291, wobei er aber für eine Integration etwa der Ausweisnummer in die öffentlichen Profildaten plädiert, um zu verhindern, dass der Dienstanbieter erst noch in Anspruch genommen werden muss (S.292). Jedenfalls im Grundsatz eine Pflicht zur Erhebung von Nutzerdaten - allerdings ohne nähere Spezifizierung der Art und des Umfangs eben jener - befürwortend Pille, NJW 2018, 3545 (3549).

2223 Eine absolute Klarnamenpflicht ließe sich nicht mit der Meinungsfreiheit vereinbar. Damit würde immerhin stets eine Äußerung auf eine bestimmte Person rückführbar sein, sodass von der Meinungsfreiheit geschützte anonyme Äußerungen nicht mehr möglich wären und insoweit erhebliche chilling effects für die Meinungsfreiheit begründet würden (Schröder, DVBl. 2018, 465 (468); Glaser, NVwZ 2012, 1432 (1437)). Zeitgleich wird auch die Attraktivität der sozialen Netzwerke als aktive Kommunikationsplattform geschmälert, was auch für die unternehmerische Freiheit der Betreiber relevant ist.

2224 So bezeichnend und ausführlicher zu der zu Grunde liegenden „irreführenden Analogie“ Schwander, ZRP 2019, $207 \mathrm{ff}$. 
durch die Betreiber so auch sichergestellt werden, dass keine (automatisierten) Fake-Accounts erstellt werden können.

Das Gesetzesvorhaben kann sich auf unterschiedliche Zielsetzungen stützen: Generalpräventiv könnte es der (angenommenen) anonymitätsbedingten Senkung der Hemmschwelle vor rechtswidrigen Verhalten entgegenwirken. ${ }^{2225}$ Damit begegnete man auch der intensiveren Rechtsverletzung, die durch die Reichweite und Geschwindigkeit, mit der sich Inhalte verbreiten, und der grundsätzlich dauerhaften Speicherung im Internet begründet wird. ${ }^{2226}$ Mit Hilfe der Auskunftsansprüche des $\mathbb{S} 14$ Abs. 2, 3 TMG könnten Täter identifiziert und eine ladungsfähige Anschrift ermittelt werden, was eine effektive Strafverfolgung und Durchsetzung zivilrechtlicher Ansprüche - hinsichtlich der Integrität entsprechender grundrechtlich geschützter Rechtsgüter trifft den Staat immerhin eine Schutzpflicht - 2227 ermöglicht. Und zuletzt kann zeitgleich und mittelbar dafür gesorgt werden, dass die Zahl von Hassnachrichten und dadurch potentiell verursachte Einschüchterungseffekt zurückgehen, die Beteiligung von Nutzern zunimmt und so die Qualität der Diskussionen und das Meinungsklima positiv beeinträchtigt wird. 2228

\section{Notwendigkeit einer entsprechende Identifizierungspflicht}

Bedenken bestehen aber schon daran, ob eine entsprechende Regelung tatsächlich notwendig ist. ${ }^{2229}$ Zwar steht dem Gesetzgeber nicht nur mit Blick auf die potentielle Eignung der Klarnamenpflicht für die relevanten Fragen ein weiter Einschätzungsspielraum zu, ${ }^{2230}$ sondern gerade auch für die Erforderlichkeit. ${ }^{2231}$ Gleichwohl bestehen hier gewisse Bedenken:

2225 BT-Drs. 19/17741, S. 35. Vgl. Krohm/Müller-Peltzer, ZD 2015, 409 mit Blick auf ein Auskunftsrecht Betroffener gegenüber den Betreibern; mit Blick auf eine Verschärfung des Äußerungsstrafrecht etwa Sabl/Bielzer, ZRP 2020, 2 (4). Zur generalpräventiven Wirkung im Kontext der Impressumspflichten etwa Heilmann, Anonymität für User-generated Content, S. 164.

2226 Vgl. EuGH, Urt. v. 03.10.2019 - Rs. C-18/18 = GRUR 2019, 1208 Rn. 28, 36 - Glawischnig-Piesczeck.

2227 Heilmann, Anonymität für User-generated Content, S. 174; Bock, Die Übertragbarkeit der Kommunikationsfreiheiten des Artikel 5 GG auf das Internet, S. 289. Im Kontext des Ehrschutzes etwa Müller-Franken, AfP 2018, 1 (11) oder Glaser, NVwZ 2012, 1432 (1436) und zum insoweit bestehenden rechtspolitischen Handlungsbedarf, ebd., S.1437 Czychowski/Nordemann, NJW 2008, 3095 (3099) sehen in dem Unmöglich-machen einer Identifizierung des Täters von Urheberrechtsverletzungen eine Verletzung der Eigentumsfreiheit des Art. 14 GG. Vgl hierzu auch Hoheisel-Guler, in: Rüdiger/Bayerl, Cyberkriminologie, S. 71 (97).

2228 Vgl. Schweiger, Der (des)informierte Bürger, S. 193 f., mit Blick auf die Aufstockung staatlicher Ermittlungstätigkeiten.

2229 Auch Art. 6 Abs. 4 DS-GVO verlangt, dass es eine „notwendige und verhältnismäßige Maßnahme“ sein muss.

2230 Zur Einschätzungsprärogative bei der Eignung etwa BVerfG NJW 2019, 3703 Rn. 166 - ALG-IISanktionen; BVerfGE 116, 202 (224) - Tariftreueerklärung.

2231 Zum „Beurteilungs- und Prognosespielraum“ bei der Erforderlichkeit etwa BVerfG, NJW 2019, 3054 Rn. 66 - Mietpreisbremse; BVerfGE 116, 202 (225) - Tariftreueerklärung; 146, 71 (126) - 


\section{1. (Faktische) Anonymität als Auslöser rechtswidriger Äußerungen?}

(Faktische) Anonymität wird immer mehr als Problem sozialer Netzwerke und internetbasierter Kommunikation angesehen. ${ }^{2232}$ Gleichwohl lässt sich eine gewisse Disbalance in der Diskussion zum einen deshalb ausmachen, weil Anonymität vielschichtiger ist und die Ermöglichung und Förderung bestimmter rechtswidriger Verhaltensweisen nur ein Aspekt von ihr ist. ${ }^{223}$ Und zum anderen wird suggeriert, dass Anonymität (im digitalen Raum) nicht wünschenswert ja gar etwas „atypisches“ sei, weshalb es eines „digitalen Vermummungsverbotes“2234 bedürfe. ${ }^{2235}$ Das vernachlässigt, dass Anonymität auch wesentliche Bedingung des Agierens in der Gesellschaft ist und nicht nur der selbstbestimmten Freiheitswahrnehmung, ${ }^{2236}$ sondern auch dem objektiven Anliegen des Kommunikationsprozesses dient. ${ }^{2237}$ Und zudem ist sie auch in der realen Welt wesentliche Grundlage der Kommunikation, insbesondere unter Abwesenden.2238 Insoweit lässt sich zumindest kritisch fragen, ob Anonymität zwangsläufig zu der Verrohung der Debattenkultur führt und ob sie maßgeblicher Auslöser für (Persönlichkeits-)Rechtsverletzungen ist. ${ }^{2239}$ Denn auch unabhängig von anonymen

Tarifeinheitsgesetz. Heilmann, Anonymität für User-generated Content, S. 171 sieht gerade die Entscheidung potentieller Identifikationssysteme als allein der Prärogative des Gesetzgebers überlassen an.

2232 Hierzu BT.-Drs. 19/17741. Hierzu auch das österreichische Gesetzesvorhaben eines „Bundesgesetz über Sorgfalt und Verantwortung im Netz“, 134/ME XXVI. GP.

2233 Ausführlicher zur „Anonymität [...] als Segen“ etwa Beater, NJ 2019, 365f, der zu Recht darauf hinweist, dass es „keine Anonymität geben [kann], die nur die Guten schützt.“, ebd. S. 372. Ebenfalls kritisch zur Fokussierung allein auf diesen Einsatzzweck, allerdings im Lichte einer unmittelbaren Klarnamenpflicht, etwa Caspar, ZRP 2015, 233 (235).

2234 So u.a. Schwander, ZRP 2019, 207.

2235 Vgl. dazu die Erläuterungen zum österreichischen Gesetzesentwurf, in denen es gleich zu Beginn heißt: „In der digitalen Welt müssen die gleichen Prinzipien gelten, wie in der real gelebten Welt. Das Internet kann und darf kein rechtsfreier Raum sein [...]. Grundprinzipien, Regeln und Gesetze müssen auch im digitalen Raum gelten. Was in der analogen Welt geahndet wird, muss auch in der digitalen Welt Folgen haben. Sich in der Anonymität des Internets verstecken zu können, darf in Fällen, in denen Straftaten begangen werden, nicht mehr möglich sein. Denn nur wenn durch eine Authentifizierung auch die Identifizierung der Täter bei Rechtsverletzungen möglich ist, werden die gleichen Maßstäbe wie auch in der analogen Welt gelten können. " Insoweit wird einerseits suggeriert, dass im Internet keinerlei Regeln gelten, was so unzutreffend ist. Vielmehr ist es schlicht deutlich schwieriger die allgemeinen Regeln durchzusetzen. Und damit einhergehend wird andererseits suggeriert, dass Anonymität und das Problem einer Identifizierung spezifisch für den Online-Bereich gelten. Dort stellen sich aber schlicht andere, technische Identifizierungsprobleme, die - zugegebener Maßen - erheblicher sein können, als in der realen Welt.

2236 Zur Bedeutung für die selbstbestimmte Wahrnehmung grundrechtlicher Freiheit siehe etwa Heilmann, Anonymität für User-generated Content, S. 86. Daneben etwa auch Caspar, ZRP 2015, 233 (235).

2237 Milker, ZUM 2017, 216 (218). Ausführlicher zu Bedeutung der „Anonymität in der liberalen Demokratie" Kersten, JuS 2017, S. $193 \mathrm{ff}$.

2238 Schwander, ZRP 2019, 207 (208). Vgl. hierzu auch Kersten, JuS 2017, 193 (194).

2239 Kritisch hierzu im Kontext des Vermummungsverbots etwa Heilmann, Anonymität für User-generated content, S. 107; und Jahn, JZ 1988, 545 (546f.), wobei er die Regelung wegen des überwiegend friedlichen Verlaufs von Versammlung zumindest eine Strafbewehrung für nicht erforderlich hält, ebd. S. 550. Mit Blick auf eine Klarnamenpflicht kritisch Caspar, ZRP 2015, 233 (235). Denn ne- 
und pseudonymen Äußerungen lässt sich durchaus der Trend beobachten, dass kommunikative Hemmschwellen gesenkt sind und auch unter dem bürgerlichen Namen - wegen der gefühlten und nicht wegen der tatsächlichen Anonymität gehetzt und verunglimpft wird. ${ }^{2240}$ Insoweit handelt es sich womöglich mehr um ein gesamtgesellschaftliches Problem.

\section{Spezifische Gefahrenlage und fehlende Effektivität bestehender Regelungen?}

Anders als in der realen Welt kann sich eine Äußerung im Internet, insbesondere in sozialen Netzwerken, wesentlich schneller und weiterverbreiten und bleibt für unbegrenzte Zeit für eine Vielzahl von Personen abrufbar. Insoweit geht mit rechtswidrigen (persönlichkeitsverletzenden) Äußerungen eine ungleich höhere Verletzungsintensität einher. ${ }^{2241}$ Bereits bestehende Regelungen könnten aber ausreichend sein, um diesen Gefahren und den Rechtsverfolgungsinteressen gerecht zu werden.

Anders als in der Offline-Welt bestehen mit $\mathbb{1} 14$ Abs. 2, $3 \mathrm{TMG}^{2242}$ bereits Vorschriften, die die Identifizierung entsprechender Nutzer erleichtern sollen. Spezifische Probleme bei der Durchsetzung des Auskunftsersuchens ${ }^{2243}$ werden durch einen entsprechenden bereits verabschiedeten Änderungsentwurf adressiert. Nicht nur die explizite Einführung eines (gesetzlichen) Auskunftsanspruchs in $\$ 14$ Abs. 3 S. TMG ist geplant, ${ }^{2244}$ sondern auch eine vereinfachte gerichtliche Durchsetzung des Anspruchs. ${ }^{2245} \mathbb{\$} 14$ Abs. 3, 4 TMG n.F. soll einen unmittelba-

ben diesem Erklärungsansatz für die Verrohung der Debattenkultur, wird dies durchaus auch damit erklärt, „dass schriftliche Kommunikation bestimmte intrinsische Mängel besonders bei der Kommunikation von Gefühlen habe, die fast zwangsläufig zu Missverständnissen und Konflikten führten[...].“, Wünschner, in: Mühlhoff/Breljak/Slaby, Affekt, Macht, Netz, S. 247.

2240 So etwa auch Caspar, ZRP 2015, 233 (235) und Schunicht, Informationelle Selbstbestimmung in sozialen Netzwerken, S. 259. Siehe auch Sick, in: Möller, Was tun gegen Fake News und Hate Speech?, S. 23 und Hegelich, zit. nach Grandjean, ZUM 2017, 565 (571). Kritisch u.a. deshalb mit Blick auf den österreichischen Gesetzesentwurf, der Chaos Computer Club Wien in seiner Stellungnahme, 96/SN-134/ME XXVI. GP. Kritisch zu diesem Argument aber OLG München, Urt. v. 08.12.2020 - 18 U 2822/19 Pre = BeckRS 2020, 34203 Rn. 56.

2241 So letztlich auch Hoven, ZStW 219 (2017), 718 (743); G. Wagner, GRUR 2020, 447 (455) und Reinlicher, Neue Kriminalpolitik 2020, 186 (191).

2242 Zur datenschutzrechtlichen Vereinbarkeit mit der DSGVO ausführlich BGH ZD 2020, 152 ff. Auskunftsbegehren zu Bestandsdaten.

2243 Zu diesen Problemen ausführlicher etwa Bohlen, NJW 2020, 1999 (2000 ff.).

2244 Zuvor handelte es sich nur um einen vertraglichen Auskunftsanspruch, sodass eine doppelte gerichtliche Geltendmachung notwendig gewesen ist. Nicht nur die grundsätzliche Anordnung war gerichtlich - nach den verfahrensrechtlichen Vorgaben des FamFG ( $\$ 14$ Abs. 4 S. 5 TMG) - geltend zu machen $(\mathbb{1} 14$ Abs. 4 TMG), sondern auch der Anspruch nach $\mathbb{2} 242$ BGB bei Verweigerung durch den Dienstanbieter - hier allerdings nach dem allgemeinen Verfahrensrecht der ZPO. Hierzu siehe auch BT-Drs. 19/18972, S. 55 oder Bohlen, NJW 2020, 1999 (2002). Eine solche Änderung aus diesen Gründen befürwortend Sahl/Bielzer, ZRP 2020, 2 (3).

2245 Hierzu ausführlich Art. 2 des Änderungsentwurfes, BT-Drs. 19/18972, S. 15 und die Begründung, S. 55. Das Gericht entscheidet nämlich grundsätzlich dann nicht nur über die Zulässigkeit, sondern auch über die Verpflichtung des Betreibers. Dadurch wird eine zweite gerichtliche Durchsetzung des Auskunftsanspruchs vermieden. Kritisch mit Blick auf den dennoch nur beschränkten, weil auf $\mathbb{} 1$ 
ren gerichtlich geltend zumachenden Auskunftsanspruch gewähren, der auf die Herausgabe der Bestandsdaten gerichtet ist. Sofern der jeweilige Nutzer ohnehin mit zutreffenden Angaben registriert ist, können also diese Daten ohne Weiteres herausverlangt werden. Gestützt auf $\mathbb{} 14$ Abs. 3 TMG hat zudem das KG Berlin die Weitergabe des Namens, der registrierten E-Mail-Adresse und der IP-Adressen und den Zeitangaben der Uploads angeordnet. ${ }^{2246}$ Inwieweit letztere Informationen bei Fehlen eines bürgerlichen Namens auch tatsächlich für die Durchsetzung der Rechte hilfreich sind, bleibt allerdings abzuwarten. ${ }^{2247}$ Und zudem ist zweifelhaft, ob das Kammergericht nicht den Anwendungsbereich der Norm überschritten hat. Denn bei den IP-Adressen und den Uploadzeitpunkten handelt es sich tendenziell eher um Nutzungsdaten i.S.d. $\$ 15$ Abs. 1 Nr. 1, 2 TMG. ${ }^{2248}$

Abseits dessen bleibt zwar nach wie vor die Einleitung eines strafrechtlichen Ermittlungsverfahrens. Die staatlichen Ermittlungsbehörden haben auch deutlich mehr Kompetenzen ${ }^{2249}$ und sollen bald auch Nutzungsdaten abfragen können. ${ }^{2250}$ Über die Akteneinsicht ( $\mathbb{S} \int 385$ Abs. 3, 406e StPO) könnten dann auch die Daten des Beschuldigten in Erfahrung gebracht werden. Allerdings hilft das kaum weiter, um eine zeitnahe Löschung persönlichkeitsverletzender Inhalte zu bewirken. ${ }^{2251}$ Hierfür besteht aber die Möglichkeit auch die Betreiber selbst von einem Inhalt in Kenntnis zu setzen und nicht nur diesen, sondern auch (zukünftige) wortgleiche Äußerungen gerichtlich sperren zu lassen. ${ }^{2252}$ Und mit $\mathbb{3}$ NetzDG werden entsprechende Ansprüche bereits vor einer gerichtlichen Geltendmachung effektuiert. Gleichwohl ermöglicht das keine Inanspruchnahme des eigentlichen Täters. ${ }^{2253}$

Abs. 3 NetzDG abstellenden, Anwendungsbereich und Schutz vor anonymer Äußerung etwa Beater, NJ 2019, 365 (371f.).

2246 KG Berlin, Beschl. v. 11.03.2020 - $10 \mathrm{~W} 13 / 20$ - juris.

2247 Rein theoretisch wird die (dynamische) IP-Adresse erst dann mit Inhalt gefüllt, wenn ein Abgleich mit den Daten des Access-Providers erfolgt. Telekommunikationsrechtlich bestehen insoweit aber nur Ermächtigungen für staatliche Stellen. Anders ist das etwa mit Blick auf das Urheberrecht, \$101 Abs. 2 Nr. 3 UrhG. Kritisch weist etwa Boblen NJW 2020, 1999 (2001) daraufhin, dass entsprechende Daten aber nicht verpflichtend bereitgehalten werden müssen und dort, wo dies der Fall ist (Access-Provider auch den Vorgaben des TKG), handelt es sich um eine recht kurzfristige Speicherung. Auch deshalb kritisch zu einem solchen Auskunftsanspruch Bock, Die Übertragbarkeit der Kommunikationsfreiheiten des Artikel 5 GG auf das Internet, S. 290 f.

2248 \101 Abs. 9 UrhG nennt hier explizit Verkehrsdaten und knüpft an die Herausgabe ein vorheriges gerichtliches Verfahren.

2249 Etwa das Auskunftsverlangen an den Access-Provider, den hinter einer IP-Adresse agierenden An-

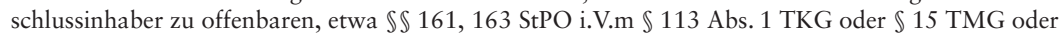
auch $\$ 100 \mathrm{~g}$ Abs. 1, 2 StPO.

2250 Siehe hierzu der geplante Entwurf eines $\$ 15$ a TMG, BT-Drs. 19/17741.

2251 Vgl. G. Wagner, GRUR 2020, 447 (455).

2252 Zur Zulässigkeit umfangreicher Sperr- bzw. Löschanordnungen EuGH, Urt. v. 03.10.2019Rs. C-18/18 = MMR 2019, 798 ff. - Glawischnig-Piesczek.

2253 Vgl. zu diesem Aspekt auch Heilmann, Anonymität für User-generated Content, S. 169. Im Kontext der Verfolgung von Urheberrechtsverletzungen weisen Czychowski/Nordemann, NJW 2008, 3095 (3098f.) auch darauf hin, dass es ,[k]ein Argument [sei], die unmittelbaren Täter vor einer Verfolgung zu schützen, [...] [durch] die Verantwortlichkeit der Internetzugangsprovider [...].“. Siehe 
Wegen derlei Schwierigkeiten und Unzulänglichkeiten kann ein generelles Bedürfnis einer Effektuierung des Auskunftsersuchens und damit u.a. der privaten Rechtsdurchsetzung jedenfalls nicht von der Hand gewiesen werden. ${ }^{2254}$

\section{Kostenrisiko als eigentliches Problem?}

In Österreich wurde allerdings darauf hingewiesen, dass nicht die Identifizierbarkeit das Problem sei, sondern das Kostenrisiko, welches den Rechtssuchenden aufgebürdet wird. ${ }^{2255}$ Ähnliches mag auch für Deutschland gelten, weil der Kläger schon für das gerichtliche Auskunftsersuchen ( $\mathbb{1} 14$ Abs. 4 S. 1 TMG) die Kosten zu tragen hat ( $\$ 14$ Abs. 4 S. 6 TMG), und diese nur in einem zweiten Verfahren vom Schädiger erstattet bekommen kann. Aber auch hinsichtlich der Geltendmachung der Ansprüche innerhalb des zweiten Verfahrens trägt der Kläger ein Kostenrisiko, was faktisch eine Vielzahl von Personen davon abhalten könnte, gegen Äußerungen vorzugehen.

\section{Verhältnismäßigkeitserwägungen}

Nur weil im Internet weitreichendere Möglichkeiten bestehen, Täter zu ermitteln, heißt das nicht, dass davon auch Gebrauch gemacht werden darf. ${ }^{2256}$ Auch bei der Offline-Kommunikation ist grundsätzlich keine Identifikationspflicht vorgesehen. ${ }^{2257}$ Und dort, wo eine spezifische Gefahr besteht - so etwa bei Versammlungen ( $\mathbb{1 7}$ Abs. 2 Nr. 2 VersG) -, wird entweder untersagt, eine potentielle Identifizierung zu erschweren, ${ }^{2258}$ oder anderweitige Schutzvorkehrungen getroffen, die - wie die presserechtlichen Gegendarstellungsrechten $-^{2259}$ die Intensität

auch Beater, NJ 2019, 365 (370). Pille, NJW 2018, 3545 (3548) zweifelt generell an der Wirksamkeit des Verfahrens, u.a. weil es dem Nutzer „häufig schon nicht gelingen [wird], den Diensteanbieter so konkret zu informieren, dass dieser überhaupt zu einer Prüfung veranlasst ist. " Deshalb priorisiert auch er, a.a.O. (3549) die Inanspruchnahme des Täters.

2254 So auch schon mit Blick auf die Notwendigkeit der Impressumspflichten des $\$ 5$ Abs. 1 TMG bzw. $\$ 55$ Abs. 1 RStV, Heilmann, Anonymität für User-generated Content, S. 168 f. Allgemein für eine vorzugswürdige zivilrechtliche Durchsetzung von Persönlichkeitsrechtsverletzungen und der Notwendigkeit eines effektiven Auskunftsanspruchs hierfür, Bohlen, NJW 2020, 1999 ff. Anders aber mit Blick auf den ursprünglichen $\mathbb{1 4}$ Abs. 3 TMG Steinbach, JZ 2017, 653 (661).

2255 Statt vieler Österreichische Rechtsanwaltskammer, Stellungnahme 81/SN-134/ME XXVI. GP, S. 5. In der Diskussion um das NetzDG weisen auch Fehling/Leymann, AfP 2020, 110 Rn. 21 daraufhin, dass wegen des hohen Aufwandes eine gerichtliche Kontrolle unterbleibt.

2256 Ähnlich auch das BVerfG NJW 2012, 1419 Rn. 141; BVerfGE 125, 260 (323 f.) - Vorratsdatenspeicherung.

2257 Zu diesem Aspekt Schwander, ZRP 2019, 207 (208).

2258 So letztlich auch Schwander, ebd. Kritisch deshalb hinsichtlich des Vergleichs von analogen und digitalen Regeln im Kontext des österreichischen Entwurfes, Weißer Ring, Stellungnahme 90/ SN-134/ME XXVI. GP, S. 2 ebenso wie die Österreichische Rechtsanwaltskammer, Stellungnahme 81/SN-134/ME XXVI. GP, S. 6.

2259 Siehe etwa $\mathbb{1} 10$ PresseG MV. Dieser findet sich auch für Telemedien mit journalistisch-redaktionellem Angebot in $\$ 56 \mathrm{RStV}$. 
von Verletzungen zumindest kompensieren sollen. Im Internet ${ }^{2260}$ bestand lange Zeit auch dieses Grundsatz-Ausnahme-Verhältnis, obgleich das Bedürfnis einer Zurücknahme der Anonymität auch für eine effektive Rechtsverfolgung mit den Impressumspflichten anerkannt ist. ${ }^{2261}$ Private Kommunikation war bislang aber

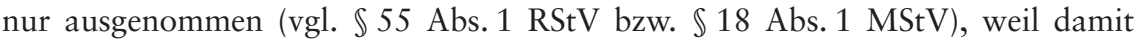
eine absolute Klarnamenpflicht eingeführt worden wäre. Die dadurch für die Meinungsäußerungsfreiheit befürchteten chilling effects werden durch die vorgeschlagene Identifizierungspflicht aber insoweit relativiert, ${ }^{2262}$ als dass eine Offenbarung der Identität der Nutzer primär nur im Verhältnis zum Betreiber erfolgt ${ }^{2263}$ und gegenüber Dritten nur im konkreten Fall und nur durch eine gerichtliche Anordnung ( $\mathbb{S} 14$ Abs. 3, Abs. 4 S.1 TMG), wofür eine vorherige Überprüfung der Rechtswidrigkeit notwendig ist. Insoweit wird also die Meinungsäußerungsfreiheit ebenso verfahrensrechtlich abgesichert ${ }^{2264}$ wie die datenschutzrechtlichen Interessen der Betroffenen. ${ }^{2265}$ Eine Weitergabe erfolgt nur dort, wo das Rechtsverfolgungsinteresse prinzipiell höher $\mathrm{zu}$ bewerten ist. ${ }^{2266}$ Derartige Fälle privilegiert aber schon die DSGVO (vgl. Art. 23 Abs. 1 lit.d), j) DS-GVO). ${ }^{2267}$ Und im internen Verhältnis ist nur das zu offenbaren, was schon jetzt verlangt werden darf (Art. 6 Abs. 1 lit. b) DS-GVO).

Tendenziell mag es aber einen Unterschied machen, ob Betreiber eine Klarnamenpflicht statuieren, diese aber nicht präventiv und effektiv durchsetzen, oder

2260 Zutreffend ist zwar, dass auch in der realen Welt keine vorherige Identifizierung notwendig ist, bevor eine Äußerung getätigt wird (so etwa Österreichische Rechtsanwaltskammer, Stellungnahme 81/ SN-134/ME XXVI. GP, S. 6), allerdings ließe sich der Unterschied hier gerade durch die erschwerte Rechtsverfolgung erklären, die technisch bedingt in der offline Welt nicht in demselben Umfang besteht, weil sich der Einzelne als Person in der Öffentlichkeit bewegt und deshalb jedenfalls durch andere identifiziert werden kann.

2261 Ausführlich zu diesem und den weiteren Aspekten der Impressumspflichten etwa Heilmann, Anonymität für User-generated content, S. $158 \mathrm{ff}$.

2262 Anders mit Blick auf den österreichischen Entwurf allerdings die Österreichische Rechtsanwaltskammer, Stellungnahme 81 /SN -134/ME XXVI. GP, S. 6 die wegen der Notwendigkeit einer Authentisierung und Authentifizierung einen gravierenden Eingriff in die Meinungsfreiheit sehen.

2263 In diese Richtung etwa auch Pille, NJW 2018, 3545 (3549f.). In so einem Fall bestünden dann nämlich deutlich stärkere Bedenken, weil die anonyme bzw. genauer gesagt pseudonyme Äußerung gar nicht mehr möglich wäre. Deshalb befürwortet Boblen, NJW 2020, 1999 (2004) etwa auch die Regelung eines Auskunftsanspruchs anstelle einer Klarnamenpflicht. Dennoch kritisch zum ursprünglichen Entwurf des $\mathbb{} 14$ Abs. 3 TMG Steinbach, JZ 2017, 653 (661).

2264 Beater, NJ 2019, 365 (372) weist daraufhin, dass „[d]er Einschüchterungsaspekt [...] kein generelles Auskunftsverbot legitimieren [kann, sondern] [...] der sachgerechten Ausgestaltung [bedarf], wenn man den Schutz vor Äußerungen nicht komplett abschaffen will." Insoweit fordert er eine Abwägung im Einzelfall zwischen „Informationsinteresse, der Schwere der Beeinträchtigung und dem Anonymitätsinteresse“.

2265 Kritisch mit Blick auf eine Offenbarung entsprechender Daten durch den Auskunftssuchenden und der damit einhergehenden öffentlichen Prangerwirkung Müller-Franken, AfP 2018, 1 (11).

2266 In diese Richtung auch, wenn auch kritisch zu der bloßen Beschränkung auf rechtswidrige Inhalte i.S.d. $\$ 1$ Abs. 3 NetzDG, Beater, NJ 2019, 365 (371f.). Ebenfalls kritisch zu dieser Einschränkung Boblen, NJW 2020, 1999 (2003).

2267 Ausführlicher zur Vereinbarkeit des Auskunftsanspruch mit der DS-GVO BGH, NJW 2020, 536 Rn. $31 \mathrm{ff}$ 
ob der Staat dies von den Betreibern verpflichtend fordert, sodass eine anonyme Nutzung gar nicht möglich ist. Aus unternehmerischer Sicht führt das immerhin dazu, dass Nutzer, die entsprechende Daten nicht zur Verfügung stellen wollen, abgeschreckt werden könnten. ${ }^{2268} \mathrm{Ob}$ die Netzwerk- und Lock-In-Effekte ${ }^{2269}$ so stark sind, dass sie diese Wirkung reduzieren, ist ungewiss. Damit verlören auch die Nutzer eine zentrale Möglichkeit partizipativ am Kommunikationsprozess teilzunehmen. ${ }^{270}$ Dies bleibt trotz der potentiellen Nachteile und Risiken sozialer Netzwerke ein wesentlicher kommunikativer Fortschritt. ${ }^{2271}$

Anders als das BVerfG hinsichtlich einer ähnlichen Regelung für Telekommunikationsanbieter $(\mathbb{S} 111 \mathrm{TKG})^{2272}$ hat der EuGH in der Rechtssache Tele2 Sverige eine umfangreiche Vorratsdatenspeicherung als „die Grenzen des absolut Notwendigen “ überschreitend angesehen. ${ }^{2273}$ Allerdings ging es dort um die umfassende Erhebung nicht nur von Bestandsdaten, sondern auch von Verkehrs- und Standortdaten, sodass ein wesentlich intensiverer Eingriff vorlag ${ }^{2274}$ als etwa bei \111 TKG und bei dem geplanten $\mathbb{\$} 3$ a NetzDG. Anders als in den telekommunikationsrechtlichen Fällen, in denen die Anbieter nur begrenzten Zugriff auf technische Verbindungsdaten haben, speichern und erheben die Anbieter sozialer Netzwerke aber bereits jetzt deutlich umfangreicher Nutzungsdaten ihrer Nutzer,

2268 Schon hinsichtlich der Löschung von rechtswidrigen Inhalten meinte König AcP 219 (2019), 611 (637): „Schon einzelne Nutzer zu vergraulen, ergibt betriebswirtschaftlich in der Regel keinen Sinn. Erst recht müssen sich die Plattformbetreiber vor dem drohenden Zensurvorwurf fürchten, der gerade in den Augen der Internetgemeinschaft besonders schwer wiegt und der Reputation einer Plattform erheblichen Schaden zufügen kann." Vgl. zum Zusammenhang zwischen ökonomischen Wert und Nutzerzahl auch BKartA, Beschl. v. 06.02.2019 - B6-22/16 = BeckRS 2019, 4895 Rn. 405.

2269 Statt vieler BKartA, Beschl. v. 06.02.2019 - B6-22/16 = BeckRS 2019, 4895 Rn. 460 ff. zu den Lock-in-Effekten und Rn. $441 \mathrm{ff}$. zu den Netzwerkeffekten.

2270 Vgl. hierzu auch die Stellungnahme des Weißen Rings zum österreichischen Entwurf, 90/ SN-134/ME XXVI. GP., S. 1 f.

2271 Reinbacher, JZ 2020, 558 sieht sie als „gewichtigen Beitrag zur Demokratisierung der Informationsbeschaffung “ an und - allerdings mit Blick auf das Internet insgesamt - spricht Nolte, ZUM 2017, 552 (553) von einer „Demokratisierung des Kommunikationsprozesses.

2272 Das BVerfG hat die Regelung explizit als nicht gravierenden Eingriff bewertet, u.a. weil es sich um wenig aufschlussreiche Daten handelte und die Datenerhebungszwecke, ähnlich wie auch bei $\mathbb{} 14$ Abs. 2, 3 TMG, eng begrenzt waren, BVerfGE 130, 151 (189) - Bestandsdatenspeicherung.

2273 EuGH, Urt. v. 21.12.2016 - Rs. C-203/15 u. C-698/15, ECLI:EU:C:2016:970, Rn. 105 - Tele2 Sverige $A B u$. Secretary of State for the Home Department: „Zum anderen sieht eine nationale Regelung [...], die sich allgemein auf alle Teilnehmer und registrierten Nutzer erstreckt und alle elektronischen Kommunikationsmittel sowie sämtliche Verkehrsdaten erfasst, keine Differenzierung, Einschränkung oder Ausnahme in Abhängigkeit von dem verfolgten Ziel vor. Sie betrifft pauschal sämtliche Personen, die elektronische Kommunikationsdienste nutzen, ohne dass sich diese Personen auch nur mittelbar in einer Lage befinden, die Anlass zur Strafverfolgung geben könnte. Sie gilt also auch für Personen, bei denen keinerlei Anhaltspunkt dafür besteht, dass ihr Verhalten in einem auch nur mittelbaren oder entfernten Zusammenhang mit schweren Straftaten stehen könnte. Zudem sieht sie keine Ausnahme vor, so dass sie auch für Personen gilt, deren Kommunikationsvorgänge nach den nationalen Rechtsvorschriften dem Berufsgeheimnis unterliegen." Diese restriktive Haltung zur Vorratsdatenspeicherung bestätigte der EuGH jüngst in den Urteilen v. 06.10.2020, C-623/17 = ECLI:EU:C:2020:790 - Privacy International und C-511/18 u.a., ECLI:EU:C:2020:791 - Quadrature du Net u.a.

2274 Ausführlicher hierzu EuGH, Urt. v. 21.12.2016 - Rs. C-203/15 u. C-698/15, ECLI:EU:C:2016:970, Rn. 93 ff. - Tele 2 Sverige AB u. Secretary of State for the Home Department. 
die sodann über die Klarnamen zu einem personenspezifischen Persönlichkeitsund Verhaltensprofil zusammengefügt und mit der Anschrift noch weiter ergänzt werden können. ${ }^{2275}$ Die bisherigen Datensätze der Profilbildung erhalten mit dem Klarnamen und der Anschrift des Nutzers eine noch größere (wirtschaftlich) Bedeutung. ${ }^{2276}$ Insoweit besteht also die Gefahr, dass man die Markt- bzw. Datenmacht bestehender Anbieter intensiviert. ${ }^{2277}$ Zudem schafft man dadurch neue spezifische Gefahren für so gespeicherte Daten. Denn es ist nicht sichergestellt, dass entsprechende Daten nur zu diesem Zweck verwendet, ${ }^{2278}$ und vor allem, dass sie sicher gespeichert werden. ${ }^{2279}$ Immerhin bestehen schon jetzt erhebliche Bedenken an der Datenverarbeitungspraxis der Betreiber. ${ }^{2280}$

Für die Betreiber selbst dürften die Pflichten vergleichsweise leicht zu erfüllen sein, weil sich ein automatisierter Abgleich entsprechender Daten mit amtlichen Dokumenten weniger aufwendig gestaltet als etwa eine umfangreiche Überwachung von Netzwerkdaten. ${ }^{2281}$ Aus Sicht der Betreiber entspricht die gesetzliche Regelung zudem ihren potentiellen, wenn auch bislang nicht durchgesetzten Interessen einer genauen Identifizierung der Nutzer. ${ }^{2282}$

2275 Mit dem Fehlen dieser Profile hat das BVerfG, a.a.O., Rn. 139 aber gerade die nur geringe Eingriffsintensität begründet. Kritisch mit Blick auf die Ergänzung dieser Daten auch Richter, MMR 2014, 517 (520) und mit Blick auf den österreichischen Entwurf Weißer Ring, Stellungnahme 90 / SN-134/ME XXVI. GP, S. 2.

2276 So auch Caspar, ZRP 2015, 233 (234f.). Ziebarth, ZD 2013, 375 (377) spricht insoweit von einer „Vergoldung“ der Profilbildung. Vgl. auch Hegelich, zit. nach Grandjean, ZUM 2017, 565 (571).

2277 Ziebarth, ebd. weist auf die Möglichkeit der netzwerkübergreifenden Verknüpfung von Profildaten hin. Allerdings wurde dies vom Bundeskartellamt jedenfalls beim Facebook-Konzern für kartellrechtlich unzulässig gehalten Beschl. v. 06.02.2019 - B6-22/16 = BeckRS 2019, 4895.

2278 Kritisch etwa Caspar, ZRP 2015, 233 (236); Nebel, K\&R 2019, 148 (152) und Oehmer/Pedrazzi, UFITA 2020, 7 (22). Der Weiße Ring weist in seiner Stellungnahme zum österreichischen Entwurf, 90/SN-134/ME XXVI. GP., S. 1 allgemein „,auf Szenarien von Datenpannen und Datendiebstählen“ hin. Allgemein auf „privacy concerns“ hinweisend Abulaish/Fazil, IEEE Technology and Society Magazine 2020, 52 (59).

2279 Vor allem in Hinblick auf die Datenweiterleitung in die USA bestehen nach wie vor erhebliche Bedenken. Der Privacy Shield wurde jüngst vom EuGH, Urt. v. 16.07.2020 - Rs. C-311/18, ECLI:EU:C:2020:559, - Data Protection Commissioner/Facebook Ireland Limited und Schrems, ebenso wie zuvor die Safe-Harbour-Regelung (hierzu EuGH, Urt. v. 06.10.2015 - Rs. C-362/14 = NJW 2015, 3151 Rn. 67 ff. - Schrems/Digital Rights Ireland) angegriffen, wobei Maßstab hierbei nicht mehr die DatenschutzRL 1995/46, sondern die DS-GVO war, ebd., Rn. 79.

2280 Ausführlich hierzu BKartA, Beschl. v. 06.02.2019 - B6-22/16 = BeckRS 2019, 4895 Rn. 629 ff., und speziell zu fehlenden Schutzmaßnahmen Rn. $787 \mathrm{ff}$.

2281 Kritisch aber zu den Belastungen Nietsch, Anonymität und die Durchsetzung urheberrechtlicher Ansprüche im Internet, S. 253.

2282 Dieses berechtigte Interesse sieht u.a. auch Heberlein, Datenschutz im Social Web, S. 164 f. Zu der besseren Vermarktung solcher Daten siehe etwa Caspar, ZRP 2015, 233 (234f.). Daraus leitet auch Nietsch, ebd., die geringer Eingriffsintensität ab. 
III. Fazit: Einführung einer relativen Klarnamenpflicht als rechtspolitisches Problem

Auch wenn sich eine Klarnamenpflicht allein zur Bekämpfung von Meinungsrobotern zurzeit nicht anbietet, ${ }^{2283}$ kann sie (mittelbar) dadurch eingeführt werden, dass die effektive Strafverfolgung und Rechtsdurchsetzung grundrechtlich geschützter Güter über bereits bestehende Regelungen hinaus gestärkt wird. Weil aber mit dem (angepassten) $\mathbb{} 14$ Abs. 2, 3 TMG, den strafrechtlichen Befugnissen, der Störerhaftung und dem Beschwerdeverfahren des $\mathbb{3}$ NetzDG jedenfalls ein nicht offensichtlich unwirksames Schutzkonzept besteht, ${ }^{2284}$ obliegt es dem Gesetzgeber im Rahmen seiner Prärogative eine darüber hinausgehende relative Klarnamenpflicht einzuführen, um seiner grundrechtlichen Schutzpflicht nachzukommen. Das Übermaßverbot steht dem jedenfalls nicht per se im Weg, ${ }^{2285}$ weil ein vertretbarer Ausgleich zwischen den kollidierenden Interessen bewirkt wird und - jedenfalls sofern das Auskunftsrecht allein auf strafrechtswidrige Inhalte i.S.d. $\mathbb{S} 1$ Abs. 3 NetzDG gestützt ist - die Rechtsverfolgungsinteressen überwiegen. ${ }^{2286}$ Gegebenenfalls müssten mit Blick auf die Datenspeicherung gewisse Sicherheitsvorkehrungen getroffen werden. ${ }^{2287}$ Im Wesentlichen dürfte es daher eine rechtspolitische Frage sein, ob man einerseits eine flächendeckende Identifizierbarkeit einfordert, obwohl bereits jetzt abseits anonymer Äußerungen dieselben Probleme bestehen, oder ob man andererseits bei der Effektuierung der Rechtsdurchsetzung bzw. -Verfolgung erst auf staatlicher Seite ansetzt, ${ }^{2288}$ und die weitere Entwicklung beobachtet. Immerhin werden auch andere Modelle vorgeschlagen, die eine undifferenzierte wenn auch nur mittelbare Offenlegung der Identität vermeiden. 2289

2283 In diese Richtung, allerdings vor allem mit Blick auf datenschutzrechtliche Erwägungen Milker, ZUM 2017, 216 (221).

2284 Vgl. hierzu im Kontext der Impressumspflichten, Heilmann, Anonymität für User-generated Content, S. $176 \mathrm{f}$.

2285 So auch - allerdings zu einem etwas anderen Modell - Bock, Die Übertragbarkeit der Kommunikationsfreiheiten des Art. 5 GG auf das Internet, S. 295, 297.

2286 Teilweise wird dafür plädiert, das Auskunftsrecht auch auf Fälle schwerwiegender, persönlichkeitsrechtsverletzender Äußerungen auszuweiten, die nicht dem $\$ 1$ Abs. 3 NetzDG unterfallen, so etwa Beater, NJ 2019, 365 (372) und Boblen, NJW 2020, 1999 (2003).

2287 In der Literatur wurde schon zuvor vorgeschlagen, dass etwa eine unabhängige neutrale Stelle entsprechende für die Rechtsverfolgung relevante Daten der Nutzer und von den Netzwerken getrennt speichert, so etwa Richter, MMR 2014, 557 (520) oder auch Nietsch, Anonymität und die Durchsetzung urheberrechtlicher Ansprüche im Internet, S. 252.

2288 Dies fordern mit Blick auf das österreichische Unterfangen Chaos Computer Club Wien, Stellungnahme 96/SN-134/ME XXVI. GP., S. 2. Ein entsprechender Regierungsentwurf sieht etwa die Verschärfung von äußerungsrechtlichen Straftatbeständen für Äußerungen, die im Internet getätigt werden vor, BT.-Drs. 87/20. Zum Entwurf siehe auch Reinbacher, Neue Kriminalpolitik 2020, S. $186 \mathrm{ff}$.

2289 Vorgeschlagen wurde etwa auch ein an das Gewaltschutzgesetz angelegtes Modell, dass zu gerichtlich angeordneten Accountsperrungen führen kann, siehe hierzu Hoheisel-Guler, in: Rüdiger/Bayerl, Cyberkriminologie, S. 71 (97 ff.). Daneben siehe auch Oehmer/Pedrazzi, UFITA 2020, 7 (22) in Anlehnung an die estländische e-ID Lösung. 


\section{F. Medienkompetenz}

Wenn vor allem auch die Medienkompetenz der Nutzer essentielle Voraussetzung dafür ist, Informationen kritisch zu rezipieren und damit vorsichtig in digitalen Diskursräumen zu agieren, so muss kritisch beobachtet werden, ob die bisher ergriffenen und geplanten Maßnahmen sowohl der KMK im schulischen Bereich als auch die (freiwilligen) Maßnahmen der Betreiber und anderer Akteure (hierzu oben 2. Teil, 3. Kap C.I.3.b) die notwendige Sensibilisierungswirkung erreichen und den Aufbau einer Medienkompetenz tatsächlich fördern ${ }^{2290}$. Denn auf schulischer Ebene mag zwar der integrative, fächerübergreifende Ansatz der KMK durchaus Sinn machen, weil Fragen der Digitalisierung und der Medienkompetenz und -pädagogik weitreichende interdisziplinäre Bezugspunkte aufweisen.2291 Allerdings wird damit die Aufgabe einer sachgerechten Bildung auf viele spezifizierte Lehrkräfte aufgeteilt, denen möglicherweise das notwenige Detailwissen fehlt. ${ }^{2292}$ Daher kann es durchaus sinnvoll ${ }^{2293}$ sein, ein spezielles Unterrichtsfach zu entwickeln, ${ }^{2294}$ das sich zentralen Fragen widmet und welches dann durch die anderen Fächer sinnvoll ergänzt wird. ${ }^{2295}$ Damit würde immerhin gewährleistet,

2290 Freilich ist dabei zu berücksichtigen, dass die Maßnahmen eine Zielvorgabe sind, also nur danach zu fragen ist, ob die ergriffenen Maßnahmen und die konkrete Umsetzung geeignet sind, die Ziele zu verwirklichen, denn „bei dem Versuch, die Voraussetzungen von demokratisch-liberalen Einstellungsmustern und Lebensformen pädagogisch zu fördern [...] [gibt es] keine Erfolgsgarantien [...].“, Drerup, in: Binder/ders., Demokratieerziehung und Bildung digitaler Öffentlichkeit, S. 29 (47f.).

2291 Hierzu KMK, Bildung in der digitalen Welt - Strategie der Kultusministerkonferenz, S. 12. Einen integrativen Ansatz befürwortend etwa Dengel, Medienpädagogik 2018, 11 (23 f.). Mit besonderem Blick auf Social Bots Knorr, Social Bots im Unterricht, S. 24 inklusive entsprechender Vorschläge für Unterrichtseinheiten im Fach Informatik und Geschichte, S. 29 ff. Mit Blick auf Social Bots aber zumindest offenlassend, ob das die geeignete Strategie ist, Kind u.a., Social Bots, S. 66.

2292 Kritisch insoweit auch Schweiger, Der (des)informierte Bürger, S. 190; Graber/Lindemann, in: Sachs-Hombach/Zywietz, Fake News Hashtags \& Social Bots, S. 51 (65); Schmid/Stock/Walter, a.a.O., S. 69 (88), die zudem auch auf den erheblichen Aufwand hinweisen, der mit der Erstellung von Lernmaterialien verbunden ist; Dengel, Medienpädagogik 2018, 11 (24).

2293 Das auch deshalb, weil länderübergreifend Unterschiede bei dem Fachangebot bestehen. Anders als in MV findet man in NRW (Pflicht-)Fächer wie Informatik und Sozialkunde nämlich nicht. Deshalb unterscheiden sich auch die zu vermittelnden Lerninhalte etwa mit Blick auf die Algorithmen. Sozialkunde und Informatik betrachten neben der technischen Seite auch Fragen der Transparenz und der Auswirkungen. In den entsprechenden vorschlagen der fachspezifischen Umsetzung des Medienkompetenzrahmens NRW fehlen diese Aspekte deshalb etwa, abrufbar unter https://www.sc hulentwicklung.nrw.de/lehrplaene/upload/klp_SI/GY19/KLP_SI_MKR_Formulierungen_finalb_doc x.pdf, (Stand: 30.05.2021).

2294 Schweiger, Der (des)informierte Bürger, S. 190 sieht auch die Möglichkeit diese Kompetenz und Sensibilisierungswirkung durch externe Personen im Rahmen von „Workshops“ zu vermitteln.

2295 In diese Richtung auch Gesellschaft für Informatik, Dagstuhl-Erklärung, S. 1, die darauf hinweist, dass die Vermittlung von Lehrinhalten eine umfangreiche Betrachtung der verschiedenen Erscheinungsformen erfordert, die technologische (Wie funktioniert das?), gesellschaftlich-kulturelle (wie wirkt das?) und eine anwendungsbezogene Perspektive (Wie nutze ich das?) kombiniert, dazu ebd., S. 3. Diese drei Perspektiven hingen aber zwingend zusammen und müssten daher auch gemeinsam und nicht in unterschiedlichen Fächern betrachtet werden, a.a.O. S. 4. Auch Schweiger, Der (des)informierte Bürger, S. 190 sieht die Notwendigkeit eines multipolaren Ansatzes, nämlich jungen Menschen die „Funktionsweisen personalisierter Aggregatoren und die Gefahren von Filterblasen und 
dass die unterschiedlichen Sphären der digitalen Welt zusammenhängend beleuchtet werden können. So ließe sich auch vermeiden, dass durch die ohnehin umfangreichen fachspezifischen Themenkomplexe, die die Lehrpläne seit jeher vorgeben, ein zu geringer zeitlicher Rahmen bleibt, ${ }^{2296}$ was sich dann noch verschärft, wenn Lehrkräfte die neuen Fragen um die digitale Medienkompetenz weniger priorisieren. ${ }^{2297}$ Und ganz generell muss gewährleistet werden, dass die Lehrkräfte selbst die notwendige Kompetenz und das Wissen haben, diese Themen $\mathrm{zu}$ vermitteln. Die Bereitstellung von Unterrichtsmaterialien ist allenfalls eine Hilfestellung für sie. Insoweit muss in der Aus- und Fortbildung der Lehrkräfte noch ein deutlicherer Fokus auf Fragen der Digitalisierung gelegt werden. Sowohl im schulischen als auch im Bereich der Lehrerausbildung besteht jedenfalls die Möglichkeit sich an den nach dem MStV verpflichtenden Informationen $\mathrm{zu}$ orientieren und anhand dieser das eigentliche Wissen sinnvoll zu vermitteln.

Zudem wird es notwendig bleiben, auch außerhalb der Schulen für eine entsprechende Kompetenzbildung und Sensibilisierung Sorge zu tragen. Dafür kann eine staatliche Einrichtung hilfreich sein, die ein entsprechendes Angebot wie die Bundeszentrale für politische Bildung bereithält. ${ }^{2298}$ So könnten auch die Schulen und Lehrkräfte durch entsprechende Materialien und Inhalte unterstützt werden. Auch kann der weitere Ausbau der Präsenz öffentlich-rechtlicher Medien hilfreich sein. Dies muss aber durch entsprechende Angebote ergänzt werden, die die Betreiber selbst vorhalten (müssen). ${ }^{2299}$ Eine Berichtspflicht ist nur ein erster Schritt einer Aufklärungs- und Sensibilisierungskampagne. Vor allem sollten Betreiber weiterhin ermutigt werden, eigenständige Ansätze zu entwickeln und beizubehalten und dies gegebenenfalls von staatlicher Seite aus zu fördern und zeitgleich auch für ein Minimum an Kontrolle zu sorgen.

\section{G. Ergänzender Schutz vor Desinformation}

Nach wie vor unberücksichtigt bleibt das spezielle Problem der Desinformation, das freilich kein singuläres Phänomen von Meinungsrobotern ist, sondern generell Risiken aufwirft. Dabei geht es nicht nur um die individuelle Wirkungssphäre

Echokammern erklärlich [zu] machen“, „die individuellen und gesellschaftlichen Folgen einer extrem verzerrten Meinungsklimawahrnehmung in Aggregatoren nachvollziehbar [zu] machen und zu ermitteln, dass der Journalismus [...] die einzige Instanz in unserer Gesellschaft ist, deren Existenz darauf aufbaut, wahre und ausgewogene Nachrichten mit einer gewissen Qualität zu verbreiten.“.

2296 Vgl. hierzu die Studienergebnisse einer Lehrerbefragung, Institut für Demoskopie Allensbach, Die Vermittlung von Nachrichtenkompetenz in der Schule, S. $40 \mathrm{ff}$.

2297 Siehe zu dieser unterschiedlichen Gewichtung auch im Ost-West-Vergleich Institut für Demoskopie Allensbach, a.a.O., S. $34 \mathrm{ff}$.

2298 Vgl. hierzu BT-Drs. 19/18729.

2299 Die Verantwortung der Betreiber bei der Vermittlung der Kompetenzen betonen auch Oehmer/ Pedrazzi, UFITA 2020, 7 (20), und speziell mit Blick auf Meinungsroboter S. 23. 
von Desinformation, sondern auch um ihre kollektive, destabilisierende und segregierende Wirkung. ${ }^{2300}$

I. Wahlrechtliche Maßnahmen nach dem Vorbild des „loi relative à la lutte contre la manipulation de l'information" Frankreichs

Zur Bekämpfung von Desinformation hat der französische Gesetzgeber mit dem „loi relative à la lutte contre la manipulation de l'information“ den Code electorale ergänzt. ${ }^{2301}$ Besonders nennenswert ist hierbei neben den Transparenzvorgaben des Art. L 163-1 für Plattformbetreiber, die in den drei Monaten vor der Wahl gelten, ${ }^{2302}$ vor allem Art. L 163-2 Abs. 1. Dieser ermöglicht es einem Richter auf Antrag, Anordnungen zu erlassen, um in demselben Zeitraum die vorsätzliche, künstliche oder automatische und massive Verbreitung falscher Darstellungen oder Anschuldigungen an die Öffentlichkeit, die geeignet sind, die Integrität der Wahl zu gefährden, zu unterbinden. Damit geht Frankreich einen mit der ECommerce-RL kompatiblen Weg. ${ }^{2303}$ Zwar käme eine Umsetzung etwa auch in \32 BWahlG durchaus in Betracht. Allerdings bleiben Falschinformationen nicht nur unmittelbar vor Wahlen ein Problem, sondern auch unabhängig davon können sie das politische Klima und damit eine später stattfindende Wahl beeinflussen. Und ohnehin wird die Durchsetzung einerseits durch das Antragserfordernis $^{2304}$, andererseits auch dadurch erschwert, dass auf jeden Inhalt einzeln reagiert werden muss. Zumindest aber sieht das Gesetz eine Entscheidungsfrist von 48 Stunden vor (Art. L-163-2-II, III), sodass vergleichsweise schnell auf entsprechende Inhalte reagiert werden kann. Und mit dem Richtervorbehalt ${ }^{2305}$ wird ein hoher Schutz für die betroffene Äußerung gewährleistet.

2300 Hierzu auch Hoven, ZStW 129 (2017), 718 (737).

2301 LOI n $^{\circ}$ 2018-1202 du 22 décembre 2018 relative à la lutte contre la manipulation de l'information.

2302 U.a. identitätsbezogene Informationen anderer Nutzer, Informationen zur Verwendung personenbezogener Daten im Kontext der Förderung bestimmter Inhalte und Informationen über Vergütungen, die für bestimmte Werbung bezahlt wurden.

2303 Denn es wird einzelfallbezogen gerichtlich eine bestimmte Anordnung getroffen, was nach Art. 14 Abs. 3 der Richtlinie ohne Weiteres möglich ist. Und seit EuGH, Urt. v. 03.10.2019 - Rs. C-18/18 = MMR 2019, 798 ff. - Glawischnig-Piesczek, kann eine solche Anordnung ja auch inhaltsgleiche Beiträge Dritter umfassen.

2304 Nach den französischen Vorgaben ist neben den Wahlkandidaten, einer Partei oder einer politischen Gruppe auch ein Staatsanwalt oder jede andere Person, die ein Interesse an dem Antrag hat, antragsbefugt. Ob das auch, angesichts der vordergenannten Gruppen, jeder Wahlberechtigte sein kann, wäre jedenfalls diskutabel.

2305 Letztlich zeigt der französische Gesetzgeber damit auch, dass es eine staatliche Aufgabe ist, Desinformation zu begegnen, die hier - anders als etwa bei Hassrede (hierzu Loi visant à lutter contre les contenus haineux sur internet, dass aber durch den Conseil Constitutionnel als partiell verfassungswidrig angesehen wurde, Décision n²020-801 DC du 18 juin 2020) - nicht auf die privaten Anbieter übertragen werden soll. 


\section{Strafrechtliches Verbot von Desinformation?}

De lege lata werden desinformative Inhalte strafrechtlich an verschiedenen Stellen punktuell erfasst, ${ }^{2306}$ weshalb schon länger über die Ergänzung bestehender bzw. über die Einführung neuer Straftatbestände nachgedacht wird. Besonders hervorzuheben sind dabei zwei Ansätze: ein in Ergänzung der $\mathbb{S} \mathbb{S} 108,108$ a StGB individualschützender, wahlrechtlicher und ein allgemeiner, auf die kollektive Sphäre des Kommunikationsprozesses gerichteter Schutz. ${ }^{2307}$ In beiden Fällen bestünde dann jedenfalls durch Aufnahme in den Katalog des $\mathbb{1}$ Abs. 3 NetzDG die Möglichkeit, dass die Nutzergemeinschaft den Schutz auch wirksam gegenüber den Betreibern sozialer Netzwerke einfordern könnte. ${ }^{2308}$ Mit beiden Ansätzen sind allerdings spezifische Probleme verbunden, die hier zumindest kurz skizziert werden sollen.

\section{Wablrechtspezifischer Schutz}

Zentrales Problem eines individualorientierten wahlrechtlichen Schutzes ${ }^{2309}$ ist letztlich die Verantwortungsverteilung zwischen Äußerndem und Rezipienten. Denn auch wenn die Äußerung einer wahlrechtsrelevanten Information moralisch verwerflich sein mag - das sind auch „Wahlkampflügen“ der Kandidaten -, ist damit nicht per se ein strafrechtlich relevantes Unrecht verbunden. ${ }^{2310}$ Wesentliche fehlerhafte Informationen für die Wahlmodalitäten sind mit $\mathbb{S} 108 \mathrm{a}$ StGB bereits erfasst. Daneben würden wohl nur besonders krasse Fälle strafrechtliche Relevanz haben. Abgesehen davon spielt aber auch die Informationsaufnahme eine nicht zu vernachlässigende Rolle, wenn es um das Ausmaß der Wirkung einer Desinformation geht. So weist etwa Rostalski auf die Mündigkeit der Wahlberechtigten hin. ${ }^{2311}$ Ihnen obliege die Aufgabe, Informationen kritisch zu hinterfragen. ${ }^{2312}$ Auch wenn ihr - angesichts der fehlenden Medienkompetenz -

2306 Siehe hierzu bereits oben, 3. Teil, 3. Kapitel, C.I.2.d.bb. Ausführlich hierzu auch Hoven, ZStW 129 (2017), 718 ff.; dies., JuS 2017, 1167 ff. und Rostalski, Rechtswissenschaft 2017, 436 ff.

2307 In diese Richtung auch Mafi-Gudarzi, ZRP 2019, 65 (68), zum wahlrechtlichen Ansatz siehe auch Rostalski, Rechtswissenschaft 2017, 436 (444ff.). Speziell zu Deep-Fakes siehe etwa Lantwin, MMR 2020, 78 ff.; zur Ergänzung bestehender Regelungen siehe Rostalski, a.a.O., 436 ff. Ein spezifischer Fall hat auch Kalifornien nun geregelt, nämlich die Verbreitung sog. Deep-Fakes von Kandidaten, um zu verhindern, dass ihre Reputation unmittelbar vor der Wahl beeinträchtigt wird oder dadurch Einfluss auf die Wähler genommen werden soll, Assembly Bill Nr. 730 und hierzu MMRAktuell 2019, 421493.

2308 Dazu auch Mafi-Gudarzi, ZRP 2019, 65 (68).

2309 Hierzu umfassender Rostalski, Rechtswissenschaft 2017, 436 (444 ff.). Siehe auch Steinebach u.a., Desinformation aufdecken und bekämpfen, S. $181 \mathrm{f}$.

2310 Insoweit wird richtiger Weise darauf hingewiesen, dass gezielte Desinformation und Propaganda schon immer verwendet wurde, und insoweit nur die „Durchschlagskraft“ im Internet neu ist, Holznagel, MMR 2018, 18 (19).

2311 Rostalski, Rechtswissenschaft 2017, 436 (444ff.).

2312 Rostalski, a.a.O. (445). 
für den Bereich der sozialen Netzwerke nicht ohne Weiteres zuzustimmen sein mag, so liegt das zentrale Problem doch eher in der verzerrten Informationsaufnahme als in der Falsch-Nachricht an sich. So weist Rückert nicht nur darauf hin, dass die Wahlentscheidung in erster Linie unabhängig von desinformativen Inhalten erfolgt, sondern - und das ist das eigentlich entscheidende - nicht die Desinformation kausal für eine bestimmte Meinungsbildung und Wahlentscheidung, sondern umgekehrt die vorgeprägte Meinung kausal für die Auswahl und Aufnahme desinformativer Inhalte ist (confirmation bias). ${ }^{2313}$ Insofern liegt das Problem eher auf Rezipientenseite, dem man durch die Entwicklung einer „Medienkompetenz" durchaus begegnen kann. Strafrechtlich relevant ist dies allerdings nicht.

\section{Kollektiver Schutz des Kommunikationsprozesses}

Überzeugender könnte daher ein Schutz des kollektiven Kommunikationsprozesses und damit des (kommunikativen) öffentlichen Friedens ${ }^{2314}$ sein. Bereits der Tatbestand der Volksverhetzung zeigt, dass äußerungsrechtliche Grenzen auch eine kollektive und gemeinwohlorientierte Schutzwirkung entfalten, sodass ein ergänzender strafrechtlicher Schutz nicht von vornherein abgelehnt werden kann. ${ }^{2315}$ Weil damit auch keine spezifische Meinung als solche verboten würde, wäre eine Pönalisierung auch im Lichte des Schrankenvorbehalts der allgemeinen Gesetze (Art. 5 Abs. 2 GG) möglich. ${ }^{2316}$ Schwierig gestaltet sich allein die Suche nach einem hinreichend bestimmten, effektiven und verhältnismäßigen Straftatbestand. ${ }^{2317}$

Vergleichsweise unproblematisch wäre die Sanktionierung der vorsätzlichen Verbreitung unwahrer Tatsachen, da dies schon nicht von der Meinungsfreiheit geschützt ist. ${ }^{2318}$ Damit erfasst man aber oftmals nur den ursprünglichen Urheber eines Inhalts. ${ }^{2319}$ Nicht strafbar wäre die Weiterleitung durch Dritte, jedenfalls dann, wenn die Informationen ohne kritische Überprüfung von anderen für richtig bewertet, geteilt, und als Äußerung zu eigen gemacht werden und damit

2313 Siehe hierzu Rückert, in: Albrecht u.a., Strafrecht und Politik, S. 167 (177ff.). Daher sei auch die Einführung eines entsprechenden Straftatbestandes abzulehnen, ebd., S. 181.

2314 So auch Mafi-Gudarzi, ZRP 2019, 65 (68).

2315 Vgl. Zu der Notwendigkeit der Berührung dieser kollektiven Sphäre Hoven, ZStW 129 (2017), 718 $(740,741)$.

2316 Ausführlicher zum allgemeinen Gesetz etwa BVerfGE 124, 300 (321 ff.) - Wunsiedel.

2317 Ausführlicher hierzu auch Hoven, ZStW 129 (2017), 718 (738 ff.).

2318 So auch Ferreau, in: Möller/Hamaleers/ders., Typen von Desinformation und Misinformation, S. 44 (69).

2319 Und zudem nur den Lügner, nicht aber den Leugner, also denjenigen, sich auf seine subjektive Wahrnehmung verlässt statt auf objektive Fakten. „Nicht der Lügner, wohl aber der Leugner handelt wahrhaftig, weil er von der Wahrheit seiner Aussage subjektiv überzeugt ist. Leugnen ist demnach auch das gutgläubige Inabredestellen einer Tatsache.“, Steinbach, JZ 2017, 653 (656). 
fahrlässig gehandelt wird. ${ }^{2320}$ Und zudem werden damit nur offensichtliche Fälle erfasst, die lediglich einen Bruchteil der problematischen Fälle ausmachen. ${ }^{2321}$

Die Lösung könnte eine Erweiterung auf Äußerungen, deren Unwahrheit im Zeitpunkt der Äußerung dem Äußernden (noch) nicht bekannt ist, selbst dann sein, wenn die Tatsache mit einer Meinung verbunden ist. Denn das BVerfG hat im Kontext persönlichkeitsbezogener Äußerungen betont, dass die tatsächliche Wahrheit einer mit einer Meinung verbundenen Tatsache durchaus auf Abwägungsebene berücksichtigt werden kann. ${ }^{2322}$ Insoweit knüpft man dann also ähnlich wie bei $\mathbb{S} 186$ StGB $\_^{2323}$ an eine Art „Sorgfaltspflicht“ an, die dem Äußernden auferlegt wird. ${ }^{2324}$ Dabei ist fraglich, wie weit diese reichen soll. Eine absolute Wahrheitspflicht kann schon angesichts des für journalistisch-redaktionelle Angebote deutlich niedrigeren Sorgfaltsmaßstabs kaum überzeugen, ${ }^{2325}$ weil damit erhebliche „chilling effects“ hervorgerufen ${ }^{2326}$ und insbesondere Spekulationen über bestimmte Hintergründe, alternative Erklärungsansätze aber auch Satire u.v.m. Gefahr laufen würden, in den strafbaren Bereich zu fallen. ${ }^{2327}$ Insoweit müssten also die Anforderungen an die Sorgfaltspflicht stark zurückgenommen werden. Ob diese - ähnlich wie bei personenbezogenen Äußerungen - situationsabhängig variieren, oder ob allgemein nur die leichtfertige Weitergabe erfasst sein soll, ist zweifelhaft. Weil es aber um strafrechtliche Sanktionen geht und zwischen herkömmlichen Medien und privaten Akteuren ein Unterschied besteht, ${ }^{2328}$ spräche einiges für einen Leichtfertigkeitsvorwurf. ${ }^{2329}$

2320 Hierzu auch Hoven, ZStW 129 (2017), 718 (743).

2321 So auch Steinebach u.a., Desinformation aufdecken und bekämpfen, S. 182.

2322 BVerfGE 85, 1 (17) - Bayer-Aktionäre; 99, 185 (197) - Scientology.

$2323 \mathrm{Zu}$ diesem teilweise vertretenen Ansatz im Kontext der Unerweislichkeit der Tatsache im Tatbestand des $\$ 186$ StGB statt vieler Regge/Pegel, in: MüKo, StGB, Bd. 4, $\mathbb{1} 186$ Rn. 28 m.w.N.

2324 Zu dieser Möglichkeit vgl. auch BVerfGE 99, 185 (197f.) - Scientology. Eine Erweiterung auf Tatsachenbehauptungen die keinerlei Drittbetroffenheit auslösen, wie bei persönlichkeitsbezogenen Äußerungen, steht etwa Schimmele, Staatliche Verantwortung für diskursive Integrität, S. 121, kritisch gegenüber.

2325 Denn sowohl nach den presserechtlichen (etwa $\$ 5$ S. 1 PresseG MV, $\mathbb{6}$ S. 1 PresseG NRW oder Art. 12 Abs. 2 S. 1 PresseG Bay) als auch nach den rundfunkrechtlichen Vorgaben ( $\$ 10$ Abs. 1 S. 2 RStV bzw. $\$ 54$ Abs. 2 S. 2 RStV) haben die Verantwortlichen nur die nach den Umständen gebotene Sorgfalt bei der Prüfung der Inhalte vor der Verbreitung einzuhalten. Dass Medien mehr abverlangt werden kann, betont das BVerfG, BVerfGE 85, 1 (22) - Bayer-Aktionäre.

2326 Vgl. BVerfGE 99, 185 (197) - Scientology. So letztlich auch Holznagel, MMR 2018, 18 (20).

2327 Illustrativ hierzu Hoven, ZStW 129 (2017), 718 (744). Ablehnend auch Schimmele, Staatliche Verantwortung für diskursive Integrität, S. 121.

2328 Vgl. BVerfGE 85, 1 (22) - Bayer-Aktionäre.

2329 Im Ergebnis ebenso Hoven, a.a.O. (743). Mit Blick auf die grundrechtlichen Implikationen einer „Wahrheitspflicht“ etwa BVerfGE 85, 1 (17, 22) - Bayer-Aktionäre m.w.N. Kritisch zur Berücksichtigung einer fehlenden Recherche als verfassungsrechtliches Abwägungskriterium Schimmele, Staatliche Verantwortung für diskursive Integrität, S. $127 \mathrm{f}$. Berücksichtigt man zudem, dass gerade die Medienkompetenz im kritischen Umgang mit Informationen fehlt, macht es auch deshalb (zunächst) Sinn, die Sorgfaltsanforderungen nicht zu überspannen. Denn die notwendigen Prüfkriterien im Umgang mit digitalen Inhalten sind oftmals gerade nicht bekannt. 
Daneben bedürfte es wegen der Ausweitung der Strafbarkeit auf Fälle fehlenden Unrechtsbewusstseins und der Beschränkung der Meinungsfreiheit schon aus Gründen der Verhältnismäßigkeit eines einschränkenden Elements. ${ }^{2330}$ Das bisherige persönlichkeitsbezogene Äußerungsstrafrecht löst das mit der „Ausnahme“2331 der „Wahrnehmung berechtigter Interessen“ in $\mathbb{} 193$ StGB. Eine entsprechende Ausnahmeregelung käme auch hier in Betracht. ${ }^{2332}$ Vor allem aber wäre (kumulativ) denkbar, qualifizierte Anforderungen an den Inhalt zu stellen. Konkret könnte - ähnlich wie bei $\mathbb{S} 130$ Abs. 1 StGB $\_^{2333}$ die Strafbarkeit von der Störungseignung für den Kommunikationsprozess bzw. den „öffentlichen kommunikativen Frieden“ abhängig gemacht werden. ${ }^{2334}$ Erfasst wären dann solche fehlerhaften Informationen, die geeignet sind, nicht nur die öffentliche Sicherheit, sondern auch den gesellschaftlichen Frieden als ein nicht durch allgemeine Unruhe, Unsicherheit und Angst vergiftetes psychisches Klima zu gefährden. ${ }^{2335}$ Für die Eignung der Störung sind letztlich dann wie auch schon bei $\mathbb{S} 130$ Abs. 1 StGB die konkreten Umstände relevant, sodass sich daraus ein „abstrakt-konkretes“ bzw. „potenzielles Gefährdungsdelikt“ ergäbe. ${ }^{2336}$ Insoweit wären auch wenn man sie nicht tatbestandlich vorsieht $-{ }^{2337}$ eine Differenzierung anhand des Verbreitungsweges bzw. -Umfanges möglich. ${ }^{2338}$ Insgesamt wird dadurch - eben-

2330 So auch Hoven, a.a.O. (743) mit Blick auf die Rücknahme des Unrechtsvorwurfes. Mit Blick auf die Meinungsfreiheit vgl. etwa BVerfGE 85, 1 (17, 21) - Bayer-Aktionäre; und umfangreicher im Kontext des $\$ 130$ Abs. 4 StGB insbesondere BVerfGE 124, 300 (331 ff., 337) - Wunsiedel. Im Kontext sog. Deep-Fakes etwa Lantwin, MMR 2020, 78 (82).

2331 Insgesamt ist streitig, ob $\mathbb{} 193$ ein Rechtfertigungs-, ein Schuldauschließungs- oder Strafunrechtsauschließungsgrund ist, hierzu statt vieler Joecks/Pegel/Regge, in: MüKo, StGB, Bd. 4, $\$ 193$ Rn. 1 m.w.N.

2332 Das schlägt Lantwin, MMR 2020, 78 (82) etwa mit Blick auf Deep-Fakes vor.

2333 Ausführlich zur Eignung bei $\mathbb{S} 130$ Abs. 1 StGB etwa Schäfer, in: MüKo StGB, Bd. 2, $\mathbb{} 130$ Rn. $22 \mathrm{ff}$.

2334 Denn durch die Restriktion auf die Störungseignung werden nur Inhalte erfasst, bei denen eine gewisse Sorgfalt der Rezipienten jedenfalls zu erwarten wäre. Zu der Zulässigkeit eines solche Ansatzes siehe auch Hoven, ZStW 129 (2017), 718 (743). Eine vergleichbare Restriktion fordert auch Ferreau, in: Möller/Hamaleers/ders., Typen von Desinformation und Misinformation, S. 44 (69) allerdings nur hinsichtlich bewusster Falschinformation. Zu der verfassungsrechtlichen Notwendigkeit eines solchen Sorgfaltspflichtverstoßes, um Sanktionen an eine nachträglich unwahre Tatsachenäußerung zu knüpfen etwa BVerfGE 99, 185 (197f.) - Scientology. Und zu den Fragen einer hinreichenden Bestimmtheit des Merkmals des „öffentlichen Friedens“ BVerfGE 124, 300 (339 ff.) - Wunsiedel. Das BVerfG, a.a.O (340) sieht dann keine Bedenken, wenn es - wie hier - als „Korrektiv“" verwendet wird.

2335 Schon mit Blick auf $₫ 130$ StGB hat der Gesetzgeber immerhin betont, dass Regelungsziel sei, „eine Vergiftung des politischen Klimas [...] [zu] verhindern [...]”, BT-Drs. 12/8588, S. 8. Das „psychische Klima" betont auch der BGH, NJW 1978, 58 (59) hinsichtlich $\$ 140$ StGB und NJW 1987, 1898; NStZ 2010, 570 mit Blick auf $\mathbb{} 126$ StGB. Zu den verfassungsrechtlichen Implikationen des Begriffs, BVerfGE 124, 300 (334, 339 ff.) - Wunsiedel. Demnach sind allein unliebsame oder lediglich beunruhigende Äußerungen hiervon nicht erfasst, ebd. (334). Daneben vgl. etwa Schramm, NJW 2002, 419 (420).

2336 Vgl. zu $\$ 130$ StGB BGH NJW 2001, 624 (626) m.w.N.

2337 Dies zumindest als überlegenswert aufwerfend Mafi-Gudarzi, ZRP 2019, 65 (68). Hoven, ZStW 129 (2017), 718 (743) betont das „besondere Strafbedürfnis“ der Verbreitung auf digitalen Wegen.

2338 Vgl. hierzu etwa BGH, NJW 1987, 1898; NStZ 2010, 570. Tendenziell kann etwa die uneingeschränkte Weitergabe an das soziale Netzwerk dafür ausreichen, sofern auch die inhaltlichen Anfor- 
so wie bei der Wahrnehmung berechtigter Interessen - auch eine Prüfung der verschiedenen Inhalte erforderlich, womit gerade auch die Meinungsfreiheit Berücksichtigung finden kann. ${ }^{2339}$ So könnte Desinformation insbesondere in Krisen etwa bei Attentaten ${ }^{2340}$ oder während Pandemien ${ }^{2341}$ - oder in gesellschaftspolitisch besonders wichtigen Themenfeldern - so wie etwa während der Flüchtlingskrise $^{2342}$ oder im Kontext des Impfschutzes ${ }^{2343}$ - strafrechtlich sanktioniert werden.

\section{Fazit: Strafrechtlicher Schutz vor allem eine rechtspolitische Frage}

Prinzipiell bleibt dem Gesetzgeber nicht der Weg versperrt, auch strafrechtliche Sanktionen an das Verbreiten desinformativer Inhalte zu knüpfen. ${ }^{2344}$ Sofern es um bewusst unwahre Tatsachenbehauptungen geht, wäre das auch vergleichsweise unproblematisch. Aus Effektivitätsgründen wäre aber eine zweifache Ausdehnung sinnvoll: Einerseits müssten auch verbundene Äußerungen erfasst sein, andererseits auch nicht vorsätzliches Handeln. Um allerdings selbst negative Auswirkungen auf den Diskurs und das Verbot einer jeden Lüge zu vermeiden, wäre eine (tatbestandliche) Restriktion erforderlich. In Anlehnung an $\mathbb{1} 130 \mathrm{StGB}$ könnte etwa eine Störungseignung für den „öffentlichen kommunikativen Frieden“ vorausgesetzt werden. Die genaue Ausgestaltung obliegt aber ohnehin der kriminalpolitischen Einschätzungsprärogative des Gesetzgebers, ${ }^{2345}$ dessen wichtigste Aufgabe es bleibt, den strafrechtlichen Schutz sachgerecht und verhältnis-

derungen erfüllt sind, Uecker, MMR 2014, 206, vgl. auch Schäfer, in: MüKo, StGB, Bd. 2, $\mathbb{1} 130$ Rn. 26. Kritisch allerdings mit Blick auf die ,inflationären Einstellung fast jeder Nachricht in das Internet“ BGH NStZ 2007, 216 Rn. 11. Ausführlicher zur Störungseignung etwa Schäfer, a.a.O., \$130 Rn. $22 \mathrm{ff}$.

2339 Diese Notwendigkeit zeigt auch BVerfGE 124, 300 (334) - Wunsiedel, auf.

2340 Hierzu etwa Weiss, Hass und Fake-News nach Attentaten, FAZ v. 25.02.2020. Und allgemeiner mit Blick auf die Angst vor Terrorismus etwa Georgi, Wie Falschmeldungen die Terrorangst schüren, FAZ v. 25.07.2016.

2341 Zur Verbreitung von Desinformation währen der Covid19-Pandemie etwa Ferrara, First Monday Vol. 25 (2020) Nr. 6 oder Cinelli u.a., Scientific Reports 10 (2020), 16598.

$2342 \mathrm{Zu}$ einer rechtlichen Auseinandersetzung mit dem Betreiber eines sozialen Netzwerks führte etwa die dort gespeicherte und weitergeleitete Aussage, dass ein Flüchtling, der sich mit Angela Merkel fotografierte, am Berliner-Attentat beteiligt gewesen sein soll, siehe hierzu LG Würzburg, ZUM 2017, $437 \mathrm{ff}$. Allgemeiner zu Desinformation im Kontext der „Flüchtlingskrise“ sind etwa die Informationen der Beauftragten der Bundesregierung für Migration, Flüchtlinge und Intergration, abrufbar unter https:/www.integrationsbeauftragte.de/ib-de/service/daten-und-fakten/fakten/fakten-stattfake-news, (Stand 30.05.2021).

$2343 \mathrm{Zu}$ Desinformation in diesem Zusammenhang siehe etwa Burki, Digital Health Vol. 1 (2019), e258 f. Auch Varol/Uluturk, First Monday Vol. 23 Nr. 5 (2018), bezeichnen die Diskussion um Impfungen als „important topic“ und verweisen ebenfalls auf die Bedeutung von Desinformation und entsprechende weitere Nachweise hierzu.

2344 So neben Mafi-Gudarzi, ZRP 2019, 65 (68), u.a. auch Hoven, ZStW 129 (2017), 718 (744).

2345 Vgl. BVerfGE 109, 133 (157) - Langfristige Sicherungsverwahrung. 
mäßig auszugestalten. Das aber wird ein „Ritt auf der Rasierklinge“2346 und vor allem zu einer rechtspolitischen Frage werden: Will man es riskieren, durch weitreichendere Eingriffe in den Diskurs u.a. auch das gesellschaftliche Vertrauen in die Neutralität und die Freiheit des Kommunikationsprozesses noch stärker zu erschüttern, als es momentan - Stichwort „Lügenpresse“ - der Fall ist? ${ }^{2347}$ Vieles spricht dafür, dass hier wie auch im Übrigen strafrechtliche Sanktionen die ultima ratio $\operatorname{sein}^{2348}$ und erst dann ergriffen werden sollten, wenn Interventionsversuche auf anderer Ebene scheitern.

\section{Alternative (medienrechtliche) Ansätze}

Daneben sind auch alternative Ansätze denkbar. ${ }^{2349}$ Aus medienrechtlicher Sicht kennen etwa die Pressegesetze der Länder (etwa $\$ 10$ PresseG MV) ebenso wie der Medienstaatsvertrag (etwa $\$ 20 \mathrm{MStV}$ ) Gegendarstellungsrechte. Auch wenn das erforderliche journalistisch-redaktionelle Angebot durch die Inhalte-Moderation noch anzunehmen sein könnte, so sind die Rechte bislang allein persönlichkeits- und damit individualschützend ausgestaltet. Es gibt aber bereits Vorschläge, dieses System auch auf Fälle desinformativer Inhalte zu übertragen, um auch dort Medien zu einer Berichtigung verpflichten zu können. ${ }^{2350}$ Dieses System entspräche auch der momentanen Ausrichtung der Haftungsprivilegierungen der ECommerce-Richtlinie, sodass auch eine Erweiterung auf Medienintermediäre in Betracht käme, was letztlich dem Ansatz der EU im Kampf gegen terroristische Inhalte entspräche. ${ }^{2351}$ Hier könnten dann neben den Fact-Checkern auch Nutzer und staatsferne Stellen wie die Medienanstalten ${ }^{2352}$ Inhalte melden, die dann intern oder durch unabhängige Stellen überprüft und sodann berichtigt oder durch entsprechende sachliche und richtige Informationen ergänzt weitergegeben werden müssten.

2346 So Lantwin, MMR 2020, 78 (8) mit Blick auf ein Verbot von (politischen) Deep-FakeS. Und von der Weth/Vachery/Kankanhalli, in: Proceedings of the International Conference on Multimedia \& Expo, S. 1, bezeichnen ein staatliches Verbot als „hard paternalism“. Das erscheint aber vor der obigen Differenzierung unsachgerecht, weil es hier primär gerade nicht um den Schutz vor sich selbst geht.

2347 Ähnlich mit Blick auf die strafrechtliche Regulierung von Deep-Fakes Lantwin, MMR 2019, 574 (578).

2348 Vgl. BVerfGE 39, 1 (45) - Schwangerschaftsabbruch I. Ausführlicher zu einem Ultima-ratio-Prinzip Jahn/Brodowski, ZStW 129 (2017), 363 (366 ff.).

2349 Siehe zu solchen etwa auch Oehmer/Pedrazzi, UFITA 2020, 7 (16 ff.).

2350 Hierzu etwa Ferreau, in: Möller/Hamaleers/ders. Typen von Desinformation und Misinformation, S. $44(65)$.

2351 Auch nach dem Kommissionsvorschlag $\operatorname{KOM}(2018) 640$ final können staatliche Stellen Löschanordnungen an die Betreiber richten, die dann die terroristischen Inhalte innerhalb einer Stunde zu entfernen haben (Art. 4 Abs. 1, 2).

2352 Das ist etwa der Vorschlag von Ferreau, in: Möller/Hamaleers/ders. Typen von Desinformation und Misinformation, S. 44 (65). 
Wissenschaftlich werden auch Methoden eines sog. Nudgings diskutiert. So existiert etwa eine Erweiterung für den Webbrowser (ShareAware), die beim Teilen von Inhalten auf deren fragwürdigen oder widersprüchlichen Aussagegehalt hinweist. ${ }^{2353}$ Sofern dies nicht als verpflichtendes Element vorgesehen wird, ist dies als Teil der gesellschaftlichen Selbstregulierung unproblematisch. Ansonsten begäbe man sich in eine Tiefe Diskussion um die Zulässigkeit paternalistischen, staatlichen Nudgings. ${ }^{2354}$

Daneben wäre darüber nachzudenken, ob auch externen Stellen ein Zugang gewährt werden muss, um eigenständig Desinformation herausfiltern und melden zu können. Letztlich beruht dieses System aber auf den Ansätzen, die die Netzwerkbetreiber mittlerweile - wenn auch nicht unbedingt freiwillig - selbstregulierend vorgesehen haben. Das sollte den Gesetzgeber aus den bisher bereits genannten Gründen zwar nicht von einer staatlichen Regulierung abhalten dürfen. Immerhin erlangen Medienintermediäre dadurch einen noch stärkeren Einfluss auf den Kommunikationsprozess. ${ }^{2355}$ Tendenziell problematisch kann ein solcher Ansatz deshalb sein, weil nicht klar ist, ob durch die im Rahmen der Gegendarstellung erfolgte Replikation des ursprünglichen Inhaltes nicht doch eine Intensivierung seines Aussagegehaltes verursacht wird (Illusory-Truth-Effect) ${ }^{2356}$ und besonders gefährdete Nutzergruppen, deren confirmation bias durch falsche Nachrichten aktiviert wird, werden durch eine Korrektur in ihrer Ansicht bestärkt (sog. „Backfire-Effekt“). ${ }^{2357}$ Insoweit ist besondere Vorsicht sowohl bei der Darstellung als auch der Formulierung etwaiger Korrekturhinweise geboten. ${ }^{2358}$ Deshalb und auch wegen der gebotenen Staatsferne des Kommunikationsprozesses sollten bei einer gesetzlichen Regelung allein prozedurale und strukturelle Fragen beantwortet werden, ${ }^{2359}$ und Fragen des Inhalts und der Darstellung den für die Korrektur zuständigen Akteuren (der Zivilgesellschaft) überlassen bleiben. Das Diskriminierungsverbot des $\mathbb{S} 94$ Abs. $1 \mathrm{MStV}$ erlaubt jedenfalls, dass

2353 Hierzu von der Weth/Vachery/Kankanhalli, in: Proceedings of the International Conference on Multimedia \& Expo Workshops, S. 2.

2354 Hierzu neben Hufen, JuS 2020, 93 ff. etwa auch Honer, DÖV 2019, 940 ff. oder in Ansätzen auch Weber/Schäfer, Staat 56 (2017), S. $561 \mathrm{ff}$.

2355 Das ist für Ferreau, a.a.O., S. 58 f., 67 der maßgebliche Grund einer Regulierung. Kritisch hierzu auch Oermann, in: Hofmann-Riem, Big Data - Regulative Herausforderungen, S. 145 (154).

2356 Darauf ebenfalls hinweisend Holznagel, MMR 2018, 18 (19); Brodnig, Hass im Netz, S. 138. Vgl. auch Steinebach u.a., Desinformation aufdecken und bekämpfen, S. 82f, $87 \mathrm{f}$ zur (Un-)Wirksamkeit der Gegendarstellungsmöglichkeiten bei Desinformation und zur Wirkung von Korrekturen speziell S. 93. Aus empirischer Sicht zu diesem Problem in Ansätzen etwa Zollo u.a., PLoS ONE 12 (7): e0181821, S. 4 ff. und ausführlicher zu den Auswirkungen von Korrekturen ursprünglicher, desinformativer Inhalte Schaewitz/Krämer, in: van Dujin u.a., Desinformation in open online media, MISDOOM 2020, S. $233 \mathrm{ff}$.

2357 Appel, in: ders., Die Psychologie des Postfaktischen, S. 205 (206). So etwa bei einer Studie Aufklärungsversuchen hinsichtlich der MMR-Impfung, wenn es um den Zusammenhang dieser kindlichen Schutzimpfung mit Autismus geht, Nyhan u.a., Pediatrics Vol. 133 (2014), S. e835 ff.

2358 Hierzu etwa Appel, ebd.; Brodnig, Hass im Netz, S. $141 \mathrm{f}$.

2359 Mit Blick auf diese Staatsferne Ferreau, in: Möller/Hamaleers/ders., Typen von Desinformation und Misinformation, S. 44 (67). 
Netzwerkbetreiber selbstregulatorische, algorithmenbasierte Maßnahmen zur Verhinderung der Verbreitung ergreifen. Sofern dieser Umstand transparent gemacht wird, kann diese „Diskriminierung“ immerhin gerechtfertigt werden. ${ }^{2360}$

Kritisch - besonders mit Blick auf Art. 14, 15 ECRL - zu bewerten, wären Versuche, den Netzwerkbetreibern analog zu den presse- bzw. rundfunkrechtlichen Sorgfaltsanforderungen (etwa $\ 5$ S. 1 PresseG MV, $\mathbb{6}$ S. 1 PresseG NRW

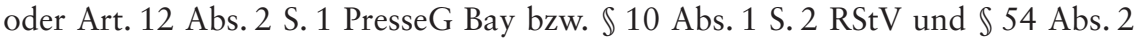
S. 2 RStV) auch $\mathbb{S} 93$ Abs. $4 \mathrm{MStV}$ entsprechende proaktive Prüfpflichten aufzuerlegen. Soweit dies als Reaktion auf die Beeinflussung des Kommunikationsprozesses - und damit zu demokratischen Zwecken - erfolgt, wäre Art. 1 Abs. 6 der Richtlinie wohl eher nicht einschlägig. Anders als im Falle der Meinungsroboter entfernt man sich hier nämlich von dem normativen Merkmal des „Schutzes des Pluralismus“ im Sinne einer Rezeptionsvielfalt, weil es hier eher um die Abwehr von Gefahren für den Kommunikationsprozess durch falsche Informationen ginge. Insoweit macht es durchaus Sinn, zunächst nur die reaktiven Pflichten zu stärken und eine Novellierung der Safe-Harbor-Regelungen für Host-Provider abzuwarten. Soweit die Richtlinie selbst eine gewisse proaktive Inpflichtnahme in Abkehr von Artt. 14 Abs. 1, 15 Abs. 1 gestatten oder zumindest eine „Good-Samaritan-Protection“2361 einführen würde, könnte die größte Hürde dann bewältigt werden. ${ }^{2362}$

Freilich kommen neben staatlich regulatorischen Maßnahmen auch weiterhin selbstregulierende Maßnahmen in Betracht, auf die auch der Staat hinwirken kann. Der EU-Verhaltenskodex bildet hier den Anfang einer dienstübergreifenden Lösung. Eine solche Lösung käme auch mit Blick auf desinformative Inhalte in Betracht. So ließe sich etwa eine gemeinsame Datenbank ${ }^{2363}$ für desinformative Inhalte erstellen, auf die die unterschiedlichen Anbieter zurückgreifen können. So könnten etwa Seitenlinks, Bilder und Videos (insbesondere Deep-Fakes) aber auch Texte einheitlich gespeichert werden, sobald sie als Desinformation klassifiziert wurden, um allen Anbietern die Möglichkeit eines (automatisierten) Abgleichs zu geben. ${ }^{2364}$

2360 Ferreau, a.a.O., S. 60.

2361 Vgl. hierzu 47 U.S. Code $\$ 230$ (c). Kritisch zu dieser Klausel als Vorbild einer europäischen Lösung etwa Kuczerawy, The EU Commission on voluntary monitoring: Good Samaritan 2.0 or Good Samaritan 0.5 ?. Der Kommissionsentwurf $\operatorname{COM}(2020) 825$ final sieht eine solche im Entwurf des Digital Service Acts in Art. 6 jedenfalls vor.

2362 Diskutiert wird insoweit etwa die Normierung einer lege-artis-Verpflichtung. Mit Blick auf die zu beachtenden europäischen Grundrechte, führt aber ein allgemeiner Pflichtenkanon erneut zu Problemen, weil damit die wirtschaftliche Stellung großer Anbieter zementiert würde. Kleinere oder neuartige Anbieter wären immerhin auch an diese Vorgaben gebunden, sofern nicht ein De-minimis-Vorbehalt - ähnlich wie $\ 1$ Abs. 2 NetzDG und $\$ 91$ Abs. 2 Nr. 1 MStV - vorgesehen wird.

2363 Eine solche gibt es bereits für terroristische Inhalte, siehe hierzu Oermann, in: Hofmann-Riem, Big Data - Regulative Herausforderungen, S. 145 (153 f.).

2364 Zu den Möglichkeiten einer automatischen Erkennung anhand von Merkmalen in Bezug auf Bilder siehe etwa Steinebach u.a., Desinformation aufdecken und bekämpfen, S. $109 \mathrm{f}$. 
IV. Fazit: Kein auswegloser, aber ein zurückhaltender Kampf ist möglich

Speziell der Kampf gegen Desinformation wird ein entscheidendes Thema in der digitalen Welt bleiben, wobei man allerdings besondere Vorsicht zu walten lassen hat, um einen zu intensiven Eingriff in die Meinungsfreiheit zu verhindern. Strafrechtliche Sanktionen sind dabei nicht ausgeschlossen, müssten aber besonders restriktiv gehandhabt werden. Besonders der bisherige selbstregulative Ansatz, die Verbreitung von Fake News netzwerkintern zu verringern und durch Hinweise und alternative Informationsangebote zu entkräften, hat einiges für sich. Einerseits verhindert man dadurch „chilling effects“, andererseits gewährleistet man, dass zutreffende Informationen wahrgenommen werden können. Dieses reaktive Konzept könnte durchaus wegweisend im Umgang mit Desinformation sein und strafrechtliche Sanktionen - vorerst - obsolet machen. Denn der Fokus sollte nicht allein auf der Entfernung und Sanktion der Verbreitung liegen, vielmehr ist es entscheidend, Nutzer zu sensibilisieren und sie im Umgang mit den Inhalten zu stärken und so eine faktenbasierte Meinungsbildung zu gewährleisten. Insoweit geht es auch hier eher darum, den Nutzern Instrumente an die Hand zu geben, derer sie sich selbst bemächtigen können. Dabei kann es durchaus sinnvoll sein, die Netzwerkbetreiber jedenfalls hinsichtlich der Ausübung der Möglichkeiten einzuschränken und gewisse Vorgaben zu machen. Und zuletzt öffnet möglicherweise schon die geplante Verordnung der EU zur Verhinderung der Verbreitung terroristischer Inhalte den Weg, dass die Mitgliedstaaten auch zukünftig selbständig gewisse Inhalte an die Betreiber melden. Freilich muss das vor allem durch eine staatsferne Stelle erfolgen. Allen voran die Medienanstalten kämen hierfür in Betracht.

\section{H. Handlungsbedarf auf europäischer Ebene}

Bedeutsam wird auf lange Sicht aber auch eine europäische Lösung. Das gilt nicht allein wegen der damit verbundenen deutlich größeren Signalwirkung. Die Notwendigkeit einer Regulierung folgt hier neben den Problemen, die die ECommerce-RL in einer veränderten digitalen Welt schafft, ${ }^{2365}$ vor allem aus den Bestrebungen der Mitgliedstaaten, eigenständige Regelungen zu treffen. In Deutschland sind neben dem MStV vor allem auch das NetzDG zu nennen. Und in Frankreich hat man neben den entsprechenden wahlspezifischen Regeln auch versucht - mit dem NetzDG durchaus vergleichbar -, die Verantwortung der Betreiber im Umgang mit bestimmten rechtswidrigen Inhalten zu stärken. Auch wenn das Gesetz wegen Verstoßes gegen die Meinungsfreiheit vom Conseil Con-

2365 Dazu bereits oben, A.I.1.a.aa(I.)(1.)(b.)(bb.). 
stitutionnel kassiert wurde, ${ }^{2366}$ bestehen damit nicht unerhebliche Schwierigkeiten für den europäischen Binnenmarkt, die vor allem im Wege der Rechtsangleichung (Art. 114 AEUV) zu lösen sein werden. Soweit es um den Umgang mit rechtswidrigen Inhalten geht, wird bereits auf EU-Ebene ein punktuelles, auf terroristische Online-Inhalte abzielendes Konzept diskutiert. ${ }^{2367}$ Dies könnte durchaus auch auf andere Inhalte ausgedehnt werden. Durch die Erstreckung der Regulierung auf digitale Medien durch den MStV besteht nochmal mehr das Bedürfnis einer europäischen Lösung. Die P2B-Verordnung sieht jedenfalls ein ähnliches Transparenz-Konzept im Verhältnis von Plattform und kommerziellem Nutzer vor, das auch darüber hinaus ausgebaut werden könnte. Angesichts der vorrangig mitgliedstaatlichen Kompetenz im Bereich der Medien dürften aber ohnehin nur gewisse Mindestvorgaben gemacht werden; den Mitgliedstaaten müssten weitreichende Umsetzungsspielräume gewährt werden, um die nationalrechtlichen Vorstellungen weiterhin verwirklichen zu können, was nicht zuletzt schon aus Art. 167 AEUV folgt. ${ }^{2368}$

Die größte Herausforderung stellt aber nach wie vor eine zeitgemäße aber gleichwohl sachgerechte Novellierung der Haftungsprivilegierungen der Art. 14, 15 ECRL dar. Die EU befindet sich hier am Anfang eines Reformprozesses, den die Kommission mit dem Vorschlag eines Digital Sevice Acts (DSA) begonnen hat. ${ }^{2369}$ Mit ihm kann die Grundlage dafür geschaffen werden, die Verantwortlichkeit bestimmter (zentraler) Anbieter über die technische Bereitstellung hinaus auch für inhaltliche Fragen zu begründen. Dabei hat die Kommission insbesondere versucht, einen Kompromiss zu finden, sodass die Betreiber eine unterstützende Rolle einnehmen können, ohne dass dabei aber die Rückzugsmöglichkeit in den „sicheren Hafen“ wegen potentieller Haftungsrisiken weiterhin attraktiv bleibt. ${ }^{2370}$ In Art. 6 DSA findet sich eine „Good-Samaritan-Protection“ nach USamerikanischem Vorbild. Hiernach wird eine Haftung nach den Art. 3, 4 und 5 DSA nicht allein deshalb begründet, weil sie freiwillig Inhalte der Nutzer überwachen oder kontrollieren. Daneben umfasst der Vorschlag auch nähere Transparenzvorgaben hinsichtlich ihrer Nutzungsbedingungen, die die Content-Moderation betreffen (Art. 12, 13 Abs. 1 lit. c DSA). Für „sehr große Online-Plattformen“ i.S.d. Art. 25 Abs. 1 DSA gelten weitergehende Beschränkungen. Das entspricht insoweit letztlich auch der nach dem nationalen Recht gebotenen Diffe-

\footnotetext{
2366 Décision du Conseil constitutionnel n 2020-801 DC du 18 juin 2020. Hierzu Heldt, JuWiss-Blog Nr. 96/2020.

$2367 \operatorname{COM}(2018) 640$ final, wobei sich hier gerade auf Art. 114 AEUV berufen wird, ebd. S. 5 f.

2368 So auch mit Blick auf eine europäische Intermediärsregulierung Ferreau, in: Möller/Hammeleers/ ders., Typisierung von Misinformation und Desinformation, S. 44 (61).

2369 Proposal for a Regulation on a Single Market For Digital Services (Digital Services Act) and amending Directive 2000/31/EC, COM(2020) 825 final. Ausführlicher zu dem Vorschlag und seinen Regelungen etwa Spindler, GRUR 2021, $545 \mathrm{ff}$ und ders, a.a.O., $653 \mathrm{ff}$.

2370 Im Kontext von Deep-Fakes weist etwa Lantwin, MMR 2019, 574 (578) auf die Notwendigkeit einer Bereichsausnahme von der „Überwachungsbefreiung“ des Art. 15 ECRL hin.
} 
renzierung aufgrund der deutlich stärkeren Marktmacht und Meinungsrelevanz (vgl. Erwgr. 53 S. 1). Gerade mit Blick auf diese Anbieter haben auch die Risiken der Meinungsroboter Eingang in den Entwurf gefunden. Nach Art. 26 DSA müssen Anbieter gewisse systemische Risiken mindestens einmal jährlich analysieren und bewerten, wozu nach Art. 26 Abs. 1 lit. c auch die „intentional manipulation of their service, including by means of inauthentic use or automated exploitation of the service, with an actual or foreseeable negative effect on the protection of public health, minors, civic discourse, or actual or foreseeable effects related to electoral processes and public security “ gehört. Die Kommmission hatte hierbei ausweislich des Erwägungsgrundes 57 S. 7 DSA „die Einrichtung von Scheinkonten, die Nutzung von Bots und anderen automatisierten oder teilautomatisierten Verhaltensweisen“ im Blick. Nach Art. 26 Abs. 2 sollen sie hierbei auch die Zusammenhänge zwischen den eigenen Diensten und seinen Maßnahmen mit den Risiken berücksichtigt werden. Art. 27 Abs. 1 S. 1 DSA sieht sodann eine Risikominderungspflicht vor. Hiernach haben die Anbieter „,angemessene, verhältnismäßige und wirksame Risikominderungsmaßnahmen, die auf die gemäß Artikel 26 ermittelten besonderen systemischen Risiken zugeschnitten sind[,]“ zu ergreifen. Insoweit folgt die Kommission bisher einen ganz ähnlichen Ansatz der regulierten Selbstregulierung so wie auch Deutschland zuvor, einzig mit dem Unterschied, dass kein verbindliches Mindestmaß normativ festgelegt ist, sondern den Betreibern ein deutlich weitreichenderer Spielraum zukommt als unter dem Reglement des MStV. Der Digital Service Act gewährleistet aber, dass externen Dritten Zugang zu den Daten erhalten können. Art. 28 DSA sieht nämlich eine unabhängige Prüfung durch externe Stellen vor, die gewisse Anforderungen erfüllen müssen (Art. 28 Abs. 2 DSA). Dadurch schaltet man also eine weitere sachkundige Ebene ein, die letztlich für den Staat als „Helfer“ dienen soll, um eine wirksame Kontrolle gewährleisten zu können. Bemerkenswert ist insoweit auch, dass der DSA erstmals eine Verpflichtung enthält, Forschergruppen den Zugang zu den Netzwerkdaten auf Verlangen zu gewähren (Art. 31 Abs. 2 DSA), die - aus den bereits genannten Gründen - ${ }^{2371}$ besondere Voraussetzungen zu erfüllen haben: Sie müssen „mit akademischen Einrichtungen verbunden sein, unabhängig von gewerblichen Interessen sein, nachweislich über Sachkenntnis auf den Gebieten verfügen, die mit den untersuchten Risiken oder den diesbezüglichen Forschungsmethoden zusammenhängen, und sich verpflichten und in der Lage sein, die mit jedem Verlangen verbundenen besonderen Anforderungen an die Datensicherheit und die Vertraulichkeit einzuhalten“ (Art. 31 Abs. 4 DSA). Der DSA knüpft also - wenn auch nicht in derselben Stringenz - an wesentliche bereits im MStV oder - mit Blick auf die Transparenzvorgaben - anderswo bereits vorhandene Regelungen an und schließt dabei auch Lücken, die der MStV

2371 S. dazu oben, 4. Kap. D. I. 
noch offengelassen hat. Insoweit könnte der Digital Service Act tatsächlich die längst überfällige zeitgemäße Anpassung des Rechts digitaler Medien innerhalb der EU sein, einen einheitlichen Rechtsrahmen schaffen und auf lange Sicht die Regelungen des MStV ablösen. Bis dahin dürfte aber noch etwas Zeit vergehen.

\section{Fazit: Stärkung der Eigenverantwortung als vorrangiges Regulierungsziel}

Schon mit dem NetzDG hat ein Prozess des Staates begonnen, die Kontrolle über „rechtsfreien digitalen Raum“ (zurück-) zu erobern. Der MStV hat diesen Prozess fortgesetzt und sorgt den Risiken vor, die von Anbieterseite für die Meinungsvielfalt drohen. Vor allem aber wird auch den Risiken der Meinungsroboter vorgebeugt. Bestehende Schutzlücken führen zwar nicht zu einem grundrechtlichen Problem, gleichwohl wird es zukünftige Regulierungsaufgabe sein, den eingeschlagenen Weg nicht nur im Rahmen der bestehenden Möglichkeiten ( $\mathbb{S} 96$ $\mathrm{MStV}$ ) zu konkretisieren, sondern ihn vor allem kritisch zu überprüfen und gegebenenfalls nachzujustieren. Handlungsziel bleibt hierbei auch weiterhin die staatliche Schutzverantwortung für den politischen Willensbildungsprozess. Will man den Weg der Transparenz auch zukünftig weiter beschreiten, so böte es sich an wie schon beim NetzDG - u.a. auch die Sorgetragungspflicht des $\mathbb{S} 93$ Abs. 4 MStV durch eine öffentliche Berichtspflicht zu ergänzen. Vor allem aber ist die Stärkung der medienwissenschaftlichen Forschung notwendige Voraussetzung, für die zukünftige, faktenbasierte Medienpolitik und eine wirksame Kontrolle der Einhaltung gesetzlicher Vorgaben. Das erfordert ggf. einen verpflichtenden Forschungszugang. Hauptaugenmerk sollte daneben weiterhin und noch stärker auf der Medienkompetenz der Nutzer liegen. Insoweit bedürfen die schulpolitischen Maßnahmen einer kritischen Evaluation insbesondere mit Blick auf die personellen und organisatorischen Voraussetzungen einer kompetenten und vor allem zeitnahen Inhaltsvermittlung. Insbesondere mit Blick auf Desinformation sollte - trotz prinzipieller, wenn auch enger verfassungsrechtlicher Zulässigkeit weiterhin Zurückhaltung gewahrt werden, soweit es um strafrechtliche Sanktionen geht. Vor allem die bereits durch die Netzwerkbetreiber vorgehaltenen präventiven Maßnahmen könnten ausgebaut und die Betreiber hierbei auch zukünftig stärker reguliert werden, soweit es jedenfalls um verfahrensrechtliche Aspekte geht. Die EU-Verordnung zur Verhinderung der Verbreitung terroristischer Inhalte hat zumindest aufgezeigt, dass vor allem auch staatlichen Behörden eine entscheidende Verantwortung zukommt, die nicht allein auf die Betreiber delegiert werden kann. Auch das könnte ein zukünftiger Ansatzpunkt sein. Vor allem die Landesmedienanstalten als staatsferne Stellen können hierzu berufen werden. Allerdings setzt das einen erheblichen Ausbau organisatorischer und personeller Art voraus. Im Übrigen werden Versuche, die Betreiber stärker und verpflichtend 
in die präventive (proaktive) Bekämpfung einzubinden, zurzeit wohl noch an den Vorgaben der E-Commerce-RL scheitern. Dem könnte man dadurch aus dem Weg gehen, dass ein Zugang für staatliche oder unabhängige Stellen geschaffen wird, eigenständig entsprechende Inhalte ausfindig zu machen und zu melden. Das entspricht weitestgehend der momentanen Praxis der Anbieter. Letztlich wird der geplante Digital Service Act wohl auch entscheidende Weichen für die Zukunft stellen.

Auch eine relative Klarnamenpflicht könnte zur Stärkung der Diskursqualität und zur Verhinderung von ungewünschten, moralisch verwerflichen Verhaltensweisen beitragen. Denn damit schafft man keine neuen Restriktionen, sondern stärkt das Prinzip der Eigenverantwortung der Nutzergemeinschaft. Auch dadurch könnte die Zivilgesellschaft für einen angemessenen sachlichen Diskurs in der digitalen Welt sensibilisiert werden und vor allem selbst zu ihm beitragen.

Die Zukunft der Regulierung sozialer Netzwerke und der Meinungsroboter liegt also vor allem in der Stärkung der Eigenverantwortung der Nutzer, sei es durch die Stärkung ihrer Medienkompetenz durch die ergriffenen Transparenzverpflichtungen des MStV und zukünftig zu ergreifender Transparenz mit Blick auf desinformative Inhalte, oder aber auch durch die Stärkung der rechtlichen Verantwortung für das eigene Verhalten. Damit wird man nicht zuletzt auch dem Grundgedanken des autonomen und vor allem mündigen Bürgers gerecht. Dieses Primärziel sollte - allerdings nur nach vorsichtiger und behutsamer Evaluation potentieller Risiken - durch unterstützende Maßnahmen abgesichert werden. Im Kern bleibt es aber weiterhin Aufgabe jedes Einzelnen und der Zivilgesellschaft insgesamt, den demokratischen Kommunikationsprozess am Leben zu erhalten und vor nachhaltigen und schadhaften Manipulationen und Beeinflussungen zu schützen. ${ }^{2372}$

$2372 \mathrm{Zu}$ dieser primär gesellschaftlichen Verantwortung siehe auch Ingold, in: Oppelland, Propaganda als neue außen- und sicherheitspolitische Herausforderung, S. 81 (11). Vgl. auch BVerfGE 8, 104 (113) - Volksbefragung, dass die öffentliche Meinungsbildung als „Erscheinung des gesellschaftlichpolitischen und nicht des staatsorganschaftlichen Bereichs“ begreift. 


\section{Teil: Zusammenfassung}

Die digitalen und sozialen Medien bringen vielfach neue Herausforderungen mit sich, die bereits vor einigen Jahren Eingang in die rechtswissenschaftliche Literatur gefunden haben. Dabei geht es vor allem um betreiberseitige Verzerrungen der Informationsvielfalt und anderen Risiken, die für die (informationelle) Selbstbestimmung der Nutzer bestehen. Eine vergleichsweise junge Diskussion hat sich hinsichtlich des hier als „Meinungsroboter“ bezeichneten Phänomens (teil-)automatisiert gesteuerter Nutzeraccounts ergeben, die verdeckt die politische Kommunikation und Willensbildung der Nutzer in sozialen Netzwerken beeinflussen könnten. ${ }^{2373}$ Insoweit stellen sich (zwangsläufig) zwei vorliegend näher untersuchte Fragen: Ist der Staat dafür verantwortlich, den Prozess der politischen Willensbildung der Nutzer sozialer Netzwerke vor Beeinflussungen durch verdeckt agierende, automatisierte Nutzerprofilen zu schützen? Und kommt er - so denn eine solche Pflicht besteht - dieser Pflicht auch in geeigneter, effektiver und angemessener Weise nach? Die kurze Antwort lautet: Ja, der Staat ist verpflichtet und ja, er kommt dieser Schutzpflicht zumindest mit den neueren Entwicklungen im Medienrecht auch in der beschriebenen Weise nach.

Diese Verantwortung offenbart das Grundgesetz aber - anders als in anderen Fällen einer Regelungs-, Ausgestaltungs- oder Gewährleistungsverantwortung - nicht unmittelbar. Zweierlei Ansatzpunkte sind hierfür dennoch theoretisch denkbar: der rundfunkrechtliche Regelungsauftrag und die grundrechtliche Schutzpflicht. ${ }^{2374}$ Praktisch und rechtsdogmatisch überzeugend ist aber nur der zuletzt genannte Ansatzpunkt. Der rundfunkrechtliche Regelungsauftrag wurde nicht nur unter Prämissen entwickelt, die in der heutigen Zeit nicht mehr dieselbe Geltungskraft haben. Er führt auch zu einem Bruch mit der üblichen Grundrechtsdogmatik, weil objektiv-rechtliche Überlegungen das subjektiv-rechtliche Element und damit den Wesenskern des Grundrechts überlagern. Überzeugende Argumente hierfür konnten bislang nicht vorgetragen werden. Denn auch die Pressefreiheit hat eine dienende objektiv-rechtliche Funktion ebenso wie die anderen Kommunikationsfreiheiten. Entsprechende Einschränkungen werden hier aber gerade nicht vorgesehen. Mit den grundrechtlichen Schutzpflichten hingegen besteht eine für alle Freiheitsrechte anerkannte grundrechtsdogmatische Figur, die den Staat als Garanten der Grundrechte in Konstellationen verpflichtet, in denen die grundrechtsbeschränkende Wirkung (sog. Übergriff) von nicht-staatlicher Seite, insbesondere von privaten Dritten, droht. Anders als im abwehrrechtlichen Bereich (Unterlassung des Eingriffs) ist die Rechtsfolge der Schutzpflicht unbestimmt. Aus ihr folgt grundsätzlich eine abstrakte Schutzver- 
antwortung, die der Konkretisierung vor allem durch den Gesetzgeber als primärer Adressat bedarf. Ihm kommt hierbei ein weiter Einschätzungsspielraum zu, um u.a. einen sachgerechten Ausgleich zwischen den Schutzbedürfnissen und dem sog. Untermaßverbot auf der einen und den damit kollidierenden abwehrrechtlichen Freiheitsrechten und dem Übermaßverbot auf der anderen Seite herzustellen. Das Bundesverfassungsgericht überprüft die Wahrnehmung der Schutzverantwortung nur eingeschränkt. Solange das Untermaßverbot nicht in evidenter Weise verletzt wird - also man nicht untätig bleibt, völlig ungeeigneten Schutz bietet, wenn ein offensichtlich vorzugswürdigerer Schutz nicht gewährt wird oder man sonst in offensichtlicher Weise hinter dem gebotenen Schutzziel zurückbleibt -, ist die Entscheidung verfassungsrechtlich nicht angreifbar. ${ }^{2375}$

Wie lässt sich vor diesem Hintergrund die Schutzpflicht des Staates im Falle der Meinungsroboter begründen?2376 Ansatzpunkt hierfür sind die Kommunikationsgrundrechte des Art. 5 Abs. 1 S. 1 GG, die vorrangig den individuellen Willensbildungsprozess schützen, angefangen mit der Informationsaufnahme über deren Verarbeitung und den internen Meinungsbildungsprozess (Art. 5 Abs. 1 S. 1, 2. Hs. GG) bis hin zur Äußerung und Verbreitung der so gebildeten Meinung (Art. 5 Abs. 1 S. 1, 1. Hs. GG). Hierdurch wird gleichzeitig auch der für die demokratische Grundordnung konstitutive Prozess einer „öffentlichen Meinungsbildung " mitgeschützt. Die Meinungsroboter erlangen hier deshalb Bedeutung, weil schon die Informationsaufnahme kein rationaler Prozess ist, der Rezipient also nicht dem Idealbild eines homo oeconomicus entspricht. Bereits die Informationsauswahl ist angesichts nur begrenzt vorhandener kognitiver Ressourcen (bounded rationality) von Verzerrungen geprägt, für die auch das menschliche Denksystem verantwortlich ist: Man bevorzugt meinungskonforme Inhalte (confirmation bias) oder bewertet die Glaubwürdigkeit von Inhalten anhand von statistischen Daten der Netzwerke, insbesondere Likes und Follower (Prinzip der sozialen Bewährtheit). Dies setzt sich bei der Informationsverarbeitung fort: $\mathrm{Ne}-$ gative Informationen werden höher gewichtet (Negativity Bias) ebenso wie Informationen, die in Anordnungen oder Reihenfolgen priorisiert angezeigt werden (Primacy Effect). Und Informationen, mit denen man öfters konfrontiert wird, werden eher als zutreffend eingeordnet (Illusory Truth Effect). ${ }^{2377}$ Indem Meinungsroboter zahlreich Inhalte verbreiten oder diese „liken“, verändern sie nicht nur die Reichweite, sondern auch wesentliche, für die Bewertung der Glaubwürdigkeit und Relevanz durch die Netzwerkalgorithmen und Nutzer maßgebliche Parameter, was zu einer priorisierten Weiterleitung, Aufnahme und Verarbeitung der Informationen führen kann. Dadurch verändern sie die Rezeptionswahr-

2375 2. Teil, 1. Kap., B.

2376 2. Teil, 2. Kapitel.

2377 Zum Willensbildungsprozess ausführlich 1. Teil, B. 
scheinlichkeit und die „kommunikative Chancengleichheit“ der Inhalte. Diese ist fundamentales Prinzip des demokratischen Kommunikationsprozesses und liegt nicht nur der demokratischen Wahl (Art. 38 Abs. 1 S. 1, Var. 4 GG, Art. 3 Abs. 1 bzw. Art. 38 Abs. 1 S. 1, Var. 4 GG i.V.m. Art. 21 Abs. 1 S. 1 GG) zugrunde. Auch die Kommunikationsfreiheiten basieren auf diesem Prinzip. Zwar ist dem Kommunikationsprozess eine gewisse Divergenz der Rezeptionswahrscheinlichkeit eigen. Die Rezeptionswahrscheinlichkeit der Inhalte in Medien, von Politikern, Parteien oder Influencern ist per se höher als die der Inhalte eines normalen Nutzers. Das ist aber solange kein Problem, wie diese Divergenz kommunikativ also durch die geistige Überzeugungskraft der Urheber bzw. Inhalte erklärt werden kann. Im Falle der Meinungsroboter folgt diese Überzeugungskraft aber allein aus der „technischen Manipulation“ der für die priorisierte Weiterleitung relevanten Parameter und Metadaten (Likes, Follower etc.) der Inhalte. Insoweit ist die Situation vergleichbar mit der Debatte um die Netzneutralität von AccessProvidern. Und zuletzt wirken die Meinungsroboter auch auf die Meinungsäußerungsfreiheit ein. Indem sie als Teil der Netzwerköffentlichkeit wahrgenommen werden und insoweit das Meinungsklima durch entsprechende Inhalte beeinflussen, können sie psychologische Effekte ausnutzen, die Nutzer davon abhalten, meinungskonträre Inhalte zu veröffentlichen (Theorie der Schweigespirale). Das führt letztlich auch wieder dazu, dass derlei Inhalte gar nicht als Information für andere zur Verfügung stehen. Insoweit können Meinungsroboter die Informationsfreiheit, die „kommunikative Chancengleichheit“ und die Meinungsäußerungsfreiheit anderer Nutzer beeinträchtigen und, insbesondere durch die wechselseitige Verstärkung der Effekte, die Willensbildung beeinflussen. ${ }^{2378}$

Gleichwohl sollen Schutzpflichten nach verbreiteter Ansicht nicht nur einen „Übergriff“ voraussetzen. Dieser müsse daneben auch - in Analogie zum Polizeirecht - eine Gefahrenschwelle überschreiten: Risikovorsorge sei fakultativ, Gefahrenvorsorge obligatorisch. Das kann im Falle der Meinungsroboter deshalb zu Problemen führen, weil aus empirischer Sicht noch nicht hinreichend geklärt ist, welche Auswirkungen die Meinungsroboter tatsächlich haben. Theoretisch herausgearbeitete Einflüsse und Wirkungsmöglichkeiten konnten bislang noch nicht hinreichend verifiziert werden. Allein der Einsatz eigener Meinungsroboter $\mathrm{zu}$ wissenschaftlichen Zwecken konnte belegen, dass ein Einsatz und damit ein Einfluss durchaus möglich ist. Insoweit bewegt man sich eher auf Ebene der Risikovorsorge. Allerdings ist die Gefahrenschwelle beweglich: Je höher die betroffenen Rechtsgüter und je größer der drohende Schaden, desto geringer sind die Anforderungen an die Eintrittswahrscheinlichkeit. Die fundamentale Bedeutung der betroffenen Kommunikationsgrundrechte und die einzelnen Einflüsse sowie die wechselseitige Verstärkung der Effekte zwischen kommunikativer Chancen-

2378 2. Teil, 2. Kapitel, A. 
gleichheit, Meinungsäußerungs- und Informationsfreiheit erlaubt eine Herabsenkung der Anforderungen an die Eintrittswahrscheinlichkeit, sodass bereits die abstrakte Gefahr der Meinungsroboter ausreicht, um eine Schutzpflicht zu begründen. Auch unabhängig hiervon sprechen gewichtige Argumente gegen eine tatbestandliche Differenzierung zwischen fakultativer Risikovorsorge und obligatorischer Gefahrenabwehr. Allein der Spielraum mag ein anderer sein: Im Bereich der „Risikovorsorge“ sind die kollidierenden Freiheitsrechte Dritter tendenziell höher zu gewichten, sodass sich der Staat auch eher auf eine Beobachterrolle zurückziehen darf als im Bereich der „Gefahrenabwehr“.2379

Sofern auch die Netzwerkbetreiber conditio sine qua non für einen erfolgreichen Einsatz werden, indem ihre Rankingalgorithmen das Verhalten der Nutzergemeinschaft analysieren und so Inhalte u.a. auch anhand der „sozialen Bewährtheit" priorisiert und vor allem auch personalisiert in Abhängigkeit vom ermittelten Interessensprofil des Empfängers anordnen, ${ }^{2380}$ trifft den Staat auch ihnen gegenüber eine Schutzpflicht, um Manipulationen und Verzerrungen vorzubeugen. ${ }^{2381}$

Hier wie auch im Falle der Meinungsroboter folgt daraus aber nicht zwingend eine Pflicht, regulative Maßnahmen zu ergreifen, immerhin sind damit zwangsläufig auch Eingriffe in die Grundrechte der Urheber, der Nutzer und der Betreiber verbunden und der Gesetzgeber ist auch durch das Übermaßverbot in seinem Handlungsspielraum beschränkt: So genießen die Urheber der Meinungsroboter den Schutz der Meinungsfreiheit. Weder steht die Automatisierung einer Zurechnung der Kommunikate im Weg - immerhin ist diese Automatisierung Ausdruck menschlichen Denkens und Handelns und steht unter menschlicher Kontrolle noch können die typischen Charakteristika der Meinungsroboter - Identitätstäuschung, Manipulation der quantitativen Legitimität von Meinungen oder manipulative Absicht - hieran etwas ändern. Maßgeblich hierfür ist trotz durchaus berechtigter Zweifel, dass die verbreiteten Inhalte selbst den Schutz der Meinungsfreiheit genießen. Insofern würde man Modalitäten höher gewichten als die Inhalte selbst. Erhebliche Probleme entstehen ohnehin nur dort, wo die Urheber manipulative Zwecke verfolgen, etwa Desinformation streuen, um Verunsicherung und Hass zu schüren, oder Meinungen unterdrücken wollen, also im Falle einer manipulativen Absicht. Eine Differenzierung zwischen „malicous“ und „benign“ führt aber zu erheblichen Beweisschwierigkeiten, die - anders als in anderen Konstellationen - nicht überzeugend gelöst werden können, ohne in Widerspruch mit gängigen Beweislastverteilungsregeln oder den grundrechtlichen Wertungen zu geraten. ${ }^{2382}$ Daneben sind auch die Grundrechte der Netzwerkbe-

2379 Hierzu 2. Teil, 2. Kapitel, A.III.

2380 Hierzu 1. Teil, A.

2381 2. Teil, 2. Kapitel, B.

2382 Hierzu 2. Teil, 3. Kapitel A.I. 
treiber, insbesondere ihre unternehmerische Freiheit (Art.12 Abs. 1 GG), sowie insbesondere die Meinungs- und Informationsfreiheit der Nutzer selbst zu berücksichtigen.

Staatlicher Schutz ist zudem nur soweit zu gewähren, wie der Nutzer auch schutzbedürftig ist, sich also nicht selbst schützen kann. Berücksichtigt man, dass wesentliche Wirkungsvoraussetzungen der Meinungsroboter auch nutzerseitig bedingt sind, so wird es vor allem darum gehen, die Nutzerautonomie zu stärken. Aus Sicht der Meinungsäußerungsfreiheit wäre hierfür erforderlich, dass verhindert wird, dass sich Nutzer von den künstlich erzeugten digitalen Zwängen der Meinungsroboter einschüchtern lassen. Im Falle der Informations- und Meinungsbildungsfreiheit wird es vor allem darum gehen, der Informationsasymmetrie zu begegnen, die durch die nicht erkennbare Identitätstäuschung geschaffen wird. Insoweit ist der Bedarf hier vergleichbar mit dem in Fällen, in denen einer Informationsasymmetrie zwischen Verbrauchern und Unternehmern bzw. sonstigen kommerziell handelnden Personen begegnet werden soll: Es geht folglich um die Schaffung von einem Mindestmaß an Transparenz. ${ }^{2383}$ Gleichzeitig ist es notwendig, die Medienkompetenz der Nutzer zu erhöhen, um sie nicht nur für die Verzerrungen selbst zu sensibilisieren, sondern auch um zu ermöglichen, dass Nutzer reflektiert in sozialen Netzwerken agieren können. Damit kann man der Wirkkraft desinformativer Inhalte, aber auch dem einseitigen Rezeptionsverhalten von Nutzern begegnen, die sich auch selbst in eine „Filterblase“ begeben. Daneben gilt es im Falle der kommunikativen Chancengleichheit zu verhindern, dass Meinungsroboter die Rezeptionswahrscheinlichkeit zu ihren Gunsten verändern können. Und zuletzt kann es aus gesellschaftlicher Sicht (Makro-Ebene des Willensbildungsprozesses) im Falle desinformativer Inhalte notwendig sein, spezifischen Schutz vor der Verbreitung zu gewähren. Denn die Stärkung der Nutzerautonomie verhindert nicht, dass Inhalte ihren Weg in den Diskurs finden und dort ihre schadhafte Wirkung dadurch entfalten, dass sie Verunsicherung schaffen, eine Spaltung begünstigen und so den Prozess der öffentlichen Meinungsbildung erschweren. ${ }^{2384}$

Betrachtet man unter diesen Prämissen die bisher ergriffenen Maßnahmen und das bisherige einfache Recht, so wurde der Staat seiner Schutzverantwortung lange Zeit nicht hinreichend gerecht. Zwar wird die Meinungsäußerungsfreiheit allgemein durch das Strafrecht ( $\mathbb{S} 240$ StGB) aber auch spezifisch mit Blick auf die Wahl ( $\mathbb{S} 108,108$ a StGB, $\mathbb{S} 32$ Abs. 1 BWG) geschützt, gleichwohl liegt in der künstlichen Herbeiführung psychologisch wirkender Zwänge keine straf- oder wahlrechtsrelevante Handlung. Aus verfassungsrechtlicher Sicht kann allein durch den Einsatz durch politische Parteien eine Grenze erreicht sein, die sich aus

2383 Hierzu 3. Teil, 3. Kapitel, B.II.1.c.aa.

2384 Zum Schutzbedarf 2. Teil, 3. Kapitel, B.II. 
der Funktion der Parteien als dienende Institutionen der Willensbildung ergibt. Ihre Betätigung muss daher besonderen Transparenzanforderungen genügen. Mit

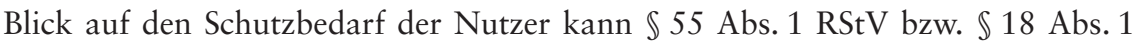
$\mathrm{MStV}$ zwar zu einer Bereithaltung eines Impressums verpflichten, gleichwohl muss gerade nicht der automatisierte, identitätstäuschende Charakter und damit die wesentliche Wirkungsvoraussetzung offenbart werden. ${ }^{2385}$ Und eine priorisierte und personalisierte Weiterleitung manipulierter Inhalte wird durch das Datenschutzrecht ebenfalls nicht verhindert. Vor allem die Kultusministerkonferenz hat die Bedeutung der Medienkompetenz zum Schutz der Nutzer in der digitalen Umgebung erkannt und versucht zumindest im schulischen Bereich diese zu stärken. Gleichwohl befindet man sich hier erst am Anfang eines langwierigen Prozesses, der zurzeit insbesondere noch organisatorische Fragen offenlässt. ${ }^{2386}$ Weitestgehend wird die Gewährleistung des Schutzes allein der freiwilligen Selbstregulierung der Betreiber überlassen. Sofern der Einsatz der Meinungsroboter straf- oder urheberrechtlich unzulässig ist, sind allein die Betreiber dazu berufen, ihre Rechte auch gegenüber den Urhebern durchzusetzen. Zwar sehen sie hierfür durchaus entsprechende präventive Vorgaben (Klarnamenzwang, Verbot eines unautorisierten automatisierten Zugriffs o.ä.) und repressive Maßnahmen (Kündigung des Nutzungsverhältnisses, Sperrung bzw. Löschung von Beiträgen und Nutzerprofilen) in den Nutzungsbedingungen vor. Allerdings setzen sie diese Vorgaben freiwillig kaum effektiv um. Erst durch öffentlichen oder wirtschaftlichen Druck werden Betreiber dazu angehalten, Maßnahmen auch tatsächlich zu ergreifen. Hieran hat der EU-Verhaltenskodex, der neben den Meinungsrobotern auch desinformative Inhalte und weitere Aspekte adressiert, nichts geändert. Auch hierbei handelt es sich allein um eine freiwillige Verpflichtung, deren Einhaltung nur durch die Betreiber selbst kontrolliert werden kann. Ein staatlicher Kontrollzugriff - und damit die eigenständige Gewährleistung von Schutz - ist so kaum möglich. Das hätte freilich aus verfassungsrechtlicher Sicht zu einer Verletzung des Untermaßverbotes und damit der Grundrechte führen können, weil der Staat damit tendenziell evident hinter dem Schutzziel zurückbleibt. ${ }^{2387}$

Allerdings ist am 7. November 2020 der Medienstaatsvertrag in Kraft getreten. Dieser sieht nicht nur transparenzerhöhende Maßnahmen hinsichtlich der Netzwerke und ihrer Rankingalgorithmen selbst ( $\mathbb{9} 93$ Abs. 1-3 MStV) und ein Verbot von Diskriminierung von Inhalten und damit von systematischen Verzerrungen bei der Vermittlung von Inhalten vor $(\mathbb{S} 94 \mathrm{MStV})$. Er führt mit $\mathbb{1 8}$ Abs. $3 \mathrm{MStV}$ auch eine eigenständige Kennzeichnungspflicht für Meinungsroboter ein, wonach gerade der identitätstäuschende Charakter zu offenbaren ist. ${ }^{2388}$

2385 2. Teil, 3. Kapitel, C.I.3.c.

2386 Ausführlich zur Strategie der KMK und den offenen Fragen 2. Teil, 3. Kapitel, C.I.3.b.

2387 Hierzu ausführlich 2. Teil, 3. Kapitel, C.I. und insbesondere das Fazit, C.I.5.

2388 2. Teil, 3. Kapitel, C.II.1. 
Mit dieser Pflicht der Urheber korrespondiert in $\mathbb{\int} 93$ Abs. $4 \mathrm{MStV}$ auch eine Pflicht der Betreiber sozialer Netzwerke, für diese Kennzeichnung „Sorge zu tragen“. Damit wird aber nicht nur die Schaffung einer Kennzeichnungsmöglichkeit für die Urheber erforderlich. Vielmehr begründet $\mathbb{\$} 93$ Abs. 4 MStV auch eine eigene infrastrukturelle Verantwortung der Betreiber, die vergleichbar mit einer Verkehrssicherungspflicht oder einer Garantenstellung ist. Sie müssen selbst Maßnahmen ergreifen, um einen verdeckten Einfluss von Meinungsrobotern zu verhindern. Hierbei handelt es sich vor allem um eine Zielvorgabe, die den Betreibern einen gewissen Umsetzungsspielraum gewährt. Gleichwohl reduziert sich dieser Spielraum bei meinungsstarken, marktmächtigen Anbietern erheblich. Von ihnen kann wesentlich mehr insbesondere auch eine proaktive Überwachung erwartet werden. Das führt auch nicht zu Konflikten mit den europäischen Vorgaben der E-Commerce-RL 2000/31/EG. Auch wenn es sich auf den ersten Blick um einen Verstoß gegen Art. 15 Abs. 1 ECRL handeln mag, weil proaktive Überwachungsmaßnahmen notwendig werden, so gilt dieses Verbot aber nicht im Bereich der allein den Mitgliedstaaten überlassenen medienrechtlichen Vielfaltssicherung (Art. 1 Abs. 6 ECRL). Um diese geht es aber auch im Falle der Meinungsroboter: Indem Transparenz geschaffen und die Betreiber hierfür in die Verantwortung genommen werden, soll der Einfluss der Meinungsroboter auf die Informationsvermittlung verringert und die Nutzer bei der Rezeption von Inhalten unterstützt werden, sodass es im Idealfall zu einer kritischeren Rezeption und dadurch bedingt $\mathrm{zu}$ einer Rezeptionsvielfalt kommt. ${ }^{2389}$ Und Art. 1 Abs. 6 ECRL erlaubt zu diesem Zweck auch ein Abweichen vom Herkunftslandprinzip (Art. 3 Abs. 1 ECRL), sodass auch die im EU-Ausland niedergelassenen Betreiber verpflichtet werden können. ${ }^{2390}$ Der Medienstaatsvertrag sieht daneben auch ein aufsichtsrechtliches Korsett vor, dass die Einhaltung der Vorgaben durch Kontroll- und Ermittlungsrechte der Landesmedienastalten absichert ( $\mathbb{S} 95 \mathrm{MStV}$ ) und ihnen zudem die Möglichkeit gibt, die abstrakten Vorgaben betreiberspezifisch näher zu konkretisieren ( $\$ 96 \mathrm{MStV}$ ).

Größtenteils bewegen sich die Länder damit innerhalb ihres verfassungsrechtlich nicht angreifbaren Handlungsspielraums. Mit dem Transparenzgebot und dem Diskriminierungsverbot adressiert man spezifische Risiken, die im ersten Fall aus einer Informationsasymmetrie in Bezug auf die Informationsvermittlung und im zweiten Fall aus der technischen Einwirkungsmöglichkeit auf den Vermittlungsprozess resultiert. Hierbei werden die Betreiber nicht über Gebühr in Anspruch genommen, vielmehr entsprechen die zu veröffentlichenden Informationen dem Informationsinteresse der Allgemeinheit. Und das Diskriminierungs-

2389 Zu $\int 93$ Abs. 4 MStV, seiner Bedeutung und den europarechtlichen Problemen 2. Teil, 3. Kapitel, C.II.2.c.

2390 2. Teil, 3. Kapitel, C.II.2.f.bb. 
verbot erlaubt es weiterhin Inhalte priorisiert weiterzuleiten, sofern dieser Umstand und die zentralen Parameter hierfür transparent gemacht werden. ${ }^{2391} \mathrm{Im}$ Falle der Meinungsroboter verhindert $\mathbb{1} 18$ Abs. 3 MStV gerade nicht die Verbreitung der von der Meinungsfreiheit geschützten Inhalte, sondern versucht nur den Vorteil zu neutralisieren, die die Urheber sich durch die multiplen Identitätstäuschungen selbst verschafft haben und der wesentliche Grundlage für die Beeinträchtigung der Meinungsäußerungsfreiheit, der kommunikativen Chancengleichheit und der Informations- und Meinungsbildungsfreiheit ist. Deutlichere Probleme wirft demgegenüber $\mathbb{\$} 93$ Abs. $4 \mathrm{MStV}$ auf. Denn die notwendigen proaktiven und automatisierten Erkennungsverfahren führen - auch bei menschlicher Überwachung - zu falsch-positiven Ergebnissen. Auch echte Nutzer könnten also als „Meinungsroboter" klassifiziert und sodann als solche gekennzeichnet werden. Weil diese Gefahr gerade durch den Medienstaatsvertrag und nicht allein privatautonom durch die Betreiber begründet wird, liegt ein mittelbar-faktischer Eingriff in die Meinungsfreiheit betroffener Nutzer und eine Veränderung der kommunikativen Chancengleichheit vor. Als Ausgleich müsste man zumindest eine einfache Möglichkeit vorsehen, durch die sich betroffene Nutzer gegen entsprechende Maßnahmen wehren können. Allerdings schweigt der MStV hierzu. Die Satzungsbefugnis der Landesmedienanstalten ist ungeeignet, um diesen Schutz nachträglich als Konkretisierung der Sorgetragungspflicht einführen zu können, weil es hierbei um eine wesentliche Frage gehen dürfte, deren Beantwortung dem Gesetzgeber selbst vorbehalten ist. Die Landesmedienanstalten könnten lediglich durch eine Übergangsfrist den Eintritt der Sorgetragungspflicht hinausschieben. Dadurch könnte sichergestellt werden, dass die Betreiber sachgerechte und nicht übereilte Maßnahmen ergreifen und sich hierbei - ähnlich wie bei desinformativen Inhalten - von externer unabhängiger Seite beraten lassen. Zukünftig führt aber kein Weg daran vorbei, die Sorgetragungspflicht der Betreiber auch auf notwendige Vorkehrung zum Schutz vor irrtümlichen Kennzeichnungen zu erstrecken. Solange dies nicht geschieht, sind verfassungsrechtliche Zweifel anzumelden.

Die interdisziplinäre Diskussion um die Meinungsroboter ist also schon deshalb noch lange nicht an einem Ende angekommen. Es gilt zudem nicht nur die Umsetzung der neuen Vorgaben kritisch zu überwachen, sondern auch auf sich verändernde Umstände sachgerecht aber eben auch zeitlich deutlich schneller zu reagieren als das mit der Transformation des Rundfunk- zum Medienstaatsvertrag oder der Realisierung der Kennzeichnungspflicht des $\mathbb{1} 18$ Abs. $3 \mathrm{MStV}$, die bereits unmittelbar nach Bekanntwerden des Phänomens gefordert wurde, erfolgt ist. Aus der grundrechtlichen Schutzpflicht folgt insoweit eine Beobachtungsund ggf. Nachbesserungspflicht nicht nur der Länder. Auch der Bund kann inner-

2391 2. Teil, 3. Kapitel, C.II.3.a. 
halb seines Kompetenzbereiches dazu berufen sein, ergänzenden Schutz - soweit er notwendig wird - zu gewähren. Inwieweit dieses Bedürfnis besteht, ist vor allem auch von empirischen Forschungsergebnissen abhängig, weshalb sie zu unterstützen ist. Das kann auf lange Sicht vor allem einen verpflichtenden Zugang zu den Netzwerkdaten notwendig machen, den die Netzwerkbetreiber bislang nur unzureichend gewähren. Das Informationsfreiheitsgesetz des Bundes könnte hierfür als Orientierungshilfe dienen.2392 Vor allem muss auch überlegt werden, wie bestimmte Einsatzstrategien präventiv oder repressiv verhindert werden können. Weil die Netzwerkeffekte und Nutzungsoberflächen conditio sine qua non u.a. für einen (erfolgreichen) Einfluss von Meinungsrobotern und desinformativen Inhalten sind, könnten Veränderungen in der Nutzeroberfläche oder den Algorithmen sinnvoll sein. Ob und wie dies erfolgt, bleibt vorerst aber eine Frage, die die Betreiber selbst beantworten müssen. ${ }^{2393}$

Im Falle desinformativer Inhalte ist es zwar möglich, strafrechtliche Sanktionen für eklatante und besonders schädliche Inhalte vorzusehen. Und seit geraumer Zeit wird zudem über eine mittelbare Klarnamenpflicht im Internet und sozialen Netzwerken debattiert. ${ }^{2394}$ Auch wenn beides verfassungsrechtlich zulässig sein kann, so ist gleichwohl zur Vorsicht zu raten: $\mathrm{Zu}$ ungewiss sind die Folgen, die aus derlei Maßnahmen resultieren. Der Staat kann den Kommunikationsprozess dadurch noch erheblicher stören, als dies zur Zeit der Fall ist, weil etwa bereits vorhandene staatskritische Strömungen (Stichwort „Lügenpres-

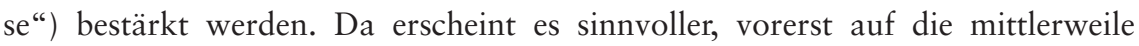
ergriffenen Maßnahmen der Betreiber zu blicken und hier - ähnlich wie mit dem NetzDG - gewisse verfahrenstechnische und organisatorische Vorgaben zu machen, um einerseits staatlichen Schutz gewährleisten zu können und andererseits die gewonnene Meinungsmacht der Betreiber sinnvoll zu kanalisieren.2395 Und letztlich sind Meinungsroboter nicht nur ein nationalstaatliches Problem. Die EU hat mit ihrem Verhaltenskodex bereits aufgezeigt, dass auch ein gemeineuropäisches Interesse an einer Regulierung besteht. Die bisherigen europäischen Vorgaben (ECRL) stammen aber noch aus dem Jahr 2001. Sie gewähren den Betreibern einen sicheren Hafen, in den sie sich zurückziehen können und vor einer Inanspruchnahme (grundsätzlich) geschützt sind. Nicht nur sieht aber die EU in jüngerer Zeit die Notwendigkeit diesen sicheren Hafen punktuell zu verlassen. Auch die Mitgliedstaaten versuchen diesen Rückzugsraum der Betreiber zu schmälern. Das macht auf lange Sicht eine europäische einheitliche Lösung notwendig. ${ }^{2396}$ Der MStV zeigt jedenfalls eine Richtung hierfür auf.

2396 2. Teil, 4. Kapitel, H. 
Insoweit mag der Medienstaatsvertrag schon wegen der weiter fortschreitenden technischen Entwicklung nicht der Weisheit letzter Schluss sein, um die Online-Gefahren für die politische Willensbildung abzuwehren. Doch versucht der Staat nun (endlich) seinen Herrschaftsanspruch auch in der virtuellen Welt durchzusetzen, ohne dabei eigenständig erhebliche neue Gefahren zu schaffen. Insoweit hat man vorerst einen Weg gefunden, der vergleichsweise sicher an Skylla - der weiteren fehlenden (Mindest-)Regulierung entsprechender Risiken und Charybdis - eine übereilte, grundrechtsinvasive Regulierung der Risiken vorbeiführt. ${ }^{2397}$

2397 Zu diesem Vergleich von Skylla und Charybdis siehe Martini, Blackbox Algorithmus, S. 103. 


\section{Literaturverzeichnis}

Abokhodair, Norah/ Yoo, Daisy/McDonald, David W., Dissecting a Social Botnet: Growth, Content and Influence in Twitter, in: Association for Computing Machinery (Hrsg.), Proceedings of the 18th ACM Conference on Computer Supported Cooperative Work \& Social Computing, New York 2015, S. 839-851

Abolaish, Muhammad/Fazil, Mohd, Socialbots: Impacts, Threat-Dimensons and Defense Challenges, IEEE Technology and Society Magazine Vol. 39 (2020), S. 52-61

Achilles, Nastasia, Vom Homo Oeconomicus zum Differenzierten Verbraucher - Analyse von Begriff, Entwicklung und neuen Herausforderungen des verbrauchervertragsrechtlichen Leitbildes auf EU-Ebene, Baden-Baden 2020.

Adelberg, Philipp Nikolaus, Rechtspflichten und -grenzen der Betreiber sozialer Netzwerke - Zum Umgang mit nutzergenerierten Inhalten, Wiesbaden 2020

Ahmed, Wasim/Vidal-Alabal, Josep/Downing, Joseph/López Seguí, Francesc, COVID-19 and the 5G Conspiracy Theory: Social Network Analysis of Twitter Data, Journal of Medical Research Vol. 22 (2020), Iss. 5, e19458, doi: 10.2196/19458

Abrens, Hans-Jürgen, Influencer-Marketing - Regulierungsrahmen und Konsequenzen seiner Anwendung (Teil 1), GRUR 2018, S. 1211-1218

Aiello, Luca Maria/Deplano, Martina/Schifanella, Rossano/Ruffo, Giancarlo, People are Strange when you're a Stranger: Impact and Influence of Bots on SocialNetworks, in: Proceedings of the Sixth International AAAI Conference on Weblogs and Social Media, Palo Alto 2012, S. 10-17

Albrecht, Jan Philipp/Jotzo, Florian, Das neue Datenschutzrecht der EU - Grundlagen, Gesetzgebungsverfahren, Synopse, 1. Auflage, Baden-Baden, 2017

Alexy, Robert, Theorie der Grundrechte, 1. Aufl., Frankfurt a.M. 1986

ANGA der Breitbandverband e.V., Statement by The Broadband Association ANGA (Germany) on the German draft Inter-State Treaty on modernising the media regulation (Medienstaatsvertrag - MStV) in Germany (TRIS Notification 2020/26/D), abrufbar unter https://ec.europa.eu/growth/tools-databases/tris/de/search/?trisaction=s earch.detail\&year=2020\&num=26 (Stand: 30.03 .2021 )

Antonio Amadeu Stiftung, Stellungnahme zum erweiterten Entwurf des NetzDG, abrufbar unter https://www.amadeu-antonio-stiftung.de/stellungnahme-der-amadeu-anton io-stiftung-zum-entwurf-eines-gesetzes-zur-bekaempfung-von-rechtsextremismus-und -hasskriminalitaet-54029/, (Stand: 30.03.2021)

Appel, Markus, Wie lässt sich das Postfaktische eindämmen?, in: ders. (Hrsg.), Die Psychologie des Postfaktischen - Über Fake News, Lügenpresse Click und Co., Springer, Wiesbaden 2020, S. 205-210

Appel, Markus/Doser, Nicole, Fake News, in: Markus Appel (Hrsg.), Die Psychologie des Postfaktischen - Über Fake News, Lügenpresse Click und Co., Springer, Wiesbaden 2020, S. 9-20

v. Arnauld, Andreas, Freiheit der Kunst, in: Josef Isensee/Paul Kirchhof (Hrsg.), Band 7, $\$ 162,3$. Auflage, Heidelberg 2009

Arndt, Nina/Droege, Michael, Versammlungsfreiheit versus Sonn- und Feiertagsschutz?, NVwZ 2003, S. 906-913

Auer-Reinsdorff, Astrid/Conrad, Isabell, Handbuch IT- und Datenschutzrecht, 3. Auflage, München 2019 
Ausloos, Jef/Leerssen, Paddy/ten Thije, Pim, Operationalizing Research Acce in Platform Governance - What to learn from other industries?, abrufbar unter https://algorithm watch.org/wp-content/uploads/2020/06/GoverningPlatforms_IViR_study_June2020 -AlgorithmWatch-2020-06-24.pdf, (Stand: 30.03.2021)

Baade, Björnstjern, Informationskriegsführung und Fake News, in: Sebastian Graf von Kielmansegg/Heike Krieger/Stefan Sohm, Die Wiederkehr der Landes- und Bündnisverteidigung - Neue Rechtsfragen eines alten Szenarios, Baden-Baden 2020, S. 161-189

Badura, Peter, Zur grundrechtlichen, gebühren- und europarechtlichen Auslegung des Rundfunkbegriffs, in: Dittmann, Armin/Fechner, Frank/Sander, Gerald G., Der Rundfunkbegriff im Wandel der Medien - Symposium zum 65. Geburtstag von Professor Dr. iur. Dr. h. c. Thomas Oppermann, Berlin 1997, S. 116-119

Bakshy, Eytan/Messing, Solomon/Adamic, Lada, Exposure to ideologically diverse news and opinion on Facebook, Science 348 (2015), S. 1130-1132, DOI: https://doi.org/10. 1126/science.aaa1160

Dies., Exposure to Diverse Information on Facebook, Facebook Research v. 07.05.2015, abrufbar unter https://research.fb.com/exposure-to-diverse-information-on-facebook -2/, (Stand 30.03.2021)

Balmas, Meital, When Fake News Becomes Real: Combined Exposure to Multiple News Sources and Political Attitudes of Inefficacy, Alienation, and Cynicism, Communication Research Vol. 41 (2014), S. 430-454

Bastos, Marco T./Mercea/Dan, The Brexit Botnet and User-Generated Hyperpartisan News, Social Science Computer Review Vol. 37 (2019), S. 38-54

Bauer, Marc, Kartellrecht 4.0? - Herausforderungen der Plattformökonomie für die Regulierung des Meinungsbildungsprozesses, WRP 2020, S. 171-176

Beater, Axel, Verbraucherschutz und Schutzzweckdenken im Wettbewerbsrecht, Tübingen. 2000

Ders., Medienrecht, 2. Auflage, Tübingen 2016

Ders., Zivil- und medienrechtlicher Schutz von und vor anonymen Äußerungen, NJ 2019, S. 365-373

Beck, Hanno, Behavioral Economics - Eine Einführung, Wiesbaden 2014

Beck, Klaus, Kommunikationswissenschaft, 6. Auflage, Stuttgart 2020

Beck, Wolfgang, Nachhaltige Regulierung sozialer Netzwerke - Ein Beitrag zur Regulierungsdebatte, DVBl. 2020, S. 253-259

Beckedahl, Moritz, Facebook: Datenabgriff von 87 Millionen Nutzern ist nur Spitze des Eisberges, veröffentlicht am 05.04.2018 auf netzpolitik.org, abrufbar unter https://net zpolitik.org/2018/facebook-datenabgriff-von-87-millionen-nutzern-ist-nur-spitze-des -eisberges/, (Stand 30.03.2021)

Becker, Alexander, Medienforscher über Social Media und Wahlkampf: „Lärmende Rhetorik im Netz für Demokratie brandgefährlich“, meedia.de, v 7.11.2016 http://meedi a.de/2016/11/07/medienforscher-ueber-social-media-und-wahlkampf-laermende-rheto rik-im-netz-fuer-demokratie-brandgefaehrlich/, (Stand: 30.03.2021)

Becker, Florian, Öffentliches und Privates Recht, NVwZ 2019, S. 1385-1392

Bedford-Strohm, Jonas, Verletzliche Öffentlichkeit. Eine Kritik (der Kritik) der Filterblase, in: ders./ Florian Höhne/Julian Zeyer-Quattender (Hrsg.), Digitaler Strukturwandel der Öffentlichkeit - Interdisziplinäre Perspektiven auf politische Partizipation im Wandel, Baden-Baden 2019, S. 201-217 
Benda, Ernst/Klein, Eckart, Verfassungsprozeßrecht - Ein Lehr- und Handbuch, 3. Auflage, Heidelberg 2001

Benjamin, Stuart Minor, Algorithms and Speech, University of Pennsylvania Law Review, Volume 161 (2013), S. 1445-1493

Bendel, Oliver, 300 Keywords Informationstechnik - Grundwissen aus Computer-, Netz- und Neue-Medien-Ethik sowie Maschinenethik, Wiesbaden 2016

Benreuther, Friedrich, Zur Interessenabwägung bei anonymen Meinungsäußerungen im Internet, Archiv für Presserecht 2011, S. 218-223

Berberich, Matthias, Der Content „gehört” nicht Facebook! - AGB-Kontrolle der Rechteeinräumung an nutzergenerierten Inhalten, MMR 2010, S. 736-741

Berg, Wilfried, Berufsfreiheit und verbotene Berufe, GewArch 1977, S. 249-254

Ders., Die wehrhafte Demokratie des Grundgesetzes im 21. Jahrhundert, in: Gerhard Dannecker (Hrsg.), Festschrift für Harro Otto: zum 70. Geburtstag 1. April 2007

Bessi, Alessandro/Ferrara, Emilio, Social Bots distort the U.S. Presidential election online discussion, First Monday Volume 21 Nr. 11, 7. November 2016, DOI: http://dx.doi.o $\mathrm{rg} / 10.5210 / \mathrm{fm} . v 21 \mathrm{i} 11.7090$

Betzalel, Noa/Ishai, Paul Ben/Feldman, Yuri, The human skin as a sub-THz receiver Does 5G pose a danger to it or not?, Environmental Research Vol. 163 (2018), S. 208-216

Beyers, Eva, Privatheit in der Waagschale - Instrumente des datenschutzrechtlichen Interessenausgleichs im Kontext sozialer Netzwerke, in: Heckmann (Hrsg.), Internetrecht und Digitale Gesellschaft, Band 9, 2018.

Bickenbach, Christian, Subjektiv-öffentliches Recht auf Klimaschutz? Die Erderwärmung vor den Gerichten, JZ 2020, S. 168-177

Bilski, Nico/Schmid, Thomas, Verantwortungsfindung beim Einsatz maschinell lernender Systeme, NJOZ 2019, S. 657-661

Bilz, Christopher, Margin of Appreciation der EU-Mitgliedstaaten: Eine Untersuchung im Anwendungsbereich der Grundrechtecharta am Beispiel des Datenschutzgrundrechts, der Religionsfreiheit, der unternehmerischen Freiheit und des Rechts auf einen wirksamen Rechtsbehelf, Tübingen 2020

Binder, Reinhart/Vesting, Thomas, Beck'scher Kommentar zum Rundfunkrecht, 4. Auflage, München 2018

Birnbacher, Dieter, Paternalismus im Strafrecht - ethisch vertretbar?, in: Andreas von Hirsch/Ulfrid Neumann/Kurt Seelmann (Hrsg.), Paternalismus im Strafrecht - Die Kriminalisierung von selbstschädigendem Verhalten, Baden-Baden 2010

Blenk, Josef, „Streaming“ - Eine Urheberrechtsverletzung? AfP 2014, S. 220-223

Bock, Michael, Die Übertragung der Kommunikationsfreiheiten des Art. 5 GG auf das Internet, Wiesbaden 2018

Böckenförde, Ernst-Wolfgang, Grundrechte als Grundsatznormen, Der Staat 29 (1990), S. 1-31

Ders., Schutzbereich, Eingriff, verfassungsimmanente Schranken - Zur Kritik gegenwärtiger Grundrechtsdogmatik, Der Staat 42 (2003), S. 165-192

Bodewig, Theo, Urhebervertragsrecht in ausgewählten Ländern - USA, in: FriedrichKarl Beier/Horst-Peter Götting/Michael Lehmann/Rainer Moufang (Hrsg.), Urhebervertragsrecht - Festgabe für Gerhard Schricker zum 60. Geburtstag, München 1995, S. 833-883

Boehme-Neßler, Volker, Facebook-Demokratie? Überlegungen zur Demokratie im digitalen Zeitalter, UFITA 2015, S. 655-685 
Ders., Gekaufte Aufmerksamkeit? Verfassungs- und parteienrechtliche Überlegungen zum Sponsoring von politischen Parteien, NVwZ 2017, S. 528-531

Ders., Das Ende der Demokratie? Effekte der Digitalisierung aus rechtlicher, politologischer und psychologischer Sicht, Berlin 2018

Boblen, Marc, Der zivilrechtliche Auskunftsanspruch bei der Bekämpfung von Hass im Internet, NJW 2020, S. 1999-2004

Boichak, Olga/ Jackson, Sam/Hemsley, Jeff/Taunupabrungsun, Sikana, Automated Diffusion? Bots and Their Influence During the 2016 U.S. Presidential Election, in: Gobinda Chowdhury/Julie McLeod/Val Gillet/Peter Willett (Hrsg.), Proceedings of the $13^{\text {th }}$ International Conference, iConference, Sheffield 2018, S. 17-26D

Borges, Georg, Rechtliche Rahmenbedingungen für autonome Systeme, NJW 2018, S. 977-982

Bortnikov, Vyacheslav, Netzneutralität und Bedingungen kommunikativer Selbstbestimmung - Pflichten des freiheitlichen Verfassungsstaates zur Gewährleistung der Neutralität des Internets im Lichte der grundrechtlichen Schutzpflichtenlehre, Schriftenreihe des Instituts für Rundfunkrecht an der Universität zu Köln, Band 109, C. H. Beck, München, 2013

Boshmaf, Yazan/Muslukhov, ldar/Beznosov, Konstantin/Ripeanu, Matei, The Socialbot Network: When Bots Socialize for Fame and Money, in: Association of Computing Machinery (Hrsg.), Proceedings of the $27^{\text {th }}$ annual ComputerSecurity Applications Conference, New York 2011, S. 93-102

Dies., Design and analysis of a social botnet, Computer Networks 57 (2013) S. 556-578

Bötticher, Astrid, Extremistische Social Bots, Kriminalistik 2017, S. 16-18

Brachten, Florian/Stieglitz, Stefan/Hofeditz, Lennart/Kloppenborg, Katharina/Reimann, Annette, Strategies and Influence of Social Bots in a 2017 German state election - A case study on Twitter, 2017, arXiv:1710.07562 [cs.CY]

Brandom, Russell, Facebook-backed lawmakers are pushing to gut privacy law, veröffentlicht bei The Verge, am 10.4.2018, abrufbar unter https://www.theverge.com/ 2018/4/10/17218756/facebook-biometric-privacy-lobbying-bipa-illinois, zuletzt abgerufen am 01.02.2019.

Bräutigam, Peter, Das Nutzungsverhältnis bei sozialen Netzwerken - Zivilrechtlicher Austausch von IT-Leistungen gegen personenbezogene Daten, MMR 2012, S. 635-641

Brehm, Wolfgang, Zur automatisierten Willenserklärung, in: Erik Jayme/Adolf Laufs/ Karlheinz Misera/Gert Reinhart/Rolf Serick (Hrsg.), Festschrift für Hubert Niederländer zum siebzigsten Geburtstag am 10. Februar 1991, Heidelberg 1991

Breithut, Jörg, Facebook blendet likes aus, spiegel.de v. 27.09.2019, abrufbar unter https://www.spiegel.de/netzwelt/netzpolitik/facebook-blendet-anzahl-der-likes-in-aust ralien-aus-a-1288993.html, Stand: 18.08.2020

Bremer, Eckhard/Esser, Michael/Hoffmann, Martin, Der Rundfunkbegriff in der Verfassungs- und Wirtschaftsordnung in Deutschland, Baden-Baden 1992

Brener, Rüdiger, Freiheit des Berufs, in: Josef Isensee/Paul Kirchhof (Hrsg.), Handbuch des Staatsrechts, Band IIX, $\mathbb{1} 170,3$. Auflage, Heidelberg 2010

Brings-Wiesen, Tobias, Meinungskampf mit allen Mitteln und ohne Regeln? - Eine Replik auf Jens Milkers „'Bot-Armeen’ als Meinungsmacher im Wahlkampf“, JuWissBlog Nr. 93/2016, abrufbar unter http://www.juwiss.de/93-2016/, (Stand 30.03.2021)

Brinkmann, Tomas, Medienrechtliche Herausforderungen im digitalen Strukturwandel der Öffentlichkeit, UFITA 2019, S. 364-394 
Bröckling, Marie, Facebook behindert Forschung zu „Fake News“, netzpolitik.org v. 12.03.2018, abrufbar unter: https://netzpolitik.org/2018/facebook-behindert-forschu ng-zu-fake-news/, (Stand 30.03.2021)

Brodnig, Ingrid, Hass im Netz - Was wir gegen Hetze Mobbing und Lügen tun können, Wien 2016

Brodowski, Dominik, Anonyme Ehrverletzungen in Internetforen: Ein Lehrstück strafrechtlicher (Fehl-)Regulierung, Juristische Rundschau 2013, S. 511-523

Brüning, Christoph, Möglichkeiten einer unionsrechtlichen Regulierung des Glücksspiels im europäischen Binnenmarkt, NVwZ 2013, S. 23-28

Bruns, Axel, After the 'APIcalypse': social media platforms and their fight against critical scholarly research, Information, Communication \& Society 2019, S. 1544-1566

Brunst, Phillip W., Umsetzungsprobleme der Impressumspflicht bei Webangeboten, MMR 2004, S. 8-13

Buchner, Benedikt, Grundsätze und Rechtmäßigkeit der Datenverarbeitung unter der DS-GVO, DuD 2016, S. 155-161

Buiten, Miriam C./de Streel, Alexandre/Peitz, Martin, Rethinking Liability Rules for Online Hosting Platforms (March 11, 2019). Rheinische Friedrich-Wilhelms-Universität Bonn - Universität Mannheim, Discussion Paper Series - CRC TR 224 (2019). DOI: http://dx.doi.org/10.2139/ssrn.3350693

Bullinger, Martin, Der Rundfunkbegriff in der Differenzierung kommunikativer Dienste, AfP 1996, S. 1-8

Ders., Freiheit von Presse, Rundfunk und Film, in: Josef Isensee/Paul Kirchhof (Hrsg.), Handbuch des Staatsrechts, Band VII, $\mathbb{1} 163$, 3. Auflage, Heidelberg 2009

Bunge, Felix, Über die kollektive Schutzrichtung des Rechts auf informationelle Selbstbestimmung, ZD-Aktuell 2015, 04635

Burggraf, Jürgen/ Gerlach, Christine/Wiesner, Jan, EuropäischeMedienregulierung im Spannungsfeld zwischen EU- und mitgliedstaatlicher Kompetenz, Media Perspektiven 2018, S. 496-510

Burki, Thala, Vaccine misinformation and social media, Digital Health Vol. 1 (2019), S. e258-e259, DOI: https://doi.org/10.1016/S2589-7500(19)30136-0

Büro für Technikfolgenabschätzung beim Deutschen Bundestag (TAB), Social Bots - Die potenziellen Meinungsmacher, TAB-Fokus Nr.16 zum Horizon-Screenings Nr.3, April 2017

Busch, Christoph, Mehr Fairness und Transparenz in der Plattformökonomie? - Die neue P2B-Verordnung im Überblick, GRUR 2019, S. 788-796

Busch, Thomas, Zur urheberrechtlichen Einordnung der Nutzung von Streamingangeboten, GRUR 2011, S. 496-503

Buser, Andreas, Ein Grundrecht auf Klimaschutz? Möglichkeiten und Grenzen grundrechtlicher Klimaklagen in Deutschland, DVBl. 2020, S. 1389-1396

Butterwegge, Christoph/Hentges, Gudrun/Wiegel, Gerd, Rechtspopulisten im Parlament: Polemik, Agitation und Propaganda der AfD, Frankfurt a.M. 2018

Cadwalladr, Carol/Graham-Harrison, Emma, Revealed: 50 million Facebook profiles harvested for Cambridge Analytica in major data breach, in: The Guardian, 17.3.2018, abrufbar unter https://www.theguardian.com/news/2018/mar/17/cam bridge-analytica-facebook-influence-us-election (Stand: 30.03.2021)

Calliess, Christian, Die grundrechtliche Schutzpflicht im mehrpoligen Verfassungsrechtsverhältnis, JZ 2006, S. 321-330 
Ders., Die Leistungsfähigkeit des Untermaßverbotes als Kontrollmaßstab grundrechtlicher Schutzpflichten, in: Rainer Grote/Ines Härtel/Karl-E. Hain/Thorsten Ingo Schmidt/ Thomas Schmitz/Gunnar Folie Schuppert/Christian Winterhoff (Hrsg.), Die Ordnung der Freiheit - Festschrift für Christian Starck zum siebzigsten Geburtstag, Tübingen 2007

Camilo de Oliveira, Renata, Zur Kritik der Abwägung in der Grundrechtsdogmatik Beitrag zu einem liberalen Grundrechtsverständnis im demokratischen Rechtsstaat, Berlin 2013

Canaris, Claus-Wilhelm, Grundrechte und Privatrecht, AcP 184 (1984), S. 201-246

Caspar, Johannes, Klarnamenpflicht versus Recht auf pseudonym Nutzung, Zeitschrift für Rechtspolitik 2015, S. 233-236

Castendyk, Oliver/Böttcher, Kathrin, Ein neuer Rundfunkbegriff für Deutschland? - Die Richtline für audiovisuelle Mediendienste und der deutsche Rundfunkbegriff, MMR 2008, S. 13-18

Castillo, Mariano, 43 killed in Mexican shootout, CNN v. 23.05.2015, abrufbar unter: https://edition.cnn.com/2015/05/22/americas/mexico-tanhuato-michoacan-shootout/i ndex.html, (Stand 30.03.2021)

Chavoshi, Nikan/Hamooni, Hossein/Mueen, Abdullah, DeBot: Twitter Bot Detection via Warped Correlation, in: Proceedings of the $16^{\text {th }}$ International Conference on Data Mining (ICDM), Barcelona 2016, S. 817-822, DOI: 10.1109/ICDM.2016.0096

Cheng, Chun/Luo, Yun/Yu, Changbin, Dynamic mechanism of social bots interfering with public opinion network, Physica A 2020, 124163, DOI: https://doi.org/10.1016/ j.physa.2020.124163

Cinelli, Matteo/Quattrociocchi, Walter/Galeazzi, Alessandro/Valensise, Carlo Michele/Brugnoli, Emanuele/Schmidt, Ana LucialZola, Paola/Zollo, Fabiana/Scala, Antonio, The Covid-19 social media Infodemic, Scientific Reports 10 (2020), 16598, DOI: https://doi.org/10.1038/s41598-020-73510-5

Classen, Claus Dieter, Staatsrecht - Grundrechte, 1. Auflage, München 2018

Ders., Die Drittwirkung der Grundrechte in der Rechtsprechung des Bundesverfassungsgerichts, AöR 1997, S. 65-107

Ders., Legitime Stärkung des Bundestages oder verfassungsrechtliches Prokrustesbett? Zum Urteil des BVerfG zum Vertrag von Lissabon, JZ 2009, S. 881-889

Clausen, Christoph, Das Verhältnis von Achtungs- und Schutzpflichten in Ausnahmesituationen, Berlin 2018

Claussen, Victor, Fighting Hate Speech and Fake News. The Network Enforcement Act (NetzDG) in Germany in the context of European legislation, Mediales - Revista di Dritto dei Media 2018, S. 128-136, abrufbar unter http://www.medialaws.eu/rivista/fi ghting-Hate Speech-and-fake-news-the-network-enforcement-act-netzdg-in-germanyin-the-context-of-european-legislation/, (Stand: 30.03.2021)

Clemens, Rudolf, Die elektronische Willenserklärung - Chancen und Gefahren, NJW 1985, S. 1998-2005

Cobbe, Jennifer/Singh, Jatinder, Regulating Recommending: Motivations, Considerations, and Principles, European Journal of Law and Technology, Vol. 10 (2019), Nr. 3, abrufbar unter https://ejlt.org/index.php/ejlt/article/view/686, (Stand: 30.03.2021)

Cohn, Cindy, Code is free speech, Time Magazine v. 17.03.2016, abrufbar unter https://t ime.com/4248928/code-is-free-speech/, (Stand: 30.03.2021) 
Cole, Mark D./Etteldorf, Christina/Ullrich, Carsten, Cross-Border Dissemination of Online Content - Current and Possible Future Regulation of the Online Environment with a Focus on the EU E-Comerce-Directive, Baden-Baden 2020

Coleman, Miles C., Bots, Social Capital and the Need for Civility, Journal of Media Ethics Vol. 33 (2018), S. 120-132

Comisión Nacional de los Derechos humanos, Recomendación Nr. 4VG /2016 sobre violaciones graves a los derechos humanos, por el uso excesivo de la fuerza que derivó en la ejecución arbitraria de 22 civiles y la privación de la vida de 4 civiles; la tortura de dos personas detenidas; el trato cruel, inhumano y degradante en perjuicio de una persona detenida y la manipulación del lugar de los hechos, atribuida a la policía federal, con motivo de los hechos ocurridos el 22 mayo de 2015 en el „Rancho del sol“, municipio de Tanhuato, Michoacán, abrufbar unter https:/www.cndh.org.mx/sites/al 1/doc/Recomendaciones/ViolacionesGraves/RecVG_004_.pdf, (Stand: 30.03.2021)

Conraths, Timo/Krüger, Stefan, Das virtuelle Hausrecht des Online-Spiel-Betreibers Wirksame Rechtsschutzmöglichkeit für Online-Spiel-Anbieter abseits des Vertragsrechts, MMR 2016, S. 310-313

Constant, Sarah A., The Computer Fraud and Abuse Act: A Prosecutor's Dream and a Hacker's Worst Nightmare - The Case Against Aaron Swartz and the Need To Reform the CFAA, Tulane Journal of Technology \& IP Vol. 16 (2013), S. 231-248

Cornils, Matthias, Die Ausgestaltung der Grundrechte - Untersuchungen zur Grundrechtsbindung des Ausgestaltungsgesetzgebers, Tübingen 2005

Ders., Vielfaltssicherung bei Telemedien, AfP 2018, S. 377-387

Ders., Die Perspektive der Wissenschaft: AVMD-Richtlinie, der 22. Rundfunkänderungsstaatsvertrag und der »Medienstaatsvertrag « - Angemessene Instrumente für die Regulierungsherausforderungen?, ZUM 2019, S. 89-103

Ders., Designing Platform Governance: A normative perspective on needs, strategies, and tools to regulate intermediaries, abrufbar unter https://algorithmwatch.org/wp-co ntent/uploads/2020/05/Governing-Platforms-legal-study-Cornils-May-2020-Algorith mWatch.pdf, (Stand: 30.03.2021)

Cornils, Matthias/Liesem, Kerstin, Stellungnahme zum Diskussionsentwurf eines Medienstaatsvertrages der Rundfunkkommission der Länder, abrufbar unter https://www. mainzer-medieninstitut.de/wp-content/uploads/Mainzer-Medieninstitut-Stellungnahm e-zum-Medienstaatsvertrag-1.pdf, (Stand: 30.03.2021)

Costera Meijer, Irene/Groot Kormelink, Tim, Checking, Sharing, Clicking and Linking Changing patterns of news use between 2004 and 2014, Digital Journalism Vol. 3 (2015) Iss. 5, S. 664-679

Cresci, Stefano, A Decade of Social Bot Detection, Communications of the ACM Vol. 63 (2020), S. 72-83

Czauderna, André, Lernen als soziale Praxis im Internet - Objektiv hermeneutische Rekonstruktionen aus einem Forum zum Videospiel Pokémon, Wiesbaden 2014

Czychowski, Christian/Nordemann, Jan Bernd, Vorratsdaten und Urheberrecht - Zulässige Nutzung gespeicherter Daten, NJW 2008, S. 3095-3099

Dachwitz, Ingo/Rudi, Tomas/Rebiger, Simon, FAQ: Was wir über den Skandal um Facebook und Cambridge Analytica wissen [UPDATE], netzpolitik.org, v. 21.3.2018, https://netzpolitik.org/2018/cambridge-analytica-was-wir-ueber-das-groesste-datenlec k-in-der-geschichte-von-facebook-wissen/, (Stand 30.03.2021)

Daniel, Florian/Cappiello/Cinzia/Ben, Boualem, Bots Acting Like Humans: Understanding and Preventing Harm, IEEE Internet computing online 2019, S. 40-49 
Dankert, Kevin, Verfälschung von Datenbeständen durch Social Bots, in: Wolfgang Hoffmann-Riem (Hrsg.), Big Data - Regulative Herausforderungen, Baden-Baden 2018, S. 157-165

Dankert, Kevin/Dreyer, Stephan, Social Bots - Grenzenloser Einfluss auf den Meinungsbildungsprozess?, Kommunikation und Recht 2017, 73-78

Dauses, Manfred A./Ludwigs, Markus, Handbuch des EU-Wirtschaftsrechts, Loseblattsammlung, Stand des Gesamtwerks 50. Ergänzungslieferung, März 2020

Denga, Michael, Gemengelage privaten Datenrechts, NJW 2018, S. 1371-1376

Dengel, Andreas, Digitale Bildung: ein interdisziplinäres Verständnis zwischen Medienpädagogik und Informatik, Medienpädagogik 2018, S. 11-26

Denninger, Erhard, Vom Elend des Gesetzgebers zwischen Übermaßverbot und Untermaßverbot, in: Herta Däublein-Gmelin/Klaus Klinkel/Hans Meyer/Helmut Simon (Hrsg.), Gegenrede: Aufklärung - Kritik ... Öffentlichkeit, Festschrift für Ernst Gottfried Mahrenholz, Baden-Baden 1994, S. 561-572

Derin, Benjamin/ Golla, Sebastian J., Der Staat als Manipulant und Saboteur der IT-Sicherheit? - Die Zulässigkeit von Begleitmaßnahmen zu „Online-Durchsuchung“ und Quellen-TKÜ, NJW 2019, S. 1111-1116

Dernbach, Beatrice, Was schwarz auf weiß gedruckt ist... Vertrauen in Journalismus, Medien und Journalisten, in: dies/ Michael Meyer (Hrsg.), Vertrauen und Glaubwürdigkeit - Interdisziplinäre Perspektiven, 1. Auflage, Wiesbaden 2005

Determann, Lothar, Gegen Eigentumsrechte an Daten - Warum Gedanken und andere Informationen frei sind und es bleiben sollten, ZD 2018, S. 503-508

Di Ciaula, Agostino, Towards 5G communication systems: Are there health implications?, International Journal of Hygiene and Environmental Health Vol. 221 (2018), S. 367-375

Diederichsen, Uwe, Zur Rechtsnatur und systematischen Stellung von Beweislast und Anscheinsbeweis, Versicherungsrecht 1966, S. 211-222

Dietlein, Johannes, Die Lehre von den grundrechtlichen Schutzpflichten, Münsterische Beiträge zur Rechtswissenschaft, Band 58, Berlin 1992

Ders., Das Untermaßverbot - Bestandsaufnahme und Entwicklungschancen einer neuen Rechtsfigur, ZG 1995 S. 131-141

Dietrich, Nico/Gersin, Enrico/Herweg, Alan, Analysemöglichkeit der Online-Kommunikation auf Social Network Sites am Beispiel PEGIDA und Facebook, in: Wolfgang Frindte/Nico Dietrich, Muslime, Flüchtlinge und Pegida Sozialpsychologische und kommunikationswissenschaftliche Studien in Zeiten globaler Bedrohungen, Wiesbaden 2017, S. 235-266

DigitalEurope, Comment on the draft Inter-State Treaty on modernising the media regulation (MStV) in Germany (TRIS Notification 2020/26/D), abrufbar unter https:/ /ec.europa.eu/growth/tools-databases/tris/de/search/?trisaction=search.detail\&year=2 020\&num=26, (Stand: 30.03.2021)

Diggelmann, Oliver/ Hadorn, Nina, Gewalt- und Interventionsverbot bei Cyberangriffen: Ausgewählte Schlüsselfragen, in: Christian Schubel/ Stephan Kirste/ Peter-Christian Müller-Graff/ Oliver Diggelmann/ Ulrich Häufelt (Hrsg.), Jahrbuch für Vergleichende Staats- und Rechtswissenschaften - 2016/2017, Baden-Baden 2017, S. 255-274

Diretoria de Análise de Politicas Públicas, Bots and Brazil's Electoral Legal System in the 2018 Elections, Policy Paper 3, Rio de Janeiro 2018, abrufbar unter http://bibliotecad igital.fgv.br/dspace/handle/10438/26229, (Stand 30.03.2021) 
Dittmann, Armin, Der Rundfunkbegriff im deutschen Recht - ein Kulturgut im Multimedialen Wande, in: ders./Fechner, Frank/Sander, Gerald G. (Hrsg.), Der Rundfunkbegriff im Wandel der Medien - Symposium zum 65. Geburtstag von Professor Dr. iur. Dr. h. c. Thomas Oppermann, Berlin 1997, S. 19-40

Dogruel, Leyla/Stark, Birgit/Facciorusso, Dominique/Liesem, Kerstin, Transparenz und Diskriminierungsfreiheit - zur Vielfaltssicherung im neuen Medienstaatsvertrag. Die Regulierung von Algorithmen aus Expertensicht, Media Perspektiven 2020, S. $139-148$

Dolde, Klaus-Peter, Grundrechtsschutz durch einfaches Verfahrensrecht?, NVwZ 1982, S. 65-71

Dolderer, Michael, Objektive Grundrechtsgehalte, Berlin 2000

Dörr, Dieter, Informationsfreiheit, in: Detlef Merten/Hans-Jürgen Papier (Hrsg.), Handbuch der Grundrechte, Band IV, Heidelberg 2011, $\$ 103$

ders., Gutachten: Die regulatorische Relevanz der Organisation massenhafter Individualkommunikation, unter besonderer Berücksichtigung der Sicherung der Meinungsvielfalt, erstellt im Auftrag von „Die Medienanstalten“, abrufbar unter https://www.die -medienanstalten.de/fileadmin/user_upload/die_medienanstalten/Themen/Intermediae re/2019_06_04_Gutachten_Relevanz_Organisation_massenhafte_Individualkommuni kation.pdf, (Stand 30.03.2021)

Ders., Die Meinungsmacht der Intermediäre und der neue Medienstaatsvertrag, WRP 2021, S. 168-173

Dörr, Dieter/Holznagel, Bernd/Picot, Arnold, Legitimation und Auftrag des öffentlichrechtlichen Fernsehens in Zeiten der Cloud, ZUM 2016, S. $920-947$

Dörr, Dieter/Natt, Alexander, Suchmaschinen und Meinungsvielfalt - Ein Beitrag zum Einfluss von Suchmaschinen auf die demokratische Willensbildung, ZUM 2014, S. 829-847

Dörr, Dieter/Schuster, Simon, Suchmaschinen im Spannungsfeld zwischen Nutzung und Regulierung Rechtliche Bestandsaufnahme und Grundstrukturen einer Neuregelung, in: Birgit Stark/Dieter Dörr/Stefan Aufenanger, Die Googleisierung der Informationssuche, Berlin/Boston 2014, S. 262-323

Dörr, Oliver/Grote, Rainer/Marauhn, Thilo, Konkordanzkommentar zum europäischen und deutschen Grundrechtsschutz, 2. Auflage, Tübingen 2013

Dreier, Horst, Grundgesetz Kommentar, Band 1, Art. 1-19, 3. Auflage, Tübingen 2013

Ders., Grundgesetz Kommentar Band 2, Art. 20-82, 3. Auflage, Tübingen 2015

Dreier, Thomas/Schulze, Gernot, Kommentar zum Urhebergesetz, 6. Auflage 2018

Drerup, Johannes, Demokratische Bildung in und für digitale Öffentlichkeiten. Zeitdiagnosen - Problemvorgaben - Herausforderungen, in: Ulrich Binder/ders., Demokratieerziehung und die Bildung digitaler Öffentlichkeiten, Wiesbaden 2020, S. 29-53

Drexl, Josef, Bedrohung der Meinungsvielfalt durch Algorithmen, ZUM 2017, S. $529-543$

Duh, Andrej/Rupnik, Marjan Slap/Korošak, Dean, Collective Behavior of Social Bots Is Encoded in Their Temporal Twitter Activity, Big Data Vol. 6 (2018, S. 113-123

Duttge, Gunnar, Die „geschäftsmäßige Suizidassistenz“ (\$2 217 StGB): Paradebeispiel für illegitimen Paternalismus!, ZStW 129 (2017), S. 448-466

Ebers, Martin, Beeinflussung und Manipulation von Kunden durch Behavioral Microtargeting - Verhaltenssteuerung durch Algorithmen aus Sicht des Zivilrechts, MMR 2018, S. 423-428 
Edwards, Chad/Edwards, Atumn/Spence, Patric R./Shelton, Ashleigh K., Is that a bot running the social media feed? Testing the differences in perceptions of communication quality for a human agent and a bot agent on Twitter, Computers in Human Behaviour Vol. 33 (2014), S. 372-376

Eidenmüller, Horst, Liberaler Paternalismus, JZ 2011, S. 814-821

Eifert, Martin, Rechenschaftspflichten für soziale Netzwerke und Suchmaschinen - Zur Veränderung des Umgangs von Recht und Politik mit dem Internet, NJW 2017, S. $1450-1454$

Ders., Das Netzwerkdurchsetzungsgesetz und Plattformregulierung, in: ders./Tobias Gostomzyk (Hrsg.), Netzwerkrecht - Die Zukunft des NetzDG und seine Folgen für die Netzwerkkommunikation, Baden-Baden 2018

El Difraoui, Asiem, Die Rolle der neuen Medien im Arabischen Frühling, Bundeszentrale für politische Bildung, abrufbar unter https://www.bpb.de/internationales/afrika/arabi scher-fruehling/52420/die-rolle-der-neuen-medien? $\mathrm{p}=$ all., (Stand: 30.03.2021)

Elsaß, Lennart/Labusga, Jan-Hendrik/Tichy, Rolf, Löschungen und Sperrungen von Beiträgen und Nutzerprofilen durch die Betreiber sozialer Netzwerke - Rechtliche Möglichkeiten des Vorgehens vor dem Hintergrund von Hate Speech, Fake News und Social Bots, CR 2017, S. 234-241

Engel, Martin/Stark, Johanna, Verbraucherrecht ohne Verbraucher?, ZEuP2015, S. 32-51

Engeler, Malte, Das überschätzte Kopplungsverbot - Die Bedeutung des Art. 7 Abs. 4 DS-GVO in Vertragsverhältnissen, ZD 2018, 55-62

Epping, Volker, Grundrechte 8. Auflage, Wiesbaden 2019

Ders./Hillgruber, Christian, Beck'scher Onlinekommentar zum Grundgesetz, 45. Edition, Stand des Gesamtwerks 15.11.2020

Erichsen, Hans-Uwe, Grundrechtliche Schutzpflichten in der Rechtsprechung des Bundesverfassungsgerichts, Jura 1997, S. 85-89

Ernst, Stefan, Die Einwilligung nach der Datenschutz-Grundverordnung - Anmerkungen zur Definition nach Art. 4 Nr. 11 DS-GVO, ZD 2017, S. 110-114

Eßer, Martin/Kramer, Philipp/v. Lewinski, Kai/Auernhammer, Herbert, Kommentar zur Datenschutz-Grundverordnung, zum Bundesdatenschutzgesetz und Nebengesetzen, 6. Auflage, Köln 2018

Etzkorn, Philipp, Bedeutung der „Entwicklungslücke“ bei selbstlernenden Systemen Rechtliche Fragen zur fortdauernden Softwareentwicklung durch maschinelles Lernen im Praxiseinsatz, MMR 2020, S. 360-365

EURACTIV, The Sounding Board's unanimous final opinion on the so-called Code of Practice, v. 24.09.2018, abrufbar unter https://www.euractiv.com/wp-content/upload s/sites/2/2018/10/3OpinionoftheSoundingboard-1.pdf, (Stand: 30.03.2021)

European Parliament Research Service, Reform of the EU liability regime for online intermediaries, abrufbar unter https://www.europarl.europa.eu/RegData/etudes/IDA N/2020/649404/EPRS_IDA(2020)649404_EN.pdf, (Stand: 30.03.2021)

European Regulators Group for Audio-visual Media Services (ERGA), ERGA Report on Disinformation: Assessment of the Implementation of the Code of Practice, 2020, abrufbar unter https://erga-online.eu/wp-content/uploads/2020/05/ERGA-2019-repor t-published-2020-LQ.pdf, (Stand: 30.03.2021) 
Facebook, Positionspapier zum Diskussionsentwurf zu den Bereichen Rundfunkbegriff, Plattformregulierung und Intermediäre „Medienstaatsvertrag“, 2018, abrufbar unter: https://www.rlp.de/fileadmin/rlp-stk/pdf-Dateien/Medienpolitik/Eingaben_Mediensta atsvertag/Verbaende_Intermediaere_/Facebook.pdf, (Stand 30.03.2021)

dies., Positionspapier zum Diskussionsentwurf für einen „Medienstaatsvertrag“, 2019, abrufbar unter https://www.rlp.de/fileadmin/rlp-stk/pdf-Dateien/Medienpolitik/Einga ben_Medienstaatsvertrag_2019/Facebook_MStV_2019.pdf, (Stand: 30.03.2021)

Fanta, Alexander, Ob Nutzer oder nicht: Facebook legt Schattenprofile über alle an, netzpolitik. org, v. 29.03.2018, abrufbar unter https://netzpolitik.org/2018/ob-nutzer -oder-nicht-facebook-legt-schattenprofile-ueber-alle-an/, (Stand: 30.03.2021)

Fechner, Frank, Medienrecht, 20. Auflage, Tübingen 2019

Fechner, Frank/Arnold, Johannes, Medienrecht 4.0, in: Walter Frenz (Hrsg.), Handbuch Industrie 4.0: Recht, Technik, Gesellschaft, Heidelberg 2020, S. 133-154

Fehling, Michael/ Leymann, Matthias, Der neue Strukturwandel der Öffentlichkeit: Wie lassen sich die sozialen Medien regulieren?, AfP 2020, S. 110-119

Ferrara, Emilio, Disinformation and Social Bots Operations in the Run up of the 2017 French Presidential Election, 2017, First Monday Volume 22 (August 2017) Number 8, DOI: https://doi.org/10.5210/fm.v22i8.8005

Ders., What types of COVID-19 conspiracies are populated by Twitter bots?, First Monday Vol. 25 (2020) Nr. 6, DOI: https://doi.org/10.5210/fm.v25i6.10633

Ders., What types of COVID-19 conspiracies are populated by Twitter bots?, 2020, arXiv:2004.09531 [cs.SI], DOI: 10.5210/fm.v25i6.10633

Ferrara, Emilio/Varol, Onur/Davis, Clayton/Menczer, Filippo/Flammini, Alessandro, The Rise of Social Bots, Communication of the ACM 59 (7), S. 96-104

Ferreau, Frederik, Rundfunkbegriff und Rundfunkregulierung - Revision erforderlich?, ZUM 2017, S. 632-639

Ders., Desinformation aus rechtswissenschaftlicher Perspektive, in: Judith Möller/ Michael Hameleers/ders., Typen von Desinformation und Misinformation - Verschiedene Formen von Desinformation und ihre Verbreitung aus kommunikationswissenschaftlicher und rechtswissenschaftlicher Perspektive, Berlin 2020, S. 44-78

Feuz, Martin/Fuller, Matthew/Stadler, Felix, Personal Web searching in the age of semantic capitalization - Diagnosing the mechanism of personalization, in: First Monday 16 (2), Februar 2016, DOI: https://doi.org/10.5210/fm.v16i2.3344

Fezer, Karl-Heinz/Büscher, Wolfgang/Obergfell, Eva Inés, Lauterkeitsrecht: Kommentar zum Gesetz gegen den unlauteren Wettbewerb (UWG), Band 1, 3. Auflage, München 2016

Fichtner, Ullrich, Manipulation durch Reporter - SPIEGEL legt Betrugsfall im eigenen Haus offen, SPIEGEL-Online v. 19.12.2018, abrufbar unter https://www.spiegel.de/k ultur/gesellschaft/fall-claas-relotius-spiegel-legt-betrug-im-eigenen-haus-offen-a-12445 79.html, (Stand 30.03.2021)

Fiegerman, Seth, Facebook is shutting down trending topics feature, CNN Business v. 01.06.2018, abrufbar unter https://money.cnn.com/2018/06/01/technology/facebook -trending-topics/index.html, (Stand 30.03.2021)

Fink, Udo, Wem dient die Rundfunkfreiheit?, DÖV 1992, S. 805-813 
Fischer, Joerg K., Medienrecht und Medienmärkte, Heidelberg 2008

Fisher, Marc/Woodrow Cox, John/Hermann, Peter, Pizzagate: From rumor, to hashtag, to gunfire in D.C., The Washington Post (online), veröffentlicht am 6.12.2016, abrufbar unter https://www.washingtonpost.com/local/pizzagate-from-rumor-to-hashtag-t o-gunfire-in-dc/2016/12/06/4c7def50-bbd4-11e6-94ac-3d324840106c_story.html?no redirect=on, (Stand 30.03.2021)

Fitzner, Julia, Sind Haftungsbeschränkungen für Host-Provider noch zeitgemäß? - Der „Safe Harbor” gem. $\$ 512$ (c) Copyright Act und die Haftungsbeschränkungen gem. Art. 14 E-Commerce-Richtlinie bzw. \$10 TMG, GRURInt 2012, S. 109-117

Flaxman, Seth/Goel, Sharad/Rao, Justin M., Filter Bubbles, Echo Chambers, and Online News Consumption, Public Opinion Quarterly Vol. 80 (2016), S. 298-320

Forelle, Michelle/Howard, Phil/Monroy-Hernández, Andrés/Savage, Saiph, Political Bots and the Manipulation of the Public Opinion in Venezuela, 2015, arXiv:1507.07109 [cs.SI]

Franck, Johannes/Müller-Peltzer, Philipp, Grüß Bot! Aktuelle Rechtsfragen zum Einsatz von Chatbots, in: Jürgen Taeger (Hrsg.), Recht 4.0 - Innovationen aus den rechtswissenschaftlichen Laboren, Tagungsband der Herbstakademie 2017, DSRTI, S. 241-258

Freitas, Carlos/Benevenuto, Fabrício/Veloso, Adriano/Gosh, Saptarshi, An empirical study of socialbot infiltration strategies in the Twitter social network, Social Network Analysis and Mining 2016, 6:23, DOI: https://doi.org/10.1007/s13278-016-0331-3

Friauf, Karl Heinrich/Höfling, Wolfram, Berliner Kommentar zum Grundgesetz, Loseblattsammlung, Stand des Gesamtwerks 2020

Friehe, Matthias, Löschen und Sperren in sozialen Netzwerken, NJW 2020, S. $1697-1702$

Fries, Peter J., Influencer-Marketing - Informationspflichten bei Werbung durch Meinungsführer in Social Media, Wiesbaden 2019

Frosio, Giancarlo/ Mendis, Sunimal, Monitoring and Filtering: European Reform or Global Trend?, in: Giancarlo Frosio (Hrsg.), The Oxford Handbook of Online Intermediary Liability, Chapter 28, Oxford 2019, S. 544-565

Fuchs, Dieter/Pfetsch, Barbara, Die Beobachtung der öffentlichen Meinung durch das Regierungssystem, in: Wolfgang van den Daele/Friedhelm Neidhardt (Hrsg.), Kommunikation und Entscheidung - Politische Funktionen öffentlicher Meinungsbildung und diskursiver Verfahren. WZB-Jahrbuch, Berlin 1996, S. 103-137

Fuchs, Martin, Automatisierte Trolle - Warum Social Bots unsere Demokratie gefährden, Neue Züricher Zeitung v. 12.09.2016, https://www.nzz.ch/digital/automatisierte-troll e-warum-social-bots-unsere-demokratie-gefaehrden-ld.116166, (Stand 30.03.2021)

Fuchs, Thomas/Hahn, Caroline, Was sind die medienrechtlichen Rahmenbedingungen des Influencer-Marketings? Kennzeichnung, Jugendschutz und Aufsicht, in: Marlis Jahnke (Hrsg.), Influencer-Marketing - Für Unternehmen und Influencer: Strategien, Plattformen, Instrumente, rechtlicher Rahmen. Mit vielen Beispielen, Wiesbaden 2018

Fublrott, Michael/Oltmanns, Sönke, Social Media im Arbeitsverhältnis - Der schmale Grat zwischen Meinungsfreiheit und Pflichtverletzung, NZA 2016, S. 785 - 791

Galetzka, Christian/Krätschmer, Manuel, Rassismus und Terrorismus im Netz - Strafrechtliche Verantwortlichkeit der Betreiber von sozialen Netzwerken, MMR 2016, S. 518-523 
Gallotti, Riccardo/Valle, Francesco/Costaldo, Nicola/Sacco, Pierluigi/De Dominico, Manilo, Assessing the risks of "infodemics" in response to COVID-19 epidemics, Nature Human Behaviour 2020, DOI: https://doi.org/10.1038/s41562-020-00994-6

Gallwitz, Florian/Kreil, Michael, Die Mär von „Social Bots“, veröffentlicht am 3.06.2019 auf Tagesspiegel. De, abrufbar unter https://background.tagesspiegel.de /die-maer-von-social-bots, (Stand 30.03.2021)

Dies., The Rise and Fall of „Social Bot“ Research, abrufbar unter https://ssrn.com/abstra $\mathrm{ct}=3814191,($ Stand 30.03.2021)

Gasser, Lucas/Kraatz, Matthias, Social Bots: Wegbereiter der Maschinokratie, VerfBlog, 2017/1/16, abrufbar unter: http://verfassungsblog.de/social-bots-wegbereiter-der-mas chinokratie/, Stand: 30.03.2021

Geiger, Rudolf, Staatsrecht III: Bezüge des Grundgesetzes zum Völker- und Europarecht, 7. Auflage, München 2018.

Georgi, Oliver, Gerüchte im Internet: Wie Falschmeldungen die Terrorangst schüren, FAZ v. 25.07.2016, abrufbar unter https://www.faz.net/aktuell/politik/inland/geruech te-im-internet-wie-falschmeldungen-die-terrorangst-schueren-14356204.html (Stand: 30.03.2021).

Geppert, Martin/Schütz, Raimund, Beck'scher TKG-Kommentar, 4. Auflage, München 2013

Gerbig, Maria, Grundrecht auf staatlichen Schutz - Ein Vergleich von Grundgesetz für die Bundesrepublik Deutschland und Verfassung der Vereinigten Staaten von Amerika, Berlin 2014

Gercke, Marco, Die Entwicklung des Internetstrafrechts 2018/2019, ZUM 2019, S. 798-807

Gerecke, Martin, Kennzeichnung von werblichen Beiträgen im Online-Marketing, GRUR 2018, S. 153-159

Gerpott, Torsten J., Artikel 17 der neuen EU-Urheberrechtsrichtlinie: Fluch oder Segen? - Einordnung des Streits um „Upload-Filter“ auf Online-Sharing-Plattformen, MMR 2019, S. 420-426.

Ders., Datenschutzerklärungen - Materiell fundierte Einwilligungen nach der DS-GVO: Empirischer Forschungsstand und Verbesserungsfelder, MMR 2020, S. 739-744

Gersdorf, Hubertus, Das Grundrecht der Rundfunkfreiheit als „Supergrundrecht“? Zur Notwendigkeit einer dogmatischen Weiterentwicklung des verfassungsrechtlichen Begriffsbildes, in: Dittmann, Armin/Fechner, Frank/Sander, Gerald G. (Hrsg.), Der Rundfunkbegriff im Wandel der Medien - Symposium zum 65. Geburtstag von Professor Dr. iur. Dr. h. c. Thomas Oppermann, Berlin 1997, S. 137-144

Ders., Multi-Media: Der Rundfunkbegriff im Umbruch? - Insbesondere zur verfassungsrechtlichen Einordnung der Zugriffs- und Abrufdienste, AfP 1995, S. 565-575

Ders., Der verfassungsrechtliche Rundfunkbegriff im Lichte der Digitalisierung der Telekommunikation - Ein Rechtsgutachten im Auftrag der Hamburgischen Anstalt für neue Medien, in: Hamburgische Anstalt für neue Medien (Hrsg.), Schriftenreihe der HAM, Band 11, Berlin 1995

Ders., Grundzüge des Rundfunkrechts - Nationaler und europäischer Regulierungsrahmen, München 2003

Ders., Legitimation und Limitierung von Onlineangeboten des öffentlich-rechtlichen Rundfunks - Konzeption der Kommunikationsverfassung des 21. Jahrhunderts, Berlin 2009 
Ders., Hate Speech in sozialen Netzwerken - Verfassungswidrigkeit des NetzDG-Entwurfs und grundrechtliche Einordnung der Anbieter sozialer Netzwerke, MMR 2017, S. $439-447$

Gersdorf, Hubertus/Paal, Boris P., Beck'scher Online Kommentar zum Informationsund Medienrecht, Stand des Gesamtwerks 30. Edition, 01.11.2020

Gertler, Martin, Zwei Paradigmen nebeneinander: Meinungsbildung durch klassische vs. Interaktive Medien, in: Mike Friedrichsen/Roland A. Kohn (Hrsg.), Digitale Politikvermittlung, 2. Auflage, Wiesbaden 2015, S. 83-103

Gesellschaft für Informatik e.V., Dagstuhl-Erklärung - Bildung in der digitalen vernetzten Welt, 2016, abrufbar unter https://gi.de/fileadmin/GI/Hauptseite/Themen/Dagstuh 1-Erkla_rung_2016-03-23.pdf, (Stand: 30.03.2021)

Gielen/Tiessen, Die neue Plattformhaftung nach der Richtlinie über das Urheberrecht im digitalen Binnenmarkt, EuZW 2019, S. 639-646

Gierschmann, Sibylle, Was bringt deutschen Unternehmen die DS-GVO? - Mehr Pflichten, aber die Rechtsunsicherheit bleibt, ZD 2016, S. 51-55

Dies., Gestaltungsmöglichkeiten bei Verwendung von personenbezogenen Daten in der Werbung Auslegung des Art. 6 Abs. 1 lit.f DS-GVO und Lösungsvorschläge, MMR 2018, S. 7-12

Glaser, Andreas, Grundrechtlicher Schutz der Ehre im Internetzeitalter, NVwZ 2012, S. $1432-1438$

Gläser, Isabel, Anwendbares Recht auf Plattformverträge - Fragen des IPR bei sozialen Netzwerken am Beispiel von Facebook, MMR 2015, S. 699-702

Gleß, Sabine/Weigend, Thomas, Intelligente Agenten und das Strafrecht, ZStW 2014, S. 561-591

Golland, Alexander, Das Kopplungsverbot in der Datenschutz-Grundverordnung - Anwendungsbereich, ökonomische Auswirkungen auf Web 2.0-Dienste und Lösungsvorschlag, MMR 2018, S. 130-135

Golba, Lukasz, Consent for personal Data Processing in digital Environment according to GDPR, Annuals of Admisistration and Law Vo. 17 (2), S. 253-265

Golding, Peter/Sousa, Helenalvan Zoonen, Liesbet, Trust and the media, EJoC Vol. 27 (2012), S. 3-6

Gomille, Christian, Die Verteidigung gegen unerwünschte Werbung, GRUR 2017, S. 241- 248

Görres-Gesellschaft, Staatslexikon, 8. Auflage, Freiburg 2018

Gorwa, Robert/Guilbeault, Douglas, Unpacking the Social Media Bot: A Typology to Guide Research and Policy, Policy \& Internet Vol. 12 (2020), S. 225-248

Götting, Horst-Peter, Urheberrechtliche und vertragsrechtliche Grundlagen, in: Friedrich-Karl Beier/Horst-Peter Götting/Michael Lehmann/Rainer Moufang (Hrsg.), Urhebervertragsrecht - Festgabe für Gerhard Schricker zum 60. Geburtstag, München 1995, S. 53-75

Graber, Robin/Lindemann, Thomas, Neue Propaganda im Internet. Social Bots und das Prinzip sozialer Bewährtheit als Instrumente der Propaganda, in: Klaus Sachs-Hombach/Bernd Zywietz (Hrsg.), Fake News, Hashtags \& Social Bots - Neue Methoden populistischer Propaganda, Wiesbaden 2018, S. 51-68

Grabitz, Eberhard/Hilf, Meinhard, Das Recht der Europäischen Union, Band IV Sekundärrecht, Loseblattsammlung, Stand 40. Ergänzungslieferung, Oktober 2009

Grabitz, Eberhard/Hilf, Meinhard/Nettesheim, Martin, Das Recht der Europäischen Union, Band 1-3, AEUV/EUV, Loseblattsammlung, Stand des Gesamtwerks 71. Ergänzungslieferung, August 2020 
Gräfe, Hans-Christian, Webtracking und Microtargeting als Gefahr für Demokratie und Medien, PinG 2019, S. 5-12

Graham, Timothy/Ackland, Robert, Do Socialbots Dream of Popping the Filter Bubble? The Role of Socialbots in Promoting Deliberative Democracy in Social Media, in: Robert W. Gehl/Maria Bakardjieva (Hrsg.), Socialbots and their friends - Digital Media and the automation of sociality, S. 187-206

Greve, Holger/Schärdel, Florian, Der digitale Pranger - Bewertungsportale im Internet, MultiMedia und Recht 2008, S. 644-650

Griesbaum, Joachim/Bekavac, Bernard/Rittberger, Marc, Typologie der Suchdienste im Internet, in: Lewandowski (Hrsg.), Handbuch Internetsuchmaschinen - Nutzerorientierung in Wissenschaft und Praxis, S. 1852.

Griess, Oliver, Klarnamenspflicht im Internet: Verfassungsrechtliche Rahmenbedingungen einer staatlichen Schutzpflicht, Göttingen 2016

Grimm, Dieter, Politische Parteien, in: Ernst Benda/Werner Maihofer/Hans-Jochen Vogel (Hrsg.), Handbuch des Verfassungsrechts, 2. Auflage, Berlin/New York 2012, $\mathbb{1 4}$

Grimme, Christian/Assenmacher, Dennis/Preuss, Mike/Adam, Lena/Lütke-Stokdiek, Justus Frederik Hermann, PropStop Technischer Bericht Bundestagswahl 2017: SocialMedia-Angriff auf das \#kanzlerduell?, abrufbar unter http:/www.propstop.de/wp-co ntent/uploads/2017/09/bundestagswahl-2017-social-media.pdf, (Stand: 30.03.2021)

Grimme, Christian/Preuss, Mike/Adam, Lena/Trautmann, Heike, SocialBots: HumanLike by Means of Human Control?, Big Data Vol. 5 (2017) Nr. 4, S. 279-293

Gröpl, Christoph, Das verfassungsrechtliche Konzept der informationellen Selbstbestimmung und des Datenschutzes, in: Helmut Rüßmann (Hrsg.), Festschrift für Gerhard Käfer, Saarbrücken 2009

Grunenberg, Benedikt, Suchmaschinen als Rundfunk - Die Haftung für Suchergebnisse nach Maßgabe der Rundfunkfreiheit, Baden-Baden 2017

Gumm, Heinz-Peter/Sommer, Manfred, Einführung in die Informatik, 10. Auflage, München 2013

Gumz, Jan Dennis/Kar, Resa Mohabbat, Social Bots, in: Mike Weber (Hrsg.), ÖFIT Trendschau: Öffentliche Informationstechnologie in der digitalisierten Gesellschaft, Berlin: Kompetenzzentrum Öffentliche IT, https://www.oeffentliche-it.de/-/social-bots, (Stand 30.03.2021)

Gundel, Jörg, Die Fortentwicklung der europäischen Medienregulierung: Zur Neufassung der AVMD-Richtlinie, ZUM 2019, S. 131-139

Günther, Jens/Böglmüller, Matthias, Künstliche Intelligenz und Roboter in der Arbeitswelt, BB 2017, S. 53-58

Gutmann, Thomas, Gesetzgeberischer Paternalismus ohne Grenzen? Zum Beschluß des Bundesverfassungsgerichts zur Lebendspende von Organen, NJW 1999, S. 3387-3389

Habel, Dominic, Roboterjournalismus, Baden-Baden 2019

Habermas, Jürgen, Political Communication in Media Society: Does Democracy Still Enjoy an Epistemic Dimension? The Impact of Normative Theory on Empirical Research, Communication Theory 16 (2006), S. 411-426

Haeg, Jonas, The Ethics of Political Bots: Should We Allow Them for Personal Use?, Journal of Practical Ethics 2017, S. 85-104

Hagen, Leonie/Neely,Stephen,Keller, Thomas E./Scharf, Ryan/Espinoza Vasquez, Fatima, Rise of the Machines? Examining the Influence of Social Bots on a Political Discussion Network, Social Science Computer Review, 2020, bislang nur online veröffentlicht, DOI: https://doi.org/10.1177/0894439320908190, (Stand: 30.03.2021) 
Hagen, Lutz, Nachrichtenjournalismus in der Vertrauenskrise - „Lügenpresse“ wissenschaftlich betrachtet: Journalismus zwischen Ressourcenkrise und entfesseltem Publikum, Communication Socialis 48 (2015), S. 152-163

Hain, Karl-Eberhard, Rundfunkfreiheit und Rundfunkordnung, in: Christian Starck (Hrsg.), Studien und Materialien zur Verfassungsgerichtsbarkeit, Band 55, Baden-Baden 1993

Ders., Der Gesetzgeber in der Klemme zwischen Übermaß- und Untermaßverbot, DVBl. 1993, S. 982-984

Ders., Sicherung des publizistischen Pluralismus auf europäischer Ebene?, AfP 2007, S. 527-534

Ders., Die zweite Gebührenentscheidung des Bundesverfassungsgerichts - Kontinuität in den Zeiten der Konvergenz, JZ 2008, S. 128-135

Ders., Ist die Etablierung einer Internetdienstefreiheit sinnvoll?, K\&R 2012, S. 98-103

Ders., Vielfaltssicherung am Ende? - Zugleich Kommentar zu BVerwG, Urteil vom 29. 1. 2014 - 6 C 2.13, K\&R 2014, S. 492-496

Hain, Karl-Eberhard/Ferreau, Frederik/Brings-Wiesen, Regulierung sozialer Netzwerke revisited, K\&R 2017, S. 433-438

Haim, Mario/Graefe, Andreas/Brosius, Hans-Bernd, Burst of the Filter Bubble? Effects of personalization on the diversity of Google News, Digital Journalism Vol. 6 (2018), S. 330-343

Halavais, Alexander, Overcoming terms of service: a proposal for ethical distributed research, Information, Communication \& Society, Vol. 22 (2019), S. 1567-1581

Hampton, Keith N./Rainie, Lee/Lu, Weixu/Dwyer, Maria/Shin, Inyoung/Purcell, Kristen, Social Media and the ,Spiral of Silence', Pew Research Center (Hrsg.), Washington D.C. 2014, abrufbar unter https://www.pewresearch.org/internet/2014/08/26/social -media-and-the-spiral-of-silence/, letzter Zugriff: 29.11.2019

Hanfeld, Michael, Brüssel sorgt für Unruhe - Killt die EU die Medienpolitik?, FAZ v. 24.04.2020, abrufbar unter https://www.faz.net/aktuell/feuilleton/medien/die-e u-kommission-koennte-den-medienstaatsvertrag-kippen-16738781.html, (Stand 30.03.2021)

Ders., EU billigt Mediengesetz - Im digitalen Sandkasten, FAZ.NET v. 29.04.2020, abrufbar unter https://www.faz.net/aktuell/feuilleton/medien/eu-kommission-billigt-d en-deutschen-medienstaatsvertrag-16745740.html, (Stand 30.03.2021)

Harding, Nicolas, Die Charakterisierung staatlicher Accounts in sozialen Netzwerken, NJW 2019, S. 1910-1915

Hartl, Korbinian, Suchmaschinen, Algorithmen und Meinungsmacht - Eine verfassungsund einfachrechtliche Betrachtung, Wiesbaden 2017.

Hartmann, Alexander, Unterlassungsansprüche im Internet - Störerhaftung für nutzergenerierte Inhalte, 2009

Hartmann, Matthias, Telemedienrecht, in: Artur-Axel Wandtke/Claudia Ohst (Hrsg.), Praxishandbuch Medienrecht, Band 5: IT-Recht, 3. Auflage, Berlin 2014, Kapitel 1

Haucap, Justus/Kehder, Christian, Suchmaschinen zwischen Wettbewerb und Monopol: Der Fall Google, DICE Ordnungspolitische Perspektiven Nr. 44

Haug, Thomas, Informationspflichten bei Social Media-Präsenzen von Rechtsanwälten, NJW 2015, S. 661-665

Hadert, Tommy/Hoffacker, Gabriele, Überschätzte Bots? - Eine Untersuchung von Twitter-Debatten - und was Redaktionen daraus lernen können, Journalistik 2019, S. $140-153$ 
He, Yukun/Zhang, Guangyan/Wu, Jie/Li, Qiang, Understanding a prospective approach to designing malicious social bots, Security and Communication Networks Vol.9 (2016), S. 2157-2172

Hege, Hans/Flecken, Eva, Suchmaschinen: auf der Suche nach einem Korrektiv, in: Kappes, Krone, Novy (Hrsg.), Medienwandel kompakt 2011-2013 - Netzwerkveröffentlichungen zu Medienökonomie, Medienpolitik \& Journalismus, Wiesbaden 2014, S. 377414

Hegelich, Simon, Social Bots, Trolle, Fake-News - Wie gefährlich sind sie für die Demokratie?, Die Politische Meinung, Ausgabe 543 (März 2017), S. 32-36

Hegelich, Simon/Janetzko, Dietmar, Are Social Bots on Twitter Political Actors? Empirical Evidence from a Ukrainian Social Botnet, in: AAAI (Hrsg.), Proceedings of the Tenth International AAAI Conference on Web and Social Media (ICWSM 2016), Palo Alto (CA) 2016, S. 579-582

Hegemann, Lisa, „Was YouTube macht, ist eine Art Enteignung“ Interview mit Axel Voss, Zeit-online, v. 25.3.2019, abrufbar unter https://www.zeit.de/digital/internet/20 19-03/axel-voss-artikel-13-uploadfilter-urheberrechtsreform, (Stand 30.03.2021)

Heidtke, Aaron, Meinungsbildung und Medienintermediäre - Vielfaltssichernde Regulierung zur Gewährleistung der Funktionsbedingungen freier Meinungsbildung im Zeitalter der Digitalisierung, Baden-Baden 2020

Heilmann, Dorothea, Regulierung von Suchmaschinen - Macht im Netz III: Plädoyer für ein Aufsichtsinstrumentarium zur medienrechtlichen Vielfaltssicherung, MMR 2020, S. $162-166$

Heilmann, Stefan, Anonymität für User generated Content? - Verfassungsrechtliche und einfach-gesetzliche Analyse der Informationspflichten für journalistisch-redaktionelle Angebote und andere Telemedien in $\mathbb{S} \$ 5 \mathrm{TMG}, 55 \mathrm{RStV}$, in: Schulz (Hrsg.), Hamburger Schriften zum Medien-, Urheber- und Telekommunikationsrecht, Band 5, 1. Auflage 2012

v. Heintschel-Heinegg, Bernd, Beck'scher Online Kommentar zum Strafgesetzbuch, 48. Edition, Stand des Gesamtwerks 1.11.2020

Heldt, Amélie Pia, Transparenz bei algorithmischen Entscheidungen - Food of Thoughts. Ein vergleichender Blick auf die Kennzeichnungspflichten im Lebensmittelrecht, CR 2018, S. 494-500

Dies., Loi Avia: Frankreichs Verfassungsrat kippt Gesetz gegen Hass im Netz, JuWissBlog Nr. 96/2020 abrufbar unter https://www.juwiss.de/96-2020/, (Stand: 30.03.2021)

Dies., Pflicht zu weltweiter Löschung: konsequente oder ausufernde Auslegung? - Anmerkung zum Urteil des EuGH v. 03.10.2019, Rs. C-18/18 (Glawischnig-Piesczeck), EuR 2020, S. 238-246

Hellenkemper, Mona, The Perfect Fit: Wie jedes Unternehmen passgenaue und hochwertige Influencer identifiziert, in: Annika Schach/Timo Lommatzsch, Influencer Relations - Marketing und PR mit digitalen Meinungsführern, Wiesbaden 2018

Hennemann, Moritz, Personalisierte Medienagebote im Datenschutz- und Vertragsrecht, ZUM 2017, S. 544-552

Hennemann, Moritz/Paal, Boris P., Meinungsvielfalt im Internet, ZRP 2017, S. 76-79

Hennemann, Moritz/Paal, Boris P., Personalisierte Medienangebote im Datenschutz- und Vertragsrecht, ZUM 2017, S. 544-552

Herberger, Maximilian, „Künstliche Intelligenz“ und Recht - ein Orientierungsversuch, NJW 2018, S. 2825-2829 
Hermes, Georg, Das Grundrecht auf Schutz von Leben und Gesundheit - Schutzpflicht und Schutzanspruch aus Art. 2 Abs. 2 Satz 1 GG, Freiburger Rechts- und Staatswissenschaftliche Abhandlungen, Band 46, Heidelberg 1987

Hesse, Konrad, Grundzüge des Verfassungsrechts der Bundesrepublik Deutschland, Neudruck der 20. Auflage (1995), Heidelberg 1999

Ders., Die verfassungsgerichtliche Kontrolle der Wahrnehmung grundrechtlicher Schutzpflichten des Gesetzgebers, in: Herta Däublein-Gmelin/Klaus Klinkel/Hans Meyer/ Helmut Simon (Hrsg.), Gegenrede: Aufklärung - Kritik - Öffentlichkeit, Festschrift für Ernst Gottfried Mahrenholz, Baden-Baden 1994, S. 541- 559

Hessel, Stefan, Soziale Netzwerke im Fokus von Phishing-Angriffen - Eine Analyse aus technischer und rechtlicher Sicht, JurPC, WebDok 137/2016, Abs. 1-106

Hener-James, Jens-Uwe/Chibanguza, Kunya J./Stücken, Benedikt, Industrie 4.0 - vertrags- und haftungsrechtliche Fragestellungen, BB 2018, S. 2818-2832

Heun, Werner, Staatliche Risikosteuerung und Verfassung, Rechtswissenschaft 2011, S. 376-399

Hill, Hermann, „Bounded Rationality“ im digitalen Zeitalter - Die Kunst des Wahrnehmens und der Informationsverarbeitung, DÖV 2020, S. 205-216

Hillgruber, Christian, Die Bedeutung der staatlichen Schutzpflicht für das menschliche Leben und der Garantie der Menschenwürde für eine gesetzliche Regelung zur Suizidbeihilfe, in: Thomas Sören Hoffmann/Marcus Knaup (Hrsg.), Was heißt: In Würde sterben? - Wider die Normalisierung des Tötens, Wiesbaden 2015

Ders., Grundrechtlicher Schutzbereich, Grundrechtsgestaltung und Grundrechtseingriff, in: Josef Isensee/Paul Kirchhof (Hrsg.), Handbuch des Staatsrechts, Band 9, $\mathbb{S} 200,3$. Auflage, Heidelberg 2011

Hindelang, Steffen, Freiheit und Kommunikation - Zur verfassungsrechtlichen Sicherung kommunikativer Selbstbestimmung der vernetzten Gesellschaft, Heidelberg 2019

Hines, Matthew, I smell a Bot: California's S.B. 1001, Free Speech and the Future of Bot Regulation, Houston Law Review Vol. 57 (2019), S. 405-435

von Hirsch, Andreas, Direkter Paternalismus im Strafrecht - Sollte selbstschädigendes Verhalten kriminalisiert werden?, in: Ders. /Ulfrid Neumann/Kurt Seelmann (Hrsg.), Paternalismus im Strafrecht - Die Kriminalisierung von selbstschädigendem Verhalten, Baden-Baden 2010, S. 57-70

von Hirsch, Andreas/Neumann, Ulfrid, „Indirekter“ Paternalismus im Strafrecht - am Beispiel der Tötung auf Verlangen (\$216 StGB), in: Dies./Kurt Seelmann (Hrsg.), Paternalismus im Strafrecht - Die Kriminalisierung von selbstschädigendem Verhalten, Baden-Baden 2010, S. 71-98

Dies., Indirekter Paternalismus und $\$ 216$ StGB: Weitere Bemerkungen zur Bedeutung und Reichweite des Paternalismus-Begriffs, in: Dies./Kurt Seelmann (Hrsg.), Paternalismus im Strafrecht - Die Kriminalisierung von selbstschädigendem Verhalten, Baden-Baden 2010, S. 99-110

Hitaj, Dorian/Hitaj, Briland/Jajodia, Sushil/Mancini, Luigi V., Capture the Bot: Using Adversarial Examples to Improve CAPTCHA Robustness to Bot Attacks, 2020, arXiv:2010.16204 [cs.CR]

Höch, Dominik/Kadelbach, Philipp, Hat der Nutzer seine Recht ein sozialen Netzwerken selbst in der Hand? - Aktuelle (persönlichkeits-) rechtliche Fragen zu Facebook, WRP 2012, S. 1060-1068 
Hoeren, Thomas, Das Telemediengesetz, NJW 2007, S. 801-806

Hoeren, Thomas/Sieber, Ulrich/Holznagel, Bernd, Handbuch Multimedia-Recht Rechtsfragen des elektronischen Geschäftsverkehrs, Stand: 53. Ergänzungslieferung, August 2020

Hoffmann, Christian/Luch, Anika D./Schulz, Sönke E./Borchers, Kim Corinna, Die digitale Dimension der Grundrechte - Das Grundgesetz im digitalen Zeitalter, in: Deutsches Institut für Vertrauen und Sicherheit im Internet (Hrsg.), DIVSI Perspektiven, Band 2, Baden-Baden 2015

Hoffmann-Riem, Wolfgang, Chancengleichheit in zukünftigen Kommunikationssystemen - Rechtspolitische Erwägungen zur Weiterentwicklung elektronischer Medien, insbesondere des Kabelfernsehens, ZRP 1976, S. 291-299

Ders., Freiheitsschutz in den globalen Kommunikationsinfrastrukturen, JZ 2014, S. 53-63

Hofmann, Franz, Die Plattformverantwortlichkeit nach dem neuen europäischen Urheberrecht - »Much Ado About Nothing «?, ZUM 2019, S. 617-627

Hoffmanns, Sebastian, Die „Lufthansa-Blockade“ 2001 - eine (strafbare) Online- Demonstration?, ZIS 2012, S. 409-414

Hoheisel-Guler, Roland, Der digitale Raum ist kein (grund-)rechtsfreier Raum, in: Thomas-Gabriel Rüdiger/Petra Saskia Bayern (Hrsg.), Cyberkriminologie - Kriminologie für das digitale Zeitalter, S. 71-108

Hölig, Sascha/Hasebrink, Uwe, Analsyis by Country - Germany, in: Reuters Institute, Digital News Report 2018, S. 80-81, abrufbar unter http://media.digitalnewsreport.or g/wp-content/uploads/2018/06/digital-news-report-2018.pdf, letzter Zugriff 3.06.2019

Hönig d'Orville, Melanie, Die Perspektive der Länder: AVMD-Richtlinie, der 22. Rundfunkänderungsstaatsvertrag und der »Medienstaatsvertrag « - Angemessene Instrumente für die Regulierungsherausforderungen?, ZUM 2019, S. 104-109

Holznagel, Bernd, Meinungsbildung im Internet, Zeitschrift für öffentliches Recht in Norddeutschland 2011, S. 205-212

Ders., Internetdienstefreiheit und Netzneutralität, AfP 2011, S. 532-539

Ders., Die Zukunft der Mediengrundrechte in Zeiten der Konvergenz, MMR 2011, S. $1-2$

Ders., Der Konflikt zwischen Art. 15 E-Commerce-RL und pro-aktiven Verhinderungspflichten von Host-Providern, ZUM 2018, S. 350-357

Ders., Phänomen „Fake News“ - Was ist zu tun? Ausmaß und Durchschlagskraft von Desinformationskampagnen, MMR 2018, S. 18-22

Ders./Schumacher, Pascal, Netzpolitik reloaded, ZRP 2011, S. 74-78

Honer, Mathias, Nudging: Keine Herausforderung für die Grundrechtsdogmatik - Zugleich zur sogenannten doppelten Widerspruchslösung für die Organspende - DÖV 2019, S. $940-949$

Hörne, Tatjana, Aktuelle Probleme aus dem materiellen Strafrecht bei rechtsextremistischen Delikten, NStZ 2002, S. 113-118

Hornung, Gerrit/Müller-Terpitz, Ralf, Rechtshandbuch Social Media, Heidelberg 2015

Hostacna, Kristyna, Social Bots und freie Meinungsbildung, Ad Legendum 2018, S. 1-5

Hoven, Elisa, Zur Strafbarkeit von Fake News - de lege lata und de lege ferenda, ZStW 129 (2017), S. 718-744 
Dies., Die Strafbarkeit der Verbreitung von „Fake News“, JuS 2017, S. 1167-1170

Howard, Philipp N./Bolsover, Gilian/Kollanyi, Bence, Junk News and Bots during the U.S. Election: What Were Michigan Voters Sharing Over Twitter ?, Data Memo 2017.1, Project on Computational Propaganda, Oxford (UK), abrufbar unter http://c omprop.oii.ox.ac.uk/wp-content/uploads/sites/89/2017/03/What-Were-Michigan-Vot ers-Sharing-Over-Twitter-v2.pdf, (Stand 30.03.2021)

Howard, Phillipp N./Kollanyi, Bence, Bots, \#StrongerIn, and \#Brexit: Computational Propaganda during the UK-EU Referendum, COMPROP Research Note 2016.1, abrufbar unter https://papers.ssrn.com/sol3/papers.cfm?abstract_id=2798311, Stand: 20.10.2020

Howard, Phillip N./Wooley, Samuel/Calo, Ryan, Algorithms, bots, and political communication in the US 2016 election: The challenge of automated political communication for election law and administration, Journal of Information technology \& Politics Vol. 15 (2018), S. 81-93

Hufen, Friedhelm, Staatsrecht II - Grundrechte, 8. Auflage, München 2020

Ders., Nudging - Rechtsformen, Möglichkeiten und Grenzen der sanften Beeinflussung des Menschen durch den Staat, JuS 2020, S. 193-199

Huster, Stefan, Das Verbot der „Auschwitzlüge“, die Meinungsfreiheit und das Bundesverfassungsgericht, NJW 1996, S. 487-491

Iben, Alexander, GreifRecht erklärt: Staatliche Schutzpflichten des Gesetzgebers in der Fallbearbeitung, GreifRecht 29 (2020), S. 36-42

Ders., Staatlicher Schutz des Meinungsbildungsprozesses in sozialen Netzwerken gegen potentielle Beeinträchtigungen durch Meinungsroboter, in: Ruth Greve, Benjamin Gwiasda, Thomas Kemper, Joshua Moir, Sabrina Müller, Arno Schönberger, Sebastian Stöcker, Julia Wagner, Lydia Wolff (Hrsg.), Der digitalisierte Staat - Chancen und Herausforderungen für den modernen Staat, S. 153-176

Indenhuck, Moritz/Britz, Thomas, Vom Datenschutzrecht zum Datenschuldrecht - Neue Leitlinie zur Verarbeitung personenbezogener Daten bei Online-Dienstleistungen, BB 2019, S. 1091-1096

Infratest dimap, Glaubwürdigkeit der Medien - Umfrage im Auftrag des WDR, 2015, abrufbar unter https://www.infratest-dimap.de/umfragen-analysen/bundesweit/umfra gen/aktuell/glaubwuerdigkeit-der-medien/, (Stand: 30.03.2021)

Infratest dimap, Glaubwürdigkeit der Medien - Eine Studie im Auftrag des WDR, 2018, abrufbar unter https://www1.wdr.de/unternehmen/der-wdr/unternehmen/glaubwuerd igkeitsstudie-2018-100.pdf (Stand 30.03.2021)

Ingold, Albert, Digitalisierung demokratischer Öffentlichkeiten, Der Staat 56 (2017), S. 491-533

Ders., Propaganda als Herausforderung des Kommunikationsrechts, in: Thomas Oppelland (Hrsg.), Propaganda aus (neue) außen- und sicherheitspolitische Herausforderung, Berlin 2018, S. 81-104

Ders., Governance of Algorithms - Kommunikationskontrolle durch „Content Curation “ in sozialen Netzwerken, in: Sebastian Unger/Antje von Ungern-Sternberg (Hrsg.), Demokratie und künstliche Intelligenz, Tübingen 2019, S. 183-213

Ders., Meinungsmacht des Netzes - Macht im Netz II: Rechtliche Herausforderungen aktueller Regulierungsvorstellungen für digitalisierte Öffentlichkeiten, MMR 2020, S. 82-86 
Institut für Demoskopie Allensbach, Die Vermittlung von Nachrichtenkompetenz in der Schule - Ergebnisse einer repräsentativen Befragung von Lehrkräften im Februar/März 2020, abrufbar unter https://www.bdzv.de/nachrichten-und-service/presse/pr essemitteilungen/artikel/detail/allensbacher-studie-zur-nachrichtenkompetenz/, Stand: 30.03.2021

Institute for Strategic Dialogue, Make Germany Great Again - Der Kreml, die Alt-right und die internationale Einflussnahme auf die Bundestagswahl 2017, abrufbar unter https://www.isdglobal.org/wp-content/uploads/2017/12/Make-Germany-Great-Again -DE-151217.pdf, (Stand 30.03.2021)

Isensee, Josef, Das Grundrecht als Abwehrrecht und staatliche Schutzpflicht, in: ders./ Kirchhof (Hrsg.), Handbuch des Staatsrechts, Band IX, 3. Auflage, Heidelberg 2011, $\$ 191$

Isensee, Josef, Anwendung der Grundrechte auf juristische Personen, in: Isensee/Kirchhof (Hrsg.), Handbuch des Staatsrechts Band IX, 3. Auflage, Heidelberg 2011, $\mathbb{1 9 9}$

Jäckel, Michael/Fröhlich, Gerrit/Röder, Daniel, Medienwirkungen kompakt - Einführung in ein dynamisches Forschungsfeld, 2. Auflage, Wiesbaden 2019

Jackson, Jasper, Eli Pariser : activist whose filter bubble warnings presaged Trump and Brexit, the guardian online, 8. Januar 2017, abrufbar unter https://www.theguardian. $\mathrm{com} / \mathrm{media} / 2017 / \mathrm{jan} / 08 /$ eli-pariser-activist-whose-filter-bubble-warnings-presaged-tru mp-and-brexit, (Stand 30.03.2021)

Jäckel, Liv, Die Simulation des Urknalls vor dem Bundesverfassungsgericht - Zur Reichweite staatlicher Schutzpflichten, DVBl. 2011, S. 13-20

Jahn, Ralf, Verfassungsrechtliche Probleme eines strafbewehrten Vermummungsverbotes, JZ 1988, S. 545-551

Jahn, Matthias/Brodowski, Dominik, Das Ultima Ratio-Prinzip als strafverfassungsrechtliche Vorgabe zur Frage der Entbehrlichkeit von Straftatbeständen, ZStW 29 (2017), S. 363-381

Jahnke, Marlis, Ist Influencer-Marketing wirklich neu?. in: dies. (Hrsg.), Influencer-Marketing - Für Unternehmen und Influencer: Strategien, Plattformen, Instrumente, rechtlicher Rahmen. Mit vielen Beispielen, Wiesbaden 2018

Janal, Ruth/Isikay, Okan, Der Einsatz von Socialbots im geschäftlichen Verkehr, GRURPrax 2018, S. 393-395

Jandt. Silke, Spezifischer Datenschutz für Telemedien und DS-GVO - Zwischen Rechtsetzung und Rechtsanwendung, ZD 2018, S. 405-408

Jandt, Silke/Steidle, Roland, Datenschutz im Internet - Rechtshandbuch zu DSGVO und BDSG, Baden-Baden 2018

Jarass, Hans D., Rundfunkbegriff im Zeitalter des Internet - Zum Anwendungsbereich der Rundfunkfreiheit, des Rundfunkstaatsvertrags und des Mediendienste-Staatsvertrags, Archiv für Presserecht 1998, S. 133-141

Jarass, Hans D., Die Grundrechte: Abwehrrechte und objektive Grundsatznormen Objektive Grundrechtsgehalte, insbes. Schutzpflichten und privatrechtsgestaltende Wirkung, in: Peter Badura/ Horst Dreier (Hrsg.), Festschrift 50 Jahre Bundesverfassungsgericht, Band 2, Tübingen 2001

Jarass, Hans D./Pieroth, Bodo, Grundgesetz für die Bundesrepublik Deutschland - Kommentar, 14. Auflage, München 2016

Jarren, Ottfried/Donges, Patrick, Politische Kommunikation in der Mediengesellschaft, 3. Auflage, Wiesbaden 2011 
Jestaedt, Matthias, Meinungsfreiheit, in: Detlef Merten/Hans-Jürgen Papier (Hrsg.), Handbuch der Grundrechte, Band IV, \$102, Heidelberg 2011

ders., Schule und außerfamiliäre Erziehung, in: Josef Isensee/Paul Kirchhof, Handbuch des Staatsrechts, band VI, 3. Auflage 2009, \$ 156

Jobst, Simon, Konsequenzen einer unmittelbaren Grundrechtsbindung Privater, NJW 2020, S. 11-16

Jorgensen, Rikke Frank/Desai, Tariq, Rights to Privacy Meets Online Platforms: Exploring Privacy Complaints against Facebook and Google, Nordic Journal of Human Rights 35 (2017), Nr. 2, S. 106-126

Jurgenson, Nathan, Facebook fair and balanced, The Society Pages v. 07.05.2015, abrufbar unter https://thesocietypages.org/cyborgology/2015/05/07/facebook-fair-and-bala nced/, Stand: 30.03.2021

Jürlicher, Tim/Röttgen, Charlotte, Bots im Kontext von Wirtschaftsrecht und Cybercrime, Innovations- und Technikrecht (InTeR) 2018, S. 15-19

Dies., Der Bot, das unbekannte Wesen - Ein rechtlicher Überblick, in: Jürgen Taeger (Hrsg.), Recht 4.0 - Innovationen aus den rechtswissenschaftlichen Laboren, S. 227-239

Kaerlein, Timo, Social Bots und die Formalisierung von Sozialität auf Online-Plattformen, in: Theo Hug/Günther Pallaver (Hrsg.), Talk with the Bots - Gesprächsroboter und Social Bots im Diskurs, Innsbruck 2018, S. 17-28

Kahl, Wolfgang, Vom weiten Schutzbereich zum engen Gewährleistungsgehalt - Kritik einer neuen Richtung der deutschen Grundrechtsdogmatik, Der Staat 43 (2004), S. $167-202$

Kahl, Wolfgang/Waldhoff Christian/Walter, Christian, Kommentar zum Bonner Grundgesetz, Loseblattsammlung, Heidelberg, Stand des Gesamtwerks Oktober 2020 (208. Aktualisierung)

Kaiser, Anna-Bettina/Reiling, Ines, Meinungsfilter durch soziale Medien - und das demokratische Ideal der Meinungsvielfalt?, in: Sebastian Unger/Antje von Ungern-Sternberg (Hrsg.), Demokratie und künstliche Intelligenz, Tübingen 2019, S. 85-110

Kalbhenn, Jan Christopher/ Hemmert-Halswick, Maximilian, Der Regierungsentwurf zur Änderung des NetzDG - Vom Compliance-Ansatz zu Designvorgaben, MMR 2020, S. 518-522

Kalscheuer, Fiete/Hornung, Christian, Das Netzwerkdurchsetzungsgesetz - Ein verfassungswidriger Schnellschuss, NVwZ 2017, S. 1721-1725

Kalscheuer, FietelJacobsen, Annika, Das digitale Hausrecht von Hoheitsträgern - Unter welchen Voraussetzungen darf der Staat Twitter-Nutzer blockieren?, NJW 2018, S. 2358-2362

Kalwey, Melina Jasmine, Medienkompetenzvermittlung an Schulen gegen Fake News, in: Christian Möller (Hrsg.), Was tun gegen Hate Speech und Fake News? Strategien und Ausblicke, Kiel 2019, S. 139-150

Kämmerer, Jörn Axel, Privatisierung - Typologie- Determinanten - Rechtspraxis - Folgen, Tübingen 2001

Kamp, Meike/Rost, Martin, Kritik an der Einwilligung - Ein Zwischenruf zu einer fiktiven Rechtsgrundlage in asymmetrischen Machtverhältnissen, DuD 2013, S. 80-84

Kampert, David, Datenschutz in sozialen Online-Netzwerken de lege lata und de lege ferenda, Hamburg 2016

Karg, Moritz/Fahl, Constantin, Rechtsgrundlagen für den Datenschutz in sozialen Netzwerken, K\&R 2011, S. 453-458 
Kaufhold, Marc-André/Reuter, Christian/Stefan, Marvin, Gesellschaftliche Herausforderungen des Missbrauchs von Bots und sozialen Medien, in: M. Burghardt/R. Wimmer/C. Wolff/C. Womser-Hacker (Hrsg.), Mensch und Computer 2017 - Workshopband, S. 51-58, DOI: 10.18420/muc2017-ws01-0386

Kastl, Graziana, Filter - Fluch oder Segen? - Möglichkeiten und Grenzen von Filtertechnologien zur Verhinderung von Rechtsverletzungen, GRUR 2016, S. 671-677

Katz, Elihu/Lazarsfeld, Paul Felix, Personal Influence - the Part Played by People in the Flow of Mass Communications, 1966

Keller, Tobias R./Klinger, Ulrike, Social Bots in Election Campaigns: Theoretical, Empirical, and Methodological Implications, Political Communication Vol. 36 (2019), S. 171-189

Kellner, Anne, Die Regulierung der Meinungsmacht von Internetintermediären, BadenBaden 2019

Keppeler, Lutz Martin, Was bleibt vom TMG-Datenschutz nach der DS-GVO? - Lösung und Schaffung von Abgrenzungsproblemen im Multimedia-Datenschutz, MMR 2015, S. 779-783

Kerr, Orin S., Are We Overprotecting Code? Thoughts on First-Generation Internet Law, Washington and Law Review Volume 57 (2000), S. 1287-1300

Kersten, Jens, Anonymität in der liberalen Demokratie, Juristische Schulung 2017, S. 193-203

Khamooshi, Arash, Breaking Down Apple's iPhone Fight With the U.S. Government, New York Times v. 21.03.2016, abrufbar unter https://www.nytimes.com/interactive/ 2016/03/03/technology/apple-iphone-fbi-fight-explained.html, (Stand: 30.03.2021)

Kiel, Heinrich/Lunk, Stefan/Oetker, Hartmut, Münchener Handbuch zum Arbeitsrecht, Band 1, Individualarbeitsrecht, 4. Auflage, München 2018

Graf von Kielmansegg, Sebastian, Grundfälle zu den allgemeinen Grundrechtslehren, JuS 2009, S. 216-221

Kind, Sonja/Bovenschulte, Marc/Ehrenberg-Silies, Simone/Jetzke, Tobias/Weide, Social Bots, in: Büro für Technikfolgen-Abschätzung beim Deutschen Bundestag (TAB) [Hrsg.], TA-Vorstudie, Horizon Scanning Nr. 3, April 2017, abrufbar unter http://ww w.tab-beim-bundestag.de/de/pdf/publikationen/berichte/TAB-Horizon-Scanning-hs00 3.pdf, (Stand 30.03.2021)

Kindhäuser, Urs/Neumann, Ulfried/Paeffgen, Hans-Ullrich, NomosKommentar zum Strafgesetzbuch, 5. Auflage, Baden-Baden 2017

Kipker, Dennis-Kenji/Voskamp, Friederike, Datenschutz in sozialen Netzwerken nach der Datenschutzgrundverordnung, DuD 2012, S. 727-742

Kirste, Stephan, Harter und weicher Paternalismus - Unter besonderer Berücksichtigung der Medizinethik, JZ 2011, S. 805-814

Kitz, Volker, Das neue Recht der elektronischen Medien in Deutschland - sein Charme, seine Fallstricke, ZUM 2007, S. 368-375

Klaas, Arne, Demokratieprinzip im Spannungsfeld mit künstlicher Intelligenz - Demokratische Entscheidungsfindung durch und mithilfe von selbstlernenden Algorithmen, MMR 2019, S. 84-90

Klaes, Roland L., Verfassungsrechtlicher Rundfunkbegriff und Internet - Eine dogmatische Bestandsaufnahme vor dem Hintergrund des 12. Rundfunkänderungsstaatsvertrages, ZUM 2009, S. 135-141

Klein, Eckart, Grundrechtliche Schutzpflichten des Staates, NJW 1989, S. 1633-1640

Klein, Hans H., Rundfunkrecht und Rundfunkfreiheit, Der Staat 20 (1981), S. 177-200 
Klein, Oliver, Das Untermaßverbot - Über die Justiziabilität grundrechtlicher Schutzpflichterfüllung, JuS 2006, S. 960-964

Klein, Oliver, Nach Trump-Eklat bei Twitter - Wie soziale Netzwerke gegen Fake News kämpfen, ZDF.de. v. 27.05.2020, abrufbar unter https:/www.zdf.de/nachrichten/digit ales/facebook-twitter-faktencheck-100.html, (Stand: 30.03.2021)

Kleinig, John, Paternalismus und Menschenwürde, in: Andreas von Hirsch /Ulfrid Neumann/Kurt Seelmann (Hrsg.), Paternalismus im Strafrecht - Die Kriminalisierung von selbstschädigendem Verhalten, Baden-Baden 2010, S. 145-171

Kleinlein, Thomas/ Rabenschlag, David, Auslandsschutz und Staatsangehörigkeit, ZaöRV 2007, S. 1277-1337

Kloepfer, Michael, Verfassungsrecht II - Grundrechte, C. H. Beck, München 2010

Kluckert, Sebastian, Das neue Infektionsschutzgesetz, 1. Auflage, Baden-Baden 2020

Kluge, Vanessa/Müller, Anne-Kathrin, Autonome Systeme, InTeR 2017, S. 24-31

Knebel, Sophie Victoria, Die Drittwirkung der Grundrechte und -freiheiten gegenüber Privaten - Regulierungsmöglichkeiten sozialer Netzwerke, in: Boris P. Paul (Hrsg.), Schriften zum Medien- und Informationsrecht, Band 35, Baden-Baden 2018

Knodel, Anne Christine, Medien und Europa - Regelungspraxis, Kompetenzen und Handlungsmöglichkeiten der Europäischen, Baden-Baden 2018

Knothe, Matthias/Nowosadtko, Volker, Sicherstellung von Rezeptionschancengleicheit, ZRP 2001, S. 33-36

Knopp, Michael, Pseudonym - Grauzone zwischen Anonymisierung und Personenbezug, DuD 2015, S. 527-530

Knorr, Benjamin, Social Bots im Unterricht, München 2018, abrufbar unter https://www .socialbotnet.de/assets/material/zulassungsarbeit.pdf, (Stand: 30.03.2021)

Köbrich, Thomas/Frotzheim, Oliver, Lass uns quatschen - werbliche Kommunikation mit Chat Bots, WRP 2017, S. 1188-1192

Köhler, Helmut/Bornkamp, Joachim/Feddersen, Jörn/Alexander, Christian, Gesetz gegen den unlauteren Wettbewerb, Kommentar, 38. Auflage, München 2020

Kohring, Matthias, Vertrauen in Medien - Vertrauen in Technologie, in: Akademie für Technikfolgenabschätzung in Baden-Württemberg (Hrsg.), Arbeitsberichte der TAAkademie, Arbeitsbericht Nr. 196/ September 2001,

Kokott, Juliane, Beweislastverteilung und Prognoseentscheidungen bei der Inanspruchnahme von Grund- und Menschenrechten, 1992

Kolbe, Frederike, Freiheitsschutz vor staatlicher Gesundheitssteuerung - Grundrechtliche Grenzen paternalistischen Staatshandelns, in: Thomas Gutmann u.a. (Hrsg.), Fundamenta Juridica, Beiträge zur rechtswissenschaftlichen Grundlagenforschung, Band 70, Baden-Baden 2017

Kollmer, Norbert/Klindt, Thomas/Schucht, Carsten, Arbeitsschutzgesetz, 3. Auflage, München 2016

Komp, Lennart/Thrun, Felix, Impfzwang als verfassungsrechtlich zulässiges Mittel gegen Masern?, JA 2020, S. 195-201

König, Carsten, Vertragliche Gestaltung der Meinungsfreiheit in sozialen Netzwerken, AcP 219 (2019), S. 611-645

Koreng, Ansgar, Netzneutralität und Meinungsmonopole, in: Birgit Stark/Dieter Dörr/ Stefan Aufenanger (Hrsg.), Die Googleisierung der Informationssuche, 2014, S. 245-261

Ders., Entwurf eines Netzwerkdurchsetzungsgesetzes: Neue Wege im Kampf gegen „Hate Speech“?, GRUR-Prax 2017, S. 203-205 
Kotsios, Andreas/Magnani, Matteo/Rossi, Luca/Shklovski, Irina/Vega, Davide, An Analysis of the Consequences of the General Data Protection Regulation on Social Network Research, 2019, arXiv:1903.03196 [cs.SI]

Krafft, Peter M./Macy, Michael/ Pentland, Alex „Sandy“, Bots as Virtual Confederates: Design and Ethics,in: in: Association for Computing Machinery (Hrsg.), Proceedings of the 2017 ACM Conference on Computer Supported Cooperative Work and Social Computing, Portland Oregon 2017, S. 183-190.

Kramer, Thorsten, Das sekundärrechtliche Herkunftslandprinzip als Regelungstechnik des Binnenmarktes, 2007, abrufbar unter https://refubium.fu-berlin.de/handle/fub188 18265, Stand: 30.09.2020.

Kreil, Michael, The Army that Never Existed: The Failure of Social Bots Research, Vortrag auf der OpenFest Conference in Sofia v. 02.11.2019, anrufbar unter https://m ichaelkreil.github.io/openbots/, (Stand: 30.03.2021)

Kreile, Johannes, Vorschläge zur Vielfaltssicherung bei Suchmaschinen im Rundfunkstaatsvertrag, ZUM 2017-278

Kresse/Heinze, Rundfunk-Dynamik am Morgen des digitalen Zeitalters - Ein Beitrag zur funktionalen Entwicklung des Rundfunkbegriffs AfP 1995, S. 574-580

Krieg, Henning, Zur Ausnahme persönlichen Zwecken dienender Telemediendienste von Pflicht zur Anbieterkennung nach $\$ 55$ Abs. 1 RStV, jurisPR-ITR 13/2011 Anm. 3d

Krings, Günter, Grund und Grenze staatlicher Schutzansprüche - Die subjektiv-rechtliche Rekonstruktion der grundrechtlichen Schutzpflichten und ihre Auswirkung auf die verfassungsrechtliche Fundierung des Verbrauchervertragsrechts, Schriften zum Öffentlichen Recht, Band 931, Berlin 2003

Krohm, Niclas/Müller-Peltzer, Philipp, Wunsch nach Identifizierung anonymer Internetnutzer - Spannungsverhältnis von Kommunikationsfreiheit und Persönlichkeitsrechten, ZD 2015, S. 409-415

Dies., Auswirkungen des Kopplungsverbots auf die Praxistauglichkeit der Einwilligung Das Aus für das Modell „Service gegen Daten“?, ZD 2017, S. 551-556

Krug, Peter, Haftung im Rahmen der Anwendung von künstlicher Intelligenz - Betrachtung unter Berücksichtigung der Besonderheiten des steuerberatenden Berufsstands, beck.digitax 2020, S. 74-80

Krupar, Felix, Die rechtliche Behandlung algorithmischen Kommunikate, in: Jürgen Jaeger (Hrsg.), Recht 4.0 - Innovationen aus den rechtswissenschaftlichen Laboren, Tagungsband Herbstakademie 2017, Oldenburg 2017, S. 275-287

Krüper, Julian, Roboter auf der Agora. Verfassungsfragen von Social Bots im digitalen Diskursraum der Moderne, in: Sebastian Unger/ Antje von Ungern-Sternberg (Hrsg.), Demokratie und künstliche Intelligenz, Tübingen 2019. S. 67-84

Kube, Hanno, Neue Medien - Internet, in: Isensee/Kirchhof (Hrsg.), Handbuch des Staatsrechts, Band IV, $\mathbb{9}$ 91, 3. Auflage, München 2006

Kuch, David, „Wohltätiger Zwang“- Zur grundrechtlichen Neuausrichtung des Erwachsenenschutzes, DÖV 2019, S. 723- 732

Kuczerawy, Aleksandra, The Proposed Regulation on Preventing the Dissemination of Terrorist Content Online: Safeguards and Risks for Freedom of Expression (December 5, 2018). For Center for Democracy and Technology, 2018. DOI: http://dx.doi.or $\mathrm{g} / 10.2139 / \mathrm{ssrn} .3296864$

Dies., The EU Commission on voluntary monitoring: Good Samaritan 2.0 or Good Samaritan 0.5?, abrufbar unter https://www.law.kuleuven.be/citip/blog/the-eu-comm ission-on-voluntary-monitoring-good-samaritan-2-0-or-good-samaritan-0-5/, (Stand: 30.03.2021) 
Dies., Fighting Online Disinformation: Did the EU Code of Practice Forget about Freedom of Expression?, in: Georgios Terzis/Dariusz Kloza/Elżbieta Kużelewska/Daniel Trottier (Hrsg.), Disinformation and digital media as a challenge for democracy, 2020, S. 291-308

Kühl, Kristian/Heger, Martin, Lackner/Kühl, Kommentar zum Strafgesetzbuch, 29. Auflage, München 2018

Kühling, Jürgen, Im Dauerlicht der Öffentlichkeit - Freifahrt für personenbezogene Bewertungsportale, Neue Juristische Wochenschrift 2015, S. 447-450

Kühling, Jürgen/Buchner, Benedikt, Datenschutz-Grundverordnung, Bundesdatenschutzgesetz: DS-GVO / BDSG, 2. Auflage, München 2018

Kull, Edgar, „Dienende Freiheit“- dienstbare Medien?, in: Peter Badura/Rupert Scholz (Hrsg.), Wege und Verfahren des Verfassungslebens : Festschrift für Peter Lerche zum 65. Geburtstag, München 1993

Künast, Renate, Regeln für Wahlkämpfe im digitalen Zeitalter - Echte Transparenz im Netz herstellen, ZRP 2019, S. 62-65

Kunig, Philip, Politische Parteien im Grundgesetz, Jura 1991, S. 247-257

Ders., Parteien, in: Isensee/Kirchhof (Hrsg.), Handbuch des Staatsrechts Band III, $\mathbb{\$} 40$, 3. Auflage 2005

Kunnert, Gerhard, Das Aus für Social-/Media-Plug-ins? - Analyse der Schlussanträge des Generalanwalts in der Rs. C-40/17 (Fashion ID) vom 19.12.2018, DuD 2019, S. 257-264

Kuntz, Wolfgang, „One-Pager“ als Muster für transparente Datenschutzhinweise vorgestellt, ZD-Aktuell 2015, 04909

Kurz, Volker, Prostitution und Sittenwidrigkeit, GewArch 2002, S. 142-145

Ladeur, Karl-Heinz, Ausschluss von Teilnehmern an Diskussionsforen im Internet - Absicherung von Kommunikationsfreiheit durch „netzwerk gerechtes” Privatrecht, MMR 2001, S. 787-792

Ladeur, Karl-Heinz/Gostomzyk, Tobias, Das Medienrecht und die Herausforderung der technologischen Hybridisierung - Eine Kommentierung zu Medienintermediären im Entwurf des Medienstaatsvertrags der Länder, K\&R 2018, S. 686-693

Lamo, Madeline/Calo, Ran, Regulation Bot Speech, UCLA Law Review Vol. 66 (2019), S. $988-1028$

von Landmann, Robert/Rohmer, Ernst, Kommentar zum Umweltrecht, Band 1, Loseblattsammlung, 93. Ergänzungslieferung, Stand August 2020

Lang, Heinrich/Wilms, Heinrich, Staatsrecht II - Grundrechte, 2. Auflage, Stuttgart 2020

Lange, Christian, Impressumspflicht in sozialen Netzwerken, ZJS 2013, 141-147

Languille, Amy N./Meyer, Carl D., Google's PageRank and Beyond: The Science of Search Engine Rankings, Princeton University Press 2006

Lantwin, Tobias, Deep-Fakes - Düstere Zeiten für den Persönlichkeitsschutz? Rechtliche Herausforderungen und Lösungsansätze, MMR 2019, S. 574-578

ders., Strafrechtliche Bekämpfung missbräuchlicher Deep Fakes - Geltendes Recht und möglicher Regelungsbedarf, MMR 2020, S. 78-82

Laude, Lennart, Aus Rundfunk- wird Medienstaatsvertrag - neuer Anstrich für neuen Durchgriff gegen Medienintermediäre?, JuWissBlog Nr. 126/2020 v. 02.11.2020, abrufbar unter https://www.juwiss.de/126-2020/ (Stand: 30.03.2021) 
Ders., Aus Rundfunk- wird Medienstaatsvertrag - neuer Anstrich für neuen Durchgriff gegen Medienintermediäre? (Teil II), JuWissBlog Nr. 127/2020 v. 02.11.2020, abrufbar unter https://www.juwiss.de/126-2020/ (Stand: 30.03.2021)

Laufer, Daniel, Der Identifizierungszwang für Gamer hat das Zeug zum Desaster, netzpolitik.org v. 14.2.2020, abrufbar unter https://netzpolitik.org/2020/identifizieru ngszwang-fuer-gamer-desaster-pistorius-caffier-klarnamenpflicht-halle/, (Stand 30.03.2021)

Lawrence, Page/Brin, Sergey/Motwani, Rajeev/Winogard, Terry, The PageRank Citation Ranking: Bringing Order to the Web, Technical Report, Stanford InfoLab, 1999, abrufbar unter http://ilpubs.stanford.edu:8090/422/, (Stand 30.03.2021)

Lee, Clarissa Ai Ling/Kerr, Eric, Trolls at the polls - What cyberharassment, online political activism, and baiting algorithms can show us about the rise and fall of Pakatan Harapan, First Monday Vol. 25 (2020) Nr. 6, DOI: https://doi.org/10.5210/fm.v25i6. 10704, (Stand: 30.03.2021)

Lehmann, Jens/Thellmann, Klaudia, Gastbeitrag: Zum technischen Hintergrund von Social Bots, in: PriceWaterhouseCoopers (Hrsg.), Social Bots: Gefahr für de Demokratie?, August 2017, S. 13-16

Leistert, Oliver, Social Bots als algorithmische Piraten und Boten einer techno-environmentalen Handlungskraft, in: Robert Seyfert/Jonathan Roberge (Hrsg.), Algorithmuskulturen - Über die rechnerische Konstruktion der Wirklichkeit, Bielefeld 2017

Leistner, Matthias, Grundlagen und Perspektiven der Haftung für Urheberrechtsverletzungen im Internet, ZUM 2012, S. 722-740

Leistner, Matthias/Metzger, Axel, The EU Copyright Package: A Way Out of the Dilemma in Two Stages, International Review of Intellectual Property and Competition Law (IIC) 2017, S. 381-384

Lenski, Sophie-Charlotte, Personenbezogene Massenkommunikation als verfassungsrechtliches Problem - Das allgemeine Persönlichkeitsrecht in Konflikt mit Medien, Kunst und Wissenschaft, Berlin 2007

Lettl, Tobias, Die Datenschutz-Grundverordnung (DSGVO), WM 2018, S. 1149-1157

Lettmann, Sabine, Schleichwerbung durch Influencer-Marketing - Das Erscheinungsbild der Influencer, GRUR 2018, S. 1206-1211

Leupold, Andreas/Glossner, Silke, Münchener Anwaltshandbuch IT.Recht, 3. Auflage, München 2013

Lewandowski, Dirk, Suchmaschinen verstehen, Berlin 2015

Lewke, Christian, "...aber das kann ich nicht tun!": Künstliche Intelligenz und ihre Beteiligung am öffentlichen Diskurs, Innovations- und Technikrecht 2017, S. 207-216

Li, Mei/Wang, Xiang/Gao, Kai/Zhang, Shanshan, A survey on Information Diffusion in Online Social Networks: Models and Methods, Information 2017, 8, 118, DOI: https://doi.org/10.3390/info8040118.

Li, Shuhao /Yun, Xiaochun /Hao, Zhiyu /Zhang, Yongzheng /Cui, Xiang /Wang, Yipeng, Modeling Social Engineering Botnet Dynamics across Multiple Social Networks, in: Dimitris Gritzalis/Steven Furnell/Marianthi Theoharidou (Hrsg.), Proceedings of the 27th Information Security and Privacy Conference, 2012, S. 261-272

Liesching, Marc, Die Durchsetzung von Verfassungs- und Europarecht gegen das NetzDG - Überblick über die wesentlichen Kritikpunkte, MMR 2018, S. 26-30

Ders., Das Herkunftslandprinzip nach E-Commerce- und AVMD-Richtlinie - Anwendbarkeit von NetzDG, JuSchG, MStV und JMStV auf oziale Netzwerke mit Sitz in anderen EU-Mitgliedstaaten, MMR-Beilage 2020, S. 3-27 
Ders., Netzwerkdurchsetzungsgesetz, NomosBundesrecht Erläuterung, 1. Online-Auflage 2018

Ders., Das Herkunftslandprinzip der E-Commerce-Richtlinie und seine Auswirkung auf die aktuelle Mediengesetzgebung in Deutschland, Medienrecht \& Medientheorie, Band 1, hrsgg. v. Gabriele Hooffacker /ders., München 2020

Liesem, Kerstin, Computational Propaganda: Einsatz von Algorithmen zur Beeinflussung der öffentlichen Meinung, in: Michael Litschka/Larissa Krainer (Hrsg.), Der Mensch im digitalen Zeitalter - Zum Zusammenhang von Ökonomisierung, Digitalisierung und Mediatisierung, Wiesbaden 2019, S. 183-197

Dies., Opening the Black Box „Algorithmus“ - Die Medienintermediärsregulierung im Entwurf zum Medienstaatsvertrag, K\&R 2019, S. 687-692

Dies., Regulierungsarchitektur für Medienintermediäre: Interdisziplinäre Überlegungen zu den Entwürfen des Medienstaatsvertrages, UFITA 2019, S. 395-427

Dies., Neulandvermessung - Die Regulierung von Medienintermediären im neuen Medienstaatsvertrag, ZUM 2020, S. 377-382.

Dies., Pionierleistung mit Signalwirkung: Die regulative Einhegung von Medienintermediären im Medienstaatsvertrag, AfP 2020, S. 277-283

Lindner, Josef Franz, Grundrechtsfestigkeit des arbeitsrechtlichen Kündigungsschutzes?, RdA 2005, S. 166-170

Lischka, Konrad, Wie Algorithmen Öffentlichkeit strukturieren - Grundlagen, Folgen, Lösungsansätze, AfP 2018, S. 388-391

Lischka, Konrad/Stöcker, Christian, Bertelsmann Stiftung (Hrsg.), Digitale Öffentlichkeit - Wie algorithmische Prozesse den gesellschaftlichen Diskurs beeinflussen, Arbeitspapier, 2017, DOI: 10.11586/2017028

Löber, Lena Isabel/Roßnagel, Alexander, Das Netzwerkdurchsetzungsgesetz in der Umsetzung - Bilanz nach den ersten Transparenzberichten, MMR 2019, S. 71-76

Dies., Kennzeichnung von Social Bots - Transparenzpflichten zum Schutz integrer Kommunikation, MMR 2019, S. 493-498

Lorenz, Bernd, Informationspflichten bei eBay, VuR 2008, S. 321-327

Ders., Auswirkungen der Dienstleistungs-Informationspflichten-Verordnung auf Internetangebote, VuR 2010, S. 323-329

Ders., Anonymität im Internet? - zur Abgrenzung von Diensteanbietern und Nutzern, VuR 2014, 83-90

Lorenz, Dieter, Aktuelle Verfassungsfragen der Euthanasie, JZ 2009, S. 57-67

Lou, Xiadan/Flammini, Alessandro/Menczer, Filippo, Information Pollution by Social Bots, 2019, arXiv:1907.06130 [cs.CY]

Lüdemann, Jörn, Grundrechtliche Vorgaben für die Löschung von Beiträgen in sozialen Netzwerken - Private Ordnung digitaler Kommunikation unter dem Grundgesetz, MMR 2019, S. 279-284

Ders., Privatisierung der Rechtsdurchsetzung in sozialen Netzwerken?, in: Martin Eifert/ Tobias Gostomzyk (Hrsg.), Netzwerkrecht - Die Zukunft des NetzDG und seine Folgen für die Netzwerkkommunikation, Baden-Baden 2018, S. 153-168

Madjarov, Peter, GreifRecht merkt an: Äußerungsbefugnisse von Bundespräsident und Regierungsmitgliedern - Anmerkung zu BVerfG, Urteil vom 10.06.2014 - 2 BvE 4/13 sowie Urteil vom 16.12.2014 - 2 BvE 2/14, GreifRecht 2015, S. 59-64

Mafi-Gudarzi, Nima, Desinformation: Herausforderung für die wehrhafte Demokratie, ZRP 2019, S. 65-68 
Maier, Michaela, Kapitel 1- Gegenstandsbereich und Grundzüge der Nachrichtenwerttheorie, in: Patrick Rössler/ Hans-Bernd Brosius (Hrsg.), Konzepte. Ansätze der Medien- und Kommunikationswissenschaft, Band 2 - Nachrichtenwerttheorie, 2. Auflage, Baden-Baden 2018, S. 13-28

Mallick, Rani/Weller, David, Aktuelle Entwicklungen im Influencer-Marketing - Ein Blick aus der Praxis, WRP 2018, S. 155-161

v. Mangoldt, Hermann/Klein, Friedrich/Starck, Christian, Grundgesetz, Kommentar, Band 1 (Art. 1-19 GG), 7. Auflage, München 2018

Dies., Grundgesetz, Kommentar, Band 2 (Art. 20- 82 GG), 7. Auflage, München 2018

Markwalder, Nora/Simmler, Monika, Roboterstrafrecht, AJP/PJA 2017, S. 171-182

Martini, Mario, Blackbox Algorithmus - Grundfragen einer Regulierung künstlicher Intelligenz, Wiesbaden 2019

Marvany, Markus, Digitaler Hausfriedensbruch - Allheilmittel oder bittere Pille?, ZRP 2016, S. 221-223

Maschewski, Felix/Nosthoff, Anna-Verena, Netzwerkeffekte - Über Facebook als kybernetische Regierungsmaschine und das Verschwinden des Subjekts, in: Rainer Mühlhoff/Anja Breljak/Jan Slaby (Hrsg.), Affekt, Macht, Netz - Auf dem Weg zu einer Sozialtheorie der Digitalen Gesellschaft, S. 55-80

Massaro, Toni M./Norton, Helen, Siri-ously? - Free Speech Rights and Artificial Intelligence, Northwestern University Law Reciew Vol. 110, S. 1169-1194

Massaro, Toni M./Norton, Helen/Kaminski, Margot E., Seriously 2.0: What Artificial Intelligence Reveals About the First Amendment, Minnesota Law Review 101 (2017), S. 2481-2525

Matthes, Jörg/Knoll, Johannes/von Sikorsky, Christian, The „Spiral of Silence“ revisited: A Meta-Analysis on the relationship between perceptions of opinion support and political opinion expression, Communication Research Vol. 45 (2018), S. 3-33

Maume, Philipp, Bestehen und Grenzen des virtuellen Hausrechts, MMR 2007, S. 620-625

Maunz, Theodor/Dürig, Günter, Kommentar zum Grundgesetz, Loseblattsammlung, Stand des Gesamtwerks 91. Ergänzungslieferung, April 2020

Maunz, Theodor/Schmidt-Bleibtreu, Bruno/Klein, Franz/Bethge, Herbert, Kommentar zum Bundesverfassungsgerichtsgesetz, Loseblattsammlung, Stand des Gesamtwerks 60. Ergänzungslieferung, Juli 2020

Mayer, Franz C., Recht und Cyberspace, NJW 1996, S. 1782-1791

Mayer, Matthias, Untermaß, Übermaß und Wesensgehaltgarantie - Die Bedeutung staatlicher Schutzpflichten für den Gestaltungsspielraum des Gesetzgebers im Grundrechtsbereich, in: Christian Starck (Hrsg.), Studien und Materialien zur Verfassungsgerichtsbarkeit, Band 97, Baden-Baden 2005

Medicus, Dieter/Lorenz, Stephan, Schuldrecht I, Allgemeiner Teil: ein Studienbuch, 21. Auflage, München 2015

Meffert, Michael F., Informationsverarbeitung und Entscheidungsfindung, in: Sonja Zmerli/Ofer Feldman (Hrsg.), Politische Psychologie - Handbuch für Studium und Wissenschaft, Baden-Baden 2015

Mehrens, Anastasia, Wenn Maschinen Meinung machen - Social Bots und Manipulationen, in: Michael Steinbrecher/Günther Rager (Hrsg.), Wenn Maschinen Meinung machen - Journalsimuskrise, Social Bots und der Angriff auf die Demokratie, Frankfurt 2018 
Messingschlager, Tanja/Holtz, Peter, Filter Bubbles und Echo Chambers, in: Markus Appel (Hrsg.), Die Psychologie des Postfaktischen - Über Fake News, „Lügenpresse“, Clickbaiting und Co., 2020, S. 91-102

Metzger, Axel, Dienst gegen Daten: Ein synallagmatischer Vertrag, AcP 216 (2016), S. $817-865$

Metzger, Miriam J./Flanagin, Andrew J./Medders, Ryan B., Social and heuristic approaches to credibility evaluation online, Journal of Communication Vol. 60 (2010), S. 413-439

Meyer, Michelle N., Misjudgements will drive social trials underground, Nature Vol. 511 (2014), S. 265

Meyer, Stephan, Grundrechtsschutz in Sachen Klimawandel, NJW 2020, S. 894-900

Michael, Lothar/Morlok, Martin, Grundrechte, 7. Auflage, Baden-Baden 2020

Milker, Jens, Bot-Armeen “ als Meinungsmacher im Wahlkampf, JuWissBlog Nr. 91/2016 v. 29.11.2016, abrufbar unter http://www.juwiss.de/91-2016/, (Stand 30.03.2021)

Ders., „Social Bots“ im Meinungskampf, Zeitschrift für Urheber- und Medienrecht 2017, S. 216-222

Ders., Social Bots: Gesetzgeberische Maßnahmen auf dem Prüfstand, InTeR 2017, S. $199-207$

Ders., Äußerungen von Hoheitsträgern im Wahlkampf und darüber hinaus, JA 2017, S. 647-654

Milstein, Alexander/Lippold, Matthias, Suchmaschinenergebnisse im Lichte der Meinungsfreiheit der nationalen und europäischen Grund- und Menschenrechte, Neue Zeitschrift für Verwaltungsrecht 2013, S. 182-187

Mitsch, Lukas, Soziale Netzwerke und der Paradigmenwechsel des öffentlichen Meinungsbildungsprozesses, DVBl. 2019, S. 811-818

Möller, Judith/Hameleers, Michael, Typen von Desinformation und Misinformation, in: dies./Frederik Ferreau, Typen von Desinformation und Misinformation- Verschiedene Formen von Desinformation und ihre Verbreitung aus kommunikationswissenschaftlicher und rechtswissenschaftlicher Perspektive, Berlin 2020, S. 10-43

Moritz, Susanne, Staatliche Schutzpflichten gegenüber pflegebedürftigen Menschen, Peter Axer u.a. (Hrsg.), Schriften zum Sozialrecht, Band 29, Baden-Baden 2013

Mueller, Robert S., Report On The Investigation Into Russian Interference In The 2016 Presidential Election, Volume I, Washington D.C. 2019, abrufbar unter https://www.j ustice.gov/storage/report.pdf, (Stand: 30.03.2021)

Muble, Florian/Ackland, Robert/Graham, Timothy, Socialbots in politischen OnlineKonversationen. Eine (überschätzte) Gefahr für demokratische Willensbildung im Internet?, ZParl 2018, S. 618-638

Müller, Jürgen, Selbstlernende Algorithmen - Wie maschinelles Lernen Unternehmen intelligent macht, Computerwoche v. 10.02.2017, abrufbar unter https://www.comp uterwoche.de/a/wie-maschinelles-lernen-unternehmen-intelligent-macht,3329733, (Stand: 30.03.2021)

Müller, Philipp/Denner, Nora, Was tun gegen „Fake News“? - Eine Analyse anhand der Entstehungsbedingungen und Wirkweisen gezielter Falschmeldungen im Internet, in: Friedrich-Naumann-Stiftung für die Freiheit (Hrsg.), Kurzgutachten, 2017; abrufbar unter https://shop.freiheit.org/\#!/Publikation/792, (Stand 30.11.2019)

Müller-Broich, Jan D., NomosKommentar zum Telemediengesetz, 1. Auflage, Baden-Baden 2012 
Müller-Franken, Sebastian, Netzwerkdurchsetzungsgesetz: Selbstbehauptung des Rechts oder erster Schritt in die selbstregulierte Vorzensur? - Verfassungsrechtliche Fragen, AfP 2018, S. 1-14

Ders., Bindung Privater an Grundrechte? - Zur Wirkung der Grundrechte auf Privatrechtsbeziehungen, in: Steffen Detterbeck/Jochen Rozek/Christian von Coolen (Hrsg.), Recht als Medium der Staatlichkeit - Festschrift für Herbert Bethge zum 70. Geburtstag, Berlin 2009, S. 223-250

Müller-Hengstenberg, Claus D./Kirn, Stefan, Intelligente (Software-)Agenten: Eine neue Herausforderung unseres Rechtssystems - Rechtliche Konsequenzen der „Verselbstständigung “ technischer Systeme, MMR 2014, S. 307-311

Müller-Terpitz, Ralf, Internet-Kommunikationsdienste wie Facebook, Google \& Co. Regulierungs- und/oder Kartellrecht?, in: Jürgen Kühling/Daniel Zimmer (Hrsg.), Neue Gemeinwohlherausforderungen - Konsequenzen für Wettbewerbsrecht und Regulierung,, Baden-Baden 2020, S. 177-196

Ders., Soziale Netzwerke als Gegenstand des geltenden Rechts. Eine rechtssystematische Einordnung, in: Martin Eifert/ Tobias Gostomzyk (Hrsg.), netzwerkrecht - Die Zukunft des NetzDG und seine Folgen für die Netzwerkkommunikation, Baden-Baden 2018, S. 45-60

Ders., Filter als Gefahr für die Meinungspluralität? - Verfassungsrechtliche Erwägungen zum Einsatz von Filtertechnologien, ZUM 2020, S. 365-374

Murthy, Dhiraj/Powell, Allison B./Tinati, Ramine/Anstead, Nick/Carr, Leslie/Halford, Susan J./Weal, Mark, Bots and Political Influence: A Socioethnical Investigation of Social Network Capital, International Journal of Communication 10 (2016), S. 4952-4971.

Murswiek, Dietrich, Die staatliche Verantwortung für die Risiken der Technik - Verfassungsrechtliche Grundlagen und immissionsschutzrechtliche Ausformung, Schriften zum Umweltrecht, Band 3, Berlin 1995

v. Münch, Ingo/Kunig, Philip, Grundgesetz Kommentar, Band 1, 6. Auflage, München 2012

Münchener Kommentar zum Bürgerlichen Gesetzbuch, Band 1, Allgemeiner Teil, hrsgg. v. Franz Jürgen Säcker/Roland Rixecker/Hartmut Oetker/Bettina Limperg, 8. Auflage, München 2018

Ders., Band 2, Schuldrecht - Allgemeiner Teil, hrsgg. v. Franz Jürgen Säcker/Roland Rixecker/Hartmut Oetker/Bettina Limperg, 8. Auflage, München 2019

Ders., Band 8, $\mathbb{S} \mathbb{S} 854,1296$, WEG, ErbbauRG, hrsgg. v. Franz Jürgen Säcker/Roland Rixecker/Hartmut Oetker/Bettina Limperg, 8. Auflage, München 2020

Ders., Band 13, IPR II, Internationales Wirtschaftsrecht, Art. 50-283 EGBGB, hrsgg. v. Franz Jürgen Säcker/Roland Rixecker/Hartmut Oetker/Bettina Limperg, 8. Auflage 2020

Münchener Kommentar zum Lauterkeitsrecht, Band 1, $\mathbb{S}$ 1-7 UWG, hrsgg. v. Peter W. Heermann/Jochen Schlingloff, 3. Auflage, München 2020

Münchener Kommentar zum Strafgesetzbuch, Band 1, $\mathbb{S}$ 1-37 StGB, hrsgg. v. Wolfgang Joecks/Klaus Miebach, 4. Auflage, München 2020

Ders., Band 3, $\mathbb{S}$ 80-184j StGB, hrsgg. v. Wolfgang Joecks/Klaus Miebach, 3. Auflage, München 2017

Ders., Band 4, $\mathbb{S}$ 185-262 StGB, hrsgg. v. Wolfgang Joecks/Klaus Miebach, 3. Auflage, München 2017

Ders., Band 5, $\mathbb{S}$ 263-356 StGB, hrsgg. v. Wolfgang Joecks/Klaus Miebach, 3. Auflage, München 2019 
Ders., Band 6, Nebenstrafrecht, hrsgg. v. Wolfgang Joecks/Klaus Miebach, 3. Auflage, München 2017

Münchener Kommentar zur Zivilprozessordnung, Band 1, $\mathbb{S} 1-354$ ZPO, hrsgg. v. Thomas Rauscher/Wolfgang Krüger, 6. Auflage, München 2020

Nagler, Georg, Dogmatische Strukturen der Beweislast im öffentlichen Recht, München 1989

Nebel, Maxi, Die Zulässigkeit der Erhebung des Klarnamens nach den Vorgaben der Datenschutz-Grundverordnung, K\&R 2019, S. 148-152

Neidhardt, Friedhelm, Öffentlichkeit und öffentliche Meinung, in: Birgit Krause/Benjamin Fretwurst/Jens Vogelgesang (Hrsg.), Fortschritte der Kommunikationsforschung - Festschrift für Lutz Erbring, Wiesbaden 2007, S. 19-33

Neis, Martina/Mara, Martina, Social Bots - Meinungsroboter im Netz, in: Markus Appel (Hrsg.), Die Psychologie des Postfaktischen: Über Fake News, „Lügenpresse“, Clickbait \& Co., Wiesbaden 2020, S. 189-203

Neubaum, German/Krämer, Nicole C., What do we fear? Expected sanctions for expressing Minority Opinions in offline and online Communication, Communication Research Vol. 45 (2018), S. 139-164

Neubert, Carl-Wendelin, Grundrechtliche Schutzpflicht des Staates gegen grundrechtsbeeinträchtigende Maßnahmen fremder Staaten am Beispiel der Überwachung durch ausländische Geheimdienste, AöR 140 (2015), S. 267-304

Neudert, Lisa Maria, Future elections may be swayed by intelligent, weaponised chatbots, MIT Technology Review 2018/ 121, abrufbar unter https://www.technologyrevi ew.com/2018/08/22/104087/future-elections-may-be-swayed-by-intelligent-weaponiz ed-chatbots/, (Stand: 30.03.2021)

Neudert, Lisa-Maria /Kollanyi, Bence/Howard, Philip N., Junk News und Bots bei der Bundespräsidentenwahl 2017: Was haben Deutsche Wähler auf Twitter geteilt?, 2017, abrufbar unter http://comprop.oii.ox.ac.uk/wp-content/uploads/sites/89/2017/03/Ger man-What-Were-German-Voters-Sharing-Over-Twitter-v9.pdf, (Stand 30.03.2021)

Niehaus, Michael, Beweismaß und Beweislast - Untersuchungsgrundsatz und Beteiligtenmitwirkung im Verwaltungsprozeß, München 1989

Nietsch, Thomas, Anonymität und die Durchsetzung urheberrechtlicher Ansprüche im Internet - Grundrechtliche Positionen im Spannungsfeld, Tübingen 2014

Nocun, Katharina, Datenschutz unter Druck: Fehlender Wettbewerb bei sozialen Netzwerken alsRisiko für den Verbraucherschutz, in Roßnagel/Friedewald/Hansen (Hrsg.), Die Fortentwicklung des Datenschutzes - Zwischen Systemgestaltung und Selbstregulierung, Wiesbaden 2018, S. 39-58

Noelle-Neumann, Elisabeth, Die Schweigespirale: Öffentliche Meinung - unsere soziale Haut, 6. Auflage, München 2001

Nölscher, Patrick, Das Netzwerkdurchsetzungsgesetz und seine Vereinbarkeit mit dem Unionsrecht, ZUM 2020, S. 301-312

Nolte, Georg, Drei Thesen zur aktuellen Debatte über Haftung und Verteilungsgerechtigkeit bei Hosting-Diensten mit nutzergenerierten Inhalten (sog. »Value-Gap«-Debatte), ZUM 2017, S. 304-312

Ders., Hate Speech, Fake-News, das »Netzwerkdurchsetzungsgesetz « und Vielfaltsicherung durch Suchmaschinen, ZUM 2017, S. 552-565 
Noyb (Europäisches Zentrum für digitale Rechte), Beschwerde nach Art. 77 Abs. 1 DSGVO gegen Facebook an die österreichische Datenschutzbehörde, abrufbar unter https://noyb.eu/sites/default/files/2020-05/complaint-facebook.pdf, (Stand: 30.03.2021)

Nyhan, Brendan/Reifler, Jason/Richey, Sean/Freed, Gary L., Effective Messages in Vaccine Promotion: A Randomized Trial, Pediatrics Vol. 133 (2014), e835-e842

Oberer, Birgit/Erkollar, Alpetkin/Stein, Anna, Social Bots - Act Like a Human, Think Like a Bot, in: Marcus Stumpf (Hrsg.), Digitalisierung und Kommunikation - Konsequenzen der digitalen Transformation für die Wirtschaftskommunikation, Wiesbaden 2019, S. 311-327

Oehmer, Franziska/Pedrazzi, Stefano, Was schützt (vor) Social Bots? Vorschläge zur Governance von computergenerierten Softwareagenten im Internet, UFITA 2020, S. 7-35

Oermann, Rechts(durch)setzung durch Informationsintermediäre: Big Data als Entscheidungs- und Handlungsressource, in: Wolfgang Hoffmann-Riem (Hrsg.), Big Data Regulative Herausforderungen, Baden-Baden 2018, S. 145-155

Oppermann, Thomas/Classen, Claus Dieter/Nettesheim, Martin, Europarecht, 8. Auflage, München 2018

Ohly, Ansgar/Sosnitza, Olaf, Gesetz gegen den Unlauteren Wettbewerb, Kommentar, 7. Auflage, München 2018

Ohrtmann, Jan-Peter/Schwiering, Sebastian, Big Data und Datenschutz - Rechtliche Herausforderungen und Lösungsansätze, NJW 2014, S. 2984-2989

Ory, Stephan, Herausforderungen der Medienfreiheit - oder - Der Rundfunk als Endprodukt der Konvergenz?, AfP 2011, S. 19-22

Ders., Der Medienstaatsvertrag - Neuer Wein in neuen Schläuchen?, ZUM 2019, S. $139-152$.

Ostermann, Georg-Julius, Transparenz und öffentlicher Meinungsbildungsprozess - Eine verfassungsrechtliche Untersuchung, Tübingen 2019

Ott, Stephan, Impressumspflicht für Webseiten - Die Neuregelungen nach $\$ 5$ TMG, \$5 55 RStV, MMR 2007, S. 354-359

Paal, Boris P., Personenbezogene Bewertungsportale im Internet - Spickmich.de und die Folgen, Recht der Jugend und des Bildungswesens 2010, S. 459-471

Ders., Vielfaltssicherung im Suchmaschinensektor, Zeitschrift für Rechtspolitik 2015, S. 34-38

Ders., Big Data im Recht - Wettbewerbs- und daten(schutz)rechtliche Herausforderungen, Neue Juristische Wochenschrift 2017, S. 1697-1701

Ders., Vielfaltssicherung bei Intermediären Fragen der Regulierung von sozialen Netzwerken, Suchmaschinen, Instant-Messengern und Videoportalen, MMR 2018, S. $567-572$

Ders., Intermediäre: Regulierung und Vielfaltssicherung, Rechtsgutachten im Auftrag der Landesanstalt für Medien Nordrhein-Westfalen, 2018, abrufbar unter https://www.m edienanstalt-nrw.de/fileadmin/user_upload/lfm-nrw/Foerderung/Forschung/Dateien_F orschung/Paal_Intermediaere_Regulierung-und-Vielfaltssicherung_Gutachten-2018.p df, (Stand 30.03.2021)

Paal, Boris P./Heidtke, Aron, Vielfaltssichernde Regulierung der Medienintermediäre nach den Vorschriften des Medienstaatsvertrags der Länder, ZUM 2020, S. 230- 240 
Paal, Boris P./Hennemann, Moritz, Meinungsbildung im digitalen Zeitalter, JZ 2017, S. 641-652

Paal, Boris P. /Pauly, Daniel A., Beck'scher Kompakt-Kommentar zur Datenschutzgrundverordnung und zum Bundesdatenschutzgesetz, 2. Auflage, München 2018

Papier, Hans-Jürgen, Rechtsstaatlichkeit und Grundrechtsschutz in der digitalen Gesellschaft, NJW 2017, S. 3025-3031

Papier, Hans-Jürgen/Schröder, Meinhard, „Gebiet des Rundfunks“ - Gutachten von H.-J. Papier und M. Schröder zu „Presseähnlichen Angeboten“, epd Medien, Nr. 60 aus 2010, S. 16-33

Paschke, Marian/Berlit, Wolfgang/Meyer, Claus/Kröner, Lars, Hamburger Kommentar Gesamtes Medienrecht, 4. Auflage, Baden-Baden 2021

Pariser, Eli, The filter bubble - what the internet is hiding from you, London 2012

Paulus, Andreas/Nölscher, Patrick, Rundfunkbegriff und Staatsferne im Konvergenzzeitalter, ZUM 2017, S. 177-186

Paulus, David/Matzke, Robin, Smart Contracts und das BGB - Viel Lärm um nichts?, ZfPW 2018, S. 431-465

Payandeh, Mehrdad, Die Neutralitätspflicht staatlicher Amtsträger im öffentlichen Meinungskampf, Der Staat 55 (2016), S. 519-550

Peine, Franz-Joseph, Gefahr, Risiko, Restrisiko - Begriffsbestimmungen und Probleme aus juristischer, in: Braunschweigische Wissenschaftliche Gesellschaft (Hrsg.), Jahrbuch 1999, Braunschweig 2000, S. 151-164

Peukert, Alexander, Gewährleistung der Meinungs- und Informationsfreiheit in sozialen Netzwerken - Vorschlag für eine Ergänzung des NetzDG um sog. Put-back-Verfahren, MMR 2018, S. 572-578

Ders., Faktenchecks auf Facebook aus lauterkeitsrechtlicher Sicht, WRP 2020, S. 391-398

Pewestorf, Adrian/Söllner, Sebastian/Tölle, Oliver, Polizei und Ordnungsrecht, Kommentar, 2. Auflage, Köln 2017

Pfaffenberg, Fabian, Twitter als Basis wissenschaftlicher Studien - Eine Bewertung gängiger Erhebungs- und Analysemethoden der Twitter-Forschung, Wiesbaden 2016

Pfaffenberg, Fabian/Adrian, Christoph/Heinrich, Philipp, Was bin ich - und wenn ja wie viele? - Identifikation und Analyse von Political Bots während des Bundestagswahlkampfs 2017 auf Twitter, in: Christina Holtz-Bacha (Hrsg.), Die (Massen-) Medien im Wahlkampf - Die Bundestagswahl 2017, Wiesbaden 2019

Pfeifer, Karl-Nikolaus, Influencer-Marketing - Rechtlicher Rahmen und Regulierungsbedürfnis (Teil 2), GRUR 2018, S. 1218-1225

Pfengler, Christian, Entparlamentarisierung zwischen Schein und Sein, GreifRecht 2020, S. 1-12

Pierrot, Jarass/Hartmann, Bernd J., Grundrechtsschutz gegen wirtschaftliche Betätigung der öffentlichen Hand, DVBl. 2002, S. 421-428

Pießkalla, Michael, Zur Reichweite der Impressumspflicht in sozialen Netzwerken, ZUM 2014, S. 368-374

Pille, Jens-Ullrich, Meinungsmacht sozialer Netzwerke, 1. Auflage, Baden-Baden 2016

Ders., Der Grundsatz der Eigenverantwortlichkeit im Internet, NJW 2018, S.35453550

Plath, Kai Uwe, Kommentar zu DSGVO, BDSG und den Datenschutzbestimmungen des TMG und TKG, 3. Auflage 2018 
Policy Department for Citizens' Rights and Constitutional Affairs, Disinformation and propaganda - impact on the functioning of the rule of law in the EU and its Member States, abrufbar unter https://www.europarl.europa.eu/RegData/etudes/STUD/2019/6 08864/IPOL_STU(2019)608864_EN.pdf, (Stand 30.03.2021)

Pomana, Andrea, Wettbewerbsrecht und Datenschutz: Facebook im Visier des Bundeskartellamts, BB 2018, S. 965-975

Ponce, Marcelo F., Noticias falsas, hechos alternativos y democracia en la sociedad postfactual, 2018, DOI: https://dx.doi.org/10.2139/ssrn.3280432

Pravemann, Timm, Art. 17 der Richtlinie zum Urheberrecht im digitalen Binnenmarkt Eine Analyse der neuen europäischen Haftungsregelung für Diensteanbieter für das Teilen von Online-Inhalten, GRUR 2019, S. 783-788

Price WaterhouseCoopers GmbH (PwC), Social Bots: Gefahr für die Demokratie? - Whitepaper mit Handlungsempfehlungen für Unternehmen, Medien und Politik, August 2017, abrufbar unter https://www.pwc.de/de/technologie-medien-und-telekommunik ation/social-bots-gefahr-fuer-die-demokratie.pdf, (Stand 30.03.2021)

Prochazka, Fabian/Schweiger, Wolfgang, Erwartungen an Medien und die Wahrnehmung ihrer Umsetzung Vertrauen in Journalismus in Deutschland: Eine Typologie der Skeptiker, Media Perspektiven 2020, S. 196-206

Prütting, Hanns, Gegenwartsprobleme der Beweislast, München 1983

Pürer, Heinz, Kommunikationswissenschaft als Sozialwissenschaft, 1. Auflage, Stuttgart 2015

Quandt, Thorsten, What's left of trust in a network society? An evolutionary model and critical discussion of trust and societal communication, European Journal of Communication Vol. 27 (2012), Issue 1, S. 7-21

Rauchfleisch, Adrian/Kaiser, Jonas, The False Positive Problem of Automatic Bot Detection in Social Science Research, The Berkman Klein Center for Internet \& Society Research Publication Series, Research Publication Nr. 2020-3, März 2020, abrufbar unter https://cyber.harvard.edu/news/2020-03/false-positive-problem-automatic-bot-d etection-social-science-research, (Stand 30.03.2021)

Rau, Jan Philipp/Stier, Sebastian, Die Echokammer-Hypothese: Fragmentierung der Öffentlichkeit und politische Polarisierung durch digitale Medien?, Zeitschrift für vergleichende Politikwissenschaft 2019, S. 399-417

Raue, Benjamin, Meinungsfreiheit in sozialen Netzwerken - Ansprüche von Nutzern sozialer Netzwerke gegen die Löschung ihrer Beiträge, JZ 2018, S. 961-970

Raue, Benjamin/v. Ungern-Sternberg, Antje, Ethische und rechtliche Grundsätze der Datenverwendung, ZRP 2020, S. 49-52

Redeker, Helmut, IT-Recht, 6. Auflage, 2017

Rehfeld, Nina, Verleumdungsaktion „Pizzagate“ - In Amerika herrscht die Lüge, FAZ, v. 09.12.2016, abrufbar unter https:/www.faz.net/aktuell/feuilleton/debatten/wie-sich-i n-amerika-die-herrschaft-der-luege-festigt-14565557.html, (Stand 30.03.2021)

Reinbacher, Tobias, Die Beleidigung im Internet - Der Regierungsentwurf eines Gesetzes zur Bekämpfung des Rechtsextrimismus und der Hasskriminalität, Neue Kriminalpolitik 2020, S. 186-198

Ders., Die „Weiterverbreitung“ von Hate Speech in sozialen Medien - Fragen der Beteiligung an einer gemäß $\$ 185$ StGB strafbaren Beleidigung, JZ 2020, S. 558-563

Reinemann, Susanne/Remmertz, Frank, Urheberrechte an User-generated Content, ZUM 2012, S. 216-227 
Remmertz, Frank, DSGVO ante portas: Aktuelle Brennpunkte im Online-Marketing, GRUR-Prax 2018, 254 -256

Rengier, Rudolf, Strafrecht Besonderer Teil I, 22. Auflage, München 2020

Rhiem, Thomas, Von Drohnen, Google-Cars und Software-Agenten - Rechtliche Herausforderungen autonomer Systeme, ITRB 2014, S. 113-115

Richardt, Johannes, Freiheit statt Paternalismus - Zur Kritik des Lotteriemonopols, ZfWG 2017, Sonderbeilage 3/2017, S. 31-34

Richter, Philipp, Ein anonymes Impressum? - Profile in sozialen Netzwerken zwischen Anbieterkenn-zeichnung und Datenschutz, MMR 2014, S. 517-521

Ders., Das NetzDG - Wunderwaffe gegen „Hate Speech“ und „Fake News“ oder ein neues Zensurmittel?, ZD-Aktuell 2017, 05623

Ricker, Reinhart, Digitalisierung und interaktive Medienangebote - Grenzen für ARD und ZDF?, Archiv für Presserecht 1998, S. 437-446

Riedel, Ann Cathrin, Wir brauchen eine faktenbasierte Digital- und Medienpolitik, medium.com v. 19.10.2019, abrufbar unter https://medium.com/@anncathrin87/wir -brauchen-eine-faktenbasierte-digital-und-medienpolitik-ef1da1bede3f, (Stand: 30.03.2021)

Rieger, Diana/Dippold, Jessica/Appel, Markus, Trolle gibt es nicht nur im Märchen Das Phänomen Trolling im Internet, in: Markus Appel (Hrsg.), Die Psychologie des Postfaktischen - Über Fake News, „Lügenpresse“,Clickbait \& Co., Wiesbaden 2020, S. $45-58$

Riis, Thomas/Schwermer, Felix, Leaving the European Safe Harbour Sailing Toward Algorithmic Content Regulation, Journal of Internet Law Vol. 22 (2019) Number 7, S. 1-21.

Rippender, Konstantin/Appel, Markus, Word-of-mouth: Von Mund-zu-Mund-Propaganda zu viralem Marketing, in: Markus Appel (Hrsg.), Die Psychologie des Postfaktischen: Über Fake News, „Lügenpresse“, Clickbait \& Co., Wiesbaden 2020, S. 167-176

Robbers, Gerhard, Sicherheit als Menschenrecht - Aspekte der Geschichte, Begründung und Wirkung einer Grundrechtsfunktion, Baden-Baden 1987

Rockstroh, Sebastian, Impressumspflicht auf Facebook-Seiten - Wann werden Telemedien „in der Regel gegen Entgelt” angeboten?, MMR 2013, S. 627-630

Rogg, Arne, Demokratie und Internet - Der Einfluss von computervermittelter Kommunikation auf Macht, Repräsentation, Legitimation und Öffentlichkeit, Opladen 2003

Roggenkamp, Jan Dirk/Krieg, Henning, Rechtliche Aspekte sozialer Medien, in: JanHinrik Schmidt/Monika Taddicken (Hrsg.), Handbuch Soziale Medien, Wiesbaden 2017, S. 343-366

Roos, Philipp/Schumacher, Phillipp, Botnetze als Herausforderung für Recht und Gesellschaft - Zombies außer Kontrolle?, MMR 2014, S. 377-383

Ross, Björn/Pilz, Laura/Cabrera, Benjamin/Brachten,Florian/Neubaum, German/Stieglitz, Stefan, Are social bots a real threat? An agent-based model of the spiral of silence to analyse the impact of manipulative actors in social networks, European Journal of Information Systems, Vol. 28 (2019), S. 394-412

Roßnagel, Alexander/Scholz, Philipp, Datenschutz durch Anonymität und Pseudonymität - Rechtsfolgen der Verwendung anonymer und pseudonymer Daten, MMR 2000, S. 721-730

Rostalski, Frauke, „Fake News“ und die „Lügenpresse“ - ein (neuer) Fall für das Strafu. Ordnungswidrigkeitenrecht ?, Rechtswissenschaft 2017, S. 436-460. 
Rothmann, Robert, Ungewollte Einwilligung? Die Rechtswirklichkeit der datenschutzrechtlichen Willenserklärung im Fall von Facebook, in: Alexander Roßnagel/ Michael Friedewald/Marit Hansen (Hrsg.), Die Fortentwicklung des Datenschutzes - Zwischen Systemgestaltung und Selbstregulierung, Wiesbaden 2018

Rothmann, Robert/Buchner, Benedikt, Der typische Facebook-Nutzer zwischen Recht und Realität - Zugleich eine Anmerkung zu LG Berlin v. 16.01.2018, DuD 2018, S. 342-346

Rücker, Daniel/Kugler, Tobias, New European General Data Protection Regulation - A Practitioner's Guide, 1 Auflage 2018

Rückert, Christian, Herausforderungen der Digitalisierung für das Strafverfahren, in: Elisa Hoven/ Hans Kudlich (Hrsg.), Digitalisierung des Strafverfahrens, Baden-Baden 2020, S. 9-38

Ruffert, Matthias, Vorrang der Verfassung und Eigenständigkeit des Privatrechts, Jus Publicum - Schriften zum öffentlichen Recht, Band 74, Tübingen 2001

Ders., Privatrechtswirkung der Grundrechte - Von Lüth bis zum Stadionverbot - und darüber hinaus ?, JuS 2020, S. 1-6.

Rundfunkkommission der Länder, Diskussionsentwurf für einen „Medienstaatsvertrag“, Stand Juli 2019, abrufbar unter https://www.rlp.de/fileadmin/rlp-stk/pdf-Dateien/Me dienpolitik/MStV-E_Synopse_2019-07_Online_.pdf, (Stand 30.03.2021)

Rusteberg, Benjamin, Der grundrechtliche Gewährleistungsgehalt, Tübingen 2009

Sachs, Michael, Kommentar zum Grundgesetz, 8. Auflage, München 2018

Saenger, Ingo, Zivilprozessordnung, Handkommentar, 8. Auflage, Baden-Baden 2019

Sahl, Jan Christian/Bielzer, Nils, NetzDG 2.0 - Ein Update für weniger Hass im Netz, ZRP 2020, S. 2-5

Sander, Günther M./Hollering, Jörg, Strafrechtlicher Verantwortlichkeit im Zusammenhang mit automatisiertem Fahren, NStZ 2017, S. 193-206.

Sandvig, Christian, The Facebook „It's Not Our Fault“ Study, Social Media Collective v. 07.05.2015, abrufbar unter https://socialmediacollective.org/2015/05/07/the-faceboo k-its-not-our-fault-study/, (Stand 30.03.2021)

Schaal, Gary S., Hybrid influencing und die Vulnerabilität digital eingebetteter demokratischer Öffentlichkeiten, in: Jonas Bedford-Strohm/Florian Höhne/Julian ZeyherQuattlender (Hrsg.), Digitaler Strukturwandel der Öffentlichkeit - Interdisziplinäre Perspektiven auf politische Partizipation im Wandel, Baden-Baden 2019, S. 121- 134

Schaewitz, Leonie/Krämer, Nicole C., Combating Disinformation: Effects of Timing and Correction Format on Factual Knowledge and Personal Beliefs, in: Max van Duin/ Mike Preuss/Viktoria Spaiser/Frank Takes/Suzan Verbrene (Hrsg.), Disinformation in Open Online Media, Second Multidisciplinary International Symposium, MISDOOM 2020, S. 233-245

Schäfer, Fabian/Evert, Stefan/Henrich/Philipp, Japan's 2014 General Election: Political Bots, Right-Wing Internet Activism and Prime Minister Shinzo Abe's Hidden Nationalist Agenda, Big Data, Volume 5 Number 4 (2017), S. 294-309

Schäfer, Svenja, Illusion of knowledge through Facebook news? Effects of snack news in a news feed on perceived knowledge, attitude strength, and willingness for discussion, Computers in Human Behavior Vol. 103 (2020), S. 1-12

Schantz, Peter, Die Datenschutz-Grundverordnung - Eine neue Zeitrechnung im Datenschutz, NJW 2016, S. 1841-1847 
Schaub, Renate, Interaktionen Mensch und Maschine, JZ 2017, S. 342-349

Schede, Christian/Schuldt, Frederik, Verfassungswidrigkeit landesrechtlicher Mietendeckel, NVwZ 2019, S. 1572-1577

Scheibel, Henrik, Weiterempfehlungen - Eine wettbewerbsrechtliche Betrachtung unter besonderer Berücksichtigung des viralen Marketings, Baden-Baden 2015

Scherer, Inge, Rezeption kommerzieller Kommunikation in sozialen Netzwerken durch minderjährige Nutzer, WRP 2018, S. 277-283

Scheufele, Betram, Kommunikation und Medien: Grundbegriffe, Theorien und Konzepte, in: Ansgar zerfaß/Manfred Piwinger, Handbuch Unternehmenskommunikation, Wiesbaden 2014, S. 105-143

Schiff, Alexander, Meinungsfreiheit in mediatisierten digitalen Räumen - Das NetzDG auf dem Prüfstand des Verfassungsrechts, MMR 2018, S. 366-371

Schimmele, Tanja, Staatliche Verantwortung für diskursive Integrität in öffentlichen Räumen, Berlin 2020

Schladebach, Marcus/ Simantiras, Nikolaos, Grundstrukturen des unionalen Rundfunkrechts, EuR 2011, S. 784-808

Schliesky, Utz, Digitalisierung - Herausforderung für den demokratischen Verfassungsstaat - Ein Beitrag zur Zukunftsfähigkeit des Grundgesetzes am Vorabend des 70. Geburtstages, NVwZ 2019, S. 693-701

Schliesky, Utz/Hoffmann, Christian/Luch, Anika D./Schulz, Sönke E./Borchers, Kim Corinna, Schutzpflichten und Drittwirkung im Internet - Das Grundgesetz im digitalen Zeitalter, in: Deutsches Institut für Vertrauen und Sicherheit im Internet (DIVSI) (Hrsg.), DIVSI-Perspektiven, Band 1, Baden-Baden 2014

Schmalenbach, Kirsten, Wahrheit und Lüge unter der Herrschaft der Grundrechte, JA 2005, S. 749-752

Dies., Wenn der Staat lügt: Desinformation im demokratischen Rechtsstaat, NVwZ 2005, S. 1357-1361

Schmid, Claudia Eva/Stock, Lennart/Walter, Svenja, Der strategische Einsatz von Fake News zur Propaganda im Wahlkampf, in: Klaus Sachs-Hombach/Bernd Zywietz (Hrsg.), Fake News, Hashtags \& Social Bots - Neue Methoden populistischer Propaganda, Wiesbaden 2018, S. 69-96

Schmid, Thomas/Braam, Laura/Mischke, Julia, Gegen Meinungsmacht - Reformbedürfnisse aus Sicht eines Regulierers - Macht im Netz I: Herausforderungen für die Sicherung der Meinungs- und Medienvielfalt, MMR 2020, S. 19-23

Schmidl, Michael, Zum virtuellen Hausrecht als Abwehrrecht, K\&R 2006, S. 563-565

Schmidt, Hannah, Aufzeichnungen aus der Echokammer - Leben in personalisierten Welten: Sind wir noch demokratiefähig?, in: Michael Steinbrecher/Günther Rager (Hrsg.), Wenn Maschinen Meinung machen - Journalismuskrise, Social Bots und der Angriff auf die Demokratie, S. 102-117

Schmidt, Jan-Hinrik, Social Media, Wiesbaden 2018

Schmidt, Jan-Hinrik/Merten, Lisa/Hasebrink, Uwe/Petrich, Isabelle/Rolfs, Amelie, Zur Relevanz von Online-Intermediären für die Meinungsbildung, in: Hans-Bredow-Institut (Hrsg.), Arbeitspapiere des Hans-Bredow-Instituts, Nr. 40, Hamburg 2017

Schmidt, Walter, Rundfunkfreiheit und Rundfunkgewährleistung, ZRP 1980, S. 132-137

Schmidt-Aßmann, Eberhard, Grundrechte als Organisations- und Verfahrensgarantien, in: D. Merten/H.-J. Papier (Hrsg.), Handbuch der Grundrechte, Band 2, Heidelberg $2009, \mathbb{S} 45$ 
Ders., Anwendungsprobleme des Art. 2 Abs. 2 GG im Immissionsschutzrecht, AöR 106 (1981), S. 205-217

Schmidt-Bleibtreu, Bruno/Hofmann, Hans/Henneke, Hans-Günter, Kommentar zum Grundgesetz, 13. Auflage, Köln 2014

Schmidt-Jortzig, Edzard, Meinungs- und Informationsfreiheit, in: Josef Isensee/Paul Kirchhof (Hrsg.), Handbuch des Staatsrechts, Band 7, $\mathbb{1} 162$, 3. Auflage, Heidelberg 2009

Schmitt, Friedrich, Die Rundfunkfreiheit: Ein Kind ihrer Zeit - aus der Zeit gefallen?, in: Philipp B. Donath/Sebastian Bretthauer/Marie-Dickel-Görig u.a. (Hrsg.), Verfassungen - ihre Rolle im Wandel der Zeit, 59. Assistententagung Öffentliches Recht, S. 203-222

Schmitz, Claus, Grundrechtskollisionen zwischen politischen Parteien und Bürgern, Baden-Baden 1995

Schmitz, Sandra/Berndt, Christian M., The German Act on Improving Law Enforcement on Social Networks: A Blunt Sword, 2018, DOI: http://dx.doi.org/10.2139/ssrn.3306 964

Schneider, Jochen, Schließt Art. 9 DS-GVO die Zulässigkeit der Verarbeitung von BigData aus? - Überlegungen, wie weit die Untersagung bei besonderen Datenkategorien reicht, ZD 2017, S. 303-308

Schneiders, Pascal, Das ist doch Diskriminierung. Eine kommunikationswissenschaftliche Perspektive zum zweiten Diskussionsentwurf eines Medienstaatsvertrags der Rundfunkkommission der Länder, abrufbar unter https://www.mainzer-medieninstit ut.de/wp-content/uploads/Diskussion-Medienstaatsvertrag_P_Schneiders.pdf, (Stand 30.03.2021)

Schoch, Friedrich/Schneider, Jens-Peter/Bier, Wolfgang, Verwaltungsgerichtsordnung Kommentar, Loseblattsammlung, Stand des Gesamtwerks Juli 2020, 39. Ergänzungslieferung

Schoen, Harald, Der demokratische Musterbürger als Normalfall? - Kognitionspsychologische Einblicke in die black box politischer Meinungsbildung, Politische Vierteljahresschrift Jahrgang 47 (2016), S. 89-101

Schomberg, Sabrina/Reubert, Patricia, Regulierung von Nudging durch Private im geplanten Medienstaatsvertrag?, ZD-Aktuell 2018, 06877

Schönke, Adolf/Schröder, Horst, Strafgesetzbuch, Kommentar, 30. Auflage, München 2019

Schramm, Edward, Zur Strafbarkeit des Versendens von Pseudo-Milzbrandbriefen, NJW 2002, S. 419-421

Schreiber, Wolfgang, BWahlG - Kommentar zum Bundeswahlgesetz unter Einbeziehung des Wahlprüfungsgesetzes, des Wahlstatistikgesetzes, der Bundeswahlordnung und sonstiger wahlrechtlicher Nebenvorschriften, 10. Aufl., Köln 2017

Schröder, Meinhard, Rahmenbedingungen der staatlichen Regulierung von Social Bots, DVB1. 2018, S. 465-472

Schulz, Wolfgang/Dreyer, Stephan, Stellungnahme zum Diskussionsentwurf eines Medienstaatsvertrags der Länder, Leibnitz Institut für Medienforschung - Hans-BredowInstitut (Hrsg.), Hamburg, 26.09.2018, abrufbar unter https://www.rlp.de/fileadmin/r lp-stk/pdf-Dateien/Medienpolitik/Eingaben_Medienstaatsvertag/Verbaende_Interme diaere_/Hans-Bredow-Institut.pdf, (Stand 30.03.2021) 
Dies., Schriftliche Stellungnahme zum zweiten Diskussionsentwurf eines Medienstaatsvertrages der Länder vom Juli 2019, Leibnitz Institut für Medienforschung - HansBredow-Institut (Hrsg.), abrufbar unter https://www.hans-bredow-institut.de/upl oads/media/default/cms/media/lez8f8q_HBI_Stellungnahme2MStV.pdf, (Stand 30.03.2021)

Schulz, Wolfgang/Grothe, Torsten, Caution, Loose Cornerstone: The Country of Origin Principle under Pressure, LSE Media Blog vom 4.07.2016, abrufbar unter https://blog s.lse.ac.uk/medialse/2016/07/04/caution-loose-cornerstone-the-country-of-origin-prin ciple-under-pressure/, (Stand 30.03.2021)

Schulz, Wolfgang/Dankert, Kevin, Die Macht der Informationsintermediäre - Erscheinungsformen, Strukturen und Regulierungsoptionen, Friedrich-Ebert-Stiftung (Hrsg.), Bonn 2016

Schunicht, Barbara Elisabeth, Informationelle Selbstbestimmung in sozialen Netzwerken: Mehrseitige Rechtsbeziehungen und arbeitsteilige Verantwortungsstrukturen als Herausforderung für das europäisierte Datenschutzrecht, Hamburg 2018, DOI: 10.17176/20180202-080848

Schuster, Frank Peter, Strafrechtliche Verantwortlichkeit der Hersteller beim automatisierten Fahren, DAR 2019, S. 6-11

Schütz, Raimund, Regulierung in der digitalen Medienwelt - Fünf aktuelle Herausforderungen, MMR 2018, S. 36-39.

Schwander, Timo, Das digitale Vermummungsverbot - eine irreführende Analogie, ZRP 2019, S. 207-209

Schwartmann, Rolf, Praxishandbuch Medien-, IT- und Urheberrecht, 4. Auflage, Heidelberg 2018

Schwartmann, Rolf/ Hermann, Maximilian/ Mühlenbeck, Robin L., Transparenz bei Medienintermediären, Leipzig 2020, abrufbar unter https://www.ma-hsh.de/infothek /publikationen/ma-hsh-gutachten-transparenz-bei-medienintermediaren.html, (Stand 30.03.2021)

Dies., Eine Medienordnung für Intermediäre - Das Zwei-Säulen-Modell zur Sicherung der Vielfalt im Netz, MMR 2019, S. 498-503

Schwarze, Jürgen, Medienfreiheit und Medienvielfalt im Europäischen Gemeinschaftsrecht, ZUM 2000, S. 779-801

Schwenke, Thomas, Das virtuelle Hausrecht als Abwehrmaßnahme gegen "Shitstorms“ innerhalb von Social Media Plattformen, K\&R 2012, S. 305-309

Schweiger, Wolfgang, Der (des)informierte Bürger im Netz - Wie soziale Medien die Meinungsbildung verändern, Wiesbaden 2017

Seehafer, Astrid/Kohler, Joel, Künstliche Intelligenz: Updates für das Produkthaftungsrecht? -Mögliche Anpassungen der europäischen Produkthaftungsrichtlinie für neue Technologien, EuZW 2020, S. 213-218

Seidel, Lore, „Fernsehen ohne Grenzen” - Zum Erlaß der EG-Rundfunkrichtlinie, NVwZ 1991, S. 120-125

Shao, Chengcheng/ Pik-Mai, Huil Pengshuai, Cuil Xinwen, Jiang/ Yuxing, Peng, Tracking and Characterizing the Competition of Fact Checking and Misinformation: Case Studies, IEEE Access Vol. 6 (2018), S. 75327 - 75341

Shao, Chengcheng/Ciampaglia, Giovanni Luca/Varol, Onur/Yang, Kai-Cheng/Flammini, Alessandro/Menczer, Filippo, The spread of low-credibility content by social bots, Nature Communications (2018)9:4787, DOI: 10.1038/s41467-018-06930-7 
Shirado, Hirokazu/Christakis, Nicholas A., Locally noisy autonomous agents improve global human coordination in network experiments, Nature Vol. 545 (2017), S. 370-374

Shirvani, Foroud, Das „scharfe Schwert“ des parteienrechtlichen Transparenzgebots, NVwZ 2017, S. 1321-1325

Silva, Bruno Castanho/ Proksch, Sven-Oliver, Fake It 'Til You Make It: A Natural Experiment to Identify European Politicians' Benefit from Twitter Bots, American Political Science Review 2020, S. 1-7, DOI: https://doi.org/10.1017/S0003055420000817

Silverman, Craig/Mac, Ryan/Dixit, Pranav, "I Have Blood on My Hands“: A Whistleblower Says Facebook Ignored Global Political Manipulation, BuzzFeed News v. 14.09.2020, abrufbar unter https://www.buzzfeednews.com/article/craigsilverman/fac ebook-ignore-political-manipulation-whistleblower-memo, (Stand 30.03.2021)

Simitis, Spiros/Hornung, Gerrit/Spieker gen. Döhmann, Indra, Datenschutzrecht DSGVO mit BDSG, NomosKommentar, 1. Auflage 2019

Siri, Jasmin, Digitalisierung der politischen Kommunikation, in: Akademie der Wissenschaften zu Göttingen (Hrsg.), Digitalisierung, Privatheit und öffentlicher Raum, Göttingen 2020, S. 95-106

Smets, Christoph, Staatsgleiche Grundrechtsbindung Privater aus Funktionsnachfolge? Zur Aufhebung eines Hausverbots für eine Versammlung auf privatem Grund, NVwZ 2016, S. 35-38

Söbbing, Thomas, Der Datenskandal bei Facebook und die rechtliche Zulässigkeit von künstlicher Intelligenz (KI) zur Beeinflussung der politischen Willensbildung (sog. Microtargeting), InTeR 2018, S. 182-188

Solmecke, Christian/Dam, Annika, Wirksamkeit der Nutzungsbedingungen sozialer Netzwerke - Rechtskonforme Lösung nach dem AGB- und dem Urheberrecht, MMR 2012, S. 71-74

Sommer, Denise/Fretwurst, Benjamin/Sommer, Katharina/Gehrau, Volker, Nachrichtenwert und Gespräche über Medienthemen, Publizistik 57 (2012), S. 381-401

Specht, Louisa/Herold, Sophie, Roboter als Vertragspartner - Gedanken zu Vertragsabschlüssen unter Einbeziehung automatisiert und autonom agierender Systeme, MMR 2018, S. 40-44

Spiegel, Johanna/Heymann, Britta, Ein Minenfeld für Anbieter sozialer Netzwerke Zwischen NetzDG, Verfassungsrecht und Vertragsfreiheit, K\&R 2020, S. 344-350

Spindler, Gerald, Digitale Wirtschaft - analoges Recht: Braucht das BGB ein Update?, JZ 2016, S. 805-816

Ders., Der Regierungsentwurf zum Netzwerkdurchsetzungsgesetz - europarechtswidrig?, ZUM 2017, S. 473-487

Ders., Herkunftslandprinzip und Kollisionsrecht - Binnenmarktintegration ohne Harmonisierung? Die Folgen der Richtlinie im elektronischen Geschäftsverkehr für das Kollisionsrecht, RabelsZ Band 66 (2002), S. 633-709

Ders., Legal Expertise commissioned by BITKOM concerning the notified German Act to Improve Enforcement of the Law in Social Networks (Netzwerkdurchsetzungsgesetz), abrufbar unter https://www.bitkom.org/sites/default/files/file/import/Legal-Expe rtise-Official-2-0.pdf, (Stand 30.03.2021)

Ders., Art. 17 DSM-RL und dessen Vereinbarkeit mit primärem Europarecht - Zugleich ein Beitrag zu Umsetzungsmöglichkeiten, GRUR 2020, S. 253-261

Ders., Die neue Urheberrechts-Richtlinie der EU, insbesondere „Upload-Filter"-Bittersweet? - Analyse der Änderungen beim Text- und Datamining, Leistungsschutz für Presseerzeugnisse und Pflichtenkreis für Hostprovider, CR 2019, S. 277-291 
Ders., Der Vorschlag für ein neues Haftungsregime für Internetprovider - der EU-Digital Services Act (Teil 1), GRUR 2021, S. 545-553

Ders., Der Vorschlag für ein neues Haftungsregime für Internetprovider - der

EU-Digital Services Act - Teil 2: Große und besonders große Plattformen

Spindler, Gerald/Schuster, Fabian, Recht der elektronischen Medien, 4. Auflage, München 2019

Stadler, Thomas, Haftung für Informationen im Internet, Berlin 2002

Ders., Verstoßen Facebook und Google Plus gegen deutsches Recht? - Ausschluss von Pseudonymen auf Social-Media Plattformen, ZD 2011, S. 57-59

Stalla-Bourdillon, Sophie/ Thorburn, Robert, The scandal of intermediary: Acknowledging the both/and dispensation for regulating hybrid actors, 2019, abrufbar unter https://papers.ssrn.com/sol3/papers.cfm?abstract_id=3317486, (Stand: 30.03.2021)

Statistisches Bundesamt (Destatis), Private Haushalte in der Informationsgesellschaft Nutzung von Informations- und Kommunikationstechnologien, 2018, abrufbar unter https://www.destatis.de/DE/Themen/Gesellschaft-Umwelt/Einkommen-Konsum-Lebe nsbedingungen/IT-Nutzung/_inhalt.html, (Stand 30.03.2021)

Staudinger, Kommentar zum Bürgerlichen Gesetzbuch, Buch 1. Allgemeiner Teil, $\mathbb{S}$ S0-124, $\mathbb{S}$ 130-133 (Sachen und Tiere; Geschäftsfähgkeit; Willenserklärung), Berlin 2017

Ders., Kommentar zum Bürgerlichen Gesetzbuch, Buch 2. Recht der Schuldverhältnisse, S $\$$ 255-304 (Leistungsstörungsrecht 1), Berlin 2019

Ders., Kommentar zum Bürgerlichen Gesetzbuch, $\mathbb{S}$ 305-310; UKlaG (AGB-Recht 1 und Unterlassungsklagengesetz), Berlin 2019

Ders., Kommentar zum Bürgerlichen Gesetzbuch, Einleitung zur Rom I-VO; Art 1-10 Rom I-VO (Internationales Vertragsrecht 1), Berlin 2016

Stiege, Hans, Autonomes Fahren und die staatliche Durchsetzung des Verbots der Rechtswidrigkeit, NZV 2019, S. 459-467

Stein, Tao/Chen, Roger/Mangla, Karan, Facebook Immune System, 2011, abrufbar unter https://allfacebook.de/wp-content/uploads/2011/10/FacebookImmuneSystem.pdf, (Stand 30.03.2021)

Steinbach, Armin, Meinungsfreiheit im postfaktischen Umfeld, Juristen Zeitung 2017, S. 653-661

Ders., Social Bots im Wahlkampf, Zeitschrift für Rechtspolitik 2017, S. 101-105

Steinmaurer, Thomas, Digitale Resilienz im Zeitalter der Datafiction, in: Michale Litschka/Larissa Krainer (Hrsg.), Der Mensch im digitalen Zeitalter, Zum Zusammenhang von Ökonomisierend, Digitalisierung und Mediatisierung, S. 31-48

Stella, Massimo/Ferrara, Emilio/de Domenico, Manlio, Bots increase exposure to negative and inflammatory content in online social systems, PNAS Vol. 115 (2018), S. $12435-12440$

Stern, Klaus, Die Schutzpflichtenfunktion der Grundrechte: Eine juristische Entdeckung, DÖV 2010, S. 241-249

Steinebach, Martin/Bader, Katarina,/Rinsdorf, Lars/Krämer, Nicole/Roßnagel, Alexander, Desinformation aufdecken und bekämpfen - Interdisziplinäre Ansätze gegen Desinformationskampagnen und für Meinungspluralität, Baden-Baden 2020

Stickelbrock, Barbara, "Impressumspflicht” im Internet - eine kritische Analyse der neueren Rechtsprechung zur Anbieterkennzeichnung nach \$6 TDG, GRUR 2004, S. $111-117$ 
Stieglitz, Stefan/Brachten, Florian/Ross, Björn/Jung, Anna-Katharina, Do Social Bots Dream of Electric Sheep? A Categorisation of Social Media Bot Accounts, 2017, arXiv: 1710.04044 [cs.HC]

Störring, Lars Peter, Das Untermaßverbot in der Diskussion - Untersuchung einer umstrittenen Rechtsfigur, Schriften zum Öffentlichen Recht, Band 1145, Band 1145, Berlin 2009

Stricke, Barry, People v. Robots: A Rodmap for Enforcing California's New Online Bot Disclosure Act, Vanderbilt Journal of Entertainment \& Technology Law Vol. 22 (2020), S. 839-894

Stroebe, Wolfgang/Hewstone, Miles/Jonas, Klaus, Einführung in die Sozialpsychologie, in: dies., Sozialpsychologie, 6. Auflage, Wiesbaden 2014

Stück, Volker, Der Anscheinsbeweis, JuS 1996, S. 153-158

Suárez-Serrato, P./Roberts, Margaret. E./Davis, Clayton/Menczer, Filippo, On the influence of social bots in online protests - Preliminary findings of a Mexican case study, in: Emma Spiro/ Yong-Yeol Ahn (Hrsg.), Social Informatics 8th International Conference, SocInfo 2016 Bellevue, WA, USA, November 11-14, 2016 Proceedings, Part II 2016, S. 269-278.

Sumaila, Nigo/Wakamiya, Shoko/Aramaki, Eiji, Making Twitter Safer: Uncovering Social-Bot on Twitter through User's Content Approach, DBSJ Journal Vol. 18 (2020), S. 1-7

Sunstein, Cass R., \#republic - Dividing Democracy in the Age of Social Media, Princeton New Jersey 2018.

Suwelack, Felix, Schleichwerbung als Boombranche? - Geltung und Wirksamkeit werberechtlicher Grundsätze beim Influencer-Marketing, MMR 2017, S. 661-665

Ders., Leistungsschutzrecht und Upload-Filter aus ökonomischer Perspektive - Werden die Reform-Vorschläge der EU-Kommission ihrem eigenen Legitimationsmodell gerecht?, MMR 2018, S. 582-586

Sydow, Gernot, Europäische Datenschutzgrundverordnung, Handkommentar, 2. Auflage 2018

Szczekalla, Peter, Die sogenannten grundrechtlichen Schutzpflichten im deutschen und europäischen Recht - Inhalt und Reichweite einer „gemeineuropäischen Grundrechtsfunktion“, Schriften zum Europäischen Recht, Band 87, Berlin 2002

Tandoc Jr., Edson C./Lim, Zheng Wei/Ling, Richard, Defining „Fake News“ - A typology of scholarly definitions, Digital Journalism Vol. 6 (2018), S. 137-153

Teubner, Gunther, Digitale Rechtssubjektee?, AcP 218 (2018), S. 155-205

Thalhofer, Thomas, Recht an Daten in der Smart Factory, GRUR-Prax 2017, S. 225-227

Thielbörger, Pierre, Propaganda-blindes Völkerrecht?, in: Thomas Oppelland (Hrsg.), Propaganda als (neue) außen- und sicherheitspolitische Herausforderung, Berlin 2017

Thieltges, Andree/Papakyriakopoulos, Orestes/Medina Serrano, Juan Carlos/Hegelich, Simon, Effects of Social Bots in the Iran-Debate on Twitter, arXiv:1805.10105 [cs.SI]

Thieltges, Andree/Hegelich, Simon, Manipulation in sozialen Netzwerken - Risikopotenziale und Risikoeinschätzungen, ZfP 2017, S. 493-511

Dies., Falschinformationen und Manipulationen durch social bots in sozialen Netzwerken, in: Andreas Blätte, Joachim Behnke, Kai-Uwe Schnapp, Claudius Wagemann (Hrsg.), Computational Social Science - Die Analyse von Big Data, 1. Auflage, Baden-Baden 2018 
Thomale, Philipp-Christian, Anforderungen an eine Medienregulierung der Zukunft Stellungnahme zum Entwurf des „Medienstaatsvertrags" aus Sicht der Praxis (Axel Springer), ZUM 2019, S. 122-126

Thum, Kai, Das Grundrecht der Rundfunkfreiheit nach deutschem und europäischem Recht- Eine Bestandsaufnahme, DÖV 2008, S. 653-661

Tian, Lichun, Objektive Grundrechtsfunktionen im Vergleich - Eine Untersuchung anhand des Grundgesetzes und der Europäischen Menschenrechtskonvention, Schriften zum Öffentlichen Recht, Band 1204, Berlin 2012

Tief, Samira, Kommunikation auf Facebook, Twitter \& YouTube - Verfassungsrechtlicher Schutz der Informationsintermediäre und ihrer Nutzer durch die Medienfreiheiten, Berlin 2020

Towfigh, Emanuel V., Das Parteien-Paradox: ein Beitrag zur Bestimmung des Verhältnisses von Demokratie und Parteien, Tübingen 2015

Treré, Emiliano, The Dark Side of Digital Politics: Understanding the Algorithmic Manufacturing of Consent and the Hindering of Online Dissidence, Institute of Development Studies Bulletin Vol. 47 (2016), Opening Governance, S. 127-138

Ders., Distorsiones tecnopolíticas: represión y resistencia algorítmica del activismo ciudadano en la era del 'big data', Trípodos Número 39 (2016), S. 35-51

Tufekci, Zeynep, How Facebooks Algorithm Suppresses Content Diversity (Modestly) and How the Newsfeed Rules Your Clicks, The Message v. 07.05.2015, abrufbar unter https://medium.com/message/how-facebook-s-algorithm-suppresses-conte nt-diversity-modestly-how-the-newsfeed-rules-the-clicks-b5f8a4bb7bab, (Stand 30.03.2021)

Uecker, Philipp, Anmerkung zum Urteil des AG Wolfratshausen: Amoklauf-Ankündigung bei Facebook, MMR 2014, S. 206

Uhlemann, Ingrid Andrea, Der Nachrichtenwert im situativen Kontext - Eine Studie zur Auswahlwahrscheinlichkeit von Nachrichten, 1. Auflage, Wiesbaden 2012

Ukrow, Jörg, Entwicklung des europäischen Medienrechts zwischen Verfassungsrecht, Völkerrecht und Europarecht, in: Jürgen Bröhmer (Hrsg.), Europa und die Welt -Kolloquium zu aktuellen europa-, völker- und menschenrechtlichen Themen aus Anlass des 80. Geburtstages von Prof. Dr. Dr. Dr. h.c. mult. Georg Ress, Baden-Baden 2016, S. 197-236

von Ungern-Sternberg, Antje, Demokratische Meinungsbildung und künstliche Intelligenz, in: Sebastian Unger/Dies. (Hrsg.), Demokratie und künstliche Intelligenz, Tübingen 2019, S. 3-31

Unruh, Peter, Zur Dogmatik der grundrechtlichen Schutzpflichten, Schriften zum Öffentlichen Recht, Band 709, Berlin 1996

Uyheng, J., Carley, K.M., Bots and online hate during the COVID-19 pandemic: case studies in the United States and the Philippines, Journal of Computational Social Sciences (2020). DOI: https://doi.org/10.1007/s42001-020-00087-4

Varol, Onur/ Ferrara. Emilia/Davis, Clayton A./Menczer, Filippo/Flammini, Alessandro, Online human-bot interactions: Detection, estimation and characterisation, in: AAAI (hrsg.), in: Proceedings of the Eleventh International AAAI Conference on Web and Social Media, 2017, S. 280-289

Varol, Onur/Uluturk, Ismail, Deception strategies and threats for online discussions, First Monday Vol. 23 Nr. 5 (2018), DOI: https://doi.org/10.5210/fm.v22i5.7883 
Veale, Tony/Cook, Mike, Twitterbots: making machines that make meaning, Cambridge (MA) 2018

Veil, Winfried, Einwilligung oder berechtigtes Interesse? - Datenverarbeitung zwischen Skylla und Charybdis, NJW 2018, S. 3337-3344

Velásquez, E. /Yazdani, M./Suárez-Serrato, Socialbots Supporting Human Rights, 2017, arXiv:1710.11346 [cs.CY]

Vesting, Thomas, Die Veränderung der Öffentlichkeit durch künstliche Intelligenz, in: Sebastian Unger/Antje von Ungern-Sternberg (Hrsg.), Demokratie und künstliche Intelligenz, Tübingen 2019, S. 33- 49

Ders., Die Rundfunkfreiheit und die neue Logik der „Content Curation“ in elektronischen Netzwerken, JZ 2020, S. 975-982

Voigt, Paul/Reuter, Wiebke, Platform-to-Business-Verordnung - Neue Anforderungen für Anbieter von Online-Vermittlungsdiensten und Online-Suchmaschinen ab Juli 2020, MMR 2019, S. 783-787

Roland, Thomas, Zur Reichweite von Menschenrechten im Klimaschutz - Wäre die „Urgenda-Entscheidung “ auch im deutschen Recht zu erwarten?, NVwZ 209, S. 114-120

Volkmann, Uwe, Der manipulierbare Wähler und das Ideal der autonomen Wahlentscheidung, in: Sebastian Unger/ Antje von Ungern-Sternberg (Hrsg.), Demokratie und künstliche Intelligenz, Tübingen 2019, S. 51-66

Volkmann, Viktor, Hate Speech durch Social Bots - Strafrechtliche Zurechnung von Volksverhetzungen gem. \$130 Abs. 1 StGB, MMR 2018, S. 58-63

Vosoughi, Soroush/Roy, Deb/Arai, Sinan, The spread of true and false news online, Science journal 359 (2018), S. 1146-1151

Voßkuble, Andreas, Umweltschutz und Grundgesetz, NVwZ 2013, S. 1-8

Wabnitz, Heinz-Bernd/Janovsky, Thomas/Schmitt, Lothar, Handbuch Wirtschafts- und Steuerstrafrecht, 5. Auflage, München 2020

Wagner, Bernd, Verträge als Rechtsgrundlage für Datenverarbeitungen: Werden Einwilligungen bald obsolet?, ZD-Aktuell 2018, 06103

Wagner, Christoph, Rundfunk- und Tendenzfreiheit von Pay-TV-Plattformen, AfP 2020, S. 281-286

Wagner, Gerhard, Haftung von Plattformen für Rechtsverletzungen (Teil 2), GRUR 2020, S. 447-457

Wagner, Stephan, Substituierte Attribute - Kognitive Störungen im Recht des digitalisierten Staates, in: Ruth Greve, Benjamin Gwiasda, Thomas Kemper, Joshua Moir, Sabrina Müller, Arno Schönberger, Sebastian Stöcker, Julia Wagner, Lydia Wolff (Hrsg.), Der digitalisierte Staat - Chancen und Herausforderungen für den modernen Staat, S. 17-38

Ders., Substituierte Attribute - Kognitive Störungen im Recht des digitalisierten Staates, RW 2020, S. 344-376

De Wall, Heinrich/ Muckel, Stefan, Kirchenrecht, 4. Auflage, München 2014

Wandtke, Artur-Axel, Grundsätze der Richtlinie über das Urheberrecht im digitalen Binnenmarkt, NJW 2019, S. 1841-1847

Wandtke, Artur-Axel/Hauck, Ronny, Art. 17 DSM-Richtlinie - Ein neues Haftungssystem im Urheberrecht, ZUM 2019, S. 627-636

Wandtke, Artur-Axel/Ohst, Claudia, Praxishandbuch Medienrecht, Band 5: IT-Recht, 3. Auflage, Berlin/Boston 2014 
Wardle, Claire/Derakhshan, Hossein, Information Disorder: Toward an interdisciplinary framework for research and policy making, Council of Europe report DGI(2017)09, abrufbar unter https://rm.coe.int/information-disorder-report-version-august-2018/16 808c9c77, (Stand: 30.03.2021)

Weaver, John Frank, Everything is not Terminator: We Need the California Bot Bill, But We Need It to Be Better, The Journal of Robotics, Artificial Intelligence \& Law Vol. 1 (2018), S. 431-436

Weaver, Russell L., Robotic Speech and The First Amendment, International Journal of Digital and Data Law Vol. 6 (2020), S. 21-33

Weber, Stefan, Roboterjournalismus, Chatbots \& Co. - Wie Algorithmen Inhalte produzieren und unser Denken beeinflussen, 2018

Weber, Franziska/Schäfer, Hans-Bernd, „Nudging“, Ein Spross der Verhaltensökonomie - Überlegungen zum liberalen Paternalismus auf gesetzgeberischer Ebene, Der Staat 56 (2017), S. 561-592

Weber, Peter, Nachrichtenwert, in: Jessica Heesen (Hrsg.), Handbuch Medien- und Informationsethik, J. B. Metzler, Stuttgart 2016, S. 114-121

Weber, Silvana/Knorr, Elena, Kognitive Verzerrungen und die Irrationalität des Denkens, in: Markus Appel (Hrsg.), Die Psychologie des Postfaktischen - Über Fake News, „Lügenpresse“, Clickbaiting und Co., Wiesbaden 2020, S. 103-115

Webster, James G., User information Regimes: How social media shape patterns of consumption, Northwestern Law Review Vol. 104 (2010), S. 593-612

Weidel, Fabian, Die Grenzen der Aufmerksamkeit: Mentale Überlastung in einer mediatisierten Gesellschaft, in: Michael Litschka/Larissa Krainer (Hrsg.), Der Mensch im digitalen Zeitalter - Zum Zusammenhang von Ökonomisierung, Digitalisierung und Mediatisierung, Wiesbaden 2019, S. 49-851

Wiss, Theresa, Soziale Netzwerke: Hass und Fake News nach Attentaten, FAZ v. 25.02.2020, abrufbar unter https://www.faz.net/aktuell/gesellschaft/wie-attentate -im-internet-zu-fake-news-und-hass-fuehren-16651057.html, (Stand: 30.03.2021).

Weiss, Wolfgang, Privatisierung und Staatsaufgaben - Privatisierungsentscheidungen im Lichte einer grundrechtlichen Staatsaufgabenlehre unter dem Grundgesetz, Tübingen 2002

Weiß, Wolfgang, Der Gefahrenerforschungseingriff bei Altlasten - Versuch einer Neubestimmung, NVwZ 1997, S. 737-744

Wendehorst, Christiane/Graf von Westphalen, Friedrich, Das Verhältnis zwischen Datenschutz-Grundverordnung und AGB-Recht, NJW 2016, S. 3745-3750

Werner, Wibke, Schutz durch das Grundgesetz im Zeitalter der Digitalisierung, NJOZ 2019, S. 1041-1046

Westphal, Manon, Digitale Öffentlichkeiten und politische Handeln - Überlegungen zu einer radikaldemokratischen politischen Bildung, in: Ulrich Binder/Johannes Drerup (Hrsg.), Demokratieerziehung und die Bildung digitaler Öffentlichkeit, Wiesbaden 2020, S. 1-27

von der Weth, Christian/Vachery, Jithin/Kankanhalli, Mohan, Nudging users to slow down the spread of fake news in social media, in: IEEE (Hrsg.), Proceedings of the International Conference on Multimedia \& Expo Workshops (ICMEW), London 2020, S. 1-6, DOI: 10.1109/ICMEW46912.2020.9106003

Graf von Westphalen, Friedrich, Nutzungsbedingungen von Facebook - Kollision mit europäischem und deutschem AGB-Recht, Verbraucher und Recht 2017, S. 323-332

Ders., Das erschöpfte liberale Recht - Unentgeltliche Nutzung von Daten - Manipulation vs. Fiktion der Vertragsfreiheit, IWRZ 2019, S. 61-65 
Wiebe, Gerhard, Das neue Tabakerzeugnisgesetz: verbrämter Paternalismus oder Stärkung des Verbraucherschutzes?, GewerbeArchiv 2016, S. 456-462

Wiese, Günther, Bewertungsportale und allgemeines Persönlichkeitsrecht, JZ 2011, S. 608-617

Wimmers, Jörg/Barudi, Malek, Der Mythos vom Value Gap - Kritik zur behaupteten Wertschöpfungslücke bei der Nutzung urheberrechtlich geschützter Inhalte auf Hosting-Diensten, GRUR 2017, S. 327-338

Woitke, Thomas, Das „Wie“ der Anbieterkennzeichnung gemäß $\$ 6$ TDG, NJW 2003, S. 871-873

Wolf, Oliver, Social Bots im Wahlkampf - Das UrhG als Handhabe gegen „Meinungsroboter"?, WRP 2019, S. 440-44

Wolff, Heinrich Amadeus/Brink, Stefan, Beck'scher Onlinekommentar zum Datenschutzrecht, 34. Edition, Stand: 01.11.2020

Woolley, Samuel C., Automating Power: Social bot interference in global politics, First Monday Vol. 21 (2016) Nr. 4, DOI: https://doi.org/10.5210/fm.v21i4.6161

Ders./ Howard, Phil, Bots Unite to Automate the Presidential Election, wired.com v. 15.05.2016, abrufbar unter https://www.wired.com/2016/05/twitterbots-2/, (Stand: 30.03.2021)

Wunden, Wolfgang, Öffentlichkeit und Kommunikationskultur, Münster 2005

Wünschner, Philipp, The Internet is Dead - Long Live the Internet: Soziale Medien und idiosynkratisches Aufbegehren, in: Rainer Mühlhoff/Anja Breljak/Jan Slaby (Hrsg.), Affekt, Macht, Netz - Auf dem Weg zu einer Sozialtheorie der Digitalen Gesellschaft, Bielefeld 2019, S. 247-268

Yuan, Xiaoyi/Schuchard, Ross J./Crooks, Andrew T., Examining Emergent Communities and Social Bots Within the Polarized Online Vaccination Debate in Twitter, Social Media + Society 2019, S. 1-12

Zagouras, Georgios, Pluralismus in Europa - Zur Sicherung der Meinungsvielfalt durch die Europäische Gemeinschaft, Baden-Baden 2009

Zech, Herbert,Künstliche Intelligenz und Haftungsfragen, ZfPW 2019, S. 198-219

Zeitelhack, Alexander, Die Welt steht auf dem Kopf - Wie Social Media das Paradigma von Öffentlichkeit verändert, in: Mike Friedrichsen/oland A. Kohn (Hrsg.), Digitale Politikvermittlung, Wiesbaden 2014, S. 169-178

Zhang, Jinxue/Zhang, Rui/Zhang, Yanchao/Yan, Guanhua, On the Impact of Social Botnets for Spam Distribution and Digital-influence Manipulation, in: IEEE (Hrsg.), 2013 IEEE Conference on Communications and Network Security (CNS), S. 46-54

Ziebarth, Wolfgang, Das Datum als Geisel - Klarnamenspflicht und Nutzeraussperrung bei Facebook, ZD 2013, S. 375-379

Zippelius, Reinhold, Allgemeine Staatsrechtslehre - Politikwissenschaft ein Studienbuch, 16. Auflage, München 2010

Zoll, Patrick, Verschwörungstheoretische Medienkritik:Wächter oder Totengräber der Demokratie? - Plädoyer für einen neuen Realismus in der Medienkritik, Communicatio Socialis Vol. 48 (2015), S. 126-137

Zollo, Fabiana/Bessi, Allessandro/Del Vicario, Michaela/Scala, Antonio, Caldarelli, Guido/ Shekhtman, Louis/Havlin, Shlomo/Quattrociocchi, Walter, Debunking in a world of tribes, PLoS ONE 12 (7): e0181821 (2017), DOI: https://doi.org/10.1371/journal. pone. 0181821 
Zuck, Rüdiger, Auf dem Weg zur Nikotin-Prohibition? - Die Rolle des paternalistischen Schutzstaats, ZRP 2008, S. 165-166

Ders., Gesetzlicher Masern-Impfzwang, ZRP 2017, S. 118-121

Zumkeller-Quast, Florian, Die Nutzung von Social Bots als Identitätstäuschung?, JuWissBlog Nr. 2/2017 v. 05.01.2017, abrufbar unter https://www.juwiss.de/2-2017/, (Stand 30.03.2021) 